Title:

The Skin Barrier Defects Caused by Keratinocyte-Specific Deletion of ADAM17 or EGFR are Based on Highly Similar Proteome and Degradome Alterations

\title{
Authors and affilitions:
}

Stefan Tholen ${ }^{1}$, Cristina Wolf ${ }^{2,3}$, Bettina Mayer ${ }^{1}$, Julia D. Knopf ${ }^{1,4}$, Stefanie Löffek ${ }^{2,5}$, Yawen Qian ${ }^{2}$, Jayachandran N. Kizhakkedathu' ${ }^{6}$, Martin L. Biniossek ${ }^{1}$, Claus-Werner Franzke ${ }^{2,9,{ }^{*}}$, Oliver Schilling, ${ }^{1,7,8,9,{ }^{*}}$ *equal contribution Institute of Molecular Medicine and Cell Research, University of Freiburg, Germany University Medical Center Freiburg, Department of Dermatology, Freiburg, Germany present address: Luxembourg Centre for Systems Biomedicine, University of Luxembourg, Luxembourg present address: Centre for Molecular Biology, University of Heidelberg (ZMBH), DKFZ-ZMBH Allianz, Heidelberg, Germany

present address:, Skin Cancer Unit of the Dermatology Department, Medical Faculty, University Duisbug-Essen, Germany

Department of Pathology and Laboratory Medicine and Department of Chemistry, Centre for Blood Research, University of British Columbia, Vancouver, British Columbia, Canada

BIOSS Centre for Biological Signaling Studies, University of Freiburg, D-79104 Freiburg, Germany German Cancer Consortium (DKTK) and German Cancer Research Center (DKFZ), Heidelberg, Germany to whom correspondence should be addressed:

PD Dr Oliver Schilling; Institute of Molecular Medicine and Cell Research; University of Freiburg; Stefan Meier Strasse 17; 79104 Freiburg, Germany, Tel: +49 761203 9615, oliver.schilling@mol-med.uni-freiburg.de PD Dr Claus-Werner Franzke; University Freiburg Medical Center; Department of Dermatology; Hauptstr. 7; 79104 Freiburg, Germany; Tel: +49 761270 67850; claus-werner.franzke@uniklinik-freiburg.de

\section{Table of contents:}

\section{Supplementary tables 1-15}


Supplementary table 1: List of all identified and quantified proteins in the quantitative proteome comparison of wild-type and $A d a m 17^{\Delta K C}$ epidermis at P3 (replicate 1).

\begin{tabular}{|c|c|c|c|c|}
\hline Protein & Uniprot & Gene name & $\begin{array}{c}\text { Fold change of } \\
\text { XPRESS ratio } \\
\left.\text { (Adam } 17^{\Delta K C} / \mathrm{WT}\right)\end{array}$ & $\begin{array}{c}\text { Sequence } \\
\text { coverage [\%] }\end{array}$ \\
\hline 10 kDa heat shock protein, mitochondrial; & Q64433 & Hspe1 & $-0,3$ & 54,9 \\
\hline 14-3-3 protein beta/alpha; & Q9CQV8 & Ywhab & 0,2 & 17,9 \\
\hline 14-3-3 protein epsilon; & P62259 & Ywhae & 0,0 & 45,5 \\
\hline 14-3-3 protein eta; & P68510 & Ywhah & 1,2 & 13 \\
\hline 14-3-3 protein gamma; & P61982 & Ywhag & 0,3 & 13 \\
\hline 14-3-3 protein sigma; & 070456 & Sfn & 0,4 & 40,7 \\
\hline 14-3-3 protein theta; & P68254 & Ywhaq & 0,0 & 38,8 \\
\hline 14-3-3 protein zeta/delta; & P63101 & Ywhaz & 0,3 & 41,6 \\
\hline 182 kDa tankyrase-1-binding protein; & P58871 & Tnks1bp1 & $-0,1$ & 43,6 \\
\hline 1-phosphatidylinositol 4,5-bisphosphate phosphodiesterase beta-3; & P51432 & Plcb3 & $-0,7$ & 1 \\
\hline $26 \mathrm{~S}$ protease regulatory subunit $10 \mathrm{~B}$ & P62334 & Psmc6 & 1,1 & 3,6 \\
\hline 265 protease regulatory subunit 4 & P62192 & Psmc1 & 0,7 & 5 \\
\hline $26 S$ protease regulatory subunit $6 \mathrm{~A}$; & 088685 & Psmc3 & 0,2 & 10,3 \\
\hline $26 \mathrm{~S}$ protease regulatory subunit $6 \mathrm{~B}$; & P54775 & Psmc4 & 0,0 & 5,3 \\
\hline 265 protease regulatory subunit 7 & P46471 & Psmc2 & $-0,2$ & 9,9 \\
\hline $26 \mathrm{~S}$ proteasome non-ATPase regulatory subunit $1 ;$ & Q3TXS7 & Psmd1 & $-1,2$ & 19,4 \\
\hline $26 \mathrm{~S}$ proteasome non-ATPase regulatory subunit $13 ;$ & Q9WVJ2 & Psmd13 & $-1,0$ & 5 \\
\hline $26 \mathrm{~S}$ proteasome non-ATPase regulatory subunit 2 ; & Q8VDM4 & Psmd2 & $-1,0$ & 31,7 \\
\hline 265 proteasome non-ATPase regulatory subunit 4 & 035226 & Psmd4 & 0,0 & 29,3 \\
\hline $26 \mathrm{~S}$ proteasome non-ATPase regulatory subunit 4 & F7Al87 & Psmd4 & $-0,2$ & 10,3 \\
\hline 265 proteasome non-ATPase regulatory subunit 7; & P26516 & Psmd7 & 1,0 & 17,8 \\
\hline $26 \mathrm{~S}$ proteasome non-ATPase regulatory subunit 9; & Q9CR00 & Psmd9 & $-0,2$ & 31,5 \\
\hline 28 kDa heat- and acid-stable phosphoprotein; & Q3UHX2 & Pdap1 & $-0,4$ & 45,9 \\
\hline $28 \mathrm{~S}$ ribosomal protein S34, mitochondrial; & Q9JIK9 & Mrps34 & $-0,1$ & 19,7 \\
\hline $28 \mathrm{~S}$ ribosomal protein $\mathrm{S} 36$, mitochondrial; & Q9CQX8 & Mrps36 & $-0,4$ & 69,6 \\
\hline $28 \mathrm{~S}$ ribosomal protein S36, mitochondrial; & Q9D6T9 & Mrps36 & $-0,6$ & 69 \\
\hline $28 \mathrm{~S}$ ribosomal protein $\mathrm{S} 6$, mitochondrial; & P58064 & Mrps6 & 1,0 & 19,2 \\
\hline $28 \mathrm{~S}$ ribosomal protein S7, mitochondrial; & Q80X85 & Mrps7 & 0,1 & 5 \\
\hline 2-amino-3-ketobutyrate coenzyme A ligase, mitochondrial; & 088986 & Gcat & 0,2 & 2,9 \\
\hline 2'-deoxynucleoside 5'-phosphate N-hydrolase 1; & Q80VJ3 & Dnph1 & $-0,4$ & 16,2 \\
\hline $39 S$ ribosomal protein L12, mitochondrial; & Q9DB15 & Mrpl12 & $-0,3$ & 17,9 \\
\hline 395 ribosomal protein L17, mitochondrial; & Q9D8P4 & Mrpl17 & 0,2 & 11,4 \\
\hline $39 S$ ribosomal protein L40, mitochondrial; & Q9Z2Q5 & Mrpl40 & 2,4 & 10,5 \\
\hline $39 S$ ribosomal protein L41, mitochondrial; & Q9CQN7 & Mrpl41 & 0,1 & 22,9 \\
\hline $39 S$ ribosomal protein $L 43$, mitochondrial; & Q99N89 & Mrpl43 & 0,4 & 7,5 \\
\hline $39 S$ ribosomal protein L49, mitochondrial; & Q9CQ40 & Mrpl49 & 0,8 & 8,4 \\
\hline $39 S$ ribosomal protein L50, mitochondrial; & Q8VDT9 & Mrpl50 & 0,3 & 17,6 \\
\hline 3-hydroxyisobutyrate dehydrogenase, mitochondrial; & Q99L13 & Hibadh & $-0,3$ & 3 \\
\hline 3-ketoacyl-CoA thiolase, mitochondrial; & Q8BWT1 & Acaa2 & 0,9 & 31,2 \\
\hline 40 S ribosomal protein S10; & P63325 & Rps10 & 0,2 & 23 \\
\hline $40 S$ ribosomal protein $\mathrm{S} 11$ & P62281 & Rps11 & 0,0 & 4,4 \\
\hline $40 S$ ribosomal protein $\mathrm{S} 12$ & Q6ZWZ6 & Rps12 & 0,5 & 53 \\
\hline 40 S ribosomal protein $\mathrm{S} 13$ & P62301 & Rps13 & $-0,4$ & 29,1 \\
\hline 40 S ribosomal protein $\mathrm{S} 14$ & P62264 & Rps14 & 0,3 & 15,2 \\
\hline 40 S ribosomal protein S15; & P62843 & Rps15 & 0,9 & 10,2 \\
\hline 40 S ribosomal protein S16; & P14131 & Rps16 & $-0,1$ & 14,4 \\
\hline 40 S ribosomal protein S17; & P63276 & Rps17 & 0,5 & 7,4 \\
\hline 40 S ribosomal protein S18; & P62270 & Rps18 & 0,6 & 32,9 \\
\hline 40 S ribosomal protein S19; & Q9CZX8 & Rps19 & 0,0 & 27,5 \\
\hline 40 S ribosomal protein $\mathrm{S} 2$ & P25444 & Rps2 & 0,2 & 15,4 \\
\hline 40 S ribosomal protein S20; & P60867 & Rps20 & 0,7 & 25,2 \\
\hline 40 S ribosomal protein S21; & Q9CQR2 & Rps21 & 0,1 & 39,8 \\
\hline $40 S$ ribosomal protein $\mathrm{S} 25$ & P62852 & Rps25 & $-0,1$ & 22,4 \\
\hline $40 S$ ribosomal protein $\mathrm{S} 27$ & Q6ZWU9 & Rps27 & $-0,3$ & 13,1 \\
\hline 40 S ribosomal protein S28; & P62858 & $\operatorname{Rps} 28$ & $-0,3$ & 37,5 \\
\hline 40 S ribosomal protein S29; & P62274 & Rps29 & 1,0 & 12,5 \\
\hline 40 S ribosomal protein S3; & P62908 & Rps3 & $-0,4$ & 5,3 \\
\hline 40 S ribosomal protein S30; & P62862 & Fau & 0,2 & 16,9 \\
\hline $40 S$ ribosomal protein $\mathrm{S} 3 \mathrm{a}$ & P97351 & Rps3a & 0,9 & 24,6 \\
\hline $40 \mathrm{~S}$ ribosomal protein $\mathrm{S} 4, \mathrm{X}$ isoform; & P62702 & $\operatorname{Rps} 4 x$ & $-0,1$ & 7,3 \\
\hline 40S ribosomal protein S5; & D3YYM6 & Rps5 & 0,4 & 30,2 \\
\hline $40 \mathrm{~S}$ ribosomal protein $\mathrm{S} 8$ & P62242 & Rps8 & 0,1 & 5,3 \\
\hline 40S ribosomal protein SA; & P14206 & Rpsa & 0,7 & 34,2 \\
\hline 45 kDa calcium-binding protein; & Q61112 & Sdf4 & 0,2 & 2,8 \\
\hline 4F2 cell-surface antigen heavy chain; & P10852 & Slc3a2 & $-0,3$ & 18,4 \\
\hline 5'(3')-deoxyribonucleotidase, cytosolic type; & Q9JM14 & $\mathrm{Nt5c}$ & 0,4 & 20 \\
\hline 5-hydroxyisourate hydrolase; & Q9CRB3 & Urah & 1,7 & 37,3 \\
\hline $60 \mathrm{kDa}$ heat shock protein, mitochondrial; & P63038 & Hspd1 & 0,8 & 39,3 \\
\hline $60 \mathrm{~S}$ acidic ribosomal protein $\mathrm{PO}$ & P14869 & Rplp0 & $-0,1$ & 15,2 \\
\hline 605 acidic ribosomal protein $\mathrm{P} 1$ & P47955 & Rplp1 & $-0,1$ & 14 \\
\hline
\end{tabular}




\begin{tabular}{|c|c|c|c|c|}
\hline Protein & Uniprot & Gene name & $\begin{array}{c}\text { Fold change of } \\
\text { XPRESS ratio } \\
\left.\text { (Adam } 17^{\Delta K C} / \mathrm{WT}\right)\end{array}$ & $\begin{array}{c}\text { Sequence } \\
\text { coverage [\%] }\end{array}$ \\
\hline 605 acidic ribosomal protein $\mathrm{P} 2$ & P99027 & Rplp2 & $-0,5$ & 67 \\
\hline $60 \mathrm{~S}$ ribosomal protein L10a; & P53026 & Rpl10a & $-0,1$ & 6 \\
\hline $60 S$ ribosomal protein L12; & P35979 & Rpl12 & 0,1 & 45,5 \\
\hline 60S ribosomal protein L13; & P47963 & Rpl13 & 0,7 & 19,9 \\
\hline $60 S$ ribosomal protein L14; & Q9CR57 & Rpl14 & $-0,9$ & 6 \\
\hline 60S ribosomal protein L17; & Q9CPR4 & Rpl17 & 0,1 & 26,1 \\
\hline $60 S$ ribosomal protein L22; & P67984 & Rpl22 & 0,9 & 18,8 \\
\hline 60 S ribosomal protein L22-like 1 & Q9D7S7 & Rpl22I1 & $-0,6$ & 9,8 \\
\hline 60 S ribosomal protein L23; & P62830 & Rpl23 & $-2,5$ & 20 \\
\hline 60S ribosomal protein L23a; & P62751 & Rpl23a & $-0,3$ & 14,7 \\
\hline $60 S$ ribosomal protein L24; & Q8BP67 & Rpl24 & 0,8 & 16,5 \\
\hline $60 S$ ribosomal protein L26; & P61255 & Rpl26 & 0,0 & 8,7 \\
\hline $60 S$ ribosomal protein L31; & P62900 & Rpl31 & 0,3 & 24,8 \\
\hline 60S ribosomal protein L38; & Q9Jग18 & Rpl38 & 0,1 & 10 \\
\hline 60S ribosomal protein L39; & P62892 & Rpl39 & 0,6 & 19,6 \\
\hline $60 S$ ribosomal protein L4; & Q9D8E6 & Rpl4 & 0,3 & 5 \\
\hline $60 S$ ribosomal protein $L 5$ & P47962 & Rpl5 & 0,1 & 7,1 \\
\hline 60S ribosomal protein L6; & P47911 & Rpl6 & $-0,3$ & 11,5 \\
\hline 60S ribosomal protein L7; & P14148 & Rpl7 & $-0,3$ & 7,8 \\
\hline $60 S$ ribosomal protein $\mathrm{L7a}$ & P12970 & Rpl7a & $-0,3$ & 3,4 \\
\hline $60 S$ ribosomal protein L8; & P62918 & Rpl8 & 0,1 & 6,2 \\
\hline 6-phosphogluconolactonase; & Q9CQ60 & Pgls & 1,2 & 34,6 \\
\hline 78 kDa glucose-regulated protein; & P20029 & Hspa5 & 0,0 & 40,8 \\
\hline 85/88 kDa calcium-independent phospholipase A2; & P97819 & Pla2g6 & $-5,6$ & 1,6 \\
\hline Abl interactor 1 & Q8CBW3 & Abi1 & 0,1 & 26 \\
\hline Acid ceramidase; & Q9WV54 & Asah1 & 0,7 & 15,7 \\
\hline Acid sphingomyelinase-like phosphodiesterase $3 a$; & P70158 & Smpdl3a & $-3,1$ & 3,4 \\
\hline Acidic leucine-rich nuclear phosphoprotein 32 family member $A$; & 035381 & Anp32a & 0,1 & 28,8 \\
\hline Acidic leucine-rich nuclear phosphoprotein 32 family member B; & Q9EST5 & Anp32b & 0,4 & 14,3 \\
\hline Acidic leucine-rich nuclear phosphoprotein 32 family member E; & P97822 & Anp32e & 0,2 & 23,4 \\
\hline Aconitate hydratase, mitochondrial; & Q99KI0 & Aco2 & $-0,5$ & 4 \\
\hline Actin-related protein $2 / 3$ complex subunit 3 ; & Q9JM76 & Arpc3 & 1,2 & 10,6 \\
\hline Actin-related protein $2 / 3$ complex subunit 5 & Q9CPW4 & Arpc5 & 0,5 & 29,1 \\
\hline Actin-related protein $2 / 3$ complex subunit 5 -like protein; & Q9D898 & Arpc5I & $-0,3$ & 28,8 \\
\hline Activated RNA polymerase II transcriptional coactivator $\mathrm{p} 15$; & P11031 & Sub1 & $-0,3$ & 29,9 \\
\hline Acyl-CoA dehydrogenase family member 9, mitochondrial; & Q8JZN5 & Acad9 & $-0,4$ & 1,6 \\
\hline Acyl-CoA-binding domain-containing protein 5 & Q5XG73 & Acbd5 & $-0,4$ & 3,3 \\
\hline Acyl-CoA-binding protein; & P31786 & Dbi & 0,2 & 39,1 \\
\hline Acyl-CoA-binding protein; & D3Z563 & Dbi & $-0,4$ & 41,3 \\
\hline Acylphosphatase-1; & P56376 & Acyp1 & 0,6 & 48,5 \\
\hline Acyl-protein thioesterase 1 & P97823 & Lypla1 & 1,9 & 24,6 \\
\hline Acyl-protein thioesterase 2 & Q9WTL7 & Lypla2 & 2,4 & 7,8 \\
\hline Adapter molecule crk; & Q64010 & Crk & 1,0 & 8,5 \\
\hline Adenosylhomocysteinase; & P50247 & Ahcy & $-0,6$ & 11,6 \\
\hline Adenylate kinase 2, mitochondrial; & Q9WTP6 & Ak2 & 1,4 & 38,1 \\
\hline Adenylate kinase isoenzyme 1 & Q9R0Y5 & Ak1 & 0,8 & 12,9 \\
\hline Adenylyl cyclase-associated protein 1 ; & P40124 & Cap1 & $-1,2$ & 3,8 \\
\hline Adiponectin; & Q60994 & Adipoq & $-0,4$ & 11,3 \\
\hline ADP/ATP translocase 2 & P51881 & Slc25a5 & $-0,4$ & 16,1 \\
\hline ADP-ribosylation factor 3 & P61205 & Arf3 & 0,0 & 21,5 \\
\hline ADP-ribosylation factor GTPase-activating protein 1 ; & Q9EPJ9 & Arfgap1 & $-0,3$ & 7,1 \\
\hline ADP-ribosylation factor GTPase-activating protein 2; & Q99K28 & Arfgap2 & $-0,4$ & 9 \\
\hline ADP-sugar pyrophosphatase; & Q9JKX6 & Nudt5 & 0,4 & 28,9 \\
\hline Adrenodoxin, mitochondrial; & P46656 & $\mathrm{Fdx1}$ & $-0,5$ & 9,6 \\
\hline Adrenodoxin-like protein, mitochondrial; & Q9CPW2 & Fdx1l & $-0,3$ & 5,7 \\
\hline Afadin; & Q9QZQ1 & Mllt4 & $-0,4$ & 3,8 \\
\hline Aftiphilin; & Q80WT5 & Aftph & 0,0 & 8,5 \\
\hline A-kinase anchor protein 12 & Q9WTQ5 & Akap12 & $-0,2$ & 6,2 \\
\hline A-kinase anchor protein 2 & A2API8 & Akap2 & $-1,0$ & 2,5 \\
\hline A-kinase anchor protein 8; & Q9DBR0 & Akap8 & $-0,4$ & 1,9 \\
\hline Alanine aminotransferase 1 & Q8QZR5 & Gpt & 0,2 & 6,9 \\
\hline Alcohol dehydrogenase 1 & P00329 & Adh1 & $-0,9$ & 4,5 \\
\hline Aldehyde dehydrogenase; & E9Q3E1 & Aldh3b2 & 0,5 & 19,8 \\
\hline Aldehyde oxidase 4; & Q3TYQ9 & Aox4 & $-3,9$ & 0,9 \\
\hline $\begin{array}{l}\text { Allergen dl chain C2A; Androgen binding protein beta; Androgen-binding } \\
\text { protein; Protein Scgb2b27; }\end{array}$ & Q8R1E9 & Scgb2b27 & 0,7 & 20,5 \\
\hline Allograft inflammatory factor 1 -like; & Q9EQX4 & Aif1l & $-0,9$ & 22,7 \\
\hline $\begin{array}{l}\text { Alpha globin 1; Alpha globin 2; Alpha-globin; Hemoglobin alpha, adult chain 1; } \\
\text { Hemoglobin subunit alpha; Protein Hba-a2; }\end{array}$ & Q91VB8 & Hba a1 & 0,4 & 62 \\
\hline Alpha/beta hydrolase domain-containing protein 14B; & E9QN99 & Abhd14b & 1,2 & 21 \\
\hline Alpha-1-antitrypsin 1-1; & P07758 & Serpina1a & $-0,2$ & 19,2 \\
\hline Alpha-1-antitrypsin 1-2; & P22599 & Serpina1b & $-0,2$ & 23,2 \\
\hline Alpha-1-antitrypsin 1-4; & Q00897 & Serpina1d & 1,3 & 20,8 \\
\hline
\end{tabular}




\begin{tabular}{|c|c|c|c|c|}
\hline Protein & Uniprot & Gene name & $\begin{array}{c}\text { Fold change of } \\
\text { XPRESS ratio } \\
\text { (Adam17 } \\
\text { (AKC / WT) }\end{array}$ & $\begin{array}{c}\text { Sequence } \\
\text { coverage [\%] }\end{array}$ \\
\hline Alpha-1-antitrypsin 1-5; & Q00898 & Serpina1e & 7,5 & 14,3 \\
\hline Alpha-2-antiplasmin; & Q61247 & Serpinf2 & $-10,0$ & 2,9 \\
\hline Alpha-2-HS-glycoprotein; & P29699 & Ahsg & 0,2 & 25,2 \\
\hline Alpha-2-macroglobulin receptor-associated protein; & P55302 & Lrpap1 & $-0,1$ & 20,8 \\
\hline Alpha-2-macroglobulin; & Q61838 & $\mathrm{A} 2 \mathrm{~m}$ & $-0,2$ & 2 \\
\hline Alpha-actinin-4; & P57780 & Actn4 & 0,7 & 23,4 \\
\hline Alpha-adducin; & Q9QYC0 & Add1 & $-0,5$ & 7,5 \\
\hline Alpha-endosulfine; & P60840 & Ensa & 0,0 & 40,5 \\
\hline Alpha-enolase; & P17182 & Eno1 & 0,8 & 14,3 \\
\hline Alpha-fetoprotein; & P02772 & Afp & $-0,3$ & 39 \\
\hline Alpha-synuclein; & 055042 & Snca & 0,0 & 9,3 \\
\hline Alpha-taxilin; & Q6PAM1 & Txlna & 0,1 & 16,8 \\
\hline Aminoacyl tRNA synthase complex-interacting multifunctional protein $1 ;$ & P31230 & Aimp1 & $-0,8$ & 21,6 \\
\hline Aminoacyl tRNA synthase complex-interacting multifunctional protein 2; & Q8R010 & Aimp2 & 0,4 & 7,8 \\
\hline Amyloid beta A4 protein; & P12023 & App & $-0,9$ & 1,2 \\
\hline AN1-type zinc finger protein 6; & Q9DCH6 & Zfand6 & $-1,3$ & 9,4 \\
\hline Anamorsin; & Q8WTY4 & Ciapin1 & 0,2 & 20,1 \\
\hline Anaphase-promoting complex subunit 13; & Q8R034 & Anapc13 & $-0,4$ & 13,5 \\
\hline Anaphase-promoting complex subunit 16; & Q9CPV2 & Anapc16 & 0,4 & 46,9 \\
\hline Anaphase-promoting complex subunit CDC26; & Q99JP4 & Cdc26 & $-0,1$ & 12,9 \\
\hline Androgen binding protein alpha; Androgen-binding protein; Protein Scgb1b27; & Q91WB5 & Scgb1b27 & 0,7 & 19,6 \\
\hline $\begin{array}{l}\text { Androgen binding protein gamma; MCG17480; Protein Scgb2b26; Salivary } \\
\text { androgen-binding protein gamma subunit; }\end{array}$ & Q8JZX1 & Scgb2b26 & 0,6 & 40,2 \\
\hline $\begin{array}{l}\text { Androgen-binding protein; Androgen-binding protein eta; Lacrimal androgen- } \\
\text { binding protein eta; Protein Scgb1b2; }\end{array}$ & 035176 & Scgb1b2 & 0,1 & 9,7 \\
\hline Androgen-binding protein; Protein Scgb1b29; Protein Scgb1b7; & D2XZ31 & Abpa29_a7 & $-0,3$ & 11,8 \\
\hline Angiotensin-converting enzyme 2 ; & Q8R0I0 & Ace2 & $-1,0$ & 1,2 \\
\hline Ankyrin repeat domain-containing protein 49 ; & Q8VE42 & Ankrd49 & 0,6 & 8,8 \\
\hline Ankyrin-3; & G5E8K5 & Ank3 & 0,1 & 4,9 \\
\hline Annexin A1; & P10107 & Anxa1 & 0,0 & 41 \\
\hline Annexin A2; & P07356 & Anxa2 & 0,4 & 47,5 \\
\hline Annexin A4; & P97429 & Anxa4 & $-1,2$ & 7,7 \\
\hline Annexin A5; & P48036 & Anxa5 & 0,6 & 31,7 \\
\hline Annexin A6; & P14824 & Anxa6 & 0,0 & 6,3 \\
\hline Annexin A7; & Q07076 & Anxa7 & $-0,5$ & 3,5 \\
\hline Annexin A8; & O35640 & Anxa8 & $-0,1$ & 12,6 \\
\hline Antithrombin-III; & P32261 & Serpinc1 & 0,1 & 9,7 \\
\hline AP2-associated protein kinase 1 & Q3UHJO & Aak1 & $-0,6$ & 3,4 \\
\hline Apolipoprotein A-I; & Q00623 & Apoa1 & 0,4 & 38,6 \\
\hline Apolipoprotein A-II; & P09813 & Apoa2 & 0,2 & 9,8 \\
\hline Apolipoprotein A-IV; & P06728 & Apoa4 & 0,4 & 56,7 \\
\hline Apolipoprotein C-I; & P34928 & Apoc1 & 1,1 & 20,5 \\
\hline Apolipoprotein C-III; & P33622 & Apoc3 & 1,5 & 19,2 \\
\hline Apolipoprotein E; & P08226 & Apoe & $-1,0$ & 28,6 \\
\hline Apolipoprotein O; & Q9DCZ4 & Apoo & 0,5 & 11 \\
\hline Apoptosis inhibitor 5; & 035841 & Api5 & 0,5 & 3,8 \\
\hline Apoptosis regulator BAX; & Q07813 & Bax & 0,2 & 17,2 \\
\hline Apoptosis-associated speck-like protein containing a CARD; & Q9EPB4 & Pycard & 0,1 & 37,8 \\
\hline Apoptotic chromatin condensation inducer in the nucleus; & Q9JIX8 & Acin1 & 0,0 & 11,6 \\
\hline Arachidonate 12-lipoxygenase, 12R-type; & 070582 & Alox12b & 0,5 & 3,6 \\
\hline Arachidonate 12-lipoxygenase, 12S-type; & P39655 & Alox12 & $-0,4$ & 5,6 \\
\hline Arf-GAP domain and FG repeat-containing protein 1 ; & Q8K2K6 & Agfg1 & $-0,1$ & 11,9 \\
\hline Arfip1 protein; Protein Arfip1; & A2RSX9 & Arfip1 & $-0,7$ & 6,7 \\
\hline Arginine and glutamate-rich protein 1 & Q3UL36 & Arglu1 & 0,2 & 3 \\
\hline $\begin{array}{l}\text { Arginine-rich, mutated in early stage tumors, isoform CRA_b; Mesencephalic } \\
\text { astrocyte-derived neurotrophic factor; }\end{array}$ & Q3TMX5 & Manf & 0,4 & 35,8 \\
\hline Arpin; & Q9D0A3 & Arpin & 0,2 & 11,9 \\
\hline Ashwin; & Q922M7 & & 0,9 & 6,5 \\
\hline Asparagine synthetase domain-containing protein 1 ; & MoQW65 & Asnsd1 & $-0,2$ & 45,2 \\
\hline Aspartate aminotransferase, mitochondrial; & P05202 & Got2 & 1,4 & 39,8 \\
\hline Aspartyl/asparaginyl beta-hydroxylase; & Q8BSY0 & Asph & 0,0 & 24,7 \\
\hline Astrocytic phosphoprotein PEA-15; & Q62048 & Pea15 & 0,1 & 18,5 \\
\hline Ataxin-2-like protein; & Q3TGG2 & Atxn2l & $-0,3$ & 10,5 \\
\hline Ataxin-2-like protein; & Q7TQHO & Atxn2l & 0,5 & 11,3 \\
\hline AT-hook DNA-binding motif-containing protein 1 ; & Q6PAL7 & Ahdc1 & 0,6 & 0,8 \\
\hline ATP synthase protein 8 & P03930 & Mtatp8 & 0,3 & 14,9 \\
\hline ATP synthase subunit alpha, mitochondrial; & Q03265 & Atp5a1 & $-0,3$ & 15 \\
\hline ATP synthase subunit beta, mitochondrial; & P56480 & Atp5b & 0,1 & 23,8 \\
\hline ATP synthase subunit $d$, mitochondrial; & Q9DCX2 & Atp5h & 0,3 & 46,3 \\
\hline ATP synthase subunit delta, mitochondrial; & Q9D3D9 & Atp5d & $-0,2$ & 13,7 \\
\hline
\end{tabular}




\section{Protein}

ATP synthase subunit e, mitochondrial;

ATP synthase subunit epsilon, mitochondrial;

ATP synthase subunit $f$, mitochondrial;

ATP synthase subunit $g$, mitochondrial;

ATP synthase subunit $O$, mitochondrial;

ATP synthase-coupling factor 6 , mitochondrial;

ATPase inhibitor, mitochondrial;

ATP-binding cassette sub-family E member 1 ;

ATP-binding cassette sub-family $F$ member 1 ;

ATP-citrate synthase;

ATP-dependent (S)-NAD(P)H-hydrate dehydratase;

ATP-dependent RNA helicase DDX1;

ATP-dependent RNA helicase DDX39A;

ATP-dependent RNA helicase DDX3X;

Atrial natriuretic peptide-converting enzyme;

AT-rich interactive domain-containing protein $1 \mathrm{~A}$;

Aurora kinase B;

Autophagy-related protein 13;

Baculoviral IAP repeat-containing protein 6;

BAG family molecular chaperone regulator 1 ;

BAG family molecular chaperone regulator 2 ;

BAG family molecular chaperone regulator 3 ;

Band 4.1-like protein 2;

Band 4.1-like protein 3;

Barrier-to-autointegration factor;

Basal cell adhesion molecule;

Basigin;

B-cell CLL/lymphoma 7 protein family member A;

B-cell CLL/lymphoma 7 protein family member $C_{\text {; }}$

B-cell linker protein

B-cell receptor-associated protein 31;

$\mathrm{Bcl} 2$ antagonist of cell death;

BCL2/adenovirus E1B 19 kDa protein-interacting protein 3-like;

$\mathrm{Bcl}$-2/adenovirus E1B $19 \mathrm{kDa}$-interacting protein 2-like protein;

Bcl-2-associated transcription factor 1;

Bcl-2-like protein 13;

BET1 homolog;

BET1-like protein;

Beta-2-glycoprotein 1;

Beta-actin-like protein 2;

Beta-catenin-interacting protein 1;

Beta-galactosidase;

Beta-globin; Protein Hbb-bs; Protein Hbb-bt;

Beta-hexosaminidase subunit beta;

BH3-interacting domain death agonist;

Bicaudal D-related protein 2;

Bifunctional glutamate/proline--tRNA ligase;

Biogenesis of lysosome-related organelles complex 1 subunit 2;

Biorientation of chromosomes in cell division protein 1 ;

Bis(5'-adenosyl)-triphosphatase;

Bleomycin hydrolase;

BolA-like protein 1;

BolA-like protein 2;

BPI fold-containing family A member 2;

Brain acid soluble protein 1;

Brain-specific angiogenesis inhibitor 1-associated protein 2;

BRI3-binding protein;

Bridging integrator 2;

Bromodomain-containing protein 4;

BSD domain-containing protein 1;

BTB/POZ domain-containing protein KCTD5;

BUB3-interacting and GLEBS motif-containing protein ZNF207;

Cadherin-1;

Cadherin-13;

Cadherin-3;

Calcineurin B homologous protein 1;

Calcineurin B homologous protein 3;

Calcineurin subunit B type 1 ;

Calcium/calmodulin-dependent protein kinase type II subunit delta;

Calcium-activated chloride channel regulator 2;

Calcium-activated chloride channel regulator 2; Chloride channel calcium

activated 2; Endothelial chloride channel; MCG120735;

Calcium-binding mitochondrial carrier protein SCaMC-1;

\begin{tabular}{|c|c|c|c|}
\hline Uniprot & Gene name & 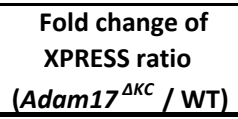 & $\begin{array}{c}\text { Sequence } \\
\text { coverage [\%] }\end{array}$ \\
\hline Q06185 & Atp5i & $-0,1$ & 32,4 \\
\hline P56382 & Atp5e & 0,8 & 15,4 \\
\hline P56135 & Atp5j2 & $-0,2$ & 30,3 \\
\hline Q9CPQ8 & Atp5I & 0,0 & 27,2 \\
\hline Q9DB20 & Atp5o & 1,5 & 46,5 \\
\hline P97450 & Atp5j & $-0,2$ & 41,7 \\
\hline E9PV44 & Atpif1 & 0,0 & 21,6 \\
\hline P61222 & Abce1 & $-0,1$ & 3,7 \\
\hline Q6P542 & Abcf1 & $-1,2$ & 1,2 \\
\hline Q91V92 & Acly & $-1,4$ & 1,1 \\
\hline Q9CZ42 & Carkd & 2,2 & 12,9 \\
\hline Q91VR5 & Ddx1 & $-0,3$ & 2,4 \\
\hline Q8VDW0 & Ddx39a & $-0,3$ & 5,2 \\
\hline Q62167 & $\operatorname{Ddx} 3 x$ & $-0,8$ & 2,1 \\
\hline Q97319 & Corin & 0,3 & 1,1 \\
\hline $\mathrm{A} 2 \mathrm{BH} 40$ & Arid1a & $-0,8$ & 0,9 \\
\hline E9PVP1 & Aim1 & $-0,7$ & 11,7 \\
\hline Q91YI1 & Atg13 & $-0,2$ & 2,5 \\
\hline 088738 & Birc6 & $-1,1$ & 0,2 \\
\hline Q60739 & Bag1 & 1,2 & 14,1 \\
\hline Q91YN9 & Bag2 & 0,6 & 9,5 \\
\hline Q9JLV1 & Bag3 & $-0,1$ & 32,1 \\
\hline 070318 & Epb41/2 & $-0,3$ & 1 \\
\hline Q9WV92 & Epb4113 & $-0,5$ & 8,5 \\
\hline 054962 & Banf1 & 0,8 & 34,8 \\
\hline Q9R069 & Bcam & 0,2 & 7,7 \\
\hline P18572 & Bsg & 0,2 & 33 \\
\hline Q9CXE2 & $\mathrm{Bcl} 7 \mathrm{a}$ & $-0,4$ & 8,6 \\
\hline 008664 & $\mathrm{Bcl} 7 \mathrm{c}$ & $-1,2$ & 10,1 \\
\hline Q9QUN3 & Blnk & 0,0 & 11,5 \\
\hline Q61335 & Bcap31 & 1,5 & 10,6 \\
\hline Q61337 & Bad & $-0,3$ & 29,6 \\
\hline Q9Z2F7 & Bnip3I & 0,2 & 12,3 \\
\hline Q99JU7 & Bnipl & $-0,5$ & 10,4 \\
\hline Q8K019 & Bclaf1 & $-0,2$ & 3,3 \\
\hline P59017 & $\mathrm{Bcl} 2 \mathrm{I} 13$ & $-0,2$ & 2,5 \\
\hline O35623 & Bet1 & 0,4 & 9,3 \\
\hline 035153 & Bet1l & 0,2 & 11,7 \\
\hline Q01339 & Apoh & 0,8 & 33,3 \\
\hline Q8BFZ3 & Actbl2 & 0,5 & 14,4 \\
\hline Q9JJN6 & Ctnnbip1 & 0,0 & 29,6 \\
\hline P23780 & Glb1 & $-0,4$ & 1,9 \\
\hline A8DUK4 & Hbbt1 & $-0,4$ & 76,9 \\
\hline P20060 & Hexb & $-0,7$ & 5 \\
\hline P70444 & Bid & 3,8 & 9,7 \\
\hline Q8CHW5 & Ccdc64b & 0,5 & 7,7 \\
\hline Q8CGC7 & Eprs & $-2,1$ & 1,2 \\
\hline Q9CWG9 & Bloc1s2 & $-0,2$ & 5,6 \\
\hline Q5SQY2 & Bod1 & $-0,2$ & 19,1 \\
\hline 089106 & Fhit & 0,7 & 12,9 \\
\hline Q8R016 & Blmh & $-0,2$ & 23,7 \\
\hline Q9D8S9 & Bola1 & $-0,1$ & 44,5 \\
\hline Q8BGS2 & Bola2 & 0,0 & 32,6 \\
\hline P07743 & Bpifa2 & 0,7 & 19,4 \\
\hline Q91XV3 & Basp1 & $-0,3$ & 32,3 \\
\hline Q8BKX1 & Baiap2 & 0,5 & 14 \\
\hline Q8BXV2 & Bri3bp & 1,2 & 3,6 \\
\hline D3Z6Q9 & Bin2 & $-0,2$ & 3,2 \\
\hline Q9ESU6 & Brd4 & $-0,2$ & 0,8 \\
\hline Q80Y55 & Bsdc1 & 0,0 & 5,9 \\
\hline Q8VC57 & Kctd5 & 0,3 & 5,1 \\
\hline E9PW12 & Znf207 & $-1,0$ & 5,6 \\
\hline P09803 & Cdh1 & 0,0 & 18 \\
\hline Q9WTR5 & Cdh13 & 0,0 & 12,2 \\
\hline P10287 & Cdh3 & $-1,2$ & 3 \\
\hline P61022 & Chp1 & 0,0 & 26,7 \\
\hline Q9JKL5 & Tesc & $-0,6$ & 7 \\
\hline Q63810 & Ppp3r1 & 0,2 & 15,3 \\
\hline Q6PHZ2 & Camk2d & $-0,1$ & 19,2 \\
\hline Q8BG22 & Clca2 & 0,9 & 0,8 \\
\hline Q9EQR4 & Clca2 & 0,1 & 8,2 \\
\hline Q8BMD8 & Slc25a24 & 0,5 & 11,8 \\
\hline
\end{tabular}




\begin{tabular}{|c|c|c|c|c|}
\hline Protein & Uniprot & Gene name & $\begin{array}{c}\text { Fold change of } \\
\text { XPRESS ratio } \\
\left(\text { Adam } 17^{\Delta K C} / \mathrm{WT}\right)\end{array}$ & $\begin{array}{c}\text { Sequence } \\
\text { coverage [\%] }\end{array}$ \\
\hline Calcium-binding protein $39 ;$ & Q06138 & Cab39 & 0,0 & 12,9 \\
\hline Calcium-independent phospholipase A2-gamma; & Q8K1N1 & Pnpla8 & 0,0 & 3,9 \\
\hline Calcium-regulated heat stable protein 1 ; & Q9CR86 & Carhsp1 & 0,2 & 10,8 \\
\hline Calcyclin-binding protein; & Q9CXW3 & Cacybp & 0,2 & 3,1 \\
\hline Calmodulin; & G3UZ90 & Calm3 & $-0,2$ & 13,8 \\
\hline Calmodulin; & P62204 & Calm3 & $-0,6$ & 32,9 \\
\hline Calmodulin-4; & Q9JM83 & Calm4 & $-0,2$ & 55,4 \\
\hline Calmodulin-like protein 3; & Q9D6P8 & Calml3 & $-0,6$ & 28,2 \\
\hline Calnexin; & P35564 & Canx & 0,3 & 14,7 \\
\hline Calpain small subunit 2 & Q9D7J7 & Capns2 & $-0,1$ & 13,8 \\
\hline Calpain-1 catalytic subunit; & 035350 & Capn1 & $-0,2$ & 8 \\
\hline Calpastatin; & P51125 & Cast & $-0,4$ & 34,7 \\
\hline Calponin-2; & Q08093 & Cnn2 & 0,6 & 23,9 \\
\hline Calponin-3; & Q9DAW9 & Cnn3 & $-0,5$ & 10 \\
\hline Calreticulin; & P14211 & Calr & 0,2 & 28,8 \\
\hline Calumenin; & 035887 & Calu & $-0,2$ & 30,5 \\
\hline cAMP-dependent protein kinase inhibitor alpha; & P63248 & Pkia & 1,2 & 27,6 \\
\hline cAMP-dependent protein kinase inhibitor gamma; & 070139 & Pkig & $-0,6$ & 28,6 \\
\hline cAMP-regulated phosphoprotein 19 & P56212 & Arpp19 & $-0,3$ & 38,4 \\
\hline CAP-Gly domain-containing linker protein 1 ; & Q922J3 & Clip1 & $-0,1$ & 30,8 \\
\hline $\begin{array}{l}\text { Capping protein (Actin filament), gelsolin-like; Capping protein (Actin filament), } \\
\text { gelsolin-like, isoform CRA_a; Macrophage-capping protein; }\end{array}$ & Q99LB4 & Capg & 1,1 & 22,1 \\
\hline Caprin-1; & Q60865 & Caprin1 & 0,0 & 7,5 \\
\hline Carbonic anhydrase 3; & P16015 & Ca3 & 0,3 & 11,5 \\
\hline Carbonic anhydrase 6; & P18761 & Ca6 & 0,4 & 10,7 \\
\hline Carbonyl reductase [NADPH] 1 ; & P48758 & Cbr1 & 1,2 & 4,7 \\
\hline Carboxylesterase 1C; & P23953 & Ces1c & 0,0 & 3,4 \\
\hline Carboxylesterase $5 \mathrm{~A}$; & Q6AW46 & Ces5a & $-3,9$ & 6,1 \\
\hline Carboxypeptidase A4; & Q6P8K8 & Cpa4 & 0,6 & 10,2 \\
\hline Carboxypeptidase E; & Q00493 & Cpe & $-0,3$ & 5,3 \\
\hline Carboxypeptidase M; & Q80V42 & $\mathrm{Cpm}$ & $-0,6$ & 7 \\
\hline Casein kinase II subunit alpha'; & 054833 & Csnk2a2 & 0,3 & 7,1 \\
\hline Caspase-14; & 089094 & Casp14 & $-0,1$ & 37,7 \\
\hline Catalase; & P24270 & Cat & $-0,2$ & 32,6 \\
\hline Catenin alpha-1; & P26231 & Ctnna1 & 0,6 & 29,9 \\
\hline Catenin beta-1; & Q02248 & Ctnnb1 & $-0,2$ & 4,3 \\
\hline Catenin delta-1; & P30999 & Ctnnd1 & 0,5 & 5,9 \\
\hline Cathelin-related antimicrobial peptide; & P51437 & Camp & 2,7 & 19,7 \\
\hline Cathepsin B; & P10605 & Ctsb & $-0,1$ & 16,8 \\
\hline Cathepsin D; & P18242 & Ctsd & 0,7 & 23,9 \\
\hline Cathepsin L1; & P06797 & Ctsl & 0,7 & 18,6 \\
\hline Cation-dependent mannose-6-phosphate receptor; & P24668 & M6pr & $-0,2$ & 7,9 \\
\hline Caveolin-1; & P49817 & Cav1 & 0,1 & 24,7 \\
\hline Caveolin-2; & Q9WVC3 & Cav2 & 0,0 & 7,4 \\
\hline CCAAT/enhancer-binding protein beta; & P28033 & Cebpb & 1,0 & 7,4 \\
\hline CD109 antigen; & Q8R422 & Cd109 & $-0,1$ & 8 \\
\hline CD166 antigen; & Q61490 & Alcam & $-0,1$ & 18,9 \\
\hline CD276 antigen; & Q8VE98 & $\mathrm{Cd} 276$ & 0,2 & 3,2 \\
\hline CD2-associated protein; & Q9JLQ0 & Cd2ap & $-0,4$ & 31,6 \\
\hline CD44 antigen; & P15379 & $\mathrm{Cd} 44$ & $-0,7$ & 9,1 \\
\hline CD63 antigen; & P41731 & Cd63 & 0,3 & 7,6 \\
\hline CD81 antigen; & P35762 & Cd81 & 2,1 & 19,1 \\
\hline CD82 antigen; & P40237 & $\mathrm{Cd} 82$ & $-0,6$ & 6,7 \\
\hline CD9 antigen; & P40240 & Cd9 & 0,8 & 3,1 \\
\hline Cdc42 effector protein 4 & Q9JM96 & Cdc42ep4 & $-0,4$ & 8,9 \\
\hline Cdc42 effector protein 5 & Q9Z0X0 & Cdc42ep5 & $-3,2$ & 16,5 \\
\hline CDGSH iron-sulfur domain-containing protein 1 ; & Q91WS0 & Cisd1 & 0,4 & 25,9 \\
\hline CDGSH iron-sulfur domain-containing protein 2; & Q9CQB5 & Cisd2 & $-0,2$ & 22,2 \\
\hline CDKN2AIP N-terminal-like protein; & Q9D211 & Cdkn2aipnl & 0,5 & 8,6 \\
\hline Cell adhesion molecule 1; & Q8R5M8 & Cadm1 & 0,3 & 2,3 \\
\hline Cell cycle control protein $50 \mathrm{~A}$; & Q8VEKO & Tmem30a & 3,1 & 3 \\
\hline Cell death regulator Aven; & Q9D9K3 & Aven & $-0,2$ & 8,2 \\
\hline Cellular nucleic acid-binding protein; & P53996 & Cnbp & 0,5 & 30,9 \\
\hline Cellular nucleic acid-binding protein; & D3YYF6 & Cnbp & 0,5 & 29,5 \\
\hline Centrin-2; & Q9R1K9 & Cetn2 & $-0,3$ & 14,4 \\
\hline CGG triplet repeat-binding protein 1 ; & Q8BHG9 & Cggbp1 & 0,4 & 20,4 \\
\hline Charged multivesicular body protein $1 \mathrm{a}$; & Q921W0 & Chmp1a & 0,1 & 12,8 \\
\hline Charged multivesicular body protein $2 \mathrm{~b}$; & Q8BJF9 & Chmp2b & $-0,5$ & 12,7 \\
\hline Charged multivesicular body protein $4 \mathrm{~b}$; & Q9D8B3 & Chmp4b & $-0,3$ & 25,9 \\
\hline Charged multivesicular body protein $4 \mathrm{c}$; & Q9D7F7 & Chmp4c & $-0,3$ & 10,8 \\
\hline Charged multivesicular body protein 5 ; & Q9D7S9 & Chmp5 & $-0,1$ & 20,1 \\
\hline Chitinase-3-like protein 1 ; & Q61362 & Chi3l1 & 2,0 & 31,9 \\
\hline Chitotriosidase-1; & Q9D7Q1 & Chit1 & 0,5 & 13,4 \\
\hline
\end{tabular}




\begin{tabular}{|c|c|c|c|c|}
\hline Protein & Uniprot & Gene name & $\begin{array}{l}\text { Fold change of } \\
\text { XPRESS ratio } \\
\text { (Adam17 } \\
\text { AKC } / \mathrm{WT})\end{array}$ & $\begin{array}{c}\text { Sequence } \\
\text { coverage [\%] }\end{array}$ \\
\hline Chloride intracellular channel protein 1 & Q9Z1Q5 & Clic1 & 0,5 & 12,4 \\
\hline Chloride intracellular channel protein 3; & Q9D7P7 & Clic3 & 0,3 & 19,4 \\
\hline Choline transporter-like protein 2 & Q8BY89 & Slc44a2 & 0,7 & 4,5 \\
\hline Chromatin complexes subunit BAP18; & Q9DCT6 & Bap18 & $-0,3$ & 70,4 \\
\hline Chromatin modification-related protein MEAF6; & Q2VPQ9 & Meaf6 & 0,9 & 12,7 \\
\hline Chromatin target of PRMT1 protein; & Q9CY57 & Chtop & 0,8 & 6,4 \\
\hline Chromobox protein homolog 1 & P83917 & $\mathrm{Cbx} 1$ & $-0,3$ & 21,1 \\
\hline Chromobox protein homolog 3 ; & P23198 & $\mathrm{Cb} \times 3$ & 0,1 & 29 \\
\hline Chromobox protein homolog 5 & Q61686 & Cbx5 & 0,3 & 17,3 \\
\hline Chromogranin-A; & P26339 & Chga & $-0,7$ & 1,9 \\
\hline Cingulin; & D3YUW7 & Cgn & $-0,4$ & 13,3 \\
\hline Citrate synthase, mitochondrial; & Q9CZU6 & Cs & 1,5 & 22,4 \\
\hline Clathrin interactor 1; MCG22297, isoform CRA_b; & Q5SUH6 & Clint1 & $-0,1$ & 24,1 \\
\hline Clathrin light chain $A$; & B1AWE0 & Clta & $-0,3$ & 28,2 \\
\hline Clathrin light chain B; & Q6IRU5 & Cltb & $-0,3$ & 26,2 \\
\hline Claudin-1; & 088551 & Cldn1 & 1,6 & 7,1 \\
\hline Claudin-5; & 054942 & Cldn5 & $-6,3$ & 7,3 \\
\hline Cleavage stimulation factor subunit 2 & Q8BIQ5 & Cstf2 & $-1,0$ & 11,6 \\
\hline Clusterin; & Q06890 & $\mathrm{Clu}$ & 0,1 & 29,7 \\
\hline C-Myc-binding protein; & Q9EQS3 & Mycbp & $-0,5$ & 62,1 \\
\hline Coactosin-like protein; & Q9CQ16 & Cotl1 & $-0,1$ & 5,6 \\
\hline Cob(I)yrinic acid a,c-diamide adenosyltransferase, mitochondrial; & Q9D273 & Mmab & $-0,4$ & 11,1 \\
\hline Cofilin-1; & P18760 & Cfl1 & 0,6 & 39,2 \\
\hline Coiled-coil and C2 domain-containing protein 1B; & Q8BRN9 & $\mathrm{Cc} 2 \mathrm{~d} 1 \mathrm{~b}$ & $-0,5$ & 2,5 \\
\hline Coiled-coil domain-containing protein 115 & Q8VE99 & Ccdc115 & 0,4 & 18,9 \\
\hline Coiled-coil domain-containing protein 12 & Q8R344 & Ccdc12 & $-0,1$ & 28,3 \\
\hline Coiled-coil domain-containing protein 124 & Q9D8X2 & Ccdc124 & $-0,5$ & 20,3 \\
\hline Coiled-coil domain-containing protein 23 & Q99LQ4 & Ccdc23 & $-0,2$ & 12,1 \\
\hline Coiled-coil domain-containing protein 43 ; & Q9CR29 & Ccdc43 & $-0,2$ & 11,3 \\
\hline Coiled-coil domain-containing protein 50 ; & Q810U5 & Ccdc50 & $-0,3$ & 19,1 \\
\hline Coiled-coil domain-containing protein 58 ; & Q8R3Q6 & Ccdc58 & 1,1 & 9,7 \\
\hline Coiled-coil domain-containing protein 6; & D3YZP9 & Ccdc6 & $-0,8$ & 7,5 \\
\hline Coiled-coil domain-containing protein 86 & Q9JJ89 & Ccdc86 & 0,4 & 1,9 \\
\hline Coiled-coil domain-containing protein 9; & Q8VC31 & Ccdc9 & 0,0 & 8,3 \\
\hline Coiled-coil domain-containing protein 91 & Q9D8L5 & Ccdc91 & 0,1 & 2 \\
\hline Coiled-coil-helix-coiled-coil-helix domain-containing protein 2, mitochondrial; & Q9D1L0 & Chchd2 & 0,1 & 30,1 \\
\hline Coiled-coil-helix-coiled-coil-helix domain-containing protein 3, mitochondrial; & Q9CRB9 & Chchd3 & 0,0 & 15,9 \\
\hline Coiled-coil-helix-coiled-coil-helix domain-containing protein 5; & Q9CQP3 & Chchd5 & 0,0 & 27,3 \\
\hline Cold-inducible RNA-binding protein; & P60824 & Cirbp & 1,5 & 14,5 \\
\hline Collagen alpha-1(I) chain; & P11087 & Col1a1 & 0,6 & 5,6 \\
\hline Collagen alpha-1(III) chain; & P08121 & Col3a1 & 0,8 & 1,3 \\
\hline Collagen alpha-1(VII) chain; & Q63870 & Col7a1 & 0,2 & 0,4 \\
\hline Collagen alpha-1(XVII) chain; & Q07563 & Col17a1 & 0,2 & 12 \\
\hline Collagen alpha-2(I) chain; & Q01149 & Col1a2 & 0,4 & 7,7 \\
\hline $\begin{array}{l}\text { Collagen type V alpha } 3 \text { chain; Collagen, type V, alpha 3; Procollagen, type V, } \\
\text { alpha 3, isoform CRA_b; Protein Col5a3; }\end{array}$ & Q9JLI2 & Col5a3 & $-0,2$ & 0,9 \\
\hline COMM domain-containing protein 3 ; & Q63829 & Commd3 & 1,1 & 11,3 \\
\hline COMM domain-containing protein 8 & Q9CZG3 & Commd8 & 1,1 & 6 \\
\hline $\begin{array}{l}\text { Complement component } 1 \mathrm{Q} \text { subcomponent-binding protein, mitochondrial; } \\
\text { Complement component } 1 \text {, q subcomponent binding protein; Complement } \\
\text { component 1, q subcomponent binding protein, isoform CRA_b; p32-RACK; }\end{array}$ & Q8R5L1 & C1qbp & 0,1 & 11,8 \\
\hline Complement factor D; & P03953 & $\mathrm{Cfd}$ & $-0,2$ & 11,6 \\
\hline Complexin-2; & P84086 & Cplx2 & $-0,1$ & 11,2 \\
\hline Constitutive coactivator of PPAR-gamma-like protein 1 ; & Q6A0A9 & FAM120A & $-0,2$ & 1,8 \\
\hline COP9 signalosome complex subunit 8 & Q8VBV7 & Cops8 & 0,6 & 20,1 \\
\hline Copper transport protein ATOX1; & 008997 & Atox1 & $-0,2$ & 83,8 \\
\hline Core histone macro-H2A.1; & Q9QZQ8 & H2afy & $-0,1$ & 5,4 \\
\hline Corneodesmosin; & Q7TPC1 & Cdsn & 0,1 & 30,1 \\
\hline Cornifin-A; & Q62266 & Sprr1a & $-1,0$ & 46,5 \\
\hline Cornifin-B; & Q62267 & Sprr1b & 1,1 & 20,3 \\
\hline Coronin-1C; & Q9WUM4 & Coro1c & 0,2 & 2,1 \\
\hline Costars family protein ABRACL; & Q4KML4 & Abracl & 0,9 & 27,2 \\
\hline COX assembly mitochondrial protein 2 homolog; & Q8K199 & Cmc2 & 0,2 & 25,3 \\
\hline Coxsackievirus and adenovirus receptor homolog; & P97792 & Cxadr & 0,1 & 27,1 \\
\hline Craniofacial development protein 1 ; & 088271 & Cfdp1 & $-0,5$ & 24,4 \\
\hline Creatine kinase B-type; & Q04447 & $\mathrm{Ckb}$ & $-0,5$ & 5,5 \\
\hline Creatine kinase M-type; & P07310 & $\mathrm{Ckm}$ & 1,0 & 10,8 \\
\hline Creatine kinase U-type, mitochondrial; & P30275 & Ckmt1 & 0,8 & 29,4 \\
\hline CREB-regulated transcription coactivator 1 ; & Q68ED7 & Crtc1 & 0,4 & 1,6 \\
\hline Crk-like protein; & P47941 & Crkl & 0,7 & 17,8 \\
\hline
\end{tabular}




\begin{tabular}{|c|c|c|c|c|}
\hline Protein & Uniprot & Gene name & $\begin{array}{c}\text { Fold change of } \\
\text { XPRESS ratio } \\
\left.\text { (Adam } 17^{\Delta K C} / \mathrm{WT}\right)\end{array}$ & $\begin{array}{c}\text { Sequence } \\
\text { coverage [\%] }\end{array}$ \\
\hline cTAGE family member 5 ; & Q8R311 & Ctage5 & 0,0 & 1,4 \\
\hline C-terminal-binding protein 1 ; & 088712 & Ctbp1 & $-0,2$ & 4,5 \\
\hline C-type lectin domain family 2 member $\mathrm{D}$; & Q91V08 & Clec2d & $-0,4$ & 5,3 \\
\hline Cyclic AMP-responsive element-binding protein 1; & Q01147 & Creb1 & 0,0 & 10,5 \\
\hline Cyclin-dependent kinase 4 inhibitor B; & P55271 & Cdkn2b & 0,4 & 9,2 \\
\hline Cyclin-dependent kinase inhibitor 1B; & P46414 & Cdkn1b & $-0,3$ & 35 \\
\hline Cyclin-K; & 088874 & Ccnk & 1,3 & 3,1 \\
\hline Cystatin E/M; Cystatin M/E; Cystatin-B; & Q9D1B1 & Cst6 & $-0,1$ & 46,3 \\
\hline Cystatin-A; & P56567 & Csta & 0,1 & 37,1 \\
\hline Cystatin-B; & Q62426 & Cstb & $-0,1$ & 39,8 \\
\hline Cysteine and glycine-rich protein 1 & P97315 & Csrp1 & $-0,4$ & 7,8 \\
\hline Cysteine-rich protein 1 & P63254 & Crip1 & $-0,1$ & 9,1 \\
\hline Cysteine-rich with EGF-like domain protein 2; & Q9CYA0 & Creld2 & $-0,1$ & 12 \\
\hline Cytochrome b5 type B; & Q9CQX2 & Cyb5b & 0,2 & 27,4 \\
\hline Cytochrome b5; & P56395 & Cyb5a & $-0,2$ & 65,3 \\
\hline Cytochrome b-c1 complex subunit 1, mitochondrial; & Q9CZ13 & Uqcrc1 & $-0,2$ & 7,9 \\
\hline Cytochrome b-c1 complex subunit 6, mitochondrial; & P99028 & Uqcrh & $-0,5$ & 44,9 \\
\hline Cytochrome b-c1 complex subunit 7; & Q9D855 & Uqcrb & 0,0 & 40,5 \\
\hline Cytochrome b-c1 complex subunit 8 & Q9CQ69 & Uqcrq & 0,0 & 34,1 \\
\hline Cytochrome b-c1 complex subunit 9; & Q8R1I1 & Uqcr10 & $-0,2$ & 37,5 \\
\hline Cytochrome b-c1 complex subunit Rieske, mitochondrial; & Q9CR68 & Uqcrfs1 & $-0,3$ & 26,3 \\
\hline Cytochrome c oxidase assembly factor 6 homolog; & Q8BGD8 & Coa6 & 0,1 & 16,5 \\
\hline Cytochrome c oxidase subunit 4 isoform 1, mitochondrial; & P19783 & Cox4i1 & 0,6 & 33,1 \\
\hline Cytochrome c oxidase subunit 5A, mitochondrial; & P12787 & Cox5a & 0,2 & 21,2 \\
\hline Cytochrome c oxidase subunit 5B, mitochondrial; & P19536 & Cox5b & 0,4 & 24,2 \\
\hline Cytochrome c oxidase subunit $6 \mathrm{~B} 1$ & P56391 & Cox6b1 & 1,2 & 41,9 \\
\hline Cytochrome c oxidase subunit 6C; & Q9CPQ1 & Cox6c & 0,2 & 34,2 \\
\hline Cytochrome c oxidase subunit 7A2, mitochondrial; & P48771 & Cox7a2 & 0,3 & 27,7 \\
\hline Cytochrome c oxidase subunit 7C, mitochondrial; & P17665 & Cox7c & 0,2 & 14,3 \\
\hline Cytochrome c, somatic; & P62897 & Cycs & 0,0 & 38,1 \\
\hline Cytochrome $\mathrm{c} 1$, heme protein, mitochondrial; & Q9D0M3 & Cyc1 & 1,0 & 10,8 \\
\hline Cytoplasmic dynein 1 intermediate chain 2; & 088487 & Dync1i2 & $-1,4$ & 3,4 \\
\hline Cytoplasmic dynein 1 light intermediate chain 1 ; & Q8R1Q8 & Dync1li1 & $-0,5$ & 3,1 \\
\hline Cytoplasmic dynein 1 light intermediate chain 2 & Q6PDLO & Dync1li2 & $-0,8$ & 5,9 \\
\hline Cytoskeleton-associated protein 4; & Q8BMK4 & Ckap4 & 0,0 & 31 \\
\hline Cytosolic non-specific dipeptidase; & Q9D1A2 & Cndp2 & $-0,3$ & 6,7 \\
\hline Cytospin-B; & Q5SXY1 & Specc1 & $-0,2$ & 3 \\
\hline D-3-phosphoglycerate dehydrogenase; & Q61753 & Phgdh & 1,7 & 21,4 \\
\hline DAZ-associated protein 1 & Q9JII5 & Dazap1 & 0,8 & 7,6 \\
\hline dCTP pyrophosphatase $1 ;$ & Q9QY93 & Dctpp1 & 0,0 & 25,3 \\
\hline D-dopachrome decarboxylase; & 035215 & Ddt & 0,8 & 51,7 \\
\hline DDRGK domain-containing protein 1 ; & Q80WW9 & Ddrgk1 & 0,0 & 10,2 \\
\hline Death domain-containing membrane protein NRADD; & Q8CJ26 & Nradd & 0,5 & 11,8 \\
\hline Death-associated protein-like 1 ; & Q9D757 & Dapl1 & $-0,4$ & 19,6 \\
\hline Decorin; & P28654 & Den & 1,0 & 13,6 \\
\hline Delta(3,5)-Delta(2,4)-dienoyl-CoA isomerase, mitochondrial; & 035459 & Ech1 & 0,4 & 16,8 \\
\hline Density-regulated protein; & Q9CQJ6 & Denr & 0,3 & 18,6 \\
\hline Deoxyhypusine hydroxylase; & Q99LN9 & Dohh & 0,3 & 24,5 \\
\hline Deoxyhypusine synthase; & Q3TXU5 & Dhps & $-0,1$ & 8,1 \\
\hline Deoxyribonuclease-2-alpha; & P56542 & Dnase2 & 0,1 & 15,7 \\
\hline Deoxyuridine triphosphatase, isoform CRA_b; Dutp protein; Protein Dut; & Q8VCG1 & Dut & $-0,8$ & 16,7 \\
\hline Dermokine; & E9Q2P1 & Dmkn & $-0,3$ & 36,3 \\
\hline Desmocollin 3, isoform CRA_b; Desmocollin-3; & G5E8S6 & Dsc3 & $-0,1$ & 24,1 \\
\hline Desmocollin-1; & P55849 & Dsc1 & $-0,2$ & 31,4 \\
\hline Desmoglein-1-alpha; & Q61495 & Dsg1a & $-0,1$ & 26,1 \\
\hline Desmoglein-1-beta; & Q7TSF1 & Dsg1b & $-0,4$ & 23,2 \\
\hline Desmoglein-2; & 055111 & Dsg2 & $-0,8$ & 4,1 \\
\hline Desmoplakin; & E9Q557 & Dsp & $-0,1$ & 13,3 \\
\hline Destrin; & Q9R0P5 & Dstn & 0,2 & 12,7 \\
\hline DET1- and DDB1-associated protein 1; & Q9D9Z5 & Dda1 & 0,3 & 11,8 \\
\hline Dickkopf-like protein 1 ; & Q9QZL9 & Dkkl1 & $-0,4$ & 6,5 \\
\hline Dihydrolipoyl dehydrogenase, mitochondrial; & 008749 & Dld & $-0,4$ & 8,6 \\
\hline $\begin{array}{l}\text { Dihydrolipoyllysine-residue succinyltransferase component of 2-oxoglutarate } \\
\text { dehydrogenase complex, mitochondrial; }\end{array}$ & Q9D2G2 & Dlst & $-0,1$ & 5,7 \\
\hline Dihydropteridine reductase; & Q8BVI4 & Qdpr & 1,4 & 14,3 \\
\hline Dihydropyrimidinase-related protein 2 & 008553 & Dpysl2 & 0,3 & 3,5 \\
\hline Dimethylaniline monooxygenase [N-oxide-forming] 2; & Q8K2I3 & Fmo2 & $-0,1$ & 3,7 \\
\hline Dipeptidyl peptidase 1 & P97821 & Ctsc & 0,2 & 7,9 \\
\hline Disabled homolog 2; & P98078 & Dab2 & $-0,3$ & 7,2 \\
\hline Disco-interacting protein 2 homolog B; & Q3UH60 & Dip2b & $-0,5$ & 2,2 \\
\hline Disks large-associated protein 4; & B1AZP2 & Dlgap4 & $-0,1$ & 6,1 \\
\hline DNA fragmentation factor subunit alpha; & 054786 & Dffa & 0,0 & 22,7 \\
\hline
\end{tabular}




\begin{tabular}{|c|c|c|c|c|}
\hline Protein & Uniprot & Gene name & $\begin{array}{c}\text { Fold change of } \\
\text { XPRESS ratio } \\
\left.\text { (Adam17 }{ }^{\Delta K C} / \mathrm{WT}\right)\end{array}$ & $\begin{array}{c}\text { Sequence } \\
\text { coverage [\%] }\end{array}$ \\
\hline DNA polymerase epsilon subunit 3; & Q9JKP7 & Pole3 & $-0,2$ & 21,6 \\
\hline DNA polymerase epsilon subunit 4; & Q9CQ36 & Pole4 & $-0,3$ & 21,2 \\
\hline DNA repair protein SWI5 homolog; & Q8K3D3 & Swi5 & 0,9 & 22,5 \\
\hline DNA-(apurinic or apyrimidinic site) lyase; & P28352 & Apex1 & 0,4 & 9,6 \\
\hline DNA-directed RNA polymerase II subunit RPB4; & Q9D7M8 & Polr2d & 0,4 & 16,9 \\
\hline DNA-directed RNA polymerase II subunit RPB9; & P60898 & Polr2i & 0,4 & 21,7 \\
\hline DNA-directed RNA polymerase III subunit RPC9; & 035427 & Crcp & 0,7 & 11,5 \\
\hline DnaJ homolog subfamily B member 1 ; & Q9QYJ3 & Dnajb1 & $-0,3$ & 3,8 \\
\hline DnaJ homolog subfamily B member 2 ; & Q9QYI5 & Dnajb2 & $-0,6$ & 5,4 \\
\hline DnaJ homolog subfamily $\mathrm{C}$ member 5 ; & P60904 & Dnajc5 & 1,3 & 22 \\
\hline DnaJ homolog subfamily $\mathrm{C}$ member 8 & Q6NZBO & Dnajc8 & $-0,3$ & 15,1 \\
\hline DnaJ homolog subfamily $\mathrm{C}$ member 9 ; & Q91WN1 & Dnajc9 & $-0,3$ & 15,4 \\
\hline Dolichyl-diphosphooligosaccharide--protein glycosyltransferase subunit 1; & Q91YQ5 & Rpn1 & $-0,3$ & 1,3 \\
\hline Dr1-associated corepressor; & Q9D6N5 & Drap1 & 0,1 & 11,5 \\
\hline Drebrin-like protein; & Q62418 & Dbnl & $-0,1$ & 34,9 \\
\hline Dynactin subunit 2 ; & Q99KJ8 & Dctn2 & 0,0 & 38,3 \\
\hline Dynein light chain 1, cytoplasmic; & P63168 & Dynll1 & 0,5 & 49,4 \\
\hline Dynein light chain 2, cytoplasmic; & Q9D0M5 & Dynll2 & 0,4 & 32,6 \\
\hline Dynein light chain roadblock-type 1 ; & P62627 & Dynlrb1 & 0,1 & 51 \\
\hline Dystroglycan; & Q62165 & Dag1 & $-0,3$ & 8 \\
\hline E3 ubiquitin-protein ligase RNF126; & Q91YL2 & Rnf126 & 0,2 & 3,2 \\
\hline Early endosome antigen 1 & Q8BL66 & Eea1 & $-0,6$ & 26,4 \\
\hline EF-hand calcium-binding domain-containing protein 14 & Q8BGQ6 & Efcab14 & $-0,3$ & 6,8 \\
\hline EF-hand domain-containing protein D2; Efhd2 protein; & Q8C845 & Efhd2 & 0,1 & 28,8 \\
\hline Electron transfer flavoprotein subunit alpha, mitochondrial; & Q99LC5 & Etfa & 0,8 & 12,9 \\
\hline Electron transfer flavoprotein subunit beta; & Q9DCW4 & Etfb & $-0,1$ & 28,2 \\
\hline ELL-associated factor 1 & Q9D4C5 & Eaf1 & $-1,9$ & 6,3 \\
\hline Elongation factor 1 -alpha 1 & P10126 & Eef1a1 & 0,1 & 11,5 \\
\hline Elongation factor 1-beta; & 070251 & Eef1b & $-0,9$ & 32,4 \\
\hline Elongation factor 1-delta; & F6ZFU0 & Eef1d & 1,3 & 19 \\
\hline Elongation factor 1-gamma; & Q9D8N0 & Eef1g & $-1,1$ & 5 \\
\hline Elongation factor 2; & P58252 & Eef2 & $-0,3$ & 14,8 \\
\hline Embigin; & P21995 & Emb & $-0,6$ & 7 \\
\hline Emerin; & 008579 & Emd & 0,2 & 25,1 \\
\hline Endophilin-A2; & Q62419 & Sh3gl1 & 0,8 & 5,4 \\
\hline Endophilin-B2; & Q8R3V5 & Sh3glb2 & $-0,2$ & 9,6 \\
\hline Endoplasmic reticulum resident protein 29 ; & P57759 & Erp29 & 0,5 & 27,1 \\
\hline Endoplasmic reticulum resident protein 44 ; & Q9D1Q6 & Erp44 & 0,5 & 21,4 \\
\hline Endoplasmin; & P08113 & Hsp90b1 & $-0,1$ & 17,3 \\
\hline Endothelial differentiation-related factor 1 & Q9JMG1 & Edf1 & $-0,2$ & 32,4 \\
\hline Endothelin-converting enzyme 1 & Q4PZA2 & Ece1 & $-0,1$ & 3,3 \\
\hline Enhancer of rudimentary homolog; & P84089 & Erh & 0,9 & 26,9 \\
\hline Enoyl-CoA delta isomerase 2, mitochondrial; & Q9WUR2 & Eci2 & 2,1 & 5,9 \\
\hline Enoyl-CoA hydratase, mitochondrial; & Q8BH95 & Echs1 & 0,5 & 11,7 \\
\hline Ensconsin; & 088735 & Map7 & $-0,4$ & 3,3 \\
\hline Envoplakin; & Q9D952 & Evpl & 0,2 & 2,3 \\
\hline Eosinophil cationic-type ribonuclease 5 ; & 035292 & Ear5 & 0,4 & 5,2 \\
\hline Epidermal growth factor receptor kinase substrate 8-like protein 1; & Q8R5F8 & Eps811 & $-1,0$ & 5,7 \\
\hline Epidermal growth factor receptor kinase substrate 8-like protein 2; & Q99K30 & Eps812 & 0,1 & 4,5 \\
\hline Epidermal growth factor receptor substrate 15 & P42567 & Eps15 & $-0,7$ & 1,3 \\
\hline Epidermal growth factor receptor substrate 15 -like 1 & Q60902 & Eps15I1 & 0,2 & 5,4 \\
\hline Epididymal secretory protein E1; & Q9Z0J0 & Npc2 & 0,5 & 24,8 \\
\hline Epiplakin; & Q8RoW0 & Eppk1 & $-0,1$ & 14,1 \\
\hline Epsin-1; & Q80VP1 & Epn1 & 0,2 & 6,1 \\
\hline Epsin-2; & Q8CHU3 & Epn2 & 0,0 & 12,5 \\
\hline Epsin-3; & Q91W69 & Epn3 & $-0,6$ & 6,3 \\
\hline Eukaryotic peptide chain release factor GTP-binding subunit ERF3A; & Q8R050 & Gspt1 & $-0,6$ & 4,7 \\
\hline Eukaryotic translation initiation factor 1 & P48024 & Eif1 & 0,5 & 47,8 \\
\hline Eukaryotic translation initiation factor $1 \mathrm{~A}$; & Q60872 & Eif1a & 0,0 & 14,6 \\
\hline Eukaryotic translation initiation factor 2 subunit 2 & Q99L45 & Eif2s2 & $-0,6$ & 16,3 \\
\hline Eukaryotic translation initiation factor $2 \mathrm{~A}$ & Q8BJW6 & Eif2a & $-0,9$ & 2,4 \\
\hline Eukaryotic translation initiation factor 3 subunit A; & P23116 & Eif3a & $-0,8$ & 0,7 \\
\hline Eukaryotic translation initiation factor 3 subunit B; & Q8JZQ9 & Eif3b & $-1,3$ & 3,2 \\
\hline Eukaryotic translation initiation factor 3 subunit C; & Q8R1B4 & Eif3c & $-0,7$ & 2,1 \\
\hline Eukaryotic translation initiation factor 3 subunit G; & Q9Z1D1 & Eif3g & 0,0 & 14,1 \\
\hline Eukaryotic translation initiation factor 3 subunit J-A; & Q3UGC7 & Eif3j1 & $-0,3$ & 19,5 \\
\hline Eukaryotic translation initiation factor 3 subunit $\mathrm{K}$; & Q9DBZ5 & Eif3k & $-0,2$ & 5 \\
\hline Eukaryotic translation initiation factor 4 gamma 1 ; & Q6NZJ6 & Eif4g1 & $-0,9$ & 3,7 \\
\hline Eukaryotic translation initiation factor 4B; & Q8BGD9 & Eif4b & $-0,3$ & 17,8 \\
\hline Eukaryotic translation initiation factor $4 \mathrm{E}$ transporter; & Q9EST3 & Eif4enif1 & $-0,2$ & 3,1 \\
\hline Eukaryotic translation initiation factor $4 \mathrm{E}$ & P63073 & Eif4e & 1,0 & 18 \\
\hline Eukaryotic translation initiation factor $4 \mathrm{E}$-binding protein 2 ; & P70445 & Eif4ebp2 & 0,3 & 22,5 \\
\hline
\end{tabular}




\begin{tabular}{|c|c|c|c|c|}
\hline Protein & Uniprot & Gene name & $\begin{array}{c}\text { Fold change of } \\
\text { XPRESS ratio } \\
\text { (Adam17 } \\
\text { (AKC / WT) }\end{array}$ & $\begin{array}{c}\text { Sequence } \\
\text { coverage [\%] }\end{array}$ \\
\hline Eukaryotic translation initiation factor $4 \mathrm{H}$; & Q9WUK2 & Eif4h & 0,3 & 23,8 \\
\hline Eukaryotic translation initiation factor 5; & P59325 & Eif5 & $-0,4$ & 6,3 \\
\hline Eukaryotic translation initiation factor $5 \mathrm{~A}-1$; & P63242 & Eif5a & 0,2 & 19 \\
\hline Eukaryotic translation initiation factor $5 \mathrm{~B}$; & Q05D44 & Eif5b & $-1,4$ & 3,7 \\
\hline Eukaryotic translation initiation factor 6 & 055135 & Eif6 & 0,2 & 31 \\
\hline Extracellular matrix protein 1 & Q61508 & Ecm1 & $-0,5$ & 38,5 \\
\hline Ezrin; & P26040 & Ezr & $-0,6$ & 13,3 \\
\hline FACT complex subunit SSRP1; & Q08943 & Ssrp1 & $-2,7$ & 3,4 \\
\hline F-actin-capping protein subunit beta; & P47757 & Capzb & 0,0 & 7,3 \\
\hline Far upstream element-binding protein 1 & Q3TUE1 & Fubp1 & 0,1 & 32,9 \\
\hline Far upstream element-binding protein 2 & Q3U0V1 & Khsrp & 0,2 & 41 \\
\hline Farnesyl pyrophosphate synthase; & Q920E5 & Fdps & $-1,6$ & 3,4 \\
\hline FAS-associated death domain protein; & Q61160 & Fadd & 0,6 & 8,3 \\
\hline Fatty acid synthase; & P19096 & Fasn & $-0,1$ & 0,4 \\
\hline Fatty acid-binding protein, adipocyte; & P04117 & Fabp4 & $-1,8$ & 25,8 \\
\hline Fatty acid-binding protein, epidermal; & Q05816 & Fabp5 & 0,7 & 71,9 \\
\hline F-box only protein 50 & G3X9C2 & Nccrp1 & $-0,7$ & 22,2 \\
\hline Ferritin heavy chain; & P09528 & Fth1 & $-0,1$ & 11,5 \\
\hline Ferritin; & Q9CPX4 & Ftl1 & $-0,2$ & 30,6 \\
\hline Fetuin-B; & Q9QXC1 & Fetub & 2,5 & 38,6 \\
\hline Fibrinogen beta chain; & Q8K0E8 & $\mathrm{Fgb}$ & 2,8 & 5,2 \\
\hline Fibrinogen gamma chain; & Q8VCM7 & Fgg & 2,0 & 6,7 \\
\hline Fibroblast growth factor-binding protein 1 ; & 070514 & Fgfbp1 & 0,0 & 13,1 \\
\hline Fibronectin; & P11276 & Fn1 & $-1,9$ & 2 \\
\hline Filaggrin; & P11088 & $\mathrm{Flg}$ & 1,9 & 22,9 \\
\hline Filaggrin-2; & Q2VIS4 & Flg2 & $-1,0$ & 41,5 \\
\hline Filaggrin-2; & E9QPZ3 & Flg2 & $-0,8$ & 41,7 \\
\hline Filamin A-interacting protein 1-like; & Q6P6L0 & Filip1l & $-0,5$ & 0,9 \\
\hline Filamin-A; & Q8BTM8 & Flna & $-0,8$ & 6,5 \\
\hline Filamin-B; & Q80X90 & Flnb & $-0,5$ & 6,1 \\
\hline FK506-binding protein 15 & Q6P9Q6 & Fkbp15 & $-0,6$ & 3,2 \\
\hline Focal adhesion kinase 1 ; & P34152 & Ptk2 & $-1,2$ & 0,9 \\
\hline Follistatin-related protein 1 & Q62356 & Fstl1 & $-0,2$ & 10,8 \\
\hline Forkhead box protein $\mathrm{O3}$; & Q9WVH4 & Foxo3 & 0,2 & 30,1 \\
\hline Formin-binding protein 4 & Q6ZQ03 & Fnbp4 & $-0,4$ & 6 \\
\hline Fos-related antigen 2 & P47930 & Fosl2 & $-0,4$ & 7,1 \\
\hline Four and a half LIM domains protein 3 ; & Q9R059 & Fhl3 & $-0,3$ & 5,8 \\
\hline Frizzled-1; & 070421 & Fzd1 & 0,7 & 3,4 \\
\hline Fructose-bisphosphate aldolase A; & P05064 & Aldoa & 1,3 & 39,8 \\
\hline Fucose mutarotase; & Q8R2K1 & Fuom & 0,1 & 14,9 \\
\hline Fumarate hydratase, mitochondrial; & P97807 & $\mathrm{Fh}$ & 1,6 & 4,3 \\
\hline G patch domain-containing protein 8 & A2A6A1 & Gpatch8 & $-0,2$ & 1,5 \\
\hline Galectin; & Q9CRB1 & Lgals7 & 0,3 & 64,7 \\
\hline Galectin-1; & P16045 & Lgals1 & 0,3 & 37,8 \\
\hline Galectin-3; & P16110 & Lgals3 & 0,8 & 24,2 \\
\hline Galectin-related protein; & Q8VED9 & Lgalsl & 0,7 & 11,6 \\
\hline Gamma-glutamyl hydrolase; & Q9ZOL8 & Ggh & 0,0 & 27,1 \\
\hline Gamma-glutamylcyclotransferase; & Q9D7X8 & Ggct & $-0,1$ & 33 \\
\hline Gamma-interferon-inducible lysosomal thiol reductase; & Q9ESY9 & Ifi30 & 0,3 & 4,4 \\
\hline Gamma-soluble NSF attachment protein; & Q9CWZ7 & Napg & $-0,4$ & 4,5 \\
\hline Gamma-synuclein; & Q9Z0F7 & Sncg & $-0,1$ & 47,2 \\
\hline Ganglioside GM2 activator; & Q60648 & $\mathrm{Gm} 2 \mathrm{a}$ & 0,0 & 18,1 \\
\hline Gap junction alpha-1 protein; & P23242 & Gja1 & $-0,5$ & 9,4 \\
\hline Gasdermin-A; & Q9EST1 & Gsdma & $-0,2$ & 12,6 \\
\hline GDP-L-fucose synthase; & P23591 & Tsta3 & 1,8 & 4,4 \\
\hline Gelsolin; & P13020 & Gsn & 0,4 & 13,8 \\
\hline General transcription factor IIF subunit 1 ; & Q3THK3 & Gtf2f1 & 0,1 & 14,2 \\
\hline General transcription factor IIF subunit 2; & Q8R0A0 & Gtf2f2 & 1,5 & 32,9 \\
\hline General vesicular transport factor $\mathrm{p} 115$ & Q9Z1Z0 & Uso1 & $-0,2$ & 2,9 \\
\hline Gephyrin; & Q8BUV3 & Gphn & 0,0 & 1,3 \\
\hline Glia maturation factor beta; & Q9CQI3 & Gmfb & 0,2 & 19 \\
\hline Glucocorticoid receptor; & P06537 & $\mathrm{Nr} 3 \mathrm{c} 1$ & $-0,2$ & 2,3 \\
\hline Glucose-6-phosphate isomerase; & P06745 & Gpi & 0,0 & 7,7 \\
\hline Glucose-induced degradation protein 8 homolog; & Q9D7M1 & Gid8 & 0,3 & 5,3 \\
\hline Glucosidase 2 subunit beta; & 008795 & Prkcsh & 0,3 & 25,5 \\
\hline Glutamyl-tRNA(GIn) amidotransferase subunit C, mitochondrial; & Q8CBY0 & Gatc & $-1,5$ & 12,9 \\
\hline Glutaredoxin-1; & Q9QUHO & Glrx & 0,7 & 10,3 \\
\hline Glutaredoxin-related protein 5, mitochondrial; & Q80Y14 & Glrx5 & 0,1 & 21,7 \\
\hline Glutathione peroxidase 1 & P11352 & Gpx1 & 0,1 & 19,4 \\
\hline Glutathione reductase, mitochondrial; & P47791 & Gsr & $-0,6$ & 7 \\
\hline Glutathione S-transferase A2; & P10648 & Gsta2 & 0,4 & 9,5 \\
\hline Glutathione S-transferase kappa 1; & Q9DCM2 & Gstk1 & 0,9 & 7,1 \\
\hline Glutathione S-transferase Mu 1; & P10649 & Gstm1 & 1,3 & 26,1 \\
\hline
\end{tabular}




\begin{tabular}{|c|c|c|c|c|}
\hline Protein & Uniprot & Gene name & $\begin{array}{c}\text { Fold change of } \\
\text { XPRESS ratio } \\
\left.\text { (Adam } 17^{\Delta K C} / \mathrm{WT}\right)\end{array}$ & $\begin{array}{c}\text { Sequence } \\
\text { coverage [\%] }\end{array}$ \\
\hline Glutathione S-transferase Mu 2; & P15626 & Gstm2 & 1,2 & 28,9 \\
\hline Glutathione S-transferase omega-1; & 009131 & Gsto1 & 1,4 & 33,3 \\
\hline Glutathione S-transferase P 2; & P46425 & Gstp2 & 1,3 & 17,1 \\
\hline Glyceraldehyde-3-phosphate dehydrogenase; & P16858 & Gapdh & 0,3 & 39,3 \\
\hline Glycerophosphoinositol inositolphosphodiesterase GDPD2; & Q9ESM6 & Gdpd2 & 0,1 & 1,9 \\
\hline Glycine cleavage system $\mathrm{H}$ protein, mitochondrial; & Q91WK5 & Gcsh & $-0,5$ & 5,9 \\
\hline Glycolipid transfer protein; & Q9JL62 & Gltp & 0,6 & 31,1 \\
\hline Glyoxalase domain-containing protein 4; & Q9CPV4 & Glod4 & 0,5 & 9,3 \\
\hline Glyoxylate reductase/hydroxypyruvate reductase; & Q91Z53 & Grhpr & $-0,1$ & 18,8 \\
\hline Glypican-1; & Q9QZF2 & Gpc1 & 0,8 & 5,7 \\
\hline Golgi apparatus protein $1 ;$ & Q61543 & Glg1 & 0,6 & 1,8 \\
\hline Golgi integral membrane protein 4 ; & Q8BXA1 & Golim4 & 0,2 & 3,8 \\
\hline Golgi reassembly-stacking protein 2; & Q99JX3 & Gorasp2 & $-0,6$ & 3,7 \\
\hline Golgi SNAP receptor complex member 1 ; & 088630 & Gosr1 & $-0,4$ & 4,8 \\
\hline Golgin subfamily A member 2 ; & Q921M4 & Golga2 & 0,1 & 8,9 \\
\hline Golgin subfamily A member 5 ; & Q9QYE6 & Golga5 & $-0,4$ & 1,4 \\
\hline GPALPP motifs-containing protein 1 ; & Q69ZC8 & Gpalpp1 & 0,3 & 5,5 \\
\hline G-protein coupled receptor 56 & Q8K209 & Gpr56 & $-0,4$ & 2,5 \\
\hline Granulins; & P28798 & Grn & 0,0 & 10 \\
\hline GRB2-associated-binding protein $1 ;$ & Q9QYY0 & Gab1 & $-0,1$ & 11,5 \\
\hline Growth arrest and DNA damage-inducible proteins-interacting protein 1; & Q9CR59 & Gadd45gip1 & $-0,3$ & 4,1 \\
\hline Growth factor receptor-bound protein 2; & Q60631 & Grb2 & 0,8 & 19,7 \\
\hline GrpE protein homolog 1, mitochondrial; & Q99LP6 & Grpel1 & 0,4 & 30,9 \\
\hline GSK3-beta interaction protein; & Q8BGR8 & Gskip & $-0,1$ & 7,2 \\
\hline GTP:AMP phosphotransferase AK3, mitochondrial; & Q9WTP7 & Ak3 & 1,3 & 31,7 \\
\hline Guanine nucleotide exchange factor MSS4; & Q91X96 & Rabif & 0,1 & 16,3 \\
\hline Guanine nucleotide-binding protein $\mathrm{G}(\mathrm{I}) / \mathrm{G}(\mathrm{S}) / \mathrm{G}(\mathrm{O})$ subunit gamma-2; & P63213 & Gng2 & 0,1 & 22,5 \\
\hline H/ACA ribonucleoprotein complex subunit 1 ; & Q9CY66 & Gar1 & $-0,2$ & 4,5 \\
\hline H/ACA ribonucleoprotein complex subunit 2; & Q9CRB2 & Nhp2 & 0,5 & 10,5 \\
\hline H/ACA ribonucleoprotein complex subunit 4; & Q9ESX5 & Dkc1 & 2,1 & 5,1 \\
\hline H-2 class I histocompatibility antigen, L-D alpha chain; & P01897 & $\mathrm{H} 2 \mathrm{~L}$ & 0,7 & 6,1 \\
\hline H-2 class II histocompatibility antigen gamma chain; & P04441 & $\mathrm{Cd} 74$ & $-0,2$ & 24,7 \\
\hline $\mathrm{H}-2$ class II histocompatibility antigen, $\mathrm{A}-\mathrm{Q}$ alpha chain; & P04227 & $\mathrm{H} 2 \mathrm{Aa}$ & 0,4 & 5,4 \\
\hline H-2 class II histocompatibility antigen, A-Q beta chain; & P06342 & $\mathrm{H} 2 \mathrm{Ab} 1$ & $-0,6$ & 6 \\
\hline Haloacid dehalogenase-like hydrolase domain-containing protein 2; & Q3UGR5 & Hdhd2 & 0,0 & 3,1 \\
\hline HBS1-like protein; & Q69ZS7 & Hbs1l & $-0,4$ & 2,9 \\
\hline HD domain-containing protein 2 ; & Q3SXD3 & Hddc2 & 0,1 & 14,1 \\
\hline Heat shock 70 kDa protein $1 \mathrm{~A}$; & Q61696 & Hspa1a & 0,2 & 34 \\
\hline Heat shock 70 kDa protein 4; & Q61316 & Hspa4 & $-0,6$ & 10,1 \\
\hline Heat shock cognate 71 kDa protein; & P63017 & Hspa8 & 0,2 & 37,3 \\
\hline Heat shock factor-binding protein 1 & Q9CQZ1 & Hsbp1 & 0,1 & 14,5 \\
\hline Heat shock protein 105 kDa; & Q61699 & Hsph1 & 0,4 & 4 \\
\hline Heat shock protein beta-1; & P14602 & Hspb1 & 0,4 & 42,1 \\
\hline Heat shock protein beta-8; & Q9JK92 & Hspb8 & 0,1 & 9,7 \\
\hline Heat shock protein HSP 90-beta; & P11499 & Hsp90ab1 & $-0,7$ & 16,3 \\
\hline Heat shock-related 70 kDa protein 2 ; & P17156 & Hspa2 & 0,1 & 14,5 \\
\hline Hematological and neurological expressed 1 protein; & P97825 & Hn1 & $-0,2$ & 37 \\
\hline Hematological and neurological expressed 1-like protein; & Q6PGH2 & Hn1l & $-0,2$ & 53,7 \\
\hline Hematopoietic lineage cell-specific protein; & P49710 & Hcls1 & $-0,1$ & 9,1 \\
\hline Heme-binding protein $1 ;$ & Q9R257 & Hebp1 & 0,3 & 6,3 \\
\hline Heme-binding protein 2; & Q9WU63 & Hebp2 & 0,6 & 26,8 \\
\hline Hemoglobin subunit beta-1; & P02088 & $\mathrm{Hbb}$ b1 & 4,9 & 76,9 \\
\hline Hemoglobin subunit beta- 2 & P02089 & $\mathrm{Hbb}$ b2 & 4,2 & 83,7 \\
\hline Hemopexin; & Q91X72 & Hpx & 1,1 & 10 \\
\hline Heparanase; & Q6YGZ1 & Hpse & $-0,3$ & 2,1 \\
\hline Hepatoma-derived growth factor; & P51859 & Hdgf & $-0,2$ & 51,5 \\
\hline Hepatoma-derived growth factor-related protein 2; & Q3UMU9 & Hdgfrp2 & 0,0 & 3,9 \\
\hline Heterochromatin protein 1-binding protein 3; & Q3TEA8 & Hp1bp3 & $-0,3$ & 17,4 \\
\hline Heterogeneous nuclear ribonucleoprotein A/B; & Q99020 & Hnrnpab & 0,1 & 22,8 \\
\hline Heterogeneous nuclear ribonucleoprotein A1; & P49312 & Hnrnpa1 & 0,6 & 17,8 \\
\hline Heterogeneous nuclear ribonucleoprotein A3; & Q8BG05 & Hnrnpa3 & 0,2 & 26,7 \\
\hline Heterogeneous nuclear ribonucleoprotein D0; & Q60668 & Hnrnpd & 0,4 & 22,7 \\
\hline Heterogeneous nuclear ribonucleoprotein D-like; & Q9Z130 & Hnrnpdl & 0,4 & 14 \\
\hline Heterogeneous nuclear ribonucleoprotein F; & Q9Z2X1 & Hnrnpf & 0,2 & 3,8 \\
\hline Heterogeneous nuclear ribonucleoprotein $\mathrm{H}$; & 035737 & Hnrnph1 & $-2,4$ & 9,9 \\
\hline Heterogeneous nuclear ribonucleoprotein K; & B2M1R6 & Hnrnpk & 0,1 & 35,7 \\
\hline Heterogeneous nuclear ribonucleoprotein $\mathrm{U}$; & Q8VEK3 & Hnrnpu & $-0,8$ & 7,4 \\
\hline Heterogeneous nuclear ribonucleoprotein U-like protein 2; & Q00PI9 & Hnrnpul2 & $-0,6$ & 1,9 \\
\hline Heterogeneous nuclear ribonucleoproteins A2/B1; & 088569 & Hnrnpa2b1 & 1,0 & 21 \\
\hline Heterogeneous nuclear ribonucleoproteins $\mathrm{C} 1 / \mathrm{C} 2$; & Q9Z204 & Hnrnpc & $-0,3$ & 7,3 \\
\hline High mobility group nucleosome-binding domain-containing protein 3 ; & Q9DCB1 & Hmgn3 & $-0,1$ & 32,3 \\
\hline High mobility group nucleosome-binding domain-containing protein 5 ; & Q9JL35 & Hmgn5 & $-0,2$ & 5,7 \\
\hline
\end{tabular}




\begin{tabular}{|c|c|c|c|c|}
\hline Protein & Uniprot & Gene name & $\begin{array}{c}\text { Fold change of } \\
\text { XPRESS ratio } \\
\text { (Adam17 } \\
\text { (AKC / WT) }\end{array}$ & $\begin{array}{c}\text { Sequence } \\
\text { coverage [\%] }\end{array}$ \\
\hline High mobility group protein $20 \mathrm{~A}$; & Q9DC33 & Hmg20a & 0,2 & 3,5 \\
\hline High mobility group protein B1; & P63158 & Hmgb1 & 0,5 & 23,7 \\
\hline High mobility group protein B2; & P30681 & Hmgb2 & $-0,2$ & 31 \\
\hline High mobility group protein B3; & 054879 & Hmgb3 & $-0,8$ & 24,5 \\
\hline Hippocalcin-like protein 1 & P62748 & Hpcal1 & 0,4 & 8,1 \\
\hline HIRA-interacting protein 3 ; & Q8BLH7 & Hirip3 & $-0,6$ & 3,7 \\
\hline Histidine ammonia-lyase; & P35492 & Hal & $-0,4$ & 22,4 \\
\hline Histidine triad nucleotide-binding protein 1 ; & P70349 & Hint1 & 0,3 & 34,5 \\
\hline Histidine triad nucleotide-binding protein 2, mitochondrial; & Q9D0S9 & Hint2 & 1,4 & 29,4 \\
\hline Histidine-rich glycoprotein; & Q9ESB3 & $\mathrm{Hrg}$ & 0,3 & 1,7 \\
\hline Histone H1.1; & P43275 & Hist1h1a & $-1,9$ & 9,4 \\
\hline Histone H1.2; & P15864 & Hist1h1c & $-0,4$ & 15,1 \\
\hline Histone H1.4; & P43274 & Hist1h1e & $-0,4$ & 14,6 \\
\hline Histone $\mathrm{H} 1.5$; & P43276 & Hist1h1b & $-0,6$ & 14,3 \\
\hline Histone H2A type 1 ; & P22752 & Hist1h2ao & 0,4 & 27,1 \\
\hline Histone H2A type 2-B; & Q64522 & Hist2h2ab & 1,3 & 12,3 \\
\hline Histone $\mathrm{H} 2 \mathrm{~B}$ type $1-\mathrm{F} / \mathrm{J} / \mathrm{L}$; & P10853 & Hist1h2bn & 0,5 & 40,5 \\
\hline Histone H2B type 2-E; & Q64524 & Hist2h2be & $-0,6$ & 34,9 \\
\hline Histone H3.3C; & P02301 & $H 3 f 3 c$ & $-1,0$ & 11,8 \\
\hline Histone $\mathrm{H} 4$; & P62806 & Hist4h4 & $-0,3$ & 50,5 \\
\hline Histone-binding protein RBBP4; & Q60972 & Rbbp4 & 0,4 & 17,6 \\
\hline Histone-binding protein RBBP7; & Q60973 & Rbbp7 & 0,7 & 15,1 \\
\hline Homeodomain-only protein; & Q8R1H0 & Hopx & 0,0 & 43,8 \\
\hline Hornerin; & E9QNP3 & Hrnr & $-0,2$ & 24,7 \\
\hline Host cell factor 1 & Q61191 & Hcfc1 & $-0,3$ & 3,2 \\
\hline HRAS-like suppressor 3; & Q8R3U1 & Pla2g16 & 0,3 & 8,6 \\
\hline Hsc70-interacting protein; & Q99L47 & St13 & 0,1 & 21,3 \\
\hline Hsp90 co-chaperone Cdc37; & Q61081 & Cdc37 & 0,1 & 14,2 \\
\hline Huntingtin-interacting protein $\mathrm{K}$; & Q9CR41 & Hypk & $-0,3$ & 24,8 \\
\hline Hypoxanthine-guanine phosphoribosyltransferase; & P00493 & Hprt1 & 0,5 & 31,7 \\
\hline Ig gamma-2B chain $\mathrm{C}$ region; & P01867 & $\operatorname{lgh} 3$ & 1,1 & 2,2 \\
\hline Immunoglobulin-binding protein 1 & Q61249 & Igbp1 & $-4,6$ & 3,5 \\
\hline Importin-7; & Q9EPL8 & Ipo7 & $-1,5$ & 2,5 \\
\hline Inactive tyrosine-protein kinase 7; & Q8BKG3 & Ptk7 & $-0,4$ & 1,6 \\
\hline Inorganic pyrophosphatase 2, mitochondrial; & Q91VM9 & Ppa2 & 1,4 & 8,6 \\
\hline Inorganic pyrophosphatase; & Q9D819 & Ppa1 & 0,7 & 6,2 \\
\hline Inosine triphosphate pyrophosphatase; & Q9D892 & Itpa & 1,2 & 8,1 \\
\hline Inosine-5'-monophosphate dehydrogenase 2 & P24547 & Impdh2 & 0,5 & 2,3 \\
\hline Inositol-trisphosphate 3-kinase C; & Q7TS72 & Itpkc & $-0,1$ & 2,1 \\
\hline Insulin receptor substrate 1 & P35569 & Irs1 & 0,7 & 0,9 \\
\hline Insulin-degrading enzyme; & Q9JHR7 & Ide & $-0,3$ & 22 \\
\hline Insulin-degrading enzyme; & F6RPJ9 & Ide & 0,0 & 22,4 \\
\hline Integral membrane protein $2 \mathrm{~B}$; & 089051 & Itm2b & 0,2 & 17,3 \\
\hline Integrin alpha-3; & Q62470 & Itga3 & $-1,2$ & 5,9 \\
\hline Integrin alpha-6; & Q61739 & Itga6 & $-0,4$ & 3,4 \\
\hline Integrin beta-1; & P09055 & $\operatorname{ltgb1}$ & $-0,3$ & 19,4 \\
\hline Intercellular adhesion molecule 1 ; & P13597 & Icam1 & 0,7 & 11,2 \\
\hline Interferon regulatory factor 2-binding protein 2; & E9Q1P8 & Irf2bp2 & $-0,2$ & 7,7 \\
\hline Interferon-induced transmembrane protein 2 & Q99J93 & Ifitm2 & 0,8 & 5,8 \\
\hline Interleukin-1 alpha; & P01582 & $\| 1 a$ & $-0,2$ & 11,5 \\
\hline Interleukin-1 receptor antagonist protein; & P25085 & $\| 1 \mathrm{rn}$ & 0,8 & 17,5 \\
\hline Interleukin-6 receptor subunit beta; & Q00560 & $116 s t$ & 0,4 & 3,4 \\
\hline Intraflagellar transport protein 20 homolog; & Q61025 & Ift20 & $-0,9$ & 22 \\
\hline Inverted formin-2; & Q0GNC1 & Inf2 & $-4,9$ & 0,8 \\
\hline Involucrin; & G3X9D9 & Ivl & $-0,9$ & 57,1 \\
\hline Iron/zinc purple acid phosphatase-like protein; & Q8BX37 & Papl & $-0,1$ & 2,3 \\
\hline Iron-sulfur cluster assembly enzyme ISCU, mitochondrial; & Q9D7P6 & Iscu & 0,4 & 7,1 \\
\hline Isocitrate dehydrogenase [NAD] subunit alpha, mitochondrial; & Q9D6R2 & $\operatorname{Idh} 3 \mathrm{a}$ & 1,3 & 23,8 \\
\hline Isocitrate dehydrogenase [NADP] cytoplasmic; & 088844 & Idh1 & 1,3 & 39,6 \\
\hline Isocitrate dehydrogenase [NADP], mitochondrial; & P54071 & Idh2 & 1,6 & 38,9 \\
\hline Isopentenyl-diphosphate Delta-isomerase 1 & P58044 & Idi1 & 0,5 & 9,3 \\
\hline Junction plakoglobin; & Q02257 & Jup & 0,0 & 13 \\
\hline Junctional adhesion molecule $A$; & 088792 & F11r & 0,5 & 11,7 \\
\hline Kallikrein 1-related peptidase b5; Kallikrein related-peptidase 5; & Q9D140 & Klk5 & 0,4 & 34,1 \\
\hline Kallikrein 6, isoform CRA_a; Kallikrein-1; KIk6 protein; Neurosin; & Q91Y82 & Klk6 & 0,4 & 22,5 \\
\hline Kallikrein related-peptidase 10; Protein Klk10; & Q99M20 & Klk10 & 0,1 & 15,1 \\
\hline Kallikrein-7; & Q91VE3 & Klk7 & 0,0 & 23,3 \\
\hline Kallikrein-8; & Q61955 & Klk8 & $-0,1$ & 4,6 \\
\hline Kelch repeat and BTB domain-containing protein 3; & Q8BHI4 & Kbtbd3 & 1,2 & 1,2 \\
\hline $\begin{array}{l}\text { Keratin associated protein 13; Protein Krtap13; Putative keratin-associated } \\
\text { protein; }\end{array}$ & 088375 & Krtap13 & 1,7 & 6,1 \\
\hline Keratin, type I cytoskeletal 10; & P02535 & Krt10 & 0,7 & 37,3 \\
\hline Keratin, type I cytoskeletal 12 & Q64291 & Krt12 & $-0,3$ & 2,3 \\
\hline
\end{tabular}




\begin{tabular}{|c|c|c|c|c|}
\hline Protein & Uniprot & Gene name & $\begin{array}{c}\text { Fold change of } \\
\text { XPRESS ratio } \\
\text { (Adam17 } \\
\text { (AKC / WT) }\end{array}$ & $\begin{array}{c}\text { Sequence } \\
\text { coverage [\%] }\end{array}$ \\
\hline Keratin, type I cytoskeletal 14; & Q61781 & Krt14 & 0,5 & 53,9 \\
\hline Keratin, type I cytoskeletal 15; & Q61414 & Krt15 & 0,6 & 35,8 \\
\hline Keratin, type I cytoskeletal 16; & Q9Z2K1 & Krt16 & 3,7 & 12,6 \\
\hline Keratin, type I cytoskeletal 17; & Q9QWL7 & Krt17 & $-0,6$ & 42,3 \\
\hline Keratin, type I cytoskeletal 19; & P19001 & Krt19 & 3,1 & 18,9 \\
\hline Keratin, type I cytoskeletal 23; & Q99PSO & Krt23 & $-0,5$ & 4,7 \\
\hline Keratin, type I cytoskeletal 25; & Q8VCW2 & Krt25 & $-1,9$ & 11 \\
\hline Keratin, type I cytoskeletal 27; & Q9Z320 & Krt27 & $-1,2$ & 17,6 \\
\hline Keratin, type I cytoskeletal 28; & A6BLY7 & Krt28 & $-1,2$ & 6,3 \\
\hline Keratin, type II cytoskeletal 1; & P04104 & Krt1 & 0,2 & 37,5 \\
\hline Keratin, type II cytoskeletal 1b; & Q6IFZ6 & Krt77 & $-0,3$ & 35,8 \\
\hline Keratin, type II cytoskeletal 2 epidermal; & Q3TTY5 & Krt2 & 0,9 & 4,5 \\
\hline Keratin, type II cytoskeletal 2 oral; & Q3UV17 & Krt76 & 0,4 & 4 \\
\hline Keratin, type II cytoskeletal 5; & Q922U2 & Krt5 & 0,3 & 32,9 \\
\hline Keratin, type II cytoskeletal 6A; & P50446 & Krt6a & 0,2 & 12,3 \\
\hline Keratin, type II cytoskeletal 7; & Q9DCV7 & Krt7 & 1,2 & 11,8 \\
\hline Keratin, type II cytoskeletal 71; & Q9ROH5 & Krt71 & $-0,7$ & 13,9 \\
\hline Keratin, type II cytoskeletal 72; & Q6IME9 & Krt72 & 0,6 & 5,4 \\
\hline Keratin, type II cytoskeletal 79; & Q8VED5 & Krt79 & $-0,2$ & 33,7 \\
\hline Keratin-associated protein $16-1$ & A2A5X5 & Krtap16 1 & $-4,4$ & 3 \\
\hline Keratinocyte differentiation-associated protein; & Q3V2T4 & Krtdap & $-0,5$ & 12,7 \\
\hline Keratinocyte proline-rich protein; & B2RUR4 & Kprp & 0,2 & 1,4 \\
\hline Keratinocyte-associated transmembrane protein 2 ; & Q8K201 & Kct2 & 0,0 & 12 \\
\hline Ketimine reductase mu-crystallin; & 054983 & Crym & $-1,3$ & 4,8 \\
\hline Kinectin; & F8VQC7 & Ktn1 & 0,1 & 17 \\
\hline Kinesin heavy chain isoform $5 \mathrm{C}$; & P28738 & Kif5c & 0,5 & 1,4 \\
\hline Kininogen-1; & 008677 & Kng1 & 0,1 & 28,8 \\
\hline Krueppel-like factor 5; & Q9Z0Z7 & Klf5 & $-0,2$ & 2,5 \\
\hline Lactoylglutathione lyase; & Q9CPU0 & Glo1 & 1,3 & 34,8 \\
\hline Ladinin-1; & P57016 & Lad1 & $-0,3$ & 24,6 \\
\hline Lamina-associated polypeptide 2, isoforms beta/delta/epsilon/gamma; & Q61029 & Tmpo & 0,4 & 29 \\
\hline Laminin subunit beta-1; & E9QN70 & Lamb1 & $-0,3$ & 1,4 \\
\hline Laminin subunit gamma-2; Laminin, gamma 2; & G5E874 & Lamc2 & $-0,8$ & 4,2 \\
\hline La-related protein 1 & Q6ZQ58 & Larp1 & $-0,6$ & 0,8 \\
\hline Large proline-rich protein BAG6; & Q9Z1R2 & Bag6 & $-0,5$ & 4,6 \\
\hline LEM domain-containing protein 2 ; & Q6DVA0 & Lemd2 & $-1,1$ & 7,8 \\
\hline Leucine zipper transcription factor-like protein 1 ; & Q9JHQ5 & Lztfl1 & 1,9 & 6,9 \\
\hline $\begin{array}{l}\text { Leucine-rich HEV glycoprotein; Leucine-rich alpha-2-glycoprotein; Leucine-rich } \\
\text { alpha-2-glycoprotein 1; Protein Lrg1; }\end{array}$ & Q91XL1 & Lrg1 & 0,3 & 4,1 \\
\hline Leucine-rich repeat flightless-interacting protein 1 & Q3UZ39 & Lrrfip1 & $-0,1$ & 20,4 \\
\hline Leucine-rich repeat flightless-interacting protein 2 ; & E9QN52 & Lrrfip2 & $-0,2$ & 16,4 \\
\hline Leucine-rich repeat-containing protein $16 \mathrm{~A}$; & Q6EDY6 & Lrrc16a & $-0,6$ & 1 \\
\hline Leucine-rich repeat-containing protein 59 ; & Q922Q8 & Lrrc59 & 0,3 & 33,2 \\
\hline Leucyl-cystinyl aminopeptidase; & Q8C129 & Lnpep & $-0,1$ & 0,8 \\
\hline Leukocyte elastase inhibitor $\mathrm{A}$; & Q9D154 & Serpinb1a & $-1,3$ & 5,5 \\
\hline Leukocyte surface antigen CD47; & Q61735 & $\mathrm{Cd} 47$ & 0,3 & 8,9 \\
\hline Leukosialin; & P15702 & Spn & $-0,5$ & 4,1 \\
\hline LIM and SH3 domain protein 1 ; & Q61792 & Lasp1 & 0,0 & 20,2 \\
\hline LIM domain and actin-binding protein 1 ; & Q9ERG0 & Lima1 & 0,0 & 6,6 \\
\hline LIM domain-containing protein 1 & Q9QXD8 & Limd1 & 0,3 & 2,8 \\
\hline LIM zinc-binding domain-containing Nebulette; & Q9DC07 & Nebl & $-0,3$ & 16,7 \\
\hline Lipoma-preferred partner homolog; & Q8BFW7 & Lpp & $-0,9$ & 7 \\
\hline L-lactate dehydrogenase A chain; & P06151 & Ldha & 0,0 & 17,5 \\
\hline Lon protease homolog, mitochondrial; & Q8CGK3 & Lonp1 & 0,1 & 1,6 \\
\hline Long-chain specific acyl-CoA dehydrogenase, mitochondrial; & P51174 & Acadl & 0,3 & 4,9 \\
\hline Loricrin; & P18165 & Lor & 0,8 & 6,4 \\
\hline Luc7-like protein 3; & Q5SUF2 & Luc713 & 0,5 & 2,5 \\
\hline Lumican; & P51885 & Lum & 0,1 & 17,2 \\
\hline Ly6/PLAUR domain-containing protein 3; & Q91YK8 & Lypd3 & 0,1 & 5,2 \\
\hline Ly6/PLAUR domain-containing protein 5; & Q9D7Z7 & Lypd5 & 0,2 & 13,7 \\
\hline $\begin{array}{l}\text { Ly6g6e protein, isoform A; Lymphocyte antigen } 6 \text { complex, locus G6E; Protein } \\
\text { Ly6g6e; }\end{array}$ & Q9D7E5 & Ly6g6e & $-0,5$ & 21,6 \\
\hline Lymphocyte antigen 6 complex locus protein G6c; & Q9Z1Q4 & Ly6g6c & $-0,4$ & 14,3 \\
\hline Lymphocyte antigen 6D; & P35459 & Ly6d & 0,1 & 48 \\
\hline Lymphocyte-specific protein 1 & P19973 & Lsp1 & 0,0 & 26,4 \\
\hline Lysosomal alpha-mannosidase; & 009159 & Man2b1 & 0,7 & 1,1 \\
\hline Lysosomal protective protein; & P16675 & Ctsa & $-0,2$ & 11,6 \\
\hline Lysosome-associated membrane glycoprotein 1 & P11438 & Lamp1 & $-0,2$ & 8,4 \\
\hline Lysosome-associated membrane glycoprotein 2; & P17047 & Lamp2 & $-0,3$ & 8,2 \\
\hline Macrophage colony-stimulating factor 1 & P07141 & Csf1 & $-1,6$ & 7,4 \\
\hline Macrophage migration inhibitory factor; & P34884 & Mif & 1,1 & 7,8 \\
\hline Magnesium-dependent phosphatase 1 ; & Q9D967 & Mdp1 & 0,9 & 7,9 \\
\hline
\end{tabular}




\begin{tabular}{|c|c|c|c|c|}
\hline Protein & Uniprot & Gene name & $\begin{array}{c}\text { Fold change of } \\
\text { XPRESS ratio } \\
\left.\text { (Adam17 }^{\Delta K C} / \mathrm{WT}\right)\end{array}$ & $\begin{array}{c}\text { Sequence } \\
\text { coverage [\%] }\end{array}$ \\
\hline Major urinary protein 4; & P11590 & Mup4 & 0,2 & 10,1 \\
\hline Major urinary protein 8; Protein Mup10; & A2BIN1 & Mup10 & 0,5 & 27,2 \\
\hline Malate dehydrogenase, cytoplasmic; & P14152 & Mdh1 & $-0,1$ & 15 \\
\hline Malate dehydrogenase, mitochondrial; & P08249 & Mdh2 & 0,2 & 44,7 \\
\hline Male-enhanced antigen 1 ; & Q64327 & Mea1 & $-0,9$ & 16,1 \\
\hline MARCKS-related protein; & P28667 & Marcksl1 & $-0,2$ & 14 \\
\hline MCG116526; Protein Scgb2b7; & D3YYY1 & Scgb2b7 & 0,2 & 23,2 \\
\hline MCG117626; Odorant binding protein la; Protein Obp1a; & Q9D3H2 & Obp1a & 0,4 & 30,1 \\
\hline MCG120169; Protein 2310050C09Rik; & G5E8Z3 & 2310050C09Rik & 1,0 & 8,9 \\
\hline MCG121122; Protein 9130204L05Rik; & G3UWB8 & 9130204L05Rik & 0,7 & 12 \\
\hline $\begin{array}{l}\text { MCG127334; MCG17849, isoform CRA_a; Protein 1810009A15Rik; } \\
\text { Uncharacterized protein; }\end{array}$ & Q9D937 & 1810009A15Rik & 0,8 & 11,4 \\
\hline MCG129038; Protein Serpinb3a; & G3X9V8 & Serpinb3a & $-1,1$ & 37,7 \\
\hline MCG130175, isoform CRA_b; Protein BC100530; cDNA sequence BC100530; & Q497J0 & BC100530 & $-0,1$ & 61,9 \\
\hline MCG15081; Protein Gm10334; Trypsinogen 15; & Q792Y8 & Gm10334 & 1,8 & 4,1 \\
\hline MCG17833, isoform CRA_c; Protein Ahnak; & G5E8K8 & Ahnak & $-0,2$ & 39,3 \\
\hline MCG17975; Protein Zfyve9; & A2A8R0 & Zfyve9 & $-1,3$ & 4,8 \\
\hline MCG21235; Protein Serpinb3b; & Q9D1Q5 & Serpinb3b & $-0,5$ & 10,6 \\
\hline MCG54087; Protein Serpina3j; & D3Z451 & Serpina3j & 0,8 & 11 \\
\hline Mediator of RNA polymerase II transcription subunit 10; & Q9CXU0 & Med10 & 0,5 & 6,7 \\
\hline Mediator of RNA polymerase II transcription subunit 21; & Q9CQ39 & Med21 & 0,1 & 22,2 \\
\hline Mediator of RNA polymerase II transcription subunit 28 & Q920D3 & Med28 & 0,1 & 11,9 \\
\hline Mediator of RNA polymerase II transcription subunit 30; & Q9CQ19 & Med30 & 0,6 & 9 \\
\hline Mediator of RNA polymerase II transcription subunit 8; & Q9D7W5 & Med8 & 0,4 & 9,8 \\
\hline Mediator of RNA polymerase II transcription subunit 9; & Q8VCS6 & Med9 & 0,7 & 10,6 \\
\hline Melanoma inhibitory activity protein 3 & Q8BI84 & Mia3 & $-0,3$ & 1,8 \\
\hline Membrane-associated progesterone receptor component 1 ; & 055022 & Pgrmc1 & 0,5 & 26,2 \\
\hline Membrane-associated progesterone receptor component 2; & Q80UU9 & Pgrmc2 & 0,4 & 49,8 \\
\hline Metallothionein-2; & P02798 & Mt2 & $-0,2$ & 19,7 \\
\hline Metallothionein-4; & P47945 & Mt4 & $-0,5$ & 19,4 \\
\hline Metaxin-1; & P47802 & Mtx1 & $-1,1$ & 6 \\
\hline Methionine aminopeptidase 2 ; & 008663 & Metap2 & 0,0 & 11,4 \\
\hline Methylosome subunit pICln; & Q61189 & Clns1a & 0,0 & 31,4 \\
\hline MICAL-like protein $1 ;$ & Q8BGT6 & Micall1 & $-0,9$ & 1,7 \\
\hline Microfibrillar-associated protein 1 & Q9CQU1 & Mfap1 & 0,2 & 8,9 \\
\hline Microtubule-associated protein 1B; & P14873 & Map1b & $-0,3$ & 0,9 \\
\hline Microtubule-associated protein 2; & P20357 & Map2 & $-0,3$ & 19,1 \\
\hline Microtubule-associated protein 4; & P27546 & Map4 & 0,0 & 37 \\
\hline Microtubule-associated protein RP/EB family member 1 ; & Q61166 & Mapre1 & $-0,3$ & 17,5 \\
\hline Microtubule-associated protein tau; & P10637 & Mapt & 0,2 & 7,2 \\
\hline Microtubule-associated serine/threonine-protein kinase 4; & Q811L6 & Mast4 & $-0,6$ & 1,7 \\
\hline Mimitin, mitochondrial; & Q59J78 & Ndufaf2 & $-0,5$ & 14,3 \\
\hline Mitochondrial antiviral-signaling protein; & Q8VCF0 & Mavs & 0,8 & 5,4 \\
\hline Mitochondrial fission 1 protein; & Q9CQ92 & Fis1 & 0,1 & 7,6 \\
\hline Mitochondrial import inner membrane translocase subunit Tim13; & P62075 & Timm13 & 1,4 & 61,1 \\
\hline Mitochondrial import inner membrane translocase subunit TIM14; & Q9CQV7 & Dnajc19 & 0,6 & 15,5 \\
\hline Mitochondrial import inner membrane translocase subunit TIM16; & Q9CQV1 & Pam16 & $-0,4$ & 30,4 \\
\hline Mitochondrial import inner membrane translocase subunit TIM50; & Q9D880 & Timm50 & $-0,7$ & 7,9 \\
\hline Mitochondrial import inner membrane translocase subunit Tim8 A; & Q9WVA2 & Timm8a1 & 0,9 & 34 \\
\hline Mitochondrial import inner membrane translocase subunit Tim9; & Q9WV98 & Timm9 & 1,3 & 18 \\
\hline Mitochondrial intermembrane space import and assembly protein 40 ; & Q8VEA4 & Chchd4 & 0,0 & 16,5 \\
\hline Mitogen-activated protein kinase 1 & P63085 & Mapk1 & 0,1 & 6,7 \\
\hline MKL/myocardin-like protein 2; & P59759 & $\mathrm{Mkl2}$ & $-0,1$ & 3,2 \\
\hline MLV-related proviral Env polyprotein; & P10404 & & $-0,1$ & 2,8 \\
\hline MOB kinase activator $1 \mathrm{~B}$; & Q8BPB0 & Mob1b & 1,5 & 5,1 \\
\hline Moesin; & P26041 & Msn & $-0,8$ & 12,3 \\
\hline Molybdopterin synthase sulfur carrier subunit; & Q9Z224 & Mocs2 & 0,2 & 21,6 \\
\hline MORF4 family-associated protein 1 ; & Q9CQL7 & Mrfap1 & $-0,1$ & 18,4 \\
\hline M-phase phosphoprotein 9; & A6H5Y1 & Mphosph9 & $-3,3$ & 1,3 \\
\hline mRNA-decapping enzyme $1 \mathrm{~A}$ & Q91YD3 & Dcp1a & 0,4 & 2 \\
\hline Mth938 domain-containing protein; & Q8R0P4 & Aamdc & 0,5 & 16,4 \\
\hline Multiple epidermal growth factor-like domains protein 9; & Q8BH27 & Megf9 & $-0,2$ & 3 \\
\hline Multiple myeloma tumor-associated protein 2 homolog; & Q99LX5 & Mmtag2 & 0,3 & 8,8 \\
\hline Musculoskeletal embryonic nuclear protein 1; & Q99J11 & Mustn1 & $-0,6$ & 18,3 \\
\hline Myc box-dependent-interacting protein 1 & 008539 & Bin1 & 0,3 & 2,9 \\
\hline Myelin basic protein; & P04370 & Mbp & 0,3 & 6,7 \\
\hline Myeloid bactenecin (F1); Neutrophilic granule protein; Protein Ngp; & 008692 & Ngp & 1,9 & 35,3 \\
\hline Myosin light chain $1 / 3$, skeletal muscle isoform; & P05977 & Myl1 & 1,3 & 40,4 \\
\hline Myosin light chain 3; & P09542 & Myl3 & 1,0 & 27,5 \\
\hline Myosin light chain 6B; & Q8Cl43 & Myl6b & 0,1 & 10,1 \\
\hline Myosin light chain kinase, smooth muscle; & Q6PDN3 & Mylk & $-0,4$ & 0,6 \\
\hline Myosin light polypeptide 6; & Q60605 & Myl6 & 0,1 & 54,3 \\
\hline
\end{tabular}




\begin{tabular}{|c|c|c|c|c|}
\hline Protein & Uniprot & Gene name & $\begin{array}{c}\text { Fold change of } \\
\text { XPRESS ratio } \\
\left.\text { (Adam17 }^{\Delta K C} / \mathrm{WT}\right)\end{array}$ & $\begin{array}{c}\text { Sequence } \\
\text { coverage [\%] }\end{array}$ \\
\hline Myosin phosphatase Rho-interacting protein; & Q5SWZ5 & Mprip & 4,4 & 0,9 \\
\hline Myosin regulatory light chain $12 \mathrm{~B}$ & Q3THE2 & Myl12b & 0,3 & 40,1 \\
\hline Myosin regulatory light chain 2, skeletal muscle isoform; & P97457 & Mylpf & 1,3 & 22,5 \\
\hline Myosin-14; & Q6URW6 & Myh14 & 0,6 & 2,8 \\
\hline Myosin-6; & Q02566 & Myh6 & 0,0 & 3,3 \\
\hline Myosin-9; & Q8VDD5 & Myh9 & $-0,6$ & 19,4 \\
\hline Myotrophin; & P62774 & Mtpn & 0,0 & 20,3 \\
\hline Myristoylated alanine-rich C-kinase substrate; & P26645 & Marcks & $-0,1$ & 21,4 \\
\hline N(4)-(beta-N-acetylglucosaminyl)-L-asparaginase; & Q64191 & Aga & 0,3 & 14,2 \\
\hline $\mathrm{N}(\mathrm{G}), \mathrm{N}(\mathrm{G})$-dimethylarginine dimethylaminohydrolase 2 & Q99LD8 & Ddah2 & 0,6 & 19,3 \\
\hline $\mathrm{Na}(+) / \mathrm{H}(+)$ exchange regulatory cofactor NHE-RF1; & P70441 & Slc9a3r1 & $-0,8$ & 47,9 \\
\hline N-acetylglucosamine-6-sulfatase; & Q8BFR4 & Gns & 0,2 & 8,8 \\
\hline $\mathrm{N}$-acetylneuraminate lyase; & Q9DCJ9 & $\mathrm{Npl}$ & 0,7 & 52,8 \\
\hline $\mathrm{N}$-acylethanolamine-hydrolyzing acid amidase; & Q9D7V9 & Naaa & $-0,2$ & 3,9 \\
\hline NAD(P)H-hydrate epimerase; & Q8K4Z3 & Apoa1bp & $-0,4$ & 2,8 \\
\hline NADH dehydrogenase [ubiquinone] 1 alpha subcomplex subunit 13; & Q9ERS2 & Ndufa13 & 0,2 & 20,8 \\
\hline NADH dehydrogenase [ubiquinone] 1 alpha subcomplex subunit 2; & Q9CQ75 & Ndufa2 & 0,4 & 24,2 \\
\hline NADH dehydrogenase [ubiquinone] 1 alpha subcomplex subunit 4; & Q62425 & Ndufa4 & 0,4 & 46,3 \\
\hline NADH dehydrogenase [ubiquinone] 1 alpha subcomplex subunit 4-like 2 ; & Q4FZG9 & Ndufa4l2 & 0,2 & 28,7 \\
\hline NADH dehydrogenase [ubiquinone] 1 alpha subcomplex subunit 5; & Q9CPP6 & Ndufa5 & 0,3 & 19,8 \\
\hline NADH dehydrogenase [ubiquinone] 1 alpha subcomplex subunit 6; & Q9CQZ5 & Ndufa6 & $-5,2$ & 13 \\
\hline NADH dehydrogenase [ubiquinone] 1 alpha subcomplex subunit 7; & Q971P6 & Ndufa7 & 0,1 & 29,2 \\
\hline NADH dehydrogenase [ubiquinone] 1 alpha subcomplex subunit 8; & Q9DCJ5 & Ndufa8 & $-0,1$ & 23,8 \\
\hline NADH dehydrogenase [ubiquinone] 1 beta subcomplex subunit 10; & Q9DCS9 & Ndufb10 & $-0,1$ & 27,8 \\
\hline NADH dehydrogenase [ubiquinone] 1 beta subcomplex subunit 3; & Q9CQZ6 & Ndufb3 & $-0,1$ & 17,3 \\
\hline $\begin{array}{l}\text { NADH dehydrogenase [ubiquinone] } 1 \text { beta subcomplex subunit } 8 \text {, } \\
\text { mitochondrial; }\end{array}$ & Q9D6J5 & Ndufb8 & 0,6 & 10,8 \\
\hline NADH dehydrogenase [ubiquinone] 1 subunit $\mathrm{C} 2$ & Q9CQ54 & Ndufc2 & 0,3 & 10,8 \\
\hline NADH dehydrogenase [ubiquinone] flavoprotein 2, mitochondrial; & Q9D6J6 & Ndufv2 & 0,4 & 16,5 \\
\hline NADH dehydrogenase [ubiquinone] flavoprotein 3, mitochondrial; & Q8BK30 & Ndufv3 & $-0,4$ & 10,6 \\
\hline $\begin{array}{l}\text { NADH dehydrogenase [ubiquinone] flavoprotein 3, mitochondrial; RIKEN cDNA } \\
\text { 1500032D16, isoform CRA_a; }\end{array}$ & Q3U422 & Ndufv3 & 1,0 & 21,6 \\
\hline NADH dehydrogenase [ubiquinone] iron-sulfur protein 4, mitochondrial; & Q9CXZ1 & Ndufs4 & 0,2 & 25,1 \\
\hline NADH dehydrogenase [ubiquinone] iron-sulfur protein 5; & Q99LY9 & Ndufs5 & 0,3 & 14,3 \\
\hline NADH dehydrogenase [ubiquinone] iron-sulfur protein 6, mitochondrial; & P52503 & Ndufs6 & $-0,3$ & 42,2 \\
\hline NADH dehydrogenase [ubiquinone] iron-sulfur protein 8, mitochondrial; & Q8K3J1 & Ndufs8 & $-0,3$ & 9,4 \\
\hline NADP-dependent malic enzyme; & P06801 & Me1 & $-0,2$ & 10,1 \\
\hline Nardilysin; & Q8BHG1 & Nrd1 & $-0,4$ & 6,2 \\
\hline Nascent polypeptide-associated complex subunit alpha, muscle-specific form; & P70670 & Naca & 0,5 & 25,6 \\
\hline Nectin-1; & Q9JKF6 & Pvrl1 & $-0,4$ & 13,4 \\
\hline Nectin-2; & P32507 & Pvrl2 & $-0,1$ & 8,5 \\
\hline Nectin-4; & Q8R007 & Pvrl4 & $-0,1$ & 24,2 \\
\hline NEDD8; & P29595 & Nedd8 & $-0,3$ & 24,7 \\
\hline Negative elongation factor B; & Q8C4Y3 & Nelfb & $-1,0$ & 2,1 \\
\hline Negative elongation factor $\mathrm{E}$ & P19426 & Nelfe & $-0,3$ & 11 \\
\hline Neudesin; & Q9CQ45 & Nenf & $-0,2$ & 42,7 \\
\hline Neural cell adhesion molecule L1; & A2AFG7 & L1cam & $-0,2$ & 9,4 \\
\hline Neural cell adhesion molecule L1-like protein; & P70232 & Chl1 & $-0,2$ & 6,5 \\
\hline Neural Wiskott-Aldrich syndrome protein; & Q91YD9 & Wasl & $-0,2$ & 4,2 \\
\hline Neuroblastoma suppressor of tumorigenicity 1 & Q61477 & Nbl1 & 0,0 & 3,9 \\
\hline Neuromodulin; & P06837 & Gap43 & 0,0 & 18,1 \\
\hline Neuronal calcium sensor 1 & Q8BNY6 & Ncs1 & 0,5 & 8,9 \\
\hline Neuroplastin; & P97300 & Nptn & 0,0 & 18,1 \\
\hline Neurotensin receptor type 1 & 088319 & Ntsr1 & $-10,0$ & 1,7 \\
\hline Nexilin; & Q7TPW1 & Nexn & $-0,3$ & 1,3 \\
\hline NF-kappa-B essential modulator; & 088522 & lkbkg & 0,1 & 16,3 \\
\hline NFU1 iron-sulfur cluster scaffold homolog, mitochondrial; & Q9QZ23 & Nfu1 & 0,1 & 20,4 \\
\hline NFU1 iron-sulfur cluster scaffold homolog, mitochondrial; & D3Z285 & Nfu1 & $-0,1$ & 14,1 \\
\hline NHP2-like protein 1; & Q9D0T1 & Nhp2l1 & 0,9 & 18,8 \\
\hline NHS-like protein $1 ;$ & Q8CAF4 & Nhsl1 & $-0,3$ & 1,7 \\
\hline Nicastrin; & P57716 & Ncstn & 0,1 & 5,9 \\
\hline Niemann-Pick C1 protein; & 035604 & Npc1 & 0,9 & 1,7 \\
\hline Nitrilase homolog 1 & Q8VDK1 & Nit1 & 1,6 & 12,6 \\
\hline Non-histone chromosomal protein HMG-17; & P09602 & Hmgn2 & $-1,6$ & 17,2 \\
\hline Non-lysosomal glucosylceramidase; & Q69ZF3 & Gba2 & $-0,4$ & 4,3 \\
\hline Non-specific lipid-transfer protein; & P32020 & Scp2 & 0,3 & 10,6 \\
\hline NSFL1 cofactor $\mathrm{p} 47$ & Q9CZ44 & Nsfl1c & $-0,3$ & 42,2 \\
\hline Nuclear autoantigenic sperm protein; & Q99MD9 & Nasp & 0,0 & 21 \\
\hline
\end{tabular}




\begin{tabular}{|c|c|c|c|c|}
\hline Protein & Uniprot & Gene name & $\begin{array}{c}\text { Fold change of } \\
\text { XPRESS ratio } \\
\left.\text { (Adam } 17^{\Delta K C} / \mathrm{WT}\right)\end{array}$ & $\begin{array}{c}\text { Sequence } \\
\text { coverage [\%] }\end{array}$ \\
\hline Nuclear distribution protein nudE-like 1 & Q9ERR1 & Ndel1 & 0,2 & 10,1 \\
\hline Nuclear fragile $\mathrm{X}$ mental retardation-interacting protein 2; & Q5F2E7 & Nufip2 & $-0,1$ & 8,5 \\
\hline Nuclear migration protein nudC; & 035685 & Nudc & 0,1 & 28,3 \\
\hline Nuclear pore complex protein Nup50; & Q9JIH2 & Nup50 & $-0,1$ & 6 \\
\hline Nuclear pore complex protein Nup93; & Q8BJ71 & Nup93 & 0,0 & 1,3 \\
\hline Nuclear pore glycoprotein p62; & Q63850 & Nup62 & $-0,1$ & 16,5 \\
\hline Nuclear receptor coactivator 6 & Q9JL19 & Ncoa6 & $-0,2$ & 1,4 \\
\hline Nuclear speckle splicing regulatory protein $1 ;$ & Q5NCR9 & Nsrp1 & 0,2 & 5,2 \\
\hline Nuclear transcription factor $Y$ subunit beta; & P63139 & Nfyb & $-0,2$ & 12,3 \\
\hline Nuclear transport factor 2 & P61971 & Nutf2 & 1,0 & 11,8 \\
\hline Nuclear ubiquitous casein and cyclin-dependent kinase substrate 1 ; & Q80XU3 & Nucks1 & $-0,5$ & 12 \\
\hline Nuclease-sensitive element-binding protein 1 & P62960 & Ybx1 & 0,6 & 30,7 \\
\hline Nucleobindin-1; & Q02819 & Nucb1 & $-0,3$ & 46 \\
\hline Nucleobindin-2; & P81117 & Nucb2 & $-0,4$ & 26,4 \\
\hline Nucleolar protein 16; & Q9CPT5 & Nop16 & 0,1 & 3,9 \\
\hline Nucleolar protein 3; & Q9D1X0 & Nol3 & $-0,4$ & 8,6 \\
\hline Nucleolar protein 56 & Q9D6Z1 & Nop56 & $-0,8$ & 5,7 \\
\hline Nucleolar RNA helicase 2; & Q9JIK5 & $\mathrm{Ddx} 21$ & $-0,7$ & 4,3 \\
\hline Nucleolin; & P09405 & $\mathrm{Ncl}$ & 0,4 & 30,1 \\
\hline Nucleophosmin; & Q61937 & Npm1 & $-0,2$ & 18,7 \\
\hline Nucleoplasmin-3; & Q9СPP0 & Npm3 & 0,2 & 8,6 \\
\hline Nucleoporin NUP53; & Q8R4R6 & Nup35 & 0,1 & 10,2 \\
\hline Nucleoprotein TPR; & F6ZDS4 & Tpr & $-0,1$ & 9,8 \\
\hline Nucleoside diphosphate kinase; & E9PZF0 & Gm20390 & 0,6 & 56,9 \\
\hline Nucleosome assembly protein 1-like 1 ; & P28656 & Nap1l1 & 0,3 & 12,7 \\
\hline Nucleosome assembly protein 1-like 4; & Q78ZA7 & Nap1|4 & 1,5 & 17,9 \\
\hline OCIA domain-containing protein 1 & Q9CRD0 & Ociad1 & 0,2 & 7,3 \\
\hline OCIA domain-containing protein 2 & Q9D8W7 & Ociad2 & 0,7 & 5,8 \\
\hline Odorant-binding protein $2 \mathrm{a}$ & Q8K1H9 & Obp2a & 0,0 & 22,2 \\
\hline Oligoribonuclease, mitochondrial; & Q9D8S4 & Rexo2 & $-0,1$ & 4,2 \\
\hline Opioid growth factor receptor; & Q99PG2 & Ogfr & $-0,6$ & 3,2 \\
\hline Osteoclast-stimulating factor 1 ; & Q62422 & Ostf1 & 0,7 & 44,2 \\
\hline Palladin; & Q9ET54 & Palld & 0,6 & 0,9 \\
\hline Palmdelphin; & Q9JHU2 & Palmd & 0,0 & 26,3 \\
\hline Palmitoyltransferase ZDHHC5; & Q8VDZ4 & Zdhhc5 & $-0,7$ & 6,7 \\
\hline $\begin{array}{l}\text { Papillary renal cell carcinoma (Translocation-associated); Papillary renal cell } \\
\text { carcinoma-associated protein; Protein Prcc; }\end{array}$ & Q9EQC8 & Prcc & $-0,2$ & 8,8 \\
\hline Paralemmin-1; & Q9ZOP4 & Palm & $-0,1$ & 32,6 \\
\hline Paralemmin-2; & Q8BR92 & Palm2 & $-0,5$ & 3,2 \\
\hline Parathymosin; & Q9D0J8 & Ptms & $-0,4$ & 10,9 \\
\hline Partner of Y14 and mago; & Q8CHP5 & Wibg & $-0,6$ & 34,5 \\
\hline Paternally-expressed gene 3 protein; & Q3URU2 & Peg3 & 0,0 & 0,7 \\
\hline PAXIP1-associated glutamate-rich protein 1; & Q99L02 & PAGR1 & $-0,2$ & 7,5 \\
\hline PC4 and SFRS1-interacting protein; & Q99JF8 & Psip1 & $-0,5$ & 15,6 \\
\hline PCNA-associated factor; & Q9CQX4 & Paf & $-0,3$ & 6,4 \\
\hline PDZ and LIM domain protein 1; & 070400 & Pdlim1 & 0,2 & 40,1 \\
\hline PDZ and LIM domain protein 2; & Q8R1G6 & Pdlim2 & $-0,5$ & 19,2 \\
\hline PDZ and LIM domain protein 4; & P70271 & Pdlim4 & 0,5 & 33,6 \\
\hline PDZ and LIM domain protein 5; & Q8CI51 & Pdlim5 & $-0,7$ & 20,1 \\
\hline PDZ domain-containing protein $11 ;$ & Q9CZG9 & Pdzd11 & $-0,1$ & 17,1 \\
\hline PDZK1-interacting protein 1; & Q9CQHO & Pdzk1ip1 & 0,6 & 28,1 \\
\hline Peflin; & Q8BFY6 & Pef1 & 1,2 & 11,6 \\
\hline Peptidyl-prolyl cis-trans isomerase A; & P17742 & Ppia & 1,1 & 57,9 \\
\hline Peptidyl-prolyl cis-trans isomerase B; & P24369 & Ppib & 0,2 & 13,9 \\
\hline Peptidyl-prolyl cis-trans isomerase C; & P30412 & Ppic & 1,7 & 6,1 \\
\hline Peptidyl-prolyl cis-trans isomerase CWC27 homolog; & Q3TKY6 & Cwc27 & $-0,3$ & 4,9 \\
\hline Peptidyl-prolyl cis-trans isomerase D; & Q9CR16 & Ppid & 0,4 & 12,4 \\
\hline Peptidyl-prolyl cis-trans isomerase E; & Q9QZH3 & Ppie & 0,0 & 7,3 \\
\hline Peptidyl-prolyl cis-trans isomerase FKBP1A; & P26883 & Fkbp1a & $-0,1$ & 25 \\
\hline Peptidyl-prolyl cis-trans isomerase FKBP2; & P45878 & Fkbp2 & 0,2 & 8,6 \\
\hline Peptidyl-prolyl cis-trans isomerase FKBP3; & Q62446 & Fkbp3 & 0,1 & 31,3 \\
\hline Peptidyl-prolyl cis-trans isomerase FKBP7; & 054998 & Fkbp7 & $-0,2$ & 8,7 \\
\hline Peptidyl-prolyl cis-trans isomerase NIMA-interacting 1 ; & Q9QUR7 & Pin1 & 1,0 & 13,9 \\
\hline Peptidyl-prolyl cis-trans isomerase NIMA-interacting 4; & Q9CWW6 & Pin4 & 0,1 & 25,2 \\
\hline Perilipin-1; & Q8CGN5 & Plin1 & $-1,0$ & 5,6 \\
\hline Perilipin-2; & P43883 & Plin2 & $-0,2$ & 13,9 \\
\hline Perilipin-3; & Q9DBG5 & Plin3 & 0,0 & 33,6 \\
\hline Perilipin-4; & 088492 & Plin4 & $-0,9$ & 15,7 \\
\hline Periostin; & Q62009 & Postn & 0,0 & 21,6 \\
\hline Periplakin; & G5E898 & Ppl & $-0,2$ & 28,9 \\
\hline Peroxiredoxin-1; & P35700 & $\operatorname{Prdx1}$ & 0,5 & 60,3 \\
\hline Peroxiredoxin-2; & Q61171 & $\operatorname{Prdx} 2$ & $-0,1$ & 31,3 \\
\hline Peroxiredoxin-5, mitochondrial; & P99029 & Prdx5 & 0,7 & 44,8 \\
\hline
\end{tabular}




\begin{tabular}{|c|c|c|c|c|}
\hline Protein & Uniprot & Gene name & $\begin{array}{c}\text { Fold change of } \\
\text { XPRESS ratio } \\
\text { (Adam17 } \\
\text { (AKC / WT) }\end{array}$ & $\begin{array}{c}\text { Sequence } \\
\text { coverage [\%] }\end{array}$ \\
\hline Peroxiredoxin-6; & D3ZOY2 & Prdx6 & 1,6 & 39 \\
\hline Peroxisomal biogenesis factor 19 & Q8VCI5 & Pex19 & $-0,5$ & 30,4 \\
\hline Peroxisomal membrane protein PEX14; & Q9R0A0 & Pex14 & $-0,1$ & 13,6 \\
\hline Persulfide dioxygenase ETHE1, mitochondrial; & Q9DCM0 & Ethe1 & 0,3 & 5,5 \\
\hline PEST proteolytic signal-containing nuclear protein; & Q6P814 & Penp & $-0,3$ & 36 \\
\hline PHD finger-like domain-containing protein $5 \mathrm{~A}$; & P83870 & Phf5a & 0,0 & 6,4 \\
\hline Phosducin-like protein 3; & Q8BVF2 & Pdcl3 & 0,2 & 3,3 \\
\hline Phosphatase and actin regulator 4 & Q501J7 & Phactr4 & $-0,3$ & 18,6 \\
\hline Phosphatidylethanolamine-binding protein 1; & P70296 & Pebp1 & 0,5 & 44,9 \\
\hline Phosphatidylinositol-glycan-specific phospholipase D; & 070362 & Gpld1 & $-0,4$ & 5,3 \\
\hline Phosphoglycerate kinase 1 & P09411 & Pgk1 & 0,0 & 17,3 \\
\hline Phosphoglycerate mutase 1 & Q9DBJ1 & Pgam1 & 0,9 & 38,6 \\
\hline Phosphoglycerate mutase 2 & 070250 & Pgam2 & 1,4 & 13 \\
\hline Phospholipase B-like 1; & Q8VCI0 & Plbd1 & 1,4 & 6,4 \\
\hline Phospholipase D3; & 035405 & Pld3 & 0,0 & 6,4 \\
\hline Phosphorylated adapter RNA export protein; & Q9JJT9 & Phax & 0,0 & 13,8 \\
\hline Phosphoserine phosphatase; & Q99LS3 & Psph & 0,5 & 11,6 \\
\hline Pinin; & 035691 & Pnn & $-0,4$ & 6,1 \\
\hline PITH domain-containing protein $1 ;$ & Q8BWR2 & Pithd1 & 0,2 & 4,3 \\
\hline Placenta-expressed transcript 1 protein; & Q8VEN2 & Plet1 & $-0,3$ & 6,3 \\
\hline Plakophilin-1; & P97350 & Pkp1 & 0,7 & 23,6 \\
\hline Plakophilin-3; & Q9QY23 & Pkp3 & 0,3 & 8,7 \\
\hline Plasminogen activator inhibitor 1 RNA-binding protein; & Q9CY58 & Serbp1 & 0,2 & 29,5 \\
\hline Plasminogen activator inhibitor 2, macrophage; & P12388 & Serpinb2 & $-0,1$ & 9,9 \\
\hline Plasminogen receptor $(\mathrm{KT})$ & Q9D3P8 & Plgrkt & 0,2 & 6,8 \\
\hline Plastin-2; & Q61233 & Lcp1 & 0,5 & 9,1 \\
\hline Plastin-3; & Q99K51 & Pls3 & 0,1 & 27,1 \\
\hline Platelet glycoprotein 4; & Q08857 & $\mathrm{Cd} 36$ & $-0,4$ & 1,7 \\
\hline Platelet-activating factor acetylhydrolase IB subunit alpha; & P63005 & Pafah1b1 & 0,3 & 2,7 \\
\hline Platelet-activating factor acetylhydrolase IB subunit beta; & Q61206 & Pafah1b2 & 0,1 & 12,2 \\
\hline Platelet-activating factor acetylhydrolase IB subunit gamma; & Q61205 & Pafah1b3 & 1,1 & 20,3 \\
\hline Plectin; & Q9QXS1 & Plec & $-0,6$ & 4,4 \\
\hline Plexin-B2; & B2RXS4 & Plxnb2 & $-0,4$ & 0,8 \\
\hline Poly(rC)-binding protein 1 & P60335 & Pcbp1 & 0,3 & 13,8 \\
\hline Poly(U)-specific endoribonuclease; & Q3V188 & Endou & $-0,7$ & 21,1 \\
\hline Polyadenylate-binding protein $1 ;$ & P29341 & Pabpc1 & 0,3 & 5,5 \\
\hline Polyadenylate-binding protein 2; & Q8CCS6 & Pabpn1 & 0,8 & 12,8 \\
\hline Polyadenylate-binding protein-interacting protein $1 ;$ & Q8VE62 & Paip1 & 0,3 & 4,9 \\
\hline Polyadenylate-binding protein-interacting protein $2 \mathrm{~B}$; & Q91W45 & Paip2b & $-0,5$ & 10,3 \\
\hline Polyglutamine-binding protein 1 & Q91VJ5 & Pqbp1 & $-0,2$ & 16,7 \\
\hline Polymerase I and transcript release factor; & 054724 & Ptrf & 0,6 & 6,6 \\
\hline Polypyrimidine tract-binding protein 1 & P17225 & Ptbp1 & $-0,3$ & 7,7 \\
\hline Polyubiquitin-B; & POCG49 & Ubb & $-0,4$ & 56,4 \\
\hline Porimin; & Q91Z22 & Tmem123 & 0,2 & 4,6 \\
\hline Ppfia1 protein; Protein Ppfia1; & $\mathrm{B} 2 \mathrm{R} \times \mathrm{Q} 2$ & Ppfia1 & 1,6 & 0,7 \\
\hline PRA1 family protein 3 ; & Q8R5J9 & Arl6ip5 & 1,4 & 5,9 \\
\hline Prefoldin subunit 1 & Q9CWM4 & Pfdn1 & $-0,4$ & 23 \\
\hline Prefoldin subunit 2 & 070591 & Pfdn2 & $-0,2$ & 36,4 \\
\hline Prefoldin subunit 3 & P61759 & Vbp1 & 0,3 & 13,3 \\
\hline Prefoldin subunit 5; & Q9WU28 & Pfdn5 & 0,4 & 41,6 \\
\hline Prefoldin subunit 6; & Q03958 & Pfdn6 & 0,5 & 34,7 \\
\hline Prelamin-A/C; & P48678 & Lmna & 0,3 & 25,7 \\
\hline Pre-mRNA 3'-end-processing factor FIP1; & Q9D824 & Fip1l1 & $-0,1$ & 10 \\
\hline Pre-mRNA-splicing factor ISY1 homolog; & Q69ZQ2 & Isy1 & 0,1 & 18,9 \\
\hline Pre-mRNA-splicing factor SPF27; & Q9D287 & Bcas2 & 0,6 & 5,8 \\
\hline Pre-mRNA-splicing factor SYF2; & Q9D198 & Syf2 & 0,2 & 10,7 \\
\hline Pre-rRNA-processing protein TSR2 homolog; & Q8C8T8 & Tsr2 & 0,1 & 17,8 \\
\hline PRKC apoptosis WT1 regulator protein; & Q925B0 & Pawr & 0,0 & 13,8 \\
\hline PRKR-interacting protein 1 & Q9CWV6 & Prkrip1 & 0,2 & 13,4 \\
\hline Proactivator polypeptide-like 1 ; & Q8C1C1 & Psapl1 & 0,6 & 12,3 \\
\hline Probable ATP-dependent RNA helicase DDX17; & Q501J6 & Ddx17 & 2,9 & 2,2 \\
\hline Probable D-tyrosyl-tRNA(Tyr) deacylase 2; & Q8BHA3 & Dtd2 & $-0,7$ & 9,5 \\
\hline Probable pleckstrin homology domain-containing family $\mathrm{N}$ member 1 ; & E9QP36 & Plekhn1 & $-0,5$ & 4,7 \\
\hline Probable RNA polymerase II nuclear localization protein SLC7A6OS; & Q7TPE5 & Slc7a6os & 1,7 & 7,5 \\
\hline Probable RNA-binding protein EIF1AD; & Q3THJ3 & Eif1ad & 0,0 & 31,2 \\
\hline Profilin-1; & P62962 & $\operatorname{Pfn} 1$ & 0,2 & 37,5 \\
\hline Programmed cell death 6-interacting protein; & Q9WU78 & Pdcd6ip & 0,3 & 2,1 \\
\hline Programmed cell death protein 10 & Q8VE70 & Pdcd10 & 0,1 & 21,2 \\
\hline Programmed cell death protein 5 & P56812 & Pdcd5 & $-0,3$ & 37,3 \\
\hline Programmed cell death protein 6 & P12815 & Pdcd6 & 0,8 & 22 \\
\hline Prohibitin; & P67778 & Phb & 1,2 & 30,5 \\
\hline Proliferating cell nuclear antigen; & P17918 & Pcna & 0,3 & 19,9 \\
\hline Proliferation-associated protein $2 \mathrm{G} 4$ & P50580 & $\mathrm{Pa} 2 \mathrm{~g} 4$ & 0,6 & 17,8 \\
\hline
\end{tabular}




\begin{tabular}{|c|c|c|c|c|}
\hline Protein & Uniprot & Gene name & $\begin{array}{c}\text { Fold change of } \\
\text { XPRESS ratio } \\
\text { (Adam17 } \\
\text { (AKC / WT) }\end{array}$ & $\begin{array}{c}\text { Sequence } \\
\text { coverage [\%] }\end{array}$ \\
\hline Proline and serine-rich protein 2 & Q8C5R2 & Proser2 & $-1,7$ & 5,1 \\
\hline Proline-rich AKT1 substrate 1 & Q9D1F4 & Akt1s1 & $-0,5$ & 16,3 \\
\hline Prolyl-tRNA synthetase associated domain-containing protein 1 ; & Q9D820 & Prorsd1 & 0,4 & 15,4 \\
\hline Prominin-2; & Q3UUY6 & Prom2 & $-0,1$ & 12,2 \\
\hline Prostaglandin E synthase 3; & Q9R0Q7 & Ptges3 & 1,0 & 28,1 \\
\hline Prostaglandin reductase 1 & Q91YR9 & Ptgr1 & $-0,6$ & 8,2 \\
\hline Prostatic acid phosphatase; & Q8CE08 & Acpp & $-0,6$ & 18,1 \\
\hline Proteasome activator complex subunit 1 & P97371 & Psme1 & 0,5 & 47,1 \\
\hline Proteasome activator complex subunit 2 & P97372 & Psme2 & 0,5 & 16,5 \\
\hline Proteasome activator complex subunit 3 & P61290 & Psme3 & $-1,4$ & 20,9 \\
\hline Proteasome maturation protein; & Q9CQT5 & Pomp & 0,3 & 22 \\
\hline Proteasome subunit alpha type-1; & Q9R1P4 & Psma1 & $-0,2$ & 33,1 \\
\hline Proteasome subunit alpha type-2; & P49722 & Psma2 & 0,2 & 9 \\
\hline Proteasome subunit alpha type- 3 ; & 070435 & Psma3 & 0,0 & 12,5 \\
\hline Proteasome subunit alpha type- 4 & Q9R1P0 & Psma4 & $-0,5$ & 13 \\
\hline Proteasome subunit alpha type-5; & Q9Z2U1 & Psma5 & 0,2 & 23,7 \\
\hline Proteasome subunit alpha type- 6 & Q9QUM9 & Psma6 & 0,0 & 26 \\
\hline Proteasome subunit alpha type-7; & Q9Z2U0 & Psma7 & $-0,1$ & 41,1 \\
\hline Proteasome subunit beta type- 1 ; & 009061 & Psmb1 & 0,4 & 31,7 \\
\hline Proteasome subunit beta type- 2 & Q9R1P3 & Psmb2 & 0,3 & 5 \\
\hline Proteasome subunit beta type-3; & Q9R1P1 & Psmb3 & $-0,1$ & 18,5 \\
\hline Proteasome subunit beta type-4; & P99026 & Psmb4 & 0,1 & 10,2 \\
\hline Proteasome subunit beta type-5; & 055234 & Psmb5 & $-0,3$ & 20,8 \\
\hline Proteasome subunit beta type- 6 ; & Q60692 & Psmb6 & 0,7 & 18,1 \\
\hline Proteasome subunit beta type-7; & P70195 & Psmb7 & 0,0 & 11,2 \\
\hline Protein 1100001G20Rik; RIKEN cDNA 1100001G20; & Q8BTE6 & 1100001G20Rik & 0,6 & 36,5 \\
\hline Protein 2010109I03Rik; RIKEN cDNA 2010109I03; & Q9CQ11 & 2010109I03Rik & 0,6 & 15,3 \\
\hline Protein 4.1; & P48193 & Epb41 & $-1,0$ & 4,7 \\
\hline $\begin{array}{l}\text { Protein 8030462N17Rik; RIKEN cDNA 8030462N17, isoform CRA_a; } \\
\text { Uncharacterized protein; }\end{array}$ & QOVAW6 & 8030462N17Rik & $-0,4$ & 7,5 \\
\hline Protein AATF; & Q9JKX4 & Aatf & $-0,2$ & 1,3 \\
\hline Protein Abhd12b; & G3UZN6 & Abhd12b & 0,6 & 5,3 \\
\hline Protein Adh6a; & E9Q4Y1 & Adh6a & $-0,9$ & 8,4 \\
\hline Protein Ahnak; & E9Q616 & Ahnak & $-0,1$ & 60,9 \\
\hline Protein Ahnak2; & Е9РYB0 & Ahnak2 & $-0,3$ & 38,2 \\
\hline Protein Ahnak2; & F7CVJ5 & Ahnak2 & $-0,4$ & 49 \\
\hline Protein Ahnak2; & F7DBB3 & Ahnak2 & $-0,2$ & 35,5 \\
\hline Protein Aim1l; & B7ZCC2 & Aim1l & $-0,7$ & 3,5 \\
\hline Protein Akap13; & E9Q394 & Akap13 & $-0,9$ & 4,4 \\
\hline Protein archease; & Q505B7 & Zbtb8os & $-0,2$ & 7,4 \\
\hline Protein Arhgef5; & E9Q7D5 & Arhgef5 & $-0,8$ & 1,1 \\
\hline Protein Auts2; & J3QNT9 & Auts2 & $-5,6$ & 14,6 \\
\hline Protein BC117090; & L7N257 & BC117090 & $-0,1$ & 62,5 \\
\hline Protein Bod1l; & E9Q6J5 & Bod1l & $-0,2$ & 1,7 \\
\hline Protein Bpifb9b; & A2AJD1 & Bpifb9b & 0,4 & 25 \\
\hline Protein BRICK1; & Q91VR8 & Brk1 & 0,2 & 26,7 \\
\hline Protein C10; & 035127 & Grcc10 & $-0,2$ & 36,5 \\
\hline Protein Cald1; & D3Z6I7 & Cald1 & $-0,6$ & 17,4 \\
\hline Protein canopy homolog 2 & Q9QXT0 & Cnpy2 & $-0,3$ & 41,2 \\
\hline Protein canopy homolog 3 & Q9DAU1 & Cnpy3 & $-0,1$ & 20,7 \\
\hline Protein canopy homolog 4 & Q8BQ47 & Cnpy4 & $-0,2$ & 22 \\
\hline Protein CASC3; & Q8K3W3 & Casc3 & 0,0 & 5,9 \\
\hline Protein CDV3; & Q4VAA2 & Cdv3 & $-0,6$ & 35,6 \\
\hline Protein CLEC16A; & Q80U30 & Clec16a & $-0,2$ & 1,2 \\
\hline Protein cordon-bleu; & Q5NBX1 & Cobl & $-0,8$ & 6,8 \\
\hline Protein Crnn; & D3YUU6 & Crnn & $-0,8$ & 1,3 \\
\hline Protein CutA; & Q9CQ89 & Cuta & 0,2 & 9,1 \\
\hline Protein Daple; & Q6VGS5 & Ccdc88c & $-0,5$ & 0,5 \\
\hline Protein Dcpp2; & E9PYC2 & Dcpp2 & 0,3 & 4,1 \\
\hline Protein DEK; & Q7TNV0 & Dek & 0,0 & 17,1 \\
\hline Protein disulfide-isomerase A3; & P27773 & Pdia3 & 0,3 & 51,3 \\
\hline Protein disulfide-isomerase A4; & P08003 & Pdia4 & $-0,2$ & 7,8 \\
\hline Protein disulfide-isomerase A6; & Q922R8 & Pdia6 & 0,6 & 25 \\
\hline Protein disulfide-isomerase; & P09103 & P4hb & 0,0 & 41,3 \\
\hline Protein DJ-1; & Q99LX0 & Park7 & 0,7 & 22,3 \\
\hline Protein dpy-30 homolog; & Q99LT0 & Dpy30 & $-0,3$ & 9,1 \\
\hline Protein Dr1; & Q91WV0 & Dr1 & $-1,1$ & 15,9 \\
\hline Protein enabled homolog; & Q03173 & Enah & 0,2 & 2,4 \\
\hline Protein FAM107B; & Н9н9т1 & Fam107b & $-0,3$ & 35,3 \\
\hline Protein FAM114A2; & Q8VE88 & Fam114a2 & $-0,7$ & 4 \\
\hline Protein FAM122A; & Q9DB52 & Fam122a & 0,2 & 3,9 \\
\hline Protein FAM136A; & Q9CR98 & Fam136a & 0,7 & 18,8 \\
\hline Protein FAM162A; & Q9D6U8 & Fam162a & 0,7 & 11,6 \\
\hline
\end{tabular}




\begin{tabular}{|c|c|c|c|c|}
\hline Protein & Uniprot & Gene name & $\begin{array}{c}\text { Fold change of } \\
\text { XPRESS ratio } \\
\left.\text { (Adam17 }{ }^{\Delta K C} / \mathrm{WT}\right)\end{array}$ & $\begin{array}{c}\text { Sequence } \\
\text { coverage [\%] }\end{array}$ \\
\hline Protein FAM177A1; & Q8BR63 & Fam177a1 & 0,3 & 26,1 \\
\hline Protein FAM192A; & Q91WE2 & Fam192a & $-0,3$ & 9,8 \\
\hline Protein FAM195B; & Q3UGS4 & Fam195b & $-0,1$ & 41,2 \\
\hline Protein FAM25C; & Q8CF02 & Fam25c & $-0,6$ & 61,8 \\
\hline Protein FAM32A; & Q9CR80 & Fam32a & $-3,9$ & 11,6 \\
\hline Protein FAM50A; & Q9WV03 & Fam50a & $-0,1$ & 8 \\
\hline Protein FAM63A; & Q76LS9 & Fam63a & $-0,6$ & 4,3 \\
\hline Protein FAM83B; & QOVBM2 & Fam83b & $-0,3$ & 6,7 \\
\hline Protein FAM83H; & Q148V8 & Fam83h & 0,4 & 5,9 \\
\hline Protein Fga; & E9PV24 & Fga & 1,4 & 15,3 \\
\hline Protein Gm14743; & A2BHD2 & Gm14743 & 0,4 & 39,2 \\
\hline Protein Gm14744; & B1AVU4 & Gm14744 & 0,4 & 37,6 \\
\hline Protein Gm5938; & A2AEN9 & Gm5938 & 0,2 & 41,7 \\
\hline Protein Gm6537; & J3QPU5 & Gm6537 & $-0,5$ & 25,9 \\
\hline Protein Gm6619; & F6URP1 & Gm6619 & 0,1 & 20,8 \\
\hline Protein Golgb1; & E9PVZ8 & Golgb1 & 0,3 & 4 \\
\hline Protein HEXIM1; & Q8R409 & Hexim1 & $-0,3$ & 21,6 \\
\hline Protein Kif13b; & E9Q4K7 & Kif13b & 0,5 & 1,7 \\
\hline Protein kinase $C$ and casein kinase substrate in neurons protein 2; & Q9WVE8 & Pacsin 2 & $-1,1$ & 3,3 \\
\hline Protein kinase $\mathrm{C}$ delta-binding protein; & Q91VJ2 & Prkcdbp & 0,2 & 13,5 \\
\hline Protein Krt78; & E9Q0F0 & Krt78 & 0,0 & 44,1 \\
\hline Protein LAP2; & Q80TH2 & Erbb2ip & $-1,0$ & 1 \\
\hline Protein lin-52 homolog; & Q8CD94 & $\operatorname{Lin} 52$ & $-0,2$ & 7,8 \\
\hline Protein lin-7 homolog C; & 088952 & $\operatorname{Lin} 7 c$ & 0,3 & 5,6 \\
\hline Protein LLP homolog; & Q9D945 & Llph & 0,4 & 11,4 \\
\hline Protein Lmo7; & E9PYF4 & Lmo7 & $-0,3$ & 5,8 \\
\hline Protein LSM14 homolog A; & $\mathrm{Q} 8 \mathrm{~K} 2 \mathrm{~F} 8$ & Lsm14a & $-1,5$ & 1,7 \\
\hline Protein lunapark; & Q7TQ95 & Lnp & 0,3 & 4,3 \\
\hline Protein LYRIC; & Q80WJ7 & Mtdh & $-0,3$ & 25 \\
\hline Protein LZIC; & Q8K3С3 & Lzic & 0,1 & 17,9 \\
\hline Protein max; & P28574 & Max & 0,1 & 13,2 \\
\hline Protein Mroh8; & E9PYI4 & Mroh8 & $-0,5$ & 1,3 \\
\hline Protein NDRG1; & Q62433 & Ndrg1 & 0,3 & 41 \\
\hline Protein NDRG2; & Q9QYG0 & Ndrg2 & 0,3 & 7,3 \\
\hline Protein Neb; & A2AQA9 & $\mathrm{Neb}$ & $-0,3$ & 3,8 \\
\hline Protein Nolc1; & E9Q5C9 & Nolc1 & $-0,1$ & 10,3 \\
\hline Protein Numa1; & E9Q7G0 & Numa1 & $-0,2$ & 0,8 \\
\hline Protein Nup153; & E9Q3G8 & Nup153 & 0,6 & 5,3 \\
\hline Protein OS-9; & $\mathrm{Q} 8 \mathrm{~K} 2 \mathrm{C} 7$ & Os9 & $-0,1$ & 1,9 \\
\hline Protein Pcdh1; Protocadherin 1; & Q8CFX3 & Pcdh1 & $-1,5$ & 3,4 \\
\hline Protein Pfdn4; & E9Q6U4 & Pfdn4 & 0,0 & 20,8 \\
\hline Protein phosphatase 1 regulatory subunit 11; & Q8K1L5 & Ppp1r11 & 0,4 & 7,5 \\
\hline Protein phosphatase 1 regulatory subunit $12 \mathrm{~A}$; & Q9DBR7 & Ppp1r12a & 0,2 & 10,2 \\
\hline Protein phosphatase 1 regulatory subunit 14A; & Q91VC7 & Ppp1r14a & $-0,8$ & 7,5 \\
\hline Protein phosphatase 1 regulatory subunit 14B; & Q62084 & Ppp1r14b & 0,2 & 29,9 \\
\hline Protein phosphatase 1 regulatory subunit 14C; & Q8R4S0 & Ppp1r14c & 0,4 & 22,6 \\
\hline Protein phosphatase 1 regulatory subunit 7; & Q3UM45 & Ppp1r7 & $-0,1$ & 24,1 \\
\hline Protein phosphatase 1G; & Q61074 & Ppm1g & $-0,3$ & 2,2 \\
\hline Protein phosphatase inhibitor 2 & Q9DCL8 & Ppp1r2 & $-0,4$ & 17,9 \\
\hline Protein POF1B; & Q8K4L4 & Pof1b & 0,3 & 31 \\
\hline Protein Prol1; & E9PYQ4 & Prol1 & 0,3 & 6,4 \\
\hline Protein PRRC1; & Q3UPH1 & Prrc1 & $-1,2$ & 3,4 \\
\hline Protein PRRC2A; & Q7TSC1 & Prrc2a & $-0,2$ & 1,9 \\
\hline Protein PRRC2B; & Q7TPM1 & Prrc2b & 0,4 & 2,3 \\
\hline Protein PRRC2C; & Q3TLH4 & Prrc2c & $-0,3$ & 9,3 \\
\hline Protein Pus7; & F6XMS4 & Pus7 & 0,5 & 11,4 \\
\hline Protein QIL1; & Q8R404 & Qil1 & 0,5 & 5,9 \\
\hline Protein Rarres1; & J3QNI9 & Rarres1 & $-0,2$ & 12 \\
\hline Protein Red; & Q9Z1M8 & $\mathrm{lk}$ & 2,3 & 3,2 \\
\hline Protein Rnf113a2; Ring finger protein $113 \mathrm{~A} 2$; & Q14B01 & Rnf113a2 & $-1,4$ & 3,3 \\
\hline Protein S100-A10; & P08207 & S100a10 & $-0,8$ & 17,5 \\
\hline Protein S100-A11; & P50543 & S100a11 & 0,5 & 43,9 \\
\hline Protein S100-A13; & P97352 & S100a13 & $-0,1$ & 40,8 \\
\hline Protein S100-A14; & Q9D2Q8 & S100a14 & 0,1 & 59,6 \\
\hline Protein S100a16; & D3Z2Y6 & S100a16 & 0,6 & 47,9 \\
\hline Protein S100-A3; & P62818 & S100a3 & $-3,3$ & 10,9 \\
\hline Protein S100-A6; & P14069 & S100a6 & 0,3 & 16,9 \\
\hline Protein S100-A9; & P31725 & S100a9 & 2,8 & 30,1 \\
\hline Protein Scaf11; & E9PZM7 & Scaf11 & $-0,5$ & 1,9 \\
\hline Protein Scgb2b17; & S4R1X8 & Scgb2b17 & 1,1 & 9,9 \\
\hline Protein Sec16a; & A2AIX1 & Sec16a & $-0,4$ & 3,9 \\
\hline Protein Serpinb3c; Serine (Or cysteine) peptidase inhibitor, clade B, & A2RSF9 & Serpinb3c & 0,0 & 28,8 \\
\hline
\end{tabular}




\begin{tabular}{|c|c|c|c|c|}
\hline Protein & Uniprot & Gene name & $\begin{array}{c}\text { Fold change of } \\
\text { XPRESS ratio } \\
\left.\text { (Adam } 17^{\Delta K C} / \mathrm{WT}\right)\end{array}$ & $\begin{array}{c}\text { Sequence } \\
\text { coverage [\%] }\end{array}$ \\
\hline Protein Serpinb6c; Serpinb6c protein; & Q6P6K7 & Serpinb6c & $-1,0$ & 12,4 \\
\hline Protein Serpinb6e; & I7HJI3 & Serpinb6e & 0,1 & 4,4 \\
\hline Protein SET; & Q9EQU5 & Set & $-0,2$ & 26 \\
\hline Protein Sf3b2; Splicing factor 3b, subunit 2; & Q3UJB0 & $\mathrm{sf} 3 \mathrm{~b} 2$ & 0,0 & 22 \\
\hline Protein SON; & Q9QX47 & Son & $-0,1$ & 1,5 \\
\hline Protein Spink5; Serine peptidase inhibitor, Kazal type 5; & Q148R4 & Spink5 & $-0,1$ & 23,4 \\
\hline Protein Srsf11; & D3Z4B0 & Srsf11 & $-0,3$ & 13 \\
\hline Protein Tex264; & E9Q137 & Tex264 & 0,4 & 7,8 \\
\hline Protein Tfg; TFG protein; Trk-fused; Trk-fused protein; & Q9Z1A1 & Tfg & $-0,1$ & 6,8 \\
\hline Protein Tmsb15b2; & A2AF31 & Tmsb15b2 & $-0,2$ & 37,5 \\
\hline Protein transport protein $\operatorname{Sec} 31 \mathrm{~A}$ & Q3UPL0 & Sec31a & 0,2 & 18,2 \\
\hline Protein transport protein Sec61 subunit beta; & Q9CQS8 & Sec61b & 0,8 & 21,9 \\
\hline Protein Trip11; & E9Q512 & Trip11 & $-0,4$ & 6,5 \\
\hline Protein TSSC4; & Q9JHE7 & Tssc4 & $-0,6$ & 30,6 \\
\hline Protein Vps13d; & B1ART1 & Vps13d & $-7,2$ & 0,9 \\
\hline Protein YIPF3; & Q3UDR8 & Yipf3 & $-0,5$ & 2,9 \\
\hline Protein-glutamine gamma-glutamyltransferase E; & Q08189 & Tgm3 & $-0,3$ & 8,8 \\
\hline Protein-glutamine gamma-glutamyltransferase K; & Q9JLF6 & $\operatorname{Tgm} 1$ & $-0,3$ & 2 \\
\hline Protein-L-isoaspartate(D-aspartate) O-methyltransferase; & P23506 & Pcmt1 & 0,4 & 6,2 \\
\hline Proteolipid protein 2; & Q9R1Q7 & Plp2 & 1,4 & 7,9 \\
\hline Prothymosin alpha; & P26350 & Ptma & $-0,5$ & 20,7 \\
\hline Pterin-4-alpha-carbinolamine dehydratase 2 & Q9CZL5 & Pcbd2 & 0,7 & 21,3 \\
\hline Pumilio homolog 1 & Q80U78 & Pum1 & $-0,6$ & 0,8 \\
\hline Pumilio homolog 2; & Q80U58 & Pum2 & $-0,6$ & 9,1 \\
\hline Purine nucleoside phosphorylase; & P23492 & Pnp & 1,3 & 3,5 \\
\hline Purkinje cell protein 4-like protein 1 & Q6W8Q3 & Pcp4l1 & $-0,3$ & 20,6 \\
\hline Putative ataxin-7-like protein 3B; & Q3UD01 & Atxn7|3b & 0,2 & 10,3 \\
\hline Putative peptidyl-tRNA hydrolase PTRHD1; & D3Z4S3 & Ptrhd1 & 1,7 & 8,6 \\
\hline Putative phospholipase B-like 2; & Q3TCN2 & Plbd2 & 0,4 & 4,2 \\
\hline Putative RNA-binding protein 3; & 089086 & Rbm3 & 0,6 & 34,6 \\
\hline Pyridoxine-5'-phosphate oxidase; & Q91XF0 & Pnpo & 1,1 & 8,8 \\
\hline Pyruvate kinase PKM; & P52480 & Pkm & $-0,1$ & 10,9 \\
\hline Rab GDP dissociation inhibitor beta; & Q61598 & Gdi2 & $-0,3$ & 10,8 \\
\hline Rab GTPase-activating protein 1 ; & A2AWA9 & Rabgap1 & 0,9 & 1,5 \\
\hline Rab GTPase-binding effector protein 2; & Q91WG2 & Rabep2 & 1,1 & 6,1 \\
\hline Rab11 family-interacting protein 1; & E9Q8L9 & Rab11fip1 & $-1,1$ & 3,4 \\
\hline Rabenosyn-5; & Q80Y56 & Zfyve20 & $-0,7$ & 0,9 \\
\hline Rab-like protein 6; & Q5U3K5 & Rabl6 & $-2,5$ & 2,3 \\
\hline Radixin; & P26043 & $\operatorname{Rdx}$ & $-0,5$ & 12,9 \\
\hline Ragulator complex protein LAMTOR1; & Q9CQ22 & Lamtor1 & 0,0 & 8,1 \\
\hline Ragulator complex protein LAMTOR5; & Q9D1L9 & Lamtor5 & $-0,4$ & 44 \\
\hline RalBP1-associated Eps domain-containing protein 1; & 054916 & Reps1 & $-0,2$ & 1,6 \\
\hline Ran-binding protein 3 & Q9CT10 & Ranbp3 & $-0,7$ & 2,9 \\
\hline Ran-specific GTPase-activating protein; & P34022 & Ranbp1 & $-0,4$ & 15,3 \\
\hline Ras GTPase-activating protein-binding protein 1; & P97855 & G3bp1 & 0,1 & 23 \\
\hline Ras GTPase-activating-like protein IQGAP1; & Q9JKF1 & Iqgap1 & $-0,3$ & 0,7 \\
\hline Ras-related protein Rab-10; & P61027 & Rab10 & $-0,6$ & 5,5 \\
\hline Ras-related protein Rab-11B; & P46638 & Rab11b & 0,9 & 36,2 \\
\hline Ras-related protein Rab-27B; & Q99P58 & Rab27b & 0,8 & 10,1 \\
\hline Ras-related protein Rab-5A; & Q9CQD1 & Rab5a & 1,2 & 5,6 \\
\hline Ras-related protein Rab-7a; & P51150 & Rab7a & $-2,5$ & 6,8 \\
\hline $\begin{array}{l}\text { Receptor accessory protein 5, isoform CRA_a; Receptor expression-enhancing } \\
\text { protein 5; }\end{array}$ & G3X8R0 & Reep5 & 0,4 & 9 \\
\hline Receptor expression-enhancing protein 6; & Q9JM62 & Reep6 & $-1,0$ & 10,5 \\
\hline Regulating synaptic membrane exocytosis protein 1 & Q99NE5 & Rims1 & $-6,3$ & 1,2 \\
\hline Regulation of nuclear pre-mRNA domain-containing protein 1B; & Q9CSU0 & Rprd1b & 2,8 & 4,6 \\
\hline Regulator of nonsense transcripts 1 & Q9EPU0 & Upf1 & 0,1 & 1,2 \\
\hline RelA-associated inhibitor; & Q5I1X5 & Ppp1r13। & $-0,6$ & 7,8 \\
\hline Renin receptor; & Q9CYN9 & Atp6ap2 & 0,6 & 3,7 \\
\hline Repetin; & P97347 & Rptn & 1,2 & 9,8 \\
\hline Reticulocalbin-1; & Q05186 & Ren1 & $-0,1$ & 28,3 \\
\hline Reticulocalbin-2; & Q8BP92 & Ren2 & $-0,1$ & 8,4 \\
\hline Reticulocalbin-3; & Q8BH97 & Ren3 & $-0,6$ & 17,9 \\
\hline Reticulon-3; & Q9ES97 & Rtn3 & 0,0 & 1,1 \\
\hline Reticulon-4; & Q99P72 & Rtn4 & $-0,2$ & 13,5 \\
\hline Retinoid-inducible serine carboxypeptidase; & Q920A5 & Scpep1 & 0,1 & 11,5 \\
\hline Retrotransposon gag domain-containing protein 1 ; & Q32KG4 & Rgag1 & $-6,3$ & 1,1 \\
\hline Retroviral-like aspartic protease 1 & Q09PK2 & Asprv1 & $-0,2$ & 14,7 \\
\hline Rho GDP-dissociation inhibitor 1 ; & Q99PT1 & Arhgdia & 0,1 & 15,2 \\
\hline Rho GDP-dissociation inhibitor 2; & Q61599 & Arhgdib & 0,0 & 10,5 \\
\hline Rho guanine nucleotide exchange factor 10 -like protein; & A2AWP8 & Arhgef10l & 0,0 & 1,6 \\
\hline Rho-related GTP-binding protein RhoC; & Q62159 & Rhoc & 0,9 & 16,8 \\
\hline Ribonuclease inhibitor; & Q91VI7 & Rnh1 & 0,0 & 9,6 \\
\hline
\end{tabular}




\begin{tabular}{|c|c|c|c|c|}
\hline Protein & Uniprot & Gene name & $\begin{array}{c}\text { Fold change of } \\
\text { XPRESS ratio } \\
\text { (Adam17 }^{\Delta K C} / \mathrm{WT} \text { ) }\end{array}$ & $\begin{array}{c}\text { Sequence } \\
\text { coverage [\%] }\end{array}$ \\
\hline Ribonuclease P protein subunit p25-like protein; & Q99JH1 & Rpp25I & 1,2 & 5,5 \\
\hline Ribonuclease pancreatic; & P00683 & Rnase1 & 0,8 & 22,1 \\
\hline Ribonuclease T2; & Q9CQ01 & Rnaset2 & $-1,6$ & 7,3 \\
\hline Ribonuclease UK114; & P52760 & Hrsp12 & 0,7 & 50,4 \\
\hline Ribosomal RNA processing protein 1 homolog A; & P56183 & Rrp1 & 0,9 & 3,4 \\
\hline Ribosome-binding protein 1 & Q99PL5 & Rrbp1 & $-0,5$ & 20,8 \\
\hline Ribosome-recycling factor, mitochondrial; & Q9D6S7 & Mrrf & 0,3 & 14,1 \\
\hline RISC-loading complex subunit TARBP2; & P97473 & Tarbp2 & $-0,5$ & 20,7 \\
\hline RNA polymerase II-associated protein 3 ; & Q9D706 & Rpap3 & $-0,6$ & 1,8 \\
\hline RNA-binding motif protein, $\mathrm{X}$ chromosome; & Q9WV02 & $\mathrm{Rbmx}$ & $-2,4$ & 14,3 \\
\hline RNA-binding motif, single-stranded-interacting protein 3; & Q8BWL5 & Rbms3 & 0,0 & 7,3 \\
\hline RNA-binding protein $8 \mathrm{~A}$ & Q9CWZ3 & Rbm8a & 0,4 & 10,9 \\
\hline RNA-binding protein EWS; & Q61545 & Ewsr1 & 0,5 & 12,6 \\
\hline RNA-binding protein FUS; & P56959 & Fus & $-0,2$ & 62,3 \\
\hline RNA-binding protein Raly; & Q64012 & Raly & $-0,5$ & 10,4 \\
\hline RNMT-activating mini protein; & Q9CQY2 & Fam103a1 & 0,2 & 7,8 \\
\hline RRP15-like protein; & Q9CYX7 & Rrp15 & 0,1 & 2,5 \\
\hline RWD domain-containing protein 4; & Q9CPR1 & Rwdd4 & 0,2 & 10,1 \\
\hline SAFB-like transcription modulator; & $\mathrm{Q} 8 \mathrm{CH} 25$ & Sltm & $-1,4$ & 1,2 \\
\hline SAP domain-containing ribonucleoprotein; & Q9D1J3 & Sarnp & 0,2 & 34,3 \\
\hline SAP30-binding protein; & Q02614 & Sap30bp & $-0,3$ & 3,9 \\
\hline Scaffold attachment factor B1; & D3YXK2 & Safb & $-0,1$ & 4,6 \\
\hline Scaffold attachment factor B2; & Q80YR5 & Safb2 & $-0,1$ & 5 \\
\hline Sciellin; & Q9EQG3 & Scel & $-0,8$ & 10,9 \\
\hline Secretoglobin family 2B member 2 & Q6UGQ3 & Scgb2b2 & 0,1 & 35,1 \\
\hline Secretoglobin family 2B member 20 ; & J3QK77 & Scgb2b20 & 0,1 & 21,4 \\
\hline Secretoglobin family 2B member 24 & Q7M747 & Scgb2b24 & 0,2 & 13,4 \\
\hline Secretory carrier-associated membrane protein 1 ; & Q8K021 & Scamp1 & $-1,0$ & 8 \\
\hline Secretory carrier-associated membrane protein 2; & Q9ERN0 & Scamp2 & 0,7 & 9,7 \\
\hline Selection and upkeep of intraepithelial T-cells protein 9; & A7TZG3 & Skint9 & 0,6 & 3,1 \\
\hline Selenide, water dikinase 1 & Q8BH69 & Sephs1 & 0,9 & 3,8 \\
\hline Selenium-binding protein 1 & P17563 & Selenbp1 & 0,0 & 14,4 \\
\hline Selenoprotein M; & Q8VHC3 & Selm & 0,2 & 14,5 \\
\hline Selenoprotein S; & Q9BCZ4 & Vimp & 0,5 & 11,1 \\
\hline Sequestosome-1; & Q64337 & Sqstm1 & 1,1 & 4,2 \\
\hline Serine/arginine repetitive matrix protein 2 ; & Q8BTI8 & Srrm2 & $-0,4$ & 1,1 \\
\hline Serine/arginine-rich splicing factor 1 ; & Q6PDM2 & Srsf1 & 0,2 & 39,1 \\
\hline Serine/arginine-rich splicing factor 10 & Q9R0U0 & Srsf10 & 0,9 & 9,9 \\
\hline Serine/arginine-rich splicing factor 2 ; & Q62093 & Srsf2 & 0,9 & 6,8 \\
\hline Serine/arginine-rich splicing factor 3 ; & P84104 & Srsf3 & 0,5 & 26,2 \\
\hline Serine/arginine-rich splicing factor 4 ; & Q8VE97 & Srsf4 & 0,1 & 4,5 \\
\hline Serine/arginine-rich splicing factor 5 ; & 035326 & Srsf5 & 0,8 & 18,2 \\
\hline Serine/arginine-rich splicing factor 6 ; & Q3TWW8 & Srsf6 & $-2,5$ & 8,8 \\
\hline Serine/arginine-rich splicing factor 7 ; & Q8BL97 & Srsf7 & 0,3 & 13,1 \\
\hline Serine/arginine-rich splicing factor 9 ; & Q9D0B0 & Srsf9 & 1,1 & 9 \\
\hline Serine/threonine-protein kinase LMTK2; & Q3TYD6 & Lmtk2 & $-0,7$ & 0,9 \\
\hline Serine/threonine-protein kinase MST4; & Q99JT2 & Mst4 & $-0,1$ & 4,3 \\
\hline Serine/threonine-protein kinase OSR1; & Q6P9R2 & Oxsr1 & $-0,5$ & 2,1 \\
\hline Serine/threonine-protein kinase PAK 2; & Q8CIN4 & Pak2 & 0,7 & 6,7 \\
\hline Serine/threonine-protein kinase WNK1; & P83741 & Wnk1 & $-0,3$ & 4,4 \\
\hline $\begin{array}{l}\text { Serine/threonine-protein phosphatase } 2 \mathrm{~A} 65 \mathrm{kDa} \text { regulatory subunit } \mathrm{A} \text { alpha } \\
\text { isoform; }\end{array}$ & Q76MZ3 & Ppp2r1a & $-0,2$ & 5,1 \\
\hline Serine/threonine-protein phosphatase 2A catalytic subunit beta isoform; & P62715 & Ppp2cb & 0,0 & 3,6 \\
\hline Serine/threonine-protein phosphatase 4 regulatory subunit 2; & Q0VGB7 & Ppp4r2 & 0,0 & 3,6 \\
\hline Serine/threonine-protein phosphatase 6 regulatory subunit 3; & Q922D4 & Ppp6r3 & $-0,9$ & 1,4 \\
\hline Serine/threonine-protein phosphatase PP1-alpha catalytic subunit; & P62137 & Ppp1ca & $-0,1$ & 4,3 \\
\hline Serine-threonine kinase receptor-associated protein; & Q9Z1Z2 & Strap & $-0,1$ & 4,3 \\
\hline Serotransferrin; & Q921I1 & Tf & 0,7 & 23 \\
\hline Serpin A12; & Q7TMF5 & Serpina12 & 0,9 & 19,1 \\
\hline Serpin A9; & Q9D7D2 & Serpina9 & 0,0 & 7,2 \\
\hline Serpin B12; & Q9D7P9 & Serpinb12 & $-0,3$ & 33,3 \\
\hline Serpin B5; & P70124 & Serpinb5 & $-0,2$ & 28,3 \\
\hline Serpin B7; & Q9D695 & Serpinb7 & $-0,7$ & 12,1 \\
\hline Serpin B8; & 008800 & Serpinb8 & $-0,5$ & 7,5 \\
\hline Serpin $\mathrm{H} 1$; & P19324 & Serpinh1 & 1,5 & 2,6 \\
\hline Serrate RNA effector molecule homolog; & Q99MR6 & Srrt & $-3,6$ & 1,5 \\
\hline Serum albumin; & P07724 & Alb & 0,4 & 44,1 \\
\hline Serum deprivation-response protein; & Q63918 & Sdpr & 0,2 & 3,8 \\
\hline SH2 domain-containing protein 4B; & A6X942 & $\operatorname{Sh} 2 d 4 b$ & $-4,2$ & 1,6 \\
\hline SH3 domain-binding glutamic acid-rich-like protein 3; & Q91VW3 & Sh3bgrl3 & 0,0 & 26,9 \\
\hline SH3 domain-binding glutamic acid-rich-like protein; & Q9JJU8 & Sh3bgrl & 0,2 & 46,5 \\
\hline SH3 domain-containing kinase-binding protein 1 ; & Q8R550 & Sh3kbp1 & $-0,4$ & 15,5 \\
\hline
\end{tabular}




\begin{tabular}{|c|c|c|c|c|}
\hline Protein & Uniprot & Gene name & $\begin{array}{c}\text { Fold change of } \\
\text { XPRESS ratio } \\
\left.\text { (Adam } 17^{\Delta K C} / \mathrm{WT}\right)\end{array}$ & $\begin{array}{c}\text { Sequence } \\
\text { coverage [\%] }\end{array}$ \\
\hline Shootin-1; & Q8K2Q9 & Kiaa1598 & 0,5 & 7 \\
\hline Short-chain dehydrogenase/reductase family $9 \mathrm{C}$ member 7 ; & Q8K3P0 & Sdr9c7 & 0,6 & 17,3 \\
\hline Sickle tail protein; & A2AQ25 & Skt & $-0,3$ & 1,4 \\
\hline Sideroflexin-1; & Q99JR1 & Sfxn1 & 0,0 & 15,8 \\
\hline Sideroflexin-3; & Q91V61 & Sfxn3 & 0,2 & 17,4 \\
\hline Signal peptide peptidase-like $2 \mathrm{~A}$; & Q9JJF9 & Sppl2a & $-0,3$ & 4,6 \\
\hline Signal transducing adapter molecule 1 & P70297 & Stam & 0,0 & 4,8 \\
\hline Signal transducing adapter molecule 2 & 088811 & Stam2 & $-1,9$ & 5,9 \\
\hline Signal-induced proliferation-associated 1-like protein 1 ; & Q8C0T5 & Sipa1l1 & 0,4 & 4,6 \\
\hline Single-stranded DNA-binding protein; & Q8R2K3 & Ssbp1 & 1,8 & 22,3 \\
\hline Sister chromatid cohesion protein PDS5 homolog A; & Q6A026 & Pds5a & $-0,8$ & 2,2 \\
\hline Sister chromatid cohesion protein PDS5 homolog B; & Q4VA53 & Pds5b & 0,4 & 0,5 \\
\hline Sjoegren syndrome nuclear autoantigen 1 homolog; & Q9JJ94 & Ssna1 & 0,3 & 20,2 \\
\hline SLAIN motif-containing protein 2 & Q8CI08 & Slain2 & $-0,3$ & 12,4 \\
\hline Small acidic protein; & Q9R0P4 & Smap & 0,1 & 21,5 \\
\hline Small EDRK-rich factor 1; & 088892 & Serf1 & 0,3 & 16,7 \\
\hline Small glutamine-rich tetratricopeptide repeat-containing protein alpha; & Q8BJU0 & Sgta & $-0,5$ & 27,3 \\
\hline Small nuclear ribonucleoprotein F; & P62307 & Snrpf & $-0,6$ & 9,3 \\
\hline Small nuclear ribonucleoprotein G; & P62309 & Snrpg & 0,3 & 15,8 \\
\hline Small nuclear ribonucleoprotein Sm D2; & P62317 & Snrpd2 & 0,2 & 32,2 \\
\hline Small nuclear ribonucleoprotein Sm D3; & P62320 & Snrpd3 & 0,8 & 31,7 \\
\hline Small proline-rich protein 2D; & 070555 & Sprr2d & 1,8 & 36,5 \\
\hline Small ubiquitin-related modifier 1 ; & P63166 & Sumo1 & $-0,4$ & 18,8 \\
\hline Small ubiquitin-related modifier 2 & P61957 & Sumo2 & $-0,2$ & 16,9 \\
\hline SNARE-associated protein Snapin; & Q9Z266 & Snapin & 0,2 & 26,5 \\
\hline SNW domain-containing protein 1 ; & Q9CSN1 & Snw1 & 0,6 & 7,3 \\
\hline Sodium/hydrogen exchanger 1 & Q61165 & Slc9a1 & $-0,5$ & 3,8 \\
\hline Sodium/potassium-transporting ATPase subunit beta- 1 ; & P14094 & Atp1b1 & 0,1 & 8,2 \\
\hline Sodium/potassium-transporting ATPase subunit beta-3; & P97370 & Atp1b3 & 1,0 & 24,8 \\
\hline Solute carrier family 12 member 2 & P55012 & Slc12a2 & $-1,2$ & 1,7 \\
\hline Sorbin and SH3 domain-containing protein 1 ; & Q62417 & Sorbs1 & $-0,6$ & 3,1 \\
\hline Sorcin; & Q6P069 & Sri & 1,0 & 35,9 \\
\hline Sorting nexin-1; & Q9WV80 & Snx1 & 0,0 & 17,1 \\
\hline Sorting nexin-12; & 070493 & Snx12 & 0,5 & 20,4 \\
\hline Sorting nexin-2; & Q9CWK8 & $\operatorname{Sn} \times 2$ & 0,2 & 8,1 \\
\hline Sorting nexin-3; & 070492 & Snx3 & 1,2 & 20,4 \\
\hline SPARC; & P07214 & Sparc & 0,2 & 15,6 \\
\hline Specifically androgen-regulated gene protein; & Q8BI29 & Sarg & $-0,3$ & 41,6 \\
\hline Spectrin alpha chain, non-erythrocytic 1 ; & P16546 & Sptan1 & 0,5 & 26,9 \\
\hline Spectrin beta chain, non-erythrocytic 1 ; & Q62261 & Sptbn1 & 0,1 & 2,5 \\
\hline Sperm-associated antigen 7; & Q7TNE3 & Spag7 & 0,0 & 20,3 \\
\hline Spermatogenesis-defective protein 39 homolog; & Q8BGQ1 & Vipas39 & $-1,0$ & 3,1 \\
\hline S-phase kinase-associated protein 1 & Q9WTX5 & Skp1 & $-0,4$ & 24,5 \\
\hline Sphingomyelin phosphodiesterase; & Q04519 & Smpd1 & 0,1 & 3,2 \\
\hline Spliceosome-associated protein CWC15 homolog; & Q9JHS9 & Cwc15 & $-0,2$ & 15,7 \\
\hline Splicing factor 1 & Q64213 & Sf1 & 0,4 & 14,6 \\
\hline Splicing factor 3 A subunit 1 & Q8K4Z5 & Sf3a1 & 0,2 & 2,5 \\
\hline Splicing factor 3B subunit 1 ; & Q99NB9 & Sf3b1 & $-0,8$ & 2,1 \\
\hline Splicing factor $3 B$ subunit 4 & Q8QZY9 & Sf3b4 & 0,6 & 3,3 \\
\hline Splicing factor 3B subunit 5; & Q923D4 & Sf3b5 & 0,1 & 25,6 \\
\hline Splicing factor, proline- and glutamine-rich; & Q8VIJ6 & Sfpq & $-0,7$ & 2,1 \\
\hline Src substrate cortactin; & Q60598 & Cttn & $-0,1$ & 29,9 \\
\hline SRSF protein kinase 1 ; & 070551 & Srpk1 & $-1,8$ & 5,9 \\
\hline StAR-related lipid transfer protein 9; & Q80TF6 & Stard9 & 0,9 & 1,7 \\
\hline Stathmin; & P54227 & Stmn1 & $-0,7$ & 43,1 \\
\hline Stefin-2; & P35174 & Stfa2 & $-1,4$ & 11,7 \\
\hline Stefin-3; & P35173 & Stfa3 & 0,2 & 68,9 \\
\hline Steroid receptor RNA activator 1 & Q80VJ2 & Sra1 & $-0,1$ & 8,1 \\
\hline Stress-70 protein, mitochondrial; & P38647 & Hspa9 & 0,9 & 24,7 \\
\hline Stress-induced-phosphoprotein 1 ; & Q60864 & Stip1 & 0,0 & 38,7 \\
\hline Stromal interaction molecule 1 ; & P70302 & Stim1 & $-0,2$ & 11,2 \\
\hline Submandibular gland protein C; & Q6JHY2 & Muc19 & $-0,3$ & 28,8 \\
\hline Succinate dehydrogenase [ubiquinone] iron-sulfur subunit, mitochondrial; & Q9CQA3 & Sdhb & 0,8 & 8,5 \\
\hline Sulfatase-modifying factor 2 & Q8BPG6 & Sumf2 & 0,7 & 10,6 \\
\hline Sulfated glycoprotein 1 & Q61207 & Psap & 0,4 & 21,2 \\
\hline Sulfhydryl oxidase 1 & Q8BND5 & Qsox1 & 0,2 & 2,8 \\
\hline SUMO-conjugating enzyme UBC9; & P63280 & Ube2i & 0,9 & 18,8 \\
\hline Superoxide dismutase $[\mathrm{Cu}-\mathrm{Zn}]$ & P08228 & Sod1 & $-0,5$ & 32,5 \\
\hline Superoxide dismutase [Mn], mitochondrial; & P09671 & Sod2 & 0,8 & 29,7 \\
\hline Suppressor of tumorigenicity 14 protein homolog; & P56677 & St14 & 1,0 & 3,2 \\
\hline Suprabasin; & Е9QPB2 & Sbsn & $-0,4$ & 68,2 \\
\hline
\end{tabular}




\begin{tabular}{|c|c|c|c|c|}
\hline Protein & Uniprot & Gene name & $\begin{array}{c}\text { Fold change of } \\
\text { XPRESS ratio } \\
\left.\text { (Adam } 17^{\Delta K C} / \mathrm{WT}\right)\end{array}$ & $\begin{array}{c}\text { Sequence } \\
\text { coverage [\%] }\end{array}$ \\
\hline Survival of motor neuron-related-splicing factor 30 & Q8BGT7 & Smndc1 & 0,1 & 29,8 \\
\hline Sushi domain-containing protein 2; & Q9DBX3 & Susd2 & $-0,2$ & 3,2 \\
\hline Sushi repeat-containing protein SRPX2; & Q8R054 & Srpx2 & 4,8 & 3,4 \\
\hline SUZ domain-containing protein 1 & Q6NXN1 & Szrd1 & $-0,3$ & 41,7 \\
\hline Swi5-dependent recombination DNA repair protein 1 homolog; & Q8BP27 & Sfr1 & 0,0 & 6,9 \\
\hline Synapse-associated protein 1 & Q9D5V6 & Syap1 & $-0,3$ & 9,9 \\
\hline Synaptic vesicle membrane protein VAT-1 homolog; & Q62465 & Vat1 & $-0,4$ & 5,2 \\
\hline Synaptojanin-2-binding protein; & Q9D6K5 & Synj2bp & 0,4 & 15,9 \\
\hline Synaptosomal-associated protein 23 & 009044 & Snap23 & $-0,1$ & 35,8 \\
\hline Synaptosomal-associated protein 29 & Q9ERB0 & Snap29 & $-0,1$ & 23,1 \\
\hline Synaptotagmin-like protein 1 & A2AE94 & Sytl1 & $-0,7$ & 9,7 \\
\hline Syndecan-1; & P18828 & Sdc1 & 2,3 & 9,6 \\
\hline Syndecan-4; & 035988 & Sdc4 & 0,0 & 22,7 \\
\hline Synergin gamma; & Q5SV85 & Synrg & $-0,9$ & 6,3 \\
\hline Syntaxin-12; & Q9ER00 & Stx12 & 0,0 & 31,8 \\
\hline Syntaxin-16; & Q8BVI5 & Stx16 & 0,4 & 3,5 \\
\hline Syntaxin-4; & P70452 & Stx4 & 1,2 & 8,4 \\
\hline Syntaxin-6; & Q9JKK1 & Stx6 & $-4,2$ & 8,6 \\
\hline Syntaxin-7; & 070439 & Stx7 & $-0,1$ & 19,9 \\
\hline Syntaxin-8; & 088983 & Stx8 & 0,3 & 15,3 \\
\hline Talin-1; & P26039 & Tln1 & $-0,1$ & 3,9 \\
\hline Target of Myb protein 1 ; & 088746 & Tom1 & $-0,4$ & 8,3 \\
\hline TATA element modulatory factor; & B9EKI3 & Tmf1 & $-0,5$ & 2,8 \\
\hline Tax1-binding protein 3; & Q9DBG9 & Tax1bp3 & 0,7 & 20,2 \\
\hline T-complex protein 1 subunit beta; & P80314 & Cct2 & $-0,1$ & 3 \\
\hline T-complex protein 1 subunit delta; & P80315 & Cct4 & 0,0 & 2,6 \\
\hline Tetraspanin-9; & Q8BJU2 & Tspan9 & 0,5 & 8,7 \\
\hline Tetratricopeptide repeat protein 1 ; & Q91Z38 & Ttc1 & $-0,1$ & 10 \\
\hline Thimet oligopeptidase; & Q8C1A5 & Thop1 & $-0,1$ & 24,7 \\
\hline Thioredoxin domain-containing protein 12 & Q9CQU0 & Txndc12 & 0,3 & 22,9 \\
\hline Thioredoxin domain-containing protein 15 & Q6P6J9 & Txndc15 & $-0,1$ & 4,1 \\
\hline Thioredoxin domain-containing protein 17 & Q9CQM5 & Txndc17 & 0,1 & 26,8 \\
\hline Thioredoxin domain-containing protein 5; & Q91W90 & Txndc5 & 0,0 & 35,5 \\
\hline Thioredoxin; & P10639 & Txn & 0,6 & 32,4 \\
\hline Thioredoxin-related transmembrane protein 1 ; & Q8VBT0 & Tmx1 & 1,4 & 20,2 \\
\hline Thioredoxin-related transmembrane protein 4 ; & Q8C0L0 & $\operatorname{Tm} \times 4$ & $-0,4$ & 7,1 \\
\hline THO complex subunit 4 & 008583 & Alyref & 0,1 & 25,1 \\
\hline Three prime repair exonuclease 2 & Q9R1A9 & Trex2 & $-0,3$ & 32,6 \\
\hline THUMP domain-containing protein 1 ; & Q99J36 & Thumpd1 & 1,2 & 15,7 \\
\hline Thy-1 membrane glycoprotein; & P01831 & Thy1 & $-0,8$ & 25,3 \\
\hline Thyroid hormone receptor-associated protein 3 ; & Q569Z6 & Thrap3 & 0,9 & 2,8 \\
\hline Tight junction protein ZO-1; & P39447 & Tjp1 & 0,9 & 4,6 \\
\hline Tight junction protein ZO-2; & Q9Z0U1 & Tjp2 & $-1,0$ & 2,7 \\
\hline Tissue factor; & P20352 & F3 & $-0,2$ & 14,3 \\
\hline Torsin-1A-interacting protein 2 & Q8BYU6 & Tor1aip2 & 0,1 & 2,4 \\
\hline TP53-regulated inhibitor of apoptosis 1 & Q9D8Z2 & Triap1 & $-0,4$ & 18,4 \\
\hline TRAF family member-associated NF-kappa-B activator; & P70347 & Tank & $-5,6$ & 5,6 \\
\hline Transaldolase; & Q93092 & Taldo1 & 0,0 & 7,1 \\
\hline Transcription activator BRG1; & Q3TKT4 & Smarca4 & $-0,6$ & 0,9 \\
\hline Transcription and mRNA export factor ENY2; & Q9JIX0 & Eny2 & 0,3 & 33,7 \\
\hline Transcription cofactor vestigial-like protein 4; & Q80V24 & Vgll4 & $-2,2$ & 3,5 \\
\hline Transcription elongation factor A protein 1 ; & P10711 & Tcea1 & 0,2 & 19,3 \\
\hline Transcription elongation factor A protein-like 5; & Q8CCT4 & Tceal5 & 0,0 & 10,2 \\
\hline Transcription elongation factor B polypeptide 2 & P62869 & Tceb2 & 0,1 & 56,8 \\
\hline Transcription factor A, mitochondrial; & P40630 & Tfam & $-10,0$ & 6,3 \\
\hline Transcription factor BTF3 homolog 4; & Q9CQH7 & Btf3|4 & $-0,1$ & 24,1 \\
\hline Transcription factor BTF3; & Q64152 & Btf3 & 0,7 & 12,7 \\
\hline Transcription factor jun-D; & P15066 & Jund & 0,5 & 8,5 \\
\hline Transcription factor $\mathrm{Sp} 1$ & 089090 & Sp1 & $-1,2$ & 30,2 \\
\hline Transcription factor Sp6; & Q9ESX2 & Sp6 & $-0,6$ & 6,1 \\
\hline Transcription initiation factor IIA subunit 1 ; & Q99PM3 & Gtf2a1 & 0,0 & 2,9 \\
\hline Transcription initiation factor TFIID subunit 10; & Q8KOH5 & Taf10 & $-0,8$ & 17,9 \\
\hline Transcription initiation factor TFIID subunit 7; & Q9R1C0 & Taf7 & 0,4 & 6,2 \\
\hline Transcription initiation factor TFIID subunit 9B; & Q6NZA9 & Taf9b & 0,9 & 3,2 \\
\hline Transcription intermediary factor 1-beta; & Q62318 & Trim28 & $-2,5$ & 1,3 \\
\hline Transcriptional activator protein Pur-alpha; & P42669 & Pura & $-0,3$ & 2,8 \\
\hline Transcriptional activator protein Pur-beta; & 035295 & Purb & 0,5 & 5,2 \\
\hline Transcriptional repressor p66-beta; & Q8VHR5 & Gatad2b & $-0,6$ & 2,4 \\
\hline Transforming acidic coiled-coil-containing protein 2; & E9Q8T1 & Tacc2 & $-0,3$ & 29,2 \\
\hline Transforming acidic coiled-coil-containing protein 2 & E9Q9Z4 & Tacc2 & $-0,3$ & 20 \\
\hline Transgelin; & P37804 & Tagln & $-0,7$ & 5,5 \\
\hline Transgelin-2; & Q9WVA4 & Tagln2 & 0,6 & 55,3 \\
\hline Trans-Golgi network integral membrane protein 1 ; & Q62313 & Tgoln1 & 0,3 & 15,6 \\
\hline
\end{tabular}


Supplementary table 2: List of all identified and quantified proteins in the quantitative proteome comparison of wild-type and $A d a m 17^{\Delta K C}$ epidermis at P3 (replicate 2).

\begin{tabular}{|c|c|c|c|c|}
\hline Protein & Uniprot & Gene name & $\begin{array}{c}\text { Fold change of } \\
\text { XPRESS ratio } \\
\left.\text { (Adam } 17^{\Delta K C} / \mathrm{WT}\right)\end{array}$ & $\begin{array}{c}\text { Sequence } \\
\text { coverage [\%] }\end{array}$ \\
\hline 10 kDa heat shock protein, mitochondrial; & Q64433 & Hspe1 & $-0,1$ & 54,9 \\
\hline 14 kDa phosphohistidine phosphatase; & Q9DAK9 & Phpt1 & 1,2 & 8,9 \\
\hline 14-3-3 protein beta/alpha; & Q9CQV8 & Ywhab & $-1,3$ & 13 \\
\hline 14-3-3 protein epsilon; & P62259 & Ywhae & 0,6 & 39,2 \\
\hline 14-3-3 protein eta; & P68510 & Ywhah & 0,1 & 13 \\
\hline 14-3-3 protein gamma; & P61982 & Ywhag & 0,1 & 17 \\
\hline 14-3-3 protein sigma; & 070456 & Sfn & 0,4 & 40,7 \\
\hline 14-3-3 protein theta; & P68254 & Ywhaq & 0,4 & 20,8 \\
\hline 14-3-3 protein zeta/delta; & P63101 & Ywhaz & 0,8 & 41,6 \\
\hline 182 kDa tankyrase-1-binding protein; & P58871 & Tnks1bp1 & $-0,2$ & 42,2 \\
\hline $26 \mathrm{~S}$ protease regulatory subunit $10 \mathrm{~B}$; & P62334 & Psmc6 & 0,3 & 6,4 \\
\hline 265 protease regulatory subunit 4 & P62192 & Psmc1 & $-0,5$ & 2 \\
\hline $26 S$ protease regulatory subunit $6 \mathrm{~A} ;$ & 088685 & Psmc3 & 0,0 & 12,5 \\
\hline $26 \mathrm{~S}$ protease regulatory subunit $6 \mathrm{~B}$; & P54775 & Psmc4 & $-0,2$ & 8,9 \\
\hline 265 protease regulatory subunit 7 & P46471 & Psmc2 & $-0,1$ & 9,9 \\
\hline 265 proteasome non-ATPase regulatory subunit $1 ;$ & Q3TXS7 & Psmd1 & $-0,1$ & 19,4 \\
\hline $26 \mathrm{~S}$ proteasome non-ATPase regulatory subunit 2; & Q8VDM4 & Psmd2 & $-0,4$ & 5 \\
\hline $26 \mathrm{~S}$ proteasome non-ATPase regulatory subunit 3; & P14685 & Psmd3 & $-0,6$ & 2,1 \\
\hline $26 \mathrm{~S}$ proteasome non-ATPase regulatory subunit 4 & 035226 & Psmd4 & 0,0 & 25 \\
\hline 265 proteasome non-ATPase regulatory subunit 4; & F7Al87 & Psmd4 & 0,1 & 10,3 \\
\hline $26 \mathrm{~S}$ proteasome non-ATPase regulatory subunit 7; & P26516 & Psmd7 & 0,6 & 13,4 \\
\hline 265 proteasome non-ATPase regulatory subunit 9; & Q9CR00 & Psmd9 & $-0,1$ & 18,9 \\
\hline 28 kDa heat- and acid-stable phosphoprotein; & Q3UHX2 & Pdap1 & $-0,2$ & 45,9 \\
\hline $28 \mathrm{~S}$ ribosomal protein S34, mitochondrial; & Q9JIK9 & Mrps34 & 0,0 & 11 \\
\hline $28 \mathrm{~S}$ ribosomal protein S36, mitochondrial; & Q9CQX8 & Mrps36 & $-0,2$ & 69,6 \\
\hline $28 \mathrm{~S}$ ribosomal protein S36, mitochondrial; & Q9D6T9 & Mrps36 & $-0,1$ & 69 \\
\hline $28 \mathrm{~S}$ ribosomal protein S7, mitochondrial; & Q80X85 & Mrps7 & 0,5 & 5 \\
\hline 2-amino-3-ketobutyrate coenzyme A ligase, mitochondrial; & 088986 & Gcat & $-0,9$ & 3,4 \\
\hline 2'-deoxynucleoside 5'-phosphate N-hydrolase 1; & Q80VJ3 & Dnph1 & 1,1 & 16,2 \\
\hline $39 \mathrm{~S}$ ribosomal protein $\mathrm{L} 12$, mitochondrial; & Q9DB15 & Mrpl12 & $-0,2$ & 17,9 \\
\hline $39 S$ ribosomal protein L17, mitochondrial; & Q9D8P4 & Mrpl17 & $-0,4$ & 11,4 \\
\hline $39 S$ ribosomal protein L41, mitochondrial; & Q9CQN7 & Mrpl41 & $-0,2$ & 23,7 \\
\hline $39 S$ ribosomal protein L43, mitochondrial; & Q99N89 & Mrpl43 & $-0,2$ & 7,5 \\
\hline 3-hydroxyisobutyrate dehydrogenase, mitochondrial; & Q99L13 & Hibadh & 0,9 & 4,2 \\
\hline 3-ketoacyl-CoA thiolase A, peroxisomal; & Q921H8 & Acaa1a & 0,2 & 15,3 \\
\hline 3-ketoacyl-CoA thiolase, mitochondrial; & Q8BWT1 & Acaa2 & 0,4 & 10,6 \\
\hline $40 S$ ribosomal protein S10; & P63325 & Rps10 & $-0,2$ & 28,5 \\
\hline $40 S$ ribosomal protein $\mathrm{S} 11$ & P62281 & Rps11 & $-0,4$ & 9,5 \\
\hline $40 S$ ribosomal protein $\mathrm{S} 12$ & Q6ZWZ6 & Rps12 & 0,1 & 53 \\
\hline $40 S$ ribosomal protein $\mathrm{S} 13$ & P62301 & Rps13 & 0,9 & 25,8 \\
\hline $40 S$ ribosomal protein $\mathrm{S} 14$ & P62264 & Rps14 & 0,0 & 8,6 \\
\hline 40 S ribosomal protein S15; & P62843 & Rps15 & $-0,2$ & 10,2 \\
\hline 40 S ribosomal protein $\mathrm{S} 16$ & P14131 & Rps16 & 0,1 & 14,4 \\
\hline $40 S$ ribosomal protein S17; & P63276 & Rps17 & $-0,1$ & 7,4 \\
\hline $40 S$ ribosomal protein S18; & P62270 & Rps18 & $-0,1$ & 32,9 \\
\hline $40 S$ ribosomal protein S19; & Q9CZX8 & Rps19 & $-0,3$ & 27,5 \\
\hline 40 S ribosomal protein S2; & P25444 & Rps2 & 0,2 & 14 \\
\hline 40 S ribosomal protein S20; & P60867 & Rps20 & 0,3 & 25,2 \\
\hline 40 S ribosomal protein S21; & Q9CQR2 & Rps21 & $-0,3$ & 39,8 \\
\hline 40 S ribosomal protein S25; & P62852 & Rps25 & $-0,2$ & 22,4 \\
\hline 40S ribosomal protein S27-like; & Q6ZWY3 & Rps27l & 0,1 & 14,3 \\
\hline $40 S$ ribosomal protein S28; & P62858 & Rps28 & $-0,2$ & 37,5 \\
\hline $40 \mathrm{~S}$ ribosomal protein $\mathrm{S} 3$ & P62908 & Rps3 & $-5,3$ & 4,7 \\
\hline $40 \mathrm{~S}$ ribosomal protein $\mathrm{S} 30$ & P62862 & Fau & $-0,5$ & 16,9 \\
\hline $40 \mathrm{~S}$ ribosomal protein $\mathrm{S} 3 \mathrm{a}$ & P97351 & Rps3a & 0,2 & 21,6 \\
\hline $40 S$ ribosomal protein $\mathrm{S} 4, \mathrm{X}$ isoform; & P62702 & $\operatorname{Rps} 4 x$ & $-0,4$ & 7,3 \\
\hline $40 \mathrm{~S}$ ribosomal protein $\mathrm{S} 5$ & D3YYM6 & Rps5 & 0,0 & 30,2 \\
\hline $40 \mathrm{~S}$ ribosomal protein $\mathrm{S} 8$ & P62242 & Rps8 & $-0,6$ & 17,8 \\
\hline $40 \mathrm{~S}$ ribosomal protein $\mathrm{SA}$ & P14206 & Rpsa & 0,6 & 31,5 \\
\hline 45 kDa calcium-binding protein; & Q61112 & Sdf4 & 0,4 & 2,8 \\
\hline 4F2 cell-surface antigen heavy chain; & P10852 & Slc3a2 & $-0,3$ & 14,1 \\
\hline 5'(3')-deoxyribonucleotidase, cytosolic type; & Q9JM14 & $\mathrm{Nt5c}$ & 0,6 & 20 \\
\hline 5'-3' exoribonuclease $1 ;$ & P97789 & Xrn1 & $-0,3$ & 1,3 \\
\hline 5-hydroxyisourate hydrolase; & Q9CRB3 & Urah & $-2,0$ & 19,5 \\
\hline $60 \mathrm{kDa}$ heat shock protein, mitochondrial; & P63038 & Hspd1 & 0,2 & 31,6 \\
\hline 605 acidic ribosomal protein $\mathrm{P0}$ & P14869 & Rplp0 & 0,2 & 29,7 \\
\hline 605 acidic ribosomal protein $\mathrm{P} 1$ & P47955 & Rplp1 & 0,0 & 14 \\
\hline $60 S$ acidic ribosomal protein P2; & P99027 & Rplp2 & $-0,3$ & 67 \\
\hline $60 S$ ribosomal protein $\mathrm{L} 10 \mathrm{a}$ & P53026 & Rpl10a & $-0,4$ & 6 \\
\hline $60 S$ ribosomal protein L11; & Q9CXW4 & Rpl11 & 0,0 & 5,2 \\
\hline
\end{tabular}




\begin{tabular}{|c|c|c|c|c|}
\hline Protein & Uniprot & Gene name & $\begin{array}{c}\text { Fold change of } \\
\text { XPRESS ratio } \\
\left.\text { (Adam } 17^{\Delta K C} / \mathrm{WT}\right)\end{array}$ & $\begin{array}{c}\text { Sequence } \\
\text { coverage [\%] }\end{array}$ \\
\hline $60 \mathrm{~S}$ ribosomal protein L12; & P35979 & Rpl12 & 0,0 & 35,8 \\
\hline $60 S$ ribosomal protein L13; & P47963 & Rpl13 & 0,1 & 24,2 \\
\hline 60S ribosomal protein L14; & Q9CR57 & Rpl14 & $-0,3$ & 11,5 \\
\hline $60 S$ ribosomal protein L17; & Q9CPR4 & Rpl17 & 0,2 & 31,5 \\
\hline $60 S$ ribosomal protein L18; & P35980 & Rpl18 & $-0,2$ & 9,6 \\
\hline $60 S$ ribosomal protein L19; & P84099 & Rpl19 & 0,1 & 4,6 \\
\hline 60 S ribosomal protein $\mathrm{L} 22$ & P67984 & Rpl22 & $-0,6$ & 18,8 \\
\hline $60 \mathrm{~S}$ ribosomal protein L22-like 1 ; & Q9D7S7 & Rpl22I1 & $-1,5$ & 9,8 \\
\hline $60 S$ ribosomal protein $\mathrm{L} 23$ & P62830 & Rpl23 & 0,7 & 20 \\
\hline $60 S$ ribosomal protein L23a; & P62751 & Rpl23a & $-0,3$ & 14,7 \\
\hline $60 S$ ribosomal protein L24; & Q8BP67 & Rpl24 & $-0,3$ & 6,8 \\
\hline 60 S ribosomal protein $\mathrm{L} 26$ & P61255 & Rpl26 & $-0,4$ & 8,7 \\
\hline $60 S$ ribosomal protein $\mathrm{L} 28$ & P41105 & Rpl28 & $-0,2$ & 8 \\
\hline $60 S$ ribosomal protein $\mathrm{L} 31$ & P62900 & Rpl31 & 0,0 & 18,4 \\
\hline 60 S ribosomal protein L38; & Q9Jग18 & Rpl38 & $-0,3$ & 24,3 \\
\hline 60 S ribosomal protein L39; & P62892 & Rpl39 & 0,2 & 19,6 \\
\hline $60 S$ ribosomal protein $L 4$ & Q9D8E6 & Rpl4 & $-0,1$ & 2,1 \\
\hline $60 \mathrm{~S}$ ribosomal protein $\mathrm{L} 6$ & P47911 & Rpl6 & $-0,4$ & 9,1 \\
\hline $60 \mathrm{~S}$ ribosomal protein L7; & P14148 & Rpl7 & $-0,2$ & 7,8 \\
\hline 6-phosphogluconolactonase; & Q9CQ60 & Pgls & 0,5 & 29,2 \\
\hline 78 kDa glucose-regulated protein; & P20029 & Hspa5 & 0,2 & 36,2 \\
\hline 85/88 kDa calcium-independent phospholipase A2; & P97819 & Pla2g6 & 6,3 & 1,6 \\
\hline Abl interactor 1 & Q8CBW3 & Abi1 & 0,1 & 18,3 \\
\hline Acid ceramidase; & Q9WV54 & Asah1 & $-0,2$ & 12,2 \\
\hline Acidic leucine-rich nuclear phosphoprotein 32 family member B; & Q9EST5 & Anp32b & 0,1 & 14,3 \\
\hline Acidic leucine-rich nuclear phosphoprotein 32 family member E; & P97822 & Anp32e & 0,3 & 12,1 \\
\hline Aconitate hydratase, mitochondrial; & Q99Kı0 & Aco2 & 0,7 & 5,4 \\
\hline Actin, aortic smooth muscle; & P62737 & Acta2 & 0,3 & 35 \\
\hline Actin-binding LIM protein 1; & Q8K4G5 & Ablim1 & $-0,2$ & 24,6 \\
\hline Actin-related protein $2 / 3$ complex subunit $1 B$; & Q9WV32 & Arpc1b & 1,4 & 29,5 \\
\hline Actin-related protein $2 / 3$ complex subunit 3 & Q9JM76 & Arpc3 & 0,4 & 8,7 \\
\hline Actin-related protein $2 / 3$ complex subunit 5 ; & Q9CPW4 & Arpc5 & 0,4 & 29,1 \\
\hline Actin-related protein $2 / 3$ complex subunit 5 -like protein; & Q9D898 & Arpc5I & $-0,3$ & 33,3 \\
\hline Actin-related protein 3 & Q99JY9 & Actr3 & 0,3 & 3,3 \\
\hline Activated RNA polymerase II transcriptional coactivator $\mathrm{p} 15$; & P11031 & Sub1 & 0,3 & 15,7 \\
\hline Acyl carrier protein, mitochondrial; & Q9CR21 & Ndufab1 & 0,2 & 7,1 \\
\hline Acyl-CoA dehydrogenase family member 9 , mitochondrial; & Q8JZN5 & Acad9 & $-0,1$ & 1,6 \\
\hline Acyl-CoA-binding domain-containing protein 6 & Q9D061 & Acbd6 & $-0,6$ & 2,8 \\
\hline Acyl-CoA-binding protein; & P31786 & Dbi & $-0,1$ & 39,1 \\
\hline Acyl-CoA-binding protein; & D3Z563 & $\mathrm{Dbi}$ & 0,0 & 41,3 \\
\hline Acylphosphatase-1; & P56376 & Acyp1 & 0,5 & 48,5 \\
\hline Adapter molecule crk; & Q64010 & Crk & 0,3 & 6,3 \\
\hline $\begin{array}{l}\text { Adapter molecule crk; Crk protein; V-crk sarcoma virus CT10 oncogene homolog } \\
\text { (Avian), isoform CRA_c; }\end{array}$ & Q8JZR2 & Crk & $-0,9$ & 10,8 \\
\hline Adenosine deaminase; & P03958 & Ada & $-1,8$ & 4 \\
\hline Adenylate kinase 2, mitochondrial; & Q9WTP6 & Ak2 & 0,5 & 20,9 \\
\hline Adenylate kinase isoenzyme 1 ; & Q9R0Y5 & Ak1 & 0,9 & 12,9 \\
\hline Adenylyl cyclase-associated protein 1 ; & P40124 & Cap1 & 0,2 & 6,1 \\
\hline Adiponectin; & Q60994 & Adipoq & 1,2 & 6 \\
\hline ADP/ATP translocase $1 ;$ & P48962 & Slc25a4 & 0,0 & 19,8 \\
\hline ADP/ATP translocase 2 & P51881 & Slc25a5 & $-0,4$ & 22,8 \\
\hline ADP-ribosylation factor GTPase-activating protein 1 ; & Q9EPJ9 & Arfgap1 & $-0,3$ & 2,3 \\
\hline ADP-ribosylation factor GTPase-activating protein 2; & Q99K28 & Arfgap2 & 0,7 & 9,4 \\
\hline ADP-sugar pyrophosphatase; & Q9JKX6 & Nudt5 & 0,0 & 28,9 \\
\hline Adrenodoxin-like protein, mitochondrial; & Q9CPW2 & Fdx1l & 0,5 & 5,7 \\
\hline Afadin; & Q9QZQ1 & Mllt4 & $-0,5$ & 2,8 \\
\hline Aftiphilin; & Q80WT5 & Aftph & $-2,0$ & 3,7 \\
\hline A-kinase anchor protein 12 & Q9WTQ5 & Akap12 & 0,4 & 7,6 \\
\hline A-kinase anchor protein 2 & A2API8 & Akap2 & 0,1 & 5,2 \\
\hline Alanine aminotransferase 1 & Q8QZR5 & Gpt & 0,2 & 2,6 \\
\hline Alcohol dehydrogenase $1 ;$ & P00329 & Adh1 & 0,2 & 2,4 \\
\hline Aldehyde dehydrogenase, mitochondrial; & P47738 & Aldh2 & 0,9 & 5,4 \\
\hline Aldehyde dehydrogenase; & E9Q3E1 & Aldh3b2 & 0,1 & 15,4 \\
\hline $\begin{array}{l}\text { Allergen dl chain C2A; Androgen binding protein beta; Androgen-binding } \\
\text { protein; Protein Scgb2b27; }\end{array}$ & Q8R1E9 & Scgb2b27 & 0,6 & 20,5 \\
\hline Allograft inflammatory factor 1 -like; & Q9EQX4 & Aif1l & $-0,2$ & 16,7 \\
\hline $\begin{array}{l}\text { Alpha globin 1; Alpha globin 2; Alpha-globin; Hemoglobin alpha, adult chain 1; } \\
\text { Hemoglobin subunit alpha; Protein Hba-a2; }\end{array}$ & Q91VB8 & Hba a1 & $-0,5$ & 62 \\
\hline Alpha/beta hydrolase domain-containing protein 14B; & Q8VCR7 & Abhd14b & 0,8 & 10 \\
\hline Alpha-1-acid glycoprotein 1 & Q60590 & Orm1 & 0,7 & 15 \\
\hline Alpha-1-antitrypsin 1-1; & P07758 & Serpina1a & 0,8 & 23,8 \\
\hline Alpha-1-antitrypsin 1-2; & P22599 & Serpina1b & 0,1 & 27,8 \\
\hline Alpha-1-antitrypsin 1-4; & Q00897 & Serpina1d & 1,3 & 25,4 \\
\hline
\end{tabular}




\begin{tabular}{|c|c|c|c|c|}
\hline Protein & Uniprot & Gene name & $\begin{array}{c}\text { Fold change of } \\
\text { XPRESS ratio } \\
\left.\text { (Adam17 }^{\Delta K C} / \mathrm{WT}\right)\end{array}$ & $\begin{array}{c}\text { Sequence } \\
\text { coverage [\%] }\end{array}$ \\
\hline Alpha-2-antiplasmin; & Q61247 & Serpinf2 & 10,0 & 2,9 \\
\hline Alpha-2-HS-glycoprotein; & P29699 & Ahsg & 0,0 & 20,3 \\
\hline Alpha-2-macroglobulin receptor-associated protein; & P55302 & Lrpap1 & 0,2 & 24,7 \\
\hline Alpha-2-macroglobulin; & Q61838 & $\mathrm{A} 2 \mathrm{~m}$ & 0,2 & 4,8 \\
\hline Alpha-actinin-4; & P57780 & Actn4 & 0,6 & 25 \\
\hline Alpha-adducin; & Q9QYCo & Add1 & 0,1 & 4,1 \\
\hline Alpha-endosulfine; & P60840 & Ensa & 0,0 & 23,1 \\
\hline Alpha-enolase; & P17182 & Eno1 & 0,6 & 18,4 \\
\hline Alpha-fetoprotein; & P02772 & Afp & 0,8 & 42,8 \\
\hline Alpha-synuclein; & 055042 & Snca & $-1,6$ & 11,4 \\
\hline Alpha-taxilin; & Q6PAM1 & Txlna & $-0,2$ & 13,9 \\
\hline Aminoacyl tRNA synthase complex-interacting multifunctional protein 1; & P31230 & Aimp1 & $-0,4$ & 11,9 \\
\hline AN1-type zinc finger protein 2B; & Q91X58 & Zfand $2 b$ & $-0,3$ & 3,2 \\
\hline AN1-type zinc finger protein 5; & 088878 & Zfand5 & $-0,9$ & 17,3 \\
\hline AN1-type zinc finger protein 6; & Q9DCH6 & Zfand6 & $-1,1$ & 9,4 \\
\hline Anamorsin; & Q8WTY4 & Ciapin1 & 0,0 & 15,9 \\
\hline Anaphase-promoting complex subunit 13 ; & Q8R034 & Anapc13 & $-0,4$ & 13,5 \\
\hline Anaphase-promoting complex subunit 16 & Q9CPV2 & Anapc16 & 0,2 & 46,9 \\
\hline Anaphase-promoting complex subunit CDC26; & Q99JP4 & Cdc26 & $-0,3$ & 9,4 \\
\hline Androgen binding protein alpha; Androgen-binding protein; Protein Scgb1b27; & Q91WB5 & Scgb1b27 & $-1,7$ & 19,6 \\
\hline $\begin{array}{l}\text { Androgen binding protein gamma; MCG17480; Protein Scgb2b26; Salivary } \\
\text { androgen-binding protein gamma subunit; }\end{array}$ & Q8JZX1 & Scgb2b26 & 0,5 & 40,2 \\
\hline $\begin{array}{l}\text { Androgen-binding protein; Androgen-binding protein eta; Lacrimal androgen- } \\
\text { binding protein eta; Protein Scgb1b2; }\end{array}$ & 035176 & Scgb1b2 & 0,2 & 31,2 \\
\hline Ankyrin-3; & G5E8K5 & Ank3 & $-0,3$ & 6,4 \\
\hline Annexin A1; & P10107 & Anxa1 & 0,2 & 36,7 \\
\hline Annexin A11; & P97384 & Anxa11 & 0,4 & 3,6 \\
\hline Annexin A2; & P07356 & Anxa2 & 0,8 & 46,6 \\
\hline Annexin A5; & P48036 & Anxa5 & $-0,1$ & 35,1 \\
\hline Annexin A6; & P14824 & Anxa6 & $-0,3$ & 7,9 \\
\hline Annexin A7; & Q07076 & Anxa7 & $-0,2$ & 3,5 \\
\hline Annexin A8; & 035640 & Anxa8 & $-0,3$ & 13,3 \\
\hline Antithrombin-III; & P32261 & Serpinc1 & 0,7 & 7,5 \\
\hline AP2-associated protein kinase 1 ; & Q3UHJO & Aak1 & $-0,2$ & 4,4 \\
\hline AP-3 complex subunit beta- 2 ; & Q9JME5 & Ap3b2 & 3,7 & 3,4 \\
\hline Apolipoprotein A-I; & Q00623 & Apoa1 & 0,5 & 35,2 \\
\hline Apolipoprotein A-II; & P09813 & Apoa2 & 0,4 & 9,8 \\
\hline Apolipoprotein A-IV; & P06728 & Apoa4 & $-0,1$ & 56,7 \\
\hline Apolipoprotein C-I; & P34928 & Apoc1 & 0,2 & 20,5 \\
\hline Apolipoprotein C-III; & P33622 & Apoc3 & $-1,2$ & 19,2 \\
\hline Apolipoprotein E; & P08226 & Apoe & $-0,5$ & 28,6 \\
\hline Apolipoprotein O; & Q9DCZ4 & Apoo & $-0,4$ & 11 \\
\hline Apoptosis inhibitor 5 & 035841 & Api5 & 1,0 & 13,7 \\
\hline Apoptosis-associated speck-like protein containing a CARD; & Q9EPB4 & Pycard & 0,0 & 17,6 \\
\hline Apoptotic chromatin condensation inducer in the nucleus; & Q9JIX8 & Acin 1 & $-0,5$ & 11,8 \\
\hline Arachidonate 12-lipoxygenase, 12R-type; & 070582 & Alox12b & 0,1 & 1,6 \\
\hline Arf-GAP domain and FG repeat-containing protein 1; & Q8K2K6 & Agfg1 & $-0,4$ & 11,9 \\
\hline Arfip1 protein; Protein Arfip1; & A2RSX9 & Arfip1 & $-0,3$ & 13,8 \\
\hline Arginase-1; & Q61176 & Arg1 & 0,3 & 3,4 \\
\hline $\begin{array}{l}\text { Arginine-rich, mutated in early stage tumors, isoform CRA_b; Mesencephalic } \\
\text { astrocyte-derived neurotrophic factor; }\end{array}$ & Q3TMX5 & Manf & 0,0 & 35,8 \\
\hline Arpin; & Q9D0A3 & Arpin & 0,1 & 6,6 \\
\hline Aryl hydrocarbon receptor; & P30561 & Ahr & $-0,4$ & 1,1 \\
\hline Aspartate aminotransferase, cytoplasmic; & P05201 & Got1 & 1,1 & 3,6 \\
\hline Aspartate aminotransferase, mitochondrial; & P05202 & Got2 & 0,6 & 29,1 \\
\hline Astrocytic phosphoprotein PEA-15; & Q62048 & Pea15 & $-0,4$ & 34,6 \\
\hline Ataxin-2-like protein; & Q3TGG2 & Atxn2l & $-0,6$ & 5,8 \\
\hline ATP synthase protein 8 & Р03930 & Mtatp8 & $-0,2$ & 14,9 \\
\hline ATP synthase subunit alpha, mitochondrial; & Q03265 & Atp5a1 & 0,0 & 15,9 \\
\hline ATP synthase subunit beta, mitochondrial; & P56480 & Atp5b & 0,4 & 16,3 \\
\hline ATP synthase subunit d, mitochondrial; & Q9DCX2 & Atp5h & 0,4 & 46,3 \\
\hline ATP synthase subunit delta, mitochondrial; & Q9D3D9 & Atp5d & 0,2 & 13,7 \\
\hline ATP synthase subunit e, mitochondrial; & Q06185 & Atp5i & 0,0 & 32,4 \\
\hline ATP synthase subunit $f$, mitochondrial; & P56135 & Atp5j2 & 0,4 & 15,8 \\
\hline ATP synthase subunit g, mitochondrial; & Q9CPQ8 & Atp5I & 0,9 & 27,2 \\
\hline ATP synthase subunit $O$, mitochondrial; & Q9DB20 & Atp5o & 0,5 & 21,6 \\
\hline ATP synthase-coupling factor 6 , mitochondrial; & P97450 & Atp5j & $-0,2$ & 41,7 \\
\hline ATPase inhibitor, mitochondrial; & E9PV44 & Atpif1 & 0,3 & 21,6 \\
\hline ATP-binding cassette sub-family A member 13 ; & Q5SSE9 & Abca13 & $-1,0$ & 0,5 \\
\hline ATP-binding cassette sub-family $\mathrm{F}$ member 1 ; & Q6P542 & Abcf1 & $-0,9$ & 4,7 \\
\hline ATP-dependent Clp protease proteolytic subunit, mitochondrial; & 088696 & Clpp & 0,8 & 6,6 \\
\hline
\end{tabular}




\begin{tabular}{|c|c|c|c|c|}
\hline Protein & Uniprot & Gene name & 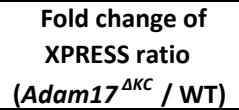 & $\begin{array}{c}\text { Sequence } \\
\text { coverage [\%] }\end{array}$ \\
\hline ATP-dependent RNA helicase DDX39A; & Q8VDW0 & Ddx39a & 0,0 & 4,8 \\
\hline ATP-dependent RNA helicase DDX3X; & Q62167 & $\operatorname{Ddx} 3 x$ & $-0,4$ & 4,5 \\
\hline Atrial natriuretic peptide-converting enzyme; & Q97319 & Corin & 0,7 & 1,1 \\
\hline AT-rich interactive domain-containing protein $1 \mathrm{~A}$; & $\mathrm{A} 2 \mathrm{BH} 40$ & Arid1a & $-0,3$ & 1,8 \\
\hline Aurora kinase B; & E9PVP1 & Aim1 & $-0,8$ & 10,2 \\
\hline Autophagy-related protein 13 & Q91YI1 & Atg13 & $-0,2$ & 2,5 \\
\hline BAG family molecular chaperone regulator 1 ; & Q60739 & Bag1 & $-0,4$ & 11,5 \\
\hline BAG family molecular chaperone regulator 3 ; & Q9JLV1 & Bag3 & $-0,8$ & 27 \\
\hline Band 4.1-like protein 1 & Q9Z2H5 & Epb4111 & $-0,1$ & 5,1 \\
\hline Band 4.1-like protein 2; & 070318 & Epb41/2 & 0,1 & 2,7 \\
\hline Band 4.1-like protein 3; & Q9WV92 & Epb4113 & 0,0 & 5,5 \\
\hline Barrier-to-autointegration factor; & 054962 & Banf1 & 0,1 & 34,8 \\
\hline Basal cell adhesion molecule; & Q9R069 & Bcam & 2,3 & 5,5 \\
\hline Basigin; & P18572 & Bsg & $-0,1$ & 27,9 \\
\hline B-cell CLL/lymphoma 7 protein family member C; & 008664 & $\mathrm{Bcl} 7 \mathrm{c}$ & $-0,1$ & 10,1 \\
\hline B-cell CLL/lymphoma 9-like protein; & Q67FY2 & Bc|9| & $-0,2$ & 3,1 \\
\hline B-cell linker protein; & Q9QUN3 & Blnk & $-0,6$ & 7,3 \\
\hline B-cell receptor-associated protein 31 & Q61335 & Bcap31 & $-0,4$ & 4,1 \\
\hline $\mathrm{Bcl} 2$ antagonist of cell death; & Q61337 & Bad & $-0,3$ & 29,6 \\
\hline BCL2/adenovirus E1B 19 kDa protein-interacting protein 3-like; & Q9Z2F7 & Bnip3l & $-0,3$ & 7,4 \\
\hline $\mathrm{Bcl}$-2/adenovirus E1B 19 kDa-interacting protein 2-like protein; & Q99JU7 & Bnipl & $-0,7$ & 8,2 \\
\hline Bcl-2-like protein 13 & P59017 & $\mathrm{Bcl} 2 \mathrm{|13}$ & $-0,4$ & 2,5 \\
\hline BET1-like protein; & 035153 & Bet1l & $-0,2$ & 11,7 \\
\hline Beta-2-glycoprotein 1; & Q01339 & Apoh & 0,7 & 11 \\
\hline Beta-actin-like protein 2; & Q8BFZ3 & Actbl2 & 0,2 & 14,4 \\
\hline Beta-catenin-interacting protein 1 ; & Q9JJN6 & Ctnnbip1 & $-0,3$ & 29,6 \\
\hline Beta-galactosidase; & P23780 & Glb1 & 0,2 & 1,9 \\
\hline Beta-globin; Protein Hbb-bs; Protein Hbb-bt; & A8DUK4 & Hbbt1 & $-0,3$ & 87,8 \\
\hline Beta-hexosaminidase subunit beta; & P20060 & Hexb & $-0,6$ & 5 \\
\hline BH3-interacting domain death agonist; & P70444 & Bid & 0,4 & 8,7 \\
\hline Bicaudal D-related protein 2; & Q8CHW5 & Ccdc64b & $-0,2$ & 6,9 \\
\hline Bifunctional glutamate/proline--tRNA ligase; & Q8CGC7 & Eprs & 0,2 & 1,2 \\
\hline Biogenesis of lysosome-related organelles complex 1 subunit 2; & Q9CWG9 & Bloc1s2 & $-0,1$ & 5,6 \\
\hline Biorientation of chromosomes in cell division protein 1 & Q5SQY2 & Bod1 & 0,0 & 27,7 \\
\hline Bis(5'-adenosyl)-triphosphatase; & 089106 & Fhit & 0,0 & 12,9 \\
\hline Bleomycin hydrolase; & Q8R016 & Blmh & $-0,2$ & 23,7 \\
\hline BolA-like protein 1 & Q9D8S9 & Bola1 & $-0,1$ & 34,3 \\
\hline BolA-like protein 2; & Q8BGS2 & Bola2 & 0,5 & 32,6 \\
\hline BPI fold-containing family A member 2 ; & P07743 & Bpifa2 & $-1,6$ & 19,4 \\
\hline Brain acid soluble protein 1 & Q91XV3 & Basp1 & 0,2 & 32,3 \\
\hline Brain-specific angiogenesis inhibitor 1-associated protein 2; & Q8BKX1 & Baiap2 & $-0,6$ & 14 \\
\hline BRI3-binding protein; & Q8BXV2 & Bri3bp & 0,2 & 3,6 \\
\hline Bridging integrator 2; & D3Z6Q9 & Bin2 & $-0,1$ & 3,2 \\
\hline Bromodomain-containing protein 4 ; & Q9ESU6 & Brd4 & $-0,4$ & 1,7 \\
\hline BSD domain-containing protein 1 ; & Q80Y55 & Bsdc1 & $-0,5$ & 5,9 \\
\hline BUB3-interacting and GLEBS motif-containing protein ZNF207; & E9PW12 & Znf207 & $-0,2$ & 5,6 \\
\hline Cadherin EGF LAG seven-pass G-type receptor 1 & 035161 & Celsr1 & $-2,0$ & 0,7 \\
\hline Cadherin EGF LAG seven-pass G-type receptor 2; & Q9R0M0 & Celsr2 & $-3,5$ & 2,4 \\
\hline Cadherin-1; & P09803 & Cdh1 & $-0,2$ & 14,5 \\
\hline Cadherin-13; & Q9WTR5 & Cdh13 & $-0,1$ & 9,9 \\
\hline Calcineurin B homologous protein 1 ; & P61022 & Chp1 & 0,2 & 26,2 \\
\hline Calcineurin subunit $B$ type 1 & Q63810 & Ppp3r1 & 0,4 & 15,3 \\
\hline Calcium/calmodulin-dependent protein kinase type II subunit delta; & Q6PHZ2 & Camk2d & 0,0 & 19,2 \\
\hline $\begin{array}{l}\text { Calcium-activated chloride channel regulator 2; Chloride channel calcium } \\
\text { activated 2; Endothelial chloride channel; MCG120735; }\end{array}$ & Q9EQR4 & Clca2 & 0,1 & 8,2 \\
\hline Calcium-binding mitochondrial carrier protein SCaMC-1; & Q8BMD8 & Slc25a24 & 0,3 & 7,2 \\
\hline Calcium-binding protein 39 & Q06138 & Cab39 & 0,3 & 12 \\
\hline Calcium-independent phospholipase A2-gamma; & Q8K1N1 & Pnpla8 & $-1,0$ & 5,9 \\
\hline Calcium-regulated heat stable protein $1 ;$ & Q9CR86 & Carhsp1 & 0,2 & 10,8 \\
\hline Calmodulin; & P62204 & Calm3 & 0,1 & 31,5 \\
\hline Calmodulin; & G3UZ90 & Calm3 & $-0,8$ & 30,8 \\
\hline Calmodulin-4; & Q9JM83 & Calm4 & $-0,4$ & 60,8 \\
\hline Calmodulin-like protein 3; & Q9D6P8 & Calml3 & $-0,1$ & 19,5 \\
\hline Calmodulin-regulated spectrin-associated protein 3; & Q80VC9 & Camsap3 & $-0,2$ & 1,7 \\
\hline Calnexin; & P35564 & Canx & $-0,1$ & 14,7 \\
\hline Calpain small subunit 2 ; & Q9D7J7 & Capns2 & 0,1 & 13,8 \\
\hline Calpain-1 catalytic subunit; & 035350 & Capn1 & $-0,3$ & 4,1 \\
\hline Calpastatin; & P51125 & Cast & $-0,2$ & 32,5 \\
\hline Calponin-2; & Q08093 & Cnn2 & 0,2 & 9,8 \\
\hline Calponin-3; & Q9DAW9 & Cnn3 & 0,6 & 9,4 \\
\hline Calreticulin; & P14211 & Calr & 0,3 & 32,5 \\
\hline Calumenin; & 035887 & Calu & 0,1 & 30,5 \\
\hline Calumenin; Calumenin, isoform CRA_b; Cardiac calumenin isoform; & Q6XLQ8 & Calu & 0,0 & 25,1 \\
\hline
\end{tabular}




\begin{tabular}{|c|c|c|c|c|}
\hline Protein & Uniprot & Gene name & $\begin{array}{c}\text { Fold change of } \\
\text { XPRESS ratio } \\
\left.\text { (Adam } 17^{\Delta K C} / \mathrm{WT}\right)\end{array}$ & $\begin{array}{l}\text { Sequence } \\
\text { coverage [\%] }\end{array}$ \\
\hline cAMP-dependent protein kinase inhibitor alpha; & P63248 & Pkia & $-0,3$ & 27,6 \\
\hline cAMP-regulated phosphoprotein 19 & P56212 & Arpp19 & $-0,4$ & 23,2 \\
\hline CAP-Gly domain-containing linker protein 1 ; & Q922J3 & Clip1 & 0,5 & 26,9 \\
\hline $\begin{array}{l}\text { Capping protein (Actin filament), gelsolin-like; Capping protein (Actin filament), } \\
\text { gelsolin-like, isoform CRA_a; Macrophage-capping protein; }\end{array}$ & Q99LB4 & Capg & 0,8 & 18,9 \\
\hline Caprin-1; & Q60865 & Caprin1 & $-0,2$ & 9,6 \\
\hline Carbonic anhydrase 3 ; & P16015 & $\mathrm{Ca} 3$ & 0,8 & 13,1 \\
\hline Carbonic anhydrase 6; & P18761 & $\mathrm{Ca} 6$ & 0,7 & 10,7 \\
\hline Carboxylesterase 1C; & P23953 & Ces1c & 1,2 & 1,6 \\
\hline Carboxypeptidase A4; & Q6P8K8 & Cpa4 & $-0,3$ & 5 \\
\hline Carboxypeptidase E; & Q00493 & Cpe & $-0,4$ & 11,3 \\
\hline Carboxypeptidase M; & Q80V42 & $\mathrm{Cpm}$ & 0,1 & 9 \\
\hline Casein kinase II subunit alpha'; & 054833 & Csnk2a2 & $-0,3$ & 3,7 \\
\hline Caspase activity and apoptosis inhibitor 1 & Q8VDY9 & Caap1 & 0,9 & 5,6 \\
\hline Caspase-14; & 089094 & Casp14 & $-0,2$ & 37,7 \\
\hline Catalase; & P24270 & Cat & 0,3 & 34,9 \\
\hline Catenin alpha-1; & P26231 & Ctnna1 & 0,6 & 15,7 \\
\hline Catenin delta-1; & P30999 & Ctnnd1 & $-0,8$ & 7,3 \\
\hline Cathelin-related antimicrobial peptide; & P51437 & Camp & 2,0 & 9,2 \\
\hline Cathepsin B; & P10605 & Ctsb & $-0,1$ & 15,3 \\
\hline Cathepsin D; & P18242 & Ctsd & $-0,1$ & 19,5 \\
\hline Cathepsin L1; & P06797 & Ctsl & 0,1 & 18,6 \\
\hline Cation-dependent mannose-6-phosphate receptor; & P24668 & M6pr & 1,6 & 7,9 \\
\hline Cation-independent mannose-6-phosphate receptor; & Q07113 & $\operatorname{lgf} 2 r$ & $-0,3$ & 0,8 \\
\hline Caveolin-1; & P49817 & Cav1 & 0,1 & 24,7 \\
\hline CCR4-NOT transcription complex subunit 3; & Q8KOV4 & Cnot3 & $-0,2$ & 4,7 \\
\hline CD109 antigen; & Q8R422 & Cd109 & 0,4 & 7,2 \\
\hline CD166 antigen; & Q61490 & Alcam & 0,2 & 21,4 \\
\hline CD2-associated protein; & Q9JLQ0 & Cd2ap & 0,0 & 24,8 \\
\hline CD44 antigen; & P15379 & $\mathrm{Cd} 44$ & 0,3 & 11,2 \\
\hline CD63 antigen; & P41731 & Cd63 & $-0,2$ & 7,6 \\
\hline CD82 antigen; & P40237 & $\mathrm{Cd} 82$ & $-0,3$ & 7,5 \\
\hline CD9 antigen; & P40240 & $\mathrm{Cd} 9$ & 0,3 & 3,1 \\
\hline Cdc42 effector protein 4 & Q9JM96 & Cdc42ep4 & 0,6 & 33,7 \\
\hline Cdc42 effector protein 5 & Q9Z0X0 & Cdc42ep5 & 0,4 & 32,9 \\
\hline CDGSH iron-sulfur domain-containing protein 2; & Q9CQB5 & Cisd2 & $-0,6$ & 31,9 \\
\hline CDKN2AIP N-terminal-like protein; & Q9D211 & Cdkn2aipnl & 0,2 & 8,6 \\
\hline Cell adhesion molecule 1 & Q8R5M8 & Cadm1 & $-0,2$ & 5,6 \\
\hline Cell cycle control protein $50 \mathrm{~A}$; & Q8VEKO & Tmem30a & 0,0 & 4,9 \\
\hline Cell death regulator Aven; & Q9D9K3 & Aven & $-0,1$ & 19,8 \\
\hline Cell surface glycoprotein MUC18; & Q8R2Y2 & Mcam & $-0,2$ & 5,1 \\
\hline Cellular nucleic acid-binding protein; & P53996 & Cnbp & 0,3 & 39,3 \\
\hline Cellular nucleic acid-binding protein; & D3YYF6 & Cnbp & 0,2 & 40,3 \\
\hline Centrin-2; & Q9R1K9 & Cetn2 & $-0,2$ & 14,4 \\
\hline Centrin-3; & 035648 & Cetn3 & 0,2 & 7,2 \\
\hline Centrosomal protein of $170 \mathrm{kDa}$ protein $\mathrm{B}$; & Q80U49 & Cep170b & 0,6 & 1,5 \\
\hline CGG triplet repeat-binding protein 1 ; & Q8BHG9 & Cggbp1 & $-0,3$ & 11,4 \\
\hline Charged multivesicular body protein $1 \mathrm{a}$; & Q921W0 & Chmp1a & 0,0 & 16,8 \\
\hline Charged multivesicular body protein $2 \mathrm{~b}$; & Q8BJF9 & Chmp2b & 0,0 & 9,4 \\
\hline Charged multivesicular body protein $4 \mathrm{~b}$; & Q9D8B3 & Chmp4b & $-0,3$ & 25,9 \\
\hline Charged multivesicular body protein 5 & Q9D7S9 & Chmp5 & $-0,2$ & 10,5 \\
\hline Chitinase-3-like protein 1 ; & Q61362 & Chi3l1 & 1,3 & 14,5 \\
\hline Chitotriosidase-1; & Q9D7Q1 & Chit1 & 0,0 & 14,2 \\
\hline Chloride intracellular channel protein 1 ; & Q9Z1Q5 & Clic1 & 0,1 & 11,2 \\
\hline Chloride intracellular channel protein 3 ; & Q9D7P7 & Clic3 & $-0,1$ & 6 \\
\hline Choline transporter-like protein 2; & Q8BY89 & Slc44a2 & 0,2 & 2,4 \\
\hline Chromatin complexes subunit BAP18; & Q9DCT6 & Bap18 & $-0,2$ & 54,3 \\
\hline Chromobox protein homolog 1; & P83917 & $\mathrm{Cb} \times 1$ & 0,5 & 12,4 \\
\hline Chromobox protein homolog 3; & P23198 & $\mathrm{Cb} \times 3$ & 0,2 & 13,1 \\
\hline Chromobox protein homolog 5; & Q61686 & $\mathrm{Cb} \times 5$ & $-3,2$ & 10,5 \\
\hline Chromogranin-A; & P26339 & Chga & $-0,3$ & 1,9 \\
\hline Cingulin; & P59242 & Cgn & $-0,6$ & 10,9 \\
\hline Citrate synthase, mitochondrial; & Q9CZU6 & Cs & 0,3 & 9,9 \\
\hline C-Jun-amino-terminal kinase-interacting protein 4 ; & Q58A65 & Spag9 & $-0,6$ & 2,5 \\
\hline Clathrin interactor 1 & Q99KN9 & Clint1 & 0,0 & 17,5 \\
\hline Clathrin light chain $A$; & B1AWEO & Clta & $-0,4$ & 28,2 \\
\hline Clathrin light chain B; & Q6IRU5 & Cltb & $-0,3$ & 26,2 \\
\hline Claudin-1; & 088551 & Cldn1 & 0,5 & 7,1 \\
\hline Cleavage stimulation factor subunit 2 & Q8BIQ5 & Cstf2 & 0,7 & 10,9 \\
\hline Clusterin; & Q06890 & $\mathrm{Clu}$ & $-0,1$ & 25,2 \\
\hline C-Myc-binding protein; & Q9EQS3 & Mycbp & 0,3 & 62,1 \\
\hline Coactosin-like protein; & Q9CQI6 & Cotl1 & 0,3 & 5,6 \\
\hline Cob(I)yrinic acid a,c-diamide adenosyltransferase, mitochondrial; & Q9D273 & Mmab & 0,4 & 14,3 \\
\hline
\end{tabular}




\begin{tabular}{|c|c|c|c|c|}
\hline Protein & Uniprot & Gene name & $\begin{array}{l}\text { Fold change of } \\
\text { XPRESS ratio } \\
\left.\text { (Adam17 }{ }^{\Delta K C} / \mathrm{WT}\right)\end{array}$ & $\begin{array}{c}\text { Sequence } \\
\text { coverage [\%] }\end{array}$ \\
\hline Cofilin-1; & P18760 & $\mathrm{Cfl1}$ & 0,5 & 39,2 \\
\hline Coiled-coil and $\mathrm{C} 2$ domain-containing protein $1 \mathrm{~B}$; & Q8BRN9 & Cc2d1b & $-0,2$ & 3,8 \\
\hline Coiled-coil domain-containing protein 12 & Q8R344 & Ccdc12 & 0,1 & 28,3 \\
\hline Coiled-coil domain-containing protein 124 & Q9D8X2 & Ccdc124 & $-0,2$ & 14,3 \\
\hline Coiled-coil domain-containing protein 43 ; & Q9CR29 & Ccdc43 & 0,0 & 5 \\
\hline Coiled-coil domain-containing protein 50 ; & Q810U5 & Ccdc50 & $-0,4$ & 19,1 \\
\hline Coiled-coil domain-containing protein 58 ; & Q8R3Q6 & Ccdc58 & $-4,9$ & 12,7 \\
\hline Coiled-coil domain-containing protein 6 & D3YZP9 & Ccdc6 & 0,5 & 7,7 \\
\hline Coiled-coil domain-containing protein 9; & Q8VC31 & Ccdc9 & 0,2 & 6,4 \\
\hline Coiled-coil-helix-coiled-coil-helix domain-containing protein 2, mitochondrial; & Q9D1L0 & Chchd2 & 0,0 & 15 \\
\hline Coiled-coil-helix-coiled-coil-helix domain-containing protein 3, mitochondrial; & Q9CRB9 & Chchd3 & $-0,2$ & 27,4 \\
\hline Coiled-coil-helix-coiled-coil-helix domain-containing protein 5 ; & Q9CQP3 & Chchd5 & 0,4 & 16,4 \\
\hline Cold-inducible RNA-binding protein; & P60824 & Cirbp & 0,9 & 6,4 \\
\hline Collagen alpha- $1(\mathrm{I})$ chain; & P11087 & Col1a1 & 1,4 & 6,4 \\
\hline Collagen alpha-1(III) chain; & P08121 & Col3a1 & 1,2 & 2,5 \\
\hline Collagen alpha-1(VII) chain; & Q63870 & Col7a1 & $-0,3$ & 0,7 \\
\hline Collagen alpha-1(XVII) chain; & Q07563 & Col17a1 & $-0,6$ & 8,3 \\
\hline Collagen alpha-2(I) chain; & Q01149 & Col1a2 & 2,1 & 10,1 \\
\hline Collagen alpha-2(IV) chain; & P08122 & Col4a2 & 0,3 & 1,5 \\
\hline $\begin{array}{l}\text { Collagen type V alpha } 3 \text { chain; Collagen, type V, alpha 3; Procollagen, type V, } \\
\text { alpha 3, isoform CRA_b; Protein Col5a3; }\end{array}$ & Q9JLI2 & Col5a3 & 0,6 & 2 \\
\hline $\begin{array}{l}\text { Complement component } 1 \mathrm{Q} \text { subcomponent-binding protein, mitochondrial; } \\
\text { Complement component } 1 \text {, q subcomponent binding protein; Complement } \\
\text { component 1, q subcomponent binding protein, isoform CRA_b; p32-RACK; }\end{array}$ & Q8R5L1 & C1qbp & 0,3 & 11,8 \\
\hline Complement factor $\mathrm{D}$; & P03953 & $\mathrm{Cfd}$ & 1,1 & 11,6 \\
\hline Consortin; & Q8CBC4 & Cnst & 0,1 & 1,7 \\
\hline COP9 signalosome complex subunit 8 & Q8VBV7 & Cops8 & 0,1 & 30,1 \\
\hline Copper transport protein ATOX1; & 008997 & Atox1 & $-0,3$ & 83,8 \\
\hline Cordon-bleu protein-like 1; & Q3UMF0 & Cobll1 & $-0,5$ & 1,5 \\
\hline Corneodesmosin; & Q7TPC1 & Cdsn & $-0,2$ & 37,8 \\
\hline Cornifin-A; & Q62266 & Sprr1a & $-1,4$ & 52,1 \\
\hline Cornifin-B; & Q62267 & Sprr1b & $-3,2$ & 20,3 \\
\hline Coronin-1A; & 089053 & Coro1a & $-1,3$ & 5,6 \\
\hline Corticosteroid-binding globulin; & Q06770 & Serpina6 & 1,9 & 2,8 \\
\hline Costars family protein ABRACL; & Q4KML4 & Abracl & 0,0 & 27,2 \\
\hline Coxsackievirus and adenovirus receptor homolog; & P97792 & Cxadr & 1,5 & 15,6 \\
\hline Craniofacial development protein $1 ;$ & 088271 & Cfdp1 & $-0,2$ & 19 \\
\hline Creatine kinase B-type; & Q04447 & $\mathrm{Ckb}$ & $-0,1$ & 2,6 \\
\hline Creatine kinase M-type; & P07310 & $\mathrm{Ckm}$ & 0,6 & 18,6 \\
\hline Creatine kinase U-type, mitochondrial; & P30275 & Ckmt1 & 0,5 & 30,9 \\
\hline Crk-like protein; & P47941 & Crkl & 2,7 & 15,8 \\
\hline CST complex subunit TEN1; & Q9D7K2 & Ten1 & 4,3 & 6,8 \\
\hline CTTNBP2 N-terminal-like protein; & Q99L0 & Cttnbp2nl & $-0,5$ & 2 \\
\hline Cullin-associated NEDD8-dissociated protein 1; & Q6ZQ38 & Cand1 & $-8,4$ & 1,1 \\
\hline $\mathrm{Cx} 9 \mathrm{C}$ motif-containing protein 4 & Q61908 & Cmc4 & $-3,8$ & 13,2 \\
\hline Cyclic AMP-responsive element-binding protein 1 ; & Q01147 & Creb1 & $-0,3$ & 10,5 \\
\hline Cyclin-dependent kinase 4 inhibitor C; & Q60772 & Cdkn2c & 0,2 & 12,5 \\
\hline Cyclin-dependent kinase inhibitor $1 \mathrm{~B}$; & P46414 & Cdkn1b & $-0,1$ & 32,5 \\
\hline Cyclin-G-associated kinase; & Q99KY4 & Gak & 0,0 & 1,3 \\
\hline Cyclin-K; & 088874 & Ccnk & $-1,4$ & 3,1 \\
\hline Cystatin E/M; Cystatin M/E; Cystatin-B; & Q9D1B1 & Cst6 & $-0,4$ & 32,9 \\
\hline Cystatin-A; & P56567 & Csta & $-0,8$ & 59,8 \\
\hline Cystatin-B; & Q62426 & Cstb & $-0,3$ & 39,8 \\
\hline Cystatin-C; & P21460 & Cst3 & 2,5 & 10,8 \\
\hline Cysteine and glycine-rich protein $1 ;$ & P97315 & Csrp1 & 0,1 & 7,8 \\
\hline Cysteine-rich protein 1 & P63254 & Crip1 & 0,3 & 19,5 \\
\hline Cysteine-rich with EGF-like domain protein 2; & Q9CYA0 & Creld2 & $-0,2$ & 12,3 \\
\hline Cytochrome b5 type B; & Q9CQX2 & Cyb5b & $-0,5$ & 27,4 \\
\hline Cytochrome b5; & P56395 & Cyb5a & 0,1 & 65,3 \\
\hline Cytochrome b-c1 complex subunit 1 , mitochondrial; & Q9CZ13 & Uqcrc1 & 0,1 & 7,9 \\
\hline Cytochrome b-c1 complex subunit 10 & Q9CPX8 & Uqcr11 & $-0,3$ & 21,4 \\
\hline Cytochrome b-c1 complex subunit 6 , mitochondrial; & P99028 & Uqcrh & 0,0 & 44,9 \\
\hline Cytochrome b-c1 complex subunit 7; & Q9D855 & Uqcrb & 0,0 & 32,4 \\
\hline Cytochrome b-c1 complex subunit 8 & Q9CQ69 & Uqcrq & $-0,1$ & 34,1 \\
\hline Cytochrome b-c1 complex subunit 9; & Q8R1I1 & Uqcr10 & $-0,1$ & 37,5 \\
\hline Cytochrome b-c1 complex subunit Rieske, mitochondrial; & Q9CR68 & Uqcrfs1 & $-0,1$ & 23,4 \\
\hline Cytochrome c oxidase assembly factor 3 homolog, mitochondrial; & Q9D2R6 & Coa3 & $-0,4$ & 18,5 \\
\hline Cytochrome c oxidase assembly factor 6 homolog; & Q8BGD8 & Coa6 & 0,1 & 16,5 \\
\hline Cytochrome c oxidase assembly factor 7; & Q921H9 & Coa7 & 0,4 & 4,3 \\
\hline Cytochrome c oxidase copper chaperone; & P56394 & Cox17 & $-0,1$ & 11,1 \\
\hline
\end{tabular}




\begin{tabular}{|c|c|c|c|c|}
\hline Protein & Uniprot & Gene name & $\begin{array}{c}\text { Fold change of } \\
\text { XPRESS ratio } \\
\left.\text { (Adam } 17^{\triangle K C} / \mathrm{WT}\right)\end{array}$ & $\begin{array}{c}\text { Sequence } \\
\text { coverage [\%] }\end{array}$ \\
\hline Cytochrome c oxidase subunit 4 isoform 1, mitochondrial; & P19783 & Cox4i1 & 0,0 & 40,2 \\
\hline Cytochrome c oxidase subunit 5A, mitochondrial; & P12787 & Cox5a & 0,1 & 32,2 \\
\hline Cytochrome c oxidase subunit 5B, mitochondrial; & P19536 & Cox5b & 0,3 & 15,6 \\
\hline Cytochrome c oxidase subunit $6 \mathrm{~B} 1$ & P56391 & Cox6b1 & 0,6 & 50 \\
\hline Cytochrome c oxidase subunit 6C; & Q9CPQ1 & Cox6c & 0,0 & 28,9 \\
\hline Cytochrome $\mathrm{c}$ oxidase subunit 7A2, mitochondrial; & P48771 & Cox7a2 & $-0,2$ & 27,7 \\
\hline Cytochrome c oxidase subunit 7C, mitochondrial; & P17665 & Cox7c & $-0,1$ & 14,3 \\
\hline Cytochrome c, somatic; & P62897 & Cycs & $-0,2$ & 38,1 \\
\hline Cytochrome $\mathrm{c} 1$, heme protein, mitochondrial; & Q9D0M3 & Cyc1 & 0,9 & 8,6 \\
\hline Cytoplasmic dynein 1 intermediate chain 2 & 088487 & Dync1i2 & 0,0 & 1,8 \\
\hline Cytoplasmic dynein 1 light intermediate chain 2; & Q6PDL0 & Dync1li2 & $-0,1$ & 5,9 \\
\hline Cytoplasmic polyadenylation element-binding protein 2 ; & Q812E0 & Cpeb2 & $-0,1$ & 1,7 \\
\hline Cytoskeleton-associated protein 4; & Q8BMK4 & Ckap4 & $-0,1$ & 25,7 \\
\hline Cytosolic 5'-nucleotidase 3A; & Q9D020 & $\mathrm{Nt5} 53 \mathrm{a}$ & 0,4 & 2,7 \\
\hline Cytosolic non-specific dipeptidase; & Q9D1A2 & Cndp2 & $-0,2$ & 4,6 \\
\hline Cytospin-B; & Q5SXY1 & Specc1 & $-0,1$ & 2,8 \\
\hline D-3-phosphoglycerate dehydrogenase; & Q61753 & Phgdh & 0,5 & 21,8 \\
\hline DAZ-associated protein 1 & Q9JII5 & Dazap1 & 0,7 & 9,4 \\
\hline dCTP pyrophosphatase 1 & Q9QY93 & Dctpp1 & $-0,5$ & 14,1 \\
\hline D-dopachrome decarboxylase; & 035215 & Ddt & 0,9 & 41,5 \\
\hline DDRGK domain-containing protein 1 ; & Q80WW9 & Ddrgk1 & 0,0 & 10,2 \\
\hline Death domain-containing membrane protein NRADD; & Q8CJ26 & Nradd & $-0,2$ & 4,8 \\
\hline Death-associated protein-like 1 & Q9D757 & Dapl1 & 0,3 & 19,6 \\
\hline Decorin; & P28654 & Den & 1,4 & 7,9 \\
\hline Delta(3,5)-Delta(2,4)-dienoyl-CoA isomerase, mitochondrial; & 035459 & Ech1 & 0,2 & 16,5 \\
\hline Density-regulated protein; & Q9CQJ6 & Denr & 0,3 & 18,6 \\
\hline Deoxyhypusine hydroxylase; & Q99LN9 & Dohh & $-0,3$ & 17,6 \\
\hline Deoxyribonuclease-2-alpha; & P56542 & Dnase2 & 0,3 & 11,7 \\
\hline Deoxyuridine triphosphatase, isoform CRA_b; Dutp protein; Protein Dut; & Q8VCG1 & Dut & 0,1 & 14,2 \\
\hline Dermokine; & E9Q2P1 & Dmkn & $-0,4$ & 36,3 \\
\hline Desmocollin 3, isoform CRA_b; Desmocollin-3; & G5E8S6 & Dsc3 & $-0,1$ & 19,4 \\
\hline Desmocollin-1; & P55849 & Dsc1 & $-0,2$ & 31,5 \\
\hline Desmoglein-1-alpha; & Q61495 & Dsg1a & $-0,3$ & 22,3 \\
\hline Desmoglein-1-beta; & Q7TSF1 & Dsg1b & $-0,5$ & 26 \\
\hline Desmoglein-2; & 055111 & Dsg2 & 0,0 & 3,3 \\
\hline Desmoglein-3; & 035902 & Dsg3 & $-0,6$ & 1,8 \\
\hline Desmoplakin; & E9Q557 & Dsp & $-0,3$ & 11 \\
\hline Destrin; & Q9R0P5 & Dstn & 0,7 & 14,5 \\
\hline DET1- and DDB1-associated protein 1; & Q9D9Z5 & Dda1 & $-0,2$ & 11,8 \\
\hline Dickkopf-like protein 1 & Q9QZL9 & Dkkl1 & $-0,9$ & 10,9 \\
\hline Dihydrolipoyl dehydrogenase, mitochondrial; & 008749 & Dld & 0,4 & 8,6 \\
\hline $\begin{array}{l}\text { Dihydrolipoyllysine-residue succinyltransferase component of 2-oxoglutarate } \\
\text { dehydrogenase complex, mitochondrial; }\end{array}$ & Q9D2G2 & Dlst & 0,2 & 4,2 \\
\hline Dihydropteridine reductase; & Q8BVI4 & Qdpr & 0,7 & 6,9 \\
\hline Dihydropyrimidinase-related protein 2 & 008553 & Dpysl2 & 0,0 & 3,5 \\
\hline Dimethylaniline monooxygenase [ $\mathrm{N}$-oxide-forming] 2; & Q8K2I3 & Fmo2 & 0,4 & 4,3 \\
\hline Dipeptidyl peptidase 1 & P97821 & Ctsc & 0,0 & 7,9 \\
\hline Disabled homolog 2; & P98078 & Dab2 & 0,2 & 11,6 \\
\hline Disco-interacting protein 2 homolog B; & Q3UH60 & Dip2b & 0,1 & 1,9 \\
\hline Disintegrin and metalloproteinase domain-containing protein 17 ; & Q9Z0F8 & Adam17 & $-3,4$ & 1,5 \\
\hline Disks large-associated protein 4 & B1AZP2 & Dlgap4 & 0,9 & 4,5 \\
\hline DNA fragmentation factor subunit alpha; & 054786 & Dffa & $-0,8$ & 17,2 \\
\hline DNA polymerase epsilon subunit 3 ; & Q9JKP7 & Pole3 & $-0,6$ & 21,6 \\
\hline DNA replication complex GINS protein PSF3; & Q9CY94 & Gins3 & $-0,5$ & 7,9 \\
\hline DNA-directed RNA polymerase II subunit RPB4; & Q9D7M8 & Polr2d & 0,2 & 16,9 \\
\hline DNA-directed RNA polymerase II subunit RPB9; & P60898 & Polr2i & $-1,6$ & 41 \\
\hline DNA-directed RNA polymerase III subunit RPC7; & Q6NXY9 & Polr3g & $-0,1$ & 5,4 \\
\hline DNA-directed RNA polymerase III subunit RPC9; & 035427 & Crcp & 0,0 & 11,5 \\
\hline DnaJ homolog subfamily B member 6 & 054946 & Dnajb6 & $-1,9$ & 4,2 \\
\hline DnaJ homolog subfamily $\mathrm{C}$ member 5 ; & P60904 & Dnajc5 & $-1,7$ & 22 \\
\hline DnaJ homolog subfamily $\mathrm{C}$ member 8 ; & Q6NZB0 & Dnajc8 & $-0,2$ & 15,1 \\
\hline DnaJ homolog subfamily $\mathrm{C}$ member 9 ; & Q91WN1 & Dnajc9 & $-0,3$ & 15,4 \\
\hline Dr1-associated corepressor; & Q9D6N5 & Drap1 & $-0,1$ & 6,8 \\
\hline Drebrin-like protein; & Q62418 & Dbnl & $-0,1$ & 34,9 \\
\hline Dynactin subunit 2 ; & Q99KJ8 & Dctn2 & $-0,2$ & 26,4 \\
\hline Dynein light chain 2, cytoplasmic; & Q9D0M5 & Dynll2 & 0,1 & 32,6 \\
\hline Dynein light chain roadblock-type 1 ; & P62627 & Dynlrb1 & $-0,1$ & 51 \\
\hline Dystroglycan; & Q62165 & Dag1 & $-0,4$ & 9,2 \\
\hline Early endosome antigen 1 ; & Q8BL66 & Eea1 & $-0,7$ & 28,5 \\
\hline EF-hand calcium-binding domain-containing protein 14 ; & Q8BGQ6 & Efcab14 & $-0,4$ & 6,2 \\
\hline EF-hand domain-containing protein D2; Efhd2 protein; & Q8C845 & Efhd2 & 1,1 & 23,3 \\
\hline EH domain-containing protein 2; & Q8BH64 & Ehd2 & 0,4 & 4,8 \\
\hline
\end{tabular}




\begin{tabular}{|c|c|c|c|c|}
\hline Protein & Uniprot & Gene name & $\begin{array}{c}\text { Fold change of } \\
\text { XPRESS ratio } \\
\left.\text { (Adam17 }^{\Delta K C} / \mathrm{WT}\right)\end{array}$ & $\begin{array}{c}\text { Sequence } \\
\text { coverage [\%] }\end{array}$ \\
\hline Electron transfer flavoprotein subunit beta; & Q9DCW4 & Etfb & $-0,1$ & 15,3 \\
\hline Elongation factor 1 -alpha 1 & P10126 & Eef1a1 & 0,0 & 21,4 \\
\hline Elongation factor 1-beta; & 070251 & Eef1b & 0,2 & 40,4 \\
\hline Elongation factor 1-delta; & F6ZFU0 & Eef1d & 1,0 & 16,7 \\
\hline Elongation factor 1-gamma; & Q9D8N0 & Eef1g & $-0,8$ & 5 \\
\hline Elongation factor 2 & P58252 & Eef2 & 0,1 & 15,4 \\
\hline Embigin; & P21995 & $\mathrm{Emb}$ & 0,2 & 5,5 \\
\hline Emerin; & 008579 & Emd & $-0,2$ & 28,6 \\
\hline Endophilin-A3; & Q62421 & Sh3gl3 & $-1,2$ & 6,1 \\
\hline Endophilin-B2; & Q8R3V5 & Sh3glb2 & 0,9 & 9,6 \\
\hline Endoplasmic reticulum resident protein 29 & P57759 & Erp29 & 0,2 & 18,3 \\
\hline Endoplasmic reticulum resident protein 44 ; & Q9D1Q6 & Erp44 & 0,3 & 20,7 \\
\hline Endoplasmin; & P08113 & Hsp90b1 & $-0,1$ & 12,5 \\
\hline Endothelial differentiation-related factor 1 ; & Q9JMG1 & Edf1 & $-0,2$ & 32,4 \\
\hline Endothelin-converting enzyme 1 & Q4PZA2 & Ece1 & 1,2 & 2 \\
\hline Enhancer of rudimentary homolog; & P84089 & Erh & 0,5 & 26,9 \\
\hline Enoyl-CoA hydratase, mitochondrial; & Q8BH95 & Echs1 & 0,2 & 11,7 \\
\hline Ensconsin; & 088735 & Map7 & $-0,7$ & 2,7 \\
\hline Envoplakin; & Q9D952 & Evpl & $-0,3$ & 2,2 \\
\hline Eosinophil cationic-type ribonuclease 5 ; & 035292 & Ear5 & 0,5 & 14,2 \\
\hline Epidermal growth factor receptor kinase substrate 8-like protein 1; & Q8R5F8 & Eps811 & $-0,5$ & 1,3 \\
\hline Epidermal growth factor receptor kinase substrate 8-like protein 2; & Q99K30 & Eps812 & $-1,2$ & 3,2 \\
\hline Epidermal growth factor receptor substrate 15 & P42567 & Eps15 & $-0,1$ & 2,9 \\
\hline Epidermal growth factor receptor substrate 15 -like 1 ; & Q60902 & Eps15l1 & $-0,6$ & 3,6 \\
\hline Epididymal secretory protein E1; & Q9Z0J0 & $\mathrm{Npc2}$ & 0,9 & 24,8 \\
\hline Epiplakin; & Q8RoW0 & Eppk1 & 0,0 & 8,1 \\
\hline Epithelial cell adhesion molecule; & Q99JW5 & Epcam & 0,3 & 2,5 \\
\hline Epsin-1; & Q80VP1 & Epn1 & $-0,3$ & 6,1 \\
\hline Epsin-2; & Q8CHU3 & Epn2 & $-0,4$ & 7,8 \\
\hline Epsin-3; & Q91W69 & Epn3 & $-0,6$ & 6,3 \\
\hline Ethylmalonyl-CoA decarboxylase; & Q9D9V3 & Echdc1 & 0,6 & 3,7 \\
\hline Eukaryotic peptide chain release factor GTP-binding subunit ERF3A; & Q8R050 & Gspt1 & $-0,5$ & 3 \\
\hline Eukaryotic translation initiation factor 1 & P48024 & Eif1 & 0,9 & 42,2 \\
\hline Eukaryotic translation initiation factor $1 \mathrm{~A}$; & Q60872 & Eif1a & 0,1 & 6,9 \\
\hline Eukaryotic translation initiation factor 2 subunit 2 ; & Q99L45 & Eif2s2 & $-0,5$ & 16,3 \\
\hline Eukaryotic translation initiation factor $2 \mathrm{~A}$ & Q8BJW6 & Eif2a & $-0,8$ & 2,4 \\
\hline Eukaryotic translation initiation factor 3 subunit $A$; & P23116 & Eif3a & $-0,8$ & 1,3 \\
\hline Eukaryotic translation initiation factor 3 subunit B; & Q8JZQ9 & Eif3b & $-1,1$ & 6,1 \\
\hline Eukaryotic translation initiation factor 3 subunit C; & Q8R1B4 & Eif3c & 3,3 & 2,1 \\
\hline Eukaryotic translation initiation factor 3 subunit G; & Q9Z1D1 & Eif3g & $-0,3$ & 6,3 \\
\hline Eukaryotic translation initiation factor 3 subunit J-A; & Q3UGC7 & Eif3j1 & $-0,1$ & 19,5 \\
\hline Eukaryotic translation initiation factor 3 subunit K; & Q9DBZ5 & Eif3k & $-0,5$ & 5 \\
\hline Eukaryotic translation initiation factor 4 gamma 1 ; & Q6NZJ6 & Eif4g1 & $-1,2$ & 3,7 \\
\hline Eukaryotic translation initiation factor $4 \mathrm{~B}$; & Q8BGD9 & Eif4b & 0,3 & 16,7 \\
\hline Eukaryotic translation initiation factor $4 \mathrm{E}$; & P63073 & Eif4e & $-0,1$ & 14,7 \\
\hline Eukaryotic translation initiation factor $4 \mathrm{E}$-binding protein 2 ; & P70445 & Eif4ebp2 & $-0,1$ & 11,7 \\
\hline Eukaryotic translation initiation factor $4 \mathrm{H}$; & Q9WUK2 & Eif4h & $-0,4$ & 25,8 \\
\hline Eukaryotic translation initiation factor 5 & P59325 & Eif5 & $-0,4$ & 6,3 \\
\hline Eukaryotic translation initiation factor $5 \mathrm{~A}-1$; & P63242 & Eif5a & 0,2 & 19 \\
\hline Eukaryotic translation initiation factor $5 \mathrm{~B}$; & Q05D44 & Eif5b & $-0,3$ & 4,4 \\
\hline Eukaryotic translation initiation factor 6 & 055135 & Eif6 & 0,1 & 48,3 \\
\hline Extracellular matrix protein 1 & Q61508 & Ecm1 & $-1,1$ & 38,1 \\
\hline Extracellular matrix protein 1 & F8WI14 & Ecm1 & $-1,1$ & 38 \\
\hline Ezrin; & P26040 & Ezr & $-0,1$ & 13,1 \\
\hline FACT complex subunit SSRP1; & Q08943 & Ssrp1 & $-0,6$ & 2,1 \\
\hline F-actin-capping protein subunit alpha-1; & P47753 & Capza1 & 0,1 & 6,3 \\
\hline F-actin-capping protein subunit beta; & P47757 & Capzb & 0,6 & 3,8 \\
\hline FAD-linked sulfhydryl oxidase ALR; & P56213 & Gfer & 0,2 & 9,1 \\
\hline Far upstream element-binding protein 1 & Q3TUE1 & Fubp1 & $-0,3$ & 34,7 \\
\hline Far upstream element-binding protein 2 & Q3U0V1 & Khsrp & 0,0 & 35,6 \\
\hline Fatty acid-binding protein, adipocyte; & P04117 & Fabp4 & 1,1 & 31,8 \\
\hline Fatty acid-binding protein, epidermal; & Q05816 & Fabp5 & $-0,2$ & 71,9 \\
\hline F-box only protein 50 & G3X9C2 & Nccrp1 & $-0,6$ & 13,9 \\
\hline Fermitin family homolog 2 & Q8CIB5 & Fermt2 & $-3,7$ & 3,5 \\
\hline Ferritin heavy chain; & P09528 & Fth1 & 0,6 & 11,5 \\
\hline Ferritin; & Q9CPX4 & Ftl1 & 0,6 & 54,1 \\
\hline Fetuin-B; & Q9QXC1 & Fetub & 2,1 & 35,1 \\
\hline FGFR1 oncogene partner 2 homolog; & Q9CRA9 & Fgfr1op2 & 0,0 & 2,8 \\
\hline Fibrinogen beta chain; & Q8K0E8 & $\mathrm{Fgb}$ & 2,1 & 17,5 \\
\hline Fibrinogen gamma chain; & Q8VCM7 & Fgg & 2,1 & 9,6 \\
\hline Fibroblast growth factor-binding protein 1; & 070514 & Fgfbp1 & 0,2 & 5,2 \\
\hline Fibronectin; & P11276 & Fn1 & 0,9 & 0,7 \\
\hline Fibulin-1; & Q08879 & Fbln1 & 0,9 & 1,4 \\
\hline
\end{tabular}




\begin{tabular}{|c|c|c|c|c|}
\hline Protein & Uniprot & Gene name & $\begin{array}{c}\text { Fold change of } \\
\text { XPRESS ratio } \\
\left.\text { (Adam17 }^{\Delta K C} / \mathrm{WT}\right)\end{array}$ & $\begin{array}{c}\text { Sequence } \\
\text { coverage [\%] }\end{array}$ \\
\hline Filaggrin; & P11088 & $\mathrm{Flg}$ & $-2,6$ & 22,9 \\
\hline Filaggrin-2; & Q2VIS4 & $\mathrm{Flg} 2$ & $-0,3$ & 42 \\
\hline Filaggrin-2; & E9QPZ3 & Flg2 & $-0,5$ & 40,8 \\
\hline Filamin-A; & Q8BTM8 & FIna & $-0,2$ & 8,1 \\
\hline Filamin-B; & Q80X90 & Flnb & 0,0 & 5,9 \\
\hline FK506-binding protein 15 & Q6P9Q6 & Fkbp15 & $-0,3$ & 1,5 \\
\hline FLYWCH family member 2 ; & Q9CQE9 & Flywch2 & $-1,4$ & 15,4 \\
\hline Follistatin-related protein 1 ; & Q62356 & Fstl1 & 0,3 & 5,9 \\
\hline Forkhead box protein $\mathrm{O} 3$ & Q9WVH4 & Foxo3 & $-0,5$ & 11,7 \\
\hline Formin-binding protein 4; & Q6ZQ03 & Fnbp4 & $-0,1$ & 4,3 \\
\hline Fos-related antigen 2 & P47930 & Fosl2 & $-0,3$ & 5,5 \\
\hline Frataxin, mitochondrial; & 035943 & Fxn & $-0,1$ & 5,8 \\
\hline Frizzled-1; & 070421 & Fzd1 & $-0,5$ & 1,9 \\
\hline Fructose-bisphosphate aldolase $A$; & P05064 & Aldoa & 0,3 & 22,5 \\
\hline G patch domain-containing protein 8 ; & A2A6A1 & Gpatch8 & $-0,3$ & 1,3 \\
\hline Galectin; & Q9CRB1 & Lgals7 & 0,3 & 64,7 \\
\hline Galectin-1; & P16045 & Lgals1 & 0,8 & 43,7 \\
\hline Galectin-3; & P16110 & Lgals3 & 0,2 & 24,2 \\
\hline Galectin-related protein; & Q8VED9 & Lgalsl & 0,7 & 5,2 \\
\hline Gamma-aminobutyric acid receptor-associated protein-like 2; & P60521 & Gabarapl2 & $-0,4$ & 6,8 \\
\hline Gamma-glutamyl hydrolase; & Q9Z0L8 & Ggh & $-0,2$ & 27,1 \\
\hline Gamma-glutamylcyclotransferase; & Q9D7X8 & Ggct & 0,1 & 33 \\
\hline Gamma-interferon-inducible lysosomal thiol reductase; & Q9ESY9 & Ifi30 & 0,4 & 9,7 \\
\hline Gamma-soluble NSF attachment protein; & Q9CWZ7 & Napg & 0,2 & 4,2 \\
\hline Gamma-synuclein; & Q9Z0F7 & Sncg & 0,3 & 47,2 \\
\hline Ganglioside GM2 activator; & Q60648 & Gm2a & $-0,4$ & 14,5 \\
\hline Gap junction alpha-1 protein; & P23242 & Gja1 & $-0,4$ & 12 \\
\hline Gasdermin-A; & Q9EST1 & Gsdma & $-0,8$ & 12,3 \\
\hline Gelsolin; & P13020 & Gsn & 0,7 & 12,6 \\
\hline General transcription factor IIF subunit 1 ; & Q3THK3 & Gtf2f1 & 0,0 & 14,2 \\
\hline General transcription factor IIF subunit 2; & Q8R0A0 & Gtf2f2 & $-0,1$ & 18,1 \\
\hline Glia maturation factor beta; & Q9CQI3 & Gmfb & 0,6 & 14,1 \\
\hline Glucocorticoid receptor; & P06537 & $\mathrm{Nr} 3 \mathrm{c} 1$ & $-0,3$ & 2,3 \\
\hline Glucose-6-phosphate isomerase; & P06745 & Gpi & 0,6 & 6,6 \\
\hline Glucosidase 2 subunit beta; & 008795 & Prkcsh & 0,1 & 15 \\
\hline Glucosylceramidase; & P17439 & Gba & $-0,4$ & 2,5 \\
\hline Glutamyl-tRNA(GIn) amidotransferase subunit C, mitochondrial; & Q8CBY0 & Gatc & $-1,3$ & 12,9 \\
\hline Glutaredoxin-1; & Q9QUHO & Glrx & 0,1 & 10,3 \\
\hline Glutaredoxin-related protein 5, mitochondrial; & Q80Y14 & Glrx5 & 0,3 & 21,7 \\
\hline Glutathione peroxidase 1; & P11352 & Gpx1 & $-0,5$ & 14,4 \\
\hline Glutathione reductase, mitochondrial; & P47791 & Gsr & 0,2 & 9,4 \\
\hline Glutathione S-transferase Mu 1; & P10649 & Gstm1 & $-0,5$ & 18,8 \\
\hline Glutathione S-transferase Mu 2; & P15626 & Gstm2 & 0,2 & 20,6 \\
\hline Glutathione S-transferase omega-1; & 009131 & Gsto1 & 0,4 & 30 \\
\hline Glutathione S-transferase P 1; & P19157 & Gstp1 & 0,4 & 9,5 \\
\hline Glyceraldehyde-3-phosphate dehydrogenase; & P16858 & Gapdh & 0,1 & 39,3 \\
\hline Glycerophosphoinositol inositolphosphodiesterase GDPD2; & Q9ESM6 & Gdpd2 & 0,1 & 1,9 \\
\hline Glycine cleavage system $\mathrm{H}$ protein, mitochondrial; & Q91WK5 & Gcsh & 0,1 & 5,9 \\
\hline Glycogenin-1; & Q9R062 & Gyg1 & 2,1 & 3 \\
\hline Glycolipid transfer protein; & Q9JL62 & Gltp & $-0,1$ & 29,5 \\
\hline Glyoxalase domain-containing protein 4; & Q9CPV4 & Glod4 & $-0,9$ & 8,5 \\
\hline Glyoxylate reductase/hydroxypyruvate reductase; & Q91753 & Grhpr & $-0,5$ & 11,2 \\
\hline Golgi integral membrane protein 4 ; & Q8BXA1 & Golim4 & $-0,3$ & 2,6 \\
\hline Golgi reassembly-stacking protein 2 & Q99JX3 & Gorasp2 & $-0,2$ & 3,7 \\
\hline Golgi SNAP receptor complex member 2; & 035166 & Gosr2 & $-0,1$ & 6,7 \\
\hline Golgin subfamily A member 2; & Q921M4 & Golga2 & $-0,5$ & 8,8 \\
\hline Golgin subfamily A member 5 ; & Q9QYE6 & Golga5 & $-0,6$ & 6,3 \\
\hline GPALPP motifs-containing protein 1 ; & Q69ZC8 & Gpalpp1 & 0,1 & 8,4 \\
\hline Granulins; & P28798 & Grn & 0,4 & 7,1 \\
\hline GRB2-associated-binding protein 1 ; & Q9QYYo & Gab1 & $-0,6$ & 17,3 \\
\hline Growth factor receptor-bound protein 2; & Q60631 & Grb2 & 0,7 & 13,3 \\
\hline GrpE protein homolog 1, mitochondrial; & Q99LP6 & Grpel1 & 0,0 & 18 \\
\hline GSK3-beta interaction protein; & Q8BGR8 & Gskip & 0,4 & 7,2 \\
\hline GTP:AMP phosphotransferase AK3, mitochondrial; & Q9WTP7 & Ak3 & 0,0 & 16,7 \\
\hline GTPase NRas; & P08556 & Nras & $-0,2$ & 6,3 \\
\hline GTP-binding protein SAR1a; & P36536 & Sar1a & $-0,2$ & 5,6 \\
\hline Guanine nucleotide exchange factor MSS4; & Q91X96 & Rabif & 0,1 & 16,3 \\
\hline $\mathrm{H} / \mathrm{ACA}$ ribonucleoprotein complex subunit 2 ; & Q9CRB2 & Nhp2 & $-0,3$ & 10,5 \\
\hline H/ACA ribonucleoprotein complex subunit 4; & Q9ESX5 & Dkc1 & 0,0 & 5,1 \\
\hline $\mathrm{H}-2$ class II histocompatibility antigen gamma chain; & P04441 & $\mathrm{Cd} 74$ & $-0,5$ & 9,7 \\
\hline Haloacid dehalogenase-like hydrolase domain-containing protein 2; & Q3UGR5 & Hdhd2 & 0,2 & 3,1 \\
\hline Haptoglobin; & Q61646 & $\mathrm{Hp}$ & 1,9 & 4,9 \\
\hline HBS1-like protein; & Q69ZS7 & Hbs1l & 0,1 & 2,9 \\
\hline
\end{tabular}




\begin{tabular}{|c|c|c|c|c|}
\hline Protein & Uniprot & Gene name & $\begin{array}{c}\text { Fold change of } \\
\text { XPRESS ratio } \\
\left.\text { (Adam } 17^{\triangle K C} / \mathrm{WT}\right)\end{array}$ & $\begin{array}{c}\text { Sequence } \\
\text { coverage [\%] }\end{array}$ \\
\hline HD domain-containing protein 2 ; & Q3SXD3 & Hddc2 & 0,2 & 14,1 \\
\hline Heat shock 70 kDa protein $1 \mathrm{~A}$; & Q61696 & Hspa1a & 0,6 & 35,9 \\
\hline Heat shock 70 kDa protein 4 & Q61316 & Hspa4 & $-0,4$ & 10,1 \\
\hline Heat shock cognate 71 kDa protein; & P63017 & Hspa8 & $-0,2$ & 33,1 \\
\hline Heat shock protein 105 kDa; & Q61699 & Hsph1 & $-0,5$ & 4 \\
\hline Heat shock protein beta-1; & P14602 & Hspb1 & 0,1 & 37,3 \\
\hline Heat shock protein beta-8; & Q9JK92 & Hspb8 & 0,0 & 4,6 \\
\hline Heat shock protein HSP 90-beta; & P11499 & Hsp90ab1 & $-0,6$ & 14,8 \\
\hline Heat shock-related 70 kDa protein 2 ; & P17156 & Hspa2 & 0,3 & 14,7 \\
\hline Hematological and neurological expressed 1 protein; & P97825 & $\mathrm{Hn} 1$ & 0,7 & 37 \\
\hline Hematological and neurological expressed 1-like protein; & Q6PGH2 & Hn1l & $-0,4$ & 53,7 \\
\hline Hematopoietic lineage cell-specific protein; & P49710 & Hcls1 & $-0,2$ & 7,2 \\
\hline Heme oxygenase 2 ; & 070252 & Hmox2 & $-0,4$ & 9,7 \\
\hline Heme-binding protein 2; & Q9WU63 & Hebp2 & 0,3 & 26,8 \\
\hline Hemoglobin subunit beta-1; & P02088 & $\mathrm{Hbb}$ b1 & 0,1 & 87,8 \\
\hline Hemoglobin subunit beta-2; & P02089 & $\mathrm{Hbb}$ b2 & $-0,5$ & 83,7 \\
\hline Hemopexin; & Q91X72 & Hpx & 1,1 & 13 \\
\hline Heparanase; & Q6YGZ1 & Hpse & $-0,8$ & 1,9 \\
\hline Hepatoma-derived growth factor; & P51859 & Hdgf & 0,1 & 44,3 \\
\hline Hepatoma-derived growth factor-related protein 2; & Q3UMU9 & Hdgfrp2 & 0,2 & 6,7 \\
\hline Hepatoma-derived growth factor-related protein 3; & Q9JMG7 & Hdgfrp3 & 0,1 & 7,9 \\
\hline Heterochromatin protein 1-binding protein 3; & Q3TEA8 & Hp1bp3 & $-0,2$ & 17,4 \\
\hline Heterogeneous nuclear ribonucleoprotein A/B; & Q99020 & Hnrnpab & 0,1 & 22,8 \\
\hline Heterogeneous nuclear ribonucleoprotein $\mathrm{A} 0$; & Q9CX86 & Hnrnpa0 & $-0,3$ & 6,6 \\
\hline Heterogeneous nuclear ribonucleoprotein $\mathrm{A} 1$; & P49312 & Hnrnpa1 & 0,6 & 15,3 \\
\hline Heterogeneous nuclear ribonucleoprotein A3; & Q8BG05 & Hnrnpa3 & 1,5 & 23,7 \\
\hline Heterogeneous nuclear ribonucleoprotein D0; & Q60668 & Hnrnpd & 0,4 & 19,4 \\
\hline Heterogeneous nuclear ribonucleoprotein D-like; & Q9Z130 & Hnrnpdl & 0,1 & 17,9 \\
\hline Heterogeneous nuclear ribonucleoprotein $\mathrm{H}$; & 035737 & Hnrnph1 & 0,9 & 9,9 \\
\hline Heterogeneous nuclear ribonucleoprotein K; & P61979 & Hnrnpk & $-0,3$ & 33,6 \\
\hline Heterogeneous nuclear ribonucleoprotein M; & Q9D0E1 & Hnrnpm & $-0,3$ & 5,3 \\
\hline Heterogeneous nuclear ribonucleoprotein Q; & Q7TMK9 & Syncrip & $-0,3$ & 9,2 \\
\hline Heterogeneous nuclear ribonucleoprotein $\mathrm{U}$; & Q8VEK3 & Hnrnpu & $-0,2$ & 7,4 \\
\hline Heterogeneous nuclear ribonucleoprotein U-like protein 2; & Q00PI9 & Hnrnpul2 & $-1,2$ & 1,9 \\
\hline Heterogeneous nuclear ribonucleoproteins A2/B1; & 088569 & Hnrnpa2b1 & 1,0 & 23,5 \\
\hline Heterogeneous nuclear ribonucleoproteins $\mathrm{C} 1 / \mathrm{C} 2$; & Q9Z204 & Hnrnpc & $-0,1$ & 7,3 \\
\hline High mobility group nucleosome-binding domain-containing protein 3; & Q9DCB1 & Hmgn3 & $-0,5$ & 32,3 \\
\hline High mobility group nucleosome-binding domain-containing protein 5 ; & Q9JL35 & Hmgn5 & $-0,2$ & 5,7 \\
\hline High mobility group protein $20 \mathrm{~A}$ & Q9DC33 & Hmg20a & $-0,2$ & 5,8 \\
\hline High mobility group protein B1; & P63158 & Hmgb1 & 0,2 & 23,7 \\
\hline High mobility group protein B2; & P30681 & Hmgb2 & 0,1 & 31 \\
\hline High mobility group protein B3; & 054879 & Hmgb3 & 0,0 & 8,2 \\
\hline Hippocalcin-like protein 1 & P62748 & Hpcal1 & 0,6 & 22,8 \\
\hline Histidine ammonia-lyase; & P35492 & $\mathrm{Hal}$ & $-0,5$ & 14,5 \\
\hline Histidine triad nucleotide-binding protein 1 ; & P70349 & Hint1 & 0,1 & 34,5 \\
\hline Histidine triad nucleotide-binding protein 2, mitochondrial; & Q9D0S9 & Hint2 & 0,1 & 17,2 \\
\hline Histidine-rich glycoprotein; & Q9ESB3 & $\mathrm{Hrg}$ & 0,7 & 1,7 \\
\hline Histone H1.1; & P43275 & Hist1h1a & $-0,2$ & 9,4 \\
\hline Histone H1.2; & P15864 & Hist1h1c & $-0,5$ & 15,1 \\
\hline Histone H1.4; & P43274 & Hist1h1e & $-0,5$ & 14,6 \\
\hline Histone H1.5; & P43276 & Hist1h1b & $-0,4$ & 14,3 \\
\hline Histone $\mathrm{H} 2 \mathrm{~A}$ type 1 ; & P22752 & Hist1h2ao & 0,1 & 27,1 \\
\hline Histone $\mathrm{H} 2 \mathrm{~A}$ type 2-B; & Q64522 & Hist2h2ab & 0,1 & 12,3 \\
\hline Histone $\mathrm{H} 2 \mathrm{~B}$ type $1-\mathrm{F} / \mathrm{J} / \mathrm{L}$; & P10853 & Hist1h2bn & 0,5 & 40,5 \\
\hline Histone H2B type 2-E; & Q64524 & Hist2h2be & $-2,3$ & 34,9 \\
\hline Histone H3.3C; & P02301 & $\mathrm{H} 3 \mathrm{f3} \mathrm{c}$ & 0,4 & 5,9 \\
\hline Histone $\mathrm{H} 4$; & P62806 & Hist4h4 & $-0,1$ & 50,5 \\
\hline Histone-binding protein RBBP4; & Q60972 & Rbbp4 & 0,8 & 11,8 \\
\hline Homeodomain-only protein; & Q8R1H0 & Hopx & $-0,2$ & 43,8 \\
\hline Hornerin; & E9QNP3 & Hrnr & $-0,1$ & 25,2 \\
\hline Host cell factor 1 & Q61191 & Hcfc1 & $-0,2$ & 4,9 \\
\hline Hsc70-interacting protein; & Q99L47 & St13 & 0,1 & 18,2 \\
\hline Hsp90 co-chaperone Cdc37; & Q61081 & Cdc37 & $-0,1$ & 10,6 \\
\hline Huntingtin-interacting protein $\mathrm{K}$; & Q9CR41 & Hypk & $-0,2$ & 24,8 \\
\hline Hypoxanthine-guanine phosphoribosyltransferase; & P00493 & Hprt1 & 0,3 & 30,7 \\
\hline Hypoxia up-regulated protein 1 & Q9JKR6 & Hyou1 & 0,0 & 3,8 \\
\hline Ig gamma-2B chain $\mathrm{C}$ region; & P01867 & $\operatorname{lgh} 3$ & 1,3 & 2 \\
\hline Ig kappa chain C region; & P01837 & & 0,7 & 13,2 \\
\hline Immunoglobulin-binding protein 1 & Q61249 & Igbp1 & $-1,3$ & 3,5 \\
\hline Inorganic pyrophosphatase 2, mitochondrial; & Q91VM9 & Ppa2 & 1,0 & 3,9 \\
\hline Inorganic pyrophosphatase; & Q9D819 & Ppa1 & 0,5 & 5,5 \\
\hline Inosine triphosphate pyrophosphatase; & Q9D892 & Itpa & $-2,8$ & 12,6 \\
\hline Inositol-trisphosphate 3-kinase C; & Q7TS72 & Itpkc & 0,2 & 2,1 \\
\hline
\end{tabular}




\begin{tabular}{|c|c|c|c|c|}
\hline Protein & Uniprot & Gene name & $\begin{array}{c}\text { Fold change of } \\
\text { XPRESS ratio } \\
\left.\text { (Adam } 17^{\Delta K C} / \mathrm{WT}\right)\end{array}$ & $\begin{array}{c}\text { Sequence } \\
\text { coverage [\%] }\end{array}$ \\
\hline Insulin receptor substrate 1 & P35569 & Irs1 & $-0,2$ & 1,7 \\
\hline Insulin-degrading enzyme; & F6RPJ9 & Ide & 0,3 & 24,5 \\
\hline Integral membrane protein $2 \mathrm{~B}$; & 089051 & $\operatorname{Itm} 2 b$ & $-1,0$ & 17,3 \\
\hline Integrator complex subunit 7; & Q7TQK1 & Ints7 & $-5,9$ & 0,9 \\
\hline Integrin alpha-3; & Q62470 & Itga3 & $-0,5$ & 3,3 \\
\hline Integrin alpha-6; & Q61739 & Itga6 & 0,4 & 4 \\
\hline Integrin beta-1; & P09055 & Itgb1 & 0,5 & 20,2 \\
\hline Integrin beta-4; & A2A863 & Itgb4 & 0,8 & 0,7 \\
\hline Inter alpha-trypsin inhibitor, heavy chain 4; & A6X935 & Itih4 & 1,6 & 1,1 \\
\hline Intercellular adhesion molecule 1 & P13597 & Icam1 & $-0,7$ & 3,5 \\
\hline Interferon-induced transmembrane protein 2; & Q99J93 & Ifitm2 & $-0,1$ & 5,8 \\
\hline Interleukin-1 alpha; & P01582 & $\| 1 a$ & $-0,3$ & 14,1 \\
\hline Interleukin-1 receptor antagonist protein; & P25085 & $\| 1 \mathrm{rn}$ & 0,3 & 17,5 \\
\hline Interleukin- 6 receptor subunit beta; & Q00560 & $\| 6 s t$ & $-0,4$ & 2,4 \\
\hline Intraflagellar transport protein 20 homolog; & Q61025 & Ift20 & 0,8 & 11,4 \\
\hline Involucrin; & G3X9D9 & $|v|$ & $-1,5$ & 67,7 \\
\hline Iron/zinc purple acid phosphatase-like protein; & Q8BX37 & Papl & 0,2 & 2,3 \\
\hline Iron-sulfur cluster assembly 2 homolog, mitochondrial; & Q9DCB8 & Isca2 & 0,4 & 15,6 \\
\hline Iron-sulfur cluster assembly enzyme ISCU, mitochondrial; & Q9D7P6 & Iscu & 0,4 & 11,9 \\
\hline Isocitrate dehydrogenase [NAD] subunit alpha, mitochondrial; & Q9D6R2 & Idh3a & 0,4 & 14,2 \\
\hline Isocitrate dehydrogenase [NADP] cytoplasmic; & 088844 & Idh1 & 1,1 & 31,4 \\
\hline Isocitrate dehydrogenase [NADP], mitochondrial; & P54071 & $\operatorname{ldh} 2$ & 1,1 & 28,1 \\
\hline Isopentenyl-diphosphate Delta-isomerase 1; & P58044 & Idi1 & $-0,6$ & 9,3 \\
\hline Junction plakoglobin; & Q02257 & Jup & 0,2 & 11,1 \\
\hline Junctional adhesion molecule $A$; & 088792 & F11r & 0,1 & 12 \\
\hline Kallikrein 1-related peptidase b5; Kallikrein related-peptidase 5; & Q9D140 & Klk5 & $-0,1$ & 29 \\
\hline Kallikrein 6, isoform CRA_a; Kallikrein-1; Klk6 protein; Neurosin; & Q91Y82 & Klk6 & $-0,5$ & 17 \\
\hline Kallikrein related-peptidase 10; Protein Klk10; & Q99M20 & Klk10 & $-0,1$ & 15,1 \\
\hline Kallikrein-11; & Q9QYN3 & Klk11 & $-0,5$ & 3,3 \\
\hline Kallikrein-7; & Q91VE3 & Klk7 & $-0,3$ & 26,1 \\
\hline Kallikrein-8; & Q61955 & Klk8 & $-0,3$ & 8,5 \\
\hline Kelch domain-containing protein 4; & Q921/2 & Klhdc4 & 0,5 & 1,9 \\
\hline $\begin{array}{l}\text { Keratin associated protein 13; Protein Krtap13; Putative keratin-associated } \\
\text { protein; }\end{array}$ & 088375 & Krtap13 & 0,8 & 17,8 \\
\hline Keratin, type I cytoskeletal 10; & P02535 & Krt10 & 0,1 & 34,8 \\
\hline Keratin, type I cytoskeletal 14; & Q61781 & Krt14 & 0,1 & 48,1 \\
\hline Keratin, type I cytoskeletal 15 & Q61414 & Krt15 & 0,8 & 37,4 \\
\hline Keratin, type I cytoskeletal 16; & Q9Z2K1 & Krt16 & $-2,9$ & 12,6 \\
\hline Keratin, type I cytoskeletal 17; & Q9QWL7 & Krt17 & $-0,6$ & 36,7 \\
\hline Keratin, type I cytoskeletal 19; & P19001 & Krt19 & $-2,1$ & 12,7 \\
\hline Keratin, type I cytoskeletal 25 & Q8VCW2 & Krt25 & 0,2 & 12,8 \\
\hline Keratin, type I cytoskeletal 27; & Q9Z320 & Krt27 & $-1,2$ & 10,7 \\
\hline Keratin, type II cytoskeletal 1 & P04104 & Krt1 & $-0,4$ & 40 \\
\hline Keratin, type II cytoskeletal $1 \mathrm{~b}$; & Q6IFZ6 & Krt77 & $-0,4$ & 27,4 \\
\hline Keratin, type II cytoskeletal 2 epidermal; & Q3TTY5 & Krt2 & 0,5 & 10,2 \\
\hline Keratin, type II cytoskeletal 5; & Q922U2 & Krt5 & $-0,3$ & 25,5 \\
\hline Keratin, type II cytoskeletal 6A; & P50446 & Krt6a & 0,7 & 13,4 \\
\hline Keratin, type II cytoskeletal 7; & Q9DCV7 & Krt7 & 0,5 & 5,9 \\
\hline Keratin, type II cytoskeletal 71; & Q9R0H5 & Krt71 & 0,7 & 5,3 \\
\hline Keratin, type II cytoskeletal 73; & Q6NXH9 & Krt73 & 0,9 & 6,5 \\
\hline Keratin, type II cytoskeletal 79; & Q8VED5 & Krt79 & $-0,3$ & 26,6 \\
\hline Keratinocyte proline-rich protein; & B2RUR4 & Kprp & $-0,7$ & 1,4 \\
\hline Keratinocyte-associated transmembrane protein 2 ; & Q8K201 & Kct2 & 0,7 & 6,9 \\
\hline Killer cell lectin-like receptor subfamily $\mathrm{G}$ member 2 ; & Q3UM83 & KIrg2 & $-3,4$ & 3,6 \\
\hline Kinectin; & Q61595 & Ktn1 & $-0,3$ & 13,9 \\
\hline Kinesin-like protein KIF21B; & Q9QXL1 & Kif21b & 1,4 & 10,3 \\
\hline Kininogen-1; & 008677 & Kng1 & 0,8 & 19,2 \\
\hline KxDL motif-containing protein 1 ; & E9Q4P0 & Kxd1 & $-0,2$ & 25,9 \\
\hline Lactotransferrin; & P08071 & Ltf & 0,6 & 3 \\
\hline Lactoylglutathione lyase; & Q9CPU0 & Glo1 & 0,7 & 34,8 \\
\hline Ladinin-1; & P57016 & Lad1 & $-0,3$ & 23,7 \\
\hline Lamina-associated polypeptide 2, isoforms beta/delta/epsilon/gamma; & Q61029 & Tmpo & 0,0 & 25 \\
\hline Lamin-B1; & P14733 & Lmnb1 & $-0,9$ & 1,9 \\
\hline Laminin subunit beta-1; & P02469 & Lamb1 & 0,2 & 0,5 \\
\hline Laminin subunit gamma-2; Laminin, gamma 2; & G5E874 & Lamc2 & 0,7 & 3,6 \\
\hline La-related protein $1 ;$ & Q6ZQ58 & Larp1 & $-0,4$ & 0,8 \\
\hline Latexin; & P70202 & Lxn & 0,3 & 4,1 \\
\hline LEM domain-containing protein 2; & Q6DVA0 & Lemd2 & $-0,4$ & 5,7 \\
\hline LETM1 and EF-hand domain-containing protein 1, mitochondrial; & Q9Z210 & Letm1 & $-0,2$ & 1,1 \\
\hline Leucine zipper protein 1 & Q8R4U7 & Luzp1 & $-0,1$ & 2,3 \\
\hline $\begin{array}{l}\text { Leucine-rich HEV glycoprotein; Leucine-rich alpha-2-glycoprotein; Leucine-rich } \\
\text { alpha-2-glycoprotein 1; Protein Lrg1; }\end{array}$ & Q91XL1 & $\operatorname{Lrg} 1$ & 0,7 & 7,9 \\
\hline
\end{tabular}




\begin{tabular}{|c|c|c|c|c|}
\hline Protein & Uniprot & Gene name & 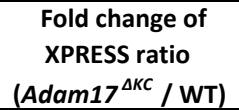 & $\begin{array}{c}\text { Sequence } \\
\text { coverage [\%] }\end{array}$ \\
\hline Leucine-rich repeat flightless-interacting protein 1 ; & Q3UZ39 & Lrrfip1 & $-0,8$ & 12,8 \\
\hline Leucine-rich repeat flightless-interacting protein 2; & E9QN52 & Lrrfip2 & $-0,1$ & 14,7 \\
\hline Leucine-rich repeat-containing protein $16 \mathrm{~A}$; & Q6EDY6 & Lrrc16a & $-0,5$ & 3,4 \\
\hline Leucine-rich repeat-containing protein 20 & Q8CI70 & Lrrc20 & $-0,4$ & 9,2 \\
\hline Leucine-rich repeat-containing protein 59; & Q922Q8 & Lrrc59 & 0,1 & 30,3 \\
\hline Leucyl-cystinyl aminopeptidase; & Q8C129 & Lnpep & $-0,1$ & 2 \\
\hline Leukocyte elastase inhibitor A; & Q9D154 & Serpinb1a & 1,3 & 9,2 \\
\hline Leukocyte surface antigen CD47; & Q61735 & $\mathrm{Cd} 47$ & $-0,6$ & 4,6 \\
\hline LIM and SH3 domain protein 1 & Q61792 & Lasp1 & $-0,3$ & 17,2 \\
\hline LIM domain and actin-binding protein $1 ;$ & Q9ERG0 & Lima1 & $-0,1$ & 4,2 \\
\hline LIM domain-containing protein 1 & Q9QXD8 & Limd1 & 0,0 & 2,8 \\
\hline LIM zinc-binding domain-containing Nebulette; & Q9DC07 & Nebl & $-0,4$ & 14,8 \\
\hline Lipolysis-stimulated lipoprotein receptor; & Q99KG5 & Lsr & $-0,1$ & 4,3 \\
\hline Lipoma-preferred partner homolog; & Q8BFW7 & Lpp & 0,4 & 7 \\
\hline Liprin-beta-2; & 035711 & Ppfibp2 & $-1,8$ & 4,5 \\
\hline L-lactate dehydrogenase A chain; & P06151 & Ldha & $-0,5$ & 17,5 \\
\hline Long-chain specific acyl-CoA dehydrogenase, mitochondrial; & P51174 & Acadl & 0,1 & 4,9 \\
\hline Long-chain-fatty-acid--CoA ligase 1 & P41216 & Acsl1 & $-0,9$ & 1,1 \\
\hline Loricrin; & P18165 & Lor & $-0,5$ & 4,1 \\
\hline Low molecular weight phosphotyrosine protein phosphatase; & Q9D358 & Acp1 & 0,5 & 5,7 \\
\hline Lumican; & P51885 & Lum & 1,2 & 19,5 \\
\hline Ly6/PLAUR domain-containing protein 3; & Q91YK8 & Lypd3 & $-0,1$ & 5,2 \\
\hline Ly6/PLAUR domain-containing protein 5; & Q9D7Z7 & Lypd5 & 0,1 & 10,9 \\
\hline $\begin{array}{l}\text { Ly6g6e protein, isoform A; Lymphocyte antigen } 6 \text { complex, locus G6E; Protein } \\
\text { Ly6g6e; }\end{array}$ & Q9D7E5 & Ly6g6e & $-0,3$ & 21,6 \\
\hline Lymphocyte antigen 6 complex locus protein G6c; & Q9Z1Q4 & Ly6g6c & $-0,8$ & 14,3 \\
\hline Lymphocyte antigen 6D; & P35459 & Ly6d & 0,2 & 48 \\
\hline Lymphocyte-specific protein 1 ; & P19973 & Lsp1 & 0,0 & 29,8 \\
\hline Lysosomal protective protein; & P16675 & Ctsa & 0,0 & 7,6 \\
\hline Lysosomal protein NCU-G1; & Q9JHJ3 & & 0,4 & 6,3 \\
\hline Lysosome membrane protein 2 & 035114 & Scarb2 & $-0,5$ & 3,3 \\
\hline Lysosome-associated membrane glycoprotein 1 ; & P11438 & Lamp1 & 0,2 & 8,4 \\
\hline Lysosome-associated membrane glycoprotein 2; & P17047 & Lamp2 & 0,2 & 6,5 \\
\hline Macrophage migration inhibitory factor; & P34884 & Mif & 0,3 & 7,8 \\
\hline Major urinary protein 20; & Q5FW60 & Mup20 & 0,1 & 6,6 \\
\hline Major urinary protein 4; & P11590 & Mup4 & 0,7 & 10,7 \\
\hline Major urinary protein 8; Protein Mup10; & A2BIN1 & Mup10 & 0,4 & 27,2 \\
\hline Malate dehydrogenase, cytoplasmic; & P14152 & Mdh1 & 0,4 & 19,2 \\
\hline Malate dehydrogenase, mitochondrial; & P08249 & Mdh2 & 0,4 & 41,1 \\
\hline Male-enhanced antigen 1 & Q64327 & Mea1 & $-0,1$ & 17,2 \\
\hline MARCKS-related protein; & P28667 & Marcksl1 & $-0,2$ & 14 \\
\hline MCG116526; Protein Scgb2b7; & D3YYY1 & Scgb2b7 & 0,8 & 23,2 \\
\hline MCG117626; Odorant binding protein la; Protein Obp1a; & Q9D3H2 & Obp1a & 0,7 & 30,1 \\
\hline MCG129038; Protein Serpinb3a; & G3X9V8 & Serpinb3a & 0,0 & 42,9 \\
\hline MCG130175, isoform CRA_b; Protein BC100530; cDNA sequence BC100530; & Q497J0 & BC100530 & $-0,3$ & 54,6 \\
\hline MCG15081; Protein Gm10334; Trypsinogen 15; & Q792Y8 & Gm10334 & $-1,6$ & 4,1 \\
\hline MCG17833, isoform CRA_c; Protein Ahnak; & G5E8K8 & Ahnak & 0,2 & 39,3 \\
\hline MCG21235; Protein Serpinb3b; & Q9D1Q5 & Serpinb3b & 0,0 & 5,9 \\
\hline MCG54087; Protein Serpina3j; & D3Z451 & Serpina3j & 0,2 & 7,6 \\
\hline Mediator of DNA damage checkpoint protein 1; & Q5PSV9 & Mdc1 & $-0,3$ & 7,3 \\
\hline Mediator of RNA polymerase II transcription subunit 21 & Q9CQ39 & Med21 & 0,0 & 22,2 \\
\hline Mediator of RNA polymerase II transcription subunit 28 & Q920D3 & Med28 & 0,1 & 11,9 \\
\hline Mediator of RNA polymerase II transcription subunit 8; & Q9D7W5 & Med8 & $-0,3$ & 9,8 \\
\hline Mediator of RNA polymerase II transcription subunit 9; & Q8VCS6 & Med9 & $-0,2$ & 10,6 \\
\hline Membrane-associated progesterone receptor component 1 & 055022 & Pgrmc1 & 0,1 & 30,3 \\
\hline Membrane-associated progesterone receptor component 2; & Q80UU9 & Pgrmc2 & $-0,1$ & 37,8 \\
\hline Metallo-beta-lactamase domain-containing protein 1 & Q8BWY4 & Mblac1 & 0,0 & 5 \\
\hline Metallothionein-2; & P02798 & Mt2 & 0,3 & 19,7 \\
\hline Metallothionein-4; & P47945 & Mt4 & 0,4 & 19,4 \\
\hline Methionine aminopeptidase 2; & 008663 & Metap2 & 0,2 & 9,2 \\
\hline Methyl-CpG-binding domain protein 2; & Q9Z2E1 & $\mathrm{Mbd} 2$ & $-1,6$ & 2,4 \\
\hline Methylosome subunit pICln; & Q61189 & Clns1a & 0,1 & 16,1 \\
\hline MICAL-like protein 1 & Q8BGT6 & Micall1 & $-0,2$ & 1,7 \\
\hline Microfibrillar-associated protein 1 & Q9CQU1 & Mfap1 & 0,5 & 16,2 \\
\hline Microtubule-associated protein $1 \mathrm{~B}$; & P14873 & Map1b & 0,3 & 0,9 \\
\hline Microtubule-associated protein 2; & P20357 & Map2 & $-0,3$ & 10,7 \\
\hline Microtubule-associated protein 4; & P27546 & Map4 & $-0,1$ & 33,2 \\
\hline Microtubule-associated protein RP/EB family member 1 ; & Q61166 & Mapre1 & $-0,1$ & 17,5 \\
\hline Microtubule-associated protein tau; & P10637 & Mapt & $-0,2$ & 4,1 \\
\hline Microtubule-associated serine/threonine-protein kinase 4; & Q811L6 & Mast4 & $-0,7$ & 1,4 \\
\hline Mimitin, mitochondrial; & Q59J78 & Ndufaf2 & $-1,3$ & 4,8 \\
\hline Mitochondrial antiviral-signaling protein; & Q8VCF0 & Mavs & $-0,3$ & 3 \\
\hline
\end{tabular}




\begin{tabular}{|c|c|c|c|c|}
\hline Protein & Uniprot & Gene name & $\begin{array}{c}\text { Fold change of } \\
\text { XPRESS ratio } \\
\left.\text { (Adam } 17^{\Delta K C} / \mathrm{WT}\right)\end{array}$ & $\begin{array}{c}\text { Sequence } \\
\text { coverage [\%] }\end{array}$ \\
\hline Mitochondrial import inner membrane translocase subunit Tim13; & P62075 & Timm13 & 0,0 & 36,8 \\
\hline Mitochondrial import inner membrane translocase subunit TIM16; & Q9CQV1 & Pam16 & $-0,3$ & 21,6 \\
\hline Mitochondrial import inner membrane translocase subunit TIM50; & Q9D880 & Timm50 & 0,5 & 7,9 \\
\hline Mitochondrial import inner membrane translocase subunit Tim8 A; & Q9WVA2 & Timm8a1 & 0,1 & 34 \\
\hline Mitochondrial intermembrane space import and assembly protein 40 ; & Q8VEA4 & Chchd4 & 0,1 & 16,5 \\
\hline MKL/myocardin-like protein 2 & P59759 & Mkl2 & $-0,4$ & 2,5 \\
\hline MLV-related proviral Env polyprotein; & P10404 & & $-0,6$ & 4,1 \\
\hline MOB kinase activator $1 \mathrm{~B}$ & Q8BPB0 & Mob1b & 0,5 & 10,6 \\
\hline Moesin; & P26041 & Msn & 0,3 & 12,1 \\
\hline Molybdopterin synthase sulfur carrier subunit; & Q9Z224 & Mocs2 & 0,5 & 11,4 \\
\hline MORF4 family-associated protein 1 & Q9CQL7 & Mrfap1 & $-0,3$ & 26,4 \\
\hline mRNA-decapping enzyme $1 \mathrm{~A}$ & Q91YD3 & Dcp1a & $-0,1$ & 2 \\
\hline Mth938 domain-containing protein; & Q8R0P4 & Aamdc & 0,5 & 21,2 \\
\hline Mucin-19; & Q6PZE0 & Muc19 & $-0,4$ & 5,8 \\
\hline Multiple epidermal growth factor-like domains protein 9; & $\mathrm{Q} 8 \mathrm{BH} 27$ & Megf9 & $-0,4$ & 1,8 \\
\hline Multiple myeloma tumor-associated protein 2 homolog; & Q99LX5 & Mmtag2 & $-0,9$ & 14,2 \\
\hline Myelin basic protein; & P04370 & Mbp & 2,1 & 8 \\
\hline Myelin protein $\mathrm{PO}$ & P27573 & $\mathrm{Mpz}$ & 4,7 & 4 \\
\hline Myeloid bactenecin (F1); Neutrophilic granule protein; Protein Ngp; & 008692 & Ngp & 2,2 & 52,7 \\
\hline Myosin light chain $1 / 3$, skeletal muscle isoform; & P05977 & Myl1 & 2,0 & 45,7 \\
\hline Myosin light chain 3; & P09542 & Myl3 & $-0,7$ & 27 \\
\hline Myosin light chain 4; & P09541 & Myl4 & $-1,4$ & 27,6 \\
\hline Myosin light chain $6 \mathrm{~B}$; & $\mathrm{Q} 8 \mathrm{Cl} 43$ & Myl6b & 0,3 & 10,1 \\
\hline Myosin light chain kinase, smooth muscle; & Q6PDN3 & Mylk & 0,5 & 0,9 \\
\hline Myosin light polypeptide 6; & Q60605 & Myl6 & 0,0 & 45,7 \\
\hline Myosin phosphatase Rho-interacting protein; & Q5SWZ5 & Mprip & $-7,4$ & 1,2 \\
\hline Myosin regulatory light chain $12 \mathrm{~B}$ & Q3THE2 & Myl12b & 0,0 & 29,7 \\
\hline Myosin regulatory light chain 2, skeletal muscle isoform; & P97457 & Mylpf & 2,6 & 36,7 \\
\hline Myosin-14; & Q6URW6 & Myh14 & $-2,3$ & 2,6 \\
\hline Myosin-4; & Q5SX39 & Myh4 & 1,7 & 7,4 \\
\hline Myosin-6; & Q02566 & Myh6 & 0,7 & 3,3 \\
\hline Myosin-8; & P13542 & Myh8 & 3,7 & 6,5 \\
\hline Myosin-9; & Q8VDD5 & Myh9 & $-0,3$ & 15,6 \\
\hline Myotrophin; & P62774 & Mtpn & 0,8 & 20,3 \\
\hline Myristoylated alanine-rich C-kinase substrate; & P26645 & Marcks & $-0,4$ & 23,6 \\
\hline $\mathrm{N}(4)$-(beta-N-acetylglucosaminyl)-L-asparaginase; & Q64191 & Aga & 0,3 & 9,8 \\
\hline $\mathrm{N}(\mathrm{G}), \mathrm{N}(\mathrm{G})$-dimethylarginine dimethylaminohydrolase 2; & Q99LD8 & Ddah2 & 0,3 & 16,1 \\
\hline $\mathrm{Na}(+) / \mathrm{H}(+)$ exchange regulatory cofactor NHE-RF1; & P70441 & Slc9a3r1 & 0,0 & 48,7 \\
\hline $\mathrm{N}$-acetylglucosamine-6-sulfatase; & Q8BFR4 & Gns & 0,8 & 8,3 \\
\hline $\mathrm{N}$-acetylneuraminate lyase; & Q9DCJ9 & $\mathrm{Npl}$ & 0,3 & 52,8 \\
\hline $\mathrm{N}$-acylethanolamine-hydrolyzing acid amidase; & Q9D7V9 & Naaa & 0,1 & 3,9 \\
\hline NADH dehydrogenase [ubiquinone] 1 alpha subcomplex subunit $12 ;$ & Q7TMF3 & Ndufa12 & 0,2 & 8,3 \\
\hline NADH dehydrogenase [ubiquinone] 1 alpha subcomplex subunit 13 ; & Q9ERS2 & Ndufa13 & 0,1 & 13,9 \\
\hline NADH dehydrogenase [ubiquinone] 1 alpha subcomplex subunit 2; & Q9CQ75 & Ndufa2 & 0,0 & 14,1 \\
\hline NADH dehydrogenase [ubiquinone] 1 alpha subcomplex subunit 4; & Q62425 & Ndufa4 & $-0,1$ & 46,3 \\
\hline NADH dehydrogenase [ubiquinone] 1 alpha subcomplex subunit 4-like 2; & Q4FZG9 & Ndufa4l2 & $-0,7$ & 28,7 \\
\hline NADH dehydrogenase [ubiquinone] 1 alpha subcomplex subunit 5; & Q9CPP6 & Ndufa5 & 0,2 & 19,8 \\
\hline NADH dehydrogenase [ubiquinone] 1 alpha subcomplex subunit 7 ; & Q9Z1P6 & Ndufa7 & $-0,4$ & 17,7 \\
\hline NADH dehydrogenase [ubiquinone] 1 alpha subcomplex subunit 8 ; & Q9DCJ5 & Ndufa8 & 0,0 & 9,9 \\
\hline NADH dehydrogenase [ubiquinone] 1 beta subcomplex subunit 10 ; & Q9DCS9 & Ndufb10 & $-0,1$ & 12,8 \\
\hline NADH dehydrogenase [ubiquinone] 1 beta subcomplex subunit 3; & Q9CQZ6 & Ndufb3 & $-0,2$ & 17,3 \\
\hline NADH dehydrogenase [ubiquinone] 1 beta subcomplex subunit 6 ; & Q3UIU2 & Ndufb6 & 0,1 & 7,2 \\
\hline $\begin{array}{l}\text { NADH dehydrogenase [ubiquinone] } 1 \text { beta subcomplex subunit } 8 \text {, } \\
\text { mitochondrial; }\end{array}$ & Q9D6J5 & Ndufb8 & 0,0 & 5,9 \\
\hline NADH dehydrogenase [ubiquinone] 1 subunit $\mathrm{C} 2$; & Q9CQ54 & Ndufc2 & 0,7 & 10,8 \\
\hline NADH dehydrogenase [ubiquinone] flavoprotein 2, mitochondrial; & Q9D6J6 & Ndufv2 & 0,5 & 12,5 \\
\hline NADH dehydrogenase [ubiquinone] flavoprotein 3 , mitochondrial; & Q8BK30 & Ndufv3 & $-0,1$ & 10,6 \\
\hline $\begin{array}{l}\text { NADH dehydrogenase [ubiquinone] flavoprotein 3, mitochondrial; RIKEN cDNA } \\
\text { 1500032D16, isoform CRA_a; }\end{array}$ & Q3U422 & Ndufv3 & $-0,2$ & 22 \\
\hline NADH dehydrogenase [ubiquinone] iron-sulfur protein 4, mitochondrial; & Q9CXZ1 & Ndufs4 & 0,4 & 25,1 \\
\hline NADH dehydrogenase [ubiquinone] iron-sulfur protein 5 ; & Q99LY9 & Ndufs5 & 0,7 & 14,3 \\
\hline NADH dehydrogenase [ubiquinone] iron-sulfur protein 6, mitochondrial; & P52503 & Ndufs6 & 0,1 & 48,3 \\
\hline NADH dehydrogenase [ubiquinone] iron-sulfur protein 8, mitochondrial; & Q8K3J1 & Ndufs8 & $-0,2$ & 9,4 \\
\hline NADP-dependent malic enzyme; & P06801 & Me1 & 0,3 & 5,8 \\
\hline Nardilysin; & Q8BHG1 & Nrd1 & $-0,2$ & 5,4 \\
\hline Nascent polypeptide-associated complex subunit alpha, muscle-specific form; & P70670 & Naca & 0,4 & 25,6 \\
\hline Nectin-1; & Q9JKF6 & Pvrl1 & $-0,2$ & 13,4 \\
\hline Nectin-4; & Q8R007 & Pvrl4 & $-0,4$ & 21,1 \\
\hline
\end{tabular}




\begin{tabular}{|c|c|c|c|c|}
\hline Protein & Uniprot & Gene name & $\begin{array}{c}\text { Fold change of } \\
\text { XPRESS ratio } \\
\left.\text { (Adam17 }^{\Delta K C} / \mathrm{WT}\right)\end{array}$ & $\begin{array}{c}\text { Sequence } \\
\text { coverage [\%] }\end{array}$ \\
\hline NEDD8; & P29595 & Nedd8 & 0,1 & 11,1 \\
\hline NEDD8-conjugating enzyme Ubc12; & P61082 & Ube $2 m$ & 0,3 & 6,5 \\
\hline Negative elongation factor B; & Q8C4Y3 & Nelfb & $-0,2$ & 4,8 \\
\hline Negative elongation factor $\mathrm{E}$; & P19426 & Nelfe & 0,5 & 5,3 \\
\hline Nesprin-1; & Q6ZWR6 & Syne1 & $-4,3$ & 0,4 \\
\hline Neudesin; & Q9CQ45 & Nenf & $-0,2$ & 42,7 \\
\hline Neural cell adhesion molecule 1 ; & P13595 & Ncam1 & 0,1 & 2,6 \\
\hline Neural cell adhesion molecule L1; & A2AFG7 & L1cam & 0,1 & 8,5 \\
\hline Neural cell adhesion molecule L1-like protein; & P70232 & Chl1 & $-0,5$ & 1,3 \\
\hline Neural Wiskott-Aldrich syndrome protein; & Q91YD9 & Wasl & $-0,9$ & 4,2 \\
\hline Neurogenic differentiation factor 2 & Q62414 & Neurod2 & 1,1 & 5 \\
\hline Neurogenic locus notch homolog protein 2; & 035516 & Notch2 & $-0,3$ & 0,3 \\
\hline Neuromodulin; & P06837 & Gap43 & 0,6 & 18,1 \\
\hline Neuronal calcium sensor 1 ; & Q8BNY6 & Ncs1 & $-0,1$ & 8,9 \\
\hline Neuroplastin; & P97300 & Nptn & 0,1 & 18,1 \\
\hline Neuroserpin; & 035684 & Serpini1 & $-1,3$ & 5,4 \\
\hline NFU1 iron-sulfur cluster scaffold homolog, mitochondrial; & Q9QZ23 & Nfu1 & $-0,6$ & 25,1 \\
\hline NHS-like protein 1 & Q8CAF4 & Nhsl1 & 0,1 & 1,7 \\
\hline Nicastrin; & P57716 & Ncstn & 0,0 & 5,9 \\
\hline Nidogen-1; & P10493 & Nid1 & 0,3 & 0,6 \\
\hline Non-histone chromosomal protein HMG-17; & P09602 & Hmgn2 & 0,6 & 17,2 \\
\hline Non-lysosomal glucosylceramidase; & Q69ZF3 & Gba2 & $-0,4$ & 4,3 \\
\hline Non-specific lipid-transfer protein; & P32020 & Scp2 & 0,2 & 9,1 \\
\hline NSFL1 cofactor p47; & Q9CZ44 & Nsfl1c & $-0,1$ & 42,4 \\
\hline Nuclear autoantigenic sperm protein; & Q99MD9 & Nasp & $-0,2$ & 10,5 \\
\hline Nuclear distribution protein nudE homolog 1 ; & Q9CZA6 & Nde1 & 0,3 & 3,2 \\
\hline Nuclear fragile X mental retardation-interacting protein 2; & Q5F2E7 & Nufip2 & $-0,6$ & 5,5 \\
\hline Nuclear migration protein nudC; & 035685 & Nudc & 0,5 & 9,6 \\
\hline Nuclear pore complex protein Nup50; & Q9JIH2 & Nup50 & 0,3 & 3 \\
\hline Nuclear pore complex protein Nup98-Nup96; & Q6PFD9 & Nup98 & $-0,2$ & 0,7 \\
\hline Nuclear pore glycoprotein p62; & Q63850 & Nup62 & $-0,2$ & 9,1 \\
\hline Nuclear receptor coactivator 6 & Q9JL19 & Ncoa6 & $-0,3$ & 0,8 \\
\hline Nuclear speckle splicing regulatory protein $1 ;$ & Q5NCR9 & Nsrp1 & 0,3 & 11,6 \\
\hline Nuclear transcription factor $Y$ subunit beta; & P63139 & Nfyb & 0,9 & 12,3 \\
\hline Nuclear ubiquitous casein and cyclin-dependent kinase substrate 1 ; & Q80XU3 & Nucks1 & 0,0 & 12 \\
\hline Nuclease-sensitive element-binding protein 1 & P62960 & Ybx1 & $-0,3$ & 30,7 \\
\hline Nucleobindin-1; & Q02819 & Nucb1 & $-0,1$ & 43,1 \\
\hline Nucleobindin-2; & P81117 & Nucb2 & $-0,4$ & 26 \\
\hline Nucleolar protein 3; & Q9D1X0 & Nol3 & $-0,1$ & 13,2 \\
\hline Nucleolar protein 56 & Q9D6Z1 & Nop56 & $-0,4$ & 5,7 \\
\hline Nucleolar RNA helicase 2; & Q9JIK5 & $\mathrm{Ddx} 21$ & $-1,1$ & 4,3 \\
\hline Nucleolin; & P09405 & $\mathrm{Ncl}$ & 0,2 & 24,9 \\
\hline Nucleophosmin; & Q61937 & Npm1 & $-0,1$ & 20,8 \\
\hline Nucleoporin NUP53; & Q8R4R6 & Nup35 & $-0,2$ & 9,1 \\
\hline Nucleoprotein TPR; & F6ZDS4 & Tpr & $-0,3$ & 9,3 \\
\hline Nucleoside diphosphate kinase; & E9PZF0 & Gm20390 & 0,3 & 62,5 \\
\hline Nucleosome assembly protein 1 -like $1 ;$ & P28656 & Nap1l1 & 0,3 & 12,7 \\
\hline Nucleosome assembly protein 1-like 4; & Q78ZA7 & Nap1/4 & 0,1 & 10,1 \\
\hline Occludin; & Q61146 & Ocln & $-0,3$ & 3,7 \\
\hline OCIA domain-containing protein 1 ; & Q9CRD0 & Ociad1 & $-0,1$ & 7,3 \\
\hline $\mathrm{OCIA}$ domain-containing protein 2 & Q9D8W7 & Ociad2 & $-0,4$ & 8,4 \\
\hline Odorant-binding protein $2 \mathrm{a}$; & Q8K1H9 & Obp2a & 0,6 & 14,8 \\
\hline Olfactory receptor; & Q7TRI2 & Olfr782 & $-0,8$ & 3,2 \\
\hline Oligoribonuclease, mitochondrial; & Q9D8S4 & Rexo2 & 0,1 & 4,2 \\
\hline Osteoclast-stimulating factor 1 & Q62422 & Ostf1 & 0,1 & 33,5 \\
\hline OTU domain-containing protein $6 \mathrm{~B}$; & $\mathrm{Q} 8 \mathrm{~K} 2 \mathrm{H} 2$ & Otud6b & 0,1 & 4,4 \\
\hline Ovostatin homolog; & Q3UU35 & Ovos & 0,0 & 2,3 \\
\hline Palladin; & Q9ET54 & Palld & 0,0 & 1,4 \\
\hline Palmdelphin; & Q9JHU2 & Palmd & $-0,3$ & 20,3 \\
\hline Palmitoyltransferase ZDHHC5; & Q8VDZ4 & Zdhhc5 & 0,0 & 14 \\
\hline $\begin{array}{l}\text { Papillary renal cell carcinoma (Translocation-associated); Papillary renal cell } \\
\text { carcinoma-associated protein; Protein Prcc; }\end{array}$ & Q9EQC8 & Prcc & 0,0 & 8,8 \\
\hline Paralemmin-1; & Q9Z0P4 & Palm & $-0,4$ & 32,6 \\
\hline Paralemmin-2; & Q8BR92 & Palm2 & 1,1 & 6,1 \\
\hline Paralemmin-3; & A2TJV2 & Palm3 & 1,3 & 1,4 \\
\hline Parathymosin; & Q9D0J8 & Ptms & $-0,1$ & 10,9 \\
\hline Partner of Y14 and mago; & Q8CHP5 & Wibg & 0,0 & 28,1 \\
\hline Parvalbumin alpha; & P32848 & Pvalb & 2,5 & 20 \\
\hline Paternally-expressed gene 3 protein; & Q3URU2 & Peg3 & 0,3 & 0,7 \\
\hline PAXIP1-associated glutamate-rich protein $1 ;$ & Q99L02 & PAGR1 & $-0,2$ & 7,5 \\
\hline PC4 and SFRS1-interacting protein; & Q99JF8 & Psip1 & 0,1 & 15,6 \\
\hline PDZ and LIM domain protein 1 & 070400 & Pdlim1 & $-0,1$ & 40,1 \\
\hline PDZ and LIM domain protein 2; & Q8R1G6 & Pdlim2 & $-0,1$ & 22,1 \\
\hline
\end{tabular}




\begin{tabular}{|c|c|c|c|c|}
\hline Protein & Uniprot & Gene name & $\begin{array}{c}\text { Fold change of } \\
\text { XPRESS ratio } \\
\left.\text { (Adam } 17^{\Delta K C} / \mathrm{WT}\right)\end{array}$ & $\begin{array}{c}\text { Sequence } \\
\text { coverage [\%] }\end{array}$ \\
\hline PDZ and LIM domain protein 4; & P70271 & Pdlim4 & 0,0 & 27,3 \\
\hline PDZ and LIM domain protein 5; & Q8CI51 & Pdlim5 & 0,1 & 16,2 \\
\hline PDZ domain-containing protein 11 & Q9CZG9 & Pdzd11 & 0,1 & 8,6 \\
\hline PDZK1-interacting protein 1 & Q9CQH0 & Pdzk1ip1 & 0,2 & 28,1 \\
\hline Peflin; & Q8BFY6 & Pef1 & 0,2 & 6,9 \\
\hline Peptidyl-prolyl cis-trans isomerase A; & P17742 & Ppia & $-0,3$ & 57,9 \\
\hline Peptidyl-prolyl cis-trans isomerase B; & P24369 & Ppib & 0,8 & 7,9 \\
\hline Peptidyl-prolyl cis-trans isomerase C; & P30412 & Ppic & 0,5 & 5,7 \\
\hline Peptidyl-prolyl cis-trans isomerase D; & Q9CR16 & Ppid & $-0,4$ & 4,6 \\
\hline Peptidyl-prolyl cis-trans isomerase E; & Q9QZH3 & Ppie & 0,1 & 7,3 \\
\hline Peptidyl-prolyl cis-trans isomerase FKBP1A; & P26883 & Fkbp1a & 0,1 & 25 \\
\hline Peptidyl-prolyl cis-trans isomerase FKBP2; & P45878 & Fkbp2 & 0,2 & 8,6 \\
\hline Peptidyl-prolyl cis-trans isomerase FKBP3; & Q62446 & Fkbp3 & 0,2 & 31,3 \\
\hline Peptidyl-prolyl cis-trans isomerase FKBP7; & 054998 & Fkbp7 & 0,6 & 8,7 \\
\hline Peptidyl-prolyl cis-trans isomerase NIMA-interacting 1 ; & Q9QUR7 & Pin1 & $-0,8$ & 13,9 \\
\hline Peptidyl-prolyl cis-trans isomerase NIMA-interacting 4; & Q9CWW6 & Pin4 & 0,1 & 25,2 \\
\hline Perilipin-1; & Q8CGN5 & Plin1 & 0,3 & 13,9 \\
\hline Perilipin-2; & P43883 & Plin2 & $-0,1$ & 13,9 \\
\hline Perilipin-3; & Q9DBG5 & Plin3 & $-0,5$ & 29,3 \\
\hline Perilipin-4; & 088492 & Plin4 & 0,0 & 14,8 \\
\hline Periostin; & Q62009 & Postn & 0,3 & 4,3 \\
\hline Periplakin; & G5E898 & Ppl & $-0,2$ & 27,3 \\
\hline Peroxiredoxin-1; & P35700 & Prdx1 & 0,4 & 60,3 \\
\hline Peroxiredoxin-2; & Q61171 & Prdx2 & 0,1 & 38,9 \\
\hline Peroxiredoxin-5, mitochondrial; & P99029 & Prdx5 & 0,6 & 36,7 \\
\hline Peroxiredoxin-6; & 008709 & Prdx6 & 1,1 & 37 \\
\hline Peroxisomal biogenesis factor 19 & Q8VCI5 & Pex19 & 0,0 & 38,1 \\
\hline Peroxisomal membrane protein PEX14; & Q9R0AO & Pex14 & $-0,9$ & 13,6 \\
\hline Persulfide dioxygenase ETHE1, mitochondrial; & Q9DCM0 & Ethe1 & 0,2 & 5,5 \\
\hline PEST proteolytic signal-containing nuclear protein; & Q6P814 & Pcnp & $-0,5$ & 36 \\
\hline Phosducin-like protein 3 & Q8BVF2 & $\mathrm{Pdcl} 3$ & $-0,4$ & 16,3 \\
\hline Phosphatase and actin regulator 4 & Q501J7 & Phactr4 & $-0,3$ & 11,7 \\
\hline Phosphatidylethanolamine-binding protein 1 ; & P70296 & Pebp1 & 0,4 & 44,9 \\
\hline Phosphatidylinositide phosphatase SAC1; & Q9EP69 & Sacm1l & $-10,0$ & 1,5 \\
\hline Phosphatidylinositol-binding clathrin assembly protein; & Q7M6Y3 & Picalm & $-0,2$ & 1,7 \\
\hline Phosphatidylinositol-glycan-specific phospholipase D; & 070362 & Gpld1 & 0,1 & 5 \\
\hline Phosphoglycerate kinase 1 ; & P09411 & Pgk1 & 0,3 & 12,5 \\
\hline Phosphoglycerate mutase 1 & Q9DBJ1 & Pgam1 & 0,3 & 46,9 \\
\hline Phosphoglycerate mutase 2 & 070250 & Pgam2 & 1,5 & 16,6 \\
\hline Phospholipase B-like 1; & Q8VCI0 & Plbd1 & 0,2 & 7,8 \\
\hline Phospholipase D3; & 035405 & Pld3 & $-0,3$ & 6,4 \\
\hline Phosphomannomutase 2; & Q9Z2M7 & Pmm2 & 0,1 & 7,4 \\
\hline Phosphorylated adapter RNA export protein; & Q9JJT9 & Phax & $-0,1$ & 13,5 \\
\hline Phosphoserine phosphatase; & Q99LS3 & Psph & $-0,2$ & 21,8 \\
\hline Pinin; & 035691 & Pnn & 0,0 & 9,5 \\
\hline Placenta-expressed transcript 1 protein; & Q8VEN2 & Plet1 & 0,0 & 6,3 \\
\hline Plakophilin-1; & P97350 & Pkp1 & $-0,3$ & 17,3 \\
\hline Plakophilin-3; & Q9QY23 & Pkp3 & 2,0 & 3,5 \\
\hline Plasminogen activator inhibitor 1 RNA-binding protein; & Q9CY58 & Serbp1 & $-0,2$ & 38,1 \\
\hline Plasminogen activator inhibitor 2 , macrophage; & P12388 & Serpinb2 & $-0,1$ & 7,7 \\
\hline Plasminogen receptor $(\mathrm{KT})$; & Q9D3P8 & Plgrkt & $-0,3$ & 6,8 \\
\hline Plastin-2; & Q61233 & Lcp1 & 0,7 & 9,3 \\
\hline Plastin-3; & Q99K51 & Pls3 & 0,1 & 23,8 \\
\hline Platelet-activating factor acetylhydrolase IB subunit alpha; & P63005 & Pafah1b1 & 0,3 & 2,7 \\
\hline Platelet-activating factor acetylhydrolase IB subunit beta; & Q61206 & Pafah1b2 & 0,2 & 3,9 \\
\hline Platelet-activating factor acetylhydrolase IB subunit gamma; & Q61205 & Pafah1b3 & 0,2 & 6,3 \\
\hline Pleckstrin homology domain-containing family A member 6; & Q7TQG1 & Plekha6 & $-1,3$ & 1,4 \\
\hline Pleckstrin homology-like domain family B member 1 & Q6PDHO & Phldb1 & 0,8 & 1,9 \\
\hline Plectin; & Q9QXS1 & Plec & $-0,4$ & 3,6 \\
\hline Plexin-B2; & B2RXS4 & Plxnb2 & 0,1 & 1,8 \\
\hline Poly(rC)-binding protein 1 & P60335 & Pcbp1 & 0,5 & 20,8 \\
\hline Poly(U)-specific endoribonuclease; & Q3V188 & Endou & 0,8 & 25,5 \\
\hline Polyadenylate-binding protein 1 ; & P29341 & Pabpc1 & $-0,7$ & 3,8 \\
\hline Polyadenylate-binding protein 2 & Q8CCS6 & Pabpn1 & 0,6 & 11 \\
\hline Polyglutamine-binding protein 1 & Q91VJ5 & Pqbp1 & $-0,2$ & 16,7 \\
\hline Polymerase I and transcript release factor; & 054724 & Ptrf & 0,5 & 7,9 \\
\hline Polypyrimidine tract-binding protein 1 ; & P17225 & Ptbp1 & 0,0 & 7,9 \\
\hline Porimin; & Q91Z22 & Tmem123 & 0,2 & 4,6 \\
\hline PRA1 family protein 3 ; & Q8R5J9 & Arl6ip5 & 1,1 & 5,9 \\
\hline Prefoldin subunit 1 ; & Q9CWM4 & Pfdn1 & 0,2 & 29,5 \\
\hline Prefoldin subunit 2 & 070591 & Pfdn2 & $-0,2$ & 29,2 \\
\hline Prefoldin subunit 3; & P61759 & Vbp1 & 0,6 & 8,7 \\
\hline Prefoldin subunit 5; & Q9WU28 & Pfdn5 & 0,1 & 21,4 \\
\hline
\end{tabular}




\begin{tabular}{|c|c|c|c|c|}
\hline Protein & Uniprot & Gene name & $\begin{array}{c}\text { Fold change of } \\
\text { XPRESS ratio } \\
\left.\text { (Adam } 17^{\Delta K C} / \mathrm{WT}\right)\end{array}$ & $\begin{array}{l}\text { Sequence } \\
\text { coverage [\%] }\end{array}$ \\
\hline Prefoldin subunit 6; & Q03958 & Pfdn6 & 0,0 & 33,7 \\
\hline Prelamin-A/C; & P48678 & Lmna & 0,0 & 27,2 \\
\hline Pre-mRNA 3'-end-processing factor FIP1; & Q9D824 & Fip1|1 & 0,1 & 12,1 \\
\hline Pre-mRNA-splicing factor ISY1 homolog; & Q69ZQ2 & Isy1 & $-0,2$ & 15,8 \\
\hline Pre-mRNA-splicing factor SYF2; & Q9D198 & Syf2 & $-0,1$ & 4,5 \\
\hline Pre-rRNA-processing protein TSR2 homolog; & Q8C8T8 & Tsr2 & $-0,2$ & 22,9 \\
\hline PRKC apoptosis WT1 regulator protein; & Q925B0 & Pawr & $-0,1$ & 9 \\
\hline Proactivator polypeptide-like 1 ; & Q8C1C1 & Psapl1 & $-0,3$ & 11,5 \\
\hline Probable pleckstrin homology domain-containing family $\mathrm{N}$ member 1 ; & E9QP36 & Plekhn1 & $-0,5$ & 8 \\
\hline Probable RNA polymerase II nuclear localization protein SLC7A6OS; & Q7TPE5 & Slc7a6os & $-0,8$ & 7,5 \\
\hline Probable RNA-binding protein EIF1AD; & Q3THJ3 & Eif1ad & $-0,1$ & 25,9 \\
\hline Profilin-1; & P62962 & Pfn1 & 0,2 & 25 \\
\hline Programmed cell death protein 10; & Q8VE70 & Pdcd10 & 0,0 & 15,6 \\
\hline Programmed cell death protein 5 & P56812 & Pdcd5 & $-0,1$ & 37,3 \\
\hline Programmed cell death protein 6 ; & P12815 & Pdcd6 & 0,0 & 14,1 \\
\hline Prohibitin; & P67778 & $\mathrm{Phb}$ & 0,5 & 27,2 \\
\hline Prohibitin-2; & 035129 & Phb2 & $-0,6$ & 14,6 \\
\hline Prolargin; & Q9JK53 & Prelp & $-0,8$ & 3,4 \\
\hline Proliferating cell nuclear antigen; & P17918 & Pcna & 0,2 & 19,9 \\
\hline Proliferation-associated protein $2 \mathrm{G} 4$ & P50580 & $\mathrm{Pa} 2 \mathrm{~g} 4$ & $-2,1$ & 11,6 \\
\hline Proline-rich AKT1 substrate 1 & Q9D1F4 & Akt1s1 & 0,1 & 13,6 \\
\hline Prolyl-tRNA synthetase associated domain-containing protein 1; & Q9D820 & Prorsd1 & 0,2 & 21,3 \\
\hline Prominin-2; & Q3UUY6 & Prom2 & 0,3 & 10,7 \\
\hline Prostaglandin E synthase 3; & Q9R0Q7 & Ptges3 & 0,7 & 28,1 \\
\hline Prostaglandin reductase 1 & Q91YR9 & Ptgr1 & 0,8 & 4,3 \\
\hline Prostatic acid phosphatase; & Q8CE08 & Acpp & 0,2 & 7,9 \\
\hline Proteasome activator complex subunit 1 & P97371 & Psme1 & 0,2 & 42 \\
\hline Proteasome activator complex subunit 2 & P97372 & Psme2 & 0,3 & 3 \\
\hline Proteasome activator complex subunit 3; & P61290 & Psme3 & $-0,1$ & 13 \\
\hline Proteasome inhibitor PI31 subunit; & Q8BHL8 & Psmf1 & 0,1 & 4,8 \\
\hline Proteasome maturation protein; & Q9CQT5 & Pomp & $-0,5$ & 7,1 \\
\hline Proteasome subunit alpha type-1; & Q9R1P4 & Psma1 & 0,0 & 28,9 \\
\hline Proteasome subunit alpha type-2; & P49722 & Psma2 & 0,1 & 9 \\
\hline Proteasome subunit alpha type-3; & 070435 & Psma3 & 0,5 & 12,5 \\
\hline Proteasome subunit alpha type-4; & Q9R1P0 & Psma4 & 0,3 & 13 \\
\hline Proteasome subunit alpha type-5; & Q9Z2U1 & Psma5 & $-0,1$ & 32,8 \\
\hline Proteasome subunit alpha type-6; & Q9QUM9 & Psma6 & 0,2 & 22 \\
\hline Proteasome subunit alpha type-7; & Q9Z2U0 & Psma7 & 0,3 & 35,5 \\
\hline Proteasome subunit beta type- 1 & 009061 & Psmb1 & 0,3 & 30,8 \\
\hline Proteasome subunit beta type- 2 & Q9R1P3 & Psmb2 & 0,2 & 14,4 \\
\hline Proteasome subunit beta type- 3 ; & Q9R1P1 & Psmb3 & 0,1 & 18,5 \\
\hline Proteasome subunit beta type- 6 & Q60692 & Psmb6 & $-0,1$ & 18,1 \\
\hline Proteasome subunit beta type-7; & P70195 & Psmb7 & $-0,1$ & 11,2 \\
\hline Protein 1100001G20Rik; RIKEN cDNA 1100001G20; & Q8BTE6 & 1100001G20Rik & 0,4 & 36,5 \\
\hline Protein 2010109I03Rik; RIKEN cDNA 2010109I03; & Q9CQ11 & 2010109I03Rik & 0,4 & 15,3 \\
\hline Protein 4.1; & P48193 & Epb41 & $-0,5$ & 6,6 \\
\hline Protein 4932418E24Rik; & Q8C5V8 & 4932418E24Rik & $-0,5$ & 1,9 \\
\hline Protein 5430401F13Rik; & E9Q328 & 5430401F13Rik & 0,4 & 21,7 \\
\hline $\begin{array}{l}\text { Protein } 8030462 \text { N17Rik; RIKEN cDNA } 8030462 \text { N17, isoform CRA_a; } \\
\text { Uncharacterized protein; }\end{array}$ & QOVAW6 & 8030462N17Rik & 0,7 & 10,5 \\
\hline Protein Abca12; & E9Q876 & Abca12 & $-0,4$ & 0,4 \\
\hline Protein Ahnak; & E9Q616 & Ahnak & $-0,4$ & 58,5 \\
\hline Protein Ahnak2; & E9PYB0 & Ahnak2 & 0,1 & 37,4 \\
\hline Protein Ahnak2; & F7CVJ5 & Ahnak2 & $-0,1$ & 49 \\
\hline Protein Ahnak2; & F7DBB3 & Ahnak2 & $-0,2$ & 36,1 \\
\hline Protein Aim1l; & B7ZCC2 & Aim1l & $-0,3$ & 2,6 \\
\hline Protein Akap13; & E9Q394 & Akap13 & $-0,3$ & 2,1 \\
\hline Protein Arhgef5; & E9Q7D5 & Arhgef5 & 0,9 & 1,1 \\
\hline Protein Bod11; & E9Q6J5 & Bod11 & 0,3 & 2 \\
\hline Protein Bpifb9b; & A2AJD1 & Bpifb9b & 0,3 & 25,4 \\
\hline Protein BRICK1; & Q91VR8 & Brk1 & $-0,5$ & 14,7 \\
\hline Protein C10; & 035127 & Grcc10 & $-0,1$ & 36,5 \\
\hline Protein Cald1; & D3Z617 & Cald1 & $-0,2$ & 16,8 \\
\hline Protein canopy homolog 2; & Q9QXT0 & Cnpy2 & 0,3 & 40,1 \\
\hline Protein canopy homolog 3 ; & Q9DAU1 & Cnpy3 & $-0,1$ & 17,8 \\
\hline Protein canopy homolog 4; & Q8BQ47 & Cnpy4 & 0,0 & 13,1 \\
\hline Protein CASC3; & Q8K3W3 & Casc3 & $-0,1$ & 5,9 \\
\hline Protein CDV3; & Q4VAA2 & Cdv3 & $-0,4$ & 30,6 \\
\hline Protein Ces2g; & E9PV38 & Ces2g & $-0,3$ & 5,2 \\
\hline Protein CLEC16A; & Q80U30 & Clec16a & $-0,1$ & 1,2 \\
\hline Protein Col22a1; & E9Q7P1 & Col22a1 & $-6,5$ & 1,6 \\
\hline Protein Col4a6; & B1AVK5 & Col4a6 & 2,0 & 0,5 \\
\hline Protein cordon-bleu; & Q5NBX1 & Cobl & $-0,8$ & 7,5 \\
\hline
\end{tabular}




\begin{tabular}{|c|c|c|c|c|}
\hline Protein & Uniprot & Gene name & $\begin{array}{c}\text { Fold change of } \\
\text { XPRESS ratio } \\
\left.\text { (Adam17 }{ }^{\Delta K C} / \mathrm{WT}\right)\end{array}$ & $\begin{array}{c}\text { Sequence } \\
\text { coverage [\%] }\end{array}$ \\
\hline Protein Crnn; & D3YUU6 & Crnn & $-0,7$ & 6 \\
\hline Protein Dcpp2; & E9PYC2 & Dcpp2 & 1,1 & 4,1 \\
\hline Protein DDI1 homolog 2; & A2ADY9 & Ddi2 & $-0,7$ & 3,5 \\
\hline Protein DEK; & Q7TNVO & Dek & $-0,1$ & 18,4 \\
\hline Protein disulfide-isomerase A3; & P27773 & Pdia3 & 0,5 & 48,3 \\
\hline Protein disulfide-isomerase $\mathrm{A} 4$; & P08003 & Pdia4 & $-0,3$ & 8,9 \\
\hline Protein disulfide-isomerase A6; & Q922R8 & Pdia6 & 0,0 & 25 \\
\hline Protein disulfide-isomerase; & P09103 & P4hb & 0,6 & 42,6 \\
\hline Protein DJ-1; & Q99LX0 & Park7 & 0,3 & 22,3 \\
\hline Protein Dnah10; & D3YYQ8 & Dnah10 & $-10,0$ & 0,4 \\
\hline Protein Dos; & Q66L44 & Dos & 0,1 & 1,4 \\
\hline Protein Dr1; & Q91WV0 & Dr1 & $-0,1$ & 11,4 \\
\hline Protein enabled homolog; & Q03173 & Enah & 0,2 & 3,9 \\
\hline Protein Fam104a; & $\mathrm{A} 2 \mathrm{~A} 6 \mathrm{P} 4$ & Fam104a & 0,4 & 13 \\
\hline Protein FAM107B; & н9н9т1 & Fam107b & $-0,3$ & 39,9 \\
\hline Protein FAM114A2; & Q8VE88 & Fam114a2 & 0,1 & 2,6 \\
\hline Protein FAM136A; & Q9CR98 & Fam136a & 0,0 & 13 \\
\hline Protein FAM162A; & Q9D6U8 & Fam162a & $-0,4$ & 7,1 \\
\hline Protein FAM168A; & Q8BGZ2 & Fam168a & $-2,4$ & 4,1 \\
\hline Protein FAM177A1; & Q8BR63 & Fam177a1 & $-0,2$ & 4,8 \\
\hline Protein FAM192A; & Q91WE2 & Fam192a & $-0,1$ & 13,4 \\
\hline Protein FAM195B; & Q3UGS4 & Fam195b & $-0,1$ & 41,2 \\
\hline Protein FAM25C; & Q8CF02 & Fam $25 c$ & $-0,4$ & 61,8 \\
\hline Protein FAM32A; & Q9CR80 & Fam 32a & 0,4 & 11,6 \\
\hline Protein FAM50A; & Q9WV03 & Fam50a & 0,0 & 8 \\
\hline Protein FAM63A; & Q76LS9 & Fam63a & $-2,0$ & 4,3 \\
\hline Protein FAM83B; & QOVBM2 & Fam83b & 0,6 & 7,6 \\
\hline Protein FAM83H; & Q148V8 & Fam83h & $-0,3$ & 5,5 \\
\hline Protein Fga; & E9PV24 & Fga & 2,5 & 18,7 \\
\hline Protein Gm14743; & A2BHD2 & Gm14743 & 0,6 & 43,3 \\
\hline Protein Gm14744; & B1AVU4 & Gm14744 & 0,6 & 37,6 \\
\hline Protein Gm15800; & E9PX61 & Gm15800 & $-0,3$ & 0,9 \\
\hline Protein Gm5849; & J3QPE5 & Gm5849 & $-0,9$ & 8,5 \\
\hline Protein Gm5938; & A2AEN9 & Gm5938 & 1,2 & 32,5 \\
\hline Protein Gm6537; & J3QPU5 & Gm6537 & $-0,5$ & 25,9 \\
\hline Protein Gm6619; & F6URP1 & Gm6619 & 0,1 & 20,8 \\
\hline Protein Golgb1; & E9PVZ8 & Golgb1 & $-0,8$ & 2,8 \\
\hline Protein HEXIM1; & Q8R409 & Hexim1 & 0,2 & 23,9 \\
\hline Protein Kif13b; & E9Q4K7 & Kif13b & $-0,1$ & 1,6 \\
\hline Protein kinase $C$ and casein kinase substrate in neurons protein 2; & Q9WVE8 & Pacsin2 & 0,0 & 3,3 \\
\hline Protein Krt78; & E9Q0F0 & Krt78 & $-0,2$ & 47,2 \\
\hline Protein LAP2; & Q80TH2 & Erbb2ip & 0,1 & 1 \\
\hline Protein lin-7 homolog C; & 088952 & $\operatorname{Lin} 7 c$ & $-0,1$ & 5,6 \\
\hline Protein LLP homolog; & Q9D945 & Llph & $-0,7$ & 11,4 \\
\hline Protein Lmo7; & E9PYF4 & Lmo7 & 0,0 & 10,3 \\
\hline Protein LSM14 homolog A; & Q8K2F8 & Lsm14a & 1,6 & 1,7 \\
\hline Protein LSM14 homolog B; & Q8CGC4 & Lsm14b & 0,4 & 3,6 \\
\hline Protein lunapark; & Q7TQ95 & Lnp & $-0,3$ & 4,3 \\
\hline Protein LYRIC; & Q80WJ7 & Mtdh & $-0,2$ & 24,9 \\
\hline Protein LZIC; & Q8K3C3 & Lzic & $-0,2$ & 15,8 \\
\hline Protein Macc1; & E9PXX8 & Macc1 & $-1,0$ & 0,8 \\
\hline Protein max; & P28574 & Max & 0,1 & 13,2 \\
\hline Protein MEMO1; & Q91VH6 & Memo1 & 0,4 & 4,7 \\
\hline Protein Mroh8; & E9PYI4 & Mroh8 & $-0,1$ & 1,3 \\
\hline Protein NDRG1; & Q62433 & Ndrg1 & 0,1 & 41 \\
\hline Protein NDRG2; & Q9QYG0 & Ndrg2 & $-0,2$ & 8,9 \\
\hline Protein Nolc1; & E9Q5C9 & Nolc1 & $-0,1$ & 12,5 \\
\hline Protein Nup153; & E9Q3G8 & Nup153 & $-0,4$ & 2,7 \\
\hline Protein PBDC1; & Q9D0B6 & $\mathrm{Pbdc1}$ & 0,4 & 5,6 \\
\hline Protein Pcdh1; & F7BJK1 & Pcdh1 & 0,3 & 2,4 \\
\hline Protein Pcdh1; Protocadherin 1; & Q8CFX3 & Pcdh1 & $-0,6$ & 2,1 \\
\hline Protein Pfdn4; & E9Q6U4 & Pfdn4 & $-0,2$ & 20,8 \\
\hline Protein phosphatase 1 regulatory subunit 11; & Q8K1L5 & Ppp1r11 & 0,1 & 7,5 \\
\hline Protein phosphatase 1 regulatory subunit $12 \mathrm{~A}$; & Q9DBR7 & Ppp1r12a & $-0,1$ & 6,3 \\
\hline Protein phosphatase 1 regulatory subunit $14 \mathrm{~A}$; & Q91VC7 & Ppp1r14a & 0,8 & 7,5 \\
\hline Protein phosphatase 1 regulatory subunit 14B; & Q62084 & Ppp1r14b & 0,2 & 29,9 \\
\hline Protein phosphatase 1 regulatory subunit $14 \mathrm{C}$; & Q8R4S0 & Ppp1r14c & $-0,9$ & 10,4 \\
\hline Protein phosphatase 1 regulatory subunit 7; & Q3UM45 & Ppp1r7 & $-0,6$ & 15,2 \\
\hline Protein phosphatase inhibitor 2 & Q9DCL8 & Ppp1r2 & $-0,2$ & 17,9 \\
\hline Protein POF1B; & Q8K4L4 & Pof1b & $-0,1$ & 23,7 \\
\hline Protein Prol1; & E9PYQ4 & Prol1 & $-0,4$ & 7,4 \\
\hline Protein PRRC1; & Q3UPH1 & Prrc1 & 0,4 & 7,7 \\
\hline Protein PRRC2A; & Q7TSC1 & Prrc2a & $-0,2$ & 1,9 \\
\hline
\end{tabular}




\begin{tabular}{|c|c|c|c|c|}
\hline Protein & Uniprot & Gene name & 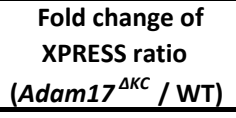 & $\begin{array}{c}\text { Sequence } \\
\text { coverage [\%] }\end{array}$ \\
\hline Protein PRRC2B; & Q7TPM1 & Prrc2b & $-0,4$ & 2,3 \\
\hline Protein PRRC2C; & Q3TLH4 & Prrc2c & $-0,3$ & 9,7 \\
\hline Protein Pus7; & F6XMS4 & Pus7 & $-1,6$ & 11,4 \\
\hline Protein Pygo2; & Q3V113 & Pygo2 & $-0,2$ & 2,2 \\
\hline Protein QIL1; & Q8R404 & Qil1 & $-2,0$ & 5,9 \\
\hline Protein Rarres1; & J3QNI9 & Rarres1 & $-0,5$ & 12 \\
\hline Protein Red; & Q9Z1M8 & $\mathrm{lk}$ & 0,6 & 3,2 \\
\hline Protein RTF2 homolog; & Q99K95 & Rtfdc1 & $-0,8$ & 6,5 \\
\hline Protein S100-A10; & P08207 & S100a10 & 1,8 & 17,5 \\
\hline Protein S100-A11; & P50543 & S100a11 & 0,3 & 34,7 \\
\hline Protein S100-A13; & P97352 & S100a13 & 0,2 & 40,8 \\
\hline Protein S100-A14; & Q9D2Q8 & S100a14 & $-0,3$ & 59,6 \\
\hline Protein S100a16; & D3Z2Y6 & S100a16 & $-0,3$ & 32,9 \\
\hline Protein S100-A3; & P62818 & S100a3 & 1,0 & 10,9 \\
\hline Protein S100-A6; & P14069 & S100a6 & 0,0 & 24,7 \\
\hline Protein S100-A9; & P31725 & S100a9 & 3,7 & 30,1 \\
\hline Protein Scgb2b12; & S4R2L0 & Scgb2b12 & 0,8 & 14,3 \\
\hline Protein Scgb2b17; & S4R1X8 & Scgb2b17 & 0,0 & 9,9 \\
\hline Protein scribble homolog; & Q80U72 & Scrib & $-0,1$ & 6,2 \\
\hline Protein SDE2 homolog; & Q8K1J5 & Sde2 & $-1,4$ & 5,1 \\
\hline Protein Sec16a; & A2AIX1 & Sec16a & $-0,4$ & 4,2 \\
\hline Protein Serpinb3c; Serine (Or cysteine) peptidase inhibitor, clade B, member 3C; & A2RSF9 & Serpinb3c & 0,2 & 18,7 \\
\hline Protein Serpinb6c; Serpinb6c protein; & Q6P6K7 & Serpinb6c & $-0,1$ & 7,7 \\
\hline Protein Serpinb6e; & I7HJI3 & Serpinb6e & 2,3 & 2,3 \\
\hline Protein SET; & Q9EQU5 & Set & 0,2 & 26 \\
\hline Protein Sf3b2; Splicing factor 3b, subunit 2; & Q3UJB0 & Sf3b2 & $-0,2$ & 17,8 \\
\hline Protein SON; & Q9QX47 & Son & $-1,6$ & 1,1 \\
\hline Protein Spink5; Serine peptidase inhibitor, Kazal type 5; & Q148R4 & Spink5 & 0,2 & 23,2 \\
\hline Protein Sptbn2; Spectrin beta 3; & Q68FG2 & Sptbn2 & 0,8 & 0,5 \\
\hline Protein Srsf11; & D3Z4B0 & Srsf11 & $-0,6$ & 7,9 \\
\hline Protein TANC1; & Q0VGY8 & Tanc1 & $-2,4$ & 1,6 \\
\hline Protein Tfg; TFG protein; Trk-fused; Trk-fused protein; & Q9Z1A1 & $\operatorname{Tfg}$ & $-0,5$ & 6,8 \\
\hline Protein Tmsb15b2; & A2AF31 & Tmsb15b2 & 0,0 & 37,5 \\
\hline Protein Tns1; & E9Q0S6 & Tns1 & 0,9 & 0,8 \\
\hline Protein Tnxb; & E9Q2T3 & Tnxb & $-0,7$ & 1,2 \\
\hline Protein transport protein Sec31A; & Q3UPL0 & Sec31a & 0,1 & 7,6 \\
\hline Protein transport protein Sec61 subunit beta; & Q9CQS8 & Sec61b & 0,2 & 21,9 \\
\hline Protein Trip11; & E9Q512 & Trip11 & $-0,3$ & 8,3 \\
\hline Protein Tsc22d2; & E9Q7M2 & $\mathrm{Tsc} 22 \mathrm{~d} 2$ & $-0,6$ & 2,1 \\
\hline Protein TSSC4; & Q9JHE7 & Tssc4 & $-0,4$ & 31,3 \\
\hline Protein Utrn; & E9Q6R7 & Utrn & $-0,5$ & 0,3 \\
\hline Protein YIPF3; & Q3UDR8 & Yipf3 & 0,0 & 2,9 \\
\hline Protein-glutamine gamma-glutamyltransferase E; & Q08189 & $\operatorname{Tgm} 3$ & 0,0 & 5,9 \\
\hline Proteolipid protein 2 & Q9R1Q7 & Plp2 & $-0,1$ & 7,9 \\
\hline Prothrombin; & P19221 & $\mathrm{F} 2$ & 1,4 & 1,5 \\
\hline Prothymosin alpha; & P26350 & Ptma & $-0,3$ & 20,7 \\
\hline Pterin-4-alpha-carbinolamine dehydratase 2 ; & Q9CZL5 & Pcbd2 & 1,5 & 21,3 \\
\hline Pumilio homolog 1; & Q80U78 & Pum1 & $-1,3$ & 2,4 \\
\hline Pumilio homolog 2; & Q80U58 & Pum2 & $-0,2$ & 9,1 \\
\hline Putative phospholipase B-like 2; & Q3TCN2 & Plbd2 & 0,1 & 2 \\
\hline Putative pre-mRNA-splicing factor ATP-dependent RNA helicase DHX15; & 035286 & Dhx15 & $-0,6$ & 1,4 \\
\hline Putative RNA-binding protein 3; & 089086 & $\mathrm{Rbm} 3$ & 0,3 & 34,6 \\
\hline Pyruvate kinase PKM; & P52480 & Pkm & 0,0 & 19,2 \\
\hline Rab GDP dissociation inhibitor alpha; & P50396 & Gdi1 & 0,3 & 4,7 \\
\hline Rab GDP dissociation inhibitor beta; & Q61598 & Gdi2 & $-0,2$ & 11,5 \\
\hline Rab GTPase-activating protein 1; & A2AWA9 & Rabgap1 & $-0,3$ & 1,5 \\
\hline Rab GTPase-binding effector protein 2; & Q91WG2 & Rabep2 & $-1,3$ & 6,1 \\
\hline Rab11 family-interacting protein 1 & E9Q8L9 & Rab11fip1 & $-0,5$ & 6,2 \\
\hline Radixin; & P26043 & $\operatorname{Rdx}$ & 0,3 & 12,7 \\
\hline Ragulator complex protein LAMTOR1; & Q9CQ22 & Lamtor1 & 0,0 & 8,1 \\
\hline Ragulator complex protein LAMTOR5; & Q9D1L9 & Lamtor5 & $-0,2$ & 44 \\
\hline Ran-binding protein 3 ; & Q9CT10 & Ranbp3 & $-0,6$ & 4,9 \\
\hline Ran-specific GTPase-activating protein; & P34022 & Ranbp1 & 0,1 & 29,1 \\
\hline Ras GTPase-activating protein-binding protein 1; & P97855 & G3bp1 & 0,0 & 28,8 \\
\hline Ras GTPase-activating-like protein IQGAP1; & Q9JKF1 & Iqgap1 & 0,0 & 1,6 \\
\hline Ras-related protein Rab-11B; & P46638 & Rab11b & 0,4 & 33 \\
\hline Ras-related protein Rab-1A; & P62821 & Rab1A & $-0,1$ & 17,4 \\
\hline Ras-related protein Rab-27B; & Q99P58 & Rab27b & 0,1 & 3,7 \\
\hline Ras-related protein Rab-5A; & Q9CQD1 & Rab5a & $-0,8$ & 5,6 \\
\hline Ras-related protein Rab-7a; & P51150 & Rab7a & 1,5 & 6,8 \\
\hline
\end{tabular}




Protein

$\begin{array}{ll} & \text { Fold change of } \\ \text { Uniprot } & \text { Gene name }\end{array}$

Sequence $\left(\right.$ Adam $\left.17^{\Delta K C} / \mathrm{WT}\right)$

coverage [\%]

Receptor accessory protein 5, isoform CRA_a; Receptor expression-enhancing protein 5;

Regucalcin;

Regulator of nonsense transcripts 1 ;

G3X8R0

Reep5

0,2

4,2

Q64374

Rgn

$-2,7$

Upf1

Q95I1X5

Repetin;

Resistin;

Reticulocalbin-1;

Reticulocalbin-2;

Reticulocalbin-3;

Reticulon-3;

Reticulon-4;

Retinoid-inducible serine carboxypeptidase;

Retroviral-like aspartic protease 1;

Rho GDP-dissociation inhibitor 1 ;

Rho GDP-dissociation inhibitor 2;

Rho-related GTP-binding protein RhoB;

Ribonuclease inhibitor;

Ribonuclease $\mathrm{P}$ protein subunit $\mathrm{p} 25$-like protein;

Ribonuclease pancreatic;

Ribonuclease T2;

Ribonuclease UK114;

Ribosomal RNA processing protein 1 homolog A;

Ribosome-binding protein 1 ;

Ribosome-recycling factor, mitochondrial;

RNA binding protein fox-1 homolog 2;

RNA-binding protein $8 \mathrm{~A}$;

RNA-binding protein EWS;

RNA-binding protein FUS;

RNA-binding protein Raly;

RNMT-activating mini protein;

RRP15-like protein;

RWD domain-containing protein 1;

RWD domain-containing protein 4;

SAFB-like transcription modulator;

Q5I1X5
P 97347

Q99P87

Q05186

Q8BP92

Q8BH97

Q9ES97

Q99P72

Q920A5

Q09PK2

Q99PT1

Q61599

P62746

Q91VI7

Q99JH1

P00683

Q9CQ01

P52760

P56183

Q99PL5

Q9D6S7

Q8BP71

Q9CWZ3

Q61545

P56959

Q64012

Q9CQY2

Q9CYX7

Q9CQK7

Q9CPR1

$\mathrm{Q} 8 \mathrm{CH} 25$

Q9D1J3

SAP domain-containing ribonucleoprotein;

Q9D1J3

D3YXK2

Scaffold attachment factor B1;

Scaffold attachment factor B2;

Sciellin;

Secretoglobin family $2 \mathrm{~B}$ member 2 ;

Secretoglobin family 2B member 20;

Secretoglobin family 2B member 24;

Secretory carrier-associated membrane protein 1;

Ppp1r13l

Rptn

Retn

Rcn1

Ren2

Ren3

Rtn3

Rtn4

Scpep1

Asprv1

Arhgdia

Arhgdib

Rhob

Rnh1

Rpp25I

Rnase1

Rnaset2

Hrsp12

Rrp1

Rrbp1

Mrrf

Rbfox2

Rbm8a

Ewsr1

Fus

Raly

Fam103a1

Rrp15

Rwdd1

Rwdd4

SItm

Sarnp

Sap30bp

Safb

Safb2

Scel

Q9EQG3

Q6UGQ3

J3QK77

Scgb2b2

Scgb2b20

Q7M747 Scgb2b24

Q8K021 Scamp1

Q9ERNo

A7TZG3

Selection and upkeep of intraepithelial T-cells protein 9;

Scamp2

Skint9

Selenbp1

$\mathrm{P} 17563$
$\mathrm{P} 07759$

Q8BTI8

Q6PDM2

Q62093

P84104

Q8VE97

O35326

Q8BL97

Q9D0B0

Q99JT2

Q6P9R2

Q8CIN4

Q8BTW9

Q9DBU3

P83741

Q3UH66

Q80W00

Serpina3k

Srrm2

Srsf1

Srsf2

Srsf3

Srsf4

Srsf5

Srsf7

Srsf9

Mst4

Oxsr1

Pak2

Pak4

Riok3

Wnk1

Wnk2

Ppp1r10

$-2,7$
0,0

9

2

10

8,1

10,5

32,3

8,4

12,8

1,1

11,9

8,6

21,5

15,2

10,5

6

4,6

5,5

8,7

7,3

50,4

3,4

20,1

6,5

3,8

10,9

9,5

51,5

13,4

22,3

6,4

3,7

10,1

1,2

34,3

3,9

2,3

4,1

10,7

35,1

21,4

13,4

11,5

3,6

3,1

10,4

8,1

1,4

29,8

3,6

17,7

Q6P1F6

Ppp2r2a

Q76MZ3

Ppp2r1a

6,8

P62715

Ppp2cb

6,1

P48453

Ppp3cb

QOVGB7

Ppp4r2

0,0

4,6 


\begin{tabular}{|c|c|c|c|c|}
\hline Protein & Uniprot & Gene name & $\begin{array}{c}\text { Fold change of } \\
\text { XPRESS ratio } \\
\left.\text { (Adam } 17^{\Delta K C} / \mathrm{WT}\right)\end{array}$ & $\begin{array}{c}\text { Sequence } \\
\text { coverage [\%] }\end{array}$ \\
\hline Serine/threonine-protein phosphatase 6 regulatory subunit 1; & Q7TSI3 & Ppp6r1 & $-0,3$ & 2,1 \\
\hline Serine/threonine-protein phosphatase 6 regulatory subunit 3; & Q922D4 & Ppp6r3 & $-0,1$ & 1,3 \\
\hline Serine/threonine-protein phosphatase PP1-alpha catalytic subunit; & P62137 & Ppp1ca & $-1,1$ & 8,2 \\
\hline Serine-threonine kinase receptor-associated protein; & Q9Z1Z2 & Strap & 0,9 & 4,3 \\
\hline Serine--tRNA ligase, cytoplasmic; & P26638 & Sars & 1,8 & 8,4 \\
\hline Serotransferrin; & Q921I1 & Tf & 0,8 & 27,1 \\
\hline Serpin A12; & Q7TMF5 & Serpina12 & 0,3 & 12,3 \\
\hline Serpin A9; & Q9D7D2 & Serpina9 & 0,5 & 6,2 \\
\hline Serpin B12; & Q9D7P9 & Serpinb12 & 0,0 & 33,3 \\
\hline Serpin B5; & P70124 & Serpinb5 & $-0,4$ & 22,9 \\
\hline Serpin B7; & Q9D695 & Serpinb7 & 0,0 & 17,1 \\
\hline Serpin B8; & 008800 & Serpinb8 & $-0,2$ & 11 \\
\hline Serum albumin; & P07724 & Alb & 1,1 & 50,2 \\
\hline Serum amyloid A-1 protein; & P05366 & Saa1 & 3,3 & 7,4 \\
\hline Serum amyloid A-2 protein; & P05367 & Saa2 & 3,5 & 7,4 \\
\hline Serum deprivation-response protein; & Q63918 & Sdpr & 0,9 & 3,8 \\
\hline SH3 domain-binding glutamic acid-rich-like protein 3; & Q91VW3 & Sh3bgrl3 & $-0,3$ & 26,9 \\
\hline SH3 domain-binding glutamic acid-rich-like protein; & Q9JJU8 & Sh3bgrl & 0,6 & 19,3 \\
\hline SH3 domain-containing kinase-binding protein 1 ; & Q8R550 & Sh3kbp1 & $-0,1$ & 16,8 \\
\hline Shootin-1; & Q8K2Q9 & Kiaa1598 & $-0,7$ & 9,5 \\
\hline Short coiled-coil protein; & Q78YZ6 & Scoc & 0,1 & 14,4 \\
\hline Short-chain dehydrogenase/reductase family $9 \mathrm{C}$ member 7 ; & Q8K3P0 & Sdr9c7 & $-0,1$ & 17,6 \\
\hline Sideroflexin-1; & Q99JR1 & Sfxn1 & $-0,3$ & 15,8 \\
\hline Sideroflexin-3; & Q91V61 & Sfxn3 & 0,4 & 14,9 \\
\hline Signal peptide peptidase-like $2 \mathrm{~A}$; & Q9JJF9 & Sppl2a & $-0,5$ & 4,6 \\
\hline Signal transducing adapter molecule 2 ; & 088811 & Stam2 & $-0,3$ & 4 \\
\hline Signal-induced proliferation-associated 1-like protein 1; & Q8C0T5 & Sipa1l1 & $-0,3$ & 5,6 \\
\hline Single-stranded DNA-binding protein, mitochondrial; & Q9CYRO & Ssbp1 & 1,1 & 5,4 \\
\hline Sister chromatid cohesion protein PDS5 homolog A; & Q6A026 & Pds5a & $-0,5$ & 1,2 \\
\hline SLAIN motif-containing protein 2 & Q8CI08 & Slain2 & 0,6 & 14,3 \\
\hline Small acidic protein; & Q9R0P4 & Smap & 0,0 & 21,5 \\
\hline Small EDRK-rich factor 2; & P84102 & Serf2 & $-0,2$ & 28,8 \\
\hline Small glutamine-rich tetratricopeptide repeat-containing protein alpha; & Q8BJU0 & Sgta & 0,8 & 26,3 \\
\hline Small nuclear ribonucleoprotein E; & P62305 & Snrpe & $-0,6$ & 21,2 \\
\hline Small nuclear ribonucleoprotein F; & P62307 & Snrpf & 0,2 & 9,3 \\
\hline Small nuclear ribonucleoprotein Sm D2; & P62317 & Snrpd2 & 0,4 & 23,7 \\
\hline Small nuclear ribonucleoprotein Sm D3; & P62320 & Snrpd3 & 0,5 & 24,6 \\
\hline Small nuclear ribonucleoprotein-associated protein $\mathrm{N}$; & P63163 & Snrpn & $-0,1$ & 9,2 \\
\hline Small ubiquitin-related modifier 1 & P63166 & Sumo1 & $-0,2$ & 18,8 \\
\hline Small ubiquitin-related modifier 2 & P61957 & Sumo2 & $-0,4$ & 16,9 \\
\hline Small VCP/p97-interacting protein; & Q3UZP4 & Svip & 3,5 & 14,3 \\
\hline Smoothelin-like protein 2; & $\mathrm{Q} 8 \mathrm{Cl} 12$ & Smtnl2 & $-0,3$ & 2,2 \\
\hline SNARE-associated protein Snapin; & Q9Z266 & Snapin & 0,1 & 16,2 \\
\hline SNW domain-containing protein 1 ; & Q9CSN1 & Snw1 & $-0,1$ & 5,8 \\
\hline Sodium/potassium-transporting ATPase subunit beta- 3 ; & P97370 & Atp1b3 & 0,4 & 11,2 \\
\hline Sorbin and $\mathrm{SH} 3$ domain-containing protein 1 ; & Q62417 & Sorbs1 & $-0,5$ & 3,1 \\
\hline Sorcin; & Q6P069 & Sri & 0,4 & 9,1 \\
\hline Sorting nexin-1; & Q9WV80 & Snx1 & $-0,4$ & 11,5 \\
\hline Sorting nexin-12; & 070493 & Snx12 & 0,3 & 9,9 \\
\hline Sorting nexin-18; & Q91ZR2 & Snx18 & $-0,4$ & 2 \\
\hline Sorting nexin-3; & 070492 & Snx3 & 0,2 & 23,5 \\
\hline Specifically androgen-regulated gene protein; & Q8BI29 & Sarg & $-0,4$ & 32,2 \\
\hline Spectrin alpha chain, non-erythrocytic 1 & P16546 & Sptan1 & 0,2 & 19,4 \\
\hline Spectrin beta chain, non-erythrocytic 1 ; & Q62261 & Sptbn1 & $-0,1$ & 3,7 \\
\hline Sperm-associated antigen 7; & Q7TNE3 & Spag7 & $-0,2$ & 14,5 \\
\hline S-phase kinase-associated protein 1 ; & Q9WTX5 & Skp1 & 0,9 & 24,5 \\
\hline Sphingomyelin phosphodiesterase; & Q04519 & Smpd1 & $-0,4$ & 1,6 \\
\hline Spliceosome-associated protein CWC15 homolog; & Q9JHS9 & Cwc15 & $-0,1$ & 11,4 \\
\hline Splicing factor 1 & Q64213 & Sf1 & 0,0 & 9,1 \\
\hline Splicing factor 3 A subunit 1 ; & Q8K4Z5 & Sf3a1 & $-0,6$ & 4,2 \\
\hline Splicing factor $3 \mathrm{~B}$ subunit 1 & Q99NB9 & Sf3b1 & $-0,5$ & 3,8 \\
\hline Splicing factor $3 \mathrm{~B}$ subunit 4 & Q8QZY9 & Sf3b4 & 0,3 & 3,3 \\
\hline Splicing factor 3B subunit 5 & Q923D4 & Sf3b5 & 0,2 & 25,6 \\
\hline Splicing factor, proline- and glutamine-rich; & Q8VIJ6 & Sfpq & $-0,8$ & 5,6 \\
\hline SRC kinase signaling inhibitor 1 ; & Q9QWI6 & $\operatorname{Srcin} 1$ & $-0,5$ & 1,9 \\
\hline Src substrate cortactin; & Q60598 & Cttn & $-0,2$ & 31,9 \\
\hline SRSF protein kinase 1 ; & 070551 & Srpk1 & $-1,1$ & 11,7 \\
\hline StAR-related lipid transfer protein 5 ; & Q9EPQ7 & Stard5 & $-0,6$ & 9,4 \\
\hline Stathmin; & P54227 & Stmn1 & $-0,4$ & 43,1 \\
\hline Stefin-1; & P35175 & Stfa1 & $-0,5$ & 66 \\
\hline Stefin-2; & P35174 & Stfa2 & $-0,7$ & 11,7 \\
\hline Stefin-3; & P35173 & Stfa3 & 0,2 & 68,9 \\
\hline
\end{tabular}




\begin{tabular}{|c|c|c|c|c|}
\hline Protein & Uniprot & Gene name & $\begin{array}{c}\text { Fold change of } \\
\text { XPRESS ratio } \\
\left.\text { (Adam } 17^{\Delta K C} / \mathrm{WT}\right)\end{array}$ & $\begin{array}{c}\text { Sequence } \\
\text { coverage [\%] }\end{array}$ \\
\hline Steroid receptor RNA activator 1 ; & Q80VJ2 & Sra1 & $-0,3$ & 8,1 \\
\hline Stress-70 protein, mitochondrial; & P38647 & Hspa9 & 0,3 & 18,4 \\
\hline Stress-induced-phosphoprotein 1; & Q60864 & Stip1 & 0,0 & 29,8 \\
\hline Stromal interaction molecule 1 & P70302 & Stim1 & 0,0 & 2,3 \\
\hline Structural maintenance of chromosomes protein $1 \mathrm{~A}$; & Q9CU62 & Smc1a & 0,6 & 1,6 \\
\hline Submandibular gland protein C; & Q6JHY2 & Muc19 & $-0,9$ & 35,9 \\
\hline Succinate dehydrogenase [ubiquinone] iron-sulfur subunit, mitochondrial; & Q9CQA3 & Sdhb & 0,1 & 3,5 \\
\hline Sulfatase-modifying factor 2 ; & Q8BPG6 & Sumf2 & 0,2 & 8,4 \\
\hline Sulfated glycoprotein 1; & Q61207 & Psap & 0,3 & 21,6 \\
\hline Sulfhydryl oxidase $1 ;$ & Q8BND5 & Qsox1 & 0,1 & 2,8 \\
\hline Sulfotransferase family cytosolic 2B member 1 ; & 035400 & Sult2b1 & $-0,9$ & 4,7 \\
\hline SUMO-conjugating enzyme UBC9; & P63280 & Ube2i & 0,2 & 9,9 \\
\hline Superoxide dismutase $[\mathrm{Cu}-\mathrm{Zn}]$ & P08228 & Sod1 & 0,1 & 32,5 \\
\hline Superoxide dismutase [Mn], mitochondrial; & P09671 & Sod2 & 0,6 & 26,1 \\
\hline Supervillin; & Q8K4L3 & Svil & 0,1 & 0,9 \\
\hline Suppressor of IKBKE 1; & Q9CPR7 & Sike1 & $-2,7$ & 9,7 \\
\hline Suppressor of tumorigenicity 14 protein homolog; & P56677 & St14 & $-0,5$ & 1,6 \\
\hline Suprabasin; & E9QPB2 & Sbsn & $-0,5$ & 55,6 \\
\hline Survival of motor neuron-related-splicing factor 30 ; & Q8BGT7 & Smndc1 & 0,1 & 29,8 \\
\hline Sushi domain-containing protein 2 & Q9DBX3 & Susd2 & 0,2 & 3,2 \\
\hline SUZ domain-containing protein 1 ; & Q6NXN1 & Szrd1 & 0,0 & 41,7 \\
\hline $\begin{array}{l}\text { SWI/SNF-related matrix-associated actin-dependent regulator of chromatin } \\
\text { subfamily E member } 1 \text {; }\end{array}$ & 054941 & Smarce1 & $-0,5$ & 6,8 \\
\hline Swi5-dependent recombination DNA repair protein 1 homolog; & Q8BP27 & Sfr1 & $-0,1$ & 3,4 \\
\hline Synapse-associated protein 1 & Q9D5V6 & Syap 1 & 0,0 & 2,2 \\
\hline Synaptic vesicle membrane protein VAT-1 homolog; & Q62465 & Vat1 & 0,2 & 5,2 \\
\hline Synaptojanin-2-binding protein; & Q9D6K5 & Synj2bp & $-0,2$ & 11,3 \\
\hline Synaptosomal-associated protein 23 & 009044 & Snap23 & $-0,4$ & 25,3 \\
\hline Synaptosomal-associated protein 29 & Q9ERB0 & Snap29 & $-0,9$ & 18,8 \\
\hline Synaptotagmin-like protein 1 & A2AE94 & Sytl1 & $-0,2$ & 9,7 \\
\hline Syndecan-4; & 035988 & Sdc4 & 0,8 & 12,1 \\
\hline Synergin gamma; & Q5SV85 & Synrg & $-0,3$ & 7,5 \\
\hline Syntaxin-12; & Q9ERO0 & Stx12 & $-0,3$ & 25,5 \\
\hline Syntaxin-7; & 070439 & Stx7 & $-0,1$ & 14,6 \\
\hline Syntaxin-8; & 088983 & Stx8 & $-0,2$ & 18,2 \\
\hline Target of Myb protein 1; & 088746 & Tom1 & $-0,3$ & 6,1 \\
\hline TATA element modulatory factor; & B9EKI3 & Tmf1 & $-0,5$ & 6,1 \\
\hline TBC1 domain family member 5 ; & Q80XQ2 & Tbc1d5 & $-0,1$ & 2,9 \\
\hline T-complex protein 1 subunit beta; & P80314 & Cct2 & 0,0 & 3 \\
\hline T-complex protein 1 subunit delta; & P80315 & Cct4 & 0,1 & 2,6 \\
\hline T-complex protein 1 subunit gamma; & P80318 & Cct3 & $-1,4$ & 12,9 \\
\hline Telomeric repeat-binding factor 2 -interacting protein 1 ; & Q91VL8 & Terf2ip & 0,4 & 4,1 \\
\hline Tetraspanin-9; & Q8BJU2 & Tspan9 & 0,4 & 8,7 \\
\hline Tetratricopeptide repeat protein 1 ; & Q91Z38 & Ttc1 & $-1,1$ & 18,4 \\
\hline Thimet oligopeptidase; & Q8C1A5 & Thop1 & 0,1 & 25 \\
\hline Thioredoxin domain-containing protein 12 & Q9CQU0 & Txndc12 & 0,5 & 22,9 \\
\hline Thioredoxin domain-containing protein 17 & Q9CQM5 & Txndc17 & 0,1 & 26,8 \\
\hline Thioredoxin domain-containing protein 5 & Q91W90 & Txndc5 & 0,6 & 43,3 \\
\hline Thioredoxin; & P10639 & Txn & 0,3 & 32,4 \\
\hline Thioredoxin-dependent peroxide reductase, mitochondrial; & P20108 & Prdx3 & $-0,3$ & 7,4 \\
\hline THO complex subunit 2 & B1AZI6 & Thoc2 & $-3,7$ & 5,5 \\
\hline THO complex subunit 4; & 008583 & Alyref & 0,4 & 18 \\
\hline Three prime repair exonuclease 2 ; & Q9R1A9 & Trex2 & $-0,5$ & 28,4 \\
\hline THUMP domain-containing protein 1 ; & Q99J36 & Thumpd1 & $-0,6$ & 8,9 \\
\hline Thy-1 membrane glycoprotein; & P01831 & Thy1 & 0,3 & 16,7 \\
\hline Tight junction protein ZO-1; & P39447 & Tjp1 & $-0,4$ & 2,4 \\
\hline Tight junction protein ZO-2; & Q9Z0U1 & Tjp2 & $-0,7$ & 2,4 \\
\hline Tissue factor; & P20352 & F3 & $-0,2$ & 14,3 \\
\hline Titin; & A2ASS6 & Ttn & 5,3 & 0,1 \\
\hline T-lymphoma invasion and metastasis-inducing protein $1 ;$ & Q60610 & Tiam1 & $-4,0$ & 0,7 \\
\hline Toll-interacting protein; & Q9QZ06 & Tollip & $-0,7$ & 6,4 \\
\hline Torsin-1A-interacting protein 1 & Q921T2 & Tor1aip1 & 0,0 & 5 \\
\hline TP53-regulated inhibitor of apoptosis 1 ; & Q9D8Z2 & Triap1 & $-0,1$ & 18,4 \\
\hline Transaldolase; & Q93092 & Taldo1 & $-0,8$ & 8,9 \\
\hline Transcription and mRNA export factor ENY2; & Q9JוX0 & Eny2 & $-0,3$ & 33,7 \\
\hline Transcription cofactor vestigial-like protein 4; & Q80V24 & Vg\|4 & 2,7 & 3,5 \\
\hline Transcription elongation factor A protein 1 ; & P10711 & Tcea1 & 0,3 & 20,9 \\
\hline Transcription elongation factor A protein-like 5; & Q8CCT4 & Tceal5 & 0,0 & 5,1 \\
\hline Transcription elongation factor B polypeptide 2 ; & P62869 & Tceb2 & 0,0 & 49,2 \\
\hline Transcription factor AP-1; & P05627 & Jun & 0,1 & 6,9 \\
\hline Transcription factor BTF3 homolog 4; & Q9CQH7 & Btf314 & 0,1 & 12 \\
\hline Transcription factor BTF3; & Q64152 & Btf3 & 0,3 & 12,7 \\
\hline
\end{tabular}




\begin{tabular}{|c|c|c|c|c|}
\hline Protein & Uniprot & Gene name & 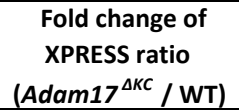 & $\begin{array}{c}\text { Sequence } \\
\text { coverage [\%] }\end{array}$ \\
\hline Transcription factor jun-D; & P15066 & Jund & 0,0 & 6,5 \\
\hline Transcription factor Sp6; & Q9ESX2 & Sp6 & $-1,0$ & 6,1 \\
\hline Transcription initiation factor IIA subunit 1 ; & Q99PM3 & Gtf2a1 & $-0,3$ & 2,9 \\
\hline Transcription initiation factor TFIID subunit 10 & Q8KOH5 & Taf10 & 3,5 & 6,4 \\
\hline Transcription initiation factor TFIID subunit 7; & Q9R1C0 & Taf7 & $-0,2$ & 6,2 \\
\hline Transcription intermediary factor 1-beta; & Q62318 & Trim28 & $-0,1$ & 1,3 \\
\hline Transcriptional activator protein Pur-alpha; & P42669 & Pura & 0,8 & 2,8 \\
\hline Transcriptional activator protein Pur-beta; & 035295 & Purb & 0,2 & 10,8 \\
\hline Transcriptional repressor p66-beta; & Q8VHR5 & Gatad2b & 0,0 & 3,5 \\
\hline Transforming acidic coiled-coil-containing protein 2; & E9Q8T1 & Tacc2 & $-0,3$ & 25,4 \\
\hline Transforming acidic coiled-coil-containing protein 2; & E9Q9Z4 & Tacc2 & $-0,4$ & 23,3 \\
\hline Transgelin-2; & Q9WVA4 & Tagln2 & 0,0 & 55,3 \\
\hline Trans-Golgi network integral membrane protein 1; & Q62313 & Tgoln1 & $-0,5$ & 16,1 \\
\hline Transitional endoplasmic reticulum ATPase; & Q01853 & Vcp & $-0,1$ & 26,4 \\
\hline Transketolase; & P40142 & Tkt & 0,1 & 4,3 \\
\hline Translation machinery-associated protein 7; & Q8K003 & Tma7 & $-0,3$ & 14,1 \\
\hline Translationally-controlled tumor protein; & P63028 & Tpt1 & 0,0 & 34,3 \\
\hline Translocon-associated protein subunit alpha; & Q9CY50 & Ssr1 & 0,0 & 11,9 \\
\hline Transmembrane emp24 domain-containing protein $10 ;$ & Q9D1D4 & Tmed10 & 0,2 & 4,1 \\
\hline Transmembrane emp24 domain-containing protein 5 & Q9CXE7 & Tmed5 & $-0,1$ & 5,2 \\
\hline Transmembrane protein 109; & Q3UBX0 & Tmem109 & 0,2 & 16,4 \\
\hline Transmembrane protein 154; & Q8C4Q9 & Tmem154 & 0,3 & 18,8 \\
\hline Transmembrane protein 263 & Q9DAM7 & Tmem263 & 0,2 & 8,7 \\
\hline Transmembrane protein 40 & Q4FJU9 & Tmem40 & $-0,8$ & 6,5 \\
\hline Transmembrane protein $79 ;$ & Q9D709 & Tmem79 & $-0,3$ & 5,9 \\
\hline Transthyretin; & P07309 & $\operatorname{Ttr}$ & 0,3 & 46,3 \\
\hline Treacle protein; & 008784 & Tcof1 & 0,2 & 8,6 \\
\hline Trichohyalin-like protein 1 & Q9D3P1 & Tchhl1 & $-1,4$ & 4,5 \\
\hline Trinucleotide repeat-containing gene $6 \mathrm{~B}$ protein; & Q8BKI2 & Tnrc6b & $-3,9$ & 1,1 \\
\hline Triosephosphate isomerase; & P17751 & Tpi1 & 0,5 & 42,5 \\
\hline Tripartite motif-containing protein 29 ; & Q8R2Q0 & Trim29 & 0,1 & 18,9 \\
\hline Tripeptidyl-peptidase 1 & 089023 & Tpp1 & 0,6 & 6,2 \\
\hline Trophoblast glycoprotein; & Q9ZOLO & Tpbg & $-0,9$ & 12 \\
\hline Tropomodulin-3; & Q9JHJ0 & Tmod3 & 0,1 & 17,3 \\
\hline Tropomyosin 1, alpha, isoform CRA_k; Tropomyosin alpha-1 chain; & G5E8R2 & Tpm1 & $-0,2$ & 33,1 \\
\hline Tropomyosin alpha-3 chain; & E9Q7Q3 & Tpm3 & $-0,2$ & 36,7 \\
\hline Tropomyosin alpha-4 chain; & Q6IRU2 & Tpm4 & $-0,1$ & 30,6 \\
\hline Tropomyosin beta chain; & A2AIM4 & Tpm2 & 0,9 & 29,9 \\
\hline Troponin C, skeletal muscle; & P20801 & Tnnc2 & 2,3 & 43,8 \\
\hline TSC22 domain family protein 1 ; & P62500 & Tsc22d1 & $-0,2$ & 22,1 \\
\hline Tubulin alpha-1B chain; & P05213 & Tuba1b & 0,5 & 30,6 \\
\hline Tubulin alpha- $4 \mathrm{~A}$ chain; & P68368 & Tuba4a & 0,9 & 29,7 \\
\hline Tubulin beta-2A chain; & Q7TMM9 & Tubb2a & 0,8 & 42,7 \\
\hline Tubulin beta- 3 chain; & Q9ERD7 & Tubb3 & 0,4 & 30,7 \\
\hline Tubulin beta-4B chain; & P68372 & Tubb4b & 0,5 & 38,9 \\
\hline Tubulin beta- 5 chain; & P99024 & Tubb5 & 0,3 & 42,8 \\
\hline Tubulin polymerization-promoting protein family member 3 ; & Q9CRB6 & Tppp3 & 0,5 & 37,5 \\
\hline Tubulin polymerization-promoting protein; & Q7TQD2 & Tppp & 0,5 & 16,5 \\
\hline Tubulin-specific chaperone A; & P48428 & Tbca & $-0,3$ & 39,8 \\
\hline Tuftelin; & 008970 & Tuft1 & $-0,2$ & 26,2 \\
\hline Tumor protein D52; & Q62393 & Tpd52 & $-1,1$ & 31,5 \\
\hline Tumor protein D54; & Q9CYZ2 & $\operatorname{Tpd} 5212$ & 0,5 & 46,6 \\
\hline Tumor protein D54; & F6VQ81 & Tpd5212 & 0,0 & 49,7 \\
\hline Tumor suppressor $\mathrm{p} 53$-binding protein 1 ; & P70399 & Tp53bp1 & $-0,3$ & 3,4 \\
\hline Tumor-associated calcium signal transducer 2 ; & Q8BGV3 & Tacstd2 & $-0,3$ & 9,8 \\
\hline Tyrosine-protein phosphatase non-receptor type substrate 1 ; & P97797 & Sirpa & $-0,2$ & 8,2 \\
\hline U1 small nuclear ribonucleoprotein 70 kDa; & Q62376 & Snrnp70 & 0,1 & 2,5 \\
\hline U1 small nuclear ribonucleoprotein $\mathrm{A}$; & Q62189 & Snrpa & $-0,2$ & 20,6 \\
\hline U1 small nuclear ribonucleoprotein C; & Q62241 & Snrpc & $-0,3$ & 7,5 \\
\hline U2 small nuclear ribonucleoprotein A'; & P57784 & Snrpa1 & 1,1 & 16,9 \\
\hline U2 small nuclear ribonucleoprotein B"; & Q9CQ17 & Snrpb2 & 0,0 & 22,7 \\
\hline U3 small nucleolar RNA-interacting protein 2; & Q91WM3 & Rrp9 & $-0,4$ & 3,2 \\
\hline U4/U6.U5 small nuclear ribonucleoprotein 27 kDa protein; & Q8K194 & Snrnp27 & 0,2 & 14,2 \\
\hline U4/U6.U5 tri-snRNP-associated protein 1; & Q97315 & Sart1 & $-0,1$ & 6,8 \\
\hline U6 snRNA-associated Sm-like protein LSm3; & P62311 & Lsm3 & 0,4 & 19,6 \\
\hline U6 snRNA-associated Sm-like protein LSm7; & Q9CQQ8 & Lsm7 & 1,1 & 15,5 \\
\hline U6 snRNA-associated Sm-like protein LSm8; & Q6ZWM4 & Lsm8 & 0,7 & 16,7 \\
\hline Ubiquilin-1; & Q8R317 & Ubqln1 & $-0,3$ & 16,5 \\
\hline Ubiquilin-2; & Q9QZMo & Ubqln 2 & $-0,4$ & 9,6 \\
\hline Ubiquitin carboxyl-terminal hydrolase 7; & Q6A4J8 & Usp7 & 0,0 & 10,1 \\
\hline Ubiquitin carboxyl-terminal hydrolase isozyme L1; & Q9R0P9 & Uchl1 & $-0,2$ & 8,1 \\
\hline Ubiquitin carboxyl-terminal hydrolase isozyme L3; & Q9JKB1 & Uchl3 & 0,3 & 12,6 \\
\hline Ubiquitin thioesterase OTUB1; & Q7TQI3 & Otub1 & 0,3 & 7,9 \\
\hline
\end{tabular}


Supplementary table 2, Tholen et al.

\begin{tabular}{lccccc}
\hline & Protein & Uniprot & Gene name & $\begin{array}{c}\text { Fold change of } \\
\text { XPRESS ratio } \\
\text { (Adam17 }\end{array}$ & $\begin{array}{c}\text { Sequence } / \text { WT) } \\
\text { coverage [\%] }\end{array}$ \\
\hline Ubiquitin-associated protein 1; & & Q8BH48 & Ubap1 & 0,0 & 4,4 \\
Ubiquitin-associated protein 2; & Q91VX2 & Ubap2 & $-0,7$ & 15,6
\end{tabular}


Supplementary table 3: List of all identified and quantified proteins in the quantitative proteome comparison of wild-type and Egfr ${ }^{\Delta K C}$ epidermis at P3 (replicate 2).

\begin{tabular}{|c|c|c|c|c|}
\hline Protein & Uniprot & Gene name & $\begin{array}{l}\text { Fold change of } \\
\text { XPRESS ratio } \\
\left(E g f r^{\Delta K C} / \mathrm{WT}\right)\end{array}$ & $\begin{array}{c}\text { Sequence } \\
\text { coverage [\%] }\end{array}$ \\
\hline 10 kDa heat shock protein, mitochondrial; & Q64433 & Hspe1 & $-0,2$ & 48 \\
\hline 14-3-3 protein epsilon; & P62259 & Ywhae & $-0,3$ & 15,3 \\
\hline 14-3-3 protein eta; & P68510 & Ywhah & $-0,8$ & 8,9 \\
\hline 14-3-3 protein gamma; & P61982 & Ywhag & 0,1 & 8,9 \\
\hline 14-3-3 protein sigma; & 070456 & Sfn & 0,2 & 36,7 \\
\hline 14-3-3 protein theta; & P68254 & Ywhaq & 0,2 & 9 \\
\hline 14-3-3 protein zeta/delta; & P63101 & Ywhaz & 0,5 & 13,9 \\
\hline 182 kDa tankyrase-1-binding protein; & P58871 & Tnks1bp1 & $-0,1$ & 29,4 \\
\hline 1-phosphatidylinositol 4,5-bisphosphate phosphodiesterase beta-3; & P51432 & Plcb3 & 0,5 & 0,9 \\
\hline $26 \mathrm{~S}$ protease regulatory subunit $6 \mathrm{~A}$; & 088685 & Psmc3 & 0,5 & 5,3 \\
\hline 265 protease regulatory subunit 7 & P46471 & Psmc2 & 0,0 & 8,1 \\
\hline $26 \mathrm{~S}$ proteasome non-ATPase regulatory subun it $1 ;$ & Q3TXS7 & Psmd1 & $-0,2$ & 19,4 \\
\hline $26 \mathrm{~S}$ proteasome non-ATPase regulatory subun it $11 ;$ & Q8BG32 & Psmd11 & 0,0 & 19,4 \\
\hline 265 proteasome non-ATPase regulatory subun it 4 & 035226 & Psmd4 & $-0,5$ & 10,1 \\
\hline $26 \mathrm{~S}$ proteasome non-ATPase regulatory subun it $9 ;$ & Q9CR00 & Psmd9 & $-0,6$ & 19,4 \\
\hline 28 kDa heat- and acid-stable phosphoprotein; & Q3UHX2 & Pdap1 & $-0,3$ & 26 \\
\hline $28 \mathrm{~S}$ ribosomal protein S36, mitochondrial; & Q9CQX8 & Mrps36 & $-0,1$ & 56,9 \\
\hline 39 S ribosomal protein L12, mitochondrial; & Q9DB15 & Mrpl12 & $-0,3$ & 10,9 \\
\hline $39 S$ ribosomal protein L41, mitochondrial; & Q9CQN7 & Mrpl41 & 0,0 & 31,1 \\
\hline 3-ketoacyl-CoA thiolase, mitochondrial; & Q8BWT1 & Acaa2 & 0,1 & 8,6 \\
\hline $40 S$ ribosomal protein S10; & P63325 & Rps10 & $-0,3$ & 23 \\
\hline $40 S$ ribosomal protein S12; & Q6ZWZ6 & Rps12 & 0,2 & 31,8 \\
\hline $40 S$ ribosomal protein S13; & P62301 & Rps13 & $-0,2$ & 19,2 \\
\hline 40 S ribosomal protein S15; & P62843 & Rps15 & 0,1 & 10,2 \\
\hline $40 S$ ribosomal protein S16; & P14131 & Rps16 & $-0,2$ & 19,9 \\
\hline $40 S$ ribosomal protein S18; & P62270 & Rps18 & $-0,3$ & 32,9 \\
\hline $40 S$ ribosomal protein S19; & Q9CZX8 & Rps19 & $-0,1$ & 27,5 \\
\hline 40 S ribosomal protein S2; & P25444 & Rps2 & $-4,6$ & 5 \\
\hline 40 S ribosomal protein $\mathrm{S} 20$; & P60867 & Rps20 & 0,0 & 10,1 \\
\hline $40 S$ ribosomal protein S21; & Q9CQR2 & Rps21 & $-0,4$ & 56,6 \\
\hline $40 S$ ribosomal protein S25; & P62852 & Rps25 & 0,0 & 22,4 \\
\hline $40 S$ ribosomal protein S28; & P62858 & Rps28 & $-0,4$ & 37,5 \\
\hline 40 S ribosomal protein S3; & P62908 & Rps3 & $-2,0$ & 11,5 \\
\hline 40 S ribosomal protein S30; & P62862 & Fau & 0,0 & 16,9 \\
\hline $40 \mathrm{~S}$ ribosomal protein $\mathrm{S} 4, \mathrm{X}$ isoform; & P62702 & $\operatorname{Rps} 4 x$ & $-0,3$ & 3,4 \\
\hline $40 S$ ribosomal protein S5; & D3YYM6 & Rps5 & $-0,1$ & 20,3 \\
\hline 40 S ribosomal protein S6; & P62754 & Rps6 & 0,0 & 4,8 \\
\hline $40 \mathrm{~S}$ ribosomal protein $\mathrm{SA}$; & P14206 & Rpsa & 0,2 & 19,3 \\
\hline 4F2 cell-surface antigen heavy chain; & P10852 & Slc3a2 & $-0,5$ & 6,7 \\
\hline 5'(3')-deoxyribonucleotidase, cytosolic type; & Q9JM14 & $\mathrm{Nt5c}$ & 0,5 & 6,7 \\
\hline 60 kDa heat shock protein, mitochondrial; & P63038 & Hspd1 & $-0,2$ & 8,9 \\
\hline $60 \mathrm{~S}$ acidic ribosomal protein $\mathrm{P} 0$ & P14869 & Rplp0 & 0,2 & 6,9 \\
\hline 605 acidic ribosomal protein $\mathrm{P} 2$ & P99027 & Rplp2 & $-0,2$ & 38,3 \\
\hline $60 \mathrm{~S}$ ribosomal protein $\mathrm{L} 10 \mathrm{a}$ & P53026 & Rpl10a & $-0,1$ & 13,8 \\
\hline $60 S$ ribosomal protein L12; & P35979 & Rpl12 & 0,1 & 44,8 \\
\hline $60 S$ ribosomal protein L13; & P47963 & Rpl13 & $-0,2$ & 8,7 \\
\hline 605 ribosomal protein L14; & Q9CR57 & Rpl14 & $-0,1$ & 6 \\
\hline $60 S$ ribosomal protein L17; & Q9CPR4 & Rpl17 & 0,0 & 16,3 \\
\hline $60 S$ ribosomal protein L21; & 009167 & Rpl21 & $-0,2$ & 4,4 \\
\hline $60 S$ ribosomal protein $\mathrm{L} 22$; & P67984 & Rpl22 & $-0,3$ & 18,8 \\
\hline $60 S$ ribosomal protein L23; & P62830 & Rpl23 & $-0,1$ & 13,3 \\
\hline $60 \mathrm{~S}$ ribosomal protein $\mathrm{L} 23 \mathrm{a}$; & P62751 & Rpl23a & $-0,1$ & 12,6 \\
\hline $60 S$ ribosomal protein L27; & P61358 & Rpl27 & $-0,3$ & 10,3 \\
\hline $60 S$ ribosomal protein $\mathrm{L} 27 \mathrm{a}$; & P14115 & Rpl27a & $-0,2$ & 7,4 \\
\hline $60 S$ ribosomal protein $\mathrm{L} 28$ & P41105 & Rpl28 & $-1,5$ & 8 \\
\hline $60 S$ ribosomal protein L31; & P62900 & Rpl31 & $-0,3$ & 7,2 \\
\hline 60 S ribosomal protein L35; & Q6ZWV7 & Rpl35 & 0,6 & 8,1 \\
\hline 605 ribosomal protein L38; & Q9JJI8 & Rpl38 & $-0,1$ & 24,3 \\
\hline $60 S$ ribosomal protein L39; & P62892 & Rpl39 & 0,7 & 19,6 \\
\hline $60 S$ ribosomal protein $\mathrm{L} 4$; & Q9D8E6 & Rpl4 & $-0,2$ & 2,1 \\
\hline $60 S$ ribosomal protein $\mathrm{L} 5$ & P47962 & Rpl5 & 0,5 & 7,1 \\
\hline $60 S$ ribosomal protein L6; & P47911 & Rpl6 & $-0,1$ & 8,8 \\
\hline $60 S$ ribosomal protein $\mathrm{L7a}$ & P12970 & Rpl7a & $-0,4$ & 3,4 \\
\hline 6-phosphogluconolactonase; & Q9CQ60 & Pgls & 0,7 & 4,8 \\
\hline 78 kDa glucose-regulated protein; & P20029 & Hspa5 & $-0,2$ & 24,7 \\
\hline Abl interactor 1 & Q8CBW3 & Abi1 & 1,0 & 7,4 \\
\hline Acetyl-CoA acetyltransferase, mitochondrial; & Q8QZT1 & Acat1 & 0,2 & 2,8 \\
\hline Acid ceramidase; & Q9WV54 & Asah1 & 0,9 & 12,7 \\
\hline
\end{tabular}




\begin{tabular}{|c|c|c|c|c|}
\hline Protein & Uniprot & Gene name & $\begin{array}{l}\text { Fold change of } \\
\text { XPRESS ratio } \\
\left.\text { (Egfr }{ }^{\Delta K C} / \mathrm{WT}\right)\end{array}$ & $\begin{array}{c}\text { Sequence } \\
\text { coverage [\%] }\end{array}$ \\
\hline Acidic leucine-rich nuclear phosphoprotein 32 family member $A$; & 035381 & Anp32a & 0,2 & 16,2 \\
\hline Acidic leucine-rich nuclear phosphoprotein 32 family member B; & Q9EST5 & Anp32b & 0,0 & 14,3 \\
\hline Aconitate hydratase, mitochondrial; & Q99KI0 & Aco2 & 0,1 & 7,8 \\
\hline Actin-binding LIM protein 1 & Q8K4G5 & Ablim1 & $-0,6$ & 18,9 \\
\hline Actin-related protein $2 / 3$ complex subun it $1 \mathrm{~B}$; & Q9WV32 & Arpc1b & 0,3 & 29,5 \\
\hline Actin-related protein $2 / 3$ complex subun it 5 & Q9CPW4 & Arpc5 & 0,2 & 29,1 \\
\hline Actin-related protein $2 / 3$ complex subun it 5 -like protein; & Q9D898 & Arpc5I & 0,6 & 16,3 \\
\hline Actin-related protein 3; & Q99JY9 & Actr3 & $-1,3$ & 5,7 \\
\hline Activated RNA polymerase II transcriptional coactivator $\mathrm{p} 15$; & P11031 & Sub1 & 0,2 & 8,7 \\
\hline Acyl-CoA-binding protein; & P31786 & Dbi & $-0,3$ & 39,1 \\
\hline Acyl-CoA-binding protein; & D3Z563 & Dbi & 0,0 & 41,3 \\
\hline Acylphosphatase-1; & P56376 & Acyp1 & 0,2 & 13,1 \\
\hline Acylphosphatase-2; & P56375 & Acyp2 & 0,0 & 12,3 \\
\hline Acylpyruvase FAHD1, mitochondrial; & Q8R0F8 & Fahd1 & $-0,2$ & 6,2 \\
\hline Adenosylhomocysteinase; & P50247 & Ahcy & 0,2 & 21,1 \\
\hline Adenylate kinase 2, mitochondrial; & Q9WTP6 & Ak2 & 0,0 & 15,3 \\
\hline Adenylate kinase isoenzyme 1 ; & Q9R0Y5 & Ak1 & 1,1 & 6,2 \\
\hline Adenylyl cyclase-associated protein 1 & P40124 & Cap1 & $-0,2$ & 2,1 \\
\hline ADP/ATP translocase 2 & P51881 & Slc25a5 & $-0,4$ & 12,4 \\
\hline ADP-ribosylation factor GTPase-activating protein 3; & Q9D8S3 & Arfgap3 & $-3,2$ & 1,9 \\
\hline ADP-sugar pyrophosphatase; & Q9JKX6 & Nudt5 & $-1,1$ & 16,1 \\
\hline Adrenodoxin-like protein, mitochondrial; & Q9CPW2 & Fdx1l & $-0,1$ & 7,5 \\
\hline Advanced glycosylation end product-specific receptor; & Q62151 & Ager & 0,3 & 10,2 \\
\hline Afadin; & Q9QZQ1 & Mllt4 & $-0,4$ & 3,3 \\
\hline Aftiphilin; & Q80WT5 & Aftph & 0,3 & 5,7 \\
\hline A-kinase anchor protein 1, mitochondrial; & 008715 & Akap1 & $-0,8$ & 1,3 \\
\hline A-kinase anchor protein 12 & Q9WTQ5 & Akap12 & 0,2 & 3,6 \\
\hline A-kinase anchor protein 2 & 054931 & Akap2 & $-0,3$ & 1,3 \\
\hline Alanine aminotransferase 1 & Q8QZR5 & Gpt & $-0,7$ & 1,8 \\
\hline Alcohol dehydrogenase $1 ;$ & P00329 & Adh1 & 0,9 & 2,4 \\
\hline Aldehyde dehydrogenase; & E9Q3E1 & Aldh3b2 & 0,4 & 20,3 \\
\hline $\begin{array}{l}\text { Allergen dl chain C2A; Androgen binding protein beta; Androgen-binding } \\
\text { protein; Protein Scgb2b27; }\end{array}$ & Q8R1E9 & Scgb2b27 & 0,9 & 20,5 \\
\hline Alpha-1-antitrypsin 1-1; & P07758 & Serpina1a & $-0,6$ & 12,1 \\
\hline Alpha-1-antitrypsin 1-2; & P22599 & Serpina1b & $-0,4$ & 12,1 \\
\hline Alpha-1-antitrypsin 1-4; & Q00897 & Serpina1d & $-0,9$ & 9 \\
\hline Alpha-2-HS-glycoprotein; & P29699 & Ahsg & $-0,2$ & 20,3 \\
\hline Alpha-2-macroglobulin receptor-associated protein; & P55302 & Lrpap1 & $-0,2$ & 10,6 \\
\hline Alpha-actinin-4; & P57780 & Actn4 & 0,3 & 8,1 \\
\hline Alpha-adducin; & Q9QYCo & Add1 & 0,5 & 3,3 \\
\hline Alpha-endosulfine; & P60840 & Ensa & 0,0 & 23,1 \\
\hline Alpha-enolase; & P17182 & Eno1 & 0,2 & 11,8 \\
\hline Alpha-fetoprotein; & P02772 & Afp & $-0,1$ & 20,2 \\
\hline Alpha-synuclein; & 055042 & Snca & $-1,3$ & 11,4 \\
\hline Alpha-taxilin; & Q6PAM1 & Txlna & $-0,6$ & 2,9 \\
\hline Aminoacyl tRNA synthase complex-interacting multifunctional protein 1; & P31230 & Aimp1 & 0,0 & 9,4 \\
\hline Aminopeptidase N; & P97449 & Anpep & $-0,2$ & 1,2 \\
\hline AN1-type zinc finger protein 2B; & Q91X58 & Zfand $2 b$ & $-0,3$ & 6,9 \\
\hline AN1-type zinc finger protein 6; & Q9DCH6 & Zfand6 & $-0,5$ & 13,5 \\
\hline Anamorsin; & Q8WTY4 & Ciapin1 & 0,7 & 23,4 \\
\hline Anaphase-promoting complex subunit 13; & Q8R034 & Anapc13 & 0,3 & 13,5 \\
\hline Anaphase-promoting complex subunit CDC26; & Q99JP4 & Cdc26 & 0,0 & 12,9 \\
\hline Androgen binding protein alpha; Androgen-binding protein; Protein Scgb1b27; & Q91WB5 & Scgb1b27 & $-0,5$ & 19,6 \\
\hline $\begin{array}{l}\text { Androgen binding protein gamma; MCG17480; Protein Scgb2b26; Salivary } \\
\text { androgen-binding protein gamma subunit; }\end{array}$ & Q8JZX1 & Scgb2b26 & 0,9 & 17,9 \\
\hline $\begin{array}{l}\text { Androgen-binding protein; Androgen-binding protein eta; Lacrimal androgen- } \\
\text { binding protein eta; Protein Scgb1b2; }\end{array}$ & 035176 & Scgb1b2 & 0,2 & 31,2 \\
\hline Ankyrin repeat domain-containing protein 17 & Q99NHO & Ankrd17 & 0,2 & 0,8 \\
\hline Ankyrin-2; & Q8C8R3 & Ank2 & 0,1 & 0,4 \\
\hline Ankyrin-3; & G5E8K5 & Ank3 & $-0,3$ & 3 \\
\hline Annexin A1; & P10107 & Anxa1 & 0,4 & 18,2 \\
\hline Annexin A2; & P07356 & Anxa2 & 1,2 & 14,5 \\
\hline Annexin A4; & P97429 & Anxa4 & 0,5 & 15,8 \\
\hline Annexin A5; & P48036 & Anxa5 & 0,5 & 19,7 \\
\hline Annexin A6; & P14824 & Anxa6 & 1,3 & 3,9 \\
\hline Annexin A8; & 035640 & Anxa8 & $-0,6$ & 8,3 \\
\hline Antithrombin-III; & P32261 & Serpinc1 & 0,2 & 2,4 \\
\hline AP2-associated protein kinase 1 ; & Q3UHJ0 & Aak1 & 0,4 & 2,6 \\
\hline Apolipoprotein A-I; & Q00623 & Apoa1 & $-0,2$ & 26,1 \\
\hline
\end{tabular}




\begin{tabular}{|c|c|c|c|c|}
\hline Protein & Uniprot & Gene name & $\begin{array}{l}\text { Fold change of } \\
\text { XPRESS ratio } \\
\left(E_{g f r}{ }^{\Delta K C} / \mathrm{WT}\right)\end{array}$ & $\begin{array}{c}\text { Sequence } \\
\text { coverage [\%] }\end{array}$ \\
\hline Apolipoprotein A-II; & P09813 & Apoa2 & 0,3 & 9,8 \\
\hline Apolipoprotein A-IV; & P06728 & Apoa4 & $-0,7$ & 34,7 \\
\hline Apolipoprotein C-I; & P34928 & Apoc1 & 0,0 & 20,5 \\
\hline Apolipoprotein E; & P08226 & Apoe & $-1,3$ & 17,4 \\
\hline Apolipoprotein O; & Q9DCZ4 & Apoo & $-2,2$ & 6,7 \\
\hline Apoptosis regulator BAX; & Q07813 & Bax & 0,1 & 6,8 \\
\hline Apoptotic chromatin condensation inducer in the nucleus; & Q9JIX8 & Acin 1 & $-0,2$ & 4 \\
\hline Arachidonate 12-lipoxygenase, 12S-type; & P39655 & Alox12 & 0,2 & 4,1 \\
\hline Arf-GAP domain and FG repeat-containing protein 1; & Q8K2K6 & Agfg1 & 0,6 & 7,8 \\
\hline Arfip1 protein; Protein Arfip1; & A2RSX9 & Arfip1 & 0,6 & 10 \\
\hline Arginase-1; & Q61176 & Arg1 & 0,9 & 3,4 \\
\hline Arginine and glutamate-rich protein 1 & Q3UL36 & Arglu1 & $-0,6$ & 3 \\
\hline $\begin{array}{l}\text { Arginine-rich, mutated in early stage tumors, isoform CRA_b; Mesencephalic } \\
\text { astrocyte-derived neurotrophic factor; }\end{array}$ & Q3TMX5 & Manf & 0,1 & 14,5 \\
\hline Arpin; & Q9D0A3 & Arpin & 0,1 & 5,3 \\
\hline Aryl hydrocarbon receptor; & P30561 & Ahr & 0,3 & 1,1 \\
\hline Aspartate aminotransferase, cytoplasmic; & P05201 & Got1 & 0,9 & 6,8 \\
\hline Aspartate aminotransferase, mitochondrial; & P05202 & Got2 & $-0,5$ & 8,1 \\
\hline Aspartyl aminopeptidase; & Q9Z2W0 & Dnpep & 4,2 & 3 \\
\hline Astrocytic phosphoprotein PEA-15; & Q62048 & Pea15 & 0,5 & 7,7 \\
\hline Ataxin-2-like protein; & Q7TQHO & Atxn2l & 0,5 & 4,8 \\
\hline ATP synthase protein 8 & P03930 & Mtatp8 & $-0,1$ & 14,9 \\
\hline ATP synthase subunit alpha, mitochond rial; & Q03265 & Atp5a1 & $-0,6$ & 10,8 \\
\hline ATP synthase subunit beta, mitochondrial; & P56480 & Atp5b & 0,2 & 7,4 \\
\hline ATP synthase subunit d, mitochondrial; & Q9DCX2 & Atp5h & 0,0 & 44,9 \\
\hline ATP synthase subunit delta, mitochond rial; & Q9D3D9 & Atp5d & 1,5 & 13,7 \\
\hline ATP synthase subunit e, mitochondrial; & Q06185 & Atp5i & 0,2 & 28,9 \\
\hline ATP synthase subunit $f$, mitochondrial; & P56135 & Atp5j2 & 0,0 & 15,8 \\
\hline ATP synthase subunit $O$, mitochondrial; & Q9DB20 & Atp5o & 0,3 & 6,2 \\
\hline ATP synthase-coupling factor 6 , mitochondrial; & P97450 & Atp5j & $-0,1$ & 24,1 \\
\hline ATPase inhibitor, mitochondrial; & 035143 & Atpif1 & 0,0 & 9,5 \\
\hline ATP-binding cassette sub-family E member 1 ; & P61222 & Abce1 & 0,5 & 1,7 \\
\hline ATP-binding cassette sub-family $\mathrm{F}$ member 1 ; & Q6P542 & Abcf1 & 0,1 & 3,5 \\
\hline ATP-citrate synthase; & Q91V92 & Acly & 0,0 & 0,9 \\
\hline ATP-dependent RNA helicase A; & 070133 & Dhx9 & 0,4 & 0,6 \\
\hline ATP-dependent RNA helicase DDX19A; & Q61655 & Ddx19a & $-0,1$ & 3,1 \\
\hline ATP-dependent RNA helicase DDX3X; & Q62167 & $D d x 3 x$ & $-0,4$ & 1,1 \\
\hline ATP-dependent RNA helicase DDX42; & Q810A7 & $\mathrm{Ddx} 42$ & $-0,4$ & 1,4 \\
\hline Atrial natriuretic peptide-converting enzyme; & Q9Z319 & Corin & $-0,1$ & 1,1 \\
\hline AT-rich interactive domain-containing protein $1 \mathrm{~A}$; & $\mathrm{A} 2 \mathrm{BH} 40$ & Arid1a & 0,0 & 0,9 \\
\hline Atrophin-1; & 035126 & Atn1 & 0,0 & 1 \\
\hline Aurora kinase B; & E9PVP1 & Aim1 & $-0,5$ & 5,7 \\
\hline BAG family molecular chaperone regulator 3 ; & Q9JLV1 & Bag3 & $-0,5$ & 15,6 \\
\hline Band 4.1-like protein 2; & 070318 & Epb41/2 & 0,8 & 6 \\
\hline Band 4.1-like protein 3; & Q9WV92 & Epb4113 & 0,5 & 6,2 \\
\hline Barrier-to-autointegration factor; & 054962 & Banf1 & 0,1 & 34,8 \\
\hline Basigin; & P18572 & Bsg & 0,0 & 27,9 \\
\hline B-cell CLL/lymphoma 7 protein family member A; & Q9CXE2 & $\mathrm{Bcl} 7 \mathrm{a}$ & 0,2 & 8,6 \\
\hline B-cell CLL/lymphoma 7 protein family member C; & 008664 & $\mathrm{Bcl} 7 \mathrm{c}$ & $-1,1$ & 10,1 \\
\hline B-cell CLL/lymphoma 9-like protein; & Q67FY2 & Bc|9l & $-0,7$ & 4,6 \\
\hline B-cell linker protein; & Q9QUN3 & Blnk & $-0,3$ & 2,6 \\
\hline B-cell receptor-associated protein 31 & Q61335 & Bcap31 & 0,4 & 4,1 \\
\hline $\mathrm{BCL} 2 /$ adenovirus E1B 19 kDa protein-interacting protein 3-like; & Q9Z2F7 & Bnip3l & 0,0 & 4,9 \\
\hline Bcl-2/adenovirus E1B 19 kDa-interacting protein 2-like protein; & Q99JU7 & Bnipl & $-0,1$ & 3 \\
\hline Bcl2-associated agonist of cell death; & Q61337 & Bad & $-1,0$ & 22,8 \\
\hline Bcl-2-associated transcription factor 1 & Q8K019 & Bclaf1 & $-0,3$ & 3,6 \\
\hline Bcl-2-like protein 13; & P59017 & $\mathrm{Bcl} 2 \mathrm{I} 13$ & 0,1 & 2,5 \\
\hline BET1-like protein; & 035153 & Bet1l & $-0,1$ & 11,7 \\
\hline Beta-catenin-interacting prote in 1 ; & Q9JJN6 & Ctnnbip1 & $-0,5$ & 29,6 \\
\hline Beta-galactosidase-1-like protein; & Q8VC60 & Glb1l & $-1,9$ & 3,4 \\
\hline Beta-globin; Protein Hbb-bs; Protein Hbb-bt; & A8DUK4 & Hbbt1 & $-0,7$ & 68,7 \\
\hline Beta-hexosaminidase subunit beta; & P20060 & Hexb & 0,0 & 8,8 \\
\hline Bifunctional glutamate/proline--tRNA ligase; & Q8CGC7 & Eprs & 0,1 & 0,5 \\
\hline Biogenesis of lysosome-related organelles complex 1 subun it 2; & Q9CWG9 & Bloc1s2 & 0,1 & 5,6 \\
\hline Bis(5'-adenosyl)-triphosphatase; & 089106 & Fhit & 0,2 & 12,9 \\
\hline Bleomycin hydrolase; & Q8R016 & Blmh & 0,1 & 20,9 \\
\hline BolA-like protein 1; & Q9D8S9 & Bola1 & 0,0 & 32,1 \\
\hline BolA-like protein 2; & Q8BGS2 & Bola2 & 0,3 & 32,6 \\
\hline BPI fold-containing family A member 2; & P07743 & Bpifa2 & 0,9 & 5,2 \\
\hline BPI fold-containing family C protein; & Q8C186 & Bpifc & 0,0 & 13,4 \\
\hline Brain acid soluble protein 1 & Q91XV3 & Basp1 & $-0,3$ & 15 \\
\hline
\end{tabular}




\begin{tabular}{|c|c|c|c|c|}
\hline Protein & Uniprot & Gene name & $\begin{array}{l}\text { Fold change of } \\
\text { XPRESS ratio } \\
\left(\text { Egfr }^{\triangle K C} / \mathrm{WT}\right)\end{array}$ & $\begin{array}{c}\text { Sequence } \\
\text { coverage [\%] }\end{array}$ \\
\hline Brain-specific angiogenesis inhibitor 1-associated protein 2; & Q8BKX1 & Baiap2 & 0,0 & 2,1 \\
\hline Breast carcinoma-amplified sequence 3 homolog; & Q8CCN5 & Bcas3 & 0,4 & 3,3 \\
\hline Bridging integrator 2; & D3Z6Q9 & Bin2 & 0,2 & 3,2 \\
\hline BUB3-interacting and GLEBS motif-containing protein ZNF207; & E9PW12 & Znf207 & $-0,3$ & 3,8 \\
\hline Cadherin-1; & P09803 & Cdh1 & 0,0 & 7 \\
\hline Cadherin-13; & Q9WTR5 & Cdh13 & 0,4 & 8,4 \\
\hline Calcineurin B homologous protein 1 ; & P61022 & Chp1 & $-0,2$ & 16,2 \\
\hline Calcium homeostasis endoplasmic reticulum protein; & Q8CGZO & Cherp & 0,0 & 1,5 \\
\hline $\begin{array}{l}\text { Calcium-activated chloride channel regulator 2; Chloride channel calcium } \\
\text { activated 2; Endothelial chloride channel; MCG120735; }\end{array}$ & Q9EQR4 & Clca2 & 0,1 & 6,2 \\
\hline Calcium-regulated heat stable protein 1 & Q9CR86 & Carhsp1 & 0,3 & 10,8 \\
\hline Calcyclin-binding protein; & Q9CXW3 & Cacybp & $-3,3$ & 8,7 \\
\hline Calmodulin; & P62204 & Calm3 & $-0,3$ & 34,2 \\
\hline Calmodulin; & G3UZ90 & Calm3 & $-0,4$ & 30,8 \\
\hline Calmodulin-4; & Q9JM83 & Calm4 & 0,0 & 41,9 \\
\hline Calmodulin-like protein 3; & Q9D6P8 & Calml3 & 0,5 & 22,1 \\
\hline Calmodulin-regulated spectrin-associated protein 3 ; & Q80VC9 & Camsap3 & $-0,2$ & 1,1 \\
\hline Calnexin; & P35564 & Canx & 0,2 & 5,4 \\
\hline Calpain small subunit 2 & Q9D7J7 & Capns2 & $-0,2$ & 9,7 \\
\hline Calpain-1 catalytic subunit; & 035350 & Capn1 & $-0,1$ & 7,4 \\
\hline Calpain-15; & Q9JLG8 & Capn15 & $-0,1$ & 1 \\
\hline Calpastatin; & P51125 & Cast & $-0,3$ & 24 \\
\hline Calponin-2; & Q08093 & Cnn2 & 0,7 & 7,2 \\
\hline Calponin-3; & Q9DAW9 & Cnn3 & 0,8 & 11,5 \\
\hline Calreticulin; & P14211 & Calr & 0,4 & 13,5 \\
\hline Calumenin; & 035887 & Calu & $-0,3$ & 22,9 \\
\hline Calumenin; Calumenin, isoform CRA_b; Cardiac calumenin isoform; & Q6XLQ8 & Calu & $-0,2$ & 20 \\
\hline cAMP-dependent protein kinase inhibitor gamma; & 070139 & Pkig & $-0,3$ & 28,6 \\
\hline cAMP-regulated phosphoprotein 19 & P56212 & Arpp19 & 0,0 & 38,4 \\
\hline CAP-Gly domain-containing linker protein 1 ; & Q922J3 & Clip1 & 0,0 & 23,6 \\
\hline Caprin-1; & Q60865 & Caprin1 & $-0,2$ & 11,6 \\
\hline Carbonyl reductase $[\mathrm{NADPH}] 1$ & P48758 & Cbr1 & $-10,0$ & 4,7 \\
\hline Carbonyl reductase [NADPH] 2; & P08074 & Cbr2 & 2,2 & 6,5 \\
\hline Carboxylesterase $1 \mathrm{C}$ & P23953 & Ces1c & 0,1 & 2,2 \\
\hline Carboxypeptidase A4; & Q6P8K8 & Cpa4 & 0,4 & 5 \\
\hline Carboxypeptidase E; & Q00493 & Cpe & 0,5 & 9,7 \\
\hline Carboxypeptidase M; & Q80V42 & $\mathrm{Cpm}$ & 0,4 & 5,4 \\
\hline Caspase activity and apoptosis inhibitor 1 ; & Q8VDY9 & Caap1 & 0,0 & 7,3 \\
\hline Caspase-14; & 089094 & Casp14 & 0,0 & 30 \\
\hline Catalase; & P24270 & Cat & 0,9 & 29,6 \\
\hline Catenin alpha-1; & P26231 & Ctnna1 & 0,8 & 3,4 \\
\hline Catenin delta-1; & P30999 & Ctnnd1 & $-0,3$ & 5,8 \\
\hline Cathepsin B; & P10605 & Ctsb & $-0,7$ & 15,3 \\
\hline Cathepsin D; & P18242 & Ctsd & $-0,3$ & 14,1 \\
\hline Cathepsin L1; & P06797 & Ctsl & 0,3 & 13,8 \\
\hline Cation-dependent mannose-6-phosphate receptor; & P24668 & M6pr & 0,2 & 12,9 \\
\hline Caveolin-1; & P49817 & Cav1 & 0,2 & 24,7 \\
\hline Caveolin-2; & Q9WVC3 & Cav2 & $-0,3$ & 7,4 \\
\hline CCR4-NOT transcription complex subunit 3; & Q8K0V4 & Cnot3 & $-0,1$ & 2,6 \\
\hline CD109 antigen; & Q8R422 & Cd109 & 0,2 & 1,7 \\
\hline CD166 antigen; & Q61490 & Alcam & 0,0 & 8,3 \\
\hline CD2 antigen cytoplasmic tail-binding protein 2 ; & Q9CWK3 & $\mathrm{Cd} 2 \mathrm{bp} 2$ & 0,4 & 4,1 \\
\hline CD2-associated protein; & Q9JLQ0 & Cd2ap & 0,4 & 15,4 \\
\hline CD44 antigen; & P15379 & $\mathrm{Cd} 44$ & 0,7 & 9,1 \\
\hline CD63 antigen; & P41731 & Cd63 & 0,3 & 7,6 \\
\hline CD82 antigen; & P40237 & Cd82 & 0,2 & 12,8 \\
\hline CD9 antigen; & P40240 & Cd9 & 0,6 & 3,1 \\
\hline Cdc42 effector protein 4 & Q9JM96 & Cdc42ep4 & 0,2 & 10,8 \\
\hline Cdc42 effector protein 5 & Q9Z0X0 & Cdc42ep5 & $-0,2$ & 16,5 \\
\hline CDGSH iron-sulfur domain-containing protein 2; & Q9CQB5 & Cisd2 & $-0,2$ & 18,5 \\
\hline Cell adhesion molecule 1 ; & Q8R5M8 & Cadm1 & $-0,3$ & 3,3 \\
\hline Cell death regulator Aven; & Q9D9K3 & Aven & $-0,3$ & 16,1 \\
\hline Cell growth regulator with EF hand domain protein 1 ; & Q8R1U2 & Cgref1 & $-0,5$ & 3,9 \\
\hline Cellular nucleic acid-binding protein; & P53996 & Cnbp & 0,1 & 22,5 \\
\hline Centrin-2; & Q9R1K9 & Cetn2 & $-0,1$ & 14,4 \\
\hline CGG triplet repeat-binding protein 1 ; & Q8BHG9 & Cggbp1 & $-0,4$ & 6 \\
\hline Charged multivesicular body protein $1 \mathrm{a}$; & Q921W0 & Chmp1a & 0,6 & 4,6 \\
\hline Charged multivesicular body protein $2 \mathrm{~b}$; & Q8BJF9 & Chmp2b & 0,0 & 4,7 \\
\hline Charged multivesicular body protein $4 \mathrm{~b}$; & Q9D8B3 & Chmp4b & 0,0 & 30,4 \\
\hline Chitinase-3-like protein 1 & Q61362 & Chi3l1 & $-0,1$ & 9,8 \\
\hline Chitotriosidase-1; & Q9D7Q1 & Chit1 & 0,0 & 12,3 \\
\hline
\end{tabular}




\begin{tabular}{|c|c|c|c|c|}
\hline Protein & Uniprot & Gene name & $\begin{array}{l}\text { Fold change of } \\
\text { XPRESS ratio } \\
\left.\text { (Egfr }{ }^{\Delta K C} / \mathrm{WT}\right)\end{array}$ & $\begin{array}{c}\text { Sequence } \\
\text { coverage [\%] }\end{array}$ \\
\hline Choline transporter-like prote in 2; & Q8BY89 & Slc44a2 & 0,0 & 2,7 \\
\hline Chromatin complexes subun it BAP18; & Q9DCT6 & Bap18 & 0,0 & 17,3 \\
\hline Chromobox protein homolog 1 ; & P83917 & $\mathrm{Cbx} 1$ & 0,4 & 12,4 \\
\hline Chromobox protein homolog 3 & P23198 & $\mathrm{Cbx3}$ & 0,3 & 6,6 \\
\hline Chromobox protein homolog 5 & Q61686 & Cbx5 & $-1,8$ & 19 \\
\hline Chromogranin-A; & P26339 & Chga & 0,1 & 2,4 \\
\hline Cingulin; & P59242 & Cgn & $-0,5$ & 7,7 \\
\hline Citrate synthase, mitochondrial; & Q9CZU6 & Cs & 0,8 & 2,4 \\
\hline Clathrin interactor 1 & Q99KN9 & Clint1 & $-0,2$ & 13,3 \\
\hline Clathrin light chain $A$; & 008585 & Clta & $-0,3$ & 18,5 \\
\hline Clathrin light chain B; & Q6IRU5 & Cltb & $-0,1$ & 26,2 \\
\hline Cleavage stimulation factor subunit 2 ; & Q8BIQ5 & Cstf2 & $-0,1$ & 9 \\
\hline Clusterin; & Q06890 & $\mathrm{Clu}$ & $-0,5$ & 24,3 \\
\hline C-Myc-binding protein; & Q9EQS3 & Mycbp & 0,1 & 62,1 \\
\hline Coactosin-like protein; & Q9CQI6 & Cotl1 & 0,3 & 5,6 \\
\hline Cob(I)yrinic acid a,c-diamide adenosyltransferase, mitochondrial; & Q9D273 & Mmab & 0,2 & 5,6 \\
\hline Cofilin-1; & P18760 & Cfl1 & 0,3 & 19,9 \\
\hline Coiled-coil and $\mathrm{C} 2$ domain-containing protein $1 \mathrm{~B}$; & Q8BRN9 & $\mathrm{Cc} 2 \mathrm{~d} 1 \mathrm{~b}$ & 0,1 & 2,5 \\
\hline Coiled-coil domain-containing protein 12 & Q8R344 & Ccdc12 & 0,0 & 28,3 \\
\hline Coiled-coil domain-containing protein 124 & Q9D8X2 & Ccdc124 & $-1,0$ & 10,6 \\
\hline Coiled-coil domain-containing protein 186 & Q8C9S4 & Ccdc186 & 0,3 & 1,5 \\
\hline Coiled-coil domain-containing protein 43 ; & Q9CR29 & Ccdc43 & $-0,1$ & 5 \\
\hline Coiled-coil domain-containing protein 50 ; & Q810U5 & Ccdc50 & $-0,5$ & 19,1 \\
\hline Coiled-coil domain-containing protein 58 & Q8R3Q6 & Ccdc58 & $-1,7$ & 19,6 \\
\hline Coiled-coil domain-containing protein 86 & Q9JJ89 & Ccdc86 & $-1,1$ & 2,6 \\
\hline Coiled-coil domain-containing protein 9; & Q8VC31 & Ccdc9 & $-0,2$ & 8,3 \\
\hline Coiled-coil domain-containing protein 91 & Q9D8L5 & Ccdc91 & $-0,3$ & 2 \\
\hline Coiled-coil-helix-coiled-coil-helix domain-containing protein 2, mitochondrial; & Q9D1L0 & Chchd2 & $-0,4$ & 15 \\
\hline Coiled-coil-helix-coiled-coil-helix domain-containing protein 3, mitochondrial; & Q9CRB9 & Chchd3 & $-0,3$ & 17,9 \\
\hline Coiled-coil-helix-coiled-coil-helix domain-containing protein 5; & Q9CQP3 & Chchd5 & 0,3 & 10,9 \\
\hline Cold-inducible RNA-binding protein; & P60824 & Cirbp & 0,0 & 10,2 \\
\hline Collagen alpha-1(I) chain; & P11087 & Col1a1 & $-0,7$ & 9,7 \\
\hline Collagen alpha-1(II) chain; & P28481 & Col2a1 & $-0,6$ & 2,8 \\
\hline Collagen alpha-1(III) chain; & P08121 & Col3a1 & $-0,3$ & 7,5 \\
\hline Collagen alpha-1(VII) chain; & Q63870 & Col7a1 & $-0,5$ & 0,7 \\
\hline Collagen alpha-1(XVII) chain; & Q07563 & Col17a1 & $-0,6$ & 4,4 \\
\hline Collagen alpha-2(I) chain; & Q01149 & Col1a2 & $-0,5$ & 18 \\
\hline Complement component $1 \mathrm{Q}$ subcomponent-binding protein, mitochondrial; & 035658 & C1qbp & 0,3 & 8,6 \\
\hline Complement factor $\mathrm{D}$; & P03953 & $\mathrm{Cfd}$ & $-0,2$ & 8,1 \\
\hline Complexin-2; & P84086 & Cplx2 & $-0,1$ & 11,2 \\
\hline COP9 signalosome complex subunit 8 & Q8VBV7 & Cops8 & 0,8 & 13,9 \\
\hline Copine-1; & Q8C166 & Cpne1 & 0,2 & 5,4 \\
\hline Copine-3; & Q8BT60 & Cpne3 & 0,4 & 3,8 \\
\hline Copper transport protein ATOX1; & 008997 & Atox1 & 0,0 & 57,4 \\
\hline Corneodesmosin; & Q7TPC1 & Cdsn & $-0,1$ & 18,4 \\
\hline Cornifin-A; & Q62266 & Sprr1a & $-1,7$ & 37,5 \\
\hline Coronin-1C; & Q9WUM4 & Coro1c & $-0,3$ & 2,1 \\
\hline Costars family protein ABRACL; & Q4KML4 & Abracl & $-0,5$ & 27,2 \\
\hline Coxsackievirus and adenovirus receptor homolog; & P97792 & Cxadr & $-0,6$ & 16,4 \\
\hline Craniofacial development protein 1 & 088271 & Cfdp1 & $-0,1$ & 13,9 \\
\hline Creatine kinase B-type; & Q04447 & $\mathrm{Ckb}$ & $-0,8$ & 2,9 \\
\hline Creatine kinase U-type, mitochondrial; & P30275 & Ckmt1 & $-0,2$ & 18,7 \\
\hline C-terminal-binding protein 1 & 088712 & Ctbp1 & 0,3 & 4,5 \\
\hline CTTNBP2 N-terminal-like protein; & Q99L0 & Cttnbp2nl & $-0,1$ & 2 \\
\hline Cyclic AMP-responsive element-binding protein 1; & Q01147 & Creb1 & $-0,4$ & 10,5 \\
\hline Cyclic GMP-AMP synthase; & Q8C6L5 & $\mathrm{Mb} 21 \mathrm{~d} 1$ & $-4,5$ & 2,2 \\
\hline Cyclin-dependent kinase inhibitor 1B; & P46414 & Cdkn1b & $-0,3$ & 24,9 \\
\hline Cyclin-dependent kinase inhibitor 1C; & P49919 & Cdkn1c & $-0,6$ & 4,9 \\
\hline Cystatin E/M; Cystatin M/E; Cystatin-B; & Q9D1B1 & Cst6 & $-0,7$ & 32,9 \\
\hline Cystatin-A; & P56567 & Csta & 0,2 & 37,1 \\
\hline Cystatin-B; & Q62426 & Cstb & $-0,3$ & 39,8 \\
\hline Cystatin-C; & P21460 & Cst3 & 0,4 & 26,5 \\
\hline Cysteine and glycine-rich protein $1 ;$ & P97315 & Csrp1 & $-0,7$ & 7,8 \\
\hline Cysteine-rich protein 1; & P63254 & Crip1 & 0,6 & 9,1 \\
\hline Cysteine-rich with EGF-like domain protein 2; & Q9CYAO & Creld2 & 0,2 & 5,1 \\
\hline Cytochrome b5 type B; & Q9CQX2 & Cyb5b & 0,2 & 8,2 \\
\hline Cytochrome b5; & P56395 & Cyb5a & 0,4 & 65,3 \\
\hline Cytochrome b-c1 complex subunit 1, mitochondrial; & Q9CZ13 & Uqcrc1 & 0,1 & 8,8 \\
\hline
\end{tabular}




Protein

Uniprot Gene name

Fold change of

Cytochrome b-c1 complex subunit 2, mitochondrial;

Q9DB77

Q9DB77

P99028

Cytochrome b-c1 complex subunit 6, mitochondrial;

Cytochrome b-c1 complex subunit 7;

Cytochrome b-c1 complex subunit 8;

Cytochrome b-c1 complex subunit 9;

Cytochrome b-c1 complex subunit Rieske, mitochondrial;

Cytochrome c oxidase assembly factor 3 homolog, mitochondrial;

Cytochrome c oxidase assembly factor 6 homolog;

Cytochrome c oxidase subunit 4 isoform 1, mitochondrial;

Q9D855

Q9CQ69

Q9CR68

Q9D2R6

Q8BGD8

$\mathrm{P} 19783$

Cytochrome c oxidase subunit 5A, mitochond rial;

Cytochrome c oxidase subunit 6B1;

Cytochrome c oxidase subunit 6C;

P12787

P56391

Q9CPQ1

Cytochrome c oxidase subunit 7A2, mitochondrial;

P48771

Cytochrome c oxidase subunit $7 \mathrm{C}$, mitochondrial;

P17665

Cytochrome c, somatic;

Cytoplasmic dynein 1 light intermediate chain 2;

P62897

Q6PDL0

Cytoskeleton-associated protein 4;

Cytosol aminopeptidase;

Cytosolic 5'-nucleotidase 3A;

Q8BMK4

Q9CPY7

Cytosolic non-specific dipeptidase;

Q9D020

Q9D1A2

Cytospin-B;

D-3-phosphoglycerate dehydrogenase;

Q5SXY1

Q61753

035215

Q8CJ26

Death domain-containing membrane protein NRADD;

Death-associated protein-like 1 ;

Delta-aminolevulinic acid dehydratase;

Q9D757

P10518

Q9CQJ6

055070

Deoxyribonuclease gamma;

Deoxyribonuclease-2-alpha;

Dermokine;

Desmocollin 1; Desmocollin 1, isoform CRA_a; Desmocollin-1;

P56542

E9Q2P1

Q32ME9

G5E8S6

Desmoglein-1-alpha;

Desmoplakin;

Q61495

E9Q557

Q9R0P5

Destrin;

Dickkopf-related protein 3;

Dihydrolipoyl dehydrogenase, mitochondrial;

Dihydrolipoyllysine-residue succinyltransferase component of 2-oxoglutarate

Q9QUN9

008749

dehydrogenase complex, mitochondrial;

Dihydropyrimidinase-related protein 3;

Dimethylaniline monooxygenase [N-oxide-forming] 2;

Q9D2G2

Q62188

Q8K2I3

Dipeptidyl peptidase 1;

Disabled homolog 2;

Disco-interacting protein 2 homolog B;

Disks large-associated protein 4;

DNA fragmentation factor subunit alpha;

DNA polymerase delta subunit 4;

DNA polymerase epsilon subunit 3;

DNA-directed RNA polymerase III subunit RPC7;

DnaJ homolog subfamily A member 1 ;

DnaJ homolog subfamily A member 4;

DnaJ homolog subfamily B member 1 ;

DnaJ homolog subfamily B member 12 ;

DnaJ homolog subfamily B member 2;

DnaJ homolog subfamily B member 6;

DnaJ homolog subfamily $\mathrm{C}$ member 5 ;

DnaJ homolog subfamily $\mathrm{C}$ member 8 ;

DnaJ homolog subfamily $\mathrm{C}$ member 9 ;

Dolichyl-diphosphooligosaccharide--protein glycosyltransferase subunit 1;

P97821

P98078

Q3UH60

B1AZP2

054786

Q9CWP8

Q9JKP7

Q6NXY9

P63037

Q9JMC3

Q9QYJ3

Q9QY14

Q9QYI5

054946

P60904

Q6NZB0

Q91WN1

Uqcrc2
Uqcrh
Uqcrb
Uqcrq
Uqcr10
Uqcrfs1
Coa3
Coa6

XPRESS ratio

Sequence

Egfr $^{\Delta K C}$ / WT)

coverage [\%]

$-0,2 \quad 3,1$

$0,3 \quad 28,1$

0,1

28,1

0,1
$0,3 \quad 9,8$

$0,3 \quad 26,6$

$0,1 \quad 17,2$

$0,3 \quad 9,3$

$0,0 \quad 16,5$

Cox4i1

Cox5a

Cox6b1

Cox6c

Cox7a2

Cox7c

Cycs

Dync1li2

Ckap4

Lap3

$\mathrm{Nt5c3a}$

Cndp2

Specc1

Phgdh

Ddt

Nradd

Dapl1

Alad

Denr

Dnase1/3

Dnase2

Dmkn

Dsc1

Dsc3

Dsg1a

Dsp

Dstn

Dkk3

Dld

0,4

16,5
23,7

32,2

52,3

0,3

$-0,2$

19,7

27,7

14,3

38,1

9,1

17

2,3

Fmo2

Ctsc

Dab2

Dip2b

Dlgap4

Dffa

Pold4

Pole3

Polr3g

Dnaja1

Dnaja4

Dnajb1

Dnajb12

Dnajb2

Dnajb6

Dnajc5

Dnajc8

Dnajc9

Q91YQ5

Rpn1

Q9D6N5

Q62418

Q99KJ8

Q9D0M5

P62627

Q62165

Q8BL66

Q3UMY5

Q9D8Y0

Q9DCW4

EF-hand domain-containing protein D2;

Electron transfer flavoprotein subunit beta;

Elongation factor 1-alpha 1;

P10126

Drap1
Dbnl
Dctn2
Dynll2
Dynlrb1
Dag1
Eea1
Eml4
Efhd2
Etfb
Eef1a1




\begin{tabular}{|c|c|c|c|c|}
\hline Protein & Uniprot & Gene name & $\begin{array}{l}\text { Fold change of } \\
\text { XPRESS ratio } \\
\left.\text { (Egfr }{ }^{\Delta K C} / \mathrm{WT}\right)\end{array}$ & $\begin{array}{c}\text { Sequence } \\
\text { coverage [\%] }\end{array}$ \\
\hline Elongation factor 1-beta; & 070251 & Eef1b & 0,4 & 32,4 \\
\hline Elongation factor 1-delta; & F6ZFU0 & Eef1d & 0,6 & 14,1 \\
\hline Elongation factor 2 ; & P58252 & Eef2 & $-0,2$ & 7,8 \\
\hline Embryonal Fyn-associated substrate; & Q64355 & Efs & $-6,2$ & 1,8 \\
\hline Emerin; & 008579 & Emd & 3,2 & 7,6 \\
\hline Endophilin-A2; & Q62419 & Sh3gl1 & $-1,2$ & 3,3 \\
\hline Endophilin-A3; & Q62421 & Sh3gl3 & $-0,7$ & 6,1 \\
\hline Endoplasmic reticulum resident protein 29; & P57759 & Erp29 & 0,3 & 11,8 \\
\hline Endoplasmic reticulum resident protein 44 & Q9D1Q6 & Erp44 & 0,0 & 2,2 \\
\hline Endoplasmin; & $\mathrm{P} 08113$ & Hsp90b1 & 0,2 & 7,9 \\
\hline Endothelial differentiation-related factor 1 & Q9JMG1 & Edf1 & 0,1 & 16,9 \\
\hline Enhancer of rudimentary homolog; & P84089 & Erh & 0,3 & 26,9 \\
\hline Enoyl-CoA hydratase, mitochondrial; & Q8BH95 & Echs1 & $-0,9$ & 5,8 \\
\hline Ensconsin; & 088735 & Map7 & $-0,2$ & 3,3 \\
\hline Envoplakin; & Q9D952 & Evpl & $-0,2$ & 1,6 \\
\hline Eosinophil cationic-type ribonuclease 5; & 035292 & Ear5 & 0,5 & 14,2 \\
\hline Epidermal growth factor receptor substrate 15 & P42567 & Eps15 & 0,3 & 7,1 \\
\hline Epidermal growth factor receptor substrate 15 -like 1 ; & Q60902 & Eps15|1 & 0,2 & 1,2 \\
\hline Epididymal secretory protein E1; & Q9Z0J0 & Npc2 & 0,1 & 18,8 \\
\hline Epiplakin; & Q8R0W0 & Eppk1 & $-0,6$ & 2,5 \\
\hline Epithelial cell adhesion molecule; & Q99JW5 & Epcam & 0,0 & 8,9 \\
\hline Epsin-2; & Q8CHU3 & Epn2 & $-0,1$ & 3,5 \\
\hline Erlin-2; & Q8BFZ9 & Erlin2 & 0,2 & 3,5 \\
\hline Eukaryotic peptide chain release factor GTP-bind ing subunit ERF3A; & Q8R050 & Gspt1 & $-0,2$ & 3 \\
\hline Eukaryotic translation initiation factor $1 \mathrm{~A}$ & Q60872 & Eif1a & $-0,1$ & 7,6 \\
\hline Eukaryotic translation initiation factor 2 subunit 2 & Q99L45 & Eif2s2 & $-0,3$ & 18,6 \\
\hline Eukaryotic translation initiation factor 3 subunit $B$; & Q8JZQ9 & Eif3b & $-0,2$ & 4,7 \\
\hline Eukaryotic translation initiation factor 3 subunit C; & Q8R1B4 & Eif3c & $-0,6$ & 2,1 \\
\hline Eukaryotic translation initiation factor 3 subu nit G; & Q9Z1D1 & Eif3g & $-0,7$ & 4,1 \\
\hline Eukaryotic translation initiation factor 3 subu nit J-A; & Q3UGC7 & Eif3j1 & $-0,6$ & 16,5 \\
\hline Eukaryotic translation initiation factor 4 gamma 1 ; & Q6NZJ6 & Eif4g1 & $-0,3$ & 7,3 \\
\hline Eukaryotic translation initiation factor 4B; & Q8BGD9 & Eif4b & $-0,2$ & 16,7 \\
\hline Eukaryotic translation initiation factor $4 \mathrm{H}$; & Q9WUK2 & Eif4h & 0,0 & 14,5 \\
\hline Eukaryotic translation initiation factor 5 & P59325 & Eif5 & $-0,3$ & 4,4 \\
\hline Eukaryotic translation initiation factor $5 \mathrm{~A}-1$; & P63242 & Eif5a & 0,3 & 24 \\
\hline Eukaryotic translation initiation factor 5B; & Q05D44 & Eif5b & $-0,5$ & 4 \\
\hline Eukaryotic translation initiation factor 6 & 055135 & Eif6 & 0,1 & 23,7 \\
\hline Extracellular matrix protein 1 & Q61508 & Ecm1 & $-0,4$ & 18,3 \\
\hline Ezrin; & P26040 & Ezr & $-0,1$ & 10,6 \\
\hline F-actin-capping protein subunit alpha-1; & P47753 & Capza1 & $-0,7$ & 5,7 \\
\hline F-actin-capping protein subunit beta; & P47757 & Capzb & 0,0 & 7,3 \\
\hline Far upstream element-binding protein 1 & Q91WJ8 & Fubp1 & $-0,3$ & 24,9 \\
\hline Far upstream element-binding protein 2; & Q3U0V1 & Khsrp & 0,0 & 24,6 \\
\hline Fatty acid-binding protein, epidermal; & Q05816 & Fabp5 & 0,0 & 63 \\
\hline Fatty aldehyde dehydrogenase; & P47740 & Aldh3a2 & 0,1 & 2,5 \\
\hline F-box only protein 50 & G3X9C2 & Nccrp1 & $-0,1$ & 4,9 \\
\hline Ferritin heavy chain; & P09528 & Fth1 & $-0,4$ & 5,5 \\
\hline Ferritin; & Q9CPX4 & Ftl1 & $-0,1$ & 37,7 \\
\hline Fetuin-B; & Q9QXC1 & Fetub & $-0,1$ & 16,6 \\
\hline Fibrinogen gamma chain; & Q8VCM7 & Fgg & 0,6 & 1,8 \\
\hline Fibronectin type III domain-containing protein 9; & Q8BJN4 & Fndc9 & 0,5 & 4,9 \\
\hline Filaggrin-2; & Q2VIS4 & Flg2 & $-0,2$ & 35,1 \\
\hline Filaggrin-2; & E9QPZ3 & Flg2 & $-0,3$ & 34,2 \\
\hline Filamin-A; & Q8BTM8 & Flna & $-0,1$ & 3,6 \\
\hline Filamin-B; & Q80X90 & Flnb & 0,0 & 4,6 \\
\hline Follistatin-related protein 1 & Q62356 & Fstl1 & $-0,3$ & 5,6 \\
\hline Forkhead box protein $\mathrm{O} 3$ & Q9WVH4 & Foxo3 & 0,0 & 11,7 \\
\hline Formin-binding protein 4 & Q6ZQ03 & Fnbp4 & 0,0 & 3,2 \\
\hline Fos-related antigen 2; & P47930 & Fosl2 & $-0,5$ & 7,4 \\
\hline Four and a half LIM domains protein 3; & Q9R059 & Fhl3 & $-1,9$ & 5,8 \\
\hline Frataxin, mitochondrial; & 035943 & Fxn & 0,0 & 11,1 \\
\hline Frizzled-1; & 070421 & Fzd1 & $-0,4$ & 1,9 \\
\hline Fructose-bisphosphate aldolase A; & P05064 & Aldoa & $-0,7$ & 16,2 \\
\hline Fucose mutarotase; & Q8R2K1 & Fuom & $-0,2$ & 9 \\
\hline FXYD domain-containing ion transport regulator 3 ; & Q61835 & Fxyd3 & 0,5 & 16,7 \\
\hline G patch domain and KOW motifs-containing protein; & Q56A08 & Gpkow & 0,3 & 1,8 \\
\hline G patch domain-containing protein 8; & A2A6A1 & Gpatch8 & $-0,4$ & 1,5 \\
\hline Galectin; & Q9CRB1 & Lgals7 & 0,0 & 36,8 \\
\hline Galectin-1; & P16045 & Lgals1 & 0,2 & 31,9 \\
\hline Galectin-3; & P16110 & Lgals3 & 0,6 & 18,6 \\
\hline Gamma-aminobutyric acid receptor-associated protein-like 2; & P60521 & Gabarapl2 & $-2,3$ & 6,8 \\
\hline
\end{tabular}




\begin{tabular}{|c|c|c|c|c|}
\hline Protein & Uniprot & Gene name & $\begin{array}{l}\text { Fold change of } \\
\text { XPRESS ratio } \\
\left.\text { (Egfr }{ }^{\Delta K C} / \mathrm{WT}\right)\end{array}$ & $\begin{array}{c}\text { Sequence } \\
\text { coverage [\%] }\end{array}$ \\
\hline Gamma-glutamyl hydrolase; & Q9Z0L8 & Ggh & 0,5 & 21,5 \\
\hline Gamma-glutamylcyclotransferase; & Q9D7X8 & Ggct & 0,3 & 27,1 \\
\hline Gamma-interferon-inducible lysosomal thiol reductase; & Q9ESY9 & Ifi30 & 0,2 & 9,7 \\
\hline Gamma-soluble NSF attachment protein; & Q9CWZ7 & Napg & 0,3 & 4,2 \\
\hline Gamma-synuclein; & Q9Z0F7 & Sncg & $-0,3$ & 17,9 \\
\hline Ganglioside GM2 activator; & Q60648 & Gm2a & 0,3 & 18,1 \\
\hline Gasdermin-A; & Q9EST1 & Gsdma & $-0,2$ & 9,6 \\
\hline Gastrin; & P48757 & Gast & $-1,4$ & 15,8 \\
\hline Gelsolin; & P13020 & Gsn & 0,7 & 10,3 \\
\hline General transcription factor IIF subunit 1 ; & Q3THK3 & Gtf2f1 & $-0,4$ & 7,1 \\
\hline General vesicular transport factor $\mathrm{p} 115$ & Q9Z1Z0 & Uso1 & $-0,3$ & 3,4 \\
\hline Glia maturation factor beta; & Q9CQ।3 & Gmfb & 0,5 & 7,7 \\
\hline Glucocorticoid receptor; & P06537 & $\mathrm{Nr3c1}$ & 0,2 & 5,1 \\
\hline Glucosidase 2 subunit beta; & 008795 & Prkcsh & $-0,6$ & 10,2 \\
\hline Glucosylceramidase; & P17439 & Gba & 0,7 & 6,6 \\
\hline Glutaredoxin-1; & Q9QUHO & Glrx & 0,6 & 10,3 \\
\hline Glutaredoxin-3; & Q9CQM9 & Glrx3 & 0,0 & 3,3 \\
\hline Glutathione peroxidase 1; & P11352 & Gpx1 & $-1,0$ & 18,4 \\
\hline Glutathione S-transferase omega-1; & 009131 & Gsto1 & $-0,2$ & 15,8 \\
\hline Glyceraldehyde-3-phosphate dehydrogenase; & P16858 & Gapdh & 0,5 & 34,3 \\
\hline Glycerol-3-phosphate acyltransferase 3; & Q8CON2 & Agpat9 & 0,3 & 3 \\
\hline Glycerophosphodiester phosphodiesterase domain-containing protein 3; & Q99LY2 & Gdpd3 & 0,2 & 3,6 \\
\hline Glycine cleavage system $\mathrm{H}$ protein, mitochondrial; & Q91WK5 & Gcsh & $-0,2$ & 5,9 \\
\hline Glycolipid transfer protein; & Q9JL62 & Gltp & 0,7 & 25,8 \\
\hline Glycylpeptide N-tetradecanoyltransferase 1; & 070310 & Nmt1 & $-0,5$ & 2,6 \\
\hline Glyoxylate reductase/hydroxypyruva te reductase; & Q91Z53 & Grhpr & 0,5 & 11,2 \\
\hline Golgin subfamily A member 2 ; & Q921M4 & Golga2 & $-0,6$ & 4,5 \\
\hline Golgin subfamily A member 5 ; & Q9QYE6 & Golga5 & $-1,2$ & 3,8 \\
\hline GPALPP motifs-containing protein 1 ; & Q69ZC8 & Gpalpp1 & 0,1 & 6,6 \\
\hline Grainyhead-like protein 1 homolog; & Q921D9 & Grhl1 & $-1,3$ & 1,9 \\
\hline Granulins; & P28798 & Grn & 0,1 & 4,8 \\
\hline GRB2-associated-binding protein 1; & Q9QYYO & Gab1 & 0,5 & 11,5 \\
\hline GRIP1-associated protein 1; & Q8VD04 & Gripap1 & 0,1 & 2,6 \\
\hline Group IIF secretory phospholipase A2; & Q9QZT4 & Pla2g2f & 0,3 & 4,2 \\
\hline Growth arrest and DNA damage-ind ucible proteins-interacting protein 1; & Q9CR59 & Gadd45gip1 & 0,0 & 8,6 \\
\hline Growth factor receptor-bound protein 2; & Q60631 & Grb2 & $-1,3$ & 31 \\
\hline GTPase NRas; & P08556 & Nras & $-0,2$ & 12,7 \\
\hline GTP-binding nuclear protein Ran; & P62827 & Ran & $-0,6$ & 5,1 \\
\hline GTP-binding protein SAR1a; & P36536 & Sar1a & $-0,7$ & 5,6 \\
\hline Guanine nucleotide exchange factor MSS4; & Q91X96 & Rabif & 0,0 & 7,3 \\
\hline H/ACA ribonucleoprotein complex subunit 2; & Q9CRB2 & Nhp2 & $-0,5$ & 17,6 \\
\hline H-2 class II histocompatibility antigen gamma chain; & P04441 & $\mathrm{Cd} 74$ & 0,3 & 8,6 \\
\hline $\mathrm{H}-2$ class II histocompatibility antigen, A-K alpha chain; & P01910 & $\mathrm{H} 2 \mathrm{Aa}$ & 1,2 & 5,2 \\
\hline Haloacid dehalogenase-like hydrolase domain-containing protein 2; & Q3UGR5 & Hdhd2 & 0,0 & 3,1 \\
\hline HD domain-containing protein 2 & Q3SXD3 & Hddc2 & $-0,2$ & 11,1 \\
\hline Heat shock 70 kDa protein 1B; & P17879 & Hspa1b & $-0,1$ & 12,8 \\
\hline Heat shock 70 kDa protein 4 & Q61316 & Hspa4 & 0,0 & 3,9 \\
\hline Heat shock cognate 71 kDa protein; & P63017 & Hspa8 & $-0,1$ & 19,3 \\
\hline Heat shock protein beta-1; & P14602 & Hspb1 & 0,0 & 41,5 \\
\hline Heat shock protein HSP 90-beta; & P11499 & Hsp90ab1 & $-0,1$ & 11,9 \\
\hline Heat shock-related 70 kDa protein 2; & P17156 & Hspa2 & $-0,4$ & 9,5 \\
\hline Hematological and neurological expressed 1 protein; & P97825 & Hn1 & $-0,1$ & 37 \\
\hline Hematological and neurological expressed 1-like protein; & Q6PGH2 & Hn1l & $-0,2$ & 53,7 \\
\hline Hematopoietic lineage cell-specific protein; & P49710 & Hcls1 & 1,3 & 10,9 \\
\hline Heme-binding protein 2 & Q9WU63 & Hebp2 & 0,3 & 26,8 \\
\hline Hemoglobin subunit alpha; & P01942 & $\mathrm{Hba}$ & $-0,6$ & 41,5 \\
\hline Hemoglobin subunit beta-1; & P02088 & $\mathrm{Hbb}$ b1 & 1,6 & 68,7 \\
\hline Hemoglobin subunit beta-2; & P02089 & $\mathrm{Hbb}$ b2 & 2,1 & 51,7 \\
\hline Hemoglobin subunit epsilon-Y2; & P02104 & $\mathrm{Hbb}$ y & $-0,7$ & 21,8 \\
\hline Hemopexin; & Q91X72 & $\mathrm{Hpx}$ & 0,8 & 2 \\
\hline Heparanase; & Q6YGZ1 & Hpse & 0,2 & 2,1 \\
\hline Hepatoma-derived growth factor; & P51859 & Hdgf & $-0,1$ & 37,1 \\
\hline Hepatoma-derived growth factor-related protein 2 ; & Q3UMU9 & Hdgfrp2 & $-0,4$ & 4,3 \\
\hline Heterochromatin protein 1-binding protein 3; & Q3TEA8 & Hp1bp3 & 0,0 & 17,4 \\
\hline Heterogeneous nuclear ribon ucleoprotein A/B; & Q99020 & Hnrnpab & 0,1 & 22,8 \\
\hline Heterogeneous nuclear ribonucleoprotein $\mathrm{A} 0$; & Q9CX86 & HnrnpaO & $-0,3$ & 11,5 \\
\hline Heterogeneous nuclear ribon ucleoprotein A1; & P49312 & Hnrnpa1 & 0,5 & 12,8 \\
\hline Heterogeneous nuclear ribonucleoprotein A3; & Q8BG05 & Hnrnpa3 & 1,5 & 20 \\
\hline Heterogeneous nuclear ribon ucleoprotein D0; & Q60668 & Hnrnpd & 0,2 & 22,7 \\
\hline
\end{tabular}




\begin{tabular}{|c|c|c|c|c|}
\hline Protein & Uniprot & Gene name & $\begin{array}{l}\text { Fold change of } \\
\text { XPRESS ratio } \\
\left.\text { (Egfr }{ }^{\Delta K C} / \mathrm{WT}\right)\end{array}$ & $\begin{array}{c}\text { Sequence } \\
\text { coverage [\%] }\end{array}$ \\
\hline Heterogeneous nuclear ribonucleoprotein K; & P61979 & Hnrnpk & 0,0 & 13,2 \\
\hline Heterogeneous nuclear ribonucleoprotein M; & Q9D0E1 & Hnrnpm & 1,1 & 3,1 \\
\hline Heterogeneous nuclear ribon ucleoprotein U; & Q8VEK3 & Hnrnpu & $-0,3$ & 3,5 \\
\hline Heterogeneous nuclear ribon ucleoprotein U-like protein 1; & Q8VDM6 & Hnrnpul1 & 0,0 & 1,3 \\
\hline Heterogeneous nuclear ribon ucleoprotein U-like protein 2; & Q00PI9 & Hnrnpul2 & $-0,6$ & 1,9 \\
\hline Heterogeneous nuclear ribonucleoproteins A2/B1; & 088569 & Hnrnpa2b1 & 0,5 & 21,2 \\
\hline Heterogeneous nuclear ribon ucleoproteins $\mathrm{C} 1 / \mathrm{C} 2$; & Q9Z204 & Hnrnpc & $-0,4$ & 7,3 \\
\hline High mobility group nucleosome-binding domain-containing protein 5 ; & Q9JL35 & Hmgn5 & $-0,1$ & 3,7 \\
\hline High mobility group protein B1; & P63158 & Hmgb1 & 0,0 & 23,7 \\
\hline High mobility group protein $\mathrm{B} 2$; & P30681 & Hmgb2 & 0,0 & 31 \\
\hline High mobility group protein B3; & 054879 & Hmgb3 & 0,3 & 16,4 \\
\hline High mobility group protein HMG-I/HMG-Y; & P17095 & Hmga1 & $-0,6$ & 8,4 \\
\hline High mobility group protein HMGI-C; & P52927 & Hmga2 & $-0,4$ & 16,7 \\
\hline Histidine ammonia-lyase; & P35492 & Hal & 0,2 & 11,6 \\
\hline Histidine triad nucleotide-binding protein 1 ; & P70349 & Hint1 & 0,2 & 34,5 \\
\hline Histone H1.1; & P43275 & Hist1h1a & $-0,6$ & 9,4 \\
\hline Histone H1.2; & P15864 & Hist1h1c & $-0,3$ & 15,1 \\
\hline Histone H1.4; & P43274 & Hist1h1e & $-0,3$ & 14,6 \\
\hline Histone H1.5; & P43276 & Hist1h1b & $-0,6$ & 14,3 \\
\hline Histone $\mathrm{H} 2 \mathrm{~B}$ type $1-\mathrm{F} / \mathrm{J} / \mathrm{L}$; & P10853 & Hist1h2bn & 0,4 & 14,3 \\
\hline Histone H2B type 2-E; & Q64524 & Hist2h2be & 0,2 & 14,3 \\
\hline Histone H3.3C; & P02301 & $H 3 f 3 c$ & 0,6 & 11,8 \\
\hline Histone H4; & P62806 & Hist4h4 & 0,2 & 42,7 \\
\hline Histone-binding protein RBBP4; & Q60972 & Rbbp4 & $-0,1$ & 10,5 \\
\hline HIV Tat-specific factor 1 homolog; & Q8BGC0 & Htatsf1 & $-0,6$ & 1,5 \\
\hline Homeodomain-only protein; & Q8R1H0 & Hopx & $-0,1$ & 43,8 \\
\hline Hornerin; & E9QNP3 & Hrnr & 0,0 & 20,6 \\
\hline Host cell factor 1 & Q61191 & Hcfc1 & 0,1 & 1,5 \\
\hline Hsc70-interacting protein; & Q99L47 & St13 & 0,4 & 6,4 \\
\hline Huntingtin-interacting protein $\mathrm{K}$; & Q9CR41 & Hypk & $-0,3$ & 13,2 \\
\hline Hydroxyacyl-coenzyme A dehydrogenase, mitochondrial; & Q61425 & Hadh & 0,8 & 3,2 \\
\hline Hydroxysteroid dehydrogenase-like protein 2 & Q2TPA8 & Hsdl2 & $-0,1$ & 3 \\
\hline Hypoxanthine-guanine phosphoribosyltransferase; & P00493 & Hprt1 & 1,5 & 25,2 \\
\hline Hypoxia up-regulated protein 1 & Q9JKR6 & Hyou1 & 0,7 & 3,1 \\
\hline Importin-7; & Q9EPL8 & Ipo7 & 0,9 & 2,8 \\
\hline Induced myeloid leukemia cell differentiation protein $\mathrm{Mcl}-1$ homolog; & P97287 & Mcl1 & 1,7 & 3,9 \\
\hline Inhibitor of nuclear factor kappa-B kinase subunit alpha; & Q60680 & Chuk & $-5,4$ & 1,1 \\
\hline Inositol-trisphosphate 3-kinase C; & Q7TS72 & Itpkc & 0,5 & 2,4 \\
\hline Insulin receptor substrate 1 & P35569 & Irs1 & 0,4 & 1,9 \\
\hline Insulin-degrading enzyme; & F6RPJ9 & Ide & 0,2 & 20,3 \\
\hline Insulin-like growth factor-binding protein 4; & P47879 & Igfbp4 & 0,4 & 11,5 \\
\hline Integrin alpha-6; & Q61739 & Itga6 & 0,6 & 1,9 \\
\hline Integrin beta-1; & P09055 & Itgb1 & 1,0 & 10 \\
\hline Intercellular adhesion molecule 1 & P13597 & Icam1 & 0,4 & 3,5 \\
\hline Interleukin-1 alpha; & P01582 & $\| 1 a$ & $-0,3$ & 4,1 \\
\hline Interleukin-1 receptor antagon ist protein; & P25085 & $\| 1 \mathrm{rn}$ & 0,5 & 9,4 \\
\hline Interleukin-6 receptor subun it beta; & Q00560 & $116 s t$ & $-0,1$ & 2,7 \\
\hline Involucrin; & G3X9D9 & $|v|$ & $-0,8$ & 41 \\
\hline Iron/zinc purple acid phosphatase-like protein; & Q8BX37 & Papl & 1,0 & 2,3 \\
\hline Iron-sulfur cluster assembly 2 homolog, mitochondrial; & Q9DCB8 & Isca2 & 0,4 & 15,6 \\
\hline Iron-sulfur cluster assembly enzyme ISCU, mitochondrial; & Q9D7P6 & Iscu & 0,0 & 6,6 \\
\hline Isocitrate dehydrogenase [NADP] cytoplasmic; & 088844 & Idh1 & 0,2 & 22,5 \\
\hline Isocitrate dehydrogenase [NADP], mitochondrial; & P54071 & Idh2 & 0,3 & 8,4 \\
\hline Isopentenyl-diphosphate Delta-isomerase 1; & P58044 & Idi1 & $-1,5$ & 5,3 \\
\hline Junction plakoglobin; & Q02257 & Jup & 0,3 & 9,5 \\
\hline Junctional protein associated with coronary artery disease; & Q5DTX6 & Jcad & 10,0 & 0,8 \\
\hline Kallikrein 1-related peptidase b5; Kallikrein related-peptidase 5; & Q9D140 & Klk5 & 0,5 & 23,5 \\
\hline Kallikrein 6, isoform CRA_a; Kallikrein-1; KIk6 protein; Neurosin; & Q91Y82 & Klk6 & $-0,5$ & 7,5 \\
\hline Kallikrein related-peptidase 10; Protein Klk10; & Q99M20 & Klk10 & 0,6 & 4,3 \\
\hline Kallikrein-7; & Q91VE3 & Klk7 & 0,6 & 14,1 \\
\hline Kallikrein-8; & Q61955 & Klk8 & 0,1 & 4,6 \\
\hline Keratin, type I cuticular Ha3-II; & Q61897 & Krt33b & $-2,3$ & 7,2 \\
\hline Keratin, type I cytoskeletal 10; & P02535 & Krt10 & $-0,3$ & 30,7 \\
\hline Keratin, type I cytoskeletal 14 & Q61781 & Krt14 & 0,0 & 39,9 \\
\hline Keratin, type I cytoskeletal 15; & Q61414 & Krt15 & 0,2 & 37,4 \\
\hline Keratin, type I cytoskeletal 16; & Q9Z2K1 & Krt16 & $-0,3$ & 12,6 \\
\hline Keratin, type I cytoskeletal 17; & Q9QWL7 & Krt17 & $-0,8$ & 40,9 \\
\hline Keratin, type I cytoskeletal 19; & P19001 & Krt19 & $-5,2$ & 14,4 \\
\hline Keratin, type I cytoskeletal 25; & Q8VCW2 & Krt25 & $-1,2$ & 10,3 \\
\hline Keratin, type I cytoskeletal 26; & Q3TRJ4 & Krt26 & $-1,8$ & 8,7 \\
\hline Keratin, type I cytoskeletal 27; & Q97320 & Krt27 & $-3,2$ & 15 \\
\hline
\end{tabular}




\begin{tabular}{|c|c|c|c|c|}
\hline Protein & Uniprot & Gene name & $\begin{array}{l}\text { Fold change of } \\
\text { XPRESS ratio } \\
\left(E_{g f r}{ }^{\Delta K C} / \mathrm{WT}\right)\end{array}$ & $\begin{array}{c}\text { Sequence } \\
\text { coverage [\%] }\end{array}$ \\
\hline Keratin, type I cytoskeletal 28 & A6BLY7 & Krt28 & $-3,7$ & 6,3 \\
\hline Keratin, type II cuticular Hb6; & P97861 & Krt86 & $-6,3$ & 1,7 \\
\hline Keratin, type II cytoskeletal 1; & P04104 & Krt1 & 0,0 & 34,4 \\
\hline Keratin, type II cytoskeletal 1b; & Q6IFZ6 & Krt77 & $-0,2$ & 22 \\
\hline Keratin, type II cytoskeletal 5; & Q922U2 & Krt5 & $-0,2$ & 27,2 \\
\hline Keratin, type II cytoskeletal 6A; & P50446 & Krt6a & $-0,2$ & 15,9 \\
\hline Keratin, type II cytoskeletal 7; & Q9DCV7 & Krt7 & 1,5 & 11,2 \\
\hline Keratin, type II cytoskeletal 71; & Q9ROH5 & Krt71 & $-0,8$ & 10,9 \\
\hline Keratin, type II cytoskeletal 75; & Q8BGZ7 & Krt75 & $-0,6$ & 10,3 \\
\hline Keratin, type II cytoskeletal 79; & Q8VED5 & Krt79 & $-0,4$ & 33,1 \\
\hline Keratin-associated protein $19-5$ & F8VQ65 & Krtap19 5 & 1,4 & 19,4 \\
\hline Keratin-associated protein 6-5; & Q925H3 & Krtap6 5 & $-3,9$ & 19,2 \\
\hline Keratinocyte-associated transmembrane protein 2; & Q8K201 & Kct2 & $-0,4$ & 6,9 \\
\hline Kininogen-1; & 008677 & Kng1 & 0,3 & 11,3 \\
\hline KxDL motif-containing protein 1 ; & E9Q4P0 & Kxd1 & 0,1 & 17,6 \\
\hline Lactoylglutathione lyase; & Q9CPU0 & Glo1 & 0,8 & 31 \\
\hline Ladinin-1; & P57016 & Lad1 & 0,0 & 14,2 \\
\hline Lamina-associated polypeptide 2, isoforms beta/delta/epsilon/gamma; & Q61029 & Tmpo & 0,0 & 18,1 \\
\hline Lamin-B1; & P14733 & Lmnb1 & $-0,6$ & 9 \\
\hline Latent-transforming growth factor beta-binding protein 4; & Q8K4G1 & Ltbp4 & 0,1 & 5,3 \\
\hline L-dopachrome tautomerase; & P29812 & Dct & $-0,3$ & 2,1 \\
\hline LEM domain-containing protein 2; & Q6DVA0 & Lemd2 & 0,1 & 2,2 \\
\hline $\begin{array}{l}\text { Leucine rich repeat (In FLII) interacting protein 1, isoform CRA_e; Leucine-rich } \\
\text { repeat flightless-interacting protein } 1 \text {; }\end{array}$ & G5E8E1 & Lrrfip1 & $-0,7$ & 5,8 \\
\hline $\begin{array}{l}\text { Leucine-rich HEV glycoprotein; Leucine-rich alpha-2-glycoprotein; Leucine-rich } \\
\text { alpha-2-glycoprotein 1; Protein Lrg1; }\end{array}$ & Q91XL1 & $\operatorname{Lrg1}$ & 0,3 & 4,1 \\
\hline Leucine-rich repeat flightless-interacting protein 1 & Q3UZ39 & Lrrfip1 & $-1,0$ & 10,7 \\
\hline Leucine-rich repeat flightless-interacting protein 2 ; & E9QN52 & Lrrfip2 & $-0,4$ & 14,5 \\
\hline Leucine-rich repeat-containing protein $16 \mathrm{~A}$ & Q6EDY6 & Lrrc16a & 0,1 & 2,1 \\
\hline Leucine-rich repeat-containing protein 59; & Q922Q8 & Lrrc59 & $-0,1$ & 22,1 \\
\hline Leucine--tRNA ligase, cytoplasmic; & Q8BMJ2 & Lars & $-0,8$ & 0,9 \\
\hline Leukocyte elastase inhibitor A; & Q9D154 & Serpinb1a & $-0,1$ & 6,6 \\
\hline LIM and SH3 domain protein 1; & Q61792 & Lasp1 & $-0,2$ & 24,7 \\
\hline LIM domain and actin-bind ing protein 1 ; & Q9ERG0 & Lima1 & $-0,4$ & 5,8 \\
\hline LIM domain-containing protein 1 & Q9QXD8 & Limd1 & 0,3 & 4,8 \\
\hline LIM zinc-binding domain-containing Nebulette; & Q9DC07 & Nebl & $-0,4$ & 18,9 \\
\hline Lipolysis-stimulated lipoprotein receptor; & Q99KG5 & Lsr & 0,2 & 4,3 \\
\hline Lipoma-preferred partner homolog; & Q8BFW7 & Lpp & 0,3 & 3,6 \\
\hline L-lactate dehydrogenase A chain; & P06151 & Ldha & 0,1 & 9,3 \\
\hline Long-chain specific acyl-CoA dehydrogenase, mitochondrial; & P51174 & Acadl & $-0,3$ & 2,6 \\
\hline Loricrin; & P18165 & Lor & $-2,0$ & 4,1 \\
\hline Low molecular weight phosphotyrosine protein phosphatase; & Q9D358 & Acp1 & 0,1 & 7 \\
\hline Lumican; & P51885 & Lum & 0,1 & 14,2 \\
\hline Ly6/PLAUR domain-containing protein 5; & Q9D7Z7 & Lypd5 & 0,1 & 13,7 \\
\hline Ly6g6e protein, isoform B; Protein Ly6g6e; & Q8K1T6 & Ly6g6e & 1,0 & 10,4 \\
\hline Lymphocyte antigen 6 complex locus protein G6c; & Q9Z1Q4 & Ly6g6c & $-0,1$ & 14,3 \\
\hline Lymphocyte antigen 6D; & P35459 & Ly6d & 0,2 & 21,3 \\
\hline Lymphocyte-specific protein 1 & P19973 & Lsp1 & 0,1 & 29,8 \\
\hline LysM and putative peptidoglycan-bind ing domain-containing protein 2 ; & Q9D7V2 & Lysmd2 & $-0,1$ & 5,1 \\
\hline Lysosomal protective protein; & P16675 & Ctsa & 0,6 & 7,6 \\
\hline Lysosome membrane protein 2 & 035114 & Scarb2 & 0,7 & 1,7 \\
\hline Lysosome-associated membrane glycoprotein $1 ;$ & P11438 & Lamp1 & 0,2 & 2,7 \\
\hline Lysosome-associated membrane glycoprotein 2; & P17047 & Lamp2 & $-0,1$ & 6,5 \\
\hline Lysozyme C-2; & P08905 & Lyz2 & 2,6 & 14,9 \\
\hline Macrophage migration inhibitory factor; & P34884 & Mif & 0,5 & 7,8 \\
\hline Macrophage-capping protein; & P24452 & Capg & 0,4 & 9,7 \\
\hline Magnesium-dependent phosphatase 1 ; & Q9D967 & Mdp1 & 0,4 & 17,7 \\
\hline Major prion protein; & P04925 & Prnp & $-3,4$ & 4,7 \\
\hline Major urinary protein 17; & B5X0G2 & Mup17 & 1,0 & 13,2 \\
\hline Major urinary protein 4; & P11590 & Mup4 & 1,1 & 4,5 \\
\hline Malate dehydrogenase, cytoplasmic; & P14152 & Mdh1 & 0,0 & 6,6 \\
\hline Malate dehydrogenase, mitochond rial; & P08249 & Mdh2 & $-0,1$ & 45 \\
\hline Male-enhanced antigen 1; & Q64327 & Mea1 & 0,6 & 7,5 \\
\hline MAP7 domain-containing protein 1 ; & A2AJIO & Map7d1 & 2,3 & 2,2 \\
\hline MARCKS-related protein; & P28667 & Marcksl1 & $-0,3$ & 6,5 \\
\hline MCG116526; Protein Scgb2b7; & D3YYY1 & Scgb2b7 & 0,3 & 23,2 \\
\hline MCG117626; Odorant binding protein la; Protein Obp1a; & Q9D3H2 & Obp1a & 1,0 & 19 \\
\hline MCG120169; Protein 2310050C09Rik; & G5E8Z3 & 2310050C09Rik & $-1,9$ & 5,7 \\
\hline MCG129038; Protein Serpinb3a; & G3X9V8 & Serpinb3a & $-0,6$ & 26,1 \\
\hline MCG17975; Protein Zfyve9; & A2A8R0 & Zfyve9 & $-0,7$ & 2,7 \\
\hline
\end{tabular}




\begin{tabular}{|c|c|c|c|c|}
\hline Protein & Uniprot & Gene name & $\begin{array}{l}\text { Fold change of } \\
\text { XPRESS ratio } \\
\left.\text { (Egfr }{ }^{\Delta K C} / \mathrm{WT}\right)\end{array}$ & $\begin{array}{c}\text { Sequence } \\
\text { coverage [\%] }\end{array}$ \\
\hline MCG20280; OTTMUSG00000000712 protein; Protein Serpinb6d; & Q3UWK8 & Serpinb6d & 1,0 & 2,1 \\
\hline Mediator of RNA polymerase II transcription subunit 21; & Q9CQ39 & Med21 & 0,2 & 22,2 \\
\hline Mediator of RNA polymerase II transcription subunit 9; & Q8VCS6 & Med9 & $-0,1$ & 10,6 \\
\hline Melanoma inhibitory activity protein 3 ; & Q8BI84 & Mia3 & $-0,3$ & 2,8 \\
\hline Membrane-associated progesterone receptor component 1; & 055022 & Pgrmc1 & 0,2 & 15,9 \\
\hline Membrane-associated progesterone receptor component 2; & Q80UU9 & Pgrmc2 & 0,1 & 30 \\
\hline Metallothionein-2; & P02798 & Mt2 & $-0,5$ & 19,7 \\
\hline Metallothionein-4; & P47945 & Mt4 & 0,3 & 19,4 \\
\hline Methionine aminopeptidase 2 ; & 008663 & Metap2 & $-0,5$ & 10,3 \\
\hline Methyl-CpG-binding domain protein 2; & Q9Z2E1 & Mbd2 & $-4,1$ & 2,4 \\
\hline Methylosome subunit plCln; & Q61189 & Clns1a & 0,2 & 16,1 \\
\hline Microfibrillar-associated protein 1 ; & Q9CQU1 & Mfap1 & $-0,2$ & 8 \\
\hline Microtubule-associated protein 1B; & P14873 & Map1b & 0,1 & 1,5 \\
\hline Microtubule-associated protein 2; & P20357 & Map2 & $-0,4$ & 2,6 \\
\hline Microtubule-associated protein 4; & P27546 & Map4 & $-0,2$ & 20,4 \\
\hline Microtubule-associated protein RP/EB family member 1 ; & Q61166 & Mapre1 & 0,0 & 17,5 \\
\hline Microtubule-associated protein tau; & P10637 & Mapt & $-0,3$ & 9 \\
\hline Microtubule-associated serine/threon ine-protein kinase 4; & Q811L6 & Mast4 & 0,3 & 0,5 \\
\hline Migration and invasion enhancer 1 & Q9CQ86 & Mien1 & 0,2 & 9,6 \\
\hline Mitochondrial antiviral-signaling protein; & Q8VCF0 & Mavs & 0,3 & 3 \\
\hline Mitochondrial import inner membrane translocase subunit Tim13; & P62075 & Timm13 & 0,1 & 26,3 \\
\hline Mitochondrial import inner membrane translocase subu nit TIM16; & Q9CQV1 & Pam16 & $-0,5$ & 30,4 \\
\hline Mitochondrial import inner membrane translocase subunit Tim8 A; & Q9WVA2 & Timm8a1 & 0,0 & 34 \\
\hline Mitochondrial intermembrane space import and assembly protein 40 ; & Q8VEA4 & Chchd4 & 0,2 & 36,7 \\
\hline Mitogen-activated protein kinase 13 & Q9Z1B7 & Mapk13 & 0,9 & 3 \\
\hline MKL/myocardin-like protein 2 & P59759 & Mkl2 & $-0,5$ & 3,6 \\
\hline MOB kinase activator $1 \mathrm{~B}$ & Q8BPB0 & Mob1b & 0,7 & 5,1 \\
\hline Moesin; & P26041 & Msn & $-0,1$ & 13,3 \\
\hline MORF4 family-associated protein 1 & Q9CQL7 & Mrfap1 & 0,0 & 18,4 \\
\hline Mucin-15; & Q8C6Z1 & Muc15 & 0,2 & 3,5 \\
\hline Mucin-19; & Q6PZE0 & Muc19 & $-3,4$ & 3,5 \\
\hline Multiple coagulation factor deficiency protein 2 homolog; & Q8K5B2 & Mcfd2 & 0,1 & 9 \\
\hline Multiple epidermal growth factor-like domains protein 9; & Q8BH27 & Megf9 & $-1,2$ & 1,8 \\
\hline Multiple myeloma tumor-associated protein 2 homolog; & Q99LX5 & Mmtag2 & $-0,5$ & 9,2 \\
\hline Myeloid bactenecin (F1); Neutrophilic granule protein; Protein Ngp; & 008692 & Ngp & 1,2 & 12 \\
\hline Myosin light chain $1 / 3$, skeletal muscle isoform; & P05977 & Myl1 & $-0,7$ & 19,3 \\
\hline Myosin light chain 6B; & Q8Cl43 & Myl6b & 0,0 & 10,1 \\
\hline Myosin light polypeptide 6; & Q60605 & Myl6 & 0,2 & 37,7 \\
\hline Myosin regulatory light chain $12 \mathrm{~B}$; & Q3THE2 & Myl12b & $-0,2$ & 29,7 \\
\hline Myosin regulatory light chain 2, ventricular/cardiac muscle isoform; & P51667 & Myl2 & $-0,7$ & 14 \\
\hline Myosin-14; & Q6URW6 & Myh14 & $-0,1$ & 0,9 \\
\hline Myosin-3; & P13541 & Myh3 & $-0,7$ & 1,1 \\
\hline Myosin-9; & Q8VDD5 & Myh9 & $-0,3$ & 13,9 \\
\hline Myristoylated alanine-rich C-kinase substrate; & P26645 & Marcks & $-0,2$ & 11 \\
\hline$N(G), N(G)$-dimethylarginine dimethylaminohydrolase 2 & Q99LD8 & Ddah2 & 0,5 & 11,5 \\
\hline $\mathrm{Na}(+) / \mathrm{H}(+)$ exchange regulatory cofactor NHE-RF1; & P70441 & Slc9a3r1 & $-0,2$ & 28,7 \\
\hline $\mathrm{N}$-acetylglucosamine-6-sulfatase; & Q8BFR4 & Gns & 1,0 & 3,9 \\
\hline $\mathrm{N}$-acetylneuraminate lyase; & Q9DCJ9 & $\mathrm{Npl}$ & 0,8 & 43,1 \\
\hline $\mathrm{NAD}(\mathrm{P}) \mathrm{H}$-hydrate epimerase; & Q8K4Z3 & Apoa1bp & $-1,9$ & 2,8 \\
\hline NADH dehydrogenase [ubiquinone] 1 alpha subcomplex subunit 13; & Q9ERS2 & Ndufa13 & $-0,1$ & 6,3 \\
\hline NADH dehydrogenase [ubiquinone] 1 alpha subcomplex subunit 2; & Q9CQ75 & Ndufa2 & 0,2 & 14,1 \\
\hline NADH dehydrogenase [ubiquinone] 1 alpha subcomplex subunit 4; & Q62425 & Ndufa4 & $-0,1$ & 46,3 \\
\hline NADH dehydrogenase [ubiquinone] 1 alpha subcomplex subunit 4-like 2; & Q4FZG9 & Ndufa4l2 & 0,5 & 28,7 \\
\hline NADH dehydrogenase [ubiquinone] 1 alpha subcomplex subunit 7; & Q9Z1P6 & Ndufa7 & 0,2 & 9,7 \\
\hline NADH dehydrogenase [ubiquinone] 1 beta subcomplex subunit 3; & Q9CQZ6 & Ndufb3 & 0,0 & 10,6 \\
\hline $\begin{array}{l}\text { NADH dehydrogenase [ubiquinone] } 1 \text { beta subcomplex subunit } 8 \text {, } \\
\text { mitochondrial; }\end{array}$ & Q9D6J5 & Ndufb8 & 0,1 & 10,8 \\
\hline NADH dehydrogenase [ubiquinone] flavoprotein 2, mitochondrial; & Q9D6J6 & Ndufv2 & 0,3 & 8,1 \\
\hline NADH dehydrogenase [ubiquinone] flavoprotein 3 , mitochondrial; & Q8BK30 & Ndufv3 & 0,2 & 10,6 \\
\hline $\begin{array}{l}\text { NADH dehydrogenase [ubiquinone] flavoprotein 3, mitochondrial; RIKEN cDNA } \\
\text { 1500032D16, isoform CRA_a; }\end{array}$ & Q3U422 & Ndufv3 & 0,0 & 26,3 \\
\hline NADH dehydrogenase [ubiquinone] iron-sulfur protein 4, mitochondrial; & Q9CXZ1 & Ndufs4 & 0,1 & 19,4 \\
\hline NADH dehydrogenase [ubiquinone] iron-sulfur protein 5; & Q99LY9 & Ndufs5 & 0,2 & 27,5 \\
\hline NADH dehydrogenase [ubiquinone] iron-sulfur protein 6 , mitochondrial; & P52503 & Ndufs6 & 0,0 & 42,2 \\
\hline NADH dehydrogenase [ubiquinone] iron-sulfur protein 8, mitochondrial; & Q8K3J1 & Ndufs8 & 0,3 & 5,2 \\
\hline NADP-dependent malic enzyme; & P06801 & Me1 & $-0,1$ & 5,8 \\
\hline Nardilysin; & Q8BHG1 & Nrd1 & 0,4 & 4,5 \\
\hline
\end{tabular}




\begin{tabular}{|c|c|c|c|c|}
\hline Protein & Uniprot & Gene name & $\begin{array}{l}\text { Fold change of } \\
\text { XPRESS ratio } \\
\left.\text { (Egfr }{ }^{\Delta K C} / \mathrm{WT}\right)\end{array}$ & $\begin{array}{c}\text { Sequence } \\
\text { coverage [\%] }\end{array}$ \\
\hline
\end{tabular}

Nascent polypeptide-associated complex subunit alpha, muscle-specific form;

Nectin-1;

Nectin-4;

NEDD8;

Negative elongation factor $E$;

Neudesin;

Neural cell adhesion molecule 1;

Neural cell adhesion molecule L1;

Neuroblastoma suppressor of tumorigenicity 1 ;

Neuroplastin;

NFU1 iron-sulfur cluster scaffold homolog, mitochondrial;

NHS-like protein 1 ;

Nicastrin;

Niemann-Pick C1 protein;

Nitrilase homolog 1;

Non-specific lipid-transfer protein;

NSFL1 cofactor p47;

Nuclear autoantigenic sperm protein;

Nuclear distribution protein nudE-like 1;

Nuclear fragile $\mathrm{X}$ mental retardation-interacting protein 2;

Nuclear migration protein nudC;

Nuclear pore complex protein Nup214;

Nuclear pore complex protein Nup50;

Nuclear pore glycoprotein p62;

Nuclear receptor coactivator 6;

Nuclear speckle splicing regulatory protein 1;

Nuclear transcription factor $Y$ subunit beta;

Nuclear ubiquitous casein and cyclin-dependent kinase substrate 1;

P70670

Q9JKF6

Q8R007

P29595

P19426

Q9CQ45

P13595

A2AFG7

Q61477

P97300

Q9QZ23

Q8CAF4

P57716

035604

Q8VDK1

P32020

Q9CZ44

Q99MD9

Q9ERR1

Q5F2E7

035685

Q80U93

Q9JIH2

Q63850

Q9JL19

Q5NCR9

P63139

Q80XU3

P62960

Q02819

P81117

Q9CPT5

Q9D6Z1

P09405

Q61937

F6ZDS4

E9PZF0

Q78ZA7

Q61146

Q9CRD0

Q8K1H9

Q9D8S4

Q9JHW2

Q62422

Q9ET54

Q9JHU2

Q8VDZ4

Q9EQC8

Papillary renal cell carcinoma (Translocation-associated); Papillary renal cell carcinoma-associated protein; Protein Prcc;

Paralemmin-1;

Parathymosin;

Partner of Y14 and mago;

Parvalbumin alpha;

Paternally-expressed gene 3 protein;

Paxillin;

PC4 and SFRS1-interacting protein;

PDZ and LIM domain protein 1;

PDZ and LIM domain protein 2;

PDZ and LIM domain protein 4;

PDZ and LIM domain protein 5;

PDZ domain-containing protein 11;

PDZK1-interacting protein 1;

Peflin;

Peptidyl-prolyl cis-trans isomerase A;

Peptidyl-prolyl cis-trans isomerase $\mathrm{B}$;

Peptidyl-prolyl cis-trans isomerase C;

Peptidyl-prolyl cis-trans isomerase CWC27 homolog;

Peptidyl-prolyl cis-trans isomerase FKBP1A;

Peptidyl-prolyl cis-trans isomerase FKBP2;

Peptidyl-prolyl cis-trans isomerase FKBP3;

Naca
Pvrl1
Pvrl4
Nedd8
Nelfe

$0,1 \quad 12,6$

$-0,2 \quad 10,7$

$-0,3 \quad 13,6$

$-0,1 \quad 11,1$

$-0,1 \quad 6,7$

$\begin{array}{lll}\text { Nenf } & 0,0 & 32,2\end{array}$

Ncam1 $\quad 0,6 \quad 2,6$

L1cam $0,2 \quad 3$

Nbl1 $\quad 0,2 \quad 3,9$

Nptn $\quad 0,0 \quad 18,1$

Nfu1 $\quad-0,3 \quad 15,3$

Nhsl1 $\quad-0,1 \quad 1,4$

Ncstn $\quad-0,7 \quad 3,2$

Npc1 - $0,7 \quad 0,9$

Nit1 $\quad-3,2 \quad 12,6$

$\begin{array}{lll}\text { Scp2 } & 0,3 & 7,3\end{array}$

Nsfl1c $\quad 0,0 \quad 28,1$

Nasp $\quad-0,4 \quad 4,4$

Ndel1 $\quad 0,3 \quad 9,9$

Nufip2 $-1,2 \quad 3,3$

Nudc $\quad 0,0 \quad 2,4$

Nup214 0,2 0,6

Nup50 $\quad-0,2 \quad 2,8$

Nup62 $\quad-0,4 \quad 6,5$

Ncoa6 $\quad-0,5 \quad 0,5$

Nsrp1 $\quad-0,2 \quad 2,8$

Nfyb $\quad 0,0 \quad 12,3$

$\begin{array}{lll}\text { Nucks1 } & -0,1 & 3,8\end{array}$

$\begin{array}{lll}\text { Ybx1 } & 0,0 & 7,8\end{array}$

$\begin{array}{lll}\text { Nucb1 } & -0,1 & 37\end{array}$

$\begin{array}{lll}\text { Nucb2 } & -0,5 & 15,7\end{array}$

Nop16 $\quad-0,1 \quad 8,4$

Nop56 $\quad 0,0 \quad 4,4$

$\begin{array}{lll}\mathrm{Ncl} & 0,6 & 19,9\end{array}$

Npm1 $\quad 0,0 \quad 16$

$\begin{array}{lll}\mathrm{Tpr} & 0,3 & 3\end{array}$

Gm20390 $\quad 0,0 \quad 54,3$

Nap1|4 $\quad 0,4 \quad 2,9$

$\begin{array}{lll}\text { Ocln } & -0,3 & 7,7\end{array}$

Ociad1 $\quad 0,0 \quad 3,2$

Obp2a $\quad 1,5 \quad 8$

Rexo2 $0,3 \quad 8,9$

Nit2 $0,2 \quad 3,3$

$\begin{array}{lll}\text { Ostf1 } & 0,0 & 29,8\end{array}$

Palld $\quad 0,9 \quad 1,6$

$\begin{array}{lll}\text { Palmd } & -0,4 & 17,6\end{array}$

$\begin{array}{lll}\text { Zdhhc5 } & 0,4 & 5,7\end{array}$

Prcc 0,0

Fkbp1a

Fkbp2

Fkbp3 


\begin{tabular}{|c|c|c|c|c|}
\hline Protein & Uniprot & Gene name & $\begin{array}{l}\text { Fold change of } \\
\text { XPRESS ratio } \\
\left.\text { (Egfr }{ }^{\Delta K C} / \mathrm{WT}\right)\end{array}$ & $\begin{array}{c}\text { Sequence } \\
\text { coverage [\%] }\end{array}$ \\
\hline Peptidyl-prolyl cis-trans isomerase FKBP7; & 054998 & Fkbp7 & 0,3 & 12,8 \\
\hline Peptidyl-prolyl cis-trans isomerase NIMA-interacting 1 ; & Q9QUR7 & Pin1 & 0,9 & 4,8 \\
\hline Peptidyl-prolyl cis-trans isomerase NIMA-interacting 4; & Q9CWW6 & Pin4 & 0,8 & 9,2 \\
\hline Perilipin-1; & Q8CGN5 & Plin1 & $-1,3$ & 2,9 \\
\hline Perilipin-2; & P43883 & Plin2 & 0,2 & 2,6 \\
\hline Perilipin-3; & Q9DBG5 & Plin3 & $-0,6$ & 10,8 \\
\hline Perilipin-4; & 088492 & Plin4 & $-0,6$ & 5,6 \\
\hline Periostin; & Q62009 & Postn & $-0,5$ & 5,7 \\
\hline Periplakin; & G5E898 & Ppl & $-0,2$ & 18,9 \\
\hline Peroxiredoxin-1; & P35700 & $\operatorname{Prdx1}$ & 0,6 & 48,2 \\
\hline Peroxiredoxin-2; & Q61171 & $\operatorname{Prdx2}$ & 0,5 & 14,6 \\
\hline Peroxiredoxin-5, mitochondrial; & P99029 & Prdx5 & 0,2 & 28,6 \\
\hline Peroxiredoxin-6; & 008709 & Prdx6 & 0,9 & 24,5 \\
\hline Peroxisomal biogenesis factor 19 & Q8VCl5 & Pex19 & 0,0 & 19,4 \\
\hline Peroxisomal membrane protein PEX14; & Q9R0A0 & Pex14 & $-1,2$ & 16 \\
\hline PEST proteolytic signal-containing nuclear protein; & Q6P814 & Pcnp & $-0,4$ & 34,7 \\
\hline Phosphatase and actin regulator 4 & Q501J7 & Phactr4 & 0,2 & 6,9 \\
\hline Phosphatidylethanolamine-binding protein 1 ; & P70296 & Pebp1 & 0,7 & 30,5 \\
\hline Phosphatidylinositol-glycan-specific phospholipase D; & 070362 & Gpld1 & 0,6 & 6,7 \\
\hline Phosphoglycerate mutase 1 & Q9DBJ1 & Pgam1 & 0,2 & 11 \\
\hline Phospholipase B-like 1; & Q8VCI0 & Plbd1 & 0,1 & 6 \\
\hline Phospholipase D3; & 035405 & Pld3 & $-0,2$ & 13,3 \\
\hline Phosphorylated adapter RNA export protein; & Q9JJT9 & Phax & $-0,5$ & 8,5 \\
\hline Phosphoserine phosphatase; & Q99LS3 & Psph & 0,1 & 15 \\
\hline Pinin; & 035691 & Pnn & $-0,1$ & 5 \\
\hline Plakophilin-1; & P97350 & Pkp1 & $-0,1$ & 6,6 \\
\hline Plakophilin-3; & Q9QY23 & Pkp3 & 1,1 & 4,5 \\
\hline Plasminogen activator inhibitor 1 RNA-bind ing protein; & Q9CY58 & Serbp1 & $-0,4$ & 19,9 \\
\hline Plasminogen activator inhibitor 2, macrophage; & P12388 & Serpinb2 & $-0,1$ & 3,7 \\
\hline Plasminogen receptor (KT); & Q9D3P8 & Plgrkt & 0,3 & 6,8 \\
\hline Plastin-2; & Q61233 & Lcp1 & 0,5 & 5,7 \\
\hline Plastin-3; & Q99K51 & Pls3 & 0,3 & 13,5 \\
\hline Platelet glycoprotein 4; & Q08857 & $\mathrm{Cd} 36$ & $-0,2$ & 2,5 \\
\hline Platelet-activating factor acetylhydrolase IB subunit alpha; & P63005 & Pafah1b1 & 0,4 & 4,4 \\
\hline Platelet-activating factor acetylhydrolase IB subunit beta; & Q61206 & Pafah1b2 & 0,1 & 3,9 \\
\hline Platelet-activating factor acetylhydrolase IB subu nit gamma; & Q61205 & Pafah1b3 & 0,7 & 6,3 \\
\hline Platelet-activating factor acetylhydrolase; & Q60963 & Pla2g7 & $-0,3$ & 2,7 \\
\hline Pleckstrin homology-like domain family B member 1 ; & Q6PDH0 & Phldb1 & $-1,7$ & 3,2 \\
\hline Plectin; & Q9QXS1 & Plec & $-0,1$ & 3,4 \\
\hline Poly(ADP-ribose) glycohydrolase; & 088622 & Parg & 0,4 & 8 \\
\hline Poly(rC)-binding protein 1 & P60335 & Pcbp1 & 0,7 & 3,7 \\
\hline Poly(U)-specific endoribonuclease; & Q3V188 & Endou & 0,6 & 16,3 \\
\hline Polyadenylate-binding protein 2 ; & Q8CCS6 & Pabpn1 & 0,8 & 6,7 \\
\hline Polyadenylate-binding protein-interacting protein $2 \mathrm{~B}$; & Q91W45 & Paip2b & $-0,4$ & 15,4 \\
\hline Polyglutamine-binding protein 1 & Q91VJ5 & Pqbp1 & $-0,1$ & 16,7 \\
\hline Polymerase I and transcript release factor; & 054724 & Ptrf & 0,8 & 4,1 \\
\hline Porimin; & Q91Z22 & Tmem123 & 0,5 & 4,6 \\
\hline PRA1 family protein 3 ; & Q8R5J9 & Arl6ip5 & 1,4 & 5,9 \\
\hline Prefoldin subunit 1 & Q9CWM4 & Pfdn1 & 1,1 & 9 \\
\hline Prefoldin subunit 2 & 070591 & Pfdn2 & $-0,2$ & 29,2 \\
\hline Prefoldin subunit 5; & Q9WU28 & Pfdn5 & $-0,2$ & 14,9 \\
\hline Prefoldin subunit 6; & Q03958 & Pfdn6 & $-0,1$ & 20,8 \\
\hline Prelamin-A/C; & P48678 & Lmna & $-0,1$ & 17,9 \\
\hline Pre-mRNA 3'-end-processing factor FIP1; & Q9D824 & Fip1l1 & 0,0 & 4,5 \\
\hline Pre-mRNA-splicing factor ISY1 homolog; & Q69ZQ2 & Isy1 & $-1,9$ & 11,6 \\
\hline Pre-rRNA-processing protein TSR2 homolog; & Q8C8T8 & Tsr2 & 0,0 & 17,8 \\
\hline PRKC apoptosis WT1 regulator protein; & Q925B0 & Pawr & $-0,5$ & 9 \\
\hline Proactivator polypeptide-like 1 ; & Q8C1C1 & Psapl1 & 0,4 & 11,5 \\
\hline Probable RNA polymerase II nuclear localization protein SLC7A6OS; & Q7TPE5 & Slc7a6os & $-1,9$ & 3,3 \\
\hline Probable RNA-binding protein EIF1AD; & Q3THJ3 & Eif1ad & 0,0 & 12,9 \\
\hline Programmed cell death 6-interacting protein; & Q9WU78 & Pdcd6ip & $-0,5$ & 2,2 \\
\hline Programmed cell death protein 5 ; & P56812 & Pdcd5 & $-0,2$ & 37,3 \\
\hline Programmed cell death protein 6 ; & P12815 & Pdcd6 & 0,0 & 5,2 \\
\hline Prohibitin; & P67778 & $\mathrm{Phb}$ & $-0,2$ & 8,1 \\
\hline Prohibitin-2; & 035129 & Phb2 & $-0,1$ & 7,6 \\
\hline Proline-rich AKT1 substrate 1 ; & Q9D1F4 & Akt1s1 & $-0,3$ & 3,1 \\
\hline Proline-rich protein 9; & Q8BV84 & Prr9 & $-3,7$ & 16,4 \\
\hline Prolyl-tRNA synthetase associated domain-containing protein 1 ; & Q9D820 & Prorsd1 & 0,0 & 15,4 \\
\hline Prominin-2; & Q3UUY6 & Prom2 & 0,4 & 6,5 \\
\hline Prostaglandin E synthase 3; & Q9R0Q7 & Ptges3 & 0,0 & 29,2 \\
\hline Prostate stem cell antigen; & P57096 & Psca & $-1,5$ & 8,1 \\
\hline
\end{tabular}




\begin{tabular}{|c|c|c|c|c|}
\hline Protein & Uniprot & Gene name & $\begin{array}{l}\text { Fold change of } \\
\text { XPRESS ratio } \\
\left(E_{g f r}{ }^{\Delta K C} / \mathrm{WT}\right)\end{array}$ & $\begin{array}{c}\text { Sequence } \\
\text { coverage [\%] }\end{array}$ \\
\hline Prostatic acid phosphatase; & Q8CE08 & Acpp & 0,3 & 7,9 \\
\hline Proteasome activator complex sub unit 1 ; & P97371 & Psme1 & 0,3 & 29 \\
\hline Proteasome subunit alpha type- 1 ; & Q9R1P4 & Psma1 & $-0,1$ & 18,3 \\
\hline Proteasome subunit alpha type- 2 ; & P49722 & Psma2 & 0,5 & 12 \\
\hline Proteasome subunit alpha type- 3 ; & 070435 & Psma3 & $-0,2$ & 8,6 \\
\hline Proteasome subunit alpha type- 4 ; & Q9R1P0 & Psma4 & 0,0 & 22,6 \\
\hline Proteasome subunit alpha type- 5 ; & Q9Z2U1 & Psma5 & $-0,1$ & 23,7 \\
\hline Proteasome subunit alpha type- 6 ; & Q9QUM9 & Psma6 & $-0,1$ & 26,4 \\
\hline Proteasome subunit alpha type- 7 ; & Q9Z2U0 & Psma7 & 0,2 & 16,9 \\
\hline Proteasome subunit beta type-1; & 009061 & Psmb1 & 0,0 & 13,3 \\
\hline Proteasome subunit beta type- 2 & Q9R1P3 & Psmb2 & 0,3 & 16,9 \\
\hline Proteasome subunit beta type- 4 & P99026 & Psmb4 & 0,0 & 7,2 \\
\hline Proteasome subunit beta type- 5 & 055234 & Psmb5 & $-0,2$ & 13,3 \\
\hline Proteasome subunit beta type- 6 ; & Q60692 & Psmb6 & $-0,3$ & 18,1 \\
\hline Proteasome subunit beta type-7; & P70195 & Psmb7 & $-0,5$ & 7,6 \\
\hline Proteasome subunit beta type- 8 & P28063 & Psmb8 & 0,3 & 4,3 \\
\hline Proteasome-associated protein ECM29 homolog; & Q6PDI5 & Ecm29 & $-0,7$ & 0,5 \\
\hline Protein 2010109I03Rik; RIKEN cDNA 2010109I03; & Q9CQ11 & 2010109I03Rik & 0,1 & 15,3 \\
\hline Protein 4930438A08Rik; & Q5SPH3 & 4930438A08Rik & $-4,2$ & 3,6 \\
\hline Protein 4932418E24Rik; & Q8C5V8 & 4932418E24Rik & 0,2 & 0,8 \\
\hline Protein 5430401F13Rik; & E9Q328 & 5430401F13Rik & 0,8 & 24 \\
\hline $\begin{array}{l}\text { Protein } 8030462 \text { N17Rik; RIKEN cDNA 8030462N17, isoform CRA_a; } \\
\text { Uncharacterized protein; }\end{array}$ & QOVAW6 & 8030462N17Rik & 0,7 & 7,5 \\
\hline Protein Abca12; & E9Q876 & Abca12 & 0,0 & 0,4 \\
\hline Protein Adh6a; & E9Q4Y1 & Adh6a & 1,3 & 2,7 \\
\hline Protein Ahnak; & E9Q616 & Ahnak & $-0,5$ & 46,3 \\
\hline Protein Ahnak2; & F7DBB3 & Ahnak2 & 0,4 & 19,2 \\
\hline Protein Ahnak2; & Е9РYB0 & Ahnak2 & 0,1 & 19,8 \\
\hline Protein Aim1l; & B7ZCC2 & Aim1l & 0,2 & 2 \\
\hline Protein Akap13; & E9Q394 & Akap13 & 0,3 & 1,8 \\
\hline Protein Ankrd35; & E9Q9D8 & Ankrd35 & 0,0 & 1,2 \\
\hline Protein BC117090; & L7N257 & BC117090 & $-0,2$ & 44,8 \\
\hline Protein Bod1l; & E9Q6J5 & Bod11 & 0,2 & 3,1 \\
\hline Protein Bpifb9b; & A2AJD1 & Bpifb9b & 0,2 & 17,8 \\
\hline Protein BRICK1; & Q91VR8 & Brk1 & 0,8 & 14,7 \\
\hline Protein C10; & 035127 & Grcc10 & $-0,2$ & 10,3 \\
\hline Protein Cald1; & D3Z6I7 & Cald1 & $-0,9$ & 25,3 \\
\hline Protein canopy homolog 2; & Q9QXT0 & Cnpy2 & 0,1 & 29,1 \\
\hline Protein canopy homolog 3 ; & Q9DAU1 & Cnpy3 & 0,0 & 4,3 \\
\hline Protein canopy homolog 4 & Q8BQ47 & Cnpy4 & 0,2 & 13,1 \\
\hline Protein CASC3; & Q8K3W3 & Casc3 & $-0,1$ & 5,4 \\
\hline Protein CDV3; & Q4VAA2 & Cdv3 & $-0,5$ & 32,7 \\
\hline Protein CLEC16A; & Q80U30 & Clec16a & 0,0 & 1,2 \\
\hline Protein Col4a6; & B1AVK5 & Col4a6 & $-0,5$ & 0,5 \\
\hline Protein cordon-bleu; & Q5NBX1 & Cobl & 0,0 & 2,1 \\
\hline Protein Crnn; & D3YUU6 & Crnn & $-3,3$ & 6,3 \\
\hline Protein CutA; & Q9CQ89 & Cuta & 0,2 & 9,1 \\
\hline Protein Daple; & Q6VGS5 & Ccdc88c & 0,4 & 1 \\
\hline Protein DEK; & Q7TNVO & Dek & 0,2 & 11,8 \\
\hline Protein disulfide-isomerase $\mathrm{A} 3$; & P27773 & Pdia3 & 0,4 & 36,4 \\
\hline Protein disulfide-isomerase $\mathrm{A} 4$; & P08003 & Pdia4 & 0,0 & 6,3 \\
\hline Protein disulfide-isomerase A6; & Q3TML0 & Pdia6 & 0,3 & 22,2 \\
\hline Protein disulfide-isomerase; & P09103 & P4hb & $-0,2$ & 26,5 \\
\hline Protein DJ-1; & Q99LX0 & Park7 & 0,4 & 13,7 \\
\hline Protein dpy-30 homolog; & Q99LT0 & Dpy30 & $-0,6$ & 9,1 \\
\hline Protein Dr1; & Q91WV0 & Dr1 & $-0,3$ & 7,4 \\
\hline Protein FAM107B; & н9н9т1 & Fam107b & $-0,3$ & 32 \\
\hline Protein FAM134B; & Q8VE91 & Fam134b & 0,2 & 3,4 \\
\hline Protein FAM136A; & Q9CR98 & Fam136a & 0,0 & 13 \\
\hline Protein FAM162A; & Q9D6U8 & Fam162a & 0,6 & 7,1 \\
\hline Protein FAM168A; & Q8BGZ2 & Fam168a & $-1,4$ & 4,1 \\
\hline Protein FAM177A1; & Q8BR63 & Fam177a1 & 0,0 & 14,5 \\
\hline Protein FAM192A; & Q91WE2 & Fam192a & 0,0 & 7,1 \\
\hline Protein FAM195B; & Q3UGS4 & Fam195b & $-0,1$ & 41,2 \\
\hline Protein FAM25C; & Q8CF02 & Fam 25c & $-0,2$ & 53,9 \\
\hline Protein FAM83B; & QOVBM2 & Fam83b & 0,2 & 1,8 \\
\hline Protein FAM83H; & Q148V8 & Fam83h & 0,8 & 3,3 \\
\hline Protein Gm14744; & B1AVU4 & Gm14744 & 0,9 & 32,9 \\
\hline Protein Gm20425; & E9Q035 & Gm20425 & 0,4 & 9,9 \\
\hline Protein Gm5849; & J3QPE5 & Gm5849 & $-0,9$ & 8,5 \\
\hline Protein Gm5938; & A2AEN9 & Gm5938 & 0,8 & 27,6 \\
\hline
\end{tabular}




\begin{tabular}{|c|c|c|c|c|}
\hline Protein & Uniprot & Gene name & $\begin{array}{l}\text { Fold change of } \\
\text { XPRESS ratio } \\
\left(E_{g f r}{ }^{\Delta K C} / \mathrm{WT}\right)\end{array}$ & $\begin{array}{c}\text { Sequence } \\
\text { coverage [\%] }\end{array}$ \\
\hline Protein Gm6537; & J3QPU5 & Gm6537 & 0,6 & 25,9 \\
\hline Protein Golgb1; & E9PVZ8 & Golgb1 & $-0,1$ & 1,5 \\
\hline Protein HEXIM1; & Q8R409 & Hexim1 & 0,0 & 25,6 \\
\hline Protein Krt78; & E9Q0F0 & Krt78 & 0,2 & 41,2 \\
\hline Protein Krtap11-1; & E9Q2E9 & Krtap11 1 & $-5,9$ & 7,7 \\
\hline Protein Lmo7; & E9PYF4 & Lmo7 & 0,2 & 3,4 \\
\hline Protein LSM14 homolog A; & Q8K2F8 & Lsm14a & 0,9 & 1,7 \\
\hline Protein LYRIC; & Q80WJ7 & Mtdh & $-1,0$ & 9,8 \\
\hline Protein LZIC; & Q8K3C3 & Lzic & 0,0 & 13,2 \\
\hline Protein max; & P28574 & Max & $-0,4$ & 6,6 \\
\hline Protein Mroh8; & E9PYI4 & Mroh8 & $-1,2$ & 1,3 \\
\hline Protein NLRC3; & Q5DU56 & Nlrc3 & 0,2 & 0,8 \\
\hline Protein Nolc1; & E9Q5C9 & Nolc1 & $-0,4$ & 7,8 \\
\hline Protein Nup153; & E9Q3G8 & Nup153 & $-0,3$ & 4,7 \\
\hline Protein Obp1b; & A2AEPO & Obp1b & 0,6 & 45 \\
\hline Protein Pcdh1; Protocadherin 1; & Q8CFX3 & Pcdh1 & $-0,3$ & 5,2 \\
\hline Protein Pfdn4; & E9Q4Q8 & Pfdn4 & 0,0 & 25,5 \\
\hline Protein phosphatase 1 regulatory subunit 11; & Q8K1L5 & Ppp1r11 & $-0,1$ & 7,5 \\
\hline Protein phosphatase 1 regulatory subunit 12A; & Q9DBR7 & Ppp1r12a & 0,0 & 8,7 \\
\hline Protein phosphatase 1 regulatory subunit 14A; & Q91VC7 & Ppp1r14a & 0,3 & 7,5 \\
\hline Protein phosphatase 1 regulatory subunit 14B; & Q62084 & Ppp1r14b & 0,4 & 13,6 \\
\hline Protein phosphatase 1G; & Q61074 & Ppm1g & $-0,4$ & 2,2 \\
\hline Protein phosphatase inhibitor 2 & Q9DCL8 & Ppp1r2 & $-0,2$ & 17,9 \\
\hline Protein POF1B; & Q8K4L4 & Pof1b & 0,0 & 22,3 \\
\hline Protein Prol1; & E9PYQ4 & Prol1 & 1,0 & 2,2 \\
\hline Protein PRRC1; & Q3UPH1 & Prrc1 & 0,5 & 3,4 \\
\hline Protein PRRC2A; & Q7TSC1 & Prrc2a & $-0,1$ & 6 \\
\hline Protein PRRC2B; & Q7TPM1 & Prrc2b & $-0,3$ & 6,5 \\
\hline Protein PRRC2C; & Q3TLH4 & Prrc2c & $-0,2$ & 5,7 \\
\hline Protein QIL1; & Q8R404 & Qil1 & $-0,2$ & 5,9 \\
\hline Protein Red; & Q9Z1M8 & $\mathrm{lk}$ & 1,1 & 3,2 \\
\hline Protein Rnf113a2; Ring finger protein $113 \mathrm{~A} 2$; & Q14B01 & Rnf113a2 & $-0,4$ & 5,6 \\
\hline Protein S100-A10; & P08207 & S100a10 & 2,0 & 10,3 \\
\hline Protein S100-A11; & P50543 & S100a11 & 0,5 & 11,2 \\
\hline Protein S100-A13; & P97352 & S100a13 & $-0,1$ & 23,5 \\
\hline Protein S100-A14; & Q9D2Q8 & S100a14 & 0,0 & 59,6 \\
\hline Protein S100a16; & D3Z2Y6 & S100a16 & $-0,4$ & 31,5 \\
\hline Protein S100-A3; & P62818 & S100a3 & $-2,8$ & 10,9 \\
\hline Protein S100-A6; & P14069 & S100a6 & $-0,3$ & 9 \\
\hline Protein S100-A9; & P31725 & S100a9 & 1,5 & 12,4 \\
\hline Protein Scaf11; & E9PZM7 & Scaf11 & $-0,7$ & 0,7 \\
\hline Protein SCAF8; & Q6DID3 & Scaf8 & 0,9 & 0,9 \\
\hline Protein Scgb2b12; & S4R2LO & Scgb2b12 & 0,4 & 14,3 \\
\hline Protein Scgb2b17; & S4R1X8 & Scgb2b17 & 0,5 & 9,9 \\
\hline Protein Sec16a; & A2AIX1 & Sec16a & 0,0 & 1,5 \\
\hline Protein Sec24b; & F6VJC5 & $\operatorname{Sec} 24 b$ & $-2,8$ & 3,9 \\
\hline $\begin{array}{l}\text { Protein Serpinb3c; Serine (Or cysteine) peptidase in hibitor, clade B, member } \\
\text { 3C; }\end{array}$ & A2RSF9 & Serpinb3c & 0,2 & 9,8 \\
\hline Protein Serpinb6c; Serpinb6c protein; & Q6P6K7 & Serpinb6c & 0,3 & 4,8 \\
\hline Protein SET; & Q9EQU5 & Set & 0,0 & 28,4 \\
\hline Protein Sf3b2; Splicing factor $3 b$, subunit 2; & Q3UJB0 & $\mathrm{Sf} 3 \mathrm{~b} 2$ & $-0,3$ & 13 \\
\hline Protein SON; & Q9QX47 & Son & 0,3 & 0,4 \\
\hline Protein Spink5; Serine peptidase inhibitor, Kazal type 5; & Q148R4 & Spink5 & 0,2 & 16,6 \\
\hline Protein Srsf11; & D3Z4B0 & Srsf11 & 0,0 & 7,9 \\
\hline $\begin{array}{l}\text { Protein Taf15; TAF15 RNA polymerase II, TATA box binding protein (TBP)- } \\
\text { associated factor; }\end{array}$ & Q8BQ46 & Taf15 & $-0,5$ & 6,3 \\
\hline Protein Tfg; TFG protein; Trk-fused; Trk-fused protein; & Q9Z1A1 & Tfg & 0,1 & 6,8 \\
\hline Protein Tmsb15b1; & A2AF33 & Tmsb15b1 & 0,0 & 62,2 \\
\hline Protein transport protein Sec31A; & Q3UPL0 & Sec31a & 0,7 & 3,4 \\
\hline Protein transport protein Sec61 subunit alpha isoform 1; & P61620 & Sec61a1 & 0,3 & 2,3 \\
\hline Protein transport protein Sec61 subunit beta; & Q9cQS8 & Sec61b & $-0,2$ & 11,5 \\
\hline Protein Trip11; & E9Q512 & Trip11 & 0,0 & 6,8 \\
\hline Protein TSSC4; & Q9JHE7 & Tssc4 & $-0,4$ & 24 \\
\hline Protein-arginine deiminase type-1; & Q9Z185 & Padi1 & 0,0 & 1,4 \\
\hline Protein-glutamine gamma-glutamyltransferase E; & Q08189 & Tgm3 & 0,2 & 8,5 \\
\hline Proteolipid protein 2; & Q9R1Q7 & Plp2 & 0,4 & 7,9 \\
\hline Prothymosin alpha; & P26350 & Ptma & 0,4 & 9 \\
\hline Pumilio homolog 1; & Q80U78 & Pum1 & 0,3 & 0,8 \\
\hline Pumilio homolog 2; & Q80U58 & Pum2 & 0,2 & 9,1 \\
\hline Purine nucleoside phosphorylase; & P23492 & Pnp & $-0,3$ & 3,5 \\
\hline Putative phospholipase B-like 2; & Q3TCN2 & Plbd2 & $-0,4$ & 3,2 \\
\hline
\end{tabular}


Protein

Uniprot

Gene name $\quad$ Fold change of

Sequence

coverage [\%]

Putative pre-mRNA-splicing factor ATP-dependent RNA helicase DHX15;

035286

Putative RNA-binding protein 3;

Pyrethroid hydrolase Ces2e;

Pyruvate kinase PKM;

Rab GDP dissociation inhibitor alpha;

Rab GDP dissociation inhibitor beta;

Rab GTPase-binding effector protein 2;

Rab11 family-interacting protein 1;

Rab-like protein 6;

Radixin;

Ragulator complex protein LAMTOR1;

Ragulator complex protein LAMTOR5

Ran-specific GTPase-activating protein;

Ras GTPase-activating protein-binding protein 1;

Ras GTPase-activating-like protein IQGAP1;

Ras-related protein Rab-10;

Ras-related protein Rab-11B;

Ras-related protein Rab-14;

Ras-related protein Rab-1A;

Ras-related protein Rab-2A;

Ras-related protein Rab-5A;

Regulator of cell cycle RGCC;

Regulator of nonsense transcripts 1 ;

RelA-associated inhibitor;

Repetin;

Reticulocalbin-1;

Reticulocalbin-2;

Reticulocalbin-3;

Reticulon-1;

Reticulon-3;

Reticulon-4;

Retinoid-inducible serine carboxypeptidase;

Retroviral-like aspartic protease 1;

Ribonuclease inhibitor;

Ribonuclease pancreatic;

Ribonuclease T2;

Ribonuclease UK114;

Ribosome-binding protein 1;

Ribosome-recycling factor, mitochondrial;

RNA polymerase-associated protein LEO1;

RNA polymerase-associated protein RTF1 homolog;

RNA-binding protein $8 \mathrm{~A}$;

RNA-binding protein EWS;

RNA-binding protein FUS;

RNA-binding protein Raly;

RRP15-like protein;

RWD domain-containing protein 1;

RWD domain-containing protein 4;

Ryanodine receptor 3;

SAFB-like transcription modulator;

SAP domain-containing ribonucleop rotein;

SAP30-binding protein;

Scaffold attachment factor B1;

Scaffold attachment factor B2

Sciellin;

Sclerostin domain-containing protein 1;

Secretoglobin family 2B member 2;

Secretoglobin family 2B member 20;

Secretoglobin family 2B member 24;

Selenium-binding protein 1 ;

Selenoprotein S;

Sepiapterin reductase;

Septin-11;

Serine protease inhibitor A3M;

Serine/arginine-rich splicing factor 1 ;

Serine/arginine-rich splicing factor 10 ;

Serine/arginine-rich splicing factor 2;

Serine/arginine-rich splicing factor 3 ;

Serine/arginine-rich splicing factor 4;

Serine/arginine-rich splicing factor 5 ;
089086

Q8BK48

P52480

P50396

Q61598

Q91WG2

E9Q8L9

Q5U3K5

P26043

Q9CQ22

Q9D1L9

P34022

P97855

Q9JKF1

P61027

P46638

Q91V41

P62821

P53994

Q9CQD1

Q9DBX1

Q9EPUO

Q5I1X5

P97347

Q05186

Q8BP92

Q8BH97

Q8K0T0

Q9ES97

Q99P72

Q920A5

Q09PK2

Q91VI7

P00683

Q9CQ01

P52760

Q99PL5

Q9D6S7

Q5XJE5

A2AQ19

Q9CWZ3

Q61545

P56959

Q64012

Q9CYX7

Q9CQK7

Q9CPR1

A2AGL3

$\mathrm{Q} 8 \mathrm{CH} 25$

Q9D1J3

Q02614

D3YXK2

Q80YR5

Q9EQG3

Q9CQN4

Q6UGQ3

J3QK77

Q7M747

P17563

Q9BCZ4

Q64105

Q8C1B7

Q03734

Q6PDM2

Q9R0U0

Q62093

P84104

Q8VE97

035326

$\begin{array}{cc}\text { Dhx15 } & 0 \\ \text { Rbm3 } & -0, \\ \text { Ces2e } & -0,2 \\ \text { Pkm } & 0,2 \\ \text { Gdi1 } & -0,1 \\ \text { Gdi2 } & 0,2 \\ \text { Rabep2 } & -4,2 \\ \text { Rab11fip1 } & 0,1 \\ \text { Rabl6 } & 0,1 \\ \text { Rdx } & -0,7 \\ \text { Lamtor1 } & -0,7 \\ \text { Lamtor } & 0,0\end{array}$

$0,0 \quad 1,4$

$-0,2 \quad 22,9$

$-0,2 \quad 2,5$

$0,2 \quad 14,1$

$-0,1 \quad 4,7$

$0,2 \quad 13,7$

$-4,2 \quad 1,8$

$0,1 \quad 4,5$

$0,1 \quad 5,4$

$-0,7 \quad 12$

$-0,7 \quad 8,1$

Lamtor5 $0,0 \quad 22$

Ranbp1 $\quad-0,1 \quad 18,7$

G3bp1 $\quad 0,0 \quad 8,6$

lqgap1 $\quad 0,2 \quad 1,3$

Rab10 0,2 $\quad 16,5$

Rab11b $\quad-1,0 \quad 21,5$

Rab14 $0,3 \quad 11,6$

Rab1A $\quad 0,8 \quad 14,9$

Rab2a $\quad 0,6 \quad 6,6$

$\begin{array}{lll}\text { Rab5a } & 0,4 & 5,6\end{array}$

$\begin{array}{lll}\text { Rgcc } & -0,3 & 8\end{array}$

$\begin{array}{lll}\text { Upf1 } & -0,1 & 2\end{array}$

$\begin{array}{lll}\text { Ppp1r131 } & 0,3 & 3,8\end{array}$

$\begin{array}{lll}\text { Rptn } \quad 0,4 & 3,8\end{array}$

$\begin{array}{lll}\text { Rcn1 } & 0,0 & 18,8\end{array}$

$\begin{array}{lll}\text { Rcn2 } & -0,4 & 3,8\end{array}$

Rcn3 $\quad-0,3 \quad 6,9$

$\begin{array}{lll}\text { Rtn1 } & -0,7 & 3,7\end{array}$

Rtn3 $0,5 \quad 1,1$

$\begin{array}{lll}\text { Rtn4 } & -0,1 & 11,5\end{array}$

Scpep1 $\quad 0,5 \quad 8,6$

Asprv1 $\quad 0,1 \quad 4,4$

Rnh1 $\quad 1,5 \quad 2,2$

$\begin{array}{lll}\text { Rnase1 } & 0,3 & 8,7\end{array}$

Rnaset2 $\quad-0,6 \quad 3,5$

$\begin{array}{lll}\text { Hrsp12 } & -0,1 & 34,8\end{array}$

$\begin{array}{lll}\text { Rrbp1 } & -0,5 & 13,7\end{array}$

Mrrf $\quad-0,5 \quad 11,1$

$\begin{array}{lll}\text { Leo1 } & -0,3 & 4,8\end{array}$

$\begin{array}{lll}\text { Rtf1 } & 0,3 & 1,5\end{array}$

$\begin{array}{lll}\text { Rbm8a } & 0,6 & 6,3\end{array}$

Ewsr1 - $0,2 \quad 4,5$

Fus $\quad-0,3 \quad 13,1$

Raly $\quad-0,4 \quad 14,2$

Rrp15 $\quad-0,3 \quad 2,5$

$\begin{array}{lll}\text { Rwdd1 } & 0,0 & 3,7\end{array}$

$\begin{array}{lll}\text { Rwdd4 } & 0,1 & 4,8\end{array}$

Ryr3 $1,2 \quad 0,5$

Sltm $\quad-0,3 \quad 3,1$

Sarnp $\quad-0,3 \quad 25,2$

Sap30bp $\quad-0,1 \quad 3,9$

Safb $\quad-0,3 \quad 3,8$

Safb2 $-0,9 \quad 5$

Scel $\quad 0,1 \quad 5,2$

Sostdc1 $\quad-0,4 \quad 6,8$

Scgb2b2 $\quad 0,6 \quad 24,6$

Scgb2b20 0,3 21,4

Scgb2b24 $\quad 0,7 \quad 13,4$

Selenbp1 $\quad-0,1 \quad 12,3$

$\begin{array}{lll}\text { Vimp } & -0,2 & 5,8\end{array}$

$\begin{array}{lll}\text { Spr } & 0,7 & 8,7\end{array}$

Sept11 - $-0,7 \quad 2,6$

Serpina3m $\quad-0,1 \quad 5,5$

$\begin{array}{lll}\text { Srsf1 } & 0,4 & 25\end{array}$

$\begin{array}{lll}\text { Srsf10 } & 0,4 & 6\end{array}$

$\begin{array}{lll}\text { Srsf2 } & -0,1 & 6,8\end{array}$

Srsf3 $\quad 0,2 \quad 21,3$

Srsf4 $\quad-0,1 \quad 4,5$

Srsf5 0,2

13 


Protein

Serine/arginine-rich splicing factor 7;
Serine/arginine-rich splicing factor 9;
Serine/threonine-protein kinase PAK 2;
Serine/threonine-protein kinase WNK1;
Serine/threonine-protein kinase WNK2;
Serine/threonine-protein phosphatase 2A 55 kDa regulatory subunit B alpha
isoform;
Serine/threonine-protein phosphatase 2A 65 kDa regulatory subunit A alpha
isoform;

\begin{tabular}{lccc} 
Uniprot & Gene name & $\begin{array}{c}\text { Fold change of } \\
\text { XPRESS ratio } \\
\text { (Egfr }\end{array}$ (KC $/$ WT) $^{\text {Sequence }}$ & $\begin{array}{c}\text { Sequerage [\%] } \\
\text { covera }\end{array}$ \\
\hline Q8BL97 & Srsf7 & 0,2 & 13,1 \\
Q9D0B0 & Srsf9 & $-0,1$ & 5 \\
Q8CIN4 & Pak2 & 0,3 & 1,9 \\
P83741 & Wnk1 & $-0,1$ & 1 \\
Q3UH66 & Wnk2 & 0,3 & 1,4 \\
Q6P1F6 & Ppp2r2a & $-0,6$ & 2,5 \\
Q76MZ3 & Ppp2r1a & 0,0 & 5,1
\end{tabular}

Serine/threonine-protein phosphatase 4 regulatory subunit 2;

Serine/threonine-protein phosphatase 6 regulatory subunit 3;

Serine/threonine-protein phosphatase CPPED1;

Serine--tRNA ligase, cytoplasmic;

Serpin A12;

Serpin A9;

Serpin B12;

Serpin B5;

Serpin B7;

Serpin B8;

Serrate RNA effector molecule homolog;

Serum albumin;

P6271

Ppp2r1a

0,0

P62715

Ppp2cb

$-0,2$

3,6

Q0VGB7

Q8BFS6

P26638

Q7TMF5

Q9D7D2

Q9D7P9

P70124

Q9D695

008800

Q99MR6

P07724

Q63918

Serum deprivation-response protein

SH3 domain-binding glutamic acid-rich-like protein 3;

SH3 domain-binding glutamic acid-rich-like protein;

$\mathrm{SH} 3$ domain-containing kinase-binding protein 1;

Shootin-1;

Short-chain dehydrogenase/reductase family $9 \mathrm{C}$ member 7;

Sickle tail protein;

Sideroflexin-1;

Sideroflexin-3;

Signal peptide peptidase-like $2 \mathrm{~A}$;

Signal transducing adapter molecule 2;

Signal-induced proliferation-associated 1-like protein 1;

Single-stranded DNA-binding protein, mitochondrial;

Sjoegren syndrome nuclear autoantigen 1 homolog;

SLAIN motif-containing protein 2;

Small acidic protein;

Small nuclear ribonucleoprotein Sm D2;

Small ubiquitin-related modifier 2;

Small VCP/p97-interacting protein;

SNARE-associated protein Snapin;

SNW domain-containing protein 1;

Sodium/potassium-transporting ATPase subunit beta-1;

Sodium/potassium-transporting ATPase subunit beta-3;

Sodium-dependent phosphate transport protein 2B;

Solute carrier family 12 member 2 ;

Sorcin;

Sorting nexin-1;

Sorting nexin-12;

Sorting nexin-18;

Sorting nexin-3;

SPARC;

Specifically androgen-regulated gene protein;

Ppp4r2

Cpped1

Sars

Serpina12

Serpina9

Serpinb12

Serpinb5

Serpinb7

Serpinb8

Srrt

Alb

Sdpr

Q91VW3

Q9JJU8

Q8R550

Q8K2Q9

Q8K3P0

A2AQ25

Q99JR1

Q91V61

Q9JJF9

088811

Q8C0T5

Q9CYRO

Q9JJ94

Q8CI08

Q9R0P4

P62317

P61957

Q3UZP4

Q9Z266

Q9CSN1

P14094

P97370

Q9DBP0

P55012

Q6P069

Q9WV80

070493

Q91ZR2

070492

P07214

Q8BI29

P16546

Q62261

Spectrin beta chain, non-erythrocytic 1 ;

Sperm-associated antigen 7;

S-phase kinase-associated protein 1;

Q7TNE3

Q9WTX5

Q04519

Q9JHS9

Spliceosome-associated protein CWC15 homolog;

Splicing factor 1 ;

Splicing factor $3 B$ subunit 1 ;

Splicing factor 3B subunit 4;

Splicing factor, proline- and glutamine-rich;

Squamous cell carcinoma antigen recognized by T-cells 3;

Src substrate cortactin;

SRSF protein kinase 1 ;

SRSF protein kinase 2;

Stathmin;

Q64213

Q99NB9

Q8QZY9

Q8VIJ6

Q9JLI8

Q60598

070551

054781

P54227

Stefin-1;

P35175

Sh3bgrl3

Sh3bgrl

Sh3kbp1

Kiaa1598

Sdr9c7

Skt

Sfxn1

Sfxn3

Sppl2a

Stam2

Sipa1I1

Ssbp1

Ssna1

Slain2

Smap

Snrpd2

Sumo2

Svip

Snapin

Snw1

Atp1b1

Atp1b3

Slc34a2

Slc12a2

Sri

Snx1

Snx12

Snx18

Snx3

Sparc

Sarg

Sptan1

Sptbn1

Spag7

Skp1

Smpd1

Cwc15

Sf1

Sf3b1

Sf3b4

Sfpq

Sart3

Cttn

Srpk1

Srpk2

Stmn1

Stfa1

$-0,4$

0,2

$-0,1$

0,3

1,2

$-0,1$

$-0,2$

0,0

0,2

0,0

$-0,3$

0,4

$-0,3$

0,3




\begin{tabular}{|c|c|c|c|c|}
\hline Protein & Uniprot & Gene name & $\begin{array}{l}\text { Fold change of } \\
\text { XPRESS ratio } \\
\left.\text { (Egfr }{ }^{\Delta K C} / \mathrm{WT}\right)\end{array}$ & $\begin{array}{c}\text { Sequence } \\
\text { coverage [\%] }\end{array}$ \\
\hline Stefin-3; & P35173 & Stfa3 & $-0,3$ & 68,9 \\
\hline Stress-70 protein, mitochondrial; & P38647 & Hspa9 & 0,0 & 7,5 \\
\hline Stress-induced-phosphoprotein 1 & Q60864 & Stip1 & $-0,1$ & 21,4 \\
\hline Stromal interaction molecule 1 ; & P70302 & Stim1 & 1,4 & 1,9 \\
\hline Submandibular gland protein C; & Q6JHY2 & Muc19 & 0,2 & 31,4 \\
\hline Sulfated glycoprotein 1 & Q61207 & Psap & 0,2 & 19,4 \\
\hline Sulfhydryl oxidase 1 & Q8BND5 & Qsox1 & 0,7 & 1,5 \\
\hline Superoxide dismutase [Cu-Zn]; & P08228 & Sod1 & $-0,1$ & 25,3 \\
\hline Superoxide dismutase [Mn], mitochondrial; & P09671 & Sod2 & 0,7 & 19,8 \\
\hline Suppressor of tumorigenicity 14 protein homolog; & P56677 & St14 & $-0,2$ & 1,6 \\
\hline Suprabasin; & E9QPB2 & Sbsn & $-0,2$ & 46,9 \\
\hline Survival of motor neuron-related-splicing factor 30 ; & Q8BGT7 & Smndc1 & $-0,5$ & 13,9 \\
\hline Sushi domain-containing protein 2 ; & Q9DBX3 & Susd2 & 0,5 & 2,4 \\
\hline Sushi repeat-containing protein SRPX2; & Q8R054 & Srpx2 & $-7,6$ & 3,4 \\
\hline SUZ domain-containing protein 1 & Q6NXN1 & Szrd1 & $-0,1$ & 25 \\
\hline $\begin{array}{l}\text { SWI/SNF-related matrix-associated actin-dependent regulator of chromatin } \\
\text { subfamily E member } 1 \text {; }\end{array}$ & 054941 & Smarce1 & $-0,6$ & 3,2 \\
\hline Swi5-dependent recombination DNA repair protein 1 homolog; & Q8BP27 & Sfr1 & 0,1 & 3,4 \\
\hline Synapse-associated protein 1 & Q9D5V6 & Syap1 & $-0,2$ & 4,1 \\
\hline Synaptic vesicle membrane protein VAT-1 homolog; & Q62465 & Vat1 & $-0,1$ & 2,2 \\
\hline Synaptojanin-2-binding protein; & Q9D6K5 & Synj2bp & 0,2 & 11,3 \\
\hline Synaptosomal-associated protein 23 & 009044 & Snap23 & $-0,5$ & 34 \\
\hline Synaptosomal-associated protein 29 & Q9ERB0 & Snap29 & $-0,4$ & 26,5 \\
\hline Synaptotagmin-like protein 1 & Q99N80 & Sytl1 & 0,5 & 9,5 \\
\hline Syndecan-4; & 035988 & Sdc4 & $-0,4$ & 5,1 \\
\hline Synergin gamma; & Q5SV85 & Synrg & 1,7 & 3,5 \\
\hline Syntaxin-12; & Q9ER00 & Stx12 & 0,2 & 15 \\
\hline Syntaxin-7; & 070439 & Stx7 & 0,3 & 10,7 \\
\hline Syntaxin-8; & 088983 & Stx8 & 0,0 & 14 \\
\hline Target of Myb protein 1 ; & 088746 & Tom1 & 0,0 & 8,1 \\
\hline TATA element modulatory factor; & B9EКI3 & Tmf1 & 0,0 & 6,1 \\
\hline Telomeric repeat-binding factor 2 -interacting protein 1 ; & Q91VL8 & Terf2ip & 0,2 & 4,1 \\
\hline Tetraspanin-8; & Q8R3G9 & Tspan8 & $-0,2$ & 4,3 \\
\hline Tetraspanin-9; & Q8BJU2 & Tspan9 & 0,6 & 8,7 \\
\hline Tetratricopeptide repeat protein $1 ;$ & Q91738 & Ttc1 & $-0,7$ & 6,8 \\
\hline Thimet oligopeptidase; & Q8C1A5 & Thop1 & $-0,3$ & 26,3 \\
\hline Thioredoxin domain-conta ining protein 12 & Q9CQU0 & Txndc12 & 0,2 & 14,1 \\
\hline Thioredoxin domain-containing protein 17 & Q9CQM5 & Txndc17 & 0,2 & 26,8 \\
\hline Thioredoxin domain-containing protein 5 & Q91W90 & Txndc5 & 0,0 & 11,6 \\
\hline Thioredoxin; & P10639 & Txn & 0,3 & 32,4 \\
\hline THO complex subunit 4; & 008583 & Alyref & $-0,3$ & 15,3 \\
\hline Three prime repair exonuclease 2 ; & Q9R1A9 & Trex2 & 0,6 & 28,4 \\
\hline Thy-1 membrane glycoprotein; & P01831 & Thy1 & $-0,4$ & 16 \\
\hline Tight junction protein ZO-1; & P39447 & Tjp1 & 0,0 & 4,5 \\
\hline Tissue factor; & P20352 & F3 & 0,3 & 13,6 \\
\hline Titin; & A2ASS6 & Ttn & $-2,5$ & 0,1 \\
\hline T-lymphoma invasion and metastasis-inducing protein $1 ;$ & Q60610 & Tiam1 & 1,5 & 0,7 \\
\hline TP53-regulated inhibitor of apoptosis 1 & Q9D8Z2 & Triap1 & 0,4 & 27,6 \\
\hline TPA-induced transmembrane protein homolog; & Q8C5C9 & & 0,0 & 3,2 \\
\hline Transcription and mRNA export factor ENY2; & Q9JIX0 & Eny2 & 0,1 & 33,7 \\
\hline Transcription cofactor vestigial-like protein 4 & Q80V24 & $\operatorname{Vg\| 4}$ & 0,8 & 7 \\
\hline Transcription elongation factor A protein 1 & P10711 & Tcea1 & 0,2 & 19,3 \\
\hline Transcription elongation factor B polypeptide 2 ; & P62869 & Tceb2 & 0,2 & 43,2 \\
\hline Transcription factor AP-1; & P05627 & Jun & 0,3 & 4,5 \\
\hline Transcription factor BTF3 homolog 4; & Q9CQH7 & Btf314 & $-0,1$ & 20,9 \\
\hline Transcription factor BTF3; & Q64152 & Btf3 & 0,1 & 12,7 \\
\hline Transcription factor ETV6; & P97360 & Etv6 & 1,0 & 5,1 \\
\hline Transcription factor jun-D; & P15066 & Jund & $-0,4$ & 4,4 \\
\hline Transcription initiation factor IIA subunit 1 ; & Q99PM3 & Gtf2a1 & $-0,2$ & 2,9 \\
\hline Transcription initiation factor TFIID subunit 7; & Q9R1C0 & Taf7 & $-0,5$ & 13,2 \\
\hline Transcription initiation factor TFIID subunit 9B; & Q6NZA9 & Taf9b & 0,2 & 3,2 \\
\hline Transcription intermediary factor 1-beta; & Q62318 & Trim28 & 1,2 & 1,3 \\
\hline Transcriptional activator protein Pur-alpha; & P42669 & Pura & 0,4 & 2,8 \\
\hline Transcriptional activator protein Pur-beta; & 035295 & Purb & $-0,3$ & 14,8 \\
\hline Transforming acidic coiled-coil-containing protein 2 ; & E9Q8T1 & Tacc2 & 0,1 & 17,1 \\
\hline Transforming acidic coiled-coil-containing protein 2 ; & E9Q9Z4 & Tacc2 & 0,2 & 17,3 \\
\hline Transgelin-2; & Q9WVA4 & Tagln2 & 0,1 & 51,8 \\
\hline Trans-Golgi network integral membrane protein 1 ; & Q62313 & Tgoln1 & $-0,5$ & 11 \\
\hline Transitional endoplasmic reticulum ATPase; & Q01853 & $V_{c p}$ & 0,0 & 10,7 \\
\hline Translation initiation factor elF-2B subun it delta; & Q61749 & Eif2b4 & $-2,7$ & 2,9 \\
\hline Translation machinery-associated protein 7; & Q8К003 & Tma7 & $-0,2$ & 14,1 \\
\hline
\end{tabular}




\begin{tabular}{|c|c|c|c|c|}
\hline Protein & Uniprot & Gene name & $\begin{array}{l}\text { Fold change of } \\
\text { XPRESS ratio } \\
\left.\text { (Egfr }{ }^{\Delta K C} / \mathrm{WT}\right)\end{array}$ & $\begin{array}{c}\text { Sequence } \\
\text { coverage [\%] }\end{array}$ \\
\hline Translationally-controlled tumor protein; & P63028 & Tpt1 & 0,5 & 23,3 \\
\hline Translocon-associated protein subunit alpha; & Q9CY50 & Ssr1 & 0,3 & 6,6 \\
\hline Transmembrane emp24 domain-containing protein 5; & Q9CXE7 & Tmed5 & 0,4 & 5,2 \\
\hline Transmembrane protein 109; & Q3UBX0 & Tmem109 & $-0,7$ & 16,4 \\
\hline Transmembrane protein 154; & Q8C4Q9 & Tmem154 & 0,6 & 6,1 \\
\hline Transmembrane protein 263 & Q9DAM7 & Tmem263 & $-0,3$ & 8,7 \\
\hline Transmembrane protein 79 & Q9D709 & Tmem79 & $-0,3$ & 3,1 \\
\hline Transmembrane protein $87 \mathrm{~A}$ & Q8BXN9 & Tmem87a & 0,8 & 2,4 \\
\hline Transthyretin; & P07309 & $\operatorname{Ttr}$ & $-0,3$ & 30,6 \\
\hline Treacle protein; & 008784 & Tcof1 & $-1,1$ & 8,9 \\
\hline Trichohyalin-like protein 1; & Q9D3P1 & Tchhl1 & $-2,1$ & 9,9 \\
\hline Triosephosphate isomerase; & P17751 & Tpi1 & 0,1 & 34,8 \\
\hline Tripartite motif-containing protein 29 & Q8R2Q0 & Trim29 & 0,8 & 17,7 \\
\hline tRNA methyltransferase 112 homolog; & Q9DCG9 & Trmt112 & $-0,7$ & 12 \\
\hline Tropomodulin-3; & Q9JHJO & Tmod3 & $-0,8$ & 8 \\
\hline Tropomyosin alpha-1 chain; & E9Q450 & Tpm1 & $-1,2$ & 27,1 \\
\hline Tropomyosin alpha-3 chain; & D3Z6I8 & Tpm3 & 0,1 & 25,9 \\
\hline Tropomyosin alpha-3 chain; & E9Q5J9 & Tpm3 & $-0,3$ & 20,1 \\
\hline Tropomyosin alpha-4 chain; & Q6IRU2 & Tpm4 & $-0,2$ & 34,7 \\
\hline Tropomyosin beta chain; & A2AIM4 & Tpm2 & $-1,1$ & 22,9 \\
\hline Tryptophan--tRNA ligase, cytoplasmic; & P32921 & Wars & $-0,6$ & 3,3 \\
\hline TSC22 domain family protein $1 ;$ & P62500 & $\mathrm{Tsc} 22 \mathrm{~d} 1$ & 0,0 & 26,2 \\
\hline Tubulin alpha-1B chain; & P05213 & Tuba1b & 0,2 & 7,5 \\
\hline Tubulin alpha-4A chain; & P68368 & Tuba4a & $-0,1$ & 7,6 \\
\hline Tubulin beta-2A chain; & Q7TMM9 & Tubb2a & 0,1 & 16,9 \\
\hline Tubulin beta-4B chain; & P68372 & Tubb4b & 0,2 & 15,1 \\
\hline Tubulin beta- 5 chain; & P99024 & Tubb5 & $-4,4$ & 18,9 \\
\hline Tubulin polymerization-promoting protein family member 3 ; & Q9CRB6 & Tppp3 & 0,4 & 14,2 \\
\hline Tubulin polymerization-promoting protein; & Q7TQD2 & Tppp & 0,3 & 6,4 \\
\hline Tubulin-specific chaperone A; & P48428 & Tbca & 0,0 & 24,1 \\
\hline Tudor domain-containing protein 1 & Q99MV1 & Tdrd1 & 0,1 & 0,9 \\
\hline Tuftelin; & 008970 & Tuft1 & $-0,2$ & 14,4 \\
\hline Tumor protein D52; & Q62393 & Tpd52 & $-0,7$ & 22,2 \\
\hline Tumor protein D54; & Q9CYZ2 & Tpd5212 & $-0,1$ & 30,1 \\
\hline Tumor suppressor p53-binding protein 1 ; & P70399 & Tp53bp1 & 0,1 & 24,7 \\
\hline Tyrosine-protein phosphatase non-receptor type 12 & P35831 & Ptpn12 & $-0,1$ & 1,7 \\
\hline Tyrosine-protein phosphatase non-receptor type substrate 1 & P97797 & Sirpa & 0,2 & 8,6 \\
\hline Tyrosine--tRNA ligase, cytoplasmic; & Q91WQ3 & Yars & $-0,3$ & 1,7 \\
\hline U1 small nuclear ribonucleoprotein A; & Q62189 & Snrpa & 0,0 & 16 \\
\hline U1 small nuclear ribonucleoprotein C; & Q62241 & Snrpc & 0,0 & 18,9 \\
\hline U3 small nucleolar RNA-interacting protein 2; & Q91WM3 & Rrp9 & $-0,3$ & 3,2 \\
\hline U4/U6.U5 tri-snRNP-associated protein 1; & Q97315 & Sart1 & $-0,5$ & 1,9 \\
\hline U6 snRNA-associated Sm-like protein LSm3; & P62311 & Lsm3 & 0,8 & 11,8 \\
\hline U6 snRNA-associated Sm-like protein LSm7; & Q9CQQ8 & Lsm7 & 0,4 & 7,8 \\
\hline U6 snRNA-associated Sm-like protein LSm8; & Q6ZWM4 & Lsm8 & 0,3 & 9,4 \\
\hline Ubiquilin-1; & Q8R317 & Ubqln1 & $-0,3$ & 8,8 \\
\hline Ubiquilin-4; & Q99NB8 & Ubqln4 & $-0,4$ & 5,4 \\
\hline Ubiquitin carboxyl-terminal hydrolase 7; & Q6A4J8 & Usp7 & 0,2 & 4,1 \\
\hline Ubiquitin carboxyl-terminal hydrolase 8; & Q80U87 & Usp8 & $-0,3$ & 1,4 \\
\hline Ubiquitin-associated protein 2 & Q91VX2 & Ubap2 & $-0,4$ & 7,2 \\
\hline Ubiquitin-associated protein 2-like; & Q80X50 & Ubap2l & $-0,3$ & 11,7 \\
\hline Ubiquitin-conjugating enzyme E2 A; & Q9Z255 & Ube2a & $-1,9$ & 6,6 \\
\hline Ubiquitin-conjugating enzyme E2 L3; & P68037 & Ube2l3 & 0,5 & 47,4 \\
\hline Ubiquitin-conjugating enzyme E2 N; & P61089 & Ube2n & 0,5 & 18,4 \\
\hline Ubiquitin-conjugating enzyme E2 variant 1; & Q9CZY3 & Ube2v1 & 0,2 & 9,5 \\
\hline Ubiquitin-like modifier-activating enzyme 1 ; & Q02053 & Uba1 & 0,3 & 2,2 \\
\hline UBX domain-containing protein 1 & Q922Y1 & Ubxn1 & $-0,7$ & 12,5 \\
\hline UBX domain-containing protein 7 ; & Q6P5G6 & Ubxn7 & 0,2 & 4,1 \\
\hline UMP-CMP kinase; & Q9DBP5 & Cmpk1 & 0,0 & 21,4 \\
\hline Uncharacterized protein C10orf35 homolog; & Q9D882 & & 3,2 & 14,1 \\
\hline Uncharacterized protein C19orf43 homolog; & Q9D735 & & $-0,8$ & 12,1 \\
\hline Uncharacterized protein C1orf198 homolog; & Q8C3W1 & & $-0,4$ & 23 \\
\hline Uncharacterized protein C1orf21 homolog; & Q8K207 & & $-0,2$ & 19 \\
\hline Uncharacterized protein C6orf132 homolog; & Q91Z58 & & $-0,3$ & 13,8 \\
\hline Uncharacterized protein C7orf57 homolog; & Q5SS90 & Gm11992 & $-0,1$ & 21 \\
\hline Uncharacterized protein C9orf78 homolog; & Q3TQ17 & & $-0,2$ & 10,4 \\
\hline Uncharacterized protein FU45252 homolog; & Q6PIU9 & & 0,1 & 5,4 \\
\hline Uncharacterized protein KIAA1522; & A2A7S8 & Kiaa1522 & $-0,1$ & 3,8 \\
\hline Unconventional myosin-IXa; & Q8C170 & Myo9a & $-4,6$ & 0,4 \\
\hline Unconventional myosin-VI; & Q64331 & Myo6 & $-1,5$ & 0,9 \\
\hline Unconventional myosin-XVIIIa; & Q9JMH9 & Myo18a & $-0,4$ & 4,5 \\
\hline
\end{tabular}




\begin{tabular}{|c|c|c|c|c|}
\hline Protein & Uniprot & Gene name & $\begin{array}{l}\text { Fold change of } \\
\text { XPRESS ratio } \\
\left.\text { (Egfr }{ }^{\Delta K C} / \mathrm{WT}\right)\end{array}$ & $\begin{array}{c}\text { Sequence } \\
\text { coverage [\%] }\end{array}$ \\
\hline Unconventional prefoldin RPB5 interactor; & Q3TLD5 & Uri1 & $-0,2$ & 2,1 \\
\hline UPF0184 protein C9orf16 homolog; & P58686 & & $-0,5$ & 10,8 \\
\hline UPF0449 protein C19orf25 homolog; & Q9D7E4 & & $-0,7$ & 33 \\
\hline UPF0688 protein C1orf174 homolog; & Q80WR5 & & $-0,5$ & 5,2 \\
\hline UPF0690 protein C1orf52 homolog; & Q9CWU4 & & 0,0 & 17,2 \\
\hline UPF0693 protein C10orf32 homolog; & Q9CRC6 & & $-0,3$ & 16,2 \\
\hline Up-regulated during skeletal muscle growth protein 5; & Q78IK2 & Usmg5 & 0,2 & 25,9 \\
\hline Uteroglobin; & Q06318 & Scgb1a1 & 1,6 & 10,4 \\
\hline UV excision repair protein RAD23 homolog B; & P54728 & Rad23b & $-0,5$ & 13,7 \\
\hline Vacuolar ATPase assembly integral membrane protein Vma21; & Q78T54 & Vma21 & $-0,7$ & 11,9 \\
\hline Vacuolar protein sorting-associated protein VTA1 homolog; & Q9CR26 & Vta1 & 0,1 & 3,2 \\
\hline Vacuolar protein-sorting-associated protein 25 & Q9CQ80 & Vps25 & $-0,2$ & 7,8 \\
\hline Vasodilator-stimulated phosphoprotein; & P70460 & Vasp & 0,3 & 5,9 \\
\hline Vesicle transport protein USE1; & Q9CQ56 & Use1 & $-3,0$ & 4,4 \\
\hline Vesicle transport through interaction with t-SNAREs homolog 1B; & 088384 & Vti1b & $-0,4$ & 17,2 \\
\hline Vesicle-associated membrane protein 3 & P63024 & Vamp3 & $-0,2$ & 15,5 \\
\hline Vesicle-associated membrane protein 4 & 070480 & Vamp4 & 0,2 & 9,9 \\
\hline Vesicle-associated membrane protein 8; & 070404 & Vamp8 & $-0,1$ & 30,7 \\
\hline Vesicle-associated membrane protein-associated protein A; & Q9WV55 & Vapa & 0,1 & 10,4 \\
\hline Vesicle-associated membrane protein-associated protein B; & Q9QY76 & Vapb & 0,1 & 18,5 \\
\hline Vesicle-fusing ATPase; & P46460 & Nsf & 0,0 & 5,2 \\
\hline Vimentin; & P20152 & Vim & 0,4 & 30,9 \\
\hline Vinculin; & Q64727 & Vcl & $-0,1$ & 18,5 \\
\hline Voltage-dependent anion-selective channel protein 1 ; & Q60932 & Vdac1 & 0,1 & 24,3 \\
\hline Voltage-dependent anion-selective channel protein 2 ; & Q60930 & Vdac2 & $-0,2$ & 3,5 \\
\hline Vomeromodulin; & Q80XI7 & & 0,7 & 13,9 \\
\hline V-set and immunoglobulin domain-containing protein 10 ; & D3YX43 & Vsig10 & $-0,5$ & 4,3 \\
\hline V-type proton ATPase subunit E 1; & P50518 & Atp6v1e1 & 0,0 & 4 \\
\hline V-type proton ATPase subunit G 1 ; & Q9CR51 & Atp6v1g1 & $-0,3$ & 23,7 \\
\hline V-type proton ATPase subunit S1; & Q9R1Q9 & Atp6ap1 & $-0,1$ & 13,2 \\
\hline WAS protein family homolog 1 ; & Q8VDD8 & Wash1 & $-0,2$ & 4,8 \\
\hline WAS/WASL-interacting protein family member 1 ; & Q8K1I7 & Wipf1 & 0,1 & 4,7 \\
\hline WAS/WASL-interacting protein family member 2 ; & Q6PEV3 & Wipf2 & $-0,2$ & 27,5 \\
\hline WASH complex subunit FAM21; & Q6PGL7 & Fam21 & $-0,3$ & 6,9 \\
\hline WD repeat-containing protein 44 & Q6NVE8 & Wdr44 & 0,2 & 1,2 \\
\hline WD repeat-containing protein 7 & Q92019 & Wdr7 & 1,4 & 1,2 \\
\hline Wiskott-Aldrich syndrome protein family member 2 ; & Q8BH43 & Wasf2 & 0,1 & 8,7 \\
\hline WW domain-binding protein 11 & Q923D5 & Wbp11 & 0,1 & 9,2 \\
\hline Xanthine dehydrogenase/oxidase; & Q00519 & Xdh & $-0,9$ & 1 \\
\hline Y-box-binding protein 3; & Q9JKB3 & Ybx3 & $-0,3$ & 10 \\
\hline Yorkie homolog; & P46938 & Yap1 & $-0,3$ & 15,2 \\
\hline YTH domain-containing family protein 2; & Q91YT7 & Ythdf2 & 0,2 & 2,1 \\
\hline YTH domain-containing family protein 3; & Q8BYK6 & Ythdf3 & $-0,5$ & 1,9 \\
\hline Zinc finger $\mathrm{CCCH}$ domain-containing protein 15 ; & Q3TIV5 & Zc3h15 & $-0,4$ & 4 \\
\hline Zinc finger $\mathrm{CCCH}$ domain-containing protein 18 ; & Q0P678 & Zc3h18 & $-0,7$ & 11,5 \\
\hline Zinc finger $\mathrm{CCCH}$ domain-containing protein 4; & Q6ZPZ3 & Zc3h4 & $-0,1$ & 7,3 \\
\hline Zinc finger protein 185 & $\mathrm{~A} 2 \mathrm{~B} 137$ & Zfp185 & 0,2 & 8,2 \\
\hline Zinc finger protein 426 & Q8R1D1 & Znf426 & 10,0 & 2,7 \\
\hline Zinc finger protein 428 & $\mathrm{Q} 8 \mathrm{C} 1 \mathrm{M} 2$ & Znf428 & $-0,1$ & 7,2 \\
\hline Zinc finger protein 593 & Q9DB42 & Znf593 & $-0,2$ & 17,2 \\
\hline Zinc finger protein 750 & Q8BH05 & Znf750 & $-0,6$ & 5,3 \\
\hline Zinc finger protein 830 & Q8R1N0 & Znf830 & $-0,2$ & 3,6 \\
\hline Zinc finger protein ZPR1; & Q62384 & Zpr1 & 0,1 & 5,4 \\
\hline Zinc finger Ran-binding domain-containing protein 2; & Q9R020 & Zranb2 & $-0,6$ & 11,8 \\
\hline Zinc transporter ZIP10; & Q6P5F6 & Slc39a10 & $-0,4$ & 3,2 \\
\hline Zinc-binding alcohol dehydrogenase domain-containing protein 2; & Q8BGC4 & Zadh2 & 0,5 & 2,4 \\
\hline \multirow[t]{6}{*}{ Zyxin; } & Q62523 & Zyx & $-0,2$ & 12,1 \\
\hline & D3YW41 & & 0,6 & 20,2 \\
\hline & E9Q019 & & $-0,4$ & 23,6 \\
\hline & J3QMA3 & & $-0,3$ & 27,5 \\
\hline & F6TL02 & & $-0,3$ & 31,3 \\
\hline & J3QNE6 & & $-0,2$ & 32 \\
\hline
\end{tabular}


Supplementary table 4: List of all identified and quantified proteins in the quantita tive proteome comparison of wild-type and Egfr ${ }^{\Delta K C}$ epidermis at $\mathrm{P} 3$ (replicate 1).

\begin{tabular}{|c|c|c|c|c|}
\hline Protein & Uniprot & Gene name & $\begin{array}{l}\text { Fold change of } \\
\text { XPRESS ratio } \\
\left(\text { Egfr }^{\Delta K C} / \mathrm{WT}\right)\end{array}$ & $\begin{array}{c}\text { Sequence } \\
\text { coverage [\%] }\end{array}$ \\
\hline $10 \mathrm{kDa}$ heat shock protein, mitochondrial; & Q64433 & Hspe1 & 0,2 & 54,9 \\
\hline 14-3-3 protein epsilon; & P62259 & Ywhae & 0,6 & 20,8 \\
\hline 14-3-3 protein eta; & P68510 & Ywhah & 0,8 & 13 \\
\hline 14-3-3 protein gamma; & P61982 & Ywhag & 0,3 & 13 \\
\hline 14-3-3 protein sigma; & 070456 & Sfn & 0,3 & 37,5 \\
\hline 14-3-3 protein zeta/delta; & P63101 & Ywhaz & 0,8 & 41,6 \\
\hline 182 kDa tankyrase-1-binding protein; & P58871 & Tnks1bp1 & 0,0 & 28,8 \\
\hline $26 \mathrm{~S}$ protease regulatory subunit $10 \mathrm{~B}$; & P62334 & Psmc6 & 0,4 & 2,8 \\
\hline $26 \mathrm{~S}$ protease regulatory subunit $6 \mathrm{~A}$ & 088685 & Psmc3 & 0,3 & 2,5 \\
\hline $26 \mathrm{~S}$ protease regulatory subunit 7 & P46471 & Psmc2 & $-0,1$ & 5,1 \\
\hline 265 proteasome non-ATPase regulatory subun it $1 ;$ & Q3TXS7 & Psmd1 & $-0,1$ & 19,4 \\
\hline $26 \mathrm{~S}$ proteasome non-ATPase regulatory subun it 3 ; & P14685 & Psmd3 & $-0,4$ & 2,1 \\
\hline $26 \mathrm{~S}$ proteasome non-ATPase regulatory subun it 4 ; & 035226 & Psmd4 & $-0,1$ & 13,8 \\
\hline 265 proteasome non-ATPase regulatory subun it $7 ;$ & P26516 & Psmd7 & $-0,4$ & 3,7 \\
\hline $26 \mathrm{~S}$ proteasome non-ATPase regulatory subun it $9 ;$ & Q9CR00 & Psmd9 & 0,5 & 19,4 \\
\hline 28 kDa heat- and acid-stable phosphoprotein; & Q3UHX2 & Pdap1 & 0,0 & 36,5 \\
\hline $28 \mathrm{~S}$ ribosomal protein S36, mitochondrial; & Q9CQX8 & Mrps36 & 1,2 & 56,9 \\
\hline $39 S$ ribosomal protein L12, mitochondrial; & Q9DB15 & Mrpl12 & 0,2 & 17,9 \\
\hline $39 S$ ribosomal protein L49, mitochondrial; & Q9CQ40 & Mrpl49 & 0,2 & 7,2 \\
\hline 3-hydroxyisobutyrate dehydrogenase, mitochondrial; & Q99L13 & Hibadh & 0,3 & 3,9 \\
\hline 3-ketoacyl-CoA thiolase, mitochondrial; & Q8BWT1 & Acaa2 & $-0,5$ & 29,2 \\
\hline 40 S ribosomal protein S10; & P63325 & Rps10 & 0,4 & 28,5 \\
\hline $40 S$ ribosomal protein $\mathrm{S} 11$ & P62281 & Rps11 & 0,4 & 4,4 \\
\hline $40 \mathrm{~S}$ ribosomal protein $\mathrm{S} 12$ & Q6ZWZ6 & Rps12 & 0,0 & 45,5 \\
\hline $40 \mathrm{~S}$ ribosomal protein $\mathrm{S} 13$ & P62301 & Rps13 & $-0,3$ & 25,8 \\
\hline 40 S ribosomal protein $\mathrm{S} 14$ & P62264 & Rps14 & 0,3 & 8,6 \\
\hline $40 \mathrm{~S}$ ribosomal protein S15; & P62843 & Rps15 & 0,4 & 10,2 \\
\hline 40 S ribosomal protein S16; & P14131 & Rps16 & $-0,1$ & 12,3 \\
\hline 40 S ribosomal protein S17; & P63276 & Rps17 & 0,8 & 23,7 \\
\hline 40 S ribosomal protein S18; & P62270 & Rps18 & 0,6 & 32,9 \\
\hline $40 S$ ribosomal protein S19; & Q9CZX8 & Rps19 & 0,0 & 34,1 \\
\hline $40 S$ ribosomal protein S2; & P25444 & Rps2 & 0,4 & 15,4 \\
\hline 40 S ribosomal protein S20; & P60867 & Rps20 & 0,3 & 25,2 \\
\hline 40 S ribosomal protein $\mathrm{S} 21$ & Q9CQR2 & Rps21 & 0,1 & 39,8 \\
\hline 40 S ribosomal protein S25; & P62852 & Rps25 & 0,2 & 22,4 \\
\hline 40 S ribosomal protein S27; & Q6ZWU9 & Rps27 & 0,3 & 22,6 \\
\hline $40 S$ ribosomal protein S28; & P62858 & Rps28 & 0,2 & 37,5 \\
\hline 40 S ribosomal protein S3; & P62908 & Rps3 & 1,5 & 9,1 \\
\hline $40 \mathrm{~S}$ ribosomal protein $\mathrm{S} 30$ & P62862 & Fau & 0,9 & 16,9 \\
\hline $40 \mathrm{~S}$ ribosomal protein $\mathrm{S} 3 \mathrm{a}$ & P97351 & Rps3a & 0,3 & 27,3 \\
\hline $40 S$ ribosomal protein $\mathrm{S} 4, \mathrm{X}$ isoform; & P62702 & Rps4x & 0,3 & 7,3 \\
\hline $40 \mathrm{~S}$ ribosomal protein $\mathrm{S} 5$; & D3YYM6 & Rps5 & 0,2 & 25,8 \\
\hline $40 S$ ribosomal protein S6; & P62754 & Rps6 & 0,4 & 4,8 \\
\hline $40 S$ ribosomal protein S8; & P62242 & Rps8 & 0,0 & 14,9 \\
\hline $40 S$ ribosomal protein SA; & P14206 & Rpsa & 0,7 & 34,2 \\
\hline 4F2 cell-surface antigen heavy chain; & P10852 & Slc3a2 & 0,2 & 5,1 \\
\hline 5'(3')-deoxyribonucleotidase, cytosolic type; & Q9JM14 & $\mathrm{Nt5c}$ & $-0,6$ & 6,7 \\
\hline 5-hydroxyisourate hydrolase; & Q9CRB3 & Urah & 0,1 & 37,3 \\
\hline $60 \mathrm{kDa}$ heat shock protein, mitochondrial; & P63038 & Hspd1 & 0,6 & 17,5 \\
\hline 605 acidic ribosomal protein $\mathrm{P} 0$ & P14869 & Rplp0 & $-0,3$ & 20,5 \\
\hline 605 acidic ribosomal protein $\mathrm{P} 1$ & P47955 & Rplp1 & 0,4 & 14 \\
\hline $60 S$ acidic ribosomal protein $\mathrm{P} 2$; & P99027 & Rplp2 & 0,1 & 67 \\
\hline $60 S$ ribosomal protein $\mathrm{L} 11$ & Q9CXW4 & Rpl11 & $-0,5$ & 13,2 \\
\hline $60 S$ ribosomal protein $\mathrm{L} 12$ & P35979 & Rpl12 & 0,1 & 30,3 \\
\hline $60 \mathrm{~S}$ ribosomal protein L13; & P47963 & Rpl13 & 0,5 & 19,9 \\
\hline $60 S$ ribosomal protein L14; & Q9CR57 & Rpl14 & 0,0 & 10,1 \\
\hline 60 S ribosomal protein $\mathrm{L} 17$ & Q9CPR4 & Rpl17 & 0,4 & 31,5 \\
\hline 60S ribosomal protein L18; & P35980 & Rpl18 & 0,1 & 22,3 \\
\hline $60 S$ ribosomal protein L19; & P84099 & Rpl19 & 0,4 & 13,4 \\
\hline $60 S$ ribosomal protein L21; & 009167 & Rpl21 & $-0,1$ & 13,8 \\
\hline $60 S$ ribosomal protein L22; & P67984 & Rpl22 & 0,7 & 18,8 \\
\hline $60 S$ ribosomal protein L22-like 1 ; & Q9D7S7 & Rpl22|1 & 0,6 & 9,8 \\
\hline $60 S$ ribosomal protein L23; & P62830 & Rpl23 & 0,5 & 33,3 \\
\hline $60 S$ ribosomal protein L23a; & P62751 & Rpl23a & 0,9 & 21,7 \\
\hline $60 S$ ribosomal protein L24; & Q8BP67 & Rpl24 & 0,9 & 16,5 \\
\hline 60S ribosomal protein L26; & P61255 & Rpl26 & 0,5 & 8,7 \\
\hline 60 S ribosomal protein L27; & P61358 & Rpl27 & 0,8 & 12,5 \\
\hline $60 S$ ribosomal protein $\mathrm{L} 27 \mathrm{a}$ & P14115 & Rpl27a & $-0,1$ & 6,8 \\
\hline
\end{tabular}




\begin{tabular}{|c|c|c|c|c|}
\hline Protein & Uniprot & Gene name & $\begin{array}{l}\text { Fold change of } \\
\text { XPRESS ratio } \\
\left(\text { Egfr }^{\triangle K C} / \mathrm{WT}\right)\end{array}$ & $\begin{array}{c}\text { Sequence } \\
\text { coverage [\%] }\end{array}$ \\
\hline $60 S$ ribosomal protein L28; & P41105 & Rpl28 & 0,3 & 16,1 \\
\hline $60 \mathrm{~S}$ ribosomal protein L31; & P62900 & Rpl31 & 0,9 & 24,8 \\
\hline $60 S$ ribosomal protein $\mathrm{L} 35$ & Q6ZWV7 & Rpl35 & 0,7 & 8,1 \\
\hline $60 S$ ribosomal protein L38; & Q9JJI8 & Rpl38 & 0,3 & 24,3 \\
\hline $60 S$ ribosomal protein L39; & P62892 & Rpl39 & 1,0 & 19,6 \\
\hline $60 S$ ribosomal protein $\mathrm{L} 4$ & Q9D8E6 & Rpl4 & $-0,1$ & 19,8 \\
\hline $60 \mathrm{~S}$ ribosomal protein $\mathrm{L} 5$; & P47962 & Rpl5 & $-0,3$ & 7,1 \\
\hline $60 S$ ribosomal protein L6; & P47911 & Rpl6 & 0,3 & 6,4 \\
\hline $60 \mathrm{~S}$ ribosomal protein $\mathrm{L7}$; & P14148 & Rpl7 & $-0,3$ & 10,7 \\
\hline $60 \mathrm{~S}$ ribosomal protein $\mathrm{L} 7 \mathrm{a}$; & P12970 & Rpl7a & 0,5 & 4,1 \\
\hline $60 S$ ribosomal protein L8; & P62918 & Rpl8 & 1,7 & 10,5 \\
\hline 6-phosphogluconolactonase; & Q9CQ60 & Pgls & $-0,2$ & 25,6 \\
\hline 78 kDa glucose-regulated protein; & P20029 & Hspa5 & 0,5 & 34,7 \\
\hline Abl interactor 1 & Q8CBW3 & Abi1 & 0,1 & 9,2 \\
\hline Acid ceramidase; & Q9WV54 & Asah1 & $-0,7$ & 16 \\
\hline Acidic leucine-rich nuclear phosphoprotein 32 family member $A$; & 035381 & Anp32a & $-0,6$ & 24,2 \\
\hline Acidic leucine-rich nuclear phosphoprotein 32 family member B; & Q9EST5 & Anp32b & 0,0 & 16,9 \\
\hline Acidic leucine-rich nuclear phosphoprotein 32 family member E; & E9PZF5 & Anp32e & $-0,1$ & 20,6 \\
\hline Aconitate hydratase, mitochondrial; & Q99KI0 & Aco2 & $-1,1$ & 3,5 \\
\hline Actin filament-associated protein 1-like 2; & Q5DTU0 & Afap1/2 & 0,3 & 2,4 \\
\hline Actin, aortic smooth muscle; & P62737 & Acta2 & 0,4 & 34,3 \\
\hline Actin-binding LIM protein 1; & Q8K4G5 & Ablim1 & $-0,2$ & 11,2 \\
\hline Actin-related protein $2 / 3$ complex subun it $1 \mathrm{~B}$; & Q9WV32 & Arpc1b & $-0,2$ & 17,1 \\
\hline Actin-related protein $2 / 3$ complex subunit 3 ; & Q9JM76 & Arpc3 & 0,0 & 24,7 \\
\hline Actin-related protein $2 / 3$ complex subunit 5 & Q9CPW4 & Arpc5 & 0,2 & 20,5 \\
\hline Actin-related protein $2 / 3$ complex subun it 5 -like protein; & Q9D898 & Arpc5I & 0,0 & 16,3 \\
\hline Activated RNA polymerase II transcriptional coactivator $\mathrm{p} 15$ & P11031 & Sub1 & $-0,5$ & 8,7 \\
\hline Acyl carrier protein, mitochondrial; & Q9CR21 & Ndufab1 & $-0,3$ & 7,1 \\
\hline Acyl-CoA-binding protein; & P31786 & Dbi & $-0,8$ & 39,1 \\
\hline Acylphosphatase-1; & P56376 & Acyp1 & 0,0 & 21,2 \\
\hline Adenylate kinase 2, mitochondrial; & Q9WTP6 & Ak2 & 0,6 & 15,3 \\
\hline Adenylate kinase isoenzyme 1 ; & Q9R0Y5 & Ak1 & 0,6 & 12,9 \\
\hline ADP/ATP translocase 2 & P51881 & Slc25a5 & $-0,4$ & 20,1 \\
\hline ADP-ribosylation factor 3 ; & P61205 & Arf3 & $-0,2$ & 5,6 \\
\hline ADP-ribosylation factor GTPase-activating protein 2; & Q99K28 & Arfgap2 & 0,1 & 1,9 \\
\hline ADP-sugar pyrophosphatase; & Q9JKX6 & Nudt5 & 0,4 & 18,8 \\
\hline Afadin; & Q9QZQ1 & Mllt4 & $-0,5$ & 2,7 \\
\hline A-kinase anchor protein 12 & Q9WTQ5 & Akap12 & 0,5 & 2,7 \\
\hline Aldehyde dehydrogenase; & E9Q3E1 & Aldh3b2 & 0,3 & 9,2 \\
\hline Aldehyde oxidase 4; & Q3TYQ9 & Aox4 & $-0,4$ & 3,4 \\
\hline $\begin{array}{l}\text { Allergen dl chain C2A; Androgen binding protein beta; Androgen-binding } \\
\text { protein; Protein Scgb2b27; }\end{array}$ & Q8R1E9 & Scgb2b27 & $-0,3$ & 11,6 \\
\hline Alpha-1-antitrypsin 1-1; & P07758 & Serpina1a & 1,2 & 13,8 \\
\hline Alpha-1-antitrypsin 1-2; & P22599 & Serpina1b & 1,3 & 13,8 \\
\hline Alpha-1-antitrypsin 1-4; & Q00897 & Serpina1d & 2,0 & 13,8 \\
\hline Alpha-2-HS-glycoprotein; & P29699 & Ahsg & 1,5 & 23,2 \\
\hline Alpha-2-macroglobulin receptor-associated protein; & P55302 & Lrpap1 & 0,3 & 17,2 \\
\hline Alpha-2-macroglobulin; & Q61838 & $\mathrm{A} 2 \mathrm{~m}$ & 0,4 & 0,7 \\
\hline Alpha-actinin-4; & P57780 & Actn4 & 0,5 & 17,2 \\
\hline Alpha-enolase; & P17182 & Eno1 & 0,0 & 22,8 \\
\hline Alpha-fetoprotein; & P02772 & Afp & 1,0 & 45 \\
\hline Alpha-synuclein; & 055042 & Snca & 1,6 & 11,4 \\
\hline Aminoacyl tRNA synthase complex-interacting multifunctional protein 1; & P31230 & Aimp1 & $-0,5$ & 11,9 \\
\hline AN1-type zinc finger protein 6; & Q9DCH6 & Zfand6 & $-0,4$ & 22 \\
\hline Anamorsin; & Q8WTY4 & Ciapin1 & 0,0 & 14,6 \\
\hline $\begin{array}{l}\text { Androgen binding protein gamma; MCG17480; Protein Scgb2b26; Salivary } \\
\text { androgen-binding protein gamma subunit; }\end{array}$ & Q8JZX1 & Scgb2b26 & $-0,6$ & 23,2 \\
\hline $\begin{array}{l}\text { Androgen-binding protein; Androgen-binding protein eta; Lacrimal androgen- } \\
\text { binding protein eta; Protein Scgb1b2; }\end{array}$ & 035176 & Scgb1b2 & $-0,5$ & 21,5 \\
\hline Androgen-binding protein; Protein Scgb1b29; Protein Scgb1b7; & D2XZ31 & Abpa29_a7 & $-0,6$ & 11,8 \\
\hline Ankyrin-3; & G5E8K5 & Ank3 & 1,2 & 3 \\
\hline Annexin A1; & P10107 & Anxa1 & 0,2 & 22,5 \\
\hline Annexin A2; & P07356 & Anxa2 & 0,5 & 33,3 \\
\hline Annexin A5; & P48036 & Anxa5 & 0,0 & 19,4 \\
\hline Annexin A8; & 035640 & Anxa8 & 0,1 & 18,6 \\
\hline Antithrombin-III; & P32261 & Serpinc1 & 1,3 & 2,4 \\
\hline AP2-associated protein kinase 1 ; & Q3UHJ0 & Aak1 & 0,3 & 3,1 \\
\hline Apolipoprotein A-I; & Q00623 & Apoa1 & $-0,2$ & 35,2 \\
\hline Apolipoprotein A-II; & P09813 & Apoa2 & 1,1 & 9,8 \\
\hline Apolipoprotein A-IV; & P06728 & Apoa4 & 0,1 & 46,1 \\
\hline
\end{tabular}




Protein

\section{Protein}

Uniprot Gene name

Fold change of

Apolipoprotein C-I;

Apolipoprotein E;

Apoptosis-associated speck-like protein containing a CARD;

Apoptotic chromatin condensation inducer in the nucleus;

Arachidonate 12-lipoxygenase, 12R-type;

Arachidonate 12-lipoxygenase, 12S-type;

Arf-GAP domain and FG repeat-containing protein 1;

Arfip1 protein; Protein Arfip1;

Arginine-rich, mutated in early stage tumors, isoform CRA_b; Mesencephalic astrocyte-derived neurotrophic factor;

Aspartate aminotransferase, mitochondrial;

Aspartyl/asparaginyl beta-hydroxylase;

Ataxin-2-like protein;

ATP synthase protein 8;

ATP synthase subunit alpha, mitochond rial;

ATP synthase subunit beta, mitochond rial;

ATP synthase subunit $d$, mitochondrial;

ATP synthase subunit delta, mitochond rial;

ATP synthase subunit e, mitochondrial;

ATP synthase subunit epsilon, mitochondrial;

ATP synthase subunit $f$, mitochondrial;

ATP synthase subunit $g$, mitochondrial;

ATP synthase subunit $O$, mitochondrial;

ATP synthase-coupling factor 6 , mitochondrial;

ATP-binding cassette sub-family F member 1 ;

ATP-dependent (S)-NAD(P)H-hydrate dehydratase;

ATP-dependent RNA helicase DDX39A;

ATP-dependent RNA helicase DDX3X;

AT-rich interactive domain-containing protein $1 A$;

Aurora kinase B;

BAG family molecular chaperone regulator 3 ;

Band 4.1-like protein 2;

Band 4.1-like protein 3;

Barrier-to-autointegration factor;

Basal cell adhesion molecule;

Basigin;

B-cell CLL/lymphoma 7 protein family member C;

B-cell linker protein;

B-cell receptor-associated protein 31;

$\mathrm{Bcl}$-2-like protein 13;

Beta-actin-like protein 2;

Beta-catenin-interacting protein 1;

Beta-galactosidase;

Beta-globin; Protein Hbb-bs; Protein Hbb-bt;

Beta-hexosaminidase subunit beta;

Bicaudal D-related protein 2;

Bifunctional glutamate/proline--tRNA ligase;

Biorientation of chromosomes in cell division protein 1;

Bis(5'-adenosyl)-triphosphatase;

Bleomycin hydrolase;

BPI fold-containing family A member 2;

BPI fold-containing family $\mathrm{C}$ protein;

Brain acid soluble protein 1 ;

Brain-specific angiogenesis inhibitor 1-associated protein 2;

BUB3-interacting and GLEBS motif-containing protein ZNF207;

Cadherin-1;

Cadherin-13;

Calcineurin subunit B type 1;

Calcium-activated chloride channel regulator 2; Chloride channel calcium

activated 2; Endothelial chloride channel; MCG120735;

Calcium-binding mitochondrial carrier protein SCaMC-1;

Calcium-binding protein 39;

Calcium-regulated heat stable protein 1 ;

Calmodulin;

Calmodulin;

Calmodulin-4;

Calnexin;

Calpain small subunit 2;

Calpain-1 catalytic subunit;

Calpastatin;

Calponin-2;

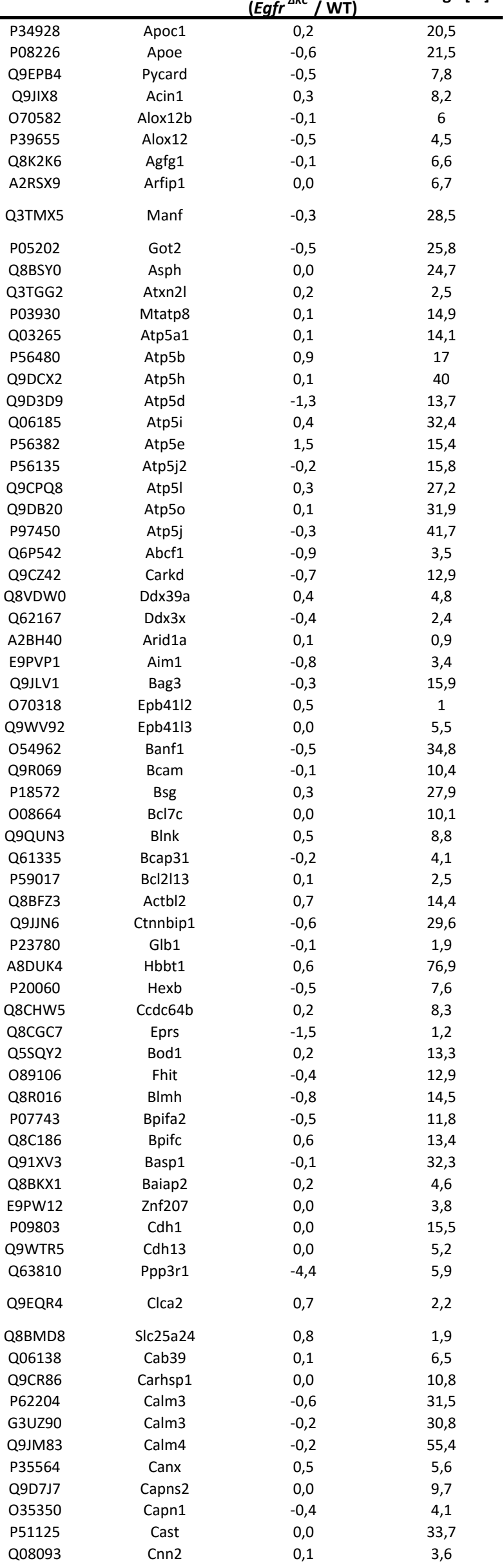




\begin{tabular}{|c|c|c|c|c|}
\hline Protein & Uniprot & Gene name & $\begin{array}{l}\text { Fold change of } \\
\text { XPRESS ratio } \\
\left(\text { Egfr }^{\Delta K C} / \mathrm{WT}\right)\end{array}$ & $\begin{array}{c}\text { Sequence } \\
\text { coverage [\%] }\end{array}$ \\
\hline Calreticulin; & P14211 & Calr & 0,2 & 25 \\
\hline Calumenin; & 035887 & Calu & 0,0 & 18,4 \\
\hline cAMP-regulated phosphoprotein 19 & P56212 & Arpp19 & 0,1 & 15,5 \\
\hline CAP-Gly domain-containing linker protein 1 ; & Q922J3 & Clip1 & 0,3 & 16,1 \\
\hline $\begin{array}{l}\text { Capping protein (Actin filament), gelsolin-like; Capping protein (Actin } \\
\text { filament), gelsolin-like, isoform CRA_a; Macrophage-capping protein; }\end{array}$ & Q99LB4 & Capg & $-0,1$ & 14 \\
\hline Caprin-1; & Q60865 & Caprin1 & 0,2 & 12 \\
\hline Carbonic anhydrase 3; & P16015 & Ca3 & $-0,3$ & 4,6 \\
\hline Carboxypeptidase A4; & Q6P8K8 & Cpa4 & $-0,9$ & 5 \\
\hline Carboxypeptidase E; & Q00493 & Cpe & 0,5 & 5,3 \\
\hline Carboxypeptidase M; & Q80V42 & $\mathrm{Cpm}$ & $-0,7$ & 9 \\
\hline Caspase-14; & 089094 & Casp14 & $-0,2$ & 37,7 \\
\hline Catalase; & P24270 & Cat & $-0,9$ & 30,2 \\
\hline Catenin alpha-1; & P26231 & Ctnna1 & 0,8 & 3,4 \\
\hline Cathelin-related antimicrobial peptide; & P51437 & Camp & 2,2 & 10,4 \\
\hline Cathepsin B; & P10605 & Ctsb & $-0,8$ & 12,1 \\
\hline Cathepsin D; & P18242 & Ctsd & $-0,4$ & 18,8 \\
\hline Cathepsin L1; & P06797 & Ctsl & $-0,5$ & 20,4 \\
\hline Cation-dependent mannose-6-phosphate receptor; & P24668 & M6pr & $-1,0$ & 14,4 \\
\hline Caveolin-1; & P49817 & Cav1 & 0,4 & 24,7 \\
\hline CD109 antigen; & Q8R422 & Cd109 & 0,2 & 3,4 \\
\hline CD166 antigen; & Q61490 & Alcam & $-0,3$ & 7,2 \\
\hline CD2-associated protein; & Q9JLQ0 & Cd2ap & 0,2 & 11,6 \\
\hline CD44 antigen; & P15379 & $\mathrm{Cd} 44$ & $-1,7$ & 6,3 \\
\hline CD63 antigen; & P41731 & Cd63 & $-1,0$ & 4,6 \\
\hline CD9 antigen; & P40240 & Cd9 & $-0,6$ & 3,1 \\
\hline Cdc42 effector protein 4 & Q9JM96 & Cdc42ep4 & $-0,7$ & 10 \\
\hline Cdc42 effector protein 5 & Q9Z0X0 & Cdc42ep5 & $-0,6$ & 16,5 \\
\hline CDGSH iron-sulfur domain-containing protein 1 ; & Q91Wso & Cisd1 & $-0,2$ & 13,9 \\
\hline Cell division control protein 42 homolog; & P60766 & $\mathrm{Cdc} 42$ & 0,0 & 8,9 \\
\hline Cellular nucleic acid-binding protein; & D3YYF6 & Cnbp & 0,0 & 29,5 \\
\hline Cellular nucleic acid-binding protein; & P53996 & Cnbp & $-0,6$ & 22,5 \\
\hline Charged multivesicular body protein $1 \mathrm{a}$; & Q921W0 & Chmp1a & $-0,2$ & 12,8 \\
\hline Charged multivesicular body protein $2 \mathrm{~b}$; & Q8BJF9 & Chmp2b & 0,4 & 4,7 \\
\hline Charged multivesicular body protein $4 \mathrm{~b}$; & Q9D8B3 & Chmp4b & 0,2 & 25,9 \\
\hline Charged multivesicular body protein 5; & Q9D7S9 & Chmp5 & 0,2 & 20,1 \\
\hline Chitinase-3-like protein 1 & Q61362 & Chi3l1 & 0,7 & 12,7 \\
\hline Chitotriosidase-1; & Q9D7Q1 & Chit1 & 0,1 & 6 \\
\hline Chloride intracellular channel protein 3; & Q9D7P7 & Clic3 & 0,3 & 7,7 \\
\hline Chromobox protein homolog 3 & P23198 & $\mathrm{Cb} \times 3$ & 0,1 & 12,6 \\
\hline Cingulin; & P59242 & Cgn & 0,2 & 3,5 \\
\hline Citrate synthase, mitochondrial; & Q9CZU6 & Cs & 0,2 & 8,8 \\
\hline Clathrin interactor 1; MCG22297, isoform CRA_b; & Q5SUH6 & Clint1 & $-0,1$ & 11,6 \\
\hline Clathrin light chain $A$; & B1AWEO & Clta & 0,3 & 28,2 \\
\hline Clathrin light chain B; & Q6IRU5 & Cltb & $-0,2$ & 26,2 \\
\hline Claudin-1; & 088551 & Cldn1 & $-0,1$ & 7,1 \\
\hline Cleavage stimulation factor subunit 2 ; & Q8BIQ5 & Cstf2 & $-3,4$ & 6,1 \\
\hline Clusterin; & Q06890 & $\mathrm{Clu}$ & $-0,8$ & 26,1 \\
\hline C-Myc-binding protein; & Q9EQS3 & Mycbp & $-1,2$ & 45,6 \\
\hline Cob(I)yrinic acid a,c-diamide adenosyltransferase, mitochondrial; & Q9D273 & Mmab & 0,1 & 5,9 \\
\hline Cofilin-1; & P18760 & Cfl1 & 0,1 & 39,2 \\
\hline Coiled-coil domain-containing protein 12 & Q8R344 & Ccdc12 & 0,0 & 14,5 \\
\hline Coiled-coil domain-containing protein 124 & Q9D8X2 & Ccdc124 & 0,5 & 6 \\
\hline Coiled-coil domain-containing protein 43 & Q9CR29 & Ccdc43 & 0,3 & 5 \\
\hline Coiled-coil domain-containing protein 50 ; & Q810U5 & Ccdc50 & 0,4 & 14,8 \\
\hline Coiled-coil domain-containing protein 9; & Q8VC31 & Ccdc9 & 0,1 & 4,2 \\
\hline Coiled-coil-helix-coiled-coil-helix domain-containing protein 3, mitochondrial; & Q9CRB9 & Chchd3 & 0,0 & 15,9 \\
\hline Collagen alpha-1(I) chain; & P11087 & Col1a1 & 0,5 & 6,4 \\
\hline Collagen alpha-1(III) chain; & P08121 & Col3a1 & 1,2 & 0,8 \\
\hline Collagen alpha-1(XVI) chain; & Q8BLX7 & Col16a1 & 0,5 & 1,6 \\
\hline Collagen alpha-1(XVII) chain; & Q07563 & Col17a1 & 0,7 & 6,6 \\
\hline Collagen alpha-2(I) chain; & Q01149 & Col1a2 & 0,5 & 9,3 \\
\hline \multicolumn{5}{|l|}{ Complement component $1 \mathrm{Q}$ subcomponent-binding protein, mitochondrial; } \\
\hline $\begin{array}{l}\text { Complement component 1, q subcomponent binding protein; Complement } \\
\text { component 1, q subcomponent binding protein, isoform CRA_b; p32-RACK; }\end{array}$ & Q8R5L1 & C1qbp & 0,0 & 11,8 \\
\hline Complexin-2; & P84086 & Cplx2 & 0,9 & 11,2 \\
\hline COP9 signalosome complex subunit 8 & Q8VBV7 & Cops8 & 0,0 & 23,4 \\
\hline Copine-3; & Q8BT60 & Cpne3 & $-0,1$ & 1,7 \\
\hline Copper transport protein ATOX1; & 008997 & Atox1 & 0,0 & 57,4 \\
\hline
\end{tabular}




\begin{tabular}{|c|c|c|c|c|}
\hline Protein & Uniprot & Gene name & $\begin{array}{l}\text { Fold change of } \\
\text { XPRESS ratio } \\
\left(\text { Egfr }^{\triangle K C} / \mathrm{WT}\right)\end{array}$ & $\begin{array}{c}\text { Sequence } \\
\text { coverage [\%] }\end{array}$ \\
\hline Corneodesmosin; & Q7TPC1 & Cdsn & $-0,2$ & 24,2 \\
\hline Cornifelin; & Q6PCW6 & Cnfn & 0,1 & 19,8 \\
\hline Cornifin-A; & Q62266 & Sprr1a & $-1,6$ & 37,5 \\
\hline Cornifin-B; & Q62267 & Sprr1b & $-1,7$ & 9,8 \\
\hline Coronin-1A; & 089053 & Coro1a & 1,6 & 3,5 \\
\hline Coxsackievirus and adenovirus receptor homolog; & P97792 & Cxadr & 0,1 & 9,9 \\
\hline Craniofacial development protein 1 & 088271 & Cfdp1 & 0,3 & 19,3 \\
\hline Creatine kinase U-type, mitochondrial; & P30275 & Ckmt1 & 0,0 & 18,9 \\
\hline Crk-like protein; & P47941 & Crkl & $-5,0$ & 8,3 \\
\hline CTTNBP2 N-terminal-like protein; & Q99LJ0 & Cttnbp2nl & $-0,1$ & 4,1 \\
\hline Cyclin-dependent kinase inhibitor 1B; & P46414 & Cdkn1b & $-0,1$ & 17,3 \\
\hline Cystatin E/M; Cystatin M/E; Cystatin-B; & Q9D1B1 & Cst6 & $-0,6$ & 40,3 \\
\hline Cystatin-A; & P56567 & Csta & 1,3 & 37,1 \\
\hline Cystatin-B; & Q62426 & Cstb & $-0,5$ & 32,7 \\
\hline Cystatin-C; & P21460 & Cst3 & $-1,4$ & 10,8 \\
\hline Cysteine and glycine-rich protein 1 ; & P97315 & Csrp1 & 0,0 & 16,6 \\
\hline Cysteine-rich protein 1 & P63254 & Crip1 & 0,0 & 9,1 \\
\hline Cytochrome b5 type B; & Q9CQX2 & Cyb5b & 0,0 & 10,3 \\
\hline Cytochrome b5; & P56395 & Cyb5a & 0,2 & 56,1 \\
\hline Cytochrome b-c1 complex subunit 1, mitochondrial; & Q9CZ13 & Uqcrc1 & $-0,4$ & 5 \\
\hline Cytochrome b-c1 complex subunit 2, mitochondrial; & Q9DB77 & Uqcrc2 & $-1,0$ & 9,1 \\
\hline Cytochrome b-c1 complex subunit 6, mitochondrial; & P99028 & Uqcrh & 0,0 & 44,9 \\
\hline Cytochrome b-c1 complex subunit 7; & Q9D855 & Uqcrb & $-0,1$ & 32,4 \\
\hline Cytochrome b-c1 complex subunit 8; & Q9CQ69 & Uqcrq & 0,4 & 34,1 \\
\hline Cytochrome b-c1 complex subunit 9; & Q8R1I1 & Uqcr10 & $-0,2$ & 37,5 \\
\hline Cytochrome b-c1 complex subunit Rieske, mitochondrial; & Q9CR68 & Uqcrfs1 & 0,3 & 14,2 \\
\hline Cytochrome c oxidase assembly factor 3 homolog, mitochondrial; & Q9D2R6 & Coa3 & 0,6 & 18,5 \\
\hline Cytochrome c oxidase assembly factor 4 homolog, mitochondrial; & Q8BT51 & COA4 & $-0,4$ & 16,1 \\
\hline Cytochrome c oxidase assembly factor 6 homolog; & Q8BGD8 & Coa6 & 0,1 & 16,5 \\
\hline Cytochrome c oxidase subunit 4 isoform 1 , mitochondrial; & P19783 & Cox4i1 & 0,7 & 33,1 \\
\hline Cytochrome c oxidase subunit $5 \mathrm{~A}$, mitochond rial; & P12787 & Cox5a & 0,0 & 27,4 \\
\hline Cytochrome c oxidase subunit 5B, mitochondrial; & P19536 & Cox5b & 0,0 & 24,2 \\
\hline Cytochrome c oxidase subunit $6 \mathrm{~B} 1$ & P56391 & Cox6b1 & $-0,5$ & 36 \\
\hline Cytochrome c oxidase subunit $6 \mathrm{C}$; & Q9CPQ1 & Cox6c & 0,0 & 18,4 \\
\hline Cytochrome c oxidase subunit 7A2, mitochondrial; & P48771 & Cox7a2 & $-0,3$ & 27,7 \\
\hline Cytochrome c oxidase subunit 7C, mitochond rial; & P17665 & $\operatorname{Cox} 7 \mathrm{c}$ & $-0,4$ & 14,3 \\
\hline Cytochrome c, somatic; & P62897 & Cycs & 0,0 & 38,1 \\
\hline Cytochrome c1, heme protein, mitochond rial; & Q9D0M3 & Cyc1 & $-0,3$ & 10,8 \\
\hline Cytoplasmic dynein 1 intermediate chain 2; & 088487 & Dync1i2 & $-1,2$ & 3,4 \\
\hline Cytoplasmic dynein 1 light intermediate chain 2; & Q6PDLO & Dync1li2 & 0,4 & 3,5 \\
\hline Cytoskeleton-associated protein 4 & Q8BMK4 & Ckap4 & $-0,1$ & 27,3 \\
\hline Cytosol aminopeptidase; & Q9CPY7 & Lap3 & 0,1 & 5,4 \\
\hline Cytosolic non-specific dipeptidase; & Q9D1A2 & Cndp2 & $-0,2$ & 6,7 \\
\hline Cytospin-B; & Q5SXY1 & Specc1 & 0,6 & 1,3 \\
\hline D-3-phosphoglycerate dehydrogenase; & Q61753 & Phgdh & 0,0 & 15,6 \\
\hline D-dopachrome decarboxylase; & 035215 & Ddt & $-0,4$ & 32,2 \\
\hline DDRGK domain-containing protein 1 ; & Q80WW9 & Ddrgk1 & 0,2 & 4,8 \\
\hline Death-associated protein-like 1; & Q9D757 & Dapl1 & $-0,3$ & 7,5 \\
\hline Decorin; & P28654 & Den & 0,8 & 9 \\
\hline Delta-aminolevulinic acid dehydratase; & P10518 & Alad & $-1,3$ & 5,8 \\
\hline Density-regulated protein; & Q9CQJ6 & Denr & $-0,5$ & 8,7 \\
\hline Deoxyhypusine hydroxylase; & Q99LN9 & Dohh & $-0,8$ & 6,9 \\
\hline Deoxyribonuclease-2-alpha; & P56542 & Dnase2 & $-0,3$ & 11,7 \\
\hline Deoxyuridine triphosphatase, isoform CRA_b; Dutp protein; Protein Dut; & Q8VCG1 & Dut & 0,3 & 9,3 \\
\hline Dermokine; & E9Q2P1 & Dmkn & $-0,3$ & 35 \\
\hline Desmocollin 3, isoform CRA_b; Desmocollin-3; & G5E8S6 & Dsc3 & $-0,1$ & 19,4 \\
\hline Desmocollin-1; & P55849 & Dsc1 & $-0,2$ & 28,7 \\
\hline Desmoglein-1-alpha; & Q61495 & Dsg1a & 0,2 & 18 \\
\hline Desmoglein-1-beta; & Q7TSF1 & Dsg1b & $-0,2$ & 16,8 \\
\hline Desmoplakin; & E9Q557 & Dsp & 0,0 & 6,9 \\
\hline Dickkopf-like protein 1; & Q9QZL9 & Dkkl1 & $-1,0$ & 6,5 \\
\hline $\begin{array}{l}\text { Dihydrolipoyllysine-residue succinyltransferase component of 2-oxoglutarate } \\
\text { dehydrogenase complex, mitochondrial; }\end{array}$ & Q9D2G2 & Dlst & 0,0 & 6,2 \\
\hline Dihydropyrimidinase-related protein 2 & 008553 & Dpysl2 & 0,4 & 8,2 \\
\hline Dimethylaniline monooxygenase [N-oxide-forming] 2; & Q8K213 & Fmo2 & $-0,6$ & 6 \\
\hline Dipeptidyl peptidase 1 & P97821 & Ctsc & $-0,4$ & 7,9 \\
\hline Disabled homolog 2; & P98078 & Dab2 & 0,5 & 6,1 \\
\hline Disks large-associated protein 4; & B1AZP2 & Dlgap4 & 0,0 & 10,7 \\
\hline DNA fragmentation factor subu nit alpha; & 054786 & Dffa & 2,8 & 7,3 \\
\hline DNA replication licensing factor MCM6; & P97311 & Mcm6 & $-7,0$ & 1,2 \\
\hline
\end{tabular}




\begin{tabular}{|c|c|c|c|c|}
\hline Protein & Uniprot & Gene name & $\begin{array}{l}\text { Fold change of } \\
\text { XPRESS ratio } \\
\left(\text { Egfr }^{\triangle K C} / \mathrm{WT}\right)\end{array}$ & $\begin{array}{c}\text { Sequence } \\
\text { coverage [\%] }\end{array}$ \\
\hline DNA-directed RNA polymerase III subunit RPC7; & Q6NXY9 & Polr3g & 0,2 & 5,4 \\
\hline DnaJ homolog subfamily $\mathrm{C}$ member 5 ; & P60904 & Dnajc5 & 1,3 & 12,1 \\
\hline DnaJ homolog subfamily C member 8 & Q6NZB0 & Dnajc8 & 0,3 & 11,4 \\
\hline Drebrin-like protein; & Q62418 & Dbnl & 0,0 & 29,8 \\
\hline Dynactin subunit 2 ; & Q99KJ8 & Dctn2 & 0,1 & 41 \\
\hline Dynein light chain 1, cytoplasmic; & P63168 & Dynll1 & $-2,4$ & 12,4 \\
\hline Dynein light chain roadblock-type 1 & P62627 & Dynlrb1 & 0,3 & 51 \\
\hline Dystroglycan; & Q62165 & Dag1 & $-0,3$ & 2,1 \\
\hline E3 ubiquitin-protein ligase RNF126; & Q91YL2 & Rnf126 & $-0,3$ & 7,7 \\
\hline Early endosome antigen 1 & Q8BL66 & Eea1 & 0,0 & 19,8 \\
\hline EF-hand domain-containing protein D2; Efhd2 protein; & Q8C845 & Efhd2 & 0,1 & 28,8 \\
\hline Electron transfer flavoprotein subunit alpha, mitochondrial; & Q99LC5 & Etfa & 0,0 & 21,3 \\
\hline Electron transfer flavoprotein subunit beta; & Q9DCW4 & Etfb & 0,0 & 7,5 \\
\hline Elongation factor 1-alpha 1 & P10126 & Eef1a1 & 0,6 & 14,7 \\
\hline Elongation factor 1-beta; & 070251 & Eef1b & $-0,4$ & 27,6 \\
\hline Elongation factor 1-delta; & F6ZFU0 & Eef1d & $-0,1$ & 14,5 \\
\hline Elongation factor 1-delta; & P57776 & Eef1d & $-0,4$ & 33,9 \\
\hline Elongation factor 1-gamma; & Q9D8N0 & Eef1g & $-0,1$ & 5 \\
\hline Elongation factor 2; & P58252 & Eef2 & $-0,1$ & 10,5 \\
\hline Emerin; & 008579 & Emd & $-0,3$ & 20,1 \\
\hline Endoplasmic reticulum resident protein 29 ; & P57759 & Erp29 & 0,7 & 8 \\
\hline Endoplasmin; & P08113 & Hsp90b1 & 0,4 & 4,2 \\
\hline Endothelial differentiation-related factor 1 ; & Q9JMG1 & Edf1 & 0,3 & 21,6 \\
\hline Enhancer of rudimentary homolog; & P84089 & Erh & $-0,1$ & 16,3 \\
\hline Enoyl-CoA delta isomerase 2, mitochondrial; & Q9WUR2 & Eci2 & 0,6 & 5,9 \\
\hline Ensconsin; & 088735 & Map7 & $-0,1$ & 1,8 \\
\hline Eosinophil cationic-type ribonuclease 5; & 035292 & Ear5 & $-0,3$ & 5,2 \\
\hline Epididymal secretory protein E1; & Q9Z0J0 & Npc2 & $-0,7$ & 18,8 \\
\hline Epiplakin; & Q8ROW0 & Eppk1 & 0,1 & 4,8 \\
\hline Epsin-3; & Q91W69 & Epn3 & $-0,1$ & 3,1 \\
\hline Eukaryotic peptide chain release factor GTP-binding subunit ERF3A; & Q8R050 & Gspt1 & 0,1 & 1,6 \\
\hline Eukaryotic translation initiation factor 1 & P48024 & Eif1 & 0,1 & 27,3 \\
\hline Eukaryotic translation initiation factor $1 \mathrm{~A}$; & Q60872 & Eif1a & 0,5 & 6,9 \\
\hline Eukaryotic translation initiation factor 2 subunit 2 & Q99L45 & Eif2s2 & $-0,2$ & 31,4 \\
\hline Eukaryotic translation initiation factor 3 subunit B; & Q8JZQ9 & Eif3b & $-0,2$ & 6,1 \\
\hline Eukaryotic translation initiation factor 3 subu nit G; & Q9Z1D1 & Eif3g & $-0,2$ & 7,2 \\
\hline Eukaryotic translation initiation factor 3 subunit J-A; & Q3UGC7 & Eif3j1 & $-0,1$ & 16,1 \\
\hline Eukaryotic translation initiation factor 4 gamma 1; & Q6NZJ6 & Eif4g1 & 0,9 & 4,9 \\
\hline Eukaryotic translation initiation factor $4 \mathrm{~B}$ & Q8BGD9 & Eif4b & $-0,1$ & 11 \\
\hline Eukaryotic translation initiation factor $4 \mathrm{H}$; & Q9WUK2 & Eif4h & $-0,1$ & 20,2 \\
\hline Eukaryotic translation initiation factor 5 & P59325 & Eif5 & 0,0 & 4,2 \\
\hline Eukaryotic translation initiation factor $5 \mathrm{~A}-1$ & P63242 & Eif5a & 0,1 & 19 \\
\hline Eukaryotic translation initiation factor $5 \mathrm{~B}$; & Q05D44 & Eif5b & $-0,7$ & 1,5 \\
\hline Eukaryotic translation initiation factor 6 & 055135 & Eif6 & $-0,3$ & 17,2 \\
\hline Extracellular matrix protein 1 & Q61508 & Ecm1 & $-0,4$ & 25,8 \\
\hline Ezrin; & P26040 & Ezr & 0,1 & 6,3 \\
\hline F-actin-capping protein subunit alpha- 1 ; & P47753 & Capza1 & 0,3 & 9,8 \\
\hline F-actin-capping protein subunit alpha-2; & P47754 & Capza2 & $-1,4$ & 16,1 \\
\hline Far upstream element-binding protein 1 & Q3TUE1 & Fubp1 & 0,0 & 27,1 \\
\hline Far upstream element-binding protein 2 & Q3U0V1 & Khsrp & 0,1 & 30,1 \\
\hline Fatty acid-binding protein, epidermal; & Q05816 & Fabp5 & $-0,6$ & 71,9 \\
\hline F-box only protein 50 & G3X9C2 & Nccrp1 & $-0,3$ & 22,2 \\
\hline Ferritin heavy chain; & P09528 & Fth1 & 0,2 & 6 \\
\hline Ferritin; & Q9CPX4 & Ftl1 & 0,3 & 34,4 \\
\hline Fetuin-B; & Q9QXC1 & Fetub & 1,6 & 12 \\
\hline Fibrinogen gamma chain; & Q8VCM7 & Fgg & 1,5 & 1,8 \\
\hline Fibroblast growth factor-binding protein 1 ; & 070514 & Fgfbp1 & 0,6 & 13,1 \\
\hline Fibronectin type III domain-containing protein 9; & Q8BJN4 & Fndc9 & $-1,3$ & 6,2 \\
\hline Filaggrin; & P11088 & Flg & $-0,5$ & 16,7 \\
\hline Filaggrin-2; & Q2VIS4 & Flg2 & $-0,6$ & 31,1 \\
\hline Filaggrin-2; & E9QPZ3 & Flg2 & $-0,2$ & 31,4 \\
\hline Filamin-A; & Q8BTM8 & Flna & $-0,3$ & 2,8 \\
\hline Filamin-B; & Q80X90 & Flnb & $-0,1$ & 1,2 \\
\hline FK506-binding protein 15 & Q6P9Q6 & Fkbp15 & 0,5 & 1,5 \\
\hline Follistatin-related protein 1 ; & Q62356 & Fstl1 & 0,4 & 7,8 \\
\hline Forkhead box protein $\mathrm{O3}$; & Q9WVH4 & Foxo3 & 0,5 & 30,1 \\
\hline Formin-binding protein 4; & Q6ZQ03 & Fnbp4 & $-0,1$ & 1,9 \\
\hline Fructose-bisphosphate aldolase $A$; & P05064 & Aldoa & $-0,7$ & 28,6 \\
\hline Galectin; & Q9CRB1 & Lgals7 & 0,1 & 64,7 \\
\hline Galectin-1; & P16045 & Lgals1 & 0,1 & 37,8 \\
\hline Galectin-3; & P16110 & Lgals3 & 0,2 & 18,9 \\
\hline
\end{tabular}




\begin{tabular}{|c|c|c|c|c|}
\hline Protein & Uniprot & Gene name & $\begin{array}{l}\text { Fold change of } \\
\text { XPRESS ratio } \\
\left(\text { Egfr }^{\Delta K C} / \mathrm{WT}\right)\end{array}$ & $\begin{array}{c}\text { Sequence } \\
\text { coverage [\%] }\end{array}$ \\
\hline Gamma-glutamyl hydrolase; & Q9ZOL8 & Ggh & $-0,3$ & 27,1 \\
\hline Gamma-glutamylcyclotransferase; & Q9D7X8 & Ggct & $-0,5$ & 17 \\
\hline Gamma-interferon-inducible lysosomal thiol reductase; & Q9ESY9 & Ifi30 & $-0,8$ & 9,7 \\
\hline Gamma-soluble NSF attachment protein; & Q9CWZ7 & Napg & $-0,2$ & 4,5 \\
\hline Gamma-synuclein; & Q9Z0F7 & Sncg & 0,3 & 47,2 \\
\hline Ganglioside GM2 activator; & Q60648 & Gm2a & $-0,6$ & 14,5 \\
\hline Gap junction alpha-1 protein; & P23242 & Gja1 & $-0,3$ & 5,2 \\
\hline Gasdermin-A; & Q9EST1 & Gsdma & $-0,1$ & 9,4 \\
\hline Gelsolin; & P13020 & Gsn & 0,5 & 14,1 \\
\hline General transcription factor IIF subunit 1 ; & Q3THK3 & Gtf2f1 & 0,5 & 2,4 \\
\hline General transcription factor IIF subunit 2; & Q8R0A0 & Gtf2f2 & 0,6 & 3,6 \\
\hline Gephyrin; & Q8BUV3 & Gphn & $-0,2$ & 2,2 \\
\hline Glia maturation factor beta; & Q9CQ।3 & Gmfb & 0,2 & 7,7 \\
\hline Glucocorticoid-induced transcript 1 protein; & Q8K3।9 & Glcci1 & $-0,9$ & 3,9 \\
\hline Glucosidase 2 subunit beta; & 008795 & Prkcsh & $-0,2$ & 8,8 \\
\hline Glucosylceramidase; & P17439 & Gba & $-0,2$ & 8,2 \\
\hline Glutaredoxin-1; & Q9QUH0 & Glrx & $-0,6$ & 10,3 \\
\hline Glutaredoxin-related protein 5, mitochondrial; & Q80Y14 & Glrx5 & 0,2 & 21,7 \\
\hline Glutathione peroxidase 1 & P11352 & Gpx1 & 0,4 & 23,9 \\
\hline Glutathione S-transferase Mu 2; & P15626 & Gstm2 & 0,5 & 13,8 \\
\hline Glutathione S-transferase omega-1; & 009131 & Gsto1 & $-0,8$ & 28,8 \\
\hline Glyceraldehyde-3-phosphate dehydrogenase; & P16858 & Gapdh & 0,1 & 36,4 \\
\hline Glycerophosphoinositol inositolphosphodiesterase GDPD2; & Q9ESM6 & Gdpd2 & 2,3 & 4,6 \\
\hline Glycine cleavage system $\mathrm{H}$ protein, mitochondrial; & Q91WK5 & Gcsh & $-0,1$ & 5,9 \\
\hline Glycolipid transfer protein; & Q9JL62 & Gltp & $-1,0$ & 21,1 \\
\hline Glyoxylate reductase/hydroxypyruvate reductase; & Q91Z53 & Grhpr & 0,3 & 11,2 \\
\hline Golgi reassembly-stacking protein 2 & Q99JX3 & Gorasp2 & 0,4 & 3,7 \\
\hline Golgin subfamily A member 2 ; & Q921M4 & Golga2 & 0,6 & 2,9 \\
\hline Golgin subfamily A member 5 ; & Q9QYE6 & Golga5 & 2,2 & 1,6 \\
\hline GPALPP motifs-containing protein 1 ; & Q69ZC8 & Gpalpp1 & $-1,0$ & 5,5 \\
\hline GRB2-associated-binding protein 1; & Q9QYYo & Gab1 & $-0,6$ & 5 \\
\hline Growth factor receptor-bound protein 2; & Q60631 & Grb2 & 0,3 & 5,9 \\
\hline GrpE protein homolog 1, mitochondrial; & Q99LP6 & Grpel1 & $-0,3$ & 14,3 \\
\hline GTP-binding nuclear protein Ran; & P62827 & Ran & $-0,3$ & 5,1 \\
\hline Guanine nucleotide-binding protein $\mathrm{G}(\mathrm{I}) / \mathrm{G}(\mathrm{S}) / \mathrm{G}(\mathrm{O})$ subunit gamma-12; & Q9DAS9 & Gng12 & 1,0 & 19,4 \\
\hline H/ACA ribonucleoprotein complex subunit 4 & Q9ESX5 & Dkc1 & 1,9 & 13,6 \\
\hline Haloacid dehalogenase-like hydrolase domain-containing protein 2 ; & Q3UGR5 & Hdhd2 & 0,3 & 3,1 \\
\hline Heat shock 70 kDa protein $1 \mathrm{~A}$; & Q61696 & Hspa1a & 0,3 & 22,8 \\
\hline Heat shock 70 kDa protein 4; & Q61316 & Hspa4 & 0,1 & 3,3 \\
\hline Heat shock cognate 71 kDa protein; & P63017 & Hspa8 & 0,7 & 24,9 \\
\hline Heat shock protein beta-1; & P14602 & Hspb1 & 0,2 & 38,3 \\
\hline Heat shock protein HSP 90-beta; & P11499 & Hsp90ab1 & 0,2 & 9 \\
\hline Hematological and neurological expressed 1 protein; & P97825 & Hn1 & $-0,4$ & 37 \\
\hline Hematological and neurological expressed 1-like protein; & Q6PGH2 & Hn1l & 0,2 & 32,1 \\
\hline Hematopoietic lineage cell-specific protein; & P49710 & Hcls1 & 0,4 & 2,1 \\
\hline Heme-binding protein 2 ; & Q9WU63 & Hebp2 & 0,2 & 26,8 \\
\hline Hemoglobin subunit alpha; & P01942 & $\mathrm{Hba}$ & 0,7 & 41,5 \\
\hline Hemoglobin subunit zeta; & P06467 & $\mathrm{Hbz}$ & $-2,7$ & 12 \\
\hline Hemopexin; & Q91X72 & $\mathrm{Hpx}$ & 0,9 & 12 \\
\hline Heparanase; & Q6YGZ1 & Hpse & $-0,5$ & 2,1 \\
\hline Hepatoma-derived growth factor; & P51859 & Hdgf & $-0,1$ & 25,7 \\
\hline Hepatoma-derived growth factor-related protein 2; & Q3UMU9 & Hdgfrp2 & 0,3 & 2,7 \\
\hline Heterogeneous nuclear ribonucleoprotein A/B; & Q99020 & Hnrnpab & 0,1 & 22,8 \\
\hline Heterogeneous nuclear ribonucleoprotein $\mathrm{A} 0$; & Q9CX86 & HnrnpaO & 0,1 & 6,6 \\
\hline Heterogeneous nuclear ribon ucleoprotein A1; & P49312 & Hnrnpa1 & $-0,2$ & 15,3 \\
\hline Heterogeneous nuclear ribon ucleoprotein A3; & Q8BG05 & Hnrnpa3 & $-1,3$ & 23,7 \\
\hline Heterogeneous nuclear ribonucleoprotein D0; & Q60668 & Hnrnpd & $-0,2$ & 13,9 \\
\hline Heterogeneous nuclear ribon ucleoprotein D-like; & Q9Z130 & Hnrnpdl & $-0,4$ & 14 \\
\hline Heterogeneous nuclear ribon ucleoprotein $\mathrm{H}$; & 035737 & Hnrnph1 & $-1,2$ & 9,9 \\
\hline Heterogeneous nuclear ribon ucleoprotein $\mathrm{K}$; & P61979 & Hnrnpk & 0,2 & 18,4 \\
\hline Heterogeneous nuclear ribon ucleoprotein U; & Q8VEK3 & Hnrnpu & 0,0 & 6,2 \\
\hline Heterogeneous nuclear ribonucleoproteins A2/B1; & 088569 & Hnrnpa2b1 & $-0,1$ & 21,2 \\
\hline High mobility group nucleosome-bind ing domain-containing protein 5 ; & Q9JL35 & Hmgn5 & 0,1 & 3,7 \\
\hline High mobility group protein B1; & P63158 & Hmgb1 & 0,0 & 17,7 \\
\hline High mobility group protein $\mathrm{B} 2$; & P30681 & Hmgb2 & $-0,3$ & 23,3 \\
\hline High mobility group protein B3; & 054879 & Hmgb3 & $-0,8$ & 16,4 \\
\hline High mobility group protein $\mathrm{HMGI}-\mathrm{C}$; & P52927 & Hmga2 & 0,1 & 16,7 \\
\hline HIRA-interacting protein 3 ; & Q8BLH7 & Hirip3 & 0,3 & 2,8 \\
\hline Histidine ammonia-lyase; & P35492 & $\mathrm{Hal}$ & 0,1 & 7,2 \\
\hline Histidine triad nucleotide-binding protein 1 ; & P70349 & Hint1 & 0,6 & 34,5 \\
\hline Histidine triad nucleotide-binding protein 2 , mitochondrial; & Q9D0S9 & Hint2 & 0,2 & 9,2 \\
\hline
\end{tabular}




\begin{tabular}{|c|c|c|c|c|}
\hline Protein & Uniprot & Gene name & $\begin{array}{l}\text { Fold change of } \\
\text { XPRESS ratio } \\
\text { (Egfr }{ }^{\Delta K C ~ / ~ W T) ~}\end{array}$ & $\begin{array}{c}\text { Sequence } \\
\text { coverage [\%] }\end{array}$ \\
\hline Histone $\mathrm{H} 1.0$; & P10922 & $\mathrm{H} 1 \mathrm{fO}$ & 1,1 & 6,7 \\
\hline Histone H1.1; & P43275 & Hist1h1a & 0,4 & 9,4 \\
\hline Histone $\mathrm{H} 1.2$; & P15864 & Hist1h1c & 0,5 & 22,6 \\
\hline Histone H1.3; & P43277 & Hist1h1d & 1,1 & 21,7 \\
\hline Histone H1.4; & P43274 & Hist1h1e & 0,9 & 21,9 \\
\hline Histone H1.5; & P43276 & Hist1h1b & 0,7 & 14,3 \\
\hline Histone $\mathrm{H} 2 \mathrm{~A}$ type 1 ; & P22752 & Hist1h2ao & $-0,1$ & 27,1 \\
\hline Histone $\mathrm{H} 2 \mathrm{~B}$ type $1-\mathrm{F} / \mathrm{J} / \mathrm{L}$; & P10853 & Hist1h2bn & $-0,3$ & 40,5 \\
\hline Histone $\mathrm{H} 2 \mathrm{~B}$ type 2-E; & Q64524 & Hist2h2be & $-0,9$ & 34,9 \\
\hline Histone H3.3C; & P02301 & $H 3 f 3 c$ & $-0,5$ & 11,8 \\
\hline Histone $\mathrm{H} 4$; & P62806 & Hist4h4 & 0,1 & 50,5 \\
\hline Histone-binding protein RBBP4; & Q60972 & Rbbp4 & 0,4 & 11,8 \\
\hline Histone-binding protein RBBP7; & Q60973 & Rbbp7 & 0,8 & 12 \\
\hline Homeodomain-only protein; & Q8R1H0 & Hopx & $-0,5$ & 43,8 \\
\hline Hornerin; & E9QNP3 & Hrnr & $-0,3$ & 24,5 \\
\hline Host cell factor 1 & Q61191 & Hcfc1 & 0,0 & 1,8 \\
\hline Hsc70-interacting protein; & Q99L47 & St13 & 0,3 & 10,2 \\
\hline Hsp90 co-chaperone Cdc37; & Q61081 & Cdc37 & 0,7 & 4,5 \\
\hline Huntingtin-interacting protein $\mathrm{K}$; & Q9CR41 & Hypk & $-1,6$ & 13,2 \\
\hline Hypoxanthine-guanine phosphoribosyltransferase; & P00493 & Hprt1 & $-0,8$ & 45 \\
\hline Inorganic pyrophosphatase; & Q9D819 & Ppa1 & $-1,1$ & 3,1 \\
\hline Inosine triphosphate pyrophosphatase; & Q9D892 & Itpa & $-0,2$ & 8,6 \\
\hline Insulin receptor substrate 1 & P35569 & Irs1 & 0,5 & 0,9 \\
\hline Insulin-degrading enzyme; & F6RPJ9 & Ide & $-0,2$ & 19,7 \\
\hline Integral membrane protein 2B; & 089051 & Itm2b & 0,4 & 13,9 \\
\hline Integrator complex subu nit 12 & Q9D168 & Ints12 & 0,2 & 5 \\
\hline Integrin beta-1; & P09055 & Itgb1 & 0,3 & 9,9 \\
\hline Interferon regulatory factor 2-binding protein 2; & E9Q1P8 & Irf2bp2 & 0,1 & 3,3 \\
\hline Interferon-induced transmembrane protein 2 & Q99J93 & Ifitm2 & 0,3 & 5,8 \\
\hline Interleukin-1 alpha; & P01582 & $\| 1 a$ & $-0,2$ & 4,1 \\
\hline Interleukin-1 receptor antagon ist protein; & P25085 & ॥lrn & $-0,2$ & 17,5 \\
\hline Involucrin; & G3X9D9 & $|v|$ & $-0,4$ & 49,6 \\
\hline Iron/zinc purple acid phosphatase-like protein; & Q8BX37 & Papl & $-0,4$ & 2,3 \\
\hline Isocitrate dehydrogenase [NAD] subun it alpha, mitochondrial; & Q9D6R2 & Idh3a & 1,4 & 6,3 \\
\hline Isocitrate dehydrogenase [NADP] cytoplasmic; & 088844 & Idh1 & 0,0 & 32,9 \\
\hline Isocitrate dehydrogenase [NADP], mitochondrial; & P54071 & Idh2 & 0,2 & 19,2 \\
\hline Isopentenyl-diphosphate Delta-isomerase $1 ;$ & P58044 & Idi1 & $-0,2$ & 9,3 \\
\hline Junction plakoglobin; & Q02257 & Jup & $-0,2$ & 14,5 \\
\hline Kallikrein 1-related peptidase b5; Kallikrein related-peptidase 5; & Q9D140 & Klk5 & $-0,4$ & 21,8 \\
\hline Kallikrein related-peptidase 10; Protein KIk10; & Q99M20 & Klk10 & $-0,4$ & 8,3 \\
\hline Kallikrein-7; & Q91VE3 & Klk7 & $-0,4$ & 26,1 \\
\hline Kallikrein-8; & Q61955 & KIk8 & 0,0 & 6,9 \\
\hline $\begin{array}{l}\text { Keratin associated protein 13; Protein Krtap13; Putative keratin-associated } \\
\text { protein; }\end{array}$ & 088375 & Krtap13 & $-0,3$ & 6,1 \\
\hline Keratin, type I cuticular Ha3-II; & Q61897 & Krt33b & $-1,2$ & 9,2 \\
\hline Keratin, type I cytoskeletal 10; & P02535 & Krt10 & $-0,3$ & 31,9 \\
\hline Keratin, type I cytoskeletal 14; & Q61781 & Krt14 & $-0,2$ & 32,2 \\
\hline Keratin, type I cytoskeletal 15; & Q61414 & Krt15 & $-0,5$ & 31,6 \\
\hline Keratin, type I cytoskeletal 17; & Q9QWL7 & Krt17 & $-1,1$ & 30,7 \\
\hline Keratin, type I cytoskeletal 25 ; & Q8VCW2 & Krt25 & $-0,4$ & 10,8 \\
\hline Keratin, type I cytoskeletal 26 ; & Q3TRJ4 & Krt26 & $-0,1$ & 1,7 \\
\hline Keratin, type I cytoskeletal 27; & Q97320 & Krt27 & $-2,0$ & 6 \\
\hline Keratin, type I cytoskeletal 42 ; & Q6IFX2 & Krt42 & 1,8 & 13,9 \\
\hline Keratin, type II cytoskeletal 1 & P04104 & Krt1 & $-0,4$ & 38,5 \\
\hline Keratin, type II cytoskeletal $1 \mathrm{~b}$; & Q6IFZ6 & Krt77 & $-0,8$ & 26 \\
\hline Keratin, type II cytoskeletal 2 epidermal; & Q3TTY5 & Krt2 & 0,5 & 6,5 \\
\hline Keratin, type II cytoskeletal 2 oral; & Q3UV17 & Krt76 & 1,7 & 4 \\
\hline Keratin, type II cytoskeletal 5; & Q922U2 & Krt5 & 0,2 & 26 \\
\hline Keratin, type II cytoskeletal 71; & Q9ROH5 & Krt71 & $-2,5$ & 7,8 \\
\hline Keratin, type II cytoskeletal 72; & Q6IME9 & Krt72 & 0,1 & 3,7 \\
\hline Keratin, type II cytoskeletal 73; & Q6NXH9 & Krt73 & 1,3 & 8,5 \\
\hline Keratin, type II cytoskeletal 79; & Q8VED5 & Krt79 & 0,1 & 17,9 \\
\hline Keratin-associated protein $16-1$ & A2A5X5 & Krtap16 1 & $-5,4$ & 3 \\
\hline Keratin-associated protein $19-5$ & F8VQ65 & Krtap19 5 & $-0,3$ & 19,4 \\
\hline Keratinocyte-associated transmembrane protein 2 ; & Q8K201 & Kct2 & 0,3 & 8,9 \\
\hline Ketimine reductase mu-crystallin; & 054983 & Crym & $-3,2$ & 4,8 \\
\hline Kinectin; & F8VQC7 & Ktn1 & 0,6 & 4,7 \\
\hline Kininogen-1; & 008677 & Kng1 & 2,0 & 32,7 \\
\hline Lactoylglutathione lyase; & Q9CPU0 & Glo1 & $-0,9$ & 31 \\
\hline Ladinin-1; & P57016 & Lad1 & $-0,3$ & 16,7 \\
\hline
\end{tabular}




(2) Protein

\section{Protein}

Uniprot

Gold change of
Gene name

Sequence (Egfr $\left.^{\Delta K C} / \mathrm{WT}\right)$ coverage [\%]

Lamina-associated polypeptide 2, isoforms beta/delta/epsilon/gamma;

Q61029

Q6DVA0

LEM domain-containing protein 2 ;

Leucine-rich HEV glycoprotein; Leucine-rich alpha-2-glycoprotein; Leucine-rich alpha-2-glycoprotein 1; Protein Lrg1;

Leucine-rich repeat flightless-interacting protein 1;

Leucine-rich repeat flightless-interacting protein 2 ;

Leucine-rich repeat-containing protein 59;

Leukocyte elastase inhibitor $A$.

Leukocyte surface antigen CD47;

LIM and SH3 domain protein 1;

LIM domain and actin-bind ing protein 1;

LIM zinc-binding domain-containing Nebulette;

Lipoma-preferred partner homolog;

L-lactate dehydrogenase A chain;

Loricrin;

Lumican;

Ly6/PLAUR domain-containing protein 3

Ly6/PLAUR domain-containing protein 5;

Lymphocyte antigen 6D

Lymphocyte-specific protein 1 ;

Lysosomal protective protein;

Lysosome-associated membrane glycoprotein 1;

Lysosome-associated membrane glycoprotein 2;

Macrophage migration inhibitory factor;

Magnesium-dependent phosphatase 1 .

Major urinary protein 2;

Malate dehydrogenase, cytoplasmic

Malate dehydrogenase, mitochondrial;

MARCKS-related protein;

MCG116526; Protein Scgb2b7;

MCG117626; Odorant binding protein la; Protein Obp1a;

MCG120169; Protein 2310050C09Rik;

MCG129038; Protein Serpinb3a;

MCG130458; Protein Fubp3;

MCG130675; Protein 2410018M08Rik; SCAN domain containing 3;

MCG21235; Protein Serpinb3b;

Membrane-associated progesterone receptor component 1

Membrane-associated progesterone receptor component 2;

Metaxin-1;

Methylosome subunit pICln

Microfibrillar-associated protein 1

Microtubule-associated protein 2;

Microtubule-associated protein 4;

Microtubule-associated protein RP/EB family member 1 ;

Microtubule-associated protein RP/EB family member 2;

Microtubule-associated protein tau;

Microtubule-associated serine/threon ine-protein kinase 4;

Mimitin, mitochondrial

Mitochondrial fission 1 protein;

Mitochondrial import inner membrane translocase subunit Tim13;

Mitochondrial import inner membrane translocase subunit Tim8 A

Mitochondrial import inner membrane translocase subu nit Tim9;

Mitochondrial intermembrane space import and assembly protein 40

Moesin;

Multidrug resistance-associated protein 9;

Myb-binding protein $1 \mathrm{~A}$

Myeloid bactenecin (F1); Neutrophilic granule protein; Protein Ngp;

Myosin light chain $1 / 3$, skeletal muscle isoform;

Myosin light chain 3;

Myosin light chain kinase, smooth muscle;

Myosin light polypeptide 6;

Myosin regulatory light chain 12B;

Myosin regulatory light chain 2, skeletal muscle isoform

Myosin regulatory light chain 2, ventricular/cardiac muscle isoform

Myosin-10;

Myosin-9;

Myotrophin;

Myristoylated alanine-rich C-kinase substrate;

$N(G), N(G)$-dimethylarginine dimethylaminohydrolase 2;

$\mathrm{Na}(+) / \mathrm{H}(+)$ exchange regulatory cofactor NHE-RF1;
Q91XL1

Q3UZ39

Q91WK0

Q922Q8

Q9D154

Q61735

Q61792

Q9ERGO

Q9DC07

Q8BFW7

P06151

P18165

P51885

Q91YK8

Q9D7Z7

P35459

P19973

P16675

P11438

P17047

P34884

Q9D967

P11589

P14152

P08249

P28667

D3YYY1

Q9D3H2

G5E873

G3X9V8

A2Al72

B2RPU8

Q9D105

055022

Q80UU9

P47802

Q61189

Q9CQU1

P20357

P27546

Q61166

Q8R001

P10637

Q811L6

Q59J78

Q9CQ92

P62075

Q9WVA2

Q9WV98

Q8VEA4

P26041

Q80WJ6

Q7TPV4

008692

P05977

P09542

Q6PDN3

Q60605

Q3THE2

P97457

P51667

Q61879

Q8VDD5

P62774

P26645

Q99LD8

P70441

Tmpo

Lemd2

Lrg1

Lrrfip1

Lrrfip2

Lrrc59

Serpinb1a

Cd47

Lasp1

Lima1

Nebl

Lpp

Ldha

Lor

Lum

Lypd3

Lypd5

Ly6d

Lsp1

Ctsa

Lamp1

Lamp2

Mif

Mdp1

Mup2

Mdh1

Mdh2

Marcksl1

Scgb2b7

Obp1a

2310050C09Rik

Serpinb3a

Fubp3

2410018M08Rik

Serpinb3b

Pgrmc1

Pgrmc2

Mtx1

Clns1a

Mfap1

Map2

Map4

Mapre1

Mapre2

Mapt

Mast4

Ndufaf2

Fis1

Timm13

Timm8a1

Timm9

Chchd4

Msn

Abcc12

Mybbp1a

Ngp

Myl1

Myl3

Mylk

Myl6

Myl12b

Mylpf

Myl2

Myh10

Myh9

Mtpn

Marcks

Ddah2

Slc9a3r1

$\begin{array}{ll}-0,2 & 25,9\end{array}$

$-0,4$

1,1

$-1,0$

$-0,1$

$-0,2$

0,0

0,3

0,6

0,6

$-0,1$

$-1,5$

$-0,4$

0,2

0,8

0,0

$-0,3$

$-0,5$

0,5

$-0,3$

0,5

0,5

$-0,8$

$-0,2$

0,0

$-0,5$

0,1

0,5

$-0,3$

$-0,3$
$-0,3$

$-0,4$

$-0,4$
$-0,1$

0,6

0,6
0,0

$-0,6$

$-0,1$

0,0 


\begin{tabular}{|c|c|c|c|c|}
\hline Protein & Uniprot & Gene name & $\begin{array}{l}\text { Fold change of } \\
\text { XPRESS ratio } \\
\left(\text { Egfr }^{\Delta K C} / \mathrm{WT}\right)\end{array}$ & $\begin{array}{c}\text { Sequence } \\
\text { coverage [\%] }\end{array}$ \\
\hline $\mathrm{N}$-acetylglucosamine-6-sulfatase; & Q8BFR4 & Gns & 0,0 & 7,4 \\
\hline N-acetylneuraminate lyase; & Q9DCJ9 & $\mathrm{Npl}$ & $-0,7$ & 46,9 \\
\hline NADH dehydrogenase [ubiquinone] 1 alpha subcomplex subunit 13; & Q9ERS2 & Ndufa13 & $-0,3$ & 13,9 \\
\hline NADH dehydrogenase [ubiquinone] 1 alpha subcomplex subunit 2; & Q9CQ75 & Ndufa2 & 0,4 & 14,1 \\
\hline NADH dehydrogenase [ubiquinone] 1 alpha subcomplex subunit 4; & Q62425 & Ndufa4 & 0,4 & 46,3 \\
\hline NADH dehydrogenase [ubiquinone] 1 alpha subcomplex subunit 4-like 2; & Q4FZG9 & Ndufa4l2 & $-1,1$ & 19,5 \\
\hline NADH dehydrogenase [ubiquinone] 1 alpha subcomplex subunit 5; & Q9CPP6 & Ndufa5 & $-1,0$ & 13,8 \\
\hline NADH dehydrogenase [ubiquinone] 1 alpha subcomplex subunit 6 & Q9CQZ5 & Ndufa6 & $-2,9$ & 13 \\
\hline NADH dehydrogenase [ubiquinone] 1 alpha subcomplex subunit 7; & Q9Z1P6 & Ndufa7 & 0,4 & 17,7 \\
\hline NADH dehydrogenase [ubiquinone] 1 beta subcomplex subunit 10; & Q9DCS9 & Ndufb10 & 0,5 & 12,8 \\
\hline NADH dehydrogenase [ubiquinone] 1 beta subcomplex subunit 3; & Q9CQZ6 & Ndufb3 & 0,2 & 10,6 \\
\hline NADH dehydrogenase [ubiquinone] 1 beta subcomplex subunit 6; & Q3UIU2 & Ndufb6 & $-0,3$ & 7,2 \\
\hline $\begin{array}{l}\text { NADH dehydrogenase [ubiquinone] } 1 \text { beta subcomplex subunit } 8 \text {, } \\
\text { mitochondrial; }\end{array}$ & Q9D6J5 & Ndufb8 & 0,2 & 5,9 \\
\hline NADH dehydrogenase [ubiquinone] 1 subunit C2; & Q9CQ54 & Ndufc2 & 0,5 & 10,8 \\
\hline NADH dehydrogenase [ubiquinone] flavoprotein 2, mitochondrial; & Q9D6J6 & Ndufv2 & 0,4 & 16,1 \\
\hline $\begin{array}{l}\text { NADH dehydrogenase [ubiquinone] flavoprotein 3, mitochondrial; RIKEN cDNA } \\
\text { 1500032D16, isoform CRA_a; }\end{array}$ & Q3U422 & Ndufv3 & 0,3 & 14,1 \\
\hline NADH dehydrogenase [ubiquinone] iron-sulfur protein 4, mitochondrial; & Q9CXZ1 & Ndufs4 & $-0,5$ & 14,3 \\
\hline NADH dehydrogenase [ubiquinone] iron-sulfur protein 6, mitochondrial; & P52503 & Ndufs6 & 0,0 & 15,3 \\
\hline NADH dehydrogenase [ubiquinone] iron-sulfur protein 8, mitochondrial; & Q8K3J1 & Ndufs8 & 0,0 & 9,4 \\
\hline NADP-dependent malic enzyme; & P06801 & Me1 & 0,1 & 5,8 \\
\hline Nardilysin; & Q8BHG1 & Nrd1 & $-0,1$ & 2,1 \\
\hline Nascent polypeptide-associated complex subunit alpha, muscle-specific form; & P70670 & Naca & 0,3 & 25,6 \\
\hline Negative elongation factor $\mathrm{E}$; & P19426 & Nelfe & 0,1 & 7,9 \\
\hline Neudesin; & Q9CQ45 & Nenf & 0,2 & 31 \\
\hline Neural cell adhesion molecule L1; & A2AFG7 & L1cam & 0,3 & 4,9 \\
\hline Neuromodulin; & P06837 & Gap43 & 0,2 & 18,1 \\
\hline Neuroplastin; & P97300 & Nptn & 0,0 & 12,7 \\
\hline NFU1 iron-sulfur cluster scaffold homolog, mitochondrial; & Q9QZ23 & Nfu1 & 0,4 & 5,9 \\
\hline Nicastrin; & P57716 & Ncstn & 0,5 & 1,7 \\
\hline Non-histone chromosomal protein HMG-14; & P18608 & Hmgn1 & $-0,5$ & 34,9 \\
\hline Non-specific lipid-transfer protein; & P32020 & Scp2 & 0,0 & 9,1 \\
\hline NSFL1 cofactor $p 47$ & Q9CZ44 & Nsfl1c & $-0,2$ & 36,5 \\
\hline Nuclear autoantigenic sperm protein; & Q99MD9 & Nasp & 0,3 & 12,9 \\
\hline Nuclear fragile $\mathrm{X}$ mental retardation-interacting protein 2 ; & Q5F2E7 & Nufip2 & 0,2 & 2 \\
\hline Nuclear migration protein nudC; & 035685 & Nudc & 0,3 & 13,3 \\
\hline Nuclear pore glycoprotein p62; & Q63850 & Nup62 & $-0,4$ & 8,2 \\
\hline Nuclear speckle splicing regulatory protein 1 & Q5NCR9 & Nsrp1 & $-0,1$ & 2,6 \\
\hline Nuclear ubiquitous casein and cyclin-dependent kinase substrate 1; & Q80XU3 & Nucks1 & $-0,2$ & 12 \\
\hline Nuclease-sensitive element-binding protein 1 & P62960 & Ybx1 & 0,5 & 23,9 \\
\hline Nucleobindin-1; & Q02819 & Nucb1 & 0,1 & 39,4 \\
\hline Nucleobindin-2; & P81117 & Nucb2 & $-0,1$ & 18,8 \\
\hline Nucleolar protein 16 & Q9CPT5 & Nop16 & 1,0 & 3,9 \\
\hline Nucleolar protein 56 & Q9D6Z1 & Nop56 & $-0,5$ & 5,7 \\
\hline Nucleolin; & P09405 & $\mathrm{Ncl}$ & 0,1 & 26,6 \\
\hline Nucleophosmin; & Q61937 & Npm1 & $-0,2$ & 23,5 \\
\hline Nucleoporin GLE1; & Q8R322 & Gle1 & 1,3 & 1,7 \\
\hline Nucleoprotein TPR; & F6ZDS4 & Tpr & 0,1 & 1,7 \\
\hline Nucleoside diphosphate kinase; & E9PZF0 & Gm20390 & $-0,2$ & 54,3 \\
\hline Nucleosome assembly protein 1 -like 1 ; & P28656 & Nap1l1 & 0,5 & 12,7 \\
\hline Nucleosome assembly protein 1-like 4; & Q78ZA7 & Nap1/4 & 0,3 & 12 \\
\hline Odorant-binding protein $2 \mathrm{a}$; & Q8K1H9 & Obp2a & $-0,3$ & 8 \\
\hline Oligoribonuclease, mitochondrial; & Q9D8S4 & Rexo2 & 0,0 & 4,2 \\
\hline Opioid growth factor receptor; & Q99PG2 & Ogfr & 0,0 & 3,2 \\
\hline Osteoclast-stimulating factor 1 ; & Q62422 & Ostf1 & $-0,1$ & 29,8 \\
\hline Palmdelphin; & Q9JHU2 & Palmd & 0,0 & 17,1 \\
\hline Palmitoyl-protein thioesterase 1 & 088531 & Ppt1 & $-0,8$ & 4,9 \\
\hline $\begin{array}{l}\text { Papillary renal cell carcinoma (Translocation-associated); Papillary renal cell } \\
\text { carcinoma-associated protein; Protein Prcc; }\end{array}$ & Q9EQC8 & Prcc & 0,2 & 8,8 \\
\hline Paralemmin-1; & Q9ZOP4 & Palm & $-0,2$ & 20,6 \\
\hline Paralemmin-2; & Q8BR92 & Palm2 & 0,4 & 4,3 \\
\hline Parathymosin; & Q9D0J8 & Ptms & 0,2 & 10,9 \\
\hline Partner of Y14 and mago; & Q8CHP5 & Wibg & $-0,2$ & 28,1 \\
\hline Parvalbumin alpha; & P32848 & Pvalb & $-0,6$ & 12,7 \\
\hline Paternally-expressed gene 3 protein; & Q3URU2 & Peg3 & 1,1 & 1,5 \\
\hline
\end{tabular}




\begin{tabular}{|c|c|c|c|c|}
\hline Protein & Uniprot & Gene name & $\begin{array}{l}\text { Fold change of } \\
\text { XPRESS ratio } \\
\left(\text { Egfr }^{\Delta K C} / \mathrm{WT}\right)\end{array}$ & $\begin{array}{c}\text { Sequence } \\
\text { coverage [\%] }\end{array}$ \\
\hline PAXIP1-associated glutamate-rich protein 1; & Q99L02 & PAGR1 & $-0,3$ & 7,5 \\
\hline PC4 and SFRS1-interacting protein; & Q99JF8 & Psip1 & 0,5 & 5,8 \\
\hline PDZ and LIM domain protein 1; & 070400 & Pdlim1 & 0,1 & 33,6 \\
\hline PDZ and LIM domain protein 2 & Q8R1G6 & Pdlim2 & $-0,7$ & 10 \\
\hline PDZ and LIM domain protein 4; & P70271 & Pdlim4 & 0,2 & 16,1 \\
\hline PDZ and LIM domain protein 5; & Q8CI51 & Pdlim5 & $-0,1$ & 4,6 \\
\hline PDZ domain-containing protein 11 & Q9CZG9 & Pdzd11 & 0,3 & 25,7 \\
\hline PDZK1-interacting protein 1 ; & Q9CQHO & Pdzk1ip1 & 0,4 & 28,1 \\
\hline Peflin; & Q8BFY6 & Pef1 & 0,2 & 6,9 \\
\hline Peptidyl-prolyl cis-trans isomerase A; & P17742 & Ppia & $-0,1$ & 57,9 \\
\hline Peptidyl-prolyl cis-trans isomerase B; & P24369 & Ppib & 0,2 & 13,9 \\
\hline Peptidyl-prolyl cis-trans isomerase C; & P30412 & Ppic & 0,3 & 6,1 \\
\hline Peptidyl-prolyl cis-trans isomerase CWC27 homolog; & Q3TKY6 & Cwc27 & $-0,1$ & 2,6 \\
\hline Peptidyl-prolyl cis-trans isomerase F, mitochondrial; & Q99KR7 & Ppif & $-0,4$ & 11,7 \\
\hline Peptidyl-prolyl cis-trans isomerase FKBP1A; & P26883 & Fkbp1a & 0,1 & 25 \\
\hline Peptidyl-prolyl cis-trans isomerase FKBP3; & Q62446 & Fkbp3 & 0,0 & 25 \\
\hline Peptidyl-prolyl cis-trans isomerase FKBP7; & 054998 & Fkbp7 & 1,2 & 3,7 \\
\hline Peptidyl-prolyl cis-trans isomerase NIMA-interacting 1 ; & Q9QUR7 & Pin1 & 0,1 & 4,8 \\
\hline Peptidyl-prolyl cis-trans isomerase NIMA-interacting 4; & Q9CWW6 & Pin4 & $-0,1$ & 16,8 \\
\hline Perilipin-1; & Q8CGN5 & Plin1 & 0,0 & 3,5 \\
\hline Perilipin-2; & P43883 & Plin2 & $-0,5$ & 8,3 \\
\hline Perilipin-3; & Q9DBG5 & Plin3 & 0,3 & 16,2 \\
\hline Perilipin-4; & 088492 & Plin4 & $-0,2$ & 9,8 \\
\hline Periostin; & Q62009 & Postn & 0,5 & 1 \\
\hline Periplakin; & G5E898 & Ppl & 0,1 & 22,6 \\
\hline Peroxiredoxin-1; & P35700 & $\operatorname{Prdx1}$ & 0,5 & 48,2 \\
\hline Peroxiredoxin-2; & Q61171 & $\operatorname{Prdx2}$ & 0,4 & 22,7 \\
\hline Peroxiredoxin-5, mitochondrial; & P99029 & Prdx5 & 0,0 & 36,7 \\
\hline Peroxiredoxin-6; & 008709 & Prdx6 & $-0,2$ & 24 \\
\hline Peroxisomal biogenesis factor 19 & Q8VCI5 & Pex19 & 0,2 & 10,7 \\
\hline Peroxisomal membrane protein PEX14; & Q9R0A0 & Pex14 & 0,3 & 17,8 \\
\hline PEST proteolytic signal-containing nuclear protein; & Q6P8I4 & Pcnp & 0,5 & 34,7 \\
\hline Phosphatase and actin regulator 4 & Q501J7 & Phactr4 & 0,0 & 4,9 \\
\hline Phosphatidylethanolamine-binding protein 1 ; & P70296 & Pebp1 & 0,0 & 44,9 \\
\hline Phosphatidylinositol-glycan-specific phospholipase D; & 070362 & Gpld1 & 0,0 & 5 \\
\hline Phosphoglycerate kinase 1 & P09411 & Pgk1 & $-0,1$ & 12,5 \\
\hline Phosphoglycerate mutase 1 & Q9DBJ1 & Pgam1 & $-0,5$ & 46,9 \\
\hline Phosphoglycerate mutase 2 & 070250 & Pgam2 & $-0,8$ & 13 \\
\hline Phospholipase B-like 1; & Q8VCI0 & Plbd1 & $-0,6$ & 8,4 \\
\hline Phospholipase D3; & 035405 & Pld3 & $-0,5$ & 6,4 \\
\hline Pinin; & 035691 & Pnn & $-0,4$ & 3,4 \\
\hline Plakophilin-1; & P97350 & Pkp1 & 0,3 & 18,1 \\
\hline Plasminogen activator inhibitor 1 RNA-bind ing protein; & Q9CY58 & Serbp1 & $-0,1$ & 17,2 \\
\hline Plasminogen activator inhibitor 2, macrophage; & P12388 & Serpinb2 & $-1,1$ & 11,3 \\
\hline Plastin-2; & Q61233 & Lcp1 & 0,2 & 4,3 \\
\hline Plastin-3; & Q99K51 & Pls3 & $-0,4$ & 8,4 \\
\hline Platelet-activating factor acetylhydrolase IB subu nit beta; & Q61206 & Pafah1b2 & 1,2 & 12,2 \\
\hline Platelet-activating factor acetylhydrolase IB subu nit gamma; & Q61205 & Pafah1b3 & 0,1 & 6,3 \\
\hline Plectin; & Q9QXS1 & Plec & $-0,1$ & 2,1 \\
\hline Plexin-B2; & B2RXS4 & Plxnb2 & 0,0 & 1,8 \\
\hline Poliovirus receptor; Poliovirus receptor-related protein 2; Tage4 receptor; & Q8K094 & Pvr & 0,1 & 4,7 \\
\hline Poliovirus receptor-related protein 1 ; & Q9JKF6 & Pvrl1 & 0,1 & 4,1 \\
\hline Poliovirus receptor-related protein 4 & Q8R007 & Pvrl4 & 0,1 & 6,9 \\
\hline Poly $(\mathrm{rC})$-binding protein 1 & P60335 & Pcbp1 & 0,4 & 3,7 \\
\hline Poly(U)-specific endoribonuclease; & Q3V188 & Endou & $-0,1$ & 11,7 \\
\hline Polyadenylate-binding protein 1 & P29341 & Pabpc1 & 0,1 & 3,8 \\
\hline Polyadenylate-binding protein 2 ; & Q8CCS6 & Pabpn1 & 0,2 & 6,7 \\
\hline Polyglutamine-binding protein 1 & Q91VJ5 & Pqbp1 & 0,3 & 22,8 \\
\hline Polymerase I and transcript release factor; & 054724 & Ptrf & 0,1 & 5,9 \\
\hline Polyubiquitin-B; & P0CG49 & Ubb & 0,5 & 56,4 \\
\hline PRA1 family protein 3; & Q8R5J9 & Arl6ip5 & 0,4 & 5,9 \\
\hline Prefoldin subunit 1 & Q9CWM4 & Pfdn1 & $-1,1$ & 23 \\
\hline Prefoldin subunit 2; & 070591 & Pfdn2 & 0,3 & 22,7 \\
\hline Prefoldin subunit 5; & Q9WU28 & Pfdn5 & 0,3 & 20,1 \\
\hline Prefoldin subunit 6; & Q03958 & Pfdn6 & 0,0 & 42,6 \\
\hline Prelamin-A/C; & P48678 & Lmna & 0,2 & 20,2 \\
\hline Pre-mRNA 3'-end-processing factor FIP1; & Q9D824 & Fip1l1 & 0,1 & 7 \\
\hline PRKC apoptosis WT1 regulator protein; & Q925B0 & Pawr & $-0,3$ & 13,8 \\
\hline Proactivator polypeptide-like 1 & Q8C1C1 & Psapl1 & 0,1 & 6,3 \\
\hline Probable RNA-binding protein EIF1AD; & Q3ТНJ3 & Eif1ad & 0,1 & 30,6 \\
\hline
\end{tabular}




\begin{tabular}{|c|c|c|c|c|}
\hline Protein & Uniprot & Gene name & $\begin{array}{l}\text { Fold change of } \\
\text { XPRESS ratio } \\
\left(\text { Egfr }^{\Delta K C} / \mathrm{WT}\right)\end{array}$ & $\begin{array}{c}\text { Sequence } \\
\text { coverage [\%] }\end{array}$ \\
\hline Proenkephalin-A; & P22005 & Penk & 2,1 & 9 \\
\hline Programmed cell death protein 10 ; & Q8VE70 & Pdcd10 & 0,5 & 10,8 \\
\hline Programmed cell death protein 5 ; & P56812 & Pdcd5 & 0,3 & 37,3 \\
\hline Programmed cell death protein 6 ; & P12815 & Pdcd6 & $-0,2$ & 15,7 \\
\hline Prohibitin; & P67778 & $\mathrm{Phb}$ & 0,4 & 24,6 \\
\hline Proliferating cell nuclear antigen; & P17918 & Pcna & 0,6 & 23 \\
\hline Proline and serine-rich protein 2 & Q8C5R2 & Proser2 & 0,0 & 2,8 \\
\hline Proline-rich AKT1 substrate 1 & Q9D1F4 & Akt1s1 & $-0,3$ & 13,6 \\
\hline Prolyl-tRNA synthetase associated domain-containing protein 1 ; & Q9D820 & Prorsd1 & $-0,5$ & 8,3 \\
\hline Prominin-2; & Q3UUY6 & Prom2 & 0,3 & 8,5 \\
\hline Prostaglandin E synthase 3; & Q9R0Q7 & Ptges3 & 0,2 & 28,5 \\
\hline Proteasome activator complex subunit 1 ; & P97371 & Psme1 & 0,4 & 19,7 \\
\hline Proteasome activator complex subunit 3 & P61290 & Psme3 & $-0,3$ & 17,7 \\
\hline Proteasome subunit alpha type-1; & Q9R1P4 & Psma1 & $-0,4$ & 30 \\
\hline Proteasome subunit alpha type-2; & P49722 & Psma2 & $-0,6$ & 9 \\
\hline Proteasome subunit alpha type- 3 ; & 070435 & Psma3 & $-0,1$ & 8,6 \\
\hline Proteasome subunit alpha type- 5 ; & Q9Z2U1 & Psma5 & 0,3 & 20,3 \\
\hline Proteasome subunit alpha type- 6 ; & Q9QUM9 & Psma6 & $-0,5$ & 5,3 \\
\hline Proteasome subunit alpha type-7; & Q9Z2U0 & Psma7 & $-0,4$ & 25,4 \\
\hline Proteasome subunit beta type- 1 ; & 009061 & Psmb1 & $-0,3$ & 34,6 \\
\hline Proteasome subunit beta type- 2 ; & Q9R1P3 & Psmb2 & $-0,7$ & 10,4 \\
\hline Proteasome subunit beta type- 4 ; & P99026 & Psmb4 & $-0,6$ & 3,4 \\
\hline Proteasome subunit beta type- 5 & 055234 & Psmb5 & $-0,3$ & 5,3 \\
\hline Proteasome subunit beta type- 6 ; & Q60692 & Psmb6 & 0,5 & 13 \\
\hline Proteasome subunit beta type-7; & P70195 & Psmb7 & $-0,7$ & 7,6 \\
\hline Protein 2010109I03Rik; RIKEN cDNA 2010109I03; & Q9CQ11 & 2010109I03Rik & $-0,9$ & 15,3 \\
\hline Protein 5430401F13Rik; & E9Q328 & 5430401F13Rik & $-0,2$ & 17,1 \\
\hline Protein Ahnak; & E9Q616 & Ahnak & $-0,3$ & 53,1 \\
\hline Protein Ahnak2; & E9PYB0 & Ahnak2 & 0,2 & 34,7 \\
\hline Protein Ahnak2; & F7CVJ5 & Ahnak2 & 0,1 & 47,8 \\
\hline Protein Ahnak2; & F7DBB3 & Ahnak2 & 0,4 & 27,9 \\
\hline Protein Aim1l; & B7ZCC2 & Aim1l & $-0,4$ & 1,6 \\
\hline Protein Arhgef5; & E9Q7D5 & Arhgef5 & $-2,5$ & 2,3 \\
\hline Protein BC117090; & L7N257 & BC117090 & $-0,2$ & 32,3 \\
\hline Protein Bpifb9b; & A2AJD1 & Bpifb9b & $-0,3$ & 12,4 \\
\hline Protein C10; & 035127 & Grcc10 & $-0,2$ & 11,9 \\
\hline Protein C8orf37 homolog; & Q3UJP5 & & 0,0 & 5,7 \\
\hline Protein Cald1; & D3Z617 & Cald1 & 0,1 & 7,4 \\
\hline Protein canopy homolog 2; & Q9QXT0 & Cnpy2 & 0,1 & 21,4 \\
\hline Protein canopy homolog 3 ; & Q9DAU1 & Cnpy3 & 0,3 & 10,9 \\
\hline Protein canopy homolog 4; & Q8BQ47 & Cnpy4 & 0,2 & 12,7 \\
\hline Protein CDV3; & Q4VAA2 & Cdv3 & $-0,2$ & 40,6 \\
\hline Protein cordon-bleu; & Q5NBX1 & Cobl & $-0,5$ & 18,9 \\
\hline Protein Crnn; & D3YUU6 & Crnn & $-1,2$ & 4,8 \\
\hline Protein CutA; & Q9CQ89 & Cuta & 0,2 & 9,1 \\
\hline Protein DEK; & Q7TNV0 & Dek & 0,2 & 15,3 \\
\hline Protein disulfide-isomerase $\mathrm{A} 3$; & P27773 & Pdia3 & 0,0 & 50,1 \\
\hline Protein disulfide-isomerase A4; & P08003 & Pdia4 & 0,3 & 5,2 \\
\hline Protein disulfide-isomerase A6; & Q3TML0 & Pdia6 & 0,4 & 22,9 \\
\hline Protein disulfide-isomerase; & P09103 & P4hb & 0,9 & 34,8 \\
\hline Protein DJ-1; & Q99LX0 & Park7 & $-0,1$ & 46,3 \\
\hline Protein Dr1; & Q91WV0 & Dr1 & $-0,2$ & 11,4 \\
\hline Protein FAM107B; & Q3TGF2 & Fam107b & 0,5 & 25,2 \\
\hline Protein FAM162A; & Q9D6U8 & Fam162a & $-0,8$ & 18,1 \\
\hline Protein FAM177A1; & Q8BR63 & Fam177a1 & 0,0 & 9,7 \\
\hline Protein FAM192A; & Q91WE2 & Fam192a & 0,1 & 9,8 \\
\hline Protein FAM195B; & Q3UGS4 & Fam195b & 0,3 & 41,2 \\
\hline Protein FAM25C; & Q8CF02 & Fam 25c & 0,0 & 43,8 \\
\hline Protein FAM50B; & Q9WTJ8 & Fam50b & 0,2 & 3,9 \\
\hline Protein Fcgbp; & E9Q0B5 & Fcgbp & 0,6 & 0,6 \\
\hline Protein Fga; & E9PV24 & Fga & 1,3 & 3,6 \\
\hline Protein Gm14744; & B1AVU4 & Gm14744 & $-0,2$ & 22 \\
\hline Protein Gm5938; & A2AEN9 & Gm5938 & 0,1 & 33,7 \\
\hline Protein Gm6537; & J3QPU5 & Gm6537 & $-0,3$ & 22,6 \\
\hline Protein Golgb1; & E9PVZ8 & Golgb1 & 0,5 & 1,5 \\
\hline Protein HEXIM1; & Q8R409 & Hexim1 & $-0,2$ & 18,8 \\
\hline Protein Krt78; & E9Q0F0 & Krt78 & 0,0 & 41,2 \\
\hline Protein lin-7 homolog C; & 088952 & $\operatorname{Lin} 7 c$ & 0,1 & 5,6 \\
\hline Protein Lmo7; & E9PYF4 & Lmo7 & 0,3 & 1,7 \\
\hline Protein lunapark; & Q7TQ95 & Lnp & 0,1 & 12,5 \\
\hline Protein LYRIC; & Q80WJ7 & Mtdh & 0,6 & 15,9 \\
\hline
\end{tabular}




\begin{tabular}{|c|c|c|c|c|}
\hline Protein & Uniprot & Gene name & $\begin{array}{l}\text { Fold change of } \\
\text { XPRESS ratio } \\
\left(\text { Egfr }^{\Delta K C} / \mathrm{WT}\right)\end{array}$ & $\begin{array}{c}\text { Sequence } \\
\text { coverage [\%] }\end{array}$ \\
\hline Protein LZIC; & Q8K3C3 & Lzic & 0,2 & 5,8 \\
\hline Protein max; & P28574 & Max & 0,1 & 13,2 \\
\hline Protein Nolc1; & E9Q5C9 & Nolc1 & 0,2 & 14 \\
\hline Protein Nup153; & E9Q3G8 & Nup153 & 0,5 & 2 \\
\hline Protein Obp1b; & A2AEPO & Obp1b & 0,0 & 18,7 \\
\hline Protein Palm2Akap2; & F7AA26 & Palm2Akap2 & 0,6 & 5,3 \\
\hline Protein Pcdh1; Protocadherin 1; & Q8CFX3 & Pcdh1 & 0,2 & 3,4 \\
\hline Protein Pfdn4; & E9Q6U4 & Pfdn4 & 0,2 & 20,8 \\
\hline Protein phosphatase 1 regulatory subunit $12 \mathrm{~A}$; & Q9DBR7 & Ppp1r12a & 0,0 & 1 \\
\hline Protein phosphatase 1 regulatory subunit 14A; & Q91VC7 & Ppp1r14a & 0,3 & 7,5 \\
\hline Protein phosphatase 1 regulatory subunit 14C; & Q8R4S0 & Ppp1r14c & 0,7 & 22,6 \\
\hline Protein phosphatase 1G; & Q61074 & Ppm1g & 0,0 & 2,2 \\
\hline Protein phosphatase inhibitor 2 & Q9DCL8 & Ppp1r2 & $-0,2$ & 17,9 \\
\hline Protein POF1B; & Q8K4L4 & Pof1b & $-0,3$ & 19,8 \\
\hline Protein Prol1; & E9PYQ4 & Prol1 & 0,4 & 2,2 \\
\hline Protein PRRC1; & Q3UPH1 & Prrc1 & 0,0 & 7,7 \\
\hline Protein PRRC2A; & Q7TSC1 & Prrc2a & 1,3 & 3,1 \\
\hline Protein PRRC2C; & Q3TLH4 & Prrc2c & 0,0 & 3,9 \\
\hline Protein Rarres1; & J3QNI9 & Rarres1 & 0,3 & 6 \\
\hline Protein Red; & Q9Z1M8 & lk & $-0,1$ & 4,5 \\
\hline Protein S100-A11; & P50543 & S100a11 & 0,1 & 18,4 \\
\hline Protein S100-A13; & P97352 & S100a13 & 0,3 & 31,6 \\
\hline Protein S100-A14; & Q9D2Q8 & S100a14 & $-0,2$ & 59,6 \\
\hline Protein S100a16; & D3Z2Y6 & S100a16 & 0,4 & 16,4 \\
\hline Protein S100-A3; & P62818 & S100a3 & $-3,8$ & 10,9 \\
\hline Protein S100-A6; & P14069 & S100a6 & $-0,4$ & 16,9 \\
\hline Protein S100-A9; & P31725 & S100a9 & 1,9 & 12,4 \\
\hline Protein Scgb1b27; & G3UXN8 & Scgb1b27 & $-0,1$ & 10 \\
\hline Protein Sec16a; & A2AIX1 & Sec16a & 0,0 & 2 \\
\hline $\begin{array}{l}\text { Protein Serpinb3c; Serine (Or cysteine) peptidase in hibitor, clade B, member } \\
\text { 3C; }\end{array}$ & A2RSF9 & Serpinb3c & $-0,6$ & 18,7 \\
\hline Protein SET; & Q9EQU5 & Set & $-0,1$ & 26 \\
\hline Protein Sf3b2; Splicing factor 3b, subunit 2; & Q3UJB0 & $\mathrm{Sf} 3 \mathrm{~b} 2$ & 0,0 & 14,2 \\
\hline Protein Spink5; Serine peptidase inhibitor, Kazal type 5; & Q148R4 & Spink5 & 0,0 & 16,4 \\
\hline Protein Srsf11; & D3Z4BO & Srsf11 & 0,5 & 8,9 \\
\hline Protein Tex264; & E9Q137 & Tex264 & 0,4 & 3,2 \\
\hline Protein Tfg; TFG protein; Trk-fused; Trk-fused protein; & Q9Z1A1 & $\operatorname{Tfg}$ & 0,1 & 4,3 \\
\hline Protein transport protein Sec31A; & Q3UPL0 & Sec31a & 0,1 & 3,4 \\
\hline Protein transport protein Sec61 subunit beta; & Q9cQS8 & Sec61b & 0,0 & 11,5 \\
\hline Protein-glutamine gamma-glutamyltransferase E; & Q08189 & $\operatorname{Tgm} 3$ & $-0,3$ & 4,2 \\
\hline Proteolipid protein 2 & Q9R1Q7 & Plp2 & $-0,6$ & 7,9 \\
\hline Prothymosin alpha; & P26350 & Ptma & 0,0 & 20,7 \\
\hline Protocadherin Fat 3; & Q8BNA6 & Fat3 & $-0,1$ & 0,4 \\
\hline Pumilio homolog 1; & Q80U78 & Pum1 & 0,0 & 7,8 \\
\hline Purine nucleoside phosphorylase; & P23492 & Pnp & $-0,3$ & 7,3 \\
\hline Putative phospholipase B-like 2; & Q3TCN2 & Plbd2 & $-0,3$ & 3,2 \\
\hline Putative RNA-binding protein 3 ; & 089086 & $\mathrm{Rbm} 3$ & 0,4 & 28,1 \\
\hline Pyrethroid hydrolase Ces2e; & Q8BK48 & Ces2e & 0,2 & 2,5 \\
\hline Pyruvate kinase PKM; & P52480 & Pkm & $-0,5$ & 10 \\
\hline Rab GDP dissociation inhibitor beta; & Q61598 & Gdi2 & 0,4 & 8,5 \\
\hline Rab11 family-interacting protein 1 ; & E9Q8L9 & Rab11fip1 & 0,0 & 1,9 \\
\hline Rab-like protein 6; & Q5U3K5 & Rabl6 & 0,6 & 2,3 \\
\hline Radixin; & P26043 & $\mathrm{Rdx}$ & 0,5 & 7,5 \\
\hline Ragulator complex protein LAMTOR1; & Q9CQ22 & Lamtor1 & 0,7 & 8,1 \\
\hline Ragulator complex protein LAMTOR5; & Q9D1L9 & Lamtor5 & 0,2 & 22 \\
\hline Ran-specific GTPase-activating protein; & P34022 & Ranbp1 & $-0,8$ & 18,7 \\
\hline Rap guanine nucleotide exchange factor 2 ; & Q8CHG7 & Rapgef2 & $-1,3$ & 0,9 \\
\hline Ras GTPase-activating protein-binding protein 1 ; & P97855 & G3bp1 & 0,1 & 18,5 \\
\hline Ras GTPase-activating-like protein IQGAP1; & Q9JKF1 & Iqgap1 & $-1,3$ & 0,7 \\
\hline Ras suppressor protein 1 & Q01730 & Rsu1 & 0,6 & 6,5 \\
\hline Ras-related protein Rab-11B; & P46638 & Rab11b & 0,7 & 29,8 \\
\hline Ras-related protein Rab-1A; & P62821 & Rab1A & $-0,3$ & 17,3 \\
\hline Ras-related protein Rab-5A; & Q9CQD1 & Rab5a & $-0,5$ & 5,6 \\
\hline Ras-related protein Rab-7a; & P51150 & Rab7a & 0,0 & 6,8 \\
\hline Regulator of cell cycle RGCC; & Q9DBX1 & Rgcc & 0,6 & 8 \\
\hline Repetin; & P97347 & Rptn & $-0,1$ & 6 \\
\hline Reticulocalbin-1; & Q05186 & Ren1 & 0,1 & 10,5 \\
\hline Reticulocalbin-2; & Q8BP92 & Ren2 & $-0,4$ & 4,7 \\
\hline Reticulocalbin-3; & Q8BH97 & Ren3 & 0,5 & 13,1 \\
\hline Reticulon-3; & Q9ES97 & Rtn3 & 0,4 & 1,1 \\
\hline Reticulon-4; & Q99P72 & Rtn4 & 0,5 & 7 \\
\hline
\end{tabular}




\begin{tabular}{|c|c|c|c|c|}
\hline Protein & Uniprot & Gene name & $\begin{array}{l}\text { Fold change of } \\
\text { XPRESS ratio } \\
\left(\text { Egfr }^{\Delta K C} / \mathrm{WT}\right)\end{array}$ & $\begin{array}{c}\text { Sequence } \\
\text { coverage [\%] }\end{array}$ \\
\hline Retinoid-inducible serine carboxypeptidase; & Q920A5 & Scpep1 & $-0,3$ & 10,2 \\
\hline Retroviral-like aspartic protease 1 & Q09PK2 & Asprv1 & $-0,2$ & 15 \\
\hline Rho GDP-dissociation inhibitor 1; & Q99PT1 & Arhgdia & 0,6 & 7,8 \\
\hline Rho GDP-dissociation inhibitor 2; & Q61599 & Arhgdib & $-0,1$ & 32 \\
\hline Rho-related GTP-binding protein RhoC; & Q62159 & Rhoc & $-0,2$ & 9,4 \\
\hline Ribonuclease inhibitor; & Q91VI7 & Rnh1 & 0,2 & 3,9 \\
\hline Ribonuclease T2; & Q9CQ01 & Rnaset2 & $-2,8$ & 3,5 \\
\hline Ribonuclease UK114; & P52760 & Hrsp12 & $-0,4$ & 12,6 \\
\hline Ribosomal protein; & Q5XJF6 & Rpl10a & $-0,3$ & 17,1 \\
\hline Ribosome-binding protein 1 ; & Q99PL5 & Rrbp1 & 0,5 & 8,3 \\
\hline Ribosome-recycling factor, mitochondrial; & Q9D6S7 & Mrrf & 0,1 & 6,5 \\
\hline RNA polymerase-associated protein LEO1; & Q5XJE5 & Leo1 & $-2,0$ & 1,9 \\
\hline RNA-binding protein 8A; & Q9CWZ3 & Rbm8a & 0,1 & 6,3 \\
\hline RNA-binding protein EWS; & Q61545 & Ewsr1 & $-0,5$ & 7,6 \\
\hline RNA-binding protein FUS; & P56959 & Fus & $-0,4$ & 13,1 \\
\hline RNA-binding protein Raly; & Q64012 & Raly & $-0,1$ & 13,4 \\
\hline RNMT-activating mini protein; & Q9CQY2 & Fam103a1 & $-0,4$ & 14,6 \\
\hline RWD domain-containing protein 4 ; & Q9CPR1 & Rwdd4 & $-1,0$ & 10,1 \\
\hline SAP domain-containing ribonucleoprotein; & Q9D1J3 & Sarnp & 0,2 & 34,3 \\
\hline SAYSvFN domain-containing protein 1 & Q8K190 & Saysd1 & $-0,3$ & 10,6 \\
\hline Scaffold attachment factor B1; & D3YXK2 & Safb & $-0,1$ & 3,2 \\
\hline Scaffold attachment factor B2; & Q80YR5 & Safb2 & 0,4 & 2,5 \\
\hline Sciellin; & Q9EQG3 & Scel & 0,1 & 4,3 \\
\hline Secretoglobin family 2B member 2 ; & Q6UGQ3 & Scgb2b2 & $-0,3$ & 24,6 \\
\hline Secretoglobin family 2B member 20 ; & J3QK77 & Scgb2b20 & $-0,2$ & 21,4 \\
\hline Secretory carrier-associated membrane protein 1 ; & Q8K021 & Scamp1 & 0,1 & 14,8 \\
\hline Secretory carrier-associated membrane protein 2 ; & Q9ERNO & Scamp2 & 0,7 & 9,7 \\
\hline Selenium-binding protein 1 & P17563 & Selenbp1 & $-0,2$ & 8,5 \\
\hline Serine hydroxymethyltransferase; & Q9CZN7 & Shmt2 & $-1,1$ & 5,4 \\
\hline Serine/arginine-rich splicing factor 1 ; & Q6PDM2 & Srsf1 & $-0,1$ & 17,3 \\
\hline Serine/arginine-rich splicing factor 2 & Q62093 & Srsf2 & 0,7 & 14,5 \\
\hline Serine/arginine-rich splicing factor 3 ; & P84104 & Srsf3 & 0,4 & 14 \\
\hline Serine/arginine-rich splicing factor 4 ; & Q8VE97 & Srsf4 & 0,8 & 4,5 \\
\hline Serine/arginine-rich splicing factor 5 & 035326 & Srsf5 & 0,0 & 20,1 \\
\hline Serine/arginine-rich splicing factor 6 ; & Q3TWW8 & Srsf6 & 0,5 & 5,3 \\
\hline Serine/arginine-rich splicing factor 7 & Q8BL97 & Srsf7 & 0,2 & 8,6 \\
\hline $\begin{array}{l}\text { Serine/threonine-protein phosphatase } 2 \mathrm{~A} 55 \mathrm{kDa} \text { regulatory subunit B alpha } \\
\text { isoform; }\end{array}$ & Q6P1F6 & Ppp2r2a & 0,3 & 2,5 \\
\hline Serine/threonine-protein phosphatase 6 regulatory subunit 3; & Q922D4 & Ppp6r3 & $-0,5$ & 1,4 \\
\hline Serotransferrin; & Q921I1 & Tf & $-0,1$ & 16,1 \\
\hline Serpin A12; & Q7TMF5 & Serpina12 & $-0,1$ & 17,2 \\
\hline Serpin A9; & Q9D7D2 & Serpina9 & 0,0 & 4,5 \\
\hline Serpin B12; & Q9D7P9 & Serpinb12 & $-0,4$ & 27,4 \\
\hline Serpin B5; & P70124 & Serpinb5 & 0,1 & 21,9 \\
\hline Serpin B7; & Q9D695 & Serpinb7 & 0,1 & 16,8 \\
\hline Serpin B8; & 008800 & Serpinb8 & 0,0 & 7,5 \\
\hline Serpin $\mathrm{H} 1$; & P19324 & Serpinh1 & 1,0 & 2,6 \\
\hline Serum albumin; & P07724 & Alb & 0,9 & 44,9 \\
\hline Serum amyloid A-2 protein; & P05367 & Saa2 & 1,6 & 15,6 \\
\hline SH3 domain-binding glutamic acid-rich-like protein 3; & Q91VW3 & Sh3bgrl3 & 0,1 & 26,9 \\
\hline SH3 domain-binding glutamic acid-rich-like protein; & Q9JJU8 & Sh3bgrl & 0,1 & 36 \\
\hline SH3 domain-containing kinase-binding protein 1 ; & Q8R550 & Sh3kbp1 & 0,2 & 9,6 \\
\hline Short-chain dehydrogenase/reductase family $9 \mathrm{C}$ member 7 ; & Q8K3P0 & Sdr9c7 & $-0,3$ & 33,9 \\
\hline Sickle tail protein; & A2AQ25 & Skt & 1,7 & 1,9 \\
\hline SLAIN motif-containing protein 2 ; & Q8C108 & Slain2 & 0,5 & 8,8 \\
\hline Small acidic protein; & Q9R0P4 & Smap & 0,1 & 21,5 \\
\hline Small glutamine-rich tetratricopeptide repeat-containing protein alpha; & Q8BJU0 & Sgta & $-4,2$ & 7,3 \\
\hline Small nuclear ribonucleoprotein F; & P62307 & Snrpf & 0,1 & 9,3 \\
\hline Small nuclear ribonucleoprotein Sm D2; & P62317 & Snrpd2 & $-0,2$ & 32,2 \\
\hline Small nuclear ribonucleoprotein Sm D3; & P62320 & Snrpd3 & 0,2 & 7,1 \\
\hline Small ubiquitin-related modifier 2 & P61957 & Sumo2 & 0,1 & 16,9 \\
\hline SNW domain-containing protein 1 ; & Q9CSN1 & Snw1 & $-0,2$ & 8,4 \\
\hline Sodium/potassium-transporting ATPase subunit beta- 3 ; & P97370 & Atp1b3 & $-0,2$ & 18 \\
\hline Sorcin; & Q6P069 & Sri & $-0,2$ & 19,2 \\
\hline Sorting nexin-3; & 070492 & Snx3 & $-0,1$ & 17,9 \\
\hline SPARC; & P07214 & Sparc & $-0,6$ & 7,6 \\
\hline Specifically androgen-regulated gene protein; & Q8BI29 & Sarg & $-0,2$ & 25,2 \\
\hline Spectrin alpha chain, non-erythrocytic 1 ; & E9Q447 & Sptan1 & 0,6 & 4,6 \\
\hline Spectrin beta chain, non-erythrocytic 1 ; & Q62261 & Sptbn1 & 0,2 & 1,3 \\
\hline Sperm-associated antigen 7; & Q7TNE3 & Spag7 & 0,1 & 15,9 \\
\hline
\end{tabular}




\begin{tabular}{|c|c|c|c|c|}
\hline Protein & Uniprot & Gene name & $\begin{array}{l}\text { Fold change of } \\
\text { XPRESS ratio } \\
\left(\text { Egfr }^{\Delta K C} / \mathrm{WT}\right)\end{array}$ & $\begin{array}{c}\text { Sequence } \\
\text { coverage [\%] }\end{array}$ \\
\hline S-phase kinase-associated protein 1 & Q9WTX5 & Skp1 & $-0,7$ & 12,9 \\
\hline Sphingomyelin phosphodiesterase; & Q04519 & Smpd1 & $-0,1$ & 1,6 \\
\hline Spliceosome-associated protein CWC15 homolog; & Q9JHS9 & Cwc15 & 0,1 & 11,4 \\
\hline Splicing factor 1; & Q64213 & Sf1 & 0,3 & 7,1 \\
\hline Splicing factor, proline- and glutamine-rich; & Q8VIJ6 & Sfpq & 0,4 & 2,1 \\
\hline Src substrate cortactin; & Q60598 & Cttn & 0,0 & 30,6 \\
\hline SRSF protein kinase 1 & 070551 & Srpk1 & 0,1 & 3,4 \\
\hline StAR-related lipid transfer protein 5; & Q9EPQ7 & Stard5 & $-0,1$ & 4,4 \\
\hline Stathmin; & P54227 & Stmn1 & 0,2 & 43,1 \\
\hline Stefin-1; & P35175 & Stfa1 & 0,3 & 43,3 \\
\hline Stefin-3; & P35173 & Stfa3 & 0,4 & 68,9 \\
\hline Steryl-sulfatase; & P50427 & Sts & 0,4 & 2,6 \\
\hline Stress-70 protein, mitochondrial; & P38647 & Hspa9 & 2,5 & 6 \\
\hline Stress-induced-phosphoprotein 1 & Q60864 & Stip1 & 0,1 & 28,4 \\
\hline Submandibular gland protein C; & Q6JHY2 & Muc19 & 0,3 & 23,3 \\
\hline Succinate dehydrogenase [ubiquinone] iron-sulfur subunit, mitochondrial; & Q9CQA3 & Sdhb & 0,3 & 8,5 \\
\hline Sulfated glycoprotein 1; & Q61207 & Psap & 0,1 & 19,8 \\
\hline Sulfhydryl oxidase 1 & Q8BND5 & Qsox1 & $-0,6$ & 5,9 \\
\hline Superoxide dismutase [Cu-Zn]; & P08228 & Sod1 & 0,0 & 32,5 \\
\hline Superoxide dismutase [Mn], mitochondrial; & P09671 & Sod2 & $-0,2$ & 9,5 \\
\hline Suprabasin; & E9QPB2 & Sbsn & $-0,2$ & 39 \\
\hline Survival of motor neuron-related-splicing factor 30 ; & Q8BGT7 & Smndc1 & $-0,1$ & 9,2 \\
\hline Sushi domain-containing protein 2 & Q9DBX3 & Susd2 & 1,4 & 2,4 \\
\hline SUZ domain-containing protein 1 ; & Q6NXN1 & Szrd1 & 0,5 & 15,9 \\
\hline Synaptojanin-2-binding protein; & Q9D6K5 & Synj2bp & $-0,1$ & 11,3 \\
\hline Synaptosomal-associated protein 23 & 009044 & Snap23 & 0,3 & 19,1 \\
\hline Synaptosomal-associated protein 29 & Q9ERB0 & Snap29 & 0,1 & 15 \\
\hline Synaptotagmin-like protein 1 & A2AE94 & Sytl1 & $-0,1$ & 6,7 \\
\hline Syndecan-4; & 035988 & Sdc4 & 0,3 & 12,1 \\
\hline Syntaxin-12; & Q9ER00 & Stx12 & $-0,2$ & 26,6 \\
\hline Syntaxin-6; & Q9JKK1 & Stx6 & 0,3 & 4,3 \\
\hline Syntaxin-7; & 070439 & Stx7 & $-0,6$ & 10,7 \\
\hline Syntaxin-8; & 088983 & Stx8 & $-0,1$ & 18,2 \\
\hline Target of Myb protein 1 ; & 088746 & Tom1 & $-0,1$ & 6,3 \\
\hline TATA element modulatory factor; & B9EKI3 & Tmf1 & 0,5 & 1,6 \\
\hline Tetraspanin-9; & Q8BJU2 & Tspan9 & 0,3 & 8,7 \\
\hline Tetratricopeptide repeat protein $1 ;$ & Q91Z38 & Ttc1 & 0,7 & 6,8 \\
\hline Thimet oligopeptidase; & Q8C1A5 & Thop1 & $-0,5$ & 28,2 \\
\hline Thioredoxin domain-containing protein 12 & Q9CQU0 & Txndc12 & 0,2 & 22,9 \\
\hline Thioredoxin domain-containing protein 17 & Q9CQM5 & Txndc17 & $-0,1$ & 26,8 \\
\hline Thioredoxin domain-containing protein 5; & Q91W90 & Txndc5 & 0,9 & 18,3 \\
\hline Thioredoxin; & P10639 & Txn & 0,0 & 29,5 \\
\hline THO complex subunit 4; & 008583 & Alyref & 0,4 & 11,4 \\
\hline Three prime repair exonuclease 2 ; & Q9R1A9 & Trex2 & $-0,8$ & 28,4 \\
\hline Thy-1 membrane glycoprotein; & P01831 & Thy1 & 0,0 & 7,4 \\
\hline Tight junction protein ZO-1; & P39447 & Tjp1 & 0,2 & 2,3 \\
\hline Tissue factor; & P20352 & F3 & 0,5 & 22,4 \\
\hline TP53-regulated inhibitor of apoptosis 1; & Q9D8Z2 & Triap1 & 0,0 & 18,4 \\
\hline Transcription and mRNA export factor ENY2; & Q9JIX0 & Eny2 & 0,2 & 25,7 \\
\hline Transcription elongation factor A protein 1 ; & P10711 & Tcea1 & 0,1 & 7,6 \\
\hline Transcription elongation factor B polypeptide 2 ; & P62869 & Tceb2 & 0,0 & 22 \\
\hline Transcription factor BTF3 homolog 4; & Q9CQH7 & Btf3|4 & $-0,1$ & 20,9 \\
\hline Transcription factor BTF3; & Q64152 & Btf3 & $-0,1$ & 25 \\
\hline Transcription factor Sp1; & 089090 & Sp1 & $-0,2$ & 3,6 \\
\hline Transcription initiation factor IIA subunit 1 ; & Q99PM3 & Gtf2a1 & $-0,1$ & 2,9 \\
\hline Transcription initiation factor TFIID subunit 10; & Q8KOH5 & Taf10 & $-1,5$ & 17,9 \\
\hline Transcriptional activator protein Pur-alpha; & P42669 & Pura & $-0,3$ & 2,8 \\
\hline Transcriptional activator protein Pur-beta; & 035295 & Purb & $-0,2$ & 5,2 \\
\hline Transducin-like enhancer protein 3 & Q08122 & Tle3 & $-0,4$ & 2,7 \\
\hline Transforming acidic coiled-coil-containing protein 2 ; & E9Q8T1 & Tacc2 & 0,0 & 18,6 \\
\hline Transforming acidic coiled-coil-containing protein 2; & E9Q9Z4 & Tacc2 & 0,4 & 20,3 \\
\hline Transgelin-2; & Q9WVA4 & Tagln2 & 0,1 & 49,7 \\
\hline Trans-Golgi network integral membrane protein 1 ; & Q62313 & Tgoln1 & 0,1 & 4,5 \\
\hline Transitional endoplasmic reticulum ATPase; & Q01853 & $V_{c p}$ & 0,0 & 15,3 \\
\hline Transketolase; & P40142 & Tkt & $-1,1$ & 7,2 \\
\hline Translationally-controlled tumor protein; & P63028 & Tpt1 & 0,0 & 30,2 \\
\hline Translocon-associated protein subunit alpha; & Q9CY50 & Ssr1 & $-0,2$ & 11,9 \\
\hline Transmembrane emp24 domain-containing protein 10; & Q9D1D4 & Tmed10 & 0,3 & 9,6 \\
\hline Transmembrane protein 109; & Q3UBX0 & Tmem109 & $-0,6$ & 16,4 \\
\hline Transmembrane protein 40 ; & Q4FJU9 & Tmem40 & $-0,9$ & 6,5 \\
\hline
\end{tabular}




\begin{tabular}{|c|c|c|c|c|}
\hline Protein & Uniprot & Gene name & $\begin{array}{l}\text { Fold change of } \\
\text { XPRESS ratio } \\
\left(\text { Egfr }^{\Delta K C} / \mathrm{WT}\right)\end{array}$ & $\begin{array}{c}\text { Sequence } \\
\text { coverage [\%] }\end{array}$ \\
\hline Transmembrane protein 79; & Q9D709 & Tmem79 & $-2,5$ & 6,6 \\
\hline Transthyretin; & P07309 & $\operatorname{Ttr}$ & 0,7 & 30,6 \\
\hline Treacle protein; & 008784 & Tcof1 & 0,7 & 10,9 \\
\hline Trinucleotide repeat-containing gene 6B protein; & Q8BKI2 & Tnrc6b & $-0,1$ & 1,2 \\
\hline Triosephosphate isomerase; & P17751 & Tpi1 & 0,2 & 30,4 \\
\hline Tripartite motif-containing protein 29 & Q8R2Q0 & Trim29 & 0,0 & 14,3 \\
\hline Tripeptidyl-peptidase 1 & 089023 & Tpp1 & 0,0 & 6,2 \\
\hline tRNA methyltransferase 112 homolog; & Q9DCG9 & Trmt112 & 0,7 & 12 \\
\hline Tropomodulin-3; & Q9JHJ0 & Tmod3 & 0,1 & 19,9 \\
\hline Tropomyosin alpha-1 chain; & E9Q450 & Tpm1 & $-0,5$ & 25,7 \\
\hline Tropomyosin alpha-3 chain; & E9Q7Q3 & Tpm3 & $-0,2$ & 26,2 \\
\hline Tropomyosin alpha-4 chain; & Q6IRU2 & Tpm4 & $-0,1$ & 30,6 \\
\hline Tropomyosin beta chain; & A2AIM4 & Tpm2 & $-0,3$ & 29,9 \\
\hline Troponin C, skeletal muscle; & P20801 & Tnnc2 & $-0,2$ & 20,6 \\
\hline Troponin C, slow skeletal and cardiac muscles; & P19123 & Tnnc1 & 1,0 & 13,7 \\
\hline TSC22 domain family protein 1 & P62500 & $\mathrm{Tsc} 22 \mathrm{~d} 1$ & 0,7 & 14 \\
\hline Tubulin alpha-1B chain; & P05213 & Tuba1b & 0,1 & 24,2 \\
\hline Tubulin alpha-4A chain; & P68368 & Tuba4a & $-0,4$ & 24,6 \\
\hline Tubulin beta-2A chain; & Q7TMM9 & Tubb2a & 0,2 & 39,8 \\
\hline Tubulin beta- 3 chain; & Q9ERD7 & Tubb3 & $-0,4$ & 32,7 \\
\hline Tubulin beta-4A chain; & Q9D6F9 & Tubb4a & 0,3 & 34,7 \\
\hline Tubulin beta-4B chain; & P68372 & Tubb4b & 0,1 & 44 \\
\hline Tubulin beta- 5 chain; & P99024 & Tubb5 & 0,7 & 41,9 \\
\hline Tubulin polymerization-promoting protein family member 3 ; & Q9CRB6 & Tppp3 & 0,6 & 24,4 \\
\hline Tubulin polymerization-promoting protein; & Q7TQD2 & Tppp & 0,3 & 12,4 \\
\hline Tubulin-specific chaperone A; & P48428 & Tbca & 0,4 & 31,5 \\
\hline Tuftelin; & 008970 & Tuft1 & $-0,1$ & 9,7 \\
\hline Tumor necrosis factor alpha-induced protein 3 & Q60769 & Tnfaip3 & 0,0 & 3,2 \\
\hline Tumor protein D52; & Q62393 & Tpd52 & 0,0 & 24,1 \\
\hline Tumor protein D54; & Q9CYZ2 & $\operatorname{Tpd} 5212$ & 0,3 & 43,2 \\
\hline Tumor protein D54; & F6VQ81 & Tpd5212 & $-0,4$ & 49,7 \\
\hline Tumor-associated calcium signal transducer 2 & Q8BGV3 & Tacstd2 & $-0,3$ & 13,9 \\
\hline Tyrosine-protein phosphatase non-receptor type substrate 1 & P97797 & Sirpa & 0,2 & 4,1 \\
\hline U1 small nuclear ribonucleoprotein A; & Q62189 & Snrpa & 0,0 & 20,6 \\
\hline U1 small nuclear ribonucleoprotein C; & Q62241 & Snrpc & $-0,5$ & 18,9 \\
\hline U2 small nuclear ribonucleoprotein B'"; & Q9CQ17 & Snrpb2 & 0,7 & 10,2 \\
\hline U4/U6.U5 tri-snRNP-associated protein 1; & Q97315 & Sart1 & 0,4 & 5,5 \\
\hline U6 snRNA-associated Sm-like protein LSm3; & P62311 & Lsm3 & 0,1 & 19,6 \\
\hline U6 snRNA-associated Sm-like protein LSm6; & P62313 & Lsm6 & $-0,4$ & 22,5 \\
\hline U6 snRNA-associated Sm-like protein LSm7; & Q9CQQ8 & Lsm7 & $-0,5$ & 15,5 \\
\hline U6 snRNA-associated Sm-like protein LSm8; & Q6ZWM4 & Lsm8 & 0,2 & 10,4 \\
\hline Ubiquilin-1; & Q8R317 & Ubqln1 & $-0,1$ & 13,1 \\
\hline Ubiquilin-2; & Q9QZMO & Ubqln2 & $-0,3$ & 6,3 \\
\hline Ubiquilin-3; & Q8C5U9 & Ubqln3 & 0,2 & 1,5 \\
\hline Ubiquinol-cytochrome-c reductase complex assembly factor 2 & Q9CQY6 & Uqcc2 & 0,5 & 13,4 \\
\hline Ubiquitin-associated protein 2 & Q91VX2 & Ubap2 & 0,0 & 4,2 \\
\hline Ubiquitin-associated protein 2-like; & Q80X50 & Ubap2l & $-0,2$ & 16,3 \\
\hline Ubiquitin-conjugating enzyme E2 L3; & P68037 & Ube2l3 & $-0,1$ & 33,1 \\
\hline Ubiquitin-conjugating enzyme E2 N; & P61089 & Ube2n & 0,1 & 18,4 \\
\hline Ubiquitin-fold modifier 1 & P61961 & Ufm1 & 0,1 & 26,3 \\
\hline UBX domain-containing protein 1 & Q922Y1 & Ubxn1 & $-0,5$ & 13,8 \\
\hline UMP-CMP kinase; & Q9DBP5 & Cmpk1 & 0,1 & 21,4 \\
\hline Uncharacterized protein C10orf35 homolog; & Q9D882 & & $-0,1$ & 24,2 \\
\hline Uncharacterized protein C19orf43 homolog; & Q9D735 & & $-0,2$ & 6,9 \\
\hline Uncharacterized protein C1orf198 homolog; & Q8C3W1 & & 0,0 & 5,6 \\
\hline Uncharacterized protein C1orf50 homolog; & Q5EBG8 & & 0,2 & 8 \\
\hline Uncharacterized protein C5orf46 homolog; & Q3V2D2 & Gm94 & 0,0 & 18,3 \\
\hline Uncharacterized protein C6orf132 homolog; & Q91758 & & $-0,2$ & 13,3 \\
\hline Uncharacterized protein C7orf57 homolog; & Q5SS90 & Gm11992 & $-0,6$ & 19,2 \\
\hline Uncharacterized protein C9orf78 homolog; & Q3TQ17 & & 0,6 & 4,8 \\
\hline Uncharacterized protein; & D3Z2H9 & Tpm3 rs7 & $-0,5$ & 19,4 \\
\hline Unconventional myosin-XVIIla; & Q9JMH9 & Myo18a & $-0,4$ & 3,6 \\
\hline UPF0184 protein C9orf16 homolog; & P58686 & & 0,6 & 10,8 \\
\hline UPF0235 protein C15orf40 homolog; & Q9CRC3 & & 0,0 & 23,8 \\
\hline UPF0449 protein C19orf25 homolog; & Q9D7E4 & & 0,3 & 21,1 \\
\hline Up-regulated during skeletal muscle growth protein 5; & Q78IK2 & Usmg5 & $-0,3$ & 43,1 \\
\hline Upstream stimulatory factor 2 & Q64705 & Usf2 & 0,0 & 4,2 \\
\hline UV excision repair protein RAD23 homolog B; & P54728 & Rad23b & $-0,4$ & 20,4 \\
\hline Vacuolar ATPase assembly integral membrane protein Vma21; & Q78T54 & Vma21 & 0,8 & 11,9 \\
\hline Vacuolar protein sorting-associated protein $4 \mathrm{~B} ;$ & P46467 & Vps4b & 0,3 & 5,2 \\
\hline Vacuolar protein sorting-associated protein VTA1 homolog; & Q9CR26 & Vta1 & $-0,2$ & 6,5 \\
\hline
\end{tabular}




\begin{tabular}{|c|c|c|c|c|}
\hline Protein & Uniprot & Gene name & $\begin{array}{l}\text { Fold change of } \\
\text { XPRESS ratio } \\
\left.\text { (Egfr }{ }^{\Delta K C} / \mathrm{WT}\right)\end{array}$ & $\begin{array}{c}\text { Sequence } \\
\text { coverage [\%] }\end{array}$ \\
\hline Vacuolar protein-sorting-associated protein 25 & Q9CQ80 & Vps25 & 3,3 & 7,8 \\
\hline Vesicle transport through interaction with t-SNAREs homolog 1B; & 088384 & Vti1b & 0,1 & 18,1 \\
\hline Vesicle-associated membrane protein 2; & P63044 & Vamp2 & 0,9 & 34,5 \\
\hline Vesicle-associated membrane protein 3 ; & P63024 & Vamp3 & $-0,5$ & 38,8 \\
\hline Vesicle-associated membrane protein 4; & 070480 & Vamp4 & 0,4 & 13,5 \\
\hline Vesicle-associated membrane protein 8; & 070404 & Vamp8 & 0,0 & 30,7 \\
\hline Vesicle-associated membrane protein-associated protein A; & Q9WV55 & Vapa & 0,2 & 13,3 \\
\hline Vesicle-associated membrane protein-associated protein B; & Q9QY76 & Vapb & 0,2 & 10,7 \\
\hline Vimentin; & P20152 & Vim & 0,4 & 32,2 \\
\hline Vinculin; & Q64727 & $\mathrm{Vcl}$ & $-0,3$ & 22,4 \\
\hline Voltage-dependent anion-selective channel protein $1 ;$ & Q60932 & Vdac1 & $-0,1$ & 20,6 \\
\hline V-type proton ATPase subunit E 1 ; & P50518 & Atp6v1e1 & 0,2 & 24,3 \\
\hline V-type proton ATPase subunit F; & Q9D1K2 & Atp6v1f & $-0,6$ & 26,9 \\
\hline V-type proton ATPase subunit $\mathrm{G} 1$; & Q9CR51 & Atp6v1g1 & $-1,0$ & 23,7 \\
\hline V-type proton ATPase subunit S1; & Q9R1Q9 & Atp6ap1 & $-0,3$ & 6,5 \\
\hline WAS/WASL-interacting protein family member 2 ; & Q6PEV3 & Wipf2 & 0,4 & 13,9 \\
\hline WASH complex subunit FAM21; & Q6PGL7 & Fam21 & 0,0 & 4,4 \\
\hline Wiskott-Aldrich syndrome protein family member 2 & Q8BH43 & Wasf2 & 0,0 & 6,6 \\
\hline WW domain-binding protein 11 & Q923D5 & Wbp11 & 0,3 & 12,6 \\
\hline X-ray repair cross-complementing protein 6; & P23475 & Xrcc6 & 0,4 & 1,5 \\
\hline Y-box-binding protein 3 & Q9JKB3 & Ybx3 & $-0,5$ & 16,6 \\
\hline Yorkie homolog; & P46938 & Yap1 & 0,0 & 16,6 \\
\hline Zinc finger $\mathrm{CCCH}$ domain-containing protein 15 ; & Q3TIV5 & Zc3h15 & 0,5 & 12,2 \\
\hline Zinc finger $\mathrm{CCCH}$ domain-containing protein 18 ; & Q0P678 & Zc3h18 & $-0,1$ & 3 \\
\hline Zinc finger protein 185 & A2BI37 & Zfp185 & $-0,8$ & 10,1 \\
\hline Zinc finger Ran-binding domain-containing protein 2; & Q9R020 & Zranb2 & 0,6 & 6,5 \\
\hline Zinc transporter ZIP10; & Q6P5F6 & Slc39a10 & 0,1 & 2,3 \\
\hline \multirow[t]{6}{*}{ Zyxin; } & Q62523 & Zyx & 0,2 & 5,5 \\
\hline & D3YW41 & & 0,2 & 28,8 \\
\hline & F6TL02 & & $-0,4$ & 39,3 \\
\hline & E9Q019 & & $-0,5$ & 23,6 \\
\hline & J3QMA3 & & $-0,2$ & 27,5 \\
\hline & J3QNE6 & & $-0,3$ & 32 \\
\hline
\end{tabular}


Fold change of

XPRESS ratio

Sequence (Egfr $\left.{ }^{\Delta K C} / \mathrm{WT}\right)$ 
Supplementary table 5: List of all identified and quantified proteins in the quantitative proteome comparison of wild-type and $A d a m 17^{\Delta K C}$ epidermis at P10 (replicate 2).

\begin{tabular}{|c|c|c|c|c|}
\hline Protein & Uniprot & Gene name & $\begin{array}{c}\text { Fold change of } \\
\text { XPRESS ratio } \\
\left.\text { (Adam17 }{ }^{\Delta K C} / \mathrm{WT}\right)\end{array}$ & $\begin{array}{c}\text { Sequence } \\
\text { coverage [\%] }\end{array}$ \\
\hline 10 kDa heat shock protein, mitochondrial; & Q64433 & Hspe1 & $-0,1$ & 34,3 \\
\hline 116 kDa U5 small nuclear ribonucleoprotein component; & 008810 & Eftud2 & 0,1 & 16,7 \\
\hline 14-3-3 protein beta/alpha; & Q9CQV8 & Ywhab & 0,4 & 29,3 \\
\hline 14-3-3 protein epsilon; & P62259 & Ywhae & 0,1 & 40 \\
\hline 14-3-3 protein eta; & P68510 & Ywhah & 0,2 & 30,1 \\
\hline 14-3-3 protein gamma; & P61982 & Ywhag & 0,2 & 16,2 \\
\hline 14-3-3 protein sigma; & 070456 & Sfn & $-0,3$ & 51,2 \\
\hline 14-3-3 protein theta; & P68254 & Ywhaq & 0,2 & 29,4 \\
\hline 14-3-3 protein zeta/delta; & P63101 & Ywhaz & 0,2 & 25,3 \\
\hline 182 kDa tankyrase-1-binding protein; & P58871 & Tnks1bp1 & $-0,1$ & 14 \\
\hline 1-acylglycerol-3-phosphate O-acyltransferase ABHD5; & Q9DBL9 & Abhd5 & $-0,3$ & 4,8 \\
\hline 1-acyl-sn-glycerol-3-phosphate acyltransferase gamma; & Q9D517 & Agpat3 & 0,4 & 2,7 \\
\hline 2,4-dienoyl-CoA reductase, mitochondrial; & Q9CQ62 & Decr1 & 0,2 & 28,7 \\
\hline 2310061N02Rik protein; MCG146860; Protein 2310061N02Rik; & Q9D6S9 & 2310061N02Rik & $-2,0$ & 5,7 \\
\hline $26 \mathrm{~S}$ protease regulatory subunit $10 \mathrm{~B}$ & P62334 & Psmc6 & 0,6 & 17 \\
\hline 265 protease regulatory subunit 4 & P62192 & Psmc1 & 0,3 & 19,1 \\
\hline $26 \mathrm{~S}$ protease regulatory subunit $6 \mathrm{~A}$ & 088685 & Psmc3 & 0,6 & 26,3 \\
\hline $26 \mathrm{~S}$ protease regulatory subunit $6 \mathrm{~B}$ & P54775 & Psmc4 & 0,6 & 18,2 \\
\hline 265 protease regulatory subunit 7 & P46471 & Psmc2 & 0,5 & 32,1 \\
\hline $26 \mathrm{~S}$ protease regulatory subunit 8 & P62196 & Psmc5 & 0,1 & 17 \\
\hline $26 \mathrm{~S}$ proteasome non-ATPase regulatory subunit 1 & Q3TXS7 & Psmd1 & 0,2 & 15,4 \\
\hline $26 \mathrm{~S}$ proteasome non-ATPase regulatory subunit 11 & Q8BG32 & Psmd11 & 0,6 & 30,8 \\
\hline $26 \mathrm{~S}$ proteasome non-ATPase regulatory subunit 12 & Q9D8W5 & Psmd12 & 0,6 & 8,3 \\
\hline $26 \mathrm{~S}$ proteasome non-ATPase regulatory subunit 13 & Q9WVJ2 & Psmd13 & 0,4 & 35,1 \\
\hline $26 \mathrm{~S}$ proteasome non-ATPase regulatory subunit 14 & 035593 & Psmd14 & 0,3 & 10,6 \\
\hline $26 \mathrm{~S}$ proteasome non-ATPase regulatory subunit 2 & Q8VDM4 & Psmd2 & 0,4 & 23,5 \\
\hline $26 \mathrm{~S}$ proteasome non-ATPase regulatory subunit 3 & P14685 & Psmd3 & $-0,1$ & 22,8 \\
\hline $26 \mathrm{~S}$ proteasome non-ATPase regulatory subunit 4 & 035226 & Psmd4 & $-0,3$ & 4 \\
\hline $26 \mathrm{~S}$ proteasome non-ATPase regulatory subunit 6 & Q99JI4 & Psmd6 & 0,2 & 26,2 \\
\hline $26 \mathrm{~S}$ proteasome non-ATPase regulatory subunit 7 & P26516 & Psmd7 & 0,3 & 33,6 \\
\hline $26 \mathrm{~S}$ proteasome non-ATPase regulatory subunit 8 & Q9CX56 & Psmd8 & 0,0 & 14,2 \\
\hline $26 \mathrm{~S}$ proteasome non-ATPase regulatory subunit 9 & Q9CR00 & Psmd9 & 0,2 & 14,9 \\
\hline 28 kDa heat- and acid-stable phosphoprotein; & Q3UHX2 & Pdap1 & $-0,1$ & 17,1 \\
\hline $28 \mathrm{~S}$ ribosomal protein $\mathrm{S} 36$, mitochondrial; & Q9CQX8 & Mrps36 & $-0,2$ & 56,9 \\
\hline 2-amino-3-ketobutyrate coenzyme A ligase, mitochondrial; & 088986 & Gcat & 0,0 & 19,6 \\
\hline 2-aminoethanethiol dioxygenase; & Q6PDY2 & Ado & $-0,3$ & 12,9 \\
\hline 2'-deoxynucleoside 5'-phosphate N-hydrolase 1 ; & Q80VJ3 & Dnph1 & $-1,7$ & 8,1 \\
\hline 2-oxoglutarate dehydrogenase, mitochondrial; & Q60597 & Ogdh & $-0,4$ & 2,3 \\
\hline $3^{\prime}\left(2^{\prime}\right), 5^{\prime}$-bisphosphate nucleotidase 1 & Q9Z0S1 & Bpnt1 & $-0,5$ & 30 \\
\hline 3-beta-hydroxysteroid-Delta(8),Delta(7)-isomerase; & P70245 & Ebp & 0,4 & 5,2 \\
\hline 3-hydroxyacyl-CoA dehydrogenase type-2; & A2AFQ2 & Hsd17b10 & 0,0 & 12,6 \\
\hline 3-hydroxyisobutyrate dehydrogenase, mitochondrial; & Q99L13 & Hibadh & $-1,5$ & 12,5 \\
\hline 3-ketoacyl-CoA thiolase A, peroxisomal; & Q921H8 & Acaa1a & 0,3 & 27,5 \\
\hline 3-ketoacyl-CoA thiolase, mitochondrial; & Q8BWT1 & Acaa2 & $-0,3$ & 33,2 \\
\hline 3-oxoacyl-[acyl-carrier-protein] synthase, mitochondrial; & Q9D404 & Oxsm & 0,6 & 7,8 \\
\hline 40 S ribosomal protein S10; & P63325 & Rps10 & $-0,1$ & 37,6 \\
\hline 40 S ribosomal protein $\mathrm{S} 11$ & P62281 & Rps11 & 0,2 & 39,2 \\
\hline $40 S$ ribosomal protein S12; & Q6ZWZ6 & Rps12 & $-0,3$ & 47 \\
\hline 40 S ribosomal protein S13; & P62301 & Rps13 & $-0,3$ & 38,4 \\
\hline 40 S ribosomal protein S14; & P62264 & Rps14 & $-0,4$ & 17,6 \\
\hline 40 S ribosomal protein S15; & P62843 & Rps15 & $-0,4$ & 10,2 \\
\hline $40 \mathrm{~S}$ ribosomal protein $\mathrm{S} 15 \mathrm{a}$ & P62245 & Rps15a & $-0,1$ & 52,3 \\
\hline 40 S ribosomal protein S16; & P14131 & Rps16 & $-0,1$ & 28,1 \\
\hline 40 S ribosomal protein S17; & P63276 & Rps17 & $-0,6$ & 7,4 \\
\hline 40 S ribosomal protein S18; & P62270 & Rps18 & 0,0 & 38,2 \\
\hline 40 S ribosomal protein S19; & Q9CZX8 & Rps19 & $-0,3$ & 26,9 \\
\hline $40 S$ ribosomal protein $\mathrm{S} 2$ & P25444 & Rps2 & $-0,2$ & 31,1 \\
\hline 40 S ribosomal protein S20; & P60867 & Rps20 & $-0,3$ & 25,2 \\
\hline $40 S$ ribosomal protein $\mathrm{S} 21$ & Q9CQR2 & Rps21 & 0,0 & 39,8 \\
\hline 40 S ribosomal protein S23; & P62267 & Rps23 & $-1,5$ & 15,4 \\
\hline 40 S ribosomal protein S24; & P62849 & Rps24 & $-0,7$ & 8,3 \\
\hline 40 S ribosomal protein S25; & P62852 & Rps25 & $-0,4$ & 22,4 \\
\hline 40 S ribosomal protein S26; & P62855 & Rps26 & $-0,5$ & 31,3 \\
\hline 40 S ribosomal protein S27; & Q6ZWU9 & Rps27 & 0,0 & 38,1 \\
\hline 40 S ribosomal protein S27-like; & Q6ZWY3 & Rps27I & 0,1 & 38,1 \\
\hline 40 S ribosomal protein S28; & P62858 & Rps28 & $-0,2$ & 37,5 \\
\hline 40 S ribosomal protein S29; & P62274 & Rps29 & $-1,3$ & 32,1 \\
\hline 40 S ribosomal protein S3; & P62908 & Rps3 & $-0,1$ & 49 \\
\hline 40 S ribosomal protein $\mathrm{S} 30$; & P62862 & Fau & $-0,5$ & 16,9 \\
\hline $40 S$ ribosomal protein S3a; & P97351 & Rps3a & $-0,3$ & 36,7 \\
\hline
\end{tabular}




\begin{tabular}{|c|c|c|c|c|}
\hline Protein & Uniprot & Gene name & $\begin{array}{c}\text { Fold change of } \\
\text { XPRESS ratio } \\
\left.\text { (Adam17 }^{\Delta K C} / \mathrm{WT}\right)\end{array}$ & $\begin{array}{c}\text { Sequence } \\
\text { coverage [\%] }\end{array}$ \\
\hline $40 \mathrm{~S}$ ribosomal protein $\mathrm{S} 4, \mathrm{X}$ isoform; & P62702 & Rps4x & $-0,2$ & 43 \\
\hline 40 S ribosomal protein S5; & P97461 & Rps5 & $-0,4$ & 22 \\
\hline $40 S$ ribosomal protein S6; & P62754 & Rps6 & $-0,3$ & 8 \\
\hline $40 \mathrm{~S}$ ribosomal protein S7; & P62082 & Rps7 & $-0,2$ & 24,5 \\
\hline 40S ribosomal protein S8; & P62242 & Rps8 & $-0,3$ & 50,5 \\
\hline 40 S ribosomal protein S9; & Q6ZWN5 & Rps9 & 0,0 & 29,4 \\
\hline $40 S$ ribosomal protein SA; & P14206 & Rpsa & $-0,6$ & 45,8 \\
\hline 4F2 cell-surface antigen heavy chain; & P10852 & Slc3a2 & $-1,1$ & 11,8 \\
\hline 4-trimethylaminobutyraldehyde dehydrogenase; & Q9JL2 & Aldh9a1 & 0,0 & 20,9 \\
\hline 5'(3')-deoxyribonucleotidase, cytosolic type; & Q9JM14 & $\mathrm{Nt5c}$ & $-0,2$ & 20 \\
\hline 5'-3' exoribonuclease 2 ; & Q9DBR1 & Xrn2 & 0,1 & 9,5 \\
\hline 5-hydroxyisourate hydrolase; & Q9CRB3 & Urah & 0,3 & 44,9 \\
\hline 5'-nucleotidase domain containing 2; Protein Nt5dc2; & Q91X76 & $\mathrm{Nt5} d c 2$ & 2,0 & 2,3 \\
\hline 5'-nucleotidase; & Q61503 & $\mathrm{Nt5e}$ & $-0,5$ & 12,8 \\
\hline 5-oxoprolinase; & Q8K010 & Oplah & $-0,1$ & 2 \\
\hline 5-phosphohydroxy-L-lysine phospho-lyase; & Q8R1K4 & Phykpl & 0,3 & 4,9 \\
\hline 6.8 kDa mitochondrial proteolipid; & P56379 & Mp68 & $-0,2$ & 13,8 \\
\hline 60 kDa heat shock protein, mitochondrial; & P63038 & Hspd1 & 1,1 & 22,2 \\
\hline 60 kDa SS-A/Ro ribonucleoprotein; & 008848 & Trove2 & 0,0 & 11,9 \\
\hline 605 acidic ribosomal protein $\mathrm{PO}$ & P14869 & Rplp0 & $-0,1$ & 21,5 \\
\hline $60 S$ acidic ribosomal protein $\mathrm{P} 2$ & P99027 & Rplp2 & $-0,2$ & 27 \\
\hline $60 S$ ribosomal protein L10-like; & P86048 & Rpl10l & $-0,3$ & 33,3 \\
\hline $60 S$ ribosomal protein $\mathrm{L} 11$ & Q9CXW4 & Rpl11 & $-0,2$ & 17,2 \\
\hline $60 S$ ribosomal protein $\mathrm{L} 12$ & P35979 & Rpl12 & $-0,3$ & 44,8 \\
\hline 60S ribosomal protein $\mathrm{L} 13$ & P47963 & Rpl13 & $-0,4$ & 31,3 \\
\hline $60 S$ ribosomal protein $\mathrm{L} 13 \mathrm{a}$ & P19253 & Rpl13a & $-0,4$ & 28,1 \\
\hline 605 ribosomal protein L14; & Q9CR57 & Rpl14 & $-0,1$ & 20,3 \\
\hline 60S ribosomal protein L15; & Q9CZM2 & Rpl15 & $-0,5$ & 38,2 \\
\hline $60 S$ ribosomal protein $\mathrm{L} 17$ & Q9CPR4 & Rpl17 & $-0,4$ & 31,5 \\
\hline $60 S$ ribosomal protein L18; & P35980 & Rpl18 & $-0,4$ & 19,7 \\
\hline $60 S$ ribosomal protein $\mathrm{L} 18 \mathrm{a}$ & P62717 & Rpl18a & $-0,3$ & 27,3 \\
\hline 60S ribosomal protein L19; & P84099 & Rpl19 & $-0,8$ & 17 \\
\hline $60 S$ ribosomal protein $\mathrm{L} 21$ & 009167 & Rpl21 & $-0,4$ & 25 \\
\hline $60 S$ ribosomal protein L22; & P67984 & Rpl22 & $-0,2$ & 39,1 \\
\hline $60 \mathrm{~S}$ ribosomal protein L22-like 1; & Q9D7S7 & Rpl22I1 & 0,0 & 9,8 \\
\hline $60 S$ ribosomal protein L23; & P62830 & Rpl23 & $-0,3$ & 18,6 \\
\hline $60 S$ ribosomal protein L24; & Q8BP67 & Rpl24 & $-0,4$ & 36,1 \\
\hline $60 S$ ribosomal protein L26; & P61255 & Rpl26 & $-0,9$ & 45,6 \\
\hline $60 S$ ribosomal protein $\mathrm{L} 27$; & P61358 & Rpl27 & $-0,4$ & 36 \\
\hline $60 S$ ribosomal protein $\mathrm{L} 27 \mathrm{a}$; & P14115 & Rpl27a & $-0,5$ & 27,7 \\
\hline $60 S$ ribosomal protein L28; & P41105 & Rpl28 & $-0,2$ & 27 \\
\hline 605 ribosomal protein L29; & P47915 & Rpl29 & $-0,5$ & 19,5 \\
\hline $60 \mathrm{~S}$ ribosomal protein L3; & P27659 & Rpl3 & $-0,4$ & 29,8 \\
\hline $60 S$ ribosomal protein L30; & P62889 & Rpl30 & $-0,3$ & 43,5 \\
\hline $60 S$ ribosomal protein $\mathrm{L} 31$; & P62900 & Rpl31 & $-0,3$ & 20 \\
\hline $60 S$ ribosomal protein L32; & P62911 & Rpl32 & $-0,6$ & 43,7 \\
\hline $60 S$ ribosomal protein L34; & Q9D1R9 & Rpl34 & $-0,1$ & 12,8 \\
\hline $60 S$ ribosomal protein L35; & Q6ZWV7 & Rpl35 & $-0,5$ & 21,1 \\
\hline $60 \mathrm{~S}$ ribosomal protein $\mathrm{L} 35 \mathrm{a}$ & 055142 & Rpl35a & $-0,4$ & 12,7 \\
\hline $60 \mathrm{~S}$ ribosomal protein L36a; & P83882 & Rpl36a & $-0,2$ & 28,3 \\
\hline $60 S$ ribosomal protein $\mathrm{L} 37 \mathrm{a}$ & P61514 & Rpl37a & $-1,8$ & 21,7 \\
\hline $60 S$ ribosomal protein L38; & Q9Jग8 & Rpl38 & $-0,1$ & 24,3 \\
\hline $60 S$ ribosomal protein L39; & P62892 & Rpl39 & $-0,4$ & 19,6 \\
\hline $60 S$ ribosomal protein $\mathrm{L} 4$ & Q9D8E6 & $\mathrm{Rp} / 4$ & $-0,4$ & 30,3 \\
\hline $60 \mathrm{~S}$ ribosomal protein $\mathrm{L} 5$ & P47962 & Rpl5 & $-0,2$ & 24,2 \\
\hline $60 \mathrm{~S}$ ribosomal protein L6; & P47911 & Rpl6 & $-0,2$ & 28,4 \\
\hline $60 S$ ribosomal protein $\mathrm{L} 7$ & P14148 & Rpl7 & $-0,3$ & 34,1 \\
\hline $60 \mathrm{~S}$ ribosomal protein L8; & P62918 & Rpl8 & $-0,2$ & 38,5 \\
\hline 60S ribosomal protein L9; & P51410 & Rpl9 & $-0,4$ & 28,1 \\
\hline 6-phosphogluconate dehydrogenase, decarboxylating; & Q9DCD0 & Pgd & $-0,3$ & 12,6 \\
\hline 6-phosphogluconolactonase; & Q9CQ60 & Pgls & 0,0 & 23,7 \\
\hline 6-pyruvoyl tetrahydrobiopterin synthase; & Q9R1Z7 & Pts & $-0,4$ & 18,8 \\
\hline 78 kDa glucose-regulated protein; & P20029 & Hspa5 & 0,1 & 27,8 \\
\hline 7-methylguanosine phosphate-specific 5'-nucleotidase; & Q3UFY7 & $\mathrm{Nt5c3b}$ & 0,7 & 4,7 \\
\hline Acetolactate synthase-like protein; & Q8BU33 & Ilvbl & $-0,4$ & 4,3 \\
\hline Acetyl-CoA acetyltransferase, cytosolic; & Q8CAY6 & Acat2 & $-0,2$ & 20,3 \\
\hline Acetyl-CoA acetyltransferase, mitochondrial; & Q8QZT1 & Acat1 & 0,4 & 13 \\
\hline Acid ceramidase; & Q9WV54 & Asah1 & $-0,3$ & 13,5 \\
\hline Acid sphingomyelinase-like phosphodiesterase $3 a ;$ & P70158 & Smpdl3a & $-0,1$ & 7,6 \\
\hline Acid sphingomyelinase-like phosphodiesterase $3 b$; & P58242 & Smpdl3b & $-1,2$ & 7,5 \\
\hline Acidic leucine-rich nuclear phosphoprotein 32 family member B; & Q9EST5 & Anp32b & 0,3 & 14,3 \\
\hline Actin, alpha cardiac muscle 1 & P68033 & Actc1 & $-0,4$ & 43,5 \\
\hline Actin, aortic smooth muscle; & P62737 & Acta2 & 0,3 & 43,5 \\
\hline
\end{tabular}




\begin{tabular}{|c|c|c|c|c|}
\hline Protein & Uniprot & Gene name & $\begin{array}{c}\text { Fold change of } \\
\text { XPRESS ratio } \\
\left.\text { (Adam17 }^{\Delta K C} / \mathrm{WT}\right)\end{array}$ & $\begin{array}{c}\text { Sequence } \\
\text { coverage [\%] }\end{array}$ \\
\hline Actin-like protein $6 \mathrm{~A}$ & Q9Z2N8 & Actl6a & 0,0 & 13,5 \\
\hline Actin-related protein 10; & Q9QZB7 & Actr10 & 0,0 & 15,3 \\
\hline Actin-related protein $2 / 3$ complex subunit $1 \mathrm{~A}$; & Q9R0Q6 & Arpc1a & 0,1 & 32,4 \\
\hline Actin-related protein $2 / 3$ complex subunit $1 B$; & Q9WV32 & Arpc1b & $-0,5$ & 34,1 \\
\hline Actin-related protein $2 / 3$ complex subunit 2 ; & Q9CVB6 & Arpc2 & $-0,4$ & 28 \\
\hline Actin-related protein $2 / 3$ complex subunit 3 ; & Q9JM76 & Arpc3 & $-0,4$ & 21,2 \\
\hline Actin-related protein $2 / 3$ complex subunit 4 & P59999 & Arpc4 & $-0,2$ & 29,2 \\
\hline Actin-related protein $2 / 3$ complex subunit 5 ; & Q9CPW4 & Arpc5 & $-0,3$ & 29,1 \\
\hline Actin-related protein $2 / 3$ complex subunit 5 -like protein; & Q9D898 & Arpc5I & $-0,3$ & 28,8 \\
\hline Actin-related protein 2; & P61161 & Actr2 & 0,0 & 23,6 \\
\hline Actin-related protein 3; & Q99JY9 & Actr3 & $-0,1$ & 37,6 \\
\hline Activated RNA polymerase II transcriptional coactivator $\mathrm{p} 15$; & P11031 & Sub1 & $-0,1$ & 18,9 \\
\hline Acyl carrier protein, mitochondrial; & Q9CR21 & Ndufab1 & 0,1 & 7,1 \\
\hline Acylamino-acid-releasing enzyme; & Q8R146 & Apeh & $-0,2$ & 17,2 \\
\hline Acyl-CoA dehydrogenase family member 10 & Q8K370 & Acad10 & 0,3 & 5 \\
\hline Acyl-CoA dehydrogenase family member 9, mitochondrial; & Q8JZN5 & Acad9 & $-0,1$ & 37,1 \\
\hline Acyl-CoA desaturase 1 & P13516 & Scd1 & $-0,4$ & 9 \\
\hline Acyl-CoA-binding protein; & P31786 & Dbi & $-0,4$ & 39,1 \\
\hline Acyl-CoA-binding protein; & D3Z563 & Dbi & 1,5 & 41,3 \\
\hline Acyl-coenzyme A thioesterase 1 ; & 055137 & Acot1 & 0,0 & 7,4 \\
\hline Acyl-coenzyme A thioesterase 9, mitochondrial; & Q9R0X4 & Acot9 & 0,4 & 13,9 \\
\hline Acyl-protein thioesterase 1 & P97823 & Lypla1 & 0,7 & 10,9 \\
\hline Acyl-protein thioesterase 2 & Q9WTL7 & Lypla2 & 0,2 & 7,8 \\
\hline Acylpyruvase FAHD1, mitochondrial; & Q8R0F8 & Fahd1 & $-0,8$ & 12,8 \\
\hline Adenine phosphoribosyltransferase; & P08030 & Aprt & 0,7 & 11,1 \\
\hline Adenosine deaminase; & P03958 & Ada & $-0,3$ & 18,2 \\
\hline Adenosine kinase; & P55264 & Adk & 0,5 & 24,9 \\
\hline Adenosylhomocysteinase; & P50247 & Ahcy & $-0,2$ & 42,8 \\
\hline Adenylate kinase 2, mitochondrial; & Q9WTP6 & Ak2 & 0,4 & 27,2 \\
\hline Adenylate kinase 4, mitochondrial; & Q9WUR9 & Ak4 & 0,2 & 14,8 \\
\hline Adenylosuccinate lyase; & P54822 & Adsl & $-0,3$ & 30,4 \\
\hline Adenylosuccinate synthetase isozyme 2 ; & P46664 & Adss & $-1,0$ & 9,4 \\
\hline Adenylyl cyclase-associated protein $1 ;$ & P40124 & Cap1 & $-0,1$ & 7,8 \\
\hline Adipocyte plasma membrane-associated protein; & Q9D7N9 & Apmap & $-0,2$ & 6,5 \\
\hline Adiponectin; & Q60994 & Adipoq & $-0,2$ & 13,8 \\
\hline ADP/ATP translocase $1 ;$ & P48962 & Slc25a4 & $-0,3$ & 31,2 \\
\hline ADP/ATP translocase 2 & P51881 & Slc25a5 & $-0,1$ & 34,9 \\
\hline ADP/ATP translocase 4; & Q3V132 & Slc25a31 & $-0,2$ & 14,7 \\
\hline ADP-dependent glucokinase; & Q8VDL4 & Adpgk & 0,2 & 7,3 \\
\hline ADP-ribosylation factor 3 & P61205 & Arf3 & 0,4 & 26 \\
\hline ADP-ribosylation factor 4; & P61750 & Arf4 & 1,4 & 27,5 \\
\hline ADP-ribosylation factor 5 & P84084 & Arf5 & 0,2 & 31,7 \\
\hline ADP-ribosylation factor 6; & P62331 & Arf6 & 0,2 & 14,9 \\
\hline ADP-ribosylation factor-like protein 3 ; & Q9WUL7 & Arl3 & 0,8 & 21,4 \\
\hline ADP-ribosylation factor-like protein 6 -interacting protein 1 ; & Q9JKW0 & Arl6ip1 & $-0,8$ & 8,9 \\
\hline ADP-ribosylation factor-like protein $8 \mathrm{~A}$ & Q8VEH3 & Arl8a & $-0,3$ & 30,9 \\
\hline ADP-sugar pyrophosphatase; & Q9JKX6 & Nudt5 & 0,0 & 23,9 \\
\hline Afadin; & Q9QZQ1 & Mllt4 & $-0,4$ & 1,1 \\
\hline Agrin; & MoQWP1 & Agrn & $-0,2$ & 8,5 \\
\hline A-kinase anchor protein 12 & Q9WTQ5 & Akap12 & $-0,3$ & 4,4 \\
\hline A-kinase anchor protein 2 & A2API8 & Akap2 & 0,8 & 4,2 \\
\hline Alanine aminotransferase 1 & Q8QZR5 & Gpt & $-0,1$ & 15,5 \\
\hline Alcohol dehydrogenase $[\mathrm{NADP}(+)]$ & Q9JII6 & Akr1a1 & 0,6 & 4,9 \\
\hline Alcohol dehydrogenase 1 & P00329 & Adh1 & $-0,2$ & 13,9 \\
\hline Alcohol dehydrogenase class $4 \mathrm{mu} / \mathrm{sigma}$ chain; & Q64437 & Adh7 & $-0,4$ & 21,4 \\
\hline Alcohol dehydrogenase class-3; & P28474 & Adh5 & 0,0 & 8 \\
\hline Aldehyde dehydrogenase family 1 member A3; & Q9JHW9 & Aldh1a3 & 0,2 & 2,7 \\
\hline Aldehyde dehydrogenase family 16 member $A 1$; & Q57119 & Aldh16a1 & 0,1 & 9,2 \\
\hline Aldehyde dehydrogenase family 8 member $A 1 ;$ & Q8BH00 & Aldh8a1 & 0,3 & 6,4 \\
\hline Aldehyde dehydrogenase, cytosolic 1 & 035945 & Aldh1a7 & $-0,1$ & 4,8 \\
\hline Aldehyde dehydrogenase, dimeric NADP-preferring; & P47739 & Aldh3a1 & 0,2 & 9,9 \\
\hline Aldehyde dehydrogenase, mitochondrial; & P47738 & Aldh2 & 0,1 & 5,4 \\
\hline Aldehyde dehydrogenase; & E9Q3E1 & Aldh3b2 & 0,4 & 20,7 \\
\hline Aldehyde oxidase 4 & Q3TYQ9 & Aox4 & $-1,1$ & 11,3 \\
\hline Aldose reductase; & P45376 & Akr1b1 & 0,7 & 15,2 \\
\hline Allograft inflammatory factor 1-like; & Q9EQX4 & Aif1l & $-0,2$ & 10 \\
\hline $\begin{array}{l}\text { Alpha globin 1; Alpha globin 2; Alpha-globin; Hemoglobin alpha, adult chain 1; } \\
\text { Hemoglobin subunit alpha; Protein Hba-a2; }\end{array}$ & Q91VB8 & naemaglobin alpha & 0,3 & 62 \\
\hline Alpha/beta hydrolase domain-containing protein 14B; & Q8VCR7 & Abhd14b & 0,2 & 10 \\
\hline Alpha-1,6-mannosyl-glycoprotein 2-beta- $\mathrm{N}$-acetylglucosaminyltransferase; & Q921V5 & Mgat2 & 0,5 & 3,6 \\
\hline Alpha-1-antitrypsin 1-1; & P07758 & Serpina1a & 1,6 & 26,5 \\
\hline Alpha-1-antitrypsin 1-2; & P22599 & Serpina1b & 0,8 & 25,2 \\
\hline
\end{tabular}




\begin{tabular}{|c|c|c|c|c|}
\hline Protein & Uniprot & Gene name & $\begin{array}{l}\text { Fold change of } \\
\text { XPRESS ratio } \\
\text { (Adam17 } \\
\text { (AKC } / \mathrm{WT})\end{array}$ & $\begin{array}{c}\text { Sequence } \\
\text { coverage [\%] }\end{array}$ \\
\hline Alpha-1-antitrypsin 1-4; & Q00897 & Serpina1d & 1,4 & 26,2 \\
\hline Alpha-1-antitrypsin 1-5; & Q00898 & Serpina1e & 1,7 & 16,2 \\
\hline Alpha-2-antiplasmin; & Q61247 & Serpinf2 & $-10,0$ & 2,9 \\
\hline Alpha-2-HS-glycoprotein; & P29699 & Ahsg & 0,9 & 38,6 \\
\hline Alpha-2-macroglobulin receptor-associated protein; & P55302 & Lrpap1 & 0,4 & 14,4 \\
\hline Alpha-2-macroglobulin; & Q61838 & $\mathrm{A} 2 \mathrm{~m}$ & 0,4 & 17,5 \\
\hline Alpha-actinin-1; & Q7TPR4 & Actn1 & $-0,1$ & 31,3 \\
\hline Alpha-actinin-4; & P57780 & Actn4 & $-0,1$ & 39,6 \\
\hline Alpha-adducin; & Q9QYCo & Add1 & 0,2 & 21,6 \\
\hline Alpha-aminoadipic semialdehyde dehydrogenase; & Q9DBF1 & Aldh7a1 & 0,0 & 12,8 \\
\hline Alpha-centractin; & P61164 & Actr1a & $-0,3$ & 17,8 \\
\hline Alpha-crystallin B chain; & P23927 & Cryab & 0,1 & 4,6 \\
\hline Alpha-enolase; & P17182 & Eno1 & 0,6 & 16,6 \\
\hline Alpha-fetoprotein; & P02772 & Afp & 0,2 & 34,9 \\
\hline Alpha-galactosidase $\mathrm{A}$; & P51569 & Gla & $-1,0$ & 5 \\
\hline Alpha-ketoglutarate-dependent dioxygenase FTO; & Q8BGW1 & Fto & 0,2 & 6 \\
\hline Alpha-mannosidase 2 & P27046 & Man2a1 & 0,3 & 5,3 \\
\hline Alpha-N-acetylgalactosaminidase; & Q9QWR8 & Naga & $-0,2$ & 7,2 \\
\hline $\begin{array}{l}\text { Alpha-N-acetylglucosaminidase; Alpha-N-acetylglucosaminidase (Sanfilippo } \\
\text { disease IIIB); Alpha-N-acetylglucosaminidase (Sanfilippo disease IIIB), isoform } \\
\text { CRA_b; N-acetyl-glucosaminidase; Protein Naglu; }\end{array}$ & 088325 & Naglu & $-0,3$ & 6,2 \\
\hline Alpha-synuclein; & 055042 & Snca & 0,7 & 9,3 \\
\hline Amine oxidase [flavin-containing] $\mathrm{A}$; & Q64133 & Maoa & 0,3 & 24,7 \\
\hline Amine oxidase [flavin-containing] B; & Q8BW75 & Maob & 0,2 & 2,1 \\
\hline Aminoacyl tRNA synthase complex-interacting multifunctional protein $1 ;$ & P31230 & Aimp1 & 0,3 & 5,5 \\
\hline Aminoacylase-1; & Q99JW2 & Acy1 & $-0,1$ & 5,6 \\
\hline Aminopeptidase $\mathrm{N}$; & P97449 & Anpep & $-0,5$ & 21,1 \\
\hline AMP deaminase 2 & Q9DBT5 & Ampd2 & 0,9 & 3,6 \\
\hline Amyloid beta A4 protein; & P12023 & App & $-0,5$ & 4,9 \\
\hline Amyloid-like protein 2; & Q06335 & Aplp2 & 0,1 & 3,5 \\
\hline AN1-type zinc finger protein 2B; & Q91X58 & Zfand $2 b$ & $-0,5$ & 3,2 \\
\hline AN1-type zinc finger protein 6; & Q9DCH6 & Zfand6 & $-0,5$ & 5,4 \\
\hline Anamorsin; & Q8WTY4 & Ciapin1 & 0,1 & 23,4 \\
\hline Angiotensin-converting enzyme 2 & Q8R0I0 & Ace2 & $-0,6$ & 6,7 \\
\hline Annexin A1; & P10107 & Anxa1 & 0,1 & 23,1 \\
\hline Annexin A11; & P97384 & Anxa11 & $-0,6$ & 5,8 \\
\hline Annexin A2; & P07356 & Anxa2 & $-0,6$ & 39,2 \\
\hline Annexin A4; & P97429 & Anxa4 & $-0,1$ & 14,4 \\
\hline Annexin A5; & P48036 & Anxa5 & $-0,4$ & 26,6 \\
\hline Annexin A6; & P14824 & Anxa6 & $-0,5$ & 32,1 \\
\hline Annexin A7; & Q07076 & Anxa7 & 0,0 & 1,7 \\
\hline Annexin A8; & 035640 & Anxa8 & 0,3 & 28,7 \\
\hline Antithrombin-III; & P32261 & Serpinc1 & 0,7 & 9,7 \\
\hline AP-1 complex subunit beta-1; & 035643 & Ap1b1 & 0,1 & 2,9 \\
\hline AP-1 complex subunit mu-1; & P35585 & Ap1m1 & 0,6 & 2,4 \\
\hline AP-2 complex subunit alpha-1; & P17426 & Ap2a1 & 0,4 & 3,4 \\
\hline AP-2 complex subunit alpha-2; & P17427 & Ap2a2 & 0,5 & 3,1 \\
\hline AP-2 complex subunit mu; & P84091 & Ap2m1 & 0,2 & 4,2 \\
\hline AP-2 complex subunit sigma; & P62743 & Ap2s1 & $-0,1$ & 10,6 \\
\hline Apolipoprotein A-I; & Q00623 & Apoa1 & 0,7 & 31,4 \\
\hline Apolipoprotein A-II; & P09813 & Apoa2 & 0,7 & 9,8 \\
\hline Apolipoprotein A-IV; & P06728 & Apoa4 & 0,8 & 43,5 \\
\hline Apolipoprotein C-I; & P34928 & Apoc1 & $-0,4$ & 20,5 \\
\hline Apolipoprotein E; & P08226 & Apoe & $-2,3$ & 24,1 \\
\hline Apolipoprotein O; & Q9DCZ4 & Apoo & $-0,4$ & 11 \\
\hline Apolipoprotein O-like; & Q78IK4 & Apool & 0,3 & 27,9 \\
\hline Apoptosis regulator BAX; & Q07813 & Bax & $-0,1$ & 6,8 \\
\hline Apoptosis-inducing factor 1 , mitochondrial; & Q9Z0X1 & Aifm1 & 0,2 & 6,9 \\
\hline Apoptotic chromatin condensation inducer in the nucleus; & Q9JIX8 & Acin1 & 0,3 & 3,6 \\
\hline Aquaporin-1; & Q02013 & Aqp1 & 0,0 & 7,1 \\
\hline Arachidonate 5-lipoxygenase-activating protein; & P30355 & Alox5ap & $-0,2$ & 5,6 \\
\hline Arfip1 protein; Protein Arfip1; & A2RSX9 & Arfip1 & 0,3 & 13,2 \\
\hline Arginase-1; & Q61176 & Arg1 & $-0,1$ & 9,6 \\
\hline Arginine/serine-rich coiled-coil protein 2 & A2RTL5 & Rsrc2 & 1,3 & 4,4 \\
\hline Arginine--tRNA ligase, cytoplasmic; & Q9D019 & Rars & $-0,1$ & 5,3 \\
\hline Argininosuccinate lyase; & Q91YI0 & Asl & $-0,2$ & 11,6 \\
\hline Argininosuccinate synthase; & P16460 & Ass1 & 0,5 & 6,1 \\
\hline Arylacetamide deacetylase; & Q99PG0 & Aadac & $-0,7$ & 6,3 \\
\hline Arylsulfatase B; & P50429 & Arsb & $-0,1$ & 6 \\
\hline Asparagine synthetase domain-containing protein 1 ; & MoQW65 & Asnsd1 & $-0,1$ & 45,2 \\
\hline Asparagine--tRNA ligase, cytoplasmic; & Q8BP47 & Nars & 0,6 & 4,3 \\
\hline Aspartate aminotransferase, cytoplasmic; & P05201 & Got1 & $-0,7$ & 29,8 \\
\hline
\end{tabular}




\begin{tabular}{|c|c|c|c|c|}
\hline Protein & Uniprot & Gene name & $\begin{array}{c}\text { Fold change of } \\
\text { XPRESS ratio } \\
\text { (Adam17 } \\
\text { (AKC } / \mathrm{WT})\end{array}$ & $\begin{array}{c}\text { Sequence } \\
\text { coverage [\%] }\end{array}$ \\
\hline Aspartate aminotransferase, mitochondrial; & P05202 & Got2 & $-0,3$ & 37 \\
\hline Aspartate--tRNA ligase, cytoplasmic; & Q922B2 & Dars & $-0,1$ & 28,3 \\
\hline Aspartyl aminopeptidase; & Q9Z2W0 & Dnpep & $-0,6$ & 25,2 \\
\hline Aspartyl/asparaginyl beta-hydroxylase; & Q8BSY0 & Asph & 0,8 & 1,2 \\
\hline Asporin; & Q99MQ4 & Aspn & 0,2 & 26 \\
\hline Ataxin-2-like protein; & Q7TQHO & Atxn2l & 0,2 & 1 \\
\hline Atlastin-3; & Q91YH5 & Atl3 & 0,1 & 4,5 \\
\hline ATP synthase $F(0)$ complex subunit B1, mitochondrial; & Q9CQQ7 & Atp5f1 & $-2,4$ & 14,8 \\
\hline ATP synthase protein 8 & P03930 & Mtatp8 & $-0,2$ & 14,9 \\
\hline ATP synthase subunit alpha, mitochondrial; & Q03265 & Atp5a1 & $-0,1$ & 30,4 \\
\hline ATP synthase subunit beta, mitochondrial; & P56480 & Atp5b & $-0,2$ & 46,7 \\
\hline ATP synthase subunit d, mitochondrial; & Q9DCX2 & Atp5h & $-0,2$ & 44,4 \\
\hline ATP synthase subunit delta, mitochondrial; & Q9D3D9 & Atp5d & $-0,4$ & 13,7 \\
\hline ATP synthase subunit e, mitochondrial; & Q06185 & Atp5i & $-0,3$ & 28,9 \\
\hline ATP synthase subunit epsilon, mitochondrial; & P56382 & Atp5e & $-1,4$ & 28,8 \\
\hline ATP synthase subunit g, mitochondrial; & Q9CPQ8 & Atp5l & $-0,4$ & 8,7 \\
\hline ATP synthase subunit gamma, mitochondrial; & Q91VR2 & Atp5c1 & $-0,2$ & 42,9 \\
\hline ATP synthase subunit $O$, mitochondrial; & Q9DB20 & Atp5o & $-0,1$ & 33,8 \\
\hline ATP synthase-coupling factor 6 , mitochondrial; & P97450 & Atp5j & 0,5 & 24,1 \\
\hline ATPase Asna1; & 054984 & Asna1 & 0,1 & 21,6 \\
\hline ATP-binding cassette sub-family A member 5 ; & Q8K448 & Abca5 & 9,0 & 1,3 \\
\hline ATP-binding cassette sub-family B member 10 , mitochondrial; & Q9J139 & Abcb10 & 1,2 & 4,8 \\
\hline ATP-binding cassette sub-family E member 1 & P61222 & Abce1 & $-0,3$ & 18 \\
\hline ATP-binding cassette sub-family $F$ member 1 ; & Q6P542 & Abcf1 & 0,7 & 6,9 \\
\hline ATP-citrate synthase; & Q91V92 & Acly & 0,1 & 7,8 \\
\hline ATP-dependent (S)-NAD(P)H-hydrate dehydratase; & Q9CZ42 & Carkd & $-0,2$ & 34,6 \\
\hline ATP-dependent 6-phosphofructokinase, liver type; & P12382 & Pfkl & 0,0 & 19 \\
\hline ATP-dependent 6-phosphofructokinase, muscle type; & P47857 & Pfkm & 0,5 & 2,7 \\
\hline ATP-dependent 6-phosphofructokinase, platelet type; & Q9WUA3 & Pfkp & $-0,1$ & 16,5 \\
\hline ATP-dependent Clp protease proteolytic subunit, mitochondrial; & 088696 & Clpp & $-0,3$ & 6,6 \\
\hline ATP-dependent RNA helicase A; & E9QNN1 & Dhx9 & 0,6 & 9,2 \\
\hline ATP-dependent RNA helicase DDX1; & Q91VR5 & Ddx1 & 0,5 & 5,3 \\
\hline ATP-dependent RNA helicase DDX39A; & Q8VDW0 & Ddx39a & 0,5 & 14,3 \\
\hline ATP-dependent RNA helicase DDX3X; & Q62167 & $\operatorname{Dd} \times 3 x$ & 0,5 & 14,2 \\
\hline Atrial natriuretic peptide-converting enzyme; & Q97319 & Corin & 0,2 & 1,1 \\
\hline Aurora kinase $\mathrm{B}$; & E9PVP1 & Aim1 & 0,7 & 4,9 \\
\hline Autophagy-related protein 2 homolog B; & Q80XK6 & Atg $2 b$ & $-0,8$ & 0,9 \\
\hline BAG family molecular chaperone regulator 1 ; & Q60739 & Bag1 & 0,0 & 3,4 \\
\hline BAG family molecular chaperone regulator 3 ; & Q9JLV1 & Bag3 & 0,5 & 5,9 \\
\hline Band 4.1-like protein 2; & 070318 & Epb4112 & 1,0 & 3,6 \\
\hline Band 4.1-like protein 3; & Q9WV92 & Epb4113 & 0,2 & 9,1 \\
\hline Barrier-to-autointegration factor; & 054962 & Banf1 & $-0,3$ & 34,8 \\
\hline Basal cell adhesion molecule; & Q9R069 & Bcam & $-0,2$ & 10,6 \\
\hline Basement membrane-specific heparan sulfate proteoglycan core protein; & B1B0C7 & Hspg2 & 0,0 & 4,3 \\
\hline Basigin; & P18572 & Bsg & $-0,4$ & 20,3 \\
\hline B-cell receptor-associated protein 31 ; & Q61335 & Bcap31 & $-0,3$ & 14,3 \\
\hline Bcl-2 homologous antagonist/killer; & 008734 & Bak1 & 2,1 & 4,8 \\
\hline BCL2/adenovirus E1B 19 kDa protein-interacting protein 3-like; & Q9Z2F7 & Bnip3l & $-0,1$ & 7,4 \\
\hline $\mathrm{Bcl}-2$-associated transcription factor 1 & Q8K019 & Bclaf1 & 0,0 & 9 \\
\hline Bcl-2-like protein 13 & P59017 & $\mathrm{Bcl} 2 \mathrm{|13}$ & 0,3 & 2,5 \\
\hline BET1 homolog; & 035623 & Bet1 & $-0,1$ & 9,3 \\
\hline Beta-1,3-glucosyltransferase; & Q8BHT6 & B3galtl & $-0,1$ & 2,7 \\
\hline Beta-2-glycoprotein 1; & Q01339 & Apoh & 0,6 & 22 \\
\hline Beta-actin-like protein 2; & Q8BFZ3 & Actbl2 & $-0,2$ & 25 \\
\hline Beta-enolase; & P21550 & Eno3 & $-1,0$ & 13,4 \\
\hline Beta-galactosidase; & P23780 & Glb1 & $-0,2$ & 11,9 \\
\hline Beta-globin; Protein Hbb-bs; Protein Hbb-bt; & A8DUK4 & Hbbt1 & 0,4 & 72,1 \\
\hline Beta-glucuronidase; & P12265 & Gusb & $-0,7$ & 12,7 \\
\hline Beta-hexosaminidase subunit alpha; & P29416 & Hexa & 1,5 & 6,3 \\
\hline Beta-hexosaminidase subunit beta; & P20060 & Hexb & $-0,9$ & 22,6 \\
\hline Beta-lactamase-like protein 2 ; & Q99KR3 & Lactb2 & 0,5 & 6,3 \\
\hline Beta-mannosidase; & Q8K214 & Manba & 0,5 & 1,7 \\
\hline BH3-interacting domain death agonist; & P70444 & Bid & 0,6 & 9,7 \\
\hline Bifunctional 3'-phosphoadenosine 5'-phosphosulfate synthase 1; & Q60967 & Papss1 & 0,8 & 5,4 \\
\hline Bifunctional glutamate/proline--tRNA ligase; & Q8CGC7 & Eprs & 0,0 & 5,2 \\
\hline Bifunctional purine biosynthesis protein PURH; & Q9CWJ9 & Atic & 0,7 & 16,4 \\
\hline Biglycan; & P28653 & Bgn & 0,4 & 31,4 \\
\hline Biliverdin reductase $\mathrm{A}$; & Q9CY64 & Blvra & 0,8 & 6,8 \\
\hline Biorientation of chromosomes in cell division protein 1 ; & Q5SQY2 & Bod1 & 2,9 & 13,3 \\
\hline Biotinidase; & Q8CIF4 & Btd & $-0,2$ & 1,7 \\
\hline Bis(5'-adenosyl)-triphosphatase; & 089106 & Fhit & $-0,5$ & 24,2 \\
\hline Bisphosphoglycerate mutase; & P15327 & Bpgm & 0,1 & 11,6 \\
\hline
\end{tabular}




\begin{tabular}{|c|c|c|c|c|}
\hline Protein & Uniprot & Gene name & 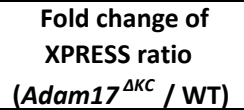 & $\begin{array}{c}\text { Sequence } \\
\text { coverage [\%] }\end{array}$ \\
\hline Bleomycin hydrolase; & Q8R016 & Blmh & $-0,5$ & 31,4 \\
\hline BolA-like protein 1; & Q9D8S9 & Bola1 & 0,2 & 12,4 \\
\hline BolA-like protein 2; & Q8BGS2 & Bola2 & $-0,5$ & 32,6 \\
\hline Bone marrow proteoglycan; & Q61878 & $\operatorname{Prg} 2$ & 0,3 & 8,5 \\
\hline Bone marrow stromal antigen 2 ; & Q8R2Q8 & Bst2 & 0,4 & 5,2 \\
\hline BPI fold-containing family C protein; & Q8C186 & Bpifc & 0,1 & 11,6 \\
\hline Brain acid soluble protein 1 & Q91XV3 & Basp1 & $-0,1$ & 9,7 \\
\hline Brain-specific angiogenesis inhibitor 1-associated protein 2; & Q8BKX1 & Baiap2 & 0,0 & 2,5 \\
\hline Branched-chain-amino-acid aminotransferase, mitochondrial; & 035855 & Bcat2 & $-1,0$ & 17,3 \\
\hline BRCA1-A complex subunit BRE; & Q8K3W0 & Bre & 0,1 & 7,3 \\
\hline BRI3-binding protein; & Q8BXV2 & Bri3bp & $-0,2$ & 3,6 \\
\hline BRISC and BRCA1-A complex member 1 ; & Q3U143 & Babam1 & $-0,5$ & 14,4 \\
\hline BRISC complex subunit Abro1; & Q3TCJ1 & Fam175b & $-0,3$ & 7,7 \\
\hline $\begin{array}{l}\text { BTB/POZ domain-containing adapter for CUL3-mediated RhoA degradation } \\
\text { protein 3; }\end{array}$ & Q922M3 & Kctd10 & $-0,2$ & 8,3 \\
\hline BTB/POZ domain-containing protein KCTD12; & Q6WVG3 & Kctd12 & $-0,1$ & 24,2 \\
\hline BUB3-interacting and GLEBS motif-containing protein ZNF207; & E9PW12 & Znf207 & $-0,3$ & 8,6 \\
\hline CAAX prenyl protease 1 homolog; & Q80W54 & Zmpste24 & $-0,3$ & 7,8 \\
\hline Cadherin-1; & P09803 & Cdh1 & $-0,6$ & 12,2 \\
\hline Cadherin-11; & P55288 & Cdh11 & 0,6 & 1,5 \\
\hline Cadherin-13; & Q9WTR5 & Cdh13 & $-0,4$ & 7,1 \\
\hline Cadherin-3; & P10287 & Cdh3 & 0,0 & 2,9 \\
\hline Cadherin-5; & P55284 & Cdh5 & 0,6 & 1,1 \\
\hline Calcineurin B homologous protein 1; & P61022 & Chp1 & $-0,3$ & 12 \\
\hline Calcium-activated chloride channel regulator 2 & Q8BG22 & Clca2 & 0,1 & 2,3 \\
\hline $\begin{array}{l}\text { Calcium-activated chloride channel regulator 2; Chloride channel calcium } \\
\text { activated 2; Endothelial chloride channel; MCG120735; }\end{array}$ & Q9EQR4 & Clca2 & $-0,1$ & 25,6 \\
\hline Calcium-activated chloride channel regulator 4 & E9PUL3 & Clca4 & 6,1 & 1,7 \\
\hline Calcium-binding mitochondrial carrier protein Aralar1; & Q8BH59 & Slc25a12 & $-0,2$ & 7,5 \\
\hline Calcium-binding mitochondrial carrier protein Aralar2; & Q9QXX4 & Slc25a13 & $-0,3$ & 12,4 \\
\hline Calcium-binding mitochondrial carrier protein SCaMC-1; & Q8BMD8 & Slc25a24 & $-0,2$ & 19,2 \\
\hline Calcium-binding protein 39 & Q06138 & Cab39 & 1,2 & 19,4 \\
\hline Calcium-regulated heat stable protein 1 & Q9CR86 & Carhsp1 & 0,1 & 18,2 \\
\hline Calcyclin-binding protein; & Q9CXW3 & Cacybp & 0,3 & 10 \\
\hline Calmodulin; & P62204 & Calm3 & $-0,6$ & 10,1 \\
\hline Calmodulin-4; & Q9JM83 & Calm4 & 1,3 & 35,8 \\
\hline Calnexin; & P35564 & Canx & 0,0 & 21,8 \\
\hline Calpain small subunit 1 & 088456 & Capns1 & $-0,5$ & 16,2 \\
\hline Calpain small subunit 2 ; & Q9D7J7 & Capns2 & 0,5 & 12,6 \\
\hline Calpain-1 catalytic subunit; & 035350 & Capn1 & $-0,2$ & 32 \\
\hline Calpain-2 catalytic subunit; & 008529 & Capn2 & 0,5 & 7,6 \\
\hline Calpain-5; & 008688 & Capn5 & 0,0 & 7,2 \\
\hline Calpastatin; & P51125 & Cast & $-0,5$ & 24 \\
\hline Calponin-2; & Q08093 & Cnn2 & 0,4 & 6 \\
\hline Calreticulin; & P14211 & Calr & $-0,1$ & 42,1 \\
\hline Calsequestrin-1; & 009165 & Casq1 & $-1,1$ & 14,1 \\
\hline Calumenin; & 035887 & Calu & 0,1 & 27 \\
\hline cAMP-dependent protein kinase type II-beta regulatory subunit; & P31324 & Prkar2b & 0,1 & 3,8 \\
\hline cAMP-regulated phosphoprotein 19 & P56212 & Arpp19 & $-1,1$ & 13,4 \\
\hline CAP-Gly domain-containing linker protein 1 & Q922J3 & Clip1 & 0,2 & 6,7 \\
\hline $\begin{array}{l}\text { Capping protein (Actin filament) muscle Z-line, beta, isoform CRA_a; F-actin- } \\
\text { capping protein subunit beta; }\end{array}$ & A2AMW0 & Capzb & $-0,1$ & 12,3 \\
\hline Caprin-1; & Q60865 & Caprin1 & 0,3 & 10,5 \\
\hline Carbonic anhydrase 3 ; & P16015 & $\mathrm{Ca} 3$ & 0,2 & 43,1 \\
\hline Carbonic anhydrase 4; & Q64444 & $\mathrm{Ca} 4$ & $-0,9$ & 9,8 \\
\hline Carbonic anhydrase 6; & P18761 & $\mathrm{Ca} 6$ & 0,2 & 21,5 \\
\hline Carbonyl reductase [NADPH] 1; & P48758 & Cbr1 & 0,2 & 7,6 \\
\hline Carbonyl reductase [NADPH] 2; & P08074 & Cbr2 & 0,7 & 5,7 \\
\hline Carbonyl reductase [NADPH] 3; & Q8K354 & Cbr3 & 0,7 & 7,6 \\
\hline Carbonyl reductase family member 4 ; & Q91VT4 & Cbr4 & 0,8 & 20,8 \\
\hline Carboxylesterase $1 \mathrm{C}$ & P23953 & Ces1c & 0,6 & 9,6 \\
\hline Carboxylesterase 1D; & Q8VCT4 & Ces1d & $-0,6$ & 8,5 \\
\hline Carboxylesterase 5A; & Q6AW46 & Ces5a & $-10,0$ & 6,1 \\
\hline Carboxypeptidase A4; & Q6P8K8 & Cpa4 & $-0,5$ & 14 \\
\hline Carboxypeptidase E; & Q00493 & Cpe & 0,4 & 12 \\
\hline Carboxypeptidase M; & Q80V42 & Cpm & $-0,4$ & 29,1 \\
\hline Cartilage intermediate layer protein 1 ; & Q66K08 & Cilp & 0,3 & 1,7 \\
\hline Cartilage-associated protein; & Q9CYD3 & Crtap & $-0,4$ & 2,3 \\
\hline Casein kinase I isoform alpha; & Q8BK63 & Csnk1a1 & 3,3 & 6,3 \\
\hline Casein kinase II subunit alpha; & Q60737 & Csnk2a1 & 0,4 & 8,4 \\
\hline Casein kinase II subunit beta; & P67871 & Csnk2b & 0,6 & 15,4 \\
\hline Caspase-14; & 089094 & Casp14 & $-0,2$ & 21,8 \\
\hline Caspase-3; & P70677 & Casp3 & $-1,3$ & 21,7 \\
\hline
\end{tabular}




\begin{tabular}{|c|c|c|c|c|}
\hline Protein & Uniprot & Gene name & $\begin{array}{c}\text { Fold change of } \\
\text { XPRESS ratio } \\
\text { (Adam17 } \\
\text { (AKC } / \mathrm{WT})\end{array}$ & $\begin{array}{c}\text { Sequence } \\
\text { coverage [\%] }\end{array}$ \\
\hline Caspase-6; & 008738 & Casp6 & $-0,1$ & 13 \\
\hline Catalase; & P24270 & Cat & 0,4 & 20,5 \\
\hline Catenin alpha-1; & P26231 & Ctnna1 & $-0,1$ & 13 \\
\hline Catenin beta-1; & Q02248 & Ctnnb1 & $-0,1$ & 36,1 \\
\hline Catenin delta-1; & P30999 & Ctnnd1 & $-0,1$ & 3,4 \\
\hline Cathepsin B; & P10605 & Ctsb & $-0,6$ & 28,6 \\
\hline Cathepsin D; & P18242 & Ctsd & $-0,6$ & 21 \\
\hline Cathepsin E; & P70269 & Ctse & $-2,1$ & 4,9 \\
\hline Cathepsin K; & P55097 & Ctsk & 0,2 & 3 \\
\hline Cathepsin L1; & P06797 & Ctsl & $-0,7$ & 19,8 \\
\hline Cathepsin S; & 070370 & Ctss & $-0,4$ & 4,1 \\
\hline Cathepsin Z; & Q9WUU7 & Ctsz & $-0,2$ & 11,1 \\
\hline Cation-dependent mannose-6-phosphate receptor; & P24668 & M6pr & 0,0 & 10,4 \\
\hline Cation-independent mannose- 6 -phosphate receptor; & Q07113 & $\operatorname{lgf} 2 r$ & 0,4 & 3,2 \\
\hline Caveolin-1; & P49817 & Cav1 & 0,0 & 28,7 \\
\hline CD109 antigen; & Q8R422 & Cd109 & $-0,4$ & 10,1 \\
\hline CD151 antigen; & 035566 & Cd151 & $-0,9$ & 4 \\
\hline CD166 antigen; & Q61490 & Alcam & $-1,0$ & 15,3 \\
\hline CD2-associated protein; & Q9JLQ0 & Cd2ap & $-0,3$ & 9,7 \\
\hline CD44 antigen; & P15379 & $\mathrm{Cd} 44$ & $-2,7$ & 6,3 \\
\hline CD59A glycoprotein; & 055186 & Cd59a & $-2,1$ & 7,3 \\
\hline CD63 antigen; & P41731 & Cd63 & $-0,4$ & 4,6 \\
\hline CD81 antigen; & P35762 & Cd81 & $-0,6$ & 19,1 \\
\hline CD9 antigen; & P40240 & $\mathrm{Cd} 9$ & $-0,7$ & 23,9 \\
\hline CD97 antigen; & Q9Z0M6 & Cd97 & 0,4 & 3,6 \\
\hline CDGSH iron-sulfur domain-containing protein 1 & Q91WS0 & Cisd1 & $-0,5$ & 34,3 \\
\hline CDP-diacylglycerol--inositol 3-phosphatidyltransferase; & Q8VDP6 & Cdipt & $-0,1$ & 10,8 \\
\hline Cell adhesion molecule 3 ; & Q99N28 & Cadm3 & $-0,3$ & 4,5 \\
\hline Cell cycle and apoptosis regulator protein 2 & Q8VDP4 & Ccar2 & 0,6 & 3,5 \\
\hline Cell division control protein 42 homolog; & P60766 & $\mathrm{Cdc} 42$ & $-0,7$ & 28,3 \\
\hline Cell division cycle 5-like protein; & Q6A068 & Cdc5I & 0,7 & 1,2 \\
\hline Cell growth regulator with EF hand domain protein 1 ; & Q8R1U2 & Cgref1 & 0,4 & 8,2 \\
\hline Cell surface glycoprotein MUC18; & Q8R2Y2 & Mcam & 0,1 & 15,3 \\
\hline Cellular nucleic acid-binding protein; & P53996 & Cnbp & $-1,9$ & 22,5 \\
\hline Cellular retinoic acid-binding protein 2 & P22935 & Crabp2 & 0,5 & 21,7 \\
\hline Centromere protein $\mathrm{O}$ & Q8K015 & Cenpo & 1,5 & 4,5 \\
\hline Ceruloplasmin; & Q61147 & $\mathrm{Cp}$ & 0,0 & 9,3 \\
\hline Charged multivesicular body protein $1 \mathrm{~b}-1$; & Q99LU0 & Chmp1b1 & $-1,4$ & 4 \\
\hline Charged multivesicular body protein 2a; & Q9DB34 & Chmp2a & 0,1 & 4,1 \\
\hline Charged multivesicular body protein $2 \mathrm{~b}$; & Q8BJF9 & Chmp2b & 0,1 & 9,4 \\
\hline Charged multivesicular body protein $4 b$; & Q9D8B3 & Chmp4b & $-0,2$ & 17 \\
\hline Chitinase-3-like protein 1 ; & Q61362 & Chi3l1 & 2,1 & 7,1 \\
\hline Chitotriosidase-1; & Q9D7Q1 & Chit1 & 0,6 & 3,7 \\
\hline Chloride channel CLIC-like protein 1 ; & Q99LI2 & Clcc1 & $-0,6$ & 3,7 \\
\hline Chloride intracellular channel protein 1 ; & Q9Z1Q5 & Clic1 & 0,3 & 14,5 \\
\hline Choline transporter-like protein 1 & A2AMH5 & Slc44a1 & $-0,1$ & 8,1 \\
\hline Choline transporter-like protein 2; & Q8BY89 & Slc44a2 & $-0,7$ & 6,1 \\
\hline Choline/ethanolamine kinase; & 055229 & Chkb & 0,9 & 7,9 \\
\hline Cholinesterase; & Q03311 & Bche & $-0,7$ & 2,8 \\
\hline Chondroitin sulfate proteoglycan 4 & Q8VHYO & Cspg4 & 0,8 & 6,1 \\
\hline Chromatin target of PRMT1 protein; & Q9CY57 & Chtop & $-0,2$ & 10,5 \\
\hline Chromobox protein homolog 3 & P23198 & $\mathrm{Cb} \times 3$ & 0,7 & 6,6 \\
\hline Chromodomain-helicase-DNA-binding protein 4; & Q6PDQ2 & Chd4 & 0,0 & 2,4 \\
\hline Cingulin; & P59242 & Cgn & 0,1 & 1,7 \\
\hline Citrate lyase subunit beta-like protein, mitochondrial; & Q8R4N0 & Clybl & 1,1 & 2,4 \\
\hline Citrate synthase, mitochondrial; & Q9CZU6 & Cs & $-0,2$ & 4,5 \\
\hline Clathrin heavy chain 1 ; & Q68FD5 & Cltc & 0,3 & 6,4 \\
\hline Clathrin interactor 1 ; & Q99KN9 & Clint1 & 0,4 & 6,6 \\
\hline Clathrin light chain A; & 008585 & Clta & 0,0 & 18,5 \\
\hline Clathrin light chain B; & Q6IRU5 & Cltb & $-0,1$ & 22,7 \\
\hline Cleavage and polyadenylation specificity factor subunit 1 ; & Q9EPU4 & Cpsf1 & 0,3 & 0,5 \\
\hline Cleavage and polyadenylation specificity factor subunit 5 & Q9CQF3 & Nudt21 & 0,6 & 11,9 \\
\hline Cleavage and polyadenylation specificity factor subunit 7; & Q8BTV2 & Cpsf7 & 0,7 & 2,3 \\
\hline Cleavage stimulation factor subunit 1 & Q99LC2 & Cstf1 & 0,0 & 7,7 \\
\hline Cleavage stimulation factor subunit 2 & Q8BIQ5 & Cstf2 & 0,3 & 6 \\
\hline Cleft lip and palate transmembrane protein 1 homolog; & Q8VBZ3 & Clptm1 & $-0,7$ & 7,2 \\
\hline Clustered mitochondria protein homolog; & Q5SW19 & Cluh & 0,5 & 2,2 \\
\hline Clusterin; & Q06890 & $\mathrm{Clu}$ & $-0,8$ & 14,5 \\
\hline CMRF35-like molecule 9; & Q1ERP8 & Cd300lg & $-0,2$ & 2,1 \\
\hline C-Myc-binding protein; & Q9EQS3 & Mycbp & $-0,1$ & 44,7 \\
\hline Coactosin-like protein; & Q9CQI6 & Cotl1 & 0,5 & 24,6 \\
\hline Coagulation factor XII; & Q80YC5 & $\mathrm{F} 12$ & 0,4 & 1,7 \\
\hline Coagulation factor XIII A chain; & Q8BH61 & F13a1 & 0,2 & 13,1 \\
\hline
\end{tabular}




\begin{tabular}{|c|c|c|c|c|}
\hline Protein & Uniprot & Gene name & $\begin{array}{c}\text { Fold change of } \\
\text { XPRESS ratio } \\
\left.\text { (Adam17 }^{\Delta K C} / \mathrm{WT}\right)\end{array}$ & $\begin{array}{c}\text { Sequence } \\
\text { coverage [\%] }\end{array}$ \\
\hline Coatomer subunit alpha; & Q8CIE6 & Copa & 0,5 & 5,1 \\
\hline Coatomer subunit beta; & Q9JIF7 & Copb1 & 0,8 & 14,5 \\
\hline Coatomer subunit beta'; & 055029 & Copb2 & 0,4 & 9,4 \\
\hline Coatomer subunit delta; & Q5XJY5 & Arcn1 & 0,8 & 12,3 \\
\hline Coatomer subunit epsilon; & 089079 & Cope & $-0,3$ & 8,8 \\
\hline Coatomer subunit gamma-1; & Q9QZE5 & Copg1 & 1,1 & 10,6 \\
\hline Cob(I)yrinic acid a,c-diamide adenosyltransferase, mitochondrial; & Q9D273 & Mmab & $-0,3$ & 5,9 \\
\hline Cochlin; & Q62507 & Coch & 0,2 & 4,9 \\
\hline Cofilin-1; & P18760 & $\mathrm{Cfl1}$ & 0,1 & 27,1 \\
\hline Cofilin-2; & P45591 & $\mathrm{Cfl} 2$ & $-0,1$ & 11,4 \\
\hline Cohesin subunit SA-1; & Q9D3E6 & Stag1 & $-0,8$ & 2,1 \\
\hline Coiled-coil domain-containing protein 115 & Q8VE99 & Ccdc115 & 0,4 & 5 \\
\hline Coiled-coil domain-containing protein 124 & Q9D8X2 & Ccdc124 & $-0,3$ & 10,6 \\
\hline Coiled-coil domain-containing protein 151 & Q8BSN3 & Ccdc151 & $-3,9$ & 2,7 \\
\hline Coiled-coil domain-containing protein $160 ;$ & Q3UYG1 & Ccdc160 & $-1,8$ & 4,3 \\
\hline Coiled-coil domain-containing protein 50; & Q810U5 & Ccdc50 & $-0,6$ & 6,3 \\
\hline Coiled-coil domain-containing protein 58 ; & Q8R3Q6 & Ccdc58 & 2,5 & 22,4 \\
\hline Coiled-coil domain-containing protein 69 & Q3TCJ8 & Ccdc69 & $-10,0$ & 10,9 \\
\hline Coiled-coil domain-containing protein 80 & Q8R2G6 & Ccdc80 & 0,7 & 2,7 \\
\hline Coiled-coil domain-containing protein 9; & Q8VC31 & Ccdc9 & 0,0 & 1,7 \\
\hline Coiled-coil-helix-coiled-coil-helix domain-containing protein 2, mitochondrial; & Q9D1L0 & Chchd2 & $-0,1$ & 15 \\
\hline Coiled-coil-helix-coiled-coil-helix domain-containing protein 3, mitochondrial; & Q9CRB9 & Chchd3 & $-0,3$ & 14,6 \\
\hline Coiled-coil-helix-coiled-coil-helix domain-containing protein 6, mitochondrial; & Q91VN4 & Chchd6 & 0,3 & 9 \\
\hline Cold shock domain-containing protein $\mathrm{C} 2$; & Q91YQ3 & Csdc2 & $-0,5$ & 28,2 \\
\hline Collagen alpha- $1(\mathrm{I})$ chain; & P11087 & Col1a1 & 0,8 & 10,5 \\
\hline Collagen alpha-1(III) chain; & P08121 & Col3a1 & 0,6 & 3,3 \\
\hline Collagen alpha-1(IV) chain; & P02463 & Col4a1 & $-0,1$ & 3,1 \\
\hline Collagen alpha-1(VI) chain; & Q04857 & Col6a1 & 0,3 & 11,4 \\
\hline Collagen alpha-1(XII) chain; & Q60847 & Col12a1 & 0,1 & 3,9 \\
\hline Collagen alpha-1(XIV) chain; & Q80X19 & Col14a1 & 1,0 & 6,2 \\
\hline Collagen alpha-1(XV) chain; & 035206 & Col15a1 & 0,2 & 15,5 \\
\hline Collagen alpha-1(XVII) chain; & Q07563 & Col17a1 & 0,5 & 0,5 \\
\hline Collagen alpha-1(XVIII) chain; & P39061 & Col18a1 & 0,0 & 2,7 \\
\hline Collagen alpha-2(I) chain; & Q01149 & Col1a2 & 0,4 & 11,9 \\
\hline Collagen alpha-2(IV) chain; & P08122 & Col4a2 & 0,2 & 4,1 \\
\hline Collagen alpha-2(VI) chain; & Q02788 & Col6a2 & 0,2 & 9,2 \\
\hline Collagen alpha-5(VI) chain; & A6H584 & Col6a5 & 3,3 & 0,6 \\
\hline Complement C3; & P01027 & $\mathrm{C} 3$ & 1,1 & 18,5 \\
\hline Complement component $\mathrm{C} 1 \mathrm{q}$ receptor; & 089103 & $\mathrm{Cd} 93$ & 1,0 & 2,8 \\
\hline Complement factor D; & P03953 & Cfd & 0,1 & 21,6 \\
\hline Complement factor I; & Q61129 & $\mathrm{Cfi}$ & 1,2 & 4 \\
\hline Constitutive coactivator of PPAR-gamma-like protein 1 ; & Q6A0A9 & FAM120A & 0,6 & 1,2 \\
\hline COP9 signalosome complex subunit 2 & P61202 & Cops2 & 0,7 & 5,9 \\
\hline COP9 signalosome complex subunit 3 ; & 088543 & Cops3 & 0,9 & 15,1 \\
\hline COP9 signalosome complex subunit 4; & 088544 & Cops4 & 0,5 & 25,2 \\
\hline COP9 signalosome complex subunit 5; & 035864 & Cops5 & 0,3 & 18,6 \\
\hline COP9 signalosome complex subunit 6; & 088545 & Cops6 & 0,6 & 19,7 \\
\hline COP9 signalosome complex subunit 7a; & Q9CZ04 & Cops7a & 0,3 & 17,7 \\
\hline COP9 signalosome complex subunit 8; & Q8VBV7 & Cops8 & $-0,1$ & 7,2 \\
\hline Copine-3; & Q8BT60 & Cpne3 & $-0,5$ & 3,4 \\
\hline Copper transport protein ATOX1; & 008997 & Atox1 & $-0,4$ & 38,2 \\
\hline Core histone macro-H2A.1; & Q9QZQ8 & H2afy & $-0,1$ & 22 \\
\hline Core histone macro-H2A.2; & Q8CCKO & H2afy2 & $-0,2$ & 12,9 \\
\hline Corneodesmosin; & Q7TPC1 & Cdsn & $-0,1$ & 7,3 \\
\hline Cornifelin; & Q6PCW6 & Cnfn & $-2,1$ & 19,8 \\
\hline Cornifin-A; & Q62266 & Sprr1a & $-1,2$ & 68,8 \\
\hline Cornifin-B; & Q62267 & Sprr1b & $-1,7$ & 9,8 \\
\hline Coronin-1C; & Q9WUM4 & Corolc & 4,9 & 3,6 \\
\hline Costars family protein ABRACL; & Q4KML4 & Abracl & $-0,3$ & 27,2 \\
\hline Coxsackievirus and adenovirus receptor homolog; & P97792 & Cxadr & $-0,4$ & 14,5 \\
\hline Craniofacial development protein 1 ; & 088271 & Cfdp1 & 0,1 & 15,9 \\
\hline Creatine kinase U-type, mitochondrial; & P30275 & Ckmt1 & $-0,2$ & 32,5 \\
\hline C-terminal-binding protein 1 & 088712 & Ctbp1 & 0,0 & 24 \\
\hline C-terminal-binding protein 2 & P56546 & Ctbp2 & 0,0 & 22,6 \\
\hline CTP synthase $1 ;$ & P70698 & Ctps1 & $-0,5$ & 37,1 \\
\hline C-type lectin domain family 11 member A; & 088200 & Clec11a & 0,3 & 4,3 \\
\hline C-type mannose receptor 2 & Q64449 & Mrc2 & 0,6 & 7 \\
\hline Cullin-1; & Q9WTX6 & Cul1 & $-0,1$ & 17,4 \\
\hline Cullin-associated NEDD8-dissociated protein 1; & Q6ZQ38 & Cand1 & 0,1 & 18,9 \\
\hline Cyclin-dependent kinase 4 inhibitor C; & Q60772 & Cdkn2c & 0,0 & 11,9 \\
\hline
\end{tabular}




\begin{tabular}{|c|c|c|c|c|}
\hline Protein & Uniprot & Gene name & $\begin{array}{c}\text { Fold change of } \\
\text { XPRESS ratio } \\
\left.\text { (Adam17 }^{\Delta K C} / \mathrm{WT}\right)\end{array}$ & $\begin{array}{c}\text { Sequence } \\
\text { coverage [\%] }\end{array}$ \\
\hline Cyclin-dependent kinase 4; & P30285 & Cdk4 & 4,5 & 6,7 \\
\hline Cyclin-dependent kinase inhibitor 1B; & P46414 & Cdkn1b & 0,0 & 18,3 \\
\hline Cystathionine beta-synthase; & Q91WT9 & Cbs & 0,1 & 21,4 \\
\hline Cystathionine gamma-lyase; & Q8VCN5 & Cth & 2,2 & 5,8 \\
\hline Cystatin E/M; Cystatin M/E; Cystatin-B; & Q9D1B1 & Cst6 & 0,9 & 32,9 \\
\hline Cystatin-A; & P56567 & Csta & 1,0 & 12,4 \\
\hline Cystatin-B; & Q62426 & Cstb & $-0,7$ & 39,8 \\
\hline Cysteine and glycine-rich protein 1 ; & P97315 & Csrp1 & $-1,1$ & 43 \\
\hline Cysteine and glycine-rich protein 2 & P97314 & Csrp2 & $-0,9$ & 28 \\
\hline Cysteine sulfinic acid decarboxylase; & Q9DBE0 & Csad & 0,3 & 15,8 \\
\hline Cysteine-rich motor neuron 1 protein; & Q9JLL0 & Crim1 & $-4,5$ & 1,4 \\
\hline Cysteine-rich protein 1; & P63254 & Crip1 & $-0,3$ & 9,1 \\
\hline Cysteine-rich protein 2 & Q9DCT8 & Crip2 & $-0,7$ & 15,4 \\
\hline Cysteine-rich with EGF-like domain protein 2; & Q9CYAO & Creld2 & $-0,1$ & 8,3 \\
\hline Cytochrome b5 type B; & Q9CQX2 & Cyb5b & $-0,5$ & 50,7 \\
\hline Cytochrome b5; & P56395 & Cyb5a & $-0,1$ & 65,3 \\
\hline Cytochrome b-c1 complex subunit 1, mitochondrial; & Q9CZ13 & Uqcrc1 & $-0,1$ & 25 \\
\hline Cytochrome b-c1 complex subunit 2, mitochondrial; & Q9DB77 & Uqcrc2 & $-0,4$ & 27,6 \\
\hline Cytochrome b-c1 complex subunit 6, mitochondrial; & P99028 & Uqcrh & $-0,4$ & 28,1 \\
\hline Cytochrome b-c1 complex subunit 7; & Q9D855 & Uqcrb & $-0,4$ & 25,2 \\
\hline Cytochrome b-c1 complex subunit 8 & Q9CQ69 & Uqcrq & $-0,3$ & 42,7 \\
\hline Cytochrome b-c1 complex subunit 9; & Q8R1I1 & Uqcr10 & $-0,3$ & 50 \\
\hline Cytochrome b-c1 complex subunit Rieske, mitochondrial; & Q9CR68 & Uqcrfs1 & 0,2 & 20,1 \\
\hline Cytochrome c oxidase assembly factor 3 homolog, mitochondrial; & Q9D2R6 & Coa3 & 0,0 & 21,1 \\
\hline Cytochrome c oxidase subunit 1 & P00397 & Mtco1 & $-1,7$ & 4,3 \\
\hline Cytochrome c oxidase subunit 2; & P00405 & Mtco2 & $-0,2$ & 16,3 \\
\hline Cytochrome c oxidase subunit 3 & P00416 & $\mathrm{mt} \mathrm{Co3}$ & $-0,6$ & 5,4 \\
\hline Cytochrome c oxidase subunit 4 isoform 1, mitochondrial; & P19783 & Cox4i1 & $-0,3$ & 35,5 \\
\hline Cytochrome c oxidase subunit $5 \mathrm{~A}$, mitochondrial; & P12787 & Cox5a & $-0,1$ & 21,9 \\
\hline Cytochrome c oxidase subunit 5B, mitochondrial; & P19536 & Cox5b & $-0,2$ & 6,3 \\
\hline Cytochrome c oxidase subunit $6 \mathrm{~B} 1$ & P56391 & Cox6b1 & $-0,1$ & 52,3 \\
\hline Cytochrome c oxidase subunit $6 \mathrm{C}$; & Q9CPQ1 & Cox6c & $-0,3$ & 18,4 \\
\hline Cytochrome c oxidase subunit 7A2, mitochondrial; & P48771 & Cox7a2 & $-0,1$ & 27,7 \\
\hline Cytochrome c oxidase subunit 7C, mitochondrial; & P17665 & Cox7c & 0,0 & 14,3 \\
\hline Cytochrome c, somatic; & P62897 & Cycs & $-0,9$ & 38,1 \\
\hline Cytochrome $\mathrm{c} 1$, heme protein, mitochondrial; & Q9D0M3 & Cyc1 & $-0,1$ & 27,4 \\
\hline Cytochrome P450 2B10; MCG7659, isoform CRA_a; & G3X8Q9 & Cyp2b10 & $-0,1$ & 6,5 \\
\hline Cytochrome P450 2F2; & P33267 & Cyp2f2 & 1,0 & 5,3 \\
\hline Cytochrome P450 2S1; & Q9DBX6 & Cyp2s1 & $-0,9$ & 5,4 \\
\hline Cytochrome P450 4B1; & Q64462 & Cyp4b1 & 0,2 & 3,2 \\
\hline Cytoglobin; & Q9CX80 & Cygb & $-0,4$ & 13,2 \\
\hline Cytoplasmic aconitate hydratase; & P28271 & Aco1 & $-0,1$ & 17,3 \\
\hline Cytoplasmic dynein 1 heavy chain 1 ; & Q9JHU4 & Dync1h1 & 0,2 & 3,6 \\
\hline Cytoplasmic dynein 1 intermediate chain 2 & 088487 & Dync1i2 & 0,1 & 8,5 \\
\hline Cytoplasmic dynein 1 light intermediate chain 1 ; & Q8R1Q8 & Dync1li1 & 0,2 & 5,2 \\
\hline Cytoplasmic dynein 1 light intermediate chain 2; & Q6PDL0 & Dync1li2 & 0,2 & 4,5 \\
\hline Cytoplasmic FMR1-interacting protein 2; & Q5SQX6 & Cyfip2 & $-1,7$ & 3,8 \\
\hline Cytoskeleton-associated protein 4; & Q8BMK4 & Ckap4 & 0,5 & 21,9 \\
\hline Cytosol aminopeptidase; & Q9CPY7 & Lap3 & 0,0 & 41,6 \\
\hline Cytosolic acyl coenzyme A thioester hydrolase; & Q91V12 & Acot7 & 0,4 & 14,2 \\
\hline Cytosolic endo-beta-N-acetylglucosaminidase; & Q8BX80 & Engase & 1,6 & 6,4 \\
\hline Cytosolic non-specific dipeptidase; & Q9D1A2 & Cndp2 & 0,0 & 13,1 \\
\hline Cytosolic phospholipase A2 beta; & B7ZCM9 & Gm28042 & 0,2 & 2,7 \\
\hline Cytosolic purine 5'-nucleotidase; & Q3V1L4 & $\mathrm{Nt5} 52$ & $-0,3$ & 14,1 \\
\hline D-3-phosphoglycerate dehydrogenase; & Q61753 & Phgdh & 0,2 & 18,2 \\
\hline DAZ-associated protein 1 & Q9JII5 & Dazap1 & 0,6 & 7,6 \\
\hline D-dopachrome decarboxylase; & 035215 & Ddt & $-0,1$ & 54,2 \\
\hline DDRGK domain-containing protein 1 & Q80WW9 & Ddrgk1 & $-0,2$ & 4,8 \\
\hline DEAH (Asp-Glu-Ala-His) box polypeptide 16, isoform CRA_a; Protein Dhx16; & G3X8X0 & Dhx16 & 0,5 & 1,6 \\
\hline Death-associated protein-like 1 ; & Q9D757 & Dapl1 & 1,3 & 7,5 \\
\hline Decorin; & P28654 & Den & 0,5 & 19,5 \\
\hline Dehydrogenase/reductase SDR family member 7; & Q9CXR1 & Dhrs7 & $-0,4$ & 5,9 \\
\hline Dehydrogenase/reductase SDR family member 7B; & Q99J47 & Dhrs7b & 0,2 & 2,8 \\
\hline Delta(24)-sterol reductase; & Q8VCH6 & Dhcr24 & 0,8 & 3,1 \\
\hline Delta(3,5)-Delta(2,4)-dienoyl-CoA isomerase, mitochondrial; & 035459 & Ech1 & $-0,2$ & 23,9 \\
\hline Delta-aminolevulinic acid dehydratase; & P10518 & Alad & 0,1 & 30,3 \\
\hline Delta-sarcoglycan; & P82347 & Sgcd & 0,3 & 5,2 \\
\hline Density-regulated protein; & Q9CQJ6 & Denr & $-0,2$ & 8,7 \\
\hline Deoxyguanosine kinase, mitochondrial; & Q9QX60 & Dguok & 0,3 & 14,8 \\
\hline Deoxyhypusine hydroxylase; & Q99LN9 & Dohh & $-5,4$ & 10,6 \\
\hline Deoxynucleoside triphosphate triphosphohydrolase SAMHD1; & Q60710 & Samhd1 & 0,2 & 12,8 \\
\hline Deoxyribonuclease-1-like 2; & Q9D1G0 & Dnase1/2 & $-1,9$ & 12,6 \\
\hline
\end{tabular}




\begin{tabular}{|c|c|c|c|c|}
\hline Protein & Uniprot & Gene name & $\begin{array}{c}\text { Fold change of } \\
\text { XPRESS ratio } \\
\left.\text { (Adam17 }^{\Delta K C} / \mathrm{WT}\right)\end{array}$ & $\begin{array}{c}\text { Sequence } \\
\text { coverage [\%] }\end{array}$ \\
\hline Deoxyribonuclease-2-alpha; & P56542 & Dnase2 & $-0,6$ & 23,8 \\
\hline Derlin-1; & Q99J56 & Derl1 & $-0,2$ & 10,8 \\
\hline Dermatopontin; & Q9QZZ6 & Dpt & $-0,4$ & 32,3 \\
\hline Dermokine; & E9Q2P1 & Dmkn & 0,0 & 20,3 \\
\hline Desmocollin-1; & P55849 & Dsc1 & 0,2 & 8,8 \\
\hline Desmocollin-3; & P55850 & Dsc3 & 0,5 & 10,6 \\
\hline Desmoglein-1-alpha; & Q61495 & Dsg1a & $-0,1$ & 3,2 \\
\hline Desmoglein-2; & 055111 & Dsg2 & $-2,7$ & 1,1 \\
\hline Desmoglein-3; & 035902 & Dsg3 & $-0,7$ & 1,8 \\
\hline Desmoplakin; & E9Q557 & Dsp & $-0,3$ & 2 \\
\hline Destrin; & Q9R0P5 & Dstn & 0,3 & 20 \\
\hline Diacylglycerol O-acyltransferase 1; & Q9Z2A7 & Dgat1 & 0,0 & 5 \\
\hline Dickkopf-related protein 3; & Q9QUN9 & Dkk3 & $-0,2$ & 3,4 \\
\hline Dihydrolipoyl dehydrogenase, mitochondrial; & 008749 & Dld & $-0,1$ & 22,8 \\
\hline $\begin{array}{l}\text { Dihydrolipoyllysine-residue acetyltransferase component of pyruvate } \\
\text { dehydrogenase complex, mitochondrial; }\end{array}$ & Q8BMF4 & Dlat & 0,5 & 6,4 \\
\hline $\begin{array}{l}\text { Dihydrolipoyllysine-residue succinyltransferase component of 2-oxoglutarate } \\
\text { dehydrogenase complex, mitochondrial; }\end{array}$ & Q9D2G2 & Dlst & $-0,3$ & 10,4 \\
\hline Dihydroorotate dehydrogenase (quinone), mitochondrial; & 035435 & Dhodh & 0,2 & 11,4 \\
\hline Dihydropteridine reductase; & Q8BVI4 & Qdpr & 0,0 & 24,9 \\
\hline Dihydropyrimidinase-related protein 1 ; & P97427 & Crmp1 & $-0,3$ & 5,2 \\
\hline Dihydropyrimidinase-related protein 2 ; & 008553 & Dpysl2 & 0,0 & 35,8 \\
\hline Dihydropyrimidinase-related protein 3 ; & E9PWE8 & Dpysl3 & 0,0 & 22 \\
\hline Di-N-acetylchitobiase; & Q8R242 & Ctbs & 0,5 & 4,1 \\
\hline Dipeptidase 1; & P31428 & Dpep1 & $-0,5$ & 12,9 \\
\hline Dipeptidyl peptidase 1 & P97821 & Ctsc & $-0,1$ & 7,4 \\
\hline Dipeptidyl peptidase 2; & Q9ET22 & Dpp7 & $-0,1$ & 2,2 \\
\hline Dipeptidyl peptidase 3; & Q99KK7 & Dpp3 & 1,2 & 2,2 \\
\hline Dipeptidyl peptidase 4; & P28843 & Dpp4 & $-0,6$ & 15,4 \\
\hline Diphosphoinositol polyphosphate phosphohydrolase 3-alpha; & P0C027 & Nudt10 & 0,3 & 5,7 \\
\hline Disabled homolog 2 & P98078 & Dab2 & $-4,9$ & 5,9 \\
\hline Disintegrin and metalloproteinase domain-containing protein $10 ;$ & 035598 & Adam10 & 1,4 & 2,4 \\
\hline Disks large-associated protein 4 & B1AZP2 & Dlgap4 & $-0,2$ & 7,3 \\
\hline DNA damage-binding protein $1 ;$ & Q3U1J4 & Ddb1 & $-0,2$ & 26,5 \\
\hline DNA ligase $1 ;$ & P37913 & Lig1 & 0,7 & 5,2 \\
\hline DNA mismatch repair protein Msh2; & P43247 & Msh2 & 0,7 & 1,2 \\
\hline DNA polymerase lambda; & Q9QXE2 & Poll & $-0,6$ & 1,6 \\
\hline DNA repair protein $\mathrm{XRCC1}$; & Q60596 & Xrcc1 & 0,3 & 2,1 \\
\hline DNA replication licensing factor $\mathrm{MCM} 2$ & P97310 & Mcm2 & 0,5 & 1,8 \\
\hline DNA replication licensing factor MCM6; & P97311 & Mcm6 & 0,5 & 3,8 \\
\hline DNA replication licensing factor $\mathrm{MCM7}$; & Q61881 & Mcm7 & 2,0 & 1,8 \\
\hline DNA topoisomerase 1 & Q04750 & Top1 & $-0,6$ & 2 \\
\hline DNA topoisomerase 2-beta; & Q64511 & Top2b & 0,3 & 4,4 \\
\hline DNA-(apurinic or apyrimidinic site) lyase; & F6QA74 & Apex1 & 0,3 & 14,5 \\
\hline DNA-directed RNA polymerase II subunit RPB1; & P08775 & Polr2a & 0,0 & 3,1 \\
\hline DNA-directed RNA polymerase II subunit RPB2; & Q8CFI7 & Polr2b & 0,2 & 7,5 \\
\hline DNA-directed RNA polymerases I, II, and III subunit RPABC1; & Q80UW8 & Polr2e & $-0,1$ & 10,5 \\
\hline DnaJ homolog subfamily A member 1 & P63037 & Dnaja1 & 0,4 & 8,1 \\
\hline DnaJ homolog subfamily A member 2 ; & Q9QYJ0 & Dnaja2 & $-0,5$ & 3,2 \\
\hline DnaJ homolog subfamily $\mathrm{C}$ member 2 & P54103 & Dnajc2 & 0,1 & 2,9 \\
\hline DnaJ homolog subfamily C member 3 ; & Q91YW3 & Dnajc3 & $-0,3$ & 1,8 \\
\hline DnaJ homolog subfamily $\mathrm{C}$ member 5 & P60904 & Dnajc5 & $-0,7$ & 17,2 \\
\hline Dolichol-phosphate mannosyltransferase subunit 1 ; & 070152 & Dpm1 & 0,0 & 9,9 \\
\hline Dolichol-phosphate mannosyltransferase subunit 3; & Q9D1Q4 & Dpm3 & 0,2 & 23,9 \\
\hline Dolichyl-diphosphooligosaccharide--protein glycosyltransferase 48 kDa subunit; & 054734 & Ddost & 0,0 & 17,7 \\
\hline Dolichyl-diphosphooligosaccharide--protein glycosyltransferase subunit 1; & Q91YQ5 & Rpn1 & 0,2 & 28,3 \\
\hline Dolichyl-diphosphooligosaccharide--protein glycosyltransferase subunit 2; & Q9DBG6 & Rpn2 & 0,1 & 16,7 \\
\hline Dolichyl-diphosphooligosaccharide--protein glycosyltransferase subunit DAD1; & P61804 & Dad1 & 0,1 & 19,5 \\
\hline Dolichyl-diphosphooligosaccharide--protein glycosyltransferase subunit STT3A; & P46978 & Stt3a & 0,1 & 7,7 \\
\hline Dolichyl-diphosphooligosaccharide--protein glycosyltransferase subunit STT3B; & Q3TDQ1 & Stt3b & $-0,2$ & 9,8 \\
\hline Double-strand-break repair protein rad21 homolog; & Q61550 & $\operatorname{Rad} 21$ & 0,2 & 2,2 \\
\hline Drebrin-like protein; & Q62418 & Dbnl & 0,3 & 2,5 \\
\hline Dual specificity protein phosphatase 14 & Q9JLY7 & Dusp14 & $-0,8$ & 16,7 \\
\hline Dual specificity protein phosphatase 23 & Q6NT99 & Dusp23 & 0,2 & 10 \\
\hline Dynactin subunit $1 ;$ & 008788 & Dctn1 & $-0,1$ & 3,6 \\
\hline Dynactin subunit 2 & Q99KJ8 & Dctn2 & 0,5 & 10 \\
\hline Dynactin subunit 4; & Q8CBY8 & Dctn4 & $-0,8$ & 10,1 \\
\hline
\end{tabular}




\begin{tabular}{|c|c|c|c|c|}
\hline Protein & Uniprot & Gene name & $\begin{array}{c}\text { Fold change of } \\
\text { XPRESS ratio } \\
\left.\text { (Adam17 }^{\Delta K C} / \mathrm{WT}\right)\end{array}$ & $\begin{array}{c}\text { Sequence } \\
\text { coverage [\%] }\end{array}$ \\
\hline Dynamin-like 120 kDa protein, mitochondrial; & P58281 & Opa1 & 0,6 & 5,5 \\
\hline Dynein light chain 1 , cytoplasmic; & P63168 & Dynll1 & $-0,3$ & 24,7 \\
\hline Dynein light chain roadblock-type 1 ; & P62627 & Dynlrb1 & $-0,1$ & 21,9 \\
\hline Dystroglycan; & Q62165 & Dag1 & $-0,1$ & 6,2 \\
\hline E3 ubiquitin-protein ligase ARIH1; & Q9Z1K5 & Arih1 & 0,4 & 4 \\
\hline E3 ubiquitin-protein ligase HUWE1; & Q7TMY8 & Huwe1 & 0,8 & 0,9 \\
\hline E3 ubiquitin-protein ligase RBX1; & P62878 & Rbx1 & $-0,3$ & 7,4 \\
\hline E3 ubiquitin-protein ligase RING1; & 035730 & Ring1 & 0,6 & 4,2 \\
\hline E3 ubiquitin-protein ligase RNF126; & Q91YL2 & Rnf126 & $-0,2$ & 7,7 \\
\hline E3 ubiquitin-protein ligase RNF181; & Q9CY62 & Rnf181 & $-0,1$ & 17,6 \\
\hline E3 ubiquitin-protein ligase RNF213; & E9Q555 & Rnf213 & 6,3 & 0,5 \\
\hline E3 ubiquitin-protein ligase TRIP12; & G5E870 & Trip12 & 0,6 & 1,6 \\
\hline E3 ubiquitin-protein ligase UBR5; & Q80TP3 & Ubr5 & $-0,7$ & 0,5 \\
\hline Early endosome antigen 1 & Q8BL66 & Eea1 & $-0,1$ & 14,3 \\
\hline Echinoderm microtubule-associated protein-like 2; & Q7TNG5 & $\mathrm{Eml2}$ & $-0,3$ & 8,2 \\
\hline Ecto-ADP-ribosyltransferase 3; & E9Q7Q8 & Art3 & 0,0 & 8,3 \\
\hline Ectonucleoside triphosphate diphosphohydrolase 1; & P55772 & Entpd1 & $-1,1$ & 39,7 \\
\hline Ectonucleoside triphosphate diphosphohydrolase 2; & 055026 & Entpd2 & 0,7 & 3,8 \\
\hline Ectonucleotide pyrophosphatase/phosphodiesterase family member 1 ; & P06802 & Enpp1 & $-1,3$ & 5,1 \\
\hline Ectonucleotide pyrophosphatase/phosphodiesterase family member 2; & Q9R1E6 & Enpp2 & 0,3 & 1,4 \\
\hline Ectonucleotide pyrophosphatase/phosphodiesterase family member 3; & Q6DYE8 & Enpp3 & $-0,1$ & 1,8 \\
\hline EF-hand domain-containing protein D2; Efhd2 protein; & Q8C845 & Efhd2 & 0,3 & 15,4 \\
\hline EGF-containing fibulin-like extracellular matrix protein 1 & Q8BPB5 & Efemp1 & 0,1 & 26,8 \\
\hline EGF-containing fibulin-like extracellular matrix protein 2; & Q9WVJ9 & Efemp2 & $-0,4$ & 23,6 \\
\hline EH domain-containing protein 1 & Q9WVK4 & Ehd1 & 0,3 & 3,7 \\
\hline EH domain-containing protein 2 & Q8BH64 & Ehd2 & 0,5 & 7,6 \\
\hline EKC/KEOPS complex subunit Tprkb; & Q8QZZ7 & Tprkb & $-0,1$ & 10,3 \\
\hline ELAV-like protein 1 & P70372 & Elavl1 & 1,4 & 11 \\
\hline Electron transfer flavoprotein subunit alpha, mitochondrial; & Q99LC5 & Etfa & $-0,2$ & 23,4 \\
\hline Electron transfer flavoprotein subunit beta; & Q9DCW4 & Etfb & $-0,1$ & 32,5 \\
\hline Elongation factor 1-alpha 1 & P10126 & Eef1a1 & 0,9 & 39,6 \\
\hline Elongation factor 1-alpha 2; & P62631 & Eef1a2 & 0,3 & 21,8 \\
\hline Elongation factor 1-beta; & 070251 & Eef1b & 0,3 & 9,8 \\
\hline Elongation factor 1-delta; & F6ZFU0 & Eef1d & 0,2 & 12,1 \\
\hline Elongation factor 1-delta; & P57776 & Eef1d & $-0,3$ & 28,6 \\
\hline Elongation factor 1-gamma; & Q9D8N0 & Eef1g & $-0,1$ & 17,8 \\
\hline Elongation factor 2; & P58252 & Eef2 & 0,0 & 37,9 \\
\hline Elongation of very long chain fatty acids protein 5 & Q8BHI7 & Elovl5 & 0,0 & 5 \\
\hline Elongator complex protein 5; & Q99L85 & Elp5 & 0,0 & 9 \\
\hline Embigin; & P21995 & Emb & $-0,3$ & 3 \\
\hline Emerin; & 008579 & Emd & 0,1 & 18,5 \\
\hline Endonuclease domain-containing 1 protein; & Q8C522 & Endod1 & $-0,3$ & 10 \\
\hline Endoplasmic reticulum aminopeptidase 1 & Q9EQH2 & Erap1 & 0,3 & 3,1 \\
\hline Endoplasmic reticulum lectin 1 & Q8VEH8 & Erlec1 & $-0,9$ & 4,3 \\
\hline Endoplasmic reticulum metallopeptidase 1 & Q3UVKо & Ermp1 & $-0,4$ & 5,3 \\
\hline Endoplasmic reticulum resident protein 29 & P57759 & Erp29 & 0,1 & 13,7 \\
\hline Endoplasmic reticulum resident protein 44 & Q9D1Q6 & Erp44 & 0,2 & 23,4 \\
\hline Endoplasmic reticulum-Golgi intermediate compartment protein 1; & Q9DC16 & Ergic1 & 0,8 & 14,1 \\
\hline Endoplasmin; & P08113 & Hsp90b1 & 0,0 & 22,2 \\
\hline Endosialin; & Q91V98 & $\mathrm{Cd} 248$ & 0,0 & 3,3 \\
\hline Endothelial differentiation-related factor 1 ; & Q9JMG1 & Edf1 & $-0,1$ & 21,6 \\
\hline Endothelin-converting enzyme 1 ; & Q4PZA2 & Ece1 & $-0,5$ & 9,2 \\
\hline Enhancer of rudimentary homolog; & P84089 & Erh & $-0,1$ & 10,6 \\
\hline Enoyl-CoA delta isomerase 1, mitochondrial; & P42125 & Eci1 & $-0,1$ & 18,3 \\
\hline Enoyl-CoA delta isomerase 2, mitochondrial; & Q9WUR2 & Eci2 & $-1,0$ & 5,6 \\
\hline Ensconsin; & 088735 & Map7 & $-0,4$ & 4,2 \\
\hline Eosinophil cationic-type ribonuclease 5 ; & 035292 & Ear5 & 0,1 & 29,7 \\
\hline Epidermal growth factor receptor; & Q01279 & Egfr & $-0,6$ & 4,4 \\
\hline Epididymal secretory protein E1; & Q9Z0J0 & $\mathrm{Npc2}$ & $-0,6$ & 16,8 \\
\hline Epiplakin; & Q8R0W0 & Eppk1 & 0,2 & 7,3 \\
\hline Epithelial cell adhesion molecule; & Q99JW5 & Epcam & $-0,4$ & 14,3 \\
\hline Epoxide hydrolase 1; & Q9D379 & Ephx1 & $-0,5$ & 22,4 \\
\hline ER lumen protein-retaining receptor 1 ; & Q99JH8 & Kdelr1 & $-1,3$ & 8 \\
\hline ER membrane protein complex subunit 1 ; & Q8C7X2 & Emc1 & $-0,2$ & 5,2 \\
\hline ER membrane protein complex subunit 2 & Q9CRD2 & Emc2 & 0,8 & 3,7 \\
\hline Erlin-2; & Q8BFZ9 & Erlin2 & $-0,2$ & 13,2 \\
\hline ERO1-like protein alpha; & Q8R180 & Ero1l & 0,0 & 4,1 \\
\hline ES1 protein homolog, mitochondrial; & Q9D172 & D10Jhu81e & $-0,5$ & 32,7 \\
\hline Ester hydrolase C11orf54 homolog; & Q91V76 & & $-0,3$ & 18,7 \\
\hline Estradiol 17-beta-dehydrogenase 11 & Q9EQ06 & Hsd17b11 & $-1,0$ & 12,8 \\
\hline
\end{tabular}




\begin{tabular}{|c|c|c|c|c|}
\hline Protein & Uniprot & Gene name & 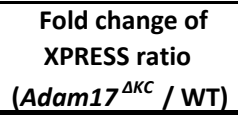 & $\begin{array}{c}\text { Sequence } \\
\text { coverage [\%] }\end{array}$ \\
\hline Estradiol 17-beta-dehydrogenase 12 & 070503 & Hsd17b12 & 0,6 & 16 \\
\hline Estradiol 17-beta-dehydrogenase 8; & P50171 & Hsd17b8 & $-0,3$ & 16,7 \\
\hline Ethylmalonyl-CoA decarboxylase; & Q9D9V3 & Echdc1 & $-0,4$ & 35,1 \\
\hline Eukaryotic initiation factor 4A-I; & P60843 & Eif4a1 & 0,2 & 21,9 \\
\hline Eukaryotic initiation factor 4A-II; & P10630 & Eif4a2 & 3,6 & 16,6 \\
\hline Eukaryotic initiation factor $4 \mathrm{~A}-\mathrm{III}$; & Q91VC3 & Eif4a3 & 0,3 & 27,5 \\
\hline Eukaryotic peptide chain release factor GTP-binding subunit ERF3A; & Q8R050 & Gspt1 & $-0,2$ & 1,4 \\
\hline Eukaryotic peptide chain release factor subunit 1 & Q8BWY3 & Etf1 & 0,2 & 5,7 \\
\hline Eukaryotic translation initiation factor $1 \mathrm{~A}$ & Q60872 & Eif1a & $-0,2$ & 6,9 \\
\hline Eukaryotic translation initiation factor 2 subunit 1 & Q6ZWX6 & Eif2s1 & 0,5 & 22,9 \\
\hline Eukaryotic translation initiation factor 2 subunit 2; & Q99L45 & Eif2s2 & 0,0 & 11,8 \\
\hline Eukaryotic translation initiation factor 2 subunit $3, \mathrm{X}$-linked; & Q9Z0N1 & Eif2s $3 x$ & 0,1 & 22,9 \\
\hline Eukaryotic translation initiation factor $2 \mathrm{~A}$; & Q8BJW6 & Eif2a & 0,4 & 7,2 \\
\hline Eukaryotic translation initiation factor 3 subunit $A$; & P23116 & Eif3a & 0,4 & 2,7 \\
\hline Eukaryotic translation initiation factor 3 subunit B; & Q8JZQ9 & Eif3b & $-1,0$ & 7,1 \\
\hline Eukaryotic translation initiation factor 3 subunit C; & Q8R1B4 & Eif3c & 0,0 & 2,2 \\
\hline Eukaryotic translation initiation factor 3 subunit $\mathrm{E}$; & P60229 & Eif3e & 0,5 & 6,5 \\
\hline Eukaryotic translation initiation factor 3 subunit F; & Q9DCH4 & Eif3f & 2,3 & 4,2 \\
\hline Eukaryotic translation initiation factor 3 subunit G; & Q971D1 & Eif3g & 0,1 & 10 \\
\hline Eukaryotic translation initiation factor 3 subunit $\mathrm{H}$; & Q91WK2 & Eif3h & 0,3 & 4 \\
\hline Eukaryotic translation initiation factor 3 subunit J-A; & Q3UGC7 & Eif3j1 & $-0,1$ & 8 \\
\hline Eukaryotic translation initiation factor 3 subunit K; & Q9DBZ5 & Eif3k & 1,3 & 11,5 \\
\hline Eukaryotic translation initiation factor 3 subunit L; & Q8QZY1 & Eif3l & $-0,3$ & 3,9 \\
\hline Eukaryotic translation initiation factor 4 gamma 1 ; & Q6NZJ6 & Eif4g1 & 0,1 & 2,9 \\
\hline Eukaryotic translation initiation factor 4B; & Q8BGD9 & Eif4b & 0,0 & 8,8 \\
\hline Eukaryotic translation initiation factor $4 \mathrm{E}$ & P63073 & Eif4e & 0,5 & 12,9 \\
\hline Eukaryotic translation initiation factor $4 \mathrm{E}$-binding protein 2 ; & P70445 & Eif4ebp2 & 0,0 & 10,8 \\
\hline Eukaryotic translation initiation factor $4 \mathrm{H}$; & Q9WUK2 & Eif4h & 0,4 & 9,3 \\
\hline Eukaryotic translation initiation factor 5 ; & P59325 & Eif5 & $-0,2$ & 1,9 \\
\hline Eukaryotic translation initiation factor $5 \mathrm{~A}-1$; & P63242 & Eif5a & 0,1 & 19 \\
\hline Eukaryotic translation initiation factor $5 \mathrm{~B}$; & Q05D44 & Eif5b & $-0,5$ & 3 \\
\hline Eukaryotic translation initiation factor 6 ; & 055135 & Eif6 & $-0,1$ & 27,3 \\
\hline Exosome complex component MTR3; & Q8BTW3 & Exosc6 & $-0,1$ & 8,8 \\
\hline Exosome complex component RRP4; & Q8VBV3 & Exosc2 & 0,1 & 6,9 \\
\hline Exosome complex component RRP41; & Q92119 & Exosc4 & 0,0 & 31 \\
\hline Exosome complex exonuclease RRP42; & Q9D0M0 & Exosc7 & 0,2 & 3,8 \\
\hline Exosome complex exonuclease RRP44; & Q9CSH3 & Dis3 & 1,4 & 1 \\
\hline Exportin-2; & Q9ERK4 & Cse1l & 3,1 & 2,6 \\
\hline Extended synaptotagmin-1; & Q3U7R1 & Esyt1 & 0,3 & 3,7 \\
\hline Extracellular matrix protein 1 & Q61508 & Ecm1 & $-0,1$ & 7,7 \\
\hline Extracellular superoxide dismutase $[\mathrm{Cu}-\mathrm{Zn}]$; & 009164 & Sod3 & 0,7 & 5,2 \\
\hline Ezrin; & P26040 & Ezr & $-1,0$ & 9,2 \\
\hline FACT complex subunit SPT16; & Q920B9 & Supt16h & $-0,5$ & 5,7 \\
\hline FACT complex subunit SSRP1; & Q08943 & Ssrp1 & 0,0 & 5,1 \\
\hline F-actin-capping protein subunit alpha-1; & P47753 & Capza1 & $-0,7$ & 36,4 \\
\hline F-actin-capping protein subunit alpha- 2 ; & P47754 & Capza2 & $-0,1$ & 31,8 \\
\hline Far upstream element-binding protein 1 ; & Q91WJ8 & Fubp1 & $-0,1$ & 26 \\
\hline Far upstream element-binding protein 2 & Q3U0V1 & Khsrp & $-0,7$ & 20,3 \\
\hline Farnesyl pyrophosphate synthase; & Q920E5 & Fdps & 0,2 & 7,1 \\
\hline FAS-associated factor 2 & Q3TDN2 & Faf2 & 0,3 & 13,6 \\
\hline Fascin; & Q61553 & Fscn1 & 0,8 & 7,7 \\
\hline Fatty acid 2-hydroxylase; & Q5MPP0 & $\mathrm{Fa} 2 \mathrm{~h}$ & $-0,7$ & 15,1 \\
\hline Fatty acid desaturase 3; & Q9JJE7 & Fads3 & $-0,7$ & 8,2 \\
\hline Fatty acid synthase; & P19096 & Fasn & 0,2 & 6,2 \\
\hline Fatty acid-binding protein, adipocyte; & P04117 & Fabp4 & 0,0 & 27,3 \\
\hline Fatty acid-binding protein, epidermal; & Q05816 & Fabp5 & 0,1 & 71,9 \\
\hline Fatty acyl-CoA reductase 2 & Q7TNT2 & Far2 & 0,6 & 2,1 \\
\hline Fatty aldehyde dehydrogenase; & P47740 & Aldh3a2 & 0,4 & 7,9 \\
\hline F-box-like/WD repeat-containing protein TBL1XR1; & Q8BHJ5 & Tbl1xr1 & 0,5 & 3,9 \\
\hline Fermitin family homolog 2 & Q8CIB5 & Fermt2 & 4,3 & 3,5 \\
\hline Ferric-chelate reductase 1 & Q8K385 & FRRS1 & $-0,5$ & 2,4 \\
\hline Ferritin heavy chain; & P09528 & Fth1 & $-0,3$ & 18,7 \\
\hline Ferritin; & Q9CPX4 & Ftl1 & $-0,1$ & 30,6 \\
\hline Ferrochelatase, mitochondrial; & P22315 & Fech & 0,2 & 11,9 \\
\hline Fetuin-B; & Q9QXC1 & Fetub & 0,1 & 9,1 \\
\hline Fibrillin-1; & A2AQ53 & Fbn1 & $-0,3$ & 5,8 \\
\hline Fibrillin-2; & Q61555 & Fbn2 & $-0,4$ & 1,8 \\
\hline Fibrinogen beta chain; & Q8K0E8 & $\mathrm{Fgb}$ & 1,2 & 11,9 \\
\hline Fibrinogen gamma chain; & Q8VCM7 & Fgg & 1,3 & 17,7 \\
\hline Fibroblast growth factor-binding protein 1 ; & 070514 & Fgfbp1 & 0,9 & 8 \\
\hline Fibroleukin; & P12804 & $\mathrm{Fgl2}$ & $-0,8$ & 6,3 \\
\hline $\begin{array}{l}\text { Fibronectin leucine rich transmembrane protein } 3 \text {; Fibronectin leucine rich } \\
\text { transmembrane protein } 3 \text { isoform CRA a: Protein Flrt3. }\end{array}$ & Q8BGT1 & Flrt3 & $-0,8$ & 2,9 \\
\hline
\end{tabular}




\begin{tabular}{|c|c|c|c|c|}
\hline Protein & Uniprot & Gene name & $\begin{array}{c}\text { Fold change of } \\
\text { XPRESS ratio } \\
\left.\text { (Adam17 }^{\Delta K C} / \mathrm{WT}\right)\end{array}$ & $\begin{array}{c}\text { Sequence } \\
\text { coverage [\%] }\end{array}$ \\
\hline Fibronectin; & P11276 & Fn1 & 0,3 & 2,3 \\
\hline Fibulin-1; & Q08879 & Fbln1 & 0,3 & 4,5 \\
\hline Fibulin-2; & P37889 & Fbln2 & 0,6 & 6,1 \\
\hline Fibulin-5; & Q9WVH9 & Fbln5 & 0,0 & 13,4 \\
\hline Filaggrin; & P11088 & Flg & $-0,6$ & 14,9 \\
\hline Filaggrin-2; & Q2VIS4 & Flg2 & $-1,6$ & 21,4 \\
\hline Filaggrin-2; & E9QPZ3 & Flg2 & $-1,2$ & 21,6 \\
\hline Filamin-A; & Q8BTM8 & FIna & 0,3 & 12,2 \\
\hline Filamin-B; & Q80X90 & Flnb & 0,4 & 7,7 \\
\hline Filamin-binding LIM protein 1 ; & Q71FD7 & Fblim1 & $-0,9$ & 20,3 \\
\hline Flotillin-1; & 008917 & Flot1 & 0,1 & 11,2 \\
\hline Flotillin-2; & Q60634 & Flot2 & $-0,1$ & 7,2 \\
\hline Follistatin-related protein 1 & Q62356 & Fstl1 & 0,9 & 6,2 \\
\hline Formin-1; & Q05860 & Fmn1 & $-5,4$ & 1,6 \\
\hline Formin-binding protein 4 & Q6ZQ03 & Fnbp4 & $-0,1$ & 0,8 \\
\hline Four and a half LIM domains protein 1 & P97447 & Fhl1 & $-0,8$ & 51,8 \\
\hline Four and a half LIM domains protein 3 & Q9R059 & Fhl3 & 0,2 & 18,7 \\
\hline Frizzled-1; & 070421 & Fzd1 & $-0,3$ & 3,3 \\
\hline Frizzled-2; & Q9JIP6 & Fzd2 & $-0,7$ & 5,6 \\
\hline Frizzled-7; & Q61090 & Fzd7 & $-0,6$ & 7,3 \\
\hline Fructose-1,6-bisphosphatase 1 & Q9QXD6 & Fbp1 & $-0,6$ & 12,7 \\
\hline Fructose-bisphosphate aldolase A; & P05064 & Aldoa & $-0,4$ & 46,7 \\
\hline Fructose-bisphosphate aldolase C; & P05063 & Aldoc & 0,3 & 12,4 \\
\hline Fumarylacetoacetase; & P35505 & Fah & $-0,2$ & 7,6 \\
\hline FUN14 domain-containing protein 2 & Q9D6K8 & Fundc2 & $-0,1$ & 7,9 \\
\hline FXYD domain-containing ion transport regulator 3 ; & Q61835 & Fxyd3 & $-0,8$ & 25 \\
\hline Galactose-1-phosphate uridylyltransferase; & Q03249 & Galt & $-0,5$ & 7,9 \\
\hline Galectin; & Q9CRB1 & Lgals7 & 0,2 & 55,1 \\
\hline Galectin-1; & P16045 & Lgals1 & 0,1 & 39,3 \\
\hline Galectin-3; & P16110 & Lgals3 & 0,2 & 24,2 \\
\hline Galectin-related protein; & Q8VED9 & Lgalsl & 0,0 & 8,1 \\
\hline Gamma-adducin; & Q9QYB5 & Add3 & 0,1 & 9,5 \\
\hline Gamma-glutamyl hydrolase; & Q9Z0L8 & Ggh & 0,5 & 27,1 \\
\hline Gamma-glutamylcyclotransferase; & Q9D7X8 & Ggct & $-1,2$ & 21,8 \\
\hline Gamma-interferon-inducible lysosomal thiol reductase; & Q9ESY9 & Ifi30 & $-0,2$ & 5,2 \\
\hline Gamma-synuclein; & Q9Z0F7 & Sncg & 1,9 & 7,3 \\
\hline Ganglioside GM2 activator; & Q60648 & Gm2a & 0,2 & 9,3 \\
\hline Gap junction alpha-1 protein; & P23242 & Gja1 & $-0,5$ & 21,7 \\
\hline Gasdermin-A; & Q9EST1 & Gsdma & $-0,7$ & 21,1 \\
\hline GDH/6PGL endoplasmic bifunctional protein; & Q8CFX1 & H6pd & 0,1 & 4,9 \\
\hline GDP-fucose protein O-fucosyltransferase 1 & Q91ZW2 & Pofut1 & 1,0 & 10,5 \\
\hline GDP-L-fucose synthase; & P23591 & Tsta3 & $-0,2$ & 14,6 \\
\hline GDP-mannose 4,6 dehydratase; & Q8K0C9 & Gmds & 0,2 & 5,4 \\
\hline Gelsolin; & P13020 & Gsn & 0,0 & 21,2 \\
\hline Gelsolin; & A6PWS5 & Gsn & $-1,0$ & 11,2 \\
\hline General transcription factor IIF subunit 1 ; & Q3THK3 & Gtf2f1 & 0,8 & 4,3 \\
\hline General transcription factor IIF subunit 2; & Q8R0A0 & Gtf2f2 & $-0,2$ & 15,7 \\
\hline General transcription factor II-I; & Q9ESZ8 & Gtf2i & $-0,6$ & 4,6 \\
\hline General vesicular transport factor $\mathrm{p} 115$ & Q9Z1Z0 & Uso1 & 1,3 & 5,3 \\
\hline Gephyrin; & Q8BUV3 & Gphn & 0,0 & 1,3 \\
\hline Gigaxonin; & Q8CA72 & Gan & 0,6 & 3,4 \\
\hline Glia maturation factor beta; & Q9CQI3 & Gmfb & $-0,3$ & 14,1 \\
\hline Glucosamine-6-phosphate isomerase 1; & 088958 & Gnpda1 & 0,3 & 27,7 \\
\hline Glucosamine-6-phosphate isomerase 2; & Q9CRC9 & Gnpda2 & $-0,6$ & 16,1 \\
\hline Glucose-6-phosphate 1-dehydrogenase X; & Q00612 & G6pdx & $-0,3$ & 5,2 \\
\hline Glucose-6-phosphate isomerase; & P06745 & Gpi & 0,7 & 9 \\
\hline Glucosidase 2 subunit beta; & 008795 & Prkcsh & 0,2 & 6,5 \\
\hline Glucosylceramidase; & P17439 & Gba & $-0,3$ & 4,9 \\
\hline Glutamate dehydrogenase 1, mitochondrial; & P26443 & Glud1 & $-0,1$ & 24,9 \\
\hline Glutamine synthetase; & P15105 & Glul & 0,3 & 12,3 \\
\hline Glutaredoxin-1; & Q9QUHO & Glrx & $-0,1$ & 10,3 \\
\hline Glutathione peroxidase 1; & P11352 & Gpx1 & $-0,2$ & 41,8 \\
\hline Glutathione peroxidase 3; & P46412 & Gpx3 & $-0,6$ & 8,5 \\
\hline Glutathione peroxidase 7; & Q99L6 & Gpx7 & 0,8 & 20,4 \\
\hline Glutathione reductase, mitochondrial; & P47791 & Gsr & 0,2 & 24,4 \\
\hline Glutathione S-transferase A2; & P10648 & Gsta2 & 0,9 & 6,1 \\
\hline Glutathione S-transferase Mu 1; & P10649 & Gstm1 & 0,3 & 23,4 \\
\hline Glutathione S-transferase Mu 2; & P15626 & Gstm2 & $-0,1$ & 39,7 \\
\hline Glutathione S-transferase Mu 3; & P19639 & Gstm3 & 0,0 & 10,6 \\
\hline Glutathione S-transferase omega-1; & 009131 & Gsto1 & 0,7 & 22,9 \\
\hline Glutathione S-transferase P 1; & P19157 & Gstp1 & 0,4 & 12,9 \\
\hline Glutathione synthetase; & P51855 & Gss & 0,1 & 5,9 \\
\hline Glyceraldehyde-3-phosphate dehydrogenase; & P16858 & Gapdh & 0,3 & 33,9 \\
\hline
\end{tabular}




\begin{tabular}{|c|c|c|c|c|}
\hline Protein & Uniprot & Gene name & $\begin{array}{c}\text { Fold change of } \\
\text { XPRESS ratio } \\
\left.\text { (Adam17 }^{\Delta K C} / \mathrm{WT}\right)\end{array}$ & $\begin{array}{l}\text { Sequence } \\
\text { coverage [\%] }\end{array}$ \\
\hline Glycerol kinase; & Q64516 & Gk & $-0,4$ & 2,4 \\
\hline Glycerol-3-phosphate dehydrogenase [NAD(+)], cytoplasmic; & P13707 & Gpd1 & 0,0 & 23,9 \\
\hline Glycerol-3-phosphate dehydrogenase, mitochondrial; & Q64521 & Gpd2 & 0,5 & 6,1 \\
\hline Glycerophosphodiester phosphodiesterase domain-containing protein 1; & Q9CRY7 & Gdpd1 & $-0,2$ & 4,1 \\
\hline Glycerophosphodiester phosphodiesterase domain-containing protein 3; & Q99LY2 & Gdpd3 & $-1,1$ & 6,4 \\
\hline Glycerophosphoinositol inositolphosphodiesterase GDPD2; & Q9ESM6 & Gdpd2 & 0,2 & 1,9 \\
\hline Glycine cleavage system $\mathrm{H}$ protein, mitochondrial; & Q91WK5 & Gcsh & $-0,7$ & 5,9 \\
\hline Glycine--tRNA ligase; & Q9CZD3 & Gars & 0,2 & 14,4 \\
\hline Glycogen phosphorylase, brain form; & Q8C194 & Pygb & 0,2 & 6,9 \\
\hline Glycogen phosphorylase, liver form; & Q9ET01 & Pygl & 0,1 & 5,1 \\
\hline Glycogen synthase kinase- 3 beta; & Q9WV60 & Gsk3b & 1,2 & 5,7 \\
\hline Glycylpeptide N-tetradecanoyltransferase 1 & 070310 & Nmt1 & 0,8 & 2,2 \\
\hline Glyoxylate reductase/hydroxypyruvate reductase; & Q91Z53 & Grhpr & 0,2 & 22,8 \\
\hline Glypican-1; & Q9QZF2 & Gpc1 & $-0,5$ & 11,5 \\
\hline Glypican-4; & P51655 & Gpc4 & $-0,5$ & 18,9 \\
\hline GMP reductase 1; & Q9DCZ1 & Gmpr & $-2,1$ & 5,2 \\
\hline GMP reductase 2 & Q99L27 & Gmpr2 & $-0,2$ & 2,6 \\
\hline GMP synthase [glutamine-hydrolyzing]; & Q3THK7 & Gmps & 0,3 & 2 \\
\hline Golgi apparatus protein 1 & Q61543 & Glg1 & 0,1 & 18,7 \\
\hline Golgi integral membrane protein 4; & D3YVW2 & Golim4 & 0,3 & 10,4 \\
\hline Golgi SNAP receptor complex member 1 ; & 088630 & Gosr1 & $-0,2$ & 4,8 \\
\hline GPI inositol-deacylase; & Q3UUQ7 & Pgap1 & $-0,6$ & 7,2 \\
\hline GPN-loop GTPase 1; & Q8VCE2 & Gpn1 & $-1,0$ & 5,4 \\
\hline G-protein coupled receptor 56 & Q8K209 & Gpr56 & $-0,1$ & 2,5 \\
\hline Granulins; & P28798 & Grn & $-1,7$ & 8,1 \\
\hline GTPase HRas; & Q61411 & Hras & $-0,3$ & 16,9 \\
\hline GTPase NRas; & P08556 & Nras & $-0,1$ & 23,3 \\
\hline GTP-binding nuclear protein Ran; & P62827 & Ran & 0,1 & 42,6 \\
\hline $\begin{array}{l}\text { GTP-binding protein SAR1a; SAR1 gene homolog A (S. cerevisiae); SAR1 gene } \\
\text { homolog A (S. cerevisiae), isoform CRA_b; }\end{array}$ & Q99JZ4 & Sar1a & $-0,7$ & 16,2 \\
\hline Guanine deaminase; & Q9R111 & Gda & 1,1 & 4,2 \\
\hline Guanine nucleotide-binding protein G(i) subunit alpha-1; & $\mathrm{B} 2 \mathrm{RSH} 2$ & Gnai1 & 1,7 & 13,3 \\
\hline Guanine nucleotide-binding protein G(i) subunit alpha-2; & P08752 & Gnai2 & 0,2 & 13,2 \\
\hline Guanine nucleotide-binding protein $\mathrm{G}(\mathrm{I}) / \mathrm{G}(\mathrm{S}) / \mathrm{G}(\mathrm{O})$ subunit gamma-12; & Q9DAS9 & Gng12 & 0,2 & 19,4 \\
\hline Guanine nucleotide-binding protein $\mathrm{G}(\mathrm{I}) / \mathrm{G}(\mathrm{S}) / \mathrm{G}(\mathrm{T})$ subunit beta-1; & P62874 & Gnb1 & 0,2 & 23,8 \\
\hline Guanine nucleotide-binding protein subunit beta-2-like 1; & P68040 & Gnb2l1 & $-0,3$ & 52,7 \\
\hline H/ACA ribonucleoprotein complex subunit 1 ; & Q9CY66 & Gar1 & $-0,2$ & 21,7 \\
\hline H/ACA ribonucleoprotein complex subunit 4; & Q9ESX5 & Dkc1 & $-0,4$ & 13,8 \\
\hline H-2 class I histocompatibility antigen, D-B alpha chain; & P01899 & H2 D1 & 0,0 & 17,7 \\
\hline H-2 class II histocompatibility antigen gamma chain; & P04441 & $\mathrm{Cd} 74$ & $-0,3$ & 9,3 \\
\hline H-2 class II histocompatibility antigen, A beta chain; & P14483 & $\mathrm{H} 2 \mathrm{Ab} 1$ & 0,0 & 16,2 \\
\hline $\mathrm{H}-2$ class II histocompatibility antigen, A-K alpha chain; & P01910 & $\mathrm{H} 2 \mathrm{Aa}$ & $-0,3$ & 5,2 \\
\hline Haloacid dehalogenase-like hydrolase domain-containing protein 2; & Q3UGR5 & Hdhd2 & $-0,1$ & 8,1 \\
\hline Haptoglobin; & Q61646 & $\mathrm{Hp}$ & 2,3 & 10,7 \\
\hline HCLS1-associated protein X-1; & 035387 & Hax1 & $-0,3$ & 4,3 \\
\hline Heat shock 70 kDa protein 1B; & P17879 & Hspa1b & 0,7 & 28,2 \\
\hline Heat shock 70 kDa protein 4 & Q61316 & Hspa4 & 0,0 & 3,2 \\
\hline Heat shock cognate 71 kDa protein; & P63017 & Hspa8 & 0,4 & 30,5 \\
\hline Heat shock protein beta-1; & P14602 & Hspb1 & 0,0 & 41,5 \\
\hline Heat shock protein HSP 90-alpha; & P07901 & Hsp90aa1 & $-0,3$ & 19,5 \\
\hline Heat shock protein HSP 90-beta; & P11499 & Hsp90ab1 & 0,3 & 23,8 \\
\hline Heat shock-related 70 kDa protein 2 ; & P17156 & Hspa2 & 0,4 & 10,9 \\
\hline Hematopoietic progenitor cell antigen CD34; & Q64314 & $\mathrm{Cd} 34$ & $-0,3$ & 6,5 \\
\hline Heme-binding protein 2 ; & Q9WU63 & Hebp2 & 1,2 & 6,3 \\
\hline Hemoglobin subunit beta- 1 & P02088 & $\mathrm{Hbb}$ b1 & 0,5 & 72,1 \\
\hline Hemoglobin subunit beta-2; & P02089 & $\mathrm{Hbb}$ b2 & 0,5 & 68 \\
\hline Hemoglobin subunit beta-H1; & P04444 & $\mathrm{Hbb}$ bh1 & 4,5 & 12,9 \\
\hline Hemopexin; & Q91X72 & Hpx & 0,6 & 17,4 \\
\hline Hepatoma-derived growth factor; & P51859 & Hdgf & 0,0 & 14,8 \\
\hline Hepatoma-derived growth factor-related protein 2 & Q3UMU9 & Hdgfrp2 & 0,7 & 2,8 \\
\hline Hepatoma-derived growth factor-related protein 3 ; & Q9JMG7 & Hdgfrp3 & $-0,8$ & 23,3 \\
\hline Hephaestin; & Q9Z0Z4 & Heph & 0,2 & 6,2 \\
\hline Hephaestin-like protein 1 ; & Q3V1H3 & Hephl1 & $-0,4$ & 4,6 \\
\hline Heterochromatin protein 1-binding protein 3 ; & Q3TEA8 & Hp1bp3 & 0,2 & 9,7 \\
\hline Heterogeneous nuclear ribonucleoprotein A/B; & Q99020 & Hnrnpab & $-0,1$ & 20 \\
\hline Heterogeneous nuclear ribonucleoprotein A0; & Q9CX86 & Hnrnpa0 & 0,5 & 23,6 \\
\hline Heterogeneous nuclear ribonucleoprotein A1; & P49312 & Hnrnpa1 & 0,6 & 35,3 \\
\hline Heterogeneous nuclear ribonucleoprotein A3; & Q8BG05 & Hnrnpa3 & $-0,4$ & 23,3 \\
\hline Heterogeneous nuclear ribonucleoprotein D0; & Q60668 & Hnrnpd & $-0,5$ & 16,2 \\
\hline Heterogeneous nuclear ribonucleoprotein F; & Q9Z2X1 & Hnrnpf & 0,7 & 22,9 \\
\hline Heterogeneous nuclear ribonucleoprotein $\mathrm{H}$; & 035737 & Hnrnph1 & 0,4 & 11,8 \\
\hline
\end{tabular}




\begin{tabular}{|c|c|c|c|c|}
\hline Protein & Uniprot & Gene name & $\begin{array}{c}\text { Fold change of } \\
\text { XPRESS ratio } \\
\left.\text { (Adam17 }^{\Delta K C} / \mathrm{WT}\right)\end{array}$ & $\begin{array}{c}\text { Sequence } \\
\text { coverage [\%] }\end{array}$ \\
\hline Heterogeneous nuclear ribonucleoprotein L; & Q8R081 & Hnrnpl & 0,0 & 14,2 \\
\hline Heterogeneous nuclear ribonucleoprotein L-like; & Q921F4 & Hnrnpll & $-0,8$ & 4,1 \\
\hline Heterogeneous nuclear ribonucleoprotein M; & Q9D0E1 & Hnrnpm & 0,6 & 19,8 \\
\hline Heterogeneous nuclear ribonucleoprotein Q; & Q7TMK9 & Syncrip & 0,1 & 14,9 \\
\hline Heterogeneous nuclear ribonucleoprotein U; & Q8VEK3 & Hnrnpu & 0,5 & 12 \\
\hline Heterogeneous nuclear ribonucleoprotein U-like protein 1; & Q8VDM6 & Hnrnpul1 & 0,3 & 6,1 \\
\hline Heterogeneous nuclear ribonucleoprotein U-like protein 2; & Q00PI9 & Hnrnpul2 & 0,1 & 11,9 \\
\hline Heterogeneous nuclear ribonucleoproteins A2/B1; & 088569 & Hnrnpa2b1 & 0,5 & 22,1 \\
\hline Heterogeneous nuclear ribonucleoproteins $\mathrm{C} 1 / \mathrm{C} 2$; & Q9Z204 & Hnrnpc & 0,4 & 10,2 \\
\hline Hexokinase-1; & P17710 & Hk1 & $-0,5$ & 8,9 \\
\hline High affinity cAMP-specific 3',5'-cyclic phosphodiesterase 7A; & P70453 & Pde7a & $-0,7$ & 8,5 \\
\hline High affinity cationic amino acid transporter 1 & Q09143 & Slc7a1 & 0,0 & 6,8 \\
\hline High mobility group nucleosome-binding domain-containing protein 3 ; & Q9DCB1 & Hmgn3 & $-0,2$ & 15,2 \\
\hline High mobility group nucleosome-binding domain-containing protein 5; & Q9JL35 & Hmgn5 & $-0,1$ & 12,6 \\
\hline High mobility group protein $20 \mathrm{~A}$ & Q9DC33 & Hmg20a & 0,2 & 10,7 \\
\hline High mobility group protein B1; & P63158 & Hmgb1 & $-0,4$ & 25,1 \\
\hline High mobility group protein $\mathrm{B} 2$; & P30681 & Hmgb2 & $-0,4$ & 31 \\
\hline High mobility group protein B3; & 054879 & Hmgb3 & 0,3 & 8,2 \\
\hline High mobility group protein HMGI-C; & P52927 & Hmga2 & $-1,0$ & 16,7 \\
\hline Hippocalcin-like protein 1 & P62748 & Hpcal1 & $-0,7$ & 18,7 \\
\hline HIRA-interacting protein 3 ; & Q8BLH7 & Hirip3 & 0,2 & 12,7 \\
\hline Histidine ammonia-lyase; & P35492 & Hal & $-0,5$ & 32,4 \\
\hline Histidine triad nucleotide-binding protein 1 ; & P70349 & Hint1 & $-0,2$ & 51,6 \\
\hline Histidine triad nucleotide-binding protein 2, mitochondrial; & Q9D0S9 & Hint2 & 0,6 & 21,5 \\
\hline Histidine triad nucleotide-binding protein 3 & Q9CPS6 & Hint3 & $-0,1$ & 9,1 \\
\hline Histidine-rich glycoprotein; & Q9ESB3 & $\mathrm{Hrg}$ & 0,4 & 3,4 \\
\hline Histidine--tRNA ligase, cytoplasmic; & Q61035 & Hars & 0,7 & 12,4 \\
\hline Histone deacetylase 1 & 009106 & Hdac1 & 0,6 & 7,1 \\
\hline Histone deacetylase complex subunit SAP25; & E9Q529 & Sap25 & $-0,7$ & 5,7 \\
\hline Histone $\mathrm{H} 1.0$ & P10922 & $\mathrm{H} 1 \mathrm{fO}$ & $-0,5$ & 20,6 \\
\hline Histone H1.1; & P43275 & Hist1h1a & $-0,2$ & 35,7 \\
\hline Histone $\mathrm{H} 1.3$; & P43277 & Hist1h1d & 0,2 & 21,7 \\
\hline Histone H1.4; & P43274 & Hist1h1e & 0,3 & 21,9 \\
\hline Histone H1.5; & P43276 & Hist1h1b & $-0,3$ & 21,5 \\
\hline Histone H2A type 1 ; & P22752 & Hist1h2ao & $-0,1$ & 32,6 \\
\hline Histone $\mathrm{H} 2 \mathrm{~A}$ type $2-\mathrm{B}$; & Q64522 & Hist2h2ab & $-0,4$ & 17,7 \\
\hline Histone H2B type $1-\mathrm{F} / \mathrm{J} / \mathrm{L}$; & P10853 & Hist1h2bn & $-0,7$ & 40,5 \\
\hline Histone H2B type 2-E; & Q64524 & Hist2h2be & 0,4 & 34,9 \\
\hline Histone H3.3C; & P02301 & $\mathrm{H} 3 \mathrm{f3c}$ & $-0,4$ & 46,2 \\
\hline Histone H4; & P62806 & Hist4h4 & $-0,5$ & 50,5 \\
\hline Histone-binding protein RBBP4; & Q60972 & Rbbp4 & 0,2 & 18,4 \\
\hline Histone-binding protein RBBP7; & Q60973 & Rbbp7 & 0,6 & 18,8 \\
\hline Homeodomain-only protein; & Q8R1H0 & Hopx & $-0,6$ & 43,8 \\
\hline Hormone-sensitive lipase; & P54310 & Lipe & 0,3 & 6,3 \\
\hline Hornerin; & E9QNP3 & Hrnr & $-1,7$ & 5,3 \\
\hline Host cell factor 1 & Q61191 & Hcfc1 & 0,7 & 4,1 \\
\hline Hsc70-interacting protein; & Q99L47 & St13 & 0,1 & 6,6 \\
\hline Huntingtin-interacting protein 1-related protein; & Q9JKY5 & Hip1r & 0,5 & 1,2 \\
\hline Huntingtin-interacting protein $\mathrm{K}$; & Q9CR41 & Hypk & 0,3 & 11,6 \\
\hline Hydroxyacid-oxoacid transhydrogenase, mitochondrial; & Q8R0N6 & Adhfe1 & 2,8 & 7,7 \\
\hline Hydroxyacyl-coenzyme A dehydrogenase, mitochondrial; & Q61425 & Hadh & $-0,2$ & 30,3 \\
\hline Hydroxyacylglutathione hydrolase, mitochondrial; & Q99KB8 & Hagh & 0,4 & 16,1 \\
\hline Hydroxymethylglutaryl-CoA synthase, cytoplasmic; & Q8JZK9 & Hmgcs1 & 0,6 & 3,7 \\
\hline Hypoxanthine-guanine phosphoribosyltransferase; & P00493 & Hprt1 & $-0,4$ & 57,8 \\
\hline Hypoxia up-regulated protein 1 & Q9JKR6 & Hyou1 & $-0,3$ & 8,8 \\
\hline Ig gamma-1 chain $\mathrm{C}$ region secreted form; & P01868 & Ighg1 & 0,7 & 8,6 \\
\hline Ig gamma-2B chain C region; & P01867 & $\operatorname{lgh} 3$ & 0,7 & 2,2 \\
\hline Ig gamma-3 chain C region; & P03987 & & 2,0 & 13,3 \\
\hline Ig heavy chain V region 102 & P01750 & & 2,4 & 21,4 \\
\hline Ig heavy chain V region AC38 205.12; & P06330 & & 1,7 & 24,6 \\
\hline Ig kappa chain V-II region 26-10; & P01631 & & 0,3 & 17,7 \\
\hline Ig kappa chain V-V region HP R16.7; & P01644 & & 2,5 & 19,4 \\
\hline Ig kappa chain $\mathrm{V}-\mathrm{V}$ region $\mathrm{K} 2$ & P01635 & & $-0,6$ & 11,3 \\
\hline Ig lambda-1 chain C region; & P01843 & & 1,9 & 16,2 \\
\hline IgG receptor FcRn large subunit p51; & Q61559 & Fcgrt & 0,2 & 5,8 \\
\hline Immediate early response 3 -interacting protein 1 ; & Q9CR20 & ler3ip1 & 0,2 & 10,1 \\
\hline Importin subunit alpha-4; & 035344 & Kpna3 & $-0,6$ & 2,7 \\
\hline Importin subunit alpha-7; & 035345 & Kpna6 & 0,4 & 4,8 \\
\hline Importin subunit beta- 1 & P70168 & Kpnb1 & 0,4 & 3,2 \\
\hline Importin-5; & Q8BKC5 & Ipo5 & $-0,2$ & 1,2 \\
\hline Importin-7; & Q9EPL8 & Ipo7 & 0,2 & 3,9 \\
\hline Inactive hydroxysteroid dehydrogenase-like protein 1 ; & Q8BTX9 & Hsdl1 & 0,4 & 8,2 \\
\hline Inactive phospholipase D5; & Q3UNN8 & Pld5 & 0,7 & 4,9 \\
\hline
\end{tabular}




\begin{tabular}{|c|c|c|c|c|}
\hline Protein & Uniprot & Gene name & $\begin{array}{l}\text { Fold change of } \\
\text { XPRESS ratio } \\
\left.\text { (Adam } 17^{\Delta K C} / \mathrm{WT}\right)\end{array}$ & $\begin{array}{c}\text { Sequence } \\
\text { coverage [\%] }\end{array}$ \\
\hline Inactive tyrosine-protein kinase 7; & Q8BKG3 & Ptk7 & 0,5 & 4,3 \\
\hline Inhibitor of carbonic anhydrase; & Q9DBD0 & Ica & 5,5 & 3,9 \\
\hline Inorganic pyrophosphatase 2, mitochondrial; & Q91VM9 & Ppa2 & $-0,2$ & 15,5 \\
\hline Inorganic pyrophosphatase; & Q9D819 & Ppa1 & $-0,3$ & 11,1 \\
\hline Inosine triphosphate pyrophosphatase; & Q9D892 & Itpa & $-0,2$ & 18,2 \\
\hline Inosine-5'-monophosphate dehydrogenase 2; & P24547 & Impdh2 & 0,6 & 9,5 \\
\hline $\begin{array}{l}\text { Inositol (Myo)-1(Or 4)-monophosphatase 1; Inositol (Myo)-1(Or 4)- } \\
\text { monophosphatase 1, isoform CRA_a; Inositol monophosphatase 1; Myo-inositol } \\
\text { monophosphatase 1; }\end{array}$ & Q924B0 & Impa1 & $-0,4$ & 27,1 \\
\hline Inositol polyphosphate 1-phosphatase; & P49442 & Inpp1 & $-0,3$ & 4,5 \\
\hline Inositol-3-phosphate synthase 1 ; & Q9JHU9 & Isyna1 & $-1,1$ & 13,6 \\
\hline Insulin-degrading enzyme; & Q9JHR7 & Ide & 0,6 & 20,3 \\
\hline Insulin-like growth factor-binding protein 4; & P47879 & Igfbp4 & 9,9 & 15,5 \\
\hline Insulin-like growth factor-binding protein 7; & Q61581 & Igfbp7 & 0,1 & 4,6 \\
\hline Integral membrane protein $2 \mathrm{~B}$ & 089051 & Itm2b & $-0,1$ & 3,4 \\
\hline Integrin alpha-1; & Q3V3R4 & Itga1 & $-0,9$ & 3,6 \\
\hline Integrin alpha-3; & Q62470 & Itga3 & $-0,6$ & 7,1 \\
\hline Integrin alpha-5; & P11688 & Itga5 & 0,2 & 6,3 \\
\hline Integrin alpha-6; & Q61739 & Itga6 & $-1,0$ & 3,9 \\
\hline Integrin alpha-7; & Q61738 & Itga7 & 0,1 & 1,9 \\
\hline Integrin alpha-8; & A2ARA8 & Itga8 & 0,5 & 1,7 \\
\hline Integrin alpha-M; & P05555 & Itgam & 3,1 & 0,8 \\
\hline Integrin alpha-V; & P43406 & Itgav & $-0,2$ & 8 \\
\hline Integrin beta-1; & P09055 & Itgb1 & $-0,5$ & 16,7 \\
\hline Integrin beta-2; & P11835 & $\operatorname{ltgb2}$ & $-0,2$ & 4,9 \\
\hline Integrin beta-5; & 070309 & Itgb5 & $-0,2$ & 3,9 \\
\hline Inter alpha-trypsin inhibitor, heavy chain 4 ; & A6X935 & Itih4 & 0,8 & 4,8 \\
\hline Inter-alpha-trypsin inhibitor heavy chain $\mathrm{H}$; & Q61703 & Itih2 & 0,6 & 5,8 \\
\hline Inter-alpha-trypsin inhibitor heavy chain H5; & Q8BJD1 & Itih5 & 1,6 & 2,5 \\
\hline Interferon regulatory factor 2-binding protein 2; & E9Q1P8 & Irf2bp2 & 0,2 & 10,4 \\
\hline Interleukin enhancer-binding factor 2 & Q9CXY6 & IIf2 & 0,6 & 15,1 \\
\hline Interleukin enhancer-binding factor 3 & Q9Z1X4 & IIf3 & 0,3 & 10 \\
\hline Interleukin-1 receptor antagonist protein; & P25085 & $\| 1 \mathrm{rn}$ & 0,1 & 11,9 \\
\hline Intersectin-1; & Q9Z0R4 & Itsn1 & $-6,5$ & 2,5 \\
\hline Intraflagellar transport protein 172 homolog; & Q6VH22 & Ift172 & $-1,3$ & 1,3 \\
\hline Intron-binding protein aquarius; & Q8CFQ3 & Aqr & 1,0 & 0,7 \\
\hline Involucrin; & P48997 & $|v|$ & $-0,8$ & 41,5 \\
\hline Iporin; & Q80U22 & Rusc2 & $-3,6$ & 1,2 \\
\hline Iron/zinc purple acid phosphatase-like protein; & Q8BX37 & Papl & $-0,5$ & 5,5 \\
\hline Isoamyl acetate-hydrolyzing esterase 1 homolog; & Q9DB29 & lah1 & 0,8 & 4,4 \\
\hline Isoaspartyl peptidase/L-asparaginase; & Q8Сом9 & Asrgl1 & 0,0 & 3,1 \\
\hline Isobutyryl-CoA dehydrogenase, mitochondrial; & Q9D7B6 & Acad8 & 0,6 & 5,1 \\
\hline Isochorismatase domain-containing protein $1 ;$ & Q91V64 & Isoc1 & 0,4 & 3,7 \\
\hline Isocitrate dehydrogenase [NAD] subunit alpha, mitochondrial; & Q9D6R2 & $\operatorname{ldh} 3 a$ & 0,3 & 33,9 \\
\hline Isocitrate dehydrogenase [NAD] subunit gamma 1, mitochondrial; & P70404 & Idh3g & 0,6 & 13,7 \\
\hline Isocitrate dehydrogenase [NADP] cytoplasmic; & 088844 & Idh1 & $-0,1$ & 41,8 \\
\hline Isocitrate dehydrogenase [NADP], mitochondrial; & P54071 & Idh2 & $-0,2$ & 30,5 \\
\hline Isocitrate dehydrogenase 3 (NAD+) beta; Protein Idh3b; Tumor-related protein; & Q91VA7 & Idh3b & 0,7 & 10,9 \\
\hline Isoleucine--tRNA ligase, mitochondrial; & Q8BIJ6 & lars2 & 0,2 & 12,5 \\
\hline Isopentenyl-diphosphate Delta-isomerase 1; & P58044 & Idi1 & $-0,3$ & 26 \\
\hline Junction plakoglobin; & Q02257 & Jup & 0,3 & 17,9 \\
\hline Junctional adhesion molecule A; & 088792 & F11r & $-0,5$ & 18,7 \\
\hline Kallikrein 1-related peptidase b5; Kallikrein related-peptidase 5; & Q9D140 & Klk5 & $-0,3$ & 18,8 \\
\hline Kallikrein 6, isoform CRA_a; Kallikrein-1; KIk6 protein; Neurosin; & Q91Y82 & Klk6 & 1,3 & 12,3 \\
\hline Kallikrein related-peptidase 10; Protein Klk10; & Q99M20 & Klk10 & 0,9 & 23 \\
\hline Kallikrein-7; & Q91VE3 & Klk7 & 0,1 & 23,3 \\
\hline Kallikrein-8; & Q61955 & Klk8 & 0,4 & 11,2 \\
\hline Keratin, type I cuticular Ha3-I; & Q8K0Y2 & Krt33a & $-1,7$ & 10,9 \\
\hline Keratin, type I cuticular Ha4; & Q9D646 & Krt34 & $-1,3$ & 14 \\
\hline Keratin, type I cytoskeletal 10; & P02535 & Krt10 & $-1,1$ & 19,6 \\
\hline Keratin, type I cytoskeletal 14 & Q61781 & Krt14 & 0,1 & 13,8 \\
\hline Keratin, type I cytoskeletal 15; & Q61414 & Krt15 & 1,8 & 7,7 \\
\hline Keratin, type I cytoskeletal 17; & Q9QWL7 & Krt17 & $-0,4$ & 20,6 \\
\hline Keratin, type I cytoskeletal 19; & P19001 & Krt19 & 9,0 & 6,7 \\
\hline Keratin, type I cytoskeletal 25; & Q8VCW2 & Krt25 & 0,0 & 14,8 \\
\hline Keratin, type I cytoskeletal 27; & Q9Z320 & Krt27 & $-1,2$ & 21,7 \\
\hline Keratin, type I cytoskeletal 28; & A6BLY7 & Krt28 & $-0,5$ & 10,8 \\
\hline Keratin, type II cuticular Hb1; & Q9ERE2 & Krt81 & 3,0 & 20,4 \\
\hline Keratin, type II cuticular Hb3; & Q6IMF0 & Krt83 & $-0,8$ & 16 \\
\hline Keratin, type II cytoskeletal 1 ; & P04104 & Krt1 & $-1,7$ & 16,2 \\
\hline Keratin, type II cytoskeletal 1b; & Q6IFZ6 & Krt77 & $-0,3$ & 18,9 \\
\hline Keratin, type II cytoskeletal 2 epidermal; & Q3TTY5 & Krt2 & 2,0 & 6,5 \\
\hline
\end{tabular}




\begin{tabular}{|c|c|c|c|c|}
\hline Protein & Uniprot & Gene name & 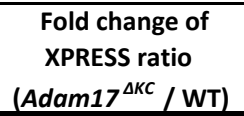 & $\begin{array}{c}\text { Sequence } \\
\text { coverage [\%] }\end{array}$ \\
\hline Keratin, type II cytoskeletal 2 oral; & Q3UV17 & Krt76 & $-1,3$ & 5,7 \\
\hline Keratin, type II cytoskeletal 5; & Q922U2 & Krt5 & 0,2 & 17,8 \\
\hline Keratin, type II cytoskeletal 71; & Q9ROH5 & Krt71 & $-0,8$ & 29,4 \\
\hline Keratin, type II cytoskeletal 72; & Q6IME9 & Krt72 & 1,0 & 9 \\
\hline Keratin, type II cytoskeletal 73; & Q6NXH9 & Krt73 & $-1,7$ & 8,3 \\
\hline Keratin, type II cytoskeletal 75; & Q8BGZ7 & Krt75 & $-0,3$ & 13,6 \\
\hline Keratin, type II cytoskeletal 79; & Q8VED5 & Krt79 & $-0,3$ & 17,9 \\
\hline Keratin-associated protein $16-1$ & A2A5X5 & Krtap16 1 & $-0,1$ & 6,2 \\
\hline Keratin-associated protein 19-3; & Q925H6 & Krtap19 3 & $-2,8$ & 16,1 \\
\hline Keratin-associated protein $19-4$ & Q925H7 & Krtap19 4 & $-1,9$ & 16,7 \\
\hline Keratin-associated protein $19-5$; & F8VQ65 & Krtap19 5 & $-1,8$ & 43,5 \\
\hline Keratin-associated protein 6-5; & Q925H3 & Krtap6 5 & $-2,2$ & 33,3 \\
\hline Keratinocyte-associated transmembrane protein 2; & Q8K201 & Kct2 & 0,3 & 3,9 \\
\hline Ketimine reductase mu-crystallin; & 054983 & Crym & $-1,7$ & 35,1 \\
\hline Ketosamine-3-kinase; & Q8K274 & Fn3krp & 0,1 & 5,2 \\
\hline Kinectin; & F8VQC7 & Ktn1 & 0,2 & 9,1 \\
\hline Kinesin-like protein KIF21B; & Q9QXL1 & Kif21b & $-0,3$ & 10,3 \\
\hline Kininogen-1; & 008677 & Kng1 & 0,4 & 24 \\
\hline Kunitz-type protease inhibitor 1 ; & Q9R097 & Spint1 & $-0,4$ & 6,1 \\
\hline Kynurenine--oxoglutarate transaminase 1 ; & Q8BTY1 & Ccbl1 & $-0,4$ & 24,7 \\
\hline Kynurenine--oxoglutarate transaminase 3 ; & Q71RI9 & Ccbl2 & $-0,3$ & 7,7 \\
\hline Lactadherin; & P21956 & Mfge8 & $-0,5$ & 4,3 \\
\hline Lactoylglutathione lyase; & Q9CPU0 & Glo1 & 0,0 & 25,5 \\
\hline Ladinin-1; & P57016 & Lad1 & 0,0 & 11,9 \\
\hline Lambda-crystallin homolog; & Q99KP3 & Cryl1 & 0,3 & 6 \\
\hline Lamina-associated polypeptide 2, isoforms beta/delta/epsilon/gamma; & Q61029 & Tmpo & 0,1 & 14,2 \\
\hline Lamin-B1; & P14733 & Lmnb1 & 0,4 & 9,4 \\
\hline Laminin subunit alpha-2; & Q60675 & Lama2 & 0,9 & 2 \\
\hline Laminin subunit alpha-4; & P97927 & Lama4 & 0,5 & 3,1 \\
\hline Laminin subunit beta-1; & P02469 & Lamb1 & 0,2 & 4,6 \\
\hline Laminin subunit gamma-1; & P02468 & Lamc1 & 0,4 & 7,6 \\
\hline LanC-like protein 1 & 089112 & Lancl1 & 1,2 & 14,3 \\
\hline Large neutral amino acids transporter small subunit 2; & Q9QXW9 & Slc7a8 & $-1,8$ & 2,6 \\
\hline Latent-transforming growth factor beta-binding protein 2 ; & E9Q1D6 & Ltbp2 & 0,0 & 1,9 \\
\hline Latent-transforming growth factor beta-binding protein 4 ; & D37598 & Ltbp4 & 0,2 & 6,7 \\
\hline Latexin; & P70202 & Lxn & $-0,6$ & 12,2 \\
\hline L-dopachrome tautomerase; & P29812 & Dct & $-0,1$ & 5,4 \\
\hline Leucine-rich repeat flightless-interacting protein 1 ; & Q3UZ39 & Lrrfip1 & $-0,3$ & 8,8 \\
\hline Leucine-rich repeat-containing protein 15 & Q80X72 & Lrrc15 & $-0,3$ & 18 \\
\hline Leucine-rich repeat-containing protein $16 \mathrm{~A}$; & Q6EDY6 & Lrrc16a & $-0,1$ & 2,6 \\
\hline Leucine-rich repeat-containing protein 27 & Q80YS5 & Lrrc27 & 10,1 & 2,8 \\
\hline Leucine-rich repeat-containing protein 47 & Q505F5 & Lrrc47 & 1,1 & 3,1 \\
\hline Leucine-rich repeat-containing protein 59; & Q922Q8 & Lrrc59 & 0,0 & 38,1 \\
\hline Leucyl-cystinyl aminopeptidase; & Q8C129 & Lnpep & $-0,2$ & 14,5 \\
\hline Leukocyte elastase inhibitor $A$; & Q9D154 & Serpinb1a & 0,7 & 12,4 \\
\hline Leukocyte surface antigen CD47; & Q61735 & Cd47 & $-0,2$ & 4,3 \\
\hline Leukotriene A-4 hydrolase; & P24527 & Lta4h & 0,3 & 10,6 \\
\hline L-fucose kinase; Protein Fuk; & Q7TMC8 & Fuk & $-2,4$ & 3,5 \\
\hline LIM and senescent cell antigen-like-containing domain protein $1 ;$ & Q99JW4 & Lims1 & $-0,7$ & 8 \\
\hline LIM and SH3 domain protein 1 & Q61792 & Lasp1 & $-0,4$ & 16,7 \\
\hline LIM domain and actin-binding protein 1 ; & Q9ERG0 & Lima1 & 0,0 & 25,6 \\
\hline LIM zinc-binding domain-containing Nebulette; & Q9DC07 & Nebl & 0,0 & 3,3 \\
\hline Lipase maturation factor 2 & Q8C3X8 & Lmf2 & 0,4 & 1,3 \\
\hline Lipoma-preferred partner homolog; & Q8BFW7 & Lpp & 0,3 & 18,4 \\
\hline Lipoprotein lipase; & P11152 & Lpl & 0,5 & 3,6 \\
\hline Liprin-alpha 4; Protein Ppfia4; & B8QI36 & Ppfia4 & $-6,5$ & 0,8 \\
\hline L-lactate dehydrogenase A chain; & P06151 & Ldha & $-0,3$ & 25 \\
\hline L-lactate dehydrogenase B chain; & P16125 & Ldhb & $-0,6$ & 19,5 \\
\hline Lon protease homolog, mitochondrial; & Q8CGK3 & Lonp1 & 1,4 & 6,2 \\
\hline Long-chain specific acyl-CoA dehydrogenase, mitochondrial; & P51174 & Acadl & $-0,2$ & 31,9 \\
\hline Long-chain-fatty-acid--CoA ligase 1 ; & P41216 & Acsl1 & 0,3 & 22,7 \\
\hline Long-chain-fatty-acid--CoA ligase 5 ; & Q8JZRO & Acsl5 & $-0,2$ & 8,9 \\
\hline Loricrin; & P18165 & Lor & 0,5 & 1,6 \\
\hline Low affinity immunoglobulin gamma Fc region receptor II; & P08101 & Fcgr2 & 0,0 & 11,9 \\
\hline Luc7-like protein 3; & Q5SUF2 & Luc713 & 0,9 & 2,5 \\
\hline Lumican; & P51885 & Lum & 0,8 & 14,2 \\
\hline Lupus La protein homolog; & P32067 & Ssb & 0,6 & 13,3 \\
\hline Ly6/PLAUR domain-containing protein 5; & Q9D7Z7 & Lypd5 & $-1,9$ & 28,1 \\
\hline Lymphocyte antigen 6 complex locus protein G6c; & Q9Z1Q4 & Ly6g6c & $-0,4$ & 20,6 \\
\hline Lymphocyte antigen 6D; & P35459 & Ly6d & $-1,3$ & 37 \\
\hline Lymphocyte-specific protein 1 & P19973 & Lsp1 & 0,0 & 17,7 \\
\hline Lys-63-specific deubiquitinase BRCC36; & P46737 & Brcc3 & $-0,2$ & 8,2 \\
\hline
\end{tabular}




\begin{tabular}{|c|c|c|c|c|}
\hline Protein & Uniprot & Gene name & $\begin{array}{c}\text { Fold change of } \\
\text { XPRESS ratio } \\
\left.\text { (Adam17 }^{\Delta K C} / \mathrm{WT}\right)\end{array}$ & $\begin{array}{c}\text { Sequence } \\
\text { coverage [\%] }\end{array}$ \\
\hline Lysine--tRNA ligase; & Q99MN1 & Kars & 0,8 & 8,7 \\
\hline Lysophosphatidic acid phosphatase type 6; & Q8BP40 & Acp6 & 0,5 & 2,6 \\
\hline Lysophospholipid acyltransferase 5; & Q91V01 & Lpcat3 & $-0,1$ & 1,8 \\
\hline Lysosomal acid phosphatase; & P24638 & Acp2 & 0,2 & 9,7 \\
\hline Lysosomal alpha-mannosidase; & 009159 & Man2b1 & $-0,2$ & 4,6 \\
\hline Lysosomal protective protein; & P16675 & Ctsa & $-0,4$ & 14,6 \\
\hline Lysosomal protein NCU-G1; & Q9JHJ3 & & $-0,3$ & 6,3 \\
\hline Lysosomal Pro-X carboxypeptidase; & Q7TMR0 & Prcp & $-0,5$ & 22 \\
\hline Lysosomal thioesterase PPT2; & 035448 & Ppt2 & $-0,4$ & 10,8 \\
\hline Lysosome membrane protein 2 & 035114 & Scarb2 & $-0,3$ & 1,7 \\
\hline Lysosome-associated membrane glycoprotein $1 ;$ & P11438 & Lamp1 & $-0,3$ & 2,7 \\
\hline Lysosome-associated membrane glycoprotein 2; & P17047 & Lamp2 & 0,0 & 6,5 \\
\hline Lysozyme g-like protein 2 ; & Q3V1I0 & Lyg2 & $-0,6$ & 12,7 \\
\hline m7GpppX diphosphatase; & Q9DAR7 & Dcps & $-0,5$ & 21,3 \\
\hline Macrophage mannose receptor 1 & Q61830 & Mrc1 & 0,4 & 7,1 \\
\hline Macrophage migration inhibitory factor; & P34884 & Mif & $-0,3$ & 7,8 \\
\hline Macrophage-capping protein; & P24452 & Capg & $-0,3$ & 17,5 \\
\hline Magnesium transporter NIPA4; & Q8BZF2 & Nipal4 & $-0,7$ & 2,5 \\
\hline Magnesium transporter protein 1 & Q9CQY5 & Magt1 & $-0,1$ & 8,3 \\
\hline Magnesium-dependent phosphatase 1 & Q9D967 & Mdp1 & 0,1 & 12,2 \\
\hline Major facilitator superfamily domain-containing protein 10 ; & Q9D2V8 & Mfsd10 & $-0,2$ & 7,2 \\
\hline Major facilitator superfamily domain-containing protein 6 ; & Q8CBH5 & Mfsd6 & 0,4 & 2,8 \\
\hline Major vault protein; & Q9EQK5 & Mvp & $-0,1$ & 12,7 \\
\hline Malate dehydrogenase, cytoplasmic; & P14152 & Mdh1 & 0,0 & 19,8 \\
\hline Malate dehydrogenase, mitochondrial; & P08249 & Mdh2 & $-0,2$ & 42 \\
\hline Maleylacetoacetate isomerase; & Q9WVL0 & Gstz1 & 0,6 & 6,5 \\
\hline Malignant T-cell-amplified sequence 2 & Q9CQ21 & Mcts2 & 0,6 & 9,9 \\
\hline Mannose-1-phosphate guanyltransferase alpha; & Q922H4 & Gmppa & 0,5 & 10,5 \\
\hline Mannose-1-phosphate guanyltransferase beta; & Q8BTZ7 & Gmppb & 0,5 & 12,5 \\
\hline Mannose-P-dolichol utilization defect 1 protein; & Q9R0Q9 & Mpdu1 & $-0,2$ & 3,6 \\
\hline Mannosyl-oligosaccharide 1,2-alpha-mannosidase IA; & P45700 & Man1a1 & $-0,2$ & 8,1 \\
\hline Mannosyl-oligosaccharide glucosidase; & Q80UM7 & Mogs & 0,1 & 7,3 \\
\hline Mas-related G-protein coupled receptor member F; & Q8VCJ6 & Mrgprf & 0,0 & 2,6 \\
\hline Matrin-3; & Q8K310 & Matr3 & 0,5 & 5,2 \\
\hline Matrix-remodeling-associated protein 7; & Q9CZH7 & Mxra7 & 1,4 & 8,4 \\
\hline MCG117626; Odorant binding protein la; Protein Obp1a; & Q9D3H2 & Obp1a & $-0,8$ & 14,1 \\
\hline MCG125471; Predicted gene, 677156; Protein Cyp4f37; & Q3V1F1 & Сyp4f37 & $-0,8$ & 7,1 \\
\hline MCG129038; Protein Serpinb3a; & G3X9V8 & Serpinb3a & 0,1 & 31,3 \\
\hline MCG13402, isoform CRA_a; Polypyrimidine tract-binding protein 1; & Q8BGJ5 & Ptbp1 & 0,5 & 16,2 \\
\hline $\begin{array}{l}\text { MCG140848; Protein Rdh1; Retinol dehydrogenase } 1 \text { (All trans); Retinol } \\
\text { dehydrogenase type 1; }\end{array}$ & Q8CGV4 & Rdh1 & $-0,4$ & 6 \\
\hline MCG142205; Protein Vmn1r210; Vomeronasal receptor V1RH10; & Q8R274 & Vmn1r210 & 0,2 & 2,6 \\
\hline MCG18410, isoform CRA_a; Protein Ddx23; & D3zoM9 & $\operatorname{Ddx} 23$ & $-0,2$ & 1 \\
\hline MCG20149, isoform CRA_a; Methyltransferase like 7A1; Protein Mett|7a1; & Q8C6B0 & Mettl7a1 & 2,2 & 6,1 \\
\hline MCG21235; Protein Serpinb3b; & Q9D1Q5 & Serpinb3b & $-0,3$ & 10,3 \\
\hline $\begin{array}{l}\text { MCG21506; Macrophage galactose } \mathrm{N} \text {-acetyl-galactosamine specific lectin } 2 \\
\text { isoform i; Protein Mgl2; }\end{array}$ & A9XX86 & $\mathrm{Mgl2}$ & 0,2 & 14,5 \\
\hline $\begin{array}{l}\text { MCG22939; Potassium channel subfamily K member 7; TWIK-2 two-pore- } \\
\text { domain K+ channel; }\end{array}$ & Q3TBV4 & Kcnk6 & $-0,1$ & 6,7 \\
\hline MCG54087; Protein Serpina3j; & D3Z451 & Serpina3j & $-0,1$ & 18,6 \\
\hline Mediator of RNA polymerase II transcription subunit 24; & Q99K74 & Med24 & 0,6 & 1,6 \\
\hline Medium-chain specific acyl-CoA dehydrogenase, mitochondrial; & P45952 & Acadm & 0,3 & 24,5 \\
\hline Melanocyte protein PMEL; & Q60696 & Pmel & 0,0 & 1,8 \\
\hline Membrane primary amine oxidase; & 070423 & Aoc3 & $-0,1$ & 21 \\
\hline Membrane-associated progesterone receptor component 1; & 055022 & Pgrmc1 & $-0,1$ & 11,8 \\
\hline Membrane-associated progesterone receptor component 2; & Q80UU9 & Pgrmc2 & 0,0 & 10,6 \\
\hline Mesencephalic astrocyte-derived neurotrophic factor; & Q9CXI5 & Manf & $-0,2$ & 32,7 \\
\hline Metalloproteinase inhibitor 2; & P25785 & Timp2 & $-0,4$ & 6,3 \\
\hline Metallothionein-4; & P47945 & Mt4 & $-3,3$ & 19,4 \\
\hline Metastasis-associated protein MTA2; & Q9R190 & Mta2 & 0,6 & 6 \\
\hline Metaxin-1; & P47802 & Mtx1 & 0,0 & 11,4 \\
\hline Metaxin-2; & 088441 & Mtx2 & 3,1 & 8,7 \\
\hline Methionine adenosyltransferase 2 subunit beta; & Q99LB6 & Mat2b & 0,2 & 18,6 \\
\hline Methionine aminopeptidase 1 ; & Q8BP48 & Metap1 & 0,4 & 10,1 \\
\hline Methionine aminopeptidase 2 & 008663 & Metap2 & $-0,2$ & 21,1 \\
\hline Methyl-CpG-binding domain protein 3; & Q9Z2D8 & Mbd3 & 0,0 & 9,7 \\
\hline Methyl-CpG-binding protein 2; & Q972D6 & Mecp2 & $-0,1$ & 6,8 \\
\hline Methylcrotonoyl-CoA carboxylase beta chain, mitochondrial; & Q3ULD5 & Mccc2 & 0,2 & 8,9 \\
\hline Methylcrotonoyl-CoA carboxylase subunit alpha, mitochondrial; & Q99MR8 & Mccc1 & $-1,1$ & 10,2 \\
\hline Methylmalonate-semialdehyde dehydrogenase [acylating], mitochondrial; & Q9EQ20 & Aldh6a1 & 0,0 & 1,5 \\
\hline Methylmalonyl-CoA epimerase, mitochondrial; & Q9D115 & Mcee & 0,1 & 10,7 \\
\hline
\end{tabular}




\begin{tabular}{|c|c|c|c|c|}
\hline Protein & Uniprot & Gene name & $\begin{array}{c}\text { Fold change of } \\
\text { XPRESS ratio } \\
\left.\text { (Adam17 }^{\Delta K C} / \mathrm{WT}\right)\end{array}$ & $\begin{array}{c}\text { Sequence } \\
\text { coverage [\%] }\end{array}$ \\
\hline Methylosome protein 50; & Q99J09 & Wdr77 & 1,0 & 13,2 \\
\hline Methylosome subunit pICln; & Q61189 & Clns1a & 0,3 & 7,2 \\
\hline Methylsterol monooxygenase 1 & Q9CRA4 & Msmo1 & $-0,7$ & 7,2 \\
\hline Methylthioribulose-1-phosphate dehydratase; & Q9WVQ5 & Apip & $-0,1$ & 23,7 \\
\hline Mevalonate kinase; & Q9R008 & Mvk & 0,5 & 19,2 \\
\hline Microfibril-associated glycoprotein 4; & Q9D1H9 & Mfap4 & 0,1 & 9,7 \\
\hline Microfibrillar-associated protein 1 & Q9CQU1 & Mfap1 & $-0,1$ & 3 \\
\hline Microsomal glutathione S-transferase 1; & Q91VS7 & Mgst1 & $-0,8$ & 18,7 \\
\hline Microtubule-actin cross-linking factor 1 & Q9QXZ0 & Macf1 & $-0,5$ & 0,3 \\
\hline Microtubule-associated protein 1B; & P14873 & Map1b & 0,4 & 0,9 \\
\hline Microtubule-associated protein 2; & P20357 & Map2 & 0,5 & 3,6 \\
\hline Microtubule-associated protein 4; & P27546 & Map4 & $-0,1$ & 21 \\
\hline Microtubule-associated protein 6; & Q7TSJ2 & Map6 & 0,4 & 10,2 \\
\hline Microtubule-associated protein RP/EB family member 1 ; & Q61166 & Mapre1 & 0,2 & 7,5 \\
\hline Midnolin; & Q3TPJ7 & Midn & 6,4 & 3,4 \\
\hline Mimecan; & Q62000 & Ogn & 0,5 & 20,1 \\
\hline Minor histocompatibility antigen $\mathrm{H} 13$; & Q9D8V0 & $\mathrm{Hm} 13$ & $-0,1$ & 4,6 \\
\hline Mitochondrial 2-oxoglutarate/malate carrier protein; & Q9CR62 & Slc25a11 & $-0,6$ & 23,6 \\
\hline Mitochondrial amidoxime reducing component 2; & Q922Q1 & Marc2 & 0,3 & 14,2 \\
\hline Mitochondrial carnitine/acylcarnitine carrier protein; & Q9Z2Z6 & Slc25a20 & $-0,3$ & 16,3 \\
\hline Mitochondrial carrier homolog 2; & Q791V5 & Mtch2 & 0,0 & 26,7 \\
\hline Mitochondrial dicarboxylate carrier; & Q9QZD8 & Slc25a10 & 0,0 & 13,2 \\
\hline Mitochondrial fission 1 protein; & Q9CQ92 & Fis1 & $-0,3$ & 17,2 \\
\hline Mitochondrial import inner membrane translocase subunit Tim13; & P62075 & Timm13 & $-0,2$ & 49,5 \\
\hline Mitochondrial import inner membrane translocase subunit TIM14; & Q9CQV7 & Dnajc19 & 0,1 & 8,2 \\
\hline Mitochondrial import inner membrane translocase subunit TIM16; & Q9CQV1 & Pam16 & $-0,1$ & 21,6 \\
\hline Mitochondrial import inner membrane translocase subunit TIM50; & Q9D880 & Timm50 & 0,1 & 12,7 \\
\hline Mitochondrial import inner membrane translocase subunit Tim8 A; & Q9WVA2 & Timm8a1 & 0,0 & 49,5 \\
\hline Mitochondrial import inner membrane translocase subunit Tim9; & Q9WV98 & Timm9 & 0,3 & 43,8 \\
\hline Mitochondrial import receptor subunit TOM22 homolog; & Q9CPQ3 & Tomm22 & $-0,6$ & 7,7 \\
\hline Mitochondrial import receptor subunit TOM40 homolog; & Q9QYA2 & Tomm40 & $-0,8$ & 7,2 \\
\hline Mitochondrial inner membrane protein OXA1L; & Q8BGA9 & Oxa1l & 0,7 & 2,1 \\
\hline Mitochondrial inner membrane protein; & Q8CAQ8 & Immt & 0,0 & 20,9 \\
\hline Mitochondrial intermembrane space import and assembly protein 40 ; & Q8VEA4 & Chchd4 & $-0,1$ & 20,1 \\
\hline Mitochondrial pyruvate carrier 1 & E9Q0V4 & Mpc1 & $-0,2$ & 9,6 \\
\hline Mitochondrial pyruvate carrier 2; & Q9D023 & Mpc2 & $-0,5$ & 22,8 \\
\hline Mitochondrial Rho GTPase 1; & Q8BG51 & Rhot1 & 1,2 & 2,9 \\
\hline Mitogen-activated protein kinase 1 & P63085 & Mapk1 & 0,7 & 25,4 \\
\hline Mitogen-activated protein kinase kinase kinase 10; & Q66L42 & Map3k10 & $-1,5$ & 0,7 \\
\hline Mitotic checkpoint protein BUB3; & Q9WVA3 & Bub3 & 0,5 & 24,2 \\
\hline MOB kinase activator $1 \mathrm{~B}$ & Q8BPB0 & Mob1b & $-0,9$ & 11,1 \\
\hline Moesin; & P26041 & Msn & 0,4 & 8,5 \\
\hline Molybdate-anion transporter; & Q921Y4 & Mfsd5 & $-0,4$ & 4,9 \\
\hline Monoacylglycerol lipase ABHD12; & Q99LR1 & Abhd12 & $-1,6$ & 3,3 \\
\hline Monocarboxylate transporter 1 & P53986 & Slc16a1 & $-0,9$ & 6,5 \\
\hline Monoglyceride lipase; & 035678 & Mg\|l & $-0,1$ & 34,9 \\
\hline MORF4 family-associated protein 1 & Q9CQL7 & Mrfap1 & 0,6 & 18,4 \\
\hline mRNA cap guanine-N7 methyltransferase; & Q9D0L8 & Rnmt & 0,4 & 19,5 \\
\hline Multidrug resistance-associated protein 9; & Q80WJ6 & Abcc12 & 4,6 & 1,6 \\
\hline Multifunctional protein ADE2; & Q9DCL9 & Paics & $-0,3$ & 11,8 \\
\hline Multimerin-2; & A6H6E2 & Mmrn2 & 1,5 & 3,3 \\
\hline Multiple inositol polyphosphate phosphatase 1 & Q9Z2L6 & Minpp1 & $-0,3$ & 12,1 \\
\hline Musculoskeletal embryonic nuclear protein 1; & Q99JI1 & Mustn1 & 2,4 & 13,4 \\
\hline Myb-binding protein $1 \mathrm{~A}$ & Q7TPV4 & Mybbp1a & 0,3 & 2,2 \\
\hline Myelin basic protein; & F6RT34 & Mbp & $-0,5$ & 32,1 \\
\hline Myelin protein PO; & E9QK82 & $\mathrm{Mpz}$ & $-0,7$ & 12,1 \\
\hline Myoferlin; & Q69ZN7 & Myof & $-0,8$ & 2,3 \\
\hline Myoglobin; & P04247 & $\mathrm{Mb}$ & $-0,2$ & 9,1 \\
\hline Myosin light chain $1 / 3$, skeletal muscle isoform; & P05977 & Myl1 & $-0,6$ & 32,9 \\
\hline Myosin light polypeptide 6; & Q60605 & Myl6 & $-0,1$ & 37,7 \\
\hline Myosin regulatory light chain $12 \mathrm{~B}$; & Q3THE2 & Myl12b & $-0,2$ & 23,3 \\
\hline Myosin regulatory light chain 2, skeletal muscle isoform; & P97457 & Mylpf & $-0,6$ & 17,2 \\
\hline Myosin regulatory light polypeptide 9; & Q9CQ19 & Myl9 & 0,2 & 28,5 \\
\hline Myosin-10; & Q61879 & Myh10 & 2,3 & 4,9 \\
\hline Myosin-14; & Q6URW6 & Myh14 & $-0,1$ & 1,3 \\
\hline Myosin-9; & Q8VDD5 & Myh9 & $-0,2$ & 11,4 \\
\hline Myristoylated alanine-rich C-kinase substrate; & P26645 & Marcks & $-0,2$ & 8,4 \\
\hline N(4)-(beta-N-acetylglucosaminyl)-L-asparaginase; & Q64191 & Aga & $-0,6$ & 7,2 \\
\hline $\mathrm{Na}(+) / \mathrm{H}(+)$ exchange regulatory cofactor NHE-RF1; & P70441 & Slc9a3r1 & 0,1 & 16,6 \\
\hline $\mathrm{N}$-acetyl-D-glucosamine kinase; & Q9QZ08 & Nagk & 0,4 & 8,6 \\
\hline N-acetylglucosamine-6-sulfatase; & Q8BFR4 & Gns & $-0,3$ & 4,4 \\
\hline $\mathrm{N}$-acetylneuraminate lyase; & Q9DCJ9 & $\mathrm{Npl}$ & $-0,3$ & 40,9 \\
\hline $\mathrm{N}$-acylethanolamine-hydrolyzing acid amidase; & Q9D7V9 & Naaa & 0,6 & 6 \\
\hline
\end{tabular}




\begin{tabular}{|c|c|c|c|c|}
\hline Protein & Uniprot & Gene name & $\begin{array}{c}\text { Fold change of } \\
\text { XPRESS ratio } \\
\left.\text { (Adam17 }^{\Delta K C} / \mathrm{WT}\right)\end{array}$ & $\begin{array}{l}\text { Sequence } \\
\text { coverage [\%] }\end{array}$ \\
\hline N-acylglucosamine 2-epimerase; & P82343 & Renbp & $-0,5$ & 4,5 \\
\hline $\mathrm{NAD}(\mathrm{P}) \mathrm{H}$-hydrate epimerase; & Q8K4Z3 & Apoa1bp & $-0,3$ & 16,3 \\
\hline NADH dehydrogenase [ubiquinone] 1 alpha subcomplex subunit 11 ; & Q9D8B4 & Ndufa11 & $-0,6$ & 11,3 \\
\hline NADH dehydrogenase [ubiquinone] 1 alpha subcomplex subunit 13; & Q9ERS2 & Ndufa13 & 0,0 & 13,2 \\
\hline NADH dehydrogenase [ubiquinone] 1 alpha subcomplex subunit 4; & Q62425 & Ndufa4 & $-0,2$ & 46,3 \\
\hline NADH dehydrogenase [ubiquinone] 1 alpha subcomplex subunit 4-like 2; & Q4FZG9 & Ndufa4l2 & 0,9 & 9,2 \\
\hline NADH dehydrogenase [ubiquinone] 1 alpha subcomplex subunit 5; & Q9CPP6 & Ndufa5 & 0,5 & 27,6 \\
\hline NADH dehydrogenase [ubiquinone] 1 alpha subcomplex subunit 7; & Q971P6 & Ndufa7 & 0,4 & 17,7 \\
\hline NADH dehydrogenase [ubiquinone] 1 alpha subcomplex subunit 8; & Q9DCJ5 & Ndufa8 & 0,5 & 17,4 \\
\hline $\begin{array}{l}\text { NADH dehydrogenase [ubiquinone] } 1 \text { beta subcomplex subunit 11, } \\
\text { mitochondrial; }\end{array}$ & 009111 & Ndufb11 & $-0,3$ & 6,6 \\
\hline NADH dehydrogenase [ubiquinone] 1 beta subcomplex subunit 6; & Q3UIU2 & Ndufb6 & $-0,2$ & 7,2 \\
\hline NADH dehydrogenase [ubiquinone] 1 subunit $\mathrm{C} 2$ & Q9CQ54 & Ndufc2 & 0,1 & 25,8 \\
\hline NADH dehydrogenase [ubiquinone] flavoprotein 1, mitochondrial; & Q91YT0 & Ndufv1 & 3,6 & 2,6 \\
\hline NADH dehydrogenase [ubiquinone] flavoprotein 2, mitochondrial; & Q9D6J6 & Ndufv2 & 0,7 & 8,1 \\
\hline $\begin{array}{l}\text { NADH dehydrogenase [ubiquinone] flavoprotein 3, mitochondrial; RIKEN cDNA } \\
\text { 1500032D16, isoform CRA_a; }\end{array}$ & Q3U422 & Ndufv3 & 0,0 & 17,3 \\
\hline NADH dehydrogenase [ubiquinone] iron-sulfur protein 2, mitochondrial; & Q91WD5 & Ndufs2 & 0,3 & 14,4 \\
\hline NADH dehydrogenase [ubiquinone] iron-sulfur protein 4, mitochondrial; & Q9CXZ1 & Ndufs4 & 0,4 & 8,6 \\
\hline NADH dehydrogenase [ubiquinone] iron-sulfur protein 5; & Q99LY9 & Ndufs5 & 0,4 & 14,3 \\
\hline NADH dehydrogenase [ubiquinone] iron-sulfur protein 6, mitochondrial; & P52503 & Ndufs6 & 0,4 & 37,8 \\
\hline NADH dehydrogenase [ubiquinone] iron-sulfur protein 7, mitochondrial; & Q9DC70 & Ndufs7 & $-0,1$ & 4 \\
\hline NADH-cytochrome b5 reductase 1 ; & Q9DB73 & Cyb5r1 & 0,3 & 15 \\
\hline NADH-cytochrome b5 reductase 3 ; & Q9DCN2 & Cyb5r3 & 0,9 & 15,6 \\
\hline NADH-ubiquinone oxidoreductase chain 5 & P03921 & Mtnd5 & 0,1 & 1,2 \\
\hline NADP-dependent malic enzyme; & P06801 & Me1 & $-0,5$ & 20,8 \\
\hline NADPH--cytochrome $\mathrm{P} 450$ reductase; & P37040 & Por & 0,0 & 6,9 \\
\hline Napsin-A; & 009043 & Napsa & $-0,3$ & 2,1 \\
\hline Nardilysin; & Q8BHG1 & Nrd1 & 0,0 & 6,2 \\
\hline Nascent polypeptide-associated complex subunit alpha, muscle-specific form; & P70670 & Naca & $-0,1$ & 12,6 \\
\hline Nectin-1; & Q9JKF6 & Pvrl1 & $-0,3$ & 4,9 \\
\hline Nectin-2; & P32507 & Pvrl2 & 0,2 & 5,1 \\
\hline Nectin-4; & Q8R007 & Pvrl4 & 0,2 & 10,6 \\
\hline NEDD8; & P29595 & Nedd8 & 0,0 & 11,1 \\
\hline NEDD8-activating enzyme E1 catalytic subunit; & Q8C878 & Uba3 & 0,6 & 1,8 \\
\hline NEDD8-conjugating enzyme Ubc12; & P61082 & Ube2m & 0,1 & 12,8 \\
\hline Neprilysin; & Q61391 & Mme & $-0,5$ & 2,5 \\
\hline Neudesin; & Q9CQ45 & Nenf & 0,2 & 10,5 \\
\hline Neural cell adhesion molecule 1 ; & P13595 & Ncam1 & 0,4 & 7,5 \\
\hline Neural cell adhesion molecule L1; & P11627 & L1cam & 0,0 & 1,5 \\
\hline Neural cell adhesion molecule L1-like protein; & P70232 & Chl1 & 0,5 & 0,7 \\
\hline Neurolysin, mitochondrial; & Q91YP2 & $\mathrm{N} l n$ & $-0,1$ & 2,1 \\
\hline Neuroplastin; & P97300 & Nptn & $-0,2$ & 13,6 \\
\hline Neutral alpha-glucosidase $A B$ & Q8BHN3 & Ganab & 0,3 & 26,7 \\
\hline Neutral amino acid transporter $\mathrm{A}$; & 035874 & Slc1a4 & $-0,4$ & 6 \\
\hline Neutral amino acid transporter $\mathrm{B}(0)$; & P51912 & Slc1a5 & $-0,3$ & 2,4 \\
\hline Neutral ceramidase; & Q9JHE3 & Asah2 & $-0,7$ & 3,6 \\
\hline Neutral cholesterol ester hydrolase $1 ;$ & Q8BLF1 & Nceh1 & 0,0 & 5,6 \\
\hline NFU1 iron-sulfur cluster scaffold homolog, mitochondrial; & Q9QZ23 & Nfu1 & 0,5 & 5,9 \\
\hline NHL repeat-containing protein 3 ; & $\mathrm{Q} 8 \mathrm{CCH} 2$ & Nhlrc3 & 0,1 & 6,1 \\
\hline NHP2-like protein 1; & Q9D0T1 & Nhp2I1 & 0,3 & 18 \\
\hline Niban-like protein 1 ; & Q8R1F1 & Fam129b & 0,6 & 1,2 \\
\hline Nicalin; & Q8VCM8 & Ncln & $-0,6$ & 8,2 \\
\hline Nicastrin; & P57716 & Ncstn & $-0,4$ & 4,4 \\
\hline Nicotinamide phosphoribosyltransferase; & Q99KQ4 & Nampt & 0,4 & 15,5 \\
\hline Nicotinate phosphoribosyltransferase; & Q8CC86 & Naprt1 & 0,2 & 5 \\
\hline Nidogen-1; & P10493 & Nid1 & $-0,1$ & 14,6 \\
\hline Nidogen-2; & 088322 & Nid2 & $-0,8$ & 3,8 \\
\hline Nischarin; & Q80TM9 & Nisch & 4,7 & 1,4 \\
\hline Nitrilase homolog 1; & Q8VDK1 & Nit1 & $-0,1$ & 27,2 \\
\hline Nodal modulator 1 & Q6GQT9 & Nomo1 & 0,4 & 2,4 \\
\hline Non-histone chromosomal protein HMG-17; & P09602 & Hmgn2 & $-0,3$ & 17,2 \\
\hline Non-POU domain-containing octamer-binding protein; & Q99K48 & Nono & 0,1 & 4,2 \\
\hline Non-specific lipid-transfer protein; & P32020 & Scp2 & $-0,2$ & 10,6 \\
\hline NSFL1 cofactor p47; & Q9CZ44 & Nsfl1c & 0,3 & 14,7 \\
\hline Nuclear autoantigenic sperm protein; & Q99MD9 & Nasp & 0,1 & 10,3 \\
\hline Nuclear factor 1 A-type; & Q02780 & Nfia & 0,2 & 13,7 \\
\hline
\end{tabular}




\begin{tabular}{|c|c|c|c|c|}
\hline Protein & Uniprot & Gene name & $\begin{array}{c}\text { Fold change of } \\
\text { XPRESS ratio } \\
\left.\text { (Adam17 }^{\Delta K C} / \mathrm{WT}\right)\end{array}$ & $\begin{array}{c}\text { Sequence } \\
\text { coverage [\%] }\end{array}$ \\
\hline Nuclear factor 1 B-type; & P97863 & Nfib & 0,1 & 10,7 \\
\hline Nuclear factor 1 X-type; & P70257 & Nfix & 0,1 & 11,7 \\
\hline Nuclear migration protein nudC; & 035685 & Nudc & 0,0 & 9,9 \\
\hline Nuclear pore glycoprotein p62; & Q63850 & Nup62 & $-0,8$ & 2,7 \\
\hline Nuclear transport factor 2 & P61971 & Nutf2 & $-0,3$ & 56,7 \\
\hline Nuclease-sensitive element-binding protein 1 & P62960 & Ybx1 & $-0,2$ & 19,6 \\
\hline Nucleobindin-1; & Q02819 & Nucb1 & 0,3 & 29 \\
\hline Nucleobindin-2; & P81117 & Nucb2 & 0,3 & 16 \\
\hline Nucleolar protein 16; & Q9CPT5 & Nop16 & $-0,2$ & 10,1 \\
\hline Nucleolar protein 56 & Q9D6Z1 & Nop56 & 0,5 & 24 \\
\hline Nucleolar protein 58 & Q6DFW4 & Nop58 & 0,3 & 13,6 \\
\hline Nucleolar RNA helicase 2; & Q9JIK5 & $\mathrm{Dd} \times 21$ & 0,3 & 1,3 \\
\hline Nucleolar transcription factor 1 ; & P25976 & Ubtf & 0,3 & 2,2 \\
\hline Nucleolin; & P09405 & $\mathrm{Ncl}$ & 0,2 & 16,7 \\
\hline Nucleolysin TIA-1; & P52912 & Tia1 & 0,0 & 8,4 \\
\hline Nucleophosmin; & Q61937 & Npm1 & $-0,3$ & 33,5 \\
\hline Nucleoplasmin-3; & Q9СPPO & Npm3 & $-0,4$ & 8,6 \\
\hline Nucleoporin Nup37; & Q9CWU9 & Nup37 & 0,5 & 5,2 \\
\hline Nucleoporin NUP53; & Q8R4R6 & Nup35 & 0,8 & 9,1 \\
\hline Nucleoporin SEH1; & Q8R2U0 & Seh1l & 0,6 & 3,6 \\
\hline Nucleoprotein TPR; & F6ZDS4 & Tpr & 1,4 & 4,9 \\
\hline Nucleoside diphosphate kinase 3 ; & Q9WV85 & Nme3 & $-1,3$ & 12,4 \\
\hline Nucleoside diphosphate kinase; & E9PZF0 & Gm20390 & $-0,4$ & 69,7 \\
\hline Nucleosome assembly protein 1-like 1 ; & P28656 & Nap1l1 & 0,1 & 7,9 \\
\hline Nurim; & Q8VC65 & $\mathrm{Nrm}$ & $-1,1$ & 3,8 \\
\hline Obg-like ATPase 1; & Q9CZ30 & Ola1 & $-1,4$ & 7,1 \\
\hline Obscurin; & A2AAJ9 & Obscn & 8,2 & 0,3 \\
\hline Occludin; & Q61146 & Ocln & $-0,6$ & 4 \\
\hline OCIA domain-containing protein 1 & Q9CRD0 & Ociad1 & $-0,1$ & 7,7 \\
\hline OCIA domain-containing protein 2 & Q9D8W7 & Ociad2 & $-0,6$ & 5,8 \\
\hline Olfactomedin-like 1; Protein Olfml1; & Q8BSH2 & Olfml1 & $-0,1$ & 3,9 \\
\hline Olfactomedin-like protein 3 & Q8BK62 & Olfml3 & 0,6 & 6,9 \\
\hline Oligoribonuclease, mitochondrial; & Q9D8S4 & Rexo2 & $-0,4$ & 7,6 \\
\hline Oligosaccharyltransferase complex subunit OSTC; & Q78XF5 & Ostc & 0,5 & 8,1 \\
\hline Omega-amidase NIT2; & Q9JHW2 & Nit2 & 0,1 & 11,6 \\
\hline Ornithine aminotransferase, mitochondrial; & P29758 & Oat & $-1,1$ & 22,6 \\
\hline Osteoclast-stimulating factor 1 & Q62422 & Ostf1 & 0,3 & 3,3 \\
\hline Oxygen-dependent coproporphyrinogen-III oxidase, mitochondrial; & P36552 & Cpox & 0,2 & 5 \\
\hline Paired amphipathic helix protein Sin3a; & Q60520 & $\sin 3 a$ & 0,5 & 1,7 \\
\hline Palmdelphin; & Q9JHU2 & Palmd & 0,1 & 2 \\
\hline Palmitoyl-protein thioesterase 1 & 088531 & Ppt1 & $-0,3$ & 8,2 \\
\hline Palmitoyltransferase ZDHHC2; & P59267 & Zdhhc2 & $-3,0$ & 3,8 \\
\hline Pantetheinase; & Q9Z0K8 & Vnn1 & $-1,9$ & 3,7 \\
\hline Parafibromin; & Q8JZM7 & Cdc73 & 1,8 & 1,9 \\
\hline Paralemmin-1; & Q9Z0P4 & Palm & $-0,7$ & 6,8 \\
\hline Parathymosin; & Q9D0J8 & Ptms & $-0,1$ & 10,9 \\
\hline Parkinson disease 7 domain-containing protein 1 ; & Q8BFQ8 & Pddc1 & $-0,2$ & 30,9 \\
\hline Partner of Y14 and mago; & Q8CHP5 & Wibg & 0,1 & 20,7 \\
\hline Paxillin; & Q8VI36 & Pxn & $-0,8$ & 6,9 \\
\hline PC4 and SFRS1-interacting protein; & Q99JF8 & Psip1 & 1,2 & 37,8 \\
\hline PDZ and LIM domain protein $1 ;$ & 070400 & Pdlim1 & $-0,7$ & 21,4 \\
\hline PDZ and LIM domain protein 2; & Q8R1G6 & Pdlim2 & 0,4 & 12,6 \\
\hline PDZ and LIM domain protein 4; & P70271 & Pdlim4 & 0,0 & 6,1 \\
\hline PDZ and LIM domain protein 5; & Q8CI51 & Pdlim5 & 0,5 & 2,5 \\
\hline PDZK1-interacting protein 1; & Q9CQHO & Pdzk1ip1 & $-0,1$ & 18,4 \\
\hline Peflin; & Q8BFY6 & Pef1 & $-0,6$ & 6,9 \\
\hline Peptidyl-prolyl cis-trans isomerase A; & P17742 & Ppia & 0,5 & 30,5 \\
\hline Peptidyl-prolyl cis-trans isomerase B; & P24369 & Ppib & 0,2 & 26,9 \\
\hline Peptidyl-prolyl cis-trans isomerase C; & P30412 & Ppic & 0,9 & 6,1 \\
\hline Peptidyl-prolyl cis-trans isomerase FKBP10; & Q61576 & Fkbp10 & 0,5 & 12,2 \\
\hline Peptidyl-prolyl cis-trans isomerase FKBP1A; & P26883 & Fkbp1a & $-0,1$ & 13 \\
\hline Peptidyl-prolyl cis-trans isomerase FKBP2; & P45878 & Fkbp2 & 0,0 & 8,6 \\
\hline Peptidyl-prolyl cis-trans isomerase FKBP3; & Q62446 & Fkbp3 & 0,0 & 21,4 \\
\hline Peptidyl-prolyl cis-trans isomerase FKBP4; & P30416 & Fkbp4 & 1,3 & 2 \\
\hline Peptidyl-prolyl cis-trans isomerase FKBP7; & 054998 & Fkbp7 & 0,9 & 13,3 \\
\hline Peptidyl-prolyl cis-trans isomerase FKBP9; & Q9Z247 & Fkbp9 & 0,6 & 5,1 \\
\hline Peptidyl-prolyl cis-trans isomerase $\mathrm{H}$; & Q9D868 & Ppih & $-0,1$ & 6 \\
\hline Peptidyl-prolyl cis-trans isomerase NIMA-interacting 4; & Q9CWW6 & Pin4 & 0,0 & 8,4 \\
\hline Peptidyl-prolyl cis-trans isomerase-like 1 & Q9D0W5 & Ppil1 & 0,8 & 14,5 \\
\hline Peptidyl-tRNA hydrolase 2, mitochondrial; & Q8R2Y8 & Ptrh2 & $-0,2$ & 32,6 \\
\hline Periaxin; & 055103 & Prx & 0,1 & 4,2 \\
\hline Pericentriolar material 1 protein; & Q9R0L6 & Pcm1 & 6,5 & 0,8 \\
\hline Perilipin-1; & Q8CGN5 & Plin1 & $-0,1$ & 22,6 \\
\hline
\end{tabular}




\begin{tabular}{|c|c|c|c|c|}
\hline Protein & Uniprot & Gene name & $\begin{array}{c}\text { Fold change of } \\
\text { XPRESS ratio } \\
\left.\text { (Adam17 }^{\Delta K C} / \mathrm{WT}\right)\end{array}$ & $\begin{array}{c}\text { Sequence } \\
\text { coverage [\%] }\end{array}$ \\
\hline Perilipin-2; & P43883 & Plin2 & $-0,9$ & 12,2 \\
\hline Perilipin-3; & Q9DBG5 & Plin3 & 0,2 & 16,5 \\
\hline Perilipin-4; & 088492 & Plin4 & 0,1 & 6,3 \\
\hline Periodic tryptophan protein 1 homolog; & Q99LL5 & Pwp1 & 0,1 & 15,6 \\
\hline Periostin; & Q62009 & Postn & 0,4 & 31,5 \\
\hline Periplakin; & Q9R269 & Ppl & $-0,1$ & 1,4 \\
\hline Peroxiredoxin-1; & P35700 & Prdx1 & 0,4 & 60,3 \\
\hline Peroxiredoxin-2; & Q61171 & Prdx2 & 0,5 & 34,3 \\
\hline Peroxiredoxin-4; & 008807 & $\operatorname{Prdx} 4$ & 0,5 & 39,1 \\
\hline Peroxiredoxin-5, mitochondrial; & P99029 & Prdx5 & 0,1 & 23,3 \\
\hline Peroxiredoxin-6; & 008709 & Prdx6 & 0,0 & 43,3 \\
\hline Peroxisomal 2,4-dienoyl-CoA reductase; & Q9WV68 & Decr2 & 0,0 & 4,1 \\
\hline Peroxisomal acyl-coenzyme A oxidase 1 ; & Q9ROHO & Acox1 & $-0,2$ & 24,5 \\
\hline Peroxisomal acyl-coenzyme A oxidase 2 ; & Q9QXD1 & Acox 2 & 0,9 & 5,3 \\
\hline Peroxisomal acyl-coenzyme A oxidase 3; & Q9EPL9 & Acox3 & $-0,6$ & 8,7 \\
\hline Peroxisomal biogenesis factor 19 & Q8VCI5 & Pex19 & 0,0 & 12 \\
\hline Peroxisomal carnitine O-octanoyltransferase; & Q9DC50 & Crot & $-0,5$ & 1,6 \\
\hline Peroxisomal coenzyme A diphosphatase NUDT7; & Q99P30 & Nudt7 & 3,0 & 4,2 \\
\hline Peroxisomal membrane protein 11B; & Q9Z210 & Pex11b & $-0,2$ & 5,1 \\
\hline Peroxisomal membrane protein 4; & Q9JJW0 & Pxmp4 & 0,4 & 16,3 \\
\hline Peroxisomal membrane protein PEX14; & Q9R0A0 & Pex14 & $-0,3$ & 16,2 \\
\hline Peroxisomal multifunctional enzyme type 2 & P51660 & Hsd17b4 & 0,1 & 8,6 \\
\hline Persulfide dioxygenase ETHE1, mitochondrial; & Q9DCMO & Ethe1 & $-1,0$ & 25,2 \\
\hline PEST proteolytic signal-containing nuclear protein; & Q6P814 & Pcnp & 0,0 & 34,7 \\
\hline PGAP2-interacting protein; & Q91YL7 & Cwh43 & $-0,1$ & 2,6 \\
\hline PHD finger-like domain-containing protein $5 \mathrm{~A}$; & P83870 & Phf5a & 0,3 & 6,4 \\
\hline Phenylalanine--tRNA ligase alpha subunit; & Q8C0C7 & Farsa & 0,4 & 7,9 \\
\hline Phenylalanine--tRNA ligase beta subunit; & Q9WUA2 & Farsb & 0,5 & 2,2 \\
\hline Phosphatase and actin regulator 4 & Q501J7 & Phactr4 & 0,0 & 4,3 \\
\hline Phosphate carrier protein, mitochondrial; & Q8VEM8 & Slc25a3 & $-0,3$ & 27,7 \\
\hline Phosphatidylethanolamine-binding protein 1 ; & P70296 & Pebp1 & 0,4 & 36,4 \\
\hline Phosphatidylinositide phosphatase SAC1; & Q9EP69 & Sacm1l & 0,2 & 3,2 \\
\hline Phosphatidylinositol transfer protein alpha isoform; & P53810 & Pitpna & 1,7 & 15,9 \\
\hline Phosphatidylinositol-glycan-specific phospholipase D; & 070362 & Gpld1 & 0,4 & 2,6 \\
\hline Phosphoacetylglucosamine mutase; & Q9CYR6 & Pgm3 & 0,7 & 12,8 \\
\hline Phosphoglucomutase-1; & Q9D0F9 & Pgm1 & 0,2 & 20,6 \\
\hline Phosphoglucomutase-2; & Q7TSV4 & Pgm2 & $-0,2$ & 13,5 \\
\hline Phosphoglucomutase-like protein 5; & Q8BZF8 & Pgm5 & 0,0 & 14,1 \\
\hline Phosphoglycerate kinase 1 & P09411 & Pgk1 & 0,6 & 15,3 \\
\hline Phosphoglycerate mutase 1 & Q9DBJ1 & Pgam1 & 0,1 & 35 \\
\hline Phosphoglycerate mutase 2 & 070250 & Pgam2 & $-0,8$ & 27,3 \\
\hline Phosphoglycolate phosphatase; & Q8CHP8 & Pgp & $-0,3$ & 6,2 \\
\hline phospholipase $\mathrm{A} 2$ inhibitor and Ly6/PLAUR domain-containing protein; & Q9CQD7 & Pinlyp & 0,0 & 9 \\
\hline Phospholipase A-2-activating protein; & P27612 & Plaa & 0,5 & 3 \\
\hline Phospholipase B-like 1; & Q8VCI0 & Plbd1 & $-0,2$ & 23,6 \\
\hline Phospholipase D3; & 035405 & Pld3 & $-0,3$ & 10,9 \\
\hline Phospholipase D4; & Q8BG07 & Pld4 & 1,1 & 4,8 \\
\hline Phospholipid hydroperoxide glutathione peroxidase, mitochondrial; & 070325 & Gpx4 & 0,2 & 21,7 \\
\hline Phospholipid scramblase 3 & Q9JIZ9 & Plscr3 & 0,0 & 4,7 \\
\hline Phospholipid transfer protein; & P55065 & Pltp & $-0,1$ & 4,5 \\
\hline Phospholipid-transporting ATPase IA; & P70704 & Atp8a1 & 0,2 & 1,6 \\
\hline Phosphoribosyl pyrophosphate synthase-associated protein 1; & Q9D0M1 & Prpsap1 & 0,3 & 13,5 \\
\hline Phosphoribosyl pyrophosphate synthase-associated protein 2; & Q8R574 & Prpsap2 & 0,6 & 16,8 \\
\hline Phosphoribosylformylglycinamidine synthase; & Q5SURO & Pfas & $-0,4$ & 1,5 \\
\hline Phosphoserine aminotransferase; & Q99K85 & Psat1 & 0,5 & 8,4 \\
\hline Phosphoserine phosphatase; & Q99LS3 & Psph & $-0,4$ & 24,4 \\
\hline Phosphotriesterase-related protein; & Q60866 & Pter & $-0,4$ & 9,5 \\
\hline Pigment epithelium-derived factor; & P97298 & Serpinf1 & 1,4 & 13,7 \\
\hline Pinin; & 035691 & Pnn & 0,2 & 5 \\
\hline PITH domain-containing protein $1 ;$ & Q8BWR2 & Pithd1 & $-0,4$ & 8,1 \\
\hline Plasma protease $\mathrm{C} 1$ inhibitor; & P97290 & Serping1 & 0,0 & 7,8 \\
\hline Plasmalemma vesicle-associated protein; & Q91VC4 & Plvap & 0,0 & 4,8 \\
\hline Plasminogen activator inhibitor 1 RNA-binding protein; & Q9CY58 & Serbp1 & $-0,2$ & 13 \\
\hline Plasminogen activator inhibitor 2 , macrophage; & P12388 & Serpinb2 & 0,3 & 8,2 \\
\hline Plasminogen receptor $(\mathrm{KT})$; & Q9D3P8 & Plgrkt & $-0,4$ & 14,3 \\
\hline Plasminogen; & P20918 & $\mathrm{Plg}$ & 1,4 & 2 \\
\hline Plastin-2; & Q61233 & Lcp1 & $-0,2$ & 28,9 \\
\hline Plastin-3; & Q99K51 & Pls3 & 0,4 & 24,9 \\
\hline Platelet endothelial cell adhesion molecule; & Q08481 & Pecam1 & $-0,3$ & 8 \\
\hline Platelet factor 4; & Q9Z126 & Pf4 & 0,2 & 8,6 \\
\hline Platelet glycoprotein 4; & Q08857 & $\mathrm{Cd} 36$ & $-0,3$ & 12,3 \\
\hline Platelet receptor Gi24; & Q9D659 & & $-4,9$ & 8 \\
\hline Platelet-activating factor acetylhydrolase IB subunit alpha; & P63005 & Pafah1b1 & $-0,7$ & 24,9 \\
\hline
\end{tabular}




\begin{tabular}{|c|c|c|c|c|}
\hline Protein & Uniprot & Gene name & $\begin{array}{c}\text { Fold change of } \\
\text { XPRESS ratio } \\
\left.\text { (Adam } 17^{\Delta K C} / \mathrm{WT}\right)\end{array}$ & $\begin{array}{c}\text { Sequence } \\
\text { coverage [\%] }\end{array}$ \\
\hline Platelet-activating factor acetylhydrolase IB subunit beta; & Q61206 & Pafah1b2 & 0,0 & 12,2 \\
\hline Platelet-activating factor acetylhydrolase IB subunit gamma; & Q61205 & Pafah1b3 & 0,3 & 6,3 \\
\hline Platelet-activating factor acetylhydrolase; & Q60963 & Pla2g7 & 0,3 & 3,6 \\
\hline Pleckstrin homology domain-containing family $\mathrm{O}$ member 2; & Q8K124 & Plekho2 & 0,4 & 6,3 \\
\hline
\end{tabular}


Supplementary table 6: List of all identified and quantified proteins in the quantitative proteome comparison of wild-type and $A d a m 17^{\Delta K C}$ epidermis at P10 (replicate 1).

\begin{tabular}{|c|c|c|c|c|}
\hline Protein & Uniprot & Gene name & $\begin{array}{c}\text { Fold change of } \\
\text { XPRESS ratio } \\
\left.\text { (Adam } 17^{\Delta K C} / \mathrm{WT}\right)\end{array}$ & $\begin{array}{c}\text { Sequence } \\
\text { coverage [\%] }\end{array}$ \\
\hline 10 kDa heat shock protein, mitochondrial; & Q64433 & Hspe1 & 0,1 & 37,3 \\
\hline 116 kDa U5 small nuclear ribonucleoprotein component; & 008810 & Eftud2 & $-0,1$ & 13,3 \\
\hline 14-3-3 protein beta/alpha; & Q9CQV8 & Ywhab & $-0,3$ & 16,7 \\
\hline 14-3-3 protein epsilon; & P62259 & Ywhae & 0,0 & 31,8 \\
\hline 14-3-3 protein eta; & P68510 & Ywhah & 0,0 & 17,1 \\
\hline 14-3-3 protein gamma; & P61982 & Ywhag & 0,0 & 11,3 \\
\hline 14-3-3 protein sigma; & 070456 & Sfn & $-0,5$ & 36,7 \\
\hline 14-3-3 protein theta; & P68254 & Ywhaq & 0,3 & 16,7 \\
\hline 14-3-3 protein zeta/delta; & P63101 & Ywhaz & $-0,5$ & 44,9 \\
\hline 182 kDa tankyrase-1-binding protein; & P58871 & Tnks1bp1 & $-0,2$ & 4,7 \\
\hline 2,4-dienoyl-CoA reductase, mitochondrial; & Q9CQ62 & Decr1 & $-0,1$ & 9 \\
\hline $26 \mathrm{~S}$ protease regulatory subunit $10 \mathrm{~B}$; & P62334 & Psmc6 & $-0,4$ & 11,1 \\
\hline 265 protease regulatory subunit 4 & P62192 & Psmc1 & $-0,6$ & 6,6 \\
\hline 265 protease regulatory subunit $6 \mathrm{~A}$; & 088685 & Psmc3 & $-0,7$ & 23,2 \\
\hline $26 \mathrm{~S}$ protease regulatory subunit $6 \mathrm{~B}$; & P54775 & Psmc4 & $-0,7$ & 3,1 \\
\hline 265 protease regulatory subunit 7 & P46471 & Psmc2 & $-0,3$ & 14,1 \\
\hline 265 protease regulatory subunit 8 & P62196 & Psmc5 & $-0,5$ & 6,7 \\
\hline $26 \mathrm{~S}$ proteasome non-ATPase regulatory subunit $1 ;$ & Q3TXS7 & Psmd1 & $-0,2$ & 8,1 \\
\hline $26 \mathrm{~S}$ proteasome non-ATPase regulatory subunit $11 ;$ & Q8BG32 & Psmd11 & $-0,4$ & 26,1 \\
\hline $26 \mathrm{~S}$ proteasome non-ATPase regulatory subunit 12 & Q9D8W5 & Psmd12 & $-0,4$ & 4,6 \\
\hline $26 \mathrm{~S}$ proteasome non-ATPase regulatory subunit 13 ; & Q9WVJ2 & Psmd13 & $-0,4$ & 11,5 \\
\hline $26 \mathrm{~S}$ proteasome non-ATPase regulatory subunit $14 ;$ & 035593 & Psmd14 & $-1,7$ & 6,5 \\
\hline $26 \mathrm{~S}$ proteasome non-ATPase regulatory subunit 2 & Q8VDM4 & Psmd2 & $-0,2$ & 12,2 \\
\hline $26 \mathrm{~S}$ proteasome non-ATPase regulatory subunit 3 ; & P14685 & Psmd3 & $-0,1$ & 17,9 \\
\hline $26 \mathrm{~S}$ proteasome non-ATPase regulatory subunit 4 & 035226 & Psmd4 & 0,0 & 4 \\
\hline 265 proteasome non-ATPase regulatory subunit 6 ; & Q99J14 & Psmd6 & $-0,3$ & 12,1 \\
\hline $26 \mathrm{~S}$ proteasome non-ATPase regulatory subunit 7; & P26516 & Psmd7 & $-0,3$ & 3,1 \\
\hline $26 \mathrm{~S}$ proteasome non-ATPase regulatory subunit 8 & Q9CX56 & Psmd8 & $-0,5$ & 10,7 \\
\hline $28 \mathrm{~S}$ ribosomal protein S15, mitochondrial; & Q9DC71 & Mrps15 & 0,0 & 2,7 \\
\hline $28 \mathrm{~S}$ ribosomal protein S36, mitochondrial; & Q9CQX8 & Mrps36 & 0,3 & 13 \\
\hline 2-amino-3-ketobutyrate coenzyme A ligase, mitochondrial; & 088986 & Gcat & 0,1 & 8,2 \\
\hline 2'-deoxynucleoside 5'-phosphate N-hydrolase $1 ;$ & Q80VJ3 & Dnph1 & 4,0 & 8,1 \\
\hline $3^{\prime}\left(2^{\prime}\right), 5^{\prime}$-bisphosphate nucleotidase 1 & Q9Z0S1 & Bpnt1 & 2,8 & 10,7 \\
\hline $39 S$ ribosomal protein L12, mitochondrial; & Q9DB15 & Mrpl12 & 0,2 & 7 \\
\hline 3-hydroxyacyl-CoA dehydrogenase type-2; & 008756 & Hsd17b10 & $-0,4$ & 3,4 \\
\hline 3-hydroxyisobutyrate dehydrogenase, mitochondrial; & Q99L13 & Hibadh & $-0,5$ & 8,7 \\
\hline 3-ketoacyl-CoA thiolase A, peroxisomal; & Q921H8 & Acaa1a & $-0,1$ & 8,8 \\
\hline 3-ketoacyl-CoA thiolase, mitochondrial; & Q8BWT1 & Acaa2 & 0,0 & 32 \\
\hline 40 S ribosomal protein S10; & P63325 & Rps10 & $-0,3$ & 15,6 \\
\hline 40 S ribosomal protein $\mathrm{S} 11$ & P62281 & Rps11 & 0,1 & 38,6 \\
\hline $40 S$ ribosomal protein $\mathrm{S} 12$ & Q6ZWZ6 & Rps12 & 0,0 & 45,5 \\
\hline 40 S ribosomal protein $\mathrm{S} 13$ & P62301 & Rps13 & $-0,1$ & 30,5 \\
\hline 40 S ribosomal protein S14; & P62264 & Rps14 & 0,0 & 22,5 \\
\hline 40 S ribosomal protein $\mathrm{S} 15$ & P62843 & Rps15 & $-0,1$ & 10,2 \\
\hline $40 \mathrm{~S}$ ribosomal protein $\mathrm{S} 15 \mathrm{a}$ & P62245 & Rps15a & 0,0 & 15,7 \\
\hline 40 S ribosomal protein S16; & P14131 & Rps16 & $-0,3$ & 22,6 \\
\hline 40 S ribosomal protein S17; & P63276 & Rps17 & 0,0 & 15,6 \\
\hline 40 S ribosomal protein S18; & P62270 & Rps18 & 0,1 & 27,1 \\
\hline 40 S ribosomal protein S19; & Q9CZX8 & Rps19 & 0,0 & 9,7 \\
\hline 40 S ribosomal protein S20; & P60867 & Rps20 & 0,2 & 25,2 \\
\hline 40 S ribosomal protein S21; & Q9CQR2 & Rps21 & 0,0 & 27,7 \\
\hline 40 S ribosomal protein S23; & P62267 & Rps23 & 2,7 & 15,4 \\
\hline 40 S ribosomal protein $\mathrm{S} 24$ & P62849 & Rps24 & 1,8 & 8,3 \\
\hline 40 S ribosomal protein S26; & P62855 & Rps26 & 0,2 & 20,9 \\
\hline 40 S ribosomal protein $\mathrm{S} 27$ & Q6ZWU9 & Rps27 & 0,0 & 22,6 \\
\hline 40S ribosomal protein S27-like; & Q6ZWY3 & Rps27l & 0,0 & 22,6 \\
\hline 40 S ribosomal protein S28; & P62858 & $\operatorname{Rps} 28$ & 0,3 & 21,4 \\
\hline 40 S ribosomal protein S29; & P62274 & Rps29 & $-0,3$ & 12,5 \\
\hline $40 \mathrm{~S}$ ribosomal protein S3; & P62908 & Rps3 & 0,0 & 30 \\
\hline $40 S$ ribosomal protein $\mathrm{S} 3 \mathrm{a}$ & P97351 & Rps3a & $-0,1$ & 27,3 \\
\hline $40 \mathrm{~S}$ ribosomal protein $\mathrm{S} 4, \mathrm{X}$ isoform; & P62702 & $\operatorname{Rps} 4 x$ & $-0,1$ & 26,2 \\
\hline 40 S ribosomal protein S5; & D3YYM6 & Rps5 & 0,1 & 18,1 \\
\hline $40 \mathrm{~S}$ ribosomal protein $\mathrm{S} 6$ & P62754 & Rps6 & 0,0 & 10,8 \\
\hline $40 \mathrm{~S}$ ribosomal protein $\mathrm{S7}$ & P62082 & Rps7 & $-0,1$ & 8,9 \\
\hline $40 \mathrm{~S}$ ribosomal protein S8; & P62242 & Rps8 & $-0,3$ & 22,1 \\
\hline $40 \mathrm{~S}$ ribosomal protein $\mathrm{S9}$ & Q6ZWN5 & Rps9 & $-0,1$ & 19,6 \\
\hline $40 \mathrm{~S}$ ribosomal protein $\mathrm{SA}$ & P14206 & Rpsa & 0,0 & 5,8 \\
\hline 4F2 cell-surface antigen heavy chain; & P10852 & Slc3a2 & $-0,5$ & 12,5 \\
\hline 4-trimethylaminobutyraldehyde dehydrogenase; & Q9JL2 & Aldh9a1 & 0,1 & 8,1 \\
\hline 5'(3')-deoxyribonucleotidase, cytosolic type; & Q9JM14 & $\mathrm{Nt} 5 \mathrm{c}$ & 0,3 & 9,3 \\
\hline
\end{tabular}




\begin{tabular}{|c|c|c|c|c|}
\hline Protein & Uniprot & Gene name & $\begin{array}{c}\text { Fold change of } \\
\text { XPRESS ratio } \\
\left.\text { (Adam } 17^{\Delta K C} / \mathrm{WT}\right)\end{array}$ & $\begin{array}{c}\text { Sequence } \\
\text { coverage [\%] }\end{array}$ \\
\hline 5'-3' exoribonuclease 2 & Q9DBR1 & Xrn2 & 0,3 & 2,1 \\
\hline 5-hydroxyisourate hydrolase; & Q9CRB3 & Urah & $-0,2$ & 27,1 \\
\hline 60 kDa heat shock protein, mitochondrial; & P63038 & Hspd1 & $-0,8$ & 19,4 \\
\hline 605 acidic ribosomal protein $\mathrm{P0}$ & P14869 & Rplp0 & 0,0 & 28,4 \\
\hline $60 \mathrm{~S}$ acidic ribosomal protein $\mathrm{P} 1 ;$ & P47955 & Rplp1 & $-0,4$ & 14 \\
\hline $60 S$ acidic ribosomal protein $\mathrm{P} 2$; & P99027 & Rplp2 & 0,1 & 67 \\
\hline 60S ribosomal protein L10-like; & P86048 & Rpl10l & 0,1 & 14,4 \\
\hline $60 S$ ribosomal protein L11; & Q9CXW4 & Rpl11 & 0,1 & 8,1 \\
\hline $60 S$ ribosomal protein L12; & P35979 & Rpl12 & 0,0 & 49,1 \\
\hline $60 S$ ribosomal protein L13; & P47963 & Rpl13 & 0,1 & 19,9 \\
\hline 60S ribosomal protein L13a; & P19253 & Rpl13a & 0,0 & 9,4 \\
\hline $60 S$ ribosomal protein L14; & Q9CR57 & Rpl14 & $-0,1$ & 15,7 \\
\hline 60S ribosomal protein L15; & Q9CZM2 & Rpl15 & $-0,2$ & 17,6 \\
\hline $60 S$ ribosomal protein L17; & Q9CPR4 & Rpl17 & 0,1 & 21,7 \\
\hline $60 \mathrm{~S}$ ribosomal protein L18; & P35980 & Rpl18 & 0,0 & 27,7 \\
\hline $60 \mathrm{~S}$ ribosomal protein L18a; & P62717 & Rpl18a & $-0,2$ & 25,6 \\
\hline 60S ribosomal protein L19; & P84099 & Rpl19 & 0,1 & 8,2 \\
\hline $60 S$ ribosomal protein L21; & 009167 & Rpl21 & 0,2 & 20,6 \\
\hline $60 S$ ribosomal protein $L 22$ & P67984 & Rpl22 & 0,2 & 8,6 \\
\hline 60 S ribosomal protein L22-like 1 ; & Q9D7S7 & Rpl22l1 & $-0,2$ & 9,8 \\
\hline $60 S$ ribosomal protein L23; & P62830 & Rpl23 & 0,1 & 49,3 \\
\hline $60 \mathrm{~S}$ ribosomal protein L23a; & P62751 & Rpl23a & 0,2 & 20,5 \\
\hline $60 S$ ribosomal protein L24; & Q8BP67 & Rpl24 & 0,0 & 28,6 \\
\hline $60 S$ ribosomal protein L26; & P61255 & Rpl26 & 0,3 & 36,9 \\
\hline $60 S$ ribosomal protein L27; & P61358 & Rpl27 & $-0,1$ & 29,4 \\
\hline $60 \mathrm{~S}$ ribosomal protein $\mathrm{L} 27 \mathrm{a}$; & P14115 & Rpl27a & 0,0 & 19,6 \\
\hline $60 S$ ribosomal protein L28; & P41105 & Rpl28 & 0,0 & 19 \\
\hline $60 S$ ribosomal protein $\mathrm{L} 29$; & P47915 & Rpl29 & 0,1 & 12,3 \\
\hline $60 S$ ribosomal protein L3; & P27659 & Rpl3 & $-0,1$ & 19,6 \\
\hline $60 \mathrm{~S}$ ribosomal protein L30; & P62889 & Rpl30 & 0,0 & 31,3 \\
\hline $60 S$ ribosomal protein $\mathrm{L} 31$ & P62900 & Rpl31 & 0,0 & 32,8 \\
\hline $60 \mathrm{~S}$ ribosomal protein L32; & P62911 & Rpl32 & 0,1 & 33,3 \\
\hline $60 S$ ribosomal protein L34; & Q9D1R9 & Rpl34 & 0,3 & 13,7 \\
\hline 60S ribosomal protein L35; & Q6ZWV7 & Rpl35 & $-0,1$ & 13,8 \\
\hline $60 \mathrm{~S}$ ribosomal protein $\mathrm{L} 35 \mathrm{a}$; & 055142 & Rpl35a & $-0,2$ & 27,3 \\
\hline $60 S$ ribosomal protein L36; & P47964 & Rpl36 & $-0,1$ & 9,6 \\
\hline $60 \mathrm{~S}$ ribosomal protein L36a; & P83882 & Rpl36a & $-1,1$ & 28,3 \\
\hline 605 ribosomal protein L37a; & P61514 & Rpl37a & 0,2 & 28,3 \\
\hline $60 \mathrm{~S}$ ribosomal protein L38; & Q9JगI8 & Rpl38 & 0,3 & 10 \\
\hline 60S ribosomal protein L39; & P62892 & Rpl39 & 0,0 & 19,6 \\
\hline $60 S$ ribosomal protein $L 4$ & Q9D8E6 & Rpl4 & 0,1 & 26,3 \\
\hline 60S ribosomal protein L5; & P47962 & Rpl5 & 0,0 & 22,6 \\
\hline 60S ribosomal protein L6; & P47911 & Rpl6 & $-0,2$ & 29,1 \\
\hline 60S ribosomal protein L7; & P14148 & Rpl7 & 0,0 & 17,4 \\
\hline 60S ribosomal protein L8; & P62918 & Rpl8 & 0,0 & 14,8 \\
\hline 60S ribosomal protein L9; & P51410 & Rpl9 & 0,0 & 26,5 \\
\hline 6-phosphogluconate dehydrogenase, decarboxylating; & Q9DCD0 & Pgd & $-0,5$ & 6,4 \\
\hline 6-phosphogluconolactonase; & Q9CQ60 & Pgls & 0,0 & 18,6 \\
\hline 78 kDa glucose-regulated protein; & P20029 & Hspa5 & $-0,2$ & 28,1 \\
\hline Acetyl-CoA acetyltransferase, cytosolic; & Q8CAY6 & Acat2 & 2,5 & 7,7 \\
\hline Acetyl-CoA acetyltransferase, mitochondrial; & Q8QZT1 & Acat1 & 0,1 & 14,6 \\
\hline Acid ceramidase; & Q9WV54 & Asah1 & $-0,5$ & 4,8 \\
\hline Acidic leucine-rich nuclear phosphoprotein 32 family member $A$; & 035381 & Anp32a & 0,1 & 19,6 \\
\hline Acidic leucine-rich nuclear phosphoprotein 32 family member E; & P97822 & Anp32e & 0,3 & 11,2 \\
\hline Actin, aortic smooth muscle; & P62737 & Acta2 & $-0,1$ & 37,7 \\
\hline Actin-related protein 10; & Q9QZB7 & Actr10 & $-0,1$ & 1,7 \\
\hline Actin-related protein $2 / 3$ complex subunit $1 A$; & Q9R0Q6 & Arpc1a & $-0,7$ & 15,1 \\
\hline Actin-related protein $2 / 3$ complex subunit $1 \mathrm{~B}$; & Q9WV32 & Arpc1b & 0,2 & 17,2 \\
\hline Actin-related protein $2 / 3$ complex subunit 2 & Q9CVB6 & Arpc2 & $-0,3$ & 15 \\
\hline Actin-related protein $2 / 3$ complex subunit 3 ; & Q9JM76 & Arpc3 & $-0,1$ & 19,3 \\
\hline Actin-related protein $2 / 3$ complex subunit 4 & P59999 & Arpc4 & 0,0 & 11,3 \\
\hline Actin-related protein $2 / 3$ complex subunit 5 & Q9CPW4 & Arpc5 & 0,0 & 12,6 \\
\hline Actin-related protein 2 & P61161 & Actr2 & $-0,1$ & 9,6 \\
\hline Actin-related protein 3; & Q99JY9 & Actr3 & $-0,4$ & 23,2 \\
\hline Activated RNA polymerase II transcriptional coactivator $\mathrm{p} 15$; & P11031 & Sub1 & 0,4 & 7,1 \\
\hline Acylamino-acid-releasing enzyme; & Q8R146 & Apeh & 0,2 & 5,6 \\
\hline Acyl-CoA dehydrogenase family member 9 , mitochondrial; & Q8JZN5 & Acad9 & $-0,3$ & 8,2 \\
\hline Acyl-CoA-binding protein; & P31786 & Dbi & 0,1 & 39,1 \\
\hline Acyl-coenzyme A thioesterase 10 , mitochondrial; & Q32MW3 & Acot10 & $-0,5$ & 6,6 \\
\hline Adenosine deaminase; & P03958 & Ada & 0,1 & 3,7 \\
\hline Adenosine kinase; & P55264 & Adk & 0,0 & 14,1 \\
\hline Adenosylhomocysteinase; & P50247 & Ahcy & 0,0 & 23,4 \\
\hline Adenylate kinase 2, mitochondrial; & Q9WTP6 & Ak2 & 0,2 & 12,1 \\
\hline
\end{tabular}




\begin{tabular}{|c|c|c|c|c|}
\hline Protein & Uniprot & Gene name & $\begin{array}{c}\text { Fold change of } \\
\text { XPRESS ratio } \\
\left.\text { (Adam } 17^{\Delta K C} / \mathrm{WT}\right)\end{array}$ & $\begin{array}{l}\text { Sequence } \\
\text { coverage [\%] }\end{array}$ \\
\hline Adenylosuccinate synthetase isozyme 2 ; & P46664 & Adss & 1,4 & 1,8 \\
\hline Adenylyl cyclase-associated protein $1 ;$ & P40124 & Cap1 & $-0,9$ & 8 \\
\hline Adipocyte plasma membrane-associated protein; & Q9D7N9 & Apmap & 0,3 & 5,1 \\
\hline Adiponectin; & Q60994 & Adipoq & 0,2 & 10,1 \\
\hline ADP/ATP translocase $1 ;$ & P48962 & Slc25a4 & 0,3 & 21,8 \\
\hline ADP/ATP translocase 2 & P51881 & Slc25a5 & 0,1 & 21,5 \\
\hline ADP-dependent glucokinase; & Q8VDL4 & Adpgk & 0,3 & 3 \\
\hline ADP-ribosylation factor 3 ; & P61205 & Arf3 & 0,0 & 28,2 \\
\hline ADP-ribosylation factor 5 & P84084 & Arf5 & $-0,3$ & 33,9 \\
\hline ADP-ribosylation factor 6 ; & P62331 & Arf6 & $-0,1$ & 14,9 \\
\hline ADP-ribosylation factor-like protein $8 \mathrm{~B}$; & Q9CQW2 & Arl8b & 0,0 & 9,7 \\
\hline ADP-sugar pyrophosphatase; & Q9JKX6 & Nudt5 & 0,3 & 12,8 \\
\hline Agrin; & A2ASQ1 & Agrn & $-0,2$ & 1,1 \\
\hline A-kinase anchor protein 2 & A2API8 & Akap2 & 10,0 & 2,5 \\
\hline Alcohol dehydrogenase [NADP(+)]; & Q9ग॥6 & Akr1a1 & 0,1 & 8,3 \\
\hline Alcohol dehydrogenase class $4 \mathrm{mu} / \mathrm{sigma}$ chain; & Q64437 & Adh7 & 0,9 & 5,6 \\
\hline Alcohol dehydrogenase class-3; & P28474 & Adh5 & $-0,1$ & 8,6 \\
\hline Aldehyde dehydrogenase family 1 member A3; & Q9JHW9 & Aldh1a3 & 0,0 & 5,1 \\
\hline Aldehyde dehydrogenase family 16 member A1; & Q57119 & Aldh16a1 & $-0,1$ & 3,7 \\
\hline Aldehyde dehydrogenase, dimeric NADP-preferring; & P47739 & Aldh3a1 & $-0,3$ & 5,5 \\
\hline Aldose reductase; & P45376 & Akr1b1 & $-0,1$ & 6,8 \\
\hline Alpha-1-antitrypsin 1-1; & P07758 & Serpina1a & 0,4 & 7 \\
\hline Alpha-1-antitrypsin 1-2; & P22599 & Serpina1b & 0,5 & 11,1 \\
\hline Alpha-1-antitrypsin 1-4; & Q00897 & Serpina1d & 1,0 & 11,6 \\
\hline Alpha-2-HS-glycoprotein; & P29699 & Ahsg & 0,6 & 22,9 \\
\hline Alpha-2-macroglobulin; & Q61838 & $\mathrm{A} 2 \mathrm{~m}$ & 0,6 & 5,2 \\
\hline Alpha-actinin-1; & Q7TPR4 & Actn1 & $-0,3$ & 14,5 \\
\hline Alpha-actinin-4; & P57780 & Actn4 & 0,3 & 27,9 \\
\hline Alpha-adducin; & Q9QYC0 & Add1 & 0,1 & 3 \\
\hline Alpha-aminoadipic semialdehyde dehydrogenase; & Q9DBF1 & Aldh7a1 & 0,1 & 6,6 \\
\hline Alpha-centractin; & P61164 & Actr1a & 0,1 & 4,8 \\
\hline Alpha-enolase; & P17182 & Eno1 & $-0,1$ & 28,3 \\
\hline Alpha-fetoprotein; & P02772 & Afp & 0,7 & 17,5 \\
\hline Amine oxidase [flavin-containing] A; & Q64133 & Maoa & $-0,5$ & 8,2 \\
\hline Aminoacyl tRNA synthase complex-interacting multifunctional protein 1; & P31230 & Aimp1 & $-0,4$ & 5,5 \\
\hline Aminoacylase-1; & Q99JW2 & Acy1 & 0,3 & 2,7 \\
\hline Aminopeptidase N; & P97449 & Anpep & $-0,4$ & 10 \\
\hline Ankyrin repeat and SOCS box protein 15 & Q8VHS6 & Asb15 & $-0,1$ & 1,4 \\
\hline Annexin A1; & P10107 & Anxa1 & $-0,1$ & 14,7 \\
\hline Annexin A2; & P07356 & Anxa2 & 0,4 & 26,3 \\
\hline Annexin A4; & P97429 & Anxa4 & 0,0 & 9,2 \\
\hline Annexin A5; & P48036 & Anxa5 & $-0,1$ & 18,5 \\
\hline Annexin A6; & P14824 & Anxa6 & 0,6 & 18,3 \\
\hline Annexin A7; & Q07076 & Anxa7 & 0,3 & 1,7 \\
\hline Annexin A8; & 035640 & Anxa8 & $-0,4$ & 7 \\
\hline AP-2 complex subunit alpha- 1 ; & P17426 & Ap2a1 & $-0,1$ & 2,2 \\
\hline AP-2 complex subunit mu; & P84091 & Ap2m1 & 1,4 & 3,9 \\
\hline Apolipoprotein A-I; & Q00623 & Apoa1 & 0,8 & 34,5 \\
\hline Apolipoprotein A-II; & P09813 & Apoa2 & 0,8 & 9,8 \\
\hline Apolipoprotein A-IV; & P06728 & Apoa4 & 0,4 & 21,5 \\
\hline Apolipoprotein E; & P08226 & Apoe & $-1,6$ & 24,4 \\
\hline Apoptotic chromatin condensation inducer in the nucleus; & Q9JIX8 & Acin 1 & $-1,4$ & 1,8 \\
\hline Aquaporin-1; & Q02013 & Aqp1 & 0,1 & 3,3 \\
\hline Arginine and glutamate-rich protein $1 ;$ & Q3UL36 & Arglu1 & 1,2 & 5,5 \\
\hline $\begin{array}{l}\text { Arginine-rich, mutated in early stage tumors, isoform CRA_b; Mesencephalic } \\
\text { astrocyte-derived neurotrophic factor; }\end{array}$ & Q3TMX5 & Manf & $-0,2$ & 10,9 \\
\hline Arginine--tRNA ligase, cytoplasmic; & Q9D019 & Rars & $-0,1$ & 1,2 \\
\hline Argininosuccinate lyase; & Q91YI0 & Asl & $-0,7$ & 4,3 \\
\hline Argininosuccinate synthase; & P16460 & Ass1 & $-0,5$ & 5,3 \\
\hline Asparagine--tRNA ligase, cytoplasmic; & Q8BP47 & Nars & $-0,6$ & 3,6 \\
\hline Aspartate aminotransferase, cytoplasmic; & P05201 & Got1 & 0,0 & 7,7 \\
\hline Aspartate aminotransferase, mitochondrial; & P05202 & Got2 & 0,1 & 16,7 \\
\hline Aspartate--tRNA ligase, cytoplasmic; & Q922B2 & Dars & 0,3 & 12,4 \\
\hline Aspartyl aminopeptidase; & Q9Z2W0 & Dnpep & 0,1 & 7 \\
\hline Aspartyl/asparaginyl beta-hydroxylase; & Q8BSY0 & Asph & 0,1 & 24,7 \\
\hline ATP synthase $F(0)$ complex subunit B1, mitochondrial; & Q9CQQ7 & Atp5f1 & 0,9 & 7,8 \\
\hline ATP synthase protein 8 & P03930 & Mtatp8 & 0,2 & 14,9 \\
\hline ATP synthase subunit alpha, mitochondrial; & Q03265 & Atp5a1 & 0,0 & 28,4 \\
\hline ATP synthase subunit beta, mitochondrial; & P56480 & Atp5b & $-0,2$ & 29,3 \\
\hline ATP synthase subunit $d$, mitochondrial; & Q9DCX2 & Atp5h & $-0,1$ & 25,4 \\
\hline ATP synthase subunit delta, mitochondrial; & Q9D3D9 & Atp5d & 3,7 & 8,3 \\
\hline ATP synthase subunit e, mitochondrial; & Q06185 & Atp5i & 0,0 & 42,3 \\
\hline
\end{tabular}




\begin{tabular}{|c|c|c|c|c|}
\hline Protein & Uniprot & Gene name & $\begin{array}{c}\text { Fold change of } \\
\text { XPRESS ratio } \\
\left.\text { (Adam } 17^{\Delta K C} / \mathrm{WT}\right)\end{array}$ & $\begin{array}{l}\text { Sequence } \\
\text { coverage [\%] }\end{array}$ \\
\hline ATP synthase subunit epsilon, mitochondrial; & P56382 & Atp5e & $-0,3$ & 28,8 \\
\hline ATP synthase subunit g, mitochondrial; & Q9CPQ8 & Atp5I & 0,4 & 18,4 \\
\hline ATP synthase subunit gamma, mitochondrial; & Q91VR2 & Atp5c1 & $-0,1$ & 18,2 \\
\hline ATP synthase subunit $O$, mitochondrial; & Q9DB20 & Atp5o & $-0,3$ & 20,2 \\
\hline ATP synthase-coupling factor 6 , mitochondrial; & P97450 & Atp5j & $-0,2$ & 20,7 \\
\hline ATPase inhibitor, mitochondrial; & 035143 & Atpif1 & 0,1 & 9,5 \\
\hline ATP-binding cassette sub-family E member 1 ; & P61222 & Abce1 & 0,4 & 10,4 \\
\hline ATP-citrate synthase; & Q91V92 & Acly & 0,2 & 2,7 \\
\hline ATP-dependent (S)-NAD(P)H-hydrate dehydratase; & Q9CZ42 & Carkd & 0,3 & 9,2 \\
\hline ATP-dependent 6-phosphofructokinase, liver type; & P12382 & Pfkl & $-0,3$ & 2,1 \\
\hline ATP-dependent 6-phosphofructokinase, muscle type; & P47857 & Pfkm & 1,0 & 2,7 \\
\hline ATP-dependent 6-phosphofructokinase, platelet type; & Q9WUA3 & Pfkp & $-0,2$ & 3,8 \\
\hline ATP-dependent Clp protease proteolytic subunit, mitochondrial; & 088696 & Clpp & $-0,1$ & 6,6 \\
\hline ATP-dependent RNA helicase A; & 070133 & Dhx9 & 0,5 & 2,3 \\
\hline ATP-dependent RNA helicase DDX24; & Q9ESV0 & $\mathrm{Ddx} 24$ & 0,9 & 2 \\
\hline ATP-dependent RNA helicase DDX39A; & Q8VDW0 & Ddx39a & 0,6 & 9,8 \\
\hline Atrial natriuretic peptide-converting enzyme; & Q97319 & Corin & 0,0 & 1,1 \\
\hline BAG family molecular chaperone regulator 3 ; & Q9JLV1 & Bag3 & $-0,3$ & 2,4 \\
\hline Barrier-to-autointegration factor; & 054962 & Banf1 & $-0,7$ & 7,9 \\
\hline Basal cell adhesion molecule; & Q9R069 & Bcam & $-0,2$ & 9,8 \\
\hline Basigin; & P18572 & Bsg & 0,0 & 10,7 \\
\hline Bcl-2-associated transcription factor 1 ; & Q8K019 & Bclaf1 & 0,1 & 2,5 \\
\hline Beta-actin-like protein 2 & Q8BFZ3 & Actbl2 & 0,4 & 14,4 \\
\hline Beta-adducin; & Q9QYB8 & Add2 & 0,4 & 2,5 \\
\hline Beta-enolase; & P21550 & Eno3 & 2,7 & 9,7 \\
\hline Beta-globin; Protein Hbb-bs; Protein Hbb-bt; & A8DUK4 & Hbbt1 & 0,4 & 73,5 \\
\hline Beta-glucuronidase; & P12265 & Gusb & 0,1 & 4,3 \\
\hline Beta-hexosaminidase subunit beta; & P20060 & Hexb & $-0,3$ & 10,3 \\
\hline Bifunctional purine biosynthesis protein $\mathrm{PURH}$; & Q9CWJ9 & Atic & $-0,3$ & 1,4 \\
\hline Biglycan; & P28653 & Bgn & $-0,3$ & 15,7 \\
\hline Bisphosphoglycerate mutase; & P15327 & Bpgm & $-0,2$ & 8,9 \\
\hline Bleomycin hydrolase; & Q8R016 & Blmh & 1,2 & 5,5 \\
\hline Brain acid soluble protein 1 ; & Q91XV3 & Basp1 & 0,3 & 22,6 \\
\hline BTB/POZ domain-containing protein KCTD12; & Q6WVG3 & Kctd12 & 0,0 & 7,3 \\
\hline BUB3-interacting and GLEBS motif-containing protein ZNF207; & E9PW12 & Znf207 & 0,3 & 5,6 \\
\hline Cadherin-1; & P09803 & Cdh1 & 0,0 & 7,5 \\
\hline Cadherin-13; & Q9WTR5 & Cdh13 & 0,5 & 5,7 \\
\hline Calcineurin B homologous protein 1; & P61022 & Chp1 & 0,4 & 5,8 \\
\hline Calcineurin subunit B type 1 ; & Q63810 & Ppp3r1 & 0,9 & 5,9 \\
\hline $\begin{array}{l}\text { Calcium-activated chloride channel regulator 2; Chloride channel calcium } \\
\text { activated 2; Endothelial chloride channel; MCG120735; }\end{array}$ & Q9EQR4 & Clca2 & $-0,3$ & 7,4 \\
\hline Calcium-binding mitochondrial carrier protein Aralar1; & Q8BH59 & Slc25a12 & 0,1 & 1,2 \\
\hline Calcium-binding protein 39 & Q06138 & Cab39 & 0,4 & 7,3 \\
\hline Calmodulin; & P62204 & Calm3 & 0,2 & 16,1 \\
\hline Calmodulin-4; & Q9JM83 & Calm4 & $-0,3$ & 19,6 \\
\hline Calnexin; & P35564 & Canx & 0,0 & 3,7 \\
\hline Calpain small subunit 1 ; & 088456 & Capns1 & $-0,1$ & 16,2 \\
\hline Calpain small subunit 2 ; & Q9D7J7 & Capns2 & $-0,4$ & 9,7 \\
\hline Calpain-1 catalytic subunit; & 035350 & Capn1 & $-0,2$ & 11,9 \\
\hline Calpain-2 catalytic subunit; & 008529 & Capn2 & $-0,3$ & 1 \\
\hline Calpastatin; & P51125 & Cast & $-0,4$ & 22,1 \\
\hline Calreticulin; & P14211 & Calr & $-0,3$ & 22,4 \\
\hline Calsequestrin-1; & 009165 & Casq1 & 2,5 & 8,3 \\
\hline Calumenin; & 035887 & Calu & 0,2 & 13,3 \\
\hline CAP-Gly domain-containing linker protein 1 ; & Q922J3 & Clip1 & 0,3 & 4,3 \\
\hline $\begin{array}{l}\text { Capping protein (Actin filament) muscle Z-line, beta, isoform CRA_a; F-actin- } \\
\text { capping protein subunit beta; }\end{array}$ & A2AMWO & Capzb & $-1,2$ & 6,9 \\
\hline Carbonic anhydrase 3; & P16015 & $\mathrm{Ca} 3$ & 0,8 & 33,1 \\
\hline Carbonic anhydrase 6; & P18761 & $\mathrm{Ca} 6$ & 0,2 & 13,8 \\
\hline Carboxylesterase 1C; & P23953 & Ces1c & 0,7 & 3,8 \\
\hline Carboxypeptidase M; & Q80V42 & Cpm & $-0,5$ & 14,4 \\
\hline Carcinoembryonic antigen-related cell adhesion molecule 16; & E9QA28 & Ceacam16 & 2,4 & 2,8 \\
\hline Casein kinase II subunit beta; & P67871 & Csnk2b & $-0,1$ & 12,2 \\
\hline Caspase-14; & 089094 & Casp14 & $-0,1$ & 10,9 \\
\hline Caspase-3; & P70677 & Casp3 & 0,5 & 7,2 \\
\hline Catalase; & P24270 & Cat & $-0,2$ & 21,3 \\
\hline Catenin alpha-1; & P26231 & Ctnna1 & $-0,2$ & 6,8 \\
\hline Catenin beta-1; & Q02248 & Ctnnb1 & $-0,4$ & 12,7 \\
\hline Cathepsin B; & P10605 & Ctsb & $-0,7$ & 15,6 \\
\hline Cathepsin D; & P18242 & Ctsd & $-0,3$ & 22,2 \\
\hline Cathepsin E; & P70269 & Ctse & $-1,6$ & 4,9 \\
\hline Cathepsin L1; & P06797 & Ctsl & $-0,8$ & 9 \\
\hline Cathepsin Z; & Q9WUU7 & Ctsz & $-0,7$ & 11,1 \\
\hline
\end{tabular}




\begin{tabular}{|c|c|c|c|c|}
\hline Protein & Uniprot & Gene name & $\begin{array}{c}\text { Fold change of } \\
\text { XPRESS ratio } \\
\left.\text { (Adam } 17^{\triangle K C} / \mathrm{WT}\right)\end{array}$ & $\begin{array}{c}\text { Sequence } \\
\text { coverage [\%] }\end{array}$ \\
\hline Cation-dependent mannose-6-phosphate receptor; & P24668 & M6pr & 0,3 & 7,9 \\
\hline Caveolin-1; & P49817 & Cav1 & 0,2 & 31,2 \\
\hline CD109 antigen; & Q8R422 & Cd109 & 0,0 & 3,1 \\
\hline CD166 antigen; & Q61490 & Alcam & $-0,6$ & 13,9 \\
\hline CD9 antigen; & P40240 & Cd9 & 0,3 & 3,1 \\
\hline CDGSH iron-sulfur domain-containing protein 1 ; & Q91WS0 & Cisd1 & 0,4 & 25,9 \\
\hline Cell division control protein 42 homolog; & P60766 & Cdc42 & 0,0 & 18,8 \\
\hline Cell surface glycoprotein MUC18; & Q8R2Y2 & Mcam & $-0,2$ & 8 \\
\hline Cellular nucleic acid-binding protein; & P53996 & Cnbp & 0,5 & 14 \\
\hline Ceruloplasmin; & Q61147 & $\mathrm{Cp}$ & 0,6 & 4 \\
\hline Charged multivesicular body protein $4 \mathrm{~b}$; & Q9D8B3 & Chmp4b & 0,2 & 6,3 \\
\hline Chloride intracellular channel protein 1 ; & Q9Z1Q5 & Clic1 & $-0,1$ & 7,5 \\
\hline Chloride intracellular channel protein 4; & Q9QYB1 & Clic4 & $-0,5$ & 7,9 \\
\hline Choline dehydrogenase, mitochondrial; & Q8BJ64 & Chdh & $-4,4$ & 1,5 \\
\hline Choline transporter-like protein 2 & Q8BY89 & Slc44a2 & $-0,3$ & 1 \\
\hline Chromobox protein homolog 1; & P83917 & $\mathrm{Cbx} 1$ & $-0,1$ & 5,9 \\
\hline Chromobox protein homolog 3 ; & P23198 & $\mathrm{Cb} \times 3$ & 0,2 & 6,6 \\
\hline Chromodomain-helicase-DNA-binding protein 4; & Q6PDQ2 & Chd4 & 0,2 & 0,8 \\
\hline Citrate lyase subunit beta-like protein, mitochondrial; & Q8R4N0 & Clybl & 0,1 & 5,9 \\
\hline Citrate synthase, mitochondrial; & Q9CZU6 & Cs & 0,4 & 2,4 \\
\hline Clathrin heavy chain $1 ;$ & Q68FD5 & Cltc & 0,2 & 1,1 \\
\hline Clathrin light chain A; & 008585 & Clta & 0,1 & 6,9 \\
\hline Clathrin light chain B; & Q6IRU5 & Cltb & 0,3 & 12,2 \\
\hline Cleavage and polyadenylation specificity factor subunit 5 ; & Q9CQF3 & Nudt21 & $-0,3$ & 3,1 \\
\hline Cleavage and polyadenylation specificity factor subunit 6 ; & Q6NVF9 & Cpsf6 & 0,1 & 4,9 \\
\hline Clusterin; & Q06890 & $\mathrm{Clu}$ & 0,0 & 3,6 \\
\hline C-Myc-binding protein; & Q9EQS3 & Mycbp & 0,0 & 10,7 \\
\hline Coagulation factor XIII A chain; & Q8BH61 & F13a1 & 0,6 & 4 \\
\hline Coatomer subunit alpha; & Q8CIE6 & Copa & 0,1 & 1,9 \\
\hline Coatomer subunit beta; & Q9JIF7 & Copb1 & 0,3 & 4,2 \\
\hline Coatomer subunit beta'; & 055029 & Copb2 & 0,9 & 3,5 \\
\hline Coatomer subunit delta; & Q5XJY5 & $\operatorname{Arcn} 1$ & $-0,7$ & 7,4 \\
\hline Coatomer subunit zeta-1; & P61924 & Copz1 & 0,5 & 10,7 \\
\hline Cochlin; & Q62507 & Coch & $-0,7$ & 2,9 \\
\hline Cofilin-1; & P18760 & $\mathrm{Cfl} 1$ & $-0,4$ & 36,7 \\
\hline Cofilin-2; & P45591 & $\mathrm{Cfl} 2$ & $-0,1$ & 9 \\
\hline Coiled-coil domain-containing protein 50 ; & Q810U5 & Ccdc50 & 0,0 & 2,7 \\
\hline Coiled-coil-helix-coiled-coil-helix domain-containing protein 2, mitochondrial; & Q9D1L0 & Chchd2 & 0,3 & 6,5 \\
\hline Coiled-coil-helix-coiled-coil-helix domain-containing protein 3, mitochondrial; & Q9CRB9 & Chchd3 & 0,1 & 15,9 \\
\hline Collagen alpha-1(I) chain; & P11087 & Col1a1 & $-1,0$ & 6,9 \\
\hline Collagen alpha-1(III) chain; & P08121 & Col3a1 & 1,2 & 3,1 \\
\hline Collagen alpha-1(IV) chain; & P02463 & Col4a1 & $-0,1$ & 2,2 \\
\hline Collagen alpha-1(VI) chain; & Q04857 & Col6a1 & $-0,2$ & 8,3 \\
\hline Collagen alpha-1(XII) chain; & Q60847 & Col12a1 & $-0,5$ & 1,3 \\
\hline Collagen alpha-1(XIV) chain; & Q80X19 & Col14a1 & $-0,2$ & 1,4 \\
\hline Collagen alpha- $1(X V)$ chain; & 035206 & Col15a1 & 0,4 & 6,8 \\
\hline Collagen alpha-1(XVIII) chain; & P39061 & Col18a1 & $-0,7$ & 3,6 \\
\hline Collagen alpha-2(I) chain; & Q01149 & Col1a2 & $-0,3$ & 6,7 \\
\hline Collagen alpha-2(VI) chain; & Q02788 & Col6a2 & 0,0 & 4,6 \\
\hline Complement $\mathrm{C} 1 \mathrm{q}$ tumor necrosis factor-related protein 3 ; & Q9ES30 & C1qtnf3 & $-0,8$ & 2,8 \\
\hline Complement C3; & P01027 & $\mathrm{C} 3$ & 0,6 & 7,6 \\
\hline Conserved oligomeric Golgi complex subunit 1; & Q97160 & $\operatorname{Cog} 1$ & 4,0 & 1,7 \\
\hline COP9 signalosome complex subunit 2 & P61202 & Cops2 & $-0,2$ & 3,5 \\
\hline COP9 signalosome complex subunit 3 ; & 088543 & Cops3 & $-0,3$ & 3,3 \\
\hline COP9 signalosome complex subunit 4 ; & 088544 & Cops4 & $-0,7$ & 12,1 \\
\hline Copper transport protein ATOX1; & 008997 & Atox1 & 0,2 & 57,4 \\
\hline Core histone macro-H2A.1; & Q9QZQ8 & H2afy & 0,1 & 22,6 \\
\hline Corneodesmosin; & Q7TPC1 & Cdsn & $-0,3$ & 2,5 \\
\hline Cornifin-A; & Q62266 & Sprr1a & $-0,5$ & 45,8 \\
\hline Corticosteroid-binding globulin; & Q06770 & Serpina6 & 0,7 & 2,8 \\
\hline Costars family protein ABRACL; & Q4KML4 & Abracl & $-0,1$ & 11,1 \\
\hline Coxsackievirus and adenovirus receptor homolog; & P97792 & Cxadr & 3,5 & 5,5 \\
\hline Creatine kinase U-type, mitochondrial; & P30275 & Ckmt1 & $-0,2$ & 12,9 \\
\hline C-terminal-binding protein 1 & 088712 & Ctbp1 & 0,2 & 2,3 \\
\hline C-terminal-binding protein 2 ; & P56546 & Ctbp2 & 0,0 & 2,4 \\
\hline CTP synthase 1 ; & P70698 & Ctps1 & 0,1 & 20,1 \\
\hline Cullin-1; & Q9WTX6 & Cul1 & $-0,1$ & 4,9 \\
\hline Cullin-associated NEDD8-dissociated protein 1; & Q6ZQ38 & Cand1 & $-0,2$ & 9,6 \\
\hline Cyclin-dependent kinase 2-associated protein 1 ; & 035207 & Cdk2ap1 & 0,0 & 11,5 \\
\hline Cystathionine beta-synthase; & Q91WT9 & Cbs & $-0,3$ & 2 \\
\hline Cystatin E/M; Cystatin M/E; Cystatin-B; & Q9D1B1 & Cst6 & 1,0 & 25,5 \\
\hline
\end{tabular}




\begin{tabular}{|c|c|c|c|c|}
\hline Protein & Uniprot & Gene name & $\begin{array}{c}\text { Fold change of } \\
\text { XPRESS ratio } \\
\left.\text { (Adam } 17^{\triangle K C} / \mathrm{WT}\right)\end{array}$ & $\begin{array}{c}\text { Sequence } \\
\text { coverage [\%] }\end{array}$ \\
\hline Cystatin-B; & Q62426 & Cstb & $-0,5$ & 28,6 \\
\hline Cysteine and glycine-rich protein 1 ; & P97315 & Csrp1 & $-0,3$ & 22,8 \\
\hline Cysteine sulfinic acid decarboxylase; & Q9DBE0 & Csad & $-0,7$ & 3,4 \\
\hline Cysteine-rich protein 1 & P63254 & Crip1 & 0,0 & 9,1 \\
\hline Cysteine-rich protein 2; & Q9DCT8 & Crip2 & $-0,4$ & 3,4 \\
\hline Cysteine-rich secretory protein $1 ;$ & Q03401 & Crisp1 & $-0,9$ & 3,7 \\
\hline Cysteine-rich with EGF-like domain protein 2; & Q9CYA0 & Creld2 & 0,5 & 4,9 \\
\hline Cysteine--tRNA ligase, cytoplasmic; & Q9ER72 & Cars & 0,9 & 1,7 \\
\hline Cytochrome b5 type B; & Q9CQX2 & Cyb5b & 0,1 & 19,2 \\
\hline Cytochrome b5; & P56395 & Cyb5a & 0,1 & 56,1 \\
\hline Cytochrome b-c1 complex subunit 1, mitochondrial; & Q9CZ13 & Uqcrc1 & 0,2 & 10 \\
\hline Cytochrome b-c1 complex subunit 2, mitochondrial; & Q9DB77 & Uqcrc2 & 0,2 & 21,6 \\
\hline Cytochrome b-c1 complex subunit 6 , mitochondrial; & P99028 & Uqcrh & 0,1 & 28,1 \\
\hline Cytochrome b-c1 complex subunit 7 & Q9D855 & Uqcrb & $-0,2$ & 25,2 \\
\hline Cytochrome b-c1 complex subunit 8 & Q9CQ69 & Uqcrq & 0,3 & 26,8 \\
\hline Cytochrome b-c1 complex subunit 9 & Q8R1I1 & Uqcr10 & 0,0 & 39,1 \\
\hline Cytochrome b-c1 complex subunit Rieske, mitochondrial; & Q9CR68 & Uqcrfs1 & 0,0 & 10,6 \\
\hline Cytochrome c oxidase subunit 2 & P00405 & Mtco2 & $-0,2$ & 3,1 \\
\hline Cytochrome c oxidase subunit 4 isoform 1, mitochondrial; & P19783 & Cox4i1 & 0,0 & 10,5 \\
\hline Cytochrome c oxidase subunit $5 \mathrm{~A}$, mitochondrial; & P12787 & Cox5a & $-0,1$ & 16,4 \\
\hline Cytochrome coxidase subunit 5B, mitochondrial; & P19536 & Cox5b & 0,2 & 9,4 \\
\hline Cytochrome c oxidase subunit 6C; & Q9CPQ1 & Cox6c & 0,3 & 18,4 \\
\hline Cytochrome c oxidase subunit 7A2, mitochondrial; & P48771 & Cox7a2 & 0,1 & 27,7 \\
\hline Cytochrome c, somatic; & P62897 & Cycs & $-0,3$ & 17,1 \\
\hline Cytochrome $\mathrm{c} 1$, heme protein, mitochondrial; & Q9D0M3 & Cyc1 & $-1,2$ & 12,6 \\
\hline Cytoplasmic aconitate hydratase; & P28271 & Aco1 & 1,2 & 2,8 \\
\hline Cytoplasmic dynein 1 intermediate chain 2; & 088487 & Dync1i2 & 0,0 & 6,7 \\
\hline Cytoskeleton-associated protein 4 & Q8BMK4 & Ckap4 & $-0,3$ & 12,2 \\
\hline Cytosol aminopeptidase; & Q9CPY7 & Lap3 & $-0,1$ & 20 \\
\hline Cytosolic acyl coenzyme A thioester hydrolase; & Q91V12 & Acot7 & 1,6 & 9,4 \\
\hline D-3-phosphoglycerate dehydrogenase; & Q61753 & Phgdh & 0,1 & 16,3 \\
\hline D-dopachrome decarboxylase; & 035215 & Ddt & $-0,2$ & 12,7 \\
\hline Death-associated protein-like $1 ;$ & Q9D757 & Dapl1 & 0,8 & 7,5 \\
\hline Decorin; & P28654 & Den & $-0,2$ & 8,2 \\
\hline Dehydrogenase/reductase SDR family member 7C; & Q8CHS7 & Dhrs7c & $-5,9$ & 4,2 \\
\hline Delta-aminolevulinic acid dehydratase; & P10518 & Alad & 0,1 & 11,5 \\
\hline Density-regulated protein; & Q9CQJ6 & Denr & $-1,0$ & 4,4 \\
\hline Deoxyribonuclease-2-alpha; & P56542 & Dnase2 & $-0,1$ & 9,3 \\
\hline Dermatopontin; & Q9QZZ6 & Dpt & $-0,3$ & 4 \\
\hline Dermokine; & E9Q2P1 & Dmkn & $-0,4$ & 25 \\
\hline Desmocollin-3; & P55850 & Dsc3 & $-0,6$ & 2,7 \\
\hline Dihydrolipoyl dehydrogenase, mitochondrial; & 008749 & Dld & 0,2 & 9,6 \\
\hline $\begin{array}{l}\text { Dihydrolipoyllysine-residue succinyltransferase component of 2-oxoglutarate } \\
\text { dehydrogenase complex, mitochondrial; }\end{array}$ & Q9D2G2 & Dlst & 0,1 & 6,2 \\
\hline Dihydropteridine reductase; & Q8BVI4 & Qdpr & $-1,7$ & 8,3 \\
\hline Dihydropyrimidinase-related protein 2 ; & 008553 & Dpys/2 & 0,1 & 21,3 \\
\hline Dihydropyrimidinase-related protein 3 ; & Q62188 & Dpysl3 & 0,0 & 10 \\
\hline Dipeptidase 1; & P31428 & Dpep1 & 2,1 & 6,3 \\
\hline Dipeptidyl peptidase 1; & P97821 & Ctsc & 0,3 & 8,4 \\
\hline Dipeptidyl peptidase 4; & P28843 & Dpp4 & $-0,3$ & 3,2 \\
\hline Disks large-associated protein 4; & B1AZP2 & Dlgap4 & $-0,2$ & 7,3 \\
\hline DNA damage-binding protein 1 & Q3U1J4 & Ddb1 & 0,3 & 5,4 \\
\hline DNA replication licensing factor MCM6; & P97311 & Mcm6 & $-0,7$ & 1 \\
\hline DNA topoisomerase 2-beta; & Q64511 & Top2b & 0,2 & 1,6 \\
\hline DNA-(apurinic or apyrimidinic site) lyase; & P28352 & Apex1 & 1,0 & 14,2 \\
\hline DNA-directed RNA polymerase II subunit RPB1; & P08775 & Polr2a & 0,3 & 0,5 \\
\hline DnaJ homolog subfamily A member 1 ; & P63037 & Dnaja1 & 0,4 & 6,5 \\
\hline Dolichyl-diphosphooligosaccharide--protein glycosyltransferase 48 kDa subunit; & 054734 & Ddost & $-0,2$ & 9,3 \\
\hline Dolichyl-diphosphooligosaccharide--protein glycosyltransferase subunit 1; & Q91YQ5 & Rpn1 & 0,4 & 10 \\
\hline Dolichyl-diphosphooligosaccharide--protein glycosyltransferase subunit 2; & Q9DBG6 & Rpn2 & $-1,1$ & 9,1 \\
\hline Dolichyl-diphosphooligosaccharide--protein glycosyltransferase subunit DAD1; & P61804 & Dad1 & 0,2 & 8,8 \\
\hline Dolichyl-diphosphooligosaccharide--protein glycosyltransferase subunit STT3A; & P46978 & Stt3a & $-0,2$ & 1 \\
\hline Dynactin subunit 2 ; & Q99KJ8 & Dctn2 & 0,0 & 5,2 \\
\hline Dynein light chain 1 , cytoplasmic; & P63168 & Dynll1 & 0,7 & 24,7 \\
\hline Dystroglycan; & Q62165 & Dag1 & 0,1 & 2,1 \\
\hline E3 ubiquitin-protein ligase RBX1; & P62878 & $\mathrm{Rb} \times 1$ & 0,6 & 7,4 \\
\hline Early endosome antigen 1 & Q8BL66 & Eea1 & 0,1 & 7,3 \\
\hline EGF-containing fibulin-like extracellular matrix protein 1 ; & Q8BPB5 & Efemp1 & 0,2 & 5,6 \\
\hline
\end{tabular}




\begin{tabular}{|c|c|c|c|c|}
\hline Protein & Uniprot & Gene name & $\begin{array}{c}\text { Fold change of } \\
\text { XPRESS ratio } \\
\left.\text { (Adam } 17^{\triangle K C} / \mathrm{WT}\right)\end{array}$ & $\begin{array}{c}\text { Sequence } \\
\text { coverage [\%] }\end{array}$ \\
\hline Elastin; & P54320 & Eln & $-0,1$ & 1,7 \\
\hline Electron transfer flavoprotein subunit alpha, mitochondrial; & Q99LC5 & Etfa & 0,0 & 41,7 \\
\hline Electron transfer flavoprotein subunit beta; & Q9DCW4 & Etfb & 0,1 & 24,7 \\
\hline Elongation factor 1-alpha 1; & P10126 & Eef1a1 & $-0,6$ & 18,2 \\
\hline Elongation factor 1-beta; & 070251 & Eef1b & $-0,6$ & 34,9 \\
\hline Elongation factor 1-delta; & P57776 & Eef1d & $-0,1$ & 18,4 \\
\hline Elongation factor 1-gamma; & Q9D8N0 & Eef1g & $-0,6$ & 7,6 \\
\hline Elongation factor 2; & P58252 & Eef2 & $-0,1$ & 18,9 \\
\hline Embigin; & P21995 & Emb & 0,6 & 2,4 \\
\hline Endoplasmic reticulum metallopeptidase 1 ; & Q3UVK0 & Ermp1 & 0,4 & 2,9 \\
\hline Endoplasmic reticulum resident protein 29 ; & P57759 & Erp29 & $-0,2$ & 21,8 \\
\hline Endoplasmic reticulum resident protein 44 ; & Q9D1Q6 & Erp44 & $-0,3$ & 10,3 \\
\hline Endoplasmin; & P08113 & Hsp90b1 & $-0,3$ & 21,3 \\
\hline Endothelial differentiation-related factor 1 & Q9JMG1 & Edf1 & 0,1 & 20,9 \\
\hline Endothelin-converting enzyme 1 & Q4PZA2 & Ece1 & $-0,1$ & 1 \\
\hline Enoyl-CoA delta isomerase 1 , mitochondrial; & P42125 & Eci1 & 0,4 & 21,1 \\
\hline Enoyl-CoA delta isomerase 2, mitochondrial; & Q9WUR2 & Eci2 & $-0,2$ & 5,9 \\
\hline Enoyl-CoA hydratase, mitochondrial; & Q8BH95 & Echs1 & $-0,9$ & 5,8 \\
\hline Eosinophil cationic-type ribonuclease 5 ; & 035292 & Ear5 & 1,8 & 20 \\
\hline Epididymal secretory protein E1; & Q9Z0J0 & Npc2 & $-0,2$ & 10,7 \\
\hline Erlin-2; & Q8BFZ9 & Erlin2 & $-0,2$ & 7,6 \\
\hline Ethylmalonyl-CoA decarboxylase; & Q9D9V3 & Echdc1 & 0,7 & 3,1 \\
\hline Eukaryotic initiation factor 4A-I; & P60843 & Eif4a1 & 0,1 & 16 \\
\hline Eukaryotic initiation factor $4 \mathrm{~A}-\mathrm{III}$; & Q91VC3 & Eif4a3 & $-0,2$ & 18 \\
\hline Eukaryotic translation initiation factor 2 subunit 1 ; & Q6ZWX6 & Eif2s1 & $-0,2$ & 27,9 \\
\hline Eukaryotic translation initiation factor 2 subunit 2 & Q99L45 & Eif2s2 & $-0,1$ & 10,3 \\
\hline Eukaryotic translation initiation factor 2 subunit 3, X-linked; & Q9Z0N1 & Eif2s3x & 0,0 & 18,6 \\
\hline Eukaryotic translation initiation factor $2 \mathrm{~A}$ & Q8BJW6 & Eif2a & $-0,3$ & 5,3 \\
\hline Eukaryotic translation initiation factor 3 subunit B; & Q8JZQ9 & Eif3b & $-0,5$ & 1,1 \\
\hline Eukaryotic translation initiation factor 3 subunit G; & Q9Z1D1 & Eif3g & $-0,2$ & 2,2 \\
\hline Eukaryotic translation initiation factor 3 subunit J-A; & Q3UGC7 & Eif3j1 & 0,3 & 11,5 \\
\hline Eukaryotic translation initiation factor $4 \mathrm{~B}$ & Q8BGD9 & Eif4b & 0,6 & 8,3 \\
\hline Eukaryotic translation initiation factor $4 \mathrm{H}$; & Q9WUK2 & Eif4h & 0,1 & 12,9 \\
\hline Eukaryotic translation initiation factor $5 \mathrm{~A}-1$; & P63242 & Eif5a & $-0,1$ & 19 \\
\hline Extracellular matrix protein 1 & Q61508 & Ecm1 & 0,3 & 5,2 \\
\hline F-actin-capping protein subunit alpha-1; & P47753 & Capza1 & $-0,3$ & 14,7 \\
\hline Far upstream element-binding protein 1 & Q91WJ8 & Fubp1 & 0,2 & 6,7 \\
\hline Far upstream element-binding protein 2 & Q3U0V1 & Khsrp & 0,1 & 13,1 \\
\hline Fascin; & Q61553 & Fscn1 & 0,6 & 2 \\
\hline Fatty acid synthase; & P19096 & Fasn & $-0,1$ & 1,8 \\
\hline Fatty acid-binding protein, adipocyte; & P04117 & Fabp4 & 0,4 & 42,4 \\
\hline Fatty acid-binding protein, epidermal; & Q05816 & Fabp5 & $-0,1$ & 31,1 \\
\hline F-box-like/WD repeat-containing protein TBL1XR1; & Q8BHJ5 & Tbl1xr1 & $-0,1$ & 1,4 \\
\hline Ferritin heavy chain; & P09528 & Fth1 & $-0,2$ & 12,6 \\
\hline Ferritin light chain 1 ; & P29391 & Ftl1 & 0,0 & 13,1 \\
\hline Fibrillin-1; & A2AQ53 & Fbn1 & 0,3 & 1,7 \\
\hline Fibrinogen beta chain; & Q8K0E8 & $\mathrm{Fgb}$ & 0,1 & 2,9 \\
\hline Fibulin-2; & P37889 & Fbln2 & 0,3 & 0,8 \\
\hline Fibulin-5; & Q9WVH9 & Fbln5 & 0,1 & 5,1 \\
\hline Filaggrin-2; & Q2VIS4 & Flg2 & $-1,7$ & 12,7 \\
\hline Filamin-A; & Q8BTM8 & Flna & $-0,4$ & 3,6 \\
\hline Filamin-B; & Q80X90 & Flnb & 0,1 & 3,5 \\
\hline Four and a half LIM domains protein 1 & P97447 & Fhl1 & 0,7 & 21,8 \\
\hline Four and a half LIM domains protein 2; & 070433 & $\mathrm{Fhl} 2$ & $-0,5$ & 2,5 \\
\hline Four and a half LIM domains protein 3 ; & Q9R059 & $\mathrm{Fhl3}$ & $-2,3$ & 6,1 \\
\hline Frizzled-2; & Q9JIP6 & Fzd2 & 0,4 & 1,2 \\
\hline Fructose-1,6-bisphosphatase 1 & Q9QXD6 & Fbp1 & $-0,2$ & 15,4 \\
\hline Fructose-bisphosphate aldolase $A$; & P05064 & Aldoa & $-0,4$ & 25,7 \\
\hline FUN14 domain-containing protein 2; & Q9D6K8 & Fundc2 & 1,6 & 7,3 \\
\hline Galactokinase; & Q9RONO & Galk1 & 0,1 & 3,1 \\
\hline Galectin; & Q9CRB1 & Lgals7 & 0,0 & 36,8 \\
\hline Galectin-1; & P16045 & Lgals1 & 0,5 & 51,1 \\
\hline Galectin-3; & P16110 & Lgals3 & $-0,2$ & 13,3 \\
\hline Gamma-adducin; & Q9QYB5 & Add3 & 0,4 & 7,8 \\
\hline Gamma-glutamyl hydrolase; & Q9Z0L8 & Ggh & $-0,6$ & 4,1 \\
\hline Gamma-glutamylcyclotransferase; & Q9D7X8 & Ggct & $-1,5$ & 10,6 \\
\hline Gamma-glutamyltransferase 7; & Q99JP7 & Ggt7 & 2,9 & 2,3 \\
\hline Gamma-synuclein; & Q9Z0F7 & Sncg & 0,6 & 10,6 \\
\hline Ganglioside GM2 activator; & Q60648 & $\mathrm{Gm} 2 \mathrm{a}$ & $-0,2$ & 5,2 \\
\hline Gap junction alpha-1 protein; & P23242 & Gja1 & 0,1 & 14,9 \\
\hline Gasdermin-A; & Q9EST1 & Gsdma & $-0,8$ & 6,5 \\
\hline Gelsolin; & P13020 & Gsn & $-0,4$ & 10,3 \\
\hline Gelsolin; & A6PWS5 & Gsn & 0,8 & 11,2 \\
\hline
\end{tabular}




\begin{tabular}{|c|c|c|c|c|}
\hline Protein & Uniprot & Gene name & $\begin{array}{c}\text { Fold change of } \\
\text { XPRESS ratio } \\
\left.\text { (Adam } 17^{\Delta K C} / \mathrm{WT}\right)\end{array}$ & $\begin{array}{c}\text { Sequence } \\
\text { coverage [\%] }\end{array}$ \\
\hline General transcription factor II-I; & Q9ESZ8 & Gtf2i & 0,5 & 3,6 \\
\hline General vesicular transport factor $\mathrm{p} 115$ & Q9Z1Z0 & Uso1 & $-0,6$ & 1,6 \\
\hline Glia maturation factor beta; & Q9CQI3 & Gmfb & $-0,2$ & 14,1 \\
\hline Glucose-6-phosphate isomerase; & P06745 & Gpi & $-1,0$ & 4,7 \\
\hline Glucosidase 2 subunit beta; & 008795 & Prkcsh & $-0,2$ & 2,3 \\
\hline Glucosylceramidase; & P17439 & Gba & $-0,4$ & 1,6 \\
\hline Glutamate dehydrogenase 1 , mitochondrial; & P26443 & Glud1 & $-0,2$ & 11,3 \\
\hline Glutathione peroxidase 1; & P11352 & Gpx1 & 1,0 & 9,5 \\
\hline Glutathione peroxidase 7; & Q99L6 & Gpx7 & $-0,3$ & 10,8 \\
\hline Glutathione reductase, mitochondrial; & P47791 & Gsr & 0,0 & 2,8 \\
\hline Glutathione S-transferase Mu 1; & P10649 & Gstm1 & $-0,9$ & 11 \\
\hline Glutathione S-transferase Mu 2; & P15626 & Gstm2 & $-2,2$ & 16,5 \\
\hline Glutathione S-transferase Mu 3; & P19639 & Gstm3 & 0,1 & 14,2 \\
\hline Glutathione S-transferase omega-1; & 009131 & Gsto1 & $-0,2$ & 7,5 \\
\hline Glutathione S-transferase P 1; & P19157 & Gstp1 & $-0,3$ & 12,9 \\
\hline Glutathione synthetase; & P51855 & Gss & $-0,1$ & 4,5 \\
\hline Glyceraldehyde-3-phosphate dehydrogenase; & P16858 & Gapdh & $-0,2$ & 16,4 \\
\hline Glycerol-3-phosphate dehydrogenase [NAD(+)], cytoplasmic; & P13707 & Gpd1 & 0,4 & 6,7 \\
\hline Glycerol-3-phosphate dehydrogenase, mitochondrial; & Q64521 & Gpd2 & $-0,1$ & 3,7 \\
\hline Glycine N-methyltransferase; & Q9QXF8 & Gnmt & $-4,2$ & 4,1 \\
\hline Glycine--tRNA ligase; & Q9CZD3 & Gars & $-0,1$ & 6 \\
\hline Glycogen phosphorylase, brain form; & Q8Cl94 & Pygb & 0,2 & 1,1 \\
\hline Glyoxylate reductase/hydroxypyruvate reductase; & Q91Z53 & Grhpr & 0,0 & 5,1 \\
\hline Glypican-4; & P51655 & Gpc4 & $-0,6$ & 9 \\
\hline GMP reductase $1 ;$ & Q9DCZ1 & Gmpr & 1,8 & 5,2 \\
\hline Golgi apparatus protein 1; & Q61543 & Glg1 & 0,0 & 5,7 \\
\hline Golgi integral membrane protein 4; & Q8BXA1 & Golim4 & $-0,3$ & 4,9 \\
\hline Golgi SNAP receptor complex member 1 ; & 088630 & Gosr1 & $-0,2$ & 7,6 \\
\hline GTP:AMP phosphotransferase AK3, mitochondrial; & Q9WTP7 & Ak3 & $-0,8$ & 4 \\
\hline GTPase HRas; & Q61411 & Hras & $-0,4$ & 4,8 \\
\hline GTP-binding nuclear protein Ran; & P62827 & Ran & 0,2 & 27,8 \\
\hline GTP-binding protein SAR1b; & Q9CQC9 & Sar1b & 2,4 & 9,6 \\
\hline Guanine nucleotide-binding protein $\mathrm{G}(\mathrm{I}) / \mathrm{G}(\mathrm{S}) / \mathrm{G}(\mathrm{T})$ subunit beta-1; & P62874 & Gnb1 & $-1,1$ & 4,7 \\
\hline Guanine nucleotide-binding protein subunit beta-2-like 1; & P68040 & Gnb2I1 & 0,0 & 42,3 \\
\hline H/ACA ribonucleoprotein complex subunit 1 ; & Q9CY66 & Gar1 & $-1,1$ & 20,2 \\
\hline H/ACA ribonucleoprotein complex subunit 2; & Q9CRB2 & Nhp2 & 0,7 & 10,5 \\
\hline H-2 class I histocompatibility antigen, D-B alpha chain; & P01899 & $\mathrm{H} 2 \mathrm{D} 1$ & 0,5 & 9,5 \\
\hline Haptoglobin; & Q61646 & $\mathrm{Hp}$ & 0,8 & 8,4 \\
\hline Heat shock 70 kDa protein 1B; & P17879 & Hspa1b & $-0,3$ & 16,4 \\
\hline Heat shock cognate 71 kDa protein; & P63017 & Hspa8 & $-0,2$ & 29,6 \\
\hline Heat shock protein beta-1; & P14602 & Hspb1 & $-0,4$ & 33,5 \\
\hline Heat shock protein HSP 90-alpha; & P07901 & Hsp90aa1 & 0,1 & 11,6 \\
\hline Heat shock protein HSP 90-beta; & P11499 & Hsp90ab1 & $-0,3$ & 16,9 \\
\hline Hematopoietic lineage cell-specific protein; & P49710 & Hcls1 & 0,7 & 2,1 \\
\hline Hematopoietic progenitor cell antigen CD34; & Q64314 & $\mathrm{Cd} 34$ & 0,2 & 4,2 \\
\hline Hemoglobin subunit alpha; & P01942 & $\mathrm{Hba}$ & 0,3 & 41,5 \\
\hline Hemoglobin subunit beta- 1 ; & P02088 & $\mathrm{Hbb}$ b1 & $-3,6$ & 67,3 \\
\hline Hemopexin; & Q91X72 & $\mathrm{Hpx}$ & 0,3 & 5 \\
\hline Hepatoma-derived growth factor; & P51859 & Hdgf & 0,3 & 11,4 \\
\hline Heterogeneous nuclear ribonucleoprotein A/B; & Q99020 & Hnrnpab & 0,2 & 9,5 \\
\hline Heterogeneous nuclear ribonucleoprotein A1; & P49312 & Hnrnpa1 & 0,1 & 19,4 \\
\hline Heterogeneous nuclear ribonucleoprotein A3; & Q8BG05 & Hnrnpa3 & 1,7 & 9,3 \\
\hline Heterogeneous nuclear ribonucleoprotein D0; & Q60668 & Hnrnpd & 0,2 & 19,6 \\
\hline Heterogeneous nuclear ribonucleoprotein D-like; & Q9Z130 & Hnrnpdl & 0,2 & 8 \\
\hline Heterogeneous nuclear ribonucleoprotein F; & Q9Z2X1 & Hnrnpf & 0,6 & 11,3 \\
\hline Heterogeneous nuclear ribonucleoprotein $\mathrm{H}$; & 035737 & Hnrnph1 & 0,5 & 15,6 \\
\hline Heterogeneous nuclear ribonucleoprotein $\mathrm{K}$; & P61979 & Hnrnpk & 0,1 & 27,3 \\
\hline Heterogeneous nuclear ribonucleoprotein L; & Q8R081 & Hnrnpl & $-0,1$ & 3,7 \\
\hline Heterogeneous nuclear ribonucleoprotein $\mathrm{M}$; & Q9D0E1 & Hnrnpm & 0,1 & 8,9 \\
\hline Heterogeneous nuclear ribonucleoprotein Q; & Q7TMK9 & Syncrip & $-0,1$ & 4,1 \\
\hline Heterogeneous nuclear ribonucleoprotein U; & Q8VEK3 & Hnrnpu & 0,2 & 4 \\
\hline Heterogeneous nuclear ribonucleoproteins A2/B1; & 088569 & Hnrnpa2b1 & 0,0 & 15,9 \\
\hline Heterogeneous nuclear ribonucleoproteins $\mathrm{C} 1 / \mathrm{C} 2$; & Q9Z204 & Hnrnpc & 0,1 & 7,3 \\
\hline Hexokinase-1; & P17710 & Hk1 & 1,1 & 2,1 \\
\hline High mobility group protein $\mathrm{B} 1$ & P63158 & Hmgb1 & 0,2 & 12,1 \\
\hline High mobility group protein B2; & P30681 & Hmgb2 & 0,5 & 12,4 \\
\hline High mobility group protein B3; & 054879 & Hmgb3 & 0,4 & 8,2 \\
\hline High mobility group protein HMG-I/HMG-Y; & P17095 & Hmga1 & 0,1 & 8,4 \\
\hline Histidine ammonia-lyase; & P35492 & $\mathrm{Hal}$ & $-0,8$ & 10,4 \\
\hline Histidine triad nucleotide-binding protein 1 ; & P70349 & Hint1 & 0,4 & 23 \\
\hline Histone deacetylase complex subunit SAP25; & Q1EHW4 & Sap25 & 0,5 & 5,8 \\
\hline Histone H1.0; & P10922 & H1fo & 0,0 & 11,3 \\
\hline Histone H1.1; & P43275 & Hist1h1a & 0,4 & 9,4 \\
\hline
\end{tabular}




\begin{tabular}{|c|c|c|c|c|}
\hline Protein & Uniprot & Gene name & $\begin{array}{c}\text { Fold change of } \\
\text { XPRESS ratio } \\
\left.\text { (Adam } 17^{\Delta K C} / \mathrm{WT}\right)\end{array}$ & $\begin{array}{c}\text { Sequence } \\
\text { coverage [\%] }\end{array}$ \\
\hline Histone H1.2; & P15864 & Hist1h1c & 0,1 & 26,9 \\
\hline Histone H1.3; & P43277 & Hist1h1d & 0,2 & 25,8 \\
\hline Histone H1.4; & P43274 & Hist1h1e & 0,2 & 26 \\
\hline Histone H1.5; & P43276 & Hist1h1b & $-0,2$ & 21,5 \\
\hline Histone $\mathrm{H} 2 \mathrm{~A}$ type 1 ; & P22752 & Hist1h2ao & $-0,1$ & 27,1 \\
\hline Histone H2A type 2-B; & Q64522 & Hist2h2ab & $-0,3$ & 12,3 \\
\hline Histone H2B type 1-F/J/L; & P10853 & Hist1h2bn & 0,1 & 28,6 \\
\hline Histone H3.3C; & P02301 & $H 3 f 3 c$ & 0,1 & 19,3 \\
\hline Histone $\mathrm{H} 4$; & P62806 & Hist4h4 & 0,1 & 50,5 \\
\hline Histone-binding protein RBBP4; & Q60972 & Rbbp4 & 0,2 & 9,3 \\
\hline Homeodomain-only protein; & Q8R1H0 & Hopx & 0,7 & 43,8 \\
\hline Host cell factor 1 & Q61191 & Hcfc1 & 0,3 & 1,8 \\
\hline Hydroxyacylglutathione hydrolase, mitochondrial; & Q99KB8 & Hagh & 0,0 & 5,5 \\
\hline Hydroxymethylglutaryl-CoA synthase, cytoplasmic; & Q8JZK9 & Hmgcs1 & 0,4 & 6,2 \\
\hline Hypoxanthine-guanine phosphoribosyltransferase; & P00493 & Hprt1 & $-0,2$ & 17,9 \\
\hline Ig gamma-1 chain $\mathrm{C}$ region secreted form; & P01868 & Ighg1 & 1,3 & 3,7 \\
\hline Ig gamma-2B chain C region; & P01867 & $\operatorname{lgh} 3$ & 0,4 & 4,2 \\
\hline Inactive tyrosine-protein kinase 7; & Q8BKG3 & Ptk7 & $-0,1$ & 0,8 \\
\hline Inorganic pyrophosphatase 2, mitochondrial; & Q91VM9 & Ppa2 & 2,0 & 8,6 \\
\hline Inorganic pyrophosphatase; & Q9D819 & Ppa1 & 0,6 & 9 \\
\hline Inosine-5'-monophosphate dehydrogenase 2 & P24547 & Impdh2 & $-0,2$ & 2,3 \\
\hline Inositol monophosphatase 1 & 055023 & Impa1 & 0,1 & 12,3 \\
\hline Inositol-3-phosphate synthase 1 & Q9JHU9 & Isyna1 & $-0,3$ & 6,3 \\
\hline Insulin-degrading enzyme; & F6RPJ9 & Ide & $-1,7$ & 11,9 \\
\hline Insulin-like growth factor-binding protein 5; & Q07079 & Igfbp5 & $-0,9$ & 7,7 \\
\hline Integrin beta-1; & P09055 & Itgb1 & 0,0 & 8,4 \\
\hline Inter alpha-trypsin inhibitor, heavy chain 4 ; & A6X935 & Itih4 & 0,2 & 0,9 \\
\hline Interleukin enhancer-binding factor 3 ; & Q9Z1X4 & IIf3 & 0,0 & 3 \\
\hline Involucrin; & G3X9D9 & $|v|$ & $-0,8$ & 42,1 \\
\hline Isocitrate dehydrogenase [NAD] subunit alpha, mitochondrial; & Q9D6R2 & $\operatorname{Idh} 3 a$ & 0,2 & 4,9 \\
\hline Isocitrate dehydrogenase [NAD] subunit gamma 1, mitochondrial; & P70404 & $\operatorname{ldh} 3 g$ & $-0,2$ & 5,3 \\
\hline Isocitrate dehydrogenase [NADP] cytoplasmic; & 088844 & Idh1 & 0,1 & 22,5 \\
\hline Isocitrate dehydrogenase [NADP], mitochondrial; & P54071 & Idh2 & $-0,4$ & 23,7 \\
\hline Isocitrate dehydrogenase $3(\mathrm{NAD}+)$ beta; Protein Idh3b; Tumor-related protein; & Q91VA7 & $\operatorname{Idh} 3 b$ & $-0,2$ & 6,8 \\
\hline Isoleucine--tRNA ligase, mitochondrial; & Q8BIJ6 & lars2 & 0,3 & 3 \\
\hline Isopentenyl-diphosphate Delta-isomerase 1 & P58044 & Idi1 & 0,2 & 12,8 \\
\hline Junction plakoglobin; & Q02257 & Jup & $-0,4$ & 2,6 \\
\hline Kallikrein related-peptidase 10; Protein Klk10; & Q99M20 & Klk10 & 1,5 & 15,5 \\
\hline Kallikrein-7; & Q91VE3 & Klk7 & $-0,4$ & 10 \\
\hline Kelch repeat and BTB domain-containing protein 3; & Q8BHI4 & Kbtbd3 & $-1,7$ & 2,5 \\
\hline Keratin, type I cytoskeletal 10; & P02535 & Krt10 & $-0,5$ & 9,8 \\
\hline Keratin, type I cytoskeletal 17; & Q9QWL7 & Krt17 & $-0,8$ & 26,3 \\
\hline Keratin, type I cytoskeletal 25; & Q8VCW2 & Krt25 & $-0,3$ & 21,3 \\
\hline Keratin, type I cytoskeletal 27; & Q9Z320 & Krt27 & $-2,8$ & 18,1 \\
\hline Keratin, type I cytoskeletal 28; & A6BLY7 & Krt28 & $-0,3$ & 5 \\
\hline Keratin, type II cuticular Hb3; & Q6IMF0 & Krt83 & $-0,6$ & 3,6 \\
\hline Keratin, type II cytoskeletal 1b; & Q6IFZ6 & Krt77 & $-1,6$ & 5,2 \\
\hline Keratin, type II cytoskeletal 5; & Q922U2 & Krt5 & 0,3 & 6 \\
\hline Keratin, type II cytoskeletal 71; & Q9R0H5 & Krt71 & $-1,0$ & 28,6 \\
\hline Keratin, type II cytoskeletal 73; & Q6NXH9 & Krt73 & 0,0 & 8,5 \\
\hline Keratin, type II cytoskeletal 79; & Q8VED5 & Krt79 & 0,2 & 3,6 \\
\hline Keratin-associated protein $16-1$ & A2A $5 \times 5$ & Krtap16 1 & $-1,3$ & 3 \\
\hline Ketimine reductase mu-crystallin; & 054983 & Crym & $-0,8$ & 30,4 \\
\hline Kininogen-1; & 008677 & Kng1 & 0,7 & 10,2 \\
\hline Kynurenine--oxoglutarate transaminase 1 ; & Q8BTY1 & Ccbl1 & 0,3 & 3,5 \\
\hline Kynurenine--oxoglutarate transaminase 3 ; & Q71RI9 & Ccbl2 & 0,1 & 2,6 \\
\hline Lactoylglutathione lyase; & Q9CPU0 & Glo1 & 0,3 & 11,4 \\
\hline Ladinin-1; & P57016 & Lad1 & $-0,4$ & 6,4 \\
\hline Lamina-associated polypeptide 2, isoforms beta/delta/epsilon/gamma; & Q61029 & Tmpo & 0,4 & 19,9 \\
\hline Lamin-B receptor; & Q3U9G9 & Lbr & 0,1 & 3,2 \\
\hline Lamin-B1; & P14733 & Lmnb1 & 0,5 & 3,4 \\
\hline Laminin subunit alpha-4; & P97927 & Lama4 & $-0,7$ & 1,2 \\
\hline Laminin subunit beta-1; & P02469 & Lamb1 & $-0,1$ & 2,3 \\
\hline Laminin subunit gamma-1; & P02468 & Lamc1 & 1,0 & 1,7 \\
\hline LanC-like protein 1 & 089112 & Lancl1 & $-0,1$ & 11,6 \\
\hline Latent-transforming growth factor beta-binding protein 4; & Q8K4G1 & Ltbp4 & 0,2 & 0,7 \\
\hline L-dopachrome tautomerase; & P29812 & Dct & 1,3 & 1,9 \\
\hline Leucine-rich repeat flightless-interacting protein 1 ; & Q3UZ39 & Lrrfip1 & $-0,2$ & 4,1 \\
\hline Leucine-rich repeat-containing protein 47 ; & Q505F5 & Lrrc47 & $-0,3$ & 2,4 \\
\hline Leucine-rich repeat-containing protein 59; & Q922Q8 & Lrrc59 & 0,3 & 16,6 \\
\hline Leucyl-cystinyl aminopeptidase; & Q8C129 & Lnpep & 0,2 & 0,9 \\
\hline
\end{tabular}




\begin{tabular}{|c|c|c|c|c|}
\hline Protein & Uniprot & Gene name & $\begin{array}{c}\text { Fold change of } \\
\text { XPRESS ratio } \\
\left.\text { (Adam } 17^{\triangle K C} / \mathrm{WT}\right)\end{array}$ & $\begin{array}{c}\text { Sequence } \\
\text { coverage [\%] }\end{array}$ \\
\hline LIM and senescent cell antigen-like-containing domain protein 1 ; & Q99JW4 & Lims1 & 0,3 & 3,7 \\
\hline LIM and SH3 domain protein 1; & Q61792 & Lasp1 & $-0,5$ & 14,5 \\
\hline LIM domain and actin-binding protein 1 ; & Q9ERG0 & Lima1 & $-0,7$ & 14,3 \\
\hline Lipoma-preferred partner homolog; & Q8BFW7 & Lpp & 1,0 & 3,6 \\
\hline Liprin-alpha 4; Protein Ppfia4; & B8QI36 & Ppfia4 & 10,0 & 0,8 \\
\hline L-lactate dehydrogenase A chain; & P06151 & Ldha & 0,2 & 26,8 \\
\hline L-lactate dehydrogenase B chain; & P16125 & Ldhb & 0,2 & 14,1 \\
\hline Long-chain specific acyl-CoA dehydrogenase, mitochondrial; & P51174 & Acadl & 0,0 & 9,5 \\
\hline Long-chain-fatty-acid--CoA ligase 1 & P41216 & Acsl1 & 0,5 & 9,3 \\
\hline Long-chain-fatty-acid--CoA ligase 5; & Q8JZR0 & Acsl5 & $-0,1$ & 2,8 \\
\hline Lumican; & P51885 & Lum & 0,4 & 10,9 \\
\hline Lupus La protein homolog; & P32067 & Ssb & 0,3 & 4,8 \\
\hline Ly6/PLAUR domain-containing protein 5; & Q9D7Z7 & Lypd5 & 0,3 & 9 \\
\hline Lysine--tRNA ligase; & Q99MN1 & Kars & 0,0 & 5 \\
\hline Lysosomal protective protein; & P16675 & Ctsa & $-0,8$ & 3,8 \\
\hline Lysosomal Pro-X carboxypeptidase; & Q7TMR0 & Prcp & 0,2 & 3,3 \\
\hline Lysosome-associated membrane glycoprotein $1 ;$ & P11438 & Lamp1 & 0,0 & 3 \\
\hline Lysosome-associated membrane glycoprotein 2; & P17047 & Lamp2 & 0,1 & 6,5 \\
\hline m7GpppX diphosphatase; & Q9DAR7 & Dcps & 0,4 & 16 \\
\hline Macrophage migration inhibitory factor; & P34884 & Mif & $-0,5$ & 7,8 \\
\hline Macrophage-capping protein; & P24452 & Capg & $-0,5$ & 5,8 \\
\hline Magnesium transporter protein 1 & Q9CQY5 & Magt1 & 0,0 & 5,4 \\
\hline Magnesium-dependent phosphatase 1 ; & Q9D967 & Mdp1 & 0,0 & 4,3 \\
\hline Major facilitator superfamily domain-containing protein 7; & Q8CE47 & Mfsd7 & 2,9 & 1,6 \\
\hline Malate dehydrogenase, cytoplasmic; & P14152 & Mdh1 & 0,1 & 24,3 \\
\hline Malate dehydrogenase, mitochondrial; & P08249 & Mdh2 & $-0,2$ & 36,7 \\
\hline Malignant T-cell-amplified sequence 1 ; & Q9DB27 & Mcts1 & $-0,1$ & 5 \\
\hline MARCKS-related protein; & P28667 & Marcksl1 & 0,1 & 7,5 \\
\hline Matrin-3; & Q8K310 & Matr3 & 1,7 & 2 \\
\hline MCG13402, isoform CRA_a; Polypyrimidine tract-binding protein 1; & Q8BGJ5 & Ptbp1 & 0,2 & 10,6 \\
\hline MCG18410, isoform CRA_a; Protein Ddx23; & D3Z0M9 & $\mathrm{Dd} \times 23$ & $-0,1$ & 1 \\
\hline MCG20149, isoform CRA_a; Methyltransferase like 7A1; Protein Mettl7a1; & Q8C6B0 & Mett|7a1 & $-1,9$ & 3,7 \\
\hline $\begin{array}{l}\text { MCG21506; Macrophage galactose N-acetyl-galactosamine specific lectin } 2 \\
\text { isoform i; Protein Mgl2; }\end{array}$ & A9XX86 & $\mathrm{Mgl2}$ & 0,2 & 2,4 \\
\hline Medium-chain specific acyl-CoA dehydrogenase, mitochondrial; & P45952 & Acadm & 0,0 & 10,5 \\
\hline Membrane primary amine oxidase; & 070423 & Aoc3 & 0,7 & 5,1 \\
\hline Metaxin-1; & P47802 & Mtx1 & 0,1 & 5,6 \\
\hline Methionine adenosyltransferase 2 subunit beta; & Q99LB6 & Mat2b & 0,0 & 10,2 \\
\hline Methionine aminopeptidase 2 ; & 008663 & Metap2 & 0,1 & 4,4 \\
\hline Methylcrotonoyl-CoA carboxylase beta chain, mitochondrial; & Q3ULD5 & Mccc2 & 0,4 & 1,6 \\
\hline Methylosome protein 50 & Q99J09 & Wdr77 & 0,2 & 9 \\
\hline Microtubule-associated protein 4; & P27546 & Map4 & 0,0 & 15,7 \\
\hline Microtubule-associated protein RP/EB family member 1 ; & Q61166 & Mapre1 & $-0,1$ & 7,1 \\
\hline Mimecan; & Q62000 & Ogn & $-0,4$ & 2,7 \\
\hline Mitochondrial 2-oxoglutarate/malate carrier protein; & Q9CR62 & Slc25a11 & 0,2 & 34,7 \\
\hline Mitochondrial amidoxime reducing component 2 & Q922Q1 & Marc2 & $-0,2$ & 8,9 \\
\hline Mitochondrial carnitine/acylcarnitine carrier protein; & Q9Z2Z6 & Slc25a20 & $-0,7$ & 5,3 \\
\hline Mitochondrial carrier homolog 2; & Q791V5 & Mtch2 & 0,5 & 7,5 \\
\hline Mitochondrial dicarboxylate carrier; & Q9QZD8 & Slc25a10 & 0,1 & 12,5 \\
\hline Mitochondrial fission 1 protein; & Q9CQ92 & Fis1 & $-0,1$ & 16,6 \\
\hline Mitochondrial import inner membrane translocase subunit Tim13; & P62075 & Timm13 & 0,3 & 15,8 \\
\hline Mitochondrial import inner membrane translocase subunit Tim9; & Q9WV98 & Timm9 & 0,1 & 16,9 \\
\hline Mitochondrial inner membrane protein; & Q8CAQ8 & Immt & $-0,1$ & 13,2 \\
\hline Mitochondrial intermembrane space import and assembly protein 40 ; & Q8VEA4 & Chchd4 & 0,2 & 6,5 \\
\hline Mitogen-activated protein kinase 1 & P63085 & Mapk1 & $-0,1$ & 10,3 \\
\hline Mitogen-activated protein kinase kinase kinase 10 & Q66L42 & Map3k10 & $-1,5$ & 0,7 \\
\hline Mitotic checkpoint protein BUB3; & Q9WVA3 & Bub3 & 0,2 & 12,6 \\
\hline MOB kinase activator $1 \mathrm{~B}$; & Q8BPB0 & Mob1b & $-0,1$ & 5,1 \\
\hline Monocarboxylate transporter 1 & P53986 & Slc16a1 & 0,3 & 2,6 \\
\hline Myelin basic protein; & P04370 & Mbp & 0,3 & 6,7 \\
\hline Myelin protein $\mathrm{PO}$ & P27573 & $\mathrm{Mpz}$ & 0,2 & 7,3 \\
\hline Myeloid-associated differentiation marker; & 035682 & Myadm & 0,2 & 5,3 \\
\hline Myosin light chain $1 / 3$, skeletal muscle isoform; & P05977 & Myl1 & 1,9 & 26,1 \\
\hline Myosin light chain kinase, smooth muscle; & Q6PDN3 & Mylk & 0,4 & 0,6 \\
\hline Myosin light polypeptide 6; & Q60605 & Myl6 & $-0,3$ & 27,2 \\
\hline Myosin regulatory light chain $12 \mathrm{~B}$; & Q3THE2 & Myl12b & $-0,4$ & 11,6 \\
\hline Myosin regulatory light chain 2, skeletal muscle isoform; & P97457 & Mylpf & 2,4 & 11,8 \\
\hline Myosin-9; & Q8VDD5 & Myh9 & $-0,2$ & 14,2 \\
\hline Myristoylated alanine-rich C-kinase substrate; & P26645 & Marcks & 0,1 & 16,5 \\
\hline $\mathrm{Na}(+) / \mathrm{H}(+)$ exchange regulatory cofactor NHE-RF1; & P70441 & Slc9a3r1 & 0,5 & 14,4 \\
\hline N-acetylglucosamine-6-sulfatase; & Q8BFR4 & Gns & $-0,4$ & 1,7 \\
\hline $\mathrm{N}$-acetylneuraminate lyase; & Q9DCJ9 & $\mathrm{Npl}$ & $-0,2$ & 11,6 \\
\hline
\end{tabular}




\begin{tabular}{|c|c|c|c|c|}
\hline Protein & Uniprot & Gene name & $\begin{array}{c}\text { Fold change of } \\
\text { XPRESS ratio } \\
\left.\text { (Adam17 }{ }^{\Delta K C} / \mathrm{WT}\right)\end{array}$ & $\begin{array}{c}\text { Sequence } \\
\text { coverage [\%] }\end{array}$ \\
\hline NAD(P)H-hydrate epimerase; & Q8K4Z3 & Apoa1bp & 0,1 & 6,4 \\
\hline NADH dehydrogenase [ubiquinone] 1 alpha subcomplex subunit 13; & Q9ERS2 & Ndufa13 & 0,1 & 6,9 \\
\hline NADH dehydrogenase [ubiquinone] 1 alpha subcomplex subunit 4; & Q62425 & Ndufa4 & 0,2 & 19,5 \\
\hline NADH dehydrogenase [ubiquinone] 1 alpha subcomplex subunit 4-like 2; & Q4FZG9 & Ndufa4l2 & $-0,7$ & 18,4 \\
\hline NADH dehydrogenase [ubiquinone] 1 alpha subcomplex subunit 8; & Q9DCJ5 & Ndufa8 & $-0,1$ & 9,9 \\
\hline $\begin{array}{l}\text { NADH dehydrogenase [ubiquinone] } 1 \text { beta subcomplex subunit } 11 \text {, } \\
\text { mitochondrial; }\end{array}$ & 009111 & Ndufb11 & 0,1 & 6,6 \\
\hline NADH dehydrogenase [ubiquinone] 1 beta subcomplex subunit 3; & Q9CQZ6 & Ndufb3 & 0,1 & 17,3 \\
\hline $\begin{array}{l}\text { NADH dehydrogenase [ubiquinone] } 1 \text { beta subcomplex subunit } 5 \text {, } \\
\text { mitochondrial; }\end{array}$ & Q9CQH3 & Ndufb5 & $-0,1$ & 6,7 \\
\hline NADH dehydrogenase [ubiquinone] 1 subunit $\mathrm{C} 2$ & Q9CQ54 & Ndufc2 & 0,1 & 5,8 \\
\hline NADH dehydrogenase [ubiquinone] flavoprotein 2, mitochondrial; & Q9D6J6 & Ndufv2 & $-0,2$ & 16,5 \\
\hline $\begin{array}{l}\text { NADH dehydrogenase [ubiquinone] flavoprotein 3, mitochondrial; RIKEN cDNA } \\
\text { 1500032D16, isoform CRA_a; }\end{array}$ & Q3U422 & Ndufv3 & $-0,1$ & 6,6 \\
\hline NADH dehydrogenase [ubiquinone] iron-sulfur protein 2, mitochondrial; & Q91WD5 & Ndufs2 & $-0,1$ & 3,6 \\
\hline NADH dehydrogenase [ubiquinone] iron-sulfur protein 4, mitochondrial; & Q9CXZ1 & Ndufs4 & 0,0 & 8,6 \\
\hline NADH dehydrogenase [ubiquinone] iron-sulfur protein 6, mitochondrial; & P52503 & Ndufs6 & 0,0 & 11,2 \\
\hline NADH-cytochrome b5 reductase 3 ; & Q9DCN2 & Cyb5r3 & $-0,2$ & 5,6 \\
\hline NADP-dependent malic enzyme; & P06801 & Me1 & $-0,1$ & 9,6 \\
\hline Nascent polypeptide-associated complex subunit alpha, muscle-specific form; & P70670 & Naca & 0,1 & 25,6 \\
\hline Nectin-4; & Q8R007 & Pvrl4 & 0,6 & 5,7 \\
\hline NEDD8-activating enzyme E1 catalytic subunit; & Q8C878 & Uba3 & 1,1 & 1,8 \\
\hline Neudesin; & Q9CQ45 & Nenf & 0,9 & 6,4 \\
\hline Neural cell adhesion molecule 1 ; & P13595 & Ncam1 & 0,7 & 1,5 \\
\hline Neuromodulin; & P06837 & Gap43 & 0,1 & 7,5 \\
\hline Neuroplastin; & P97300 & Nptn & 0,0 & 8,1 \\
\hline Neutral alpha-glucosidase $A B$ & Q8BHN3 & Ganab & 0,0 & 3,6 \\
\hline NFU1 iron-sulfur cluster scaffold homolog, mitochondrial; & Q9QZ23 & Nfu1 & 0,3 & 3,1 \\
\hline NHP2-like protein 1 & Q9D0T1 & Nhp2l1 & $-0,4$ & 23,4 \\
\hline Nicotinamide phosphoribosyltransferase; & Q99KQ4 & Nampt & 0,2 & 2,2 \\
\hline Nidogen-1; & P10493 & Nid1 & $-0,2$ & 2,3 \\
\hline Non-specific lipid-transfer protein; & P32020 & Scp2 & 0,0 & 2 \\
\hline NSFL1 cofactor $p 47$ & Q9CZ44 & Nsfl1c & $-0,3$ & 5 \\
\hline Nuclear autoantigenic sperm protein; & Q99MD9 & Nasp & $-0,5$ & 8,7 \\
\hline Nuclear factor 1 A-type; & Q02780 & Nfia & 0,4 & 10,2 \\
\hline Nuclear migration protein nudC; & 035685 & Nudc & $-0,6$ & 7,8 \\
\hline Nuclear transport factor 2 & P61971 & Nutf2 & 0,4 & 5,5 \\
\hline Nuclear ubiquitous casein and cyclin-dependent kinase substrate 1 ; & Q80XU3 & Nucks1 & 0,2 & 3,8 \\
\hline Nuclease-sensitive element-binding protein 1 & P62960 & Ybx1 & 0,0 & 23,9 \\
\hline Nucleobindin-1; & Q02819 & Nucb1 & 0,2 & 13,3 \\
\hline Nucleobindin-2; & P81117 & Nucb2 & $-0,1$ & 7,4 \\
\hline Nucleolar protein 56 & Q9D6Z1 & Nop56 & 0,4 & 4,3 \\
\hline Nucleolar protein 58; & Q6DFW4 & Nop58 & $-1,8$ & 2,2 \\
\hline Nucleolar RNA helicase 2; & Q9JIK5 & $\mathrm{Dd} \times 21$ & 0,5 & 2,7 \\
\hline Nucleolin; & P09405 & $\mathrm{Ncl}$ & 0,2 & 11 \\
\hline Nucleophosmin; & Q61937 & Npm1 & 0,5 & 5,1 \\
\hline Nucleoprotein TPR; & F6ZDS4 & $\mathrm{Tpr}$ & $-0,6$ & 1,2 \\
\hline Nucleoside diphosphate kinase A; & P15532 & Nme1 & $-0,2$ & 44,1 \\
\hline Nucleoside diphosphate kinase; & E9PZF0 & Gm20390 & 0,1 & 47,2 \\
\hline Nucleosome assembly protein 1-like 4; & Q78ZA7 & Nap1|4 & $-0,4$ & 2,9 \\
\hline OCIA domain-containing protein 1 & Q9CRD0 & Ociad1 & 0,3 & 3,2 \\
\hline Oligoribonuclease, mitochondrial; & Q9D8S4 & Rexo2 & 0,7 & 4,2 \\
\hline Palmitoyl-protein thioesterase 1 & 088531 & Ppt1 & $-0,2$ & 4,9 \\
\hline Paralemmin-1; & Q9Z0P4 & Palm & $-0,1$ & 2,1 \\
\hline Parathymosin; & Q9D0J8 & Ptms & 0,4 & 10,9 \\
\hline Parvalbumin alpha; & P32848 & Pvalb & 2,1 & 12,7 \\
\hline PC4 and SFRS1-interacting protein; & Q99JF8 & Psip1 & 0,4 & 7,3 \\
\hline PDZ and LIM domain protein $1 ;$ & 070400 & Pdlim1 & $-0,2$ & 11,3 \\
\hline PDZ and LIM domain protein 2; & Q8R1G6 & Pdlim2 & 0,2 & 4,6 \\
\hline PDZ and LIM domain protein 5; & Q8CI51 & Pdlim5 & $-0,7$ & 4,4 \\
\hline Peptidyl-prolyl cis-trans isomerase A; & P17742 & Ppia & $-0,2$ & 4,8 \\
\hline Peptidyl-prolyl cis-trans isomerase B; & P24369 & Ppib & $-0,2$ & 22,7 \\
\hline Peptidyl-prolyl cis-trans isomerase C; & P30412 & Ppic & 1,2 & 9,4 \\
\hline Peptidyl-prolyl cis-trans isomerase FKBP10; & Q61576 & Fkbp10 & $-0,4$ & 7,5 \\
\hline Peptidyl-prolyl cis-trans isomerase FKBP1A; & P26883 & Fkbp1a & $-0,1$ & 12 \\
\hline Peptidyl-prolyl cis-trans isomerase FKBP3; & Q62446 & Fkbp3 & 0,2 & 15,2 \\
\hline Peptidyl-prolyl cis-trans isomerase FKBP9; & Q9Z247 & Fkbp9 & $-0,4$ & 4,2 \\
\hline Peptidyl-tRNA hydrolase 2, mitochondrial; & Q8R2Y8 & Ptrh2 & 0,2 & 16 \\
\hline
\end{tabular}




\begin{tabular}{|c|c|c|c|c|}
\hline Protein & Uniprot & Gene name & $\begin{array}{c}\text { Fold change of } \\
\text { XPRESS ratio } \\
\left.\text { (Adam } 17^{\Delta K C} / \mathrm{WT}\right)\end{array}$ & $\begin{array}{c}\text { Sequence } \\
\text { coverage [\%] }\end{array}$ \\
\hline Perilipin-1; & Q8CGN5 & Plin1 & 0,4 & 11,6 \\
\hline Perilipin-2; & P43883 & Plin2 & $-0,9$ & 10,1 \\
\hline Perilipin-3; & Q9DBG5 & Plin3 & $-0,1$ & 5,5 \\
\hline Perilipin-4; & 088492 & Plin4 & 0,4 & 12,5 \\
\hline Periostin; & Q62009 & Postn & 0,1 & 13 \\
\hline Periplakin; & Q9R269 & Ppl & $-1,2$ & 0,7 \\
\hline Peroxiredoxin-1; & P35700 & Prdx1 & 0,0 & 28,2 \\
\hline Peroxiredoxin-2; & Q61171 & Prdx2 & 0,1 & 29,3 \\
\hline Peroxiredoxin-4; & 008807 & Prdx4 & $-1,1$ & 9,5 \\
\hline Peroxiredoxin-5, mitochondrial; & P99029 & Prdx5 & 0,0 & 20 \\
\hline Peroxiredoxin-6; & D3ZOY2 & $\operatorname{Prd} \times 6$ & 0,3 & 18 \\
\hline Peroxisomal acyl-coenzyme A oxidase 1 ; & Q9ROHO & Acox1 & 0,7 & 5,6 \\
\hline Peroxisomal acyl-coenzyme A oxidase 3 ; & Q9EPL9 & Acox3 & 0,0 & 2,6 \\
\hline Peroxisomal biogenesis factor 19 & Q8VCI5 & Pex19 & 0,1 & 8 \\
\hline Peroxisomal multifunctional enzyme type 2 ; & P51660 & Hsd17b4 & 0,5 & 2,6 \\
\hline Persulfide dioxygenase ETHE1, mitochondrial; & Q9DCM0 & Ethe1 & $-0,6$ & 6,7 \\
\hline PEST proteolytic signal-containing nuclear protein; & Q6P814 & Pcnp & 0,2 & 29,8 \\
\hline PHD finger-like domain-containing protein $5 \mathrm{~A}$; & P83870 & Phf5a & 0,3 & 6,4 \\
\hline Phenylalanine--tRNA ligase alpha subunit; & Q8C0C7 & Farsa & $-1,1$ & 1,4 \\
\hline Phosphate carrier protein, mitochondrial; & Q8VEM8 & Slc25a3 & 0,1 & 14,8 \\
\hline Phosphatidylethanolamine-binding protein 1; & P70296 & Pebp1 & $-0,1$ & 32,6 \\
\hline Phosphatidylinositol transfer protein alpha isoform; & P53810 & Pitpna & $-0,9$ & 10,3 \\
\hline Phosphoglucomutase-1; & Q9D0F9 & Pgm1 & 0,2 & 5,5 \\
\hline Phosphoglucomutase-2; & Q7TSV4 & Pgm2 & 0,4 & 4,2 \\
\hline Phosphoglycerate kinase 1 & P09411 & Pgk1 & 0,2 & 6 \\
\hline Phosphoglycerate mutase 1 & Q9DBJ1 & Pgam1 & $-0,2$ & 18,9 \\
\hline Phosphoglycerate mutase 2 & 070250 & Pgam2 & 1,6 & 15,4 \\
\hline Phosphoglycolate phosphatase; & Q8CHP8 & Pgp & $-0,3$ & 10,3 \\
\hline Phospholipase B-like 1; & Q8VCI0 & Plbd1 & 0,0 & 5,3 \\
\hline Phospholipase D3; & 035405 & Pld3 & $-0,5$ & 1,8 \\
\hline Phospholipid hydroperoxide glutathione peroxidase, mitochondrial; & 070325 & Gpx4 & $-0,1$ & 14,6 \\
\hline Phosphomannomutase 2 & Q9Z2M7 & Pmm2 & $-0,3$ & 7,9 \\
\hline Phosphoribosyl pyrophosphate synthase-associated protein 2; & Q8R574 & Prpsap2 & 0,0 & 8,4 \\
\hline Phosphoserine aminotransferase; & Q99K85 & Psat1 & $-0,3$ & 3,8 \\
\hline PITH domain-containing protein 1 & Q8BWR2 & Pithd1 & 0,0 & 3,8 \\
\hline Plasminogen activator inhibitor 1 RNA-binding protein; & Q9CY58 & Serbp1 & 0,9 & 11,5 \\
\hline Plasminogen; & P20918 & Plg & 0,4 & 1 \\
\hline Plastin-2; & Q61233 & Lcp1 & 0,9 & 3,5 \\
\hline Plastin-3; & Q99K51 & Pls3 & $-0,2$ & 3,7 \\
\hline Platelet glycoprotein 4; & Q08857 & $\mathrm{Cd} 36$ & 0,3 & 4,2 \\
\hline Platelet-activating factor acetylhydrolase IB subunit alpha; & P63005 & Pafah1b1 & $-0,7$ & 13,2 \\
\hline Plectin; & Q9QXS1 & Plec & 0,0 & 0,9 \\
\hline Poly(rC)-binding protein 1 & P60335 & Pcbp1 & 0,2 & 9,3 \\
\hline Poly(rC)-binding protein 2 & Q61990 & Pcbp2 & $-0,1$ & 6,6 \\
\hline Poly(U)-specific endoribonuclease; & Q3V188 & Endou & $-0,6$ & 6,8 \\
\hline Polyadenylate-binding protein 1 & P29341 & Pabpc1 & $-1,2$ & 2,4 \\
\hline Polymerase I and transcript release factor; & 054724 & Ptrf & 0,1 & 24,2 \\
\hline PRA1 family protein 3 & Q8R5J9 & Arl6ip5 & 1,5 & 3,7 \\
\hline Prefoldin subunit 2 & 070591 & Pfdn2 & 0,0 & 13,6 \\
\hline Prefoldin subunit 3; & P61759 & Vbp1 & 0,1 & 3,6 \\
\hline Prefoldin subunit 4; & E9Q6U4 & Pfdn4 & 0,0 & 11,5 \\
\hline Prefoldin subunit 5; & Q9WU28 & Pfdn5 & $-0,1$ & 17,6 \\
\hline Prelamin-A/C; & P48678 & Lmna & $-0,5$ & 27,5 \\
\hline Pre-mRNA-processing factor 19; & Q99KP6 & Prpf19 & 0,3 & 6 \\
\hline Prenylated Rab acceptor protein 1 ; & Q9Z0S9 & Rabac1 & 0,0 & 4,3 \\
\hline Prenylcysteine oxidase; & Q9CQF9 & Pcyox1 & 0,0 & 8,1 \\
\hline Probable ATP-dependent RNA helicase DDX5; & Q61656 & $\mathrm{Ddx} 5$ & $-0,1$ & 8,1 \\
\hline Probable global transcription activator SNF2L1; & Q6PGB8 & Smarca1 & 0,6 & 2,4 \\
\hline Profilin-1; & P62962 & Pfn1 & 0,0 & 26,4 \\
\hline Programmed cell death protein 10; & Q8VE70 & Pdcd10 & 0,0 & 15,2 \\
\hline Programmed cell death protein 4 ; & Q61823 & Pdcd4 & 0,8 & 2,3 \\
\hline Prohibitin; & P67778 & Phb & 0,2 & 11,8 \\
\hline Prohibitin-2; & 035129 & Phb2 & $-0,1$ & 30,8 \\
\hline Prolargin; & Q9JK53 & Prelp & $-0,5$ & 13 \\
\hline Proliferating cell nuclear antigen; & P17918 & Pcna & 0,4 & 17,2 \\
\hline Proliferation-associated protein $2 \mathrm{G} 4$ & P50580 & $\mathrm{Pa} 2 \mathrm{~g} 4$ & $-0,3$ & 17,8 \\
\hline Prolow-density lipoprotein receptor-related protein 1 ; & Q91ZX7 & Lrp1 & $-0,2$ & 1,9 \\
\hline Prolyl 4-hydroxylase subunit alpha-2; & Q60716 & P4ha2 & $-0,3$ & 5,4 \\
\hline Prostaglandin E synthase 3; & Q9R0Q7 & Ptges3 & 0,9 & 5,4 \\
\hline Prostaglandin F2 receptor negative regulator; & Q9WV91 & Ptgfrn & 0,5 & 1,1 \\
\hline Prostaglandin reductase 1 & Q91YR9 & Ptgr1 & $-0,2$ & 4 \\
\hline Proteasome activator complex subunit 1 & P97371 & Psme1 & 0,4 & 19 \\
\hline Proteasome activator complex subunit 2 & P97372 & Psme2 & $-0,2$ & 5,5 \\
\hline
\end{tabular}




\begin{tabular}{|c|c|c|c|c|}
\hline Protein & Uniprot & Gene name & $\begin{array}{c}\text { Fold change of } \\
\text { XPRESS ratio } \\
\left.\text { (Adam17 }{ }^{\Delta K C} / \mathrm{WT}\right)\end{array}$ & $\begin{array}{c}\text { Sequence } \\
\text { coverage [\%] }\end{array}$ \\
\hline Proteasome activator complex subunit 3; & P61290 & Psme3 & 0,0 & 13,4 \\
\hline Proteasome subunit alpha type-1; & Q9R1P4 & Psma1 & 0,1 & 16,3 \\
\hline Proteasome subunit alpha type-2; & P49722 & Psma2 & 0,1 & 6 \\
\hline Proteasome subunit alpha type-3; & 070435 & Psma3 & $-0,2$ & 15 \\
\hline Proteasome subunit alpha type- 4 & Q9R1P0 & Psma4 & 0,0 & 12,9 \\
\hline Proteasome subunit alpha type-5; & Q9Z2U1 & Psma5 & 0,0 & 17,4 \\
\hline Proteasome subunit alpha type-6; & Q9QUM9 & Psma6 & $-0,1$ & 15,4 \\
\hline Proteasome subunit alpha type-7; & Q9Z2U0 & Psma7 & $-0,2$ & 25,4 \\
\hline Proteasome subunit beta type- 1 ; & 009061 & Psmb1 & $-0,1$ & 25,4 \\
\hline Proteasome subunit beta type- 2 & Q9R1P3 & Psmb2 & 0,5 & 7 \\
\hline Proteasome subunit beta type-3; & Q9R1P1 & Psmb3 & $-0,1$ & 7,8 \\
\hline Proteasome subunit beta type- 4 & P99026 & Psmb4 & 0,1 & 5,7 \\
\hline Proteasome subunit beta type-5; & 055234 & Psmb5 & 0,0 & 29,9 \\
\hline Proteasome subunit beta type- 6 & Q60692 & Psmb6 & $-3,4$ & 4,2 \\
\hline Proteasome subunit beta type-7; & P70195 & Psmb7 & $-0,2$ & 14,4 \\
\hline Protein 4930438A08Rik; & Q5SPH3 & 4930438A08Rik & $-0,6$ & 5,6 \\
\hline Protein Adh6b; & Q3UQ40 & Adh6b & $-0,3$ & 4 \\
\hline Protein Ahnak; & E9Q616 & Ahnak & $-0,2$ & 46,3 \\
\hline Protein Ahnak2; & F7CVJ5 & Ahnak2 & $-1,2$ & 19 \\
\hline Protein argonaute-2; & Q8CJG0 & Ago2 & $-0,3$ & 2,8 \\
\hline Protein BC117090; & L7N257 & BC117090 & 1,2 & 32,3 \\
\hline Protein BRICK1; & Q91VR8 & Brk1 & $-0,3$ & 12 \\
\hline Protein Cald1; & E9QA16 & Cald1 & $-0,3$ & 24 \\
\hline Protein canopy homolog 2; & Q9QXT0 & Cnpy2 & $-0,1$ & 18,7 \\
\hline Protein canopy homolog 4 & Q8BQ47 & Cnpy4 & 0,3 & 9 \\
\hline Protein CDV3; & Q4VAA2 & Cdv3 & $-0,1$ & 5,7 \\
\hline Protein Col22a1; & E9Q7P1 & Col22a1 & $-6,5$ & 1,4 \\
\hline Protein Col6a3; & E9PWQ3 & Col6a3 & $-0,1$ & 5,8 \\
\hline Protein Crnn; & D3YUU6 & Crnn & $-1,5$ & 16,7 \\
\hline Protein CutA; & Q9CQ89 & Cuta & 0,2 & 9,1 \\
\hline Protein Cyp4f39; RIKEN cDNA 4732474A20, isoform CRA_b; & Q8BGU0 & Сур4f39 & 0,0 & 1,7 \\
\hline Protein disulfide-isomerase $\mathrm{A} 3$; & P27773 & Pdia3 & $-0,2$ & 22,8 \\
\hline Protein disulfide-isomerase A4; & P08003 & Pdia4 & $-0,4$ & 15,7 \\
\hline Protein disulfide-isomerase A6; & Q922R8 & Pdia6 & 0,0 & 16,6 \\
\hline Protein disulfide-isomerase TMX3; & Q8BXZ1 & $\operatorname{Tm} \times 3$ & $-0,3$ & 3,5 \\
\hline Protein disulfide-isomerase; & P09103 & P4hb & $-0,3$ & 31,2 \\
\hline Protein DJ-1; & Q99LX0 & Park7 & 0,2 & 16,7 \\
\hline Protein Dr1; & Q91WV0 & Dr1 & 1,9 & 4 \\
\hline Protein FAM192A; & Q91WE2 & Fam192a & 0,1 & 3,9 \\
\hline Protein FAM25C; & Q8CF02 & Fam 25c & $-0,5$ & 38,2 \\
\hline Protein FAM26D; & Q8CE93 & Fam26d & 0,8 & 3,5 \\
\hline Protein Fga; & E9PV24 & Fga & 1,1 & 4,8 \\
\hline Protein HEXIM1; & Q8R409 & Hexim1 & 0,3 & 9 \\
\hline Protein ITFG3; & Q8C0Z1 & Itfg3 & $-0,2$ & 9,7 \\
\hline Protein kinase $\mathrm{C}$ delta-binding protein; & Q91VJ2 & Prkcdbp & 0,2 & 18,1 \\
\hline Protein Krtap11-1; & E9Q2E9 & Krtap11 1 & $-1,1$ & 7,7 \\
\hline Protein LYRIC; & Q80WJ7 & Mtdh & 0,1 & 3,5 \\
\hline Protein LZIC; & Q8K3C3 & Lzic & 0,1 & 16,1 \\
\hline Protein NipSnap homolog 1 & 055125 & Nipsnap1 & 0,0 & 3,2 \\
\hline Protein NipSnap homolog 3B; & Q9CQE1 & Nipsnap3b & 2,0 & 4,6 \\
\hline Protein Nolc1; & E9Q5C9 & Nolc1 & 0,5 & 3,3 \\
\hline Protein Numa1; & E9Q7G0 & Numa1 & $-0,2$ & 0,9 \\
\hline Protein phosphatase 1 regulatory subunit 7; & Q3UM45 & Ppp1r7 & 0,0 & 2,5 \\
\hline Protein PRRC2C; & Q3TLH4 & Prrc2c & 0,6 & 1,5 \\
\hline Protein RCC2; & Q8BK67 & $\operatorname{Rcc} 2$ & 0,8 & 8,3 \\
\hline Protein S100-A11; & P50543 & S100a11 & $-0,1$ & 7,1 \\
\hline Protein S100-A13; & P97352 & S100a13 & 0,3 & 29,6 \\
\hline Protein S100-A14; & Q9D2Q8 & S100a14 & 0,3 & 14,4 \\
\hline Protein S100a16; & D3Z2Y6 & S100a16 & $-0,4$ & 32,9 \\
\hline Protein S100-A3; & P62818 & S100a3 & $-0,7$ & 20,8 \\
\hline Protein S100-A6; & P14069 & S100a6 & $-0,3$ & 16,9 \\
\hline Protein SET; & Q9EQU5 & Set & 0,5 & 24,2 \\
\hline Protein Sf3b2; Splicing factor 3b, subunit 2; & Q3UJB0 & $\mathrm{Sf} 3 \mathrm{~b} 2$ & $-0,4$ & 1,1 \\
\hline $\begin{array}{l}\text { Protein Slc25a1; Solute carrier family } 25 \text { (Mitochondrial carrier, citrate } \\
\text { transporter), member } 1 \text {; }\end{array}$ & Q8JZU2 & Slc25a1 & 0,6 & 5,1 \\
\hline Protein Spink5; Serine peptidase inhibitor, Kazal type 5; & Q148R4 & Spink5 & $-0,8$ & 0,8 \\
\hline Protein Tfg; Trk-fused; & B8JJG6 & Tfg & 0,2 & 20,2 \\
\hline Protein Tmed7; & D3YZZ5 & Tmed7 & $-0,2$ & 5,3 \\
\hline Protein transport protein Sec61 subunit alpha isoform 1; & P61620 & Sec61a1 & $-0,1$ & 4 \\
\hline Protein transport protein Sec61 subunit beta; & Q9CQS8 & Sec61b & 0,3 & 11,5 \\
\hline Protein tyrosine phosphatase type IVA 2; & 070274 & Ptp4a2 & 2,4 & 8,4 \\
\hline Protein Uvrag; & Q8COK8 & Uvrag & 3,3 & 3 \\
\hline Protein-glutamine gamma-glutamyltransferase $\mathrm{E}$; & Q08189 & Tgm3 & $-0,3$ & 17,3 \\
\hline
\end{tabular}




\begin{tabular}{|c|c|c|c|c|}
\hline Protein & Uniprot & Gene name & $\begin{array}{c}\text { Fold change of } \\
\text { XPRESS ratio } \\
\left.\text { (Adam } 17^{\Delta K C} / \mathrm{WT}\right)\end{array}$ & $\begin{array}{c}\text { Sequence } \\
\text { coverage [\%] }\end{array}$ \\
\hline Proteolipid protein 2; & Q9R1Q7 & Plp2 & $-0,1$ & 7,9 \\
\hline Prothymosin alpha; & P26350 & Ptma & 0,7 & 11,7 \\
\hline Pterin-4-alpha-carbinolamine dehydratase 2 ; & Q9CZL5 & Pcbd2 & 0,1 & 7,4 \\
\hline Purine nucleoside phosphorylase; & P23492 & Pnp & $-0,1$ & 9 \\
\hline Putative adenosylhomocysteinase 3 ; & Q68FL4 & Ahcyl2 & $-0,3$ & 5,5 \\
\hline Putative GTP cyclohydrolase 1 type 2 Nif3l1; & Q9EQ80 & Nif3l1 & 0,8 & 5,5 \\
\hline Putative phospholipase B-like 2; & Q3TCN2 & Plbd2 & $-0,1$ & 1,5 \\
\hline Putative pre-mRNA-splicing factor ATP-dependent RNA helicase DHX15; & 035286 & Dhx15 & 0,0 & 2,5 \\
\hline Putative RNA-binding protein 3; & 089086 & $\mathrm{Rbm} 3$ & 0,1 & 15,7 \\
\hline Putative RNA-binding protein Luc7-like 2; & Q7TNC4 & Luc712 & 1,0 & 5,8 \\
\hline Pyridoxal kinase; & Q8K183 & Pdxk & 0,4 & 8,7 \\
\hline Pyrroline-5-carboxylate reductase 3; & Q9DCC4 & Pycrl & 0,4 & 6,9 \\
\hline Pyruvate kinase PKM; & P52480 & $\mathrm{Pkm}$ & 0,0 & 23 \\
\hline Rab GDP dissociation inhibitor beta; & Q61598 & Gdi2 & 0,7 & 5,4 \\
\hline Radixin; & P26043 & $\mathrm{Rdx}$ & $-0,3$ & 5,8 \\
\hline Ragulator complex protein LAMTOR1; & Q9CQ22 & Lamtor1 & 0,0 & 8,1 \\
\hline Ragulator complex protein LAMTOR5; & Q9D1L9 & Lamtor5 & $-0,1$ & 22 \\
\hline Ran-binding protein 3; & Q9CT10 & Ranbp3 & 0,3 & 2,4 \\
\hline Ran-specific GTPase-activating protein; & P34022 & Ranbp1 & 0,2 & 13,3 \\
\hline Ras GTPase-activating protein-binding protein 1; & P97855 & G3bp1 & $-0,5$ & 1,9 \\
\hline Ras suppressor protein 1 & Q01730 & Rsu1 & $-0,1$ & 3,1 \\
\hline Ras-related $\mathrm{C} 3$ botulinum toxin substrate 1 ; & P63001 & Rac1 & 0,0 & 21,4 \\
\hline Ras-related protein Rab-10; & P61027 & Rab10 & 0,2 & 24 \\
\hline Ras-related protein Rab-11B; & P46638 & Rab11b & 0,0 & 46,8 \\
\hline Ras-related protein Rab-14; & Q91V41 & Rab14 & 0,1 & 33,5 \\
\hline Ras-related protein Rab-18; & P35293 & Rab18 & $-0,3$ & 12,6 \\
\hline Ras-related protein Rab-1A; & P62821 & Rab1A & 0,1 & 35,1 \\
\hline Ras-related protein Rab-1B; & Q9D1G1 & Rab1b & $-0,2$ & 34,3 \\
\hline Ras-related protein Rab-25; & Q9WTL2 & Rab25 & $-0,2$ & 13,8 \\
\hline Ras-related protein Rab-27B; & Q99P58 & Rab27b & $-0,2$ & 6,4 \\
\hline Ras-related protein Rab-2A; & P53994 & Rab2a & 0,1 & 13,2 \\
\hline Ras-related protein Rab-35; & Q6PHN9 & Rab35 & 0,1 & 9,5 \\
\hline Ras-related protein Rab-5A; & Q9CQD1 & Rab5a & $-0,4$ & 22,3 \\
\hline Ras-related protein Rab-5B; & P61021 & Rab5b & 0,1 & 15,8 \\
\hline Ras-related protein Rab-5C; & P35278 & Rab5c & 0,1 & 16,7 \\
\hline Ras-related protein Rab-6A; & P35279 & Rab6a & 0,0 & 12 \\
\hline Ras-related protein Rab-7a; & P51150 & Rab7a & 0,2 & 16,9 \\
\hline Ras-related protein Rab-8A; & P55258 & Rab8a & 1,3 & 23,7 \\
\hline Ras-related protein Rap-1b; & Q99JI6 & Rap1b & 0,1 & 10,9 \\
\hline Receptor expression-enhancing protein 5; & Q60870 & Reep5 & $-1,6$ & 10,3 \\
\hline Redox-regulatory protein FAM213A; & Q9CYH2 & Fam213a & $-0,3$ & 24,3 \\
\hline Regulator of nonsense transcripts 1 ; & Q9EPU0 & Upf1 & 0,9 & 6 \\
\hline Reticulocalbin-1; & Q05186 & Ren1 & $-0,2$ & 10,2 \\
\hline Reticulocalbin-2; & Q8BP92 & $\operatorname{Ren} 2$ & $-0,6$ & 4,7 \\
\hline Reticulocalbin-3; & Q8BH97 & Ren3 & 0,1 & 10,6 \\
\hline Reticulon-3; & Q9ES97 & Rtn3 & 0,3 & 1,1 \\
\hline Reticulon-4; & Q99P72 & Rtn4 & 0,1 & 7,6 \\
\hline Retinoid-inducible serine carboxypeptidase; & Q920A5 & Scpep1 & $-0,1$ & 3,8 \\
\hline Retroviral-like aspartic protease 1 & Q09PK2 & Asprv1 & 0,0 & 4,4 \\
\hline Rho GDP-dissociation inhibitor 1; & Q99PT1 & Arhgdia & $-0,1$ & 20,6 \\
\hline Rho GDP-dissociation inhibitor 2; & Q61599 & Arhgdib & $-0,3$ & 11,5 \\
\hline Rho-related GTP-binding protein RhoC; & Q62159 & Rhoc & $-0,1$ & 11,4 \\
\hline Ribose-phosphate pyrophosphokinase 1 ; & Q9D7G0 & Prps1 & 0,2 & 5,3 \\
\hline Ribose-phosphate pyrophosphokinase 2; & Q9CS42 & Prps2 & $-0,7$ & 6,3 \\
\hline Ribosomal protein; & Q5XJF6 & Rpl10a & 0,2 & 23,5 \\
\hline Ribosome-binding protein 1 ; & Q99PL5 & Rrbp1 & $-0,3$ & 9,8 \\
\hline RNA-binding protein 25 ; & B2RY56 & Rbm25 & 0,1 & 3,8 \\
\hline RNA-binding protein $39 ;$ & Q8VH51 & Rbm39 & 1,9 & 7,1 \\
\hline RNA-binding protein $8 \mathrm{~A}$; & Q9CWZ3 & Rbm8a & $-0,2$ & 4,6 \\
\hline RNA-binding protein EWS; & Q61545 & Ewsr1 & 0,1 & 3,7 \\
\hline RNA-binding protein FUS; & P56959 & Fus & 0,2 & 42,3 \\
\hline RNA-binding protein Raly; & Q64012 & Raly & 0,0 & 7,6 \\
\hline RuvB-like 1; & P60122 & Ruvbl1 & 0,1 & 11,8 \\
\hline RuvB-like 2; & Q9WTM5 & Ruvbl2 & $-0,1$ & 16,2 \\
\hline S-adenosylmethionine synthase isoform type-2; & Q3THS6 & Mat2a & 0,4 & 8,4 \\
\hline SAP domain-containing ribonucleoprotein; & Q9D1J3 & Sarnp & 0,3 & 9 \\
\hline Sarcoplasmic/endoplasmic reticulum calcium ATPase 2; & 055143 & Atp2a2 & $-0,2$ & 1 \\
\hline Scaffold attachment factor B1; & D3YXK2 & Safb & $-0,5$ & 2,2 \\
\hline Scaffold attachment factor B2; & Q80YR5 & Safb2 & 0,1 & 11,2 \\
\hline Sciellin; & Q9EQG3 & Scel & $-0,5$ & 3,4 \\
\hline Secreted frizzled-related protein $1 ;$ & Q8C4U3 & Sfrp1 & 0,3 & 3,2 \\
\hline Secretory carrier-associated membrane protein 2; & Q9ERNO & Scamp2 & $-0,4$ & 3,6 \\
\hline
\end{tabular}




\begin{tabular}{|c|c|c|c|c|}
\hline Protein & Uniprot & Gene name & $\begin{array}{c}\text { Fold change of } \\
\text { XPRESS ratio } \\
\left.\text { (Adam } 17^{\triangle K C} / \mathrm{WT}\right)\end{array}$ & $\begin{array}{c}\text { Sequence } \\
\text { coverage [\%] }\end{array}$ \\
\hline Selenium-binding protein 1 & P17563 & Selenbp1 & 0,7 & 17,6 \\
\hline Selenoprotein M; & Q8VHC3 & Selm & $-0,2$ & 4,8 \\
\hline Septin-11; & Q8C1B7 & Sept11 & 0,2 & 12,1 \\
\hline Septin-2; & P42208 & Sept2 & $-0,1$ & 22,7 \\
\hline Septin-7; & 055131 & Sept7 & 1,2 & 5 \\
\hline Septin-8; & Q8CHH9 & Sept8 & $-0,2$ & 7,7 \\
\hline Septin-9; & Q80UG5 & Sept9 & $-1,0$ & 4,1 \\
\hline Serine hydroxymethyltransferase, cytosolic; & P50431 & Shmt1 & 0,2 & 6,8 \\
\hline Serine hydroxymethyltransferase; & Q9CZN7 & Shmt2 & 0,2 & 14,1 \\
\hline Serine/arginine-rich splicing factor 1 ; & Q6PDM2 & Srsf1 & 0,1 & 18,5 \\
\hline Serine/arginine-rich splicing factor 10 ; & Q9ROU0 & Srsf10 & 0,4 & 3,8 \\
\hline Serine/arginine-rich splicing factor 2 ; & Q62093 & Srsf2 & 0,3 & 14,5 \\
\hline Serine/arginine-rich splicing factor 3 ; & P84104 & Srsf3 & 0,2 & 15,2 \\
\hline Serine/arginine-rich splicing factor 5 ; & O35326 & Srsf5 & 0,7 & 11,5 \\
\hline Serine/arginine-rich splicing factor 6 ; & Q3TWW8 & Srsf6 & 0,4 & 10,9 \\
\hline Serine/arginine-rich splicing factor 7 ; & Q8BL97 & Srsf7 & 0,1 & 3,4 \\
\hline $\begin{array}{l}\text { Serine/threonine-protein phosphatase } 2 \mathrm{~A} 55 \mathrm{kDa} \text { regulatory subunit } \mathrm{B} \text { alpha } \\
\text { isoform; }\end{array}$ & Q6P1F6 & Ppp2r2a & $-0,3$ & 1,8 \\
\hline $\begin{array}{l}\text { Serine/threonine-protein phosphatase } 2 \mathrm{~A} 65 \mathrm{kDa} \text { regulatory subunit } \mathrm{A} \text { alpha } \\
\text { isoform; }\end{array}$ & Q76MZ3 & Ppp2r1a & $-0,2$ & 4,9 \\
\hline Serine/threonine-protein phosphatase $2 \mathrm{~A}$ catalytic subunit beta isoform; & P62715 & Ppp2cb & $-0,1$ & 5,2 \\
\hline Serine/threonine-protein phosphatase 2B catalytic subunit alpha isoform; & P63328 & Ppp3ca & 0,7 & 5,6 \\
\hline Serine/threonine-protein phosphatase 4 catalytic subunit; & P97470 & Ppp4c & 0,2 & 4,2 \\
\hline Serine/threonine-protein phosphatase CPPED1; & Q8BFS6 & Cpped1 & $-0,1$ & 4,6 \\
\hline Serine/threonine-protein phosphatase PGAM5, mitochondrial; & Q8BX10 & Pgam5 & 0,1 & 8,6 \\
\hline Serine/threonine-protein phosphatase PP1-alpha catalytic subunit; & P62137 & Ppp1ca & 0,0 & 15,2 \\
\hline Serine/threonine-protein phosphatase PP1-beta catalytic subunit; & P62141 & Ppp1cb & $-0,1$ & 13,8 \\
\hline Serine/threonine-protein phosphatase PP1-gamma catalytic subunit; & P63087 & Ppp1cc & 0,1 & 12,7 \\
\hline Serine-threonine kinase receptor-associated protein; & Q9Z1Z2 & Strap & $-0,3$ & 19,4 \\
\hline Serine--tRNA ligase, cytoplasmic; & P26638 & Sars & $-0,1$ & 8,8 \\
\hline Serotransferrin; & Q921I1 & Tf & 0,5 & 30,6 \\
\hline Serpin B12; & Q9D7P9 & Serpinb12 & 0,2 & 18,9 \\
\hline Serpin B5; & P70124 & Serpinb5 & 0,0 & 18,1 \\
\hline Serpin B6; & Q60854 & Serpinb6 & $-0,5$ & 6,3 \\
\hline Serpin $\mathrm{H} 1$; & P19324 & Serpinh1 & $-0,3$ & 12 \\
\hline Serrate RNA effector molecule homolog; & Q99MR6 & Srrt & $-0,1$ & 1,5 \\
\hline Serum albumin; & P07724 & Alb & 0,5 & 42,6 \\
\hline Serum deprivation-response protein; & Q63918 & Sdpr & 0,0 & 6,5 \\
\hline S-formylglutathione hydrolase; & Q9R0P3 & Esd & $-0,1$ & 33,3 \\
\hline SH3 domain-binding glutamic acid-rich-like protein 3; & Q91VW3 & Sh3bgrl3 & $-0,2$ & 27 \\
\hline SH3 domain-binding glutamic acid-rich-like protein; & Q9JJU8 & Sh3bgrl & $-0,2$ & 8,8 \\
\hline Short-chain dehydrogenase/reductase family $16 \mathrm{C}$ member 6 & Q05A13 & Sdr16c6 & 1,6 & 3,8 \\
\hline Sideroflexin-3; & Q91V61 & Sfxn3 & $-0,4$ & 2,5 \\
\hline Sigma non-opioid intracellular receptor 1 ; & 055242 & Sigmar1 & 0,0 & 5,8 \\
\hline Signal recognition particle 9 kDa protein; & P49962 & Srp9 & 0,0 & 10,5 \\
\hline Small nuclear ribonucleoprotein F; & P62307 & Snrpf & 0,0 & 9,3 \\
\hline Small nuclear ribonucleoprotein Sm D1; & P62315 & Snrpd1 & 0,2 & 9,2 \\
\hline Small nuclear ribonucleoprotein Sm D2; & P62317 & Snrpd2 & $-0,4$ & 47,5 \\
\hline Small nuclear ribonucleoprotein Sm D3; & P62320 & Snrpd3 & $-0,4$ & 16,7 \\
\hline Small nuclear ribonucleoprotein-associated protein B; & P27048 & Snrpb & 2,5 & 12,6 \\
\hline S-methyl-5'-thioadenosine phosphorylase; & Q9CQ65 & Mtap & 0,0 & 18 \\
\hline Sodium/potassium-transporting ATPase subunit alpha-1; & Q8VDN2 & Atp1a1 & $-0,3$ & 21,6 \\
\hline Sodium/potassium-transporting ATPase subunit beta-3; & P97370 & Atp1b3 & $-0,2$ & 10,4 \\
\hline Sorcin; & Q6P069 & Sri & 0,1 & 9,1 \\
\hline Sorting nexin-3; & 070492 & Snx3 & 0,0 & 4,3 \\
\hline SPARC; & P07214 & Sparc & $-0,5$ & 11,6 \\
\hline Spectrin alpha chain, non-erythrocytic $1 ;$ & P16546 & Sptan1 & $-0,2$ & 3,3 \\
\hline Spectrin beta chain, non-erythrocytic 1 ; & Q62261 & Sptbn1 & $-0,3$ & 1,4 \\
\hline S-phase kinase-associated protein 1 ; & Q9WTX5 & Skp1 & 1,1 & 8 \\
\hline Spliceosome RNA helicase Ddx39b; & Q9Z1N5 & Ddx39b & 0,1 & 15,2 \\
\hline Splicing factor 1 & Q64213 & Sf1 & 0,1 & 2,4 \\
\hline Splicing factor 3B subunit 1 & Q99NB9 & Sf3b1 & 0,1 & 1,3 \\
\hline Splicing factor 3B subunit 3; & Q921M3 & Sf3b3 & $-0,6$ & 1,9 \\
\hline Splicing factor U2AF 35 kDa subunit; & Q9D883 & U2af1 & 0,0 & 3,3 \\
\hline Splicing factor, proline- and glutamine-rich; & Q8VIJ6 & Sfpq & 0,2 & 9,3 \\
\hline Src substrate cortactin; & Q60598 & Cttn & $-0,2$ & 16,5 \\
\hline Stathmin; & P54227 & Stmn1 & 0,5 & 20,6 \\
\hline Stefin-3; & P35173 & Stfa3 & 0,4 & 18,4 \\
\hline Stomatin-like protein 2, mitochondrial; & Q99JB2 & Stoml2 & 0,4 & 4 \\
\hline Stress-70 protein, mitochondrial; & P38647 & Hspa9 & 0,0 & 9,1 \\
\hline Stress-induced-phosphoprotein 1 & Q60864 & Stip1 & $-0,1$ & 10,5 \\
\hline
\end{tabular}




\begin{tabular}{|c|c|c|c|c|}
\hline Protein & Uniprot & Gene name & $\begin{array}{c}\text { Fold change of } \\
\text { XPRESS ratio } \\
\left.\text { (Adam } 17^{\triangle K C} / \mathrm{WT}\right)\end{array}$ & $\begin{array}{c}\text { Sequence } \\
\text { coverage [\%] }\end{array}$ \\
\hline Structural maintenance of chromosomes protein 3; & Q9CW03 & Smc3 & $-0,1$ & 1 \\
\hline Succinate dehydrogenase [ubiquinone] flavoprotein subunit, mitochondrial; & Q8K2B3 & Sdha & 0,2 & 13,4 \\
\hline Succinate dehydrogenase [ubiquinone] iron-sulfur subunit, mitochondrial; & Q9CQA3 & Sdhb & 0,1 & 3,5 \\
\hline Sulfated glycoprotein 1; & Q61207 & Psap & 0,2 & 8,3 \\
\hline SUMO-activating enzyme subunit 2 ; & Q9Z1F9 & Uba2 & $-0,4$ & 4,9 \\
\hline SUN domain-containing protein 2 & Q8BJS4 & Sun2 & 0,3 & 5,7 \\
\hline Superoxide dismutase $[\mathrm{Cu}-\mathrm{Zn}]$ & P08228 & Sod1 & 0,4 & 32,5 \\
\hline Superoxide dismutase [Mn], mitochondrial; & P09671 & Sod2 & 0,0 & 6,3 \\
\hline Suprabasin; & Q8CIT9 & Sbsn & $-0,5$ & 10 \\
\hline Sushi domain-containing protein 2 ; & Q9DBX3 & Susd2 & $-0,3$ & 4,5 \\
\hline SWI/SNF complex subunit SMARCC2; & Q6PDG5 & Smarcc2 & $-0,1$ & 2,8 \\
\hline Synaptic vesicle membrane protein VAT-1 homolog; & Q62465 & Vat1 & $-0,4$ & 9,9 \\
\hline Syndecan-1; & P18828 & Sdc1 & $-0,6$ & 3,5 \\
\hline Syndecan-4; & 035988 & Sdc4 & 0,3 & 19,7 \\
\hline Syntaxin-12; & Q9ER00 & Stx12 & 0,4 & 15 \\
\hline TAR DNA-binding protein 43 ; & Q921F2 & Tardbp & 0,2 & 6,8 \\
\hline T-complex protein 1 subunit alpha; & P11983 & Tcp1 & $-0,2$ & 13,8 \\
\hline T-complex protein 1 subunit beta; & P80314 & Cct2 & $-0,7$ & 8,8 \\
\hline T-complex protein 1 subunit delta; & P80315 & Cct4 & 0,3 & 17,4 \\
\hline T-complex protein 1 subunit epsilon; & P80316 & Cct5 & 0,1 & 17,2 \\
\hline T-complex protein 1 subunit eta; & P80313 & Cct7 & 0,6 & 13,1 \\
\hline T-complex protein 1 subunit gamma; & P80318 & Cct3 & $-0,2$ & 13,9 \\
\hline T-complex protein 1 subunit theta; & P42932 & Cct8 & $-0,1$ & 15 \\
\hline T-complex protein 1 subunit zeta; & P80317 & Cct6a & 0,2 & 9,8 \\
\hline Tetranectin; & P43025 & Clec3b & 0,8 & 5,9 \\
\hline Thimet oligopeptidase; & Q8C1A5 & Thop1 & 0,1 & 16,4 \\
\hline Thioredoxin domain-containing protein 12 ; & Q9CQU0 & Txndc12 & $-0,1$ & 8,8 \\
\hline Thioredoxin domain-containing protein 5 & Q91W90 & Txndc5 & $-0,3$ & 19,5 \\
\hline Thioredoxin; & P10639 & Txn & $-0,2$ & 21 \\
\hline Thioredoxin-dependent peroxide reductase, mitochondrial; & P20108 & Prdx3 & 0,0 & 4,3 \\
\hline THO complex subunit 4 & 008583 & Alyref & 0,2 & 5 \\
\hline Thy-1 membrane glycoprotein; & P01831 & Thy1 & 0,0 & 7,4 \\
\hline Thyroid hormone receptor-associated protein 3; & Q569Z6 & Thrap3 & 0,0 & 3,9 \\
\hline Titin; & A2ASS6 & Ttn & 2,8 & 0,1 \\
\hline Transaldolase; & Q93092 & Taldo1 & 0,3 & 9,2 \\
\hline Transcription and mRNA export factor ENY2; & Q9JIX0 & Eny2 & $-0,1$ & 25,7 \\
\hline Transcription elongation factor A protein 1 & P10711 & Tcea1 & 0,4 & 4 \\
\hline Transcription factor BTF3 homolog 4; & Q9CQH7 & Btf314 & 1,2 & 4,4 \\
\hline Transcription factor BTF3; & Q64152 & Btf3 & 0,0 & 9,3 \\
\hline Transcription intermediary factor 1-beta; & Q62318 & Trim28 & 0,6 & 4,6 \\
\hline Transcriptional activator protein Pur-beta; & 035295 & Purb & 0,1 & 17,6 \\
\hline Transcriptional repressor CTCF; & Q61164 & Ctcf & 0,1 & 1,2 \\
\hline Transducin-like enhancer protein 3; & Q08122 & Tle3 & 0,0 & 2,6 \\
\hline Transferrin receptor protein 1 & Q62351 & Tfrc & 0,5 & 5,4 \\
\hline Transformer-2 protein homolog beta; & P62996 & Tra2b & 0,0 & 4,8 \\
\hline Transforming growth factor-beta-induced protein ig-h3; & P82198 & Tgfbi & 0,0 & 3,2 \\
\hline Transgelin-2; & Q9WVA4 & Tagln2 & $-0,2$ & 29,1 \\
\hline Transitional endoplasmic reticulum ATPase; & Q01853 & $\mathrm{Vcp}$ & $-0,1$ & 31,8 \\
\hline Transketolase; & P40142 & Tkt & $-0,1$ & 23,6 \\
\hline Translationally-controlled tumor protein; & P63028 & Tpt1 & $-0,2$ & 23,3 \\
\hline Translin-associated protein $X$ & Q9QZE7 & Tsnax & 0,2 & 3,1 \\
\hline Translocation protein SEC62; & Q8BU14 & Sec62 & 0,1 & 2,3 \\
\hline Translocon-associated protein subunit alpha; & Q9CY50 & Ssr1 & $-0,2$ & 2,8 \\
\hline Translocon-associated protein subunit delta; & Q62186 & Ssr4 & $-0,1$ & 16,9 \\
\hline Transmembrane emp24 domain-containing protein $10 ;$ & Q9D1D4 & Tmed10 & $-0,1$ & 12,8 \\
\hline Transmembrane emp24 domain-containing protein 2 ; & Q9R0Q3 & Tmed2 & $-0,2$ & 8 \\
\hline Transmembrane emp24 domain-containing protein 5; & Q9CXE7 & Tmed5 & $-0,2$ & 5,2 \\
\hline Transmembrane emp24 domain-containing protein 9; & Q99KF1 & Tmed9 & $-0,1$ & 7,7 \\
\hline Transmembrane protein 14C; & Q9CQN6 & Tmem14c & 0,3 & 9,6 \\
\hline Transmembrane protein 33 & Q9CR67 & Tmem33 & $-0,6$ & 6,5 \\
\hline Transmembrane protein 43 ; & Q9DBS1 & Tmem43 & $-2,3$ & 6,3 \\
\hline Transthyretin; & P07309 & $\operatorname{Ttr}$ & 0,4 & 21,8 \\
\hline Treacle protein; & 008784 & Tcof1 & 0,3 & 5,9 \\
\hline Trichohyalin-like protein 1; & Q9D3P1 & Tchhl1 & $-1,4$ & 26,6 \\
\hline Triosephosphate isomerase; & P17751 & Tpi1 & $-0,2$ & 27,1 \\
\hline Tripartite motif-containing protein 29 ; & Q8R2Q0 & Trim29 & $-0,3$ & 7,2 \\
\hline Tripeptidyl-peptidase 1 & 089023 & Tpp1 & $-0,3$ & 3,7 \\
\hline tRNA-splicing ligase RtcB homolog; & Q99LF4 & Rtcb & 0,0 & 3,8 \\
\hline Tropomyosin alpha-1 chain; & E9Q448 & Tpm1 & $-0,2$ & 10,6 \\
\hline Tropomyosin alpha-4 chain; & Q6IRU2 & Tpm4 & $-0,2$ & 12,5 \\
\hline Tropomyosin beta chain; & A2AIM4 & Tpm2 & 0,0 & 19,7 \\
\hline
\end{tabular}




\begin{tabular}{|c|c|c|c|c|}
\hline Protein & Uniprot & Gene name & $\begin{array}{c}\text { Fold change of } \\
\text { XPRESS ratio } \\
\left.\text { (Adam } 17^{\triangle K C} / \mathrm{WT}\right)\end{array}$ & $\begin{array}{c}\text { Sequence } \\
\text { coverage [\%] }\end{array}$ \\
\hline Tryptophan--tRNA ligase, cytoplasmic; & P32921 & Wars & $-1,2$ & 5 \\
\hline Tubulin alpha-1B chain; & P05213 & Tuba1b & 0,1 & 33,7 \\
\hline Tubulin alpha-4A chain; & P68368 & Tuba4a & $-0,3$ & 30,6 \\
\hline Tubulin alpha-8 chain; & Q9JJZ2 & Tuba8 & $-0,4$ & 16,9 \\
\hline Tubulin beta-2A chain; & Q7TMM9 & Tubb2a & $-0,1$ & 24,3 \\
\hline Tubulin beta- 3 chain; & Q9ERD7 & Tubb3 & $-0,5$ & 23,3 \\
\hline Tubulin beta-4B chain; & P68372 & Tubb4b & 0,0 & 20,4 \\
\hline Tubulin beta- 5 chain; & P99024 & Tubb5 & 0,3 & 24,3 \\
\hline Tubulin polymerization-promoting protein family member 3 ; & Q9CRB6 & Tppp3 & 0,3 & 11,9 \\
\hline Tumor protein D52; & Q62393 & Tpd52 & 0,0 & 17,9 \\
\hline Tumor protein D54; & Q9CYZ2 & $\operatorname{Tpd} 5212$ & $-0,2$ & 6,3 \\
\hline Tumor-associated calcium signal transducer 2 ; & Q8BGV3 & Tacstd2 & $-0,4$ & 6,3 \\
\hline U1 small nuclear ribonucleoprotein 70 kDa; & Q62376 & Snrnp70 & 0,3 & 6,5 \\
\hline U1 small nuclear ribonucleoprotein $\mathrm{A}$; & Q62189 & Snrpa & $-0,1$ & 8,5 \\
\hline U2 small nuclear ribonucleoprotein A'; & P57784 & Snrpa1 & 2,1 & 16,2 \\
\hline U2 small nuclear ribonucleoprotein B"; & Q9CQI7 & Snrpb2 & 0,2 & 12,6 \\
\hline U4/U6.U5 tri-snRNP-associated protein 1; & Q97315 & Sart1 & 0,1 & 1,9 \\
\hline U5 small nuclear ribonucleoprotein 200 kDa helicase; & Q6P4T2 & Snrnp200 & $-0,3$ & 0,9 \\
\hline U6 snRNA-associated Sm-like protein LSm2; & 035900 & Lsm2 & 0,4 & 27,4 \\
\hline U6 snRNA-associated Sm-like protein LSm3; & P62311 & Lsm3 & 0,4 & 11,8 \\
\hline U6 snRNA-associated Sm-like protein LSm6; & P62313 & Lsm6 & 0,2 & 31,3 \\
\hline U6 snRNA-associated Sm-like protein LSm7; & Q9CQQ8 & Lsm7 & $-0,2$ & 7,8 \\
\hline U6 snRNA-associated Sm-like protein LSm8; & Q6ZWM4 & Lsm8 & 0,6 & 9,4 \\
\hline Ubiquitin carboxyl-terminal hydrolase 5; & P56399 & Usp5 & $-0,3$ & 2,2 \\
\hline Ubiquitin thioesterase OTUB1; & Q7TQI3 & Otub1 & $-0,6$ & 3,7 \\
\hline Ubiquitin-40S ribosomal protein S27a; & P62983 & Rps27a & 0,3 & 36,5 \\
\hline Ubiquitin-associated protein 2-like; & Q80X50 & Ubap2l & 0,1 & 4,8 \\
\hline Ubiquitin-conjugating enzyme E2 L3; & P68037 & Ube2l3 & 0,0 & 20,2 \\
\hline Ubiquitin-conjugating enzyme E2 N; & P61089 & Ube2n & $-0,5$ & 11,8 \\
\hline Ubiquitin-conjugating enzyme E2 R2; & Q6ZWZ2 & Ube2r2 & $-0,3$ & 3,4 \\
\hline Ubiquitin-fold modifier 1 & P61961 & Ufm1 & $-0,2$ & 26,3 \\
\hline Ubiquitin-fold modifier-conjugating enzyme 1 ; & Q9CR09 & Ufc1 & 0,0 & 18,3 \\
\hline Ubiquitin-like modifier-activating enzyme 1 ; & Q02053 & Uba1 & 0,2 & 2,5 \\
\hline Ubiquitin-like protein $4 \mathrm{~A}$; & P21126 & Ubl4a & 0,5 & 10,8 \\
\hline UDP-glucuronosyltransferase 1-6; & Q64435 & Ugt1a6 & 0,7 & 1,7 \\
\hline UMP-CMP kinase; & Q9DBP5 & Cmpk1 & $-0,1$ & 5,1 \\
\hline Uncharacterized protein C1orf21 homolog; & Q8K207 & & 0,6 & 10,7 \\
\hline Uncharacterized protein; & D3Z2H9 & Tpm3 rs7 & $-0,3$ & 11,3 \\
\hline UPF0668 protein C10orf76 homolog; & Q6PD19 & & $-2,3$ & 1,5 \\
\hline Up-regulated during skeletal muscle growth protein 5; & Q78IK2 & Usmg5 & 0,0 & 43,1 \\
\hline Uridine diphosphate glucose pyrophosphatase; & Q9D142 & Nudt14 & 0,2 & 4,1 \\
\hline UTP--glucose-1-phosphate uridylyltransferase; & Q91ZJ5 & Ugp2 & $-0,5$ & 6,1 \\
\hline UV excision repair protein RAD23 homolog B; & P54728 & $\operatorname{Rad} 23 b$ & 0,1 & 10,6 \\
\hline Vacuolar protein sorting-associated protein $29 ;$ & Q9QZ88 & Vps29 & $-0,2$ & 27,1 \\
\hline Valine--tRNA ligase; & Q971Q9 & Vars & 0,0 & 2,9 \\
\hline Versican core protein; & Q62059 & Vcan & 0,1 & 5,7 \\
\hline Very long-chain specific acyl-CoA dehydrogenase, mitochondrial; & P50544 & Acadvl & $-0,6$ & 2,1 \\
\hline Very-long-chain enoyl-CoA reductase; & Q9CY27 & Tecr & $-0,2$ & 8,1 \\
\hline Vesicle-associated membrane protein 3; & P63024 & Vamp3 & 0,3 & 23,3 \\
\hline Vesicle-associated membrane protein 8; & 070404 & Vamp8 & 0,2 & 8,9 \\
\hline Vesicle-associated membrane protein-associated protein A; & Q9WV55 & Vapa & 0,6 & 17,7 \\
\hline Vesicle-associated membrane protein-associated protein B; & Q9QY76 & Vapb & 0,1 & 5,8 \\
\hline Vesicular integral-membrane protein VIP36; & Q9DBH5 & $\operatorname{Lman} 2$ & $-0,1$ & 2,5 \\
\hline Vimentin; & P20152 & Vim & $-0,2$ & 23 \\
\hline Vinculin; & Q64727 & $\mathrm{Vcl}$ & 0,6 & 15,5 \\
\hline Vitamin D-binding protein; & P21614 & $\mathrm{Gc}$ & $-0,1$ & 2,1 \\
\hline Voltage-dependent anion-selective channel protein 1; & Q60932 & Vdac1 & 0,0 & 9,5 \\
\hline Voltage-dependent anion-selective channel protein 2; & Q60930 & Vdac2 & 0,0 & 18,7 \\
\hline Voltage-dependent anion-selective channel protein 3; & Q60931 & Vdac3 & $-0,3$ & 18,4 \\
\hline V-type proton ATPase catalytic subunit A; & P50516 & Atp6v1a & $-0,3$ & 13,1 \\
\hline V-type proton ATPase subunit B, brain isoform; & P62814 & Atp6v1b2 & 0,1 & 10,4 \\
\hline V-type proton ATPase subunit D; & P57746 & Atp6v1d & 0,2 & 4,5 \\
\hline V-type proton ATPase subunit F; & Q9D1K2 & Atp6v1f & 0,2 & 10,1 \\
\hline V-type proton ATPase subunit G 1; & Q9CR51 & Atp6v1g1 & 7,0 & 14,4 \\
\hline WD repeat-containing protein 1 ; & 088342 & Wdr1 & $-0,3$ & 14,5 \\
\hline WD repeat-containing protein 5 & P61965 & Wdr5 & 1,1 & 5,7 \\
\hline Wiskott-Aldrich syndrome protein family member 2 ; & Q8BH43 & Wasf2 & $-0,3$ & 1,6 \\
\hline WW domain-binding protein 11 ; & Q923D5 & Wbp11 & 0,6 & 1,7 \\
\hline Xaa-Pro dipeptidase; & Q11136 & Pepd & $-0,1$ & 1,4 \\
\hline Xanthine dehydrogenase/oxidase; & Q00519 & Xdh & 0,1 & 2,1 \\
\hline Y-box-binding protein 3; & Q9JKB3 & Ybx3 & $-0,1$ & 19,9 \\
\hline Yorkie homolog; & P46938 & Yap1 & $-0,2$ & 5,1 \\
\hline Zinc finger $\mathrm{CCCH}$ domain-containing protein 18 ; & Q0P678 & Zc3h18 & 0,2 & 2,4 \\
\hline
\end{tabular}




\begin{tabular}{|c|c|c|c|c|}
\hline Protein & Uniprot & Gene name & $\begin{array}{c}\text { Fold change of } \\
\text { XPRESS ratio } \\
\left.\text { (Adam } 17^{\Delta K C} / \mathrm{WT}\right)\end{array}$ & $\begin{array}{c}\text { Sequence } \\
\text { coverage [\%] }\end{array}$ \\
\hline Zinc finger homeobox protein 3; & Q61329 & Zfhx3 & $-0,2$ & 0,2 \\
\hline Zinc finger protein 185; & A2BI37 & Zfp185 & $-0,5$ & 8 \\
\hline Zinc finger protein 426; & Q8R1D1 & Znf426 & 7,0 & 2,7 \\
\hline Zinc finger Ran-binding domain-containing protein 2; & Q9R020 & Zranb2 & 0,6 & 8,2 \\
\hline \multirow[t]{5}{*}{ Zyxin; } & Q62523 & Zyx & $-0,7$ & 12,8 \\
\hline & E9Q019 & & $-1,4$ & 20,3 \\
\hline & J3QMA3 & & $-1,3$ & 27,5 \\
\hline & F6TL02 & & $-1,2$ & 27,4 \\
\hline & J3QN66 & & $-1,6$ & 27,3 \\
\hline
\end{tabular}


Supplementary table 7: List of all identified and quantified proteins in the quantitative proteome comparison of wild-type and Egfr ${ }^{\Delta K C}$ epidermis at P10 (replicate 2).

\begin{tabular}{|c|c|c|c|c|}
\hline Protein & Uniprot & Gene name & $\begin{array}{l}\text { Fold change of } \\
\text { XPRESS ratio } \\
\left(\text { Egfr }^{\triangle K C} / \mathrm{WT}\right)\end{array}$ & $\begin{array}{c}\text { Sequence } \\
\text { coverage [\%] }\end{array}$ \\
\hline 1,2-dihydroxy-3-keto-5-methylthiopentene dioxygenase; & Q99JT9 & Adi1 & 0,3 & 5,6 \\
\hline 10 kDa heat shock protein, mitochondrial; & Q64433 & Hspe1 & 0,2 & 47,1 \\
\hline 116 kDa U5 small nuclear ribonucleoprotein component; & 008810 & Eftud2 & 0,2 & 30 \\
\hline 14-3-3 protein beta/alpha; & Q9CQV8 & Ywhab & $-0,2$ & 48,4 \\
\hline 14-3-3 protein epsilon; & P62259 & Ywhae & 0,1 & 57,3 \\
\hline 14-3-3 protein eta; & P68510 & Ywhah & 0,3 & 49,6 \\
\hline 14-3-3 protein gamma; & P61982 & Ywhag & 0,5 & 31,6 \\
\hline 14-3-3 protein sigma; & 070456 & Sfn & $-0,7$ & 40,7 \\
\hline 14-3-3 protein theta; & P68254 & Ywhaq & 0,3 & 49,4 \\
\hline 14-3-3 protein zeta/delta; & P63101 & Ywhaz & 0,3 & 49,8 \\
\hline 15 kDa selenoprotein; & Q9ERR7 & Sep15 & $-0,3$ & 17,9 \\
\hline 182 kDa tankyrase-1-binding protein; & P58871 & Tnks1bp1 & 0,0 & 24,2 \\
\hline 1-acyl-sn-glycerol-3-phosphate acyltransferase beta; & Q8K3K7 & Agpat2 & $-0,7$ & 10,1 \\
\hline 1-acyl-sn-glycerol-3-phosphate acyltransferase delta; & Q8K4X7 & Agpat4 & 0,9 & 2,4 \\
\hline 1-acyl-sn-glycerol-3-phosphate acyltransferase gamma; & Q9D517 & Agpat3 & $-0,9$ & 7,4 \\
\hline 2,4-dienoyl-CoA reductase, mitochondrial; & Q9CQ62 & Decr1 & $-0,4$ & 31,6 \\
\hline $26 \mathrm{~S}$ protease regulatory subunit $10 \mathrm{~B}$ & P62334 & Psmc6 & $-0,5$ & 28 \\
\hline $26 S$ protease regulatory subunit 4 & P62192 & Psmc1 & $-0,5$ & 34,8 \\
\hline $26 S$ protease regulatory subunit $6 \mathrm{~A}$; & 088685 & Psmc3 & $-0,7$ & 34 \\
\hline $26 \mathrm{~S}$ protease regulatory subunit $6 \mathrm{~B}$; & P54775 & Psmc4 & $-0,6$ & 16,3 \\
\hline 265 protease regulatory subunit 7 & P46471 & Psmc2 & $-0,2$ & 38,1 \\
\hline $26 S$ protease regulatory subunit 8 & P62196 & Psmc5 & $-0,4$ & 21,8 \\
\hline $26 \mathrm{~S}$ proteasome non-ATPase regulatory subun it $1 ;$ & Q3TXS7 & Psmd1 & $-0,5$ & 22,1 \\
\hline $26 \mathrm{~S}$ proteasome non-ATPase regulatory subun it $11 ;$ & Q8BG32 & Psmd11 & $-0,3$ & 39,1 \\
\hline $26 \mathrm{~S}$ proteasome non-ATPase regulatory subun it $12 ;$ & Q9D8W5 & Psmd12 & $-0,9$ & 18,6 \\
\hline $26 \mathrm{~S}$ proteasome non-ATPase regulatory subun it 13 ; & Q9WVJ2 & Psmd13 & $-0,5$ & 44,7 \\
\hline $26 \mathrm{~S}$ proteasome non-ATPase regulatory subun it 14 & 035593 & Psmd14 & $-0,4$ & 17,1 \\
\hline $26 \mathrm{~S}$ proteasome non-ATPase regulatory subun it 2 & Q8VDM4 & Psmd2 & $-0,6$ & 28,1 \\
\hline $26 \mathrm{~S}$ proteasome non-ATPase regulatory subun it 3; & P14685 & Psmd3 & $-0,5$ & 32,5 \\
\hline $26 \mathrm{~S}$ proteasome non-ATPase regulatory subun it 4 ; & 035226 & Psmd4 & $-0,1$ & 27,1 \\
\hline $26 \mathrm{~S}$ proteasome non-ATPase regulatory subun it 4 ; & F7Al87 & Psmd4 & $-0,5$ & 10,3 \\
\hline $26 \mathrm{~S}$ proteasome non-ATPase regulatory subun it 5 ; & Q8BJY1 & Psmd5 & $-0,3$ & 2,6 \\
\hline $26 \mathrm{~S}$ proteasome non-ATPase regulatory subun it 6 ; & Q99J।4 & Psmd6 & $-0,4$ & 26,2 \\
\hline $26 \mathrm{~S}$ proteasome non-ATPase regulatory subun it 7 ; & P26516 & Psmd7 & $-0,6$ & 31,5 \\
\hline $26 \mathrm{~S}$ proteasome non-ATPase regulatory subun it 8 & Q9CX56 & Psmd8 & $-0,5$ & 25,3 \\
\hline $26 \mathrm{~S}$ proteasome non-ATPase regulatory subun it 9; & Q9CR00 & Psmd9 & 0,0 & 27,5 \\
\hline 28 kDa heat- and acid-stable phosphoprotein; & Q3UHX2 & Pdap1 & 0,0 & 41,4 \\
\hline $28 \mathrm{~S}$ ribosomal protein S36, mitochondrial; & Q9CQX8 & Mrps36 & 0,2 & 56,9 \\
\hline 2-amino-3-ketobutyrate coenzyme A ligase, mitochondrial; & 088986 & Gcat & $-0,2$ & 19,1 \\
\hline 2-aminoethanethiol dioxygenase; & Q6PDY2 & Ado & 1,0 & 12,9 \\
\hline 2'-deoxynucleoside 5'-phosphate N-hyd rolase 1; & Q80VJ3 & Dnph1 & 2,0 & 8,1 \\
\hline $3^{\prime}\left(2^{\prime}\right), 5^{\prime}-$ bisphosphate nucleotidase 1 & Q9Z0S1 & Bpnt1 & 0,1 & 27,6 \\
\hline 39 S ribosomal protein L12, mitochondrial; & Q9DB15 & Mrpl12 & $-0,2$ & 11,9 \\
\hline $39 S$ ribosomal protein L41, mitochondrial; & Q9CQN7 & Mrpl41 & 0,0 & 9,9 \\
\hline 3-hydroxyacyl-CoA dehydrogenase type-2; & A2AFQ2 & Hsd17b10 & 0,5 & 49,4 \\
\hline 3-hydroxyisobutyrate dehydrogenase, mitochondrial; & Q99L13 & Hibadh & 0,2 & 12,8 \\
\hline 3-ketoacyl-CoA thiolase A, peroxisomal; & Q921H8 & Acaa1a & 0,0 & 42,7 \\
\hline 3-ketoacyl-CoA thiolase, mitochondrial; & Q8BWT1 & Acaa2 & $-0,1$ & 49,1 \\
\hline $40 S$ ribosomal protein S10; & P63325 & Rps10 & $-0,3$ & 28,5 \\
\hline 40 S ribosomal protein $\mathrm{S} 11$ & P62281 & Rps11 & $-0,4$ & 45,6 \\
\hline $40 S$ ribosomal protein S12; & Q6ZWZ6 & Rps12 & $-0,1$ & 53 \\
\hline $40 S$ ribosomal protein S13; & P62301 & Rps13 & $-0,3$ & 38,4 \\
\hline 40 S ribosomal protein S14; & P62264 & Rps14 & 0,1 & 22,5 \\
\hline 40 S ribosomal protein S15; & P62843 & Rps15 & $-1,1$ & 10,2 \\
\hline $40 \mathrm{~S}$ ribosomal protein $\mathrm{S} 15 \mathrm{a}$; & P62245 & Rps15a & $-0,7$ & 51,5 \\
\hline $40 S$ ribosomal protein S16; & P14131 & Rps16 & $-0,2$ & 36,3 \\
\hline 40 S ribosomal protein S17; & P63276 & Rps17 & $-0,3$ & 15,6 \\
\hline 40 S ribosomal protein S18; & P62270 & Rps18 & $-0,5$ & 42,8 \\
\hline 40 S ribosomal protein S19; & Q9CZX8 & Rps19 & $-0,1$ & 37,9 \\
\hline $40 S$ ribosomal protein S20; & P60867 & Rps20 & $-0,5$ & 25,2 \\
\hline 40 S ribosomal protein $\mathrm{S} 21$ & Q9CQR2 & Rps21 & 0,0 & 39,8 \\
\hline $40 S$ ribosomal protein S23; & P62267 & Rps23 & $-0,9$ & 15,4 \\
\hline 40 S ribosomal protein $\mathrm{S} 24$ & P62849 & Rps24 & 0,3 & 28,6 \\
\hline 40 S ribosomal protein S25; & P62852 & Rps25 & $-0,1$ & 22,4 \\
\hline 40 S ribosomal protein S26; & P62855 & Rps26 & $-1,0$ & 31,3 \\
\hline 40 S ribosomal protein S27; & Q6ZWU9 & Rps27 & $-0,2$ & 38,1 \\
\hline $40 S$ ribosomal protein S27-like; & Q6ZWY3 & Rps27I & $-0,1$ & 38,1 \\
\hline 40 S ribosomal protein S28; & P62858 & Rps28 & 0,2 & 37,5 \\
\hline
\end{tabular}




\begin{tabular}{|c|c|c|c|c|}
\hline Protein & Uniprot & Gene name & $\begin{array}{l}\text { Fold change of } \\
\text { XPRESS ratio } \\
\left(\text { Egfr }^{\triangle K C} / \mathrm{WT}\right)\end{array}$ & $\begin{array}{c}\text { Sequence } \\
\text { coverage [\%] }\end{array}$ \\
\hline 40 S ribosomal protein S29; & P62274 & Rps29 & $-1,3$ & 12,5 \\
\hline 40 S ribosomal protein S3; & P62908 & Rps3 & $-0,5$ & 69,5 \\
\hline $40 S$ ribosomal protein $\mathrm{S} 30$ & P62862 & Fau & $-0,1$ & 16,9 \\
\hline $40 \mathrm{~S}$ ribosomal protein $\mathrm{S} 3 \mathrm{a}$ & P97351 & Rps3a & $-0,4$ & 40,2 \\
\hline $40 \mathrm{~S}$ ribosomal protein $\mathrm{S} 4, \mathrm{X}$ isoform; & P62702 & $\operatorname{Rps} 4 x$ & $-0,5$ & 58,2 \\
\hline $40 S$ ribosomal protein S5; & D3YYM6 & Rps5 & $-0,2$ & 25,8 \\
\hline $40 S$ ribosomal protein S6; & P62754 & Rps6 & $-0,5$ & 18,9 \\
\hline $40 S$ ribosomal protein S7; & P62082 & Rps7 & $-0,2$ & 30,7 \\
\hline $40 S$ ribosomal protein S8; & P62242 & Rps8 & $-0,5$ & 51 \\
\hline 40 S ribosomal protein S9; & Q6ZWN5 & Rps9 & $-0,4$ & 33,5 \\
\hline $40 \mathrm{~S}$ ribosomal protein $\mathrm{SA}$; & P14206 & Rpsa & 0,0 & 34,2 \\
\hline 4F2 cell-surface antigen heavy chain; & P10852 & Slc3a2 & $-1,0$ & 24 \\
\hline 4-trimethylaminobutyraldehyde dehydrogenase; & Q9JL2 & Aldh9a1 & 0,2 & 30,4 \\
\hline 5'(3')-deoxyribonucleotidase, cytosolic type; & Q9JM14 & $\mathrm{Nt5c}$ & 0,4 & 26 \\
\hline 5,6-dihydroxyindole-2-carboxylic acid oxidase; & P07147 & Tyrp1 & 0,0 & 8 \\
\hline 5'-3' exoribonuclease 2 & Q9DBR1 & Xrn2 & 0,2 & 12,3 \\
\hline 5-hydroxyisourate hydrolase; & Q9CRB3 & Urah & $-0,4$ & 37,3 \\
\hline 5'-nucleotidase domain containing 2; Protein $\mathrm{Nt5dc2;}$ & Q91X76 & $\mathrm{Nt} 5 \mathrm{dc} 2$ & $-0,1$ & 4,1 \\
\hline 5'-nucleotidase; & Q61503 & $\mathrm{Nt5e}$ & $-0,4$ & 19,8 \\
\hline 5-oxoprolinase; & Q8K010 & Oplah & $-1,3$ & 2,3 \\
\hline 6.8 kDa mitochondrial proteolipid; & P56379 & Mp68 & $-1,1$ & 13,8 \\
\hline $60 \mathrm{kDa}$ heat shock protein, mitochondrial; & P63038 & Hspd1 & $-0,1$ & 33 \\
\hline 60 kDa SS-A/Ro ribonucleoprotein; & 008848 & Trove2 & 0,1 & 9,1 \\
\hline 605 acidic ribosomal protein $\mathrm{P0}$ & P14869 & Rplp0 & $-0,5$ & 38,8 \\
\hline $60 S$ acidic ribosomal protein $\mathrm{P} 1$ & P47955 & Rplp1 & $-0,6$ & 14 \\
\hline $60 S$ acidic ribosomal protein P2; & P99027 & Rplp2 & $-0,4$ & 38,3 \\
\hline 60S ribosomal protein L10; & Q6ZWV3 & Rpl10 & $-0,7$ & 39,8 \\
\hline $60 S$ ribosomal protein $\mathrm{L} 11$ & Q9CXW4 & Rpl11 & 0,0 & 17,2 \\
\hline $60 S$ ribosomal protein L12; & P35979 & Rpl12 & $-0,4$ & 54,5 \\
\hline $60 S$ ribosomal protein L13; & P47963 & Rpl13 & $-0,5$ & 24,2 \\
\hline $60 S$ ribosomal protein L13a; & P19253 & Rpl13a & $-0,5$ & 28,1 \\
\hline $60 S$ ribosomal protein L14; & Q9CR57 & Rpl14 & $-0,5$ & 20,3 \\
\hline $60 \mathrm{~S}$ ribosomal protein L15; & Q9CZM2 & Rpl15 & $-0,7$ & 30,4 \\
\hline $60 S$ ribosomal protein $\mathrm{L} 17$ & Q9CPR4 & Rpl17 & $-0,4$ & 31,5 \\
\hline $60 S$ ribosomal protein L18; & P35980 & Rpl18 & $-0,3$ & 33,5 \\
\hline $60 S$ ribosomal protein L18a; & P62717 & Rpl18a & $-0,7$ & 34,7 \\
\hline $60 S$ ribosomal protein L19; & P84099 & Rpl19 & $-0,3$ & 21,1 \\
\hline $60 S$ ribosomal protein L21; & 009167 & Rpl21 & $-0,8$ & 25 \\
\hline $60 S$ ribosomal protein $\mathrm{L} 22$ & P67984 & Rpl22 & $-0,1$ & 18,8 \\
\hline $60 S$ ribosomal protein L22-like 1 & Q9D7S7 & Rpl22I1 & $-0,7$ & 9,8 \\
\hline $60 \mathrm{~S}$ ribosomal protein $\mathrm{L} 23$ & P62830 & Rpl23 & $-0,5$ & 43,6 \\
\hline $60 S$ ribosomal protein $\mathrm{L} 24$ & Q8BP67 & $\mathrm{Rpl} 24$ & $-0,5$ & 36,1 \\
\hline $60 S$ ribosomal protein $\mathrm{L} 26$ & P61255 & Rpl26 & 0,2 & 36,9 \\
\hline $60 S$ ribosomal protein $\mathrm{L} 27$ & P61358 & $\mathrm{Rpl} 27$ & $-0,7$ & 36 \\
\hline $60 S$ ribosomal protein $\mathrm{L} 27 \mathrm{a}$ & P14115 & Rpl27a & $-0,2$ & 27,7 \\
\hline $60 S$ ribosomal protein L28; & P41105 & Rpl28 & $-0,5$ & 27 \\
\hline $60 S$ ribosomal protein L29; & P47915 & Rpl29 & $-0,2$ & 19,5 \\
\hline $60 \mathrm{~S}$ ribosomal protein L3; & P27659 & Rpl3 & $-0,5$ & 35,7 \\
\hline $60 S$ ribosomal protein L30; & P62889 & Rpl30 & $-0,4$ & 57,4 \\
\hline $60 \mathrm{~S}$ ribosomal protein $\mathrm{L} 31$ & P62900 & Rpl31 & $-0,4$ & 24,8 \\
\hline $60 S$ ribosomal protein L32; & P62911 & Rpl32 & $-0,9$ & 45,9 \\
\hline $60 S$ ribosomal protein $\mathrm{L} 34$ & Q9D1R9 & Rpl34 & $-0,4$ & 12,8 \\
\hline $60 S$ ribosomal protein L35; & Q6ZWV7 & Rpl35 & $-0,2$ & 21,1 \\
\hline $60 S$ ribosomal protein $\mathrm{L} 35 \mathrm{a}$ & 055142 & Rpl35a & $-0,5$ & 20,9 \\
\hline $60 S$ ribosomal protein L36a; & P83882 & Rpl36a & $-1,1$ & 28,3 \\
\hline $60 S$ ribosomal protein $\mathrm{L} 37 \mathrm{a}$ & P61514 & Rpl37a & $-0,3$ & 41,3 \\
\hline $60 S$ ribosomal protein L38; & Q9Jग18 & Rpl38 & 0,1 & 38,6 \\
\hline $60 S$ ribosomal protein L39; & P62892 & Rpl39 & $-0,4$ & 19,6 \\
\hline $60 S$ ribosomal protein $L 4$ & Q9D8E6 & Rpl4 & $-0,6$ & 36,3 \\
\hline $60 S$ ribosomal protein $\mathrm{L} 5$ & P47962 & Rpl5 & $-0,7$ & 34 \\
\hline $60 S$ ribosomal protein L6; & P47911 & Rpl6 & $-1,0$ & 38,5 \\
\hline $60 S$ ribosomal protein $\mathrm{L} 7$ & P14148 & Rpl7 & $-0,6$ & 28,9 \\
\hline $60 S$ ribosomal protein L8; & P62918 & Rpl8 & $-0,8$ & 38,5 \\
\hline 60 S ribosomal protein L9; & P51410 & Rpl9 & $-0,4$ & 38 \\
\hline 6-phosphogluconate dehydrogenase, decarboxylating; & Q9DCDO & Pgd & 0,3 & 20,7 \\
\hline 6-phosphogluconolactonase; & Q9CQ60 & Pgls & 0,3 & 30 \\
\hline 78 kDa glucose-regulated protein; & P20029 & Hspa5 & $-0,2$ & 40,6 \\
\hline Acetolactate synthase-like protein; & Q8BU33 & llvbl & $-0,1$ & 4,6 \\
\hline Acetyl-CoA acetyltransferase, cytosolic; & Q8CAY6 & Acat2 & 0,0 & 24,7 \\
\hline Acetyl-CoA acetyltransferase, mitochondrial; & Q8QZT1 & Acat1 & $-0,2$ & 34 \\
\hline Acid ceramidase; & Q9WV54 & Asah1 & $-0,5$ & 25,4 \\
\hline
\end{tabular}




\begin{tabular}{|c|c|c|c|c|}
\hline Protein & Uniprot & Gene name & $\begin{array}{l}\text { Fold change of } \\
\text { XPRESS ratio } \\
\left(\text { Egfr }^{\triangle K C} / \mathrm{WT}\right)\end{array}$ & $\begin{array}{c}\text { Sequence } \\
\text { coverage [\%] }\end{array}$ \\
\hline Acid sphingomyelinase-like phosphodiesterase $3 \mathrm{~b}$; & P58242 & Smpdl3b & $-1,2$ & 6,4 \\
\hline Acid trehalase-like protein 1 & Q8BP56 & Athl1 & 1,2 & 6,8 \\
\hline Acidic leucine-rich nuclear phosphoprotein 32 family member $A$; & 035381 & Anp32a & 0,5 & 20,7 \\
\hline Acidic leucine-rich nuclear phosphoprotein 32 family member B; & Q9EST5 & Anp32b & 0,1 & 14,3 \\
\hline Actin, alpha cardiac muscle 1 & P68033 & Actc1 & $-0,4$ & 40,6 \\
\hline Actin, aortic smooth muscle; & P62737 & Acta2 & $-0,4$ & 40,6 \\
\hline Actin-binding LIM protein 1; & Q8K4G5 & Ablim1 & 0,3 & 4,8 \\
\hline Actin-related protein 10 & Q9QZB7 & Actr10 & $-0,3$ & 6,7 \\
\hline Actin-related protein $2 / 3$ complex subunit $1 A$ & Q9R0Q6 & Arpc1a & $-0,3$ & 32,4 \\
\hline Actin-related protein $2 / 3$ complex subun it $1 B$; & Q9WV32 & Arpc1b & 0,1 & 32,3 \\
\hline Actin-related protein $2 / 3$ complex subun it 2 & Q9CVB6 & Arpc2 & $-0,3$ & 38 \\
\hline Actin-related protein $2 / 3$ complex subunit 3 ; & Q9JM76 & Arpc3 & $-0,1$ & 21,2 \\
\hline Actin-related protein $2 / 3$ complex subunit 4 & P59999 & Arpc4 & 0,0 & 22,6 \\
\hline Actin-related protein $2 / 3$ complex subun it 5 & Q9CPW4 & Arpc5 & 0,3 & 35,8 \\
\hline Actin-related protein $2 / 3$ complex subun it 5 -like protein; & Q9D898 & Arpc5I & $-0,2$ & 28,8 \\
\hline Actin-related protein 2 & P61161 & Actr2 & $-0,5$ & 26,9 \\
\hline Actin-related protein 3 ; & Q99JY9 & Actr3 & $-0,2$ & 48,3 \\
\hline Activated RNA polymerase II transcriptional coactivator $\mathrm{p} 15$; & P11031 & Sub1 & $-0,3$ & 26 \\
\hline Acyl carrier protein, mitochondrial; & Q9CR21 & Ndufab1 & $-0,8$ & 7,1 \\
\hline Acylamino-acid-releasing enzyme; & Q8R146 & Apeh & 0,0 & 16,3 \\
\hline Acyl-CoA dehydrogenase family member $10 ;$ & Q8K370 & Acad10 & 0,1 & 7,7 \\
\hline Acyl-CoA dehydrogenase family member 9 , mitochondrial; & Q8JZN5 & Acad9 & $-0,4$ & 39 \\
\hline Acyl-CoA desaturase 1 & P13516 & Scd1 & 0,0 & 6,2 \\
\hline Acyl-CoA-binding protein; & P31786 & Dbi & $-0,4$ & 39,1 \\
\hline Acyl-coenzyme A thioesterase 1 ; & 055137 & Acot1 & 0,6 & 5,5 \\
\hline Acyl-coenzyme $\mathrm{A}$ thioesterase 13 & Q9CQR4 & Acot13 & 0,3 & 8,6 \\
\hline Acyl-coenzyme A thioesterase 9, mitochondrial; & Q9R0X4 & Acot9 & 0,5 & 17,5 \\
\hline Acylglycerol kinase, mitochondrial; & Q9ESW4 & Agk & 0,1 & 7,1 \\
\hline Acyl-protein thioesterase $1 ;$ & P97823 & Lypla1 & 0,2 & 19 \\
\hline Acyl-protein thioesterase 2 & Q9WTL7 & Lypla2 & $-0,9$ & 10,8 \\
\hline Acylpyruvase FAHD1, mitochondrial; & Q8R0F8 & Fahd1 & $-0,3$ & 13,2 \\
\hline Adenine phosphoribosyltransferase; & P08030 & Aprt & 0,5 & 22,2 \\
\hline Adenomatous polyposis coli protein 2 ; & Q9Z1K7 & Apc2 & $-3,6$ & 0,7 \\
\hline Adenosine deaminase; & P03958 & Ada & $-0,2$ & 17 \\
\hline Adenosine kinase; & P55264 & Adk & 0,6 & 30,7 \\
\hline Adenosylhomocysteinase; & P50247 & Ahcy & 0,1 & 44 \\
\hline Adenylate kinase 2, mitochondrial; & Q9WTP6 & Ak2 & 0,4 & 38,1 \\
\hline Adenylosuccinate lyase; & P54822 & Adsl & 0,0 & 16,3 \\
\hline Adenylosuccinate synthetase isozyme 2 ; & P46664 & Adss & 1,2 & 14 \\
\hline Adenylyl cyclase-associated protein $1 ;$ & P40124 & Cap1 & $-0,3$ & 13,7 \\
\hline Adipocyte enhancer-binding protein 1 & Q640N1 & Aebp1 & $-0,3$ & 1,4 \\
\hline Adipocyte plasma membrane-associated protein; & Q9D7N9 & Apmap & 0,1 & 18,6 \\
\hline Adiponectin; & Q60994 & Adipoq & 0,9 & 18,6 \\
\hline ADP/ATP translocase $1 ;$ & P48962 & Slc25a4 & 0,0 & 43,3 \\
\hline ADP/ATP translocase 2 & P51881 & Slc25a5 & $-0,2$ & 39,3 \\
\hline ADP-dependent glucokinase; & Q8VDL4 & Adpgk & $-0,3$ & 10,7 \\
\hline ADP-ribosylation factor 1 & P84078 & Arf1 & $-0,1$ & 62,4 \\
\hline ADP-ribosylation factor 4 & P61750 & Arf4 & $-1,6$ & 41,1 \\
\hline ADP-ribosylation factor 5 & P84084 & Arf5 & $-0,4$ & 63,3 \\
\hline ADP-ribosylation factor 6 & P62331 & Arf6 & 0,1 & 26,9 \\
\hline ADP-ribosylation factor GTPase-activating protein 1; & Q9EPJ9 & Arfgap1 & 0,3 & 2,3 \\
\hline ADP-ribosylation factor-like protein 1 & P61211 & Arl1 & 0,1 & 14,5 \\
\hline ADP-ribosylation factor-like protein 3 ; & Q9WUL7 & Arl3 & $-0,1$ & 44,5 \\
\hline ADP-ribosylation factor-like protein 6 -interacting protein 1 ; & Q9JKW0 & Arl6ip1 & $-0,3$ & 4,9 \\
\hline ADP-ribosylation factor-like protein $8 \mathrm{~A}$ & Q8VEH3 & Arl8a & 0,2 & 30,3 \\
\hline ADP-ribosylation factor-like protein 8B; & Q9CQW2 & Arl8b & 0,0 & 22 \\
\hline ADP-sugar pyrophosphatase; & Q9JKX6 & Nudt5 & 0,4 & 38,1 \\
\hline Afadin; & Q9QZQ1 & Mllt4 & $-0,6$ & 5,2 \\
\hline Agrin; & MoQWP1 & Agrn & $-1,8$ & 11,3 \\
\hline A-kinase anchor protein 12 & Q9WTQ5 & Akap12 & 1,6 & 10,2 \\
\hline A-kinase anchor protein 2 & 054931 & Akap2 & 0,1 & 4,9 \\
\hline A-kinase anchor protein 8 & Q9DBRO & Akap8 & $-0,7$ & 1,3 \\
\hline Alanine aminotransferase 1 & Q8QZR5 & Gpt & $-0,1$ & 13,9 \\
\hline Alanine--tRNA ligase, cytoplasmic; & Q8BGQ7 & Aars & 0,0 & 2,4 \\
\hline Alcohol dehydrogenase $[\operatorname{NADP}(+)]$; & Q9נו16 & Akr1a1 & 0,5 & 20 \\
\hline Alcohol dehydrogenase 1 ; & P00329 & Adh1 & 0,4 & 20,8 \\
\hline Alcohol dehydrogenase class $4 \mathrm{mu} / \mathrm{sigma}$ chain; & Q64437 & Adh7 & 0,2 & 21,1 \\
\hline Alcohol dehydrogenase class-3; & P28474 & Adh5 & 0,6 & 24,3 \\
\hline Aldehyde dehydrogenase family 1 member A3; & Q9JHW9 & Aldh1a3 & 1,5 & 10,4 \\
\hline Aldehyde dehydrogenase family 16 member $\mathrm{A} 1$; & Q57119 & Aldh16a1 & $-0,2$ & 7,4 \\
\hline Aldehyde dehydrogenase, cytosolic 1 ; & 035945 & Aldh1a7 & 0,7 & 2 \\
\hline
\end{tabular}




\begin{tabular}{|c|c|c|c|c|}
\hline Protein & Uniprot & Gene name & $\begin{array}{l}\text { Fold change of } \\
\text { XPRESS ratio } \\
\left(\text { Egfr }^{\Delta K C} / \mathrm{WT}\right) \\
\end{array}$ & $\begin{array}{c}\text { Sequence } \\
\text { coverage [\%] }\end{array}$ \\
\hline Aldehyde dehydrogenase, dimeric NADP-preferring; & P47739 & Aldh3a1 & 0,2 & 15,2 \\
\hline Aldehyde dehydrogenase; & E9Q3E1 & Aldh3b2 & $-0,3$ & 22,1 \\
\hline Aldehyde oxidase 4; & Q3TYQ9 & Aox4 & 0,0 & 10,7 \\
\hline Aldose reductase; & P45376 & Akr1b1 & 0,8 & 24,7 \\
\hline $\begin{array}{l}\text { Alpha globin 1; Alpha globin 2; Alpha-globin; Hemoglobin alpha, adult chain 1; } \\
\text { Hemoglobin subunit alpha; Protein Hba-a2; }\end{array}$ & Q91VB8 & raemaglobin alpha & 0,6 & 62 \\
\hline Alpha/beta hydrolase domain-containing protein 14B; & Q8VCR7 & Abhd14b & 0,3 & 10 \\
\hline Alpha-1-antitrypsin 1-1; & P07758 & Serpina1a & $-0,6$ & 26,5 \\
\hline Alpha-1-antitrypsin 1-2; & P22599 & Serpina1b & 0,4 & 25,2 \\
\hline Alpha-1-antitrypsin 1-4; & Q00897 & Serpina1d & 0,0 & 18,4 \\
\hline Alpha-2-antiplasmin; & Q61247 & Serpinf2 & 2,6 & 9,8 \\
\hline Alpha-2-HS-glycoprotein; & P29699 & Ahsg & 0,3 & 30,1 \\
\hline Alpha-2-macroglobulin receptor-associated protein; & P55302 & Lrpap1 & $-0,3$ & 17,2 \\
\hline Alpha-2-macroglobulin; & Q61838 & $\mathrm{A} 2 \mathrm{~m}$ & 0,3 & 16,9 \\
\hline Alpha-actinin-1; & Q7TPR4 & Actn1 & $-0,2$ & 41,1 \\
\hline Alpha-actinin-2; & Q9JI91 & Actn2 & $-4,4$ & 14,7 \\
\hline Alpha-actinin-4; & P57780 & Actn4 & 0,1 & 50,8 \\
\hline Alpha-adducin; & Q9QYC0 & Add1 & 0,4 & 26 \\
\hline Alpha-aminoadipic semialdehyde dehydrogenase; & Q9DBF1 & Aldh7a1 & 0,0 & 28,2 \\
\hline Alpha-centractin; & P61164 & Actr1a & $-0,7$ & 26,1 \\
\hline Alpha-endosulfine; & P60840 & Ensa & 0,8 & 11,6 \\
\hline Alpha-enolase; & P17182 & Eno1 & 0,4 & 33,4 \\
\hline Alpha-fetoprotein; & P02772 & Afp & 0,8 & 49,3 \\
\hline Alpha-galactosidase $\mathrm{A}$; & P51569 & Gla & $-0,8$ & 12,4 \\
\hline Alpha-ketoglutarate-depen dent dioxygenase FTO; & Q8BGW1 & Fto & 0,8 & 8,2 \\
\hline Alpha-mannosidase 2 & P27046 & Man2a1 & 0,0 & 7,3 \\
\hline Alpha-N-acetylgalactosaminidase; & Q9QWR8 & Naga & 0,4 & 15,4 \\
\hline $\begin{array}{l}\text { Alpha-N-acetylglucosaminidase; Alpha-N-acetylglucosaminidase (Sanfilippo } \\
\text { disease IIIB); Alpha-N-acetylglucosaminidase (Sanfilippo disease IIIB), isoform } \\
\text { CRA_b; N-acetyl-glucosaminidase; Protein Naglu; }\end{array}$ & 088325 & Naglu & $-0,4$ & 3,4 \\
\hline Alpha-parvin; & Q9EPC1 & Parva & 0,7 & 5,6 \\
\hline Alpha-soluble NSF attachment protein; & Q9DB05 & Napa & $-0,2$ & 5,1 \\
\hline Alpha-taxilin; & Q6PAM1 & Txlna & $-0,3$ & 5,1 \\
\hline Amidophosphoribosyltransferase; & Q8ClH9 & Ppat & 0,7 & 9,3 \\
\hline Amine oxidase [flavin-containing] A; & Q64133 & Maoa & $-0,1$ & 19,2 \\
\hline Aminoacyl tRNA synthase complex-interacting multifunctional protein 1; & P31230 & Aimp1 & $-0,6$ & 10,3 \\
\hline Aminoacylase-1; & Q99JW2 & Acy1 & 0,0 & 15,7 \\
\hline Aminopeptidase $\mathrm{N}$; & P97449 & Anpep & $-0,6$ & 23,8 \\
\hline AMP deaminase 2; & Q9DBT5 & Ampd2 & $-1,3$ & 7,3 \\
\hline AMP deaminase 3; & 008739 & Ampd3 & $-0,5$ & 1,7 \\
\hline Amyloid beta A4 protein; & P12023 & App & 0,0 & 4,5 \\
\hline Amyloid-like protein 2; & Q06335 & Aplp2 & $-0,4$ & 4,4 \\
\hline AN1-type zinc finger protein 2B; & Q91X58 & Zfand $2 b$ & 0,2 & 10,1 \\
\hline AN1-type zinc finger protein 5 & 088878 & Zfand5 & 0,4 & 4,7 \\
\hline AN1-type zinc finger protein 6 & Q9DCH6 & Zfand6 & 0,0 & 9,4 \\
\hline Anamorsin; & Q8WTY4 & Ciapin1 & $-0,4$ & 8,9 \\
\hline Angiotensin-converting enzyme 2 ; & Q8R0I0 & Ace2 & $-2,0$ & 9,3 \\
\hline Angiotensin-converting enzyme; & P09470 & Ace & 1,1 & 2,5 \\
\hline Angiotensinogen; & P11859 & Agt & 0,4 & 4,8 \\
\hline Ankyrin repeat and SOCS box protein 18; & Q8VHA6 & Asb18 & 5,8 & 3,9 \\
\hline Ankyrin repeat domain-containing protein 13D; & Q6PD24 & Ankrd13d & 10,0 & 4,6 \\
\hline Ankyrin repeat domain-containing protein SOWAHD; & Q8BY98 & Sowahd & 4,8 & 3,7 \\
\hline Annexin A1; & P10107 & Anxa1 & 0,4 & 51,7 \\
\hline Annexin A11; & P97384 & Anxa11 & $-0,2$ & 6 \\
\hline Annexin A2; & P07356 & Anxa2 & 0,3 & 59,3 \\
\hline Annexin A3; & 035639 & Anxa3 & $-0,3$ & 16,1 \\
\hline Annexin A4; & P97429 & Anxa4 & 0,4 & 26,3 \\
\hline Annexin A5; & P48036 & Anxa5 & 0,0 & 35,1 \\
\hline Annexin A6; & P14824 & Anxa6 & $-0,1$ & 35,2 \\
\hline Annexin A8; & 035640 & Anxa8 & 0,2 & 32,7 \\
\hline Anthrax toxin receptor 2 & Q6DFX2 & Antxr2 & 1,0 & 3,1 \\
\hline Antigen-presenting glycoprotein CD1d1; & P11609 & $\mathrm{Cd} 1 \mathrm{~d} 1$ & 0,1 & 2,1 \\
\hline Antithrombin-III; & P32261 & Serpinc1 & $-0,1$ & 11,2 \\
\hline AP-1 complex subunit beta- 1 ; & 035643 & Ap1b1 & 0,1 & 9,8 \\
\hline AP-1 complex subunit gamma-1; & P22892 & Ap1g1 & 1,4 & 2,8 \\
\hline AP-1 complex subunit mu-1; & P35585 & Ap1m1 & 0,0 & 4,3 \\
\hline AP-2 complex subunit alpha-1; & P17426 & Ap2a1 & $-0,1$ & 7,8 \\
\hline AP-2 complex subunit beta; & Q9DBG3 & Ap2b1 & 0,3 & 8 \\
\hline AP-2 complex subunit mu; & P84091 & Ap2m1 & $-0,1$ & 12 \\
\hline Apolipoprotein A-I; & Q00623 & Apoa1 & 0,6 & 38,6 \\
\hline
\end{tabular}




\begin{tabular}{|c|c|c|c|c|}
\hline Protein & Uniprot & Gene name & $\begin{array}{l}\text { Fold change of } \\
\text { XPRESS ratio } \\
\left(E g f r^{\Delta K C} / \mathrm{WT}\right)\end{array}$ & $\begin{array}{c}\text { Sequence } \\
\text { coverage [\%] }\end{array}$ \\
\hline Apolipoprotein A-II; & P09813 & Apoa2 & 0,7 & 9,8 \\
\hline Apolipoprotein A-IV; & P06728 & Apoa4 & $-0,4$ & 59,7 \\
\hline Apolipoprotein B-100; & E9Q414 & Apob & 0,5 & 0,8 \\
\hline Apolipoprotein C-III; & P33622 & Apoc3 & $-4,3$ & 19,2 \\
\hline Apolipoprotein D; & P51910 & Apod & 0,4 & 12,7 \\
\hline Apolipoprotein E; & P08226 & Apoe & $-2,6$ & 30,9 \\
\hline Apolipoprotein O; & Q9DCZ4 & Apoo & 0,2 & 20,1 \\
\hline Apolipoprotein O-like; & Q78IK4 & Apool & $-0,2$ & 31,7 \\
\hline Apoptosis inhibitor 5; & 035841 & Api5 & 0,2 & 8,5 \\
\hline Apoptosis regulator BAX; & Q07813 & Bax & 0,0 & 24 \\
\hline Apoptosis-associated speck-like protein containing a CARD; & Q9EPB4 & Pycard & $-0,2$ & 34,2 \\
\hline Apoptosis-inducing factor 1, mitochondrial; & Q9Z0X1 & Aifm1 & $-0,1$ & 16,1 \\
\hline Apoptotic chromatin condensation inducer in the nucleus; & Q9JIX8 & Acin1 & 0,0 & 5,3 \\
\hline Aquaporin-1; & Q02013 & Aqp1 & 0,3 & 14,5 \\
\hline Arachidonate 5-lipoxygenase; & P48999 & Alox5 & $-0,6$ & 4,1 \\
\hline Arachidonate 8S-lipoxygenase; & 035936 & Alox8 & $-1,1$ & 4,5 \\
\hline $\begin{array}{l}\text { Arf-GAP with Rho-GAP domain, ANK repeat and PH domain-containing protein } \\
\text { 1; }\end{array}$ & Q4LDD4 & Arap1 & 0,9 & 2,1 \\
\hline Arfip1 protein; Protein Arfip1; & A2RSX9 & Arfip1 & $-0,2$ & 22,9 \\
\hline Arginase-1; & Q61176 & Arg1 & $-1,2$ & 32,2 \\
\hline Arginase-2, mitochondrial; & 008691 & Arg2 & 1,5 & 4,2 \\
\hline Arginine and glutamate-rich protein $1 ;$ & Q3UL36 & Arglu1 & 0,4 & 3 \\
\hline Arginine/serine-rich coiled-coil protein 2; & A2RTL5 & Rsrc2 & 0,4 & 7,9 \\
\hline Arginine/serine-rich protein PNISR; & A2AJT4 & Pnisr & 0,6 & 1,9 \\
\hline $\begin{array}{l}\text { Arginine-rich, mutated in early stage tumors, isoform CRA_b; Mesencephalic } \\
\text { astrocyte-derived neurotro phic factor; }\end{array}$ & Q3TMX5 & Manf & 0,0 & 35,8 \\
\hline Arginine--tRNA ligase, cytoplasmic; & Q9D019 & Rars & $-0,3$ & 5,5 \\
\hline Argininosuccinate lyase; & Q91YI0 & Asl & $-0,8$ & 29,3 \\
\hline Argininosuccinate synthase; & P16460 & Ass1 & $-1,6$ & 8 \\
\hline Arylsulfatase B; & P50429 & Arsb & $-0,6$ & 3,7 \\
\hline Asparagine--tRNA ligase, cytoplasmic; & Q8BP47 & Nars & 0,4 & 14,1 \\
\hline Aspartate aminotransferase, cytoplasmic; & P05201 & Got1 & 0,7 & 38,5 \\
\hline Aspartate aminotransferase, mitochondrial; & P05202 & Got2 & $-0,2$ & 49,3 \\
\hline Aspartate--tRNA ligase, cytoplasmic; & Q922B2 & Dars & $-0,1$ & 23,4 \\
\hline Aspartyl aminopeptidase; & Q9Z2W0 & Dnpep & 0,3 & 36,2 \\
\hline Aspartyl/asparaginyl beta-hydroxylase; & Q8BSY0 & Asph & 0,3 & 6,5 \\
\hline Asporin; & Q99MQ4 & Aspn & $-0,7$ & 29,2 \\
\hline Ataxin-2-like protein; & Q7TQHO & Atxn2l & 0,3 & 2,1 \\
\hline Atlastin-3; & Q91YH5 & Atl3 & 0,3 & 3,9 \\
\hline ATP synthase $F(0)$ complex subunit $B 1$, mitochondrial; & Q9CQQ7 & Atp5f1 & 1,4 & 14,8 \\
\hline ATP synthase $F(0)$ complex subunit $C 2$, mitochondrial; & P56383 & Atp5g2 & $-0,4$ & 5,1 \\
\hline ATP synthase protein 8 & P03930 & Mtatp8 & $-0,4$ & 14,9 \\
\hline ATP synthase subunit alpha, mitochond rial; & Q03265 & Atp5a1 & $-0,6$ & 39,6 \\
\hline ATP synthase subunit beta, mitochond rial; & P56480 & Atp5b & $-0,8$ & 43,7 \\
\hline ATP synthase subunit $d$, mitochondrial; & Q9DCX2 & Atp5h & $-0,1$ & 50 \\
\hline ATP synthase subunit delta, mitochond rial; & Q9D3D9 & Atp5d & 1,7 & 13,7 \\
\hline ATP synthase subunit e, mitochondrial; & Q06185 & Atp5i & $-0,4$ & 52,1 \\
\hline ATP synthase subunit epsilon, mitochondrial; & P56382 & Atp5e & 0,0 & 15,4 \\
\hline ATP synthase subunit $f$, mitochondrial; & P56135 & Atp5j2 & $-0,2$ & 30,3 \\
\hline ATP synthase subunit g, mitochondrial; & Q9CPQ8 & Atp5l & $-0,3$ & 27,2 \\
\hline ATP synthase subunit gamma, mitochond rial; & Q91VR2 & Atp5c1 & $-0,7$ & 42,9 \\
\hline ATP synthase subunit $O$, mitochondrial; & Q9DB20 & Atp5o & $-0,6$ & 49,8 \\
\hline ATP synthase-coupling factor 6 , mitochondrial; & P97450 & Atp5j & $-0,7$ & 41,7 \\
\hline ATPase Asna1; & 054984 & Asna1 & 0,2 & 17 \\
\hline ATP-binding cassette sub-family A member 9; & Q8K449 & Abca9 & 1,6 & 1,8 \\
\hline ATP-binding cassette sub-family B member 10 , mitochond rial; & Q9J139 & Abcb10 & $-1,3$ & 2,4 \\
\hline ATP-binding cassette sub-family E member 1 ; & P61222 & Abce1 & 0,5 & 27,2 \\
\hline ATP-binding cassette sub-family F member 1 ; & Q6P542 & Abcf1 & $-0,6$ & 1,8 \\
\hline ATP-binding cassette sub-family $\mathrm{F}$ member 2 ; & Q99LE6 & Abcf2 & $-0,1$ & 4,5 \\
\hline ATP-binding cassette sub-family $\mathrm{G}$ member 2 ; & Q7TMS5 & Abcg2 & 0,3 & 8,7 \\
\hline ATP-citrate synthase; & Q91V92 & Acly & $-0,4$ & 6 \\
\hline ATP-dependent (S)-NAD(P)H-hydrate dehydratase; & Q9CZ42 & Carkd & 0,0 & 50,8 \\
\hline ATP-dependent 6-phosphofructokinase, liver type; & P12382 & Pfkl & $-0,1$ & 20,8 \\
\hline ATP-dependent 6-phosphofructokinase, muscle type; & P47857 & Pfkm & 0,5 & 7,2 \\
\hline ATP-dependent 6-phosphofructokinase, platelet type; & Q9WUA3 & Pfkp & $-0,7$ & 25,9 \\
\hline ATP-dependent Clp protease ATP-bin ding subunit clpX-like, mitochondrial; & Q9JHS4 & Clpx & 0,5 & 2,1 \\
\hline ATP-dependent Clp protease proteolytic subunit, mitochondrial; & 088696 & Clpp & $-0,6$ & 16,2 \\
\hline ATP-dependent RNA helicase A; & E9QNN1 & Dhx9 & 0,6 & 6,4 \\
\hline ATP-dependent RNA helicase DDX1; & Q91VR5 & Ddx1 & 0,2 & 7,8 \\
\hline ATP-dependent RNA helicase DDX19A; & Q61655 & Ddx19a & 0,3 & 2,1 \\
\hline
\end{tabular}




\begin{tabular}{|c|c|c|c|c|}
\hline Protein & Uniprot & Gene name & $\begin{array}{l}\text { Fold change of } \\
\text { XPRESS ratio } \\
\left(E g f r^{\Delta K C} / \mathrm{WT}\right)\end{array}$ & $\begin{array}{c}\text { Sequence } \\
\text { coverage [\%] }\end{array}$ \\
\hline ATP-dependent RNA helicase DDX39A; & Q8VDW0 & Ddx39a & 0,3 & 18,3 \\
\hline Atrial natriuretic peptide receptor 3 ; & P70180 & Npr3 & 0,0 & 1,7 \\
\hline Atrial natriuretic peptide-converting enzyme; & Q97319 & Corin & $-0,2$ & 1,1 \\
\hline AT-rich interactive domain-containing protein $1 \mathrm{~A}$; & $\mathrm{A} 2 \mathrm{BH} 40$ & Arid1a & $-0,2$ & 1,2 \\
\hline Aurora kinase B; & E9PVP1 & Aim1 & $-1,0$ & 3,3 \\
\hline BAG family molecular chaperone regulator 1 ; & Q60739 & Bag1 & 0,3 & 8,2 \\
\hline BAG family molecular chaperone regulator 3 ; & Q9JLV1 & Bag3 & $-0,5$ & 14,7 \\
\hline Band 4.1-like protein 2; & 070318 & Epb41/2 & 1,2 & 11 \\
\hline Band 4.1-like protein 3; & Q9WV92 & Epb4113 & 0,6 & 4,4 \\
\hline Barrier-to-autointegration factor; & 054962 & Banf1 & $-0,7$ & 34,8 \\
\hline Basal cell adhesion molecule; & Q9R069 & Bcam & $-0,5$ & 27,7 \\
\hline Basement membrane-specific heparan sulfate proteoglycan core protein; & B1B0C7 & Hspg2 & 0,8 & 7,4 \\
\hline Basic leucine zipper and W2 domain-containing protein 1; & Q9CQC6 & Bzw1 & 0,0 & 2,4 \\
\hline Basic leucine zipper and W2 domain-containing protein 2; & Q91VK1 & Bzw2 & 0,5 & 4,5 \\
\hline Basigin; & P18572 & Bsg & 0,1 & 27,9 \\
\hline Bax inhibitor 1; & Q9D2C7 & Tmbim6 & $-0,8$ & 65,6 \\
\hline B-cell receptor-associated protein 31 ; & Q61335 & Bcap31 & $-1,2$ & 14,3 \\
\hline Bcl-2 homologous antagonist/killer; & 008734 & Bak1 & 1,3 & 22,5 \\
\hline BCL2/adenovirus E1B 19 kDa protein-interacting protein 3-like; & Q9Z2F7 & Bnip3l & $-0,7$ & 7,4 \\
\hline Bcl-2/adenovirus E1B 19 kDa-interacting protein 2-like protein; & Q99JU7 & Bnipl & $-0,3$ & 8,8 \\
\hline $\mathrm{Bcl}-2$-associated transcription factor 1 & Q8K019 & Bclaf1 & 0,0 & 9,8 \\
\hline Bcl-2-like protein 1 & Q64373 & $\mathrm{Bcl} 2 \mathrm{|l}$ & $-0,1$ & 12,8 \\
\hline Bcl-2-like protein 13; & P59017 & $\mathrm{Bcl} 2 \mathrm{|13}$ & 0,0 & 3,7 \\
\hline BET1 homolog; & 035623 & Bet1 & $-0,7$ & 9,3 \\
\hline BET1-like protein; & 035153 & Bet1l & $-0,6$ & 11,7 \\
\hline Beta-2-glycoprotein 1; & Q01339 & Apoh & $-2,5$ & 17,1 \\
\hline Beta-actin-like protein 2 & Q8BFZ3 & Actbl2 & $-1,3$ & 27,1 \\
\hline Beta-catenin-like protein 1 ; & Q9CWL8 & Ctnnbl1 & 0,2 & 1,4 \\
\hline Beta-centractin; & Q8R5C5 & Actr1b & $-0,3$ & 22,3 \\
\hline Beta-enolase; & P21550 & Eno3 & 2,9 & 18,4 \\
\hline Beta-galactosidase; & P23780 & Glb1 & 0,2 & 3,1 \\
\hline Beta-galactosidase-1-like protein; & Q8VC60 & Glb1l & $-0,1$ & 1,4 \\
\hline Beta-globin; Protein Hbb-bs; Protein Hbb-bt; & A8DUK4 & Hbbt1 & 0,9 & 93,2 \\
\hline Beta-glucuronidase; & P12265 & Gusb & 0,6 & 8,2 \\
\hline Beta-hexosaminidase subunit alpha; & P29416 & Hexa & $-0,3$ & 4,5 \\
\hline Beta-hexosaminidase subunit beta; & P20060 & Hexb & $-0,9$ & 25,7 \\
\hline Beta-lactamase-like protein 2 ; & Q99KR3 & Lactb2 & 0,1 & 6,3 \\
\hline Beta-mannosidase; & Q8K214 & Manba & $-0,4$ & 3,3 \\
\hline Bifunctional 3'-phosphoadenosine 5'-phosphosulfate synthase 1; & Q60967 & Papss1 & 0,1 & 5,1 \\
\hline Bifunctional glutamate/proline--tRNA ligase; & Q8CGC7 & Eprs & $-0,5$ & 6,9 \\
\hline Bifunctional purine biosynthesis protein PURH; & Q9CWJ9 & Atic & 1,0 & 23,8 \\
\hline Biglycan; & P28653 & Bgn & $-0,4$ & 36,9 \\
\hline Biliverdin reductase $A$; & Q9CY64 & Blvra & 0,8 & 7,5 \\
\hline Biorientation of chromosomes in cell division protein 1 ; & Q5SQY2 & Bod1 & $-1,7$ & 18,5 \\
\hline Bis(5'-adenosyl)-triphosphatase; & 089106 & Fhit & 0,0 & 12,9 \\
\hline Bleomycin hydrolase; & Q8R016 & Blmh & $-0,8$ & 39,8 \\
\hline BolA-like protein 1; & Q9D8S9 & Bola1 & 0,4 & 28,5 \\
\hline BolA-like protein 2; & Q8BGS2 & Bola2 & 0,1 & 22,1 \\
\hline BolA-like protein 3; & Q8CEI1 & Bola3 & 1,4 & 18,9 \\
\hline Bone marrow proteoglycan; & Q61878 & Prg2 & 2,5 & 8,5 \\
\hline BPI fold-containing family C protein; & Q8C186 & Bpifc & 0,0 & 13,4 \\
\hline Brain acid soluble protein 1 & Q91XV3 & Basp1 & 0,3 & 37,6 \\
\hline Branched-chain-amino-acid a minotransferase, mitochondrial; & 035855 & Bcat2 & 1,3 & 8,5 \\
\hline BRCA1-A complex subunit BRE; & Q8K3W0 & Bre & 0,1 & 8,1 \\
\hline BRI3-binding protein; & Q8BXV2 & Bri3bp & $-0,2$ & 16,6 \\
\hline BRISC complex subunit Abro1; & Q3TCJ1 & Fam175b & $-0,2$ & 14,2 \\
\hline BTB/POZ domain-containing protein KCTD12; & Q6WVG3 & Kctd12 & 0,9 & 38,2 \\
\hline BTB/POZ domain-containing protein KCTD15; & Q8K0E1 & Kctd15 & 0,1 & 5,3 \\
\hline BUB3-interacting and GLEBS motif-containing protein ZNF207; & E9PW12 & Znf207 & 0,4 & 11,4 \\
\hline CAAX prenyl protease 1 homolog; & Q80W54 & Zmpste24 & $-0,4$ & 11,2 \\
\hline CAD protein; & B2RQC6 & Cad & 0,2 & 0,8 \\
\hline Cadherin-1; & P09803 & Cdh1 & $-0,2$ & 17,4 \\
\hline Cadherin-11; & P55288 & Cdh11 & $-0,2$ & 4,6 \\
\hline Cadherin-13; & Q9WTR5 & Cdh13 & 1,8 & 10,9 \\
\hline Cadherin-3; & P10287 & Cdh3 & $-0,5$ & 2,9 \\
\hline Cadherin-5; & P55284 & Cdh5 & 0,1 & 1,3 \\
\hline Calcineurin B homologous protein 1 ; & P61022 & Chp1 & $-0,6$ & 32,5 \\
\hline Calcineurin subunit B type 1 ; & Q63810 & Ppp3r1 & 0,1 & 22,4 \\
\hline $\begin{array}{l}\text { Calcium-activated chloride channel regulator 2; Chloride channel calcium } \\
\text { activated 2; Endothelial chloride channel; MCG120735; }\end{array}$ & Q9EQR4 & Clca2 & $-0,2$ & 32,9 \\
\hline
\end{tabular}




\begin{tabular}{|c|c|c|c|c|}
\hline Protein & Uniprot & Gene name & $\begin{array}{l}\text { Fold change of } \\
\text { XPRESS ratio } \\
\left(\text { Egfr }^{\Delta K C} / \mathrm{WT}\right)\end{array}$ & $\begin{array}{c}\text { Sequence } \\
\text { coverage [\%] }\end{array}$ \\
\hline Calcium-binding mitochondrial carrier protein Aralar1; & Q8BH59 & Slc25a12 & $-0,4$ & 10 \\
\hline Calcium-binding mitochondrial carrier protein Aralar2; & Q9QXX4 & Slc25a13 & $-1,2$ & 13,5 \\
\hline Calcium-binding mitochondrial carrier protein SCaMC-1; & Q8BMD8 & Slc25a24 & $-0,2$ & 13,5 \\
\hline Calcium-binding protein $39 ;$ & Q06138 & Cab39 & $-0,3$ & 25,2 \\
\hline Calcium-regulated heat stable protein $1 ;$ & Q9CR86 & Carhsp1 & 0,4 & 10,8 \\
\hline Calcyclin-binding protein; & Q9CXW3 & Cacybp & $-0,1$ & 10 \\
\hline Calmodulin; & P62204 & Calm3 & 0,1 & 31,5 \\
\hline Calmodulin-4; & Q9JM83 & Calm4 & 0,1 & 49,3 \\
\hline Calmodulin-like protein 3; & Q9D6P8 & Calml3 & $-0,9$ & 19,5 \\
\hline Calnexin; & P35564 & Canx & $-0,4$ & 28,3 \\
\hline Calpain small subunit 1 ; & 088456 & Capns1 & 0,2 & 16,2 \\
\hline Calpain small subunit 2; & Q9D7J7 & Capns2 & $-0,3$ & 17,8 \\
\hline Calpain-1 catalytic subunit; & 035350 & Capn1 & $-0,2$ & 41,4 \\
\hline Calpain-2 catalytic subunit; & 008529 & Capn2 & 0,7 & 12,4 \\
\hline Calpain-5; & 008688 & Capn5 & $-1,3$ & 5 \\
\hline Calpastatin; & P51125 & Cast & $-0,6$ & 33,4 \\
\hline Calreticulin; & P14211 & Calr & $-0,7$ & 35,8 \\
\hline Calsequestrin-1; & 009165 & Casq1 & 3,1 & 8,3 \\
\hline Calumenin; & O35887 & Calu & 0,2 & 33 \\
\hline cAMP-regulated phosphoprotein 19; & P56212 & Arpp19 & $-0,3$ & 13,4 \\
\hline CAP-Gly domain-containing linker protein 1 ; & Q922J3 & Clip1 & 0,1 & 10,1 \\
\hline $\begin{array}{l}\text { Capping protein (Actin filament) muscle Z-line, beta, isoform CRA_a; F-actin- } \\
\text { capping protein subunit beta; }\end{array}$ & A2AMWO & Capzb & $-0,4$ & 31,9 \\
\hline $\begin{array}{l}\text { Capping protein (Actin filament), gelsolin-like; Capping protein (Actin } \\
\text { filament), gelsolin-like, isoform CRA_a; Macrophage-capping protein; }\end{array}$ & Q99LB4 & Capg & $-0,1$ & 29,8 \\
\hline Caprin-1; & Q60865 & Caprin1 & 0,0 & 4,8 \\
\hline CapZ-interacting protein; & Q3UZA1 & Rcsd1 & 0,0 & 2,9 \\
\hline Carbonic anhydrase 2 ; & P00920 & $\mathrm{Ca} 2$ & $-2,1$ & 5 \\
\hline Carbonic anhydrase 3; & P16015 & $\mathrm{Ca} 3$ & 0,8 & 52,3 \\
\hline Carbonic anhydrase 4; & Q64444 & $\mathrm{Ca} 4$ & 0,2 & 14,1 \\
\hline Carbonic anhydrase 6; & P18761 & $\mathrm{Ca} 6$ & $-0,7$ & 29,3 \\
\hline Carbonyl reductase $[\mathrm{NADPH}] 2$ & P08074 & Cbr2 & $-0,1$ & 7 \\
\hline Carbonyl reductase family member 4 ; & Q91VT4 & Cbr4 & 0,3 & 14 \\
\hline Carboxylesterase $1 \mathrm{C}$ & P23953 & Ces1c & 0,3 & 13,4 \\
\hline Carboxylesterase 1D; & Q8VCT4 & Ces1d & 0,0 & 18,4 \\
\hline Carboxylesterase $5 \mathrm{~A}$; & Q6AW46 & Ces5a & 0,8 & 6,1 \\
\hline Carboxymethylenebuten olidase homolog; & Q8R1G2 & $\mathrm{Cmbl}$ & $-0,2$ & 5,2 \\
\hline Carboxypeptidase A4; & Q6P8K8 & Cpa4 & $-1,2$ & 10 \\
\hline Carboxypeptidase D; & 089001 & $\mathrm{Cpd}$ & $-0,4$ & 1,2 \\
\hline Carboxypeptidase E; & Q00493 & Cpe & 0,2 & 9,2 \\
\hline Carboxypeptidase M; & Q80V42 & $\mathrm{Cpm}$ & $-1,6$ & 38,4 \\
\hline Carboxypeptidase Q; & Q9WVJ3 & $\mathrm{Cpq}$ & $-0,2$ & 2,8 \\
\hline Cartilage-associated protein; & Q9CYD3 & Crtap & 0,4 & 6,5 \\
\hline Casein kinase I isoform alpha; & Q8BK63 & Csnk1a1 & $-0,2$ & 13,2 \\
\hline Casein kinase II subunit alpha; & Q60737 & Csnk2a1 & 0,2 & 8,2 \\
\hline Casein kinase II subunit beta; & P67871 & Csnk2b & $-0,2$ & 26,8 \\
\hline Caspase-14; & 089094 & Casp14 & $-1,4$ & 43,2 \\
\hline Caspase-3; & P70677 & Casp3 & 0,4 & 36,1 \\
\hline Caspase-6; & 008738 & Casp6 & 0,2 & 17 \\
\hline Catalase; & P24270 & Cat & $-0,1$ & 18,6 \\
\hline Catenin alpha-1; & P26231 & Ctnna1 & $-0,4$ & 31 \\
\hline Catenin beta-1; & Q02248 & Ctnnb1 & $-0,4$ & 33,7 \\
\hline Catenin delta-1; & P30999 & Ctnnd1 & 0,0 & 3,6 \\
\hline Cathepsin B; & P10605 & Ctsb & $-1,1$ & 25,4 \\
\hline Cathepsin D; & P18242 & Ctsd & $-0,7$ & 27,6 \\
\hline Cathepsin E; & P70269 & Ctse & $-1,4$ & 4,9 \\
\hline Cathepsin L1; & P06797 & Ctsl & $-1,0$ & 25,1 \\
\hline Cathepsin S; & 070370 & Ctss & 1,1 & 3,8 \\
\hline Cathepsin Z; & Q9WUU7 & Ctsz & $-0,2$ & 15 \\
\hline Cation-dependent mannose-6-phosphate receptor; & P24668 & M6pr & 0,1 & 14,4 \\
\hline Cation-independent mannose-6-phosphate receptor; & Q07113 & $\operatorname{lgf} 2 r$ & 0,3 & 7,5 \\
\hline Caveolin-1; & P49817 & Cav1 & 0,1 & 28,7 \\
\hline C-C motif chemokine 27 & Q9Z1X0 & $\mathrm{Ccl} 27$ & $-1,1$ & 11,8 \\
\hline CD109 antigen; & Q8R422 & Cd109 & $-0,1$ & 17,3 \\
\hline CD151 antigen; & 035566 & Cd151 & 0,2 & 4 \\
\hline CD166 antigen; & Q61490 & Alcam & $-1,0$ & 25,3 \\
\hline CD276 antigen; & Q8VE98 & $\mathrm{Cd} 276$ & 1,1 & 6,6 \\
\hline CD2-associated protein; & Q9JLQ0 & Cd2ap & $-0,5$ & 5,5 \\
\hline CD44 antigen; & P15379 & $\mathrm{Cd} 44$ & 2,6 & 6,3 \\
\hline CD63 antigen; & P41731 & Cd63 & $-0,4$ & 4,6 \\
\hline CD81 antigen; & P35762 & Cd81 & 0,1 & 27,5 \\
\hline
\end{tabular}




\begin{tabular}{|c|c|c|c|c|}
\hline Protein & Uniprot & Gene name & $\begin{array}{l}\text { Fold change of } \\
\text { XPRESS ratio } \\
\left(\text { Egfr }^{\triangle K C} / \mathrm{WT}\right)\end{array}$ & $\begin{array}{c}\text { Sequence } \\
\text { coverage [\%] }\end{array}$ \\
\hline CD82 antigen; & P40237 & Cd82 & $-0,5$ & 6,7 \\
\hline CD9 antigen; & P40240 & Cd9 & $-0,1$ & 23,9 \\
\hline CD97 antigen; & Q9Z0M6 & Cd97 & 2,8 & 3,6 \\
\hline Cdc42 effector protein 4 & Q9JM96 & Cdc42ep4 & 0,8 & 33,7 \\
\hline CDGSH iron-sulfur domain-containing protein 1 & Q91WS0 & Cisd1 & $-0,7$ & 25,9 \\
\hline CDGSH iron-sulfur domain-containing protein 2 & Q9CQB5 & Cisd2 & 0,4 & 38,9 \\
\hline CDP-diacylglycerol--inositol 3-phosphatidyltransferase; & Q8VDP6 & Cdipt & $-0,5$ & 16 \\
\hline Cell adhesion molecule 3; & Q99N28 & Cadm3 & 0,6 & 4,5 \\
\hline Cell cycle control protein $50 \mathrm{~A}$; & Q8VEKO & Tmem30a & 0,1 & 4,9 \\
\hline Cell differentiation protein RCD1 homolog; & Q9JKYO & Rqcd1 & $-0,1$ & 3 \\
\hline Cell division control protein 42 homolog; & P60766 & Cdc42 & 0,1 & 48,2 \\
\hline Cell division cycle 5-like protein; & Q6A068 & Cdc5I & 0,4 & 1,2 \\
\hline Cell growth regulator with EF hand domain protein 1 ; & Q8R1U2 & Cgref1 & $-1,7$ & 10,3 \\
\hline Cell growth-regulating nucleolar protein; & Q08288 & Lyar & 0,5 & 6,2 \\
\hline Cell surface glycoprotein MUC18; & Q8R2Y2 & Mcam & 0,1 & 22,1 \\
\hline Cellular nucleic acid-binding protein; & P53996 & Cnbp & 0,7 & 30,9 \\
\hline Cellular nucleic acid-binding protein; & D3YYF6 & Cnbp & 0,7 & 29,5 \\
\hline Centrin-1; & P41209 & Cetn1 & 0,1 & 8,1 \\
\hline Centrosomal protein of $170 \mathrm{kDa}$; & Q6A065 & Cep170 & 0,7 & 1,8 \\
\hline Ceruloplasmin; & Q61147 & $\mathrm{Cp}$ & 1,4 & 14,2 \\
\hline Charged multivesicular body protein $1 \mathrm{~b}-1$; & Q99LU0 & Chmp1b1 & 0,5 & 4 \\
\hline Charged multivesicular body protein $2 \mathrm{a}$; & Q9DB34 & Chmp2a & 0,2 & 8,1 \\
\hline Charged multivesicular body protein $2 \mathrm{~b}$; & Q8BJF9 & Chmp2b & 0,2 & 8 \\
\hline Charged multivesicular body protein $4 \mathrm{~b}$; & Q9D8B3 & Chmp4b & $-0,1$ & 21,9 \\
\hline Charged multivesicular body protein 5 ; & Q9D7S9 & Chmp5 & 0,2 & 10,5 \\
\hline Chitinase-3-like protein 1; & Q61362 & Chi3l1 & 3,1 & 12,7 \\
\hline Chitotriosidase-1; & Q9D7Q1 & Chit1 & 0,2 & 3,7 \\
\hline Chloride channel CLIC-like protein 1 ; & Q99LI2 & Clcc1 & 0,3 & 8,5 \\
\hline Chloride intracellular channel protein 1 ; & Q9Z1Q5 & Clic1 & 0,6 & 25,3 \\
\hline Chloride intracellular channel protein 3; & Q9D7P7 & Clic3 & $-1,0$ & 6,8 \\
\hline Choline transporter-like prote in 1 ; & Q6X893 & Slc44a1 & $-0,2$ & 5,1 \\
\hline Choline transporter-like prote in 2 & Q8BY89 & Slc44a2 & $-0,1$ & 5 \\
\hline Choline/ethanolamine kinase; & 055229 & Chkb & 0,6 & 14,2 \\
\hline Chondroitin sulfate proteoglycan 4; & Q8VHYO & Cspg4 & 0,1 & 6,6 \\
\hline Chromatin modification-related protein MEAF6; & Q2VPQ9 & Meaf6 & 0,2 & 15,2 \\
\hline Chromatin target of PRMT1 protein; & Q9CY57 & Chtop & $-0,6$ & 12,8 \\
\hline Chromobox protein homolog 1 & P83917 & Cbx1 & 0,2 & 5,9 \\
\hline Chromobox protein homolog 3 ; & P23198 & $\mathrm{Cb} \times 3$ & $-0,4$ & 13,7 \\
\hline Chromobox protein homolog 5 & Q61686 & $\mathrm{Cbx} 5$ & $-0,2$ & 6,8 \\
\hline Chromodomain-helicase-DNA-binding protein 4; & Q6PDQ2 & Chd4 & 0,1 & 2,1 \\
\hline Ciliary neurotrophic factor receptor subunit alpha; & 088507 & Cntfr & $-1,6$ & 2,4 \\
\hline Cingulin; & P59242 & Cgn & $-0,4$ & 2,5 \\
\hline Citrate lyase subunit beta-like protein, mitochondrial; & Q8R4N0 & Clybl & $-0,1$ & 16,9 \\
\hline Citrate synthase, mitochondrial; & Q9CZU6 & Cs & 0,3 & 6,5 \\
\hline C-Jun-amino-terminal kinase-interacting protein 4; & Q58A65 & Spag9 & 0,2 & 2,8 \\
\hline Clathrin heavy chain 1 ; & Q68FD5 & Cltc & 0,6 & 9,1 \\
\hline Clathrin interactor 1 ; & Q99KN9 & Clint1 & $-0,2$ & 8,3 \\
\hline Clathrin light chain A; & B1AWE0 & Clta & 0,3 & 28,2 \\
\hline Clathrin light chain B; & Q6IRU5 & Cltb & 0,4 & 26,2 \\
\hline Claudin-1; & 088551 & Cldn1 & $-0,1$ & 7,1 \\
\hline Claudin-4; & 035054 & Cldn4 & $-1,5$ & 5,7 \\
\hline Cleavage and polyadenylation specificity factor subunit 5 & Q9CQF3 & Nudt21 & 0,2 & 30 \\
\hline Cleavage and polyadenylation specificity factor subunit 6; & Q6NVF9 & Cpsf6 & $-0,1$ & 8,8 \\
\hline Cleavage stimulation factor subunit 2 & Q8BIQ5 & Cstf2 & 0,1 & 6 \\
\hline Cleft lip and palate transmembrane protein 1 homolog; & Q8VBZ3 & Clptm1 & $-0,1$ & 11,4 \\
\hline Clusterin; & Q06890 & $\mathrm{Clu}$ & $-1,9$ & 28,1 \\
\hline C-Myc-binding protein; & Q9EQS3 & Mycbp & 0,5 & 62,1 \\
\hline Coagulation factor XIII A chain; & Q8BH61 & F13a1 & 0,4 & 15,6 \\
\hline Coatomer subunit alpha; & Q8CIE6 & Copa & 0,7 & 4,9 \\
\hline Coatomer subunit beta; & Q9JIF7 & Copb1 & $-0,2$ & 20,7 \\
\hline Coatomer subunit beta'; & 055029 & Copb2 & $-0,2$ & 11,9 \\
\hline Coatomer subunit delta; & Q5XJY5 & Arcn1 & 0,2 & 16,2 \\
\hline Coatomer subunit epsilon; & 089079 & Cope & $-0,9$ & 8,8 \\
\hline Coatomer subunit gamma-1; & Q9QZE5 & Copg1 & 0,2 & 15,6 \\
\hline Coatomer subunit zeta-1; & P61924 & Copz1 & 0,5 & 22,6 \\
\hline Coatomer subunit zeta-2; & Q9JHH9 & Copz2 & 0,4 & 9,3 \\
\hline Cochlin; & Q62507 & Coch & $-0,8$ & 8,7 \\
\hline Cofilin-1; & P18760 & $\mathrm{Cfl1}$ & 0,3 & 39,2 \\
\hline Cofilin-2; & P45591 & $\mathrm{Cfl} 2$ & 0,2 & 16,3 \\
\hline Coiled-coil domain-containing protein $102 \mathrm{~A}$; & Q3TMW1 & Ccdc102a & 0,0 & 4,7 \\
\hline Coiled-coil domain-containing protein 151 & Q8BSN3 & Ccdc151 & 3,1 & 5,6 \\
\hline
\end{tabular}




\begin{tabular}{|c|c|c|c|c|}
\hline Protein & Uniprot & Gene name & $\begin{array}{l}\text { Fold change of } \\
\text { XPRESS ratio } \\
\left(\text { Egfr }^{\Delta K C} / \mathrm{WT}\right) \\
\end{array}$ & $\begin{array}{c}\text { Sequence } \\
\text { coverage [\%] }\end{array}$ \\
\hline Coiled-coil domain-containing protein 160 & Q3UYG1 & Ccdc160 & 2,8 & 6,5 \\
\hline Coiled-coil domain-containing protein 43 ; & Q9CR29 & Ccdc43 & 0,4 & 6,3 \\
\hline Coiled-coil domain-containing protein 50 & Q810U5 & Ccdc50 & 1,7 & 9 \\
\hline Coiled-coil domain-containing protein 58 ; & Q8R3Q6 & Ccdc58 & $-1,5$ & 19,4 \\
\hline Coiled-coil domain-containing protein 6; & D3YZP9 & Ccdc6 & 0,8 & 5,1 \\
\hline Coiled-coil domain-containing protein 9; & Q8VC31 & Ccdc9 & 0,0 & 1,7 \\
\hline Coiled-coil domain-containing protein 90B, mitochondrial; & Q8C3X2 & Ccdc90b & $-0,8$ & 2,7 \\
\hline Coiled-coil domain-containing protein 91 & Q9D8L5 & Ccdc91 & $-1,4$ & 1,8 \\
\hline Coiled-coil-helix-coiled-coil-helix domain-containing protein 2, mitochondrial; & Q9D1L0 & Chchd2 & 0,0 & 30,1 \\
\hline Coiled-coil-helix-coiled-coil-helix domain-containing protein 3, mitochondrial; & Q9CRB9 & Chchd3 & $-0,6$ & 17,2 \\
\hline Cold-inducible RNA-binding protein; & P60824 & Cirbp & 0,6 & 14,5 \\
\hline Collagen alpha-1(I) chain; & P11087 & Col1a1 & $-1,5$ & 19 \\
\hline Collagen alpha-1(II) chain; & P28481 & Col2a1 & $-1,9$ & 3 \\
\hline Collagen alpha-1(III) chain; & P08121 & Col3a1 & $-0,7$ & 12,6 \\
\hline Collagen alpha-1(IV) chain; & P02463 & Col4a1 & $-0,7$ & 1,3 \\
\hline Collagen alpha-1(V) chain; & 088207 & Col5a1 & $-0,2$ & 2,3 \\
\hline Collagen alpha-1(VI) chain; & Q04857 & Col6a1 & $-0,2$ & 15,7 \\
\hline Collagen alpha-1(VII) chain; & Q63870 & Col7a1 & $-0,6$ & 2,3 \\
\hline Collagen alpha-1(XII) chain; & Q60847 & Col12a1 & $-0,1$ & 9,3 \\
\hline Collagen alpha-1(XIV) chain; & Q80X19 & Col14a1 & $-0,2$ & 20,2 \\
\hline Collagen alpha-1(XV) chain; & 035206 & Col15a1 & 0,2 & 7,7 \\
\hline Collagen alpha-1(XVI) chain; & Q8BLX7 & Col16a1 & $-0,4$ & 1,1 \\
\hline Collagen alpha-1(XVII) chain; & Q07563 & Col17a1 & 0,3 & 2,2 \\
\hline Collagen alpha- 1 (XVIII) chain; & P39061 & Col18a1 & $-0,3$ & 4,8 \\
\hline Collagen alpha-2(I) chain; & Q01149 & Col1a2 & $-1,2$ & 24,6 \\
\hline Collagen alpha-2(IV) chain; & P08122 & Col4a2 & 0,3 & 2,2 \\
\hline Collagen alpha-2(VI) chain; & Q02788 & Col6a2 & $-0,2$ & 15,9 \\
\hline Collagen alpha-6(VI) chain; & Q8C6K9 & Col6a6 & $-0,7$ & 0,4 \\
\hline $\begin{array}{l}\text { Collagen type V alpha } 3 \text { chain; Collagen, type V, alpha 3; Procollagen, type V, } \\
\text { alpha 3, isoform CRA_b; Protein Col5a3; }\end{array}$ & Q9JLI2 & Col5a3 & 0,9 & 2 \\
\hline Collectin-12; & Q8K4Q8 & Colec12 & 1,0 & 0,9 \\
\hline Complement C3; & P01027 & $\mathrm{C} 3$ & $-0,2$ & 29 \\
\hline Complement C4-B; & P01029 & $\mathrm{c} 4 \mathrm{~b}$ & 0,7 & 1,1 \\
\hline $\begin{array}{l}\text { Complement component } 1 \mathrm{Q} \text { subcomponent-binding protein, mitochondrial; } \\
\text { Complement component } 1 \text {, q subcomponent binding protein; Complement } \\
\text { component } 1 \text {, q subcomponent binding protein, isoform CRA_b; p32-RACK; }\end{array}$ & Q8R5L1 & C1qbp & $-0,1$ & 7,2 \\
\hline Complement component C1q receptor; & 089103 & $\mathrm{Cd} 93$ & 0,2 & 1,7 \\
\hline Complement component receptor 1 -like protein; & Q64735 & Cr11 & 0,3 & 2,1 \\
\hline Complement factor B; & P04186 & $\mathrm{Cfb}$ & $-0,2$ & 4,2 \\
\hline Complement factor D; & P03953 & $\mathrm{Cfd}$ & 0,6 & 20,1 \\
\hline Complement factor $\mathrm{H}$; & P06909 & Cfh & $-0,5$ & 3,2 \\
\hline Complement factor I; & Q61129 & $\mathrm{Cfi}$ & 0,5 & 1,3 \\
\hline Constitutive coactivator of PPAR-gamma-like protein 1 ; & Q6A0A9 & FAM120A & 0,2 & 6,9 \\
\hline COP9 signalosome complex subunit 1 & Q99LD4 & Gps1 & 0,3 & 10,4 \\
\hline COP9 signalosome complex subunit 2 ; & P61202 & Cops2 & 0,1 & 10,6 \\
\hline COP9 signalosome complex subunit 3 ; & 088543 & Cops3 & 1,0 & 9,2 \\
\hline COP9 signalosome complex subunit 4 ; & 088544 & Cops4 & 0,3 & 25,6 \\
\hline COP9 signalosome complex subunit 5 & 035864 & Cops5 & 0,1 & 13,8 \\
\hline COP9 signalosome complex subunit 6; & 088545 & Cops6 & 0,2 & 6,7 \\
\hline COP9 signalosome complex subunit 7a; & Q9CZO4 & Cops7a & 0,1 & 21,1 \\
\hline COP9 signalosome complex subunit 7b; & Q8BV13 & Cops7b & 0,1 & 6,4 \\
\hline COP9 signalosome complex subunit 8 & Q8VBV7 & Cops8 & 0,3 & 7,2 \\
\hline Copine-3; & Q8BT60 & Cpne3 & 0,0 & 2,8 \\
\hline Copper transport protein ATOX1; & 008997 & Atox 1 & 0,7 & 57,4 \\
\hline Cordon-bleu protein-like 1 ; & Q3UMF0 & Cobll1 & $-0,1$ & 0,7 \\
\hline Core histone macro-H2A.1; & Q9QZQ8 & H2afy & $-0,2$ & 39,8 \\
\hline Core histone macro-H2A.2; & Q8CCK0 & H2afy2 & 0,0 & 21 \\
\hline Corneodesmosin; & Q7TPC1 & Cdsn & 0,1 & 13,5 \\
\hline Cornifin-A; & Q62266 & Sprr1a & $-0,8$ & 63,2 \\
\hline Cornifin-B; & Q62267 & Sprr1b & $-1,1$ & 20,3 \\
\hline Coronin-1A; & 089053 & Coro1a & 1,4 & 5,6 \\
\hline Corticosteroid-binding globulin; & Q06770 & Serpina6 & $-3,4$ & 2,8 \\
\hline Costars family protein ABRACL; & Q4KML4 & Abracl & 0,7 & 27,2 \\
\hline Coxsackievirus and adenovirus receptor homolog; & P97792 & Cxadr & $-0,5$ & 19,5 \\
\hline Craniofacial development protein 1 ; & 088271 & Cfdp1 & 0,7 & 16,6 \\
\hline Creatine kinase B-type; & Q04447 & $\mathrm{Ckb}$ & $-0,5$ & 5,5 \\
\hline Creatine kinase U-type, mitochondrial; & P30275 & Ckmt1 & $-0,4$ & 33,3 \\
\hline cTAGE family member 5 & Q8R311 & Ctage5 & $-0,1$ & 2,7 \\
\hline
\end{tabular}




\begin{tabular}{|c|c|c|c|c|}
\hline Protein & Uniprot & Gene name & $\begin{array}{l}\text { Fold change of } \\
\text { XPRESS ratio } \\
\left(\text { Egfr }^{\Delta K C} / \mathrm{WT}\right)\end{array}$ & $\begin{array}{c}\text { Sequence } \\
\text { coverage [\%] }\end{array}$ \\
\hline C-terminal-binding protein 1 & 088712 & Ctbp1 & 0,3 & 26,3 \\
\hline C-terminal-binding protein 2 & P56546 & Ctbp2 & 0,2 & 23,3 \\
\hline CTP synthase 1 ; & P70698 & Ctps1 & $-0,7$ & 35,9 \\
\hline CTP synthase 2 & P70303 & Ctps2 & 0,7 & 10,4 \\
\hline C-type lectin domain family 11 member A; & 088200 & Clec11a & 0,2 & 4,3 \\
\hline C-type mannose receptor 2 & Q64449 & Mrc2 & 0,0 & 5,1 \\
\hline Cullin-1; & Q9WTX6 & Cul1 & 0,5 & 14,9 \\
\hline Cullin-3; & Q9JLV5 & Cul3 & 2,0 & 3,8 \\
\hline Cullin-4B; & A2A432 & Cul4b & 0,1 & 2,7 \\
\hline Cullin-associated NEDD8-dissociated protein 1; & Q6ZQ38 & Cand1 & 0,2 & 27,8 \\
\hline Cyclic AMP-dependent transcription factor ATF-7; & Q8R0S1 & Atf7 & $-0,3$ & 2,2 \\
\hline Cyclic AMP-responsive element-binding protein 1; & Q01147 & Creb1 & 0,4 & 7,3 \\
\hline Cyclin-dependent kinase 2 & P97377 & Cdk2 & 0,8 & 4,6 \\
\hline Cyclin-dependent kinase 6; & Q64261 & Cdk6 & 1,1 & 8,6 \\
\hline Cyclin-dependent kinase inhibitor 1B; & P46414 & Cdkn1b & $-0,3$ & 10,7 \\
\hline Cystathionine beta-synthase; & Q91WT9 & Cbs & $-0,9$ & 14,4 \\
\hline Cystathionine gamma-lyase; & Q8VCN5 & Cth & $-0,7$ & 11,1 \\
\hline Cystatin E/M; Cystatin M/E; Cystatin-B; & Q9D1B1 & Cst6 & 0,2 & 55 \\
\hline Cystatin-A; & P56567 & Csta & $-1,5$ & 24,7 \\
\hline Cystatin-B; & Q62426 & Cstb & $-0,7$ & 39,8 \\
\hline Cystatin-C; & P21460 & Cst3 & 0,4 & 10,8 \\
\hline Cysteine and glycine-rich protein 1 ; & P97315 & Csrp1 & $-0,7$ & 48,2 \\
\hline Cysteine and glycine-rich protein 2; & P97314 & Csrp2 & 0,8 & 21,8 \\
\hline Cysteine sulfinic acid decarboxylase; & Q9DBE0 & Csad & 0,5 & 17,6 \\
\hline Cysteine-rich protein 1 & P63254 & Crip1 & 1,0 & 19,5 \\
\hline Cysteine-rich protein 2; & Q9DCT8 & Crip2 & $-0,2$ & 18,8 \\
\hline Cysteine-rich secretory protein 1 ; & Q03401 & Crisp1 & $-2,5$ & 14,3 \\
\hline Cysteine-rich with EGF-like domain protein 2; & Q9CYA0 & Creld2 & $-0,2$ & 9,4 \\
\hline Cysteine--tRNA ligase, cytoplasmic; & Q9ER72 & Cars & $-0,4$ & 9,5 \\
\hline Cytochrome b; & P00158 & Mt Cyb & $-0,2$ & 2,4 \\
\hline Cytochrome b5 type B; & Q9CQX2 & Cyb5b & 0,0 & 50,7 \\
\hline Cytochrome b5; & P56395 & Cyb5a & 0,3 & 65,3 \\
\hline Cytochrome b-c1 complex subunit 1, mitochondrial; & Q9CZ13 & Uqcrc1 & $-0,8$ & 30,8 \\
\hline Cytochrome b-c1 complex subunit 2, mitochondrial; & Q9DB77 & Uqcrc2 & $-0,7$ & 40,2 \\
\hline Cytochrome b-c1 complex subunit 6, mitochondrial; & P99028 & Uqcrh & $-0,3$ & 28,1 \\
\hline Cytochrome b-c1 complex subunit 7; & Q9D855 & Uqcrb & $-0,5$ & 25,2 \\
\hline Cytochrome b-c1 complex subunit 8; & Q9CQ69 & Uqcrq & $-0,3$ & 26,8 \\
\hline Cytochrome b-c1 complex subunit 9; & Q8R1I1 & Uqcr10 & $-0,3$ & 37,5 \\
\hline Cytochrome b-c1 complex subunit Rieske, mitochondrial; & Q9CR68 & Uqcrfs1 & $-0,1$ & 32,8 \\
\hline Cytochrome c oxidase assembly factor 3 homolog, mitochondrial; & Q9D2R6 & Сoa3 & $-0,1$ & 17,6 \\
\hline Cytochrome c oxidase copper chaperone; & P56394 & Cox17 & 0,4 & 11,1 \\
\hline Cytochrome c oxidase subunit 2; & P00405 & Mtco2 & $-1,2$ & 23,3 \\
\hline Cytochrome c oxidase subunit 3; & P00416 & $\mathrm{mt} \mathrm{Co3}$ & $-0,3$ & 5,4 \\
\hline Cytochrome c oxidase subunit 4 isoform 1, mitochondrial; & P19783 & Cox4i1 & $-0,8$ & 35,5 \\
\hline Cytochrome c oxidase subunit $5 \mathrm{~A}$, mitochond rial; & P12787 & Cox5a & $-1,1$ & 21,2 \\
\hline Cytochrome c oxidase subunit $5 \mathrm{~B}$, mitochondrial; & P19536 & Cox5b & $-0,8$ & 24,2 \\
\hline Cytochrome c oxidase subunit 6B1; & P56391 & Cox6b1 & $-0,6$ & 41,9 \\
\hline Cytochrome c oxidase subunit 6C; & Q9CPQ1 & Cox6c & $-0,4$ & 18,4 \\
\hline Cytochrome c oxidase subunit 7A2, mitochondrial; & P48771 & Cox7a2 & $-0,4$ & 27,7 \\
\hline Cytochrome c oxidase subunit 7C, mitochondrial; & P17665 & Cox7c & 0,1 & 14,3 \\
\hline Cytochrome c, somatic; & P62897 & Cycs & 0,3 & 38,1 \\
\hline Cytochrome c1, heme protein, mitochond rial; & Q9D0M3 & Cyc1 & $-0,9$ & 27,1 \\
\hline Cytochrome P450 2B10; MCG7659, isoform CRA_a; & G3X8Q9 & Cyp2b10 & 1,1 & 4,1 \\
\hline Cytochrome P450 2F2; & P33267 & Cyp2f2 & 2,1 & 5,7 \\
\hline Cytochrome P450 2S1; & Q9DBX6 & Cyp2s1 & $-1,1$ & 7,2 \\
\hline $\begin{array}{l}\text { Cytochrome P450, family 2, subfamily g, polypeptide 1; Olfactory-specific } \\
\text { steroid hydroxylase; Protein Cyp2g1; }\end{array}$ & Q9WV19 & Cyp2g1 & $-1,2$ & 14,6 \\
\hline Cytoglobin; & Q9CX80 & Cygb & 2,2 & 12,6 \\
\hline Cytoplasmic aconitate hydratase; & P28271 & Aco1 & 0,6 & 17,1 \\
\hline Cytoplasmic dynein 1 heavy chain 1 ; & Q9JHU4 & Dync1h1 & 0,1 & 4,6 \\
\hline Cytoplasmic dynein 1 intermediate chain 2; & 088487 & Dync1i2 & $-0,1$ & 11,9 \\
\hline Cytoplasmic dynein 1 light intermediate chain 1 ; & Q8R1Q8 & Dync1li1 & $-3,1$ & 2,3 \\
\hline Cytoskeleton-associated protein 4; & Q8BMK4 & Ckap4 & $-0,5$ & 36 \\
\hline Cytosol aminopeptidase; & Q9CPY7 & Lap3 & $-0,4$ & 49,3 \\
\hline Cytosolic acyl coenzyme A thioester hydrolase; & Q91V12 & Acot7 & $-0,3$ & 23,9 \\
\hline Cytosolic endo-beta- $\mathrm{N}$-acetylglucosaminidase; & Q8BX80 & Engase & $-0,1$ & 8,2 \\
\hline Cytosolic non-specific dipeptidase; & Q9D1A2 & Cndp2 & 0,0 & 19,4 \\
\hline Cytosolic phospholipase A2 beta; & P0C871 & Pla2g4b & $-2,0$ & 4,2 \\
\hline Cytosolic purine 5'-nucleotidase; & Q3V1L4 & $\mathrm{Nt5} 22$ & $-1,3$ & 5,7 \\
\hline D-3-phosphoglycerate dehydrogenase; & Q61753 & Phgdh & 0,4 & 25,5 \\
\hline DAZ-associated protein 1 & Q9JII5 & Dazap1 & 0,2 & 18,7 \\
\hline
\end{tabular}




\begin{tabular}{|c|c|c|c|c|}
\hline Protein & Uniprot & Gene name & $\begin{array}{l}\text { Fold change of } \\
\text { XPRESS ratio } \\
\left(\text { Egfr }^{\Delta K C} / \mathrm{WT}\right)\end{array}$ & $\begin{array}{c}\text { Sequence } \\
\text { coverage [\%] }\end{array}$ \\
\hline D-dopachrome decarboxylase; & 035215 & Ddt & 0,2 & 51,7 \\
\hline DDRGK domain-containing protein 1 ; & Q80WW9 & Ddrgk1 & 0,0 & 7,9 \\
\hline DEAH (Asp-Glu-Ala-His) box polypeptide 16, isoform CRA_a; Protein Dhx16; & G3X8X0 & Dhx16 & 0,6 & 1,6 \\
\hline Death domain-containing membrane protein NRADD; & Q8CJ26 & Nradd & $-0,2$ & 11,8 \\
\hline Death-associated protein-like 1 & Q9D757 & Dapl1 & 0,4 & 7,5 \\
\hline Decorin; & P28654 & Den & $-0,2$ & 32,5 \\
\hline Dehydrogenase/reductase SDR family member 1 & Q99L04 & Dhrs1 & 0,1 & 3,8 \\
\hline Dehydrogenase/reductase SDR family member 7; & Q9CXR1 & Dhrs7 & 0,1 & 8,3 \\
\hline Dehydrogenase/reductase SDR family member 7B; & Q99J47 & Dhrs7b & $-0,1$ & 11,8 \\
\hline Delta(24)-sterol reductase; & Q8VCH6 & Dhcr24 & $-0,7$ & 4,5 \\
\hline Delta(3,5)-Delta(2,4)-dienoyl-CoA isomerase, mitochondrial; & 035459 & Ech1 & $-0,1$ & 25,7 \\
\hline Delta-aminolevulinic acid dehydratase; & P10518 & Alad & $-0,3$ & 28,2 \\
\hline Delta-sarcoglycan; & P82347 & Sgcd & $-0,2$ & 23,4 \\
\hline Density-regulated protein; & Q9CQJ6 & Denr & 0,1 & 18,6 \\
\hline Deoxynucleoside triphosphate triphosphohydrolase SAMHD1; & Q60710 & Samhd1 & 0,7 & 16,3 \\
\hline Deoxyribonuclease-1-like 2; & Q9D1G0 & Dnase1l2 & 0,3 & 7,2 \\
\hline Deoxyribonuclease-2-alpha; & P56542 & Dnase2 & $-0,3$ & 18,1 \\
\hline Derlin-1; & Q99J56 & Derl1 & $-0,6$ & 7,6 \\
\hline Dermatopontin; & Q9QZZ6 & Dpt & 0,2 & 32,3 \\
\hline Dermokine; & E9Q2P1 & Dmkn & 0,7 & 29,9 \\
\hline Desmocollin-1; & P55849 & Dsc1 & 0,0 & 22 \\
\hline Desmocollin-2; & P55292 & Dsc2 & $-0,4$ & 2,7 \\
\hline Desmocollin-3; & P55850 & Dsc3 & $-0,1$ & 14,3 \\
\hline Desmoglein-1-alpha; & Q61495 & Dsg1a & $-0,4$ & 7,9 \\
\hline Desmoglein-2; & 055111 & Dsg2 & $-0,3$ & 6,4 \\
\hline Desmoglein-3; & 035902 & Dsg3 & 1,9 & 1,4 \\
\hline Desmoplakin; & E9Q557 & Dsp & $-0,3$ & 2,5 \\
\hline Destrin; & Q9R0P5 & Dstn & 0,2 & 34,5 \\
\hline Developmentally-regulated GTP-binding protein 1; & P32233 & Drg1 & 0,6 & 14,7 \\
\hline Diacylglycerol O-acyltransferase 1 & Q9Z2A7 & Dgat1 & 0,2 & 5 \\
\hline Dihydrolipoyl dehydrogenase, mitochondrial; & 008749 & Dld & 0,0 & 24,2 \\
\hline $\begin{array}{l}\text { Dihydrolipoyllysine-residue acetyltransferase component of pyruvate } \\
\text { dehydrogenase complex, mitochondrial; }\end{array}$ & Q8BMF4 & Dlat & $-0,4$ & 4,2 \\
\hline $\begin{array}{l}\text { Dihydrolipoyllysine-residue succinyltransferase component of 2-oxoglutarate } \\
\text { dehydrogenase complex, mitochondrial; }\end{array}$ & Q9D2G2 & Dlst & $-0,6$ & 19,6 \\
\hline Dihydroorotate dehydrogenase (quinone), mitochondrial; & 035435 & Dhodh & 0,4 & 8,6 \\
\hline Dihydropteridine reductase; & Q8BVI4 & Qdpr & 0,3 & 32,3 \\
\hline Dihydropyrimidinase-related protein 2 ; & 008553 & Dpysl2 & 0,7 & 48,1 \\
\hline Dihydropyrimidinase-related protein 3 ; & E9PWE8 & Dpysl3 & 0,4 & 27,4 \\
\hline Dipeptidase 1; & P31428 & Dpep1 & 0,4 & 19,3 \\
\hline Dipeptidyl peptidase 1 & P97821 & Ctsc & $-0,4$ & 15,4 \\
\hline Dipeptidyl peptidase 2; & Q9ET22 & Dpp7 & $-0,2$ & 10,7 \\
\hline Dipeptidyl peptidase 4; & P28843 & Dpp4 & $-0,7$ & 11,2 \\
\hline Diphosphoinositol polyphosphate phosphohydrolase 1 ; & Q9J146 & Nudt3 & 0,3 & 23,9 \\
\hline Diphthamide biosynthesis protein 7 & Q9CYU6 & Dph7 & $-0,5$ & 2,7 \\
\hline Disabled homolog 2; & P98078 & Dab2 & 0,7 & 5,9 \\
\hline Disintegrin and metalloproteinase domain-containing protein $10 ;$ & 035598 & Adam10 & 0,4 & 3,7 \\
\hline Disintegrin and metalloproteinase domain-containing protein 9; & Q61072 & Adam9 & 0,6 & 1,7 \\
\hline Disks large-associated protein 4 & B1AZP2 & Dlgap4 & 0,2 & 10,7 \\
\hline DNA (cytosine-5)-methyltransferase $3 \mathrm{~A}$; & 088508 & Dnmt3a & 0,4 & 7,5 \\
\hline DNA damage-binding protein 1 ; & Q3U1J4 & Ddb1 & 0,1 & 25,8 \\
\hline DNA mismatch repair protein Msh2; & P43247 & Msh2 & 0,8 & 1,2 \\
\hline DNA polymerase delta catalytic subunit; & P52431 & Pold1 & 0,8 & 2 \\
\hline DNA polymerase epsilon subunit 4 & Q9CQ36 & Pole4 & 0,6 & 21,2 \\
\hline DNA polymerase lambda; & Q9QXE2 & Poll & 0,1 & 1,6 \\
\hline DNA replication licensing factor $\mathrm{MCM} 3$; & P25206 & Mcm3 & 0,7 & 1,6 \\
\hline DNA replication licensing factor MCM4; & P49717 & Mcm4 & 0,1 & 2,6 \\
\hline DNA replication licensing factor MCM6; & P97311 & Mcm6 & 0,0 & 3 \\
\hline DNA replication licensing factor MCM7; & Q61881 & Mcm7 & 0,5 & 6,1 \\
\hline DNA topoisomerase 1 & Q04750 & Top1 & 0,3 & 2,9 \\
\hline DNA topoisomerase 2-beta; & Q64511 & Top2b & $-0,2$ & 7,6 \\
\hline DNA-(apurinic or apyrimidinic site) lyase; & P28352 & Apex1 & 0,4 & 15,3 \\
\hline DNA-directed RNA polymerase II subunit RPB1; & P08775 & Polr2a & $-0,1$ & 2,2 \\
\hline DNA-directed RNA polymerase II subunit RPB2; & Q8CFI7 & Polr2b & $-0,4$ & 5,4 \\
\hline DNA-directed RNA polymerase II subunit RPB4; & Q9D7M8 & Polr2d & 0,5 & 23,2 \\
\hline DNA-directed RNA polymerase II subunit RPB7; & P62488 & Polr2g & 0,1 & 20,9 \\
\hline DNA-directed RNA polymerases I, II, and III subunit RPABC1; & Q80UW8 & Polr2e & 0,2 & 10,5 \\
\hline DNA-directed RNA polymerases I, II, and III subunit RPABC3; & Q923G2 & Polr2h & 0,2 & 8,7 \\
\hline DnaJ homolog subfamily A member 1 ; & P63037 & Dnaja1 & 1,6 & 11,6 \\
\hline DnaJ homolog subfamily A member 2 & Q9QYJ0 & Dnaja2 & 0,1 & 2,4 \\
\hline
\end{tabular}


Protein

DnaJ homolog subfamily B member 1;

DnaJ homolog subfamily B member 11 ;

DnaJ homolog subfamily B member 6 ;

DnaJ homolog subfamily $\mathrm{C}$ member 10 ;

DnaJ homolog subfamily $\mathrm{C}$ member 3 ;

DnaJ homolog subfamily $\mathrm{C}$ member 8 ;

DnaJ homolog subfamily $\mathrm{C}$ member 9 ;

Dolichol-phosphate mannosyltransferase subunit 1 ;

Dolichol-phosphate mannosyltransferase subunit 3;

Dolichyl-diphosphooligosaccharide--protein glycosyltransferase 48 kDa

subunit;

Dolichyl-diphosphooligosaccharide--protein glycosyltransferase subunit 1;

Dolichyl-diphosphooligosaccharide--protein glycosyltransferase subunit 2;

Dolichyl-diphosphooligosaccharide--protein glycosyltransferase subunit DAD1;

Dolichyl-diphosphooligosaccharide--protein glycosyltransferase subunit STT3A;

Dolichyl-diphosphooligosaccharide--protein glycosyltransferase subunit STT3B;

Dolichyl-phosphate beta-glucosyltransferase;

Double-strand-break repair protein rad21 homolog;

Dr1-associated corepressor

Drebrin-like protein;

Dual specificity protein kinase CLK1;

Dual specificity protein phosphatase 14 ;

Dynactin subunit 1 ;

Dynactin subunit 2;

Dynactin subunit 4;

Dynactin subunit 5;

Dynactin subunit 6 ;

Dynamin-like 120 kDa protein, mitochondrial;

Dynein light chain 2, cytoplasmic;

Dynein light chain roadblock-type 1;

Dyslexia-associated protein KIAA0319-like protein;

Dystroglycan

Dystrophin;

E3 ubiquitin-protein ligase ARIH1;

E3 ubiquitin-protein ligase HUWE1;

E3 ubiquitin-protein ligase RBX1;

E3 ubiquitin-protein ligase RNF126;

E3 ubiquitin-protein ligase RNF181;

Early endosome antigen 1 ;

Echinoderm microtubule-associated protein-like 1;

Echinoderm microtubule-associated protein-like 2;

Ectonucleoside triphosphate diphosphohydrolase 1 ;

Ectonucleoside triphosphate diphosphohydrolase 2;

Ectonucleoside triphosphate diphosphohydrolase 5;

Ectonucleotide pyrophosphatase/phosphodiesterase family member 1;

Ectonucleotide pyrophosphatase/phosphodiesterase family mem ber 3 ;

EF-hand domain-containing protein D2;

EGF domain-specific O-linked $\mathrm{N}$-acetylglucosamine transferase;

EGF-containing fibulin-like extracellular matrix protein 1;

EGF-containing fibulin-like extracellular matrix protein 2;

EH domain-containing protein 1;

EH domain-containing protein 2;

EH domain-containing protein 4 ;

Elastin;

ELAV-like protein 1;

Electron transfer flavoprotein subunit alpha, mitochondrial;

Electron transfer flavoprotein subunit beta;

Electron transfer flavoprotein-ubiquinone oxidoreductase, mitochondrial;

Elongation factor 1-alpha 1;

Elongation factor 1-beta;

Elongation factor 1-delta;

Elongation factor 1-gamma;

Elongation factor 2;

\begin{tabular}{lccc} 
Uniprot & Gene name & $\begin{array}{c}\text { Fold change of } \\
\text { XPRESS ratio } \\
\text { (Egfr }{ }^{\Delta K C} \text { / WT) }\end{array}$ & $\begin{array}{c}\text { Sequence } \\
\text { coverage [\%] }\end{array}$ \\
\hline Q9QYJ3 & Dnajb1 & 0,1 & 5,9 \\
Q99KV1 & Dnajb11 & $-0,5$ & 6,1 \\
O54946 & Dnajb6 & 0,3 & 6,3 \\
Q9DC23 & Dnajc10 & $-1,1$ & 2,8 \\
Q91YW3 & Dnajc3 & $-0,1$ & 3,8 \\
Q6NZB0 & Dnajc8 & 0,1 & 4,1 \\
Q91WN1 & Dnajc9 & 0,4 & 4,6 \\
O70152 & Dpm1 & 0,0 & 13,5 \\
Q9D1Q4 & Dpm3 & 0,1 & 23,9 \\
O54734 & Ddost & 0,0 & 14,3 \\
Q91Y05 & Rpn1 & $-0,1$ & 34,4 \\
Q9DBG6 & Rpn2 & 0,0 & 19,8
\end{tabular}

Dad1

$-0,4$

P46978

Stt3a

0,1

7,2

Q3TDQ1

Stt3b

0,1

5

Q9DB25

Q61550

Q9D6N5

Q62418

P22518

Q9JLY7

008788

Q99KJ8

Q8CBY8

Q9QZB9

Q9WUB4

P58281

Q9D0M5

P62627

Q8K135

Q62165

P11531

Q9Z1K5

Q7TMY8

P62878

Q91YL2

Q9CY62

Q8BL66

Q05BC3

Q7TNG5

P55772

055026

Q9WUZ9

P06802

Alg5

Rad21

Drap1

Dbnl

Clk1

Dusp14

Dctn1

Dctn2

Dctn4

Dctn5

Dctn6

Opa1

Dynll2

Dynlrb1

Kiaa0319l

Dag1

Dmd

Arih1

Huwe1

Rbx1

Rnf126

Rnf181

Eea1

Eml1

Eml2

Entpd1

Entpd2

Entpd5

Enpp1

Q6DYE8

Enpp3

Q9D8Y0

Q8BYW9

Q8BPB5

Q9WVJ9

Q9WVK4

Q8BH64

Q9EQP2

P54320

P70372

Q99LC5

Q9DCW4

Q921G7

P10126

070251

P57776

Q9D8N0

P58252
Efhd2

Eogt

Efemp1

Efemp2

Ehd1

Ehd2

Ehd4

Eln

Elavl1

Etfa

Etfb

Etfdh

Eef1a1

Eef1b

Eef1d

Eef1g

Eef2 


\begin{tabular}{|c|c|c|c|c|}
\hline Protein & Uniprot & Gene name & $\begin{array}{l}\text { Fold change of } \\
\text { XPRESS ratio } \\
\left(\text { Egfr }^{\Delta K C} / \mathrm{WT}\right)\end{array}$ & $\begin{array}{c}\text { Sequence } \\
\text { coverage [\%] }\end{array}$ \\
\hline Elongator complex protein 4 & Q9ER73 & Elp4 & $-0,2$ & 3,2 \\
\hline Embigin; & P21995 & $\mathrm{Emb}$ & $-1,3$ & 7 \\
\hline Emerin; & 008579 & Emd & $-0,5$ & 25,5 \\
\hline EMILIN-2; & Q8K482 & Emilin2 & 1,6 & 1,2 \\
\hline Endonuclease domain-containing 1 protein; & Q8C522 & Endod1 & $-0,2$ & 5,4 \\
\hline Endophilin-A2; & Q62419 & Sh3gl1 & 0,2 & 2,2 \\
\hline Endoplasmic reticulum aminopeptidase 1 & Q9EQH2 & Erap1 & 0,0 & 4,4 \\
\hline Endoplasmic reticulum metallopeptidase 1 ; & Q3UVK0 & Ermp1 & $-0,4$ & 5,1 \\
\hline Endoplasmic reticulum resident protein 29 & P57759 & Erp29 & $-0,5$ & 17,6 \\
\hline Endoplasmic reticulum resident protein 44 & Q9D1Q6 & Erp44 & $-0,3$ & 23,4 \\
\hline Endoplasmic reticulum-Golgi intermediate compartment protein 1; & Q9DC16 & Ergic1 & 0,6 & 13,8 \\
\hline Endoplasmic reticulum-Golgi intermediate compartment protein 2; & Q9CR89 & Ergic2 & $-0,1$ & 3,4 \\
\hline Endoplasmin; & P08113 & Hsp90b1 & $-0,4$ & 30 \\
\hline Endosialin; & Q91V98 & $\mathrm{Cd} 248$ & 1,2 & 0,9 \\
\hline Endothelial differentiation-related factor 1 & Q9JMG1 & Edf1 & 0,3 & 21,6 \\
\hline Endothelin-converting enzyme 1 & Q4PZA2 & Ece1 & $-0,9$ & 4,2 \\
\hline Enhancer of rudimentary homolog; & P84089 & Erh & $-0,3$ & 26,9 \\
\hline Enoyl-CoA delta isomerase 1 , mitochondrial; & P42125 & Eci1 & 0,1 & 29,1 \\
\hline Enoyl-CoA delta isomerase 2, mitochondrial; & Q9WUR2 & Eci2 & 0,2 & 38,8 \\
\hline Enoyl-CoA hydratase, mitochondrial; & Q8BH95 & Echs1 & 0,0 & 14,1 \\
\hline Ensconsin; Microtubule-associated protein 7, isoform CRA_b; & D3YWN7 & Map7 & $-0,9$ & 8,8 \\
\hline Envoplakin; & Q9D952 & Evpl & $-2,0$ & 1,2 \\
\hline Eosinophil cationic-type ribonuclease 5; & 035292 & Ear5 & 2,3 & 29,7 \\
\hline Eosinophil peroxidase; & P49290 & Epx & $-0,1$ & 1,1 \\
\hline Ephrin-B1; & P52795 & Efnb1 & 0,6 & 5,2 \\
\hline Epididymal secretory protein E1; & Q9Z0J0 & $\mathrm{Npc2}$ & $-0,1$ & 24,8 \\
\hline Epiplakin; & Q8R0W0 & Eppk1 & 0,4 & 4,8 \\
\hline Epithelial cell adhesion molecule; & Q99JW5 & Epcam & $-0,5$ & 40,3 \\
\hline Epoxide hydrolase 1 & Q9D379 & Ephx1 & 0,1 & 29 \\
\hline Epsilon-sarcoglycan; & 070258 & Sgce & 0,6 & 8,8 \\
\hline Epsin-3; & Q91W69 & Epn3 & $-0,3$ & 1,6 \\
\hline Equilibrative nucleoside transporter 1 ; & Q9JIM1 & Slc29a1 & 1,1 & 9,9 \\
\hline ER membrane protein complex subunit 1 & Q8C7X2 & Emc1 & $-0,5$ & 6,6 \\
\hline ER membrane protein complex subunit 10 ; & Q3TAS6 & Emc10 & $-0,4$ & 3,9 \\
\hline Erlin-2; & Q8BFZ9 & Erlin2 & $-0,5$ & 16,5 \\
\hline ERO1-like protein alpha; & Q8R180 & Ero1l & $-0,3$ & 9,7 \\
\hline Erythrocyte band 7 integral membrane protein; & P54116 & Stom & $-0,4$ & 9,2 \\
\hline ES1 protein homolog, mitochondrial; & Q9D172 & D10Jhu81e & 0,2 & 35,7 \\
\hline Ester hydrolase C11orf54 homolog; & Q91V76 & & 0,6 & 18,4 \\
\hline Estradiol 17 beta-dehydrogenase 5 & P70694 & Akr1c6 & $-10,0$ & 3,1 \\
\hline Estradiol 17-beta-dehydrogenase 11 & Q9EQ06 & Hsd17b11 & 0,7 & 3,7 \\
\hline Estradiol 17-beta-dehydrogenase 12 & 070503 & Hsd17b12 & $-0,2$ & 21,2 \\
\hline Estradiol 17-beta-dehydrogenase 8; & P50171 & Hsd17b8 & 0,3 & 20,1 \\
\hline Ethylmalonyl-CoA decarboxylase; & Q9D9V3 & Echdc1 & 0,9 & 30,1 \\
\hline Etoposide-induced protein 2.4 & Q61070 & Ei24 & 0,2 & 5,6 \\
\hline ETS-related transcription factor Elf-5; & Q8VDK3 & Elf5 & 0,3 & 4,5 \\
\hline Eukaryotic initiation factor 4A-I; & P60843 & Eif4a1 & 0,3 & 25,6 \\
\hline Eukaryotic initiation factor $4 \mathrm{~A}-\mathrm{II}$; & P10630 & Eif4a2 & 0,2 & 21,5 \\
\hline Eukaryotic initiation factor 4A-III; & Q91VC3 & Eif4a3 & $-0,2$ & 36,3 \\
\hline Eukaryotic peptide chain release factor GTP-bind ing subunit ERF3A; & Q8R050 & Gspt1 & $-0,5$ & 9,3 \\
\hline Eukaryotic peptide chain release factor subunit 1 & Q8BWY3 & Etf1 & $-0,6$ & 5,7 \\
\hline Eukaryotic translation elongation factor 1 epsilon- 1 ; & Q9D1M4 & Eef1e1 & $-0,3$ & 5,2 \\
\hline Eukaryotic translation initiation factor 1 & P48024 & Eif1 & 0,5 & 42,2 \\
\hline Eukaryotic translation initiation factor $1 \mathrm{~A}$; & Q60872 & Eif1a & 0,2 & 14,6 \\
\hline Eukaryotic translation initiation factor 2 subunit 1 ; & Q6ZWX6 & Eif2s1 & 0,1 & 48,6 \\
\hline Eukaryotic translation initiation factor 2 subunit 2 & Q99L45 & Eif2s2 & 0,1 & 18,7 \\
\hline Eukaryotic translation initiation factor 2 subu nit 3, X-linked; & Q9Z0N1 & Eif2s $3 x$ & $-0,1$ & 39,2 \\
\hline Eukaryotic translation initiation factor $2 \mathrm{~A}$; & Q8BJW6 & Eif2a & 0,2 & 13,6 \\
\hline Eukaryotic translation initiation factor 3 subunit $A$; & P23116 & Eif3a & $-0,7$ & 3,1 \\
\hline Eukaryotic translation initiation factor 3 subunit $B$ & Q8JZQ9 & Eif3b & $-0,4$ & 9,7 \\
\hline Eukaryotic translation initiation factor 3 subunit $C$; & Q8R1B4 & Eif3c & 0,1 & 1,1 \\
\hline Eukaryotic translation initiation factor 3 subu nit G; & Q9Z1D1 & Eif3g & $-1,0$ & 7,8 \\
\hline Eukaryotic translation initiation factor 3 subu nit $\mathrm{H}$; & Q91WK2 & Eif3h & $-0,3$ & 11,6 \\
\hline Eukaryotic translation initiation factor 3 subu nit J-A; & Q3UGC7 & Eif3j1 & $-0,2$ & 19,5 \\
\hline Eukaryotic translation initiation factor 3 subunit K; & Q9DBZ5 & Eif3k & $-0,3$ & 5 \\
\hline Eukaryotic translation initiation factor 3 subunit L; & Q8QZY1 & Eif3l & $-0,1$ & 1,2 \\
\hline Eukaryotic translation initiation factor 4 gamma 1 ; & Q6NZJ6 & Eif4g1 & 0,0 & 4 \\
\hline Eukaryotic translation initiation factor 4B; & Q8BGD9 & Eif4b & 0,4 & 12,9 \\
\hline Eukaryotic translation initiation factor $4 \mathrm{E}$; & P63073 & Eif4e & $-1,1$ & 9,7 \\
\hline Eukaryotic translation initiation factor $4 \mathrm{H}$; & Q9WUK2 & Eif4h & $-0,3$ & 20,2 \\
\hline Eukaryotic translation initiation factor 5 ; & P59325 & Eif5 & $-2,4$ & 2,3 \\
\hline
\end{tabular}




\begin{tabular}{|c|c|c|c|c|}
\hline Protein & Uniprot & Gene name & $\begin{array}{l}\text { Fold change of } \\
\text { XPRESS ratio } \\
\left(\text { Egfr }^{\Delta K C} / \mathrm{WT}\right)\end{array}$ & $\begin{array}{c}\text { Sequence } \\
\text { coverage [\%] }\end{array}$ \\
\hline Eukaryotic translation initiation factor $5 \mathrm{~A}-1$ & P63242 & Eif5a & $-0,5$ & 19 \\
\hline Eukaryotic translation initiation factor $5 \mathrm{~B}$; & Q05D44 & Eif $5 b$ & $-0,5$ & 2,4 \\
\hline Eukaryotic translation initiation factor 6; & 055135 & Eif6 & $-0,4$ & 41,6 \\
\hline Excitatory amino acid transporter 1 ; & P56564 & Slc1a3 & $-0,3$ & 3,7 \\
\hline Exocyst complex component 5 & Q3TPX4 & Exoc5 & 3,2 & 3,1 \\
\hline Exosome complex component MTR3; & Q8BTW3 & Exosc6 & 0,4 & 12,5 \\
\hline Exosome complex component RRP4; & Q8VBV3 & Exosc2 & $-0,1$ & 6,1 \\
\hline Exosome complex component RRP41; & Q92119 & Exosc4 & 0,6 & 9,8 \\
\hline Exosome complex component RRP43; & Q9D753 & Exosc8 & 0,4 & 16,7 \\
\hline Exosome complex component RRP45; & Q9JHI7 & Exosc9 & 0,2 & 8,9 \\
\hline Exosome complex component RRP46; & Q9CRA8 & Exosc5 & 0,6 & 6,8 \\
\hline Exosome complex exonuclease RRP42; & Q9D0M0 & Exosc7 & $-0,3$ & 13,7 \\
\hline Exportin-1; & Q6P5F9 & Xpo1 & 0,3 & 2,1 \\
\hline Exportin-2; & Q9ERK4 & Cse1l & $-0,3$ & 2,9 \\
\hline Extended synaptotagmin-1; & Q3U7R1 & Esyt1 & $-0,1$ & 5 \\
\hline Extracellular matrix protein 1 & Q61508 & Ecm1 & $-0,3$ & 11,3 \\
\hline Extracellular superoxide dismutase $[\mathrm{Cu}-\mathrm{Zn}]$ & 009164 & Sod3 & $-0,9$ & 5,2 \\
\hline Ezrin; & P26040 & Ezr & $-0,1$ & 8 \\
\hline FACT complex subunit SSRP1; & Q08943 & Ssrp1 & $-0,4$ & 4,2 \\
\hline F-actin-capping protein subunit alpha-1; & P47753 & Capza1 & 0,0 & 46,9 \\
\hline F-actin-capping protein subunit alpha-2; & P47754 & Capza2 & 0,2 & 41,3 \\
\hline FAD-linked sulfhydryl oxidase ALR; & P56213 & Gfer & 0,3 & 10,6 \\
\hline Far upstream element-bind ing protein 1 & Q3TUE1 & Fubp1 & 0,2 & 29,3 \\
\hline Far upstream element-binding protein 2 & Q3U0V1 & Khsrp & 0,6 & 28,7 \\
\hline Farnesyl pyrophosphate synthase; & Q920E5 & Fdps & 0,0 & 7,6 \\
\hline FAS-associated factor 1 & P54731 & Faf1 & $-0,1$ & 2,5 \\
\hline FAS-associated factor 2 & Q3TDN2 & Faf2 & $-0,9$ & 13,6 \\
\hline Fascin; & Q61553 & Fscn1 & 0,2 & 9,7 \\
\hline Fatty acid 2-hydroxylase; & Q5MPP0 & $\mathrm{Fa} 2 \mathrm{~h}$ & 0,2 & 15,6 \\
\hline Fatty acid desaturase 3 ; & Q9JJE7 & Fads3 & $-1,2$ & 2,9 \\
\hline Fatty acid synthase; & P19096 & Fasn & 0,3 & 5 \\
\hline Fatty acid-binding protein, adipocyte; & P04117 & Fabp4 & $-0,1$ & 42,4 \\
\hline Fatty acid-binding protein, epidermal; & Q05816 & Fabp5 & $-1,4$ & 71,9 \\
\hline Fatty aldehyde dehydrogenase; & P47740 & Aldh3a2 & 0,1 & 6,8 \\
\hline F-box only protein 50 & G3X9C2 & Nccrp1 & $-1,1$ & 8,3 \\
\hline F-box-like/WD repeat-containing protein TBL1XR1; & Q8BHJ5 & Tbl1xr1 & $-0,1$ & 3,9 \\
\hline Fermitin family homolog 2 & Q8CIB5 & Fermt2 & $-10,0$ & 3,5 \\
\hline Ferritin heavy chain; & P09528 & Fth1 & 1,7 & 18,7 \\
\hline Ferritin; & Q9CPX4 & Ftl1 & 0,3 & 54,1 \\
\hline Ferrochelatase, mitochondrial; & P22315 & Fech & 0,6 & 5,2 \\
\hline Fetuin-B; & Q9QXC1 & Fetub & 0,9 & 2,9 \\
\hline Fibrillin-1; & A2AQ53 & Fbn1 & 0,7 & 11,3 \\
\hline Fibrillin-2; & Q61555 & Fbn2 & 0,1 & 2 \\
\hline Fibrinogen beta chain; & Q8K0E8 & $\mathrm{Fgb}$ & 0,1 & 10,2 \\
\hline Fibrinogen gamma chain; & Q8VCM7 & Fgg & $-0,2$ & 22 \\
\hline Fibroblast growth factor-binding protein $1 ;$ & 070514 & Fgfbp1 & $-0,6$ & 13,1 \\
\hline Fibronectin leucine rich transmembrane protein 1; MCG52071; Protein FIrt1; & Q6RKD8 & Flrt1 & $-0,4$ & 1,2 \\
\hline Fibronectin; & P11276 & Fn1 & $-0,4$ & 8,2 \\
\hline Fibulin-1; & Q08879 & Fbln1 & 0,2 & 9,4 \\
\hline Fibulin-2; & P37889 & Fbln2 & 1,0 & 8,8 \\
\hline Fibulin-5; & Q9WVH9 & Fbln5 & $-0,1$ & 18,8 \\
\hline Filaggrin; & P11088 & $\mathrm{Flg}$ & $-0,3$ & 12,8 \\
\hline Filaggrin-2; & Q2VIS4 & Flg2 & $-1,6$ & 16,3 \\
\hline Filaggrin-2; & E9QPZ3 & Flg2 & $-1,5$ & 15,7 \\
\hline Filamin-A; & Q8BTM8 & Flna & $-0,1$ & 18,8 \\
\hline Filamin-B; & Q80X90 & Flnb & $-0,3$ & 18,1 \\
\hline Filamin-binding LIM protein 1; & Q71FD7 & Fblim1 & 0,1 & 11,5 \\
\hline Filamin-C; & Q8VHX6 & Flnc & $-1,0$ & 2,9 \\
\hline Flap endonuclease $1 ;$ & P39749 & Fen1 & 0,3 & 2,9 \\
\hline Flotillin-1; & 008917 & Flot1 & 0,2 & 9,3 \\
\hline Flotillin-2; & Q60634 & Flot2 & 0,1 & 15,4 \\
\hline Follistatin-related protein 1 & Q62356 & Fstl1 & 0,7 & 8,8 \\
\hline Formin-1; & Q05860 & Fmn1 & $-4,8$ & 1,5 \\
\hline Fos-related antigen 2; & P47930 & Fosl2 & $-0,4$ & 10,7 \\
\hline Four and a half LIM domains protein 1 ; & P97447 & Fhl1 & 0,3 & 45 \\
\hline Four and a half LIM domains protein 2; & 070433 & $\mathrm{Fhl} 2$ & $-0,8$ & 21,5 \\
\hline Four and a half LIM domains protein 3; & Q9R059 & $\mathrm{Fhl3}$ & $-0,3$ & 16,3 \\
\hline Frizzled-1; & 070421 & Fzd1 & 1,1 & 5 \\
\hline Frizzled-2; & Q9JIP6 & Fzd2 & $-0,1$ & 4,7 \\
\hline Frizzled-7; & Q61090 & Fzd7 & $-0,3$ & 4,7 \\
\hline
\end{tabular}




\begin{tabular}{|c|c|c|c|c|}
\hline Protein & Uniprot & Gene name & $\begin{array}{l}\text { Fold change of } \\
\text { XPRESS ratio } \\
\left(\text { Egfr }^{\Delta K C} / \mathrm{WT}\right)\end{array}$ & $\begin{array}{c}\text { Sequence } \\
\text { coverage [\%] }\end{array}$ \\
\hline Fructose-1,6-bisphosphatase 1; & Q9QXD6 & Fbp1 & $-0,6$ & 23,7 \\
\hline Fructose-bisphosphate aldolase $A$; & P05064 & Aldoa & $-0,4$ & 56,9 \\
\hline Fructose-bisphosphate aldolase C; & P05063 & Aldoc & $-0,3$ & 20,1 \\
\hline Fumarylacetoacetase; & P35505 & Fah & 0,7 & 7,6 \\
\hline FUN14 domain-containing protein 2; & Q9D6K8 & Fundc2 & 0,6 & 25,8 \\
\hline Furin; & P23188 & Furin & $-0,4$ & 1,3 \\
\hline G patch domain-containing protein 8; & A2A6A1 & Gpatch8 & $-0,1$ & 1,5 \\
\hline G protein-activated inward rectifier potassium channel 3; & P48543 & Kcnj9 & $-6,0$ & 6,6 \\
\hline Galactokinase; & Q9RONO & Galk1 & 0,6 & 8,7 \\
\hline Galactose-1-phosphate uridylyltransferase; & Q03249 & Galt & $-0,5$ & 6,4 \\
\hline Galectin; & Q9CRB1 & Lgals7 & 0,2 & 64,7 \\
\hline Galectin-1; & P16045 & Lgals1 & 0,1 & 43,7 \\
\hline Galectin-3; & P16110 & Lgals3 & $-0,5$ & 18,6 \\
\hline Galectin-related protein; & Q8VED9 & Lgalsl & $-0,2$ & 11,6 \\
\hline Gamma-adducin; & Q9QYB5 & Add3 & 0,9 & 16,7 \\
\hline Gamma-butyrobetaine dioxygenase; & Q924Y0 & Bbox1 & 0,0 & 2,6 \\
\hline Gamma-enolase; & P17183 & Eno2 & $-10,0$ & 17,1 \\
\hline Gamma-glutamyl hydrolase; & Q9Z0L8 & Ggh & $-0,8$ & 24,6 \\
\hline Gamma-glutamylcyclotransferase; & Q9D7X8 & Ggct & $-1,9$ & 42 \\
\hline Gamma-glutamyltransferase 5; & Q9Z2A9 & Ggt5 & $-2,7$ & 2,8 \\
\hline Gamma-interferon-inducible lysosomal thiol reductase; & Q9ESY9 & Ifi30 & $-0,7$ & 5,2 \\
\hline Gamma-synuclein; & Q9Z0F7 & Sncg & $-0,1$ & 47,2 \\
\hline Ganglioside GM2 activator; & Q60648 & Gm2a & $-0,2$ & 18,1 \\
\hline Gap junction alpha-1 protein; & P23242 & Gja1 & $-1,3$ & 22,3 \\
\hline Gap junction beta-3 protein; & P28231 & Gjb3 & 0,1 & 5,6 \\
\hline Gasdermin-A; & Q9EST1 & Gsdma & $-1,2$ & 20,6 \\
\hline Gasdermin-D; & Q9D8T2 & Gsdmdc1 & 0,9 & 2,1 \\
\hline GDP-L-fucose synthase; & P23591 & Tsta3 & 0,6 & 20,6 \\
\hline GDP-mannose 4,6 dehydratase; & Q8K0C9 & Gmds & 0,4 & 5,4 \\
\hline Gelsolin; & P13020 & Gsn & 0,0 & 23,8 \\
\hline Gelsolin; & A6PWS5 & Gsn & $-0,4$ & 11,2 \\
\hline General transcription factor $3 C$ polypeptide 1 ; & Q8K284 & Gtf3c1 & $-0,7$ & 1,4 \\
\hline General transcription factor IIF subunit 1 ; & Q3THK3 & Gtf2f1 & 0,2 & 10,8 \\
\hline General transcription factor IIF subunit 2; & Q8R0A0 & Gtf2f2 & $-0,1$ & 6,4 \\
\hline General vesicular transport factor $\mathrm{p} 115$ & Q9Z1Z0 & Uso1 & 0,0 & 5,1 \\
\hline Gephyrin; & Q8BUV3 & Gphn & $-0,1$ & 7,4 \\
\hline Geranylgeranyl pyrophosphate synthase; & Q9WTNO & Ggps1 & 0,2 & 4 \\
\hline Glia maturation factor beta; & Q9CQ।3 & $\mathrm{Gmfb}$ & 0,4 & 14,1 \\
\hline Glucosamine-6-phosphate isomerase 1; & 088958 & Gnpda1 & 0,0 & 15,6 \\
\hline Glucose-6-phosphatase 3; & Q6NSQ9 & G6pc3 & $-0,9$ & 6,1 \\
\hline Glucose-6-phosphate 1-dehydrogenase X; & Q00612 & G6pdx & $-0,1$ & 7,8 \\
\hline Glucose-6-phosphate isomerase; & P06745 & Gpi & 0,2 & 19,7 \\
\hline Glucosidase 2 subunit beta; & 008795 & Prkcsh & $-0,6$ & 15,7 \\
\hline Glucosylceramidase; & P17439 & Gba & $-0,7$ & 13,6 \\
\hline Glutamate dehydrogenase 1, mitochondrial; & P26443 & Glud1 & $-0,1$ & 44,8 \\
\hline Glutamyl aminopeptidase; & P16406 & Enpep & 1,8 & 2,1 \\
\hline Glutaredoxin-1; & Q9QUH0 & Glrx & $-0,2$ & 10,3 \\
\hline Glutaredoxin-related protein 5, mitochondrial; & Q80Y14 & Glrx5 & 0,7 & 9,2 \\
\hline Glutathione peroxidase 1; & P11352 & Gpx1 & 0,4 & 41,3 \\
\hline Glutathione peroxidase 3; & P46412 & Gpx3 & 0,4 & 23,1 \\
\hline Glutathione peroxidase 7; & Q99L6 & Gpx7 & 0,4 & 24,7 \\
\hline Glutathione reductase, mitochond rial; & P47791 & Gsr & $-0,1$ & 19,6 \\
\hline Glutathione S-transferase Mu 1; & P10649 & Gstm1 & 0,2 & 27,1 \\
\hline Glutathione S-transferase Mu 2; & P15626 & Gstm2 & 0,4 & 41,3 \\
\hline Glutathione S-transferase omega-1; & 009131 & Gsto1 & 0,8 & 19,6 \\
\hline Glutathione S-transferase P 1; & P19157 & Gstp1 & 0,0 & 28,1 \\
\hline Glutathione synthetase; & P51855 & Gss & 0,3 & 6,2 \\
\hline Glyceraldehyde-3-phosphate dehydrogenase; & P16858 & Gapdh & 0,2 & 33,9 \\
\hline Glycerol-3-phosphate dehydrogenase [NAD(+)], cytoplasmic; & P13707 & Gpd1 & 0,2 & 19,9 \\
\hline Glycerol-3-phosphate dehydrogenase, mitochondrial; & Q64521 & Gpd2 & 0,0 & 5,6 \\
\hline Glycerophosphodiester phosphodiesterase domain-containing protein 3; & Q99LY2 & Gdpd3 & $-1,7$ & 7 \\
\hline Glycine cleavage system $\mathrm{H}$ protein, mitochondrial; & Q91WK5 & Gcsh & 0,6 & 5,9 \\
\hline Glycine--tRNA ligase; & Q9CZD3 & Gars & $-0,1$ & 21,7 \\
\hline Glycogen phosphorylase, brain form; & Q8C194 & Pygb & 0,0 & 3,8 \\
\hline Glycogen phosphorylase, liver form; & Q9ET01 & Pygl & 0,1 & 2 \\
\hline Glycogen synthase kinase-3 beta; & Q9WV60 & Gsk3b & 0,8 & 9,8 \\
\hline Glycolipid transfer protein; & Q9JL62 & Gltp & $-0,3$ & 16,3 \\
\hline Glycylpeptide N-tetradecanoyltransferase 1; & 070310 & Nmt1 & 0,0 & 6,3 \\
\hline Glyoxalase domain-containing protein 4 & Q9CPV4 & Glod4 & $-0,4$ & 6,5 \\
\hline Glyoxylate reductase/hydroxypyruvate reductase; & Q91Z53 & Grhpr & 0,1 & 27,9 \\
\hline
\end{tabular}




\begin{tabular}{|c|c|c|c|c|}
\hline Protein & Uniprot & Gene name & $\begin{array}{l}\text { Fold change of } \\
\text { XPRESS ratio } \\
\left(\text { Egfr }^{\triangle K C} / \mathrm{WT}\right)\end{array}$ & $\begin{array}{c}\text { Sequence } \\
\text { coverage [\%] }\end{array}$ \\
\hline Glypican-1; & Q9QZF2 & Gpc1 & 0,3 & 8,4 \\
\hline Glypican-4; & P51655 & Gpc4 & $-0,3$ & 23 \\
\hline GMP reductase 2 & Q99L27 & Gmpr2 & 0,4 & 4,6 \\
\hline GMP synthase [glutamine-hydrolyzing]; & Q3THK7 & Gmps & 0,5 & 4,9 \\
\hline Golgi apparatus protein 1; & Q61543 & Glg1 & 0,0 & 15,8 \\
\hline Golgi integral membrane protein 4; & D3YVW2 & Golim4 & $-0,4$ & 16,1 \\
\hline Golgi membrane protein 1 & Q91XA2 & Golm1 & 0,8 & 4,6 \\
\hline Golgi phosphoprotein 3; & Q9CRA5 & Golph3 & 0,2 & 4,4 \\
\hline Golgi reassembly-stacking protein 2; & Q99JX3 & Gorasp2 & 0,7 & 8,6 \\
\hline Golgi SNAP receptor complex member 1 ; & 088630 & Gosr1 & $-0,1$ & 4,8 \\
\hline Golgin subfamily A member 5 ; & Q9QYE6 & Golga5 & $-1,0$ & 4,5 \\
\hline Golgin subfamily A member 7; & Q91W53 & Golga7 & $-0,4$ & 10,9 \\
\hline GPALPP motifs-containing protein 1 ; & Q69ZC8 & Gpalpp1 & 0,7 & 3,5 \\
\hline GPI inositol-deacylase; & Q3UUQ7 & Pgap1 & $-1,0$ & 6,1 \\
\hline GPN-loop GTPase 1; & Q8VCE2 & Gpn1 & 0,3 & 4,3 \\
\hline G-protein coupled receptor 56 & Q8K209 & Gpr56 & $-0,5$ & 1,5 \\
\hline G-protein coupled receptor family C group 5 member D; & Q9JIL6 & Gprc5d & $-7,2$ & 4,9 \\
\hline Granulins; & P28798 & Grn & 0,2 & 4,6 \\
\hline Growth arrest-specific protein 1; & Q01721 & Gas1 & $-0,1$ & 8,5 \\
\hline GTP:AMP phosphotransferase AK3, mitochondrial; & Q9WTP7 & Ak3 & 0,9 & 10,6 \\
\hline GTPase HRas; & Q61411 & Hras & $-0,3$ & 31,2 \\
\hline GTPase NRas; & P08556 & Nras & 0,3 & 31,2 \\
\hline GTP-binding nuclear protein Ran; & P62827 & Ran & 0,3 & 42,6 \\
\hline GTP-binding protein 1 & 008582 & Gtpbp1 & 1,4 & 2,1 \\
\hline $\begin{array}{l}\text { GTP-binding protein SAR1a; SAR1 gene homolog A (S. cerevisiae); SAR1 gene } \\
\text { homolog A (S. cerevisiae), isoform CRA_b; }\end{array}$ & Q99JZ4 & Sar1a & 0,3 & 11,6 \\
\hline Guanine deaminase; & Q9R111 & Gda & 0,8 & 4,8 \\
\hline Guanine nucleotide-binding protein G(i) subunit alpha-1; & B2RSH2 & Gnai1 & 0,5 & 15,5 \\
\hline Guanine nucleotide-binding protein G(i) subunit alpha-2; & P08752 & Gnai2 & 0,8 & 16,9 \\
\hline Guanine nucleotide-binding protein $\mathrm{G}(\mathrm{I}) / \mathrm{G}(\mathrm{S}) / \mathrm{G}(\mathrm{O})$ subunit gamma-12; & Q9DAS9 & Gng12 & $-0,4$ & 41,7 \\
\hline Guanine nucleotide-binding protein $\mathrm{G}(\mathrm{I}) / \mathrm{G}(\mathrm{S}) / \mathrm{G}(\mathrm{O})$ subunit gamma-2; & P63213 & Gng2 & 1,6 & 22,5 \\
\hline Guanine nucleotide-binding protein $\mathrm{G}(\mathrm{I}) / \mathrm{G}(\mathrm{S}) / \mathrm{G}(\mathrm{O})$ subunit gamma-5; & Q80SZ7 & Gng5 & 0,4 & 40,3 \\
\hline Guanine nucleotide-binding protein $\mathrm{G}(\mathrm{I}) / \mathrm{G}(\mathrm{S}) / \mathrm{G}(\mathrm{T})$ subunit beta-1; & P62874 & Gnb1 & 0,2 & 25,6 \\
\hline Guanine nucleotide-binding protein $\mathrm{G}(\mathrm{I}) / \mathrm{G}(\mathrm{S}) / \mathrm{G}(\mathrm{T})$ subunit beta-2; & P62880 & Gnb2 & 1,0 & 34 \\
\hline Guanine nucleotide-binding protein G(s) subunit alpha isoforms short; & P63094 & Gnas & 0,7 & 14 \\
\hline Guanine nucleotide-binding protein subunit alpha-11; & P21278 & Gna11 & $-0,7$ & 2,5 \\
\hline Guanine nucleotide-binding protein subunit beta-2-like 1; & P68040 & Gnb2l1 & $-0,1$ & 63,7 \\
\hline Guanylate cyclase 2G; & Q6TL19 & Gucy2g & 10,0 & 1,2 \\
\hline H/ACA ribonucleoprotein complex subunit 1 ; & Q9CY66 & Gar1 & $-0,5$ & 20,7 \\
\hline H/ACA ribonucleoprotein complex subunit 2; & Q9CRB2 & Nhp2 & 0,1 & 21,6 \\
\hline H/ACA ribonucleoprotein complex subunit 4; & Q9ESX5 & Dkc1 & $-0,2$ & 23,6 \\
\hline $\mathrm{H} 1$ histone family, member $\mathrm{X}$; Protein $\mathrm{H} 1 \mathrm{fx}$; & Q80ZM5 & $H 1 f x$ & $-0,1$ & 11,7 \\
\hline H-2 class I histocompatibility antigen, $\mathrm{K}-\mathrm{B}$ alpha chain; & P01901 & $\mathrm{H} 2 \mathrm{~K} 1$ & $-1,6$ & 16,4 \\
\hline H-2 class I histocompatibility antigen, L-D alpha chain; & P01897 & $\mathrm{H} 2 \mathrm{~L}$ & 0,8 & 23,8 \\
\hline H-2 class II histocompatibility antigen, A beta chain; & P14483 & $\mathrm{H} 2 \mathrm{Ab} 1$ & $-4,2$ & 16,2 \\
\hline H-2 class II histocompatibility antigen, A-Q alpha chain; & P04227 & $\mathrm{H} 2 \mathrm{Aa}$ & 1,3 & 11,8 \\
\hline $\mathrm{H}-2$ class II histocompatibility antigen, $\mathrm{A}-\mathrm{Q}$ beta chain; & P06342 & $\mathrm{H} 2 \mathrm{Ab} 1$ & 6,8 & 19,6 \\
\hline Haloacid dehalogenase-like hydrolase domain-containing protein 2; & Q3UGR5 & Hdhd2 & 0,2 & 8,1 \\
\hline Haloacid dehalogenase-like hydrolase domain-containing protein 3 ; & Q9CYW4 & Hdhd3 & 0,0 & 4,4 \\
\hline Haptoglobin; & Q61646 & $\mathrm{Hp}$ & 0,5 & 6,3 \\
\hline HD domain-containing protein 2 ; & Q3SXD3 & Hddc2 & 0,4 & 11,1 \\
\hline Heat shock 70 kDa protein $1 \mathrm{~A}$; & Q61696 & Hspa1a & 0,4 & 36,7 \\
\hline Heat shock 70 kDa protein 4 & Q61316 & Hspa4 & 0,3 & 5,6 \\
\hline Heat shock cognate 71 kDa protein; & P63017 & Hspa8 & $-0,1$ & 45,2 \\
\hline Heat shock protein beta-1; & P14602 & Hspb1 & $-0,5$ & 37,8 \\
\hline Heat shock protein HSP 90-alpha; & P07901 & Hsp90aa1 & $-0,4$ & 31,5 \\
\hline Heat shock protein HSP 90-beta; & P11499 & Hsp90ab1 & $-0,3$ & 31,5 \\
\hline Heat shock-related 70 kDa protein 2; & P17156 & Hspa2 & 0,9 & 19,7 \\
\hline Hematological and neurological expressed 1 protein; & P97825 & Hn1 & 0,7 & 9,7 \\
\hline Hematological and neurological expressed 1-like protein; & Q6PGH2 & Hn1l & 0,1 & 40,5 \\
\hline Hematopoietic progenitor cell antigen CD34; & Q64314 & $\mathrm{Cd} 34$ & 0,1 & 14,1 \\
\hline Heme-binding protein 2 & Q9WU63 & Hebp2 & $-0,6$ & 13,7 \\
\hline Hemoglobin subunit beta- 1 & P02088 & $\mathrm{Hbb}$ b1 & $-2,9$ & 78,9 \\
\hline Hemopexin; & Q91X72 & $\mathrm{Hpx}$ & 0,1 & 24,6 \\
\hline Heparan-alpha-glucosaminide $\mathrm{N}$-acetyltransferase; & Q3UDW8 & Hgsnat & 0,9 & 2 \\
\hline Hepatoma-derived growth factor; & P51859 & Hdgf & 0,4 & 37,1 \\
\hline Hepatoma-derived growth factor-related protein 2 ; & Q3UMU9 & Hdgfrp2 & 0,2 & 5,5 \\
\hline Hepatoma-derived growth factor-related protein 3 ; & Q9JMG7 & Hdgfrp3 & 0,2 & 7,9 \\
\hline Hephaestin; & Q9Z0Z4 & Heph & $-0,1$ & 0,8 \\
\hline Hephaestin-like protein 1 ; & Q3V1H3 & Hephl1 & $-1,7$ & 2,8 \\
\hline Heterochromatin protein 1-binding protein 3; & Q3TEA8 & Hp1bp3 & 0,5 & 15 \\
\hline
\end{tabular}




\begin{tabular}{|c|c|c|c|c|}
\hline Protein & Uniprot & Gene name & $\begin{array}{l}\text { Fold change of } \\
\text { XPRESS ratio } \\
\left(\text { Egfr }^{\Delta K C} / \mathrm{WT}\right)\end{array}$ & $\begin{array}{c}\text { Sequence } \\
\text { coverage [\%] }\end{array}$ \\
\hline Heterogeneous nuclear ribon ucleoprotein A/B; & Q99020 & Hnrnpab & 0,4 & 15,8 \\
\hline Heterogeneous nuclear ribonucleoprotein $\mathrm{A} 0$; & Q9CX86 & HnrnpaO & 0,3 & 16,7 \\
\hline Heterogeneous nuclear ribon ucleoprotein A1; & P49312 & Hnrnpa1 & 0,2 & 25 \\
\hline Heterogeneous nuclear ribonucleoprotein A3; & Q8BG05 & Hnrnpa3 & 0,5 & 32 \\
\hline Heterogeneous nuclear ribon ucleoprotein D0; & Q60668 & Hnrnpd & 0,6 & 17,1 \\
\hline Heterogeneous nuclear ribon ucleoprotein D-like; & Q9Z130 & Hnrnpdl & 0,9 & 14 \\
\hline Heterogeneous nuclear ribonucleoprotein F; & Q9Z2X1 & Hnrnpf & $-0,1$ & 28,4 \\
\hline Heterogeneous nuclear ribonucleoprotein $\mathrm{H}$; & 035737 & Hnrnph1 & 0,7 & 22,7 \\
\hline Heterogeneous nuclear ribon ucleoprotein K; & B2M1R6 & Hnrnpk & 0,1 & 40,2 \\
\hline Heterogeneous nuclear ribonucleoprotein L; & Q8R081 & Hnrnpl & 0,3 & 18,4 \\
\hline Heterogeneous nuclear ribonucleoprotein L-like; & Q921F4 & Hnrnpll & 0,2 & 2,2 \\
\hline Heterogeneous nuclear ribonucleoprotein M; & Q9D0E1 & Hnrnpm & 0,9 & 14,5 \\
\hline Heterogeneous nuclear ribon ucleoprotein $Q ;$ & Q7TMK9 & Syncrip & 0,5 & 20,5 \\
\hline Heterogeneous nuclear ribonucleoprotein R; Protein Hnrnpr; & Q8VHM5 & Hnrnpr & 0,2 & 12 \\
\hline Heterogeneous nuclear ribon ucleoprotein $\mathrm{U}$; & Q8VEK3 & Hnrnpu & $-0,1$ & 19 \\
\hline Heterogeneous nuclear ribon ucleoprotein U-like protein 2; & Q00PI9 & Hnrnpul2 & 1,2 & 7,9 \\
\hline Heterogeneous nuclear ribon ucleoproteins A2/B1; & 088569 & Hnrnpa2b1 & 0,2 & 25,8 \\
\hline Heterogeneous nuclear ribon ucleoproteins $\mathrm{C} 1 / \mathrm{C} 2$; & Q9Z204 & Hnrnpc & 0,0 & 18,5 \\
\hline Hexaprenyldihydroxybenzoate methyltransferase, mitochondrial; & Q8BMS4 & Coq3 & 0,2 & 4,3 \\
\hline Hexokinase-1; & P17710 & Hk1 & $-0,4$ & 14 \\
\hline High affinity cAMP-specific 3',5'-cyclic phosphodiesterase 7A; & P70453 & Pde7a & $-0,3$ & 17,6 \\
\hline High affinity cationic amino acid transporter 1 ; & Q09143 & Slc7a1 & $-1,8$ & 11,7 \\
\hline High mobility group nucleosome-binding domain-containing protein 3 ; & Q9DCB1 & Hmgn3 & $-0,9$ & 32,3 \\
\hline High mobility group nucleosome-bind ing domain-containing protein 5 ; & Q9JL35 & Hmgn5 & $-1,2$ & 3,7 \\
\hline High mobility group protein B1; & P63158 & Hmgb1 & 0,5 & 17,7 \\
\hline High mobility group protein B2; & P30681 & Hmgb2 & 0,1 & 24,8 \\
\hline High mobility group protein B3; & 054879 & Hmgb3 & 0,6 & 16,4 \\
\hline High mobility group protein HMG-I/HMG-Y; & P17095 & Hmga1 & 0,0 & 8,4 \\
\hline High mobility group protein $\mathrm{HMGI-C}$ & P52927 & Hmga2 & 1,2 & 16,7 \\
\hline Hippocalcin-like protein 1 & P62748 & Hpcal1 & 0,1 & 31,6 \\
\hline HIRA-interacting protein 3; & Q8BLH7 & Hirip3 & 1,0 & 12,7 \\
\hline Histidine ammonia-lyase; & P35492 & $\mathrm{Hal}$ & $-1,1$ & 35,2 \\
\hline Histidine triad nucleotide-binding protein 1 ; & P70349 & Hint1 & 0,5 & 51,6 \\
\hline Histidine triad nucleotide-binding protein 2, mitochondrial; & Q9D0S9 & Hint2 & 0,2 & 21,5 \\
\hline Histidine triad nucleotide-binding protein 3 & Q9CPS6 & Hint3 & 0,1 & 9,1 \\
\hline Histidine-rich glycoprotein; & Q9ESB3 & $\mathrm{Hrg}$ & $-0,1$ & 4,6 \\
\hline Histidine--tRNA ligase, cytoplasmic; & Q61035 & Hars & 0,4 & 12,8 \\
\hline Histone deacetylase 1 & 009106 & Hdac1 & 0,0 & 4,4 \\
\hline Histone H1.0; & P10922 & $\mathrm{H} 1 \mathrm{fO}$ & $-0,5$ & 20,6 \\
\hline Histone H1.1; & P43275 & Hist1h1a & $-0,5$ & 31,5 \\
\hline Histone H1.2; & P15864 & Hist1h1c & 0,2 & 22,6 \\
\hline Histone H1.3; & P43277 & Hist1h1d & 0,4 & 24,9 \\
\hline Histone $\mathrm{H} 1.4$; & P43274 & Hist1h1e & 0,1 & 25,1 \\
\hline Histone H1.5; & P43276 & Hist1h1b & 0,4 & 24,7 \\
\hline Histone H2A type 1 ; & P22752 & Hist1h2ao & $-0,4$ & 32,6 \\
\hline Histone H2A type 2-B; & Q64522 & Hist2h2ab & $-0,6$ & 35,4 \\
\hline Histone $\mathrm{H} 2 \mathrm{~B}$ type $1-\mathrm{F} / \mathrm{J} / \mathrm{L}$; & P10853 & Hist1h2bn & 0,2 & 40,5 \\
\hline Histone $\mathrm{H} 2 \mathrm{~B}$ type 2-E; & Q64524 & Hist2h2be & $-0,8$ & 34,9 \\
\hline Histone H3.3C; & P02301 & $\mathrm{H} 3 \mathrm{f} 3 \mathrm{c}$ & $-0,4$ & 19,3 \\
\hline Histone $\mathrm{H} 4$; & P62806 & Hist4h4 & $-0,7$ & 50,5 \\
\hline Histone-binding protein RBBP4; & Q60972 & Rbbp4 & 0,3 & 26,8 \\
\hline Histone-binding protein RBBP7; & Q60973 & Rbbp7 & 0,8 & 15,1 \\
\hline Homeodomain-only protein; & Q8R1H0 & Hopx & 1,2 & 43,8 \\
\hline Hormone-sensitive lipase; & P54310 & Lipe & 0,3 & 2,1 \\
\hline Hornerin; & E9QNP3 & Hrnr & $-2,0$ & 13,2 \\
\hline Host cell factor 1 & Q61191 & Hcfc1 & 0,0 & 0,9 \\
\hline Hsc70-interacting protein; & Q99L47 & St13 & 0,4 & 20,2 \\
\hline Hsp90 co-chaperone Cdc37; & Q61081 & Cdc37 & $-0,2$ & 11,3 \\
\hline Huntingtin-interacting protein $\mathrm{K}$; & Q9CR41 & Hypk & 0,7 & 24,8 \\
\hline Hydroxyacid-oxoacid transhydrogenase, mitochondrial; & Q8R0N6 & Adhfe1 & 1,0 & 1,7 \\
\hline Hydroxyacyl-coenzyme A dehydrogenase, mitochondrial; & Q61425 & Hadh & $-0,4$ & 19,4 \\
\hline Hydroxyacylglutathione hydrolase, mitochondrial; & Q99KB8 & Hagh & 0,7 & 16,1 \\
\hline Hydroxymethylglutaryl-CoA synthase, cytoplasmic; & Q8JZK9 & Hmgcs1 & 0,8 & 11,7 \\
\hline Hypoxanthine-guanine phosphoribosyltransferase; & P00493 & Hprt1 & $-0,2$ & 49,5 \\
\hline Hypoxia up-regulated protein 1 & Q9JKR6 & Hyou1 & $-0,4$ & 20,3 \\
\hline Ig gamma-1 chain $C$ region secreted form; & P01868 & Ighg1 & 0,7 & 3,7 \\
\hline Ig gamma-2B chain C region; & P01867 & Igh 3 & 0,2 & 14,9 \\
\hline Ig gamma-3 chain C region; & P03987 & & 0,0 & 15,6 \\
\hline Ig heavy chain $V$ region 6.96 & P18528 & & 0,3 & 19,4 \\
\hline Ig heavy chain $V$ region MOPC $104 \mathrm{E}$ & P01756 & & 0,4 & 16,2 \\
\hline Ig kappa chain C region; & P01837 & & 0,2 & 13,2 \\
\hline
\end{tabular}




\begin{tabular}{|c|c|c|c|c|}
\hline Protein & Uniprot & Gene name & $\begin{array}{l}\text { Fold change of } \\
\text { XPRESS ratio } \\
\left(\text { Egfr }^{\Delta K C} / \mathrm{WT}\right)\end{array}$ & $\begin{array}{c}\text { Sequence } \\
\text { coverage [\%] }\end{array}$ \\
\hline Ig kappa chain V-II region 26-10; & P01631 & & 0,9 & 17,7 \\
\hline IgG receptor FcRn large subunit p51; & Q61559 & Fcgrt & 1,0 & 5,8 \\
\hline Immediate early response 3 -interacting protein 1 ; & Q9CR20 & ler3ip1 & $-0,6$ & 34,1 \\
\hline Immunoglobulin superfa mily containing leucine-rich repeat protein; & Q6GU68 & Islr & 1,8 & 8,2 \\
\hline Importin subunit beta-1; & P70168 & Kpnb1 & 0,4 & 8,2 \\
\hline Importin-5; & Q8BKC5 & Ipo5 & 0,1 & 4,8 \\
\hline Importin-7; & Q9EPL8 & Ipo7 & $-0,4$ & 2,8 \\
\hline Inactive hydroxysteroid dehydrogenase-like protein 1 ; & Q8BTX9 & Hsdl1 & $-0,1$ & 3,9 \\
\hline Inactive tyrosine-protein kinase 7 & Q8BKG3 & Ptk7 & 0,3 & 7,3 \\
\hline Inhibitor of carbonic anhydrase; & Q9DBD0 & Ica & $-3,6$ & 7,4 \\
\hline Inhibitor of nuclear factor kappa-B kinase-interacting protein; & Q9DBZ1 & Ikbip & 0,2 & 8,6 \\
\hline Inorganic pyrophosphatase 2, mitochondrial; & Q91VM9 & Ppa2 & $-0,2$ & 22,5 \\
\hline Inorganic pyrophosphatase; & Q9D819 & Ppa1 & 0,7 & 32,9 \\
\hline Inosine triphosphate pyrophosphatase; & Q9D892 & Itpa & 0,5 & 33,8 \\
\hline $\begin{array}{l}\text { Inosine-5'-monophosphate dehydrogenase 2; } \\
\text { Inositol (Myo)-1(Or 4)-monophosphatase 1; Inositol (Myo)-1(Or 4)- }\end{array}$ & P24547 & Impdh2 & 0,3 & 15,6 \\
\hline $\begin{array}{l}\text { monophosphatase 1, isoform CRA_a; Inositol monophosphatase 1; Myo- } \\
\text { inositol monophosphatase 1; }\end{array}$ & Q924B0 & Impa1 & 1,0 & 34,7 \\
\hline Inositol monophosphatase 2; & Q91UZ5 & Impa2 & 1,2 & 13,8 \\
\hline Inositol polyphosphate 1-phosphatase; & P49442 & Inpp1 & 0,8 & 11,6 \\
\hline Inositol-3-phosphate synthase 1 & Q9JHU9 & Isyna1 & 0,5 & 12,6 \\
\hline Insulin-degrading enzyme; & F6RPJ9 & Ide & $-1,0$ & 24,5 \\
\hline Insulin-like growth factor-binding protein 4; & P47879 & Igfbp4 & 1,6 & 11,4 \\
\hline Insulin-like growth factor-binding protein 7; & Q61581 & Igfbp7 & 0,8 & 4,6 \\
\hline Integral membrane protein $2 \mathrm{~B}$; & 089051 & Itm2b & $-0,2$ & 14,3 \\
\hline Integrator complex subu nit 12 & Q9D168 & Ints12 & 0,7 & 2,2 \\
\hline Integrator complex subu nit 3; & Q7TPD0 & Ints3 & 0,7 & 1,7 \\
\hline Integrin alpha-1; & Q3V3R4 & Itga1 & 1,1 & 4,7 \\
\hline Integrin alpha-11; & P61622 & Itga11 & $-0,2$ & 0,8 \\
\hline Integrin alpha-3; & Q62470 & Itga3 & $-0,7$ & 3,8 \\
\hline Integrin alpha-5; & P11688 & $\operatorname{ltg} 5$ & 1,0 & 6 \\
\hline Integrin alpha-6; & Q61739 & Itga6 & 0,5 & 10,8 \\
\hline Integrin alpha-M; & P05555 & Itgam & 1,2 & 0,9 \\
\hline Integrin alpha-V; & P43406 & Itgav & 0,7 & 16,7 \\
\hline Integrin beta-1; & P09055 & Itgb1 & 0,0 & 16,3 \\
\hline Integrin beta-2; & P11835 & $\operatorname{ltgb} 2$ & 2,7 & 5,6 \\
\hline Integrin beta-3; & 054890 & $\operatorname{ltg} 33$ & 0,7 & 3 \\
\hline Integrin beta-5; & 070309 & Itgb5 & 1,0 & 1,8 \\
\hline Inter alpha-trypsin inhibitor, heavy chain 4 ; & A6X935 & Itih4 & $-0,3$ & 0,9 \\
\hline Inter-alpha-trypsin inhibitor heavy chain $\mathrm{H} 1$; & Q61702 & Itih1 & 2,8 & 2,8 \\
\hline Inter-alpha-trypsin inhibitor heavy chain $\mathrm{H} 2$; & Q61703 & Itih2 & $-0,3$ & 10,6 \\
\hline Inter-alpha-trypsin inhibitor heavy chain H3; & Q61704 & Itih3 & $-0,5$ & 6 \\
\hline Intercellular adhesion molecule 1 & P13597 & Icam1 & 0,8 & 7,1 \\
\hline Interferon regulatory factor 2-binding protein 2; & E9Q1P8 & Irf2bp2 & 1,7 & 6,1 \\
\hline Interferon-induced transmembrane protein 2 & Q99J93 & Ifitm2 & $-0,1$ & 5,8 \\
\hline Interferon-related developmental regulator 1 ; & P19182 & Ifrd1 & 0,9 & 1,8 \\
\hline Interleukin enhancer-binding factor 2 & Q9CXY6 & Ilf2 & 0,2 & 20,8 \\
\hline Interleukin enhancer-binding factor 3 ; & Q9Z1X4 & Ilf3 & $-0,1$ & 15,2 \\
\hline Interleukin-1 receptor accessory protein; & Q61730 & IIrap & 0,0 & 5,5 \\
\hline Interleukin-1 receptor antagon ist protein; & P25085 & $\| 1 \mathrm{rn}$ & $-0,3$ & 20 \\
\hline Intraflagellar transport protein 25 homolog; & Q9D6H2 & Hspb11 & 0,3 & 11,9 \\
\hline Involucrin; & G3X9D9 & $|v|$ & $-1,3$ & 54,5 \\
\hline Iron/zinc purple acid phosphatase-like protein; & Q8BX37 & Papl & $-1,2$ & 7,1 \\
\hline Iron-sulfur cluster assembly enzyme ISCU, mitochondrial; & Q9D7P6 & Iscu & 0,4 & 6,6 \\
\hline Isoamyl acetate-hydrolyzing esterase 1 homolog; & Q9DB29 & lah1 & 0,3 & 16,1 \\
\hline Isoaspartyl peptidase/L-asparaginase; & Q8С0M9 & Asrgl1 & 0,7 & 6,4 \\
\hline Isobutyryl-CoA dehydrogenase, mitochondrial; & Q9D7B6 & Acad8 & $-0,6$ & 11,1 \\
\hline Isochorismatase domain-containing protein 1 & Q91V64 & Isoc1 & 0,3 & 4,7 \\
\hline Isocitrate dehydrogenase [NAD] subunit alpha, mitochondrial; & Q9D6R2 & Idh3a & 0,4 & 29,8 \\
\hline Isocitrate dehydrogenase [NAD] subun it gamma 1, mitochondrial; & P70404 & Idh3g & 0,5 & 19,8 \\
\hline Isocitrate dehydrogenase [NADP] cytoplasmic; & 088844 & Idh1 & 0,1 & 48,3 \\
\hline Isocitrate dehydrogenase [NADP], mitochondrial; & P54071 & Idh2 & 0,9 & 36,5 \\
\hline $\begin{array}{l}\text { Isocitrate dehydrogenase } 3(\mathrm{NAD}+) \text { beta; Protein Idh3b; Tumor-related } \\
\text { protein; }\end{array}$ & Q91VA7 & $\operatorname{Idh} 3 b$ & $-0,3$ & 20,8 \\
\hline Isoleucine--tRNA ligase, cytoplasmic; & Q8BU30 & lars & $-0,5$ & 1,3 \\
\hline Isoleucine--tRNA ligase, mitochondrial; & Q8BIJ6 & lars2 & 0,2 & 11,9 \\
\hline Isopentenyl-diphosphate Delta-isomerase 1; & P58044 & Idi1 & 0,4 & 21,6 \\
\hline Isovaleryl-CoA dehydrogenase, mitochond rial; & Q9JHI5 & Ivd & $-0,7$ & 2,4 \\
\hline Junction plakoglobin; & Q02257 & Jup & $-0,5$ & 22,1 \\
\hline Junctional adhesion molecule $\mathrm{A}$; & 088792 & F11r & $-0,2$ & 28,7 \\
\hline Kallikrein 1-related peptidase b5; Kallikrein related-peptidase 5; & Q9D140 & Klk5 & $-0,2$ & 4,8 \\
\hline
\end{tabular}




\begin{tabular}{|c|c|c|c|c|}
\hline Protein & Uniprot & Gene name & $\begin{array}{l}\text { Fold change of } \\
\text { XPRESS ratio } \\
\left(E g f r^{\Delta K C} / \mathrm{WT}\right)\end{array}$ & $\begin{array}{c}\text { Sequence } \\
\text { coverage [\%] }\end{array}$ \\
\hline Kallikrein 6, isoform CRA_a; Kallikrein-1; KIk6 protein; Neurosin; & Q91Y82 & Klk6 & 2,2 & 19,8 \\
\hline Kallikrein related-peptidase 10; Protein KIk10; & Q99M20 & Klk10 & 0,9 & 32,4 \\
\hline Kallikrein-7; & Q91VE3 & Klk7 & $-0,2$ & 30,1 \\
\hline Kelch-like protein 7; & Q8BUL5 & $\mathrm{Klhl7}$ & $-4,1$ & 3,8 \\
\hline Keratin, type I cuticular Ha3-I; & Q8KOY2 & Krt33a & $-10,0$ & 25 \\
\hline Keratin, type I cuticular Ha4; & Q9D646 & Krt34 & $-2,9$ & 28,8 \\
\hline Keratin, type I cytoskeletal 10; & P02535 & Krt10 & $-1,1$ & 26 \\
\hline Keratin, type I cytoskeletal 14; & Q61781 & Krt14 & 0,1 & 22,1 \\
\hline Keratin, type I cytoskeletal 15; & Q61414 & Krt15 & $-0,2$ & 15,7 \\
\hline Keratin, type I cytoskeletal 17; & Q9QWL7 & Krt17 & $-1,0$ & 34,4 \\
\hline Keratin, type I cytoskeletal 23; & Q99PS0 & Krt23 & $-0,7$ & 5,2 \\
\hline Keratin, type I cytoskeletal 25; & Q8VCW2 & Krt25 & $-2,5$ & 37,9 \\
\hline Keratin, type I cytoskeletal 27; & Q9Z320 & Krt27 & $-5,1$ & 36,2 \\
\hline Keratin, type I cytoskeletal 28 ; & A6BLY7 & Krt28 & $-1,9$ & 23,8 \\
\hline Keratin, type II cuticular Hb6; & P97861 & Krt86 & $-3,8$ & 24,7 \\
\hline Keratin, type II cytoskeletal 1; & P04104 & Krt1 & $-1,8$ & 22,9 \\
\hline Keratin, type II cytoskeletal 1b; & Q6IFZ6 & Krt77 & $-1,0$ & 31,6 \\
\hline Keratin, type II cytoskeletal 2 epidermal; & Q3TTY5 & Krt2 & 0,0 & 5,8 \\
\hline Keratin, type II cytoskeletal 2 oral; & Q3UV17 & Krt76 & 0,7 & 5,7 \\
\hline Keratin, type II cytoskeletal 5; & Q922U2 & Krt5 & $-0,5$ & 18,4 \\
\hline Keratin, type II cytoskeletal 6A; & P50446 & Krt6a & 0,8 & 12,3 \\
\hline Keratin, type II cytoskeletal 71; & Q9R0H5 & Krt71 & $-3,4$ & 44,1 \\
\hline Keratin, type II cytoskeletal 72; & Q6IME9 & Krt72 & $-3,1$ & 15,8 \\
\hline Keratin, type II cytoskeletal 73; & Q6NXH9 & Krt73 & $-0,9$ & 25,6 \\
\hline Keratin, type II cytoskeletal 75; & Q8BGZ7 & Krt75 & $-1,8$ & 19,4 \\
\hline Keratin, type II cytoskeletal 79; & Q8VED5 & Krt79 & $-0,3$ & 19 \\
\hline Keratin-associated protein $15-1$ & Q9QZU5 & Krtap15 1 & $-3,1$ & 24,7 \\
\hline Keratin-associated protein $16-1$ & A2A5X5 & Krtap16 1 & $-2,4$ & 3 \\
\hline Keratin-associated protein 19-5; & F8VQ65 & Krtap19 5 & $-2,7$ & 19,4 \\
\hline Keratin-associated protein $3-1$; & A2A591 & Krtap3 1 & $-1,6$ & 17,3 \\
\hline Keratinocyte-associated transmembrane protein 2 ; & Q8K201 & Kct2 & 0,1 & 3,9 \\
\hline Ketimine reductase mu-crystallin; & 054983 & Crym & $-1,7$ & 39,3 \\
\hline Ketosamine-3-kinase; & Q8K274 & Fn3krp & 0,1 & 2,6 \\
\hline KH domain-containing, RNA-binding, signal transduction-associated protein 1; & Q60749 & Khdrbs1 & 0,4 & 4,5 \\
\hline Kinectin; & Q61595 & Ktn1 & $-0,4$ & 6,8 \\
\hline Kinesin-1 heavy chain; & Q61768 & Kif5b & 1,3 & 4,9 \\
\hline Kininogen-1; & 008677 & Kng1 & 1,1 & 29,2 \\
\hline Kunitz-type protease inhibitor 1 ; & Q9R097 & Spint1 & $-0,7$ & 8,9 \\
\hline Kynurenine--oxoglutarate transaminase 1 ; & Q8BTY1 & Ccbl1 & 0,3 & 20,9 \\
\hline Kynurenine--oxoglutarate transaminase 3; & Q71RI9 & Ccbl2 & $-0,1$ & 8,4 \\
\hline Lactadherin; & P21956 & Mfge8 & $-0,7$ & 6 \\
\hline Lactotransferrin; & P08071 & Ltf & 2,3 & 1,4 \\
\hline Lactoylglutathione lyase; & Q9CPU0 & Glo1 & $-0,9$ & 49,5 \\
\hline Ladinin-1; & P57016 & Lad1 & $-0,6$ & 19,7 \\
\hline Lamina-associated polypeptide 2, isoforms beta/delta/epsilon/gamma; & Q61029 & Tmpo & 0,1 & 29 \\
\hline Lamin-B receptor; & Q3U9G9 & Lbr & $-0,4$ & 5 \\
\hline Lamin-B1; & P14733 & Lmnb1 & 0,3 & 16,5 \\
\hline Laminin subunit alpha-2; & Q60675 & Lama2 & 0,7 & 2 \\
\hline Laminin subunit alpha-3; & Q61789 & Lama3 & $-2,3$ & 1,2 \\
\hline Laminin subunit alpha-4; & P97927 & Lama4 & 0,4 & 5,8 \\
\hline Laminin subunit alpha-5; & Q61001 & Lama5 & $-1,7$ & 0,3 \\
\hline Laminin subunit beta-1; & P02469 & Lamb1 & 0,1 & 9,7 \\
\hline Laminin subunit beta-2; & Q61292 & Lamb2 & 0,5 & 0,5 \\
\hline Laminin subunit gamma-1; & F8VQJ3 & Lamc1 & 0,0 & 14,4 \\
\hline LanC-like protein $1 ;$ & 089112 & Lancl1 & 0,3 & 12 \\
\hline LanC-like protein 2; & Q9JJK2 & Lancl2 & 0,3 & 2 \\
\hline Large neutral amino acids transporter small subunit 2; & Q9QXW9 & Slc7a8 & $-3,0$ & 5,5 \\
\hline Large proline-rich protein BAG6; & Q9Z1R2 & Bag6 & $-0,1$ & 1,8 \\
\hline Latent-transforming growth factor beta-binding protein 2; & 008999 & Ltbp2 & $-0,6$ & 1,2 \\
\hline Latent-transforming growth factor beta-binding protein 4; & D3Z598 & Ltbp4 & 0,0 & 20 \\
\hline Latexin; & P70202 & Lxn & 0,6 & 12,2 \\
\hline Latrophilin-2; & E9Q6C7 & Lphn2 & $-0,1$ & 3,3 \\
\hline LDLR chaperone MESD; & Q9ERE7 & Mesdc2 & $-1,3$ & 13 \\
\hline L-dopachrome tautomerase; & P29812 & Dct & 2,1 & 5,6 \\
\hline Leptin receptor gene-related protein; & 089013 & Leprot & $-0,4$ & 9,9 \\
\hline Leucine zipper protein 1 & Q8R4U7 & Luzp1 & $-0,8$ & 0,7 \\
\hline Leucine zipper transcription factor-like protein 1 ; & Q9JHQ5 & Lztfl1 & 0,2 & 11,9 \\
\hline $\begin{array}{l}\text { Leucine-rich HEV glycoprotein; Leucine-rich alpha-2-glycoprotein; Leucine-rich } \\
\text { alpha-2-glycoprotein 1; Protein Lrg1; }\end{array}$ & Q91XL1 & $\operatorname{Lrg} 1$ & $-0,2$ & 4,1 \\
\hline
\end{tabular}




\begin{tabular}{|c|c|c|c|c|}
\hline Protein & Uniprot & Gene name & $\begin{array}{l}\text { Fold change of } \\
\text { XPRESS ratio } \\
\left(E g f r^{\Delta K C} / \mathrm{WT}\right)\end{array}$ & $\begin{array}{c}\text { Sequence } \\
\text { coverage [\%] }\end{array}$ \\
\hline Leucine-rich repeat flightless-interacting protein 1 ; & Q3UZ39 & Lrrfip1 & $-0,5$ & 13 \\
\hline Leucine-rich repeat flightless-interacting protein 2 ; & Q91WKO & Lrrfip2 & 0,3 & 8,2 \\
\hline Leucine-rich repeat-containing protein 15 & Q80X72 & Lrrc15 & $-1,2$ & 11,9 \\
\hline Leucine-rich repeat-containing protein 16A; & Q6EDY6 & Lrrc16a & $-0,3$ & 2,5 \\
\hline Leucine-rich repeat-containing protein 47 ; & Q505F5 & Lrrc47 & 0,4 & 10,2 \\
\hline Leucine-rich repeat-containing protein 59 ; & Q922Q8 & Lrrc59 & 0,0 & 41,4 \\
\hline Leucine--tRNA ligase, cytoplasmic; & Q8BMJ2 & Lars & $-0,8$ & 1,4 \\
\hline Leucyl-cystinyl aminopeptidase; & Q8C129 & Lnpep & 0,3 & 10,5 \\
\hline Leukocyte elastase inhibitor A; & Q9D154 & Serpinb1a & 0,6 & 9,8 \\
\hline Leukocyte surface antigen CD47; & Q61735 & $\mathrm{Cd} 47$ & $-0,2$ & 8,9 \\
\hline Leukotriene A-4 hydrolase; & P24527 & Lta4h & 0,2 & 14,2 \\
\hline L-fucose kinase; Protein Fuk; & Q7TMC8 & Fuk & 0,4 & 4,4 \\
\hline LIM and senescent cell antigen-like-containing domain protein $1 ;$ & Q99JW4 & Lims1 & 0,2 & 15,1 \\
\hline LIM and SH3 domain protein 1 & Q61792 & Lasp1 & $-1,0$ & 16,3 \\
\hline LIM domain and actin-bind ing protein $1 ;$ & Q9ERG0 & Lima1 & $-0,9$ & 27,9 \\
\hline LIM zinc-binding domain-containing Nebulette; & Q9DC07 & Nebl & 0,4 & 8,4 \\
\hline Lipid phosphate phosphohydrolase 3 & Q99JY8 & Ppap2b & 0,6 & 7,1 \\
\hline Lipoma HMGIC fusion partner-like 2 protein; & Q8BGA2 & Lhfpl2 & 0,6 & 6,5 \\
\hline Lipoma-preferred partner homolog; & Q8BFW7 & Lpp & 0,1 & 16 \\
\hline Lipoprotein lipase; & P11152 & Lpl & $-0,9$ & 7,4 \\
\hline Liprin-beta-2; & 035711 & Ppfibp2 & $-1,1$ & 1 \\
\hline L-lactate dehydrogenase A chain; & P06151 & Ldha & 0,2 & 30,4 \\
\hline L-lactate dehydrogenase B chain; & P16125 & Ldhb & $-0,1$ & 26,9 \\
\hline Lon protease homolog, mitochondrial; & Q8CGK3 & Lonp1 & 0,2 & 9,7 \\
\hline Long-chain specific acyl-CoA dehydrogenase, mitochondrial; & P51174 & Acadl & $-0,2$ & 33,5 \\
\hline Long-chain-fatty-acid--CoA ligase 1 & P41216 & Acsl1 & 0,2 & 16,6 \\
\hline Low affinity immunoglobulin gamma Fc region receptor II; & P08101 & Fcgr2 & 0,7 & 3,2 \\
\hline Low-density lipoprotein receptor-related protein 2 & A2ARV4 & Lrp2 & 0,0 & 0,4 \\
\hline Luc7-like protein 3; & Q5SUF2 & Luc7l3 & 0,0 & 2,5 \\
\hline Lumican; & P51885 & Lum & $-0,2$ & 38,8 \\
\hline Lupus La protein homolog; & P32067 & Ssb & 0,3 & 15,7 \\
\hline Ly6/PLAUR domain-containing protein 3; & Q91YK8 & Lypd3 & 0,4 & 5,2 \\
\hline Ly6/PLAUR domain-containing protein 5; & Q9D7Z7 & Lypd5 & $-1,7$ & 13,7 \\
\hline Lymphatic vessel endothelial hyaluronic acid receptor 1 ; & Q8BHCO & Lyve1 & $-0,5$ & 3,8 \\
\hline Lymphocyte antigen 6 complex locus protein G6c; & Q9Z1Q4 & Ly6g6c & $-0,6$ & 21,4 \\
\hline Lymphocyte antigen 6 complex locus protein G6d; & Q9Z1Q3 & Ly6g6d & $-2,5$ & 8,1 \\
\hline Lymphocyte antigen 6D; & P35459 & Ly6d & 0,4 & 21,3 \\
\hline Lymphocyte-specific protein 1 ; & P19973 & Lsp1 & 0,7 & 7,5 \\
\hline Lys-63-specific deubiquitinase BRCC36; & P46737 & Brcc3 & $-0,9$ & 12 \\
\hline Lysine-specific histone demethylase $1 \mathrm{~A}$ & Q6ZQ88 & Kdm1a & 0,3 & 6,4 \\
\hline Lysine--tRNA ligase; & Q99MN1 & Kars & $-0,5$ & 11,4 \\
\hline Lysophosphatidic acid phosphatase type 6; & Q8BP40 & Acp6 & 0,6 & 5,7 \\
\hline Lysophosphatidylcholine acyltransferase 2; & Q8BYI6 & Lpcat2 & $-0,1$ & 1,7 \\
\hline Lysophospholipid acyltransferase 5; & Q91V01 & Lpcat3 & $-0,3$ & 1,8 \\
\hline Lysophospholipid acyltransferase 7; & Q8CHK3 & Mboat7 & $-0,4$ & 3,5 \\
\hline Lysosomal alpha-mannosidase; & 009159 & Man2b1 & $-0,2$ & 4,4 \\
\hline Lysosomal protective protein; & P16675 & Ctsa & $-0,4$ & 12,9 \\
\hline Lysosomal protein NCU-G1; & Q9JHJ3 & & $-0,5$ & 6,3 \\
\hline Lysosomal Pro-X carboxypeptidase; & Q7TMRO & Prcp & 2,1 & 11,4 \\
\hline Lysosomal thioesterase PPT2; & 035448 & Ppt2 & $-0,5$ & 22,5 \\
\hline Lysosome membrane protein 2 & 035114 & Scarb2 & $-0,2$ & 6,1 \\
\hline Lysosome-associated membrane glycoprotein $1 ;$ & P11438 & Lamp1 & $-0,1$ & 11,8 \\
\hline Lysosome-associated membrane glycoprotein 2; & P17047 & Lamp2 & 0,1 & 8,4 \\
\hline Lysozyme C-2; & P08905 & Lyz2 & 1,8 & 14,9 \\
\hline Lysozyme g-like protein 2 & Q3V110 & Lyg2 & $-0,9$ & 13,6 \\
\hline m7GpppX diphosphatase; & Q9DAR7 & Dcps & 0,7 & 32 \\
\hline Macrophage mannose receptor 1 ; & Q61830 & Mrc1 & 0,6 & 6,4 \\
\hline Macrophage migration inhibitory factor; & P34884 & Mif & $-0,4$ & 7,8 \\
\hline Magnesium transporter NIPA4; & Q8BZF2 & Nipal4 & $-0,7$ & 3,4 \\
\hline Magnesium transporter protein 1 & Q9CQY5 & Magt1 & 0,3 & 8,3 \\
\hline Magnesium-dependent phosphatase 1; & Q9D967 & Mdp1 & 0,4 & 28,7 \\
\hline Major vault protein; & Q9EQK5 & Mvp & 0,1 & 7,2 \\
\hline Malate dehydrogenase, cytoplasmic; & P14152 & Mdh1 & 0,3 & 31,4 \\
\hline Malate dehydrogenase, mitochond rial; & P08249 & Mdh2 & $-0,1$ & 61,2 \\
\hline Malectin; & Q6ZQI3 & Mlec & $-0,4$ & 13,4 \\
\hline Maleylacetoacetate isomerase; & Q9WVL0 & Gstz1 & 0,5 & 11,6 \\
\hline Malignant T-cell-amplified sequence 1 ; & Q9DB27 & Mcts1 & $-0,8$ & 45,9 \\
\hline Manganese-transporting ATPase $13 \mathrm{~A} 1$; & Q9EPE9 & Atp13a1 & $-0,1$ & 0,9 \\
\hline Mannose-1-phosphate guanyltransferase alpha; & Q922H4 & Gmppa & 0,1 & 11,9 \\
\hline Mannose-1-phosphate guanyltransferase beta; & Q8BTZ7 & Gmppb & $-0,1$ & 20,6 \\
\hline Mannose-P-dolichol utilization defect 1 protein; & Q9R0Q9 & Mpdu1 & $-0,5$ & 8,1 \\
\hline
\end{tabular}




\begin{tabular}{|c|c|c|c|c|}
\hline Protein & Uniprot & Gene name & $\begin{array}{l}\text { Fold change of } \\
\text { XPRESS ratio } \\
\left(\text { Egfr }^{\Delta K C} / \mathrm{WT}\right)\end{array}$ & $\begin{array}{c}\text { Sequence } \\
\text { coverage [\%] }\end{array}$ \\
\hline Mannosyl-oligosaccharide 1,2-alpha-mannosidase IB; & P39098 & Man1a2 & 0,1 & 7 \\
\hline Mannosyl-oligosaccharide glucosidase; & Q80UM7 & Mogs & $-0,3$ & 10,3 \\
\hline MARCKS-related protein; & P28667 & Marcksl1 & 0,0 & 17,5 \\
\hline MARVEL domain-containing protein 2 & Q3UZPO & Marveld2 & $-0,2$ & 1,8 \\
\hline Matrin-3; & Q8K310 & Matr3 & 0,1 & 4,4 \\
\hline Matrix-remodeling-associated protein 7; & Q9CZH7 & Mxra7 & 0,5 & 15,9 \\
\hline Matrix-remodeling-associated protein 8; & Q9DBV4 & Mxra8 & 0,2 & 3,5 \\
\hline MCG117626; Odorant binding protein la; Protein Obp1a; & Q9D3H2 & Obp1a & 0,0 & 9,2 \\
\hline MCG125471; Predicted gene, 677156; Protein Cyp4f37; & Q3V1F1 & Cyp4f37 & 1,4 & 5,7 \\
\hline $\begin{array}{l}\text { MCG127334; MCG17849, isoform CRA_a; Protein 1810009A15Rik; } \\
\text { Uncharacterized protein; }\end{array}$ & Q9D937 & 1810009A15Rik & 0,0 & 11,4 \\
\hline MCG129038; Protein Serpinb3a; & G3X9V8 & Serpinb3a & 1,2 & 14 \\
\hline MCG13402, isoform CRA_a; Polypyrimidine tract-binding protein 1; & Q8BGJ5 & Ptbp1 & 0,1 & 18,6 \\
\hline MCG14442; Protein Eif2b3; & B1AUN2 & Eif2b3 & $-0,6$ & 7,7 \\
\hline MCG148436; Protein 1700014D04Rik; & J3QMS2 & 1700014D04Rik & 2,9 & 17,2 \\
\hline MCG18410, isoform CRA_a; Protein Ddx23; & D3Z0M9 & Ddx23 & 0,3 & 1,3 \\
\hline MCG20149, isoform CRA_a; Methyltransferase like 7A1; Protein MettI7a1; & Q8C6B0 & Mettl7a1 & 1,1 & 14,8 \\
\hline MCG20280; OTTMUSG00000000712 protein; Protein Serpinb6d; & Q3UWK8 & Serpinb6d & 10,0 & 2,1 \\
\hline $\begin{array}{l}\text { MCG21506; Macrophage galactose N-acetyl-galactosamine specific lectin } 2 \\
\text { isoform i; Protein Mgl2; }\end{array}$ & A9XX86 & $\mathrm{Mgl2}$ & 0,7 & 19,9 \\
\hline MCG54087; Protein Serpina3j; & D3Z451 & Serpina3j & 0,9 & 10,5 \\
\hline Mediator of RNA polymerase II transcription subunit 28 ; & Q920D3 & Med28 & $-1,4$ & 11,9 \\
\hline Mediator of RNA polymerase II transcription subunit 4; & Q9CQA5 & Med4 & 0,4 & 3,3 \\
\hline Medium-chain specific acyl-CoA dehydrogenase, mitochondrial; & P45952 & Acadm & 0,4 & 18,8 \\
\hline Melanocyte protein PMEL; & Q60696 & Pmel & 0,7 & 3,2 \\
\hline Melanoma inhibitory activity protein 3 ; & Q8BI84 & Mia3 & $-0,4$ & 3,5 \\
\hline Membrane magnesium transporter 1 ; & Q8K273 & Mmgt1 & $-0,7$ & 6,9 \\
\hline Membrane primary amine oxidase; & 070423 & Aoc3 & 0,2 & 18,3 \\
\hline Membrane-associated progesterone receptor component 1 ; & 055022 & Pgrmc1 & 0,3 & 26,2 \\
\hline Membrane-associated progesterone receptor component 2; & Q80UU9 & Pgrmc2 & $-0,1$ & 38,2 \\
\hline Mesoderm-specific transcript protein; & Q07646 & Mest & 1,4 & 3,3 \\
\hline Metalloreductase STEAP3; & Q8CI59 & Steap3 & $-0,3$ & 8,1 \\
\hline Metallothionein-2; & P02798 & $\mathrm{Mt2}$ & 2,8 & 19,7 \\
\hline Metallothionein-4; & P47945 & Mt4 & 1,2 & 19,4 \\
\hline Metal-response element-binding transcription factor 2 & Q02395 & Mtf2 & $-0,3$ & 1,5 \\
\hline Metastasis-associated protein MTA3; & Q924K8 & Mta3 & 0,3 & 6,3 \\
\hline Metaxin-1; & P47802 & Mtx1 & 0,1 & 11,4 \\
\hline Methionine adenosyltransferase 2 subunit beta; & Q99LB6 & Mat2b & 0,4 & 22,2 \\
\hline Methionine aminopeptidase 1 & Q8BP48 & Metap1 & $-0,3$ & 15,5 \\
\hline Methionine aminopeptidase 2 ; & 008663 & Metap2 & $-0,2$ & 23,4 \\
\hline Methyl-CpG-binding domain protein 2; & Q9Z2E1 & Mbd2 & 0,1 & 3,6 \\
\hline Methyl-CpG-binding domain protein 3; & Q9Z2D8 & Mbd3 & 0,2 & 8,1 \\
\hline Methyl-CpG-binding protein 2; & Q9Z2D6 & Mecp2 & 0,5 & 5,8 \\
\hline Methylcrotonoyl-CoA carboxylase beta chain, mitochondrial; & Q3ULD5 & Mccc2 & $-0,2$ & 19,7 \\
\hline Methylmalonate-semialdehyde dehydrogenase [acylating], mitochondrial; & Q9EQ20 & Aldh6a1 & 0,6 & 8,8 \\
\hline Methylmalonyl-CoA epimerase, mitochondrial; & Q9D1I5 & Mcee & $-2,5$ & 10,7 \\
\hline Methylosome protein 50; & Q99J09 & Wdr77 & 0,2 & 13,5 \\
\hline Methylosome subunit pICln; & Q61189 & Clns1a & 0,3 & 12,7 \\
\hline Methylsterol monooxygenase 1 & Q9CRA4 & Msmo1 & $-0,7$ & 2,4 \\
\hline Methylthioribose-1-phosphate isomerase; & Q9CQT1 & Mri1 & 1,3 & 7,9 \\
\hline Methylthioribulose-1-phosphate dehydratase; & Q9WVQ5 & Apip & 0,5 & 3,3 \\
\hline Mevalonate kinase; & Q9R008 & Mvk & 0,0 & 14,9 \\
\hline Microfibril-associated glycoprotein 4; & Q9D1H9 & Mfap4 & $-0,3$ & 9,7 \\
\hline Microfibrillar-associated protein 1; & Q9CQU1 & Mfap1 & 0,5 & 3 \\
\hline Microfibrillar-associated protein 5; & Q9QZJ6 & Mfap5 & $-0,5$ & 9,8 \\
\hline Microsomal glutathione S-transferase 1; & Q91VS7 & Mgst1 & 1,0 & 18,7 \\
\hline Microsomal glutathione S-transferase 3; & Q9CPU4 & Mgst3 & $-0,3$ & 9,2 \\
\hline Microtubule-associated protein $1 \mathrm{~A}$ & Q9QYR6 & Map1a & 0,5 & 2,6 \\
\hline Microtubule-associated protein 1B; & P14873 & Map1b & 0,2 & 2,4 \\
\hline Microtubule-associated protein 2; & P20357 & Map2 & 0,0 & 3,6 \\
\hline Microtubule-associated protein 4; & P27546 & Map4 & $-0,1$ & 30,7 \\
\hline Microtubule-associated protein 6; & Q7TSJ2 & Map6 & 0,3 & 2,9 \\
\hline Microtubule-associated protein RP/EB family member 1 ; & Q61166 & Mapre1 & $-0,2$ & 10,4 \\
\hline Mimecan; & Q62000 & Ogn & $-1,7$ & 12,1 \\
\hline Minor histocompatibility antigen $\mathrm{H} 13$; & Q9D8V0 & $\mathrm{Hm} 13$ & $-0,3$ & 11 \\
\hline Mitochondrial 2-oxoglutarate/malate carrier protein; & Q9CR62 & Slc25a11 & 0,0 & 27,4 \\
\hline Mitochondrial amidoxime reducing component 2; & Q922Q1 & Marc2 & $-0,4$ & 16,3 \\
\hline Mitochondrial carnitine/acylcarnitine carrier protein; & Q9Z2Z6 & Slc25a20 & $-0,1$ & 12,6 \\
\hline Mitochondrial carrier homolog 2; & Q791V5 & Mtch2 & $-0,3$ & 16,8 \\
\hline
\end{tabular}




\begin{tabular}{|c|c|c|c|c|}
\hline Protein & Uniprot & Gene name & $\begin{array}{l}\text { Fold change of } \\
\text { XPRESS ratio } \\
\left(E g f r^{\Delta K C} / \mathrm{WT}\right)\end{array}$ & $\begin{array}{c}\text { Sequence } \\
\text { coverage [\%] }\end{array}$ \\
\hline Mitochondrial chaperone BCS1; & Q9CZP5 & Bcs1l & 0,3 & 2,2 \\
\hline Mitochondrial dicarboxylate carrier; & Q9QZD8 & Slc25a10 & 0,2 & 16 \\
\hline Mitochondrial fission 1 protein; & Q9CQ92 & Fis1 & $-0,6$ & 24,8 \\
\hline Mitochondrial import inner membrane translocase subunit Tim10 B; & Q9WV96 & Timm10b & 0,3 & 27,9 \\
\hline Mitochondrial import inner membrane translocase subunit Tim13; & P62075 & Timm13 & $-0,4$ & 61,1 \\
\hline Mitochondrial import inner membrane translocase subu nit TIM14; & Q9CQV7 & Dnajc19 & 0,3 & 15,5 \\
\hline Mitochondrial import inner membrane translocase subunit TIM16; & Q9CQV1 & Pam16 & 0,0 & 21,6 \\
\hline Mitochondrial import inner membrane translocase subunit Tim17-B; & Q9Z0V7 & Timm17b & $-0,5$ & 8,7 \\
\hline Mitochondrial import inner membrane translocase subunit Tim23; & Q9WTQ8 & Timm23 & $-0,4$ & 8,1 \\
\hline Mitochondrial import inner membrane translocase subu nit Tim8 A; & Q9WVA2 & Timm8a1 & 0,2 & 34 \\
\hline Mitochondrial import inner membrane translocase subu nit Tim8 B; & P62077 & Timm8b & 0,0 & 21,7 \\
\hline Mitochondrial import inner membrane translocase subunit Tim9; & Q9WV98 & Timm9 & $-0,2$ & 56,2 \\
\hline Mitochondrial import receptor subunit TOM22 homolog; & Q9CPQ3 & Tomm22 & $-1,6$ & 16,2 \\
\hline Mitochondrial import receptor subunit TOM40 homolog; & Q9QYA2 & Tomm40 & $-0,7$ & 11,6 \\
\hline Mitochondrial inner membrane organizing system protein 1 ; & Q7TNS2 & Minos1 & $-0,4$ & 10,5 \\
\hline Mitochondrial inner membrane protein OXA1L; & Q8BGA9 & Oxa1l & 0,2 & 2,1 \\
\hline Mitochondrial inner membrane protein; & Q8CAQ8 & Immt & $-0,5$ & 30,9 \\
\hline Mitochondrial pyruvate carrier 2 & Q9D023 & Mpc2 & $-0,3$ & 28,3 \\
\hline Mitogen-activated protein kinase 1 & P63085 & Mapk1 & 0,5 & 31,6 \\
\hline Mitogen-activated protein kinase 14 & P47811 & Mapk14 & $-0,2$ & 11,8 \\
\hline Mitogen-activated protein kinase 3 ; & Q63844 & Mapk3 & $-1,5$ & 10,3 \\
\hline Mitogen-activated protein kinase kinase kinase MLT; & Q9ESL4 & Zak & $-0,1$ & 2,7 \\
\hline Mitotic checkpoint protein BUB3; & Q9WVA3 & Bub3 & 0,5 & 29,4 \\
\hline MKI67 FHA domain-interacting nucleolar phosphoprotein; & Q91VE6 & Nifk & $-0,1$ & 3,8 \\
\hline MOB kinase activator $1 \mathrm{~B}$ & Q8BPB0 & Mob1b & 0,5 & 10,6 \\
\hline Moesin; & P26041 & Msn & 0,8 & 15,6 \\
\hline Molybdenum cofactor biosynthesis protein 1 ; & Q5RKZ7 & Mocs1 & 3,4 & 2,5 \\
\hline Monoacylglycerol lipase ABHD12; & Q99LR1 & Abhd12 & 1,2 & 3,3 \\
\hline Monocarboxylate transporter 1 & P53986 & Slc16a1 & 0,8 & 8,5 \\
\hline Monoglyceride lipase; & 035678 & Mgll & 1,3 & 16,2 \\
\hline mRNA cap guanine-N7 methyltransferase; & Q9D0L8 & Rnmt & 0,4 & 24,9 \\
\hline Multifunctional protein ADE2; & Q9DCL9 & Paics & 0,5 & 21,6 \\
\hline Multimerin-2; & A6H6E2 & Mmrn2 & 1,0 & 1,7 \\
\hline Multiple inositol polyphosphate phosphatase 1; & Q9Z2L6 & Minpp1 & $-0,8$ & 13,1 \\
\hline Myelin basic protein; & F6RT34 & $\mathrm{Mbp}$ & $-0,1$ & 25,9 \\
\hline Myelin protein $\mathrm{PO}$ & P27573 & Mpz & $-0,2$ & 12,1 \\
\hline Myelin protein $\mathrm{PO}$ & E9QK82 & Mpz & $-0,2$ & 12,1 \\
\hline Myelin-associated glycoprotein; & P20917 & Mag & 0,0 & 1,6 \\
\hline Myeloid bactenecin (F1); Neutrophilic granule protein; Protein Ngp; & 008692 & Ngp & 2,6 & 19,2 \\
\hline Myeloid-associated differentiation marker; & 035682 & Myadm & 0,3 & 8,4 \\
\hline Myoferlin; & Q69ZN7 & Myof & 0,4 & 4,2 \\
\hline Myosin light chain $1 / 3$, skeletal muscle isoform; & P05977 & Myl1 & 1,7 & 32,9 \\
\hline Myosin light chain kinase, smooth muscle; & Q6PDN3 & Mylk & $-0,4$ & 1,2 \\
\hline Myosin light polypeptide 6; & Q60605 & Myl6 & $-0,5$ & 54,3 \\
\hline Myosin regulatory light chain 10 & E9QNY3 & Myl10 & 0,3 & 6,1 \\
\hline Myosin regulatory light chain $12 \mathrm{~B}$ & Q3THE2 & Myl12b & $-0,6$ & 40,1 \\
\hline Myosin regulatory light chain 2, skeletal muscle isoform; & P97457 & Mylpf & 2,8 & 7,7 \\
\hline Myosin regulatory light polypeptide 9 & Q9CQ19 & Myl9 & $-1,1$ & 33,7 \\
\hline Myosin-10; & Q61879 & Myh10 & $-0,4$ & 9,1 \\
\hline Myosin-11; & E9QPE7 & Myh11 & $-5,1$ & 5,7 \\
\hline Myosin-14; & Q6URW6 & Myh14 & 0,6 & 5,8 \\
\hline Myosin-9; & Q8VDD5 & Myh9 & $-0,2$ & 28,6 \\
\hline Myotrophin; & P62774 & Mtpn & 0,5 & 14,4 \\
\hline Myristoylated alanine-rich C-kinase substrate; & P26645 & Marcks & 0,8 & 23,6 \\
\hline $\mathrm{N}(4)$-(beta-N-acetylglucosaminyl)-L-asparaginase; & Q64191 & Aga & 0,6 & 9,2 \\
\hline$N(G), N(G)$-dimethylarginine dimethylaminohydrolase 2 ; & Q99LD8 & Ddah2 & 1,1 & 39,6 \\
\hline $\mathrm{Na}(+) / \mathrm{H}(+)$ exchange regulatory cofactor NHE-RF1; & P70441 & Slc9a3r1 & $-0,4$ & 35,5 \\
\hline $\mathrm{Na}(+) / \mathrm{H}(+)$ exchange regulatory cofactor NHE-RF2; & Q9JHL1 & Slc9a3r2 & 0,1 & 3,9 \\
\hline $\mathrm{N}$-acetylated-alpha-linked acidic dipeptidase 2 & Q9CZR2 & Naalad2 & 2,5 & 17,4 \\
\hline $\mathrm{N}$-acetyl-D-glucosamine kinase; & Q9QZ08 & Nagk & 0,3 & 8,6 \\
\hline $\mathrm{N}$-acetylglucosamine-6-sulfatase; & Q8BFR4 & Gns & 0,2 & 10,5 \\
\hline $\mathrm{N}$-acetylneuraminate lyase; & Q9DCJ9 & $\mathrm{Npl}$ & $-0,3$ & 51,6 \\
\hline $\mathrm{N}$-acylethanolamine-hydrolyzing acid amidase; & Q9D7V9 & Naaa & 0,3 & 6,1 \\
\hline $\mathrm{N}$-acylglucosamine 2-epimerase; & P82343 & Renbp & 0,5 & 6,2 \\
\hline $\mathrm{NAD}(\mathrm{P}) \mathrm{H}$ dehydrogenase [quinone] $1 ;$ & Q64669 & Nqo1 & $-0,2$ & 3,6 \\
\hline NAD(P)H-hydrate epimerase; & Q8K4Z3 & Apoa1bp & $-0,1$ & 22 \\
\hline NADH dehydrogenase [ubiquinone] 1 alpha subcomplex subunit $11 ;$ & Q9D8B4 & Ndufa11 & 1,0 & 24,1 \\
\hline NADH dehydrogenase [ubiquinone] 1 alpha subcomplex subunit 13 ; & Q9ERS2 & Ndufa13 & $-0,2$ & 20,8 \\
\hline NADH dehydrogenase [ubiquinone] 1 alpha subcomplex subunit 4; & Q62425 & Ndufa4 & $-0,1$ & 46,3 \\
\hline NADH dehydrogenase [ubiquinone] 1 alpha subcomplex subunit 4-like 2; & Q4FZG9 & Ndufa4I2 & $-0,7$ & 19,5 \\
\hline
\end{tabular}




\begin{tabular}{|c|c|c|c|c|}
\hline Protein & Uniprot & Gene name & $\begin{array}{l}\text { Fold change of } \\
\text { XPRESS ratio } \\
\left(\text { Egfr }^{\triangle K C} / \mathrm{WT}\right)\end{array}$ & $\begin{array}{c}\text { Sequence } \\
\text { coverage [\%] }\end{array}$ \\
\hline NADH dehydrogenase [ubiquinone] 1 alpha subcomplex subunit 5; & Q9CPP6 & Ndufa5 & 0,1 & 19,8 \\
\hline NADH dehydrogenase [ubiquinone] 1 alpha subcomplex subunit 7; & Q9Z1P6 & Ndufa7 & $-0,3$ & 17,7 \\
\hline NADH dehydrogenase [ubiquinone] 1 alpha subcomplex subunit 8 & Q9DCJ5 & Ndufa8 & $-0,1$ & 9,9 \\
\hline NADH dehydrogenase [ubiquinone] 1 beta subcomplex subunit 10; & Q9DCS9 & Ndufb10 & $-0,2$ & 31,5 \\
\hline $\begin{array}{l}\text { NADH dehydrogenase [ubiquinone] } 1 \text { beta subcomplex subunit } 11 \text {, } \\
\text { mitochondrial; }\end{array}$ & 009111 & Ndufb11 & $-1,0$ & 17,9 \\
\hline NADH dehydrogenase [ubiquinone] 1 beta subcomplex subunit 3; & Q9CQZ6 & Ndufb3 & $-0,3$ & 10,6 \\
\hline $\begin{array}{l}\text { NADH dehydrogenase [ubiquinone] } 1 \text { beta subcomplex subunit } 5 \text {, } \\
\text { mitochondrial; }\end{array}$ & Q9CQH3 & Ndufb5 & $-0,4$ & 16 \\
\hline NADH dehydrogenase [ubiquinone] 1 beta subcomplex subunit 6; & Q3UIU2 & Ndufb6 & $-0,6$ & 25,8 \\
\hline NADH dehydrogenase [ubiquinone] 1 beta subcomplex subunit 7; & Q9CR61 & Ndufb7 & $-0,4$ & 7,3 \\
\hline $\begin{array}{l}\text { NADH dehydrogenase [ubiquinone] } 1 \text { beta subcomplex subunit } 8 \text {, } \\
\text { mitochondrial; }\end{array}$ & Q9D6J5 & Ndufb8 & 0,2 & 6,5 \\
\hline NADH dehydrogenase [ubiquinone] 1 subunit $C 2$; & Q9CQ54 & Ndufc2 & 0,4 & 24,2 \\
\hline NADH dehydrogenase [ubiquinone] flavoprotein 1, mitochondrial; & Q91YT0 & Ndufv1 & 0,4 & 9,1 \\
\hline NADH dehydrogenase [ubiquinone] flavoprotein 2, mitochondrial; & Q9D6J6 & Ndufv2 & 0,7 & 12,5 \\
\hline NADH dehydrogenase [ubiquinone] flavoprotein 3 , mitochondrial; & Q8BK30 & Ndufv3 & 0,1 & 10,6 \\
\hline $\begin{array}{l}\text { NADH dehydrogenase [ubiquinone] flavoprotein 3, mitochondrial; RIKEN cDNA } \\
\text { 1500032D16, isoform CRA_a; }\end{array}$ & Q3U422 & Ndufv3 & $-0,8$ & 18,2 \\
\hline NADH dehydrogenase [ubiquinone] iron-sulfur protein 2, mitochondrial; & Q91WD5 & Ndufs2 & $-0,2$ & 13,8 \\
\hline NADH dehydrogenase [ubiquinone] iron-sulfur protein 3, mitochondrial; & Q9DCT2 & Ndufs3 & $-0,9$ & 14,8 \\
\hline NADH dehydrogenase [ubiquinone] iron-sulfur protein 4, mitochondrial; & Q9CXZ1 & Ndufs4 & 0,3 & 14,3 \\
\hline NADH dehydrogenase [ubiquinone] iron-sulfur protein 5; & Q99LY9 & Ndufs5 & $-0,1$ & 13,2 \\
\hline NADH dehydrogenase [ubiquinone] iron-sulfur protein 6, mitochondrial; & P52503 & Ndufs6 & $-0,3$ & 15,3 \\
\hline NADH dehydrogenase [ubiquinone] iron-sulfur protein 7, mitochondrial; & Q9DC70 & Ndufs7 & 0,3 & 4 \\
\hline NADH-cytochrome b5 reductase 3 ; & Q9DCN2 & Cyb5r3 & $-0,3$ & 16,6 \\
\hline NADP-dependent malic enzyme; & P06801 & Me1 & $-0,7$ & 32,9 \\
\hline NADPH--cytochrome $\mathrm{P} 450$ reductase; & P37040 & Por & 0,1 & 3,4 \\
\hline N-alpha-acetyltransferase 50 & Q6PGB6 & Naa50 & 0,7 & 5,9 \\
\hline Nascent polypeptide-associated complex subunit alpha, muscle-specific form; & P70670 & Naca & $-0,1$ & 25,6 \\
\hline Nectin-1; & Q9JKF6 & Pvrl1 & 0,0 & 9,1 \\
\hline Nectin-2; & P32507 & Pvrl2 & $-0,2$ & 9,6 \\
\hline Nectin-4; & Q8R007 & Pvrl4 & $-0,1$ & 12 \\
\hline NEDD8; & P29595 & Nedd8 & 0,3 & 11,1 \\
\hline NEDD8-activating enzyme E1 catalytic subunit; & Q8C878 & Uba3 & $-0,1$ & 6,7 \\
\hline NEDD8-conjugating enzyme Ubc12; & P61082 & Ube2m & 0,2 & 6,5 \\
\hline Neprilysin; & Q61391 & Mme & $-0,7$ & 4,8 \\
\hline Neudesin; & Q9CQ45 & Nenf & $-0,3$ & 36,8 \\
\hline Neural cell adhesion molecule 1 ; & P13595 & Ncam1 & 0,2 & 6,5 \\
\hline $\begin{array}{l}\text { Neurogenic locus notch homolog protein 2; Notch gene homolog } 2 \\
\text { (Drosophila), isoform CRA_a; }\end{array}$ & G5E8J0 & Notch2 & 1,6 & 1,3 \\
\hline Neurolysin, mitochondrial; & Q91YP2 & $\mathrm{N} \ln$ & 0,5 & 3,4 \\
\hline Neuromodulin; & P06837 & Gap43 & 0,1 & 7,5 \\
\hline Neuronal proto-oncogene tyrosine-protein kinase Src; & P05480 & Src & 0,8 & 1,6 \\
\hline Neuroplastin; & P97300 & Nptn & 0,0 & 18,1 \\
\hline Neutral alpha-glucosidase $A B$ & Q8BHN3 & Ganab & $-0,5$ & 20,2 \\
\hline Neutral amino acid transporter A; & 035874 & Slc1a4 & $-1,5$ & 13,5 \\
\hline $\begin{array}{l}\text { Neutral amino acid transporter ASCT2; Neutral amino acid transporter } \mathrm{B}(0) \text {; } \\
\text { Solute carrier family } 1 \text { (Neutral amino acid transporter), member } 5 \text {; }\end{array}$ & Q9ESU7 & Slc1a5 & 1,3 & 7,6 \\
\hline Neutral cholesterol ester hydrolase $1 ;$ & Q8BLF1 & Nceh1 & 0,1 & 5,6 \\
\hline NFU1 iron-sulfur cluster scaffold homolog, mitochondrial; & Q9QZ23 & Nfu1 & 0,9 & 6,5 \\
\hline NHP2-like protein 1; & Q9D0T1 & Nhp2l1 & 0,2 & 27,3 \\
\hline Niban-like protein 1 ; & Q8R1F1 & Fam129b & 0,2 & 1,9 \\
\hline
\end{tabular}


Supplementary table 8: List of all identified and quantified proteins in the quantitative proteome comparison of wild-type and Egfr ${ }^{\Delta K C}$ epidermis at P10 (replicate 1).

\begin{tabular}{|c|c|c|c|c|}
\hline Protein & Uniprot & Gene name & $\begin{array}{l}\text { Fold change of } \\
\text { XPRESS ratio } \\
\left(\text { Egfr }^{\triangle K C} / \mathrm{WT}\right)\end{array}$ & $\begin{array}{c}\text { Sequence } \\
\text { coverage [\%] }\end{array}$ \\
\hline 10 kDa heat shock protein, mitochondrial; & Q64433 & Hspe1 & 0,0 & 54,9 \\
\hline 116 kDa U5 small nuclear ribonucleoprotein component; & 008810 & Eftud2 & 0,5 & 27,1 \\
\hline 14-3-3 protein beta/alpha; & Q9CQV8 & Ywhab & 0,0 & 48,4 \\
\hline 14-3-3 protein epsilon; & P62259 & Ywhae & 0,3 & 57,3 \\
\hline 14-3-3 protein eta; & P68510 & Ywhah & 0,0 & 49,6 \\
\hline 14-3-3 protein gamma; & P61982 & Ywhag & 0,1 & 31,6 \\
\hline 14-3-3 protein sigma; & 070456 & Sfn & $-0,4$ & 40,7 \\
\hline 14-3-3 protein theta; & P68254 & Ywhaq & $-0,2$ & 38 \\
\hline 14-3-3 protein zeta/delta; & P63101 & Ywhaz & 0,1 & 49,8 \\
\hline 15 kDa selenoprotein; & Q9ERR7 & Sep15 & $-0,9$ & 17,9 \\
\hline 182 kDa tankyrase-1-binding protein; & P58871 & Tnks1bp1 & 0,1 & 23,6 \\
\hline 1-acyl-sn-glycerol-3-phosphate acyltransferase beta; & Q8K3K7 & Agpat2 & $-0,1$ & 9,7 \\
\hline 1-acyl-sn-glycerol-3-phosphate acyltransferase delta; & Q8K4X7 & Agpat4 & 0,1 & 2,4 \\
\hline 1-acyl-sn-glycerol-3-phosphate acyltransferase gamma; & Q9D517 & Agpat3 & $-1,2$ & 9,8 \\
\hline 2,4-dienoyl-CoA reductase, mitochondrial; & Q9CQ62 & Decr1 & $-0,4$ & 34 \\
\hline $26 \mathrm{~S}$ protease regulatory subunit $10 \mathrm{~B}$; & P62334 & Psmc6 & $-0,3$ & 41,6 \\
\hline $26 \mathrm{~S}$ protease regulatory subunit 4 & P62192 & Psmc1 & $-0,1$ & 34,5 \\
\hline $26 \mathrm{~S}$ protease regulatory subunit $6 \mathrm{~A}$; & 088685 & Psmc3 & $-0,4$ & 38,8 \\
\hline $26 \mathrm{~S}$ protease regulatory subunit $6 \mathrm{~B}$; & P54775 & Psmc4 & $-0,4$ & 21,3 \\
\hline $26 S$ protease regulatory subunit 7 & P46471 & Psmc2 & $-0,5$ & 40,2 \\
\hline $26 S$ protease regulatory subunit 8 & P62196 & Psmc5 & $-0,5$ & 31,3 \\
\hline $26 \mathrm{~S}$ proteasome non-ATPase regulatory subun it $1 ;$ & Q3TXS7 & Psmd1 & $-0,5$ & 26,9 \\
\hline $26 \mathrm{~S}$ proteasome non-ATPase regulatory subun it $11 ;$ & Q8BG32 & Psmd11 & $-0,4$ & 38,9 \\
\hline $26 \mathrm{~S}$ proteasome non-ATPase regulatory subun it $12 ;$ & Q9D8W5 & Psmd12 & $-0,4$ & 13,8 \\
\hline $26 \mathrm{~S}$ proteasome non-ATPase regulatory subun it 13 ; & Q9WVJ2 & Psmd13 & $-0,5$ & 43,4 \\
\hline $26 \mathrm{~S}$ proteasome non-ATPase regulatory subun it $14 ;$ & O35593 & Psmd14 & $-0,4$ & 13,9 \\
\hline $26 \mathrm{~S}$ proteasome non-ATPase regulatory subun it 2; & Q8VDM4 & Psmd2 & $-0,4$ & 29,5 \\
\hline $26 \mathrm{~S}$ proteasome non-ATPase regulatory subun it 3 ; & P14685 & Psmd3 & $-0,9$ & 34,2 \\
\hline $26 \mathrm{~S}$ proteasome non-ATPase regulatory subun it 4 ; & 035226 & Psmd4 & $-0,5$ & 10,6 \\
\hline $26 \mathrm{~S}$ proteasome non-ATPase regulatory subun it 4; & F7AI87 & Psmd4 & 0,4 & 4,1 \\
\hline $26 \mathrm{~S}$ proteasome non-ATPase regulatory subun it 5 ; & Q8BJY1 & Psmd5 & $-0,2$ & 2,6 \\
\hline $26 \mathrm{~S}$ proteasome non-ATPase regulatory subun it 6 ; & Q99J।4 & Psmd6 & $-0,6$ & 30,6 \\
\hline $26 \mathrm{~S}$ proteasome non-ATPase regulatory subun it 7; & P26516 & Psmd7 & $-0,5$ & 31,5 \\
\hline $26 \mathrm{~S}$ proteasome non-ATPase regulatory subun it 8 & Q9CX56 & Psmd8 & $-0,2$ & 24,9 \\
\hline $26 \mathrm{~S}$ proteasome non-ATPase regulatory subun it 9; & Q9CR00 & Psmd9 & 0,3 & 22,1 \\
\hline 28 kDa heat- and acid-stable phosphoprotein; & Q3UHX2 & Pdap1 & $-0,1$ & 41,4 \\
\hline $28 \mathrm{~S}$ ribosomal protein $\mathrm{S} 36$, mitochondrial; & Q9CQX8 & Mrps36 & 0,1 & 56,9 \\
\hline 2-amino-3-ketobutyrate coenzyme A ligase, mitochondrial; & 088986 & Gcat & $-0,3$ & 19,9 \\
\hline 2-aminoethanethiol dioxygenase; & Q6PDY2 & Ado & $-0,1$ & 16,4 \\
\hline 2'-deoxynucleoside 5'-phosphate N-hydrolase 1; & Q80VJ3 & Dnph1 & $-0,2$ & 26,6 \\
\hline 2-oxoglutarate and iron-dependent oxygenase domain-containing protein 3; & Q9D136 & Ogfod3 & 0,3 & 7,9 \\
\hline $3^{\prime}\left(2^{\prime}\right), 5^{\prime}$-bisphosphate nucleotidase 1 ; & Q9Z0S1 & Bpnt1 & 0,2 & 30 \\
\hline $39 S$ ribosomal protein L12, mitochondrial; & Q9DB15 & Mrpl12 & $-0,1$ & 7 \\
\hline 3-hydroxyacyl-CoA dehydrogenase type-2; & A2AFQ2 & Hsd17b10 & $-0,1$ & 55,9 \\
\hline 3-hydroxyisobutyrate dehydrogenase, mitochondrial; & Q99L13 & Hibadh & 0,0 & 22,1 \\
\hline 3-ketoacyl-CoA thiolase A, peroxisomal; & Q921H8 & Acaa1a & $-0,1$ & 36,1 \\
\hline 3-ketoacyl-CoA thiolase, mitochondrial; & Q8BWT1 & Acaa2 & 0,1 & 45,8 \\
\hline 40 S ribosomal protein S10; & P63325 & Rps10 & $-0,1$ & 37,6 \\
\hline $40 S$ ribosomal protein S11; & P62281 & Rps11 & $-0,3$ & 50 \\
\hline $40 \mathrm{~S}$ ribosomal protein $\mathrm{S} 12$ & Q6ZWZ6 & Rps12 & $-0,1$ & 53 \\
\hline 40 S ribosomal protein S13; & P62301 & Rps13 & $-0,3$ & 38,4 \\
\hline 40 S ribosomal protein S14; & P62264 & Rps14 & $-0,5$ & 22,5 \\
\hline 40 S ribosomal protein S15; & P62843 & Rps15 & $-0,3$ & 10,2 \\
\hline $40 \mathrm{~S}$ ribosomal protein $\mathrm{S} 15 \mathrm{a}$; & P62245 & Rps15a & $-0,5$ & 42,6 \\
\hline 40S ribosomal protein S16; & P14131 & Rps16 & $-0,2$ & 36,3 \\
\hline 40 S ribosomal protein S17; & P63276 & Rps17 & $-0,2$ & 15,6 \\
\hline 40 S ribosomal protein S18; & P62270 & Rps18 & $-0,1$ & 37,5 \\
\hline 40S ribosomal protein S19; & Q9CZX8 & Rps19 & $-0,2$ & 37,9 \\
\hline $40 S$ ribosomal protein $\mathrm{S} 2$ & P25444 & Rps2 & $-0,4$ & 40,6 \\
\hline 40 S ribosomal protein S20; & P60867 & Rps20 & $-0,4$ & 25,2 \\
\hline $40 S$ ribosomal protein $\mathrm{S} 21$ & Q9CQR2 & Rps21 & $-0,2$ & 39,8 \\
\hline 40 S ribosomal protein S23; & P62267 & Rps23 & $-1,3$ & 15,4 \\
\hline 40 S ribosomal protein S24; & P62849 & Rps24 & $-1,3$ & 19,5 \\
\hline 40 S ribosomal protein S25; & P62852 & Rps25 & $-0,1$ & 22,4 \\
\hline 40 S ribosomal protein S26; & P62855 & Rps26 & $-0,2$ & 31,3 \\
\hline 40 S ribosomal protein S27; & Q6ZWU9 & Rps27 & $-0,3$ & 38,1 \\
\hline 40 S ribosomal protein S27-like; & Q6ZWY3 & Rps27I & $-0,3$ & 38,1 \\
\hline
\end{tabular}




\begin{tabular}{|c|c|c|c|c|}
\hline Protein & Uniprot & Gene name & $\begin{array}{l}\text { Fold change of } \\
\text { XPRESS ratio } \\
\left(\text { Egfr }^{\triangle K C} / \mathrm{WT}\right)\end{array}$ & $\begin{array}{c}\text { Sequence } \\
\text { coverage [\%] }\end{array}$ \\
\hline $40 S$ ribosomal protein S28; & P62858 & Rps28 & $-0,1$ & 37,5 \\
\hline $40 S$ ribosomal protein S29; & P62274 & Rps29 & $-0,3$ & 32,1 \\
\hline 40 S ribosomal protein $\mathrm{S} 3$ & P62908 & Rps3 & $-0,4$ & 72,8 \\
\hline 40 S ribosomal protein $\mathrm{S} 30$ & P62862 & Fau & $-1,0$ & 16,9 \\
\hline $40 S$ ribosomal protein S3a; & P97351 & Rps3a & $-0,2$ & 36 \\
\hline $40 \mathrm{~S}$ ribosomal protein $\mathrm{S} 4, \mathrm{X}$ isoform; & P62702 & $\operatorname{Rps} 4 x$ & $-0,1$ & 55,5 \\
\hline 40 S ribosomal protein S5; & D3YYM6 & Rps5 & $-0,1$ & 30,2 \\
\hline 40 S ribosomal protein S6; & P62754 & Rps6 & $-0,3$ & 18,9 \\
\hline 40 S ribosomal protein S7; & P62082 & Rps7 & $-0,4$ & 30,7 \\
\hline 40 S ribosomal protein $\mathrm{S} 8$ & P62242 & Rps8 & $-0,3$ & 51 \\
\hline 40 S ribosomal protein S9; & Q6ZWN5 & Rps9 & $-0,6$ & 37,6 \\
\hline $40 \mathrm{~S}$ ribosomal protein $\mathrm{SA}$ & P14206 & Rpsa & $-0,1$ & 42 \\
\hline 45 kDa calcium-binding protein; & Q61112 & Sdf4 & 0,1 & 8,6 \\
\hline 4F2 cell-surface antigen heavy chain; & P10852 & Slc3a2 & $-1,1$ & 22,4 \\
\hline 4-trimethylaminobutyraldehyde dehydrogenase; & Q9JLJ2 & Aldh9a1 & 0,6 & 32,8 \\
\hline 5'(3')-deoxyribonucleotidase, cytosolic type; & Q9JM14 & $\mathrm{Nt5c}$ & 0,2 & 11 \\
\hline 5,6-dihydroxyindole-2-carboxylic acid oxidase; & P07147 & Tyrp1 & 1,2 & 4,3 \\
\hline 5'-3' exoribonuclease 2 & Q9DBR1 & Xrn2 & 0,3 & 15,7 \\
\hline 5-hydroxyisourate hydrolase; & Q9CRB3 & Urah & $-0,1$ & 37,3 \\
\hline 5'-nucleotidase domain containing 2; Protein $\mathrm{Nt5dc2;}$ & Q91X76 & $\mathrm{Nt5} \mathrm{dc} 2$ & 0,9 & 4,9 \\
\hline 5'-nucleotidase; & Q61503 & $\mathrm{Nt5e}$ & $-0,7$ & 24,3 \\
\hline 5-oxoprolinase; & Q8K010 & Oplah & $-1,5$ & 5,4 \\
\hline 6.8 kDa mitochondrial proteolipid; & P56379 & Mp68 & $-0,2$ & 13,8 \\
\hline 60 kDa heat shock protein, mitochondrial; & P63038 & Hspd1 & $-0,1$ & 36 \\
\hline 60 kDa SS-A/Ro ribonucleoprotein; & 008848 & Trove2 & 0,4 & 7,4 \\
\hline $60 \mathrm{~S}$ acidic ribosomal protein $\mathrm{PO}$ & P14869 & Rplp0 & $-0,5$ & 42,9 \\
\hline $60 \mathrm{~S}$ acidic ribosomal protein $\mathrm{P} 1$; & P47955 & Rplp1 & $-0,3$ & 14 \\
\hline $60 \mathrm{~S}$ acidic ribosomal protein $\mathrm{P} 2$ & P99027 & Rplp2 & $-0,4$ & 38,3 \\
\hline $60 S$ ribosomal protein L10; & Q6ZWV3 & Rpl10 & $-0,4$ & 39,8 \\
\hline $60 S$ ribosomal protein L11; & Q9cxW4 & Rpl11 & $-0,1$ & 17,2 \\
\hline $60 S$ ribosomal protein L12; & P35979 & Rpl12 & $-0,4$ & 54,5 \\
\hline $60 S$ ribosomal protein L13; & P47963 & Rpl13 & $-0,2$ & 27 \\
\hline $60 \mathrm{~S}$ ribosomal protein L13a; & P19253 & Rpl13a & $-0,5$ & 28,1 \\
\hline $60 S$ ribosomal protein L14; & Q9CR57 & Rpl14 & $-0,5$ & 20,3 \\
\hline $60 S$ ribosomal protein L15; & Q9CZM2 & Rpl15 & $-0,4$ & 30,4 \\
\hline $60 S$ ribosomal protein L17; & Q9CPR4 & Rpl17 & $-0,2$ & 31,5 \\
\hline $60 S$ ribosomal protein L18; & P35980 & Rpl18 & $-0,4$ & 33,5 \\
\hline $60 \mathrm{~S}$ ribosomal protein L18a; & P62717 & Rpl18a & $-0,6$ & 30,1 \\
\hline 60S ribosomal protein L19; & P84099 & Rpl19 & $-0,1$ & 22,2 \\
\hline $60 S$ ribosomal protein $\mathrm{L} 21$ & 009167 & Rpl21 & $-0,1$ & 25,6 \\
\hline $60 S$ ribosomal protein L22; & P67984 & Rpl22 & $-0,1$ & 18,8 \\
\hline $60 \mathrm{~S}$ ribosomal protein L22-like 1 & Q9D7S7 & Rpl22I1 & $-0,3$ & 9,8 \\
\hline $60 S$ ribosomal protein $\mathrm{L} 23$; & P62830 & Rpl23 & $-0,4$ & 37,9 \\
\hline $60 S$ ribosomal protein L24; & Q8BP67 & Rpl24 & $-0,3$ & 36,1 \\
\hline $60 S$ ribosomal protein $\mathrm{L} 26$ & P61255 & Rpl26 & $-0,6$ & 36,9 \\
\hline $60 S$ ribosomal protein $\mathrm{L} 27$; & P61358 & Rpl27 & $-0,5$ & 36 \\
\hline $60 S$ ribosomal protein $\mathrm{L} 27 \mathrm{a}$ & P14115 & Rpl27a & $-0,3$ & 27,7 \\
\hline 605 ribosomal protein L28; & P41105 & Rpl28 & 0,0 & 32,1 \\
\hline $60 S$ ribosomal protein $\mathrm{L} 29$; & P47915 & Rpl29 & $-0,1$ & 12,3 \\
\hline $60 \mathrm{~S}$ ribosomal protein $\mathrm{L} 3$; & P27659 & $\mathrm{Rpl3}$ & $-0,6$ & 29,8 \\
\hline $60 \mathrm{~S}$ ribosomal protein L30; & P62889 & Rpl30 & $-0,4$ & 57,4 \\
\hline $60 S$ ribosomal protein L31; & P62900 & Rpl31 & $-0,3$ & 31,2 \\
\hline $60 S$ ribosomal protein L32; & P62911 & Rpl32 & $-0,3$ & 35,6 \\
\hline $60 S$ ribosomal protein L34; & Q9D1R9 & Rpl34 & 0,0 & 12,8 \\
\hline $60 \mathrm{~S}$ ribosomal protein $\mathrm{L} 35$ & Q6ZWV7 & Rpl35 & $-0,1$ & 21,1 \\
\hline $60 \mathrm{~S}$ ribosomal protein $\mathrm{L} 35 \mathrm{a}$; & 055142 & Rpl35a & $-0,6$ & 20,9 \\
\hline $60 \mathrm{~S}$ ribosomal protein $\mathrm{L} 36 \mathrm{a}$ & P83882 & Rpl36a & 0,5 & 28,3 \\
\hline $60 \mathrm{~S}$ ribosomal protein $\mathrm{L} 37 \mathrm{a}$ & P61514 & Rpl37a & $-0,3$ & 41,3 \\
\hline $60 S$ ribosomal protein L38; & Q9Jग18 & Rpl38 & $-0,3$ & 38,6 \\
\hline $60 S$ ribosomal protein L39; & P62892 & Rpl39 & $-0,1$ & 19,6 \\
\hline $60 S$ ribosomal protein $\mathrm{L} 4$; & Q9D8E6 & Rpl4 & $-0,5$ & 36,3 \\
\hline $60 \mathrm{~S}$ ribosomal protein L5; & P47962 & Rpl5 & $-0,1$ & 34 \\
\hline 60 S ribosomal protein L6; & P47911 & Rpl6 & $-0,4$ & 38,5 \\
\hline $60 S$ ribosomal protein L7; & P14148 & Rpl7 & $-0,5$ & 24,1 \\
\hline $60 S$ ribosomal protein $\mathrm{L} 8$ & P62918 & Rpl8 & $-0,2$ & 38,5 \\
\hline $60 S$ ribosomal protein L9; & P51410 & Rpl9 & $-0,1$ & 38 \\
\hline 6-phosphogluconate dehydrogenase, decarboxylating; & Q9DCD0 & Pgd & $-0,3$ & 32,5 \\
\hline 6-phosphogluconolactonase; & Q9CQ60 & Pgls & 0,4 & 30 \\
\hline 6-pyruvoyl tetrahydrobiopterin synthase; & Q9R1Z7 & Pts & 0,0 & 18,1 \\
\hline 78 kDa glucose-regulated protein; & P20029 & Hspa5 & $-0,2$ & 43,1 \\
\hline 7-dehydrocholesterol reductase; & 088455 & Dhcr7 & $-1,1$ & 2,8 \\
\hline
\end{tabular}




\begin{tabular}{|c|c|c|c|c|}
\hline Protein & Uniprot & Gene name & $\begin{array}{l}\text { Fold change of } \\
\text { XPRESS ratio } \\
\left(\text { Egfr }^{\Delta K C} / \mathrm{WT}\right)\end{array}$ & $\begin{array}{c}\text { Sequence } \\
\text { coverage [\%] }\end{array}$ \\
\hline 7-methylguanosine phosphate-specific 5'-nucleotidase; & Q3UFY7 & $\mathrm{Nt5} 53 \mathrm{~b}$ & 0,3 & 4,7 \\
\hline Acetolactate synthase-like protein; & Q8BU33 & Ilvbl & $-0,4$ & 9,8 \\
\hline Acetyl-CoA acetyltransferase, cytosolic; & Q8CAY6 & Acat2 & 0,1 & 30,5 \\
\hline Acetyl-CoA acetyltransferase, mitochondrial; & Q8QZT1 & Acat1 & 0,4 & 29 \\
\hline Acid ceramidase; & Q9WV54 & Asah1 & $-0,2$ & 22,3 \\
\hline Acid sphingomyelinase-like phosphodiesterase 3a; & P70158 & Smpdl3a & 0,0 & 4,9 \\
\hline Acid sphingomyelinase-like phosphodiesterase 3b; & P58242 & Smpdl3b & $-0,9$ & 4,6 \\
\hline Acid trehalase-like protein 1 & Q8BP56 & Athl1 & $-0,5$ & 4,1 \\
\hline Acidic leucine-rich nuclear phosphoprotein 32 family member $A$; & 035381 & Anp32a & $-0,2$ & 16,2 \\
\hline Acidic leucine-rich nuclear phosphoprotein 32 family member B; & Q9EST5 & Anp32b & 0,3 & 14,3 \\
\hline Actin, alpha cardiac muscle 1 & P68033 & Actc1 & $-0,3$ & 48 \\
\hline Actin, aortic smooth muscle; & P62737 & Acta2 & $-0,6$ & 48 \\
\hline Actin-like protein $6 \mathrm{~A}$; & Q9Z2N8 & Actl6a & $-0,6$ & 15,7 \\
\hline Actin-related protein 10; & Q9QZB7 & Actr10 & $-0,9$ & 18,9 \\
\hline Actin-related protein $2 / 3$ complex subun it $1 \mathrm{~A}$; & Q9R0Q6 & Arpc1a & 0,0 & 32,4 \\
\hline Actin-related protein $2 / 3$ complex subun it $1 \mathrm{~B}$; & Q9WV32 & Arpc1b & 0,0 & 32,3 \\
\hline Actin-related protein $2 / 3$ complex subun it 2 & Q9CVB6 & Arpc2 & $-0,1$ & 35 \\
\hline Actin-related protein $2 / 3$ complex subun it 3 ; & Q9JM76 & Arpc3 & 0,0 & 21,2 \\
\hline Actin-related protein $2 / 3$ complex subun it 4 & P59999 & Arpc4 & 0,2 & 22,6 \\
\hline Actin-related protein $2 / 3$ complex subun it 5 & Q9CPW4 & Arpc5 & $-0,3$ & 35,8 \\
\hline Actin-related protein $2 / 3$ complex subun it 5 -like protein; & Q9D898 & Arpc5I & $-0,1$ & 28,8 \\
\hline Actin-related protein 2 & P61161 & Actr2 & 0,0 & 26,9 \\
\hline Actin-related protein 3; & Q99JY9 & Actr3 & 0,1 & 50,7 \\
\hline Activated RNA polymerase II transcriptional coactivator $\mathrm{p} 15$; & P11031 & Sub1 & 0,1 & 26 \\
\hline Activator of $90 \mathrm{kDa}$ heat shock protein ATPase homolog 1 ; & Q8BK64 & Ahsa1 & $-1,2$ & 3,6 \\
\hline Acyl carrier protein, mitochondrial; & Q9CR21 & Ndufab1 & $-0,1$ & 18,1 \\
\hline Acylamino-acid-releasing enzyme; & Q8R146 & Apeh & 0,1 & 18 \\
\hline Acyl-CoA dehydrogenase family member $10 ;$ & Q8K370 & Acad10 & $-0,1$ & 9,5 \\
\hline Acyl-CoA dehydrogenase family member 9 , mitochondrial; & Q8JZN5 & Acad9 & $-0,1$ & 32,2 \\
\hline Acyl-CoA-binding protein; & P31786 & Dbi & 0,1 & 39,1 \\
\hline Acyl-coenzyme A thioesterase 1 ; & 055137 & Acot1 & $-0,3$ & 5,5 \\
\hline Acyl-coenzyme A thioesterase 13 & Q9CQR4 & Acot13 & $-0,6$ & 15,7 \\
\hline Acyl-coenzyme A thioesterase 9, mitochondrial; & Q9R0X4 & Acot9 & 0,3 & 12,3 \\
\hline Acyl-protein thioesterase 1 & P97823 & Lypla1 & 0,8 & 14,5 \\
\hline Acyl-protein thioesterase 2 & Q9WTL7 & Lypla2 & $-0,2$ & 15,6 \\
\hline Acylpyruvase FAHD1, mitochondrial; & Q8R0F8 & Fahd1 & 0,4 & 19,8 \\
\hline Adenine phosphoribosyltransferase; & P08030 & Aprt & 0,1 & 12,8 \\
\hline Adenosine deaminase; & P03958 & Ada & $-0,1$ & 12,5 \\
\hline Adenosine kinase; & P55264 & Adk & 0,7 & 38,8 \\
\hline Adenosylhomocysteinase; & P50247 & Ahcy & 0,3 & 51,2 \\
\hline Adenylate kinase 2, mitochondrial; & Q9WTP6 & Ak2 & 0,3 & 38,1 \\
\hline Adenylate kinase 4, mitochondrial; & Q9WUR9 & Ak4 & $-1,7$ & 10,4 \\
\hline Adenylosuccinate lyase; & P54822 & Adsl & 0,0 & 25 \\
\hline Adenylosuccinate synthetase isozyme 1 ; & P28650 & Adssl1 & $-2,0$ & 5,7 \\
\hline Adenylosuccinate synthetase isozyme 2 ; & P46664 & Adss & $-0,3$ & 21,7 \\
\hline Adenylyl cyclase-associated protein 1 ; & P40124 & Cap1 & $-0,5$ & 13,3 \\
\hline Adipocyte enhancer-binding protein 1 & Q640N1 & Aebp1 & $-0,1$ & 2,4 \\
\hline Adipocyte plasma membrane-associated protein; & Q9D7N9 & Apmap & 0,3 & 12 \\
\hline Adiponectin; & Q60994 & Adipoq & 0,5 & 18,6 \\
\hline ADP/ATP translocase $1 ;$ & P48962 & Slc25a4 & 0,1 & 45,6 \\
\hline ADP/ATP translocase 2 & P51881 & Slc25a5 & $-0,3$ & 38,6 \\
\hline ADP-dependent glucokinase; & Q8VDL4 & Adpgk & 0,2 & 13,7 \\
\hline ADP-ribosylation factor 3 & P61205 & Arf3 & 0,2 & 45,9 \\
\hline ADP-ribosylation factor 4 & P61750 & Arf4 & 0,6 & 41,7 \\
\hline ADP-ribosylation factor 5 & P84084 & Arf5 & 0,2 & 47,8 \\
\hline ADP-ribosylation factor 6 & P62331 & Arf6 & $-0,1$ & 32,6 \\
\hline ADP-ribosylation factor-like protein 1 ; & P61211 & Arl1 & $-0,1$ & 23,9 \\
\hline ADP-ribosylation factor-like protein 3 ; & Q9WUL7 & Arl3 & 0,4 & 53,3 \\
\hline ADP-ribosylation factor-like protein 6 -interacting protein 1 ; & Q9JKW0 & Arl6ip1 & 0,1 & 4,9 \\
\hline ADP-ribosylation factor-like protein 6 -interacting protein 4 & Q9JM93 & Arl6ip4 & 0,3 & 8 \\
\hline ADP-ribosylation factor-like protein $8 \mathrm{~A}$; & Q8VEH3 & Arl8a & $-0,1$ & 30,3 \\
\hline ADP-ribosylation factor-like protein $8 \mathrm{~B}$; & Q9CQW2 & Arl8b & $-0,1$ & 22 \\
\hline ADP-sugar pyrophosphatase; & Q9JKX6 & Nudt5 & 0,4 & 27,1 \\
\hline Afadin; & Q9QZQ1 & Mllt4 & $-0,6$ & 4,9 \\
\hline Afamin; & 089020 & Afm & 0,4 & 3,6 \\
\hline Agrin; & MOQWP1 & Agrn & $-1,4$ & 7,6 \\
\hline A-kinase anchor protein 12 & Q9WTQ5 & Akap12 & 1,4 & 12,2 \\
\hline A-kinase anchor protein 2; & 054931 & Akap2 & 0,7 & 6,2 \\
\hline Alanine aminotransferase 1 & Q8QZR5 & Gpt & $-0,8$ & 23,4 \\
\hline Alanine--tRNA ligase, cytoplasmic; & Q8BGQ7 & Aars & $-0,3$ & 1,2 \\
\hline Alcohol dehydrogenase $[\operatorname{NADP}(+)]$; & Q9JII6 & Akr1a1 & 0,6 & 11,7 \\
\hline
\end{tabular}




\begin{tabular}{|c|c|c|c|c|}
\hline Protein & Uniprot & Gene name & $\begin{array}{l}\text { Fold change of } \\
\text { XPRESS ratio } \\
\left(\text { Egfr }^{\Delta K C} / \mathrm{WT}\right) \\
\end{array}$ & $\begin{array}{c}\text { Sequence } \\
\text { coverage [\%] }\end{array}$ \\
\hline Alcohol dehydrogenase 1; & P00329 & Adh1 & 0,1 & 12,5 \\
\hline Alcohol dehydrogenase class 4 mu/sigma chain; & Q64437 & Adh7 & $-0,7$ & 30,2 \\
\hline Alcohol dehydrogenase class-3; & P28474 & Adh5 & 0,4 & 21,1 \\
\hline Aldehyde dehydrogenase family 1 member A3; & Q9JHW9 & Aldh1a3 & 1,1 & 19,7 \\
\hline Aldehyde dehydrogenase family 16 member $\mathrm{A} 1$; & Q57119 & Aldh16a1 & 0,0 & 5,2 \\
\hline Aldehyde dehydrogenase, cytosolic 1 & 035945 & Aldh1a7 & 1,0 & 6,4 \\
\hline Aldehyde dehydrogenase, dimeric NADP-preferring; & P47739 & Aldh3a1 & $-0,3$ & 18,1 \\
\hline Aldehyde dehydrogenase, mitochondrial; & P47738 & Aldh2 & $-0,1$ & 3,5 \\
\hline Aldehyde dehydrogenase; & E9Q3E1 & Aldh $3 b 2$ & $-0,2$ & 20,9 \\
\hline Aldehyde oxidase 4 & Q3TYQ9 & Aox4 & $-1,5$ & 7,6 \\
\hline Aldo-keto reductase family 1 member $\mathrm{C} 18$; & Q8K023 & Akr1c18 & 6,0 & 5,9 \\
\hline Aldose reductase; & P45376 & Akr1b1 & 0,3 & 32 \\
\hline $\begin{array}{l}\text { Alpha globin 1; Alpha globin 2; Alpha-globin; Hemoglobin alpha, adult chain 1; } \\
\text { Hemoglobin subunit alpha; Protein Hba-a2; }\end{array}$ & Q91VB8 & raemaglobin alpha & 1,0 & 66,9 \\
\hline Alpha/beta hydrolase domain-containing protein 14B; & E9QN99 & Abhd14b & 1,1 & 24,8 \\
\hline Alpha-1-antitrypsin 1-1; & P07758 & Serpina1a & 0,7 & 31,1 \\
\hline Alpha-1-antitrypsin 1-2; & P22599 & Serpina1b & 0,2 & 29,8 \\
\hline Alpha-1-antitrypsin 1-4; & Q00897 & Serpina1d & 1,1 & 28,3 \\
\hline Alpha-2-antiplasmin; & Q61247 & Serpinf2 & $-1,7$ & 12,1 \\
\hline Alpha-2-HS-glycoprotein; & P29699 & Ahsg & 0,8 & 30,1 \\
\hline Alpha-2-macroglobulin receptor-associated protein; & P55302 & Lrpap1 & 0,0 & 20,8 \\
\hline Alpha-2-macroglobulin; & Q61838 & $\mathrm{A} 2 \mathrm{~m}$ & 1,2 & 21,4 \\
\hline Alpha-actinin-1; & Q7TPR4 & Actn1 & $-0,7$ & 45,8 \\
\hline Alpha-actinin-4; & P57780 & Actn4 & $-0,9$ & 52 \\
\hline Alpha-adducin; & Q9QYCo & Add1 & 1,1 & 25,3 \\
\hline Alpha-aminoadipic semialdehyde dehydrogenase; & Q9DBF1 & Aldh7a1 & 0,6 & 27,1 \\
\hline Alpha-centractin; & P61164 & Actr1a & 0,1 & 26,1 \\
\hline Alpha-crystallin B chain; & P23927 & Cryab & $-0,5$ & 4,6 \\
\hline Alpha-endosulfine; & P60840 & Ensa & $-0,5$ & 11,6 \\
\hline Alpha-enolase; & P17182 & Eno1 & 0,3 & 35,9 \\
\hline Alpha-fetoprotein; & P02772 & Afp & 1,2 & 49,3 \\
\hline Alpha-galactosidase A; & P51569 & Gla & $-0,7$ & 8,1 \\
\hline Alpha-ketoglutarate-depen dent dioxygenase FTO; & Q8BGW1 & Fto & 0,5 & 12,5 \\
\hline Alpha-mannosidase 2 & P27046 & Man2a1 & 0,0 & 5,8 \\
\hline Alpha-N-acetylgalactosaminidase; & Q9QWR8 & Naga & $-0,3$ & 17,6 \\
\hline $\begin{array}{l}\text { Alpha-N-acetylglucosaminidase; Alpha-N-acetylglucosaminidase (Sanfilippo } \\
\text { disease IIIB); Alpha-N-acetylglucosaminidase (Sanfilippo disease IIIB), isoform } \\
\text { CRA_b; N-acetyl-glucosaminidase; Protein Naglu; }\end{array}$ & 088325 & Naglu & 0,8 & 2,8 \\
\hline Alpha-soluble NSF attachment protein; & Q9DB05 & Napa & $-0,4$ & 13,9 \\
\hline Alpha-synuclein; & 055042 & Snca & 1,2 & 20,7 \\
\hline Alpha-taxilin; & Q6PAM1 & Txlna & 0,0 & 1,8 \\
\hline Amidophosphoribosyltransferase; & Q8ClH9 & Ppat & 1,2 & 7 \\
\hline Amine oxidase [flavin-containing] A; & Q64133 & Maoa & 0,1 & 24,5 \\
\hline Amine oxidase [flavin-containing] B; & Q8BW75 & Maob & 0,3 & 2,1 \\
\hline Aminoacyl tRNA synthase complex-interacting multifunctional protein $1 ;$ & P31230 & Aimp1 & $-0,6$ & 10,3 \\
\hline Aminoacylase-1; & Q99JW2 & Acy1 & 0,3 & 11 \\
\hline Aminopeptidase $\mathrm{N}$; & P97449 & Anpep & $-0,1$ & 21,2 \\
\hline AMP deaminase 3; & 008739 & Ampd3 & $-0,6$ & 1,7 \\
\hline Amyloid beta A4 protein; & P12023 & App & $-0,6$ & 2,9 \\
\hline Amyloid-like protein 2; Amyloid-like protein 2, isoform 751; & Q60709 & Aplp2 & $-0,5$ & 1,3 \\
\hline AN1-type zinc finger protein 2B; & Q91X58 & Zfand $2 b$ & $-0,1$ & 3,2 \\
\hline AN1-type zinc finger protein 6 & Q9DCH6 & Zfand6 & $-0,5$ & 5,4 \\
\hline Anamorsin; & Q8WTY4 & Ciapin1 & $-0,1$ & 15,9 \\
\hline Angiotensin-converting enzyme 2 ; & Q8R0I0 & Ace2 & $-1,4$ & 6,2 \\
\hline Angiotensin-converting enzyme; & P09470 & Ace & 0,7 & 2,5 \\
\hline Angiotensinogen; & P11859 & Agt & 0,7 & 4,8 \\
\hline Annexin A1; & P10107 & Anxa1 & 0,3 & 47,7 \\
\hline Annexin A11; & P97384 & Anxa11 & $-0,4$ & 4,2 \\
\hline Annexin A2; & P07356 & Anxa2 & $-0,4$ & 59,3 \\
\hline Annexin A3; & 035639 & Anxa3 & $-0,3$ & 14,2 \\
\hline Annexin A4; & P97429 & Anxa4 & 0,0 & 28,8 \\
\hline Annexin A5; & P48036 & Anxa5 & $-0,1$ & 35,1 \\
\hline Annexin A6; & P14824 & Anxa6 & $-0,6$ & 36,6 \\
\hline Annexin A7; & Q07076 & Anxa7 & 0,0 & 5,4 \\
\hline Annexin A8; & 035640 & Anxa8 & $-0,5$ & 32,7 \\
\hline Antigen-presenting glycoprotein CD1d1; & P11609 & $\mathrm{Cd} 1 \mathrm{~d} 1$ & $-0,2$ & 2,1 \\
\hline Antithrombin-III; & P32261 & Serpinc1 & 0,2 & 7,3 \\
\hline AP-1 complex subunit beta-1; & 035643 & Ap1b1 & $-0,3$ & 13,2 \\
\hline AP-1 complex subunit mu-1; & P35585 & Ap1m1 & 0,0 & 4,7 \\
\hline AP-2 complex subunit alpha-1; & P17426 & Ap2a1 & $-0,5$ & 8,3 \\
\hline
\end{tabular}




\begin{tabular}{|c|c|c|c|c|}
\hline Protein & Uniprot & Gene name & $\begin{array}{l}\text { Fold change of } \\
\text { XPRESS ratio } \\
\left(E g f r^{\triangle K C} / \mathrm{WT}\right)\end{array}$ & $\begin{array}{c}\text { Sequence } \\
\text { coverage [\%] }\end{array}$ \\
\hline AP-2 complex subunit alpha- 2 ; & P17427 & Ap2a2 & 0,4 & 9,1 \\
\hline AP-2 complex subunit beta; & Q9DBG3 & Ap2b1 & 0,5 & 9,4 \\
\hline AP-2 complex subunit mu; & P84091 & Ap2m1 & 0,0 & 13,4 \\
\hline AP-2 complex subunit sigma; & P62743 & Ap2s1 & $-0,4$ & 4,9 \\
\hline Apolipoprotein A-I; & Q00623 & Apoa1 & 0,5 & 38,6 \\
\hline Apolipoprotein A-II; & P09813 & Apoa2 & 0,5 & 9,8 \\
\hline Apolipoprotein A-IV; & P06728 & Apoa4 & 0,2 & 53,7 \\
\hline Apolipoprotein B-100; & E9Q414 & Apob & 0,9 & 1,1 \\
\hline Apolipoprotein C-I; & P34928 & Apoc1 & $-1,9$ & 20,5 \\
\hline Apolipoprotein C-III; & P33622 & Apoc3 & 0,4 & 19,2 \\
\hline Apolipoprotein D; & P51910 & Apod & 0,3 & 6,3 \\
\hline Apolipoprotein E; & P08226 & Apoe & $-2,3$ & 28,6 \\
\hline Apolipoprotein O; & Q9DCZ4 & Apoo & 0,2 & 20,1 \\
\hline Apolipoprotein O-like; & Q78IK4 & Apool & 0,6 & 37,7 \\
\hline Apoptosis inhibitor 5; & 035841 & Api5 & 0,3 & 6,2 \\
\hline Apoptosis regulator BAX; & Q07813 & Bax & 0,7 & 29,7 \\
\hline Apoptosis-associated speck-like protein containing a CARD; & Q9EPB4 & Pycard & $-0,9$ & 34,2 \\
\hline Apoptosis-inducing factor 1, mitochondrial; & Q9Z0X1 & Aifm1 & $-0,1$ & 11 \\
\hline Apoptotic chromatin condensation inducer in the nucleus; & Q9JIX8 & Acin1 & 0,6 & 4,2 \\
\hline Aquaporin-1; & Q02013 & Aqp1 & 0,3 & 14,5 \\
\hline Arachidonate 5-lipoxygenase; & P48999 & Alox5 & 1,2 & 6,4 \\
\hline Arachidonate 8S-lipoxygenase; & 035936 & Alox8 & 2,7 & 4,5 \\
\hline $\begin{array}{l}\text { Arf-GAP with Rho-GAP domain, ANK repeat and PH domain-containing protein } \\
1 ;\end{array}$ & Q4LDD4 & Arap1 & 0,8 & 2,1 \\
\hline Arfip1 protein; Protein Arfip1; & A2RSX9 & Arfip1 & 0,0 & 15,5 \\
\hline Arginase-1; & Q61176 & Arg1 & $-0,5$ & 19,8 \\
\hline Arginase-2, mitochondrial; & 008691 & Arg2 & $-0,5$ & 4,2 \\
\hline Arginine and glutamate-rich protein $1 ;$ & Q3UL36 & Arglu1 & 0,2 & 3 \\
\hline Arginine/serine-rich coiled-coil protein 2; & A2RTL5 & Rsrc2 & 0,1 & 7,9 \\
\hline $\begin{array}{l}\text { Arginine-rich, mutated in early stage tumors, isoform CRA_b; Mesencephalic } \\
\text { astrocyte-derived neurotrophic factor; }\end{array}$ & Q3TMX5 & Manf & $-0,1$ & 35,8 \\
\hline Arginine--tRNA ligase, cytoplasmic; & Q9D019 & Rars & $-0,6$ & 4,2 \\
\hline Argininosuccinate lyase; & Q91YI0 & Asl & $-0,6$ & 18,9 \\
\hline Argininosuccinate synthase; & P16460 & Ass1 & $-0,6$ & 12,9 \\
\hline Arylacetamide deacetylase; & Q99PG0 & Aadac & $-1,1$ & 6 \\
\hline Arylsulfatase B; & P50429 & Arsb & 0,5 & 5,1 \\
\hline Asparagine--tRNA ligase, cytoplasmic; & Q8BP47 & Nars & 0,4 & 14,8 \\
\hline Aspartate aminotransferase, cytoplasmic; & P05201 & Got1 & 0,2 & 39,2 \\
\hline Aspartate aminotransferase, mitochondrial; & P05202 & Got2 & 0,1 & 49,3 \\
\hline Aspartate--tRNA ligase, cytoplasmic; & Q922B2 & Dars & 0,0 & 31,9 \\
\hline Aspartyl aminopeptidase; & Q9Z2W0 & Dnpep & 0,1 & 31,5 \\
\hline Aspartyl/asparaginyl beta-hydroxylase; & Q8BSY0 & Asph & 0,4 & 5,2 \\
\hline Asporin; & Q99MQ4 & Aspn & $-0,6$ & 22,5 \\
\hline Ataxin-2-like protein; & Q7TQH0 & Atxn2l & 0,0 & 1 \\
\hline Atlastin-3; & Q91YH5 & Atl3 & $-0,4$ & 6,2 \\
\hline ATP synthase $F(0)$ complex subunit $B 1$, mitochondrial; & Q9CQQ7 & Atp5f1 & $-2,0$ & 14,8 \\
\hline ATP synthase $\mathrm{F}(0)$ complex subunit $C 2$, mitochondrial; & P56383 & Atp5g2 & $-0,8$ & 5,1 \\
\hline ATP synthase protein 8 & P03930 & Mtatp8 & $-0,2$ & 14,9 \\
\hline ATP synthase subunit alpha, mitochond rial; & Q03265 & Atp5a1 & $-0,2$ & 35,8 \\
\hline ATP synthase subunit beta, mitochond rial; & P56480 & Atp5b & $-0,1$ & 43,5 \\
\hline ATP synthase subunit $d$, mitochondrial; & Q9DCX2 & Atp5h & $-0,8$ & 50 \\
\hline ATP synthase subunit delta, mitochond rial; & Q9D3D9 & Atp5d & $-1,2$ & 13,7 \\
\hline ATP synthase subunit e, mitochondrial; & Q06185 & Atp5i & $-0,4$ & 50,7 \\
\hline ATP synthase subunit epsilon, mitochondrial; & P56382 & Atp5e & $-0,2$ & 28,8 \\
\hline ATP synthase subunit $f$, mitochondrial; & P56135 & Atp5j2 & $-0,3$ & 15,8 \\
\hline ATP synthase subunit g, mitochondrial; & Q9CPQ8 & Atp5I & $-0,7$ & 41,7 \\
\hline ATP synthase subunit gamma, mitochondrial; & Q91VR2 & Atp5c1 & $-0,4$ & 42,9 \\
\hline ATP synthase subunit $O$, mitochondrial; & Q9DB20 & Atp5o & $-0,3$ & 54,5 \\
\hline ATP synthase-coupling factor 6 , mitochondrial; & P97450 & Atp5j & $-0,2$ & 41,7 \\
\hline ATPase Asna1; & 054984 & Asna1 & 0,1 & 19 \\
\hline ATP-binding cassette sub-family A member 9; & Q8K449 & Abca9 & 1,7 & 2,3 \\
\hline ATP-binding cassette sub-family E member 1 ; & P61222 & Abce1 & 0,0 & 24,5 \\
\hline ATP-binding cassette sub-family $\mathrm{F}$ member 1 ; & Q6P542 & Abcf1 & $-0,6$ & 1,2 \\
\hline ATP-binding cassette sub-family $\mathrm{G}$ member 2 ; & Q7TMS5 & Abcg2 & 0,2 & 8,7 \\
\hline ATP-citrate synthase; & Q91V92 & Acly & $-0,1$ & 9,5 \\
\hline ATP-dependent (S)-NAD(P)H-hydrate dehydratase; & Q9CZ42 & Carkd & $-0,1$ & 37 \\
\hline ATP-dependent 6-phosphofructokinase, liver type; & P12382 & Pfkl & $-0,3$ & 26,3 \\
\hline ATP-dependent 6-phosphofructokinase, muscle type; & P47857 & Pfkm & 0,3 & 9,5 \\
\hline ATP-dependent 6-phosphofructokinase, platelet type; & Q9WUA3 & Pfkp & $-0,9$ & 20,2 \\
\hline ATP-dependent Clp protease ATP-bin ding subunit clpX-like, mitochondrial; & Q9JHS4 & Clpx & 0,2 & 2,1 \\
\hline
\end{tabular}


Protein

Uniprot Gene name

Fold change of

XPRESS ratio Sequence

ATP-dependent Clp protease proteolytic subunit, mitochondrial;

088696

$\left(\right.$ Egfr $\left.^{\Delta K C} / \mathrm{WT}\right)$

coverage [\%]

ATP-dependent RNA helicase A;

ATP-dependent RNA helicase DDX1;

ATP-dependent RNA helicase DDX19A;

ATP-dependent RNA helicase DDX39A;

E9QNN1

Q91VR5

Q61655

Q8VDW0

ATP-dependent RNA helicase DDX3X;

Q62167

Q97319

Aurora kinase B;

BAG family molecular chaperone regulator 1 ;

E9PVP1

Q60739

BAG family molecular chaperone regulator 3 ;

Q9JLV1

Band 3 anion transport protein;

Band 4.1-like protein 2;

Band 4.1-like protein 3;

Barrier-to-autointegration factor;

Basal cell adhesion molecule;

P04919

070318

Q9WV92

054962

Q9R069

Clpp

Dhx9

Ddx1

Ddx19a

Ddx39a

Ddx3x

Corin

Aim1

Bag1

Bag3

Slc4a1

Epb4112

Epb4113

Banf1

Bcam

$\begin{array}{lc}0,2 & 12,1 \\ 0,5 & 8,3\end{array}$

Basement membrane-specific heparan sulfate proteoglycan core protein;

B1B0C7

Hspg2

$\begin{array}{rr}0,0 & 2,1 \\ -0,3 & 23\end{array}$

$-0,3$

Q91VK1
P18572

Basic leucine zipper and W2 domain-containing protein 2;

BC035947 protein; Protein BC035947;

B2RQY6

Q61335

B-cell receptor-associated protein 31;

$\mathrm{BCL}$ /adenovirus E1B $19 \mathrm{kDa}$ protein-interacting protein 3-like;

$\mathrm{Bcl}$-2/adenovirus E1B $19 \mathrm{kDa}$-interacting protein 2-like protein;

$\mathrm{Bcl}$-2-associated transcription factor 1 ;

Bcl-2-like protein 1;

Bcl-2-like protein 13;

Beta-2-microglobulin;

Beta-actin-like protein 2;

Beta-centractin;

Beta-enolase;

Beta-galactosidase;

Beta-globin; Protein Hbb-bs; Protein Hbb-bt;

Beta-glucuronidase;

Beta-hexosaminidase subunit beta;

Beta-lactamase-like protein 2;

Beta-mannosidase;

Bifunctional glutamate/proline--tRNA ligase;

Bifunctional purine biosynthesis protein $\mathrm{PURH}$;

Bzw2

0,2

$$
\begin{gathered}
\text { Bzw2 } \\
\text { Bsg }
\end{gathered}
$$

BC035947

Q9Z2F7

Q99JU7

Q8K019

Q64373

P59017

P01887

Q8BFZ3

Q8R5C5

P21550

P23780

A8DUK4

P12265

P20060

Q99KR3

Q8K214

Q8CGC7

Q9CWJ9

P28653

Q9CY64

Q8R016

Q9D8S9

Q8BGS2

Q61878

Q8C186

Q91XV3

O35855

Q8K3W0

Q8BXV2

D3Z6Q9

Q3TCJ1

Bcap31

Bnip3l

Bnipl

Bclaf1

Bcl2I1

Bcl2l13

B2m

Actbl2

Actr1b

Eno3

Glb1

Hbbt1

Gusb

Hexb

Lactb2

Manba

Eprs

Atic

Bgn

Blvra

Blmh

Bola1

Bola2

Prg2

Bpifc

Basp1

Bcat2

Bre

Bri3bp

Bin2

Fam175b

4,8

12,1

7,1

2,8

12,4

8,2

34,8

22,2

5,4

4,5

$\begin{array}{cc}-1,4 & 4,5 \\ 0,5 & 23,9\end{array}$

14,3

$-0,2$

$-0,5$

$-0,6$

0,6

$-0,2$

0,4

0,1

$-0,1$

$-0,2$

1,5

0,7

1,0

0,2

$-0,9$

0,6

BRI3-binding protein;

Bridging integrator 2;

BTB/POZ domain-containing adapter for CUL3-mediated RhoA degradation

Q922M3

Kctd10

BTB/POZ domain-containing protein KCTD12;

$\mathrm{BTB} / \mathrm{POZ}$ domain-containing protein KCTD15;

BUB3-interacting and GLEBS motif-containing protein ZNF207;

CAAX prenyl protease 1 homolog;

Cadherin-1;

Cadherin-11;

Cadherin-13;

Cadherin-3;

Cadherin-5;

Calcineurin B homologous protein 1;

Calcineurin subunit B type 1 ;

Calcium-activated chloride channel regulator 2;

Calcium-activated chloride channel regulator 2; Chloride channel calcium

activated 2; Endothelial chloride channel; MCG120735;

Calcium-binding mitochondrial carrier protein Aralar1;

Calcium-binding mitochondrial carrier protein Aralar2;

Calcium-binding mitochondrial carrier protein SCaMC-1;

Calcium-binding protein 39;

Q6WVG3

Q8K0E1

E9PW12

Q80W54

P09803

P55288

Q9WTR5

P10287

P55284

P61022

Q63810

Q8BG22

Q9EQR4

Q8BH59

Q9QXX4

Q8BMD8

Q06138
Kctd12

Kctd15

Znf207

Zmpste24

Cdh1

Cdh11

Cdh13

Cdh3

Cdh5

Chp1

Ppp3r1

Clca2

Clca2

Slc25a12

Slc25a13

Slc25a24

Cab39 


\begin{tabular}{|c|c|c|c|c|}
\hline Protein & Uniprot & Gene name & $\begin{array}{l}\text { Fold change of } \\
\text { XPRESS ratio } \\
\left(\text { Egfr }^{\Delta K C} / \mathrm{WT}\right)\end{array}$ & $\begin{array}{c}\text { Sequence } \\
\text { coverage [\%] }\end{array}$ \\
\hline Calcium-regulated heat stable protein $1 ;$ & Q9CR86 & Carhsp1 & $-0,1$ & 10,8 \\
\hline Calcium-transporting ATPase type $2 \mathrm{C}$ member 2 ; & A7L9Z8 & Atp2c2 & $-0,4$ & 3,2 \\
\hline Calcyclin-binding protein; & Q9CXW3 & Cacybp & $-0,2$ & 17 \\
\hline Calmodulin; & P62204 & Calm3 & $-0,3$ & 31,5 \\
\hline Calmodulin-4; & Q9JM83 & Calm4 & $-0,6$ & 49,3 \\
\hline Calnexin; & P35564 & Canx & $-0,4$ & 18,6 \\
\hline Calpain small subunit 1 & 088456 & Capns1 & $-0,2$ & 21,6 \\
\hline Calpain small subunit 2 & Q9D7J7 & Capns2 & $-0,5$ & 17,8 \\
\hline Calpain-1 catalytic subunit; & 035350 & Capn1 & $-0,6$ & 43,5 \\
\hline Calpain-2 catalytic subunit; & 008529 & Capn2 & 0,7 & 14,3 \\
\hline Calpain-5; & 008688 & Capn5 & $-0,3$ & 4,7 \\
\hline Calpastatin; & P51125 & Cast & $-0,8$ & 30,8 \\
\hline Calreticulin; & P14211 & Calr & $-0,3$ & 29,1 \\
\hline Calsequestrin-1; & 009165 & Casq1 & 2,7 & 8,3 \\
\hline Calsequestrin-2; & 009161 & Casq2 & 1,9 & 4,2 \\
\hline Calumenin; & 035887 & Calu & 0,2 & 33 \\
\hline Calumenin; Calumenin, isoform CRA_b; Cardiac calumenin isoform; & Q6XLQ8 & Calu & $-0,1$ & 27,6 \\
\hline CAP-Gly domain-containing linker protein 1 ; & Q922J3 & Clip1 & $-0,2$ & 12,6 \\
\hline $\begin{array}{l}\text { Capping protein (Actin filament) muscle Z-line, beta, isoform CRA_a; F-actin- } \\
\text { capping protein subunit beta; }\end{array}$ & A2AMWO & Capzb & $-0,1$ & 31,9 \\
\hline $\begin{array}{l}\text { Capping protein (Actin filament), gelsolin-like; Capping protein (Actin } \\
\text { filament), gelsolin-like, isoform CRA_a; Macrophage-capping protein; }\end{array}$ & Q99LB4 & Capg & $-0,1$ & 25,8 \\
\hline Caprin-1; & Q60865 & Caprin1 & $-0,1$ & 11,5 \\
\hline CapZ-interacting protein; & Q3UZA1 & Rcsd1 & 0,0 & 2,9 \\
\hline Carbonic anhydrase 2; & P00920 & $\mathrm{Ca} 2$ & 0,7 & 15 \\
\hline Carbonic anhydrase 3; & P16015 & $\mathrm{Ca} 3$ & 0,1 & 57,3 \\
\hline Carbonic anhydrase 4; & Q64444 & $\mathrm{Ca} 4$ & $-1,2$ & 11,5 \\
\hline Carbonic anhydrase 6; & P18761 & Ca6 & 0,1 & 24,9 \\
\hline Carbonyl reductase [NADPH] 1; & P48758 & Cbr1 & 0,1 & 4,7 \\
\hline Carbonyl reductase [NADPH] 2; & P08074 & Cbr2 & 1,0 & 9,8 \\
\hline Carbonyl reductase family member 4 ; & Q91VT4 & Cbr4 & 1,9 & 16,5 \\
\hline Carboxylesterase 1C; & P23953 & Ces1c & 0,4 & 16,1 \\
\hline Carboxylesterase 1D; & Q8VCT4 & Ces1d & $-0,4$ & 18,6 \\
\hline Carboxylesterase $5 \mathrm{~A}$; & Q6AW46 & Ces5a & $-0,1$ & 6,1 \\
\hline Carboxymethylenebuten olidase homolog; & Q8R1G2 & $\mathrm{Cmbl}$ & 0,6 & 5,2 \\
\hline Carboxypeptidase A4; & Q6P8K8 & Cpa4 & $-0,8$ & 7,1 \\
\hline Carboxypeptidase D; & 089001 & Cpd & $-0,5$ & 3,2 \\
\hline Carboxypeptidase E; & Q00493 & Cpe & 0,0 & 2,5 \\
\hline Carboxypeptidase M; & Q80V42 & $\mathrm{Cpm}$ & $-0,7$ & 33,9 \\
\hline Carboxypeptidase Q; & Q9WVJ3 & Cpq & 0,7 & 4,9 \\
\hline Cartilage-associated protein; & Q9CYD3 & Crtap & 0,7 & 4,8 \\
\hline Casein kinase II subunit alpha; & Q60737 & Csnk2a1 & 0,2 & 5,1 \\
\hline Casein kinase II subunit beta; & P67871 & Csnk2b & 0,1 & 20,3 \\
\hline Caspase-14; & 089094 & Casp14 & $-1,5$ & 34,2 \\
\hline Caspase-3; & P70677 & Casp3 & 0,7 & 30,7 \\
\hline Caspase-6; & 008738 & Casp6 & $-0,1$ & 11,2 \\
\hline Catalase; & P24270 & Cat & $-1,2$ & 19,9 \\
\hline Catenin alpha-1; & P26231 & Ctnna1 & $-0,4$ & 37,7 \\
\hline Catenin beta-1; & Q02248 & Ctnnb1 & $-0,4$ & 35,5 \\
\hline Catenin delta-1; & P30999 & Ctnnd1 & $-0,7$ & 4,6 \\
\hline Cathepsin B; & P10605 & Ctsb & $-0,7$ & 29,5 \\
\hline Cathepsin D; & P18242 & Ctsd & $-0,7$ & 27,6 \\
\hline Cathepsin E; & P70269 & Ctse & $-2,2$ & 4,9 \\
\hline Cathepsin L1; & P06797 & Ctsl & $-0,9$ & 25,1 \\
\hline Cathepsin Z; & Q9WUU7 & Ctsz & $-0,5$ & 15,4 \\
\hline Cation-dependent mannose-6-phosphate receptor; & P24668 & M6pr & $-0,1$ & 16,9 \\
\hline Cation-independent mannose-6-phosphate receptor; & Q07113 & $\operatorname{lgf} 2 r$ & 0,4 & 7,3 \\
\hline Caveolin-1; & P49817 & Cav1 & 0,5 & 28,7 \\
\hline C-C motif chemokine 27; & Q9Z1X0 & $\mathrm{Ccl} 27$ & $-1,9$ & 11,8 \\
\hline CCAAT/enhancer-binding protein beta; & P28033 & Cebpb & $-0,1$ & 4,4 \\
\hline CD109 antigen; & Q8R422 & Cd109 & $-0,6$ & 11,9 \\
\hline CD151 antigen; & 035566 & Cd151 & $-0,7$ & 4 \\
\hline CD166 antigen; & Q61490 & Alcam & $-0,7$ & 18,8 \\
\hline CD44 antigen; & P15379 & $\mathrm{Cd} 44$ & $-2,1$ & 6,3 \\
\hline CD63 antigen; & P41731 & Cd63 & 0,0 & 4,6 \\
\hline CD81 antigen; & P35762 & Cd81 & 0,6 & 27,5 \\
\hline CD82 antigen; & P40237 & $\mathrm{Cd} 82$ & $-1,2$ & 6,7 \\
\hline CD9 antigen; & P40240 & $\mathrm{Cd} 9$ & $-0,4$ & 12,8 \\
\hline CD97 antigen; & Q9Z0M6 & Cd97 & 1,5 & 1,2 \\
\hline Cdc42 effector protein 4 & Q9JM96 & Cdc42ep4 & 0,2 & 17,4 \\
\hline CDGSH iron-sulfur domain-containing protein 1 ; & Q91WS0 & Cisd1 & $-0,1$ & 34,3 \\
\hline
\end{tabular}




\begin{tabular}{|c|c|c|c|c|}
\hline Protein & Uniprot & Gene name & $\begin{array}{l}\text { Fold change of } \\
\text { XPRESS ratio } \\
\left(\text { Egfr }^{\triangle K C} / \mathrm{WT}\right)\end{array}$ & $\begin{array}{c}\text { Sequence } \\
\text { coverage [\%] }\end{array}$ \\
\hline CDGSH iron-sulfur domain-containing protein 2; & Q9CQB5 & Cisd2 & 0,5 & 26,9 \\
\hline CDKN2A-interacting protein; & Q8BI72 & Cdkn2aip & 1,1 & 6 \\
\hline CDP-diacylglycerol--inositol 3-phosphatidyltransferase; & Q8VDP6 & Cdipt & $-0,9$ & 10,8 \\
\hline Cell adhesion molecule 3 & Q99N28 & Cadm3 & 0,4 & 4,5 \\
\hline Cell cycle control protein $50 A ;$ & Q8VEKO & Tmem30a & $-0,3$ & 6,9 \\
\hline Cell differentiation protein RCD1 homolog; & Q9JKYO & Rqcd1 & $-0,3$ & 3 \\
\hline Cell division control protein 42 homolog; & P60766 & Cdc42 & 0,1 & 41,4 \\
\hline Cell division cycle 5-like protein; & Q6A068 & Cdc5I & 0,5 & 2,2 \\
\hline Cell division cycle protein 16 homolog; & Q8R349 & Cdc16 & 0,1 & 1,3 \\
\hline Cell growth regulator with EF hand domain protein 1 ; & Q8R1U2 & Cgref1 & $-0,9$ & 10,3 \\
\hline Cell growth-regulating nucleolar protein; & Q08288 & Lyar & 0,6 & 10,7 \\
\hline Cell surface glycoprotein MUC18; & Q8R2Y2 & Mcam & 0,2 & 22,1 \\
\hline Cellular nucleic acid-binding protein; & P53996 & Cnbp & 0,6 & 30,9 \\
\hline Cellular nucleic acid-binding protein; & D3YYF6 & Cnbp & 0,6 & 29,5 \\
\hline Centrin-1; & P41209 & Cetn1 & 0,0 & 8,1 \\
\hline Centromere protein $\mathrm{V}$; & Q9CXS4 & Cenpv & 0,0 & 4 \\
\hline Ceroid-lipofuscinosis neuronal protein 5 homolog; & Q3UMW8 & $C \ln 5$ & $-4,8$ & 5,3 \\
\hline Ceruloplasmin; & Q61147 & $\mathrm{Cp}$ & 0,7 & 14,2 \\
\hline CGG triplet repeat-binding protein 1 ; & Q8BHG9 & Cggbp1 & $-0,1$ & 8,4 \\
\hline Charged multivesicular body protein 1a; & Q921W0 & Chmp1a & $-0,5$ & 4,1 \\
\hline Charged multivesicular body protein $1 b-1 ;$ & Q99LU0 & Chmp1b1 & $-0,7$ & 4 \\
\hline Charged multivesicular body protein $2 \mathrm{a}$; & Q9DB34 & Chmp2a & $-0,1$ & 8,1 \\
\hline Charged multivesicular body protein $2 \mathrm{~b}$; & Q8BJF9 & Chmp2b & 0,1 & 8 \\
\hline Charged multivesicular body protein $4 \mathrm{~b}$; & Q9D8B3 & Chmp4b & 0,5 & 33,9 \\
\hline Charged multivesicular body protein 5 ; & Q9D7S9 & Chmp5 & 0,2 & 10,5 \\
\hline Chitinase-3-like protein 1; & Q61362 & Chi3l1 & 1,0 & 14,2 \\
\hline Chloride channel CLIC-like protein 1; & Q99LI2 & Clcc1 & $-0,5$ & 10,9 \\
\hline Chloride intracellular channel protein 1; & Q9Z1Q5 & Clic1 & $-0,1$ & 23,7 \\
\hline Chloride intracellular channel protein 3; & Q9D7P7 & Clic3 & $-0,9$ & 6,8 \\
\hline Choline transporter-like protein 1 & Q6X893 & Slc44a1 & 0,1 & 5,1 \\
\hline Choline transporter-like prote in 2 & Q8BY89 & Slc44a2 & 0,0 & 4 \\
\hline Choline/ethanolamine kinase; & 055229 & Chkb & $-4,1$ & 8,9 \\
\hline Chondroitin sulfate proteoglycan 4; & Q8VHYO & Cspg4 & 0,6 & 6,4 \\
\hline Chromatin complexes subun it BAP18; & Q9DCT6 & Bap18 & 0,4 & 42 \\
\hline Chromatin target of PRMT1 protein; & Q9CY57 & Chtop & 0,3 & 20,7 \\
\hline Chromobox protein homolog 1 & P83917 & Cbx1 & $-0,2$ & 14,6 \\
\hline Chromobox protein homolog 3 ; & P23198 & $\mathrm{Cb} \times 3$ & 1,3 & 22,4 \\
\hline Chromobox protein homolog 5 & Q61686 & $\mathrm{Cbx} 5$ & 0,2 & 6,8 \\
\hline Chromodomain Y-like protein; & Q9WTK2 & Cdyl & 0,4 & 2,5 \\
\hline Chromodomain-helicase-DNA-binding protein 4; & Q6PDQ2 & Chd4 & 0,2 & 1,3 \\
\hline Cingulin; & P59242 & Cgn & $-0,8$ & 0,8 \\
\hline Citrate lyase subunit beta-like protein, mitochondrial; & Q8R4N0 & Clybl & 0,0 & 3,6 \\
\hline Citrate synthase, mitochondrial; & Q9CZU6 & Cs & $-0,2$ & 8,8 \\
\hline C-Jun-amino-terminal kinase-interacting protein 4; & Q58A65 & Spag9 & $-0,3$ & 2,8 \\
\hline Clathrin heavy chain 1 & Q68FD5 & Cltc & 0,2 & 10,2 \\
\hline Clathrin interactor 1 & Q99KN9 & Clint1 & 0,0 & 12,2 \\
\hline Clathrin light chain A; & B1AWE0 & Clta & 0,4 & 28,2 \\
\hline Clathrin light chain B; & Q6IRU5 & Cltb & 0,1 & 26,2 \\
\hline Claudin-1; & 088551 & Cldn1 & $-0,4$ & 7,1 \\
\hline Claudin-4; & 035054 & Cldn4 & $-0,9$ & 5,7 \\
\hline Cleavage and polyadenylation specificity factor subunit 4 & Q8BQZ5 & Cpsf4 & 0,1 & 4,3 \\
\hline Cleavage and polyadenylation specificity factor subunit 5 & Q9CQF3 & Nudt21 & 0,3 & 13,7 \\
\hline Cleavage and polyadenylation specificity factor subunit 6; & Q6NVF9 & Cpsf6 & 1,3 & 4,9 \\
\hline Cleavage and polyadenylation specificity factor subunit 7; & Q8BTV2 & Cpsf7 & 0,1 & 2,3 \\
\hline Cleavage stimulation factor subunit 2 & Q8BIQ5 & Cstf2 & 0,3 & 6 \\
\hline Cleft lip and palate transmembrane protein 1 homolog; & Q8VBZ3 & Clptm1 & $-0,4$ & 8,4 \\
\hline Clusterin; & Q06890 & Clu & $-1,6$ & 24,3 \\
\hline CMRF35-like molecule 9; & Q1ERP8 & Cd300lg & $-2,4$ & 10,6 \\
\hline C-Myc-binding protein; & Q9EQS3 & Mycbp & $-0,2$ & 55,3 \\
\hline Coactosin-like protein; & Q9CQI6 & Cotl1 & 0,9 & 11,3 \\
\hline Coagulation factor XIII A chain; & Q8BH61 & F13a1 & 0,7 & 15,6 \\
\hline Coatomer subunit alpha; & Q8CIE6 & Copa & $-0,2$ & 6,9 \\
\hline Coatomer subunit beta; & Q9JIF7 & Copb1 & $-0,1$ & 27,8 \\
\hline Coatomer subunit beta'; & 055029 & Copb2 & $-0,1$ & 15,8 \\
\hline Coatomer subunit delta; & Q5XJY5 & Arcn1 & 0,1 & 23,1 \\
\hline Coatomer subunit epsilon; & 089079 & Cope & 0,0 & 18,5 \\
\hline Coatomer subunit gamma-1; & Q9QZE5 & Copg1 & 0,1 & 15,6 \\
\hline Coatomer subunit gamma-2; & Q9QXK3 & Copg2 & $-0,1$ & 2,8 \\
\hline Coatomer subunit zeta-1; & P61924 & Copz1 & 0,0 & 22,6 \\
\hline Coatomer subunit zeta-2; & Q9JHH9 & Copz2 & $-0,3$ & 9,3 \\
\hline Cochlin; & Q62507 & Coch & $-1,0$ & 10,7 \\
\hline
\end{tabular}




\begin{tabular}{|c|c|c|c|c|}
\hline Protein & Uniprot & Gene name & $\begin{array}{l}\text { Fold change of } \\
\text { XPRESS ratio } \\
\left(\text { Egfr }^{\triangle K C} / \mathrm{WT}\right)\end{array}$ & $\begin{array}{c}\text { Sequence } \\
\text { coverage [\%] }\end{array}$ \\
\hline Cofilin-1; & P18760 & $\mathrm{Cfl} 1$ & 0,0 & 39,2 \\
\hline Cofilin-2; & P45591 & $\mathrm{Cfl} 2$ & 0,1 & 28,3 \\
\hline Coiled-coil domain-containing protein 58 ; & Q8R3Q6 & Ccdc58 & 3,6 & 32,8 \\
\hline Coiled-coil domain-containing protein 86 & Q9JJ89 & Ccdc86 & $-0,1$ & 1,9 \\
\hline Coiled-coil domain-containing protein $88 \mathrm{~B}$; & Q4QRL3 & Ccdc88b & $-6,4$ & 0,7 \\
\hline Coiled-coil domain-containing protein 9; & Q8VC31 & Ccdc9 & $-0,1$ & 1,7 \\
\hline Coiled-coil domain-containing protein 90B, mitochondrial; & Q8C3X2 & Ccdc90b & 0,1 & 14,5 \\
\hline Coiled-coil domain-containing protein 91 & Q9D8L5 & Ccdc91 & $-0,1$ & 1,8 \\
\hline Coiled-coil-helix-coiled-coil-helix domain-containing protein 2, mitochondrial; & Q9D1L0 & Chchd2 & $-0,6$ & 30,1 \\
\hline Coiled-coil-helix-coiled-coil-helix domain-containing protein 3, mitochondrial; & Q9CRB9 & Chchd3 & $-0,2$ & 31 \\
\hline Coiled-coil-helix-coiled-coil-helix domain-containing protein 6, mitochondrial; & Q91VN4 & Chchd6 & 0,4 & 5,7 \\
\hline Cold shock domain-containing protein $\mathrm{C} 2$ & Q91YQ3 & Csdc2 & 0,5 & 13 \\
\hline Cold shock domain-containing protein E1; & Q91W50 & Csde1 & $-2,2$ & 2,3 \\
\hline Cold-inducible RNA-binding protein; & P60824 & Cirbp & 0,2 & 14,5 \\
\hline Collagen alpha- $1(I)$ chain; & P11087 & Col1a1 & $-0,6$ & 17,8 \\
\hline Collagen alpha-1(III) chain; & P08121 & Col3a1 & 0,0 & 12 \\
\hline Collagen alpha-1(IV) chain; & P02463 & Col4a1 & 0,7 & 3,5 \\
\hline Collagen alpha-1(V) chain; & 088207 & Col5a1 & 0,3 & 1 \\
\hline Collagen alpha-1(VI) chain; & Q04857 & Col6a1 & 0,1 & 14,1 \\
\hline Collagen alpha-1(VII) chain; & Q63870 & Col7a1 & $-0,1$ & 0,8 \\
\hline Collagen alpha-1(XII) chain; & Q60847 & Col12a1 & $-0,1$ & 7,4 \\
\hline Collagen alpha-1(XIV) chain; & Q80X19 & Col14a1 & 0,0 & 21,5 \\
\hline Collagen alpha-1(XV) chain; & 035206 & Col15a1 & 0,8 & 33,8 \\
\hline Collagen alpha-1(XVII) chain; & Q07563 & Col17a1 & $-1,4$ & 0,9 \\
\hline Collagen alpha- 1 (XVIII) chain; & P39061 & Col18a1 & 0,6 & 6,8 \\
\hline Collagen alpha-2(I) chain; & Q01149 & Col1a2 & $-0,6$ & 23,3 \\
\hline Collagen alpha-2(IV) chain; & P08122 & Col4a2 & 1,3 & 2,2 \\
\hline Collagen alpha-2(VI) chain; & Q02788 & Col6a2 & 0,0 & 12,8 \\
\hline Collagen alpha-6(VI) chain; & Q8C6K9 & Col6a6 & $-0,2$ & 0,9 \\
\hline $\begin{array}{l}\text { Collagen type V alpha } 3 \text { chain; Collagen, type V, alpha 3; Procollagen, type V, } \\
\text { alpha 3, isoform CRA_b; Protein Col5a3; }\end{array}$ & Q9JLI2 & Col5a3 & 0,9 & 4,1 \\
\hline Collectin-12; & Q8K4Q8 & Colec12 & 0,4 & 3,2 \\
\hline Complement C1q subcomponent subunit $A$; & P98086 & C1qa & 0,2 & 4,5 \\
\hline Complement C3; & P01027 & $\mathrm{C} 3$ & 0,8 & 30,1 \\
\hline Complement C4-B; & P01029 & $\mathrm{C} 4 \mathrm{~b}$ & 0,4 & 0,5 \\
\hline $\begin{array}{l}\text { Complement component } 1 \mathrm{Q} \text { subcomponent-binding protein, mitochondrial; } \\
\text { Complement component } 1 \text {, q subcomponent binding protein; Complement } \\
\text { component 1, q subcomponent binding protein, isoform CRA_b; p32-RACK; }\end{array}$ & Q8R5L1 & C1qbp & 0,2 & 7,2 \\
\hline Complement component receptor 1-like protein; & Q64735 & Cr1l & 0,0 & 4,1 \\
\hline Complement factor B; & P04186 & $\mathrm{Cfb}$ & 0,2 & 4,2 \\
\hline Complement factor D; & Р03953 & $\mathrm{Cfd}$ & 0,5 & 11,6 \\
\hline Complement factor $\mathrm{H}$; & P06909 & Cfh & 0,7 & 6,9 \\
\hline Complement factor I; & Q61129 & $\mathrm{Cfi}$ & 0,9 & 7,5 \\
\hline Complexin-2; & P84086 & Cplx2 & 1,2 & 11,2 \\
\hline Constitutive coactivator of PPAR-gamma-like protein 1 ; & Q6A0A9 & FAM120A & 0,2 & 4,9 \\
\hline COP9 signalosome complex subunit 1 & Q99LD4 & Gps1 & 0,1 & 14 \\
\hline COP9 signalosome complex subunit 2 & P61202 & Cops2 & 0,4 & 10,6 \\
\hline COP9 signalosome complex subunit 3; & 088543 & Cops3 & 0,2 & 16,8 \\
\hline COP9 signalosome complex subunit 4; & 088544 & Cops4 & 0,2 & 11,1 \\
\hline COP9 signalosome complex subunit 5; & 035864 & Cops5 & 0,2 & 24,6 \\
\hline COP9 signalosome complex subunit 6; & 088545 & Cops6 & 0,0 & 19,9 \\
\hline COP9 signalosome complex subunit $7 a$; & Q9CZ04 & Cops7a & 0,0 & 17,5 \\
\hline COP9 signalosome complex subunit 8; & Q8VBV7 & Cops8 & 0,0 & 7,2 \\
\hline Copine-1; & Q8C166 & Cpne1 & 0,2 & 8,7 \\
\hline Copper transport protein ATOX1; & 008997 & Atox1 & 0,4 & 57,4 \\
\hline Cordon-bleu protein-like 1 & Q3UMF0 & Cobll1 & $-1,5$ & 3,5 \\
\hline Core histone macro-H2A.1; & Q9QZQ8 & H2afy & $-0,2$ & 39,8 \\
\hline Core histone macro-H2A.2; & Q8CCK0 & H2afy2 & 0,3 & 14 \\
\hline Corneodesmosin; & Q7TPC1 & Cdsn & $-0,3$ & 14,6 \\
\hline Cornifin-A; & Q62266 & Sprr1a & $-0,6$ & 68,8 \\
\hline Coronin-1A; & 089053 & Corola & $-0,2$ & 5,6 \\
\hline Costars family protein ABRACL; & Q4KML4 & Abracl & $-0,3$ & 27,2 \\
\hline Coxsackievirus and adenovirus receptor homolog; & P97792 & Cxadr & $-0,7$ & 15,6 \\
\hline Craniofacial development protein 1 ; & 088271 & Cfdp1 & $-0,7$ & 20 \\
\hline Creatine kinase B-type; & Q04447 & $\mathrm{Ckb}$ & 0,0 & 6,8 \\
\hline Creatine kinase M-type; & P07310 & $\mathrm{Ckm}$ & 0,1 & 2,6 \\
\hline Creatine kinase U-type, mitochondrial; & P30275 & Ckmt1 & $-0,6$ & 33,3 \\
\hline
\end{tabular}




\begin{tabular}{|c|c|c|c|c|}
\hline Protein & Uniprot & Gene name & $\begin{array}{l}\text { Fold change of } \\
\text { XPRESS ratio } \\
\left(\text { Egfr }^{\Delta K C} / \mathrm{WT}\right)\end{array}$ & $\begin{array}{c}\text { Sequence } \\
\text { coverage [\%] }\end{array}$ \\
\hline cTAGE family member 5 & E9PY90 & Ctage5 & $-0,5$ & 4,6 \\
\hline C-terminal-binding protein 1 & 088712 & Ctbp1 & 0,2 & 26,3 \\
\hline C-terminal-binding protein 2 & P56546 & Ctbp2 & 0,7 & 20,7 \\
\hline CTP synthase 1 & P70698 & Ctps1 & $-0,5$ & 38,9 \\
\hline C-type mannose receptor 2 & Q64449 & Mrc2 & 0,3 & 7,2 \\
\hline Cullin-1; & Q9WTX6 & Cul1 & 0,1 & 11 \\
\hline Cullin-3; & Q9JLV5 & Cul3 & $-0,9$ & 6,3 \\
\hline Cullin-associated NEDD8-dissociated protein 1; & Q6ZQ38 & Cand1 & 0,5 & 28 \\
\hline Cyclic AMP-responsive element-binding protein 1 ; & Q01147 & Creb1 & $-0,3$ & 7,3 \\
\hline Cyclic GMP-AMP synthase; & Q8C6L5 & Mb21d1 & 1,5 & 2,2 \\
\hline Cyclin-dependent kinase 6; & Q64261 & Cdk6 & 0,1 & 7,4 \\
\hline Cyclin-dependent kinase inhibitor 1B; & P46414 & Cdkn1b & 0,6 & 4,1 \\
\hline Cystathionine beta-synthase; & Q91WT9 & Cbs & $-0,2$ & 11,8 \\
\hline Cystathionine gamma-lyase; & Q8VCN5 & Cth & 0,3 & 9 \\
\hline Cystatin E/M; Cystatin M/E; Cystatin-B; & Q9D1B1 & Cst6 & 0,4 & 55 \\
\hline Cystatin-B; & Q62426 & Cstb & $-0,9$ & 39,8 \\
\hline Cystatin-C; & P21460 & Cst3 & $-0,2$ & 26,5 \\
\hline Cysteine and glycine-rich protein $1 ;$ & P97315 & Csrp1 & 0,0 & 48,2 \\
\hline Cysteine and glycine-rich protein 2 & P97314 & Csrp2 & 0,8 & 23,3 \\
\hline Cysteine and histidine-rich domain-containing protein $1 ;$ & Q9D1P4 & Chordc1 & 3,0 & 4,8 \\
\hline Cysteine sulfinic acid decarboxylase; & Q9DBE0 & Csad & 0,8 & 18,9 \\
\hline Cysteine-rich protein 1 & P63254 & Crip1 & 0,3 & 9,1 \\
\hline Cysteine-rich protein 2; & Q9DCT8 & Crip2 & $-0,8$ & 22,6 \\
\hline Cysteine-rich secretory protein 1 & Q03401 & Crisp1 & $-0,7$ & 3,7 \\
\hline Cysteine-rich with EGF-like domain protein 2; & Q9CYA0 & Creld2 & 0,0 & 9,4 \\
\hline Cysteine--tRNA ligase, cytoplasmic; & Q9ER72 & Cars & $-0,7$ & 6 \\
\hline Cytochrome b; & P00158 & Mt Cyb & $-0,9$ & 2,4 \\
\hline Cytochrome b5 reductase 4; & Q3TDX8 & Cyb5r4 & 0,4 & 3,3 \\
\hline Cytochrome b5 type B; & Q9CQX2 & Cyb5b & $-0,3$ & 50,7 \\
\hline Cytochrome b5; & P56395 & Cyb5a & 0,2 & 65,3 \\
\hline Cytochrome b-c1 complex subunit 1, mitochondrial; & Q9CZ13 & Uqcrc1 & $-0,4$ & 32,5 \\
\hline Cytochrome b-c1 complex subunit 2, mitochondrial; & Q9DB77 & Uqcrc2 & $-0,5$ & 37,1 \\
\hline Cytochrome b-c1 complex subunit 6, mitochondrial; & P99028 & Uqcrh & $-0,6$ & 28,1 \\
\hline Cytochrome b-c1 complex subunit 7; & Q9D855 & Uqcrb & $-0,5$ & 25,2 \\
\hline Cytochrome b-c1 complex subunit 8; & Q9CQ69 & Uqcrq & $-0,3$ & 26,8 \\
\hline Cytochrome b-c1 complex subunit 9; & Q8R1I1 & Uqcr10 & $-0,6$ & 37,5 \\
\hline Cytochrome b-c1 complex subunit Rieske, mitochondrial; & Q9CR68 & Uqcrfs1 & $-0,3$ & 31,4 \\
\hline Cytochrome c oxidase assembly factor 3 homolog, mitochondrial; & Q9D2R6 & Coa3 & 0,2 & 26,9 \\
\hline Cytochrome c oxidase assembly factor 6 homolog; & Q8BGD8 & Coa6 & $-0,2$ & 13,9 \\
\hline Cytochrome c oxidase assembly factor 7 & Q921H9 & Coa7 & 0,1 & 3 \\
\hline Cytochrome c oxidase copper chaperone; & P56394 & Cox17 & $-0,7$ & 11,1 \\
\hline Cytochrome c oxidase protein 20 homolog; & Q9D7J4 & Cox20 & 0,0 & 12 \\
\hline Cytochrome c oxidase subunit 2; & P00405 & Mtco2 & $-0,4$ & 16,3 \\
\hline Cytochrome c oxidase subunit 3; & P00416 & $\mathrm{mt} \mathrm{Co3}$ & $-1,2$ & 5,4 \\
\hline Cytochrome c oxidase subunit 4 isoform 1 , mitochondrial; & P19783 & Cox4i1 & $-0,6$ & 40,2 \\
\hline Cytochrome c oxidase subunit 5A, mitochond rial; & P12787 & Cox5a & $-0,6$ & 32,2 \\
\hline Cytochrome c oxidase subunit 5B, mitochondrial; & P19536 & Cox5b & $-0,9$ & 24,2 \\
\hline Cytochrome c oxidase subunit 6B1; & P56391 & Cox6b1 & $-0,4$ & 41,9 \\
\hline Cytochrome c oxidase subunit 6C; & Q9CPQ1 & Cox6c & $-0,6$ & 18,4 \\
\hline Cytochrome c oxidase subunit 7A2, mitochondrial; & P48771 & Cox7a2 & $-0,2$ & 27,7 \\
\hline Cytochrome c oxidase subunit 7C, mitochondrial; & P17665 & Cox7c & $-0,4$ & 14,3 \\
\hline Cytochrome c, somatic; & P62897 & Cycs & $-0,2$ & 38,1 \\
\hline Cytochrome c1, heme protein, mitochondrial; & Q9D0M3 & Cyc1 & $-0,2$ & 22,2 \\
\hline Cytochrome P450 2B10; & P12791 & Cyp2b10 & 0,4 & 2,9 \\
\hline Cytochrome P450 2F2; & P33267 & Сур2f2 & 0,7 & 13,8 \\
\hline Cytochrome P450 2S1; & Q9DBX6 & Cyp2s1 & $-1,1$ & 11,4 \\
\hline $\begin{array}{l}\text { Cytochrome P450, family 2, subfamily g, polypeptide 1; Olfactory-specific } \\
\text { steroid hydroxylase; Protein Cyp2g1; }\end{array}$ & Q9WV19 & Cyp2g1 & $-0,7$ & 2,8 \\
\hline Cytoglobin; & Q9CX80 & Cygb & 1,9 & 13,7 \\
\hline Cytoplasmic aconitate hydratase; & P28271 & Aco1 & 0,5 & 21,5 \\
\hline Cytoplasmic dynein 1 heavy chain 1 ; & Q9JHU4 & Dync1h1 & $-0,3$ & 4,6 \\
\hline Cytoplasmic dynein 1 intermediate chain 2; & 088487 & Dync1i2 & $-0,1$ & 15,5 \\
\hline Cytoplasmic dynein 1 light intermediate chain 1 ; & Q8R1Q8 & Dync1li1 & 2,3 & 2,3 \\
\hline Cytoplasmic dynein 1 light intermediate chain 2; & Q6PDL0 & Dync1li2 & $-0,1$ & 3,3 \\
\hline Cytoskeleton-associated protein 4 & Q8BMK4 & Ckap4 & $-0,1$ & 35,8 \\
\hline Cytosol aminopeptidase; & Q9CPY7 & Lap3 & 0,1 & 49,3 \\
\hline Cytosolic acyl coenzyme A thioester hydrolase; & Q91V12 & Acot7 & 0,9 & 21,5 \\
\hline Cytosolic endo-beta-N-acetylglucosaminidase; & Q8BX80 & Engase & $-0,2$ & 7,1 \\
\hline Cytosolic non-specific dipeptidase; & Q9D1A2 & Cndp2 & $-0,4$ & 21,9 \\
\hline Cytosolic phospholipase A2 beta; & P0C871 & Pla2g4b & $-0,6$ & 2,8 \\
\hline Cytosolic purine 5'-nucleotidase; & Q3V1L4 & $\mathrm{Nt} 5 \mathrm{c} 2$ & 4,1 & 2,7 \\
\hline
\end{tabular}




\begin{tabular}{|c|c|c|c|c|}
\hline Protein & Uniprot & Gene name & $\begin{array}{l}\text { Fold change of } \\
\text { XPRESS ratio } \\
\left(\text { Egfr }^{\Delta K C} / \mathrm{WT}\right)\end{array}$ & $\begin{array}{c}\text { Sequence } \\
\text { coverage [\%] }\end{array}$ \\
\hline Cytospin-B; & Q5SXY1 & Specc1 & 0,1 & 1,5 \\
\hline D-3-phosphoglycerate dehydrogenase; & Q61753 & Phgdh & 0,1 & 27,4 \\
\hline DAZ-associated protein 1 & Q9JII5 & Dazap1 & 0,5 & 18,7 \\
\hline D-dopachrome decarboxylase; & 035215 & Ddt & 0,2 & 51,7 \\
\hline Death domain-containing membrane protein NRADD; & Q8CJ26 & Nradd & 0,2 & 11,8 \\
\hline Death-associated protein-like 1 & Q9D757 & Dapl1 & 0,0 & 12,1 \\
\hline Decorin; & P28654 & Den & $-0,1$ & 23,7 \\
\hline Dehydrogenase/reductase SDR family member 4; & Q99LB2 & Dhrs4 & $-0,8$ & 4,7 \\
\hline Dehydrogenase/reductase SDR family member 7; & Q9CXR1 & Dhrs7 & $-0,3$ & 5,9 \\
\hline Dehydrogenase/reductase SDR family member 7B; & Q99J47 & Dhrs7b & 0,1 & 6,2 \\
\hline Dehydrogenase/reductase SDR family member on chromosome $\mathrm{X}$ homolog; & Q8VBZ0 & Dhrsx & $-6,4$ & 9,6 \\
\hline Delta(24)-sterol reductase; & Q8VCH6 & Dhcr24 & $-0,3$ & 1,7 \\
\hline Delta(3,5)-Delta(2,4)-dienoyl-CoA isomerase, mitochondrial; & 035459 & Ech1 & 0,3 & 25,4 \\
\hline Delta-aminolevulinic acid dehydratase; & P10518 & Alad & $-0,1$ & 25,5 \\
\hline Delta-sarcoglycan; & P82347 & Sgcd & 0,9 & 12,5 \\
\hline Density-regulated protein; & Q9CQJ6 & Denr & $-1,3$ & 21,2 \\
\hline Deoxyguanosine kinase, mitochondrial; & Q9QX60 & Dguok & 0,9 & 10,6 \\
\hline Deoxyhypusine synthase; & Q3TXU5 & Dhps & 0,4 & 8,1 \\
\hline Deoxynucleoside triphosphate triphosphohydrolase SAMHD1; & Q60710 & Samhd1 & 0,4 & 22,3 \\
\hline Deoxyribonuclease-1-like 2; & Q9D1G0 & Dnase1/2 & $-2,2$ & 17,1 \\
\hline Deoxyribonuclease-2-alpha; & P56542 & Dnase2 & $-0,5$ & 18,1 \\
\hline Deoxyuridine triphosphatase, isoform CRA_b; Dutp protein; Protein Dut; & Q8VCG1 & Dut & 0,7 & 17,9 \\
\hline Derlin-1; & Q99J56 & Derl1 & $-0,5$ & 7,6 \\
\hline Dermatopontin; & Q9QZZ6 & Dpt & $-0,2$ & 32,3 \\
\hline Dermokine; & E9Q2P1 & Dmkn & $-0,5$ & 21,3 \\
\hline Desmocollin-1; & P55849 & Dsc1 & $-0,6$ & 12,9 \\
\hline Desmocollin-3; & P55850 & Dsc3 & $-0,2$ & 14,6 \\
\hline Desmoglein-1-alpha; & Q61495 & Dsg1a & $-0,8$ & 8,2 \\
\hline Desmoglein-2; & 055111 & Dsg2 & 1,0 & 2,3 \\
\hline Desmoglein-3; & 035902 & Dsg3 & $-0,7$ & 2,5 \\
\hline Desmoplakin; & E9Q557 & Dsp & $-0,7$ & 2,1 \\
\hline Destrin; & Q9R0P5 & Dstn & $-0,1$ & 34,5 \\
\hline Developmentally-regulated GTP-binding protein 1; & P32233 & Drg1 & 0,1 & 12,5 \\
\hline Diacylglycerol O-acyltransferase 1 & Q9Z2A7 & Dgat1 & 0,1 & 5 \\
\hline Dihydrofolate reductase; & P00375 & Dhfr & 0,6 & 8,6 \\
\hline Dihydrolipoyl dehydrogenase, mitochondrial; & 008749 & Dld & 0,1 & 26,3 \\
\hline $\begin{array}{l}\text { Dihydrolipoyllysine-residue acetyltransferase component of pyruvate } \\
\text { dehydrogenase complex, mitochondrial; }\end{array}$ & Q8BMF4 & Dlat & $-0,1$ & 4,2 \\
\hline $\begin{array}{l}\text { Dihydrolipoyllysine-residue succinyltransferase component of 2-oxoglutarate } \\
\text { dehydrogenase complex, mitochondrial; }\end{array}$ & Q9D2G2 & Dlst & $-0,2$ & 15 \\
\hline Dihydropteridine reductase; & Q8BVI4 & Qdpr & 0,3 & 39,2 \\
\hline Dihydropyrimidinase-related protein 2 ; & 008553 & Dpysl2 & 0,9 & 46,9 \\
\hline Dihydropyrimidinase-related protein 3 ; & E9PWE8 & Dpysl3 & 0,7 & 29,6 \\
\hline Dipeptidase 1; & P31428 & Dpep1 & 0,6 & 19,3 \\
\hline Dipeptidyl peptidase 1; & P97821 & Ctsc & $-0,1$ & 12,3 \\
\hline Dipeptidyl peptidase 2 & Q9ET22 & Dpp7 & $-0,3$ & 5,5 \\
\hline Dipeptidyl peptidase 4; & P28843 & Dpp4 & $-0,6$ & 12,5 \\
\hline Diphosphoinositol polyphosphate phosphohydrolase 1 ; & Q9J146 & Nudt3 & 0,4 & 30,2 \\
\hline Disks large-associated protein 4 & B1AZP2 & Dlgap4 & 0,0 & 16,8 \\
\hline DNA damage-binding protein $1 ;$ & Q3U1J4 & Ddb1 & 0,5 & 30,7 \\
\hline DNA mismatch repair protein Msh6; & P54276 & Msh6 & 6,4 & 0,7 \\
\hline DNA polymerase delta catalytic subunit; & P52431 & Pold1 & 0,5 & 1 \\
\hline DNA polymerase lambda; & Q9QXE2 & Poll & 0,2 & 1,6 \\
\hline DNA repair protein SWI5 homolog; & Q8K3D3 & Swi5 & $-0,3$ & 15,3 \\
\hline DNA replication licensing factor $\mathrm{MCM} 3$; & P25206 & Mcm3 & 0,5 & 1,5 \\
\hline DNA replication licensing factor MCM4; & P49717 & Mcm4 & 0,7 & 2,4 \\
\hline DNA replication licensing factor $\mathrm{MCM} 5$; & P49718 & Mcm5 & 1,0 & 3,5 \\
\hline DNA replication licensing factor MCM6; & P97311 & Mcm6 & 0,8 & 6,1 \\
\hline DNA replication licensing factor $\mathrm{MCM7}$; & Q61881 & Mcm7 & 0,6 & 3,3 \\
\hline DNA topoisomerase 1 ; & Q04750 & Top1 & 0,2 & 1,6 \\
\hline DNA topoisomerase 2-beta; & Q64511 & Top2b & 0,4 & 6 \\
\hline DNA-(apurinic or apyrimidinic site) lyase; & P28352 & Apex1 & 0,5 & 15,3 \\
\hline DNA-directed RNA polymerase II subunit RPB1; & P08775 & Polr2a & 0,1 & 3,6 \\
\hline DNA-directed RNA polymerase II subunit RPB2; & Q8CFI7 & Polr2b & 0,0 & 5,6 \\
\hline DNA-directed RNA polymerase II subunit RPB3; & P97760 & Polr2c & 0,1 & 5,5 \\
\hline DNA-directed RNA polymerase II subunit RPB7; & P62488 & Polr2g & 0,1 & 20,9 \\
\hline DNA-directed RNA polymerases I, II, and III subunit RPABC3; & Q923G2 & Polr $2 \mathrm{~h}$ & $-0,1$ & 8,7 \\
\hline DnaJ homolog subfamily A member 1 ; & P63037 & Dnaja1 & 0,0 & 20,7 \\
\hline DnaJ homolog subfamily A member 2 & Q9QYJ0 & Dnaja2 & 0,1 & 5,1 \\
\hline
\end{tabular}




\begin{tabular}{|c|c|c|c|c|}
\hline Protein & Uniprot & Gene name & $\begin{array}{l}\text { Fold change of } \\
\text { XPRESS ratio } \\
\left(\text { Egfr }^{\triangle K C} / \mathrm{WT}\right)\end{array}$ & $\begin{array}{c}\text { Sequence } \\
\text { coverage [\%] }\end{array}$ \\
\hline DnaJ homolog subfamily B member 1 & Q9QYJ3 & Dnajb1 & 0,1 & 5,9 \\
\hline DnaJ homolog subfamily B member 11 ; & Q99KV1 & Dnajb11 & $-0,1$ & 7,5 \\
\hline DnaJ homolog subfamily B member 4 ; & Q9D832 & Dnajb4 & 0,0 & 3 \\
\hline DnaJ homolog subfamily B member 6 & 054946 & Dnajb6 & $-0,4$ & 4,3 \\
\hline DnaJ homolog subfamily $\mathrm{C}$ member 3 ; & Q91YW3 & Dnajc3 & $-0,4$ & 1,8 \\
\hline DnaJ homolog subfamily $\mathrm{C}$ member 8 ; & Q6NZB0 & Dnajc8 & 0,2 & 4,1 \\
\hline Dolichol-phosphate mannosyltransferase subunit 1 ; & 070152 & Dpm1 & 0,0 & 13,5 \\
\hline Dolichol-phosphate mannosyltransferase subunit 3; & Q9D1Q4 & Dpm3 & $-0,1$ & 13 \\
\hline $\begin{array}{l}\text { Dolichyl-diphosphooligosaccharide--protein glycosyltransferase } 48 \text { kDa } \\
\text { subunit; }\end{array}$ & 054734 & Ddost & $-0,1$ & 20,2 \\
\hline Dolichyl-diphosphooligosaccharide--protein glycosyltransferase subunit 1; & Q91YQ5 & Rpn1 & $-0,2$ & 32,4 \\
\hline Dolichyl-diphosphooligosaccharide--protein glycosyltransferase subunit 2; & Q9DBG6 & Rpn2 & $-0,2$ & 22,8 \\
\hline Dolichyl-diphosphooligosaccharide--protein glycosyltransferase subunit DAD1; & P61804 & Dad1 & 0,3 & 19,5 \\
\hline Dolichyl-diphosphooligosaccharide--protein glycosyltransferase subunit STT3A; & P46978 & Stt3a & $-0,5$ & 10,8 \\
\hline Dolichyl-diphosphooligosaccharide--protein glycosyltransferase subunit STT3B; & Q3TDQ1 & Stt3b & $-0,4$ & 7,8 \\
\hline Dolichyl-phosphate beta-glucosyltransferase; & Q9DB25 & Alg5 & 0,1 & 12,7 \\
\hline Double-strand-break repair protein rad21 homolog; & Q61550 & $\operatorname{Rad} 21$ & $-0,5$ & 2,2 \\
\hline Dr1-associated corepressor; & Q9D6N5 & Drap1 & 0,5 & 11,5 \\
\hline Drebrin-like protein; & Q62418 & Dbnl & $-0,3$ & 17,9 \\
\hline Dual specificity protein phosphatase 14 & Q9JLY7 & Dusp14 & $-3,1$ & 10,6 \\
\hline Dynactin subunit 2 & Q99KJ8 & Dctn2 & $-0,2$ & 19,2 \\
\hline Dynactin subunit 3 ; & Q9Z0Y1 & Dctn3 & 0,6 & 5,7 \\
\hline Dynactin subunit 4 & Q8CBY8 & Dctn4 & 0,1 & 12,4 \\
\hline Dynactin subunit 5; & Q9QZB9 & Dctn5 & $-0,4$ & 6 \\
\hline Dynactin subunit 6; & Q9WUB4 & Dctn6 & 2,4 & 13,6 \\
\hline Dynamin-like 120 kDa protein, mitochondrial; & P58281 & Opa1 & $-0,8$ & 7,4 \\
\hline Dynein light chain 1 , cytoplasmic; & P63168 & Dynll1 & $-0,7$ & 49,4 \\
\hline Dynein light chain 2, cytoplasmic; & Q9D0M5 & Dynll2 & $-0,1$ & 32,6 \\
\hline Dynein light chain roadblock-type 1 ; & P62627 & Dynlrb1 & 0,1 & 34,4 \\
\hline Dystroglycan; & Q62165 & Dag1 & $-0,4$ & 7,1 \\
\hline E3 ubiquitin-protein ligase ARIH1; & Q9Z1K5 & Arih1 & 0,2 & 2,5 \\
\hline E3 ubiquitin-protein ligase HUWE1; & Q7TMY8 & Huwe1 & $-0,3$ & 1,5 \\
\hline E3 ubiquitin-protein ligase NEDD4; & P46935 & Nedd4 & 0,0 & 0,9 \\
\hline E3 ubiquitin-protein ligase RBX1; & P62878 & Rbx1 & 0,1 & 7,4 \\
\hline E3 ubiquitin-protein ligase RNF114; & Q9ET26 & Rnf114 & 0,6 & 31,6 \\
\hline E3 ubiquitin-protein ligase RNF126; & Q91YL2 & Rnf126 & $-0,1$ & 7,7 \\
\hline E3 ubiquitin-protein ligase RNF13; & 054965 & Rnf13 & $-0,6$ & 6,3 \\
\hline E3 ubiquitin-protein ligase RNF181; & Q9CY62 & Rnf181 & 0,1 & 19,2 \\
\hline E3 ubiquitin-protein ligase UHRF1; & Q8VDF2 & Uhrf1 & 0,6 & 2,8 \\
\hline Early endosome antigen 1 & Q8BL66 & Eea1 & 0,0 & 20,4 \\
\hline Echinoderm microtubule-associated protein-like 1; & Q05BC3 & Eml1 & $-0,1$ & 1,6 \\
\hline Echinoderm microtubule-associated protein-like 2; & Q7TNG5 & Eml2 & $-0,3$ & 8,8 \\
\hline Ecto-ADP-ribosyltransferase 3; & E9Q7Q8 & Art3 & 0,0 & 16,9 \\
\hline Ectonucleoside triphosphate diphosphohydrolase $1 ;$ & P55772 & Entpd1 & $-0,5$ & 4,7 \\
\hline Ectonucleoside triphosphate diphosphohydrolase 2; & 055026 & Entpd2 & 1,8 & 7,1 \\
\hline Ectonucleotide pyrophosphatase/phosphodiesterase family member 1 ; & P06802 & Enpp1 & 0,2 & 2,4 \\
\hline Ectonucleotide pyrophosphatase/phosphodiesterase family member 3; & Q6DYE8 & Enpp3 & 0,8 & 0,9 \\
\hline EF-hand domain-containing protein D2; Efhd2 protein; & Q8C845 & Efhd2 & $-0,1$ & 21,7 \\
\hline EGF-containing fibulin-like extracellular matrix protein 1 ; & Q8BPB5 & Efemp1 & 0,3 & 27,6 \\
\hline EGF-containing fibulin-like extracellular matrix protein 2 ; & Q9WVJ9 & Efemp2 & 0,4 & 6 \\
\hline EH domain-containing protein 1 ; & Q9WVK4 & Ehd1 & $-0,4$ & 4,9 \\
\hline EH domain-containing protein 2 ; & Q8BH64 & Ehd2 & 0,6 & 10,7 \\
\hline EH domain-containing protein 4 ; & Q9EQP2 & Ehd4 & 0,0 & 1,5 \\
\hline EKC/KEOPS complex subunit Lage3; & Q9CR70 & Lage3 & $-1,3$ & 10,8 \\
\hline EKC/KEOPS complex subunit Tprkb; & Q8QZZ7 & Tprkb & 0,2 & 10,3 \\
\hline Elastin; & P54320 & Eln & 0,1 & 3 \\
\hline ELAV-like protein 1; & P70372 & Elavl1 & 0,3 & 17,5 \\
\hline Electron transfer flavoprotein subunit alpha, mitochondrial; & Q99LC5 & Etfa & 0,1 & 48 \\
\hline Electron transfer flavoprotein subunit beta; & Q9DCW4 & Etfb & 0,2 & 51,4 \\
\hline Elongation factor 1-alpha 1 & P10126 & Eef1a1 & $-0,2$ & 39,6 \\
\hline Elongation factor 1 -alpha 2 & P62631 & Eef1a2 & 1,9 & 18,1 \\
\hline Elongation factor 1-beta; & 070251 & Eef1b & $-0,3$ & 24,4 \\
\hline Elongation factor 1-delta; & P57776 & Eef1d & $-0,3$ & 34,3 \\
\hline Elongation factor 1-gamma; & Q9D8N0 & Eef1g & $-0,9$ & 24,7 \\
\hline
\end{tabular}




\begin{tabular}{|c|c|c|c|c|}
\hline Protein & Uniprot & Gene name & $\begin{array}{l}\text { Fold change of } \\
\text { XPRESS ratio } \\
\left(\text { Egfr }^{\triangle K C} / \mathrm{WT}\right)\end{array}$ & $\begin{array}{c}\text { Sequence } \\
\text { coverage [\%] }\end{array}$ \\
\hline Elongation factor 2; & P58252 & Eef2 & 0,1 & 42,5 \\
\hline Elongator complex protein 5 & Q99L85 & Elp5 & 0,6 & 15,7 \\
\hline Embigin; & P21995 & Emb & $-0,6$ & 7 \\
\hline Emerin; & 008579 & Emd & 0,2 & 18,9 \\
\hline EMILIN-2; & Q8K482 & Emilin2 & 1,6 & 1,2 \\
\hline Endoglin; & Q63961 & Eng & 0,4 & 2,5 \\
\hline Endonuclease domain-contain ing 1 protein; & Q8C522 & Endod1 & $-0,3$ & 7,6 \\
\hline Endophilin-A2; & Q62419 & Sh3gl1 & $-0,3$ & 2,2 \\
\hline Endoplasmic reticulum aminopeptidase 1 ; & Q9EQH2 & Erap1 & $-0,5$ & 11 \\
\hline Endoplasmic reticulum mannosyl-oligosaccharide 1,2-alpha-mannosidase; & A2AJ15 & Man1b1 & $-0,2$ & 2 \\
\hline Endoplasmic reticulum metallopeptidase 1 & Q3UVKо & Ermp1 & $-0,3$ & 2,8 \\
\hline Endoplasmic reticulum resident protein 29 & P57759 & Erp29 & $-0,4$ & 23,3 \\
\hline Endoplasmic reticulum resident protein 44 & Q9D1Q6 & Erp44 & $-0,5$ & 25,9 \\
\hline Endoplasmic reticulum-Golgi intermediate compartment protein 1 ; & Q9DC16 & Ergic1 & $-0,3$ & 7,9 \\
\hline Endoplasmic reticulum-Golgi intermediate compartment protein 3; & Q9CQE7 & Ergic3 & 0,1 & 8,1 \\
\hline Endoplasmin; & P08113 & Hsp90b1 & $-0,4$ & 31,5 \\
\hline Endosialin; & Q91V98 & $\mathrm{Cd} 248$ & 0,0 & 3,8 \\
\hline Endothelial differentiation-related factor 1 ; & Q9JMG1 & Edf1 & 0,0 & 21,6 \\
\hline Endothelin-converting enzyme 1 & Q4PZA2 & Ece1 & $-0,5$ & 6,5 \\
\hline Enhancer of rudimentary homolog; & P84089 & Erh & 0,2 & 26,9 \\
\hline Enoyl-CoA delta isomerase 1, mitochondrial; & P42125 & Eci1 & 0,2 & 32,5 \\
\hline Enoyl-CoA delta isomerase 2, mitochondrial; & Q9WUR2 & Eci2 & $-0,4$ & 14,3 \\
\hline Enoyl-CoA hydratase, mitochondrial; & Q8BH95 & Echs1 & 0,5 & 8,3 \\
\hline Ensconsin; Microtubule-associated protein 7, isoform CRA_b; & D3YWN7 & Map7 & $-0,7$ & 8,5 \\
\hline Envoplakin; & Q9D952 & Evpl & $-2,4$ & 0,4 \\
\hline Eosinophil cationic-type ribonuclease 5 ; & 035292 & Ear5 & 0,5 & 29,7 \\
\hline Eosinophil peroxidase; & P49290 & Epx & 0,9 & 5 \\
\hline Ephrin-B1; & P52795 & Efnb1 & 1,1 & 5,2 \\
\hline Epidermal growth factor receptor; & Q01279 & Egfr & $-0,6$ & 3,2 \\
\hline Epididymal secretory protein E1; & Q970J0 & Npc2 & $-0,5$ & 24,8 \\
\hline Epiplakin; & Q8ROW0 & Eppk1 & $-0,1$ & 6,2 \\
\hline Epithelial cell adhesion molecule; & Q99JW5 & Epcam & $-0,9$ & 25,7 \\
\hline Epoxide hydrolase 1 & Q9D379 & Ephx1 & $-0,2$ & 18,4 \\
\hline Epoxide hydrolase 3; MCG14254; & G3XA19 & Ephx3 & $-6,4$ & 4,8 \\
\hline Epsin-2; & Q8CHU3 & Epn2 & 0,8 & 3,5 \\
\hline Equilibrative nucleoside transporter 1 ; & Q9JIM1 & Slc29a1 & 0,6 & 8 \\
\hline ER membrane protein complex subunit 1 & Q8C7X2 & Emc1 & $-0,6$ & 4,3 \\
\hline ER membrane protein complex subunit 10 ; & Q3TAS6 & Emc10 & $-0,3$ & 3,9 \\
\hline ER membrane protein complex subunit 7; & Q9EP72 & Emc7 & $-0,6$ & 5,4 \\
\hline Erlin-2; & Q8BFZ9 & Erlin2 & $-0,4$ & 18,2 \\
\hline ERO1-like protein alpha; & Q8R180 & Ero1l & $-0,2$ & 7,1 \\
\hline Erythrocyte band 7 integral membrane protein; & P54116 & Stom & $-0,4$ & 7 \\
\hline ES1 protein homolog, mitochondrial; & Q9D172 & D10Jhu81e & $-0,4$ & 24,4 \\
\hline Ester hydrolase C11orf54 homolog; & Q91V76 & & 0,5 & 31,4 \\
\hline Estradiol 17-beta-dehydrogenase 11 & Q9EQ06 & Hsd17b11 & 0,6 & 6,4 \\
\hline Estradiol 17-beta-dehydrogenase 12 & 070503 & Hsd17b12 & 0,0 & 24 \\
\hline Estradiol 17-beta-dehydrogenase 8; & P50171 & Hsd17b8 & $-0,3$ & 20,1 \\
\hline Ethylmalonyl-CoA decarboxylase; & Q9D9V3 & Echdc1 & 0,7 & 35,1 \\
\hline ETS-related transcription factor Elf-5; & Q8VDK3 & Elf5 & 0,4 & 4,5 \\
\hline Eukaryotic initiation factor $4 \mathrm{~A}-\mathrm{I}$; & P60843 & Eif4a1 & $-0,4$ & 30,8 \\
\hline Eukaryotic initiation factor $4 \mathrm{~A}-\mathrm{II}$; & P10630 & Eif4a2 & $-0,8$ & 23,5 \\
\hline Eukaryotic initiation factor $4 \mathrm{~A}-\mathrm{III}$; & Q91VC3 & Eif4a3 & 0,2 & 34,3 \\
\hline Eukaryotic peptide chain release factor GTP-bind ing subunit ERF3A; & Q8R050 & Gspt1 & $-0,6$ & 7,4 \\
\hline Eukaryotic peptide chain release factor subunit 1 & Q8BWY3 & Etf1 & $-0,7$ & 8,5 \\
\hline Eukaryotic translation initiation factor 1 & P48024 & Eif1 & 0,8 & 42,2 \\
\hline Eukaryotic translation initiation factor $1 \mathrm{~A}$; & Q60872 & Eif1a & $-0,5$ & 14,6 \\
\hline Eukaryotic translation initiation factor 2 subunit 1 ; & Q6ZWX6 & Eif2s1 & $-0,3$ & 52,1 \\
\hline Eukaryotic translation initiation factor 2 subunit 2 & Q99L45 & Eif2s2 & $-0,3$ & 21,1 \\
\hline Eukaryotic translation initiation factor 2 subu nit $3, \mathrm{X}$-linked; & Q9Z0N1 & Eif2s $3 x$ & 0,0 & 43,9 \\
\hline Eukaryotic translation initiation factor $2 \mathrm{~A}$; & Q8BJW6 & Eif2a & 0,2 & 21,7 \\
\hline Eukaryotic translation initiation factor 3 subunit $A$; & P23116 & Eif3a & 0,2 & 4,4 \\
\hline Eukaryotic translation initiation factor 3 subunit B; & Q8JZQ9 & Eif3b & 0,3 & 8,8 \\
\hline Eukaryotic translation initiation factor 3 subunit C; & Q8R1B4 & Eif3c & $-0,7$ & 2,1 \\
\hline Eukaryotic translation initiation factor 3 subunit F; & Q9DCH4 & Eif3f & 0,8 & 9,1 \\
\hline Eukaryotic translation initiation factor 3 subunit G; & Q9Z1D1 & Eif3g & $-0,4$ & 12,8 \\
\hline Eukaryotic translation initiation factor 3 subu nit $\mathrm{H}$; & Q91WK2 & Eif3h & 0,2 & 8 \\
\hline Eukaryotic translation initiation factor 3 subu nit J-A; & Q3UGC7 & Eif3j1 & $-0,1$ & 15,3 \\
\hline Eukaryotic translation initiation factor 3 subunit $\mathrm{K}$; & Q9DBZ5 & Eif3k & $-0,3$ & 5 \\
\hline Eukaryotic translation initiation factor 3 subu nit $M$; & Q99JX4 & Eif3m & $-1,0$ & 7,8 \\
\hline Eukaryotic translation initiation factor 4 gamma 1 ; & Q6NZJ6 & Eif4g1 & 0,2 & 2 \\
\hline
\end{tabular}




\begin{tabular}{|c|c|c|c|c|}
\hline Protein & Uniprot & Gene name & $\begin{array}{l}\text { Fold change of } \\
\text { XPRESS ratio } \\
\left(\text { Egfr }^{\triangle K C} / \mathrm{WT}\right)\end{array}$ & $\begin{array}{c}\text { Sequence } \\
\text { coverage [\%] }\end{array}$ \\
\hline Eukaryotic translation initiation factor $4 \mathrm{~B}$; & Q8BGD9 & Eif4b & $-0,2$ & 15,2 \\
\hline Eukaryotic translation initiation factor $4 \mathrm{E}$ & P63073 & Eif4e & 0,4 & 11,5 \\
\hline Eukaryotic translation initiation factor $4 \mathrm{E}$-binding protein 2 ; & P70445 & Eif4ebp2 & 0,4 & 10,8 \\
\hline Eukaryotic translation initiation factor $4 \mathrm{H}$ & Q9WUK2 & Eif4h & $-0,3$ & 20,2 \\
\hline Eukaryotic translation initiation factor 5 & P59325 & Eif5 & $-0,9$ & 4 \\
\hline Eukaryotic translation initiation factor $5 \mathrm{~A}-1$ & P63242 & Eif5a & $-0,5$ & 19 \\
\hline Eukaryotic translation initiation factor $5 \mathrm{~B}$; & Q05D44 & Eif5b & 1,2 & 2,4 \\
\hline Eukaryotic translation initiation factor 6 & 055135 & Eif6 & 0,5 & 36,7 \\
\hline Exosome complex component RRP4; & Q8VBV3 & Exosc2 & 0,2 & 6,9 \\
\hline Exosome complex component RRP41; & Q92119 & Exosc4 & 0,2 & 9,8 \\
\hline Exosome complex component RRP45; & Q9JHI7 & Exosc9 & 1,1 & 8,4 \\
\hline Exosome complex exonuclease RRP42; & Q9D0M0 & Exosc7 & 1,0 & 13,7 \\
\hline Exportin-1; & Q6P5F9 & Xpo1 & 0,1 & 3 \\
\hline Exportin-2; & Q9ERK4 & Cse1l & 0,3 & 2,6 \\
\hline Extended synaptotagmin-1; & Q3U7R1 & Esyt1 & 1,5 & 4,6 \\
\hline Extracellular matrix protein 1 & Q61508 & Ecm1 & 0,3 & 6,3 \\
\hline Extracellular superoxide dismutase [Cu-Zn]; & 009164 & Sod3 & 1,2 & 5,2 \\
\hline Ezrin; & P26040 & Ezr & $-1,5$ & 10,8 \\
\hline F-actin-capping protein subunit alpha-1; & P47753 & Capza1 & $-0,6$ & 40,9 \\
\hline F-actin-capping protein subunit alpha-2; & P47754 & Capza2 & $-0,4$ & 38,1 \\
\hline Far upstream element-binding protein 1 & Q3TUE1 & Fubp1 & 0,3 & 31,3 \\
\hline Far upstream element-binding protein 2 & Q3U0V1 & Khsrp & 0,1 & 30,3 \\
\hline Farnesyl pyrophosphate synthase; & Q920E5 & Fdps & $-0,1$ & 7,6 \\
\hline FAS-associated factor 2 & Q3TDN2 & Faf2 & $-0,6$ & 13,6 \\
\hline Fascin; & Q61553 & Fscn1 & $-0,1$ & 11,6 \\
\hline Fatty acid 2-hydroxylase; & Q5MPP0 & $\mathrm{Fa} 2 \mathrm{~h}$ & $-0,3$ & 11 \\
\hline Fatty acid desaturase 3 & Q9JJE7 & Fads3 & $-1,3$ & 6 \\
\hline Fatty acid synthase; & P19096 & Fasn & $-0,2$ & 7,5 \\
\hline Fatty acid-binding protein, adipocyte; & P04117 & Fabp4 & $-0,3$ & 48,5 \\
\hline Fatty acid-binding protein, epidermal; & Q05816 & Fabp5 & $-0,6$ & 71,9 \\
\hline Fatty aldehyde dehydrogenase; & P47740 & Aldh3a2 & $-0,5$ & 10,3 \\
\hline F-box only protein 50 & G3X9C2 & Nccrp1 & $-1,0$ & 17,3 \\
\hline F-box only protein 6; & Q9QZN4 & Fbxo6 & $-0,2$ & 15,6 \\
\hline F-box-like/WD repeat-containing protein TBL1XR1; & Q8BHJ5 & Tbl1xr1 & 0,5 & 4,5 \\
\hline Ferritin heavy chain; & P09528 & Fth1 & 1,1 & 6 \\
\hline Ferritin; & Q9CPX4 & Ftl1 & 0,2 & 47 \\
\hline Ferrochelatase, mitochondrial; & P22315 & Fech & $-2,1$ & 5,7 \\
\hline Fetuin-B; & Q9QXC1 & Fetub & 0,7 & 16,6 \\
\hline Fibrillin-1; & A2AQ53 & Fbn1 & 1,2 & 13,9 \\
\hline Fibrillin-2; & Q61555 & Fbn2 & 1,0 & 1,9 \\
\hline Fibrinogen beta chain; & Q8K0E8 & $\mathrm{Fgb}$ & 0,7 & 12,7 \\
\hline Fibrinogen gamma chain; & Q8VCM7 & Fgg & $-0,3$ & 20,2 \\
\hline Fibroblast growth factor-binding protein 1 ; & 070514 & Fgfbp1 & $-0,7$ & 5,2 \\
\hline Fibroleukin; & P12804 & $\mathrm{Fgl2}$ & $-1,4$ & 1,9 \\
\hline Fibronectin leucine rich transmembrane protein 1; MCG52071; Protein Flrt1; & Q6RKD8 & Flrt1 & $-0,6$ & 1,2 \\
\hline Fibronectin; & P11276 & Fn1 & 0,2 & 4,2 \\
\hline Fibulin-1; & Q08879 & Fbln1 & 0,3 & 11,8 \\
\hline Fibulin-2; & P37889 & Fbln2 & 0,9 & 10,4 \\
\hline Fibulin-5; & Q9WVH9 & Fbln5 & 0,3 & 21,2 \\
\hline Filaggrin-2; & E9QPZ3 & Flg2 & $-2,5$ & 13,4 \\
\hline Filaggrin-2; & Q2VIS4 & Flg2 & $-0,7$ & 12,5 \\
\hline Filamin-A; & Q8BTM8 & Flna & $-0,2$ & 18,8 \\
\hline Filamin-B; & Q80X90 & Flnb & $-0,6$ & 16,3 \\
\hline Filamin-binding LIM protein 1 & Q71FD7 & Fblim1 & $-0,4$ & 8,8 \\
\hline Filamin-C; & Q8VHX6 & Flnc & 4,4 & 2,7 \\
\hline Flotillin-1; & 008917 & Flot1 & 0,0 & 11,2 \\
\hline Flotillin-2; & Q60634 & Flot2 & 0,0 & 12,9 \\
\hline Focal adhesion kinase 1 ; & P34152 & Ptk2 & $-3,4$ & 1,7 \\
\hline Follistatin-related protein 1 & Q62356 & Fstl1 & 1,2 & 10,5 \\
\hline Forkhead box protein $\mathrm{O} 3$; & Q9WVH4 & Foxo3 & $-1,7$ & 30,1 \\
\hline Fos-related antigen 2 & P47930 & Fosl2 & $-1,2$ & 10,7 \\
\hline Four and a half LIM domains protein 1 ; & P97447 & Fhl1 & 0,9 & 47,1 \\
\hline Four and a half LIM domains protein 2; & 070433 & Fhl2 & $-0,1$ & 17,9 \\
\hline Four and a half LIM domains protein 3 ; & Q9R059 & Fhl3 & 1,3 & 12,5 \\
\hline Frizzled-1; & 070421 & Fzd1 & 0,0 & 3,4 \\
\hline Frizzled-2; & Q9JIP6 & Fzd2 & 0,1 & 4,7 \\
\hline Frizzled-7; & Q61090 & Fzd7 & 0,0 & 4,7 \\
\hline Fructose-1,6-bisphosphatase 1 & Q9QXD6 & Fbp1 & $-0,3$ & 19,8 \\
\hline Fructose-bisphosphate aldolase A; & P05064 & Aldoa & $-0,1$ & 56,9 \\
\hline Fructose-bisphosphate aldolase C; & P05063 & Aldoc & $-0,2$ & 15,2 \\
\hline
\end{tabular}




\begin{tabular}{|c|c|c|c|c|}
\hline Protein & Uniprot & Gene name & $\begin{array}{l}\text { Fold change of } \\
\text { XPRESS ratio } \\
\left(\text { Egfr }^{\Delta K C} / \mathrm{WT}\right)\end{array}$ & $\begin{array}{c}\text { Sequence } \\
\text { coverage [\%] }\end{array}$ \\
\hline Fumarylacetoacetase; & P35505 & Fah & 0,4 & 18,1 \\
\hline FUN14 domain-containing protein 2; & Q9D6K8 & Fundc2 & $-0,9$ & 15,2 \\
\hline Furin; & P23188 & Furin & $-0,2$ & 1,3 \\
\hline G patch domain-containing protein 8 & $\mathrm{~A} 2 \mathrm{~A} 6 \mathrm{~A} 1$ & Gpatch8 & 0,0 & 0,7 \\
\hline G1/S-specific cyclin-D3; & P30282 & Ccnd3 & 0,7 & 15,8 \\
\hline Galactokinase; & Q9R0NO & Galk1 & 0,8 & 18,6 \\
\hline Galactose-1-phosphate uridylyltransferase; & Q03249 & Galt & $-0,9$ & 10,6 \\
\hline Galectin; & Q9CRB1 & Lgals7 & $-0,5$ & 64,7 \\
\hline Galectin-1; & P16045 & Lgals1 & 0,4 & 51,1 \\
\hline Galectin-3; & P16110 & Lgals3 & $-0,4$ & 18,6 \\
\hline Galectin-related protein; & Q8VED9 & Lgalsl & $-0,2$ & 18,6 \\
\hline Gamma-adducin; & Q9QYB5 & Add3 & 0,6 & 19,8 \\
\hline Gamma-glutamyl hydrolase; & Q9ZOL8 & Ggh & $-0,3$ & 21,5 \\
\hline Gamma-glutamylcyclotransferase; & Q9D7X8 & Ggct & $-2,5$ & 42 \\
\hline Gamma-glutamyltranspeptidase 1 ; & Q60928 & Ggt1 & $-0,1$ & 6 \\
\hline Gamma-interferon-inducible lysosomal thiol reductase; & Q9ESY9 & Ifi30 & $-1,0$ & 9,7 \\
\hline Gamma-synuclein; & Q9Z0F7 & Sncg & $-0,2$ & 47,2 \\
\hline Ganglioside GM2 activator; & Q60648 & Gm2a & $-0,4$ & 14,5 \\
\hline Gap junction alpha-1 protein; & P23242 & Gja1 & $-1,1$ & 29,8 \\
\hline Gap junction beta-3 protein; & P28231 & Gjb3 & $-0,6$ & 5,6 \\
\hline Gasdermin-A; & Q9EST1 & Gsdma & $-1,5$ & 22,9 \\
\hline GDH/6PGL endoplasmic bifunctional protein; & Q8CFX1 & H6pd & 0,5 & 5,8 \\
\hline GDNF family receptor alpha-2; & 008842 & Gfra2 & 0,7 & 1,9 \\
\hline GDP-fucose protein O-fucosyltransferase 1 & Q91ZW2 & Pofut1 & $-0,7$ & 10,5 \\
\hline GDP-L-fucose synthase; & P23591 & Tsta3 & 0,4 & 6,9 \\
\hline GDP-mannose 4,6 dehydratase; & Q8K0C9 & Gmds & 0,3 & 5,4 \\
\hline Gelsolin; & P13020 & Gsn & 0,1 & 26,2 \\
\hline Gelsolin; & A6PWS5 & Gsn & $-0,8$ & 11,2 \\
\hline General transcription factor IIF subunit 1 ; & Q3THK3 & Gtf2f1 & $-0,2$ & 10 \\
\hline General transcription factor IIF subunit 2; & Q8R0A0 & Gtf2f2 & $-0,1$ & 2,8 \\
\hline General vesicular transport factor $\mathrm{p} 115$ & Q9Z1Z0 & Uso1 & 1,5 & 5,6 \\
\hline Gephyrin; & Q8BUV3 & Gphn & $-0,2$ & 4,7 \\
\hline Geranylgeranyl pyrophosphate synthase; & Q9WTN0 & Ggps1 & 0,0 & 7,3 \\
\hline Glia maturation factor beta; & Q9CQ13 & $\mathrm{Gmfb}$ & $-0,3$ & 14,1 \\
\hline Glucocorticoid receptor; & P06537 & $\mathrm{Nr3c1}$ & 1,2 & 2,9 \\
\hline Glucosamine-6-phosphate isomerase 1; & 088958 & Gnpda1 & 0,7 & 17 \\
\hline Glucose-6-phosphate 1-dehydrogenase X; & Q00612 & G6pdx & $-0,6$ & 11,8 \\
\hline Glucose-6-phosphate isomerase; & P06745 & Gpi & 0,1 & 19,7 \\
\hline Glucosidase 2 subunit beta; & 008795 & Prkcsh & $-0,2$ & 17,1 \\
\hline Glucosylceramidase; & P17439 & Gba & 0,0 & 8,5 \\
\hline Glutamate dehydrogenase 1, mitochondrial; & P26443 & Glud1 & 0,0 & 39,8 \\
\hline Glutamine synthetase; & P15105 & Glul & $-0,2$ & 7 \\
\hline Glutamyl aminopeptidase; & P16406 & Enpep & 3,2 & 4,3 \\
\hline Glutaredoxin-1; & Q9QUHO & Glrx & 0,2 & 10,3 \\
\hline Glutaredoxin-3; & Q9CQM9 & Glrx3 & $-0,2$ & 9,8 \\
\hline Glutathione peroxidase 1; & P11352 & Gpx1 & 0,2 & 60,2 \\
\hline Glutathione peroxidase 3; & P46412 & Gpx3 & 0,3 & 23,1 \\
\hline Glutathione peroxidase 7; & Q99L6 & Gpx7 & 0,3 & 20,4 \\
\hline Glutathione reductase, mitochondrial; & P47791 & Gsr & 0,3 & 13,4 \\
\hline Glutathione S-transferase Mu 1; & P10649 & Gstm1 & $-1,1$ & 33,9 \\
\hline Glutathione S-transferase Mu 2; & P15626 & Gstm2 & 0,0 & 44 \\
\hline Glutathione S-transferase Mu 3; & P19639 & Gstm3 & 0,1 & 10,6 \\
\hline Glutathione S-transferase Mu 5; & P48774 & Gstm5 & $-0,4$ & 11,4 \\
\hline Glutathione S-transferase omega-1; & 009131 & Gsto1 & 0,4 & 25,8 \\
\hline Glutathione S-transferase P 1; & P19157 & Gstp1 & 0,4 & 34,3 \\
\hline Glutathione synthetase; & P51855 & Gss & 0,4 & 15,8 \\
\hline Glyceraldehyde-3-phosphate dehydrogenase; & P16858 & Gapdh & 0,6 & 33,3 \\
\hline Glycerol-3-phosphate dehydrogenase [NAD(+)], cytoplasmic; & P13707 & Gpd1 & $-0,1$ & 26,7 \\
\hline Glycerol-3-phosphate dehydrogenase, mitochondrial; & Q64521 & Gpd2 & 0,0 & 5 \\
\hline Glycerophosphodiester phosphodiesterase domain-containing protein 3; & Q99LY2 & Gdpd3 & $-1,1$ & 7 \\
\hline Glycine cleavage system $\mathrm{H}$ protein, mitochondrial; & Q91WK5 & Gcsh & $-0,2$ & 5,9 \\
\hline Glycine N-methyltransferase; & Q9QXF8 & Gnmt & $-1,8$ & 4,1 \\
\hline Glycine--tRNA ligase; & Q9CZD3 & Gars & 0,3 & 21,7 \\
\hline Glycogen phosphorylase, brain form; & Q8C194 & Pygb & $-0,9$ & 4 \\
\hline Glycogen phosphorylase, liver form; & Q9ET01 & Pygl & $-1,2$ & 4,5 \\
\hline Glycogen synthase kinase-3 beta; & Q9WV60 & Gsk3b & 0,4 & 11,7 \\
\hline Glycylpeptide N-tetradecanoyltransferase 1; & 070310 & Nmt1 & 0,1 & 6,3 \\
\hline Glyoxalase domain-containing protein 4 & Q9CPV4 & Glod4 & 0,3 & 12,9 \\
\hline Glyoxylate reductase/hydroxypyruvate reductase; & Q91Z53 & Grhpr & 0,1 & 25,3 \\
\hline Glypican-1; & Q9QZF2 & Gpc1 & 0,3 & 5,9 \\
\hline
\end{tabular}




(20)

\section{Protein}

Uniprot Gene name

Fold change of

Glypican-4;

P51655

P51655

Q99L27

GMP reductase 2;

Q61543

Golgi integral membrane protein 4;

Golgi phosphoprotein 3;

Golgi SNAP receptor complex member 1 ;

Golgin subfamily A member 5;

GPALPP motifs-containing protein 1;

GPI inositol-deacylase;

G-protein coupled receptor 56 ;

G-protein coupled receptor family C group 5 member D;

Granulins;

Growth factor receptor-bound protein 2;

Growth hormone-inducible transmembrane protein;

GTP:AMP phosphotransferase AK3, mitochondrial;

GTPase HRas;

GTPase NRas;

GTP-binding nuclear protein Ran;

D3YVW2

088630

Q9QYE6

Q69ZC8

Q3UUQ7

Q8K209

Q9JIL6

P28798

Q60631

Q91VC9

Q9WTP7

Q61411

P08556

P62827

GTP-binding protein 1;

GTP-binding protein SAR1a; SAR1 gene homolog A (S. cerevisiae); SAR1 gene

008582

homolog A (S. cerevisiae), isoform CRA_b;

Guanine nucleotide-binding protein $\mathrm{G}(\mathrm{i})$ subunit alpha-1;

Guanine nucleotide-binding protein $\mathrm{G}(\mathrm{i})$ subunit alpha-2;

Guanine nucleotide-binding protein $\mathrm{G}(\mathrm{I}) / \mathrm{G}(\mathrm{S}) / \mathrm{G}(\mathrm{O})$ subunit gamma-12;

Guanine nucleotide-binding protein $\mathrm{G}(\mathrm{I}) / \mathrm{G}(\mathrm{S}) / \mathrm{G}(\mathrm{O})$ subunit gamma-2;

Guanine nucleotide-binding protein $\mathrm{G}(\mathrm{I}) / \mathrm{G}(\mathrm{S}) / \mathrm{G}(\mathrm{O})$ subunit gamma-5;

Guanine nucleotide-binding protein $G(I) / G(S) / G(T)$ subunit beta-1;

Guanine nucleotide-binding protein $\mathrm{G}(\mathrm{I}) / \mathrm{G}(\mathrm{S}) / \mathrm{G}(\mathrm{T})$ subunit beta-2;

Guanine nucleotide-binding protein $\mathrm{G}(\mathrm{k})$ subunit alpha;

Guanine nucleotide-binding protein $\mathrm{G}(\mathrm{s})$ subunit alpha isoforms short;

Guanine nucleotide-binding protein subunit alpha-13;

Guanine nucleotide-binding protein subunit beta-2-like 1;

H/ACA ribonucleoprotein complex subunit 1;

H/ACA ribonucleoprotein complex subunit 2;

H/ACA ribonucleoprotein complex subunit 4;

$\mathrm{H}-2$ class I histocompatibility antigen, D-B alpha chain;

$\mathrm{H}-2$ class I histocompatibility antigen, K-B alpha chain;

$\mathrm{H}-2$ class I histocompatibility antigen, L-D alpha chain;

$\mathrm{H}-2$ class II histocompatibility antigen gamma chain;

$\mathrm{H}-2$ class II histocompatibility antigen, A beta chain;

$\mathrm{H}-2$ class II histocompatibility antigen, $\mathrm{A}-\mathrm{B}$ alpha chain;

$\mathrm{H}-2$ class II histocompatibility antigen, A-Q alpha chain;

$\mathrm{H}-2$ class II histocompatibility antigen, $\mathrm{A}-\mathrm{Q}$ beta chain;

Haloacid dehalogenase-like hydrolase domain-containing protein 2;

Haloacid dehalogenase-like hydrolase domain-containing protein 3;

Haptoglobin;

HD domain-containing protein 2;

HEAT repeat-containing protein 6 ;

Heat shock $70 \mathrm{kDa}$ protein $12 \mathrm{~A}$;

Heat shock $70 \mathrm{kDa}$ protein $1 \mathrm{~A}$;

Heat shock 70 kDa protein 1-like;

Heat shock 70 kDa protein 4;

Heat shock cognate 71 kDa protein;

Heat shock factor-binding protein 1;

Heat shock protein beta-1;

Heat shock protein HSP 90-alpha;

Heat shock protein HSP 90-beta;

Heat shock-related $70 \mathrm{kDa}$ protein 2;

Hematological and neurological expressed 1 protein;

Hematological and neurological expressed 1-like protein;

Q99JZ4

B2RSH2

P08752

Q9DAS9

P63213

Q80SZ7

P62874

P62880

Q9DC51

P63094

P27601

P68040

Q9CY66

Q9CRB2

Q9ESX5

P01899

P01901

P01897

P04441

P14483

P14434

P04227

P06342

Q3UGR5

Q9CYW4

Q61646

Q3SXD3

Q6P1G0

Q8K0U4

Q61696

P16627

Q61316

P63017

Q9CQZ1

P14602

P07901

P11499

P17156

P97825

Q6PGH2

P49710

Q64314

Hematopoietic progenitor cell antigen CD34;

A2AJ76

P04444

Hemoglobin subunit beta-H1;

Hemopexin;

Hepatoma-derived growth factor;

Hepatoma-derived growth factor-related protein 2;

Hepatoma-derived growth factor-related protein 3;

Hephaestin;

Hephaestin-like protein 1 ;

Heterochromatin protein 1-binding protein 3;

Q91X72

P51859

Q3UMU9

Q9JMG7

Q9Z0Z4

Q3V1H3

Q3TEA8

Gpc4

XPRESS ratio

Sequence

coverage [\%]

$\begin{array}{ccc}\text { Gpc4 } & -0,5 & 24,4 \\ \text { Gmpr2 } & 0,5 & 4,6\end{array}$

Glg1

Golim4

Golph3

Gosr1

Golga5

Gpalpp1

Pgap1

Gpr56

Gprc5d

Grn

Grb2

Ghitm

Ak3

Hras

Nras

Ran

Gtpbp1

0,0

0,0

0,3

$-0,2$

0,1

0,5

$-1,7$

$-0,1$

$-4,8$

$-0,9$

$-0,9$
0,0

$-0,4$

$-0,1$

$-0,1$
$-0,1$

0,0

0,4

Sar1a

1,5

0,9

Gnai1

Gnai2

Gng12

Gng2

Gng5

Gnb1

Gnb2

Gnai3

Gnas

Gna13

Gnb2l1

Gar1

Nhp2

Dkc1

H2 D1

H2 K1

$\mathrm{H} 2 \mathrm{~L}$

Cd74

H2 Ab1

$\mathrm{H} 2 \mathrm{Aa}$

$\mathrm{H} 2 \mathrm{Aa}$

$\mathrm{H} 2 \mathrm{Ab} 1$

Hdhd2

Hdhd3

$\mathrm{Hp}$

Hddc2

Heatr6

Hspa12a

Hspa1a

Hspa1l

Hspa4

Hspa8

Hsbp1

Hspb1

Hsp90aa1

Hsp90ab1

Hspa2

$\mathrm{Hn} 1$

Hn1l

Hcls1

Cd34

Hmcn2

Hbb bh1

$\mathrm{Hpx}$

Hdgf

Hdgfrp2

Hdgfrp3

Heph

Hephl1

Hp1bp3 


\begin{tabular}{|c|c|c|c|c|}
\hline Protein & Uniprot & Gene name & $\begin{array}{l}\text { Fold change of } \\
\text { XPRESS ratio } \\
\left(\text { Egfr }^{\triangle K C} / \mathrm{WT}\right)\end{array}$ & $\begin{array}{c}\text { Sequence } \\
\text { coverage [\%] }\end{array}$ \\
\hline Heterogeneous nuclear ribon ucleoprotein A/B; & Q99020 & Hnrnpab & 0,3 & 20 \\
\hline Heterogeneous nuclear ribonucleoprotein $\mathrm{A} 0$; & Q9CX86 & Hnrnpa0 & 0,4 & 23,6 \\
\hline Heterogeneous nuclear ribon ucleoprotein A1; & P49312 & Hnrnpa1 & 0,4 & 30 \\
\hline Heterogeneous nuclear ribon ucleoprotein A3; & Q8BG05 & Hnrnpa3 & $-0,3$ & 32 \\
\hline Heterogeneous nuclear ribon ucleoprotein D0; & Q60668 & Hnrnpd & $-0,2$ & 22,7 \\
\hline Heterogeneous nuclear ribon ucleoprotein D-like; & Q9Z130 & Hnrnpdl & 0,4 & 14 \\
\hline Heterogeneous nuclear ribonucleoprotein F; & Q9Z2X1 & Hnrnpf & 0,3 & 24,3 \\
\hline Heterogeneous nuclear ribon ucleoprotein $\mathrm{H}$; & 035737 & Hnrnph1 & 0,4 & 22,7 \\
\hline Heterogeneous nuclear ribonucleoprotein K; & B2M1R6 & Hnrnpk & 0,3 & 40,2 \\
\hline Heterogeneous nuclear ribonucleoprotein L; & Q8R081 & Hnrnpl & 0,3 & 18,4 \\
\hline Heterogeneous nuclear ribon ucleoprotein L-like; & Q921F4 & Hnrnpll & 0,6 & 2,5 \\
\hline Heterogeneous nuclear ribon ucleoprotein M; & Q9D0E1 & Hnrnpm & 0,5 & 15,6 \\
\hline Heterogeneous nuclear ribon ucleoprotein Q; & Q7TМК9 & Syncrip & $-0,1$ & 19,9 \\
\hline Heterogeneous nuclear ribon ucleoprotein R; Protein Hnrnpr; & Q8VHM5 & Hnrnpr & 0,3 & 13,8 \\
\hline Heterogeneous nuclear ribon ucleoprotein U; & Q8VEK3 & Hnrnpu & 0,3 & 17,9 \\
\hline Heterogeneous nuclear ribon ucleoprotein U-like protein 2; & Q00PI9 & Hnrnpul2 & $-0,1$ & 12,8 \\
\hline Heterogeneous nuclear ribon ucleoproteins A2/B1; & 088569 & Hnrnpa2b1 & 0,2 & 23,5 \\
\hline Heterogeneous nuclear ribon ucleoproteins $\mathrm{C} 1 / \mathrm{C} 2$; & Q9Z204 & Hnrnpc & 0,1 & 22,7 \\
\hline Hexokinase-1; & P17710 & Hk1 & $-0,2$ & 7,5 \\
\hline High affinity cationic amino acid transporter 1 & Q09143 & Slc7a1 & 0,7 & 5,5 \\
\hline High mobility group nucleosome-binding domain-containing protein 3 ; & Q9DCB1 & Hmgn3 & 1,7 & 32,3 \\
\hline High mobility group nucleosome-bind ing domain-containing protein 5 ; & Q9JL35 & Hmgn5 & $-0,4$ & 5,7 \\
\hline High mobility group protein $\mathrm{B} 1$ & P63158 & Hmgb1 & $-0,1$ & 17,7 \\
\hline High mobility group protein B2; & P30681 & Hmgb2 & 0,1 & 31 \\
\hline High mobility group protein B3; & 054879 & Hmgb3 & 0,0 & 24,5 \\
\hline High mobility group protein HMG-I/HMG-Y; & P17095 & Hmga1 & $-0,6$ & 8,4 \\
\hline Hippocalcin-like protein 1 & P62748 & Hpcal1 & $-0,2$ & 23,8 \\
\hline HIRA-interacting protein 3 ; & Q8BLH7 & Hirip3 & 0,7 & 12,7 \\
\hline Histidine ammonia-lyase; & P35492 & $\mathrm{Hal}$ & $-1,2$ & 35,2 \\
\hline Histidine triad nucleotide-binding protein 1 & P70349 & Hint1 & 0,5 & 51,6 \\
\hline Histidine triad nucleotide-binding protein 2 , mitochondrial; & Q9D0S9 & Hint2 & 0,6 & 21,5 \\
\hline Histidine triad nucleotide-binding protein 3 ; & Q9CPS6 & Hint3 & $-0,2$ & 15,2 \\
\hline Histidine-rich glycoprotein; & Q9ESB3 & $\mathrm{Hrg}$ & 0,3 & 3,4 \\
\hline Histidine--tRNA ligase, cytoplasmic; & Q61035 & Hars & 0,4 & 17,9 \\
\hline Histone deacetylase 1 & 009106 & Hdac1 & $-1,1$ & 7,9 \\
\hline Histone deacetylase complex subunit SAP25; & E9Q529 & Sap25 & $-1,9$ & 8,4 \\
\hline Histone H1.0; & P10922 & H1fo & 0,9 & 20,6 \\
\hline Histone H1.1; & P43275 & Hist1h1a & 0,4 & 35,7 \\
\hline Histone H1.2; & P15864 & Hist1h1c & 0,1 & 30,2 \\
\hline Histone H1.3; & P43277 & Hist1h1d & 1,1 & 29 \\
\hline Histone $\mathrm{H} 1.4$; & P43274 & Hist1h1e & 0,8 & 29,2 \\
\hline Histone H1.5; & P43276 & Hist1h1b & 1,0 & 28,7 \\
\hline Histone H2A type 1 ; & P22752 & Hist1h2ao & $-0,3$ & 32,6 \\
\hline Histone $\mathrm{H} 2 \mathrm{~A}$ type $2-\mathrm{B}$; & Q64522 & Hist2h2ab & $-0,8$ & 17,7 \\
\hline Histone $\mathrm{H} 2 \mathrm{~B}$ type $1-\mathrm{A}$; & P70696 & Hist1h2ba & $-0,5$ & 39,4 \\
\hline Histone $\mathrm{H} 2 \mathrm{~B}$ type $1-\mathrm{F} / \mathrm{J} / \mathrm{L}$; & P10853 & Hist1h2bn & $-0,4$ & 46,8 \\
\hline Histone H2B type 2-E; & Q64524 & Hist2h2be & 0,4 & 41,3 \\
\hline Histone H3.3C; & P02301 & $H 3 f 3 c$ & $-0,2$ & 19,3 \\
\hline Histone H4; & P62806 & Hist4h4 & $-0,3$ & 50,5 \\
\hline Histone lysine demethylase PHF8; & Q80TJ7 & Phf8 & 4,5 & 2,8 \\
\hline Histone-binding protein RBBP4; & Q60972 & Rbbp4 & 0,0 & 24 \\
\hline Histone-binding protein RBBP7; & Q60973 & Rbbp7 & 0,3 & 19 \\
\hline HIV Tat-specific factor 1 homolog; & Q8BGC0 & Htatsf1 & $-0,3$ & 1,1 \\
\hline Homeodomain-only protein; & Q8R1H0 & Hopx & 0,2 & 43,8 \\
\hline Hormone-sensitive lipase; & P54310 & Lipe & 0,4 & 7 \\
\hline Hornerin; & E9QNP3 & Hrnr & $-2,8$ & 13,2 \\
\hline Host cell factor 1 ; & Q61191 & Hcfc1 & 0,4 & 3,3 \\
\hline Hsc70-interacting protein; & Q99L47 & St13 & $-0,6$ & 14,4 \\
\hline Hsp90 co-chaperone Cdc37; & Q61081 & Cdc37 & $-0,3$ & 11,3 \\
\hline Huntingtin-interacting protein $\mathrm{K}$; & Q9CR41 & Hypk & $-2,8$ & 24,8 \\
\hline Hydroxyacyl-coenzyme A dehydrogenase, mitochondrial; & Q61425 & Hadh & $-0,2$ & 23,9 \\
\hline Hydroxyacylglutathione hydrolase, mitochondrial; & Q99KB8 & Hagh & 0,3 & 22,1 \\
\hline Hydroxymethylglutaryl-CoA synthase, cytoplasmic; & Q8JZK9 & Hmgcs1 & 0,4 & 18,3 \\
\hline Hydroxysteroid dehydrogenase-like protein 2; & Q2TPA8 & $\mathrm{Hsdl} 2$ & 0,6 & 2,4 \\
\hline Hypoxanthine-guanine phosphoribosyltransferase; & Р00493 & Hprt1 & 0,1 & 49,5 \\
\hline Hypoxia up-regulated protein 1 & Q9JKR6 & Hyou1 & $-0,3$ & 17,7 \\
\hline Ig gamma-1 chain $C$ region secreted form; & P01868 & lghg1 & 0,0 & 3,7 \\
\hline Ig gamma-2A chain $C$ region secreted form; & P01864 & & 0,0 & 9,9 \\
\hline Ig gamma-2B chain $\mathrm{C}$ region; & P01867 & $\operatorname{lgh} 3$ & 0,3 & 14,9 \\
\hline Ig gamma-3 chain C region; & P03987 & & 0,2 & 15,6 \\
\hline Ig heavy chain $V$ region 6.96 & P18528 & & 0,0 & 19,4 \\
\hline
\end{tabular}




Protein

\section{Protein}

Uniprot Gene name

Fold change of
XPRESS ratio

Sequence $\left(\right.$ Egfr $\left.^{\Delta K C} / \mathrm{WT}\right)$ coverage [\%]

Ig heavy chain $\mathrm{V}$ region AC38 205.12;

P06330

P01837

Ig kappa chain $C$ region;

Ig kappa chain $\mathrm{V}$-II region 26-10;

IgG receptor FcRn large subunit p51;

Immediate early response 3 -interacting protein 1 ;

Immunoglobulin superfamily containing leucine-rich repeat protein;

P01631

Q61559

Q9CR20

Q6GU68

Importin subunit alpha-3;

035343

Importin subunit alpha-7;

Importin subunit beta-1;

035345

P70168

Importin-5;

Inactive hydroxysteroid dehydrogenase-like protein 1;

Q8BKC5

Q8BTX9

Inactive tyrosine-protein kinase 7;

Inhibitor of carbonic anhydrase;

Inhibitor of nuclear factor kappa-B kinase-interacting protein;

Q8BKG3

Q9DBD0

Q9DBZ1

Inorganic pyrophosphatase 2, mitochondrial;

Q91VM9

Inorganic pyrophosphatase;

Inosine triphosphate pyrophosphatase;

Inosine-5'-monophosphate dehydrogenase 2;

Q9D819

Q9D892

P24547

Inositol (Myo)-1(Or 4)-monophosphatase 1; Inositol (Myo)-1(Or 4)-

monophosphatase 1, isoform CRA_a; Inositol monophosphatase 1; Myo-

Q924B0

0,5

0,0

$-1,7$

Fcgrt

ler3ip1

0,7

0,7
$-0,2$

$-0,2$
1,1

Kpna4

Kpna6

Kpnb1

Ipo5

Hsdl1

Ptk7

Ica

Ikbip

Ppa2

Ppa1

Itpa

Impdh2

1,1
$-0,3$

$-0,3$

0,1

$-0,1$

$-0,4$

$-0,4$

0,3

1,9

0,3

$-0,1$

$-0,1$

$-0,1$
0,4

Impa1

0,0

Q91UZ5

Q80V26

P49442

Q9JHU9

F6RPJ9

P47879

Q61581

089051

Q3V3R4

P61622

Q62470

P11688

Q61739

P43406

P09055

P11835

054890

070309

055222

A6X935

Q61702

Q61703

Q61704

Q8BJD1

P13597

E9Q1P8

Q99J93

Q9CXY6

Q9Z1X4

E9QLA5

G3X9D9

Q8BX37

Q9DB29

Q8C0M9

Q9D7B6

P85094

Q9D6R2

P70404

088844

P54071

Impa2

Impad1

Inpp1

Isyna1

Ide

Igfbp4

Igfbp7

Itm2b

Itga1

Itga11

Itga3

Itga5

Itga6

Itgav

Itgb1

Itgb2

Itgb3

Itgb5

Ilgb5

Itih4

Itih1

Itih2

Itih3

Itih5

Icam 1

Irf2bp2

Ifitm2

IIf2

IIf3

Inf2

Ivl

Papl

lah1

Asrgl1

Acad8

Isoc2a

Idh3a

Idh3g

Idh1

Idh2

Q91VA7

Idh3b

Q8BIJ6

P58044

Q9JHI5

Q02257

088792

Q9D140

Q91Y82

Q99M20

lars2

Idi1

Ivd

Jup

F11r

KIk5

KIk6

Kallikrein 6, isoform CRA a; Kallikrein-1; Klk6 protein; Neurosin;

Kallikrein related-peptidase 10; Protein Klk10;

24,6

13,2

13,2

17,7

34,1

6,1

2,7

4,8

7,2

5,9

3,9

11,8

9,1

9,7

20,4

38,1

33,8

13,4

34,7

$-2,2$

0,1

0,2

$-0,1$

$-0,9$

1,0

0,8

$-0,6$

0,5

$-1,2$

$-0,9$

0,9

0,3

0,3
0,6

0,2

0,3

$-2,8$

0,2

$-4,4$

$-0,1$

$-3,4$

$-0,2$

$-0,3$

2,6

0,1

$-0,6$

1,2 


\begin{tabular}{|c|c|c|c|c|}
\hline Protein & Uniprot & Gene name & $\begin{array}{l}\text { Fold change of } \\
\text { XPRESS ratio } \\
\left(E g f r^{\Delta K C} / \mathrm{WT}\right)\end{array}$ & $\begin{array}{c}\text { Sequence } \\
\text { coverage [\%] }\end{array}$ \\
\hline Kallikrein-7; & Q91VE3 & Klk7 & $-0,5$ & 27,3 \\
\hline Keratin, type I cuticular Ha1; & Q61765 & Krt31 & $-3,1$ & 15,4 \\
\hline Keratin, type I cytoskeletal 10; & P02535 & Krt10 & $-2,1$ & 27,3 \\
\hline Keratin, type I cytoskeletal 14 & Q61781 & Krt14 & $-0,2$ & 17,8 \\
\hline Keratin, type I cytoskeletal 15 ; & Q61414 & Krt15 & $-1,1$ & 17,5 \\
\hline Keratin, type I cytoskeletal 17; & Q9QWL7 & Krt17 & $-1,2$ & 27,3 \\
\hline Keratin, type I cytoskeletal 25 ; & Q8VCW2 & Krt25 & $-1,8$ & 36,3 \\
\hline Keratin, type I cytoskeletal 26; & Q3TRJ4 & Krt26 & $-4,4$ & 6,7 \\
\hline Keratin, type I cytoskeletal 27 & Q9Z320 & Krt27 & $-3,2$ & 36,6 \\
\hline Keratin, type I cytoskeletal 28; & A6BLY7 & Krt28 & $-0,9$ & 22,3 \\
\hline Keratin, type II cuticular Hb6; & P97861 & Krt86 & $-3,4$ & 18,7 \\
\hline Keratin, type II cytoskeletal 1; & P04104 & Krt1 & $-2,6$ & 21,4 \\
\hline Keratin, type II cytoskeletal 1b; & Q6IFZ6 & Krt77 & $-1,2$ & 31,6 \\
\hline Keratin, type II cytoskeletal 2 epidermal; & Q3TTY5 & Krt2 & 0,9 & 5,4 \\
\hline Keratin, type II cytoskeletal 2 oral; & Q3UV17 & Krt76 & $-0,8$ & 5,7 \\
\hline Keratin, type II cytoskeletal 5; & Q922U2 & Krt5 & $-1,1$ & 20,3 \\
\hline Keratin, type II cytoskeletal 6A; & P50446 & Krt6a & $-1,4$ & 12,3 \\
\hline Keratin, type II cytoskeletal 71; & Q9ROH5 & Krt71 & $-2,6$ & 40,6 \\
\hline Keratin, type II cytoskeletal 72; & Q6IME9 & Krt72 & $-3,8$ & 15,8 \\
\hline Keratin, type II cytoskeletal 73; & Q6NXH9 & Krt73 & $-0,7$ & 21,2 \\
\hline Keratin, type II cytoskeletal 75; & Q8BGZ7 & Krt75 & $-1,5$ & 17,8 \\
\hline Keratin, type II cytoskeletal 79; & Q8VED5 & Krt79 & $-0,7$ & 17,1 \\
\hline Keratin-associated protein $15-1$ & Q9QZU5 & Krtap15 1 & $-4,1$ & 24,7 \\
\hline Keratin-associated protein $16-1$ & A2A5X5 & Krtap16 1 & $-3,4$ & 6,2 \\
\hline Keratin-associated protein $19-5$ & F8VQ65 & Krtap19 5 & 0,0 & 19,4 \\
\hline Keratin-associated protein $3-1$; & A2A591 & Krtap3 1 & $-2,2$ & 17,3 \\
\hline Keratinocyte-associated transmembrane protein 2; & Q8K201 & Kct2 & 0,3 & 3,9 \\
\hline Ketimine reductase mu-crystallin; & 054983 & Crym & $-1,5$ & 41,5 \\
\hline Ketosamine-3-kinase; & Q8K274 & Fn3krp & 0,2 & 2,6 \\
\hline KH domain-containing, RNA-binding, signal transduction-associated protein 1; & Q60749 & Khdrbs1 & 0,5 & 4,5 \\
\hline Kinectin; & Q61595 & Ktn1 & $-0,4$ & 7,8 \\
\hline Kinesin-1 heavy chain; & Q61768 & Kif5b & $-1,9$ & 4,7 \\
\hline Kinesin-like protein KIF3B; & Q61771 & Kif3b & $-0,3$ & 2,1 \\
\hline Kininogen-1; & 008677 & Kng1 & 1,2 & 29,2 \\
\hline Kunitz-type protease inhibitor 1 ; & Q9R097 & Spint1 & $-0,7$ & 10,8 \\
\hline Kynurenine--oxoglutarate transaminase 1 ; & Q8BTY1 & Ccbl1 & $-0,1$ & 14,4 \\
\hline Kynurenine--oxoglutarate transaminase 3 ; & Q71RI9 & $\mathrm{Ccbl} 2$ & $-0,2$ & 11,2 \\
\hline Lactadherin; & P21956 & Mfge8 & $-0,9$ & 2,2 \\
\hline Lactoylglutathione lyase; & Q9CPU0 & Glo1 & $-0,8$ & 49,5 \\
\hline Ladinin-1; & P57016 & Lad1 & $-0,9$ & 18,9 \\
\hline Lamina-associated polypeptide 2 , isoforms alpha/zeta; & Q61033 & Tmpo & 0,2 & 10,1 \\
\hline Lamina-associated polypeptide 2, isoforms beta/delta/epsilon/gamma; & Q61029 & Tmpo & 0,3 & 29 \\
\hline Lamin-B receptor; & Q3U9G9 & Lbr & 0,3 & 4,5 \\
\hline Lamin-B1; & P14733 & Lmnb1 & $-0,2$ & 15,3 \\
\hline Laminin subunit alpha-2; & Q60675 & Lama2 & 0,8 & 1,4 \\
\hline Laminin subunit alpha-4; & P97927 & Lama4 & 0,8 & 6,8 \\
\hline Laminin subunit alpha-5; & Q61001 & Lama5 & $-1,9$ & 0,3 \\
\hline Laminin subunit beta-1; & P02469 & Lamb1 & 0,1 & 7,9 \\
\hline Laminin subunit beta-2; & Q61292 & Lamb2 & 0,2 & 0,5 \\
\hline Laminin subunit gamma-1; & F8VQJ3 & Lamc1 & 0,1 & 12,5 \\
\hline LanC-like protein 1; & 089112 & Lancl1 & 0,2 & 10,5 \\
\hline Large neutral amino acids transporter small subunit 1 ; & Q9Z127 & Slc7a5 & 0,0 & 5,3 \\
\hline Large neutral amino acids transporter small subunit 2 ; & Q9QXW9 & Slc7a8 & $-2,8$ & 3 \\
\hline Large proline-rich protein BAG6; & Q9Z1R2 & Bag6 & $-0,2$ & 2,6 \\
\hline Latent-transforming growth factor beta-binding protein 2 ; & 008999 & Ltbp2 & 0,2 & 1,5 \\
\hline Latent-transforming growth factor beta-binding protein 4; & D3Z598 & Ltbp4 & 0,2 & 20,3 \\
\hline Latexin; & P70202 & Lxn & 0,1 & 17,6 \\
\hline LDLR chaperone MESD; & Q9ERE7 & Mesdc2 & 0,1 & 7,1 \\
\hline L-dopachrome tautomerase; & P29812 & Dct & 0,8 & 4,1 \\
\hline Leptin receptor gene-related protein; & 089013 & Leprot & $-0,4$ & 9,9 \\
\hline LETM1 and EF-hand domain-containing protein 1, mitochondrial; & Q9Z210 & Letm1 & $-0,2$ & 1,1 \\
\hline Leucine zipper protein 1 ; & Q8R4U7 & Luzp1 & 0,3 & 0,7 \\
\hline Leucine-rich repeat flightless-interacting protein 1 ; & Q3UZ39 & Lrrfip1 & $-0,6$ & 5,9 \\
\hline Leucine-rich repeat-containing protein 15 & Q80X72 & Lrrc15 & $-0,6$ & 13,6 \\
\hline Leucine-rich repeat-containing protein $16 \mathrm{~A}$; & Q6EDY6 & Lrrc16a & $-0,6$ & 1 \\
\hline Leucine-rich repeat-containing protein 20 ; & Q8CI70 & Lrrc20 & $-0,2$ & 9,2 \\
\hline Leucine-rich repeat-containing protein 47 ; & Q505F5 & Lrrc47 & $-0,1$ & 9,3 \\
\hline Leucine-rich repeat-containing protein 59 ; & Q922Q8 & Lrrc59 & 0,1 & 28,3 \\
\hline Leucine--tRNA ligase, cytoplasmic; & Q8BMJ2 & Lars & $-0,3$ & 2,5 \\
\hline
\end{tabular}




\begin{tabular}{|c|c|c|c|c|}
\hline Protein & Uniprot & Gene name & $\begin{array}{l}\text { Fold change of } \\
\text { XPRESS ratio } \\
\left(E g f r^{\Delta K C} / \mathrm{WT}\right)\end{array}$ & $\begin{array}{c}\text { Sequence } \\
\text { coverage [\%] }\end{array}$ \\
\hline Leucyl-cystinyl aminopeptidase; & Q8C129 & Lnpep & 0,0 & 12,3 \\
\hline Leukocyte elastase inhibitor A; & Q9D154 & Serpinb1a & 0,7 & 6,1 \\
\hline Leukocyte surface antigen CD47; & Q61735 & $\mathrm{Cd} 47$ & 0,1 & 8,9 \\
\hline Leukotriene A-4 hydrolase; & P24527 & Lta4h & 0,3 & 14,2 \\
\hline LIM and senescent cell antigen-like-containing domain protein $1 ;$ & Q99JW4 & Lims1 & 0,4 & 26,2 \\
\hline LIM and SH3 domain protein 1 & Q61792 & Lasp1 & $-0,4$ & 16,7 \\
\hline LIM domain and actin-binding protein 1 ; & Q9ERG0 & Lima1 & $-0,3$ & 23 \\
\hline Lipid phosphate phosphohydrolase 3 ; & Q99JY8 & Ppap2b & 0,3 & 7,1 \\
\hline Lipoma-preferred partner homolog; & Q8BFW7 & Lpp & 0,1 & 24,1 \\
\hline Lipoprotein lipase; & P11152 & Lpl & $-0,1$ & 5,3 \\
\hline L-lactate dehydrogenase A chain; & P06151 & Ldha & 0,2 & 30,4 \\
\hline L-lactate dehydrogenase B chain; & P16125 & Ldhb & $-0,3$ & 32 \\
\hline Lon protease homolog, mitochondrial; & Q8CGK3 & Lonp1 & $-0,5$ & 14,2 \\
\hline Long-chain specific acyl-CoA dehydrogenase, mitochond rial; & P51174 & Acadl & 0,0 & 36,5 \\
\hline Long-chain-fatty-acid--CoA ligase 1 & P41216 & Acsl1 & 0,1 & 26,3 \\
\hline Long-chain-fatty-acid--CoA ligase 5; & Q8JZRO & Acsl5 & $-0,9$ & 4,2 \\
\hline Luc7-like protein 3; & Q5SUF2 & Luc7l3 & 0,1 & 4,2 \\
\hline Lumican; & P51885 & Lum & 0,1 & 38,8 \\
\hline Lupus La protein homolog; & P32067 & Ssb & 0,1 & 21,4 \\
\hline L-xylulose reductase; & Q91X52 & Dcxr & 0,1 & 4,7 \\
\hline Ly6/PLAUR domain-containing protein 3; & Q91YK8 & Lypd3 & $-1,4$ & 5,2 \\
\hline Ly6/PLAUR domain-containing protein 5; & Q9D7Z7 & Lypd5 & $-2,2$ & 13,7 \\
\hline Lymphocyte antigen 6 complex locus protein G6c; & Q9Z1Q4 & Ly6g6c & $-0,4$ & 21,4 \\
\hline Lymphocyte antigen 6D; & P35459 & Ly6d & 0,1 & 48 \\
\hline Lymphocyte-specific protein 1 ; & P19973 & Lsp1 & 0,2 & 10,2 \\
\hline Lys-63-specific deubiquitinase BRCC36; & P46737 & Brcc3 & 0,0 & 12,7 \\
\hline Lysine-specific histone demethylase $1 \mathrm{~A}$; & Q6ZQ88 & Kdm1a & 0,2 & 8,7 \\
\hline Lysine--tRNA ligase; & Q99MN1 & Kars & $-0,1$ & 13,1 \\
\hline Lysophosphatidic acid phosphatase type 6; & Q8BP40 & Acp6 & 0,4 & 8,4 \\
\hline Lysophospholipid acyltransferase 5 & Q91V01 & Lpcat3 & $-0,7$ & 4,5 \\
\hline Lysophospholipid acyltransferase 7; & Q8CHK3 & Mboat7 & $-0,3$ & 3,5 \\
\hline Lysosomal acid phosphatase; & P24638 & Acp2 & 0,5 & 7,1 \\
\hline Lysosomal alpha-glucosidase; & P70699 & Gaa & 0,1 & 1,9 \\
\hline Lysosomal alpha-mannosidase; & 009159 & Man2b1 & 0,0 & 4,2 \\
\hline Lysosomal protective protein; & P16675 & Ctsa & $-0,5$ & 15,2 \\
\hline Lysosomal protein NCU-G1; & Q9JHJ3 & & $-0,2$ & 6,3 \\
\hline Lysosomal Pro-X carboxypeptidase; & Q7TMRO & Prcp & $-1,6$ & 14,1 \\
\hline Lysosomal thioesterase PPT2; & 035448 & Ppt2 & $-0,3$ & 22,5 \\
\hline Lysosome membrane protein 2 & 035114 & Scarb2 & $-1,2$ & 6,1 \\
\hline Lysosome-associated membrane glycoprotein 1 ; & P11438 & Lamp1 & $-0,6$ & 11,8 \\
\hline Lysosome-associated membrane glycoprotein 2; & P17047 & Lamp2 & $-0,3$ & 6,5 \\
\hline Lysozyme g-like protein 2 & Q3V1I0 & Lyg2 & $-2,0$ & 6,6 \\
\hline m7GpppX diphosphatase; & Q9DAR7 & Dcps & 0,3 & 35,8 \\
\hline Macrophage mannose receptor 1 & Q61830 & Mrc1 & 1,0 & 10 \\
\hline Macrophage migration inhibitory factor; & P34884 & Mif & 0,0 & 7,8 \\
\hline Magnesium transporter NIPA4; & Q8BZF2 & Nipal4 & $-0,7$ & 3,4 \\
\hline Magnesium transporter protein 1; & Q9CQY5 & Magt1 & $-0,2$ & 11,5 \\
\hline Magnesium-dependent phosphatase 1; & Q9D967 & Mdp1 & 1,2 & 17,7 \\
\hline Major facilitator superfamily domain-containing protein 10 ; & Q9D2V8 & Mfsd10 & $-0,2$ & 4,2 \\
\hline Major urinary protein 2 & P11589 & Mup2 & 2,3 & 14,4 \\
\hline Major vault protein; & Q9EQK5 & Mvp & $-0,1$ & 5 \\
\hline Malate dehydrogenase, cytoplasmic; & P14152 & Mdh1 & 0,3 & 31,4 \\
\hline Malate dehydrogenase, mitochond rial; & P08249 & Mdh2 & 0,0 & 61,2 \\
\hline Malectin; & Q6ZQI3 & Mlec & 0,1 & 6,9 \\
\hline Malignant T-cell-amplified sequence 1 ; & Q9DB27 & Mcts1 & 0,3 & 35,9 \\
\hline Malonyl-CoA-acyl carrier protein transacylase, mitochondrial; & Q8R3F5 & Mcat & 2,0 & 3,4 \\
\hline Manganese-transporting ATPase $13 \mathrm{~A} 1$; & Q9EPE9 & Atp13a1 & $-0,2$ & 0,9 \\
\hline Mannose-1-phosphate guanyltransferase alpha; & Q922H4 & Gmppa & 0,8 & 10,2 \\
\hline Mannose-1-phosphate guanyltransferase beta; & Q8BTZ7 & Gmppb & 0,1 & 14,2 \\
\hline Mannose-6-phosphate isomerase; & Q924M7 & Mpi & 0,4 & 2,6 \\
\hline Mannose-P-dolichol utilization defect 1 protein; & Q9R0Q9 & Mpdu1 & $-0,2$ & 8,1 \\
\hline Mannosyl-oligosaccharide 1,2-alpha-mannosidase IA; & P45700 & Man1a1 & $-0,9$ & 4,3 \\
\hline Mannosyl-oligosaccharide glucosidase; & Q80UM7 & Mogs & $-0,3$ & 8,9 \\
\hline MARCKS-related protein; & P28667 & Marcksl1 & 0,1 & 14 \\
\hline Matrin-3; & Q8K310 & Matr3 & 0,6 & 6 \\
\hline Matrix metalloproteinase-14; & P53690 & Mmp14 & $-1,0$ & 2,6 \\
\hline Matrix-remodeling-associated protein 7; & Q9CZH7 & Mxra7 & 0,4 & 15,9 \\
\hline MCG125471; Predicted gene, 677156; Protein Cyp4f37; & Q3V1F1 & Cyp4f37 & $-1,0$ & 1,5 \\
\hline $\begin{array}{l}\text { MCG127334; MCG17849, isoform CRA_a; Protein 1810009A15Rik; } \\
\text { Uncharacterized protein; }\end{array}$ & Q9D937 & 1810009A15Rik & 0,0 & 8,1 \\
\hline MCG129038; Protein Serpinb3a; & G3X9V8 & Serpinb3a & 0,9 & 14,5 \\
\hline
\end{tabular}




\begin{tabular}{l} 
Protein \\
\hline MCG13402, isoform CRA_a; Polypyrimidine tract-binding protein 1; \\
MCG14442; Protein Eif2b3;
\end{tabular}

\begin{tabular}{lccc} 
Uniprot & Gene name & $\begin{array}{c}\text { Fold change of } \\
\text { XPRESS ratio } \\
\left.\text { (Egfr }^{\Delta K C} / \mathbf{W T}\right)\end{array}$ & $\begin{array}{c}\text { Sequence } \\
\text { coverage [\%] }\end{array}$ \\
\hline Q8BGJ5 & Ptbp1 & 0,4 & 23,5 \\
B1AUN2 & Eif2b3 & $-0,8$ & 10
\end{tabular}

MCG20149, isoform CRA_a; Methyltransferase like 7A1; Protein Mettl7a1;

MCG20280; OTTMUSG00000000712 protein; Protein Serpinb6d;

MCG21506; Macrophage galactose N-acetyl-galactosamine specific lectin 2

isoform i; Protein Mgl2;

MCG54087; Protein Serpina3j;

Medium-chain specific acyl-CoA dehydrogenase, mitochondrial;

Melanocyte protein PMEL;

Melanoma inhibitory activity protein 3;

Membrane magnesium transporter 1;

Membrane primary amine oxidase;

Membrane-associated progesterone receptor component 1 ;

Membrane-associated progesterone receptor component 2;

Mesoderm-specific transcript protein;

Metalloreductase STEAP3;

Metallothionein-4;

Metal-response element-binding transcription factor 2;

Metastasis-associated protein MTA2;

Methionine adenosyltransferase 2 subunit beta;

Methionine aminopeptidase 1;

Methionine aminopeptidase 2;

Methyl-CpG-binding domain protein 3;

Methyl-CpG-binding protein 2;

Methylcrotonoyl-CoA carboxylase beta chain, mitochondrial;

B1AUN2

Methylmalonate-semialdehyde dehydrogenase [acylating], mitochondrial;

Methylmalonyl-CoA epimerase, mitochond rial;

Methylosome protein 50;

Methylosome subunit pICln;

Methylsterol monooxygenase 1;

Methylthioribose-1-phosphate isomerase;

Methylthioribulose-1-phosphate dehydratase;

Mevalonate kinase;

Microfibrillar-associated protein 1 ;

Microfibrillar-associated protein 5;

Microsomal glutathione S-transferase 1;

Microtubule-associated protein 1A;

Microtubule-associated protein 1B;

Microtubule-associated protein 4;

Microtubule-associated protein RP/EB family member 1;

Mimecan;

Minor histocompatibility antigen $\mathrm{H} 13$;

Mitochondrial 2-oxoglutarate/malate carrier protein;

Mitochondrial amidoxime reducing component 2;

Mitochondrial antiviral-signaling protein;

Mitochondrial carnitine/acylcarnitine carrier protein;

Mitochondrial carrier homolog 2;

Mitochondrial chaperone BCS1;

Mitochondrial dicarboxylate carrier;

Mitochondrial fission 1 protein;

Mitochondrial import inner membrane translocase subunit Tim13;

Mitochondrial import inner membrane translocase subu nit TIM14;

Mitochondrial import inner membrane translocase subunit TIM16;

Mitochondrial import inner membrane translocase subu nit Tim23;

Mitochondrial import inner membrane translocase subunit TIM50;

Mitochondrial import inner membrane translocase subunit Tim8 A;

Mitochondrial import inner membrane translocase subunit Tim8 B;

Mitochondrial import inner membrane translocase subunit Tim9;

Mitochondrial import receptor subunit TOM22 homolog;

Mitochondrial import receptor subunit TOM40 homolog;

Mitochondrial inner membrane protein OXA1L;

Mitochondrial inner membrane protein;

Mitochondrial pyruvate carrier 2;

Mitogen-activated protein kinase 1;

Mitogen-activated protein kinase 14;

Mitogen-activated protein kinase kinase kinase 10;

Mitotic checkpoint protein BUB3;

MKI67 FHA domain-interacting nucleolar phosphoprotein;

$\mathrm{MOB}$ kinase activator $1 \mathrm{~B}$;
Q8C6B0

Q3UWK8

A9XX86

D3Z451

P45952

Q60696

Q8B184

Q8K273

070423

055022

Q80UU9

Q07646

Q8C159

P47945

Q02395

Q9R190

Q99LB6

Q8BP48

008663

Q9Z2D8

Q9Z2D6

Q3ULD5

Q9EQ20

Mettl7a1

$-0,3 \quad 18,9$

2,5

0,1

4,8

$\begin{array}{cc}\mathrm{Mgl2} & 0,1 \\ \text { Serpina3j } & 0,4\end{array}$

Acadm

Pmel

Mia3

Mmgt1

Aoc3

Pgrmc1

Pgrmc2

Mest

Steap3

Mt4

Mtf2

Mta2

Mat2b

Metap1

Metap2

Mbd3

Mecp2

Mccc2

Aldh6a1

Q9D115

Q99J09

Q61189

Q9CRA4

Q9CQT1

Q9WVQ5

Q9R008

Q9CQU1

Q9QZJ6

Q91VS7

Q9QYR6

P14873

P27546

Q61166

Q62000

Q9D8V0

Q9CR62

Q922Q1

Q8VCF0

Q9Z2Z6

Q791V5

Q9CZP5

Q9QZD8

Q9CQ92

P62075

Q9CQV7

Q9CQV1

Q9WTQ8

Q9D880

Q9WVA2

P62077

Q9WV98

Q9CPQ3

Q9QYA2

Q8BGA9

Q8CAQ8

Q9D023

P63085

P47811

Q66L42

Q9WVA3

Q91VE6

Q8BPB0

\section{Mcee}

Wdr77

Clns1a

Msmo1

Mri1

Apip

Mvk

Mfap1

Mfap5

Mgst1

Map1a

Map1b

Map4

Mapre1

Ogn

$\mathrm{Hm} 13$

Slc25a11

Marc2

Mavs

Slc25a20

Mtch2

Bcs1l

Slc25a10

Fis1

Timm13

Dnajc19

Pam16

Timm23

Timm50

Timm8a1

Timm8b

Timm9

Tomm22

Tomm40

Oxa1

Immt

Mpc2

Mapk1

Mapk14

Map3k10

Bub3

Nifk

Mob1b

0,4
0,2

11,9

18,8

1,8

1,3

6,9

18,3

26,2

37,8

3,3

12,4

19,4

1,5

2,7

12

5,4

27,6

8,1

5,8

11,4

3

10,7

18,4

12,7

7,2

7,6

21,2

20

4,8

9,8

18,7

0,9

3,2

30

10,4

12,1

8,9

27,7

18,3

3

14

19,8

2,2

21,3

24,8

61,1

15,5

21,6

12,9

3,1

34

13,3

48,3

33,1

18,6

4,2

26,7

42,5

28,5

7,9

0,7

29,4

3,8

10,6 


\begin{tabular}{|c|c|c|c|c|}
\hline Protein & Uniprot & Gene name & $\begin{array}{l}\text { Fold change of } \\
\text { XPRESS ratio } \\
\left(\text { Egfr }^{\Delta K C} / \mathrm{WT}\right)\end{array}$ & $\begin{array}{c}\text { Sequence } \\
\text { coverage [\%] }\end{array}$ \\
\hline Moesin; & P26041 & Msn & $-0,2$ & 11,8 \\
\hline Molybdenum cofactor biosynthesis protein 1 ; & Q5RKZ7 & Mocs1 & $-0,3$ & 1,3 \\
\hline Molybdopterin synthase sulfur carrier subunit; & Q9Z224 & Mocs2 & $-0,2$ & 12,5 \\
\hline Monocarboxylate transporter 1 & P53986 & Slc16a1 & 0,6 & 8,5 \\
\hline Monoglyceride lipase; & 035678 & Mgll & 0,0 & 24,1 \\
\hline mRNA cap guanine-N7 methyltransferase; & Q9D0L8 & Rnmt & 0,5 & 19,5 \\
\hline Multifunctional protein ADE2; & Q9DCL9 & Paics & 0,4 & 25,4 \\
\hline Multimerin-2; & A6H6E2 & Mmrn2 & 0,9 & 1,6 \\
\hline Multiple inositol polyphosphate phosphatase 1 & Q9Z2L6 & Minpp1 & $-0,5$ & 11,4 \\
\hline Murinoglobulin-1; & P28665 & Mug1 & 0,3 & 1,9 \\
\hline Muscleblind-like protein 2; & Q8C181 & Mbnl2 & 0,4 & 2,6 \\
\hline Musculoskeletal embryonic nuclear protein 1; & Q99JI1 & Mustn1 & 2,8 & 13,4 \\
\hline Myelin basic protein; & F6RT34 & $\mathrm{Mbp}$ & 0,0 & 32,1 \\
\hline Myelin protein $\mathrm{PO}$ & P27573 & $\mathrm{Mpz}$ & $-0,9$ & 17,3 \\
\hline Myelin protein P0; & E9QK82 & Mpz & 0,8 & 17,3 \\
\hline Myeloid-associated differentiation marker; & 035682 & Myadm & 1,2 & 5,3 \\
\hline Myoblast determination protein 1 & P10085 & Myod1 & $-6,4$ & 10,4 \\
\hline Myoferlin; & Q69ZN7 & Myof & 0,5 & 2,8 \\
\hline Myosin light chain $1 / 3$, skeletal muscle isoform; & P05977 & Myl1 & 2,1 & 41 \\
\hline Myosin light chain 4 & P09541 & Myl4 & 1,6 & 10,9 \\
\hline Myosin light chain 6B; & Q8CI43 & Myl6b & 1,6 & 19,8 \\
\hline Myosin light chain kinase, smooth muscle; & Q6PDN3 & Mylk & $-0,7$ & 1,2 \\
\hline Myosin light polypeptide 6; & Q60605 & Myl6 & $-0,5$ & 45,7 \\
\hline Myosin regulatory light chain 10 & E9QNY3 & Myl10 & 0,0 & 6,1 \\
\hline Myosin regulatory light chain $12 \mathrm{~B}$ & Q3THE2 & Myl12b & $-0,4$ & 40,1 \\
\hline Myosin regulatory light chain 2, skeletal muscle isoform; & P97457 & Mylpf & 2,6 & 38,5 \\
\hline Myosin regulatory light polypeptide 9 ; & Q9CQ19 & Myl9 & $-0,7$ & 33,7 \\
\hline Myosin-10; & Q61879 & Myh10 & 0,3 & 10,2 \\
\hline Myosin-14; & Q6URW6 & Myh14 & 0,5 & 4,3 \\
\hline Myosin-9; & Q8VDD5 & Myh9 & $-0,6$ & 33,1 \\
\hline Myotrophin; & P62774 & Mtpn & $-0,2$ & 14,4 \\
\hline Myotubularin-related protein 12 & Q80TA6 & Mtmr12 & 0,1 & 2,1 \\
\hline Myotubularin-related protein 2; & Q9Z2D1 & Mtmr2 & 0,0 & 1,4 \\
\hline Myristoylated alanine-rich C-kinase substrate; & P26645 & Marcks & 0,7 & 21,4 \\
\hline $\mathrm{N}(4)$-(beta- $\mathrm{N}$-acetylglucosaminyl)-L-asparaginase; & Q64191 & Aga & 0,2 & 7,2 \\
\hline$N(G), N(G)$-dimethylarginine dimethylaminohydrolase $1 ;$ & Q9CWso & Ddah1 & 0,3 & 8,8 \\
\hline $\mathrm{N}(\mathrm{G}), \mathrm{N}(\mathrm{G})$-dimethylarginine dimethylaminohydrolase 2 ; & Q99LD8 & Ddah2 & 0,6 & 37,2 \\
\hline $\mathrm{Na}(+) / \mathrm{H}(+)$ exchange regulatory cofactor NHE-RF1; & P70441 & Slc9a3r1 & $-0,5$ & 34,1 \\
\hline N-acetylated-alpha-linked acidic dipeptidase 2; & Q9CZR2 & Naalad2 & 1,7 & 9,5 \\
\hline $\mathrm{N}$-acetyl-D-glucosamine kinase; & Q9QZ08 & Nagk & 0,1 & 8,6 \\
\hline $\mathrm{N}$-acetylglucosamine-6-sulfatase; & Q8BFR4 & Gns & $-0,1$ & 8,3 \\
\hline $\mathrm{N}$-acetylneuraminate lyase; & Q9DCJ9 & $\mathrm{Npl}$ & $-0,7$ & 55 \\
\hline N-acylglucosamine 2-epimerase; & P82343 & Renbp & 0,6 & 4,5 \\
\hline NAD kinase; & P58058 & Nadk & 0,0 & 2,5 \\
\hline $\mathrm{NAD}(\mathrm{P}) \mathrm{H}$-hydrate epimerase; & Q8K4Z3 & Apoa1bp & 0,3 & 19,1 \\
\hline NADH dehydrogenase [ubiquinone] 1 alpha subcomplex subunit $11 ;$ & Q9D8B4 & Ndufa11 & $-0,8$ & 24,1 \\
\hline NADH dehydrogenase [ubiquinone] 1 alpha subcomplex subunit 13; & Q9ERS2 & Ndufa13 & $-0,3$ & 20,8 \\
\hline NADH dehydrogenase [ubiquinone] 1 alpha subcomplex subunit 4; & Q62425 & Ndufa4 & 0,1 & 46,3 \\
\hline NADH dehydrogenase [ubiquinone] 1 alpha subcomplex subunit 4-like 2; & Q4FZG9 & Ndufa4l2 & $-0,3$ & 28,7 \\
\hline NADH dehydrogenase [ubiquinone] 1 alpha subcomplex subunit 5; & Q9CPP6 & Ndufa5 & $-0,2$ & 13,8 \\
\hline NADH dehydrogenase [ubiquinone] 1 alpha subcomplex subunit 7; & Q9Z1P6 & Ndufa7 & 0,5 & 8 \\
\hline NADH dehydrogenase [ubiquinone] 1 alpha subcomplex subunit 8; & Q9DCJ5 & Ndufa8 & $-0,3$ & 17,4 \\
\hline NADH dehydrogenase [ubiquinone] 1 beta subcomplex subunit 10; & Q9DCS9 & Ndufb10 & $-1,0$ & 25,5 \\
\hline $\begin{array}{l}\text { NADH dehydrogenase [ubiquinone] } 1 \text { beta subcomplex subunit 11, } \\
\text { mitochondrial; }\end{array}$ & 009111 & Ndufb11 & $-0,5$ & 17,9 \\
\hline NADH dehydrogenase [ubiquinone] 1 beta subcomplex subunit 3; & Q9CQZ6 & Ndufb3 & $-0,5$ & 17,3 \\
\hline $\begin{array}{l}\text { NADH dehydrogenase [ubiquinone] } 1 \text { beta subcomplex subunit } 5 \text {, } \\
\text { mitochondrial; }\end{array}$ & Q9CQH3 & Ndufb5 & $-0,4$ & 6,7 \\
\hline NADH dehydrogenase [ubiquinone] 1 beta subcomplex subunit 7; & Q9CR61 & Ndufb7 & $-0,6$ & 7,3 \\
\hline $\begin{array}{l}\text { NADH dehydrogenase [ubiquinone] } 1 \text { beta subcomplex subunit } 8 \text {, } \\
\text { mitochondrial; }\end{array}$ & Q9D6J5 & Ndufb8 & $-0,4$ & 6,5 \\
\hline NADH dehydrogenase [ubiquinone] 1 beta subcomplex subunit 9; & Q9CQJ8 & Ndufb9 & $-0,7$ & 5 \\
\hline NADH dehydrogenase [ubiquinone] 1 subunit $\mathrm{C} 2$; & Q9CQ54 & Ndufc2 & $-0,9$ & 25,8 \\
\hline NADH dehydrogenase [ubiquinone] flavoprotein 1, mitochondrial; & Q91YT0 & Ndufv1 & 0,3 & 4,4 \\
\hline NADH dehydrogenase [ubiquinone] flavoprotein 2, mitochondrial; & Q9D6J6 & Ndufv2 & 0,6 & 12,5 \\
\hline NADH dehydrogenase [ubiquinone] flavoprotein 3, mitochondrial; & Q8BK30 & Ndufv3 & $-0,3$ & 10,6 \\
\hline $\begin{array}{l}\text { NADH dehydrogenase [ubiquinone] flavoprotein 3, mitochondrial; RIKEN cDNA } \\
\text { 1500032D16, isoform CRA_a; }\end{array}$ & Q3U422 & Ndufv3 & $-0,2$ & 16,2 \\
\hline NADH dehydrogenase [ubiquinone] iron-sulfur protein 2, mitochondrial; & Q91WD5 & Ndufs2 & $-0,1$ & 16,6 \\
\hline
\end{tabular}




\begin{tabular}{|c|c|c|c|c|}
\hline Protein & Uniprot & Gene name & $\begin{array}{l}\text { Fold change of } \\
\text { XPRESS ratio } \\
\left(\text { Egfr }^{\Delta K C} / \mathrm{WT}\right)\end{array}$ & $\begin{array}{c}\text { Sequence } \\
\text { coverage [\%] }\end{array}$ \\
\hline NADH dehydrogenase [ubiquinone] iron-sulfur protein 3 , mitochondrial; & Q9DCT2 & Ndufs3 & 0,1 & 19 \\
\hline NADH dehydrogenase [ubiquinone] iron-sulfur protein 4, mitochondrial; & Q9CXZ1 & Ndufs4 & $-0,2$ & 5,7 \\
\hline NADH dehydrogenase [ubiquinone] iron-sulfur protein 5; & Q99LY9 & Ndufs5 & $-0,2$ & 27,5 \\
\hline NADH dehydrogenase [ubiquinone] iron-sulfur protein 6 , mitochondrial; & P52503 & Ndufs6 & 1,2 & 15,3 \\
\hline NADH dehydrogenase [ubiquinone] iron-sulfur protein 7, mitochondrial; & Q9DC70 & Ndufs7 & $-0,3$ & 4 \\
\hline $\mathrm{NADH}$-cytochrome b5 reductase 3 ; & Q9DCN2 & Cyb5r3 & 0,4 & 29,9 \\
\hline NADP-dependent malic enzyme; & P06801 & Me1 & $-0,6$ & 29,7 \\
\hline NADPH--cytochrome $\mathrm{P} 450$ reductase; & P37040 & Por & $-0,6$ & 3,4 \\
\hline N-alpha-acetyltransferase 50; & Q6PGB6 & Naa50 & 0,3 & 5,9 \\
\hline Nardilysin; & Q8BHG1 & Nrd1 & $-1,2$ & 2,3 \\
\hline Nascent polypeptide-associated complex subunit alpha, muscle-specific form; & P70670 & Naca & 0,0 & 2,9 \\
\hline Nectin-1; & Q9JKF6 & Pvrl1 & 0,0 & 9,1 \\
\hline Nectin-2; & P32507 & Pvrl2 & $-0,3$ & 13,8 \\
\hline Nectin-4; & Q8R007 & Pvrl4 & 0,0 & 16,9 \\
\hline NEDD8; & P29595 & Nedd8 & 0,2 & 11,1 \\
\hline NEDD8-conjugating enzyme Ubc12; & P61082 & Ube2m & 0,1 & 9,3 \\
\hline Neprilysin; & Q61391 & Mme & $-0,7$ & 3,5 \\
\hline Neudesin; & Q9CQ45 & Nenf & $-0,1$ & 37,4 \\
\hline Neural cell adhesion molecule $1 ;$ & P13595 & Ncam1 & 0,3 & 7,8 \\
\hline Neuromodulin; & P06837 & Gap43 & 0,1 & 7,5 \\
\hline Neuron navigator 1 ; & Q8CH77 & Nav1 & 0,2 & 2,5 \\
\hline Neuronal proto-oncogene tyrosine-protein kinase Src; & P05480 & Src & 0,3 & 1,6 \\
\hline Neuroplastin; & P97300 & Nptn & 0,1 & 18,1 \\
\hline Neutral alpha-glucosidase $A B$ & Q8BHN3 & Ganab & $-0,5$ & 21,7 \\
\hline Neutral amino acid transporter A; & 035874 & Slc1a4 & $-0,8$ & 8,4 \\
\hline $\begin{array}{l}\text { Neutral amino acid transporter ASCT2; Neutral amino acid transporter } \mathrm{B}(0) \text {; } \\
\text { Solute carrier family } 1 \text { (Neutral amino acid transporter), member } 5\end{array}$ & Q9ESU7 & Slc1a5 & 0,9 & 10,6 \\
\hline Neutral cholesterol ester hydrolase 1 & Q8BLF1 & Nceh1 & 0,7 & 5,6 \\
\hline NFU1 iron-sulfur cluster scaffold homolog, mitochondrial; & Q9QZ23 & Nfu1 & 0,3 & 5,9 \\
\hline NHP2-like protein 1; & Q9D0T1 & Nhp2l1 & 0,0 & 27,3 \\
\hline Niban-like protein 1 & Q8R1F1 & Fam129b & 0,6 & 2,3 \\
\hline Nicalin; & Q8VCM8 & Ncln & $-0,7$ & 4,3 \\
\hline Nicastrin; & P57716 & Ncstn & $-0,6$ & 3,2 \\
\hline Nicotinamide phosphoribosyltransferase; & Q99KQ4 & Nampt & 0,3 & 25,3 \\
\hline Nidogen-1; & P10493 & Nid1 & 0,4 & 21 \\
\hline Nidogen-2; & 088322 & Nid2 & $-0,2$ & 7,5 \\
\hline Niemann-Pick C1 protein; & 035604 & Npc1 & $-0,6$ & 4,1 \\
\hline Nischarin; & Q80TM9 & Nisch & 5,1 & 0,7 \\
\hline Nitrilase homolog 1; & Q8VDK1 & Nit1 & 0,8 & 9,3 \\
\hline Nodal modulator 1 & Q6GQT9 & Nomo1 & $-1,1$ & 2,4 \\
\hline Non-histone chromosomal protein HMG-17; & P09602 & Hmgn2 & 0,9 & 17,2 \\
\hline Non-POU domain-containing octamer-binding protein; & Q99K48 & Nono & 0,2 & 10,8 \\
\hline Non-specific lipid-transfer protein; & P32020 & Scp2 & 0,0 & 14,1 \\
\hline Notchless protein homolog 1 & Q8VEJ4 & Nle1 & 1,8 & 4,7 \\
\hline NSFL1 cofactor $p 47$ & Q9CZ44 & Nsfl1c & $-0,4$ & 30 \\
\hline Nuclear autoantigenic sperm protein; & Q99MD9 & Nasp & 0,0 & 12,3 \\
\hline Nuclear cap-binding protein subunit 1 ; & Q3UYV9 & Ncbp1 & $-0,1$ & 1,3 \\
\hline Nuclear distribution protein nudE-like 1; & Q9ERR1 & Ndel1 & 0,4 & 9,9 \\
\hline Nuclear export mediator factor Nemf; & Q8CCP0 & Nemf & $-0,3$ & 0,9 \\
\hline Nuclear factor 1 A-type; & Q02780 & Nfia & 0,2 & 7,4 \\
\hline Nuclear factor 1 B-type; & P97863 & Nfib & $-0,3$ & 11,3 \\
\hline Nuclear factor 1 C-type; & P70255 & Nfic & 0,4 & 4,8 \\
\hline Nuclear factor 1 X-type; & P70257 & Nfix & 0,3 & 11,1 \\
\hline Nuclear migration protein nudC; & 035685 & Nudc & 0,3 & 39,8 \\
\hline Nuclear pore complex protein Nup85; & Q8R480 & Nup85 & 0,1 & 3,2 \\
\hline Nuclear pore glycoprotein p62; & Q63850 & Nup62 & $-0,6$ & 5,3 \\
\hline
\end{tabular}


Supplementary table 9: List of all proteins (A) altered (FC<-0.58/FC $>0.58$ ) at least in one experimental setup in both replicates and (B) identified at least in six quantitative proteome comparisons.

\begin{tabular}{|c|c|c|c|c|c|c|c|c|c|}
\hline \multirow{4}{*}{ Uniprot } & \multirow{4}{*}{ Protein } & \multicolumn{8}{|c|}{ Fold change $\left(\log _{2}\right)$ of XPRESS ratio } \\
\hline & & \multicolumn{4}{|c|}{ 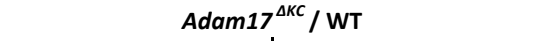 } & \multicolumn{4}{|c|}{$E g f r r^{\Delta K C} / \mathrm{WT}$} \\
\hline & & \multicolumn{2}{|c|}{ P3 } & \multicolumn{2}{|c|}{ P10 } & \multicolumn{2}{|c|}{ P3 } & \multicolumn{2}{|c|}{ P10 } \\
\hline & & Replicate 1 & Replicate 2 & Replicate 1 & Replicate 2 & Replicate 1 & Replicate 2 & Replicate 1 & Replicate 2 \\
\hline P10852 & 4F2 cell-surface antigen heavy chain; & $-0,3$ & $-0,3$ & $-1,1$ & $-0,5$ & $-0,5$ & 0,2 & $-1,0$ & $-1,1$ \\
\hline Q6ZWV7 & $60 S$ ribosomal protein L35; & & & $-0,5$ & $-0,1$ & 0,6 & 0,7 & $-0,2$ & $-0,1$ \\
\hline P62892 & $60 S$ ribosomal protein L39; & 0,6 & 0,2 & $-0,4$ & 0,0 & 0,7 & 1,0 & $-0,4$ & $-0,1$ \\
\hline Q9QZQ1 & Afadin; & $-0,4$ & $-0,5$ & $-0,4$ & & $-0,4$ & $-0,5$ & $-0,6$ & $-0,6$ \\
\hline Q9WTQ5 & A-kinase anchor protein 12 & $-0,2$ & 0,4 & $-0,3$ & & 0,2 & 0,5 & 1,6 & 1,4 \\
\hline Q00897 & Alpha-1-antitrypsin 1-4; & 1,3 & 1,3 & 1,4 & 1,0 & $-0,9$ & 2,0 & 0,0 & 1,1 \\
\hline P29699 & Alpha-2-HS-glycoprotein; & 0,2 & 0,0 & 0,9 & 0,6 & $-0,2$ & 1,5 & 0,3 & 0,8 \\
\hline P17182 & Alpha-enolase; & 0,8 & 0,6 & 0,6 & $-0,1$ & 0,2 & 0,0 & 0,4 & 0,3 \\
\hline P02772 & Alpha-fetoprotein; & $-0,3$ & 0,8 & 0,2 & 0,7 & $-0,1$ & 1,0 & 0,8 & 1,2 \\
\hline P31230 & $\begin{array}{l}\text { Aminoacyl tRNA synthase complex- } \\
\text { interacting multifunctional protein } 1 \text {; }\end{array}$ & $-0,8$ & $-0,4$ & 0,3 & $-0,4$ & 0,0 & $-0,5$ & $-0,6$ & $-0,6$ \\
\hline Q9DCH6 & AN1-type zinc finger protein 6; & $-1,3$ & $-1,1$ & $-0,5$ & & $-0,5$ & $-0,4$ & 0,0 & $-0,5$ \\
\hline Q00623 & Apolipoprotein A-I; & 0,4 & 0,5 & 0,7 & 0,8 & $-0,2$ & $-0,2$ & 0,6 & 0,5 \\
\hline P09813 & Apolipoprotein A-II; & 0,2 & 0,4 & 0,7 & 0,8 & 0,3 & 1,1 & 0,7 & 0,5 \\
\hline P08226 & Apolipoprotein E; & $-1,0$ & $-0,5$ & $-2,3$ & $-1,6$ & $-1,3$ & $-0,6$ & $-2,6$ & $-2,3$ \\
\hline P05202 & Aspartate aminotransferase, mitochondrial; & 1,4 & 0,6 & $-0,3$ & 0,1 & $-0,5$ & $-0,5$ & $-0,2$ & 0,1 \\
\hline Q6P542 & $\begin{array}{l}\text { ATP-binding cassette sub-family } F \text { member } \\
1 ;\end{array}$ & $-1,2$ & $-0,9$ & 0,7 & & 0,1 & $-0,9$ & $-0,6$ & $-0,6$ \\
\hline E9PVP1 & Aurora kinase B; & $-0,7$ & $-0,8$ & 0,7 & & $-0,5$ & $-0,8$ & $-1,0$ & $-0,9$ \\
\hline 070318 & Band 4.1-like protein 2; & $-0,3$ & 0,1 & 1,0 & & 0,8 & 0,5 & 1,2 & 0,7 \\
\hline A8DUK4 & $\begin{array}{l}\text { Beta-globin; Protein Hbb-bs; Protein Hbb- } \\
\text { bt; }\end{array}$ & $-0,4$ & $-0,3$ & 0,4 & 0,4 & $-0,7$ & 0,6 & 0,9 & 1,0 \\
\hline P20060 & Beta-hexosaminidase subunit beta; & $-0,7$ & $-0,6$ & $-0,9$ & $-0,3$ & 0,0 & $-0,5$ & $-0,9$ & $-0,9$ \\
\hline P51125 & Calpastatin; & $-0,4$ & $-0,2$ & $-0,5$ & $-0,4$ & $-0,3$ & 0,0 & $-0,6$ & $-0,8$ \\
\hline Q6P8K8 & Carboxypeptidase A4; & 0,6 & $-0,3$ & $-0,5$ & & 0,4 & $-0,9$ & $-1,2$ & $-0,8$ \\
\hline Q80V42 & Carboxypeptidase M; & $-0,6$ & 0,1 & $-0,4$ & $-0,5$ & 0,4 & $-0,7$ & $-1,6$ & $-0,7$ \\
\hline 089094 & Caspase-14; & $-0,1$ & $-0,2$ & $-0,2$ & $-0,1$ & 0,0 & $-0,2$ & $-1,4$ & $-1,5$ \\
\hline P26231 & Catenin alpha-1; & 0,6 & 0,6 & $-0,1$ & $-0,2$ & 0,8 & 0,8 & $-0,4$ & $-0,4$ \\
\hline P10605 & Cathepsin B; & $-0,1$ & $-0,1$ & $-0,6$ & $-0,7$ & $-0,7$ & $-0,8$ & $-1,1$ & $-0,7$ \\
\hline P18242 & Cathepsin D; & 0,7 & $-0,1$ & $-0,6$ & $-0,3$ & $-0,3$ & $-0,4$ & $-0,7$ & $-0,7$ \\
\hline P06797 & Cathepsin L1; & 0,7 & 0,1 & $-0,7$ & $-0,8$ & 0,3 & $-0,5$ & $-1,0$ & $-0,9$ \\
\hline Q61490 & CD166 antigen; & $-0,1$ & 0,2 & $-1,0$ & $-0,6$ & 0,0 & $-0,3$ & $-1,0$ & $-0,7$ \\
\hline P53996 & Cellular nucleic acid-binding protein; & 0,5 & 0,3 & $-1,9$ & 0,5 & 0,1 & $-0,6$ & 0,7 & 0,6 \\
\hline Q61362 & Chitinase-3-like protein 1 & 2,0 & 1,3 & 2,1 & & $-0,1$ & 0,7 & 3,1 & 1,0 \\
\hline Q06890 & Clusterin; & 0,1 & $-0,1$ & $-0,8$ & 0,0 & $-0,5$ & $-0,8$ & $-1,9$ & $-1,6$ \\
\hline P11087 & Collagen alpha-1(I) chain; & 0,6 & 1,4 & 0,8 & $-1,0$ & $-0,7$ & 0,5 & $-1,5$ & $-0,6$ \\
\hline P08121 & Collagen alpha-1(III) chain; & 0,8 & 1,2 & 0,6 & 1,2 & $-0,3$ & 1,2 & $-0,7$ & 0,0 \\
\hline Q62266 & Cornifin-A; & $-1,0$ & $-1,4$ & $-1,2$ & $-0,5$ & $-1,7$ & $-1,6$ & $-0,8$ & $-0,6$ \\
\hline Q9D1B1 & Cystatin E/M; Cystatin M/E; Cystatin-B; & $-0,1$ & $-0,4$ & 0,9 & 1,0 & $-0,7$ & $-0,6$ & 0,2 & 0,4 \\
\hline Q62426 & Cystatin-B; & $-0,1$ & $-0,3$ & $-0,7$ & $-0,5$ & $-0,3$ & $-0,5$ & $-0,7$ & $-0,9$ \\
\hline P19536 & $\begin{array}{l}\text { Cytochrome c oxidase subunit } 5 B \text {, } \\
\text { mitochondrial; }\end{array}$ & 0,4 & 0,3 & $-0,2$ & 0,2 & & 0,0 & $-0,8$ & $-0,9$ \\
\hline Q9D0M3 & $\begin{array}{l}\text { Cytochrome c1, heme protein, } \\
\text { mitochondrial; }\end{array}$ & 1,0 & 0,9 & $-0,1$ & $-1,2$ & & $-0,3$ & $-0,9$ & $-0,2$ \\
\hline 035215 & D-dopachrome decarboxylase; & 0,8 & 0,9 & $-0,1$ & $-0,2$ & 1,3 & $-0,4$ & 0,2 & 0,2 \\
\hline Q9D757 & Death-associated protein-like 1 ; & $-0,4$ & 0,3 & 1,3 & 0,8 & 0,1 & $-0,3$ & 0,4 & 0,0 \\
\hline P28654 & Decorin; & 1,0 & 1,4 & 0,5 & $-0,2$ & & 0,8 & $-0,2$ & $-0,1$ \\
\hline Q8BVI4 & Dihydropteridine reductase; & 1,4 & 0,7 & 0,0 & $-1,7$ & & & 0,3 & 0,3 \\
\hline 008553 & Dihydropyrimidinase-related protein 2 ; & 0,3 & 0,0 & 0,0 & 0,1 & & 0,4 & 0,7 & 0,9 \\
\hline Q8BL66 & Early endosome antigen 1 & $-0,6$ & $-0,7$ & $-0,1$ & 0,1 & $-0,5$ & 0,0 & $-0,1$ & 0,0 \\
\hline Q9D8N0 & Elongation factor 1-gamma; & $-1,1$ & $-0,8$ & $-0,1$ & $-0,6$ & & $-0,1$ & $-0,7$ & $-0,9$ \\
\hline Q8BJW6 & Eukaryotic translation initiation factor $2 \mathrm{~A}$; & $-0,9$ & $-0,8$ & 0,4 & $-0,3$ & & & 0,2 & 0,2 \\
\hline Q8JZQ9 & $\begin{array}{l}\text { Eukaryotic translation initiation factor } 3 \\
\text { subunit B; }\end{array}$ & $-1,3$ & $-1,1$ & $-1,0$ & $-0,5$ & $-0,2$ & $-0,2$ & $-0,4$ & 0,3 \\
\hline Q6NZJ6 & $\begin{array}{l}\text { Eukaryotic translation initiation factor } 4 \\
\text { gamma } 1 \text {; }\end{array}$ & $-0,9$ & $-1,2$ & 0,1 & & $-0,3$ & 0,9 & 0,0 & 0,2 \\
\hline P59325 & Eukaryotic translation initiation factor 5 & $-0,4$ & $-0,4$ & $-0,2$ & & $-0,3$ & 0,0 & $-2,4$ & $-0,9$ \\
\hline G3X9C2 & F-box only protein 50 & $-0,7$ & $-0,6$ & & & $-0,1$ & $-0,3$ & $-1,1$ & $-1,0$ \\
\hline P09528 & Ferritin heavy chain; & $-0,1$ & 0,6 & $-0,3$ & $-0,2$ & $-0,4$ & 0,2 & 1,7 & 1,1 \\
\hline Q9QXC1 & Fetuin-B; & 2,5 & 2,1 & 0,1 & & $-0,1$ & 1,6 & 0,9 & 0,7 \\
\hline Q8K0E8 & Fibrinogen beta chain; & 2,8 & 2,1 & 1,2 & 0,1 & & & 0,1 & 0,7 \\
\hline Q8VCM7 & Fibrinogen gamma chain; & 2,0 & 2,1 & 1,3 & & 0,6 & 1,5 & $-0,2$ & $-0,3$ \\
\hline E9Q019 & Filaggrin & $-1,0$ & $-0,8$ & & $-1,4$ & $-0,4$ & $-0,5$ & $-1,1$ & $-1,6$ \\
\hline E9QPZ3 & Filaggrin-2; & $-0,8$ & $-0,5$ & $-1,2$ & & $-0,3$ & $-0,2$ & $-1,5$ & $-2,5$ \\
\hline
\end{tabular}




\begin{tabular}{|c|c|c|c|c|c|c|c|c|c|}
\hline \multirow{4}{*}{ Uniprot } & \multirow{4}{*}{ Protein } & \multicolumn{8}{|c|}{ Fold change $\left(\log _{2}\right)$ of XPRESS ratio } \\
\hline & & \multicolumn{4}{|c|}{$\operatorname{Adam17} 7^{\Delta K C} / \mathrm{WT}$} & \multicolumn{4}{|c|}{$\mathrm{Egfr}^{\Delta K C} / \mathrm{WT}$} \\
\hline & & \multicolumn{2}{|c|}{ P3 } & \multirow{2}{*}{\multicolumn{2}{|c|}{ P10 }} & \multicolumn{2}{|c|}{ P3 } & \multicolumn{2}{|c|}{ P10 } \\
\hline & & Replicate 1 & Replicate 2 & & Replicate 2 & Replicate 1 & Replicate 2 & Replicate 1 & Replicate 2 \\
\hline Q2VIS4 & Filaggrin-2; & $-1,0$ & $-0,3$ & $-1,6$ & $-1,7$ & $-0,2$ & $-0,6$ & $-1,6$ & $-0,7$ \\
\hline Q62356 & Follistatin-related protein 1 & $-0,2$ & 0,3 & 0,9 & & $-0,3$ & 0,4 & 0,7 & 1,2 \\
\hline P05064 & Fructose-bisphosphate aldolase $A$ & 1,3 & 0,3 & $-0,4$ & $-0,4$ & $-0,7$ & $-0,7$ & $-0,4$ & $-0,1$ \\
\hline Q9D7X8 & Gamma-glutamylcyclotransferase; & $-0,1$ & 0,1 & $-1,2$ & $-1,5$ & 0,3 & $-0,5$ & $-1,9$ & $-2,5$ \\
\hline Q9ESY9 & $\begin{array}{l}\text { Gamma-interferon-inducible lysosomal } \\
\text { thiol reductase; }\end{array}$ & 0,3 & 0,4 & $-0,2$ & & 0,2 & $-0,8$ & $-0,7$ & $-1,0$ \\
\hline P23242 & Gap junction alpha-1 protein; & $-0,5$ & $-0,4$ & $-0,5$ & 0,1 & & $-0,3$ & $-1,3$ & $-1,1$ \\
\hline Q9EST1 & Gasdermin-A; & $-0,2$ & $-0,8$ & $-0,7$ & $-0,8$ & $-0,2$ & $-0,1$ & $-1,2$ & $-1,5$ \\
\hline P11499 & Heat shock protein HSP 90-beta; & $-0,7$ & $-0,6$ & 0,3 & $-0,3$ & $-0,1$ & 0,2 & $-0,3$ & $-0,4$ \\
\hline Q91X72 & Hemopexin; & 1,1 & 1,1 & 0,6 & 0,3 & 0,8 & 0,9 & 0,1 & 0,3 \\
\hline Q00PI9 & $\begin{array}{l}\text { Heterogeneous nuclear ribonucleoprotein } \\
\text { U-like protein 2; }\end{array}$ & $-0,6$ & $-1,2$ & 0,1 & & $-0,6$ & & 1,2 & $-0,1$ \\
\hline 088569 & $\begin{array}{l}\text { Heterogeneous nuclear ribonucleoproteins } \\
\text { A2/B1; }\end{array}$ & 1,0 & 1,0 & 0,5 & 0,0 & 0,5 & $-0,1$ & 0,2 & 0,2 \\
\hline P35492 & Histidine ammonia-lyase; & $-0,4$ & $-0,5$ & $-0,5$ & $-0,8$ & 0,2 & 0,1 & $-1,1$ & $-1,2$ \\
\hline E9QNP3 & Hornerin; & $-0,2$ & $-0,1$ & $-1,7$ & & 0,0 & $-0,3$ & $-2,0$ & $-2,8$ \\
\hline P01867 & Ig gamma-2B chain $\mathrm{C}$ region; & 1,1 & 1,3 & 0,7 & 0,4 & & & 0,2 & 0,3 \\
\hline Q91VM9 & $\begin{array}{l}\text { Inorganic pyrophosphatase 2, } \\
\text { mitochondrial; }\end{array}$ & 1,4 & 1,0 & $-0,2$ & 2,0 & & & $-0,2$ & $-0,1$ \\
\hline F6RPJ9 & Insulin-degrading enzyme; & 0,0 & 0,3 & & $-1,7$ & 0,2 & $-0,2$ & $-1,0$ & $-0,9$ \\
\hline G3X9D9 & Involucrin; & $-0,9$ & $-1,5$ & & $-0,8$ & $-0,8$ & $-0,4$ & $-1,3$ & $-1,2$ \\
\hline Q8BX37 & $\begin{array}{l}\text { Iron/zinc purple acid phosphatase-like } \\
\text { protein; }\end{array}$ & $-0,1$ & 0,2 & $-0,5$ & & 1,0 & $-0,4$ & $-1,2$ & $-1,8$ \\
\hline 088844 & $\begin{array}{l}\text { Isocitrate dehydrogenase [NADP] } \\
\text { cytoplasmic; }\end{array}$ & 1,3 & 1,1 & $-0,1$ & 0,1 & 0,2 & 0,0 & 0,1 & 0,4 \\
\hline P54071 & $\begin{array}{l}\text { Isocitrate dehydrogenase [NADP], } \\
\text { mitochondrial; }\end{array}$ & 1,6 & 1,1 & $-0,2$ & $-0,4$ & 0,3 & 0,2 & 0,9 & 0,2 \\
\hline Q91Y82 & $\begin{array}{l}\text { Kallikrein 6, isoform CRA_a; Kallikrein-1; } \\
\text { KIk6 protein; Neurosin; }\end{array}$ & 0,4 & $-0,5$ & 1,3 & & $-0,5$ & & 2,2 & 2,0 \\
\hline Q99M20 & $\begin{array}{l}\text { Kallikrein related-peptidase 10; Protein } \\
\text { KIk10; }\end{array}$ & 0,1 & $-0,1$ & 0,9 & 1,5 & 0,6 & $-0,4$ & 0,9 & 1,2 \\
\hline P02535 & Keratin, type I cytoskeletal 10; & 0,7 & 0,1 & $-1,1$ & $-0,5$ & $-0,3$ & $-0,3$ & $-1,1$ & $-2,1$ \\
\hline Q9QWL7 & Keratin, type I cytoskeletal 17; & $-0,6$ & $-0,6$ & $-0,4$ & $-0,8$ & $-0,8$ & $-1,1$ & $-1,0$ & $-1,2$ \\
\hline Q8VCW2 & Keratin, type I cytoskeletal 25; & $-1,9$ & 0,2 & 0,0 & $-0,3$ & $-1,2$ & $-0,4$ & $-2,5$ & $-1,8$ \\
\hline Q9Z320 & Keratin, type I cytoskeletal 27; & $-1,2$ & $-1,2$ & $-1,2$ & $-2,8$ & $-3,2$ & $-2,0$ & $-5,1$ & $-3,2$ \\
\hline A6BLY7 & Keratin, type I cytoskeletal 28 & $-1,2$ & & $-0,5$ & $-0,3$ & $-3,7$ & & $-1,9$ & $-0,9$ \\
\hline P04104 & Keratin, type II cytoskeletal 1 & 0,2 & $-0,4$ & $-1,7$ & & 0,0 & $-0,4$ & $-1,8$ & $-2,6$ \\
\hline Q6IFZ6 & Keratin, type II cytoskeletal $1 \mathrm{~b}$; & $-0,3$ & $-0,4$ & $-0,3$ & $-1,6$ & $-0,2$ & $-0,8$ & $-1,0$ & $-1,2$ \\
\hline Q9ROH5 & Keratin, type II cytoskeletal 71; & $-0,7$ & 0,7 & $-0,8$ & $-1,0$ & $-0,8$ & $-2,5$ & $-3,4$ & $-2,6$ \\
\hline Q6NXH9 & Keratin, type II cytoskeletal 73; & & 0,9 & $-1,7$ & 0,0 & & 1,3 & $-0,9$ & $-0,7$ \\
\hline A2A5X5 & Keratin-associated protein $16-1$ & $-4,4$ & & $-0,1$ & $-1,3$ & & $-5,4$ & $-2,4$ & $-3,4$ \\
\hline 054983 & Ketimine reductase mu-crystallin; & $-1,3$ & & $-1,7$ & $-0,8$ & & $-3,2$ & $-1,7$ & $-1,5$ \\
\hline 008677 & Kininogen-1; & 0,1 & 0,8 & 0,4 & 0,7 & 0,3 & 2,0 & 1,1 & 1,2 \\
\hline Q9CPUO & Lactoylglutathione lyase; & 1,3 & 0,7 & 0,0 & 0,3 & 0,8 & $-0,9$ & $-0,9$ & $-0,8$ \\
\hline Q3UZ39 & $\begin{array}{l}\text { Leucine-rich repeat flightless-interacting } \\
\text { protein 1; }\end{array}$ & $-0,1$ & $-0,8$ & $-0,3$ & $-0,2$ & $-1,0$ & $-1,0$ & $-0,5$ & $-0,6$ \\
\hline Q9D7Z7 & Ly6/PLAUR domain-containing protein 5; & 0,2 & 0,1 & $-1,9$ & 0,3 & 0,1 & $-0,3$ & $-1,7$ & $-2,2$ \\
\hline P05977 & $\begin{array}{l}\text { Myosin light chain } 1 / 3 \text {, skeletal muscle } \\
\text { isoform; }\end{array}$ & 1,3 & 2,0 & $-0,6$ & 1,9 & $-0,7$ & 0,9 & 1,7 & 2,1 \\
\hline P97457 & $\begin{array}{l}\text { Myosin regulatory light chain 2, skeletal } \\
\text { muscle isoform; }\end{array}$ & 1,3 & 2,6 & $-0,6$ & 2,4 & & 0,6 & 2,8 & 2,6 \\
\hline P26645 & $\begin{array}{l}\text { Myristoylated alanine-rich C-kinase } \\
\text { substrate; }\end{array}$ & $-0,1$ & $-0,4$ & $-0,2$ & 0,1 & $-0,2$ & $-0,2$ & 0,8 & 0,7 \\
\hline P06801 & NADP-dependent malic enzyme; & $-0,2$ & 0,3 & $-0,5$ & $-0,1$ & $-0,1$ & 0,1 & $-0,7$ & $-0,6$ \\
\hline Q9JIK5 & Nucleolar RNA helicase 2; & $-0,7$ & $-1,1$ & 0,3 & 0,5 & & & 0,2 & 0,4 \\
\hline Q9JHU2 & Palmdelphin; & 0,0 & $-0,3$ & 0,1 & & $-0,4$ & 0,0 & $-0,7$ & $-0,6$ \\
\hline P32848 & Parvalbumin alpha; & & 2,5 & & 2,1 & 0,4 & $-0,6$ & 2,5 & 0,8 \\
\hline P30412 & Peptidyl-prolyl cis-trans isomerase C; & 1,7 & 0,5 & 0,9 & 1,2 & 1,1 & 0,3 & 0,1 & 0,0 \\
\hline P43883 & Perilipin-2; & $-0,2$ & $-0,1$ & $-0,9$ & $-0,9$ & 0,2 & $-0,5$ & $-0,9$ & $-0,5$ \\
\hline P99029 & Peroxiredoxin-5, mitochondrial; & 0,7 & 0,6 & 0,1 & 0,0 & 0,2 & 0,0 & 0,0 & $-0,1$ \\
\hline Q9DCMO & $\begin{array}{l}\text { Persulfide dioxygenase ETHE1, } \\
\text { mitochondrial; }\end{array}$ & 0,3 & 0,2 & $-1,0$ & $-0,6$ & & & $-0,2$ & $-0,2$ \\
\hline 070250 & Phosphoglycerate mutase 2; & 1,4 & 1,5 & $-0,8$ & 1,6 & & $-0,8$ & 1,2 & 1,8 \\
\hline 035405 & Phospholipase D3; & 0,0 & $-0,3$ & $-0,3$ & $-0,5$ & $-0,2$ & $-0,5$ & $-0,8$ & $-0,7$ \\
\hline P63005 & $\begin{array}{l}\text { Platelet-activating factor acetylhydrolase IB } \\
\text { subunit alpha; }\end{array}$ & 0,3 & 0,3 & $-0,7$ & $-0,7$ & 0,4 & & 0,1 & $-0,2$ \\
\hline Q8R5J9 & PRA1 family protein 3 ; & 1,4 & 1,1 & 0,0 & 1,5 & 1,4 & 0,4 & 0,3 & 0,0 \\
\hline Q925BO & PRKC apoptosis WT1 regulator protein; & 0,0 & $-0,1$ & $-0,3$ & & $-0,5$ & $-0,3$ & $-0,6$ & $-0,7$ \\
\hline P17918 & Proliferating cell nuclear antigen; & 0,3 & 0,2 & 0,4 & 0,4 & & 0,6 & 0,8 & 0,8 \\
\hline Q9R0Q7 & Prostaglandin E synthase 3; & 1,0 & 0,7 & 0,3 & 0,9 & 0,0 & 0,2 & 0,2 & $-0,1$ \\
\hline
\end{tabular}




\begin{tabular}{|c|c|c|c|c|c|c|c|c|c|}
\hline \multirow{4}{*}{ Uniprot } & \multirow{4}{*}{ Protein } & \multicolumn{8}{|c|}{ Fold change $\left(\log _{2}\right)$ of XPRESS ratio } \\
\hline & & \multicolumn{4}{|c|}{$\operatorname{Adam} 17^{\Delta K C} / \mathrm{WT}$} & \multicolumn{4}{|c|}{$\mathrm{Egfr}^{\Delta K c} / \mathrm{WT}$} \\
\hline & & \multicolumn{2}{|c|}{ P3 } & \multicolumn{2}{|c|}{ P10 } & \multicolumn{2}{|r|}{ Egfr $^{\Delta \kappa}$} & \multicolumn{2}{|c|}{ P10 } \\
\hline & & Replicate 1 & Replicate 2 & Replicate 1 & Replicate 2 & Replicate 1 & Replicate 2 & Replicate 1 & Replicate 2 \\
\hline$\overline{\text { Q8CE08 }}$ & Prostatic acid phosphatase; & $-0,6$ & 0,2 & $-0,6$ & & 0,3 & & $-1,2$ & $-1,1$ \\
\hline D3YUU6 & Protein Crnn; & $-0,8$ & $-0,7$ & $-1,2$ & $-1,5$ & $-3,3$ & $-1,2$ & $-2,7$ & $-2,5$ \\
\hline E9PV24 & Protein Fga; & 1,4 & 2,5 & 1,2 & 1,1 & & 1,3 & $-0,1$ & 0,0 \\
\hline P62818 & Protein S100-A3; & $-3,3$ & 1,0 & $-0,7$ & $-0,7$ & $-2,8$ & $-3,8$ & $-1,7$ & $-1,7$ \\
\hline P31725 & Protein S100-A9; & 2,8 & 3,7 & $-0,1$ & & 1,5 & 1,9 & 1,3 & 0,4 \\
\hline Q9CZL5 & $\begin{array}{l}\text { Pterin-4-alpha-carbinolamine dehydratase } \\
2 ;\end{array}$ & 0,7 & 1,5 & 0,2 & 0,1 & & & $-0,1$ & $-0,1$ \\
\hline Q9CT10 & Ran-binding protein 3 & $-0,7$ & $-0,6$ & 0,2 & 0,3 & & & 0,4 & 0,0 \\
\hline Q62159 & Rho-related GTP-binding protein RhoC; & 0,9 & & $-0,3$ & $-0,1$ & & $-0,2$ & 0,6 & 0,9 \\
\hline Q9CQ01 & Ribonuclease T2; & $-1,6$ & $-0,8$ & 0,8 & & $-0,6$ & $-2,8$ & 1,0 & 1,1 \\
\hline P17563 & Selenium-binding protein $1 ;$ & 0,0 & $-1,1$ & 0,4 & 0,7 & $-0,1$ & $-0,2$ & 0,9 & 0,9 \\
\hline Q62093 & Serine/arginine-rich splicing factor 2 ; & 0,9 & 1,5 & 0,0 & 0,3 & $-0,1$ & 0,7 & 0,5 & 0,3 \\
\hline 035326 & Serine/arginine-rich splicing factor 5 ; & 0,8 & 0,7 & 0,1 & 0,7 & 0,2 & 0,0 & 0,5 & 0,2 \\
\hline Q921I1 & Serotransferrin; & 0,7 & 0,8 & 0,8 & 0,5 & & $-0,1$ & 0,2 & 0,7 \\
\hline P61957 & Small ubiquitin-related modifier 2 & $-0,2$ & $-0,4$ & 0,0 & & $-0,2$ & 0,1 & 0,7 & 0,6 \\
\hline Q9WTX5 & S-phase kinase-associated protein 1 & $-0,4$ & 0,9 & 0,6 & 1,1 & 1,0 & $-0,7$ & 0,2 & 0,6 \\
\hline Q8VIJ6 & Splicing factor, proline- and glutamine-rich; & $-0,7$ & $-0,8$ & 0,6 & 0,2 & $-0,3$ & 0,4 & 0,5 & $-0,1$ \\
\hline P54227 & Stathmin; & $-0,7$ & $-0,4$ & 0,1 & 0,5 & $-0,2$ & 0,2 & 0,8 & 0,6 \\
\hline Q6JHY2 & Submandibular gland protein C; & $-0,3$ & $-0,9$ & & & 0,2 & 0,3 & $-2,3$ & $-2,6$ \\
\hline Q9R1A9 & Three prime repair exonuclease 2 ; & $-0,3$ & $-0,5$ & $-0,5$ & & 0,6 & $-0,8$ & $-1,5$ & $-0,9$ \\
\hline P01831 & Thy-1 membrane glycoprotein; & $-0,8$ & 0,3 & $-0,3$ & 0,0 & $-0,4$ & 0,0 & 0,6 & 0,8 \\
\hline A2ASS6 & Titin; & & 5,3 & 5,8 & 2,8 & $-2,5$ & & 4,2 & $-2,2$ \\
\hline Q08189 & $\begin{array}{l}\text { Transglutaminase-3; Protein-glutamine } \\
\text { gamma-glutamyltransferase } \mathrm{E} \text {; }\end{array}$ & $-0,3$ & 0,0 & $-0,5$ & $-0,3$ & 0,2 & $-0,3$ & $-0,7$ & $-0,9$ \\
\hline Q9CXE7 & $\begin{array}{l}\text { Transmembrane emp24 domain-containing } \\
\text { protein } 5 \text {; }\end{array}$ & 0,2 & $-0,1$ & & $-0,2$ & 0,4 & & $-0,9$ & $-0,9$ \\
\hline Q3UBX0 & Transmembrane protein 109; & 1,6 & 0,2 & $-0,9$ & & $-0,7$ & $-0,6$ & 0,9 & $-1,2$ \\
\hline Q9D3P1 & Trichohyalin-like protein 1 & $-2,6$ & $-1,4$ & $-1,0$ & $-1,4$ & $-2,1$ & & $-3,0$ & $-2,4$ \\
\hline P20801 & Troponin C, skeletal muscle; & 2,3 & 2,3 & 1,4 & & & $-0,2$ & 1,9 & 2,3 \\
\hline Q7TMM9 & Tubulin beta-2A chain; & 0,9 & 0,8 & $-0,2$ & $-0,1$ & 0,1 & 0,2 & 0,1 & 0,0 \\
\hline P57784 & U2 small nuclear ribonucleoprotein A'; & 1,5 & 1,1 & 0,1 & 2,1 & & & 1,2 & $-0,1$ \\
\hline Q6ZWM4 & U6 snRNA-associated Sm-like protein LSm8; & 0,6 & 0,7 & $-0,3$ & 0,6 & 0,3 & 0,2 & 0,4 & 0,4 \\
\hline Q8K207 & Uncharacterized protein C1orf21 homolog; & 0,7 & $-0,1$ & 0,8 & 0,6 & $-0,2$ & & 1,0 & \\
\hline Q91Z58 & $\begin{array}{l}\text { Uncharacterized protein C6orf132 } \\
\text { homolog; }\end{array}$ & $-0,4$ & $-0,4$ & $-0,4$ & & $-0,3$ & $-0,2$ & $-2,0$ & $-1,6$ \\
\hline P20152 & Vimentin; & 0,2 & 0,9 & 0,8 & $-0,2$ & 0,4 & 0,4 & 1,6 & 1,0 \\
\hline Q60932 & $\begin{array}{l}\text { Voltage-dependent anion-selective channel } \\
\text { protein } 1 \text {; }\end{array}$ & 0,0 & 0,0 & $-0,4$ & 0,0 & 0,1 & $-0,1$ & $-0,7$ & $-0,7$ \\
\hline Q9R020 & $\begin{array}{l}\text { Zinc finger Ran-binding domain-containing } \\
\text { protein } 2 \text {; }\end{array}$ & 0,7 & 1,0 & $-0,7$ & 0,6 & $-0,6$ & 0,6 & 0,4 & $-0,2$ \\
\hline Q62523 & Zyxin; & $-0,5$ & $-0,2$ & $-0,8$ & $-0,7$ & $-0,2$ & 0,2 & $-1,0$ & $-0,3$ \\
\hline F6TL02 & & $-1,0$ & $-1,0$ & $-0,1$ & $-1,2$ & $-0,3$ & $-0,4$ & $-0,7$ & $-1,3$ \\
\hline J3QMA3 & & $-1,0$ & $-0,6$ & $-0,4$ & $-1,3$ & $-0,3$ & $-0,2$ & $-1,0$ & $-1,3$ \\
\hline
\end{tabular}


Supplementary table 10: Dimethylated (naturally unmodified) N-termini identified in the TAlLS experiment comparing wild-type and Adam $17^{4 k C}$ epidermis at P3 (replicate 1). This is a comprehensive, non redundant listing. Up to two potential protein IDS per peptide are stated.

\begin{tabular}{|c|c|c|c|c|c|c|c|c|c|c|c|}
\hline Non Prime Site & Prime Site & $\begin{array}{l}\text { Fold change of } \\
\text { XPRESS ratio } \\
\text { (Adam17 } 7^{\text {AkC } / \text { WT) }}\end{array}$ & Modifications & Hyperscore & $\begin{array}{c}\text { Mass error } \\
\text { in ppm }\end{array}$ & Charge & $\begin{array}{c}\begin{array}{c}\text { Precursor } \\
\text { neutral mass } \\
\text { in Da }\end{array} \\
\end{array}$ & Uniprot ID 1 & Uniprot ID 2 & Protein Name 1 & Protein Name 2 \\
\hline $\bar{M}$ & VDREQLVQKARLAEQAER & 10,3 & $\mathrm{~N}-\operatorname{ter}+28.03 \mathrm{Da}, \mathrm{K}+28.03 \mathrm{Da}$ & 16,4 & $-4,779$ & 3 & 2194,2 & 14336_MOUSE & & 14-3-3 protein gamma; & \\
\hline VLSSIEQKSN & EEGSEEKGPEVKEYR & $-0,7$ & $\mathrm{~N}-\operatorname{ter}+34.06 \mathrm{Da}, \mathrm{K}+34.06 \mathrm{Da}$ & 27,4 & $-4,385$ & 3 & 1867,0 & 1433S_MOUSE & & 14-3-3 protein sigma; & Stratifin; \\
\hline GEELSCEERN & LLSVAYKNVVGGQR & $-0,4$ & $\mathrm{~N}$-ter $+34.06 \mathrm{Da}, \mathrm{K}+34.06 \mathrm{Da}$ & 15,8 & $-0,064$ & 3 & 1571,0 & 14335_MOUSE & & 14-3-3 protein sigma; & Stratifin; \\
\hline RYEDMAAFMK & SAVEKGEELSCEER & $-2,0$ & $\begin{array}{l}\text { N-ter +34.06 Da, K +34.06 Da, C } \\
+57.02 \mathrm{Da}\end{array}$ & 27,6 & $-1,004$ & 3 & 1689,9 & 1433S_MOUSE & & 14-3-3 protein sigma; & Stratifin; \\
\hline DEAMADLHTL & SEDSYKDSTLMMQLLR & $-0,9$ & $\mathrm{~N}$-ter + 34.06 Da, K+34.06 Da & 39,8 & $-6,856$ & 3 & 1966,1 & 1433S_MOUSE & & 14-3-3 protein sigma; & Stratifin; \\
\hline SIEQKSNEEG & SEEKGPEVKEYR & 0,8 & $\mathrm{~N}$-ter +28.03 Da, K+28.03 Da & 15,8 & 1,756 & 4 & 1533,8 & 14335_MOUSE & & 14-3-3 protein sigma; & Stratifin; \\
\hline & SLIQKAKLAEQAER & 0,0 & $\mathrm{~N}$-ter +34.06 Da, K +34.06 Da & 24,4 & 5,269 & 3 & 1686,1 & 14335_MOUSE & & 14-3-3 protein sigma; & Stratifin; \\
\hline ELSCEERNLL & SVAYKNVVGGQR & $-0,2$ & $\mathrm{~N}$-ter +34.06 Da, K+34.06 Da & 20,5 & $-0,371$ & 3 & 1344,8 & 14335_MOUSE & & 14-3-3 protein sigma; & Stratifin; \\
\hline TFDEAMADLH & TLSEDSYKDSTLIMQLLR & 1,7 & $\mathrm{~N}$-ter +28.03 Da, K+28.03 Da & 50,2 & 3,685 & 3 & 2168,2 & 1433S_MOUSE & & 14-3-3 protein sigma; & Stratifin; \\
\hline EDMAAFMKSA & VEKGEELSCEER & $-0,6$ & $\begin{array}{l}\text { N-ter }+34.06 \mathrm{Da}, \mathrm{K}+34.06 \mathrm{Da}, \mathrm{C} \\
+57.02\end{array}$ & 22,1 & 6,320 & 3 & 1531,8 & 1433S_MOUSE & & 14-3-3 protein sigma; & Stratifin; \\
\hline M & DKNELVQKAKLAEQAER & 10,3 & N-ter +28.03 Da, K +28.03 Da & 21,6 & $-6,678$ & 4 & 2212,2 & 1433Z_MOUSE & D3YXF4_MOUSE & 14-3-3 protein zeta/delta; & Protein kinase C inhibitor protein 1; $;$ EZZ-2; \\
\hline ERYDDMAACM & KSVTEQGAELSNEER & $-1,5$ & $\mathrm{~N}$-ter $+28.03 \mathrm{Da}, \mathrm{K}+28.03 \mathrm{Da}$ & 15,9 & 7,781 & 3 & 1731,9 & 1433Z_MOUSE & D3YXF4_MOUSE & 14-3-3 protein zeta/delta; & Protein kinase C inhibitor protein 1;;EZ-2; \\
\hline TAFQAASALG & AEFASEACR & $-0,1$ & N-ter +34.06 Da, C +57.02 Da & 28,8 & $-0,837$ & 2 & 1073,5 & SEP15_MOUSE & D3Z3Y8_MOUSE & 15 kDa selenoprotein; & \\
\hline HGGSTSAVRL & ANISVPASESPR & $-0,1$ & $\mathrm{~N}$-ter $+34.06 \mathrm{Da}$ & 19,6 & 0,158 & 2 & 1260,7 & TB182_MOUSE & - & 182 kDa tankyrase-1-binding protein; & \\
\hline DQLGLRNLEV & SSCVSSEGPSEAR & $-1,2$ & N-ter $+28.03 \mathrm{Da}, \mathrm{C}+57.02 \mathrm{Da}$ & 16,9 & 4,560 & 2 & 1379,6 & TB182_MOUSE & & 182 kDa tankyrase-1-binding protein; & \\
\hline MAGARPGVH & ALQLEPPTVVETLR & 0,1 & N-ter +28.03 Da & 24,0 & 7,336 & 2 & 1592,9 & PLCB3_MOUSE & & $\begin{array}{l}\text { 1-phosphatidylinosito 4,5-bisphosphate } \\
\text { phosphodiesterase beta-3; }\end{array}$ & $\begin{array}{l}\text { Phosphoinositide phospholipase C-beta- } \\
\text { 3;Phospholipase C-beta-3; }\end{array}$ \\
\hline CRSIFGDALL & IDPLDKYPLSAGIPLPSPQDLMGR & 0,1 & $\mathrm{~N}-\operatorname{ter}+34.06 \mathrm{Da}, \mathrm{K}+34.06 \mathrm{Da}$ & 15,7 & 3,529 & 3 & 2660,5 & PLCB3_MOUSE & & $\begin{array}{l}\text { 1-phosphatidylinositol 4,5-bisphosphate } \\
\text { phosphodiesterase beta-3; }\end{array}$ & $\begin{array}{l}\text { Phosphoinositide phospholipase C-beta- } \\
\text { 3; Phospholipase C-beta-3; }\end{array}$ \\
\hline YCRSIFGDAL & LIDPLDKYPLSAGIPLPSPQDLMGR & 0,0 & $\mathrm{~N}$-ter $+28.03 \mathrm{Da}, \mathrm{K}+28.03 \mathrm{Da}$ & 14,9 & $-0,362$ & 3 & 2761,5 & PLCB3_MOUSE & & $\begin{array}{l}\text { 1-phosphatidylinositol 4,5-bisphosphate } \\
\text { phosphodiesterase beta-3; }\end{array}$ & $\begin{array}{l}\text { Phosphoinositide phospholipase C-beta- } \\
\text { 3;Phospholipase C-beta-3; } \\
\text { 265 proteasome AAA-ATPase subunit }\end{array}$ \\
\hline SRPQTGLSFL & GPEPEDLEDLYSR & 0,0 & $\mathrm{~N}$-ter $+34.06 \mathrm{Da}$ & 30,5 & $-1,222$ & 2 & 1552,8 & PRS6B_MOUSE & & 265 protease regulatory subunit $6 \mathrm{~B} ;$ & 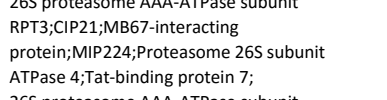 \\
\hline GLAPPALWDL & AADKQTLQSEQPLQVAR & $-0,6$ & $\mathrm{~N}$-ter $+34.06 \mathrm{Da}, \mathrm{K}+34.06 \mathrm{Da}$ & 19,2 & 5,376 & 3 & 1950,1 & PRS7_MOUSE & Q8BVQ9_MOUSE & 265 protease regulatory subunit $7 ;$ & $\begin{array}{l}265 \text { proteasome AAA-ATPase subunit } \\
\text { RPT1; Proteasome } 265 \text { subunit ATPase 2; Protein } \\
\text { MSS1; }\end{array}$ \\
\hline M & KDVPAFLQQSQSSGPGQAAVWHR & $-0,7$ & $\mathrm{~N}$-ter $+34.06 \mathrm{Da}, \mathrm{K}+34.06 \mathrm{Da}$ & 47,7 & 0,556 & 4 & 2692,4 & PSD13_MOUSE & E9Q519_MOUSE & 265 proteasome non-ATPase regulatory subunit 13; & $\begin{array}{l}265 \text { proteasome regulatory subunit RPN9;26S } \\
\text { proteasome regulatory subunit S111;265 } \\
\text { proteasome regulatory subunit } 440.5 ;\end{array}$ \\
\hline MVL & ESTMVCVDNSEYMR & $-0,4$ & $\mathrm{~N}$-ter $+34.06 \mathrm{Da}, \mathrm{C}+57.02 \mathrm{Da}$ & 31,5 & $-4,784$ & 2 & 1753,8 & PSMD4_MOUSE & & 265 proteasome non-ATPase regulatory subunit 4 & $\begin{array}{l}265 \text { proteasome regulatory subunit RPN10;26S } \\
\text { proteasome regulatory subunit } \\
\text { S5A;Multiubiquuitin chain-binding protein; }\end{array}$ \\
\hline M & VLESTMVCVDNSEYMR & $-0,3$ & $\mathrm{~N}$-ter $+34.06 \mathrm{Da}, \mathrm{C}+57.02 \mathrm{Da}$ & 52,4 & $-0,915$ & 2 & 1965,9 & PSMD4_MOUSE & & 265 proteasome non-ATPase regulatory subunit 4 & $\begin{array}{l}\text { 265 proteasome regulatory subunit RPN10;26S } \\
\text { proteasome regulatory subunit } \\
\text { S5A;Multiubiquitin chain-binding protein; }\end{array}$ \\
\hline VQNVGTVVQH & SEGKPLNVTVIR & $-0,5$ & $\mathrm{~N}$-ter $+28.03 \mathrm{Da}, \mathrm{K}+28.03 \mathrm{Da}$ & 15,9 & 0,802 & 3 & 1367,8 & PSMD9_MOUSE & & 265 proteasome non-ATPase regulatory subunit 9; & 265 proteasome regulatory subunit p27; \\
\hline SRLCLAGKRC & LLAAYVDSHOWEAR & $-0,5$ & $\mathrm{~N}$-ter +34.06 Da & 17,2 & 0,786 & 3 & 1778,9 & RT27_MOUSE & & $28 \mathrm{~S}$ ribosomal protein 527 , mitochondrial; & \\
\hline LCLAGKRCLL & & $-0,6$ & $\mathrm{~N}$-ter +34.06 Da & 24,2 & $-6,235$ & 3 & 1552,7 & RT27_MOUSE & & 285 ribosomal protein 527 , mitochondrial; & \\
\hline AGGGARWRFI & ATSPAAELSPTELTEMR & 0,4 & $\mathrm{~N}$-ter $+28.03 \mathrm{Da}$ & 18,7 & 0,928 & 2 & 1830,9 & RM39_MOUSE & & 39 S ribosomal protein L39, mitochondrial; & \\
\hline GAGGGARWRF & & 0,1 & $\mathrm{~N}$-ter +34.06 Da & 34,0 & $-6,759$ & 3 & 1950,0 & RM39_MOUSE & & 395 ribosomal protein L39, mitochondrial; & \\
\hline LGRSYGRRKL & SQTQGPPDNPGFVESVDEYQFVER & $-0,8$ & N-ter $+34.06 \mathrm{Da}$ & 38,8 & 4,201 & 3 & 2758,3 & RM49_MOUSE & & 395 ribosomal protein L49, mitochondrial; & \\
\hline PVVAETVEEV & KKEPVLVCPPLR & $-1,8$ & $\begin{array}{l}\text { N-ter +34.06 Da, K +34.06 Da, C } \\
+57.02 \mathrm{Da}\end{array}$ & 20,1 & 0,260 & 3 & 1537,0 & RM50_MOUSE & & 395 ribosomal protein L50, mitochondrial; & \\
\hline PETIDSVIVG & NVMQSSSDAAYLAR & 0,1 & $\mathrm{~N}$-ter +34.06 Da & 15,1 & $-1,292$ & 2 & 1545,8 & THIM_MOUSE & & 3-ketoacyl-CoA thiolase, mitochondrial; & $\begin{array}{l}\text { Acetyl-CoA acyltransferase; Beta- } \\
\text { ketothiolase;Mitochondrial 3-oxoacyl-CoA } \\
\text { thiolase; }\end{array}$ \\
\hline GIQYLRDYLH & LPPEIVPATLR & 0,0 & $\mathrm{~N}$-ter $+28.03 \mathrm{Da}$ & 21,4 & 0,081 & 2 & 1232,8 & RS10_MOUSE & J3QNI7_MOUSE & 40 S ribosomal protein S10; & \\
\hline NTA & ALIHDG & $-0,5$ & $\mathrm{~N}$-ter $+34.06 \mathrm{Da}$ & 24,4 & $-0,100$ & 3 & 998 & RS12_MOUSE & Q6ZWZ6_MOUSE & E 40 S ribosomal protein S12; & \\
\hline & & 1,9 & N-ter $+2 \varepsilon$ & 23,0 & 2,19 & 2 & 145 & & & 40S ril & \\
\hline & & $-0,5$ & $\mathrm{~N}-\mathrm{t}$ & 18,3 & $-2,6$ & 3 & 146 & USE & D3 & 405 & \\
\hline & QEFVR & $-0,3$ & $\mathrm{Da}$ & 28,8 & 1,5 & 3 & 16 & & & 405 & \\
\hline & & $-0,3$ & $6 \mathrm{Da}, \mathrm{K}+3$ & 25,5 & -2, & 3 & 132 & & & 40S ribosomal protein S19; & \\
\hline WVDTVKLAKH & FTTR & $-0,4$ & $3 \mathrm{Da}, \mathrm{K}+2$ & 27,7 & $-0,6$ & 3 & 1886,9 & OUSE & 3_MOUSE & 40S ribosomal protein S19; & \\
\hline ACLSLPQMPG & VTVKDVNQQEFVR & $-0,3$ & $\mathrm{~N}$-ter + 34.06 Da, K+34.06 Da & 29,1 & 1,226 & 3 & 1629,0 & RS19_MOUSE & S4R223_MOUSE & 40S ribosomal protein S19; & \\
\hline MQND & AGEFVDLYVPR & $-0,7$ & $\mathrm{~N}$-ter +34.06 Da & 34,7 & $-2,537$ & 2 & 1298,7 & RS21_MOUSE & & 40S ribosomal protein S21; & \\
\hline
\end{tabular}




\begin{tabular}{|c|c|c|c|c|c|c|c|c|c|c|c|}
\hline Non Prime Site & Prime Site & $\begin{array}{c}\text { Fold change of } \\
\text { XPRESS ratio } \\
\text { (Adam17 } 7^{\text {LKc } / W T)} \\
\end{array}$ & Modifications & Hyperscore & $\begin{array}{c}\text { Mass error } \\
\text { in ppm }\end{array}$ & Charge & $\begin{array}{c}\text { Precursor } \\
\text { neutral mass } \\
\text { in Da }\end{array}$ & Uniprot ID 1 & Uniprot ID 2 & Protein Name 1 & Protein Name 2 \\
\hline KCSASNRIIA & AKDHASIQMNVAEVDR & $-0,4$ & N-ter +28.03 Da, K+28.03 Da & 25,7 & $-7,329$ & 3 & 1838,9 & RS21_MOUSE & & 40 S ribosomal protein $521 ;$ & \\
\hline & DAGEFVDLYVPR & $-0,2$ & N-ter +34.06 Da & 38,2 & $-0,071$ & 2 & 1413,7 & RS21_MOUSE & & $40 S$ ribosomal protein S21; & \\
\hline MQNDA & GEFVDLYVPR & $-0,3$ & $\mathrm{~N}$-ter +28.03 Da & 22,1 & $-2,125$ & 2 & 1221,6 & RS21_MOUSE & & 40 r ribosomal protein S21; & \\
\hline YGICGAIRRM & GESDDSILR & 0,1 & $\mathrm{~N}$-ter +34.06 Da & 21,2 & 1,169 & 2 & 1024,5 & RS21_MOUSE & & 40 r ribosomal protein S21; & \\
\hline CSASNRIIAA & KDHASIQMNVAEVDR & $-0,4$ & $\mathrm{~N}$-ter +28.03 Da, K+28.03 Da & 32,7 & $-8,753$ & 3 & 1767,9 & RS21_MOUSE & & 40 ribosomal protein S21; & \\
\hline TYGICGAIRR & MGESDDSILR & $-0,1$ & $\mathrm{~N}$-ter +34.06 Da & 20,9 & $-1,641$ & 2 & 1155,6 & RS21_MOUSE & & 40 r ribosomal protein S21; & \\
\hline NRIIAAKDHA & SIQMNVAEVDR & $-0,1$ & $\mathrm{~N}$-ter +34.06 Da & 36,8 & 1,851 & 2 & 1294,7 & RS21_MOUSE & & 40 ribosomal protein S21; & \\
\hline SQGQCTQVRV & EFMDDTSR & $-0,4$ & $\mathrm{~N}$-ter +28.03 Da & 23,9 & 0,583 & 2 & 1027,4 & RS28_MOUSE & J3QNN8_MOUSE & 40 ribosomal protein S28; & \\
\hline IIRNVKGPVR & EGDVLTLLESER & 7,9 & $\mathrm{~N}$-ter +28.03 Da & 21,1 & $-0,072$ & 2 & 1387,7 & RS28_MOUSE & G3UYV7_MOUSE & 40 r ribosomal protein S28; & \\
\hline IRNVKGPVRE & GDVLTLLESER & $-0,7$ & $\mathrm{~N}-\operatorname{ter}+28.03 \mathrm{Da}$ & 20,1 & 6,425 & 2 & 1258,7 & RS28_MOUSE & G3UYV7_MOUSE & 40 r ribosomal protein S28; & \\
\hline TGSQGQCTQV & RVEFMDDTSR & $-0,4$ & N-ter +34.06 Da & 22,1 & $-0,387$ & 3 & 1288,6 & RS28_MOUSE & J3QNN8_MOUSE & $40 S$ ribosomal protein S28; & \\
\hline QRAKSMKFVD & GLMIHSGDPVNYYVDTAVR & $-0,7$ & $\mathrm{~N}$-ter +34.06 Da & 29,1 & 2,006 & 3 & 2140,1 & RS3_MOUSE & & 40 ribosomal protein $53 ;$ & \\
\hline IGKDIEKACQ & SIYPLHDVFVR & 1,0 & $\mathrm{~N}$-ter $+34.06 \mathrm{Da}$ & 21,8 & $-3,112$ & 3 & 1378,8 & RS3A_MOUSE & D3Z6C3_MOUSE & $\begin{array}{l}\text { 40S ribosomal protein S3a \{ECO:0000255|HAMAP- } \\
\text { Rule:MF__3122\}; }\end{array}$ & Protein TU-11; \\
\hline NTGQRAVLKF & AAATGATPIAGR & $-0,5$ & $\mathrm{~N}$-ter +28.03 Da & 24,1 & $-0,645$ & 2 & 1083,6 & RSSA_MOUSE & D3YTT7_MOUSE & $\begin{array}{l}\text { 40S ribosomal protein SA \{ECO:0000255|HAMAP- } \\
\text { Rule:MF__3016\}; }\end{array}$ & 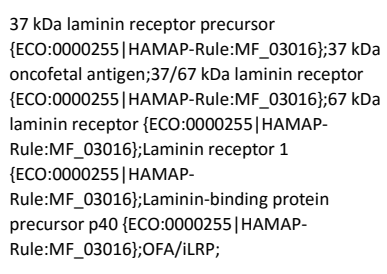 \\
\hline TWEKLLLAAR & AIVAIENPADVSVISSR & 1,6 & $\mathrm{~N}$-ter $+28.03 \mathrm{Da}$ & 28,5 & $-2,373$ & 2 & 1768,0 & RSSA_MOUSE & D3YTT7_MOUSE & $\begin{array}{l}\text { 40S ribosomal protein SA \{ECO:0000255|HAMAP- } \\
\text { Rule:MF_03016\}; }\end{array}$ & 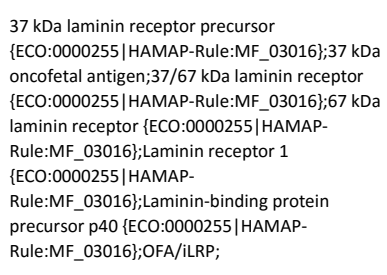 \\
\hline RTWEKLLLAA & RAIVAIENPADVSVISSR & $-0,5$ & $\mathrm{~N}$-ter +34.06 Da & 43,4 & $-1,397$ & 3 & 1930,1 & RSSA_MOUSE & D3YTT7_MOUSE & $\begin{array}{l}\text { 40S ribosomal protein SA \{ECO:0000255|HAMAP- } \\
\text { Rule:MF_03016\}; }\end{array}$ & 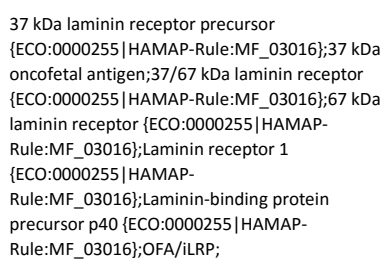 \\
\hline SVYESPGFFL & NLEPIPGALDALR & $-0,8$ & $\mathrm{~N}$-ter +34.06 Da & 20,2 & 0,212 & 2 & 1411,8 & NT5C_MOUSE & A2A9X5_MOUSE & $5^{\prime}\left(3^{\prime}\right)$-deoxyribonucleotidase, cytosolic type; & $\begin{array}{l}\text { Cytosolic 5',3'-pyrimidine nucleotidase;Deoxy- } \\
5 \text { 5'-nucleotidase } 1 \text {; } \\
60 \text { kDa chaperonin;Chaperonin 60;HSP-65;Heat }\end{array}$ \\
\hline ALAPHLTRAY & AKDVKFGADAR & 0,1 & N-ter +34.06 Da, K+34.06 Da & 26,1 & $-0,156$ & 4 & 1278,8 & CH60_MOUSE & D3Z2F2_MOUSE & 60 kDa heat shock protein, mitochondrial; & $\begin{array}{l}\text { shock protein } 60 ; \text { Mitochondrial matrix protein } \\
\text { P1; } \\
60 \mathrm{kDa} \text { chaperonin;Chaperonin 60;HSP-65;Heat }\end{array}$ \\
\hline PLVIIAEDVD & GEALSTLVLNR & $-0,2$ & N-ter+34.06 Da & 19,1 & 4,802 & 2 & 1205,7 & CH60_MOUSE & & $60 \mathrm{kDa}$ heat shock protein, mitochondrial; & $\begin{array}{l}\text { shock protein 60; Mitochondrial matrix protein } \\
\text { P1; }\end{array}$ \\
\hline $\begin{array}{l}\text { NGSIYNPEVL } \\
\text { SFGIOOVE }\end{array}$ & $\begin{array}{l}\text { DITEQALHSR } \\
\end{array}$ & 1,0 & $\mathrm{~N}-\operatorname{ter}+28.03 \mathrm{Da}$ & 16,8 & 2,334 & 3 & $\begin{array}{l}1196,6 \\
23998\end{array}$ & RLA0_MOUSE & S4R1N1_MOUSE & $60 \mathrm{~S}$ acidic ribosomal protein P0; & 60 r ribosomal protein L10E; \\
\hline $\begin{array}{l}\text { SFGLIIQQVF } \\
\text { GLIOOVENN }\end{array}$ & DNGSIYNPEVLDITEQALHSR & $-0,1$ & N-ter +28.03 Da & 34,1 & 0,541 & 3 & 2398,2 & RLAO_MOUSE & S4R1N1_MOUSE & $60 \mathrm{~S}$ acidic ribosomal protein P0; & 60 S ribosomal protein L10E; \\
\hline $\begin{array}{l}\text { GLIIOQVVEN } \\
\text { YAASYLAAL }\end{array}$ & GSIYNPEVLDITEQALHSR & $-1,2$ & N-ter +34.06 Da & 21,2 & 8,952 & 3 & 2175,2 & RLAO_MOUSE & S4R1N1_MOUSE & $60 \mathrm{~S}$ acidic ribosomal protein $\mathrm{PO}$; & 60 r ribosomal protein L10E; \\
\hline $\begin{array}{l}\text { YVASYLLAAL } \\
\text { RKPSAPKKGD }\end{array}$ & $\begin{array}{l}\text { GGNSSPSAKDIKKILDVVGIEADDDR } \\
\text { SSAEELKLATQLTGPVMPIR }\end{array}$ & $\begin{array}{l}-0,3 \\
0,8\end{array}$ & $\begin{array}{l}\text { N-ter +34.06 Da, } \mathrm{K}+34.06 \mathrm{Da} \\
\mathrm{N} \text {-ter +28.03 Da, K+28.03 Da }\end{array}$ & $\begin{array}{l}18,1 \\
31,9\end{array}$ & $\begin{array}{r}6,085 \\
-0,455\end{array}$ & $\begin{array}{l}4 \\
3\end{array}$ & $\begin{array}{l}2822,6 \\
2196,2\end{array}$ & $\begin{array}{l}\text { RLA2_MOUSE } \\
\text { RL13 MOUSE }\end{array}$ & & $\begin{array}{l}60 \text { Sacidic ribosomal protein P2; } \\
60 \text { s ribosomal protein } 133 \text {; }\end{array}$ & \\
\hline M & AEGQVLVLDGR & $-0,4$ & $\mathrm{~N}$-ter $+34.06 \mathrm{Da}$ & 37,0 & 0,168 & 2 & 1189,7 & RL13A_MOUSE & D3YY61_MOUSE & 60 ribosomal protein L13a; & $\begin{array}{l}\text { A52; } \\
\text { Transplantation antigen P198;Tum-P198 }\end{array}$ \\
\hline $\mathrm{M}$ & KFNPFVTSDR & $-0,4$ & N-ter +34.06 Da, K +34.06 Da & 34,5 & $-0,567$ & 2 & 1408,8 & RL26_MOUSE & B1ARA3_MOUSE & $60 S$ ribosomal protein L26; & $\begin{array}{l}\text { antigen; } \\
\text { Silica-induced gene } 20 \text { protein; }\end{array}$ \\
\hline
\end{tabular}




\begin{tabular}{|c|c|c|c|c|c|c|c|c|c|c|c|}
\hline Non Prime Site & Prime Site & $\begin{array}{l}\text { Fold change of } \\
\text { XPRESS ratio } \\
\text { (Adam17 } 7^{\text {AkC } / \text { WT) }}\end{array}$ & Modifications & Hyperscore & $\begin{array}{c}\text { Mass error } \\
\text { in ppm }\end{array}$ & Charge & $\begin{array}{c}\text { Precursor } \\
\text { neutral mass } \\
\text { in Da }\end{array}$ & Uniprot ID 1 & Uniprot ID 2 & Protein Name 1 & Protein Name 2 \\
\hline $\begin{array}{l}\text { CGKYYRVCTL } \\
\text { GKYYRVCTLA }\end{array}$ & $\begin{array}{l}\text { AllDPGDSDIIR } \\
\text { IIDPGDSDIIR }\end{array}$ & $\begin{array}{l}-0,4 \\
-0,3\end{array}$ & $\begin{array}{l}\text { N-ter +28.03 Da } \\
\text { N-ter +34.06 Da }\end{array}$ & $\begin{array}{l}25,2 \\
15,0\end{array}$ & $\begin{array}{c}-1,446 \\
5,526\end{array}$ & $\begin{array}{l}2 \\
2\end{array}$ & $\begin{array}{l}1311,7 \\
1246,7\end{array}$ & $\begin{array}{l}\text { RL30_MOUSE } \\
\text { RL30_MOUSE }\end{array}$ & & $\begin{array}{l}60 S \text { ribosomal protein L30; } \\
\text { 60S ribosomal protein L30; }\end{array}$ & \\
\hline MLAKTGVHHY & SGNNIELGTACGKYYR & 0,8 & $\begin{array}{l}\text { N-ter +28.03 Da, K+28.03 Da, C } \\
+57.02 \mathrm{Da}\end{array}$ & 15,6 & 1,881 & 3 & 1857,9 & RL30_MOUSE & & 60 S ribosomal protein L30; & \\
\hline $\begin{array}{l}\text { TACGKYYRVC } \\
\text { TVAGGAWTYN }\end{array}$ & $\begin{array}{l}\text { TLAIIDPGDSDIIR } \\
\text { TTSAVTVKSAIR }\end{array}$ & $\begin{array}{l}0,4 \\
1,0\end{array}$ & $\begin{array}{l}\text { N-ter +34.06 Da } \\
\text { N-ter +28.03 Da, K+28.03 Da }\end{array}$ & $\begin{array}{l}23,9 \\
36,5\end{array}$ & $\begin{array}{l}3,977 \\
4,877\end{array}$ & $\begin{array}{l}2 \\
3\end{array}$ & $\begin{array}{l}1531,9 \\
1288,8\end{array}$ & $\begin{array}{l}\text { RL30_MOUSE } \\
\text { RL37A_MOUSE }\end{array}$ & & $\begin{array}{l}\text { 60S ribosomal protein L30; } \\
\text { 60S ribosomal protein L37a; }\end{array}$ & \\
\hline M & RPLTEEETR & $-0,3$ & $\mathrm{~N}$-ter $+34.06 \mathrm{Da}$ & 17,1 & $-1,310$ & 3 & 1294,7 & NIP7_MOUSE & E9Q109_MOUSE & $\begin{array}{l}\text { 60S ribosome subunit biogenesis protein NIP7 } \\
\text { homolog. }\end{array}$ & PEachy;kDa93; \\
\hline LGVGPDGHTC & SLLPDHPLLQER & $-0,5$ & N-ter +34.06 Da & 19,4 & 0,403 & 3 & 1484,8 & 6PGL_MOUSE & D3Z4X1_MOUSE & $\begin{array}{l}\text { nombolog; } \\
\text { 6-phosphogluconolactonase; }\end{array}$ & \\
\hline NGVFEVVATN & GDTHLGGEDFDQR & $-0,4$ & $\mathrm{~N}$-ter +28.03 Da & 17,4 & $-7,111$ & 3 & 1473,6 & GRP78_MOUSE & & 78 kDa glucose-regulated protein; & $\begin{array}{l}\text { Heat shock } 70 \text { kDa protein 5:Immunoglobulin } \\
\text { heavy chain-binding protein; }\end{array}$ \\
\hline CPLTLGIETV & GGVMTKLIPR & $-0,6$ & $\mathrm{~N}-\operatorname{ter}+34.06 \mathrm{Da}, \mathrm{K}+34.06 \mathrm{Da}$ & 15,2 & $-4,292$ & 3 & 1138,8 & GRP78_MOUSE & & 78 kDa glucose-regulated protein; & $\begin{array}{l}\text { Heat shock } 70 \text { kDa protein } 5 ; \text { Immunoglobulin } \\
\text { heavy chain-binding protein; }\end{array}$ \\
\hline RPLTKDNHLL & GTFDLTGIPPAPR & $-0,6$ & $\mathrm{~N}$-ter $+34.06 \mathrm{Da}$ & 20,8 & $-5,084$ & 2 & 1374,8 & GRP78_MOUSE & & 78 kDa glucose-regulated protein; & $\begin{array}{l}\text { Heat shock } 70 \mathrm{kDa} \text { protein 5;/mmunoglobulin } \\
\text { heavy chain-binding protein; }\end{array}$ \\
\hline LIGDAAKNQL & TSNPENTVFDAKR & $-0,6$ & N-ter +34.06 Da, K+34.06 Da & 23,2 & 3,486 & 3 & 1545,9 & GRP78_MOUSE & & 78 kDa glucose-regulated protein; & $\begin{array}{l}\text { Heat shock } 70 \text { kDa protein 5;Immunoglobulin } \\
\text { heavy chain-binding protein; }\end{array}$ \\
\hline QEVRCLERSY & ASKPTLNEVIIVSAIR & $-0,4$ & $\mathrm{~N}$-ter +34.06 Da, K+34.06 Da & 60,9 & $-0,510$ & 2 & 1764,1 & THIL_MOUSE & & Acetyl-CoA acetyltransferase, mitochondrial; & Acetoacetyl-CoA thiolase; \\
\hline LNKLTVFTL & MDVTKGQFESHLR & $-0,4$ & $\mathrm{~N}$-ter +34.06 Da, K+34.06 Da & 19,2 & $-1,483$ & 4 & 1614,9 & ASAH1_MOUSE & & Acid ceramidase; & $\begin{array}{l}\text { Acylshhingosine deacylase; N-acylsphingosine } \\
\text { amidohydrolase; }\end{array}$ \\
\hline KPVLNKLTVF & TTLMDVTKGQFESHLR & $-0,8$ & $\mathrm{~N}$-ter $+34.06 \mathrm{Da}, \mathrm{K}+34.06 \mathrm{Da}$ & 30,5 & $-2,121$ & 3 & 1930,1 & ASAH1_MOUSE & & Acid ceramidase; & $\begin{array}{l}\text { Acylshingosine deacylase;N-acylsphingosine } \\
\text { amidohydrolase; }\end{array}$ \\
\hline YNDGEVDDEE & DEEEAGEEEGSQKR & $-0,7$ & $\mathrm{~N}$-ter +28.03 Da, K+28.03 Da & 29,7 & 0,545 & 3 & 1647,7 & AN32A_MOUSE & D3Z7M9_MOUSE & $\begin{array}{l}\text { Acidic leucine-rich nuclear phosphoprotein } 32 \text { family } \\
\text { member } A \text {; }\end{array}$ & $\begin{array}{l}\text { Acidic nuclear phosphoprotein pp32;Leucine- } \\
\text { rich acidic nuclear protein;Potent heat-stable } \\
\text { protein phosphatase 2A inhibitor I1PP2A; }\end{array}$ \\
\hline $\begin{array}{l}\text { VVDNGSGMCK } \\
\text { AGFAGDDAPR }\end{array}$ & $\begin{array}{l}\text { AGFAGDDAPR } \\
\text { AVFPSIVGRPR }\end{array}$ & $\begin{array}{l}1,8 \\
19\end{array}$ & $\begin{array}{l}\text { N-ter }+34.06 \mathrm{Da} \\
\mathrm{N} \text {-eter } 28.03 \mathrm{Da}\end{array}$ & $\begin{array}{l}20,3 \\
35,1\end{array}$ & $\begin{array}{l}-1,285 \\
-1,628\end{array}$ & 2 & $\begin{array}{l}1009,5 \\
1225,\end{array}$ & $\begin{array}{l}\text { ACTA_MOUSE } \\
\text { ACTA MOUSE }\end{array}$ & $\begin{array}{l}\text { ACTB_MOUSE } \\
\text { ACTB MOUSE }\end{array}$ & $\begin{array}{l}\text { Actin, aortic smooth muscle; } \\
\text { antic }\end{array}$ & Alpha-actin-2; \\
\hline MCEE & EDSTALVCDNGSGLCKAGFAGDDAPR & 1,6 & N-ter +34.06 Da, K +34.06 Da, C & 17,4 & $-1,235$ & 3 & $\begin{array}{l}1223,1 \\
2750,3\end{array}$ & $\begin{array}{l}\text { ACIA_MUUSE } \\
\text { ACTA_MOUSE }\end{array}$ & ACTB_MOUSE & $\begin{array}{l}\text { Actin, aortic smooth muscle; } \\
\text { Actin, aortic smooth muscle; }\end{array}$ & $\begin{array}{l}\text { Alpha-actin- } 2 ; \\
\text { Alpha-actin-2; }\end{array}$ \\
\hline MCE & EEDSTALVCDNGSGLCKAGFAGDDAPR & $-3,5$ & $\begin{array}{l}\text { N-ter +34.06 Da, K+34.06 Da, C } \\
+57.02 \text { Da }\end{array}$ & 29,2 & 4,684 & 3 & 2879,4 & ACTA_MOUSE & & Actin, aortic smooth muscle; & Alpha-actin-2; \\
\hline MC & EEEDSTALVCDNGSGLCKAGFAGDDAPR & 6,6 & $\begin{array}{l}\text { N-ter }+28.03 \mathrm{Da}, \mathrm{K}+28.03 \mathrm{Da}, \mathrm{C} \\
+57.02 \mathrm{Da}\end{array}$ & 19,9 & $-7,266$ & 4 & 2996,3 & ACTA_MOUSE & & Actin, aortic smooth muscle; & Alpha-actin-2; \\
\hline $\begin{array}{l}\text { SLEKSYELPD } \\
\text { RKDLYANTVL }\end{array}$ & GQVITIGNER & $-0,7$ & $\mathrm{~N}-\operatorname{ter}+34.06 \mathrm{Da}$ & 19,5 & $-0,892$ & 2 & 1119,7 & ACTA_MOUSE & ACTBL_MOUSE & Actin, aortic smooth muscle; & Alpha-actin-2; \\
\hline $\begin{array}{l}\text { RKDLYANTVL } \\
\text { VAPEEHPVLL }\end{array}$ & $\begin{array}{l}\text { SGGTTMYPGIADR } \\
\text { TEAPLNPKANR }\end{array}$ & $\begin{array}{l}-0,8 \\
-0,4\end{array}$ & $\begin{array}{l}\mathrm{N}-\operatorname{ter}+34.06 \mathrm{Da} \\
\mathrm{N} \text {-ter }+28.03 \mathrm{Da}, \mathrm{K}+28.03 \mathrm{Da}\end{array}$ & $\begin{array}{l}45,3 \\
38,5\end{array}$ & $\begin{array}{l}-0,661 \\
2,207\end{array}$ & ${ }_{3}^{2}$ & $\begin{array}{l}1358,7 \\
126,7\end{array}$ & $\begin{array}{l}\text { ACCA_M MOSE } \\
\text { ACTA MOUSE }\end{array}$ & $\begin{array}{l}\text { ACTB_MOUSE } \\
\text { ACTB MOUSE }\end{array}$ & $\begin{array}{l}\text { Actin, aortic smooth muscle; } \\
\text { Actin, aortic smooth muscle; }\end{array}$ & $\begin{array}{l}\text { Alpha-actin-2; } \\
\text { Alpha-actin-2; }\end{array}$ \\
\hline $\begin{array}{l}\text { THTVPYYGY } \\
\text { THY }\end{array}$ & ALPHALLR & $\begin{array}{l}-0,4 \\
-0,7\end{array}$ & N-ter +34.06 Da & $\begin{array}{l}30,0 \\
21,0\end{array}$ & 5,288 & 3 & $\begin{array}{l}923,6 \\
923,6\end{array}$ & ACTB_MOUSE & ACTBL_MOUSE & Actin, cytoplasmic 1; & Beta-actin; \\
\hline DRMQKEITAL & APSTMKIKIIAPPER & $-0,7$ & $\mathrm{~N}$-ter +28.03 Da, K+28.03 Da & 32,9 & 0,058 & 3 & 1735,0 & ACTB_MOUSE & ACTA_MOUSE & Actin, cytoplasmic 1; & Beta-actin; \\
\hline mfam & DDDIAALVVDNGSGMCKAGFAGDDAPR & 1,5 & $\begin{array}{l}\text { N-ter +28.03 Da, K+28.03 Da, C } \\
+57.02 \mathrm{Da}\end{array}$ & 28,3 & $-5,980$ & 3 & 2923,3 & ACTB_MOUSE & E9Q1F2_MOUSE & Actin, cytoplasmic 1; & Beta-actin; \\
\hline QQMMWISKQEY & DESGPSIVHR & $-0,5$ & $\mathrm{~N}$-ter +34.06 Da & 24,2 & 3,355 & 3 & 1129,6 & ACTB_MOUSE & ACTG_MOUSE & Actin, cytoplasmic 1; & Beta-actin; \\
\hline MDDDIAALVV & DNGSGMCKAGFAGDDAPR & $-0,4$ & N-ter +28.03 Da, K+28.03 Da, C & 33,9 & $-8,759$ & 3 & 1880,8 & ACTB_MOUSE & ACTG_MOUSE & Actin, cytoplasmic 1; & Beta-actin; \\
\hline MATAASSSSL & EKSYELPDGQVITIGNER & $-0,2$ & $\mathrm{~N}$-ter + $34.06 \mathrm{Da}, \mathrm{K}+34.06 \mathrm{Da}$ & 24,4 & 0,094 & 3 & 2115,2 & ACTB_MOUSE & ACTA_MOUSE & Actin, cytoplasmic 1; & Beta-actin; \\
\hline $\begin{array}{l}\text { GRPRHQGVMV } \\
\text { PRHGVMVGM }\end{array}$ & $\begin{array}{l}\text { GMGQKDSSVGGEAASKR } \\
\text { GGKDSYGDEASKR }\end{array}$ & $-0,5$ & $\begin{array}{l}\mathrm{N} \text { N-ter }+34.06 \mathrm{Da}, \mathrm{K}+34.06 \mathrm{Da} \\
\mathrm{N} \text {-ter }+28.03 \mathrm{Da} \mathrm{K}+28.0 \mathrm{Ba}\end{array}$ & $\begin{array}{l}52,0 \\
33,3\end{array}$ & $\begin{array}{l}7,908 \\
4,388\end{array}$ & ${ }_{4}^{3}$ & $\begin{array}{l}1957,1 \\
175,9\end{array}$ & $\begin{array}{l}\text { ACTB_MOUSE } \\
\text { ACTB MOUSE }\end{array}$ & $\begin{array}{l}\text { ACTA_MOUSE } \\
\text { ACTA MOUSE }\end{array}$ & $\begin{array}{l}\text { Actin, cytoplasmic } 1 ; \\
\text { Actin cytoplasmic } 1 ;\end{array}$ & $\begin{array}{l}\text { Beta-actin; } \\
\text { Beta-actin; }\end{array}$ \\
\hline YNELRVAPEE & HPVLLTEAPLNPKANR & $-1,1$ & $\mathrm{~N}-\operatorname{ter}+34.06 \mathrm{Da}, \mathrm{K}+34.06 \mathrm{Da}$ & 22,7 & -1,739 & 3 & 1837,1 & ACTB_MOUSE & ACTG_MOUSE & $\begin{array}{l}\text { Actin, cytoplasmic } 1 ; \\
\text { Actin, cytoplasmic } 1 \text {; }\end{array}$ & Beta-actin; \\
\hline ASLSTFQQMW & ISKQEYDESGPSIVHR & $-1,2$ & $\mathrm{~N}$-ter $+34.06 \mathrm{Da}, \mathrm{K}+34.06 \mathrm{Da}$ & 50,1 & 0,209 & 3 & 1912,0 & ACTB_MOUSE & ACTG_MOUSE & Actin, cytoplasmic 1; & Beta-actin; \\
\hline LSTFQQMWIS & KQEYDESGPSIVHR & $-0,8$ & $\mathrm{~N}-\operatorname{ter}+28.03 \mathrm{Da}, \mathrm{K}+28.03 \mathrm{Da}$ & 29,4 & $-9,102$ & 3 & 1699,8 & ACTB_MOUSE & ACTG_MOUSE & Actin, cytoplasmic 1; & Beta-actin; \\
\hline ATAASSSSLE & KSYELPDGQVITIGNER & 0,2 & $\mathrm{~N}$-ter +34.06 Da, K+34.06 Da & 20,8 & 2,76 & 3 & 1986 & ACTB_MOUSE & ACTA_MOUSE & Actin, cytoplasmic 1; & Beta-actin; \\
\hline LRVAPEEHPV & & 1,6 & $\mathrm{~N}-\mathrm{ter}+28.03 \mathrm{Da}, \mathrm{K}+28.03 \mathrm{Da}$ & 44,4 & 0,3 & 3 & 1491 & & ousE & & Beta-actin; \\
\hline STFQQMWWISK & QEYDESGPSIVHR & 0,2 & $\mathrm{~N}$-ter $+34.06 \mathrm{Da}$ & 17,0 & $-6,762$ & 3 & 1549,8 & ACTB_MOUSE & ACTG_MOUSE & Actin, cytoplasmic 1; & Beta-actin; \\
\hline DIAALVVDNG & SGMCKAGFAGDDAPR & 0,0 & $\begin{array}{l}\text { N-ter +34.06 Da, K +34.06 Da, C } \\
+57.20 \text { Da }\end{array}$ & 22,4 & $-5,591$ & 3 & 1606,8 & ACTB_MOUSE & ACTG_MOUSE & Actin, cytoplasmic 1; & Beta-actin; \\
\hline SLSTFQQMWI & SKQEYDESGPSIVHR & $-0,7$ & $\mathrm{~N}$-ter $+28.03 \mathrm{Da}, \mathrm{K}+28.03 \mathrm{Da}$ & 29 & $-1,62$ & 3 & 1786,9 & ACTE & ACTC & & Beta-actin; \\
\hline QEMATAASSS & $\begin{array}{l}\text { SLEKSYELPDGQVVITIGNER } \\
\text { SGIFYIS }\end{array}$ & 0,1 & $\mathrm{~N}$-ter +28.03 Da, K+28.03 Da & 55 & $-0,043$ & 3 & 2303,2 & ACTB_MOUSE & nOUSE & Actin, cytoplasmic 1; & Beta-actin; \\
\hline $\begin{array}{l}\text { EQEMATAASS } \\
\text { WIGGSILSL }\end{array}$ & $\begin{array}{l}\text { SSLEKSYELPDGQVITIGNER } \\
\text { STFEOMWISKOEYESGPSIVHR }\end{array}$ & $\begin{array}{r}2,5 \\
-0,5\end{array}$ & $\begin{array}{l}\mathrm{N} \text { Ner }+28.03 \mathrm{Da}, \mathrm{K}+28.03 \mathrm{Da} \\
\mathrm{N} \text {-ter }+30606 \mathrm{Da} \mathrm{K}+340\end{array}$ & 34,2 & $\begin{array}{l}0,418 \\
-0,991\end{array}-2$ & 3 & $\begin{array}{l}2390,2 \\
28204\end{array}$ & $\begin{array}{l}\text { ACTB_MOUSE } \\
\text { ACTB MOUSE }\end{array}$ & $\begin{array}{l}\text { ACTA_MOUSE } \\
\text { ATGG MOUSE }\end{array}$ & $\begin{array}{l}\text { Actin, cytoplassmic 1; } \\
\text { Actin cytoplasmic }\end{array}$ & $\begin{array}{l}\text { Beta-actin; } \\
\text { Beta-attin: }\end{array}$ \\
\hline
\end{tabular}




\begin{tabular}{|c|c|c|c|c|c|c|c|c|c|c|c|}
\hline Non Prime Site & Prime Site & 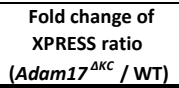 & Modifications & Hyperscore & $\begin{array}{c}\text { Mass error } \\
\text { in ppm }\end{array}$ & Charge & $\begin{array}{c}\text { Precursor } \\
\text { neutral mass } \\
\text { in Da }\end{array}$ & Uniprot ID 1 & Uniprot ID 2 & Protein Name 1 & Protein Name 2 \\
\hline $\begin{array}{l}\text { VDIRKDLYAN } \\
\text { VMDSGDGVTH }\end{array}$ & $\begin{array}{l}\text { TVLSGGTTMYPGIADR } \\
\text { TVPIYEGYALPHALR }\end{array}$ & $\begin{array}{l}0,2 \\
1,6\end{array}$ & $\begin{array}{l}\text { N-ter +34.06 Da } \\
\text { N-ter +28.03 Da }\end{array}$ & $\begin{array}{l}34,4 \\
16,3\end{array}$ & $\begin{array}{c}-1,075 \\
1,682\end{array}$ & $\begin{array}{ll}2 \\
3\end{array}$ & $\begin{array}{l}1671,9 \\
1840,0\end{array}$ & $\begin{array}{l}\text { ACTB_MOUSE } \\
\text { ACTB_MOUSE }\end{array}$ & $\begin{array}{l}\text { ACTG_MOUSE } \\
\text { ACTBL_MOUSE }\end{array}$ & $\begin{array}{l}\text { Actin, cytoplasmic 1; } \\
\text { Actin, cytoplasmic 1; }\end{array}$ & $\begin{array}{l}\text { Beta-actin; } \\
\text { Beta-actin; }\end{array}$ \\
\hline MEEEI & AALVIDNGSGMCKAGFAGDDAPR & $-0,4$ & 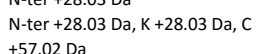 & $\begin{array}{l}10,3 \\
18,0\end{array}$ & $\begin{array}{l}1,202 \\
-2,424\end{array}$ & 3 & $\begin{array}{l}2348,1 \\
234,0\end{array}$ & ACTG_MOUSE & G3UYGO_MOUSE & Actin, cytoplasmic 2; & Gamma-actin; \\
\hline LDFEQEMATA & ASSSSLEKSYELPDGQVITIGNER & $-0,8$ & N-ter +28.03 Da, K +28.03 Da & 23,9 & $-2,388$ & 3 & 2635,3 & ACTG_MOUSE & ACTA_MOUSE & Actin, cytoplasmic 2; & Gamma-actin; \\
\hline M & EEEIAALVIDNGSGMCKAGFAGDDAPR & 4,9 & $\begin{array}{l}\text { N-ter +28.03 Da, K +28.03 Da, C } \\
+57.02 \mathrm{Da}\end{array}$ & 24,0 & 7,190 & 3 & 2848,4 & ACTG_MOUSE & G3UYGO_MOUSE & Actin, cytoplasmic 2; & Gamma-actin; \\
\hline RVAPEEHPVL & LTEAPLNPKANR & $-0,5$ & N-ter +34.06 Da, $\mathrm{K}+34.06 \mathrm{Da}$ & 33,1 & $-1,363$ & 3 & 1390,9 & ACTG_MOUSE & ACTA_MOUSE & Actin, cytoplasmic 2; & Gamma-actin; \\
\hline MEEEIAA & LVIDNGSGMCKAGFAGDDAPR & 0,5 & $\begin{array}{l}\text { N-ter +28.03 Da, K +28.03 Da, C } \\
+57.02 \mathrm{Da}\end{array}$ & 17,1 & 3,033 & 3 & 2206,1 & ACTG_MOUSE & G3UYGO_MOUSE & Actin, cytoplasmic 2; & Gamma-actin; \\
\hline $\begin{array}{l}\text { DFEQEMATAA } \\
\text { VALDFEQEMA }\end{array}$ & $\begin{array}{l}\text { SSSSLEKSYELPDGQVITIGNER } \\
\text { TAASSSSLEKSYELPDGQVITIGNER }\end{array}$ & $\begin{array}{l}-0,8 \\
0,6\end{array}$ & $\begin{array}{l}\text { N-ter +34.06 Da, } \mathrm{K}+34.06 \mathrm{Da} \\
\text { N-ter +34.06 Da, K+34.06 Da }\end{array}$ & $\begin{array}{l}38,6 \\
18,4\end{array}$ & $\begin{array}{l}6,785 \\
5,492\end{array}$ & $\begin{array}{l}3 \\
3\end{array}$ & $\begin{array}{l}2576,4 \\
2819,5\end{array}$ & $\begin{array}{l}\text { ACTG_MOUSE } \\
\text { ACTG_MOUSE }\end{array}$ & $\begin{array}{l}\text { ACTA_MOUSE } \\
\text { ACTA_MOUSE }\end{array}$ & $\begin{array}{l}\text { Actin, cytoplasmic 2; } \\
\text { Actin, cytoplasmic 2; }\end{array}$ & $\begin{array}{l}\text { Gamma-actin; } \\
\text { Gamma-actin; }\end{array}$ \\
\hline MEEEIAAL & VIDNGSGMCKAGFAGDDAPR & $-1,3$ & $\begin{array}{l}\text { N-ter +34.06 Da, K+34.06 Da, C } \\
+57.02 \mathrm{Da}\end{array}$ & 21,7 & $-3,890$ & 3 & 2105,0 & ACTG_MOUSE & G3UYGO_MOUSE & Actin, cytoplasmic 2; & Gamma-actin; \\
\hline $\mathrm{MC}$ & EEETTALVCDNGSGLCKAGFAGDDAPR & 0,3 & $\begin{array}{l}\text { N-ter +28.03 Da, } \mathrm{K}+28.03 \mathrm{Da}, \mathrm{C} \\
+57.02 \mathrm{Da}\end{array}$ & 27,7 & 8,143 & 3 & 2895,3 & ACTH_MOUSE & DЗZ2K3_MOUSE & Actin, gamma-enteric smooth muscle; & $\begin{array}{l}\text { Alpha-actin-3;Gamma-2-actin;Smooth muscle } \\
\text { gamma-actin; }\end{array}$ \\
\hline MCE & EETTALVCDNGSGLCKAGFAGDDAPR & $-10,0$ & $\begin{array}{l}\text { N-ter +34.06 Da, K }+34.06 \mathrm{Da}, \mathrm{C} \\
\text { +57.02 Da }\end{array}$ & 31,9 & 5,573 & 3 & 2778,3 & ACTH_MOUSE & D3Z2K3_MOUSE & Actin, gamma-enteric smooth muscle; & $\begin{array}{l}\text { Alpha-actin-3;Gamma-2-actin;Smooth muscle } \\
\text { gamma-actin; }\end{array}$ \\
\hline QDVRDRMIHR & STSQGSINSPVYSR & $-1,9$ & N-ter +34.06 Da & 43,0 & $-1,450$ & 2 & 1515,8 & ABLM1_MOUSE & E9QK41_MOUSE & Actin-binding LIM protein $1 ;$ & Actin-binding LIM protein family member 1 ; \\
\hline M & ILLEVNNR & $-0,4$ & N-ter $+28.03 \mathrm{Da}$ & 24,1 & 0,442 & 2 & 1128,6 & ARPC2_MOUSE & & Actin-related protein $2 / 3$ complex subunit 2 ; & Arp2/3 complex 34 kDa subunit; \\
\hline GTVTHLCRQY & SDAPPLTLDGIKDR & $-0,5$ & N-ter +34.06 Da, K +34.06 Da & 54,0 & $-0,064$ & 3 & 1564,9 & ACPM_MOUSE & F8WJ64_MOUSE & Acyl carrier protein, mitochondrial; & $\begin{array}{l}\text { Cl-SDAP;NADH-ubiquinone oxidoreductase } 9.6 \\
\text { kDa subunit; }\end{array}$ \\
\hline FAKPPVGPLR & FAPPEAPEPWSGVR & 0,2 & $\mathrm{~N}$-ter +34.06 Da & 16,7 & $-4,445$ & 2 & 1572,8 & EST2C_MOUSE & EST2E_MOUSE & Acylcarnitine hydrolase; & $\begin{array}{l}\text { Carboxylesterase 2;Peroxisome proliferator- } \\
\text { inducible acylcarnitine hydrolase; }\end{array}$ \\
\hline TGGRTTWART & NATLSVEPEGR & $-0,2$ & N-ter +28.03 Da & 20,9 & $-0,166$ & 2 & 1199,6 & ACOT2_MOUSE & & Acyl-coenzyme A thioesterase 2, mitochondrial; & $\begin{array}{l}\text { Acyl coenzyme A thioester hydrolase;MTE- } \\
\text { I;VVery-long-chain acyl-CoA thioesterase; }\end{array}$ \\
\hline SPCLLPRVHL & ASAFGSSTESLVAR & 0,0 & $\mathrm{~N}$-ter +28.03 Da & 58,9 & 0,071 & 2 & 1409,7 & ACO15_MOUSE & & Acyl-coenzyme A thioesterase THEM5; & $\begin{array}{l}\text { Acyl-coenzyme A thioesterase 15; Thioesterase } \\
\text { superfamily member } 5 \text {; }\end{array}$ \\
\hline LPRVHLASAF & GSSTESLVAR & 0,2 & $\mathrm{~N}$-ter $+28.03 \mathrm{Da}$ & 30,7 & $-0,869$ & 2 & 1033,5 & ACO15_MOUSE & & Acyl-coenzyme A thioesterase THEM5; & $\begin{array}{l}\text { Acyl-coenzyme A thioesterase 15; Thioesterase } \\
\text { superfamily member } 5 \text {; }\end{array}$ \\
\hline PCLLPRVHLA & SAFGSSTESLVAR & 0,1 & $\mathrm{~N}$-ter+34.06 Da & 42,3 & 0,000 & 2 & 1344,7 & ACO15_MOUSE & & Acyl-coenzyme A thioesterase THEM5; & $\begin{array}{l}\text { Acyl-coenzyme A thioesterase 15; Thioesterase } \\
\text { superfamily member } 5 \text {; }\end{array}$ \\
\hline NGTQRTRLLL & SDAQSMPSHTLAPGAR & $-6,4$ & $\mathrm{~N}$-ter +34.06 Da & 18,3 & 1,143 & 3 & 1658,8 & ADCY7_MOUSE & & Adenylate cyclase type 7; & $\begin{array}{l}\text { ATP pyrophosphate-lyase 7;Adenylate cyclase } \\
\text { type VII;Adenylyl cyclase 7; } \\
\text { ATP pyrophosphate-lyase 9;Adenylate cyclase }\end{array}$ \\
\hline PHQQLLHHHS & TEVSCDSSGDSNSVR & 4,6 & N-ter +28.03 Da, C+57.02 Da & 15,1 & $-1,288$ & 4 & 1626,7 & ADCY9_MOUSE & & Adenylate cyclase type 9; & $\begin{array}{l}\text { type IX;Adenylyl cyclase 9;Adenylyl cyclase type } \\
10 ; \\
\text { ATP-AMP transphosphorylase } 2 \\
\text { \{ECO:0000255|AHMAPA- }\end{array}$ \\
\hline M & APNVLASEPEIPKGIR & $-0,3$ & $\mathrm{~N}$-ter +28.03 Da, K+28.03 Da & 61,2 & 1,429 & 3 & 1746,0 & KAD2_MOUSE & & $\begin{array}{l}\text { Adenylate kinase 2, mitochondrial } \\
\{E C O: 0000255 \mid \text { HAMAP-Rule:MF_03168\}; }\end{array}$ & 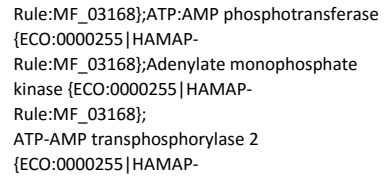 \\
\hline & NVLASEPEIPKGIR & $-0,4$ & N-ter +34.06 Da, K+34.06 Da & 36,3 & 0,502 & 3 & 1590,0 & KAD2_MOUSE & F7BP55_MOUSE & $\begin{array}{l}\text { Adenylate kinase 2, mitochondrial } \\
\text { \{ECO:0000255|HAMAP-Rule:MF_03168\}; }\end{array}$ & 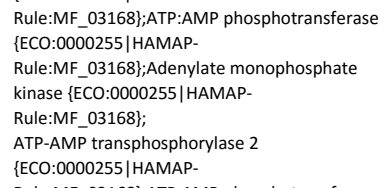 \\
\hline NVLA & SEPEIPKGIR & 0,0 & N-ter +34.06 Da, K+34.06 Da & 38,8 & $-2,258$ & 3 & 1192,7 & KAD2_MOUSE & F7BP55_MOUSE & $\begin{array}{l}\text { Adenylate kinase 2, mitochondrial } \\
\{E C O: 0000255 \text { |HAMAP-Rule:MF_03168\}; }\end{array}$ & $\begin{array}{l}\text { Rule:MF_031688;;ATP:AMP phosphotransferase } \\
\text { \{ECO:0_000255|HAMAP- } \\
\text { Rule:MF_03168\};Adenylate monophosphate } \\
\text { kinase \{ECO:0000255|HAMAP- } \\
\text { Rule:MF_03168\}; }\end{array}$ \\
\hline
\end{tabular}




\begin{tabular}{|c|c|c|c|c|c|c|c|c|c|c|c|}
\hline Non Prime Site & Prime Site & $\begin{array}{l}\text { Fold change of } \\
\text { XPRESS ratio } \\
\text { (Adam17 } 7_{\text {LKC } / W T)}\end{array}$ & Modifications & Hyperscore & $\begin{array}{l}\text { Mass error } \\
\text { in ppm }\end{array}$ & Charge & $\begin{array}{c}\text { Precursor } \\
\text { neutral mass } \\
\text { in Da }\end{array}$ & Uniprot ID 1 & Uniprot ID 2 & Protein Name 1 & Protein Name 2 \\
\hline $\mathrm{N}$ & VLASEPEIPKGIR & $-0,2$ & N-ter +28.03 Da, K+28.03 Da & 15,2 & 0,682 & 3 & 1463,9 & KAD2_MOUSE & F7BP55_MOUSE & $\begin{array}{l}\text { Adenylate kinase 2, mitochondrial } \\
\text { \{ECO:0000255|HAMAP-Rule:MF_03168\}; }\end{array}$ & 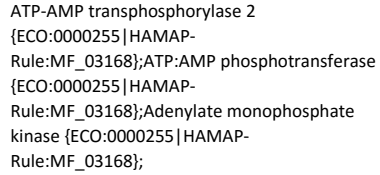 \\
\hline NTLQNISEGL & WYYPKVIER & $-0,2$ & $\mathrm{~N}$-ter +28.03 Da, K+28.03 Da & 18,6 & 4,135 & 3 & 1157,7 & PUR8_MOUSE & E9Q242_MOUSE & Adenylosuccinate lyase; & Adenylosuccinase; \\
\hline LTVGKRCCL & WIQDLCMDLQNLKR & 1,3 & $\begin{array}{l}\text { N-ter +34.06 Da, K+34.06 Da, C } \\
+57.02 \mathrm{Da}\end{array}$ & 17,8 & 0,998 & 3 & 1900,0 & PUR8_MOUSE & E9Q242_MOUSE & Adenylosuccinate lyase; & Adenylosuccinase; \\
\hline $\begin{array}{l}\text { VGSGPPPPPP } \\
\text { GIPSPPSYGS }\end{array}$ & $\begin{array}{l}\text { GPPPPPISTSSGSDDSASR } \\
\text { GPSA }\end{array}$ & 0,2 & $\mathrm{~N}$-ter $+34.06 \mathrm{Da}$ & $\begin{array}{l}41,6 \\
409\end{array}$ & $-0,217$ & 3 & $\begin{array}{l}1844,9 \\
2478,2\end{array}$ & $\begin{array}{l}\text { CAP1_MOUSE } \\
\text { CAP1 MOUSE }\end{array}$ & & Adenylyl cyclase-associated protein 1 & \\
\hline $\begin{array}{l}\text { GLPSGPSVGS } \\
\text { RTFGTTGRR }\end{array}$ & $\begin{array}{l}\text { GPPPPPPGPPPPPISTSSGSDDSASR } \\
\text { AGEEAADSPELRR }\end{array}$ & $\begin{array}{l}-0,3 \\
-0,4\end{array}$ & $\begin{array}{l}\mathrm{N}-\mathrm{ter}+28.03 \mathrm{Da} \\
\mathrm{N} \text {-ter }+28.03 \mathrm{Da}\end{array}$ & $\begin{array}{l}40,9 \\
47,5\end{array}$ & $\begin{array}{l}6,247 \\
-2,554\end{array}$ & $\begin{array}{l}3 \\
2\end{array}$ & $\begin{array}{l}2478,2 \\
1368,7\end{array}$ & $\begin{array}{l}\text { CAP1_MOUSE } \\
\text { ADXL_MOUSE }\end{array}$ & & $\begin{array}{l}\text { Adenylyl cyclase-associated protein 1; } \\
\text { Adrenodoxin-like protein, mitochondrial; }\end{array}$ & Ferredoxin-1-like protein; \\
\hline GSPAMHVASF & APEPCTDNSDSLIAKINQR & $-0,4$ & $\begin{array}{l}\text { N-ter +28.03 Da, K+28.03 Da, C } \\
+57.02 \text { Da }\end{array}$ & 29,2 & 6,173 & 3 & 2184,1 & AKAP8_MOUSE & & A-kinase anchor protein $8 ;$ & A-kinase anchor protein $95 \mathrm{kDa}$; \\
\hline AAGSPAMHVA & SFAPEPCTDNSDSLAAKINQR & 0,1 & $\begin{array}{l}\text { N-ter +28.03 Da, K+28.03 Da, C } \\
+57.02 \text { Da }\end{array}$ & 29,7 & 1,198 & 3 & 2418,2 & AKAP8_MOUSE & & A-kinase anchor protein 8 & A-kinase anchor protein $95 \mathrm{kDa}$; \\
\hline LPQYLDQSCF & AVMLGGPEETR & 0,1 & $\mathrm{~N}$-ter +34.06 Da & 22,3 & $-0,084$ & 2 & 1192,6 & E9Q3E1_MOUSE & & $\begin{array}{l}\text { Aldehyde dehydrogenase } \\
\text { \{ECO:0000256|PRRNR:PIRNR036492\}; }\end{array}$ & \\
\hline FGPILPLVTV & RSLDEAIEFINR & $-1,7$ & $\mathrm{~N}$-ter +34.06 Da & 23,3 & $-8,340$ & 3 & 1495,8 & E9Q3E1_MOUSE & & $\begin{array}{l}\text { Aldehyde dehydrogenase } \\
\text { \{ECO:0000256|PIRNR:PIRNR036492\}; }\end{array}$ & \\
\hline MSAAETG & SEPSQGAGPSEATLHSLR & 1,0 & $\mathrm{~N}$-ter +34.06 Da & 31,3 & 1,989 & 3 & 1857,0 & E9Q3E1_MOUSE & & $\begin{array}{l}\text { Aldehyde dehydrogenase } \\
\text { \{ECO:0000256|PIRNR:PIRNR036492\}; }\end{array}$ & \\
\hline INRREKPLAL & YAFSNNNQVVNGMLER & 0,4 & $\mathrm{~N}$-ter $+28.03 \mathrm{Da}$ & 34,2 & $-1,124$ & 3 & 1953,9 & E9Q3E1_MOUSE & & $\begin{array}{l}\text { Aldehyde dehydrogenase } \\
\text { \{ECO:0000256|PIRNR:PIRNR036492\}; }\end{array}$ & \\
\hline $\begin{array}{l}\text { PALLNAITRF } \\
\text { RRGPRISRLL }\end{array}$ & $\begin{array}{l}\text { YGDNPQTSPNLGR } \\
\text { SAAATSAVPAPNHQPEVFCNQIFINNEWHDAVSR }\end{array}$ & $\begin{array}{c}0,1 \\
-0,3\end{array}$ & $\begin{array}{l}\text { N-ter }+34.06 \mathrm{Da} \\
\text { N-ter }+34.06 \mathrm{Da}, \mathrm{C}+57.02 \mathrm{Da}\end{array}$ & $\begin{array}{l}22,5 \\
30,5\end{array}$ & $\begin{array}{r}1,995 \\
-1,075\end{array}$ & $\begin{array}{l}2 \\
4\end{array}$ & $\begin{array}{l}1451,7 \\
3810,8\end{array}$ & $\begin{array}{l}\text { AL3B1_MOUSE } \\
\text { ALDH2_MOUSE }\end{array}$ & $\begin{array}{l}\text { E9Q3E1_MOUSE } \\
\text { D3YYF__MOUSE }\end{array}$ & $\begin{array}{l}\text { Aldehyde dehydrogenase family } 3 \text { member B1; } \\
\text { Aldehyde dehydrogenase, mitochondrial; }\end{array}$ & $\begin{array}{l}\text { Aldehyde dehydrogenase 7; } \\
\text { AHD-M1;ALDH class 2;ALDH-E2;ALDHI; }\end{array}$ \\
\hline QMKGSGKCCM & DPDEKCLESR & 1,0 & $\begin{array}{l}\text { N-ter }+28.03 \mathrm{Da}, \mathrm{K}+28.03 \mathrm{Da}, \mathrm{C} \\
+57.02 \mathrm{Da}\end{array}$ & 15,7 & $-1,225$ & 3 & 1303,6 & Q3TYQ9_MOUSE & & Aldehyde oxidase 4; & $\begin{array}{l}\text { Aldehyde oxidase homolog 2;Azaheterocycle } \\
\text { hydroxylase 4;Retinal oxidase; }\end{array}$ \\
\hline DFFIGKPTLR & ELTSQHPYAEVFIGR & 0,5 & $\mathrm{~N}$-ter +28.03 Da & 11,3 & 1,576 & 3 & 1773,9 & MGTSA_MOUSE & & $\begin{array}{l}\text { Alpha-1,6-mannosylglycoprotein 6-beta-N- } \\
\text { acetylglucosaminyltransferase } A \text {; }\end{array}$ & $\begin{array}{l}\text { Alpha-mannoside beta-1,6-N- } \\
\text { acetylylucosaminyltransferase;Gl|cNAc-T } \\
\text { V;Mannoside actylglucosaminyyltransferase } \\
\text { 5;N-Acactylglucosaminyl-transferase V; }\end{array}$ \\
\hline DVQETDTSQK & DQSPASHEIATNLGDFALR & 0,6 & $\mathrm{~N}$-ter +28.03 Da & 21,4 & 0,000 & 3 & 2069,0 & A1AT4_MOUSE & & Alpha-1-antitrypsin 1-4; & $\begin{array}{l}\text { Alpha-1 protease inhibitor 4;Serine protease } \\
\text { inhibitor 1-4; Serine protease inhibitor A1d; }\end{array}$ \\
\hline VPTANAALPA & DPPASVVVGPVVVPR & $-0,8$ & N-ter +34.06 Da & 30,1 & 7,026 & 2 & 1520,9 & FETUA_MOUSE & & Alpha-2-HS-glycoprotein; & Countertrypin;Fetuin-A; \\
\hline DQVIASFKVL & AGDKNFITAEELR & $-1,3$ & N-ter +34.06 Da, K +34.06 Da & 32,2 & 0,130 & 3 & 1530,9 & ACTN4_MOUSE & & Alpha-actinin-4; & $\begin{array}{l}\text { F-actin cross-linking protein; Non-muscle alpha- } \\
\text { actinin 4; }\end{array}$ \\
\hline TAANRICKVL & AVNQENEHLMEDYER & 0,4 & N-ter +28.03 Da & 38,7 & 0,420 & 3 & 1903,8 & ACTN4_MOUSE & E9Q2W9_MOUSE & Alpha-actinin-4; & $\begin{array}{l}\text { F-actin cross-linking protein;Non-muscle alpha- } \\
\text { actinin 4; }\end{array}$ \\
\hline SDIKALLRKH & EAFESDLAAHQDR & $-1,5$ & N-ter +34.06 Da & 18,3 & 0,394 & 3 & 1521,7 & ACTN4_MOUSE & ACTN1_MOUSE & Alpha-actinin-4; & $\begin{array}{l}\text { F-actin cross-linking protein; Non-muscle alpha- } \\
\text { actinin 4; }\end{array}$ \\
\hline GPEEFKACLI & SLGYDVENDR & 0,5 & $\mathrm{~N}$-ter $+34.06 \mathrm{Da}$ & 16,1 & $-0,582$ & 2 & 1200,6 & ACTN4_MOUSE & & Alpha-actinin-4; & $\begin{array}{l}\text { F-actin cross-linking protein;Non-muscle alpha- } \\
\text { actinin 4; }\end{array}$ \\
\hline GWRDLGYVYL & NIDDCWIGGR & $-4,0$ & $\mathrm{~N}$-ter $+34.06 \mathrm{Da}, \mathrm{C}+57.02 \mathrm{Da}$ & 16,9 & $-5,642$ & 2 & 1238,6 & NAGAB_MOUSE & & Alpha-N-acetylgalactosaminidase; & Alpha-galactosidase B; \\
\hline SRAGLAAISR & AQGLASTSTAPSPSR & $-0,6$ & $\mathrm{~N}$-ter +28.03 Da & 25,3 & $-0,617$ & 2 & 1457,8 & ZFN2B_MOUSE & EocY18_MOUSE & AN1-type zinc finger protein 2B; & $\begin{array}{l}\text { Arsenite-inducible RNA-associated protein-like } \\
\text { protein; }\end{array}$ \\
\hline VPVRFLFLLL & GPSSANMDYHEIGR & 1,3 & $\mathrm{~N}$-ter $+28.03 \mathrm{Da}$ & 14,9 & $-0,831$ & 3 & 1560,7 & B3A2_MOUSE & & Anion exchange protein 2; & $\begin{array}{l}\text { Band 3-related protein;Non-erythroid band 3- } \\
\text { like protein;Solute carrier family } 4 \text { member 2; }\end{array}$ \\
\hline MTEEETARLR & AGALMARPNSPQSSGSGR & 0,8 & $\mathrm{~N}$-ter +34.06 Da & 20,7 & $-1,966$ & 3 & 1776,9 & ANR63_MOUSE & & $\begin{array}{l}\text { Ankyrin repeat domain-containing protein } 63 \\
\{E C O: 0000250 \mid \text { UniProtKB:C9JTQ0\}; }\end{array}$ & \\
\hline HLEEVVLAML & KTPAQFDADELR & 0,3 & $\mathrm{~N}-\operatorname{ter}+34.06 \mathrm{Da}, \mathrm{K}+34.06 \mathrm{Da}$ & 27,6 & 0,479 & 3 & 1457,8 & ANXA1_MOUSE & & Annexin A1; & 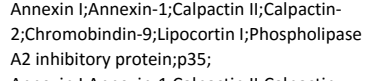 \\
\hline ELRGAMKGLG & TDEDTLEILTTR & 0,5 & $\mathrm{~N}$-ter +28.03 Da & 35,4 & $-2,583$ & 2 & 1546,8 & ANXA1_MOUSE & & Annexin A1; & 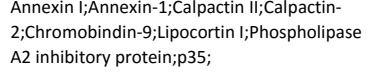 \\
\hline
\end{tabular}




\begin{tabular}{|c|c|c|c|c|c|c|c|c|c|c|c|}
\hline Non Prime Site & Prime Site & 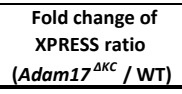 & Modifications & Hyperscore & $\begin{array}{c}\text { Mass error } \\
\text { in ppm }\end{array}$ & Charge & $\begin{array}{c}\text { Precursor } \\
\text { neutral mass } \\
\text { in Da } \\
\end{array}$ & Uniprot ID 1 & Uniprot ID 2 & Protein Name 1 & Protein Name 2 \\
\hline RELYDAGYKR & KGTDVPKWISIMTER & 4,3 & N-ter +28.03 Da, K+28.03 Da & 15,2 & $-2,815$ & 3 & 1844,0 & ANXA2_MOUSE & BOV2N5_MOUSE & Annexin A2; & $\begin{array}{l}\text { Annexin II;Annexin-2; Calpactin I heavy } \\
\text { chain;Calpactin-1 heavy chain; Chromobindin- } \\
\text { 8;ilipocortin II;Placental anticoagulant protein } \\
\text { IV;Protein I;p36; }\end{array}$ \\
\hline KLMVALAKGR & RAEDGSVIDYELIDQDAR & 5,5 & N-ter+28.03 Da & 36,7 & $-4,535$ & 3 & 2092,0 & ANXA2_MOUSE & BOV2N5_MOUSE & Annexin A2; & $\begin{array}{l}\text { Annexin II;Annexin-2; Calpactin I heavy } \\
\text { chain;Calpactin-1 heavy chain; Chromobindin- } \\
\text { 8;ilipocortin II;Placental anticoagulant protein } \\
\text { IV;Protein I;;36; }\end{array}$ \\
\hline LCKLSLEGDH & STPPSAYGSVKPYTNFDAER & $-4,0$ & N-ter +28.03 Da, K+28.03 Da & 30,9 & 0,757 & 3 & 2242,1 & ANXA2_MOUSE & BOV2N7_MOUSE & Annexin A2; & $\begin{array}{l}\text { Annexin II;Annexin-2;Calpactin I heavy } \\
\text { chain;Calpactin-1 heavy chain;Chromobindin- } \\
\text { 8;Lipocortin II;Placental anticoagulant protein } \\
\text { IV;Protein I;p36; }\end{array}$ \\
\hline DALNIETAVK & TKGVDEVTIVNILTNR & 2,2 & $\mathrm{~N}$-ter +28.03 Da, $\mathrm{K}+28.03 \mathrm{Da}$ & 30,8 & $-4,809$ & 3 & 1827,0 & ANXA2_MOUSE & BOV2N7_MOUSE & Annexin A2; & $\begin{array}{l}\text { Annexin II:Annexin-2; Calpactin I heavy } \\
\text { chain;Calpactin-1 heavy chain;Chromobindin- } \\
\text { 8;Lipocortin II;Placental anticoagulant protein } \\
\text { IV;Protein I;p36; }\end{array}$ \\
\hline DSLIEIICSR & TNQELQEINR & 3,9 & $\mathrm{~N}$-ter $+28.03 \mathrm{Da}$ & 37,9 & 0,628 & 2 & 1271,7 & ANXA2_MOUSE & BOV2N5_MOUSE & Annexin A2; & $\begin{array}{l}\text { Annexin II;Annexin-2; Calpactin I heavy } \\
\text { chain;Calpactin-1 heavy chain;Chromobindin- } \\
\text { 8;Lipocortin II;Placental anticoagulant protein } \\
\text { IV;Protein I;p36; }\end{array}$ \\
\hline CKLSLEGDHS & TPPSAYGSVKPYTNFDAER & 1,9 & N-ter +34.06 Da, K +34.06 Da & 23,1 & $-2,028$ & 3 & 2167,1 & ANXA2_MOUSE & BOV2N7_MOUSE & Annexin A2; & $\begin{array}{l}\text { Annexin II:Annexin-2; Calpactin I heavy } \\
\text { chain;Cal;pactin-1 heavy chain;Chromobindin- } \\
\text { 8;Lipocortin II;Placental anticoagulant protein } \\
\text { IV;Protein I;p36; }\end{array}$ \\
\hline ALLALKFILA & FAIPDKPR & $-0,4$ & $\mathrm{~N}$-ter +28.03 Da, K+28.03 Da & 16,2 & $-1,697$ & 3 & 998,6 & ANO10_MOUSE & & Anoctamin-10; & Transmembrane protein 16K; \\
\hline GALGCAICHG & NPVDDICIAKPR & $-0,3$ & $\begin{array}{l}\text { N-ter +34.06 Da, K +34.06 Da, C } \\
\text { +57.02 Da }\end{array}$ & 30,5 & 0,409 & 3 & 1464,8 & ANT3_MOUSE & & Antithrombin-III; & Serpin C1; \\
\hline VQMGAVDLLG & GGLDSLIGDSNFGAPSASVAAAPAPAR & $-0,1$ & $\mathrm{~N}$-ter +28.03 Da & 33,2 & $-5,402$ & 3 & 1320,7 & AP1B1_MOUSE & Q5SVG5_MOUSE & AP-1 complex subunit beta-1; & $\begin{array}{l}\text { Adaptor protein complex AP-1 subunit beta- } \\
\text { 1;Adaptor-related protein complex } 1 \text { subunit } \\
\text { beta-1;Beta-1-adaptin;Beta-adaptin 1;Clathrin } \\
\text { assembly protein complex } 1 \text { beta large } \\
\text { chain;Golgi adaptor HA1/AP1 } 1 \text { adaptin beta } \\
\text { subunit; } \\
\text { Adaptor protein complex AP-1 subunit beta- } \\
\text { 1;Adaptor-related protein complex } 1 \text { subunit } \\
\text { beta-1;Beta-1-adaptin;Beta-adaptin 1;Clathrin } \\
\text { assembly protein complex } 1 \text { beta large } \\
\text { chain; Golgi adaptor HA1/AP1 adaptin beta } \\
\text { subunit; }\end{array}$ \\
\hline KASESSSEES & SSMEDSSSESESESGSDSEPAPR & $-10,0$ & $\mathrm{~N}$-ter +34.06 Da & 13,1 & 1,659 & 4 & 2407,0 & AP3B1_MOUSE & & AP- 3 complex subunit beta- 1 ; & $\begin{array}{l}\text { Adaptor protein complex AP-3 subunit beta- } \\
\text { 1;Adaptor-related protein complex } 3 \text { subunit } \\
\text { beta-1;Beta-3A-adaptin;Clathrin assembly } \\
\text { protein complex } 3 \text { beta-1 large chain; }\end{array}$ \\
\hline QSQWDKVKDF & ANVYVDAVKDSGR & 0,4 & $\mathrm{~N}$-ter +34.06 Da, K+34.06 Da & 21,6 & 1,230 & 3 & 1460,8 & APOA1_MOUSE & & Apolipoprotein A-l; & Apolipoprotein A1; \\
\hline $\begin{array}{l}\text { GSAAWHVWQQ } \\
\text { OLLNLENW }\end{array}$ & $\begin{array}{l}\text { DEPQSQWDKVKDFANVYVDAVKDSGR } \\
\text { DTLGSTVSOLOER }\end{array}$ & $\begin{array}{l}0,2 \\
0,2\end{array}$ & $\begin{array}{l}\text { N-ter +28.03 Da, K +28.03 Da } \\
\text { N-ter+34.06 Da }\end{array}$ & $\begin{array}{l}23,9 \\
34,0\end{array}-10$ & $\begin{array}{l}-0,225 \\
-0,85\end{array}$ & $\begin{array}{l}4 \\
2\end{array}$ & $\begin{array}{l}3107,6 \\
1466,8\end{array}$ & $\begin{array}{l}\text { APPA1_MOUSE } \\
\text { APOA1 MOUSE }\end{array}$ & & $\begin{array}{l}\text { Apolipoprotein A-l; } \\
\text { Apolipoprotein A-l: }\end{array}$ & $\begin{array}{l}\text { Apoliopopopotein A1; } \\
\text { Apoliloporotein A1: }\end{array}$ \\
\hline ARQKLQELQG & RLSPVAEERR & 0,3 & $\begin{array}{l}\text { N-ter }+34.06 \mathrm{Da} \\
\text { N-ter +34.06 Da }\end{array}$ & 19,5 & $-0,403$ & 3 & 1236,7 & APOA1 MOUSE & & $\begin{array}{l}\text { Apopipoporotenin A-l; } \\
\text { Apolipoprotein A-l; }\end{array}$ & Apolipoprotein A1; \\
\hline LVTLLTGCLA & EGEPEVTDQLEWQSNQPWEQALNR & $-1,7$ & $\mathrm{~N}$-ter +34.06 Da & 47,4 & $-2,055$ & 3 & 2916,4 & APOE_MOUSE & G3UWN5_MOUSE & E Apolipoprotein E; & \\
\hline GPLVEQGRQR & TANLGAGAAQPLR & $-2,9$ & $\mathrm{~N}$-ter +34.06 Da & 31,0 & $-3,609$ & 2 & 1272,7 & APOE_MOUSE & G3UWN5_MOUSE & E Apolipoprotein E; & \\
\hline LQELAEQLQT & TKEESGAVAAAASVPAQSTAR & 4,3 & $\mathrm{~N}$-ter $+28.03 \mathrm{Da}, \mathrm{K}+28.03 \mathrm{Da}$ & 18,4 & 1,214 & 3 & 2057,1 & ASC_MOUSE & & $\begin{array}{l}\text { Apoptosis-associated speck-like protein containing a } \\
\text { CARD; }\end{array}$ & PYD and CARD domain-containing protein; \\
\hline SREAEAPTLK & QFEDEEGEER & 10,3 & N-ter +28.03 Da & 16,9 & 5,009 & 3 & 1294,5 & ACINU_MOUSE & F6RJ39_MOUSE & $\begin{array}{l}\text { Apoptotic chromatin condensation inducer in the } \\
\text { nucleus; }\end{array}$ & \\
\hline ASPHPAQLLR & SLSPLSGTTDTKAESPAGR & $-1,1$ & N-ter $+34.06 \mathrm{Da}, \mathrm{K}+34.06 \mathrm{Da}$ & 33,5 & 2,519 & 3 & 1942,1 & ACINU_MOUSE & FGRJ39_MOUSE & $\begin{array}{l}\text { Apoptotic chromatin condensation inducer in the } \\
\text { nucleus; }\end{array}$ & \\
\hline
\end{tabular}




\begin{tabular}{|c|c|c|c|c|c|c|c|c|c|c|c|}
\hline Non Prime Site & Prime Site & $\begin{array}{l}\text { Fold change of } \\
\text { XPRESS ratio } \\
\text { (Adam17 } 7^{\text {AkC } / \text { WT) }} \\
\end{array}$ & Modifications & Hyperscore & $\begin{array}{l}\text { Mass error } \\
\text { in ppm }\end{array}$ & Charge & $\begin{array}{c}\begin{array}{c}\text { Precursor } \\
\text { neutral mass } \\
\text { in Da }\end{array} \\
\end{array}$ & Uniprot ID 1 & Uniprot ID 2 & Protein Name 1 & Protein Name 2 \\
\hline PVTPPEPPPT & AGEDEGLPAAPPIPPR & $-5,3$ & N-ter +34.06 Da & 16,7 & $-9,551$ & 3 & 1619,9 & ARAP1_MOUSE & & $\begin{array}{l}\text { Arf-GAP with Rho-GAP domain, ANK repeat and PH } \\
\text { domain-containing protein 1; }\end{array}$ & $\begin{array}{l}\text { Centaurin-delta-2 } \\
\text { \{ECO:0000250|UniProtkB:Q96P48\}; }\end{array}$ \\
\hline GQIPCVLTRS & VHSVAIVGAPFSR & 0,1 & N-ter +28.03 Da & 28,3 & $-1,387$ & 3 & 1366,8 & ARGI2_MOUSE & & Arginase-2, mitochondrial; & $\begin{array}{l}\text { Kidney-type arginase;Non-hepatic } \\
\text { arginase;Type II arginase; }\end{array}$ \\
\hline $\begin{array}{l}\text { ESGWTDTESD } \\
\text { AHPDFNGGAW }\end{array}$ & $\begin{array}{l}\text { SDSEPDVPQR } \\
\text { GPAASEEEEEEEEEEEEGR }\end{array}$ & $\begin{array}{c}-6,4 \\
8,1\end{array}$ & $\begin{array}{l}\text { N-ter +34.06 Da } \\
\text { N-ter +28.03 Da }\end{array}$ & $\begin{array}{l}14,6 \\
12,9\end{array}$ & $\begin{array}{r}0,000 \\
-3,333\end{array}$ & $\begin{array}{l}3 \\
4\end{array}$ & $\begin{array}{l}1162,6 \\
2305,9\end{array}$ & $\begin{array}{l}\text { ARMX2_MOUSE } \\
\text { ARSI_MOUSE }\end{array}$ & & $\begin{array}{l}\text { Armadillo repeat-containing X-linked protein 2; } \\
\text { Arylsulfatase I; }\end{array}$ & \\
\hline IGGLAEFCKA & SAELALGENNEVLKSGR & $-0,5$ & $\mathrm{~N}$-ter $+34.06 \mathrm{Da}, \mathrm{K}+34.06 \mathrm{Da}$ & 37,2 & 2,962 & 3 & 1854,1 & AATM_MOUSE & & Aspartate aminotransferase, mitochondrial; & $\begin{array}{l}\text { Fatty acid-binding protein;Glutamate } \\
\text { oxaloacetate transaminase 2;Kynurenine } \\
\text { aminotransferase 4;Kynurenine } \\
\text { aminotransferase IV;Kynurenine--oxoglutarate } \\
\text { transaminase 4;Kynurenine--oxoglutarate } \\
\text { transaminase IV;Plasma membrane-associated } \\
\text { fatty acid-binding protein;Transaminase A; }\end{array}$ \\
\hline GLYGERVGAF & TVVCKDAEEAKR & 0,6 & $\begin{array}{l}\text { N-ter +28.03 Da, } \mathrm{K}+28.03 \mathrm{Da}, \mathrm{C} \\
+57.02 \mathrm{Da}\end{array}$ & 20,2 & $-0,804$ & 4 & 1488,8 & AATM_MOUSE & & Aspartate aminotransferase, mitochondrial; & $\begin{array}{l}\text { Fatty acid-binding protein;Glutamate } \\
\text { oxaloacetate transaminase 2;Kynurenine } \\
\text { aminotransferase 4;Kynurenine } \\
\text { aminotransferase IV;Kynurenine--oxoglutarate } \\
\text { transaminase 4;Kynurenine--oxoglutarate } \\
\text { transaminase IV;Plasma membrane-associated } \\
\text { fatty acid-binding protein; Transaminase A; }\end{array}$ \\
\hline EHHLVPILAT & AVQEELEKGTPEPGPLGATDER & 1,6 & N-ter +34.06 Da, $\mathrm{K}+34.06 \mathrm{Da}$ & 21,5 & $-1,003$ & 3 & 2390,3 & DNPEP_MOUSE & & Aspartyl aminopeptidase; & \\
\hline VLEAADGSGI & FDEDEDDLQR & $-2,5$ & N-ter+28.03 Da & 12,9 & $-1,526$ & 2 & 1308,6 & ATX3_MOUSE & Q5M8S1_MOUSE & Ataxin-3; & Machado-Joseph disease protein 1 homolog; \\
\hline PGIIPRIISVR & EPMQTGIKAVDSLVPIGR & $-0,8$ & $\mathrm{~N}$-ter $+28.03 \mathrm{Da}, \mathrm{K}+28.03 \mathrm{Da}$ & 18,3 & $-2,184$ & 3 & 1966,1 & ATPA_MOUSE & D3Z6F5_MOUSE & ATP synthase subunit alpha, mitochondrial; & \\
\hline RNLHASNTRL & QKTGTAEMSSILEER & $-0,4$ & $\mathrm{~N}$-ter $+34.06 \mathrm{Da}, \mathrm{K}+34.06 \mathrm{Da}$ & 38,5 & -1,200 & 3 & 1746,9 & ATPA_MOUSE & DGRJ16_MOUSE & ATP synthase subunit alpha, mitochondrial; & \\
\hline LTELKRGQY & SPMAIEEQVAVIYAGVR & $-1,1$ & N-ter+34.06 Da & 19,8 & $-2,943$ & 3 & 1866,0 & ATPA_MOUSE & D3Z6F5_MOUSE & ATP synthase subunit alpha, mitochondrial; & \\
\hline LIKEGDVVKR & TGAIVDVPVGEELLGR & 1,7 & $\mathrm{~N}$-ter $+28.03 \mathrm{Da}$ & 24,7 & $-5,744$ & 2 & 1651,9 & ATPA_MOUSE & D3Z6F_M_MOUSE & ATP synthase subunit alpha, mitochondrial; & \\
\hline PAGVHPARDY & AAQASAAPKAGTATGR & $-0,2$ & $\mathrm{~N}$-ter $+34.06 \mathrm{Da}$, $\mathrm{K}+34.06 \mathrm{Da}$ & 51,4 & 0,467 & 3 & 1495,9 & ATPB_MOUSE & & ATP synthase subunit beta, mitochondrial; & \\
\hline AGVHPARDYA & AQASAAPKAGTATGR & 0,0 & $\mathrm{~N}$-ter $+34.06 \mathrm{Da}, \mathrm{K}+34.06 \mathrm{Da}$ & 16,9 & 0,070 & 3 & 1424,8 & ATPB_MOUSE & & ATP synthase subunit beta, mitochondrial; & \\
\hline SVQAIYVPAD & DLTDPAPATTFAHLDATTVISR & -0,2 & $\mathrm{N}$-ter $+28.03 \mathrm{Da}$ & 33,8 & $-3,542$ & 3 & 2340,2 & ATPB_MOUSE & & ATP synthase subunit beta, mitochondrial; & \\
\hline QDIAILGMD & ELSEEDKLTVSR & $-0,2$ & $\mathrm{~N}$-ter $+34.06 \mathrm{Da}, \mathrm{K}+34.06 \mathrm{Da}$ & 19,0 & $-5,488$ & 3 & 1472,8 & ATPB_MOUSE & & ATP synthase subunit beta, mitochondrial; & \\
\hline TVLSRAIAEL & GIYPAVDPLDSTSR & $-0,1$ & N-ter +34.06 Da & 20,0 & 0,393 & 2 & 1523,8 & ATPB_MOUSE & & ATP synthase subunit beta, mitochondrial; & \\
\hline KGSITSVQAI & YVPADDLTDPAPATTFAHLDATTVLSR & $-1,8$ & $\mathrm{~N}$-ter $+34.06 \mathrm{Da}$ & 21,7 & 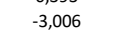 & 3 & 2891,5 & ATPB_MOUSE & & ATP synthase subunit beta, mitochondrial; & \\
\hline QEEKEDVKSC & AEFVSGSQLR & $-0,6$ & $\mathrm{~N}-\mathrm{ter}+34.06 \mathrm{Da}$ & 19,2 & -0,177 & 2 & 1126,6 & ATPSH_MOUSE & D3Z1B2_MOUSE & ATP synthase subunit d, mitochondrial; & \\
\hline SWNETTHARL & ASLSEKPPAIDWAYYR & $-0,2$ & $\mathrm{~N}$-ter $+34.06 \mathrm{Da}$, $\mathrm{K}+34.06 \mathrm{Da}$ & 27,9 & 1,600 & 3 & 1934,1 & ATPSH_MOUSE & G3X9L_MOUSE & ATP synthase subunit d, mitochondrial; & \\
\hline ETFHARLASL & $\begin{array}{l}\text { SEKPPAIDWAYYR } \\
\text { Se }\end{array}$ & $-0,4$ & $\mathrm{~N}$-ter $+34.06 \mathrm{Da}, \mathrm{K}+34.06 \mathrm{Da}$ & 32,4 & 0,960 & 3 & 1662,9 & ATPSH_MOUSE & G3X9L_MOUSE & ATP synthase subunit d, mitochondrial; & \\
\hline WNETFHARLA & SLSEKPPAIDWAYYR & -0,2 & $\mathrm{N}$-ter $+34.06 \mathrm{Da}, \mathrm{K}+34.06 \mathrm{Da}$ & 19,8 & $-4,180$ & 3 & 1863,0 & ATPSH_MOUSE & G3X9L_MOUSE & ATP synthase subunit d, mitochondrial; & \\
\hline VNADSSVQLL & AEEAVTLDMLDLGAAR & $-0,7$ & $\mathrm{~N}$-ter $+34.06 \mathrm{Da}$ & 21,8 & 7,427 & 2 & 1707,9 & ATPD_MOUSE & & ATP synthase subunit delta, mitochondrial; & F-ATPase delta subunit; \\
\hline LDMLDLGAAR & ANLEKAQSELSGAADEAAR & 1,3 & $\mathrm{~N}$-ter $+28.03 \mathrm{Da}, \mathrm{K}+28.03 \mathrm{Da}$ & 36,0 & 3,017 & 3 & 1986,0 & ATPD_MOUSE & & ATP synthase subunit delta, mitochondrial; & F-ATPase delta subunit; \\
\hline SPLIKFGRYS & ALIIGMAYGAKR & 7,6 & $\mathrm{~N}$-ter $+34.06 \mathrm{Da}, \mathrm{K}+34.06 \mathrm{Da}$ & 30,3 & 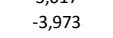 & 3 & 1330,8 & ATPSI_MOUSE & Q8BTB6_MOUSE & ATP synthase subunit e, mitochondrial; & \\
\hline M & $\begin{array}{l}\text { VPPVQVSPLIKFGR } \\
\text { VPVIN }\end{array}$ & $-0,3$ & $\mathrm{~N}$-ter $+28.03 \mathrm{Da}, \mathrm{K}+28.03 \mathrm{Da}$ & 41,9 & 0,439 & 3 & 1592,0 & ATPSI_MOUSE & Q8BTB6_MOUSE & ATP synthase subunit e, mitochondrial; & \\
\hline LVATEVWMWF & $\begin{array}{l}\text { YIGEIGKR } \\
\text { YITH }\end{array}$ & 0,5 & $\mathrm{~N}$-ter $+34.06 \mathrm{Da}, \mathrm{K}+34.06 \mathrm{Da}$ & 22,2 & $-3,307$ & 3 & 1115,7 & ATPSL_MOUSE & F2Z452_MOUSE & ATP synthase subunit g, mitochondrial; & \\
\hline M & ATLKDITR & $-0,2$ & $\mathrm{~N}$-ter +28.03 Da, $\mathrm{K}+28.03 \mathrm{Da}$ & 21,3 & $-2,050$ & 3 & 972,6 & ATPG_MOUSE & Q8C2Q8_MOUSE & ATP synthase subunit gamma, mitochondrial; & F-ATPase gamma subunit; \\
\hline RSFSTSVVRP & FAKLVRPPVQVYGIEGR & -0,5 & $\mathrm{N}$-ter $+28.03 \mathrm{Da}, \mathrm{K}+28.03 \mathrm{Da}$ & 33,7 & $-2,465$ & 4 & 1984,2 & ATPO_MOUSE & FGXVMI_MOUSE & $\begin{array}{l}\text { ATP synthase subunit O, mitochondrial; } \\
\text { a }\end{array}$ & Oligomycin sensitivity conferral protein; \\
\hline LLAENGRLGN & TQGISAFSTIMSVHR & 0,9 & $\mathrm{~N}$-ter +28.03 Da & 21,2 & $-7,031$ & 3 & 1774,9 & ATPO_MOUSE & F7D3P8_MOUSE & ATP synthase subunit $O$, mitochondrial; & Oligomycin sensitivity conferral protein; \\
\hline QTRGFVSDSS & DSMDTGAGSIR & $-0,1$ & N-ter +28.03 Da & 29,5 & $-0,527$ & 2 & 1136,5 & ATIF1_MOUSE & & ATPase inhibitor, mitochondrial; & Inhibitor of $F(1) F(0)-A T$ Pase; \\
\hline KVLQTRGFVS & DSSDSMDTGAGSIR & $\begin{array}{l}-, 11 \\
-0,6\end{array}$ & N-ter $+34.06 \mathrm{Da}$ & 53,8 & 0,907 & 2 & 1431,6 & ATIF1_MOUSE & & ATPase inhibitor, mitochondrial; & Inhibitor of $F(1) F(0)-A T P a s e ;$ \\
\hline LQTRGFVSDS & SDSMDTGAGSIR & 0,2 & $\mathrm{~N}$-ter $+28.03 \mathrm{Da}$ & 45,2 & 0,571 & 2 & 1223,6 & ATIF1_MOUSE & & ATPase inhibitor, mitochondrial; & Inhibitor of $F(1) F(0)-A T P a s e ;$ \\
\hline MKVLQTRGFV & SDSSDSMDTGAGSIR & $-0,4$ & $\mathrm{~N}$-ter +34.06 Da & 65,2 & 0,132 & 2 & 1518,7 & ATIF1_MOUSE & & ATPase inhibitor, mitochondrial; & Inhibitor of F(1)F(0)-ATPase; \\
\hline TRGFVSDSSD & SMDTGAGSIR & 0,3 & $\mathrm{~N}$-ter +34.06 Da & 30,2 & $-2,040$ & 2 & 1027,5 & ATIF1_MOUSE & & ATPase inhibitor, mitochondrial; & Inhibitor of $F(1)$ F(o)-ATPase; \\
\hline VLQTRGFVSD & SSDSMDTGAGSIR & $-0,1$ & $\mathrm{~N}$-ter +28.03 Da & 49,2 & 0,457 & 2 & 1310,6 & ATIF1_MOUSE & & ATPase inhibitor, mitochondrial; & Inhibitor of $F(1)$ F(o)-ATPase; \\
\hline GMKVLQTRGF & VSDSSDSMDTGAGSIR & $-0,5$ & $\mathrm{~N}$-ter +34.06 Da & 68,8 & $-0,432$ & 2 & 1617,7 & ATIF1_MOUSE & & ATPase inhibitor, mitochondrial; & Inhibitor of F(1)F(0)-ATPase; \\
\hline HPRLCLALLC & EAKESPPAQPTR & $-0,3$ & $\mathrm{~N}$-ter $+34.06 \mathrm{Da}, \mathrm{K}+34.06 \mathrm{Da}$ & 19,8 & $-0,797$ & 3 & 1377,8 & ABCB8_MOUSE & D3Z116_MOUSE & $\begin{array}{l}\text { ATP-binding cassette sub-family B member } 8 \text {, } \\
\text { mitochondrial; }\end{array}$ & \\
\hline & DDSEEEEEEEENR & $-0,4$ & N-ter +34.06 Da & 35,8 & 0,359 & & & & & $\begin{array}{l}\text { Mitochondrial; } \\
\text { ATP-binding cassette sub-family F member } 1 \text {; }\end{array}$ & \\
\hline GNVFEALLQD & DSEEEEEEEENR & $-0,2$ & N-ter + 34.06 Da & 14,5 & $-7,892$ & 2 & 1556,6 & ABCF1_MOUSE & & ATP-binding cassette sub-family F member 1 ; & \\
\hline GGFGSGGGGF & GGGGGGFSGGGGGGFGGGR & $-10,0$ & N-ter +34.06 Da & 20,6 & $-0,415$ & 2 & 1444,7 & DHX9_MOUSE & E9QNN1_MOUSE & ATP-dependent RNA helicase A; & DEAH box protein 9;Nuclear DNA helicase II; \\
\hline LGLDQQFAGL & DLNSSDNQSGGSTASKGR & 0,1 & N-ter +34.06 Da, K+34.06 Da & 69,5 & $-0,486$ & 3 & 1847,9 & DDX3X_MOUSE & & ATP-dependent RNA helicase DDX3X; & $\begin{array}{l}\text { D1Pas1-related sequence } 2 \text { 2DEAD box RNA } \\
\text { helicase DEAD 3;DEAD bex protein } 3, x- \\
\text { chromosomal;:Embryonic RNA helicase; }\end{array}$ \\
\hline
\end{tabular}




\begin{tabular}{|c|c|c|c|c|c|c|c|c|c|c|c|}
\hline Non Prime Site & Prime Site & $\begin{array}{l}\text { Fold change of } \\
\text { XPRESS ratio } \\
\text { (Adam17 } 7 \text { akc /WT) }\end{array}$ & Modifications & Hyperscore & $\begin{array}{l}\text { Mass error } \\
\text { in ppm }\end{array}$ & Charge & $\begin{array}{c}\text { Precursor } \\
\text { neutral mass } \\
\text { in Da } \\
\end{array}$ & Uniprot ID 1 & Uniprot ID 2 & Protein Name 1 & Protein Name 2 \\
\hline HVAVENALGL & DQQFAGLDLNSSDNQSGGSTASKGR & $-0,4$ & N-ter +34.06 Da, K+34.06 Da & 24,6 & $-0,728$ & 3 & 2607,3 & DDX3X_MOUSE & & ATP-dependent RNA helicase DDX3X; & $\begin{array}{l}\text { D1Pas1-related sequence 2; DEAD box RNA } \\
\text { helicase DEAD3; DEAD box protein 3, X- } \\
\text { chromosomal;Embryonic RNA helicase; }\end{array}$ \\
\hline KNNHNNCKKT & SNEEKPKLSIGEECR & $-2,5$ & $\begin{array}{l}\text { N-ter +34.06 Da, K +34.06 Da, C } \\
\text { +57.02 Da }\end{array}$ & 18,1 & 3,457 & 3 & 1877,1 & ARISB_MOUSE & & AT-rich interactive domain-containing protein $5 B$; & $\begin{array}{l}\text { Developmentally and sexually retarded with } \\
\text { transient immune abnormalities protein;MRF1- } \\
\text { like;Modulator recognition factor protein 2; }\end{array}$ \\
\hline RGFDKAYVVL & GQFLVLKKDEDLFR & $-0,2$ & $\mathrm{~N}$-ter +34.06 Da, $\mathrm{K}+34.06 \mathrm{Da}$ & 34,3 & 1,600 & 3 & 1809,1 & BAF_MOUSE & & Barrier-to-autointegration factor; & $\begin{array}{l}\text { Breakpoint cluster region protein 1;LAP2- } \\
\text { binding protein 1; }\end{array}$ \\
\hline KAYVVLGQFL & VLKKDEDLFR & 0,4 & N-ter +34.06 Da, K+34.06 Da & 29,3 & $-0,439$ & 3 & 1363,9 & BAF_MOUSE & & Barrier-to-autointegration factor; & $\begin{array}{l}\text { Breakpoint cluster region protein 1;LAP2- } \\
\text { binding protein 1; }\end{array}$ \\
\hline LISGYSGAQA & ELHVSVPPR & $-0,1$ & N-ter +28.03 Da & 28,0 & $-1,504$ & 3 & 1060,6 & BCAM_MOUSE & D3YTK7_MOUSE & Basal cell achesion molecule; & $\begin{array}{l}\text { B-CAM cell surface glycoprotein; Lutheran } \\
\text { antigen; }\end{array}$ \\
\hline MTSKLHSSLK & SAYHQYLQTSR & 4,8 & $\mathrm{~N}$-ter $+28.03 \mathrm{Da}$ & 17,8 & 4,629 & 2 & 1380,7 & CRBG3_MOUSE & J3QPS2_MOUSE & Beta/gamma crystallin domain-containing protein 3; & \\
\hline RPRHQGVMVG & MGQKDCYVGDEAQSKR & $-0,7$ & $\begin{array}{l}\text { N-ter +34.06 Da, K +34.06 Da, C } \\
\text { +57.02 Da }\end{array}$ & 30,0 & 7,182 & 4 & 1973,0 & ACTBL_MOUSE & & Beta-actin-like protein 2; & Kappa-actin; \\
\hline $\begin{array}{l}\text { TYAGWVSSQL } \\
M\end{array}$ & $\begin{array}{l}\text { SQLPQHSIDQGAEDVVMAFSR } \\
\text { APSQLALFSVSDKTGLVEFAR }\end{array}$ & $\begin{array}{l}-1,9 \\
-0,4\end{array}$ & $\begin{array}{l}\mathrm{N} \text {-ter +34.06 Da } \\
\mathrm{N} \text {-ter +34.06 Da, K+34.06 Da }\end{array}$ & $\begin{array}{l}33,4 \\
69,6\end{array}$ & $\begin{array}{l}1,489 \\
0,173\end{array}$ & $\begin{array}{l}3 \\
3\end{array}$ & $\begin{array}{l}2348,2 \\
2303,3\end{array}$ & $\begin{array}{l}\text { CNBP1_MOUSE } \\
\text { PUR9_MOUSE }\end{array}$ & & $\begin{array}{l}\text { Beta-catenin-interacting protein } 1 ; \\
\text { Bifunctional purine biosynthesis protein PURH; }\end{array}$ & Inhibitor of beta-catenin and TCf-4; \\
\hline LVSICLFHSL & FSFQVEDYYAPTIESLIR & 0,0 & $\mathrm{~N}$-ter +28.03 Da & 19,5 & $-0,725$ & 3 & 2205,1 & BSPH1_MOUSE & & Binder of sperm protein homolog 1 & $\begin{array}{l}\text { Bovine seminal plasma protein homolog } \\
\text { 1:Bovine seminal plasma protein-like 1: }\end{array}$ \\
\hline SLMTHAMTFT & AVSEKDNQEGTFVKWR & $-0,8$ & $\mathrm{~N}$-ter +28.03 Da, $\mathrm{K}+28.03 \mathrm{Da}$ & 38,5 & 2,929 & 3 & 1977,0 & BLMH_MOUSE & & Bleomycin hydrolase; & \\
\hline MEEIFRVVCI & CLGNPPETFTWEYR & $-0,2$ & $\mathrm{~N}$-ter +28.03 Da, C +57.02 Da & 14,8 & 3,224 & 2 & 1796,8 & BLMH_MOUSE & E9PY26_MOUSE & Bleomycin hydrolase; & \\
\hline EEFEFSQSYL & FFWDKVER & $-0,4$ & N-ter +28.03 Da, K +28.03 Da & 24,0 & $-1,013$ & 3 & 1181,6 & BLMH_MOUSE & E9PYUO_MOUSE & Bleomycin hydrolase; & \\
\hline VERCYFFLNA & FVDTAQKKEPEDGR & $-0,4$ & N-ter +28.03 Da, K +28.03 Da & 29,6 & 0,938 & 3 & 1702,9 & BLMH_MOUSE & E9PYUO_MOUSE & Bleomycin hydrolase; & \\
\hline EFEFSQSYLF & FWDKVER & $-0,8$ & N-ter +28.03 Da, $\mathrm{K}+28.03 \mathrm{Da}$ & 21,6 & $-1,253$ & 3 & 1034,6 & BLMH_MOUSE & E9PYUO_MOUSE & Bleomycin hydrolase; & \\
\hline EIFRVVCICL & GNPPETFTWEYR & $-0,3$ & N-ter +34.06 Da & 46,9 & $-2,024$ & 2 & 1529,7 & BLMH_MOUSE & E9PY26_MOUSE & Bleomycin hydrolase; & \\
\hline MNVYDHELVF & GVSLKNMNKAER & $-0,4$ & N-ter +28.03 Da, K +28.03 Da & 22,3 & $-0,976$ & 4 & 1429,8 & BLMH_MOUSE & & Bleomycin hydrolase; & \\
\hline MMEEIFRVVC & ICLGNPPETFTWEYR & $-0,1$ & N-ter +34.06 Da, C +57.02 Da & 40,8 & $-2,501$ & 3 & 1915,9 & BLMH_MOUSE & E9PY26_MOUSE & Bleomycin hydrolase; & \\
\hline EEIFRVVCIC & LGNPPETFTWEYR & $-0,1$ & N-ter +34.06 Da & 20,0 & $-1,581$ & 2 & 1642,8 & BLMH_MOUSE & E9PY26_MOUSE & Bleomycin hydrolase; & \\
\hline DKVERCYFFL & NAFVDTAQKKEPEDGR & $-0,5$ & N-ter +28.03 Da, K+28.03 Da & 21,9 & 0,793 & 4 & 1888,0 & BLMH_MOUSE & E9PYUO_MOUSE & Bleomycin hydrolase; & \\
\hline MTFTAVSEKD & NQEGTFVKWR & $-0,2$ & $\mathrm{~N}$-ter $+28.03 \mathrm{Da}, \mathrm{K}+28.03 \mathrm{Da}$ & 29,9 & $-0,151$ & 3 & 1319,7 & BLMH_MOUSE & & Bleomycin hydrolase; & \\
\hline MTHAMTFTAV & SEKDNQEGTFVKWR & $-0,3$ & $\mathrm{~N}$-ter $+28.03 \mathrm{Da}, \mathrm{K}+28.03 \mathrm{Da}$ & 26,7 & $-0,055$ & 3 & 1806,9 & BLMH_MOUSE & & Bleomycin hydrolase; & \\
\hline FCIRLRNLVH & SGATKGEISSTQDAMMEEIFR & $-1,1$ & $\mathrm{~N}$-ter $+34.06 \mathrm{Da}, \mathrm{K}+34.06 \mathrm{Da}$ & 35,2 & 6,573 & 3 & 2355,2 & BLMH_MOUSE & E9PY26_MOUSE & Bleomycin hydrolase; & \\
\hline ESLMTHAMTF & TAVSEKDNQEGTFVKWR & $-0,5$ & N-ter +34.06 Da, K +34.06 Da & 61,0 & $-0,858$ & 3 & 2096,2 & BLMH_MOUSE & & Bleomycin hydrolase; & \\
\hline FGESLMTHAM & TFTAVSEKDNQEGTFVKWR & $-1,3$ & N-ter +34.06 Da, K +34.06 Da & 23,0 & $-0,298$ & 4 & 2344,3 & BLMH_MOUSE & & Bleomycin hydrolase; & \\
\hline LMTHAMTFTA & $\begin{array}{l}\text { VSEKDNQEGTFVKWR } \\
\text { SETV }\end{array}$ & $-0,7$ & $\mathrm{~N}$-ter +34.06 Da, $\mathrm{K}+34.06 \mathrm{Da}$ & 29,8 & 0,052 & 3 & 1924,1 & BLMH_MOUSE & & Bleomycin hydrolase; & \\
\hline NVYDHELVFG & VSLKNMNKAER & $-0,7$ & $\mathrm{~N}$-ter +28.03 Da, $\mathrm{K}+28.03 \mathrm{Da}$ & 21,8 & $-3,416$ & 3 & 1372,8 & BLMH_MOUSE & & Bleomycin hydrolase; & \\
\hline VQGAQHVFQH & VVPQEGKPVTNQKSSGR & $-0,8$ & $\mathrm{~N}-\operatorname{ter}+28.03 \mathrm{Da}, \mathrm{K}+28.03 \mathrm{Da}$ & 27,7 & $-3,057$ & 3 & 1894,1 & BLMH_MOUSE & E9PZH4_MOUSE & Bleomycin hydrolase; & \\
\hline EYEQTGNEYN & NEYEVYDNENGEPR & $-0,2$ & $\mathrm{~N}$-ter $+34.06 \mathrm{Da}$ & 17,3 & 1,304 & 3 & 1760,8 & SIAL_MOUSE & & Bone sialoprotein 2; & $\begin{array}{l}\text { Bone sialoprotein II;Cell-binding } \\
\text { sialoprotein;Integrin-binding sialoprotein; }\end{array}$ \\
\hline LLWSLYIASS & QTVYPGITAR & $-0,7$ & $\mathrm{~N}$-ter $+28.03 \mathrm{Da}$ & 21,8 & 0,793 & 2 & 1132,6 & BPIFC_MOUSE & Q3V1D7_MOUSE & BPI fold-containing family C protein; & $\begin{array}{l}\text { Bactericidal/permeability-increasing protein- } \\
\text { like 2; }\end{array}$ \\
\hline PGLAIHCMQV & HKETIDAVPNAIPGR & $-0,3$ & N-ter +34.06 Da, K+34.06 Da & 18,0 & $-1,540$ & 3 & 1685,0 & Q99LA2_MOUSE & Q9JMDO_MOUSE & $\begin{array}{l}\text { BUB3-interacting and GLEBS motif-containing protein } \\
\text { ZNF207; }\end{array}$ & $\begin{array}{l}49 \mathrm{kDa} \text { zinc finger protein;Zinc finger protein } \\
207 \text {; }\end{array}$ \\
\hline M & APAGILNGKLVSAQIR & $-0,3$ & N-ter +34.06 Da, K+34.06 Da & 34,4 & 0,656 & 3 & 1675,1 & C1TC_MOUSE & & C-1-tetrahydrofolate synthase, cytoplasmic; & \\
\hline VYPGLRRQKR & DWVIPPISCPENEKGEFPKNLVQIKSNR & $-0,7$ & $\begin{array}{l}\text { N-ter +28.03 Da, K+28.03 Da, C } \\
+57.02 \mathrm{Da}\end{array}$ & 20,2 & $-0,323$ & 5 & 3405,8 & CADH1_MOUSE & & Cadherin-1; & ARC-1;Epithelial cadherin;Uvomorulin; \\
\hline ELPHKNMFTV & NRDTGVISVLTSGLDR & $-0,3$ & N-ter $+34.06 \mathrm{Da}$ & 17,9 & $\begin{array}{l}1,725 \\
-489\end{array}$ & 3 & 1736,0 & CADH1_MOUSE & & Cadherin-1; & ARC-1;Epithelial cadherin;Uvomorulin; \\
\hline $\begin{array}{l}\text { LLSQVLLVTS } \\
\text { RTSPVPRQKR }\end{array}$ & $\begin{array}{l}\text { ADDLECTPGFQR } \\
\text { SIVVSPILPENOR }\end{array}$ & $\begin{array}{l}0,1 \\
0,1\end{array}$ & $\begin{array}{l}\text { N-ter +34.06 Da, C +57.02 Da } \\
\text { N-ter +28.03 Da }\end{array}$ & $\begin{array}{l}21,5 \\
53,1\end{array}$ & $\begin{array}{c}-4,849 \\
0,188\end{array}$ & $\begin{array}{l}2 \\
2\end{array}$ & $\begin{array}{l}1449,7 \\
151,9\end{array}$ & $\begin{array}{l}\text { CAD13_MOUSE } \\
\text { CAD13 MOUSE }\end{array}$ & & $\begin{array}{l}\text { Cadherin-13; } \\
\text { Cadherin-13; }\end{array}$ & $\begin{array}{l}\text { Heart cadherin;Truncated cadherin; } \\
\text { Heart cadherin;Truncated cadherin; }\end{array}$ \\
\hline PTRLLRRRKR & EWVMPPIIVYPENGKGPFPQR & $-0,3$ & N-ter +34.06 Da, $\mathrm{K}+34.06 \mathrm{Da}$ & 29,6 & $-2,505$ & 3 & 2392,3 & CADH__MOUSE & & Cadherin-3; & $\begin{array}{l}\text { Placental cadherin; } \\
\text { Calcineurin B-like protein;Calcium-binding }\end{array}$ \\
\hline PLGDRIINAF & FSEGEDQVNFR & $-0,8$ & $\mathrm{~N}$-ter +28.03 Da & 12,6 & $-1,622$ & 2 & 1354,6 & CHP1_MOUSE & BORO91_MOUSE & Calcineurin B homologous protein 1; & $\begin{array}{l}\text { protein CHP;Calcium-binding protein p22; ;F- } \\
\text { hand calcium-binding domain-containing } \\
\text { protein p22;Sid 470;p24; }\end{array}$ \\
\hline TMVTCGGTVY & SVGGSIAPR & $-6,4$ & $\mathrm{~N}$-ter $+34.06 \mathrm{Da}$ & 13,9 & 1,024 & 2 & 876,5 & CALI_MOUSE & & Calicin; & \\
\hline FLTMMARKMK & DTDSEEEIR & $-0,6$ & $\mathrm{~N}$-ter $+34.06 \mathrm{Da}$ & 30,0 & $-1,684$ & 2 & 1126,5 & CALM_MOUSE & CALL3_MOUSE & Calmodulin; & \\
\hline TNLGEKLTD & EEVDEMIR & $-0,2$ & $\mathrm{~N}$-ter $+34.06 \mathrm{Da}$ & 16,6 & 0,663 & 2 & 1053,5 & CALM_MOUSE & Q3UKW2_MOUSE & E Calmodulin; & \\
\hline KEAFSLFDKD & GDGTITTKELGTVMR & 0,1 & N-ter +28.03 Da, K +28.03 Da & 40,9 & 1,527 & 3 & 1633,9 & CALM_MOUSE & Q3UKW2_MOUSE & E Calmodulin; & \\
\hline MINEVDADGN & GTIDFPEFLTMMAR & $-0,3$ & $\mathrm{~N}$-ter $+28.03 \mathrm{Da}$ & 16,6 & 0,241 & 2 & 1655,8 & CALM_MOUSE & Q3UKW2_MOUSE & E Calmodulin; & \\
\hline AFRVFDKDGN & GYISAAELR & $-0,2$ & $\mathrm{~N}$-ter $+34.06 \mathrm{Da}$ & 15,6 & 0,493 & 2 & 1012,6 & CALM_MOUSE & Q3UKW2_MOUSE & E Calmodulin; & \\
\hline INEVDADGNG & TIDFPEFLTMMAR & 1,9 & N-ter +28.03 Da & 19,7 & $-0,625$ & 2 & 1598,8 & CALM_MOUSE & Q3UKW2_MOUSE & E Calmodulin; & \\
\hline
\end{tabular}




\begin{tabular}{|c|c|c|c|c|c|c|c|c|c|c|c|}
\hline Non Prime Site & Prime Site & $\begin{array}{c}\text { Fold change of } \\
\text { XPRESS ratio } \\
\text { (Adam17 } 7^{\text {KKc } / W T)}\end{array}$ & Modifications & Hyperscore & $\begin{array}{l}\text { Mass error } \\
\text { in ppm }\end{array}$ & Charge & $\begin{array}{c}\text { Precursor } \\
\text { neutral mass } \\
\text { in Da }\end{array}$ & Uniprot ID 1 & Uniprot ID 2 & Protein Name 1 & Protein Name 2 \\
\hline FSLFDKDGDG & TITTKELGTVMR & 1,8 & N-ter +34.06 Da, K +34.06 Da & 21,5 & $-0,493$ & 3 & 1416,9 & CALM_MOUSE & Q3UKW2_MOUSE & E Calmodulin; & \\
\hline SEEEREAFR & VFDKDGNGYISAAELR & 1,9 & $\mathrm{~N}$-ter +28.03 Da, $\mathrm{K}+28.03 \mathrm{Da}$ & 32,0 & 0,441 & 3 & 1809,9 & CALM_MOUSE & Q3UKW2_MOUSE & E Calmodulin; & \\
\hline QEELEDMIRV & ADVDQDGKVKYEEFVR & $-0,5$ & $\mathrm{~N}$-ter +34.06 $\mathrm{Da}, \mathrm{K}+34.06 \mathrm{Da}$ & 20,6 & $-1,848$ & 3 & 1999,1 & CALM4_MOUSE & & Calmodulin-4; & Calcium-binding protein Dd112; \\
\hline MSHGFTKEEV & AEFQAAFNR & $-0,4$ & $\mathrm{~N}$-ter +34.06 Da & 25,2 & $-0,184$ & 2 & 1086,6 & CALM4_MOUSE & & Calmodulin-4; & Calcium-binding protein Dd112; \\
\hline MSHG & FTKEEVAEFQAAFNR & 0,6 & $\mathrm{~N}$-ter +34.06 $\mathrm{Da}, \mathrm{K}+34.06 \mathrm{Da}$ & 22,2 & 6,731 & 3 & 1854,0 & CALM4_MOUSE & & Calmodulin-4; & Calcium-binding protein Dd112; \\
\hline MSH & GFTKEEVAEFQAAFNR & $-0,2$ & $\mathrm{~N}$-ter +34.06 Da, $\mathrm{K}+34.06 \mathrm{Da}$ & 20,0 & $-2,456$ & 3 & 1911,0 & CALM4_MOUSE & & Calmodulin-4; & Calcium-binding protein Dd112; \\
\hline MSHGFT & KEEVAEFQAAFNR & 0,2 & $\mathrm{~N}$-ter +28.03 Da, $\mathrm{K}+28.03 \mathrm{Da}$ & 18,4 & 2,943 & 3 & 1593,8 & CALM4_MOUSE & & Calmodulin-4; & Calcium-binding protein Dd112; \\
\hline ITVDELKESL & SKLGESLSQEELEDMIR & $-0,9$ & $\mathrm{~N}$-ter +28.03 Da, $\mathrm{K}+28.03 \mathrm{Da}$ & 30,8 & $-5,687$ & 3 & 2019,0 & CALM4_MOUSE & & Calmodulin-4; & Calcium-binding protein Dd112; \\
\hline LKESLSKLGE & SLSQEELEDMIR & 0,7 & $\mathrm{~N}$-ter $+28.03 \mathrm{Da}$ & 36,1 & $-1,217$ & 2 & 1476,7 & CALM4_MOUSE & & Calmodulin-4; & Calcium-binding protein Dd112; \\
\hline MSHGF & TKEEVAEFQAAFNR & 1,1 & $\mathrm{~N}$-ter +28.03 Da, $\mathrm{K}+28.03 \mathrm{Da}$ & 31,9 & $-0,295$ & 3 & 1694,9 & CALM4_MOUSE & & Calmodulin-4; & Calcium-binding protein Dd112; \\
\hline SQEELEDMIR & VADVDQDGKVKYEEFVR & 4,7 & $\mathrm{~N}$-ter +28.03 Da, $\mathrm{K}+28.03 \mathrm{Da}$ & 23,6 & 3,071 & 4 & 2080,1 & CALM4_MOUSE & & Calmodulin-4; & Calcium-binding protein Dd112; \\
\hline SQEELEDMIR & VADVDQDGKVK & $-0,4$ & $\mathrm{~N}$-ter +28.03 Da, $\mathrm{K}+28.03 \mathrm{Da}$ & 20,8 & 8,335 & 3 & 1256,7 & CALM4_MOUSE & & Calmodulin-4; & Calcium-binding protein Dd112; \\
\hline ELEDMIRVAD & VDQDGKVKYEEFVR & $-0,2$ & $\mathrm{~N}$-ter +28.03 Da, $\mathrm{K}+28.03 \mathrm{Da}$ & 20,4 & $-4,558$ & 4 & 1794,9 & CALM4_MOUSE & & Calmodulin-4; & Calcium-binding protein Dd112; \\
\hline KEAFSLFDKD & GDGSITTQELGTVMR & 0,1 & $\mathrm{~N}$-ter +34.06 Da & 16,1 & 3,500 & 2 & 1597,8 & CALL3_MOUSE & & Calmodulin-like protein 3; & \\
\hline EFKEAFSLFD & KDGDGSITTQELGTVMR & 0,8 & $\mathrm{~N}$-ter +28.03 Da, $\mathrm{K}+28.03 \mathrm{Da}$ & 17,2 & 1,125 & 3 & 1862,9 & CALL3_MOUSE & & Calmodulin-like protein 3; & \\
\hline FPEFLTMMAR & KMKDTDSEEEIR & 0,1 & $\mathrm{~N}$-ter +28.03 Da, $\mathrm{K}+28.03 \mathrm{Da}$ & 27,0 & 1,532 & 3 & 1563,8 & CALL3_MOUSE & CALM_MOUSE & Calmodulin-like protein 3; & \\
\hline PEFLTMMARK & MKDTDSEEEIR & 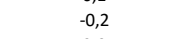 & $\mathrm{N}$-ter +34.06 Da, $\mathrm{K}+34.06 \mathrm{Da}$ & 21,5 & 3,374 & 3 & 1419,7 & CAL3_MOUSE & CALM_MOUSE & Calmodulin-like protein 3; & \\
\hline VNEIDKDGNG & TVDFPEFLTMMSR & 2,0 & $\mathrm{~N}$-ter $+28.03 \mathrm{Da}$ & 20,4 & $-0,125$ & 2 & 1600,8 & CALL3_MOUSE & & Calmodulin-like protein 3; & \\
\hline SEEEEREAFR & VFDKDGNGFVSAAELR & $-0,7$ & N-ter +28.03 Da, K+28.03 Da & 20,9 & $-4,375$ & 3 & 1779,9 & CALL3_MOUSE & & Calmodulin-like protein 3; & \\
\hline PEPPPQQQHF & TVVEASESEEVRR & 0,1 & $\mathrm{~N}$-ter $+28.03 \mathrm{Da}$ & 19,3 & $-2,104$ & 3 & 1517,8 & CPNS2_MOUSE & & Calpain small subunit 2 ; & Calcium-dependent protease small subunit 2; \\
\hline PEPPPQQQHF & TVVEASESEEVR & 0,0 & $\mathrm{~N}$-ter +34.06 Da & 45,4 & $-1,095$ & 2 & 1367,7 & CPNS2_MOUSE & & Calpain small subunit 2; & Calcium-dependent protease small subunit 2; \\
\hline PPPQQQHFTV & VEASESEEVR & $-0,2$ & $\mathrm{~N}$-ter +34.06 Da & 21,1 & 0,257 & 2 & 1167,6 & CPNS2_MOUSE & & Calpain small subunit 2; & Calcium-dependent protease small subunit 2; \\
\hline DNFKTLFSKL & AGDDMEISVKELQTILNR & 1,1 & N-ter +34.06 Da, K +34.06 Da & 35,3 & 4,995 & 3 & 2099,2 & CAN1_MOUSE & & Calpain-1 catalytic subunit; & $\begin{array}{l}\text { Calcium-activated neutral proteinase 1;Calpain } \\
\text { mu-type;Calpain-1 large subunit;:Micromolar- } \\
\text { calpain; }\end{array}$ \\
\hline IKWKRPTELM & SNPQFIVDGATR & 0,1 & $\mathrm{~N}$-ter +34.06 Da & 18,1 & 7,315 & 2 & 1337,7 & CAN1_MOUSE & & Calpain-1 catalytic subunit; & $\begin{array}{l}\text { Calcium-activated neutral proteinase 1;Calpain } \\
\text { mu-type; Calpain-1 large subunit;:Micromolar- } \\
\text { calpain; }\end{array}$ \\
\hline RIRLPPGEYI & VVPSTFEPNKEGDFLLR & 1,7 & N-ter +34.06 Da, K +34.06 Da & 28,1 & 2,428 & 3 & 2015,1 & CAN1_MOUSE & & Calpain-1 catalytic subunit; & $\begin{array}{l}\text { Calcium-activated neutral proteinase 1;Calpain } \\
\text { mu-type; Calpain-1 large subunit;:Micromolar- } \\
\text { calpain; }\end{array}$ \\
\hline GGCRNYPATF & WVNPQFKIR & 0,7 & N-ter +34.06 Da, K +34.06 Da & 16,1 & $-0,954$ & 3 & 1254,8 & CAN1_MOUSE & & Calpain-1 catalytic subunit; & $\begin{array}{l}\text { Calcium-activated neutral proteinase 1;Calpain } \\
\text { mu-type;Calpain-1 large subunit;:Micromolar- } \\
\text { calpain; }\end{array}$ \\
\hline PRASMCSIRS & APPKLASLKGVVPEDAVETLAGSLGTR & $-0,5$ & N-ter +28.03 Da, $\mathrm{K}+28.03 \mathrm{Da}$ & 16,1 & 4,161 & 4 & 2759,6 & ICAL_MOUSE & Q8CE80_MOUSE & Calpastatin; & Calpain inhibitor; \\
\hline CSIRSAPPKL & ASLKGVVPEDAVETLAGSLGTR & 3,5 & $\mathrm{~N}$-ter +34.06 Da, K+34.06 Da & 25,0 & $-1,116$ & 3 & 2237,3 & ICAL_MOUSE & Q8CE80_MOUSE & Calpastatin; & Calpain inhibitor; \\
\hline RSAPPKLASL & KGVVPEDAVETLAGSLGTR & 0,1 & N-ter +34.06 Da, K +34.06 Da & 34,3 & $-0,609$ & 3 & 1966,1 & ICAL_MOUSE & Q8CE80_MOUSE & Calpastatin; & Calpain inhibitor; \\
\hline VPRASMCSIR & SAPPKLASLKGVVPEDAVETLAGSLGTR & $-0,5$ & N-ter +28.03 Da, $\mathrm{K}+28.03 \mathrm{Da}$ & 34,6 & 1,368 & 4 & 2846,6 & ICAL_MOUSE & Q8CE80_MOUSE & Calpastatin; & Calpain inhibitor; \\
\hline SIRSAPPKLA & SLKGVVPEDAVETLAGSLGTR & $-0,2$ & $\mathrm{~N}$-ter $+28.03 \mathrm{Da}, \mathrm{K}+28.03 \mathrm{Da}$ & 54,0 & 1,298 & 3 & 2154,2 & ICAL_MOUSE & Q8CE80_MOUSE & Calpastatin; & Calpain inhibitor; \\
\hline LLGLLGLAAA & DPAIYFKEQFLDGDAWTNR & $-0,3$ & N-ter +34.06 Da, K+34.06 Da & 43,3 & $-0,552$ & 3 & 2353,2 & CALR_MOUSE & & Calreticulin; & $\begin{array}{l}\text { CRP55;Calregulin;Endoplasmic reticulum } \\
\text { resiont nortion }\end{array}$ \\
\hline LTKEEIVDKY & DLFVGSQATDFGEALVR & $-0,1$ & $\mathrm{~N}$-ter +34.06 Da & 28,8 & $-0,376$ & 3 & 1858,0 & CALU_MOUSE & Q6XLQ8_MOUSE & Calumenin; & $\begin{array}{l}\text { resident protein 60;HACBP; } \\
\text { Crocalbin; }\end{array}$ \\
\hline GETQFAPGQW & AGIVLDEPIGKNDGSVAGVR & $-0,5$ & N-ter +28.03 Da, K+28.03 Da & 30,8 & 6,518 & 3 & 2022,1 & CLIP1_MOUSE & Q8COS5_MOUSE & CAP-Gly domain-containing linker protein 1; & Restin; \\
\hline LPVMVWFPGG & AFLAGSASTYEGSELAAR & 4,3 & $\mathrm{~N}$-ter $+28.03 \mathrm{Da}$ & 13,9 & 4,096 & 3 & 1827,9 & EST4A_MOUSE & & Carboxylesterase $4 \mathrm{~A}$; & \\
\hline VTIEDLQALL & DNEDEEMQHNEGIER & $-0,4$ & $\mathrm{~N}$-ter $+34.06 \mathrm{Da}$ & 46,4 & $-3,616$ & 3 & 1877,8 & CBPA4_MOUSE & & Carboxypeptidase A4; & \\
\hline LMYPYGYTV & KKAPDAEELDDVAR & $-0,6$ & $\mathrm{~N}$-ter +28.03 Da, $\mathrm{K}+28.03 \mathrm{Da}$ & 28,0 & $-6,391$ & 3 & 1639,9 & CBPA4_MOUSE & & Carboxypeptidase A4; & \\
\hline PAAGMRRRRR & LQQEDGISFEYHR & $-0,1$ & N-ter +34.06 Da & 21,1 & $-5,188$ & 3 & 1654,8 & CBPE_MOUSE & & Carboxypeptidase E; & $\begin{array}{l}\text { Carboxypeptidase H;Enkephalin } \\
\text { convertase; Prohormone-processing } \\
\text { carboxypeptidase; }\end{array}$ \\
\hline HWIMDIPFVL & SANLHGGDLVANYPYDETR & 0,3 & $\mathrm{~N}$-ter +28.03 Da & 47,3 & 0,377 & 3 & 2119,0 & CBPE_MOUSE & & Carboxypeptidase E; & $\begin{array}{l}\text { Carboxypeptidase } \mathrm{H} \text {;Enkephalin } \\
\text { convertase; Prodormone-processing } \\
\text { carboxypeptidase; }\end{array}$ \\
\hline ETRSGTAHEY & SSCPDDAIFQSLAR & 0,1 & N-ter +28.03 Da, C +57.02 Da & 24,8 & $-0,439$ & 2 & 1593,8 & CBPE_MOUSE & & Carboxypeptidase E; & $\begin{array}{l}\text { Carboxypeptidase H;Enkephalin } \\
\text { convertase;Prohormone-processing } \\
\text { carboxypeptidase; }\end{array}$ \\
\hline VQATGTLLSR & SLTPDDDVFQHLAYTYASR & $-1,2$ & $\mathrm{~N}$-ter +34.06 Da & 16,3 & 2,998 & 3 & 2232,1 & CBPM_MOUSE & & Carboxypeptidase $\mathrm{M}$; & \\
\hline ELTEEITRLM & ANTEVMAEGKPR & 0,5 & N-ter +28.03 Da, K +28.03 Da & 37, & 3,597 & 3 & 1414,7 & CASPE_MOUSE & & Caspase-14; & Mini-ICE; \\
\hline RLALTLCVTK & AREGSEVDMEALER & $-0,2$ & N-ter +34.06 Da & 34,5 & $-1,720$ & 3 & 1624,8 & CASPE_MOUSE & & Caspase-14; & Mini-ICE; \\
\hline $\begin{array}{l}\text { MESEMS } \\
\text { CFEWG }\end{array}$ & $\begin{array}{l}\text { DPQPLQEER } \\
\text { EGEUKGEFEMYR }\end{array}$ & 0,0 & 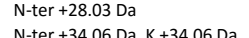 & 30,2 & $-0,263$ & 2 & 1138,6 & $\begin{array}{l}\text { CASPE_MOUSE } \\
\text { CASPE MOUSS }\end{array}$ & & $\begin{array}{l}\text { Caspase-14; } \\
\text { Cospase-14. }\end{array}$ & $\begin{array}{l}\text { Mini-ICE; } \\
\text { Min-ICE. }\end{array}$ \\
\hline CAFVVLMAHG & EEGLLKGEDEKMVR & $-0,6$ & $\mathrm{~N}$-ter +34.06 Da, K +34.06 Da & 21,2 & $-0,230$ & 3 & 1734,0 & CASPE_MOUSE & & Caspase-14; & Mini-ICE; \\
\hline
\end{tabular}




\begin{tabular}{|c|c|c|c|c|c|c|c|c|c|c|c|}
\hline Non Prime Site & Prime Site & 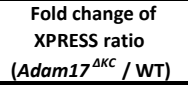 & Modifications & Hyperscore & $\begin{array}{c}\text { Mass error } \\
\text { in ppm }\end{array}$ & charge & $\begin{array}{c}\text { Precursor } \\
\text { neutral mass } \\
\text { in Da }\end{array}$ & Uniprot ID 1 & Uniprot ID 2 & Protein Name 1 & Protein Name 2 \\
\hline ALTLCVTKAR & EGSEVDMEALER & 6,7 & N-ter +28.03 Da & 33,3 & 1,579 & 2 & 1391,6 & CASPE_MOUSE & & Caspase-14; & Mini-ICE; \\
\hline TEVMQEGKPR & KVNPEVQSTLR & $-0,1$ & $\mathrm{~N}-\operatorname{ter}+34.06 \mathrm{Da}, \mathrm{K}+34.06 \mathrm{Da}$ & 18,9 & $-1,865$ & 3 & 1337,8 & CASPE_MOUSE & & Caspase-14; & Mini-ICE; \\
\hline LELTEEITRL & MANTEVMQEGKPR & 0,4 & $\mathrm{~N}$-ter +34.06 Da, K +34.06 Da & 57,1 & 1,986 & 3 & 1557,8 & CASPE_MOUSE & & Caspase-14; & Mini-ICE; \\
\hline MESE & MSDPQPLQEER & 0,0 & $\mathrm{~N}$-ter +28.03 Da & 39,2 & 0,221 & 2 & 1356,6 & CASPE_MOUSE & & Caspase-14; & Mini-ICE; \\
\hline LTEEITRLMA & NTEVMQEGKPR & 0,4 & $\mathrm{~N}$-ter +28.03 Da, K +28.03 Da & 30,3 & $-6,609$ & 3 & 1343,7 & CASPE_MOUSE & & Caspase-14; & Mini-ICE; \\
\hline LALTLCVTKA & REGSEVDMEALER & 0,2 & $\mathrm{~N}$-ter +34.06 Da & 34,2 & 3,019 & 3 & 1553,8 & CASPE_MOUSE & & Caspase-14; & Mini-ICE; \\
\hline MESEM & SDPQPLQEER & 0,2 & $\mathrm{~N}$-ter +34.06 Da & 43,9 & $-0,243$ & 2 & 1231,6 & CASPE_MOUSE & & Caspase-14; & Mini-ICE; \\
\hline ME & SEMSDPQPLQEER & 1,6 & $\mathrm{~N}$-ter +34.06 Da & 18,9 & $-1,202$ & 2 & 1578,7 & CASPE_MOUSE & & Caspase-14; & Mini-ICE; \\
\hline PTYTDTLHIY & STVEGYISYR & 0,1 & $\mathrm{~N}$-ter +34.06 Da & 34,6 & $-0,661$ & 2 & 1207,6 & CASPE_MOUSE & & Caspase-14; & Mini-ICE; \\
\hline VLNKNPVNYF & AEVEQMAFDPSNMPPGIEPSPDKMLQGR & $-0,2$ & N-ter +34.06 Da, K+34.06 Da & 32,3 & $-5,028$ & 4 & 3138,5 & CATA_MOUSE & & Catalase; & \\
\hline VNYFAEVEQM & AFDPSNMPPGIEPSPDKMLQGR & $-0,4$ & N-ter +34.06 Da, K+34.06 Da & 36,9 & $-1,467$ & 3 & 2451,3 & CATA_MOUSE & & Catalase; & \\
\hline AVYCKFHYKT & DQGIKNLPVGEAGR & 0,7 & N-ter +34.06 Da, K+34.06 Da & 18,7 & $-4,528$ & 3 & 1520,9 & CATA_MOUSE & & Catalase; & \\
\hline AGSRGPLLVQ & DVVFTDEMAHFDR & 0,3 & $\mathrm{~N}$-ter +28.03 Da & 15,9 & 1,737 & 3 & 1608,7 & CATA_MOUSE & A2AL20_MOUSE & Catalase; & \\
\hline SPDKMLQGRL & FAYPDTHR & 0,0 & $\mathrm{~N}$-ter+28.03 Da & 23,4 & $-1,158$ & 3 & 1033,5 & CATA_MOUSE & & Catalase; & \\
\hline ASQRPDVLTT & GGGNPIGDKLNIMTAGSR & 0,0 & N-ter +34.06 Da, K+34.06 Da & 28,0 & 6,838 & 3 & 1825,0 & CATA_MOUSE & A2AL20_MOUSE & Catalase; & \\
\hline ERIPERVVHA & KGAGAFGYFEVTHDITR & 0,1 & $\mathrm{~N}$-ter +34.06 Da, K+34.06 Da & 42,5 & $-0,052$ & 3 & 1936,1 & CATA_MOUSE & A2AL20_MOUSE & Catalase; & \\
\hline GKRTPIAVRF & STVTGESGSADTVR & 0,0 & N-ter +28.03 Da & 40,5 & $-2,508$ & 2 & 1393,7 & CATA_MOUSE & & Catalase; & \\
\hline MTAGSRGPLL & VQDVVFTDEMAHFDR & $-0,4$ & $\mathrm{~N}$-ter $+28.03 \mathrm{Da}$ & 27,6 & 3,589 & 3 & 1835,9 & CATA_MOUSE & A2AL20_MOUSE & Catalase; & \\
\hline GSRGPLLVQD & VVFTDEMAHFDR & 0,3 & $\mathrm{~N}$-ter +34.06 Da & 34,6 & 5,789 & 3 & 1499,7 & CATA_MOUSE & A2AL20_MOUSE & Catalase; & \\
\hline GGELAYALNN & FDKQIIVDPLSFSEER & 0,1 & $\mathrm{~N}$-ter $+34.06 \mathrm{Da}, \mathrm{K}+34.06 \mathrm{Da}$ & 19,6 & 6,773 & 3 & 1990,1 & CTNA1_MOUSE & & Catenin alpha-1; & $\begin{array}{l}102 \text { kDa cadherin-associated protein;Alpha E- } \\
\text { catenin;CAP102; }\end{array}$ \\
\hline KLPGRVAFGE & DIDLPETFDAR & $-1,3$ & $\mathrm{~N}$-ter +34.06 Da & 29,1 & $-3,467$ & 2 & 1324,7 & CATB_MOUSE & & Cathepsin B; & $\begin{array}{l}\text { Cathepsin } \mathrm{B} 1 \\
\text {; }\end{array}$ \\
\hline $\begin{array}{l}\text { PKLPGRVAFG } \\
\text { PKV }\end{array}$ & EDIDLPETFDAR & $-1,9$ & N-ter +34.06 Da & 27,6 & $-1,237$ & 2 & 1453,7 & CATB_MOUSE & & Cathepsin B; & Cathepsin B1; \\
\hline GPKLPGRVAF & GEDIDLPETFDAR & $-0,5$ & $\mathrm{~N}$-ter +34.06 Da & 21,7 & $-8,263$ & 2 & 1510,7 & CATB_MOUSE & & Cathepsin B; & Cathepsin B1; \\
\hline GDNGFFKLLR & GENHCGIESEIVAGIPR & $-0,6$ & $\mathrm{~N}$-ter $+34.06 \mathrm{Da}, \mathrm{C}+57.02 \mathrm{Da}$ & 15,7 & $-2,508$ & 3 & 1870,9 & CATB_MOUSE & & Cathepsin B; & Cathepsin B1; \\
\hline LPGRVAFGED & IDLPETFDAR & $-1,2$ & $\mathrm{~N}$-ter +34.06 Da & 22,7 & $-0,495$ & 2 & 1209,7 & CATB_MOUSE & & Cathepsin $\mathrm{B}$ & Cathepsin B1; \\
\hline KNIFSFYLNR & DPEGQPGGELMLGGTDSK & $-0,2$ & $\mathrm{~N}$-ter +28.03 Da, $\mathrm{K}+28.03 \mathrm{Da}$ & 27,8 & $-0,163$ & 2 & 1842,9 & CATD_MOUSE & FGYGL6_MOUSE & Cathepsin D; & \\
\hline GSLSGYLSQD & TVSVPCKSDQSKAR & 0,5 & $\begin{array}{l}\text { N-ter +28.03 Da, K +28.03 Da, C } \\
+57.02 \mathrm{Da}\end{array}$ & 18,4 & 5,091 & 4 & 1645,9 & CATD_MOUSE & FGYGL6_MOUSE & Cathepsin D; & \\
\hline ILGDVFIGSY & YTVFDRDNNR & 0,0 & $\mathrm{~N}$-ter +34.06 Da & 16,2 & $-0,898$ & 3 & 1332,7 & CATD_MOUSE & & Cathepsin D; & \\
\hline QWKSTHRRLY & GTNEEEWR & 0,3 & N-ter +34.06 Da & 25,9 & $-0,853$ & 2 & 1053,5 & CATL1_MOUSE & & Cathepsin L1; & $\begin{array}{l}\text { Cathepsin L;Major excreted protein;p39 } \\
\text { cysteine proteinase; }\end{array}$ \\
\hline CLFLFSVVLR & ALFQDSDPKR & $-6,4$ & $\mathrm{~N}$-ter +34.06 Da, K+34.06 Da & 14,2 & $-6,904$ & 2 & 1243,7 & CTSR1_MOUSE & & Cation channel sperm-associated protein 1 ; & \\
\hline VFKIASNKSR & SILEAEGVFHR & $-3,5$ & N-ter +34.06 Da & 24,1 & 5,338 & 2 & 1290,7 & CD109_MOUSE & & CD109 antigen; & $\begin{array}{l}\text { GPI-anchored alpha-2 macroglobulin-related } \\
\text { protein; }\end{array}$ \\
\hline EASKSQEMVH & LVNKEPSETPDQCMTADETR & 1,1 & $\begin{array}{l}\text { N-ter +28.03 Da, K +28.03 Da, C } \\
+57.02 \mathrm{Da}\end{array}$ & 22,3 & 1,723 & 3 & 2376,1 & CD44_MOUSE & Q3U8S1_MOUSE & CD44 antigen; & $\begin{array}{l}\text { Extracellular matrix receptor III;GP90 } \\
\text { lymphocyte homing/adhesion receptor;HUTCH- } \\
\text { I;Hermes antigen;Hyaluronate } \\
\text { receptor;Lymphocyte antigen 24;Phagocytic } \\
\text { glycoprotein 1;Phagocytic glycoprotein I; }\end{array}$ \\
\hline CEPVMEEKVQ & EQSSLLENGAVHQGDTSWGSSGSGSQSSQGR & 7,3 & $\mathrm{~N}$-ter +28.03 Da & 17,5 & 0,508 & 4 & 3147,4 & BORG2_MOUSE & & Cdc42 effector protein 3 ; & Binder of Rho GTPases 2; \\
\hline M & VGGEASAAVEKLVSGVR & $-0,6$ & $\mathrm{~N}$-ter $+28.03 \mathrm{Da}, \mathrm{K}+28.03 \mathrm{Da}$ & 46,3 & 0,415 & 3 & 1684,0 & C2AIL_MOUSE & & CDKN2AIP N-terminal-like protein; & $\begin{array}{l}\text { CDKN2A-interacting protein N-terminal-like } \\
\text { protein; }\end{array}$ \\
\hline VEELPNQFYR & DPNALHEILR & 10,3 & $\mathrm{~N}$-ter +28.03 Da & 23,6 & $-2,403$ & 2 & 1204,7 & RAD17_MOUSE & & Cell cycle checkpoint protein RAD17; & \\
\hline $\begin{array}{l}\text { SDRGFOFVSS } \\
\text { PAPGKLPHIS }\end{array}$ & $\begin{array}{l}\text { SLPDICYR } \\
\text { SHPLLODLAAAR }\end{array}$ & $\begin{array}{l}-0,5 \\
10,3\end{array}$ & $\begin{array}{l}\mathrm{N}-\operatorname{ter}+34.06 \mathrm{Da}, \mathrm{C}+57.02 \mathrm{Da} \\
\mathrm{N} \text {-ter }+28.03 \mathrm{Da}\end{array}$ & $\begin{array}{l}16,3 \\
18,1\end{array}$ & $\begin{array}{r}-1,323 \\
9,616\end{array}$ & $\begin{array}{l}2 \\
2\end{array}$ & $\begin{array}{l}1056,6 \\
1318,8\end{array}$ & $\begin{array}{l}\text { CNBP_MOUSE } \\
\text { C17OB_MOUSEE }\end{array}$ & D3YYF6_MOUSE & $\begin{array}{l}\text { Cellular nucleic acid-binding protein; } \\
\text { Centrosomal protein of } 170 \mathrm{kDa} \text { protein B; }\end{array}$ & $\begin{array}{l}\text { Zinc finger protein 9; } \\
\text { Centrosomal protein } 170 \mathrm{~B} \text {. }\end{array}$ \\
\hline IPTFGKSFTL & ASSENQLGAPISGEGLPGR & $\begin{array}{l}10,3 \\
1,0\end{array}$ & $\begin{array}{l}\text { N-ter }+28.03 \mathrm{Da} \\
\mathrm{N}-\operatorname{ter}+34.06 \mathrm{Da}\end{array}$ & $\begin{array}{l}18,1 \\
40,4\end{array}$ & $\begin{array}{l}9,616 \\
-2,239\end{array}$ & 3 & $\begin{array}{l}1318,8 \\
1873,0\end{array}$ & CH3L1_MOUSE & D3Z2P2 MOUSE & $\begin{array}{l}\text { Centrosomal protein of } 170 \text { kDa protein B; } \\
\text { Chitinase-3-like protein 1; }\end{array}$ & $\begin{array}{l}\text { Centrosomal protein } 1 / \text { BB; } \\
\text { BRP39 protein;Breast regression protein }\end{array}$ \\
\hline FTLASSENQL & GAPISGEGLPGR & 0,6 & N-ter +28.03 Da & 21,5 & $-8,073$ & 2 & 1137,6 & CH3L1_MOUSE & D3Z2P2_MOUSE & Chitinase-3--like protein 1; & $\begin{array}{l}\text { 39;Cartilage glycoprotein 39; } \\
\text { BRP39 protein;Breast regression protein } \\
\text { 39;Cartilage glycoprotein 39; }\end{array}$ \\
\hline MGIPTFGKSF & TLASSENQLGAPISGEGLPGR & 1,2 & N-ter +28.03 Da & 77,2 & 4,657 & 2 & 2081,1 & CH3L1_MOUSE & D3Z2P2_MOUSE & Chitinase-3-like protein 1 ; & $\begin{array}{l}\text { BRP39 protein;Breast regression protein } \\
\text { 39;Cartilage glycoprotein 39; }\end{array}$ \\
\hline TLLMVQWGSA & AKLVCYLTNWSQYR & 0,2 & $\begin{array}{l}\text { N-ter + 28.03 Da, K +28.03 Da, C } \\
+57.02 \mathrm{Da}\end{array}$ & 17,8 & $-6,721$ & 3 & 1857,0 & CHIT1_MOUSE & & Chitotriosidase-1; & Chitinase-1; \\
\hline GQPCPPCSRA & VASVGKDEYTFWVVGAGSAGCVLASR & $-1,3$ & $\begin{array}{l}\text { N-ter + } 34.06 \mathrm{Da}, \mathrm{K}+34.06 \mathrm{Da}, \mathrm{C} \\
\text { +57.02 Da }\end{array}$ & 18,3 & 4,943 & 4 & 2666,5 & CHDH_MOUSE & & Choline dehydrogenase, mitochondrial; & \\
\hline NNKGVTEILR & DGECPAVITPSKPLAQR & $-0,1$ & $\begin{array}{l}\text { N-ter +34.06 Da, K +34.06 Da, C } \\
\text { +57.02 Da }\end{array}$ & 31,0 & $-2,148$ & 3 & 1906,1 & CTL2_MOUSE & & Choline transporter-like protein 2; & Solute carrier family 44 member 2; \\
\hline $\begin{array}{l}\text { SSCLVLAARH } \\
\text { PLPEGLFWLL }\end{array}$ & $\begin{array}{l}\text { ASASSTNLKDVLSNLIPKEQAR } \\
\text { VTGOMPTEEOVSWISR }\end{array}$ & $\begin{array}{r}-0,5 \\
0,6\end{array}$ & $\begin{array}{l}\mathrm{N}-\mathrm{ter}+28.03 \mathrm{Da}, \mathrm{K}+28.03 \mathrm{Da} \\
\text { N-ter }+34.06 \mathrm{Da}\end{array}$ & $\begin{array}{l}35,3 \\
22,2\end{array}$ & $\begin{array}{r}3,005 \\
-1,964\end{array}$ & 4 & $\begin{array}{l}2425,4 \\
18810\end{array}$ & $\begin{array}{l}\text { CIIY_MOUSE } \\
\text { CISYMOUSE }\end{array}$ & & $\begin{array}{l}\text { Citrate synthase, mitochondrial; } \\
\text { Citrate snnthase, mitochondriali }\end{array}$ & $\begin{array}{l}\text { Citrate (Si)-synthase; } \\
\text { Citrate (Si)-synthase: }\end{array}$ \\
\hline PLPEGLFWLL & VTGQMPTEEQVSWLSR & 0,6 & $\mathrm{~N}$-ter +34.06 Da & 22,2 & $-1,964$ & 3 & 1881,0 & CISY_MOUSE & & Citrate synthase, mitochondrial; & \\
\hline
\end{tabular}




\begin{tabular}{|c|c|c|c|c|c|c|c|c|c|c|c|}
\hline Non Prime Site & Prime Site & $\begin{array}{l}\text { Fold change of } \\
\text { XPRESS ratio } \\
\text { (Adam17 } 7^{\text {AKC } / W T)} \\
\end{array}$ & Modifications & Hyperscore & $\begin{array}{l}\text { Mass error } \\
\text { in ppm }\end{array}$ & Charge & $\begin{array}{c}\text { Precursor } \\
\text { neutral mass } \\
\text { in Da }\end{array}$ & Uniprot ID 1 & Uniprot ID 2 & Protein Name 1 & Protein Name 2 \\
\hline IVHVKGIVLV & ALADGTLAIFHR & 10,3 & N-ter +28.03 Da & 14,2 & $-2,588$ & 2 & 1311,7 & JIP3_MOUSE & JIP4_MOUSE & C-Jun-amino-terminal kinase-interacting protein 3; & $\begin{array}{l}\text { JNK MAP kinase scaffold protein 3;JNK/SAPK- } \\
\text { associated protein 1; Mitogen-activated protein } \\
\text { kinase 8-interacting protein 3;Sunday driver 2; }\end{array}$ \\
\hline SSQSMNFSLM & STNTVGLGLPMSR & 0,2 & $\mathrm{~N}-\mathrm{ter}+34.06 \mathrm{Da}$ & 15,6 & 1,974 & 2 & 1365,8 & EPN4_MOUSE & Q5SUH7_MOUSE & Clathrin interactor 1 & Enthoprotin;:Epsin-4; Epsin-related protein; \\
\hline LIWDNGMVLG & EQEVSDNELQELSTQGSR & 0,2 & N-ter +34.06 Da & 45,8 & $-6,954$ & 3 & 2082,0 & CLUS_MOUSE & E9PXG5_MOUSE & Clusterin; & $\begin{array}{l}\text { Apolipoprotein J;Clustrin;Sulfated glycoprotein } \\
2 \text {; }\end{array}$ \\
\hline GEDKYYLRVS & TVTTHSSDSEVPSR & $-0,1$ & $\mathrm{~N}$-ter $+28.03 \mathrm{Da}$ & 36,5 & 1,631 & 3 & 1529,7 & CLUS_MOUSE & & Clusterin; & $\begin{array}{l}\text { Apolipoprotein J;Clustrin;SUlfated glycoprotein } \\
\text { 2; }\end{array}$ \\
\hline M & VLLAAAVCTKAGKAIVSR & $-0,5$ & $\begin{array}{l}\text { N-ter +28.03 Da, K+28.03 Da, C } \\
+57.02 \mathrm{Da}\end{array}$ & 19,5 & 1,201 & 4 & 1911,2 & COPD_MOUSE & & Coatomer subunit delta; & Archain;Delta-coat protein; \\
\hline M & ARPGNIALVTAR & $-0,6$ & N-ter +34.06 Da & 23,4 & 7,217 & 3 & 1271,8 & CC137_MOUSE & H3BJB7_MOUSE & Coiled-coil domain-containing protein 137; & \\
\hline HDQSKEARLL & ESPCSAIKQEQQR & $-4,8$ & $\begin{array}{l}\text { N-ter +34.06 Da, K+34.06 Da, C } \\
+57.02 \mathrm{Da}\end{array}$ & 16,3 & 3,311 & 3 & 1627,9 & CCD66_MOUSE & & Coiled-coil domain-containing protein 66 ; & \\
\hline $\begin{array}{l}\text { QARTINPPAT } \\
\text { SKAKDSEGID }\end{array}$ & $\begin{array}{l}\text { SLPKTAVSVPAGR } \\
\text { YAHVEVPESPGAEETAGEAVSR }\end{array}$ & $\begin{array}{l}8,4 \\
1,6\end{array}$ & $\begin{array}{l}\text { N-ter }+28.03 \mathrm{Da}, \mathrm{K}+28.03 \mathrm{Da} \\
\text { N-ter +28.03 Da }\end{array}$ & $\begin{array}{l}20,5 \\
17,0\end{array}$ & $\begin{array}{r}-3,953 \\
1,727\end{array}$ & $\begin{array}{l}3 \\
4\end{array}$ & $\begin{array}{l}1337,8 \\
2254,1\end{array}$ & $\begin{array}{l}\text { CCD71_MOUSE } \\
\text { CCD99_MOUSE }\end{array}$ & & $\begin{array}{l}\text { Coiled-coil domain-containing protein } 71 ; \\
\text { Coiled-coil domain-containing protein } 96 ;\end{array}$ & \\
\hline MRAAPRR & APAAQPPAAAAPSAVGSPAAAPR & $-0,8$ & N-ter +34.06 Da & 23,8 & $-2,115$ & 3 & 2030,1 & CHCH2_MOUSE & B2RPU8_MOUSE & $\begin{array}{l}\text { Coiled-coil-helix-coiled-coil-helix domain-containing } \\
\text { protein 2, mitochondrial; }\end{array}$ & \\
\hline MRAAPRRAPA & AQPPAAAAPSAVGSPAAAPR & $-1,0$ & $\mathrm{~N}$-ter +34.06 Da & 31,1 & $-4,069$ & 3 & 1791,0 & CHCH2_MOUSE & B2RPU8_MOUSE & $\begin{array}{l}\text { Coiled-coil-helix-coiled-coil-helix domain-containing } \\
\text { protein 2, mitochondrial; }\end{array}$ & \\
\hline MRAAPR & RAPAAQPPAAAAPSAVGSPAAAPR & $-0,6$ & $\mathrm{~N}$-ter +28.03 Da & 48,1 & 0,962 & 3 & 2180,2 & CHCH2_MOUSE & B2RPU8_MOUSE & $\begin{array}{l}\text { Coiled-coil-helix-coiled-coil-helix domain-containing } \\
\text { protein 2, mitochondrial; }\end{array}$ & \\
\hline QALEQVFSKY & GQISEVVVVKDR & 0,2 & N-ter +34.06 Da, K+34.06 Da & 21,9 & 4,003 & 3 & 1395,9 & CIRBP_MOUSE & K4D165_MOUSE & Cold-inducible RNA-binding protein; & $\begin{array}{l}\text { A18 hnRNP;Glycine-rich RNA-binding protein } \\
\text { CIRP; }\end{array}$ \\
\hline NFASQMSYGY & DEKSAGVSVPGPMGPSGPR & $-0,6$ & N-ter +34.06 Da, K+34.06 Da & 27,5 & 4,222 & 3 & 1892,0 & CO1A1_MOUSE & & Collagen alpha-1(I) chain; & Alpha-1 type I collagen; \\
\hline GGNFASQMSY & GYDEKSAGVSVPGPMGPSGPR & $-0,7$ & $\mathrm{~N}$-ter $+28.03 \mathrm{Da}, \mathrm{K}+28.03 \mathrm{Da}$ & 21,2 & 3,233 & 3 & 2100,0 & CO1A1_MOUSE & & Collagen alpha-1(I) chain; & Alpha-1 type I collagen; \\
\hline GLGGNFASQM & SYGYDEKSAGVSVPGPMGPSGPR & 0,8 & N-ter +34.06 Da, K+34.06 Da & 52,2 & $-5,285$ & 3 & 2362,2 & CO1A1_MOUSE & & Collagen alpha-1(I) chain; & Alpha-1 type I collagen; \\
\hline AGKHGNRGEP & GPAGSVGPVGAVGPR & $-0,1$ & $\mathrm{~N}$-ter +34.06 Da & 31,0 & $-5,256$ & 2 & 1310,8 & CO1A2_MOUSE & & Collagen alpha-2(I) chain; & Alpha-2 type I collagen; \\
\hline GLTGNFAAQY & SDKGVSSGPGPMGLMGPR & $-0,3$ & N-ter +28.03 Da, K+28.03 Da & 27,4 & $-1,958$ & 3 & 1784,9 & CO1A2_MOUSE & EOCXI2_MOUSE & Collagen alpha-2(I) chain; & Alpha-2 type I collagen; \\
\hline PSGPVGKDGR & SGQPGPVGPAGVR & 0,6 & N-ter +34.06 Da & 30,6 & 0,000 & 2 & 1211,7 & CO1A2_MOUSE & & Collagen alpha-2(I) chain; & Alpha-2 type I collagen; \\
\hline PGLTGNFAAQ & YSDKGVSSGPGPMGLMGPR & 0,3 & N-ter +34.06 Da, K+34.06 Da & 21,8 & 4,839 & 3 & 1960,0 & CO1A2_MOUSE & EOCXI2_MOUSE & Collagen alpha-2(l) chain; & Alpha-2 type I collagen; \\
\hline PGAKGPTGSA & GTSGPPGLAGPPGSPGPQGSTGPQGIR & 10,3 & $\mathrm{~N}$-ter +28.03 Da & 19,6 & 3,521 & 3 & 2411,2 & CO5A2_MOUSE & & Collagen alpha-2(V) chain; & \\
\hline RRDHVLGLAR & SELEEDIIPEEDIISR & 0,8 & $\mathrm{~N}$-ter $+28.03 \mathrm{Da}$ & 20,7 & $-1,044$ & 2 & 1914,0 & CO3_MOUSE & & Complement C3; & HSE-MSF; \\
\hline IRGSPGAPSF & AAGPPVSEGKYFSSNPIIPSR & 0,2 & N-ter +34.06 Da, K+34.06 Da & 24,0 & 1,782 & 3 & 2241,3 & CDSN_MOUSE & & Corneodesmosin; & \\
\hline RGSPGAPSFA & AGPPVSEGKYFSSNPIIPSR & $-0,2$ & $\mathrm{~N}$-ter $+34.06 \mathrm{Da}, \mathrm{K}+34.06 \mathrm{Da}$ & 62,7 & 1,242 & 3 & 2170,2 & CDSN_MOUSE & & Corneodesmosin; & \\
\hline QVKPLGPQLM & DPKVSLPQGEPQGEPLEK & 0,0 & N-ter +28.03 Da, K+28.03 Da & 19,5 & $-0,141$ & 3 & 2118,1 & CDSN_MOUSE & & Corneodesmosin; & \\
\hline SQSGSSGSSR & DRPGSGSALPTGDK & $-0,2$ & $\mathrm{~N}$-ter $+28.03 \mathrm{Da}, \mathrm{K}+28.03 \mathrm{Da}$ & 21,2 & $-1,342$ & 3 & 1412,7 & CDSN_MOUSE & & Corneodesmosin; & \\
\hline SGGKIYPVGY & FTKDNPIR & $-0,1$ & $\mathrm{~N}$-ter $+28.03 \mathrm{Da}, \mathrm{K}+28.03 \mathrm{Da}$ & 18,1 & 3,242 & 3 & 1045,6 & CDSN_MOUSE & & Corneodesmosin; & \\
\hline TKDNPIRGSP & GAPSFAAGPPVSEGKYFSSNPIIPSR & $-0,2$ & $\mathrm{~N}$-ter + 34.06 Da, K+34.06 Da & 25,1 & 1,110 & 3 & 2700,5 & CDSN_MOUSE & & Corneodesmosin; & \\
\hline GSPGAPSFAA & GPPVSEGKYFSSNPIIPSR & $-0,1$ & $\mathrm{~N}$-ter + 34.06 Da, $\mathrm{K}+34.06 \mathrm{Da}$ & 54,5 & 0,048 & 3 & 2099,2 & CDSN_MOUSE & & Corneodesmosin; & \\
\hline ILPGILAKSI & GTLSDPCKDPTR & $-2,5$ & $\begin{array}{l}\text { N-ter +34.06 Da, K +34.06 Da, C } \\
+57.02 \mathrm{Da}\end{array}$ & 18,0 & $-7,982$ & 2 & 1413,8 & CDSN_MOUSE & & Corneodesmosin; & \\
\hline TYSGGKIYPV & GYFTKDNPIR & 1,3 & N-ter +28.03 Da, K+28.03 Da & 18,7 & 2,049 & 3 & 1265,7 & CDSN_MOUSE & & Corneodesmosin; & \\
\hline AGLLPGILA & KSIGTISDPCKDPTR & $-0,2$ & N-ter +34.06 Da, K+34.06 Da, C & 24,2 & $-0,056$ & 3 & 1776,0 & CDSN_MOUSE & & Corneodesmosin; & \\
\hline FAAGPPVSEG & KYFSSNPIIPSR & $-0,7$ & $\begin{array}{l}+5.7 .02 \mathrm{Da} \\
\text { N-ter +34.06 Da, K+34.06 Da }\end{array}$ & 24,9 & $-1,690$ & 3 & 1475,9 & CDSN_MOUSE & & Corneodesmosin; & \\
\hline SSGYPVGVAF & QPVGSGGVOPCGTGSVSSKGPCSGTR & $-0,4$ & $\mathrm{~N}-\mathrm{ter}+28.03 \mathrm{Da}, \mathrm{K}+28.03 \mathrm{Da}, \mathrm{C}$ & 26,6 & $-1,951$ & 3 & 2559,2 & CDSN_MOUSE & & Corneodesmosin; & \\
\hline NSYLVPGMTY & SGGKIYPVGYFTKDNPIR & $-0,3$ & $\begin{array}{l}+5+.72 \mathrm{Da} \\
\mathrm{N} \text {-er }+34.06 \mathrm{Da}, \mathrm{K}+34.066 \mathrm{Da}\end{array}$ & 48,8 & $-1,606$ & 4 & 2113,2 & CDSN_MOUSE & & Corneodesmosin; & \\
\hline GLILPGILAK & SIGTLSDPCKDPTR & $-0,6$ & N-ter +28.03 Da, K+28.03 Da, C & 29,4 & 1,433 & 3 & 1601,8 & CDSN MOUSE & & Corneodesmosin; & \\
\hline GPPVSEGKYF & SSNPIIISR & 0,0 & $\begin{array}{l}+57.02 \mathrm{Da} \\
\text { N-ter }+28.03 \mathrm{Da}\end{array}$ & 17,2 & 1,201 & 2 & 997,6 & CDSN MOUSE & & Corneodesmosin; & \\
\hline GSOSGRWVSS & SSQWVSSSSQSGSSGSSR & 0,0 & $\mathrm{~N}$-ter $+34.06 \mathrm{Da}$ & 16,8 & $-5,431$ & 2 & 1820,8 & CDSN_MOUSE & & Corneodesmosin; & \\
\hline SGSOSGRWVS & SSSQWVYSSSSOSGSSGSSR & 0,2 & N-ter +28.03 Da & 36,1 & $-5,410$ & 2 & 1901,8 & CDSN MOUSE & & Corneodesmosin; & \\
\hline SGRWVSSSSO & WVSSSSOSGSSGSSR & 0,2 & $\mathrm{~N}$-ter $+28.03 \mathrm{Da}$ & 21,0 & 0,066 & & 1512,7 & CDSN MOUSE & & Corneodesmosin; & \\
\hline SGSSGSQSGR & WVSSSSQWVSSSSQSGSSGSSR & $-1,8$ & $\mathrm{~N}$-ter $+34.06 \mathrm{Da}$ & 38,1 & $-3,898$ & 3 & 2280,0 & CDSN_MOUSE & & Corneodesmosin; & \\
\hline PMRAIFLADG & KVFTTGFSR & 3,5 & $\mathrm{~N}$-ter $+28.03 \mathrm{Da}, \mathrm{K}+28.03 \mathrm{Da}$ & 16,8 & 0,909 & 2 & 1097,6 & COR1B_MOUSE & & Coronin-1B; & $\begin{array}{l}\text { Coronin-2; } \\
\text { Acidic-type mitochondrial creatine }\end{array}$ \\
\hline AGDEETYEVF & AELFDPVIQER & 0,2 & $\mathrm{~N}$-ter +34.06 Da & 24,0 & 3,477 & 2 & 1349,8 & KCRU_MOUSE & BOROEg_MOUSE & Creatine kinase U-type, mitochondrial; & $\begin{array}{l}\text { kinase;Ubiquitous mitochondrial creatine } \\
\text { kinase; } \\
\text { Acidic-type mitochondrial creatine }\end{array}$ \\
\hline GYDPRTMKHT & TDLDASKIR & 1,6 & N-ter +34.06 Da, K+34.06 Da & 16,4 & 5,603 & 3 & 1085,7 & KCRU_MOUSE & BOROFO_MOUSE & Creatine kinase U-type, mitochondrial; & $\begin{array}{l}\text { Acidic-type mitochondrial creatine } \\
\text { kinase;Ubiquitous mitochondrial creatine } \\
\text { kinase; }\end{array}$ \\
\hline
\end{tabular}




\begin{tabular}{|c|c|c|c|c|c|c|c|c|c|c|c|}
\hline Non Prime Site & Prime Site & $\begin{array}{l}\text { Fold change of } \\
\text { XPRESS ratio } \\
\text { (Adam17 }{ }^{\text {AKC } / \text { WT) }} \\
\end{array}$ & Modifications & Hyperscore & $\begin{array}{l}\text { Mass error } \\
\text { in ppm }\end{array}$ & Charge & $\begin{array}{c}\text { Precursor } \\
\text { neutral mass } \\
\text { in Da }\end{array}$ & Uniprot ID 1 & Uniprot ID 2 & Protein Name 1 & Protein Name 2 \\
\hline LENLRLQKRG & TGGVDTAATGSVFDISNLDR & 1,6 & $\mathrm{~N}$-ter +28.03 Da & 20,0 & $-9,131$ & 3 & 2023,0 & KCRU_MOUSE & & Creatine kinase U-type, mitochondrial; & $\begin{array}{l}\text { Acidic-type mitochondrial creatine } \\
\text { kinase;Ubiquitous mitochondrial creatine } \\
\text { kinase; } \\
\text { Acidic-type mitochondrial creatine }\end{array}$ \\
\hline RGTGGVDTAA & TGSVFDISNLDR & 0,0 & $\mathrm{~N}$-ter +34.06 Da & 38,6 & 0,515 & 2 & 1356,7 & KCRU_MOUSE & & Creatine kinase U-type, mitochondrial; & $\begin{array}{l}\text { Acidic-type mitochondrial creatine } \\
\text { kinase;Ubiquitous mitochondrial creatine } \\
\text { kinase; }\end{array}$ \\
\hline NGYDPRTMKH & TTDLDASKIR & 0,0 & N-ter +28.03 Da, K+28.03 Da & 32,8 & $-1,528$ & 3 & 1174,7 & KCRU_MOUSE & BOROFO_MOUSE & Creatine kinase U-type, mitochondrial; & $\begin{array}{l}\text { Acidic-type mitochondrial creatine } \\
\text { kinase;Ubiquitous mitochondrial creatine } \\
\text { kinase; }\end{array}$ \\
\hline IPGGLTEARP & ATAEVQEIADR & $-0,3$ & $\mathrm{~N}$-ter $+28.03 \mathrm{Da}$ & 30,6 & $-0,162$ & 2 & 1229,6 & CYTA_MOUSE & EOCZ96_MOUSE & Cystatin-A; & Cystatin-A1;Stefin-A; \\
\hline & GGLTEARPATAEVQEIADR & $-0,2$ & N-ter+34.06 Da & 60,6 & 1,287 & 3 & 2017,1 & CYTA_MOUSE & EOCZ96_MOUSE & Cystatin-A; & Cystatin-A1;Stefin-A; \\
\hline M & IPGGLTEARPATAEVQEIADR & 0,0 & $\mathrm{~N}$-ter +28.03 Da & 13,5 & 0,495 & 3 & 2221,2 & CYTA_MOUSE & EOCZ96_MOUSE & Cystatin-A; & Cystatin-A1;Stefin-A; \\
\hline M & IPGGLTEAR & $-0,5$ & $\mathrm{~N}$-ter +34.06 Da & 29,1 & 0,371 & 2 & 1077,6 & CYTA_MOUSE & EOCZ96_MOUSE & Cystatin-A; & Cystatin-A1;Stefin-A; \\
\hline MIPGGLTEA & RPATAEVQEIADR & $-0,1$ & N-ter+34.06 Da & 37,7 & $-2,279$ & 3 & 1488,8 & CYTA_MOUSE & EOCZ96_MOUSE & Cystatin-A; & Cystatin-A1;Stefin-A; \\
\hline TPKQGPRMLG & APEEADANEEGVR & 0,3 & N-ter +34.06 Da & 15,0 & $-4,080$ & 2 & 1419,7 & CYTC_MOUSE & A2APX3_MOUSE & Cystatin-C; & Cystatin-3; \\
\hline WAATPKQGPR & MLGAPEEADANEEGVRR & $-1,2$ & N-ter +34.06 Da & 24,9 & 6,117 & 3 & 1876,9 & CYTC_MOUSE & A2APX3_MOUSE & Cystatin-C; & Cystatin-3; \\
\hline GVNIGGAGSY & IYEKPQTEAPQVTGPIEVPVVR & $-1,5$ & $\mathrm{~N}$-ter +34.06 Da, K+34.06 Da & 30,3 & 3,928 & 3 & 2517,5 & CRIP2_MOUSE & & Cysteine-rich protein 2 & Heart LIM protein; \\
\hline IGGAGSYYYE & KPQTEAPQVTGPIEVPVVR & $-1,5$ & $\mathrm{~N}$-ter +34.06 Da, K+34.06 Da & 24,9 & $-4,964$ & 3 & 2112,3 & CRIP2_MOUSE & & Cysteine-rich protein 2; & Heart LIM protein; \\
\hline VNIGGAGSYI & YEKPQTEAPQVTGPIEVPVVR & $-0,8$ & $\mathrm{~N}$-ter +28.03 Da, K+28.03 Da & 50,1 & $-1,002$ & 3 & 2392,3 & CRIP2_MOUSE & & Cysteine-rich protein 2; & $\begin{array}{l}\text { Heart LIM protein; } \\
\text { Complex III subunit 1;Core protein I;Ubiquinol- }\end{array}$ \\
\hline KDLPKVVELL & ADIVQNSSLEDSQIEKER & 2,6 & $\mathrm{~N}$-ter +34.06 Da, K+34.06 Da & 22,7 & $-4,927$ & 3 & 2128,1 & QCR1_MOUSE & & Cytochrome b-c1 complex subunit 1, mitochondrial; & $\begin{array}{l}\text { cytochrome-c reductase complex core protein } \\
1 \text { Complex III subunit 1;Core protein I;Ubiquinol- }\end{array}$ \\
\hline ALRGTATFAQ & ALQSVPETQVSILDNGLR & 0,1 & N-ter +28.03 Da & 17,5 & 4,568 & 3 & 1967,1 & QCR1_MOUSE & & Cytochrome b-c1 complex subunit 1, mitochondrial; & $\begin{array}{l}\text { cytochrome-c reductase complex core protein } \\
1 ;\end{array}$ \\
\hline LLRLPALRGT & ATFAQALQSVPETQVSILDNGLR & $-0,2$ & $\mathrm{~N}$-ter+34.06 Da & 21,2 & 3,328 & 3 & 2491,4 & QCR1_MOUSE & & Cytochrome b-c1 complex subunit 1, mitochondrial; & $\begin{array}{l}\text { Complex III subunit 1; Core protein l;Ubiquinol- } \\
\text { cytochrome-c reductase complex core protein } \\
1 ;\end{array}$ \\
\hline ALLRLPALRG & TATFAQALQSVPETQVSILDNGLR & $-0,2$ & $\mathrm{~N}$-ter +28.03 Da & 60,9 & 1,274 & 3 & 2586,4 & QCR1_MOUSE & & Cytochrome b-c1 complex subunit 1 , mitochondrial; & $\begin{array}{l}\text { Complex III subunit 1;Core protein I;Ubiquinol- } \\
\text { cytochrome-c reductase complex core protein } \\
1 \text {; } \\
\text { Complex III subunit 6;Complex III subunit } \\
\text { VIIIICCytochrome c1 non-heme } 11 \mathrm{kDa}\end{array}$ \\
\hline EDERKMLTGS & GDPKEEEEEELVDPLTTVR & $-0,2$ & $\mathrm{~N}$-ter +28.03 Da, K+28.03 Da & 51,9 & 0,803 & 3 & 2240,1 & QCR6_MOUSE & & Cytochrome b-c1 complex subunit 6 , mitochondrial; & $\begin{array}{l}\text { protein;Mitochondrial hinge protein;Ubiquinol- } \\
\text { cytochrome c reductase complex } 11 \mathrm{kDa} \\
\text { protein; } \\
\text { Complex III subunit 7;Complex III subunit }\end{array}$ \\
\hline DGFRKWYYNA & AGFNKLGLMR & $-0,3$ & N-ter+34.06 Da, K+34.06 Da & 17,8 & $-1,360$ & 3 & 1173,7 & QCR7_MOUSE & Q9CQB4_MOUSE & Cytochrome b-c1 complex subunit 7; & $\begin{array}{l}\text { VII; Ubiquinol-cytochrome c reductase complex } \\
14 \text { kDa protein; } \\
\text { Complex III subunit 8;Complex III subunit } \\
\text { VIII;Ubiquinol-cytochrome c reductase complex }\end{array}$ \\
\hline M & GREFGNLAR & $-0,4$ & $\mathrm{~N}$-ter +34.06 Da & 24,0 & $-0,095$ & 3 & 1052,6 & QCR8_MOUSE & & Cytochrome b-c1 complex subunit 8; & $\begin{array}{l}9.5 \mathrm{kDa} \text { protein;Ubiquinol-cytochrome } \mathrm{C} \\
\text { reductase complex ubiquinone-binding protein } \\
\text { QP-C; }\end{array}$ \\
\hline MLSVAA & RSGPFAPVLSATSR & 0,5 & $\mathrm{~N}$-ter +34.06 Da & 34,7 & $-9,110$ & 3 & 1478,8 & UCRI_MOUSE & & $\begin{array}{l}\text { Cytochrome b-c1 complex subunit Rieske, } \\
\text { mitochondrial; }\end{array}$ & $\begin{array}{l}\text { Complex III subunit 5;Cytochrome b-cc complex } \\
\text { subunit 5; Rieske iron-sulfur protein;Ubiquinol- } \\
\text { cytochrome c reductase iron-sulfur subunit; }\end{array}$ \\
\hline GLNVPASVRF & SHTDVKVPDFSDYR & $-0,3$ & $\mathrm{~N}$-ter +28.03 Da, K+28.03 Da & 22,9 & 0,058 & 3 & 1720,8 & UCRI_MOUSE & & $\begin{array}{l}\text { Cytochrome b-c1 complex subunit Rieske, } \\
\text { mitochondrial; }\end{array}$ & $\begin{array}{l}\text { Complex III subunit 5;Cytochrome b-c1 complex } \\
\text { subunit } 5 \text {;Rieske iron-sulfur protein; Ubiquinol- } \\
\text { cytochrome c reductase iron-sulfur subunit; }\end{array}$ \\
\hline RAISTSVCLR & AHGSVVKSEDYAFPTYADR & $-0,4$ & N-ter +34.06 Da, K+34.06 Da & 31,2 & $-0,183$ & 3 & 2180,1 & COX41_MOUSE & & $\begin{array}{l}\text { Cytochrome c oxidase subunit } 4 \text { isoform } 1 \text {, } \\
\text { mitochondrial; }\end{array}$ & $\begin{array}{l}\text { Cytochrome c oxidase polypeptide } \\
\text { IV;Cytochrome coxidase subunit IV isoform 1; }\end{array}$ \\
\hline ISTSVCLRAH & GSVVKSEDYAFPTYADR & $-0,9$ & N-ter +34.06 Da, K+34.06 Da & 40,5 & 3,240 & 3 & 1972,0 & COX41_MOUSE & & $\begin{array}{l}\text { Cytochrome c oxidase subunit } 4 \text { isoform } 1 \text {, } \\
\text { mitochondrial; }\end{array}$ & $\begin{array}{l}\text { Cytochrome c oxidase polypeptide } \\
\text { IV;Cytochrome coxidase subunit IV isoform 1; }\end{array}$ \\
\hline RKGMNTLVGY & DLVPEPKIIDAALR & $-0,5$ & N-ter +28.03 Da, K+28.03 Da & 23,1 & $-3,174$ & 2 & 1605,0 & COX5A_MOUSE & & Cytochrome c oxidase subunit $5 \mathrm{~A}$, mitochondrial; & Cytochrome c oxidase polypeptide Va; \\
\hline VIQELRPTLN & ELGISTPEELGLDK & $-0,4$ & N-ter +34.06 Da, K+34.06 Da & 27,8 & $-0,959$ & 2 & 1667,0 & COX5A_MOUSE & & Cytochrome c oxidase subunit 5A, mitochondrial; & Cytochrome c oxidase polypeptide Va; \\
\hline
\end{tabular}




\begin{tabular}{|c|c|c|c|c|c|c|c|c|c|c|c|}
\hline Non Prime Site & Prime Site & 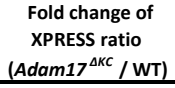 & Modifications & Hyperscore & $\begin{array}{l}\text { Mass error } \\
\text { in ppm }\end{array}$ & Charge & $\begin{array}{c}\text { Precursor } \\
\text { neutral mass } \\
\text { in Da }\end{array}$ & Uniprot ID 1 & Uniprot ID 2 & Protein Name 1 & Protein Name 2 \\
\hline EEFDARWVTY & FNKPDIDAWELR & $-0,3$ & N-ter +34.06 Da, K+34.06 Da & 15,9 & 0,763 & 2 & 1570,9 & COX5A_MOUSE & & Cytochrome c oxidase subunit 5A, mitochondrial; & Cytochrome c oxidase polypeptide Va; \\
\hline QELRPTLNEL & GISTPEELGLDK & $-0,5$ & N-ter +28.03 Da, $\mathrm{K}+28.03 \mathrm{Da}$ & 41,8 & $-1,414$ & 2 & 1412,8 & COX5A_MOUSE & & Cytochrome c oxidase subunit 5A, mitochondrial; & Cytochrome c oxidase polypeptide Va; \\
\hline EFDARWVTYF & NKPDIDAWELR & 0,7 & $\mathrm{~N}$-ter +34.06 Da, K+34.06 Da & 28,3 & $-0,561$ & 3 & 1423,8 & COX5A_MOUSE & & Cytochrome c oxidase subunit 5A, mitochondrial; & Cytochrome c oxidase polypeptide Va; \\
\hline EIYPYVIOELL & RPTLNELGISTPEELGLDK & $-0,5$ & N-ter +34.06 Da, K+34.06 Da & 36,7 & $-1,288$ & 3 & 2248,3 & COX5A_MOUSE & & Cytochrome c oxidase subunit 5A, mitochondrial; & Cytochrome c oxidase polypeptide Va; \\
\hline YPYVIQELRP & TLNELGISTPEELGLDK & 0,2 & N-ter +34.06 Da, K+34.06 Da & 24,2 & 8,258 & 3 & 1995,2 & COX5A_MOUSE & & Cytochrome c oxidase subunit 5A, mitochondrial; & Cytochrome c oxidase polypeptide Va; \\
\hline DAWELRKGMN & TLVGYDLVPEPKIIDAALR & 1,2 & N-ter +28.03 Da, K+28.03 Da & 20,5 & $-3,409$ & 3 & 2138,2 & COX5A_MOUSE & & Cytochrome c oxidase subunit 5A, mitochondrial; & Cytochrome c oxidase polypeptide Va; \\
\hline WELRKGMNTL & VGYDLVPEPKIIDAALR & $-0,4$ & $\mathrm{~N}$-ter +34.06 Da, K+34.06 Da & 42,8 & $-0,980$ & 3 & 1936,2 & COX5A_MOUSE & & Cytochrome c oxidase subunit 5A, mitochondrial; & Cytochrome c oxidase polypeptide Va; \\
\hline PRGAAVTRSM & ASGGGVPTDEEQATGLER & $-0,3$ & N-ter+34.06 Da & 53,2 & $-0,111$ & 2 & 1806,9 & COX5B_MOUSE & Q9D881_MOUSE & Cytochrome c oxidase subunit 5B, mitochondrial; & Cytochrome c oxidase polypeptide Vb; \\
\hline GPRGAAVTRS & MASGGGVPTDEEQATGLER & $-0,5$ & N-ter+34.06 Da & 56,3 & 1,031 & 2 & 1937,9 & COX5B_MOUSE & Q9D881_MOUSE & Cytochrome c oxidase subunit 5B, mitochondrial; & Cytochrome c oxidase polypeptide Vb; \\
\hline RGAAVTRSMA & SGGGVPTDEEQATGLER & $-0,2$ & N-ter+34.06 Da & 50,3 & $-0,345$ & 2 & 1735,8 & COX5B_MOUSE & Q9D881_MOUSE & Cytochrome c oxidase subunit $5 \mathrm{~B}$, mitochondrial; & Cytochrome c oxidase polypeptide $\mathrm{Vb}$; \\
\hline ALPGVGVSML & NVFLKSR & $-1,0$ & N-ter +28.03 Da, $\mathrm{K}+28.03 \mathrm{Da}$ & 21,0 & $-1,519$ & 3 & 918,6 & CX6A1_MOUSE & Q9DCW5_MOUSE & Cytochrome c oxidase subunit 6A1, mitochondrial; & Cytochrome c oxidase polypeptide Vla-liver; \\
\hline YFVALPGVGV & SMLNVFLKSR & $-2,4$ & N-ter +34.06 Da, K+34.06 Da & 26,6 & $-5,930$ & 3 & 1261,8 & CX6A1_MOUSE & Q9DCW5_MOUSE & Cytochrome c oxidase subunit 6A1, mitochondrial; & Cytochrome c oxidase polypeptide Vla-liver; \\
\hline M & SSGALLPKPQMR & $-0,4$ & $\mathrm{~N}$-ter +28.03 Da, $\mathrm{K}+28.03 \mathrm{Da}$ & 30,1 & 0,819 & 3 & 1339,8 & COX6C_MOUSE & D3Z6E1_MOUSE & Cytochrome c oxidase subunit $6 \mathrm{C}$; & $\begin{array}{l}\text { Cytochrome c oxidase polypeptide VIC; } \\
\text { Complex III subunit } 4 \text {;Complex III subunit }\end{array}$ \\
\hline AVALHSAVSA & SDLELHPPSYPWSHR & $-0,5$ & $\mathrm{~N}$-ter +34.06 Da & 36,0 & $-1,400$ & 3 & 1853,9 & CY1_MOUSE & & Cytochrome $\mathrm{c} 1$, heme protein, mitochondrial; & $\begin{array}{l}\text { IV;Cytochrome b-c1 complex subunit } \\
\text { 4;Ubiquinol-cytochrome-c reductase complex } \\
\text { cytochrome c1 subunit; }\end{array}$ \\
\hline EVEADITLAG & FHLPKGTMVLTNLTALHR & 12,1 & $\mathrm{~N}$-ter +28.03 Da, K+28.03 Da & 24,1 & $-0,190$ & 4 & 2104,2 & CP2J6_MOUSE & G3UXTO_MOUSE & Cytochrome P450 2J6; & Arachidonic acid epoxygenase;CYPIII6; \\
\hline KSIVDAYVLL & NLGDSVTTDHISPAGNIAR & 0,5 & $\mathrm{~N}$-ter +34.06 Da & 18,1 & 1,165 & 3 & 1971,0 & ACOC_MOUSE & & Cytoplasmic aconitate hydratase; & $\begin{array}{l}\text { Citrate hydro-lyase; Iron regulatory protein } \\
\text { 1; Iron-responsive element-binding protein } 1 \text {; }\end{array}$ \\
\hline PGCPPNAAQL & AVMQGANVLVTQR & $-0,6$ & $\mathrm{~N}$-ter +34.06 Da & 60,9 & 0,844 & 2 & 1419,8 & DAZP2_MOUSE & & DAZ-associated protein 2; & $\begin{array}{l}\text { Deleted in azoospermia-associated protein } \\
\text { 2;;roline-rich protein expressed in brain; }\end{array}$ \\
\hline $\begin{array}{l}\text { INDESRGHGH } \\
\text { HEQHRRNTVL }\end{array}$ & $\begin{array}{l}\text { SDEEDEEQPR } \\
\text { TTMHAFPYKTR }\end{array}$ & $\begin{array}{l}1,4 \\
2,3\end{array}$ & $\begin{array}{l}\text { N-ter +28.03 Da } \\
\text { N-ter +28.03 Da, K+28.03 Da }\end{array}$ & $\begin{array}{l}14,8 \\
15,2\end{array}$ & $\begin{array}{l}6,885 \\
7,744\end{array}$ & $\begin{array}{l}3 \\
3\end{array}$ & $\begin{array}{l}1260,5 \\
1520,8\end{array}$ & $\begin{array}{l}\text { DCAF8_MOUSE } \\
\text { DOCK8_MOUSE }\end{array}$ & & $\begin{array}{l}\text { DDB1-and CUL4-associated factor 8; } \\
\text { Dedicator of cytokinesis protein 8; }\end{array}$ & WD repeat-containing protein 42A; \\
\hline M & VAPMKGQVCVVTGASR & $-6,4$ & $\begin{array}{l}\text { N-ter +34.06 Da, } \mathrm{K}+34.06 \mathrm{Da}, \mathrm{C} \\
\text { +57.02 Da }\end{array}$ & 31,4 & 1,098 & 3 & 1727,0 & DHRS1_MOUSE & & Dehydrogenase/reductase SDR family member 1; & \\
\hline PSAIRHVRSW & SNIPFITVPLSR & $-0,6$ & N-ter +34.06 Da & 17,2 & 0,870 & 2 & 1376,8 & P5CS_MOUSE & D3Z0B4_MOUSE & Delta-1-pyrroline-5-carboxylate synthase; & $\begin{array}{l}\text { Aldehyde dehydrogenase family } 18 \text { member } \\
\mathrm{A} 1 ;\end{array}$ \\
\hline $\begin{array}{l}\text { SASNLIYPIF } \\
\text { KRPSLSEQQ }\end{array}$ & $\begin{array}{l}\text { VTDVPDDVQPIASLPGVAR } \\
\text { QAASALVETIR }\end{array}$ & $\begin{array}{r}0,1 \\
-3,0\end{array}$ & $\begin{array}{l}\text { N-ter }+28.03 \mathrm{Da} \\
\text { N-ter }+34.06 \mathrm{Da}\end{array}$ & $\begin{array}{l}15,7 \\
20,9\end{array}$ & $\begin{array}{r}-5,306 \\
2,762\end{array}$ & $\begin{array}{l}3 \\
3\end{array}$ & $\begin{array}{l}1976,1 \\
1191,7\end{array}$ & $\begin{array}{l}\text { HEM2_MOUSE } \\
\text { DEN4C_MOUSE }\end{array}$ & & $\begin{array}{l}\text { Delta-aminolevvlinic acid dehydratase; } \\
\text { DENN domain-containing protein } 4 C_{;}\end{array}$ & Porphobilinogen synthase; \\
\hline GSDLSSLQKR & AGGADQFSKPEAR & 0,2 & $\mathrm{~N}$-ter +28.03 Da, K+28.03 Da & 44,6 & $-0,647$ & 3 & 1388,7 & DMKN_MOUSE & E9Q2P1_MOUSE & Dermokine; & Epidermis-specific secreted protein $\mathrm{K} K 30 / \mathrm{Sk} 89$; \\
\hline KEAASSGIQN & ALGQGHGEEGGSTLMGSR & $-0,1$ & $\mathrm{~N}$-ter +28.03 Da & 39,7 & $-8,174$ & 3 & 1770,8 & DMKN_MOUSE & E9Q2P1_MOUSE & Dermokine; & Epidermis-specific secreted protein $\mathrm{SK} 30 / \mathrm{K} 89$; \\
\hline GSRDIETSNF & DEGYSVSR & $-0,4$ & $\mathrm{~N}$-ter+34.06 Da & 17,8 & 3,589 & 2 & 945,5 & DMKN_MOUSE & E9Q2P1_MOUSE & Dermokine; & Epidermis-specific secreted protein SK30/אK89; \\
\hline NSGNSGSGSR & DIETSNFDEGYSVSR & $-0,1$ & $\mathrm{~N}$-ter $+28.03 \mathrm{Da}$ & 49,3 & 0,286 & 2 & 1745,8 & DMKN_MOUSE & E9Q2P1_MOUSE & Dermokine; & Epidermis-specific secreted protein $\mathrm{SK} 30 / \mathrm{K} 89$; \\
\hline SSLQKRAGGA & DQFSKPEAR & 0,4 & N-ter +34.06 Da, K+34.06 Da & 35,8 & 5,058 & 2 & 1144,7 & DMKN_MOUSE & E9Q2P1_MOUSE & Dermokine; & Epidermis-specific secreted protein $\mathrm{SK} 30 / \mathrm{K} 89$; \\
\hline SGSRDIETSN & FDEGYSVSR & $-0,1$ & $\mathrm{~N}$-ter $+28.03 \mathrm{Da}$ & 17,0 & 3,215 & 2 & 1086,5 & DMKN_MOUSE & E9Q2P1_MOUSE & Dermokine; & Epidermis-specific secreted protein $\mathrm{SK30/ \$ K89;}$ \\
\hline DISSLQKRAG & GADQFSKPEAR & $-1,0$ & N-ter +34.06 Da, K+34.06 Da & 36,3 & $-3,844$ & 2 & 1272,7 & DMKN_MOUSE & E9Q2P1_MOUSE & Dermokine; & Epidermis-specific secreted protein SK30/אK89; \\
\hline SDLSSLQKRA & GGADQFSKPEAR & $-0,1$ & N-ter +34.06 Da, K+34.06 Da & 30,0 & 2,551 & 3 & 1329,7 & DMKN_MOUSE & E9Q2P1_MOUSE & Dermokine; & Epidermis-specific secreted protein $\mathrm{SK} 30 / \mathrm{KK} 89$; \\
\hline GSGSRGTGSR & GGSGGSGGSGGGNKPECNNPGNDVR & $-0,4$ & $\begin{array}{l}\text { N-ter +28.03 Da, } \mathrm{K}+28.03 \mathrm{Da}, \mathrm{C} \\
+57.02 \mathrm{Da}\end{array}$ & 70,9 & $-1,194$ & 3 & 2343,0 & DMKN_MOUSE & E9Q2P1_MOUSE & Dermokine; & Epidermis-specific secreted protein $\mathrm{SK} 30 / \mathrm{KK} 89$; \\
\hline
\end{tabular}




\begin{tabular}{|c|c|c|c|c|c|c|c|c|c|c|c|}
\hline Non Prime Site & Prime Site & 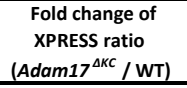 & Modifications & Hyperscore & $\begin{array}{l}\text { Mass error } \\
\text { in ppm }\end{array}$ & Charge & $\begin{array}{c}\begin{array}{c}\text { Precursor } \\
\text { neutral mass } \\
\text { in Da }\end{array} \\
\end{array}$ & Uniprot ID 1 & Uniprot ID 2 & Protein Name 1 & Protein Name 2 \\
\hline VAQPGYGTVR & GNNQNSGCTNPPPSGSHESFSNSGGSSNDGSR & $-0,2$ & N-ter +34.06 Da, C+57.02 Da & 60,6 & $-1,579$ & 3 & 3226,3 & DMKN_MOUSE & E9Q2P1_MOUSE & Dermokine; & Epidermis-specific secreted protein SK30/SK89; \\
\hline RGTGSRGGSG & GSGGSGGGNKPECNNPGNDVR & $-0,9$ & $\begin{array}{l}\text { N-ter }+34.06 \mathrm{Da}, \mathrm{K}+34.06 \mathrm{Da}, \mathrm{C} \\
+57.02 \mathrm{Da}\end{array}$ & 29,0 & $-7,381$ & 3 & 2097,0 & DMKN_MOUSE & E9Q2P1_MOUSE & Dermokine; & Epidermis-specific secreted protein SK30/SK89; \\
\hline SGSRGTGSRG & GSGGSGGSGGGNKPECNNPGNDVR & $-1,1$ & $\begin{array}{l}\text { N-ter }+34.06 \mathrm{Da}, \mathrm{K}+34.06 \mathrm{Da}, \mathrm{C} \\
+57.02 \mathrm{Da}\end{array}$ & 26,4 & $-0,087$ & 3 & 2298,1 & DMKN_MOUSE & E9Q2P1_MOUSE & Dermokine; & Epidermis-specific secreted protein SK30/SK89; \\
\hline RGNNQNSGCT & NPPPSGSHESFSNSGGSSNDGSR & $-0,1$ & N-ter +34.06 Da & 45,6 & $-3,526$ & 3 & 2294,0 & DMKN_MOUSE & E9Q2P1_MOUSE & Dermokine; & Epidermis-specific secreted protein SK30/SK89; \\
\hline QPGYGTVRGN & NQNSGCTNPPPSGSHESFSNSGGSSNDGSR & $-0,8$ & N-ter +34.06 Da, C +57.02 Da & 27,6 & $-3,041$ & 3 & 3055,3 & DMKN_MOUSE & E9Q2P1_MOUSE & Dermokine; & Epidermis-specific secreted protein SK30/SK89; \\
\hline VFEHRLGEAA & RSLGNAGNEIGR & $-0,2$ & N-ter +34.06 Da & 22,6 & $-0,625$ & 3 & 1276,7 & DMKN_MOUSE & E9Q2P1_MOUSE & Dermokine; & Epidermis-specific secreted protein SK30/SK89; \\
\hline GTGSRGGSGG & SGGSGGGNKPECNNPGNDVR & $-10,0$ & $\begin{array}{l}\text { N-ter +34.06 Da, K+34.06 Da, C } \\
\text { +57.02 Da }\end{array}$ & 18,9 & $-4,063$ & 3 & 2040,0 & DMKN_MOUSE & E9Q2P1_MOUSE & Dermokine; & Epidermis-specific secreted protein $\mathrm{SK} 30 / \mathrm{SK} 89$; \\
\hline WASGGNYGTN & SLGGSVGQGGNGGPLDYETNAQGAVAQPGYGTVR & 0,1 & N-ter +34.06 Da & 16,0 & 3,210 & 3 & 3267,6 & DMKN_MOUSE & & Dermokine; & Epidermis-specific secreted protein $5 K 30 / 5 K 89$; \\
\hline FEHRLGEAAR & SLGNAGNEIGR & 1,8 & N-ter +28.03 Da & 40,8 & 0,806 & 2 & 1114,6 & DMKN_MOUSE & E9Q2P1_MOUSE & Dermokine; & Epidermis-specific secreted protein $5 K 30 / 5 K 89$; \\
\hline SGNSGNSGSG & SRDIETSNFDEGYSVSR & 1,4 & $\mathrm{~N}$-ter +28.03 Da & 32,3 & $-1,908$ & 3 & 1988,9 & DMKN_MOUSE & E9Q2P1_MOUSE & Dermokine; & Epidermis-specific secreted protein $\mathrm{SK30/5K89;}$ \\
\hline VRGNNQNSGC & TNPPPSGSHESFSNSGGSSNDGSR & 10,3 & $\mathrm{~N}$-ter $+28.03 \mathrm{Da}$ & 28,6 & $-7,734$ & 3 & 2389,0 & DMKN_MOUSE & E9Q2P1_MOUSE & Dermokine; & Epidermis-specific secreted protein SK30/SK89; \\
\hline NGGSGSRDIE & TSNFDEGYSVSR & 0,9 & $\mathrm{~N}$-ter +28.03 Da & 18,9 & $-0,432$ & 2 & 1388,6 & DMKN_MOUSE & E9Q2P1_MOUSE & Dermokine; & Epidermis-specific secreted protein SK30/SK89; \\
\hline $\begin{array}{l}\text { PAGQELLGK } \\
\text { GQELGYKAV }\end{array}$ & $\begin{array}{l}\text { AVDPETSSGEGLR } \\
\text { DPETSSGEGLR }\end{array}$ & $\begin{array}{c}0,0 \\
-0,5\end{array}$ & $\begin{array}{l}\mathrm{N}-\operatorname{ter}+34.06 \mathrm{Da} \\
\mathrm{N} \text {-ter }+34.06 \mathrm{Da}\end{array}$ & $\begin{array}{l}33,5 \\
17,0\end{array}$ & $\begin{array}{l}-1,257 \\
1,268\end{array}$ & $\begin{array}{l}2 \\
2\end{array}$ & $\begin{array}{l}1350,7 \\
1180,6\end{array}$ & $\begin{array}{l}\text { DSC1_MOUSE } \\
\text { DSC1_MOUSE }\end{array}$ & $\begin{array}{l}\text { Q32ME9_MOUSE } \\
\text { Q32ME9_MOUSE }\end{array}$ & $\begin{array}{l}\text { Desmocollin-1; } \\
\text { Desmocollin-1; }\end{array}$ & \\
\hline AETPVGKVNL & EECLKSPSLILSSDPAFR & $-0,6$ & $\begin{array}{l}\text { N-ter +34.06 Da, K+34.06 Da, C } \\
+57.02 \text { Da }\end{array}$ & 35,4 & 1,038 & 3 & 2116,2 & DSC1_MOUSE & Q32ME9_MOUSE & Desmocollin-1; & \\
\hline PGVDKEPYNL & FYIEKDTGDIYCTR & $-0,8$ & $\begin{array}{l}\text { N-ter +34.06 Da, K +34.06 Da, C } \\
\text { +57.02 Da }\end{array}$ & 22,1 & 4,214 & 3 & 1848,0 & DSC1_MOUSE & Q32ME9_MOUSE & Desmocollin-1; & \\
\hline DGLPAGQELL & GYKAVDPETSSGEGLR & $-1,1$ & $\mathrm{~N}$-ter +34.06 Da, K+34.06 Da & 32,1 & 4,781 & 3 & 1732,9 & DSC1_MOUSE & Q32ME9_MOUSE & Desmocollin-1; & \\
\hline VDKEPYNLFY & IEKDTGDIYCTR & 0,0 & $\begin{array}{l}\text { N-ter +34.06 Da, K +34.06 Da, C } \\
+57.02 \mathrm{Da}\end{array}$ & 22,6 & $-0,325$ & 3 & 1537,8 & DSC1_MOUSE & Q32ME9_MOUSE & Desmocollin-1; & \\
\hline QIPDQPKHFS & IHPDTGVITTTPLLDR & $-0,6$ & N-ter +34.06 Da & 22,7 & $-5,196$ & 3 & 1883,1 & DSC1_MOUSE & Q32ME9_MOUSE : & Desmocollin-1; & \\
\hline LPAGQELLGY & KAVDPETSSGEGLR & 2,1 & $\mathrm{~N}$-ter +34.06 Da, K+34.06 Da & 34,2 & 3,826 & 3 & 1512,9 & DSC1_MOUSE & Q32ME9_MOUSE D & Desmocollin-1; & \\
\hline VGKVNLEECL & KSPSLILSSDPAFR & $-0,4$ & $\mathrm{~N}$-ter +34.06 Da, K+34.06 Da & 15,8 & 0,504 & 3 & 1585,0 & DSC1_MOUSE & Q32ME9_MOUSE & Desmocollin-1; & \\
\hline QQIPDQPKHF & SIHPDTGVITTTTPLLDR & 0,3 & $\mathrm{~N}$-ter +34.06 Da & 44,0 & 0,355 & 3 & 1970,1 & DSC1_MOUSE & Q32ME9_MOUSE D & Desmocollin-1; & \\
\hline NSPYFTQTSY & TTEVEENRIDVEILR & $-0,1$ & $\mathrm{~N}$-ter $+34.06 \mathrm{Da}$ & 29,9 & 0,108 & 3 & 1849,0 & DSC1_MOUSE & Q32ME9_MOUSE & Desmocollin-1; & \\
\hline GVDKEPYNLF & YIEKDTGDIYCTR & $-0,4$ & $\begin{array}{l}\text { N-ter +34.06 Da, K+34.06 Da, C } \\
\text { +57.02 Da }\end{array}$ & 27,3 & $-7,923$ & 3 & 1700,9 & DSC1_MOUSE & Q32ME9_MOUSE & Desmocollin-1; & \\
\hline GLPAgQELLG & YKAVDPETSSGEGLR & $-0,2$ & $\mathrm{~N}$-ter $+34.06 \mathrm{Da}, \mathrm{K}+34.06 \mathrm{Da}$ & 39,5 & $-0,953$ & 3 & 1675,9 & DSC1_MOUSE & Q32ME9_MOUSE & Desmocollin-1; & \\
\hline KLLRVNLCDC & THPSQCPLR & $-5,3$ & $\mathrm{~N}$-ter +34.06 Da, C +57.02 Da & 21,2 & $-1,946$ & 2 & 1128,6 & DSC3_MOUSE & G5E8S6_MOUSE & Desmocollin-3; & \\
\hline FELMDGVKTS & AAPEICQEYSGTLR & 0,4 & $\mathrm{~N}$-ter +34.06 Da, C+57.02 Da & 45,1 & $-2,332$ & 2 & 1627,8 & DSG1A_MOUSE & DSG1B_MOUSE & Desmoglein-1-alpha; & DG1;DGl;Desmosomal glycoprotein l; \\
\hline ANVIITERVI & APGSSLPTSLTIPNPQETSNVVVTER & $-0,3$ & $\mathrm{~N}$-ter +28.03 Da & 22,5 & 3,414 & 3 & 2721,4 & DSG1A_MOUSE & & Desmoglein-1-alpha; & DG1;DGl;Desmosomal glycoprotein I; \\
\hline RNPIAKIHSD & CAANQPVTYR & $-0,8$ & $\mathrm{~N}$-ter +34.06 Da, C +57.02 Da & 18,2 & $-6,916$ & 2 & 1212,6 & DSG1A_MOUSE & DSG1B_MOUSE & Desmoglein-1-alpha; & DG1;DGl;Desmosomal glycoprotein I; \\
\hline NIPYLEQSSY & DITIEENALHSQLVQIR & 0,0 & $\mathrm{~N}$-ter +34.06 Da & 45,6 & $-1,836$ & 3 & 2012,1 & DSG1A_MOUSE & & Desmoglein-1-alpha; & DG1;DGl;Desmosomal glycoprotein I; \\
\hline SRMEANHRVG & EFVATDLDTGR & 0,1 & N-ter +34.06 Da & 40,6 & $-1,748$ & 2 & 1256,6 & DSG1A_MOUSE & DSG1B_MOUSE & Desmoglein-1-alpha; & DG1;DGl;Desmosomal glycoprotein I; \\
\hline EQSSYDIEIE & ENALHSQLVQIR & 0,2 & $\mathrm{~N}$-ter $+34.06 \mathrm{Da}$ & 23,6 & $-5,749$ & 3 & 1440,8 & DSG1A_MOUSE & DSG1B_MOUSE & Desmoglein-1-alpha; & DG1;DG|;Desmosomal glycoprotein l; \\
\hline TWQTVRRQKR & EWIKFAAACR & 0,4 & $\begin{array}{l}\text { N-ter +34.06 Da, K +34.06 Da, C } \\
\text { +57.02 Da }\end{array}$ & 56,0 & 0,984 & 2 & 1318,8 & DSG1A_MOUSE & DSG1B_MOUSE & Desmoglein-1-alpha; & DG1;DG|;Desmosomal glycoprotein I; \\
\hline VTMEOYQRLN & GEYKGTVISIDDSLQR & $-0,2$ & $\begin{array}{l}+57.02 \mathrm{Da} \\
\text { N-ter } 28.03 \mathrm{Da}, \mathrm{K}+28.03 \mathrm{Da}\end{array}$ & 38,1 & 0,707 & 3 & 1836,0 & DSGIA MOUSE & DSG1B_MOUSE & Desmoglein-1-alpha; & \\
\hline $\begin{array}{l}\text { VTERVIQPTS } \\
\text { VTS }\end{array}$ & GMIGNLSMTPELSSAHNVIVTER & 0,0 & N-ter +34.06 Da & $\begin{array}{l}30,1 \\
35,8\end{array}$ & 1,926 & 3 & 2489,3 & DSGIA_MOUSE & & Desmoglein-1-alpha; & DG1;DGl;Desmosomal glycoprotein I; \\
\hline RVIIPTSGMI & GNLSMTPELSSAHNVIVTER & 0,0 & N-ter +28.03 Da & 21.5 & 5,721 & 3 & 2182,1 & DSG1A MOUSE & & Desmoglein-1-alpha; & DG1:DG1:Desmosomal glycoprotein I: \\
\hline SNVWVTERVV & GPISGADLHGMLEIPDLR & 0,0 & $\mathrm{~N}$-ter $+28.03 \mathrm{Da}$ & 31,3 & $-0,208$ & 3 & 1918,0 & DSG1A_MOUSE & & Desmoglein-1-alpha: & DG1:DGl:Desmosomal glycoprotein I: \\
\hline VVGPVPGADL & HGMLEIPDLR & $-0,2$ & $\mathrm{~N}$-ter $+28.03 \mathrm{Da}$ & 15,1 & $-1,157$ & 2 & 1207,6 & DSG1A MOUSE & DSG1B MOUSE & Desmoglein-1-alpha: & DG1:DGl:Desmosomal glycoprotein I: \\
\hline PTSGMIGNLS & MTPELSSAHNVIVTER & 0,2 & $\mathrm{~N}$-ter +34.06 Da & 53,5 & 0,76 & 3 & 1817,0 & DSG1A_MOUSE & & Desmoglein-1-alpha: & DG1:DGl:Desmosomal glycoprotein I: \\
\hline VIOPTSGMIG & NLSMTPELSSAHNVIVTER & 2,0 & $\mathrm{~N}$-ter $+28.03 \mathrm{Da}$ & 33,4 & $-4,27$ & 3 & 2125,1 & DSG1A_MOUSE & & Desmoglein-1-alpha: & DG1:DGl:Desmosomal glycoprotein I: \\
\hline TLKPSVHFHD & NQQASNVVVTER & 5,1 & $\mathrm{~N}$-ter +28.03 Da & 23,8 & $-4,077$ & 2 & 1371,7 & DSG1A MOUSE & DSG1B MOUSE & Desmoglein-1-alpha: & DG1:DGl:Desmosomal glycoprotein I: \\
\hline TTVISENAYH & SGPGVQHPVPIPDPLGYGNVTVR & 0,9 & $\mathrm{~N}$-ter +28.03 Da & 45,9 & 0,880 & 3 & 2383,3 & DSG1A MOUSE & & Desmoglein-1-alpha: & DG1:DGl:Desmosomal glycoprotein I: \\
\hline VTERVIAPGS & SLPTSLTPNPQETSNVVVTER & 1,0 & $\mathrm{~N}$-ter $+28.03 \mathrm{Da}$ & 27,6 & 1,119 & 3 & 2409,3 & DSG1A_MOUSE & & Desmoglein-1-alpha: & DG1:DGl:Desmosomal glycoprotein I: \\
\hline QPTSGMIGNL & SMTPELSSAHNVIVTER & $-0,1$ & $\mathrm{~N}$-ter $+28.03 \mathrm{Da}$ & 64,6 & 0,105 & 3 & 1898,0 & DSG1A MOUSE & & Desmoglein-1-alpha: & DG1:DGl:Desmosomal glycoprotein I: \\
\hline HMRSSSDHHF & SQTVGSASPSMAR & 0,7 & $\mathrm{~N}$-ter +28.03 Da & 20,3 & $-7,418$ & 2 & 1305,6 & DSG1A_MOUSE & & Desmoglein-1-alpha; & DG1;DGI;Desmosomal glycoprotein I; \\
\hline IVTERVIAPG & SSLPTSTIPNPQETSNVVVTER & 0,1 & $\mathrm{~N}$-ter +34.06 Da & 41,7 & $-0,479$ & 3 & 2502,3 & DSG1A_MOUSE & & Desmoglein-1-alpha; & DG1;DGl;Desmosomal glycoprotein I; \\
\hline
\end{tabular}




\begin{tabular}{|c|c|c|c|c|c|c|c|c|c|c|c|}
\hline Non Prime Site & Prime Site & $\begin{array}{c}\text { Fold change of } \\
\text { XPRESS ratio } \\
\text { (Adam17 }{ }^{\Delta K c} / \mathrm{WT} \text { ) }\end{array}$ & Modifications & Hyperscore & $\begin{array}{c}\text { Mass error } \\
\text { in ppm }\end{array}$ & Charge & $\begin{array}{c}\text { Precursor } \\
\text { neutral mass } \\
\text { in Da }\end{array}$ & Uniprot ID 1 & Uniprot ID 2 & Protein Name 1 & Protein Name 2 \\
\hline$\overline{\text { APGSSLPTSL }}$ & TIPNPQETSNVVVTER & 0,0 & $\mathrm{~N}$-ter $+28.03 \mathrm{Da}$ & 29,7 & 8,545 & 3 & 1811,0 & DSG1A_MOUSE & & Desmoglein-1-alpha; & DG1;DG|;Desmosomal glycoprotein I; \\
\hline GIGSSGLVST & TMGASGTGLNMGGTATIGHMR & $-0,2$ & $\mathrm{~N}$-ter $+28.03 \mathrm{Da}$ & 45,8 & 0,049 & 3 & 2048,0 & DSG1A_MOUSE & & Desmoglein-1-alpha; & DG1;DGl;Desmosomal glycoprotein I; \\
\hline TSGMIGNLSM & TPELSSAHNVIVTER & 0,3 & $\mathrm{~N}$-ter $+28.03 \mathrm{Da}$ & 51,4 & 2,080 & 3 & 1679,9 & DSG1A_MOUSE & & Desmoglein-1-alpha; & DG1;DGl;Desmosomal glycoprotein I; \\
\hline NIPYLEQSSY & DIEIEENALHSQLVQIR & $-0,7$ & $\mathrm{~N}$-ter $+28.03 \mathrm{Da}$ & 45,7 & $-0,540$ & 3 & 2034,1 & DSG1B_MOUSE & & Desmoglein-1-beta; & Desmoglein-5; \\
\hline LRDGTNVIVT & ERVIAPGSSLPTSLTIPNPR & $-0,6$ & $\mathrm{~N}$-ter $+28.03 \mathrm{Da}$ & 20,7 & 1,124 & 3 & 2132,2 & DSG1B_MOUSE & & Desmoglein-1-beta; & Desmoglein-5; \\
\hline PTSGMIGNLS & MTPELSSAQNVIVTER & $-0,1$ & $\mathrm{~N}$-ter $+28.03 \mathrm{Da}$ & 19,9 & 1,884 & 3 & 1801,9 & DSG1B_MOUSE & & Desmoglein-1-beta; & Desmoglein-5; \\
\hline QPTSGMIGNL & SMTPELSSAQNVIVTER & $-0,5$ & $\mathrm{~N}$-ter $+28.03 \mathrm{Da}$ & 19,8 & $-2,854$ & 3 & 1889,0 & DSG1B_MOUSE & & Desmoglein-1-beta; & Desmoglein-5; \\
\hline GIGSSGLVST & TMGAAGTGLNMGGTATIGHMR & $-0,8$ & $\mathrm{~N}$-ter $+28.03 \mathrm{Da}$ & 24,5 & 1,278 & 3 & 2032,0 & DSG1B_MOUSE & & Desmoglein-1-beta; & Desmoglein-5; \\
\hline TGFELMDGVK & TSAAPEICQEYSGTLR & $-0,1$ & N-ter +28.03 Da, C +57.02 Da & 19,8 & $-4,909$ & 3 & 1809,9 & DSG1B_MOUSE & DSG1A_MOUSE & Desmoglein-1-beta; & Desmoglein-5; \\
\hline GTALELEAQ & AATGFIVDPVSNLR & $-0,8$ & $\mathrm{~N}$-ter $+34.06 \mathrm{Da}$ & 23,7 & $-0,803$ & 2 & 1492,9 & DESP_MOUSE & E9PZWO_MOUSE & Desmoplakin; & \\
\hline TALELLEAQA & ATGFIVDPVSNLR & $-0,2$ & $\mathrm{~N}$-ter +34.06 Da & 18,0 & $-0,562$ & 2 & 1421,8 & DESP_MOUSE & E9PZWO_MOUSE & Desmoplakin; & \\
\hline HGIRLEAQI & ATGGIIDPKESHR & $-0,6$ & N-ter +28.03 Da, K +28.03 Da & 22,0 & 0,486 & 4 & 1435,8 & DESP_MOUSE & E9PZWO_MOUSE & Desmoplakin; & \\
\hline RQPVTVTELV & DSGILRPSTVNELESGQISYDEVGER & $-0,1$ & $\mathrm{~N}$-ter $+34.06 \mathrm{Da}$ & 21,0 & $-1,109$ & 3 & 2883,4 & DESP_MOUSE & E9PZWO_MOUSE & Desmoplakin; & \\
\hline VRPGTALELL & EAQAATGFIVDPVSNLR & $-1,7$ & $\mathrm{~N}$-ter $+34.06 \mathrm{Da}$ & 29,6 & 0,932 & 3 & 1821,0 & DESP_MOUSE & E9PZWO_MOUSE & Desmoplakin; & \\
\hline SGILRPSTVN & ELESGQISYDEVGER & $-0,4$ & $\mathrm{~N}$-ter $+28.03 \mathrm{Da}$ & 64,7 & $-0,402$ & 2 & 1737,8 & DESP_MOUSE & E9PZWO_MOUSE & Desmoplakin; & \\
\hline IGLVRPGTAL & ELLEAQAATGFIVDPVSNLR & $-0,3$ & $\mathrm{~N}$-ter +34.06 Da & 22,1 & $-8,031$ & 3 & 2176,2 & DESP_MOUSE & E9PZWO_MOUSE & Desmoplakin; & \\
\hline ILRPSTVNEL & ESGQISYDEVGER & 0,0 & $\mathrm{~N}$-ter $+28.03 \mathrm{Da}$ & 17,7 & 2,871 & 2 & 1495,7 & DESP_MOUSE & E9PZWO_MOUSE & Desmoplakin; & \\
\hline LELLEAQAAT & GFIVDPVSNLR & $-0,3$ & $\mathrm{~N}$-ter $+28.03 \mathrm{Da}$ & 14,8 & $-0,241$ & 2 & 1243,7 & DESP_MOUSE & E9PZWO_MOUSE & Desmoplakin; & \\
\hline ARLTEEETVC & LDLDKVEAYR & $-0,3$ & N-ter +34.06 Da, K +34.06 Da & 21,6 & 8,902 & 3 & 1288,8 & DESP_MOUSE & E9PZWO_MOUSE & Desmoplakin; & \\
\hline LVRPGTALEL & LEAQAATGFIVDPVSNLR & 0,4 & N-ter +34.06 Da & 17,7 & $-0,103$ & 3 & 1934,1 & DESP_MOUSE & E9PZWO_MOUSE & Desmoplakin; & \\
\hline FIVDPVSNLR & LPVEEAYKR & 1,1 & N-ter +28.03 Da, K +28.03 Da & 15,8 & $-1,978$ & 3 & 1159,7 & DESP_MOUSE & E9PZWO_MOUSE & Desmoplakin; & \\
\hline PGtALELLEA & QAATGFIVDPVSNLR & $-0,2$ & $\mathrm{~N}$-ter +28.03 Da & 43,0 & $-0,866$ & 2 & 1614,9 & DESP_MOUSE & E9PZWO_MOUSE & Desmoplakin; & \\
\hline KGHGIRLEAA & QIATGGIIIPKESHR & 0,5 & $\mathrm{~N}$-ter +34.06 Da, K+34.06 Da & 20,5 & $-0,118$ & 4 & 1689,0 & DESP_MOUSE & E9PZWO_MOUSE & Desmoplakin; & \\
\hline ASFERMDHLR & QLQNIIIATSRR & 2,5 & N-ter $+28.03 \mathrm{Da}$ & 22,2 & $-4,305$ & 2 & 1298,7 & DESP_MOUSE & E9PZWO_MOUSE & Desmoplakin; & \\
\hline LRPSTVNELE & SGQISYDEVGER & $-0,3$ & N-ter $+28.03 \mathrm{Da}$ & 24,8 & $-0,146$ & 2 & 1366,6 & DESP_MOUSE & E9PZWO_MOUSE & Desmoplakin; & \\
\hline PIKRTMVQSP & SGVILQEAADIHAR & $-0,3$ & N-ter +34.06 Da & 29,8 & 0,330 & 3 & 1512,9 & DESP_MOUSE & E9PZWO_MOUSE & Desmoplakin; & \\
\hline NIPIKRTMVQ & SPSGVILQEAADIHAR & 0,9 & N-ter $+28.03 \mathrm{Da}$ & 19,6 & $-1,004$ & 3 & 1690,9 & DESP_MOUSE & E9PZWO_MOUSE & Desmoplakin; & \\
\hline SIKDYELQLA & SYTSGLETLINIPIKR & $-1,2$ & $\mathrm{~N}$-ter +28.03 Da, K+28.03 Da & 14,1 & $-2,308$ & 3 & 1860,1 & DESP_MOUSE & E9PZWO_MOUSE & Desmoplakin; & \\
\hline VTKTEITHLG & TCQDVNHNKVIETNR & 0,1 & $\begin{array}{l}\text { N-ter +28.03 Da, K }+28.03 \text { Da, C } \\
+57.02 \text { Da }\end{array}$ & 25,6 & $-1,166$ & 4 & 1882,9 & DESP_MOUSE & E9PZWO_MOUSE & Desmoplakin; & \\
\hline DMLKVYEARL & TEEETVCLDLDKVEAYR & 1,8 & $\begin{array}{l}\text { N-ter +34.06 Da, K +34.06 Da, C } \\
+57.02 \text { Da }\end{array}$ & 36,6 & $-0,327$ & 3 & 2137,1 & DESP_MOUSE & E9PZWO_MOUSE & Desmoplakin; & \\
\hline ALELLEAQAA & TGFIVDPVSNLR & 0,1 & $\mathrm{~N}$-ter $+34.06 \mathrm{Da}$ & 23,6 & $-2,439$ & 2 & 1350,8 & DESP_MOUSE & E9PZWO_MOUSE & Desmoplakin; & \\
\hline GQRFLEFQFL & TGGLVDPEVHGR & 0,1 & $\mathrm{~N}$-ter $+28.03 \mathrm{Da}$ & 20,6 & $-0,868$ & 3 & 1263,7 & DESP_MOUSE & E9PZWO_MOUSE & Desmoplakin; & \\
\hline ETLLNIPIKR & TMVQSPSGVILQEAADIHAR & 0,9 & $\mathrm{~N}$-ter $+28.03 \mathrm{Da}$ & 56,5 & $-1,672$ & 3 & 2150,1 & DESP_MOUSE & E9PZWO_MOUSE & Desmoplakin; & \\
\hline LLGSPSRRSY & SLPPHQKVPLPSLSPTMQAGTIAR & $-0,3$ & $\mathrm{~N}$-ter +28.03 Da, K+28.03 Da & 37,4 & 3,520 & 4 & 2581,5 & ODP2_MOUSE & & $\begin{array}{l}\text { Dihydrolipoyllysine-residue acetyltransferase } \\
\text { component of pyruvate dehydrogenase complex, } \\
\text { mitochondrial; }\end{array}$ & $\begin{array}{l}\text { Dihydrolipoamide acetyltransferase component } \\
\text { of pyruvate dehydrogenases complex;:Prruvate } \\
\text { dehydrogenase complex component } E 2 ;\end{array}$ \\
\hline KPVSAIKPTA & APPLAEAGAAKGLR & $-0,2$ & N-ter +28.03 Da, K+28.03 Da & 22,6 & 9,784 & 3 & 1376,8 & ODO2_MOUSE & & $\begin{array}{l}\text { Dihydrolipoyllysine-residue succinyltransferase } \\
\text { component of 2-oxoglutarate dehydrogenase } \\
\text { complex, mitochondrial; }\end{array}$ & $\begin{array}{l}\text { 2-oxoglutarate dehydrogenase complex } \\
\text { component E2;iDhydrolipoamide } \\
\text { succinyttransferase component of 2- } \\
\text { oxoglutarate dehydrogenase complex; } ; 2 \text {;K; }\end{array}$ \\
\hline RFFQTTAVCK & NDVITVQTPAFAESVTEGDVR & $-0,5$ & N-ter +34.06 Da & 51,9 & 5,472 & 3 & 2281,2 & ODO2_MOUSE & & $\begin{array}{l}\text { Dihydrolipoyllysine-residue succinyltransferase } \\
\text { component of 2-oxoglutarate dehydrogenase } \\
\text { complex, mitochondrial; }\end{array}$ & $\begin{array}{l}\text { 2-oxoglutarate dehydrogenase complex } \\
\text { component E2;Dinhydrolipoamide } \\
\text { succinyltransferase component of } 2 \text { - } \\
\text { oxogglutarate dehydrogenase complex;E2K; }\end{array}$ \\
\hline KTVTPASSAK & TSPAKQQAPPVR & 0,1 & N-ter +28.03 Da, K+28.03 Da & 35,5 & 0,075 & 3 & 1334,8 & DPYL2_MOUSE & & Dihydropyrimidinase-related protein 2; & Unc-33-like phosphoprotein 2; \\
\hline GAGVSGLISL & KCCVDEGLEPTCFER & 0,0 & $\begin{array}{l}\text { N-ter +34.06 Da, K+34.06 Da, C } \\
+57.02 \mathrm{Da}\end{array}$ & 34,5 & $-1,523$ & 3 & 1966,9 & FMO2_MOUSE & D3YVR1_MOUSE & Dimethylaniline monooxygenase [N-oxide-forming] 2; & $\begin{array}{l}\text { Dimethylaniline oxidase 2;Pulmonary flavin- } \\
\text { containing monooxygenase } 2 \text {; }\end{array}$ \\
\hline GLVPQNKYLM & KEPVLNDDLPSR & $-0,4$ & $\mathrm{~N}$-ter +28.03 Da, $\mathrm{K}+28.03 \mathrm{Da}$ & 17,2 & 3,540 & 3 & 1437,8 & FMO2_MOUSE & & Dimethylaniline monooxygenase [N-oxide-forming] 2; & $\begin{array}{l}\text { Dimethylaniline oxidase 2;Pulmonary flavin- } \\
\text { containing monooxygenase } 2 \text {; }\end{array}$ \\
\hline ATGYTFSFSF & LEDSLVKVEDNR & 0,2 & N-ter +28.03 Da, K+28.03 Da & 16,0 & $-1,560$ & 3 & 1471,8 & FMO2_MOUSE & & Dimethylaniline monooxygenase [N-oxide-forming] 2; & $\begin{array}{l}\text { Dimethlylaniline oxidase 2;Pulmonary flavin- } \\
\text { containing monooxygenase } 2 \text {; }\end{array}$ \\
\hline VSGLISLKCC & VDEGLEPTCFER & 0,1 & N-ter +34.06 Da, C +57.02 Da & 17,0 & $-0,942$ & 2 & 1484,7 & FMO2_MOUSE & D3YVR1_MOUSE & Dimethylaniline monooxygenase [N-oxide-forming] 2; & $\begin{array}{l}\text { Dimethlylaniline oxidase 2;Pulmonary flavin- } \\
\text { containing monooxygenase 2; }\end{array}$ \\
\hline LYFGPCNSYQ & YRLVGPGQWEGAR & 1,1 & N-ter +34.06 Da & 20,0 & $-0,394$ & 3 & 1521,8 & FMO2_MOUSE & & Dimethylaniline monooxygenase [N-oxide-forming] 2; & $\begin{array}{l}\text { Dimethylaniline oxidase 2;Pulmonary flavin- } \\
\text { containing monooxygenase 2; }\end{array}$ \\
\hline APMTDEIQQQ & ILNLPESWDWR & $-0,8$ & N-ter +34.06 Da & 28,1 & 1,161 & 2 & 1461,8 & CATC_MOUSE & D3Z220_MOUSE & Dipeptidyl peptidase 1 & $\begin{array}{l}\text { Cathepsin C;Cathepsin J;Dipeptidyl peptidase } \\
\text { I;DipeptidyI transferase; }\end{array}$ \\
\hline FKYEVRGHTA & ISYCHETMTGWVHDVLGR & $-0,2$ & N-ter +34.06 Da, C +57.02 Da & 16,8 & $-0,319$ & 4 & 2194,1 & CATC_MOUSE & D3Z220_MOUSE & Dipeptidyl peptidase 1 ; & $\begin{array}{l}\text { Cathepsin C;Cathepsin J;Dipeptidyl peptidase } \\
\text { l;Dipeptidyl transferase; }\end{array}$ \\
\hline
\end{tabular}




\begin{tabular}{|c|c|c|c|c|c|c|c|c|c|c|c|}
\hline Non Prime Site & Prime Site & 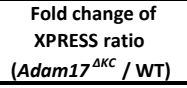 & Modifications & Hyperscore & $\begin{array}{l}\text { Mass error } \\
\text { in ppm }\end{array}$ & charge & $\begin{array}{c}\text { Precursor } \\
\text { neutral mass } \\
\text { in Da }\end{array}$ & Uniprot ID 1 & Uniprot ID 2 & Protein Name 1 & Protein Name 2 \\
\hline PMTDEIQQQI & LNLPESWDWR & $-5,3$ & N-ter +28.03 Da & 19,4 & 2,900 & 2 & 1342,7 & CATC_MOUSE & D3Z220_MOUSE & Dipeptidyl peptidase 1; & $\begin{array}{l}\text { Cathepsin C;Cathepsin J;Dipeptidyl peptidase } \\
\text { l;Dipeptidyl transferase; }\end{array}$ \\
\hline MTDEIQQQIL & NLPESWDWR & $-0,3$ & $\mathrm{~N}$-ter +28.03 Da & 17,8 & $-2,436$ & 2 & 1229,6 & CATC_MOUSE & D3Z220_MOUSE & Dipeptidyl peptidase 1; & $\begin{array}{l}\text { Cathepsin C;Cathepsin J;ipipeptidyl peptidase } \\
\text { |;Dipeptidyl transferase; }\end{array}$ \\
\hline ILNLPESWDW & RNVQGVNYVSPVR & $-0,5$ & $\mathrm{~N}$-ter +34.06 Da & 23,8 & $-3,806$ & 3 & 1520,9 & CATC_MOUSE & D3Z220_MOUSE & Dipeptidyl peptidase 1 & $\begin{array}{l}\text { Cathepsin C;Cathepsin J;Dipeptidyl peptidase } \\
\text { l;Dipeptidyl transferase; }\end{array}$ \\
\hline TGIEYWIIKN & SWGSNWGESGYFR & 0,2 & $\mathrm{~N}$-ter +34.06 Da & 27,3 & $-4,593$ & 2 & 1565,7 & CATC_MOUSE & & Dipeptidyl peptidase 1 & $\begin{array}{l}\text { Cathepsin C;Cathepsin J;iipeptidyl peptidase } \\
\text { |;Dipeptidyl transferase; }\end{array}$ \\
\hline LSETASILRH & ATAHSLVLVDELGR & 6,5 & $\mathrm{~N}$-ter +28.03 Da & 15,1 & $-2,912$ & 3 & 1507,8 & MSH6_MOUSE & & DNA mismatch repair protein Msh6; & $\begin{array}{l}\text { G/T mismatch-binding protein;Muts-alpha } 160 \\
\text { kDa subunit;p160; }\end{array}$ \\
\hline TSFHTSASLA & KDDYYQILGVPR & 0,5 & $\mathrm{~N}$-ter +28.03 Da, K+28.03 Da & 20,7 & $-0,853$ & 3 & 1521,8 & DNJA3_MOUSE & & Dnas homolog subfamily A member 3, mitochondrial; & $\begin{array}{l}\text { Dnaj protein Tid-1;Tumorous imaginal discs } \\
\text { protein Tid56 homolog: }\end{array}$ \\
\hline M & GKDYYQTLGLAR & $-0,3$ & N-ter +34.06 Da, K+34.06 Da & 27,4 & $-1,993$ & 3 & 1451,8 & DNJB1_MOUSE & & DnaJ homolog subfamily B member 1; & Heat shock 40 kDa protein 1 ; \\
\hline LIVVVVVVG & TDQNFYSLLGVSKTASSR & $-0,7$ & $\mathrm{~N}$-ter +34.06 Da, K +34.06 Da & 35,1 & 2,984 & 3 & 2041,1 & DJC10_MOUSE & & DnaJ homolog subfamily C member 10; & $\begin{array}{l}\text { Endoplasmic reticulum DNA J domain- } \\
\text { containing protein } 5 \text {; Endoplasmic reticulum } \\
\text { DnaJ-PDI fusion protein } 1 ; \text {; d domain-containing } \\
\text { protein disulfide isomerase-like protein; }\end{array}$ \\
\hline M & LLPSAAEGQGTAITHALTSASSVCQVEPVGR & $-1,4$ & N-ter +34.06 Da, C+57.02 Da & 28,3 & 3,305 & 4 & 3384,8 & DNJC2_MOUSE & Q3TRX6_MOUSE & DnaJ homolog subfamily C member 2; & Mouse Id associate 1;Zuotin-related factor 1 ; \\
\hline VILAPVHLCS & YIAWYCR & $-2,3$ & N-ter +34.06 Da, C+57.02 Da & 11,3 & 2,063 & 2 & 1064,5 & DJC25_MOUSE & & Dnas homolog subfamily $\mathrm{C}$ member 25 ; & \\
\hline LAPTPGSASS & EAPPLVNEDVKR & 0,4 & $\mathrm{~N}$-ter +28.03 Da, K+28.03 Da & 18,0 & 7,018 & 3 & 1421,8 & RPN1_MOUSE & & $\begin{array}{l}\text { Dolichyl-diphosphooligosaccharide--protein } \\
\text { glycosyltransferase subunit 1; }\end{array}$ & $\begin{array}{l}\text { Dolichyl-diphosphooligosaccharide---protein } \\
\text { glycosyltransferase } 67 \text { kDa subunit;iibophorin } \\
\text { l;RRibophorin-1; }\end{array}$ \\
\hline GALAPTPGSA & SSEAPPLVNEDVKR & 0,0 & N-ter +28.03 Da, K+28.03 Da & 55,7 & 2,627 & 3 & 1595,9 & RPN1_MOUSE & & $\begin{array}{l}\text { Dolichyl-diphosphooligosaccharide--protein } \\
\text { glycosyltransferase subunit 1; }\end{array}$ & $\begin{array}{l}\text { Dolichyl-diphosphooligosaccharide--protein } \\
\text { glycosyltransferase } 67 \text { kDa subunit;:Ribophorin } \\
\text { 1;Ribophorin-1; }\end{array}$ \\
\hline ALITIASVQA & LTPTHYLTKQDVER & 0,1 & $\mathrm{~N}$-ter +28.03 Da, K+28.03 Da & 34,8 & $-1,421$ & 3 & 1756,0 & RPN2_MOUSE & A2ACG7_MOUSE & $\begin{array}{l}\text { Dolichyl-diphosphooligosaccharide--protein } \\
\text { glycosyltransferase subunit 2; }\end{array}$ & $\begin{array}{l}\text { Dolichyl-diphosphooligosaccharide--protein } \\
\text { glycosyltransferase } 63 \text { kDa subunit;;ibophorin } \\
\text { I;;Ribophorin-2; }\end{array}$ \\
\hline LTITASVQAL & TPTHYLTKQDVER & 0,0 & $\mathrm{~N}$-ter +34.06 Da, K +34.06 Da & 22,0 & $-2,232$ & 3 & 1654,9 & RPN2_MOUSE & A2ACG7_MOUSE & $\begin{array}{l}\text { Dolichyl-diphosphooligosaccharide--protein } \\
\text { glycosyltransferase subunit 2; }\end{array}$ & $\begin{array}{l}\text { Dolichyl-diphosphoolizosaccharide--protein } \\
\text { glycosyltransferase } 63 \text { kDa subunit;Ribophorin } \\
\text { II;Ribophorin-2; }\end{array}$ \\
\hline VTSSQPGKLR & SPFLQKQLTQPETSYGR & $-0,1$ & $\mathrm{~N}$-ter +34.06 Da, K+34.06 Da & 19,2 & 5,122 & 3 & 2047,2 & DBNL_MOUSE & & Drebrin-like protein; & $\begin{array}{l}\text { Actin-binding protein 1;SH3 domain-containing } \\
\text { protein 7; }\end{array}$ \\
\hline ALQEAYVRVV & TEKSPTDWALFTYEGNSNDIR & $-1,3$ & $\mathrm{~N}$-ter +34.06 Da, K+34.06 Da & 20,9 & $-3,023$ & 3 & 2511,3 & DBNL_MOUSE & & Drebrin-like protein; & $\begin{array}{l}\text { Actin-binding protein 1;SH3 domain-containing } \\
\text { protein } 7 \text {; }\end{array}$ \\
\hline RQIVQKKQTE & NPKELDER & 10,3 & N-ter +28.03 Da, K+28.03 Da & 16,3 & $-2,648$ & 2 & 1055,6 & DMD_MOUSE & & Dystrophin; & \\
\hline NCKQCLERSA & SLISQAEHSLKENDHAR & 1,4 & $\mathrm{~N}$-ter +34.06 Da, K+34.06 Da & 17,3 & $-4,139$ & 3 & 2002,1 & TRI18_MOUSE & Q6PD02_MOUSE & E3 ubiquitin-protein ligase Midline-1; & $\begin{array}{l}\text { Midini;RING finger protein Midlline-1; Tripartite } \\
\text { motif-containing protein 18; }\end{array}$ \\
\hline LPEHVDQLLQ & VDSEDNESQGQVEGR & $-10,0$ & $\mathrm{~N}$-ter +34.06 Da & 18,4 & 3,621 & 3 & 1681,8 & RN123_MOUSE & Q05CH9_MOUSE & E3 ubiquitin-protein ligase RNF123; & $\begin{array}{l}\text { Kip1 ubiquitination-promoting complex protein } \\
\text { 1;RING finger protein 123; }\end{array}$ \\
\hline $\begin{array}{l}\text { SSFFVEVLMM } \\
\text { TFQQKIKDKK }\end{array}$ & $\begin{array}{l}\text { MNNAAVPTAEFLAVSIR } \\
\text { ESIYFSHQR }\end{array}$ & $\begin{array}{l}1,2 \\
2,2\end{array}$ & $\begin{array}{l}\text { N-ter +34.06 Da } \\
\text { N-ter +28.03 Da }\end{array}$ & $\begin{array}{l}13,7 \\
19,8\end{array}$ & $\begin{array}{l}-0,109 \\
-5,604\end{array}$ & $\begin{array}{l}3 \\
2\end{array}$ & $\begin{array}{l}1837,0 \\
1193,6\end{array}$ & $\begin{array}{l}\text { EPG5_MOUSE } \\
\text { EFHB_MOUSE }\end{array}$ & & $\begin{array}{l}\text { Ectopic } P \text { granules protein } 5 \text { homolog; } \\
\text { EF-hand domain-containing family member B; }\end{array}$ & \\
\hline LPRVAAKLNV & APVSDIIEIKSPDTFVR & $-0,6$ & N-ter +34.06 Da, K+34.06 Da & 16,3 & 3,372 & 3 & 1954,2 & ETFA_MOUSE & & $\begin{array}{l}\text { Electron transfer flavoprotein subunit alpha, } \\
\text { mittochondrial; }\end{array}$ & \\
\hline LRRAASLRF & QSTLVIAEHANDSLAPITLNTITAAGR & $-0,4$ & $\mathrm{~N}$-ter +34.06 Da & 43,0 & $-2,559$ & 3 & 2810,5 & ETFA_MOUSE & & $\begin{array}{l}\text { Electron transfer flavoprotein subunit alpha, } \\
\text { mitochondrial; }\end{array}$ & \\
\hline KCLPLCAPRC & SSTSAVPQITTHYTVHPR & $-0,3$ & $\mathrm{~N}$-ter +34.06 Da & 38,8 & 1,387 & 4 & 2015,1 & ETFD_MOUSE & & $\begin{array}{l}\text { Electron transfer flavoprotein-ubiquinone } \\
\text { oxidoreductase, mitochondrial; }\end{array}$ & $\begin{array}{l}\text { Electron-transferring-flavoprotein } \\
\text { dehydrogenase; }\end{array}$ \\
\hline SQADCAVLIV & AAGVGEFEAGISKNGQTR & $-0,6$ & $\mathrm{~N}$-ter +34.06 Da, K +34.06 Da & 19,0 & 0,215 & 3 & 1859,0 & EF1A1_MOUSE & EF1A2_MOUSE & Elongation factor 1-alpha 1; & $\begin{array}{l}\text { Elongation factor Tu:Eukaryotic elongation } \\
\text { factor } 1 \mathrm{~A}-1 \text {; }\end{array}$ \\
\hline DGPKFLKSGD & AAIVDMVPGKPMCVESFSDYPPLGR & $-0,2$ & $\begin{array}{l}\text { N-ter +28.03 Da, K+28.03 Da, C } \\
+57.02 \mathrm{Da}\end{array}$ & 42,1 & $-1,396$ & 3 & 2791,4 & EF1A1_MOUSE & & Elongation factor 1-alpha 1; & $\begin{array}{l}\text { Elongation factor Tu; Eukaryotic elongation } \\
\text { factor } 1 \mathrm{~A}-1 \text {; }\end{array}$ \\
\hline QADCAVLIVA & AGVGEFEAGISKNGQTR & 0,0 & $\mathrm{~N}$-ter +28.03 Da, K+28.03 Da & 35,0 & 0,675 & 3 & 1775,9 & EF1A1_MOUSE & EF1A2_MOUSE & Elongation factor 1-alpha 1; & $\begin{array}{l}\text { Elongation factor Tu; Eukaryotic elongation } \\
\text { factor } 1 \mathrm{~A}-1 \text {; }\end{array}$ \\
\hline KQLIVGVNKM & DSTEPPYSQKR & 0,2 & $\mathrm{~N}$-ter +34.06 Da, K+34.06 Da & 23,5 & $-1,234$ & 3 & 1374,7 & EF1A1_MOUSE & D3YZ68_MOUSE & Elongation factor 1-alpha 1; & $\begin{array}{l}\text { Elongation factor Tu; Eukaryotic elongation } \\
\text { factor } 1 \mathrm{~A}-1 \text {; }\end{array}$ \\
\hline LPLQDVYKIG & GIGTVPVGR & 0,4 & N-ter +34.06 Da & 14,4 & $-2,358$ & 2 & 888,6 & EF1A1_MOUSE & EF1A2_MOUSE & Elongation factor 1-alpha 1; & $\begin{array}{l}\text { Elongation factor Tu; Eukaryotic elongation } \\
\text { factor } 1 \mathrm{~A}-1 \text {; }\end{array}$ \\
\hline GTtLEALDC & ILPPTRPTDKPLR & $-0,6$ & $\mathrm{~N}$-ter $+34.06 \mathrm{Da}, \mathrm{K}+34.06 \mathrm{Da}$ & 15,6 & 2,349 & 4 & 1571,0 & EF1A1_MOUSE & EF1A2_MOUSE & Elongation factor 1-alpha 1; & $\begin{array}{l}\text { Elongation factor Tu; Eukaryotic elongation } \\
\text { factor } 1 \mathrm{~A}-1 ;\end{array}$ \\
\hline
\end{tabular}




\begin{tabular}{|c|c|c|c|c|c|c|c|c|c|c|c|}
\hline Non Prime Site & Prime Site & 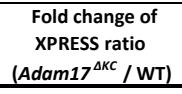 & Modifications & Hyperscore & $\begin{array}{c}\text { Mass error } \\
\text { in ppm }\end{array}$ & Charge & $\begin{array}{c}\text { Precursor } \\
\text { neutral mass } \\
\text { in Da } \\
\end{array}$ & Uniprot ID 1 & Uniprot ID 2 & Protein Name 1 & Protein Name 2 \\
\hline PLRLPLQDVY & KIGGIGTVPVGR & $-0,4$ & $\mathrm{~N}$-ter +34.06 Da, K+34.06 Da & 22,3 & $-1,798$ & 3 & 1220,8 & EF1A1_MOUSE & EF1A2_MOUSE & Elongation factor 1-alpha 1; & $\begin{array}{l}\text { Elongation factor Tu; Eukaryotic elongation } \\
\text { factor } 1 \mathrm{~A}-1 \text {; }\end{array}$ \\
\hline GVKQLIVGVN & KMDSTEPPYSQKR & 1,3 & $\mathrm{~N}$-ter $+28.03 \mathrm{Da}, \mathrm{K}+28.03 \mathrm{Da}$ & 24,4 & 3,507 & 4 & 1649,9 & EF1A1_MOUSE & D3YZ68_MOUSE & $=$ Elongation factor 1-alpha 1; & $\begin{array}{l}\text { Elongation factor Tu; Eukaryotic elongation } \\
\text { factor } 1 \mathrm{~A}-1 \text {; }\end{array}$ \\
\hline PTRPTDKPLR & LPLQDVYKIGGIGTVPVGR & 0,7 & $\mathrm{~N}$-ter +28.03 Da, K+28.03 Da & 26,9 & $-0,343$ & 3 & 2037,2 & EF1A1_MOUSE & EF1A2_MOUSE & Elongation factor 1-alpha 1; & $\begin{array}{l}\text { Elongation factor Tu; Eukaryotic elongation } \\
\text { factor } 1 \mathrm{~A}-1 \text {; }\end{array}$ \\
\hline TTLEALDCI & LPPTRPTDKPLR & $-0,3$ & N-ter +34.06 Da, K+34.06 Da & 19,1 & $-3,420$ & 4 & 1457,9 & EF1A1_MOUSE & EF1A2_MOUSE & Elongation factor 1-alpha 1; & $\begin{array}{l}\text { Elongation factor Tu; Eukaryotic elongation } \\
\text { factor } 1 \mathrm{~A}-1 \text {; }\end{array}$ \\
\hline LKSGDAAIVD & MVPGKPMCVESFSDYPPLGR & $-0,3$ & $\begin{array}{l}\text { N-ter +34.06 Da, K+34.06 Da, C } \\
\text { +57.02 Da }\end{array}$ & 29,7 & $-2,952$ & 3 & 2334,2 & EF1A1_MOUSE & & Elongation factor 1-alpha 1; & $\begin{array}{l}\text { Elongation factor Tu; Eukaryotic elongation } \\
\text { factor } 1 \mathrm{~A}-1 \text {; }\end{array}$ \\
\hline MVPGKPMCVE & SFSDYPPLGR & 0,5 & $\mathrm{~N}$-ter +28.03 Da & 15,0 & $-2,313$ & 2 & 1165,6 & EF1A1_MOUSE & & Elongation factor 1-alpha 1; & $\begin{array}{l}\text { Elongation factor Tu; Eukaryotic elongation } \\
\text { factor } 1 \mathrm{~A}-1 \text {; }\end{array}$ \\
\hline WKFETSKYYV & TIIDAPGHR & 0,1 & N-ter $+34.06 \mathrm{Da}$ & 22,1 & $-0,295$ & 3 & 1012,6 & EF1A1_MOUSE & EF1A2_MOUSE & Elongation factor 1-alpha 1; & $\begin{array}{l}\text { Elongation factor Tu; Eukaryotic elongation } \\
\text { factor } 1 \mathrm{~A}-1 \text {; }\end{array}$ \\
\hline KSGDAAIVDM & VPGKPMCVESFSDYPPLGR & 0,0 & $\begin{array}{l}\text { N-ter }+28.03 \mathrm{Da}, \mathrm{K}+28.03 \mathrm{Da}, \mathrm{C} \\
+57.02 \mathrm{Da}\end{array}$ & 24,3 & 1,139 & 3 & 2191,1 & EF1A1_MOUSE & & Elongation factor 1-alpha 1; & $\begin{array}{l}\text { Elongation factor Tu; Eukaryotic elongation } \\
\text { factor } 1 \mathrm{~A}-1 \text {; }\end{array}$ \\
\hline KPLRLPLQDV & YKIGGIGTVPVGR & $-1,5$ & $\mathrm{~N}$-ter +34.06 Da, K +34.06 Da & 18,0 & $-0,649$ & 3 & 1383,9 & EF1A1_MOUSE & EF1A2_MOUSE & Elongation factor 1-alpha 1; & $\begin{array}{l}\text { Elongation factor Tu; Eukaryotic elongation } \\
\text { factor } 1 \mathrm{~A}-1 \text {; }\end{array}$ \\
\hline SLWKFETSKY & YVTIIDAPGHR & $-0,4$ & $\mathrm{~N}$-ter +34.06 Da & 19,4 & $-9,000$ & 3 & 1274,7 & EF1A1_MOUSE & D3YZ68_MOUSE & Elongation factor 1-alpha 1; & $\begin{array}{l}\text { Elongation factor Tu; Eukaryotic elongation } \\
\text { factor } 1 \mathrm{~A}-1 \text {; }\end{array}$ \\
\hline EGYVPSQADV & AVFEAVSGPPPADLCHALR & $-0,6$ & N-ter +34.06 Da, C +57.02 Da & 55,7 & $-1,713$ & 3 & 2040,1 & EF1B_MOUSE & G3UX43_MOUSE & E Elongation factor 1-beta; & \\
\hline PSQADVAVFE & AVSGPPPADLCHALR & $-1,1$ & $\mathrm{~N}-\operatorname{ter}+34.06 \mathrm{Da}, \mathrm{C}+57.02 \mathrm{Da}$ & 26,5 & $-2,004$ & 3 & 1593,9 & EF1B_MOUSE & G3UX43_MOUSE & $\begin{array}{l}\text { E Elongation factor 1-beta; } \\
\text { E }\end{array}$ & \\
\hline DDDIDLFGSD & DEEESEEAKKLR & $-0,4$ & N-ter +34.06 Da, K +34.06 Da & 29,7 & $-4,021$ & 3 & 1563,9 & EF1B_MOUSE & & Elongation factor 1-beta; & \\
\hline SILLDVKPWD & DETDMTKLEECVR & $-1,6$ & $\begin{array}{l}\text { N-ter + }+34.06 \text { Da, K }+34.06 \text { Da, C } \\
+55702 \text { Da }\end{array}$ & 27,2 & $-9,140$ & 3 & 1692,8 & EF1B_MOUSE & & Elongation factor 1-beta; & \\
\hline DDIDLFGSDD & EEESEEAKKLR & $-0,2$ & $\mathrm{~N}$-ter +34.06 Da, K+34.06 Da & 15,7 & 0,689 & 3 & 1448,9 & EF1B_MOUSE & & Elongation factor 1-beta; & \\
\hline QADVAVFEAV & SGPPPADLCHALR & $-0,5$ & N-ter $+34.06 \mathrm{Da}, \mathrm{C}+57.02 \mathrm{Da}$ & 56,2 & $-1,682$ & 3 & 1423,7 & EF1B_MOUSE & G3UX43_MOUSE & E Elongation factor 1-beta; & \\
\hline GYVPSQADVA & VFEAVSGPPPADLCHALR & $-0,5$ & N-ter +34.06 Da, C +57.02 Da & 29,0 & 2,383 & 3 & 1969,0 & EF1B_MOUSE & G3UX43_MOUSE & E Elongation factor 1-beta; & \\
\hline LADKSYIEGY & VPSQADVAVFEAVSGPPPADLCHALR & $-0,7$ & N-ter $+34.06 \mathrm{Da}, \mathrm{C}+57.02 \mathrm{Da}$ & 19,7 & $-0,474$ & 4 & 2736,4 & EF1B_MOUSE & G3UX43_MOUSE & E Elongation factor 1-beta; & \\
\hline SQADVAVFEA & VSGPPPADLCHALR & $-0,7$ & N-ter $+34.06 \mathrm{Da}, \mathrm{C}+57.02 \mathrm{Da}$ & 36,4 & 1,311 & 3 & 1522,8 & EF1B_MOUSE & G3UX43_MOUSE & E Elongation factor 1-beta; & \\
\hline SILLDVKPWD & DETDMAQLETCVR & $-0,3$ & N-ter +34.06 Da, C +57.02 Da & 17,8 & $-2,184$ & 2 & 1600,7 & EF1D_MOUSE & Q91VK2_MOUSE & E Elongation factor 1-delta; & \\
\hline IQLDGLVWGA & SKLVPVGYGIR & $-0,6$ & N-ter +34.06 Da, K +34.06 Da & 24,2 & $-0,238$ & 3 & 125,8 & EF1D_MOUSE & Q91VK2_MOUSE & E Elongation factor 1-delta; & \\
\hline RENIIKSLAG & SSGPGASSGPGGDHSELIVR & 0,7 & $\mathrm{~N}$-ter $+28.03 \mathrm{Da}$ & 25,5 & $-4,059$ & 3 & 1893,9 & EF1D_MOUSE & Q91VK2_MOUSE & E Elongation factor 1-delta; & \\
\hline QELKARARYL & AEKYEWDVAEAR & $-0,3$ & $\mathrm{~N}$-ter +34.06 Da, K +34.06 Da & 23,2 & 5,075 & 3 & 1533,8 & EF2_MOUSE & & Elongation factor 2; & \\
\hline DGSGFLINLI & DSPGHVDFSSEVTAALR & $-0,4$ & $\mathrm{~N}$-ter $+28.03 \mathrm{Da}$ & 40,7 & 1,650 & 3 & 1814,9 & EF2_MOUSE & & Elongation factor 2; & \\
\hline PRLMEPYYLV & EIQCPEQVVGGIYGVLNR & $-1,3$ & $\mathrm{~N}$-ter +34.06 Da, C +57.02 Da & 16,9 & 4,451 & 3 & 2064,1 & EF2_MOUSE & & Elongation factor 2; & \\
\hline GDALLQMITI & HLPSPVTAQKYR & $-0,7$ & $\mathrm{~N}$-ter +34.06 Da, K +34.06 Da & 15,8 & $-0,477$ & 3 & 1463,9 & EF2_MOUSE & & Elongation factor 2; & \\
\hline KDGSGFLNL & IDSPGHVDFSSEVTAALR & $-0,2$ & $\mathrm{~N}$-ter $+34.06 \mathrm{Da}$ & 29,0 & $-4,130$ & 3 & 1934,0 & EF2_MOUSE & & Elongation factor 2; & \\
\hline & NFTVDQIR & 0,0 & $\mathrm{~N}$-ter $+28.03 \mathrm{Da}$ & 18,0 & $-0,979$ & 2 & 1019,5 & EF2_MOUSE & & Elongation factor 2; & \\
\hline GSGFLINLID & SPGHVDFSSEVTAALR & 0,8 & $\mathrm{~N}$-ter $+28.03 \mathrm{Da}$ & 34,7 & 1,820 & 3 & 1699,9 & EF2_MOUSE & & Elongation factor 2; & \\
\hline SVIAHVDHGK & STLTDSLVCKAGIIASAR & 1,0 & $\begin{array}{l}\text { N-ter }+28.03 \mathrm{Da}, \mathrm{K}+28.03 \mathrm{Da}, \mathrm{C} \\
+57.02 \mathrm{Da}\end{array}$ & 16,5 & 1,093 & 3 & 1918,1 & EF2_MOUSE & & Elongation factor 2; & \\
\hline VIAHVDHGKS & TLTDSLVCKAGIIASAR & 0,5 & $\begin{array}{l}\text { N-ter }+28.03 \mathrm{Da}, \mathrm{K}+28.03 \mathrm{Da}, \mathrm{C} \\
+57.02 \mathrm{Da}\end{array}$ & 37,9 & 2,236 & 3 & 1831,0 & EF2_MOUSE & & Elongation factor 2; & \\
\hline QPRLMEPYYL & VEIQCPEQVVGGIYGVLNR & $-0,6$ & $\begin{array}{l}+57.02 \mathrm{Da} \\
\mathrm{N} \text {-ter }+34.06 \mathrm{Da}, \mathrm{C}+57.02 \mathrm{Da}\end{array}$ & 50,1 & 0,369 & 3 & 2163,2 & EF2_MOUSE & & Elongation factor 2; & \\
\hline & VNFTVDQIR & $-0,2$ & $\mathrm{~N}-\mathrm{ter}+34.06 \mathrm{Da}$ & 15,9 & $-0,710$ & 2 & 1124,6 & EF2_MOUSE & & Elongation factor 2; & \\
\hline M & VLSGVDKMIR & $-0,1$ & N-ter $+34.06 \mathrm{Da}, \mathrm{K}+34.06 \mathrm{Da}$ & 24,1 & $-0,842$ & 3 & 1184,8 & ETUD1_MOUSE & D3Z1V3_MOUSE & $\begin{array}{l}\text { Elongation factor Tu GTP-binding domain-containing } \\
\text { protein 1; }\end{array}$ & \\
\hline AAtRPDPAEG & DPTDPTFTSLPVR & 2,6 & $\mathrm{~N}$-ter +34.06 Da & 28,1 & $-0,675$ & 2 & 1478,8 & EMB_MOUSE & & Embigin; & $\begin{array}{l}\text { Teratocarcinoma glycoprotein Gp-70; } \\
94 \text { kDa glucose-regulated protein;Endoplasmic } \\
\text { reticulum resident protein 99;Heat shock }\end{array}$ \\
\hline VLLTFGFVRA & DDEVDVDGTVEEDLGKSR & $-0,3$ & N-ter +28.03 Da, K+28.03 Da & 81,6 & 0,639 & 3 & 2033,0 & ENPL_MOUSE & & Endoplasmin; & $\begin{array}{l}\text { reticulum resident protein 99; Heat shock } \\
\text { protein } 90 \text { kDa beta member 1;Polymorphic } \\
\text { tumor rejection antigen 1; Tumor rejection } \\
\text { antigen gp96; }\end{array}$ \\
\hline LTFGFVRADD & EVDVDGTVEEDLGKSR & $-0,8$ & $\mathrm{~N}$-ter $+34.06 \mathrm{Da}, \mathrm{K}+34.06 \mathrm{Da}$ & 29,5 & 0,770 & 3 & 1815,0 & ENPL_MOUSE & & Endoplasmin; & $\begin{array}{l}94 \text { kDa glucose-regulated protein;Endoplasmic } \\
\text { reticulum resident protein } 99 ; \text {;eat shock } \\
\text { protein } 90 \text { kDa beta member 1;Polymorphic } \\
\text { tumor rejection antigen 1; Tumor rejection } \\
\text { antigen gp96; }\end{array}$ \\
\hline VPAPSSTVTV & GVVPKTSAGTTDPEEATR & $-1,3$ & N-ter $+34.06 \mathrm{Da}, \mathrm{K}+34.06 \mathrm{Da}$ & 16,6 & $-5,037$ & 3 & 1883,0 & MAP7_MOUSE & D3YWN7_MOUSE & E Ensconsin; & $\begin{array}{l}\text { Epithelial microtubule-associated protein of } \\
115 \mathrm{kDa} \text {;Microtubule-associated protein } 7 \text {; }\end{array}$ \\
\hline
\end{tabular}




\begin{tabular}{|c|c|c|c|c|c|c|c|c|c|c|c|}
\hline Non Prime Site & Prime Site & $\begin{array}{c}\text { Fold change of } \\
\text { XPRESS ratio } \\
\text { (Adam17 } 7 \text { "kC / WT) }\end{array}$ & Modifications & Hyperscore & $\begin{array}{l}\text { Mass error } \\
\text { in ppm }\end{array}$ & Charge & $\begin{array}{c}\text { Precursor } \\
\text { neutral mass } \\
\text { in Da }\end{array}$ & Uniprot ID 1 & Uniprot ID 2 & Protein Name 1 & Protein Name 2 \\
\hline YELQADTYRC & SLEPALAVSAPKR & $-1,1$ & $\mathrm{~N}$-ter +34.06 Da, $\mathrm{K}+34.06 \mathrm{Da}$ & 15,2 & 3,478 & 3 & 1405,9 & EVPL_MOUSE & & Envoplakin; & $\begin{array}{l}210 \text { kDa cornified envelope precursor } \\
\text { protein;p210; }\end{array}$ \\
\hline QKPKVQLQER & VSEIFQVLPETEQEIR & 1,2 & $\mathrm{~N}$-ter +28.03 Da & 21,2 & 4,160 & 3 & 1944,0 & EVPL_MOUSE & & Envoplakin; & $\begin{array}{l}210 \mathrm{kDa} \text { cornified envelope precursor } \\
\text { protein;p210; }\end{array}$ \\
\hline FTRYMWPDPS & GTLDPTWALQQLQQLR & 10,3 & $\mathrm{~N}$-ter +28.03 Da & 21,3 & $-4,584$ & 3 & 1895,0 & MA2B2_MOUSE & & Epididymis-specific alpha-mannosidase; & Mannosidase alpha class $2 \mathrm{~B}$ member 2; \\
\hline NRRLSVEEAV & AAGVVGGEIQEKLLSAER & $-0,1$ & N-ter +34.06 Da, K +34.06 Da & 43,8 & 0,949 & 3 & 1894,1 & EPIPL_MOUSE & EPIPL_MOUSE & Epiplakin; & \\
\hline DASLKQQDTG & ARGSGTSPDEGDAQDSSESAR & 0,5 & $\mathrm{~N}$-ter +34.06 Da & 44,1 & 0,473 & 3 & 2112,9 & EPIPL_MOUSE & EPIPL_MOUSE & Epiplakin; & \\
\hline VPKIKKNQLG & ASTPSRPQAEAALPTTAR & 0,6 & $\mathrm{~N}$-ter +28.03 Da & 26,3 & 0,539 & 3 & 1852,0 & EPIPL_MOUSE & & Epiplakin; & \\
\hline VNNRRLSVEE & AVAAGVVGGEIIEKKLSAER & 0,1 & N-ter +34.06 Da, K +34.06 Da & 43,7 & $-1,790$ & 3 & 2064,2 & EPIPL_MOUSE & EPIPL_MOUSE & Epiplakin; & \\
\hline VYAKLLSAEH & AVTGYTDPYSGEQISLFQAMQR & 4,7 & $\mathrm{~N}$-ter $+28.03 \mathrm{Da}$ & 25,5 & 3,250 & 3 & 2489,2 & EPIPL_MOUSE & & Epiplakin; & \\
\hline ETQESKPKPR & DASLKQQDTGAR & 0,3 & N-ter +28.03 Da, K+28.03 Da & 27,4 & $-1,558$ & 3 & 1344,7 & EPIPL_MOUSE & EPIPL_MOUSE & Epiplakin; & \\
\hline LRPGTALVLL & EAQAATGFIIDPVNNR & 0,0 & $\mathrm{~N}$-ter+28.03 Da & 20,2 & 0,401 & 2 & 1742,9 & EPIPL_MOUSE & EPIPL_MOUSE & Epiplakin; & \\
\hline VGREEALRLL & EVQVATGGVIDPR & 0,6 & $\mathrm{~N}$-ter +28.03 Da & 20,6 & 2,993 & 2 & 1367,8 & EPIPL_MOUSE & & Epiplakin; & \\
\hline ELAQGTKTIH & EVTEMDSVKR & 0,7 & $\mathrm{~N}-\operatorname{ter}+28.03 \mathrm{Da}, \mathrm{K}+28.03 \mathrm{Da}$ & 30,2 & 0,479 & 3 & 1248,6 & EPIPL_MOUSE & EPIPL_MOUSE & Epiplakin; & \\
\hline VLRPGTALVL & LEAQAATGFIIDPVNNR & $-0,1$ & $\mathrm{~N}$-ter +34.06 Da & 35,7 & 3,646 & 3 & 1862,0 & EPIPL_MOUSE & EPIPL_MOUSE & Epiplakin; & \\
\hline KNHGIRLEA & QIATGGVIDPVHSHR & 0,1 & $\mathrm{~N}$-ter +34.06 Da & 19,8 & $-0,493$ & 4 & 1619,9 & EPIPL_MOUSE & EPIPL_MOUSE & Epiplakin; & \\
\hline HEVTEMDSVK & RYLGGSSCIAGVLVPVQGEPGR & 0,0 & $\mathrm{~N}-\operatorname{ter}+28.03 \mathrm{Da}, \mathrm{C}+57.02 \mathrm{Da}$ & 19,5 & $-1,260$ & 3 & 2299,2 & EPIPL_MOUSE & EPIPL_MOUSE & Epiplakin; & \\
\hline LKQQDTGARG & SGTSPDEGDAQDSSESAR & 1,4 & $\mathrm{~N}$-ter +28.03 Da & 21,2 & $-0,164$ & 2 & 1822,8 & EPIPL_MOUSE & EPIPL_MOUSE & Epiplakin; & \\
\hline LSAEHAVTGY & TDPYSGEQISLFQAMQR & 2,9 & $\mathrm{~N}$-ter +28.03 Da & 30,0 & $-1,949$ & 3 & 1998,0 & EPIPL_MOUSE & & Epiplakin; & \\
\hline TMRELAQGTK & TIHEVTEMDSVKR & 0,9 & $\mathrm{~N}$-ter +34.06 Da, K+34.06 Da & 20,6 & $-0,557$ & 4 & 1611,9 & EPIPL_MOUSE & EPIPL_MOUSE & Epiplakin; & \\
\hline LGVMELVSLL & TQIIEETEER & $-1,4$ & $\mathrm{~N}$-ter +28.03 Da & 22,0 & $-1,958$ & 2 & 1274,6 & EPIPL_MOUSE & EPIPL_MOUSE & Epiplakin; & \\
\hline QQDTGARGSG & TSPDEGDAQDSSESAR & 2,5 & $\mathrm{~N}$-ter +28.03 Da & 37,5 & $-0,952$ & 2 & 1678,7 & EPIPL_MOUSE & EPIPL_MOUSE & Epiplakin; & \\
\hline $\begin{array}{l}\text { QSISVWELLF } \\
\text { VHSDRNVYPS }\end{array}$ & $\begin{array}{l}\text { YREVPESLR } \\
\text { AGVLFVHVLER }\end{array}$ & $\begin{array}{l}0,2 \\
10,9\end{array}$ & $\begin{array}{l}\mathrm{N} \text {-ter }+28.03 \mathrm{Da} \\
\mathrm{N} \text {-ter }+28.03 \mathrm{Da}\end{array}$ & $\begin{array}{l}20,4 \\
23,8\end{array}$ & $\begin{array}{r}-1,018 \\
4,017\end{array}$ & $\begin{array}{l}3 \\
3\end{array}$ & $\begin{array}{l}1175,6 \\
1266,8\end{array}$ & $\begin{array}{l}\text { EPIPL_MOUSE } \\
\text { SGCE_MOUSE }\end{array}$ & D3Z572_MOUSE & $\begin{array}{l}\text { Epiplakin; } \\
\text { Epsilon-sarcoglycan; }\end{array}$ & \\
\hline VGGSLAESVG & SPPPAATPTPTPPTR & 0,0 & $\mathrm{~N}$-ter +34.06 Da & 22,8 & $-0,197$ & 2 & 1520,8 & EPN1_MOUSE & & Epsin-1; & $\begin{array}{l}\text { EPS-15-interacting protein 1; Intersectin-EH- } \\
\text { binding protein 1; }\end{array}$ \\
\hline MSGVGGSLAE & SVGSPPPAATPTPTPPTR & $-1,1$ & $\mathrm{~N}$-ter $+34.06 \mathrm{Da}$ & 17,5 & 1,133 & 2 & 1764,0 & EPN1_MOUSE & & Epsin-1; & $\begin{array}{l}\text { EPS-15-interacting protein 1; Intersectin-EH- } \\
\text { bisding protein 1. }\end{array}$ \\
\hline $\begin{array}{l}\text { LNVFGLRSIY } \\
\text { แแLSGDAHS }\end{array}$ & $\begin{array}{l}\text { SLILGQDNAADQSR } \\
\text { SEVPGAAAEGPGGSGVGLGDR }\end{array}$ & $\begin{array}{l}0,8 \\
0,0\end{array}$ & $\begin{array}{l}\text { N-ter }+28.03 \mathrm{Da} \\
\mathrm{N} \text {-ter }+28.03 \mathrm{Da}\end{array}$ & $\begin{array}{l}20,2 \\
56,2\end{array}$ & $\begin{array}{l}-2,242 \\
0,321\end{array}$ & $\begin{array}{l}2 \\
3\end{array}$ & $\begin{array}{l}1514,8 \\
1866,9\end{array}$ & $\begin{array}{l}\text { EMC3_MOUSE } \\
\text { EMC7_MOUSE }\end{array}$ & & $\begin{array}{l}\text { ER membrane protein complex subunit } 3 ; \\
\text { ER membrane protein complex subunit } 7 \text {; }\end{array}$ & Transmembrane protein 111; \\
\hline FSPTGSPLSS & AAYSSPKFHGSLPLAPCEAR & $-10,0$ & $\begin{array}{l}\text { N-ter +34.06 Da, K+34.06 Da, C } \\
+57.02 \mathrm{Da}\end{array}$ & 20,6 & 5,425 & 4 & 2226,2 & GATA1_MOUSE & & Erythroid transcription factor; & $\begin{array}{l}\text { Eryf1;GATA-binding factor 1;NF-E1 DNA-binding } \\
\text { protein; }\end{array}$ \\
\hline RYLSPKWIKM & FVLDEADEMLSR & $-0,5$ & N-ter +34.06 Da & 13,2 & $-0,685$ & 2 & 1457,7 & IF4A1_MOUSE & IF4A2_MOUSE & Eukaryotic initiation factor 4A-I; & ATP-dependent RNA helicase elF4A-1; \\
\hline KEALRAGLNC & STETMPIIINLIAPPR & $-1,1$ & $\mathrm{~N}-\operatorname{ter}+34.06 \mathrm{Da}, \mathrm{K}+34.06 \mathrm{Da}$ & 42,7 & 5,348 & 3 & 1848,1 & IF2A_MOUSE & & Eukaryotic translation initiation factor 2 subunit 1 ; & $\begin{array}{l}\text { Eukaryotic translation initiation factor } 2 \text { subunit } \\
\text { alpha; }\end{array}$ \\
\hline M & AGGEGGVTLGQPHLSR & $-0,5$ & N-ter +34.06 Da & 21,2 & 0,064 & 3 & 1568,9 & IF2G_MOUSE & A2AAW9_MOUSE & $\begin{array}{l}\text { Eukaryotic translation initiation factor } 2 \text { subunit } 3, X \text { - } \\
\text { linked; }\end{array}$ & $\begin{array}{l}\text { Eukaryotic translation initiation factor } 2 \text { subunit } \\
\text { gamma, } X \text {-linked; }\end{array}$ \\
\hline GPEPEVRAKP & AAQSEEETATSPAASPTPQSAER & $-0,2$ & $\mathrm{~N}$-ter $+28.03 \mathrm{Da}$ & 30,4 & $-6,181$ & 3 & 2343,1 & EIF3B_MOUSE & & $\begin{array}{l}\text { Eukaryotic translation initiation factor } 3 \text { subunit B } \\
\text { \{ECO:0000255|HAMAP-Rule:MF_03001\}; }\end{array}$ & 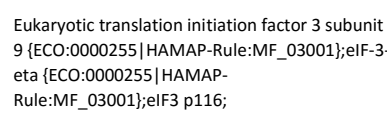 \\
\hline VGQTEDAEED & AEAGPEPEVR & $-1,1$ & $\mathrm{~N}$-ter +34.06 Da & 26,9 & $-0,092$ & 2 & 1087,6 & EIF3B_MOUSE & & $\begin{array}{l}\text { Eukaryotic translation initiation factor } 3 \text { subunit B } \\
\text { \{ECO:0000255|HAMAP-Rule:MF_03001\}; }\end{array}$ & 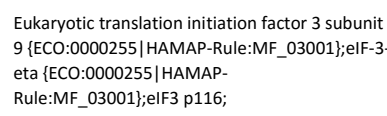 \\
\hline GGSEVGQTED & AEEDAEAGPEPEVR & $-0,3$ & $\mathrm{~N}$-ter +34.06 Da & 20,0 & 2,347 & 2 & 1531,7 & EIF3B_MOUSE & & $\begin{array}{l}\text { Eukaryotic translation initiation factor } 3 \text { subunit B } \\
\text { \{ECO:0000255|HAMAP-Rule:MF_03001\}; }\end{array}$ & 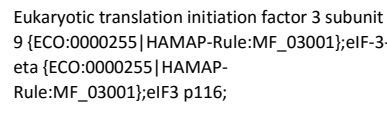 \\
\hline MQD & AENVAVPEAAEER & $-1,2$ & N-ter +34.06 Da & 25,0 & $-0,775$ & 2 & 1417,7 & EIF3B_MOUSE & & $\begin{array}{l}\text { Eukaryotic translation initiation factor } 3 \text { subunit B } \\
\text { \{ECO:0000255|HAMAP-Rule:MF_03001\}; }\end{array}$ & 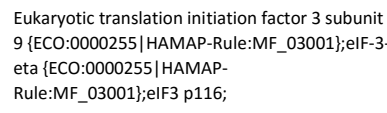 \\
\hline EVRAKPAAQS & EEETATSPAASPTPQSAER & 0,2 & $\mathrm{~N}$-ter +28.03 Da & 38,0 & 1,207 & 2 & 1985,9 & EIF3B_MOUSE & & $\begin{array}{l}\text { Eukaryotic translation initiation factor } 3 \text { subunit B } \\
\text { \{ECO:0000255|HAMAP-Rule:MF_03001\}; }\end{array}$ & 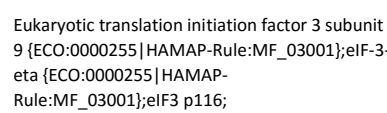 \\
\hline
\end{tabular}




\begin{tabular}{|c|c|c|c|c|c|c|c|c|c|c|c|}
\hline Non Prime Site & Prime Site & 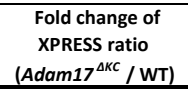 & Modifications & Hyperscore & $\begin{array}{l}\text { Mass error } \\
\text { in ppm }\end{array}$ & Charge & $\begin{array}{c}\text { Precursor } \\
\text { neutral mass } \\
\text { in Da } \\
\end{array}$ & Uniprot ID 1 & Uniprot ID 2 & Protein Name 1 & Protein Name 2 \\
\hline VRAKPAAQSE & EETATSPAASPTPQSAER & $-0,1$ & N-ter+28.03 Da & 38,9 & 6,721 & 3 & 1856,9 & EIF3B_MOUSE & & $\begin{array}{l}\text { Eukaryotic translation initiation factor } 3 \text { subunit B } \\
\{\text { BECO:0000255|HAMAP-Rule:MF_03001\}; }\end{array}$ & $\begin{array}{l}\text { Eukaryotic translation initiation factor } 3 \text { subunit } \\
9\{\text { \{ECO:0000255|HAMAP-Rule:MF_03001\};ilf-3. } \\
\text { eta \{ECO:0000255|HAMAP- } \\
\text { Rule:MF__3001\};:IF3 p116; }\end{array}$ \\
\hline RAKPAAQSEE & ETATSPAASPTPQSAER & 0,1 & $\mathrm{~N}$-ter +34.06 Da & 14,9 & $-3,800$ & 3 & 1733,9 & EIF3B_MOUSE & & $\begin{array}{l}\text { Eukaryotic translation initiation factor } 3 \text { subunit B } \\
\text { \{ECO:0000255|HAMAP-Rule:MF_03001\}; }\end{array}$ & 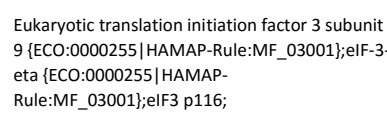 \\
\hline PEVRAKPAAQ & SEEETATSPAASPTPQSAER & 0,1 & $\mathrm{~N}$-ter +28.03 Da & 29,9 & $-2,313$ & 2 & 2073,0 & EIF3B_MOUSE & & $\begin{array}{l}\text { Eukaryotic translation initiation factor } 3 \text { subunit B } \\
\text { \{ECO:0000255|HAMAP-Rule:MF_03001\}; }\end{array}$ & $\begin{array}{l}\text { Eukaryotic translation initiation factor } 3 \text { subunit } \\
9\{\text { \{EC:0000255|HAMAP-Rule:MF_03001\};elf-3. } \\
\text { eta \{ECO:0000255|HAMAP-: } \\
\text { Rule:MF_03001\};; IF3 p116; }\end{array}$ \\
\hline M & KPILLQGHER & $-0,2$ & N-ter +34.06 Da, K +34.06 Da & 32,9 & $-1,795$ & 4 & 1388,9 & EIF3I_MOUSE & & $\begin{array}{l}\text { Eukaryotic translation initiation factor } 3 \text { subunit I } \\
\text { \{ECO:0000255|HAMAP-Rule:MF_03008\}; }\end{array}$ & 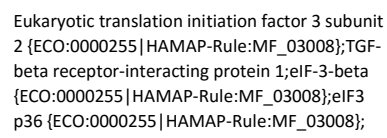 \\
\hline QGVQQFPASV & APAPVLMNOPPQIAPKR & $-0,2$ & $\mathrm{~N}$-ter +28.03 Da, K+28.03 Da & 32,4 & $-1,962$ & 3 & 1883,1 & IF4G1_MOUSE & D3YWY7_MOUSE & Eukaryotic translation initiation factor 4 gamma 1 ; & \\
\hline MIPSQISYSA & SQGAYYIPGQGR & $-0,7$ & N-ter +34.06 Da & 41,4 & $-0,225$ & 2 & 1329,7 & IF4G1_MOUSE & D3YWY7_MOUSE & Eukaryotic translation initiation factor 4 gamma 1 ; & \\
\hline PPGQTAPVVF & STPQATQMNTPSQPR & $-1,5$ & N-ter +34.06 Da & 21,2 & 5,123 & 2 & 1676,9 & IF4G1_MOUSE & D6RCH1_MOUSE & Eukaryotic translation initiation factor 4 gamma 1 ; & \\
\hline PKSPPYTAFL & GNLPYDVTEDSIKDFFR & $-0,2$ & $\mathrm{~N}$-ter $+34.06 \mathrm{Da}, \mathrm{K}+34.06 \mathrm{Da}$ & 62,9 & $-1,390$ & 3 & 2083,1 & IF4B_MOUSE & & Eukaryotic translation initiation factor $4 \mathrm{~B}$; & \\
\hline QRPRLNLKPR & SAPKEDDASASTSQSSR & 0,2 & N-ter $+34.06 \mathrm{Da}, \mathrm{K}+34.06 \mathrm{Da}$ & 36,4 & $-1,115$ & 3 & 1790,9 & IF4B_MOUSE & & Eukaryotic translation initiation factor $4 \mathrm{~B}$; & \\
\hline ASTSQSSRAA & SIFGGAKPVDTAAR & 1,7 & N-ter $+34.06 \mathrm{Da}, \mathrm{K}+34.06 \mathrm{Da}$ & 30,7 & 0,274 & 3 & 1456,9 & IF4B_MOUSE & & Eukaryotic translation initiation factor $4 \mathrm{~B}$; & \\
\hline EDLDSLLSAL & SLNEESLGNRR & 0,1 & $\mathrm{~N}$-ter $+28.03 \mathrm{Da}$ & 20,5 & 6,898 & 3 & 1301,7 & IF4B_MOUSE & & Eukaryotic translation initiation factor $4 \mathrm{~B}$; & \\
\hline EDLDSLLSAL & SLNEESLGNR & $-0,5$ & $\mathrm{~N}$-ter +34.06 Da & 28,1 & 4,768 & 2 & 1151,6 & IF4B_MOUSE & & Eukaryotic translation initiation factor $4 \mathrm{~B}$; & \\
\hline PSRAIPTRRV & ALGDGVQLPPGDYSTTPGGTLFSTTPGGTR & $-0,4$ & $\mathrm{~N}$-ter +28.03 Da & 19,3 & $-2,881$ & 3 & 2947,5 & 4EBP1_MOUSE & & $\begin{array}{l}\text { Eukaryotic translation initiation factor 4E-binding } \\
\text { protein 1; }\end{array}$ & $\begin{array}{l}\text { Phosphorylated heat- and acid-stable protein } \\
\text { regulated by insulin } 1 \text {; }\end{array}$ \\
\hline GNLPFNTVQG & DIDAIFKDLSIR & $-0,1$ & $\mathrm{~N}$-ter +34.06 Da, K+34.06 Da & 14,5 & $-0,271$ & 3 & 1472,9 & IF4H_MOUSE & & Eukaryotic translation initiation factor $4 \mathrm{H}$; & $\begin{array}{l}\text { Williams-Beuren syndrome chromosomal } \\
\text { region } 1 \text { protein homolog; }\end{array}$ \\
\hline YVEFDEVDSL & KEALTYDGALLGDR & $-0,6$ & $\mathrm{~N}$-ter +34.06 Da, $\mathrm{K}+34.06 \mathrm{Da}$ & 30,2 & 4,460 & 3 & 1588,9 & IF4H_MOUSE & & Eukaryotic translation initiation factor $4 \mathrm{H}$; & $\begin{array}{l}\text { Williams-Beuren syndrome chromosomal } \\
\text { region } 1 \text { protein homolog; }\end{array}$ \\
\hline YVGNLPFNTV & QGDIDAIFRDLSIR & $-0,3$ & N-ter +28.03 Da, K+28.03 Da & 14,0 & $-3,699$ & 3 & 1645,9 & IF4H_MOUSE & & Eukaryotic translation initiation factor $4 \mathrm{H}$; & $\begin{array}{l}\text { Williams-Beuren syndrome chromosomal } \\
\text { region } 1 \text { protein homolog; }\end{array}$ \\
\hline DEVDSLKEAL & TYDGALLGDR & $-0,5$ & $\mathrm{~N}$-ter +34.06 Da & 17,4 & $-0,628$ & 2 & 1113,6 & IF4H_MOUSE & & Eukaryotic translation initiation factor $4 \mathrm{H}$; & $\begin{array}{l}\text { Williams-Beuren syndrome chromosomal } \\
\text { region } 1 \text { protein homolog; }\end{array}$ \\
\hline NDFQLIGIQD & GYLSLLDSGEVR & $-0,1$ & N-ter $+28.03 \mathrm{Da}$ & 21,8 & $-4,093$ & 2 & 1463,8 & IF5A1_MOUSE & J3QPS8_MOUSE & Eukaryotic translation initiation factor 5A-1; & Eukaryotic initiation factor $5 \mathrm{~A}$ isoform 1; $\mathrm{elF}-4 \mathrm{D}$; \\
\hline KYEDICPSTH & NMDVPNIKR & $-0,4$ & $\mathrm{~N}$-ter +28.03 Da, $\mathrm{K}+28.03 \mathrm{Da}$ & 18,5 & $-1,136$ & 3 & 1141,6 & IF5A1_MOUSE & IF5A2_MOUSE & Eukaryotic translation initiation factor 5A-1; & Eukaryotic initiation factor $5 \mathrm{~A}$ isoform 1; $\mathrm{elF}-4 \mathrm{D}$; \\
\hline DVPNIKRNDF & QLIGIQDGYLSLQDSGEVR & $-0,6$ & N-ter +28.03 Da & 38,6 & 8,280 & 3 & 2231,2 & IF5A1_MOUSE & J3QPS8_MOUSE & Eukaryotic translation initiation factor 5A-1; & Eukaryotic initiation factor $5 \mathrm{~A}$ isoform 1; ;lF-4D; \\
\hline VGVHIADVSH & FIRPGNALDQESAR & $-6,4$ & $\mathrm{~N}$-ter +34.06 Da & 21,9 & 2,548 & 2 & 1606,9 & RRP44_MOUSE & & Exosome complex exonuclease RRP44; & $\begin{array}{l}\text { Protein DIS3 homolog;Ribosomal RNA- } \\
\text { processing protein 44: }\end{array}$ \\
\hline KDLLPLMER & FKVLLEKLMMAQDEER & 12,5 & $\mathrm{~N}$-ter +28.03 Da, K +28.03 Da & 20,2 & $-3,727$ & 3 & 2063,1 & XPOT_MOUSE & & Exportin-T; & Exportin(tRNA); tRNA exportin; \\
\hline $\begin{array}{l}\text { RLLOHHHEG } \\
\text { TNYLNNVALV }\end{array}$ & $\begin{array}{l}\text { AAPSPPPQTR } \\
\text { AGDTGNATGLGQGPTR }\end{array}$ & $\begin{array}{l}0,1 \\
-0,5\end{array}$ & $\begin{array}{l}\text { N-ter +34.06 Da } \\
\text { N-ter 28.03 Da }\end{array}$ & $\begin{array}{l}24,4 \\
35,4\end{array}$ & $\begin{array}{l}2,745 \\
5,587\end{array}$ & $\begin{array}{l}2 \\
2\end{array}$ & $\begin{array}{l}1054,6 \\
1128,8\end{array}$ & $\begin{array}{l}\text { ECM1_MOUSE } \\
\text { ECM1 MOUSE }\end{array}$ & $\begin{array}{l}\text { D3VW64_MOUSE } \\
\text { F8W14 MOUSE }\end{array}$ & $\begin{array}{l}\text { Extracellular matrix protein } 1 \text {; } \\
\text { Extracellular matrix rotein } 1\end{array}$ & $\begin{array}{l}\text { Secretory component } t 885 ; \\
\text { Seccetory component } \mathrm{p} 85 \text {; }\end{array}$ \\
\hline CFNTNYLRNV & ALVAGDTGNATGLGEQGPTR & $-0,8$ & N-ter +34.06 Da & 51,5 & 0,989 & 3 & 1918,0 & ECM1_MOUSE & F8WI14_MOUSE & Extracellular matrix protein 1 ; & Secretory component p85; \\
\hline NYLRNVALVA & GDTGNATGLGEQGPTR & $-0,3$ & N-ter $+34.06 \mathrm{Da}$ & 34,4 & 9,388 & 2 & 1563,8 & ECM1_MOUSE & F8WI14_MOUSE & Extracellular matrix protein 1 ; & Secretory component p85; \\
\hline PPGRPSPDNL & KQICLPER & $-0,7$ & 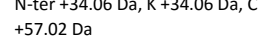 & 19,4 & $-2,514$ & 3 & 1110,7 & ECM1_MOUSE & D3YV64_MOUSE & Extracellular matrix protein 1 ; & Secretory component p85; \\
\hline SHLSRQGETL & NVLETGYSR & $-0,3$ & N-ter +34.06 Da & 24,9 & 0,559 & 2 & 1071,6 & ECM1_MOUSE & D3YV64_MOUSE & Extracellular matrix protein 1; & Secretory component p85; \\
\hline $\begin{array}{l}\text { NLPQTGYSHL } \\
\text { HTCWKAWEG }\end{array}$ & $\begin{array}{l}\text { SRQGGETLVVLETGYSR } \\
\text { TLGYGCR }\end{array}$ & $\begin{array}{r}0,5 \\
-0,5\end{array}$ & $\begin{array}{l}\mathrm{N}-\mathrm{ter}+34.06 \mathrm{Da} \\
\mathrm{N} \text {-er }+28.03 \mathrm{Ca}+5\end{array}$ & $\begin{array}{l}36,6 \\
16,8\end{array}$ & $\begin{array}{l}-2,329 \\
-1439\end{array}$ & 3 & $\begin{array}{l}1843,0 \\
1040,5\end{array}$ & $\begin{array}{l}\text { ECM1_MOUSE } \\
\text { ECM1MOUSE }\end{array}$ & $\begin{array}{l}\text { D3VV64_MOUSE } \\
\text { F8W14 MOUSE }\end{array}$ & $\begin{array}{l}\text { Extracellular matrix } \mathrm{p} \\
\text { Extracelluar matrixp }\end{array}$ & $\begin{array}{l}\text { Secretory component } 885 \text {; } \\
\text { Secretory component } 85 \text {. }\end{array}$ \\
\hline $\begin{array}{l}\text { HTCTWKAWEG } \\
\text { NTNYRNVL }\end{array}$ & $\begin{array}{l}\text { TLDGCYER } \\
\text { VAGDTGNATGLGEQGPTI }\end{array}$ & $\begin{array}{l}-0,5 \\
0,0\end{array}$ & $\begin{array}{l}\text { N-ter } 28.00 \mathrm{Da}, \mathrm{C}+57.02 \mathrm{Da} \\
\text { N-ter } 34.06 \mathrm{Da}\end{array}$ & $\begin{array}{l}16,8 \\
39,0\end{array}$ & $\begin{array}{l}-1,439 \\
-5,934\end{array}$ & 2 & $\begin{array}{r}1040,5 \\
1733,9\end{array}$ & $\begin{array}{l}\text { ECM1_MOUSE } \\
\text { ECM1 MOUSE }\end{array}$ & F8WI14_MOUSE & $\begin{array}{l}\text { Extracellular matrix protein } 1 ; \\
\text { Extracellular matrix roteein } 1 \text {. }\end{array}$ & $\begin{array}{l}\text { Secretory component } 885 \text {; } \\
\text { Secretory component } 85 \text {. }\end{array}$ \\
\hline NCFNTNYLRN & $\begin{array}{l}\text { VALVAGDTGNATGLGEQGPTR } \\
\text { VilG }\end{array}$ & $-0,4$ & $\begin{array}{l}\text { Nerer } 344.06 \text { Da } \\
\text { N-ter }+34.06 \text { Da }\end{array}$ & 20,8 & $-3,713$ & 3 & 2017,1 & $\begin{array}{l}\text { ECM1_MOUSE } \\
\text { ECM1_MOUSE }\end{array}$ & $\begin{array}{l}\text { F8WW14_MOUSE } \\
\text { F8W114 MOUSE }\end{array}$ & $\begin{array}{l}\text { Extracellular matrix rotein } 1 \text {; } \\
\text { Extracellular matrix protein } 1 \text {; }\end{array}$ & $\begin{array}{l}\text { Secretory component t } 85 \text {; } \\
\text { Secretory component } 885 \text {; }\end{array}$ \\
\hline
\end{tabular}




\begin{tabular}{|c|c|c|c|c|c|c|c|c|c|c|c|}
\hline Non Prime Site & Prime Site & $\begin{array}{l}\text { Fold change of } \\
\text { XPRESS ratio } \\
\left.\text { (Adam17 } 7^{\text {LCC }} / \mathrm{WT}\right)\end{array}$ & Modifications & Hyperscore & $\begin{array}{l}\text { Mass error } \\
\text { in ppm }\end{array}$ & Charge & $\begin{array}{c}\text { Precursor } \\
\text { neutral mass } \\
\text { in Da }\end{array}$ & Uniprot ID 1 & Uniprot ID 2 & Protein Name 1 & Protein Name 2 \\
\hline ARDENKRTHN & $\begin{array}{l}\text { DIIHNENMR } \\
\text { TCTECGOSE }\end{array}$ & 0,0 & N-ter +34.06 Da & 20,0 & 0,085 & 3 & 1174,6 & EZRI_MOUSE & & Ezrin; & Cytovillin;Villin-2;p81; \\
\hline GGLPERSCML & TGTPESVQSAKR & $-0,6$ & N-ter +34.06 Da, K +34.06 Da & 41,9 & $-0,977$ & 3 & 1327,8 & FUBP1_MOUSE & Q3TUE1_MOUSE & Far upstream element-binding protein 1; & \\
\hline GGPPPGPPPP & AGGGGGAAGAGGGPPPGPPGAGDR & 0,0 & N-ter +28.03 Da & 67,2 & 5,691 & 3 & 1841,9 & FUBP2_MOUSE & & 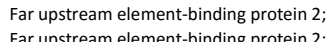 & 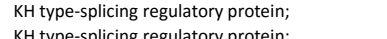 \\
\hline GPPPPAGGGG & GAAGAGGGPPPGPPGAGDR & 0,6 & N-ter +28.03 Da & 47,8 & $-0,842$ & 2 & 1542,8 & FUBP2_MOUSE & & 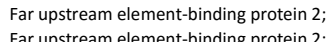 & 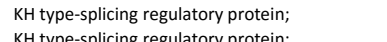 \\
\hline ERAGVKMILI & QDGSQNTNVDKPLR & $-0,3$ & N-ter +34.06 Da, K+34.06 Da & 24,0 & 7,004 & 3 & 1638,9 & FUBP2_MOUSE & & Far upstream element-binding protein 2; & $\begin{array}{l}\text { KH type-splicing regulatory protein; } \\
\text { FAS-associating death domain-containing }\end{array}$ \\
\hline EADLQVAFDI & VCDNVGR & 1,8 & N-ter +28.03 Da, C +57.02 Da & 11,7 & $-2,829$ & 2 & 846,4 & FADD_MOUSE & & FAS-associated death domain protein; & $\begin{array}{l}\text { protein;Mediator of receptor induced } \\
\text { toxicity;Protein FADD; }\end{array}$ \\
\hline M & $\begin{array}{l}\text { APAPPPAASFTPAEVQR } \\
\text { P }\end{array}$ & $-0,5$ & N-ter +34.06 Da & 34,7 & $-0,632$ & 2 & 1739,9 & FA2H_MOUSE & & Fatty acid 2-hydroxylase; & Fatty acid alpha-hydroxylase; \\
\hline SVSCGRGNAG & QTNYGFANSTMER & $-3,8$ & N-ter +34.06 Da & 14,9 & $-2,381$ & 2 & 1551,7 & FAS_MOUSE & & Fatty acid synthase; & \\
\hline KTETVCTFQD & GALVQHQQWDGKESTITR & $-0,1$ & $\mathrm{~N}$-ter +34.06 Da, K +34.06 Da & 19,4 & 4,800 & 4 & 2121,2 & FABP5_MOUSE & & Fatty acid-binding protein, epidermal; & $\begin{array}{l}\text { Epidermal-type fatty acid-binding protein;Fatty } \\
\text { acid-binding protein 5;Kkeratinocyte lipid- } \\
\text { binding proteinipPsoriasis-associated fatty acid- } \\
\text { binding protein homolog; }\end{array}$ \\
\hline STVKTTVFSC & NLGEKFDETTADGR & $-0,8$ & N-ter +34.06 Da, K+34.06 Da & 27,8 & 3,081 & 3 & 1619,9 & FABPS_MOUSE & & Fatty acid-binding protein, epidermal; & $\begin{array}{l}\text { Epidermal-type fatty acid-binding protein;Fatty } \\
\text { acid-binding protein 5;Keratinocyte lipid- } \\
\text { binding protein;Psoriasis-associated fatty acid- } \\
\text { binding protein homolog; }\end{array}$ \\
\hline TESTVKTTVF & SCNLGEKFDETTADGR & $-0,5$ & $\begin{array}{l}\text { N-ter +34.06 Da, K+34.06 Da, C } \\
+57.02 \mathrm{Da}\end{array}$ & 34,3 & 1,284 & 3 & 1866,9 & FABP5_MOUSE & & Fatty acid-binding protein, epidermal; & $\begin{array}{l}\text { Epidermal-type fatty acid-binding protein;Fatty } \\
\text { acid-binding protein 5;keratinocyte lipid- } \\
\text { bindidig protein;Psoriasis-associated fatty acid- } \\
\text { binding protein homolog; }\end{array}$ \\
\hline ITVKTESTVK & TTVFSCNLGEKFDETTADGR & 0,2 & $\begin{array}{l}\text { N-ter +34.06 Da, K+34.06 Da, C } \\
\text { +57.02 Da }\end{array}$ & 25,4 & $-0,690$ & 3 & 2315,1 & FABP5_MOUSE & & Fatty acid-binding protein, epidermal; & $\begin{array}{l}\text { Epidermal-type fatty acid-binding protein;Fatty } \\
\text { acid-binding protein 5;keratinocyte lipid- } \\
\text { binding proteinipPsoriasisisassociated fatty acid- } \\
\text { binding protein homolog; }\end{array}$ \\
\hline GNNITVKTES & TVKTTVFSCNLGEKFDETTADGR & 1,9 & $\begin{array}{l}\text { N-ter +28.03 Da, K+28.03 Da, C } \\
+57.02 \mathrm{Da}\end{array}$ & 22,0 & $-9,687$ & 4 & 2659,3 & FABP5_MOUSE & & Fatty acid-binding protein, epidermal; & $\begin{array}{l}\text { Epidermal-type fatty acid-binding protein;Fatty } \\
\text { acid-binding protein 5;Keratinocyte lipid- } \\
\text { binding protein;Psoriasis-associated fatty acid- } \\
\text { binding protein homolog; }\end{array}$ \\
\hline FSNCNLSHID & EVIYPCPVEPR & $-0,8$ & N-ter +34.06 Da, C +57.02 Da & 16,9 & $-5,812$ & 2 & 1391,7 & FACR2_MOUSE & & Fatty acyl-CoA reductase 2; & Male sterility domain-containing protein 1 \\
\hline LPPRISWQLL & FLERPLYR & 0,0 & $\mathrm{~N}$-ter +28.03 Da & 20,2 & $-0,890$ & 3 & 1120,6 & FBX50_MOUSE & & F-box only protein 50 ; & $\begin{array}{l}\text { NCC receptor protein 1;Non-specific cytotoxic } \\
\text { cell receptor protein } 1 \text { homolog; }\end{array}$ \\
\hline PPRISWQLLF & LERPLYR & 0,3 & N-ter +34.06 Da & 17,8 & $-1,323$ & 3 & 979,6 & FBX50_MOUSE & & F-box only protein 50; & $\begin{array}{l}\text { NCC receptor protein 1;Non-specific cytotoxic } \\
\text { cell receptor protein } 1 \text { homolog; }\end{array}$ \\
\hline ILSVASTVWT & TDTEDKGEFLSEGGGVR & 0,9 & $\mathrm{~N}$-ter $+28.03 \mathrm{Da}, \mathrm{K}+28.03 \mathrm{Da}$ & 37,0 & 0,431 & 3 & 1851,9 & Q99K47_MOUSE & E9PV24_MOUSE & Fibrinogen alpha chain; & \\
\hline AGSSSGSGVQ & GASAGGLAADASR & 0,2 & $\mathrm{~N}$-ter +34.06 Da & 57,9 & 0,176 & 2 & 1136,6 & FILA_MMOUSE & J3QMA3_MOUSE & Filaggrin; & \\
\hline SSNRRDRPRQ & LSPSQSSDSQVHSGVQVEGR & 4,6 & $\mathrm{~N}$-ter +28.03 Da & 18,2 & $-3,075$ & 3 & 2111,0 & FILA_MOUSE & & Filaggrin; & \\
\hline RSSNRRDRPR & QLSPSQSSDSQVHSGVQVEGR & 1,2 & $\mathrm{~N}$-ter $+28.03 \mathrm{Da}$ & 27,0 & 3,388 & 4 & 2239,1 & FILA_MOUSE & & Filaggrin; & \\
\hline SSSGSGVQGA & SAGGLAADASR & 1,3 & $\mathrm{~N}$-ter $+34.06 \mathrm{Da}$ & 23,6 & $-2,573$ & 2 & 1008,5 & FILA_MOUSE & J3QM50_MOUSE & Filaggrin; & \\
\hline RQPSASQSSD & SQVHSGVQVEAQR & 0,5 & $\mathrm{~N}$-ter $+28.03 \mathrm{Da}$ & 32,3 & $-7,218$ & 3 & 1451,7 & FILA_MOUSE & FGTLO2_MOUSE & Filaggrin; & \\
\hline DRPRQPSASQ & SSDSQVHSGVQVEAQR & 1,1 & $\mathrm{~N}$-ter +34.06 Da & 47,5 & $-2,629$ & 3 & 1746,9 & FILA_MOUSE & FGTL02_MOUSE & Filaggrin; & \\
\hline QPVRPQGPSQ & DSSSQPQASQGQPSOSGSGR & $-0,1$ & $\mathrm{~N}$-ter +34.06 Da & 45,5 & 0,944 & 3 & 2008,9 & FILA2_MOUSE & & Filaggrin-2; & Intermediate filament-associated protein; \\
\hline KKHRRGHQHQ & EEESETEEEEETPR & 0,3 & $\mathrm{~N}$-ter +34.06 Da & 50,1 & 3,812 & 2 & 1755,8 & FILA2_MOUSE & E9QPZ3_MOUSE & Filaggrin-2; & Intermediate filament-associated protein; \\
\hline KHRRGHQHQE & EESETEEEEETPR & 0,3 & $\mathrm{~N}$-ter +28.03 Da & 34,1 & 0,740 & 2 & 1620,7 & FILA2_MOUSE & E9QPZ3_MOUSE & Filaggrin-2; & Intermediate filament-associated protein; \\
\hline RRSPVHPESS & EGEEHSVIPQR & 0,6 & $\mathrm{~N}$-ter +28.03 Da & 40,9 & 0,229 & 2 & 1307,7 & FILA2_MOUSE & & Filaggrin-2; & Intermediate filament-associated protein; \\
\hline RSPRRSPVHP & ESSEGEEHSVIPQR & $-0,6$ & $\mathrm{~N}$-ter +34.06 Da & 17,4 & $-2,161$ & 3 & 1616,8 & FILA2_MOUSE & & Filaggrin-2; & Intermediate filament-associated protein; \\
\hline RGHQHQEEES & ETEEEEETPR & 0,1 & $\mathrm{~N}$-ter $+28.03 \mathrm{Da}$ & 29,0 & $-2,270$ & 2 & 1275,5 & FILA2_MOUSE & E9QPZ3_MOUSE & Filaggrin-2; & Intermediate filament-associated protein; \\
\hline RSPVHPESSE & GEEHSVIPQR & 1,3 & $\mathrm{~N}$-ter $+28.03 \mathrm{Da}$ & 30,4 & 0,508 & 2 & 1178,6 & FILA2_MOUSE & & Filaggrin-2; & Intermediate filament-associated protein; \\
\hline SSOSSRHGSY & GPQDYDYGQSGYGPSGR & $-0,4$ & $\mathrm{~N}$-ter $+28.03 \mathrm{Da}$ & 25,1 & $-2,563$ & 3 & 1830,8 & FILA2_MOUSE & E9QPZ3_MOUSE & Filaggrin-2; & Intermediate filament-associated protein; \\
\hline SVHGQPVRPQ & GPSQDSSSQPQASQGQPSQSGSGR & $-0,5$ & $\mathrm{~N}$-ter $+34.06 \mathrm{Da}$ & 25,5 & $-5,250$ & 3 & 2378,1 & FILA2_MOUSE & & Filaggrin-2; & Intermediate filament-associated protein; \\
\hline GQPVRPQGPS & QDSSSQPQASQGQPSQSGSGR & $-0,1$ & $\mathrm{~N}$-ter $+28.03 \mathrm{Da}$ & 24,3 & $-6,795$ & & 2130,9 & FILA2_MOUSE & & Filaggrin-2; & Intermediate filament-associated protein; \\
\hline QSGSRRSPRS & QPVHPESSEGEEHSV & 5,6 & $\mathrm{~N}$-ter $+28.03 \mathrm{Da}$ & 29, & $-0,741$ & 3 & 2155,0 & FILA2_MOUSE & & Filaggrin-2; & Intermediate filament-associated protein; \\
\hline GHQQRESVHG & OPVRPQGPSQDSSSQPQASQGQPSQSGSGR & 0,1 & N-ter $+34.06 \mathrm{Da}$ & 20,6 & $-5,022$ & & 3083,5 & FILA2_MOUSE & & Filaggrin-2; & Intermediate filament-associated protein; \\
\hline LSGHYGRNHF & QSTISGNQYDSSOSSR & $-0,8$ & $\mathrm{~N}$-ter +28.03 Da & 17,3 & $-2,932$ & 2 & 1771,8 & FILA2_MOUSE & E9QPZ3_MOUSE & Filaggrin-2; & Intermediate filament-associated protein; \\
\hline QRESVHGQPV & RPQGPSQDSSSQPQASQGQPS & $-0,6$ & $\mathrm{~N}$-ter+28.03 Da & 29 & $-4,8$ & 3 & 2753,3 & FILA2_MOUSE & & Filaggrin-2; & Intermediate filament-associated protein; \\
\hline PRRSPVHPES & SEGEEHSVIPQR & 0,5 & $\mathrm{~N}$-ter +28.03 Da & 23,0 & $-1,503$ & 3 & 1394,7 & FILA2_MOUSE & & Filaggrin-2; & Intermediate filament-associated protein; \\
\hline
\end{tabular}




\begin{tabular}{|c|c|c|c|c|c|c|c|c|c|c|c|}
\hline Non Prime Site & Prime Site & 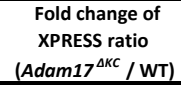 & Modifications & Hyperscore & $\begin{array}{c}\text { Mass error } \\
\text { in ppm }\end{array}$ & Charge & $\begin{array}{c}\text { Precursor } \\
\text { neutral mass } \\
\text { in Da }\end{array}$ & Uniprot ID 1 & Uniprot ID 2 & Protein Name 1 & Protein Name 2 \\
\hline QSCRTQSNCQ & SGTSGGQGYGCVSEGQSSR & 0,7 & N-ter +28.03 Da, C +57.02 Da & 13,4 & $-0,635$ & 2 & 1887,8 & FILA2_MOUSE & E9QPZ3_MOUSE & Filaggrin-2; & Intermediate filament-associated protein; \\
\hline GQGNQSCRTQ & SNCQSGTSGGQGYGCVSEGQSSR & 2,4 & N-ter +28.03 Da, C +57.02 Da & 16,5 & $-0,588$ & 3 & 2377,0 & FILA2_MOUSE & E9QPZ3_MOUSE & $\begin{array}{l}\text { Filaggrin-2; } \\
\text { Filazorin? }\end{array}$ & $\begin{array}{l}\text { Intermediate filament-associated protein; } \\
\text { intermediate filamentasscaited rontein. }\end{array}$ \\
\hline HGQPVRPQGP & SQDSSSQPQASQGQPSQSGSGR & $-0,1$ & $\mathrm{~N}$-ter +34.06 Da & 39,3 & 5,164 & 3 & 2224,0 & FILA2_MOUSE & & Filaggrin-2; & Intermediate filament-associated protein; \\
\hline RPQGPSQDSS & SQPQASQGQPSQSGSGR & 0,5 & $\mathrm{~N}$-ter+28.03 Da & 39,7 & 0,408 & 3 & 1713,8 & FILA2_MOUSE & & Filaggrin-2; & Intermediate filament-associated protein; \\
\hline SPRRSPVHPE & SSEGEEHSVIPQR & 0,5 & $\mathrm{~N}$-ter+34.06 Da & 25,9 & 0,671 & 3 & 1487,7 & FILA2_MOUSE & & Filaggrin-2; & Intermediate filament-associated protein; \\
\hline VRPQGPSQDS & SSQPQASQGQPSQSGSGR & 0,3 & $\mathrm{~N}$-ter +28.03 Da & 30,9 & $-1,220$ & 3 & 1800,8 & FILA2_MOUSE & & Filaggrin-2; & Intermediate filament-associated protein; \\
\hline PVRPQGPSQD & SSSQPQASQGQPSQSGSGR & 0,4 & $\mathrm{~N}$-ter +34.06 Da & 42,2 & 0,000 & 3 & 1893,9 & FILA2_MOUSE & & Filaggrin-2; & Intermediate filament-associated protein; \\
\hline QGQAGHQQRE & SVHGQPVRPQGPSQDSSSQPQASQGQPSQSGSGR & 0,5 & $\mathrm{~N}$-ter +28.03 Da & 26,3 & $-3,004$ & 4 & 3457,6 & FILA2_MOUSE & & Filaggrin-2; & $\begin{array}{l}\text { Intermediate filament-associated protein; } \\
\text { Actin-binding protein 280;Alpha- }\end{array}$ \\
\hline PNSPFQVTAL & AGDQPTVQTPLR & $-1,3$ & $\mathrm{~N}$-ter+34.06 Da & 30,8 & $-4,402$ & 2 & 1315,7 & FLNA_MOUSE & B7FAU9_MOUSE & Filamin-A; & $\begin{array}{l}\text { filamin;Endothelial actin-binding } \\
\text { protein;Filamin-1;Non-muscle filamin; }\end{array}$ \\
\hline RDAGYGGLSL & SIEGPSKVDINTEDLEDGTCR & 1,8 & $\begin{array}{l}\text { N-ter +34.06 Da, K +34.06 Da, C } \\
+57.02 \mathrm{Da}\end{array}$ & 19,3 & 2,910 & 3 & 2402,2 & FLNA_MOUSE & B7FAU9_MOUSE & Filamin-A; & $\begin{array}{l}\text { Actin-binding protein } 280 ; \text { Alpha- } \\
\text { filamin;Endothelial actin-binding } \\
\text { protein;Filamin-1;Non-muscle filamin; } \\
\text { Actin-binding protein } 280 \text {;Alpha- }\end{array}$ \\
\hline TAGAGMGEVE & VVIQDPTGQKGTVEPQLEAR & $-0,4$ & $\mathrm{~N}$-ter +28.03 Da, K+28.03 Da & 16,9 & 2,879 & 3 & 2220,2 & FLNA_MOUSE & B7FAU9_MOUSE & Filamin-A; & $\begin{array}{l}\text { Actin-binding protein 280;;|lapha- } \\
\text { filamin;Endothelial actin-binding } \\
\text { protein;Filamin-1;Non-muscle filamin; }\end{array}$ \\
\hline APGRYTIKVL & FASQEIPASPFR & 0,1 & N-ter +34.06 Da & 12,0 & 3,105 & 2 & 1382,8 & FLNB_MOUSE & & Filamin-B; & $\begin{array}{l}\text { ABP-280-like protein;Actin-binding-like } \\
\text { protein;Beta-filamin; }\end{array}$ \\
\hline KSNVFTVVTR & GAGIGGLGITVEGPSESKINCR & 7,3 & $\begin{array}{l}\text { N-ter +34.06 Da, K+34.06 Da, C } \\
+57.02 \mathrm{Da}\end{array}$ & 52,8 & 5,129 & 3 & 2239,2 & FLNB_MOUSE & & Filamin-B; & $\begin{array}{l}\text { ABP-280-like protein;Actin-binding-like } \\
\text { protein;Beta-filamin; }\end{array}$ \\
\hline AIKHTIAVVW & GGVNIPHSPYR & $-0,4$ & $\mathrm{~N}$-ter $+34.06 \mathrm{Da}$ & 17,6 & $-2,028$ & 3 & 1229,7 & FLNB_MOUSE & & Filamin-B; & $\begin{array}{l}\text { ABP-280-like protein;Actin-binding-like } \\
\text { protein;Beta-filamin; }\end{array}$ \\
\hline HTIAVVWGGV & NIPHSPYR & $-0,3$ & $\mathrm{~N}$-ter $+34.06 \mathrm{Da}$ & 17,5 & $-0,098$ & 3 & 1016,6 & FLNB_MOUSE & & Filamin-B; & $\begin{array}{l}\text { ABP-280-like protein;Actin-binding-like } \\
\text { protein;Beta-filamin; }\end{array}$ \\
\hline M & TVKKIAIFGATGR & 0,1 & N-ter +34.06 Da, K +34.06 Da & 32,3 & $-0,682$ & 3 & 1463,0 & BLVRB_MOUSE & E9PZC3_MOUSE & Flavin reductase (NADPH); & $\begin{array}{l}\text { Biliverdin reductase B;Biliverdin-IX beta- } \\
\text { reductase;NNDPH-dependent } \\
\text { diaphorase;NADPH-flavin reductase; }\end{array}$ \\
\hline SVCVVHLRNL & GTLDNPSSLDETAYER & $-0,1$ & $\mathrm{~N}$-ter +28.03 Da & 30,7 & 0,334 & 2 & 1794,8 & FRDA_MOUSE & E9Q2P9_MOUSE & Frataxin, mitochondrial; & \\
\hline EDRMAQWILL & AAQNGDLTELR & 1,2 & N-ter +28.03 Da & 17,3 & $-6,324$ & 3 & 1214,6 & GPAN1_MOUSE & Q6PES3_MOUSE & $\begin{array}{l}G \text { patch domain and ankyrin repeat-containing } \\
\text { protein } 1 \text {; }\end{array}$ & G5 protein;HLA-B-associated transcript 4; \\
\hline LQRLFSRQDC & CGNCSDSEEELPTR & $-10,0$ & N-ter +34.06 Da, C +57.02 Da & 15,3 & 3,255 & 3 & 1686,7 & GRK6_MOUSE & & G protein-coupled receptor kinase 6 & G protein-coupled receptor kinase GRK6; \\
\hline LLCGEEQGAD & AALHFI & 0,0 & $\mathrm{~N}$-ter +34.06 Da & 20,7 & $-0,936$ & 3 & 958,6 & Q9CRB1_MOUSE & LEG7_MOUSE & Galectin \{ECO:0000256|RuleBase:RU102079\}; & \\
\hline VNLLCGEEQG & ADAALHFNPR & $-0,1$ & $\mathrm{~N}$-ter +34.06 Da & 28,1 & $-2,178$ & 3 & 1144,6 & Q9CRB1_MOUSE & LEG7_MOUSE & Galectin \{ECO:0000256|RuleBase:RU102079\}; & \\
\hline QAGRFHVNLL & CGEEQGADAALHFNPR & $-0,2$ & $\mathrm{~N}$-ter +28.03 Da, C +57.02 Da & 73,4 & $-1,110$ & 3 & 1798,8 & Q9CRB1_MOUSE & LEG7_MOUSE & Galectin \{ECO:0000256|RuleBase:RU102079\}; & \\
\hline DAALHFNPRL & DTSEVVFNTKEQGKWGR & 0,1 & N-ter +34.06 Da, K +34.06 Da & 18,8 & $-0,815$ & 3 & 2082,2 & Q9CRB1_MOUSE & D3Z141_MOUSE & Galectin \{ECO:0000256|RuleBase:RU102079\}; & \\
\hline GRFHVNLLCG & EEQGADAALHFNPR & $-0,1$ & $\mathrm{~N}$-ter +34.06 Da & 45,4 & $-1,760$ & 3 & 1587,8 & Q9CRB1_MOUSE & LEG7_MOUSE & Galectin \{ECO:0000256|RuleBase:RU102079\}; & \\
\hline RFHVNLLCGE & EQGADAALHFNPR & $-0,6$ & $\mathrm{~N}$-ter +28.03 Da & 18,2 & $-1,30$ & 3 & 1452,7 & Q9CRB1_MOUSE & LEG7_MOUSE & Galectin \{ECO:0000256|RuleBase:RU102079\}; & \\
\hline LHFNPRLDTS & EVVFNTKEQGKWGR & $-0,4$ & N-ter +34.06 Da, K+34.06 Da & 25,2 & $-2,0$ & 3 & 1779,1 & Q9CRB1_MOUSE & D3Z141_MOUSE & Galectin \{ECO:0000256|RuleBase:RU102079\}; & \\
\hline HVNLLCGEEQ & GADAALHFNPR & $-0,2$ & N-ter +34.06 Da & 24,6 & $-2,241$ & 3 & 1201,6 & Q9CRB1_MOUSE & LEG7_M̄MOUSE & Galectin \{ECO:0000256|RuleBase:RU102079\}; & \\
\hline AGRFHVNLLC & AALHFNPR & $-0,2$ & $\mathrm{~N}$-ter +34.06 Da & 60,6 & 1,032 & 3 & 1644,8 & Q9CRB1_MOUSE & LEG7_MOUSE & Galectin \{ECO:0000256|RuleBase:RU102079\}; & \\
\hline VRVGTVMRIR & GMVPDQAGR & $-0,2$ & $\mathrm{~N}$-ter +28.03 Da & 28,2 & $-3,439$ & 2 & 957,5 & Q9CRB1_MOUSE & D3Z14ī_MOUSE & Galectin \{ECO:0000256|RuleBase:RU102079\}; & \\
\hline DQAGRFHVNL & LCGEEQG & $-0,3$ & N-ter $+34.06 \mathrm{Da}, \mathrm{C}+57.02 \mathrm{Da}$ & 73 & $-0,5$ & 3 & 1917,9 & Q9CRB1_MOUSE & LEG7_MOMUSE & Galectin \{ECO:0000256|RuleBase:RU102079\}; & \\
\hline ADAALHFNPR & LDTSEV & 7,1 & $\mathrm{~N}$-ter $+28.03 \mathrm{D}$ & 17 & $-1,3$ & 4 & 2177,2 & Q9CRB1_MOUSE & D3Z141_MOUSE & Galectin \{ECO:0000256|RuleBase:RU10 & \\
\hline PDQAGRFHVN & LLC & 0,0 & N-te & & $-1,7$ & 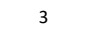 & 2025,0 & Q9CRB1_MOUSE & LEG7_MOUSE & Galectin $\{E C$ & \\
\hline VPDQAGRFHV & AALLHFNPR & 0,1 & $\mathrm{a}, \mathrm{c}+57.02 \mathrm{Da}$ & 6 & $-4,1$ & 3 & 213 & Q9CRB1_MOUSE & LEG7_MOUSE & Galectin $\{E$ & \\
\hline & & $-0,3$ & N-ter +34 & 2 & & 3 & 11 & Q9CRB1_MOUSE & D3Z141_MOUSE & Galectin $\{E$ & \\
\hline KEQGKWGREE & & $-0,5$ & N-ter +34 & 18 & & 3 & 10 & 1_MOUSE & OUSE & Galectin $\{E$ & \\
\hline 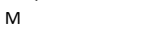 & SATQH & 1,1 & $\mathrm{~N}$-ter +2 & 2 & -0 & 4 & 15 & 1_MOUSE & DUSE & Galectin & \\
\hline LDT & KEQGKWGR & $-0,5$ & $\mathrm{~K}+34.06 \mathrm{Da}$ & 2 & 0, & 3 & 18 & DUSE & DUSE & Galectin & \\
\hline & SLPQC & 0,0 & & 1 & -1 & 2 & & & & & \\
\hline & KEQGKWGP & $0, \mathrm{C}$ & $28.03 \mathrm{Da}$ & & & 4 & 1949,0 & Q9CRB1 & D3Z14 & Galecti & \\
\hline MVPDQAGRFH & VNLLCGEEQGADAALHFNPR & $-0,2$ & $\mathrm{~N}-\operatorname{ter}+28.03 \mathrm{Da}, \mathrm{C}+57.02 \mathrm{Da}$ & 21,4 & 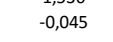 & 3 & 2238,1 & Q9CRB1_MOUSE & LEG7_MOUSE & Galectin \{ECO:0000256|RuleBase:RU102079\}; & \\
\hline MACGLV & ASNLNLKPGECLKVR & $-0,4$ & $\begin{array}{l}\text { N-ter +34.06 Da, K+34.06 Da, C } \\
+57.02 \text { Da }\end{array}$ & 23,9 & 0,388 & 4 & 1800,1 & LEG1_MOUSE & & Galectin-1; & $\begin{array}{l}14 \text { kDa lectin;Beta-galactoside-binding lectin L- } \\
14-\text { - Galaptin;Lactose-binding lectin 1;Lectin } \\
\text { galactoside-binding soluble } 1 ; \text {; }- \text {-ac lectin } 1 ;\end{array}$ \\
\hline SDAKSFVLNL & GKDSNNLCLHFNPR & $-0,2$ & $\begin{array}{l}\text { N-ter +34.06 Da, K +34.06 Da, C } \\
\text { +57.02 Da }\end{array}$ & 40,1 & $-0,574$ & 4 & 1738,9 & LEG1_MOUSE & & Galectin-1; & $\begin{array}{l}14 \mathrm{kDa} \text { lectin;Beta-galactoside-binding lectin L- } \\
14-\text { - Galaptin;:Lactose-binding lectin 1;Lectin } \\
\text { galactoside-binding soluble 1;S-Lac lectin } 1 ;\end{array}$ \\
\hline
\end{tabular}




\begin{tabular}{|c|c|c|c|c|c|c|c|c|c|c|c|}
\hline Non Prime Site & Prime Site & $\begin{array}{l}\text { Fold change of } \\
\text { XPRESS ratio } \\
\text { (Adam17 } 7_{\text {AKC } / W T)}\end{array}$ & Modifications & Hyperscore & $\begin{array}{l}\text { Mass error } \\
\text { in ppm }\end{array}$ & Charge & $\begin{array}{c}\text { Precursor } \\
\text { neutral mass } \\
\text { in Da }\end{array}$ & Uniprot ID 1 & Uniprot ID 2 & Protein Name 1 & Protein Name 2 \\
\hline VASDAKSFVL & NLGKDSNNLCLHFNPR & $-0,2$ & $\begin{array}{l}\text { N-ter +34.06 Da, K+34.06 Da, C } \\
+57.02 \mathrm{Da}\end{array}$ & 25,6 & 1,320 & 4 & 1966,1 & LEG1_MOUSE & & Galectin-1; & $\begin{array}{l}14 \text { KDa lectin;Beta-galactoside-binding lectin L- } \\
14-; ; G a l a p t i n ; L \text { Lactose-binding lectin 1;Lectin } \\
\text { galactoside-binding soluble 1;S-Lac lectin } 1 ;\end{array}$ \\
\hline MACGLVASNL & NLKPGECLKVR & $-0,3$ & $\begin{array}{l}\text { N-ter +34.06 Da, K+34.06 Da, C } \\
+57.02 \mathrm{Da}\end{array}$ & 31,0 & $-0,494$ & 3 & 1414,9 & LEG1_MOUSE & & Galectin-1; & $\begin{array}{l}14 \text { KDa lectin;Beta-galactoside-binding lectin L- } \\
14-\text { - Galaptin;LLactose-binding lectin 1;Lectin } \\
\text { galactoside-binding soluble 1;S-Lac lectin } 1 ;\end{array}$ \\
\hline MACGLVA & SNLNLKPGECLKVR & $-0,2$ & $\begin{array}{l}\text { N-ter +34.06 Da, K+34.06 Da, C } \\
+57.02 \mathrm{Da}\end{array}$ & 21,2 & $-0,519$ & 4 & 1729,1 & LEG1_MOUSE & & Galectin-1; & $\begin{array}{l}14 \text { kDa lectin;Beta-galactoside-binding lectin L- } \\
14-\text { - Galaptini-Lactose-binding lectin 1;Lectin } \\
\text { galactoside-binding soluble 1;S-Lac lectin } 1 ;\end{array}$ \\
\hline RIVLDFRRGN & DVAFHFNPR & 0,0 & $\mathrm{~N}$-ter +28.03 Da & 20,8 & $-1,413$ & 3 & 1129,6 & LEG3_MOUSE & LEG4_MOUSE & Galectin-3; & 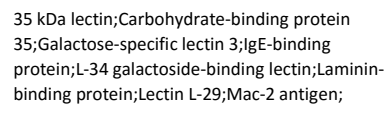 \\
\hline GMVPDQAGRF & HVNLLCGEEQGADAALHFNPR & $-3,0$ & N-ter +34.06 Da, C +57.02 Da & 11,2 & $-2,264$ & 4 & 2381,2 & LEG7_MOUSE & Q9CRB1_MOUSE & Galectin-7; & \\
\hline LGNYYIAASY & VKYIESAGAR & $-0,7$ & N-ter +28.03 Da, K+28.03 Da & 23,9 & 2,258 & 3 & 1148,7 & GGH_MOUSE & & Gamma-glutamyl hydrolase; & $\begin{array}{l}\text { Conjugase;FGPH;Foly|polyglutamate } \\
\text { hydrolase;GH;Gamma-Glu-x carboxypeptidase; }\end{array}$ \\
\hline FGKMAKLGNY & YIAASVVKYIESAGAR & $-0,6$ & N-ter +28.03 Da, K+28.03 Da & 24,9 & 1,484 & 3 & 1817,0 & GGH_MOUSE & & Gamma-glutamyl hydrolase; & $\begin{array}{l}\text { Conjugase;FGPH;Folylpolyglutamate } \\
\text { hydrolase;GH;Gamma-Glu-x carboxypeptidase; }\end{array}$ \\
\hline HAPNAVKTSF & YLAEFLVSEAR & $-0,6$ & $\mathrm{~N}$-ter $+34.06 \mathrm{Da}$ & 17,7 & 0,300 & 2 & 1330,7 & GGH_MOUSE & & Gamma-glutamyl hydrolase; & $\begin{array}{l}\text { Conjugase;FGPH;Folylpolylutamate } \\
\text { hydrolase;GH;Gamma-Glu-x carboxypeptidase; }\end{array}$ \\
\hline AQERNVSGTW & EFTCQHGELECR & $-0,2$ & $\mathrm{~N}$-ter $+34.06 \mathrm{Da}, \mathrm{C}+57.02 \mathrm{Da}$ & 14,0 & $-0,749$ & 3 & 1598,7 & GILT_MOUSE & & $\begin{array}{l}\text { Gamma-interferon-inducible lysosomal thiol } \\
\text { reductase; }\end{array}$ & $\begin{array}{l}\text { Gamma-interferon-inducible protein IP- } \\
\text { 30; Lysosomal thiol reductase IP30; }\end{array}$ \\
\hline TCKAHDVCLL & GPRPLPPSPPVR & $-0,1$ & $\mathrm{~N}$-ter +34.06 Da & 28,8 & $-2,374$ & 3 & 1302,8 & GILT_MOUSE & & $\begin{array}{l}\text { Gamma-interferon-inducible lysosomal thiol } \\
\text { reductase; }\end{array}$ & $\begin{array}{l}\text { Gamma-interferon-inducible protein IP- } \\
\text { 30; Lysosomal thiol reductase IP30; }\end{array}$ \\
\hline YGNAQERNVS & GTWEFTCQHGELECR & 0,4 & N-ter +28.03 Da, C+57.02 Da & 17,7 & 9,537 & 3 & 1936,8 & GILT_MOUSE & & $\begin{array}{l}\text { Gamma-interferon-inducible lysosomal thiol } \\
\text { reductase; }\end{array}$ & $\begin{array}{l}\text { Gamma-interferon-inducible protein IP- } \\
\text { 30; Lysosomal thiol reductase IP30; }\end{array}$ \\
\hline KAHDVCLLGP & RPLPPSPPVR & 0,1 & $\mathrm{~N}$-ter +34.06 Da & 28,2 & $-1,910$ & 3 & 1148,7 & GILT_MOUSE & & $\begin{array}{l}\text { Gamma-interferon-inducible lysosomal thiol } \\
\text { reductase; }\end{array}$ & $\begin{array}{l}\text { Gamma-interferon-inducible protein IP- } \\
\text { 30; Lysosomal thiol reductase IP30; }\end{array}$ \\
\hline PLEVPRAATA & SLSQASSEGTTCKAHDVCLLGPRPLPPSPPVR & $-0,6$ & $\begin{array}{l}\text { N-ter +34.06 Da, K+34.06 Da, C } \\
+57.02 \mathrm{Da}\end{array}$ & 24,3 & $-8,111$ & 5 & 3582,9 & GILT_MOUSE & & $\begin{array}{l}\text { Gamma-interferon-inducible lysosomal thiol } \\
\text { reductase; }\end{array}$ & $\begin{array}{l}\text { Gamma-interferon-inducible protein IP- } \\
\text { 30;Lysosomal thiol reductase IP30; }\end{array}$ \\
\hline WLMVMEIMNI & TLVPYGNAQER & 0,1 & $\mathrm{~N}$-ter +34.06 Da & 19,1 & 9,121 & 2 & 1280,7 & GILT_MOUSE & & $\begin{array}{l}\text { Gamma-interferon-inducible lysosomal thiol } \\
\text { reductase; }\end{array}$ & $\begin{array}{l}\text { Gamma-interferon-inducible protein IP- } \\
\text { 30; Lysosomal thiol reductase IP30; }\end{array}$ \\
\hline ERGENLYVVM & EVVETLQEVTLER & $-0,8$ & $\mathrm{~N}$-ter $+34.06 \mathrm{Da}$ & 46,8 & 3,102 & 2 & 1577,9 & GSDMA_MOUSE & & Gasdermin-A; & Gasdermin-1;Gasdermin-A1; \\
\hline RSSTLEVQTL & SVAPTALENLHKER & $-0,5$ & N-ter +34.06 Da, K+34.06 Da & 19,1 & 4,523 & 4 & 1632,0 & GSDMA_MOUSE & & Gasdermin-A; & Gasdermin-1;Gasdermin-A1; \\
\hline AATLHTSTAM & AAQHGMDDDGTGQKaIWR & 0,3 & $\mathrm{~N}$-ter + 34.06 Da, K+34.06 Da & 21,3 & $-1,823$ & 4 & 2081,0 & GELS_MOUSE & & Gelsolin; & Actin-depolymerizing factor;Brevin; \\
\hline DEVAASAILT & AQLDEELGGTPVQSR & $-0,3$ & N-ter +28.03 Da & 44,4 & $-3,193$ & 2 & 1626,8 & GELS_MOUSE & & Gelsolin; & Actin-depolymerizing factor;Brevin; \\
\hline AGSMSVSLVA & DENPFAQGALR & 1,0 & N-ter +34.06 Da & 22,9 & $-1,437$ & 2 & 1250,7 & GELS_MOUSE & & Gelsolin; & Actin-depolymerizing factor;Brevin; \\
\hline MIIYKGGTSR & DGGQTAPASIR & 0,4 & N-ter +28.03 Da & 25,9 & $-1,725$ & 2 & 1099,6 & GELS_MOUSE & & Gelsolin; & Actin-depolymerizing factor;Brevin; \\
\hline VVEHPEFLKA & GKEPGLQIWR & $-0,3$ & N-ter +34.06 Da, K+34.06 Da & 18,5 & $-1,595$ & 3 & 1250,8 & GELS_MOUSE & A6PWS5_MOUSE & Gelsolin; & Actin-depolymerizing factor;Brevin; \\
\hline KNWRDPDQTD & GPGLGYLSSHIANVER & 0,5 & $\mathrm{~N}$-ter +28.03 Da & 18,1 & 4,765 & 3 & 1696,9 & GELS_MOUSE & & Gelsolin; & Actin-depolymerizing factor;Brevin; \\
\hline EPPSFVGWFL & GWDDNYWSVDPLDR & $-0,2$ & N-ter +34.06 Da & 18,1 & 0,62 & 2 & 1770,8 & GELS_MOUSE & & Gelsolin; & Actin-depolymerizing factor;Brevin; \\
\hline ENPFAQGALR & SEDCFILDHGR & 0,7 & $\mathrm{~N}-\operatorname{ter}+34.06 \mathrm{Da}, \mathrm{C}+57.02 \mathrm{Da}$ & 20,5 & 4,983 & 3 & 1381,7 & GELS_MOUSE & & Gelsolin; & Actin-depolymerizing factor;Brevin; \\
\hline VSNGAGSMSV & SLVADENPFAQGALR & 0,1 & N-ter +34.06 Da & 37,2 & $-2,095$ & 2 & 1620,9 & GELS_MOUSE & & Gelsolin; & Actin-depolymerizing factor;Brevin; \\
\hline YKVSNGAGSM & SVSLVADENPFAQGALR & 1,1 & $\mathrm{~N}$-ter+34.06 Da & 15,6 & $-0,553$ & 3 & 1807,0 & GELS_MOUSE & & Gelsolin; & Actin-depolymerizing factor;Brevin; \\
\hline NGAGSMSVSL & VADENPFAQGALR & 0,1 & $\mathrm{~N}$-ter+28.03 Da & 23,6 & 0,353 & 2 & 1414,7 & GELS_MOUSE & & Gelsolin; & Actin-depolymerizing factor;Brevin; \\
\hline VLKTPSAAYL & WVGAGASEAEKTGAQELLKVLR & 0,0 & $\mathrm{~N}$-ter +28.03 Da, K+28.03 Da & 20,7 & $-4,916$ & 4 & 2396,3 & GELS_MOUSE & & Gelsolin; & Actin-depolymerizing factor;Brevin; \\
\hline YNFTPLARHR & TLTAEEAEEEWERR & 0,1 & $\mathrm{~N}-\mathrm{ter}+34.06 \mathrm{Da}$ & 19,7 & $-0,952$ & 3 & 1781,9 & T2FA_MOUSE & & General transcription factor IIF subunit 1; & Transcription initiation factor IIF subunit alpha; \\
\hline HHKILLANFL & AQTEALMKGKLPEEAR & 0,1 & $\mathrm{~N}$-ter $+34.06 \mathrm{Da}, \mathrm{K}+34.06 \mathrm{Da}$ & 22,4 & $-0,746$ & 3 & 1873,1 & G6PI_MOUSE & & Glucose-6-phosphate isomerase; & $\begin{array}{l}\text { Autocrine motility } \\
\text { factor;Neuroleukin;Phosphoglucose } \\
\text { isomerase;Phosphohexose isomerase; } \\
\text { Acid beta-glucosidase; Beta- }\end{array}$ \\
\hline HVTGWTDWNL & ALNPEGGPNWVR & $-0,1$ & $\mathrm{~N}$-ter +34.06 Da & 26,1 & $-0,744$ & 2 & 1342,7 & GLCM_MOUSE & & Glucosylceramidase; & $\begin{array}{l}\text { glucocerebrosidase; D-glucosyl-N- } \\
\text { acylsphingosine glucohydrolase; }\end{array}$ \\
\hline EFVDITATNN & TSAIQDYLQQLTGAR & 1,0 & $\mathrm{~N}$-ter +28.03 Da & 21,0 & $-0,236$ & 3 & 1691,9 & GLRX1_MOUSE & & Glutaredoxin-1; & Thioltransferase-1; \\
\hline LPFKQGLLEF & VDITATNNTSAIQDYLQQLTGAR & $-0,4$ & N-ter +34.06 Da & 58,3 & $-0,593$ & 3 & 2526,3 & GLRX1_MOUSE & & Glutaredoxin-1; & Thioltransferase-1; \\
\hline
\end{tabular}




\begin{tabular}{|c|c|c|c|c|c|c|c|c|c|c|c|}
\hline Non Prime Site & Prime Site & 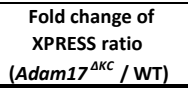 & Modifications & Hyperscore & $\begin{array}{l}\text { Mass error } \\
\text { in ppm }\end{array}$ & Charge & $\begin{array}{c}\text { Precursor } \\
\text { neutral mass } \\
\text { in Da } \\
\end{array}$ & Uniprot ID 1 & Uniprot ID 2 & Protein Name 1 & Protein Name 2 \\
\hline VCRNDIAWNF & EKFLVGPDGVPVR & 3,6 & N-ter +34.06 Da, K+34.06 Da & 22,4 & $-1,214$ & 3 & 1479,9 & GPX1_MOUSE & & Glutathione peroxidase 1; & $\begin{array}{l}\text { Cellular glutathione peroxidase;Selenium- } \\
\text { dependent glutathione peroxidase 1; }\end{array}$ \\
\hline AQSTVYAFSA & RPLTGGEPVSLGSLR & 0,2 & N-ter +34.06 Da & 22,6 & $-1,905$ & 3 & 1571,9 & GPX1_MOUSE & & Glutathione peroxidase 1; & $\begin{array}{l}\text { Cellular glutathione peroxidase; Selenium- } \\
\text { dependent glutathione peroxidase } 1 \text {; }\end{array}$ \\
\hline AAAQSTVYAF & SARPLTGGEPVSLGSLR & 0,2 & N-ter +34.06 Da & 31,4 & $-8,021$ & 3 & 1730,0 & GPX1_MOUSE & & Glutathione peroxidase $1 ;$ & $\begin{array}{l}\text { Cellular glutathione peroxidase:Selenium- } \\
\text { dependent glutathione peroxidase 1; }\end{array}$ \\
\hline TVYAFSARPL & TGGEPVSLGSLR & $-0,1$ & $\mathrm{~N}$-ter $+34.06 \mathrm{Da}$ & 18,6 & 2,815 & 2 & 1205,7 & GPX1_MOUSE & & Glutathione peroxidase 1 ; & $\begin{array}{l}\text { Cellular glutathione peroxidase; Selenium- } \\
\text { dependent glutathione peroxidase } 1 \text {; }\end{array}$ \\
\hline ARQKMTLESF & SKVPPLIASFVR & 0,2 & $\mathrm{~N}$-ter +34.06 Da, $\mathrm{K}+34.06$ Da & 14,1 & 1,445 & 3 & 1380,9 & GSTO1_MOUSE & & Glutathione S-transferase omega- $1 ;$ & $\begin{array}{l}\text { Glutathione S-transferase omega 1- } \\
\text { 1;Glutathione-dependent dehydroascorbate } \\
\text { reductase;Monomethylarsonic acid reductase;s- } \\
\text { (Phenacy)glutaththione reductase;p28; }\end{array}$ \\
\hline SEERASYILM & EKIEPEPFR & 0,4 & N-ter +28.03 Da, $\mathrm{K}+28.03 \mathrm{Da}$ & 17,1 & $-1,497$ & 3 & 1199,7 & GSHB_MOUSE & Q3UEE2_MOUSE & Glutathione synthetase; & Glutathione synthase; \\
\hline KAENGKLVIN & GKPTIIFQER & 0,6 & N-ter +28.03 Da, K+28.03 Da & 21,3 & $-3,128$ & 3 & 1243,7 & S4R257_MOUSE & G3P_MOUSE & $\begin{array}{l}\text { Glyceraldehyde-3-phosphate dehydrogenase } \\
\{\text { \{EO:000256|RuleBase:RU361160\}; }\end{array}$ & \\
\hline IVEGLMTTVH & AITATQKTVDGPSGKLWR & 0,3 & N-ter +28.03 Da, $\mathrm{K}+28.03 \mathrm{Da}$ & 47,7 & $-0,447$ & 3 & 2012,1 & G3P_MOUSE & D3YYI5_MOUSE & Glyceraldehyde-3-phosphate dehydrogenase; & Peptidyl-cysteine S-nitrosylase GAPDH; \\
\hline GLMTTVHAIT & ATQKTVDGPSGKLWR & 0,4 & $\mathrm{~N}$-ter $+28.03 \mathrm{Da}, \mathrm{K}+28.03 \mathrm{Da}$ & 25,8 & $-0,289$ & 4 & 1727,0 & G3P_MOUSE & D3YYIS_MOUSE & Glyceraldehyde-3-phosphate dehydrogenase; & Peptidyl-cysteine S-nitrosylase GAPDH; \\
\hline DNFVKLISWY & DNEYGYSNR & $-0,4$ & N-ter +28.03 Da & 26,6 & 1,221 & 2 & 1144,5 & G3P_MOUSE & G3PT_MOUSE & $\begin{array}{l}\text { Glyceraldehyde-3-phosphate dehydrogenase; } \\
\text {; }\end{array}$ & Peptidyl-cysteine S-nitrosylase GAPDH; \\
\hline ELNGKLTGMA & FRVPTPNVSVVDLTCR & 0,2 & N-ter +28.03 Da, C +57.02 Da & 47,1 & 2,116 & 3 & 1887,0 & G3P_MOUSE & G3PT_MOUSE & Glyceraldehyde-3-phosphate dehydrogenase; & Peptidyl-cysteine S-nitrosylase GAPDH; \\
\hline AITATQKTVD & GPSGKLWR & 0,1 & $\mathrm{~N}$-ter $+28.03 \mathrm{Da}, \mathrm{K}+28.03 \mathrm{Da}$ & 25,3 & 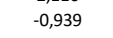 & 3 & 955,6 & G3P_MOUSE & D3YYIS_MOUSE & Glyceraldehyde-3-phosphate dehydrogenase; & Peptidyl-cysteine S-nitrosylase GAPDH; \\
\hline IALNDNFVKL & ISWYDNEYGYSNR & 0,0 & N-ter +34.06 Da & 37,7 & $-2,233$ & 2 & 1699,8 & G3P_MOUSE & D3YYIS_MOUSE & Glyceraldehyde-3-phosphate dehydrogenase; & Peptidyl-cysteine S-nitrosylase GAPDH; \\
\hline TVVHAITATQ & KTVDGPSGKLWR & 0,0 & $\mathrm{~N}$-ter + $+34.06 \mathrm{Da}$, $\mathrm{K}+34.06 \mathrm{Da}$ & 15,0 & 3,727 & 4 & 1444,9 & G3P_MOUSE & D3YYIS_MOUSE & Glyceraldehyde-3-phosphate dehydrogenase; & Peptidyl-cysteine S-nitrosylase GAPDH; \\
\hline MTTVHAITAT & QKTVDGPSGKLWR & 0,0 & $\mathrm{~N}$-ter $+28.03 \mathrm{Da}, \mathrm{K}+28.03 \mathrm{Da}$ & 17,8 & 1,475 & 4 & 1554,9 & G3P_MOUSE & D3YYIS_MOUSE & Glyceraldehyde-3-phosphate dehydrogenase; & Peptidyl-cysteine S-nitrosylase GAPDH; \\
\hline LNGKLTGMAF & RVPTPNVSVVDLTCR & $-0,1$ & $\mathrm{~N}$-ter $+28.03 \mathrm{Da}, \mathrm{C}+57.02 \mathrm{Da}$ & 40,5 & 0,115 & 3 & 1739,9 & G3P_MOUSE & G3PT_MOUSE & Glyceraldehyde-3-phosphate dehydrogenase; & Peptidyl-cysteine S-nitrosylase GAPDH; \\
\hline ALNDNFVKLI & SWYDNEYGYSNR & 0,0 & N-ter +34.06 Da & 52,6 & 0,315 & 2 & 1586,7 & G3P_MOUSE & D3YYIS_MOUSE & Glyceraldehyde-3-phosphate dehydrogenase; & Peptidyl-cysteine S-nitrosylase GAPDH; \\
\hline EGLMTTVHAI & $\begin{array}{l}\text { TATQKTVDGPSGKLWR } \\
\text { TATV }\end{array}$ & 0,0 & $\mathrm{~N}$-ter $+28.03 \mathrm{Da}, \mathrm{K}+28.03 \mathrm{Da}$ & 18,3 & 1,419 & 4 & 1828,0 & G3P_MOUSE & D3YYIS_MOUSE & Glyceraldehyde-3-phosphate dehydrogenase; & Peptidyl-cysteine S-nitrosylase GAPDH; \\
\hline LMTTVHAITA & TQKTVDGPSGKLWR & $-0,1$ & $\mathrm{~N}$-ter $+34.06 \mathrm{Da}, \mathrm{K}+34.06 \mathrm{Da}$ & 35,5 & 0,358 & 3 & 1674,0 & G3P_MOUSE & D3YYIS_MOUSE & Glyceraldehyde-3-phosphate dehydrogenase; & Peptidyl-cysteine S-nitrosylase GAPDH; \\
\hline TVHAITATQK & TVDGPSGKLWR & 0,7 & $\mathrm{~N}$-ter $+34.06 \mathrm{Da}, \mathrm{K}+34.06 \mathrm{Da}$ & 32,0 & -1,322 & 3 & 1282,8 & G3P_MOUSE & D3YYIS_MOUSE & Glyceraldehyde-3-phosphate dehydrogenase; & Peptidyl-cysteine S-nitrosylase GAPDH; \\
\hline M & $\begin{array}{l}\text { VKVGVNGFGR } \\
\text { VKG }\end{array}$ & $-0,1$ & $\mathrm{~N}$-ter $+28.03 \mathrm{Da}, \mathrm{K}+28.03 \mathrm{Da}$ & 32,3 & $-1,100$ & 3 & 1087,7 & G3P_MOUSE & D3YYIS_MOUSE & Glyceraldehyde-3-phosphate dehydrogenase; & Peptidyl-cysteine S-nitrosylase GAPDH; \\
\hline LNDNFVKLIS & WYDNEYGYSNR & 0,1 & $\mathrm{~N}$-ter $+34.06 \mathrm{Da}$ & 35,3 & $-0,333$ & 2 & 1499,7 & G3P_MOUSE & G3PT_MOUSE & Glyceraldehyde-3-phosphate dehydrogenase; & Peptidyl-cysteine S-nitrosylase GAPDH; \\
\hline NDNFVKLLSW & YDNEYGYSNR & 0,2 & N-ter +28.03 Da & 15,2 & $-0,305$ & 2 & 1307,5 & G3P_MOUSE & G3PT_MOUSE & Glyceraldehyde-3-phosphate dehydrogenase; & Peptidyl-cysteine S-nitrosylase GAPDH; \\
\hline MVRQKQNKYP & ILFLTQGKSDIYPELMDLR & 6,4 & N-ter +28.03 Da, $\mathrm{K}+28.03 \mathrm{Da}$ & 17,3 & $-3,030$ & 3 & 2307,3 & GPCP1_MOUSE & BOR058_MOUSE & Glycerophosphocholine phosphodiesterase GPCPD1; & $\begin{array}{l}\text { Glycerophosphodiester phosphodiesterase } \\
\text { 5; Preimplantation protein 4; }\end{array}$ \\
\hline RFRSPDDSCN & QLTQFLYGFCKOSR & 4,4 & $\begin{array}{l}\text { N-ter }+28.03 \mathrm{Da}, \mathrm{K}+28.03 \mathrm{Da}, \mathrm{C} \\
+57.02 \mathrm{Da}\end{array}$ & 24,6 & 6,870 & 3 & 1831,0 & GYS2_MOUSE & & Glycogen [starch] synthase, liver; & \\
\hline \multirow[t]{2}{*}{ M } & KVFVTGPLPAEGR & 0,9 & $\mathrm{~N}$-ter +34.06 Da, $\mathrm{K}+34.06 \mathrm{Da}$ & 24,0 & $-1,319$ & 3 & 1437,9 & GRHPR_MOUSE & D6REG4_MOUSE & Glyoxylate reductase/hydroxypyruvate reductase; & \\
\hline & MKVFVTGPLPAEGR & 0,0 & N-ter +34.06 Da, $\mathrm{K}+34.06 \mathrm{Da}$ & 35,3 & 0,509 & 3 & 1568,9 & GRHPR_MOUSE & DGREG4_MOUSE & Glyoxylate reductase/hydroxypyruvate reductase; & \\
\hline M & RPQQAPVSGKVFIQR & $-4,4$ & N-ter +34.06 Da, K+34.06 Da & 18,0 & $-2,666$ & 4 & 1909,1 & GOGA7_MOUSE & & Golgin subfamily A member 7; & \\
\hline M & AAAGAAATDLEVVR & $-0,2$ & N-ter +34.06 Da & 41,1 & 0,074 & 2 & 1347,8 & PIGS_MOUSE & & GPI transamidase component PIG-S; & $\begin{array}{l}\text { Phosphatidylinositol-glycan biosynthesis class S } \\
\text { protein; }\end{array}$ \\
\hline LTKLPGYPVK & EVKCDMEVSCPEGYTCCR & $-0,2$ & $\begin{array}{l}\text { N-ter +34.06 Da, K+34.06 Da, C } \\
+57.02 \mathrm{Da}\end{array}$ & 48,4 & $-0,085$ & 3 & 2347,0 & GRN_MOUSE & Q3U9N4_MOUSE & Granulins; & PC cell-derived growth factor;Proepithelin; \\
\hline LLTKLPGYPV & KEVKCDMEVSCPEGYTCCR & 0,0 & $\begin{array}{l}\text { N-ter +28.03 Da, K+28.03 Da, C } \\
+57.02 \mathrm{Da}\end{array}$ & 30,0 & $-6,733$ & 4 & 2491,1 & GRN_MOUSE & Q3U9N4_MOUSE & Granulins; & PC cell-derived growth factor;:Proepithelin; \\
\hline TKLPGYPVKE & VKCDMEVSCPEGYTCCR & 0,0 & $\begin{array}{l}\text { N-ter +34.06 Da, K+34.06 Da, C } \\
+57.02 \mathrm{Da}\end{array}$ & 30,2 & 2,521 & 3 & 2218,0 & GRN_MOUSE & Q3U9N4_MOUSE & Granulins; & PC cell-derived growth factor;:Proepithelin; \\
\hline MAALVA & AAALAAAEPAPAVPQAAGSGGPTSR & $-1,4$ & N-ter +34.06 Da & 24,7 & 5,168 & 3 & 2222,2 & GDC_MOUSE & D3YVC9_MOUSE & Graves disease carrier protein homolog; & $\begin{array}{l}\text { Mitochondrial solute carrier protein } \\
\text { homolog;Solute carrier family } 25 \text { member 16; }\end{array}$ \\
\hline MTKVK & AAPEESEAQAEGCSEER & $-1,1$ & N-ter +34.06 Da, C +57.02 Da & 26,4 & 0,795 & 3 & 1882,8 & NHP2_MOUSE & & H/ACA ribonucleoprotein complex subunit 2; & $\begin{array}{l}\text { Nucleolar protein family A member 2; snoRNP } \\
\text { protein NHP2; }\end{array}$ \\
\hline M & TKVKAAPEESEAQAEGCSEER & $-0,3$ & $\begin{array}{l}\text { N-ter +28.03 Da, K+28.03 Da, C } \\
+57.02 \mathrm{Da}\end{array}$ & 32,2 & $-1,547$ & 3 & 2389,1 & NHP2_MOUSE & & H/ACA ribonucleoprotein complex subunit 2; & $\begin{array}{l}\text { Nucleolar protein family A member 2;snoRNP } \\
\text { protein NHP2; }\end{array}$ \\
\hline M & FLQYYLNEQGDR & $-0,6$ & $\mathrm{~N}$-ter +28.03 Da & 17,1 & $-1,524$ & 2 & 1703,8 & NOP10_MOUSE & & H/ACA ribonucleoprotein complex subunit 3; & $\begin{array}{l}\text { Nucleolar protein 10;Nucleolar protein family A } \\
\text { member 3;snoRNP protein NOP10; }\end{array}$ \\
\hline AGVIAGLINVL & RIINEPTAAAIAYGLDR & 0,0 & N-ter +34.06 Da & 33,7 & 0,798 & 3 & 1877,1 & HS71A_MOUSE & HS71B_MOUSE & Heat shock 70 kDa protein $1 \mathrm{~A}$; & Heat shock 70 kDa protein 3;Hsp68; \\
\hline VEIIANDQGN & RTTPSYVAFTDTER & $-0,2$ & $\mathrm{~N}$-ter +34.06 Da & 24,6 & $-0,238$ & 3 & 1676,9 & HS71A_MOUSE & HS71B_MOUSE & Heat shock $70 \mathrm{kDa}$ protein $1 \mathrm{~A}$; & Heat shock 70 kDa protein 3;Hsp68; \\
\hline
\end{tabular}




\begin{tabular}{|c|c|c|c|c|c|c|c|c|c|c|c|}
\hline Non Prime Site & Prime Site & $\begin{array}{l}\text { Fold change of } \\
\text { XPRESS ratio } \\
\text { (Adam17 } 7_{\text {LKC } / W T)}\end{array}$ & Modifications & Hyperscore & $\begin{array}{l}\text { Mass error } \\
\text { in ppm }\end{array}$ & Charge & $\begin{array}{c}\text { Precursor } \\
\text { neutral mass } \\
\text { in Da }\end{array}$ & Uniprot ID 1 & Uniprot ID 2 & Protein Name 1 & Protein Name 2 \\
\hline TKQTQTFTTY & SDNQPGVLIQVYEGER & $-0,4$ & N-ter +34.06 Da & 40,0 & $-1,467$ & 3 & 1836,9 & HS71A_MOUSE & HS71B_MOUSE & Heat shock $70 \mathrm{kDa}$ protein $1 \mathrm{~A}$; & Heat shock 70 kDa protein 3;Hsp68; \\
\hline IEDGIFEVKS & TAGDTHLGGEDFDNR & 1,5 & N-ter +34.06 Da & 24,8 & $-7,009$ & 3 & 1637,7 & HS71A_MOUSE & HS71B_MOUSE & Heat shock 70 kDa protein $1 \mathrm{~A} ;$ & Heat shock 70 kDa protein 3; Hsp 68 ; \\
\hline VMTVLIKRNT & TIPTKQTQTFTTYSDNQPGVLIQVYEGER & 1,3 & $\mathrm{~N}$-ter +28.03 Da, $\mathrm{K}+28.03 \mathrm{Da}$ & 21,3 & $-5,573$ & 4 & 3369,7 & HS71A_MOUSE & HS71B_MOUSE & Heat shock $70 \mathrm{kDa}$ protein $1 \mathrm{~A}$; & Heat shock 70 kDa protein 3;Hsp68; \\
\hline AYFNDSQRQA & TKDAGVIAGLNVLR & 1,8 & N-ter +28.03 Da, $\mathrm{K}+28.03 \mathrm{Da}$ & 22,6 & 2,559 & 3 & 1481,9 & HS71A_MOUSE & HS71B_MOUSE & Heat shock $70 \mathrm{kDa}$ protein $1 \mathrm{~A}$; & Heat shock 70 kDa protein 3;Hsp68; \\
\hline KRNTTIPTKQ & TQTFTTYSDNQPGVLIQVYYGER & 1,7 & $\mathrm{~N}$-ter +28.03 Da & 38,0 & $-5,418$ & 3 & 2673,3 & HS71A_MOUSE & HS71B_MOUSE & Heat shock $70 \mathrm{kDa}$ protein $1 \mathrm{~A}$; & Heat shock 70 kDa protein 3;Hsp68; \\
\hline LLDVAPLSL & GLETAGGVMTVLIKR & $-0,1$ & N-ter +34.06 Da, K +34.06 Da & 22,4 & 1,115 & 3 & 1612,0 & HS71L_MOUSE & HSP7C_MOUSE & Heat shock 70 kDa protein 1 -like; & $\begin{array}{l}\text { Heat shock } 70 \text { kDa-like protein 1;Spermatid- } \\
\text { specific heat shock protein 70; }\end{array}$ \\
\hline LIGDAAKNQV & AMNPTNTVFDAKR & $-0,4$ & N-ter +34.06 Da, K+34.06 Da & 20,8 & $-4,886$ & 3 & 1531,8 & HSP7C_MOUSE & Q504P4_MOUSE & Heat shock cognate 71 kDa protein; & Heat shock 70 kDa protein 8; \\
\hline VPAYFNDSQR & QATKDAGTIAGLNVLR & 3,5 & $\mathrm{~N}$-ter $+28.03 \mathrm{Da}, \mathrm{K}+28.03 \mathrm{Da}$ & 21,3 & $-8,897$ & 3 & 1683,0 & HSPFC_MOUSE & & Heat shock cognate 71 kDa protein; & Heat shock 70 kDa protein 8 ; \\
\hline NTTIPTKQTQ & TFTTYSDNQPGVLIOVYEGER & 2,7 & $\mathrm{~N}$-ter +28.03 Da & 23,3 & 4,699 & 3 & 2444,2 & HSP7C_MOUSE & HS71A_MOUSE & Heat shock cognate 71 kDa protein; & Heat shock $70 \mathrm{kDa}$ protein 8 \\
\hline EELNADLFRG & TLDPVEKALR & 1,6 & N-ter +28.03 Da, K+28.03 Da & 32,3 & $-1,417$ & 3 & 1196,7 & HSP7C_MOUSE & Q504P4_MOUSE & Heat shock cognate 71 kDa protein; & Heat shock $70 \mathrm{kDa}$ protein 8 \\
\hline GVMTVLIKRN & TTIPTKQTQTFTYYSDNQPGVLIQVYYGER & 0,5 & N-ter +34.06 Da, K+34.06 Da & 16,6 & $-2,811$ & 4 & 3482,8 & HSP7C_MOUSE & Q504P4_MOUSE & Heat shock cognate 71 kDa protein; & Heat shock 70 kDa protein 8; \\
\hline TІРТКQTQTF & TTYSDNQPGVLIQVYEGER & 0,1 & N-ter +28.03 Da & 36,6 & $-0,955$ & 3 & 2196,1 & HSP7C_MOUSE & HS71A_MOUSE & Heat shock cognate 71 kDa protein; & Heat shock $70 \mathrm{kDa}$ protein 8; \\
\hline ASGISAGQLY & STQAAEDKEEESLHSIISNTEAVR & $-0,7$ & $\mathrm{~N}$-ter +28.03 Da, K +28.03 Da & 20,2 & 2,035 & 4 & 2699,3 & TRAP1_MOUSE & & Heat shock protein $75 \mathrm{kDa}$, mitochondrial; & $\begin{array}{l}\text { TNFR-associated protein 1;Tumor necrosis } \\
\text { factor type } 1 \text { receptor-associated protein; }\end{array}$ \\
\hline LPDEWSQWFS & AAGWPGYVRPLPAATAEGPAAVTLAAPAFSR & $-0,5$ & N-ter +34.06 Da & 26,5 & 2,148 & 3 & 3069,7 & HSPB1_MOUSE & D3YZO6_MOUSE & Heat shock protein beta-1; & $\begin{array}{l}\text { Growth-related } 25 \text { kDa protein;Heat shock } 25 \\
\text { kDa protein;Heat shock } 27 \text { kDa protein;p25; }\end{array}$ \\
\hline TAEGPAAVTL & AAPAFSR & $-0,5$ & $\mathrm{~N}$-ter +28.03 Da & 18,2 & $-0,935$ & 2 & 746,4 & HSPB1_MOUSE & D3YZ06_MOUSE & Heat shock protein beta-1; & $\begin{array}{l}\text { Growth-related } 25 \text { kDa protein;Heat shock } 25 \\
\text { kDa protein;Heat shock } 27 \text { kDa protein;;225; }\end{array}$ \\
\hline GYVRPLPAAT & AEGPAAVTLAAPAFSR & $-0,3$ & $\mathrm{~N}$-ter +28.03 Da & 56,7 & $-0,835$ & 2 & 1555,8 & HSPB1_MOUSE & D3YZ06_MOUSE & Heat shock protein beta-1; & $\begin{array}{l}\text { Growth-related } 25 \text { kDa protein;Heat shock } 25 \\
\text { kDa protein;Heat shock } 27 \text { kDa protein;;225; }\end{array}$ \\
\hline PDEWSQWFSA & AGWPGYVRPLPAATAEGPAAVTLAAPAFSR & $-0,4$ & $\mathrm{~N}$-ter $+34.06 \mathrm{Da}$ & 25,3 & 0,833 & 3 & 2998,6 & HSPB1_MOUSE & D3YZ06_MOUSE & Heat shock protein beta-1; & $\begin{array}{l}\text { Growth-related } 25 \text { kDa protein;Heat shock } 25 \\
\text { kDa protein; Heat shock } 27 \text { kDa protein;;25; }\end{array}$ \\
\hline YVRPLPAATA & EGPAAVTLAAPAFSR & $-0,4$ & $\mathrm{~N}$-ter $+28.03 \mathrm{Da}$ & 26,7 & $-8,945$ & 2 & 1484,8 & HSPB1_MOUSE & D3YZ06_MOUSE & Heat shock protein beta-1; & $\begin{array}{l}\text { Growth-related } 25 \text { kDa protein;Heat shock } 25 \\
\text { kDa protein;Heat shock } 27 \text { kDa protein;p25; }\end{array}$ \\
\hline APEELTVKTK & EGVVETTGKHEER & $-0,9$ & $\mathrm{~N}$-ter +28.03 Da, K+28.03 Da & 16,5 & 0,324 & 4 & 1537,8 & HSPB1_MOUSE & D3YZ06_MOUSE & Heat shock protein beta-1; & $\begin{array}{l}\text { Growth-related } 25 \text { kDa protein;Heat shock } 25 \\
\text { kDa protein;Heat shock } 27 \text { kDa protein;p25; }\end{array}$ \\
\hline RDWYPAHSRL & FDQAFGVPR & $-0,6$ & $\mathrm{~N}$-ter +28.03 Da & 25,5 & $-0,094$ & 2 & 1063,6 & HSPB1_MOUSE & D3YZ06_MOUSE & Heat shock protein beta-1; & $\begin{array}{l}\text { Growth-related } 25 \text { kDa protein;Heat shock } 25 \\
\text { kDa protein;Heat shock } 27 \text { kDa protein;;25; }\end{array}$ \\
\hline PEELTVKTKE & GVVEITGKHEER & $-0,5$ & N-ter +34.06 Da, K+34.06 Da & 18,2 & 4,706 & 3 & 1420,8 & HSPB1_MOUSE & D3YZ06_MOUSE & Heat shock protein beta-1; & $\begin{array}{l}\text { Growth-related } 25 \text { kDa protein;Heat shock } 25 \\
\text { kDa protein;Heat shock } 27 \text { kDa protein;p25; }\end{array}$ \\
\hline DEWSQWFSAA & GWPGYVRPLPAATAEGPAAVTLAAPAFSR & $-0,4$ & $\mathrm{~N}$-ter $+34.06 \mathrm{Da}$ & 19,8 & 0,273 & 3 & 2927,6 & HSPB1_MOUSE & D3YZ06_MOUSE & Heat shock protein beta-1; & $\begin{array}{l}\text { Growth-related } 25 \text { kDa protein;Heat shock } 25 \\
\text { kDa protein;Heat shock } 27 \text { kDa protein;p25; }\end{array}$ \\
\hline AAGWPGYVRP & LPAATAEGPAAVTLAAPAFSR & 0,6 & $\mathrm{~N}$-ter $+28.03 \mathrm{Da}$ & 19,7 & $-8,200$ & 3 & 2009,1 & HSPB1_MOUSE & D3YZ06_MOUSE & Heat shock protein beta-1; & $\begin{array}{l}\text { Growth-related } 25 \text { kDa protein;Heat shock } 25 \\
\text { kDa protein;Heat shock } 27 \text { kDa protein;;25; }\end{array}$ \\
\hline LAAPAFSRAL & NRQLSSGVSEIR & $-1,1$ & N-ter +34.06 Da & 39,6 & $-2,026$ & 3 & 1378,8 & HSPB1_MOUSE & D3YZO6_MOUSE & Heat shock protein beta-1; & $\begin{array}{l}\text { Growth-related } 25 \text { kDa protein;Heat shock } 25 \\
\text { kDa protein;Heat shock } 27 \text { kDa protein;p25; }\end{array}$ \\
\hline PFRDWYPAHS & RLFDQAFGVPR & $-0,3$ & $\mathrm{~N}$-ter $+34.06 \mathrm{Da}$ & 16,5 & $-0,596$ & 3 & 1338,8 & HSPB1_MOUSE & D3YZ06_MOUSE & Heat shock protein beta-1; & $\begin{array}{l}\text { Growth-related } 25 \text { kDa protein;Heat shock } 25 \\
\text { kDa protein;Heat shock } 27 \text { kDa protein;;25; }\end{array}$ \\
\hline TERRVPFSLL & RSPSWEPFR & $-0,4$ & N-ter +34.06 Da & 18,6 & $-0,585$ & 3 & 1194,6 & HSPB1_MOUSE & D3YZ06_MOUSE & Heat shock protein beta-1; & $\begin{array}{l}\text { Growth-related } 25 \text { kDa protein;Heat shock } 25 \\
\text { kDa protein;Heat shock } 27 \text { kDa protein;p25; }\end{array}$ \\
\hline PGYVRPLPAA & TAEGPAAVTLAAPAFSR & 0,8 & N-ter +34.06 Da & 45,6 & $-2,222$ & 2 & 1662,9 & HSPB1_MOUSE & D3YZO6_MOUSE & Heat shock protein beta-1; & $\begin{array}{l}\text { Growth-related } 25 \text { kDa protein; Heat shock } 25 \\
\text { kDa protein;HHeat shock } 27 \text { kDa protein;p25; }\end{array}$ \\
\hline KVEKVVVSNR & LVTSPCCIVTSTYGWTANMER & 3,7 & N-ter +28.03 Da, C +57.02 Da & 13,7 & $-2,181$ & 3 & 2473,1 & HS90A_MOUSE & & Heat shock protein HSP 90-alpha; & $\begin{array}{l}\text { Heat shock } 86 \text { kDa;Tumor-specific } \\
\text { transplantation } 86 \text { kDa antigen; }\end{array}$ \\
\hline LFETALLSSG & FSLEDPQTHSNR & $-0,8$ & $\mathrm{~N}$-ter $+34.06 \mathrm{Da}$ & 16,8 & $-9,204$ & 3 & 1463,7 & HS9OB_MOUSE & & Heat shock protein HSP 90-beta; & $\begin{array}{l}\text { Heat shock } 84 \text { kDa;Tumor-specific } \\
\text { transplantation } 84 \text { kDa antigen; }\end{array}$ \\
\hline
\end{tabular}




\begin{tabular}{|c|c|c|c|c|c|c|c|c|c|c|c|}
\hline Non Prime Site & Prime Site & $\begin{array}{l}\text { Fold change of } \\
\text { XPRESS ratio } \\
\text { (Adam17 } 7^{\text {NKC }} / \mathrm{WT} \text { ) }\end{array}$ & Modifications & Hyperscore & $\begin{array}{l}\text { Mass error } \\
\text { in ppm }\end{array}$ & Charge & $\begin{array}{c}\text { Precursor } \\
\text { neutral mass } \\
\text { in Da }\end{array}$ & Uniprot ID 1 & Uniprot ID 2 & Protein Name 1 & Protein Name 2 \\
\hline ELPEYYLNFI & RGVVDSEDLPLNISR & 0,0 & $\mathrm{~N}$-ter +34.06 Da & 21,5 & $-8,500$ & 3 & 1702,9 & HS9OB_MOUSE & HS90A_MOUSE & Heat shock protein HSP 90-beta; & $\begin{array}{l}\text { Heat shock } 84 \text { kDa; Tumor-specific } \\
\text { transplantation } 84 \text { kDa antigen; }\end{array}$ \\
\hline VVKKHSQFIG & YPITLYLEKER & $-0,5$ & N-ter +34.06 Da, K +34.06 Da & 22,7 & $-2,341$ & 3 & 1491,9 & HS9OB_MOUSE & & Heat shock protein HSP 90-beta; & $\begin{array}{l}\text { Heat shock } 84 \text { kDa; Tumor-specific } \\
\text { transplantation } 84 \text { kDa antigen; }\end{array}$ \\
\hline NVDTDFQANL & AQMEEKPVPAAPVPSPVAPAPVPSR & $-0,8$ & $\mathrm{~N}$-ter $+28.03 \mathrm{Da}, \mathrm{K}+28.03 \mathrm{Da}$ & 27,4 & $-0,426$ & 3 & 2577,4 & HN1_MOUSE & & Hematological and neurological expressed 1 protein; & \\
\hline TDFQANLAQM & EEKPVPAAPVPSPVAPAPVPSR & $-0,7$ & N-ter +34.06 Da, K+34.06 Da & 34,9 & 2,166 & 3 & 2259,3 & HN1_MOUSE & & Hematological and neurological expressed 1 protein; & \\
\hline PGGGSNFSLG & FDEPAEQPVR & $-0,2$ & N-ter +34.06 Da & 16,0 & $-1,636$ & 2 & 1220,6 & HN1_MOUSE & & Hematological and neurological expressed 1 protein; & \\
\hline LRPPGGGSNF & SLGFDEPAEQPVR & $-0,4$ & N-ter +34.06 Da & 41,4 & $-2,163$ & 2 & 1477,8 & HN1_MOUSE & & Hematological and neurological expressed 1 protein; & \\
\hline $\begin{array}{l}\text { SGEDKSNIIA } \\
\text { GEDKSNIKAA }\end{array}$ & $\begin{array}{l}\text { AWGKIGGHGAEYGAEALER } \\
\text { WGKIGGHGAEYGAEALER }\end{array}$ & $\begin{array}{l}-0,5 \\
-0,5\end{array}$ & $\begin{array}{l}\mathrm{N} \text {-ter }+34.06 \mathrm{Da}, \mathrm{K}+34.06 \mathrm{Da} \\
\mathrm{N} \text {-ter }+34.06 \mathrm{Da}, \mathrm{K}+34.06 \mathrm{Da}\end{array}$ & $\begin{array}{l}41,0 \\
24,2\end{array}$ & $\begin{array}{l}-0,489 \\
1,369\end{array}$ & $\begin{array}{l}4 \\
4\end{array}$ & $\begin{array}{l}2039,1 \\
1968,1\end{array}$ & $\begin{array}{l}\text { HBA_MOUSE } \\
\text { HBA_MOUSE }\end{array}$ & $\begin{array}{l}\text { Q91VB8_MOUSE } \\
\text { Q91VB8_MOUSE }\end{array}$ & $\begin{array}{l}\text { Hemoglobin subunit alpha; } \\
\text { Hemoglobin subunit alpha; }\end{array}$ & $\begin{array}{l}\text { Alpha-globin;Hemoglobin alpha chain; } \\
\text { Alpha-globin;Hemoglobin alpha chain; }\end{array}$ \\
\hline TFASLSELHC & DKLHVDPENFR & $-0,2$ & N-ter +34.06 Da, K+34.06 Da & 17,9 & $-0,625$ & 3 & 1436,8 & HBB1_MOUSE & HBB2_MOUSE & Hemoglobin subunit beta-1; & $\begin{array}{l}\text { Beta-1-globin;Hemoglobin beta-1 } \\
\text { chain;Hemoglobin beta-major chain; }\end{array}$ \\
\hline DAEKAAVSCL & WGKVNSDEVGGEALGR & 1,5 & N-ter +28.03 Da, $\mathrm{K}+28.03$ Da & 24,8 & 1,963 & 3 & 1728,9 & HBB1_MOUSE & & Hemoglobin subunit beta-1; & $\begin{array}{l}\text { Beta-1-globin;Hemoglobin beta-1 } \\
\text { chain;Hemoglobin beta-major chain; }\end{array}$ \\
\hline EKSAVSCLWA & KVNPDEVGGEALGR & 2,2 & N-ter +28.03 Da, $\mathrm{K}+28.03$ Da & 24,5 & $-1,068$ & 3 & 1495,8 & HBB2_MOUSE & & Hemoglobin subunit beta-2; & $\begin{array}{l}\text { Beta-2-globin;Hemoglobin beta-2 } \\
\text { chain;Hemoglobin beta-minor chain; }\end{array}$ \\
\hline EKVGGETLGR & LIVYPWTQR & 10,3 & N-ter +28.03 Da & 16,6 & $-1,973$ & 2 & 1315,8 & HBBO_MOUSE & HBBZ_MOUSE & Hemoglobin subunit beta-HO; & Beta-HO-globin;Hemoglobin beta-HO chain; \\
\hline CTYLCYSFLC & CCDGLGQSR & $-3,3$ & N-ter +34.06 Da, C+57.02 Da & 14,6 & 6,713 & 2 & 1085,5 & HS3S2_MOUSE & & Heparan sulfate glucosamine 3-O-sulfotransferase 2; & $\begin{array}{l}\text { Heparan sulfate D-glucosaminyy 3-O- } \\
\text { sulfotransferase 2; }\end{array}$ \\
\hline KDLKDYFTKF & GEVVDCTIKMDPNTGR & $-0,3$ & $\begin{array}{l}\text { N-ter +34.06 Da, K +34.06 Da, C } \\
+57.02 \mathrm{Da}\end{array}$ & 19,4 & 1,289 & 3 & 1859,0 & ROAA_MOUSE & Q80XR6_MOUSE & Heterogeneous nuclear ribonucleoprotein $\mathrm{A} / \mathrm{B}$; & CArG-binding factor-A; \\
\hline $\begin{array}{l}\text { PEQLRKLFIG } \\
\text { KTKEPVKKIF }\end{array}$ & $\begin{array}{l}\text { GLSFETDDSLR } \\
\text { VGGLSPDTPEEKIR }\end{array}$ & $\begin{array}{r}0,1 \\
-2,3\end{array}$ & $\begin{array}{l}\text { N-ter +28.03 Da } \\
\text { N-ter +34.06 Da, K +34.06 Da }\end{array}$ & $\begin{array}{l}16,4 \\
23,7\end{array}$ & $\begin{array}{l}-0,657 \\
-7,335\end{array}$ & $\begin{array}{l}2 \\
3\end{array}$ & $\begin{array}{l}1367,7 \\
1564,9\end{array}$ & $\begin{array}{l}\text { ROA3_MOUSE } \\
\text { HNRPD_MOUSE }\end{array}$ & $\begin{array}{l}\text { B2RXM2_MOUSE } \\
\text { G3X9W0_MOUSE }\end{array}$ & $\begin{array}{l}\text { Heterogeneous nuclear ribonucleoprotein } \mathrm{A3} \text {; } \\
\text { Heterogeneous nuclear ribonucleoprotein } \mathrm{DO} \text {; }\end{array}$ & AU-rich element RNA-binding protein 1; \\
\hline KDLTEYLSRF & GEVVDCTIKTDPVTGR & $-0,3$ & N-ter +34.06 Da, K+34.06 Da, C & 18,7 & 0,881 & 3 & 1814,0 & HNRDL_MOUSE & F6VQH5_MOUSE & Heterogeneous nuclear ribonucleoprotein D-like; & JKT41-binding protein; \\
\hline & MLGPEGGEGYVVKLR & $-0,9$ & $\mathrm{~N}$-ter +28.03 Da, K+28.03 Da & 42,1 & $-1,864$ & 3 & 1659,9 & HNRPF_MOUSE & J3QMQ5_MOUSE & Heterogeneous nuclear ribonucleoprotein F; & \\
\hline GGQRIKQIRH & ESGASIIIDEPLEGSEDR & $\begin{array}{l}-0,7 \\
-10\end{array}$ & N-ter +34.06 Da, K+34.06 Da & 21,4 & 9,740 & 3 & 1999,1 & HNRPK_MOUSE & B2MIRG_MOUSE & Heterogeneous nuclear ribonucleoprotein $\mathrm{K}$; & \\
\hline GQRIKQIRHE & SGASIKIDEPLEGSEDR & $-0,1$ & $\mathrm{~N}$-ter +34.06 Da, $\mathrm{K}+34.06 \mathrm{Da}$ & 17,6 & 1,175 & 3 & 1870,0 & HNRPK_MOUSE & B2M1R6_MOUSE & Heterogeneous nuclear ribonucleoprotein K; & \\
\hline IKQIRHESGA & SIKIDEPLEGSEDR & 0,3 & $\mathrm{~N}$-ter +28.03 Da, $\mathrm{K}+28.03 \mathrm{Da}$ & 20,1 & $-2,187$ & 3 & 1642,8 & HNRPK_MOUSE & B2M1R6_MOUSE & Heterogeneous nuclear ribonucleoprotein $\mathrm{K}$; & \\
\hline LGGPIITTQV & TIPKDLAGSIIGKGGQR & $-1,0$ & $\mathrm{~N}$-ter +34.06 Da, K+34.06 Da & 37,0 & $-5,785$ & 3 & 1812,2 & HNRPK_MOUSE & B2M1R6_MOUSE & Heterogeneous nuclear ribonucleoprotein $\mathrm{K}$; & \\
\hline HHLRDYFEEY & GKIDTIEITDR & 2,2 & N-ter +34.06 Da, K+34.06 Da & 21,0 & $-3,186$ & 3 & 1440,9 & ROA2_MOUSE & & Heterogeneous nuclear ribonucleoproteins $A 2 / B 1$; & \\
\hline GHNAEVRKAL & SRQEMQEVQSSR & $-0,9$ & N-ter +34.06 Da & 30,2 & 1,599 & 3 & 1497,7 & ROA2_MOUSE & & Heterogeneous nuclear ribonucleoproteins A2/B1; & \\
\hline ELSPKCTVSF & LLSEDGSGKGAALITAVGVR & 0,0 & $\mathrm{~N}$-ter $+28.03 \mathrm{Da}, \mathrm{K}+28.03 \mathrm{Da}$ & 24,1 & $-1,521$ & 3 & 1969,1 & HXK1_MOUSE & G3UVV4_MOUSE & Hexokinase-1; & Hexokinase type l;Hexokinase, tumor isozyme; \\
\hline MADEIAK & AQVAQPGGDTIFGKIIR & 0,5 & N-ter +34.06 Da, K+34.06 Da & 22,9 & 1,467 & 3 & 1838,1 & HINT1_MOUSE & BOR1E3_MOUSE & Histidine triad nucleotide-binding protein $1 ;$ & $\begin{array}{l}\text { Adenosine 5'-monophosphoramidase; Protein } \\
\text { kinase C inhibitor 1; Protein kinase C-interacting } \\
\text { protein 1; }\end{array}$ \\
\hline DTIFGKIIRK & EIPAKIIFEDDR & $-0,2$ & N-ter +28.03 Da, $\mathrm{K}+28.03 \mathrm{Da}$ & 17,6 & 2,926 & 3 & 1500,8 & HINT1_MOUSE & BOR1E3_MOUSE & Histidine triad nucleotide-binding protein 1; & 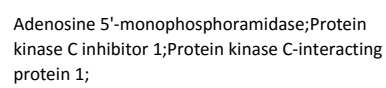 \\
\hline LVTLQCSHAL & SPTNCDASEPLAEKVLDLINKGR & 0,0 & $\begin{array}{l}\text { N-ter +28.03 Da, K+28.03 Da, C } \\
+57.02 \mathrm{Da}\end{array}$ & 47,0 & 0,689 & 4 & 2610,4 & HRG_MOUSE & & Histidine-rich glycoprotein; & Histidine-proline-rich glycoprotein; \\
\hline QLYGRSPPAV & AMQAGPR & 0,5 & N-ter +34.06 Da & 16,8 & 7,185 & 2 & 763,4 & KAT6A_MOUSE & G3X940_MOUSE & Histone acetyltransferase KATGA; & $\begin{array}{l}\text { MOZ, YBF2/SAS3, SAS2 and TIP60 protein } \\
\text { 3;Monocytic leukemia zinc finger } \\
\text { homolog; Monocytic leukemia zinc finger } \\
\text { protein; }\end{array}$ \\
\hline KVDENFDCVE & ADDVEGKIR & 2,8 & N-ter +28.03 Da, $\mathrm{K}+28.03 \mathrm{Da}$ & 17,1 & $-3,492$ & 2 & 1057,6 & HAT1_MOUSE & A2ATU9_MOUSE & Histone acetyltransferase type $B$ catalytic subunit; & Histone acetyltransferase $1 ;$ \\
\hline YMAAVLEYLT & AEILELAGNAAR & $-0,1$ & N-ter +28.03 Da & 28,2 & $-6,525$ & 2 & 1254,7 & H2A1F_MOUSE & H2A1H_MOUSE & Histone H2A type 1-F; & \\
\hline PRHLQLA & DEELNK & $-0,3$ & $\mathrm{~N}$-ter $+28.03 \mathrm{Da}, \mathrm{K}+28.03 \mathrm{Da}$ & 35 & $-3,7$, & 2 & 124 & H2A1F_MOUSE & H2A1H & 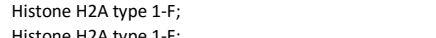 & \\
\hline RHLQLAIR & EELNKL & -0, & $\mathrm{~N}$-ter $+28.03 \mathrm{Da}, \mathrm{K}+28.03 \mathrm{Da}$ & & $-5,8$ & 3 & 112 & H2A1F_MOUSE & H2A1 & Histone H2A typ & \\
\hline MAAVLEYLTA & EILELAGNAAR & $-1,3$ & $\mathrm{~N}$-ter +34.06 Da & & & 2 & 1189,7 & H2A1F_MOUSE & H2A1H_MOUSE & Histone H2A type 1-F; & \\
\hline $\begin{array}{l}\text { AGAPVYMAAV } \\
\text { VYMAAVLEYL }\end{array}$ & $\begin{array}{l}\text { LEYLTAEILELAGNAAR } \\
\text { TAEILELAGNAAR }\end{array}$ & $\begin{array}{l}-0,1 \\
-0,3\end{array}$ & $\begin{array}{l}\mathrm{N} \text {-ter }+34.06 \mathrm{Da} \\
\mathrm{N} \text {-ter }+34.06 \mathrm{Da}\end{array}$ & $\begin{array}{l}26,1 \\
418\end{array}$ & $\begin{array}{l}-0,584 \\
-0,087\end{array}$ & $\begin{array}{l}3 \\
2\end{array}$ & $\begin{array}{l}1880,1 \\
13618\end{array}$ & $\begin{array}{l}\text { H2A1F_MOUSE } \\
\text { H2A1F_MOUSE }\end{array}$ & $\begin{array}{l}\text { H2A1H_MOUSE } \\
\text { H2A1__MOUSE }\end{array}$ & $\begin{array}{l}\text { Histone } \mathrm{H} 2 \mathrm{~A} \text { type } 1-\mathrm{F} \\
\text { Histone H2A type } 1-\mathrm{F}\end{array}$ & \\
\hline
\end{tabular}




\begin{tabular}{|c|c|c|c|c|c|c|c|c|c|c|c|}
\hline Non Prime Site & Prime Site & 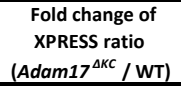 & Modifications & Hyperscore & $\begin{array}{c}\text { Mass error } \\
\text { in ppm }\end{array}$ & Charge & $\begin{array}{c}\text { Precursor } \\
\text { neutral mass } \\
\text { in Da }\end{array}$ & Uniprot ID 1 & Uniprot ID 2 & Protein Name 1 & Protein Name 2 \\
\hline$\overline{\text { GAGAPVYMAA }}$ & $\begin{array}{l}\text { VLEYLTAEILELAGNAAR } \\
\text { VFLALEGA }\end{array}$ & $-0,2$ & N-ter +28.03 Da & 28,6 & 0,455 & 3 & 1973,1 & H2A1F_MOUSE & H2A1H_MOUSE & Histone H2A type 1-F; & \\
\hline APVYMAAVLE & YLTAEILELAGNAAR & $-0,1$ & $\mathrm{~N}$-ter $+34.06 \mathrm{Da}$ & 16,2 & $-0,061$ & 3 & 1637,9 & H2A1F_MOUSE & H2A1H_MOUSE & Histone H2A type 1-F; & \\
\hline GISSKAMGIM & NSFVNDIFER & $-1,6$ & N-ter $+28.03 \mathrm{Da}$ & 21,2 & 0,000 & 2 & 1267,6 & H2B1B_MOUSE & H2B1C_MOUSE & Histone H2B type 1-B; & h2B-143; \\
\hline RKLPFQRLVR & EIAQDFKTDLR & $-0,3$ & $\mathrm{~N}$-ter +34.06 Da, K+34.06 Da & 18,7 & $-1,138$ & 3 & 1402,8 & H31_MOUSE & H32_MOUSE & Histone H3.1; & \\
\hline EASEAYLVGL & FEDTNLCAIHAKR & $-1,1$ & $\begin{array}{l}\text { N-ter +34.06 Da, K+34.06 Da, C } \\
\text { +57.02 Da }\end{array}$ & 19,2 & $-1,701$ & 4 & 1641,9 & H31_MOUSE & H32_MOUSE & Histone H3.1; & \\
\hline GAKRHRKVLR & DNIQGITKPAIR & $-0,8$ & N-ter +34.06 Da, K+34.06 Da & 21,1 & $-0,358$ & 3 & 1392,9 & H4_MOUSE & & Histone $\mathrm{H} 4$; & \\
\hline GGAKRHRKVL & RDNIQGITKPAIR & $-0,5$ & $\mathrm{~N}$-ter +34.06 Da, K +34.06 Da & 19,1 & $-1,996$ & 4 & 1549,0 & H4_MOUSE & & Histone $\mathrm{H}_{4}$; & \\
\hline RRLARRGGVK & RISGLIYEETR & $-3,8$ & $\mathrm{~N}$-ter +34.06 Da & 27,0 & 0,000 & 2 & 1369,8 & H4_MOUSE & & Histone $\mathrm{H}_{4}$; & \\
\hline LARRGGVKRI & SGLIYEETR & 0,1 & $\mathrm{~N}$-ter +34.06 Da & 22,5 & 4,081 & 2 & 1100,6 & H4_MOUSE & & Histone $\mathrm{H} 4$; & \\
\hline EFHQILKNPD & DPDTVDIIMQNLDR & $-0,3$ & $\mathrm{~N}$-ter +34.06 Da & 25,7 & $-0,357$ & 2 & 1677,9 & HORN_MOUSE & E9QNP3_MOUSE & Hornerin; & \\
\hline STQWSHGSGS & EQSSGLGHYGSTSGQTASSTR & 0,0 & N-ter +34.06 Da & 60,2 & $-0,562$ & 3 & 2131,0 & HORN_MOUSE & HORN_MOUSE & Hornerin; & \\
\hline SGQNYNSTRQ & GCGQGKSSGSEQYGASSGQSSGCSSGQSTR & $-1,3$ & $\begin{array}{l}\text { N-ter +28.03 Da, K+28.03 Da, C } \\
+57.02 \mathrm{Da}\end{array}$ & 60,0 & $-6,534$ & 3 & 2981,2 & HORN_MOUSE & EgQNP3_MOUSE & Hornerin; & \\
\hline YNSTRQGCGQ & GKSSGSEQYGASSGQSSGCSSGQSTR & 0,8 & $\begin{array}{l}\text { N-ter +34.06 Da, K +34.06 Da, C } \\
+57.02 \mathrm{Da}\end{array}$ & 47,8 & $-4,433$ & 3 & 2591,2 & HORN_MOUSE & E9QNP3_MOUSE & Hornerin; & \\
\hline QNYNSTRQGC & GQGKSSGSEQYGASSGQSSGCSSGQSTR & 0,4 & N-ter +34.06 Da, K+34.06 Da, C & 34,7 & 3,130 & 3 & 2776,3 & HORN_MOUSE & E9QNP3_MOUSE & Hornerin; & \\
\hline SQSSSTQWSH & GSGSEQSSGLGHYGSTSGQTASSTR & $-0,2$ & $\begin{array}{l}5 / .02 \mathrm{Da} \\
\mathrm{N}-\mathrm{ter}+28.03 \mathrm{Da}\end{array}$ & 44,3 & $-6,829$ & 3 & 2413,1 & HORN_MOUSE & HORN_MOUSE & Hornerin; & \\
\hline RSRSTSRESS & GSQEYSSGSSEEPGFTHGSGR & $-1,1$ & $\mathrm{~N}-\mathrm{ter}+34.06 \mathrm{Da}$ & 41,1 & $-6,196$ & 3 & 2175,9 & HORN_MOUSE & E9QNP3_MOUSE & Hornerin; & \\
\hline NSTRQGCGQG & KSSGSEQYGASSGQSSGCSSGQSTR & $-2,0$ & $\begin{array}{l}\text { N-ter +28.03 Da, } \mathrm{K}+28.03 \mathrm{Da}, \mathrm{C} \\
+57.02 \mathrm{Da}\end{array}$ & 25,9 & $-6,534$ & 3 & 2522,1 & HORN_MOUSE & E9QNP3_MOUSE & Hornerin; & \\
\hline TSGQNYNSTR & QGCGQGKSSGSEQYGASSGQSSGCSSGQSTR & 10,3 & N-ter +28.03 Da, K+28.03 Da, C & 36,9 & $-2,667$ & 3 & 3109,3 & HORN_MOUSE & E9QNP3_MOUSE & Hornerin; & \\
\hline SSTQWSHGSG & SEOSSGLGHYGSTSGQTASSTR & 0,6 & $\begin{array}{l}+57.02 \mathrm{Da} \\
\mathrm{N} \text {-ter }+28.03 \mathrm{Da}\end{array}$ & 72,0 & $-1,625$ & 3 & 2212,0 & HORN_MOUSE & HORN_MOUSE & Hornerin; & \\
\hline SSTQWSHGSG & SEQSSGLGQYGSPSGQTSSSTRQGSGQGQASGSGR & $-0,1$ & $\mathrm{~N}$-ter +28.03 Da & 37,0 & $-7,641$ & 4 & 3372,5 & HORN_MOUSE & E9QNP3_MOUSE & Hornerin; & \\
\hline QSSSTQWSHG & SGSEQSSGLGHYGSTSGQTASSTR & 1,0 & $\mathrm{~N}$-ter +28.03 Da & 73,7 & $-2,416$ & 3 & 2356,1 & HORN_MOUSE & HORN_MOUSE & Hornerin; & \\
\hline RRSRSTSRES & SGSQEYSSGSSEEPGFTHGSGR & 0,1 & $\mathrm{~N}$-ter $+28.03 \mathrm{Da}$ & 63,1 & $-2,832$ & 3 & 2257,0 & HORN_MOUSE & E9QNP3_MOUSE & Hornerin; & \\
\hline KKTHVSGSGH & SSSTGKYTSTSGQNYNSTR & 0,1 & $\mathrm{~N}$-ter +28.03 Da, K+28.03 Da & 46,3 & $-3,983$ & 3 & 2081,0 & HORN_MOUSE & E9QNP3_MOUSE & Hornerin; & \\
\hline TISKIKGLVR & APQVYILPPPAEQLSR & $-0,1$ & N-ter +34.06 Da & 33,5 & 5,072 & 2 & 1812,1 & IGG2B_MOUSE & & Ig gamma-2B chain C region; & \\
\hline RTISKIKGLV & RAPQVYILPPPAEQLSR & 0,2 & $\mathrm{~N}$-ter +28.03 Da & 42,3 & $-3,715$ & 3 & 1962,1 & IGG2B_MOUSE & & Ig gamma-2B chain C region; & \\
\hline ERTISKIKGL & VRAPQVYILPPPAEQLSR & 0,3 & $\mathrm{~N}$-ter +34.06 Da & 43,0 & $-8,453$ & 3 & 2067,2 & IGG2B_MOUSE & & Ig gamma-2B chain C region; & \\
\hline & IVLTQSPASLAVSLGQR & 0,3 & $\mathrm{~N}$-ter +34.06 Da & 45,4 & 0,053 & 3 & 1888,1 & KV3A1_MOUSE & KV3A2_MOUSE I & Ig kappa chain V-III region PC 2880/PC 1229; & \\
\hline LLFWIPASRG & DILLTQSPAILSVSPGER & $-1,5$ & $\mathrm{~N}$-ter +34.06 Da & 15,5 & $-0,880$ & 3 & 1929,1 & KV5A9_MOUSE & & Ig kappa chain V-V region L7; & \\
\hline D & IQMTQTTSSLSASLGDR & 0,3 & N-ter +34.06 Da & $\begin{array}{l}19,0 \\
19,0\end{array}$ & $\begin{array}{llll}-1,640 \\
-1,64\end{array}$ & 2 & 1944,0 & KV5AA_MOUSE & KV5AB_MOUSE I & $\begin{array}{l}\text { Ig kappa chain V-V region MOPC 173; } \\
\text { a }\end{array}$ & \\
\hline LLWFPGARC & DIQMTQSPSSLSASLGER & 0,1 & $\mathrm{~N}$-ter +34.06 Da & 22,7 & 0,000 & 2 & 1940,0 & KV5A7_MOUSE & KVSA8_MOUSE I & Ig kappa chain V-V region MOPC 41; & \\
\hline LLPLLAGAQA & AIVFIKEPSSQDALQGR & $-0,3$ & $\mathrm{~N}$-ter +34.06 Da, K+34.06 Da & 62,6 & $-0,052$ & 3 & 1926,1 & PTK7_MOUSE & & Inactive tyrosine-protein kinase 7; & $\begin{array}{l}\text { Protein chuzhoi;Protein-tyrosine kinase } \\
\text { 7;Pseudo tyrosine kinase receptor 7; Tyrosine- } \\
\text { protein kinase-like 7; }\end{array}$ \\
\hline SAVFSLLKAN & NPWPAGSSCCVPTAR & 0,4 & $\mathrm{~N}$-ter $+28.03 \mathrm{Da}, \mathrm{C}+57.02 \mathrm{Da}$ & 21,0 & 7,220 & 3 & 1686,8 & INHBE_MOUSE & & Inhibin beta Echain; & Activin beta-E chain; \\
\hline GFLAGRFSLF & GLGSEPAAGEAEVASDGGTVDLR & $-1,0$ & $\mathrm{~N}$-ter +34.06 Da & 23,4 & 2,780 & 3 & 2191,1 & IMPA3_MOUSE & & Inositol monophosphatase 3; & $\begin{array}{l}\text { Golgi 3-prime phosphoadenosine 5-prime } \\
\text { phosphate 3-prime phosphatase; inositol } \\
\text { monophosphatase domain-containing protein } \\
\text { 1;Inositol-1(or 4)-monophosphatase 3;Myo- } \\
\text { inositol monophosphatase A3; }\end{array}$ \\
\hline SIGDVFAGFR & AAAGVDSASAR & 1,4 & $\mathrm{~N}$-ter +34.06 Da & 16,6 & 2,474 & 2 & 1008,5 & IRS4_MOUSE & & Insulin receptor substrate 4; & Phosphoprotein of $160 \mathrm{kDa}$; \\
\hline FPSQNDINLS & EAPPLPQPEVIHNMTEFKR & 0,6 & $\mathrm{~N}$-ter +28.03 Da, K+28.03 Da & 20,5 & 5,322 & 4 & 2288,2 & IDE_MOUSE & F6RPJ9_MOUSE I & Insulin-degrading enzyme; & Insulin protease;|Insulysin; \\
\hline TMSNPAIQRI & EDQIVKSPEDKR & $-0,2$ & $\mathrm{~N}-\operatorname{ter}+34.06 \mathrm{Da}$, K +34.06 Da & 19,3 & $-5,233$ & 3 & 1544,9 & IDE_MOUSE & FGRPJ9_MOUSE II & Insulin-degrading enzyme; & Insulin protease:Insulysin; \\
\hline STSENMFLEL & FCQIISEPCFNTLR & -0,1 & $\mathrm{N}-\operatorname{ter}+28.03 \mathrm{Da}, \mathrm{C}+57.02 \mathrm{Da}$ & 22,4 & $-6,888$ & 3 & 1811,9 & IDE_MOUSE & FGRPJ9_MOUSE II & Insulin-degrading enzyme; & Insulin protease;|'Insulysin; \\
\hline $\begin{array}{l}\text { DDLTNLVYKL } \\
\text { DEL }\end{array}$ & FSEVENKNVPLPEFPEHPFQEEHLR & 0,1 & $\mathrm{~N}$-ter +34.06 Da, K +34.06 Da & 42,5 & $-1,955$ & 5 & 3115,6 & IDE_MOUSE & FGRPJ9_MOUSE II & Insulin-degrading enzyme; & Insulin protease;|IInulysin; \\
\hline $\begin{array}{l}\text { RANGIQGLRF } \\
\text { Res }\end{array}$ & 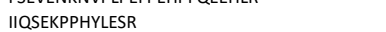 & 0,3 & $\mathrm{~N}$-ter +34.06 Da, K +34.06 Da & 21,7 & 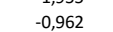 & 4 & 1764,0 & IDE_MOUSE & FGRPJ9_MOUSE II & Insulin-degrading enzyme; & Insulin protease;|IInulysin; \\
\hline ANGIQGLRFI & IQSEKPPHYLESR & 0,4 & $\mathrm{~N}$-ter $+34.06 \mathrm{Da}, \mathrm{K}+34.06 \mathrm{Da}$ & $\begin{array}{l}21,7 \\
17,7\end{array}$ & $\begin{array}{l}-3,686 \\
-306\end{array}$ & 4 & $\begin{array}{ll}1650,9 \\
1650,9\end{array}$ & IDE_MOUSE & FGRPJ9_MOUSE II & Insulin-degrading enzyme; & Insulin protease,'Insulysin; \\
\hline FQEEHLRQLY & KIVPIKDIR & 0,7 & $\mathrm{~N}$-ter +28.03 Da, K+28.03 Da & 18,5 & $\begin{array}{l}-2,398 \\
-2,398\end{array}-1$ & 3 & 1164,8 & IDE MOUSE & FGRPJ9 MOUSE II & Insulin-degrading enzyme; & Insulin protease:IInsulysin; \\
\hline $\begin{array}{l}\text { NGIQGLRFII } \\
\text { N }\end{array}$ & QSEKPPHYLESR & $-0,5$ & $\mathrm{~N}$-ter $+34.06 \mathrm{Da}, \mathrm{K}+34.06 \mathrm{Da}$ & $\begin{array}{l}10,2 \\
17,2\end{array}$ & $\begin{array}{l}-1,297 \\
-1,297\end{array}$ & 4 & $\begin{array}{l}1537,9 \\
1537,9\end{array}$ & IDE MOUSE & FGRPJ9 MOUSE II & Insulin-degrading enzyme; & Insulin protease; Insulysin; \\
\hline PLLPSQLVRY & REVQLPDR & 0,9 & $\mathrm{~N}$-ter $+34.06 \mathrm{Da}$ & 20,8 & 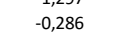 & 3 & 1045,6 & IDE MOUSE & FGRPJ9 MOUSE II & Insulin-degrading enzyme; & Insulin protease; Insulysin; \\
\hline EFPSQNDINL & SEAPPLPQPEVIHNMTEFKR & 0,3 & $\mathrm{~N}$-ter $+34.06 \mathrm{Da}, \mathrm{K}+34.06 \mathrm{Da}$ & $\begin{array}{l}2,0 \\
32,2\end{array}$ & $\begin{array}{l}-3,471 \\
-3,200\end{array}$ & 4 & 2387,3 & IDE MOUSE & FGRPJ9 MOUSE II & Insulin-degrading enzyme; & Insulin protease; Insulysin; \\
\hline 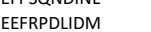 & VLDKLRPENVR & 0,1 & $\mathrm{~N}$-ter $+28.03 \mathrm{Da}, \mathrm{K}+28.03 \mathrm{Da}$ & 20,8 & $\begin{array}{l}-1,574 \\
-1,474\end{array}$ & 4 & $\begin{array}{l}1393,8 \\
1393,3\end{array}$ & IDE MOUSE & FGRPJ9 MOUSE II & Insulin-degrading enzyme; & Insulin protease; Insulysin; \\
\hline TLTKDDIIRF & YQEMLAVDAPR & 0,1 & N-ter +28.03 Da & 24,9 & $-0,227$ & 2 & 1319,7 & IDE_MOUSE & FGRPJ9_MOUSE I & Insulin-degrading enzyme; & Insulin protease;IInsulysin; \\
\hline $\begin{array}{l}\text { LLRGPPVARA } \\
\text { L }\end{array}$ & GAGAVGAGPVVR & 0,1 & $\mathrm{~N}$-ter $+28.03 \mathrm{Da}$ & 22,4 & $-0,289$ & 2 & 1037,6 & IBP3_MOUSE & & Insulin-like growth factor-binding protein 3; & \\
\hline LAAGPRPSLG & DEAIHCPPCSEEKLAR & $-0,5$ & $\begin{array}{l}\text { N-ter + 34.06 Da, K+34.06 Da, C } \\
\text { +57.02 Da }\end{array}$ & 27,2 & $-8,472$ & 4 & 1979,0 & IBP4_MOUSE & & Insulin-like growth factor-binding protein 4; & \\
\hline
\end{tabular}




\begin{tabular}{|c|c|c|c|c|c|c|c|c|c|c|c|}
\hline Non Prime Site & Prime Site & $\begin{array}{c}\text { Fold change of } \\
\text { XPRESS ratio } \\
\text { (Adam17 } 7^{\text {AKC } / W T)} \\
\end{array}$ & Modifications & Hyperscore & $\begin{array}{l}\text { Mass error } \\
\text { in ppm }\end{array}$ & Charge & $\begin{array}{c}\text { Precursor } \\
\text { neutral mass } \\
\text { in Da } \\
\end{array}$ & Uniprot ID 1 & Uniprot ID 2 & Protein Name 1 & Protein Name 2 \\
\hline RETIRGIQKR & EASNCFTIR & $-0,1$ & N-ter $+28.03 \mathrm{Da}, \mathrm{C}+57.02 \mathrm{Da}$ & 28,5 & 0,621 & 2 & 1124,5 & ITM2B_MOUSE & & Integral membrane protein $2 \mathrm{~B}$; & $\begin{array}{l}\text { Immature BRI2; Protein E25B; Transmembrane } \\
\text { protein BRI; }\end{array}$ \\
\hline DVYYCGLKYI & KDDVILNEPSADAPAAR & 0,4 & N-ter +34.06 Da, K+34.06 Da & 32,9 & 3,240 & 3 & 1849,0 & ITM2B_MOUSE & & Integral membrane protein 2B; & $\begin{array}{l}\text { Immature BRI2; Protein E25B; Transmembrane } \\
\text { protein BRI; }\end{array}$ \\
\hline $\begin{array}{l}\text { INSKNYFTSA } \\
\text { KRRLSFSETF }\end{array}$ & $\begin{array}{l}\text { AYPELFIATKEQSR } \\
\text { TEDDLOSITHDLEETIQPR }\end{array}$ & $\begin{array}{l}0,7 \\
0,0\end{array}$ & $\begin{array}{l}\mathrm{N} \text {-ter }+34.06 \mathrm{Da}, \mathrm{K}+34.06 \mathrm{Da} \\
\mathrm{N} \text {-ter +28.03 Da }\end{array}$ & $\begin{array}{l}37,5 \\
59,2\end{array}$ & $\begin{array}{r}8,416 \\
-0,529\end{array}$ & $\begin{array}{l}3 \\
3\end{array}$ & $\begin{array}{l}1720,0 \\
2267,1\end{array}$ & $\begin{array}{l}\text { IL1A_MOUSE } \\
\text { IL1A_MOUSE }\end{array}$ & & $\begin{array}{l}\text { Interleukin-1 alpha; } \\
\text { Interleukin-1 alpha; }\end{array}$ & \\
\hline MSKEEDSIWF & RPTLLQDSGLYACVIR & 1,0 & $\mathrm{~N}$-ter $+28.03 \mathrm{Da}, \mathrm{C}+57.02 \mathrm{Da}$ & 17,2 & $-0,793$ & 3 & 1889,0 & IRPL1_MOUSE & B1ASUO_MOUSE I & Interleukin-1 receptor accessory protein-like 1 ; & $\begin{array}{l}\text { X-linked interleukin-1 receptor accessory } \\
\text { protein-like 1: }\end{array}$ \\
\hline PQEPQGLLCL & EQQQQQEPQMQEQHLR & $-0,8$ & $\mathrm{~N}$-ter $+34.06 \mathrm{Da}$ & 47,6 & $-2,380$ & 3 & 2098,0 & INVO_MOUSE & G3X9D9_MOUSE । & Involucrin; & \\
\hline LAAHVAPRRS & ISSQQTIPPSAKYGGR & 0,2 & N-ter +28.03 Da, $\mathrm{K}+28.03 \mathrm{Da}$ & 16,4 & 1,602 & 3 & 1745,0 & IDHG1_MOUSE & & $\begin{array}{l}\text { Isocitrate dehydrogenase [NAD] subunit gamma 1, } \\
\text { mitochondrial; }\end{array}$ & $\begin{array}{l}\text { Isocitric dehydrogenase subunit gamma; } \mathrm{NAD}(+) \text {, } \\
\text { specific ICDH subunit gamma; }\end{array}$ \\
\hline EKLLLPYVEL & DLHSYDLGIENR & 0,3 & N-ter +28.03 Da & 19,2 & $-8,552$ & 3 & 1458,7 & IDHC_MOUSE & D3YVY3_MOUSE I & Isocitrate dehydrogenase [NADP] cytoplasmic; & $\begin{array}{l}\text { Cytosolic NADP-isocitrate } \\
\text { dehydrogenase,jIDPFNADP(+)-specific } \\
\text { ICDH;Oxalosuccinate decarboxylase; } \\
\text { Cytosolic NADP-isocitrate }\end{array}$ \\
\hline LLPYVELDL & HSYDLGIENR & 0,1 & N-ter +34.06 Da & 17,9 & 5,405 & 3 & 1236,6 & IDHC_MOUSE & D3YVY__MOUSE & Isocitrate dehydrogenase [NADP] cytoplasmic; & $\begin{array}{l}\text { dehydrogenase;:IDP;NADPP(+)-specific } \\
\text { ICDH;Oxalosuccinate decarboxylase; } \\
\text { Cytosolic NADP-isocitrate }\end{array}$ \\
\hline TSVLICPDGK & TVEAEAAHGTVTR & $-0,4$ & $\mathrm{~N}$-ter +28.03 Da & 18,1 & $-1,385$ & 3 & 1368,7 & IDHC_MOUSE & & Isocitrate dehydrogenase [NADP] cytoplasmic; & $\begin{array}{l}\text { dehydrogenase;IDP;NADP(+)-specific } \\
\text { ICDH;Oxalosuccinate decarboxylase; }\end{array}$ \\
\hline LLHRAFSVFL & FNTENKLLLQQR & $-0,7$ & $\mathrm{~N}$-ter +34.06 Da, $\mathrm{K}+34.06 \mathrm{Da}$ & 19,7 & 7,306 & 3 & 1571,0 & IDI__MOUSE & G3XA48_MOUSE & Isopentenyl-diphosphate Delta-isomerase 1; & Isopentenyl pyrophosphate isomerase 1 ; \\
\hline VEALIHAILR & AGDKDDITEPAVCALR & $-1,6$ & $\begin{array}{l}\text { N-ter +34.06 Da, K+34.06 Da, C } \\
+57.02 \mathrm{Da}\end{array}$ & 28,7 & $-0,389$ & 3 & 1798,0 & PLAK_MOUSE & & Junction plakoglobin; & Desmoplakin III;Desmoplakin-3; \\
\hline ELAQDKEAAD & AIDAEGASAPLMELLHSR & 0,0 & N-ter+28.03 Da & 24,7 & 0,157 & 3 & 1908,0 & PLAK_MOUSE & & Junction plakoglobin; & Desmoplakin III;Desmoplakin-3; \\
\hline KATIGLIRNL & $\begin{array}{l}\text { ALCPANHAPLQEAAVIPR } \\
\text { A }\end{array}$ & $-0,4$ & N-ter +28.03 Da, C+57.02 Da & 24,5 & 3,422 & 3 & 195,1 & PLAK_MOUSE & & Junction plakoglobin; & Desmoplakin III;Desmoplakin-3; \\
\hline LVKATIGLIR & NLALCPANHAPLQEAAVIPR & 0,8 & N-ter +28.03 Da, C+57.02 Da & 37,4 & 0,641 & 3 & 2182,2 & PLAK_MOUSE & & Junction plakoglobin; & Desmoplakin III;Desmoplakin-3; \\
\hline AADAIDAEGA & SAPLMELLHSR & 0,6 & N-ter +34.06 Da & 22,2 & $-2,714$ & 3 & 1286,7 & PLAK_MOUSE & & Junction plakoglobin; & Desmoplakin III;Desmoplakin-3; \\
\hline ASAPLMELLH & SRNEGTATYAAAVLFR & $-0,2$ & N-ter +28.03 Da & 37,5 & $-7,684$ & 3 & 1753,9 & PLAK_MOUSE & & Junction plakoglobin; & Desmoplakin III;Desmoplakin-3; \\
\hline YDSGIHSGVN & TCVPSVSSKGIMDEDDACGR & 0,8 & $\begin{array}{l}\text { N-ter +34.06 Da, K+34.06 Da, C } \\
+57.02 \mathrm{Da}\end{array}$ & 30,7 & 1,020 & 3 & 2251,1 & PLAK_MOUSE & & Junction plakoglobin; & Desmoplakin III;Desmoplakin-3; \\
\hline SGEDSSLLLA & TQVEGQTTNLQR & 0,3 & N-ter $+28.03 \mathrm{Da}$ & 26,1 & $-0,570$ & 2 & 1401,7 & PLAK_MOUSE & & Junction plakoglobin; & Desmoplakin III;Desmoplakin-3; \\
\hline HCRKPHYVIL & LGEHNLEKTDGCEQR & $-0,8$ & $\begin{array}{l}\text { N-ter +34.06 Da, K +34.06 Da, C } \\
+57.02 \mathrm{Da}\end{array}$ & 17,7 & $-3,716$ & 4 & 1852,9 & KLK11_MOUSE & & Kallikrein-11; & Hippostasin;Serine protease 20; \\
\hline DVTFPSDLMC & SDVKLISSR & 0,5 & $\mathrm{~N}$-ter +34.06 Da, K +34.06 Da & 19,2 & 5,025 & 3 & 1071,7 & KLK7_MOUSE & & Kallikrein-7; & $\begin{array}{l}\text { Serine protease 6;Stratum corneum } \\
\text { chymotryptic enzyme; Thymopsin; }\end{array}$ \\
\hline ATKSFRHPGY & STKTHVNDIMLVR & 0,2 & $\mathrm{~N}$-ter $+28.03 \mathrm{Da}, \mathrm{K}+28.03 \mathrm{Da}$ & 18,7 & $-8,588$ & 3 & 1568,9 & KLK7_MOUSE & & Kallikrein-7; & $\begin{array}{l}\text { Serine protease 6;Stratum corneum } \\
\text { chymotryptic enzyme;Thymopsin; }\end{array}$ \\
\hline GTSCTVSGWG & TTTSPDVTFPSDLMCSDVKLISSR & 0,8 & $\begin{array}{l}\text { N-ter +28.03 Da, K+28.03 Da, C } \\
+57.02 \mathrm{Da}\end{array}$ & 13,6 & 1,031 & 3 & 2712,3 & KLK7_MOUSE & & Kallikrein-7; & $\begin{array}{l}\text { Serine protease 6;Stratum corneum } \\
\text { chymotryptic enzyme; Thymopsin; }\end{array}$ \\
\hline SGWGTTTSPD & VTFPSDLMCSDVKLISSR & $-1,3$ & $\begin{array}{l}\text { N-ter +34.06 Da, K+34.06 Da, C } \\
+57.02 \mathrm{Da}\end{array}$ & 18,4 & 1,835 & 3 & 2122,2 & KLK7_MOUSE & & Kallikrein-7; & $\begin{array}{l}\text { Serine protease 6;Stratum corneum } \\
\text { chymotryptic enzyme; Thymopsin; }\end{array}$ \\
\hline VNTLRCQLGD & RLNVEVDAAPTVDLNR & $-4,8$ & $\mathrm{~N}-\operatorname{ter}+34.06 \mathrm{Da}$ & 31,0 & 1,045 & 3 & 1815,0 & K1H1_MOUSE & KRT34_MOUSE & Keratin, type I cuticular Ha1; & HKA-1; Hair keratin, type I Ha1;Keratin-31; \\
\hline QVVSSSEQLQ & SCQAEIIELR & $-0,8$ & N-ter +34.06 Da, C+57.02 Da & 22,9 & $-5,584$ & 2 & 1251,7 & K1H1_MOUSE & KRT34_MOUSE & Keratin, type I cuticular Ha1; & HKA-1; Hair keratin, type I Ha1;Keratin-31; \\
\hline ELQAQHELRN & SLENTLTESEAR & $-6,4$ & $\mathrm{~N}$-ter +34.06 Da & 35,6 & $-0,433$ & 2 & 1382,7 & K1H1_MOUSE & KRT34_MOUSE & Keratin, type I cuticular Ha1; & HKA-1; Hair keratin, type I Ha1;Keratin-31; \\
\hline STGDVNVEMN & AAPGVDLTQLLNNMR & 0,1 & N-ter +34.06 Da & 68,4 & $-1,032$ & 2 & 1645,9 & K1C10_MOUSE & A2A513_MOUSE & Keratin, type I cytoskeletal 10; & $\begin{array}{l}56 \text { kDa cytokeratin;Cytokeratin-10; Keratin, type } \\
\text { c cytoskeletal } 59 \text { kDa;Keratin-10; }\end{array}$ \\
\hline QIsalasals & ALEEQLQQIR & $-0,1$ & N-ter +34.06 Da & 38,5 & $-0,317$ & 2 & 1260,7 & K1C10_MOUSE & A2A513_MOUSE & Keratin, type I cytoskeletal 10; & $\begin{array}{l}56 \text { kDa cytokeratin;Cytokeratin-10; Keratin, type } \\
\text { cytoskeletal } 59 \text { kDa;Keratin-10; }\end{array}$ \\
\hline GQILTLTDN & ANVLLQIDNAR & 0,1 & $\mathrm{~N}-\mathrm{ter}+28.03 \mathrm{Da}$ & 45,7 & $-0,080$ & 2 & 1253,7 & K1C10_MOUSE & K1C14_MOUSE & Keratin, type I cytoskeletal 10; & $\begin{array}{l}56 \text { kDa cytokeratin;Cytokeratin-10;Keratin, type } \\
\text { I cytoskeletal } 59 \text { kDa;keratin-10; }\end{array}$ \\
\hline TGDVNVEMNA & APGVDLTQLLNNMR & 0,1 & N-ter +28.03 Da & 49,5 & $-0,573$ & 2 & 1568,8 & K1C10_MOUSE & A2A513_MOUSE & Keratin, type I cytoskeletal 10; & $\begin{array}{l}56 \text { kDa cytokeratin;Cytokeratin-10;Keratin, type } \\
\text { I cytoskeletal } 59 \text { kDa;Keratin-10; }\end{array}$ \\
\hline NVEMNAAPGV & DLTQLLNNMR & 0,0 & $\mathrm{~N}$-ter $+34.06 \mathrm{Da}$ & 28,5 & $-0,160$ & 2 & 1250,7 & K1C10_MOUSE & A2A513_MOUSE & Keratin, type I cytoskeletal 10; & $\begin{array}{l}56 \text { kDa cytokeratin; Cytokeratin-10;Keratin, type } \\
\text { I cytoskeletal } 59 \mathrm{kDa} \text {;:eratin-10; }\end{array}$ \\
\hline LKGQILTLT & DNANVLLQIDNAR & $-0,4$ & N-ter +34.06 Da & 50,5 & $-1,878$ & 2 & 1488,8 & K1C10_MOUSE & K1C14_MOUSE & Keratin, type I cytoskeletal 10; & $\begin{array}{l}56 \text { kDa cytokeratin; Cytokeratin-10;Keratin, type } \\
\text { I cytoskeletal } 59 \mathrm{kDa} \text {; Keratin-10; }\end{array}$ \\
\hline ENEVTLRQSV & EADINGLRR & 0,0 & N-ter +34.06 Da & 17,9 & $-1,297$ & 3 & 1076,6 & K1C10_MOUSE & K1C12_MOUSE & Keratin, type I cytoskeletal 10; & $\begin{array}{l}56 \text { kDa cytokeratin; Cytokeratin-10;Keratin, type } \\
\text { I cytoskeletal } 59 \mathrm{kDa} \text {; Keratin-10; }\end{array}$ \\
\hline QSQLALKRSL & EASLAETEGR & $-0,6$ & $\mathrm{~N}$-ter $+28.03 \mathrm{Da}$ & 34,4 & $-0,825$ & 2 & 1089,5 & K1C10_MOUSE & A2A513_MOUSE & Keratin, type I cytoskeletal 10; & $\begin{array}{l}56 \text { kDa cytokeratin; Cytokeratin-10;Keratin, type } \\
\text { I cytoskeletal } 59 \text { kDa;Keratin-10; }\end{array}$ \\
\hline SQIQSQISAL & EEQLQQIR & $-0,4$ & N-ter +34.06 Da & 20,4 & $-4,450$ & 2 & 1076,6 & K1C10_MOUSE & K1C26_MOUSE & Keratin, type I cytoskeletal 10; & $\begin{array}{l}56 \text { kDa cytokeratin;Cytokeratin-10;Keratin, type } \\
\text { I cytoskeletal } 59 \text { kDa;Keratin-10; }\end{array}$ \\
\hline
\end{tabular}




\begin{tabular}{|c|c|c|c|c|c|c|c|c|c|c|}
\hline Non Prime Site & Prime Site & 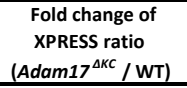 & Modifications & Hyperscore & $\begin{array}{c}\text { Mass error } \\
\text { in ppm }\end{array}$ & Charge & $\begin{array}{c}\begin{array}{c}\text { Precursor } \\
\text { neutral mass } \\
\text { in Da }\end{array} \\
\end{array}$ & Uniprot ID 1 & Protein Name 1 & Protein Name 2 \\
\hline FNQKSKELT & EIDSNIEOMSSHKSEITELR & 0,0 & $\mathrm{~N}$-ter +34.06 Da, K+34.06 Da & 42,3 & 3,061 & 4 & 2413,3 & K1C10_MOUSE & A2A513_MOUSE Keratin, type I cytoskeletal 10; & $\begin{array}{l}56 \text { kDa cytokeratin; Cytokeratin-10;Keratin, type } \\
\text { I cytoskeletal } 59 \text { kDa;Keratin-10; }\end{array}$ \\
\hline QNVSTGDVNV & EMNAAPGVDLTQLLNNMR & 0,0 & N-ter +34.06 Da & 43,8 & $-0,494$ & 3 & 2020,0 & K1C10_MOUSE & A2A513_MOUSE Keratin, type I cytoskeletal 10; & $\begin{array}{l}56 \text { kDa cytokeratin; Cytokeratin-10; Keratin, type } \\
\text { I cytoskeletal } 59 \text { kDa;Keratin-10; }\end{array}$ \\
\hline QQLLDIKTRL & ENEIOTYR & $-0,3$ & $\mathrm{~N}$-ter +34.06 Da & 25,8 & $-1,011$ & 2 & 1085,6 & K1C10_MOUSE & A2A513_MOUSE Keratin, type I cytoskeletal 10; & $\begin{array}{l}56 \text { kDa cytokeratin; Cytokeratin-10; Keratin, type } \\
\text { I cytoskeletal } 59 \text { kDa;Keratin-10; }\end{array}$ \\
\hline GGYGGGSSSG & GAGGHGGSSGGGYGGGSSSGGQGGSGGFKSSGGGL & $-0,8$ & N-ter +34.06 Da, K+34.06 Da & 27,7 & $-6,432$ & 5 & 3586,7 & K1C10_MOUSE & A2A513_MOUSE Keratin, type I cytoskeletal 10; & $\begin{array}{l}56 \text { kDa cytokeratin; Cytokeratin-10; Keratin, type } \\
\text { I cytoskeletal } 59 \text { kDa;Keratin-10; }\end{array}$ \\
\hline GHGGSSGGGY & GGGSSSGGQGGSGGFKSSGGGDQSSKGPR & 0,2 & $\mathrm{~N}$-ter +34.06 Da, K+34.06 Da & 37,4 & $-0,386$ & 4 & 2585,3 & K1C10_MOUSE & A2A513_MOUSE Keratin, type I cytoskeletal 10; & $\begin{array}{l}56 \text { kDa cytokeratin;Cytokeratin-10;Keratin, type } \\
\text { I cytoskeletal } 59 \text { kDa;Keratin-10; }\end{array}$ \\
\hline GGAGGHGGSS & GGGYGGGSSSGGQGGSGGFKSSGGGDQSSKGPR & 0,2 & $\mathrm{~N}$-ter $+34.06 \mathrm{Da}, \mathrm{K}+34.06 \mathrm{Da}$ & 36,8 & $-2,976$ & 4 & 2919,4 & K1C10_MOUSE & A2A513_MOUSE Keratin, type I cytoskeletal 10; & $\begin{array}{l}56 \text { kDa cytokeratin;Cytokeratin-10;Keratin, type } \\
\text { I cytoskeletal } 59 \text { kDa;Keratin-10; }\end{array}$ \\
\hline VRVSSTRGSL & GGGYSSGGFSGGSFSR & 0,1 & $\mathrm{~N}$-ter $+28.03 \mathrm{Da}$ & 65,2 & $-0,468$ & 2 & 1493,7 & K1C10_MOUSE & A2A513_MOUSE Keratin, type I cytoskeletal 10; & $\begin{array}{l}56 \mathrm{kDa} \text { cytokeratin;Cytokeratin-10;Keratin, type } \\
\text { I cytoskeletal } 59 \mathrm{kDa} \text {;Keratin-10; }\end{array}$ \\
\hline HGGSSGGGYG & GGSSSGGQGGSGGFKSSGGGDQSSKGPR & 0,1 & $\mathrm{~N}$-ter $+28.03 \mathrm{Da}, \mathrm{K}+28.03 \mathrm{Da}$ & 22,8 & 7,637 & 4 & 2510,2 & K1C10_MOUSE & A2A513_MOUSE Keratin, type I cytoskeletal 10; & $\begin{array}{l}56 \text { kDa cytokeratin;Cytokeratin-10;Keratin, type } \\
\text { I cytoskeletal } 59 \mathrm{kDa} \text {;Keratin-10; }\end{array}$ \\
\hline GAGGHGGSSG & GGYGGGSSSGGQGGSGGFKSSGGGDQSSKGPR & 0,2 & $\mathrm{~N}$-ter $+34.06 \mathrm{Da}, \mathrm{K}+34.06 \mathrm{Da}$ & 34,0 & 0,977 & 4 & 2862,4 & K1C10_MOUSE & A2A513_MOUSE Keratin, type I cytoskeletal 10; & $\begin{array}{l}56 \text { kDa cytokeratin;Cytokeratin-10;Keratin, type } \\
\text { I cytoskeletal } 59 \mathrm{kDa} \text {;Keratin-10; }\end{array}$ \\
\hline RVSSTRGSLG & GGYSSGGFSGGSFSR & 0,0 & N-ter +34.06 Da & 55,5 & $-0,623$ & 2 & 1442,7 & K1C10_MOUSE & A2A513_MOUSE Keratin, type I cytoskeletal 10; & $\begin{array}{l}56 \text { kDa cytokeratin;Cytokeratin-10;Keratin, type } \\
\text { I cytoskeletal } 59 \mathrm{kDa} \text {;Keratin-10; }\end{array}$ \\
\hline GGSVRVSSTR & GSLGGGYSSGGFSGGSFSR & 0,6 & N-ter +34.06 Da & 93,3 & $-0,967$ & 2 & 1756,8 & K1C10_MOUSE & A2A513_MOUSE Keratin, type I cytoskeletal 10; & $\begin{array}{l}56 \text { kDa cytokeratin;Cytokeratin-10;Keratin, type } \\
\text { I cytoskeletal } 59 \mathrm{kDa} \text {;Keratin-10; }\end{array}$ \\
\hline SSSGGAGGHG & GSSGGGYGGGSSSGGQGGSGGFKSSGGGDQSSKGP & 0,3 & $\mathrm{~N}$-ter $+28.03 \mathrm{Da}, \mathrm{K}+28.03 \mathrm{Da}$ & 36,9 & 1,626 & 4 & 3132,4 & K1C10_MOUSE & A2A513_MOUSE Keratin, type I cytoskeletal 10; & $\begin{array}{l}56 \text { kDa cytokeratin;Cytokeratin-10;Keratin, type } \\
\text { I cytoskeletal } 59 \mathrm{kDa} \text { KKeratin-10; }\end{array}$ \\
\hline GGSSGGGYGG & GSSSGGQGGSGGFKSSGGGDQSSKGPR & 0,2 & $\mathrm{~N}$-ter $+28.03 \mathrm{Da}, \mathrm{K}+28.03 \mathrm{Da}$ & 28,9 & 0,244 & 4 & 2453,2 & K1C10_MOUSE & A2A513_MOUSE Keratin, type I cytoskeletal 10; & $\begin{array}{l}56 \text { kDa cytokeratin;Cytokeratin-10; Keratin, type } \\
\text { I cytoskeletal } 59 \mathrm{kDa} \text { Keratin-10; }\end{array}$ \\
\hline DVNVEMNAAP & GVDLTQLLNNMR & 0,3 & $\mathrm{~N}$-ter $+28.03 \mathrm{Da}$ & 44,2 & $-3,707$ & 2 & 1400,7 & K1C10_MOUSE & A2A513_MOUSE Keratin, type I cytoskeletal 10; & $\begin{array}{l}56 \text { kDa cytokeratin;Cytokeratin-10; Keratin, type } \\
\text { I cytoskeletal } 59 \mathrm{kDa} \text { KKeratin-10; }\end{array}$ \\
\hline AGGHGGSSGG & GYGGGSSSGGQGGSGGFKSSGGGDQSSKGPR & 0,1 & $\mathrm{~N}$-ter $+28.03 \mathrm{Da}, \mathrm{K}+28.03 \mathrm{Da}$ & 24,6 & 3,475 & 4 & 2787,3 & K1C10_MOUSE & A2A513_MOUSE Keratin, type I cytoskeletal 10; & $\begin{array}{l}56 \text { kDa cytokeratin;Cytokeratin-10; Keratin, type } \\
\text { I cytoskeletal } 59 \mathrm{kDa} \text { KKeratin-10; }\end{array}$ \\
\hline vsSTRGSLGG & GYSSGGFSGGSFSR & 0,3 & $\mathrm{~N}$-ter +34.06 Da & 24,8 & 1,874 & 2 & 1385,7 & K1C10_MOUSE & A2A513_MOUSE Keratin, type I cytoskeletal 10; & $\begin{array}{l}56 \text { kDa cytokeratin;Cytokeratin-10;Keratin, type } \\
\text { cytoskeletal } 59 \mathrm{kDa} \text { KReratin-10; }\end{array}$ \\
\hline NQKSKELTTE & IDSNIEQMSSHKSEITELR & $-0,1$ & $\mathrm{~N}$-ter $+34.06 \mathrm{Da}, \mathrm{K}+34.06 \mathrm{Da}$ & 22,8 & $-3,758$ & 4 & 2284,2 & K1C10_MOUSE & A2A513_MOUSE Keratin, type I cytoskeletal 10; & $\begin{array}{l}56 \text { kDa cytokeratin;Cytokeratin-10; Keratin, type } \\
\text { cytoskeletal } 59 \mathrm{kDa} \text { KReratin-10; }\end{array}$ \\
\hline KELTTEIDSN & IEQMSSHKSEITELR & 0,1 & $\mathrm{~N}$-ter +28.03 Da, $\mathrm{K}+28.03 \mathrm{Da}$ & 52,3 & $-0,108$ & 3 & 1843,0 & K1C10_MOUSE & A2A513_MOUSE Keratin, type I cytoskeletal 10; & $\begin{array}{l}56 \text { kDa cytokeratin;Cytokeratin-10; Keratin, type } \\
\text { c cytoskeletal } 59 \mathrm{kDa} \text { KReratin-10; }\end{array}$ \\
\hline YKTIEDLKGQ & ILTLTTDNANVLLQIDNAR & $-0,5$ & $\mathrm{~N}$-ter +34.06 Da & 35,7 & 0,797 & 3 & 2131,2 & K1C10_MOUSE & A2A513_MOUSE Keratin, type I cytoskeletal 10; & $\begin{array}{l}56 \text { kDa cytokeratin;Cytokeratin-10; Keratin, type } \\
\text { c cytoskeletal } 59 \mathrm{kDa} \text { KReratin-10; }\end{array}$ \\
\hline EGRYCVQLSQ & IQSQISALEEQLQQIR & $-0,2$ & $\mathrm{~N}$-ter +28.03 Da & 47,8 & $-2,456$ & 3 & 1911,0 & K1C10_MOUSE & A2A513_MOUSE Keratin, type I cytoskeletal 10; & $\begin{array}{l}56 \text { kDa cytokeratin;Cytokeratin-10;Keratin, type } \\
\text { cytoskeletal } 59 \mathrm{kDa} \text { KReratin-10; }\end{array}$ \\
\hline ISALEEQLQQ & IRAETECQNAEYQQLLDIKTR & $-0,3$ & $\begin{array}{l}\text { N-ter +34.06 Da, K+34.06 Da, C } \\
+57.02 \mathrm{Da}\end{array}$ & 37,4 & 1,245 & 4 & 2646,4 & K1C10_MOUSE & A2A513_MOUSE Keratin, type I cytoskeletal 10; & $\begin{array}{l}56 \text { kDa cytokeratin; Cytokeratin-10; Keratin, type } \\
\text { I cytoskeletal } 59 \mathrm{kDa} \text {;Keratin-10; }\end{array}$ \\
\hline DAEEWFNOKS & KELTTEIDSNIEQMSSHKSEITELR & $-0,2$ & $\mathrm{~N}$-ter $+28.03 \mathrm{Da}, \mathrm{K}+28.03 \mathrm{Da}$ & 23,8 & 0,898 & 4 & 3001,5 & K1C10_MOUSE & A2A513_MOUSE Keratin, type I cytoskeletal 10; & $\begin{array}{l}56 \text { kDa cytokeratin;Cytokeratin-10;Keratin, type } \\
\text { I cytoskeletal } 59 \mathrm{kDa} \text {;Keratin-10; }\end{array}$ \\
\hline LaSQLALKQS & LEASLAETEGR & $-1,4$ & $\mathrm{~N}$-ter +28.03 Da & 24,6 & 0,498 & 2 & 1202,6 & K1C10_MOUSE & A2A513_MOUSE Keratin, type I cytoskeletal 10; & $\begin{array}{l}56 \text { kDa cytokeratin; Cytokeratin-10; Keratin, type } \\
\text { I cytoskeletal } 59 \mathrm{kD} \text {; Keratin-10; }\end{array}$ \\
\hline YQQLLIIKTR & LENEIQTYR & 2,4 & $\mathrm{~N}$-ter+28.03 Da & 33,5 & 0,670 & 2 & 1192,6 & K1C10_MOUSE & A2A513_MOUSE Keratin, type I cytoskeletal 10; & $\begin{array}{l}56 \text { kDa cytokeratin; Cytokeratin-10; Keratin, type } \\
\text { I cytoskeletal } 59 \mathrm{kD} \text {; Keratin-10; }\end{array}$ \\
\hline LENEIQTYRS & LLEGEGSSSGGGGGR & 0,1 & $\mathrm{~N}$-ter $+28.03 \mathrm{Da}$ & 44,6 & $-0,964$ & 2 & 1346,6 & K1C10_MOUSE & A2A513_MOUSE Keratin, type I cytoskeletal 10; & $\begin{array}{l}56 \text { kDa cytokeratin; Cytokeratin-10; Keratin, type } \\
\text { I cytoskeletal } 59 \text { kDa;Keratin-10; }\end{array}$ \\
\hline AETEGRYCVQ & LSQIQSQISALEEQLQQIR & $-0,6$ & $\mathrm{~N}$-ter $+28.03 \mathrm{Da}$ & 52,3 & $-4,192$ & 3 & 2239,2 & K1C10_MOUSE & A2A513_MOUSE Keratin, type I cytoskeletal 10; & $\begin{array}{l}56 \text { kDa cytokeratin; Cytokeratin-10; Keratin, type } \\
\text { I cytoskeletal } 59 \text { kDa;Keratin-10; }\end{array}$ \\
\hline KTIEDLKGQI & LTLTTDNANVLLQIDNAR & $-0,2$ & $\mathrm{~N}$-ter $+28.03 \mathrm{Da}$ & 32,8 & $-6,203$ & 3 & 2012,1 & K1C10_MOUSE & A2A513_MOUSE Keratin, type I cytoskeletal 10; & $\begin{array}{l}56 \text { kDa cytokeratin;Cytokeratin-10;Keratin, type } \\
\text { I cytoskeletal } 59 \text { kDa;Keratin-10; }\end{array}$ \\
\hline IEDLKGQILT & LTTDNANVLLQIDNAR & 0,0 & N-ter +34.06 Da & 44,2 & $-0,443$ & 3 & 1804,0 & K1C10_MOUSE & A2A513_MOUSE Keratin, type I cytoskeletal 10; & $\begin{array}{l}56 \text { kDa cytokeratin;Cytokeratin-10;Keratin, type } \\
\text { I cytoskeletal } 59 \mathrm{kDa} \text {;Keratin-10; }\end{array}$ \\
\hline NVSTGDVNVE & MNAAPGVDLTQLLNNMR & $-1,5$ & N-ter +34.06 Da & 44,0 & 0,370 & 3 & 1891,0 & K1C10_MOUSE & A2A513_MOUSE Keratin, type I cytoskeletal 10; & $\begin{array}{l}56 \text { kDa cytokeratin;Cytokeratin-10;Keratin, type } \\
\text { I cytoskeletal } 59 \mathrm{kDa} \text { k'Keratin-10; }\end{array}$ \\
\hline VSTGDVNVEM & NAAPGVDLTQLLNNMR & 0,1 & $\mathrm{~N}$-ter $+28.03 \mathrm{Da}$ & 60,9 & 6,094 & 2 & 1753,9 & K1C10_MOUSE & A2A513_MOUSE Keratin, type I cytoskeletal 10; & $\begin{array}{l}56 \text { KDa cytokeratin; Cytokeratin-10;Keratin, type } \\
\text { I cytoskeletal } 59 \text { kDa;Keratin-10; }\end{array}$ \\
\hline KGQILTLTTD & NANVLLQIDNAR & 0,9 & $\mathrm{~N}$-ter $+28.03 \mathrm{Da}$ & 51,8 & $-1,825$ & 2 & 1367,8 & K1C10_MOUSE & K1C14_MOUSE Keratin, type I cytoskeletal 10; & $\begin{array}{l}56 \text { kDa cytokeratin;Cytokeratin-10;Keratin, type } \\
\text { I cytoskeletal } 59 \mathrm{kDa} \text {;Keratin-10; }\end{array}$ \\
\hline
\end{tabular}




\begin{tabular}{|c|c|c|c|c|c|c|c|c|c|c|}
\hline Non Prime Site & Prime Site & $\begin{array}{l}\text { Fold change of } \\
\text { XPRESS ratio } \\
\text { (Adam17 } 7^{\text {LKC } / W T)}\end{array}$ & Modifications & Hyperscore & $\begin{array}{l}\text { Mass error } \\
\text { in ppm }\end{array}$ & Charge & $\begin{array}{c}\text { Precursor } \\
\text { neutral mass } \\
\text { in Da } \\
\end{array}$ & Uniprot ID 1 & Protein Name 1 & Protein Name 2 \\
\hline SKELTTEIDS & NIEQMSSHKSEITELR & 1,1 & $\mathrm{~N}$-ter $+28.03 \mathrm{Da}, \mathrm{K}+28.03 \mathrm{Da}$ & 31,4 & 0,153 & 4 & 1957,0 & K1C10_MOUSE & A2A513_MOUSE Keratin, type I cytoskeletal 10; & $\begin{array}{l}56 \text { kDa cytokeratin;Cytokeratin-10;Keratin, type } \\
\text { I cytoskeletal } 59 \text { kDa;Keratin-10; }\end{array}$ \\
\hline DLQNVSTGDV & NVEMNAAPGVDLTQLLNNMR & $-0,3$ & N-ter +34.06 Da & 21,9 & 2,281 & 3 & 2233,2 & K1C10_MOUSE & A2A513_MOUSE Keratin, type I cytoskeletal 10; & $\begin{array}{l}56 \text { kDa cytokeratin; Cytokeratin-10;keratin, type } \\
\text { I cytoskeletal } 59 \text { kDa;Keratin-10; }\end{array}$ \\
\hline QILTLTTDNA & NVLLQIDNAR & $-0,4$ & $\mathrm{~N}$-ter +34.06 Da & 33,0 & $-0,336$ & 2 & 1188,7 & K1C10_MOUSE & K1C14_MOUSE Keratin, type I cytoskeletal 10; & $\begin{array}{l}56 \text { kDa cytokeratin; Cytokeratin-10;keratin, type } \\
\text { I cytoskeletal } 59 \text { kDa;keratin-10; }\end{array}$ \\
\hline TEGRYCVQLS & QIQSQISALEEQLQQIR & 0,1 & $\mathrm{~N}$-ter +34.06 Da & 42,2 & 2,051 & 3 & 2045,1 & K1C10_MOUSE & A2A513_MOUSE Keratin, type I cytoskeletal 10; & $\begin{array}{l}56 \text { kDa cytokeratin; Cytokeratin-10;keratin, type } \\
\text { I cytoskeletal } 59 \text { kDa;keratin-10; }\end{array}$ \\
\hline revalsalas & QISALEEQLQQIR & $-0,3$ & $\mathrm{~N}$-ter +34.06 Da & 53,7 & $-1,006$ & 2 & 1588,9 & K1C10_MOUSE & A2A513_MOUSE Keratin, type I cytoskeletal 10; & $\begin{array}{l}56 \text { kDa cytokeratin; Cytokeratin-10;Keratin, type } \\
\text { I cytoskeletal } 59 \mathrm{kDa} \text {;Keratin-10; }\end{array}$ \\
\hline LAETEGRYCV & QLSQIOSQISALEEQLQQIR & $-0,2$ & $\mathrm{~N}$-ter +28.03 Da & 61,1 & 0,464 & 3 & 2367,3 & K1C10_MOUSE & A2A513_MOUSE Keratin, type I cytoskeletal 10; & $\begin{array}{l}56 \text { kDa cytokeratin; Cytokeratin-10;Keratin, type } \\
\text { I cytoskeletal } 59 \mathrm{kDa} \text {;Keratin-10; }\end{array}$ \\
\hline IELQSQLALK & QSLEAsLAETEGR & $-0,4$ & $\mathrm{~N}$-ter +34.06 Da & 45,9 & $-1,122$ & 2 & 1423,7 & K1C10_MOUSE & A2A513_MOUSE Keratin, type I cytoskeletal 10; & $\begin{array}{l}56 \text { kDa cytokeratin; Cytokeratin-10;keratin, type } \\
\text { I cytoskeletal } 59 \mathrm{kDa} \text {;Keratin-10; }\end{array}$ \\
\hline GRYcralsal & QSQISALEEQLQQIR & $-0,2$ & $\mathrm{~N}$-ter $+28.03 \mathrm{Da}$ & 44,4 & 5,164 & 3 & 1798,0 & K1C10_MOUSE & A2A513_MOUSE Keratin, type I cytoskeletal 10; & $\begin{array}{l}56 \text { kDa cytokeratin;Cytokeratin-10;Keratin, type } \\
\text { I cytoskeletal } 59 \mathrm{kDa} \text {;Keratin-10; }\end{array}$ \\
\hline LKYENEVTLR & QSVEADINGLR & 0,3 & N-ter $+28.03 \mathrm{Da}$ & 36,8 & $-0,894$ & 2 & 1228,6 & K1C10_MOUSE & K1C13_MOUSE Keratin, type I cytoskeletal 10; & $\begin{array}{l}56 \text { kDa cytokeratin;Cytokeratin-10;Keratin, type } \\
\text { I cytoskeletal } 59 \mathrm{kDa} \text {; Keratin-10; }\end{array}$ \\
\hline SALEEQLQQI & RAETECQNAEYQQLLDIKTR & $-0,3$ & $\begin{array}{l}\text { N-ter +34.06 Da, K+34.06 Da, C } \\
\text { +57.02 Da }\end{array}$ & 29,1 & 2,916 & 4 & 2533,3 & K1C10_MOUSE & A2A513_MOUSE Keratin, type I cytoskeletal 10; & $\begin{array}{l}56 \text { kDa cytokeratin; Cytokeratin-10;Keratin, type } \\
\text { I cytoskeletal } 59 \mathrm{kDa} \text {;Keratin-10; }\end{array}$ \\
\hline ANVLLIDNA & RLAADDFR & $-0,2$ & $\mathrm{~N}$-ter +34.06 Da & 22,1 & $-2,201$ & 3 & 996,6 & K1C10_MOUSE & K1C13_MOUSE Keratin, type I cytoskeletal 10; & $\begin{array}{l}56 \text { kDa cytokeratin;Cytokeratin-10;Keratin, type } \\
\text { I cytoskeletal } 59 \mathrm{kDa} \text {;Keratin-10; }\end{array}$ \\
\hline EYQQLLDIKT & RLENEIOTYR & $-0,1$ & N-ter +34.06 Da & 21,4 & $-0,884$ & 3 & 1354,7 & K1C10_MOUSE & A2A513_MOUSE Keratin, type I cytoskeletal 10; & $\begin{array}{l}56 \text { kDa cytokeratin;Cytokeratin-10;Keratin, type } \\
\text { I cytoskeletal } 59 \mathrm{kDa} \text {; } \text { eratin-10; }\end{array}$ \\
\hline valsalasal & SALEEQLQQIR & 0,4 & N-ter +34.06 Da & 45,7 & $-2,815$ & 2 & 1347,8 & K1C10_MOUSE & A2A513_MOUSE Keratin, type I cytoskeletal 10; & $\begin{array}{l}56 \text { kDa cytokeratin; Cytokeratin-10;Keratin, type } \\
\text { I cytoskeletal } 59 \mathrm{kDa} \text {;Keratin-10; }\end{array}$ \\
\hline SNIEQMSSHK & SEITELRR & 0,6 & N-ter $+28.03 \mathrm{Da}$ & 25,7 & $-0,290$ & 3 & 1030,6 & K1C10_MOUSE & A2A513_MOUSE Keratin, type I cytoskeletal 10; & $\begin{array}{l}56 \text { kDa cytokeratin;Cytokeratin-10;Keratin, type } \\
\text { I cytoskeletal } 59 \mathrm{kDa} \text {; Keratin-10; }\end{array}$ \\
\hline TRGSLGGGYS & SGGFSGGSFSR & 1,1 & N-ter +34.06 Da & 22,6 & $-1,018$ & 2 & 1078,5 & K1C10_MOUSE & A2A513_MOUSE Keratin, type I cytoskeletal 10; & $\begin{array}{l}56 \text { kDa cytokeratin;Cytokeratin-10;Keratin, type } \\
\text { I cytoskeletal } 59 \mathrm{kDa} \text {; Keratin-10; }\end{array}$ \\
\hline SGGAGGHGGS & SGGGYGGGSSSGGQGGSGGFKSSGGGDQSSKGPR & 1,4 & N-ter +28.03 Da, K+28.03 Da & 62,4 & 4,179 & 3 & 2988,4 & K1C10_MOUSE & A2A513_MOUSE Keratin, type I cytoskeletal 10; & $\begin{array}{l}56 \text { kDa cytokeratin;Cytokeratin-10;Keratin, type } \\
\text { I cytoskeletal } 59 \mathrm{kDa} \text {;eratin-10; }\end{array}$ \\
\hline KDAEEWFNQK & SKELTTEIDSNIEQMSSHKSEITELR & 1,3 & $\mathrm{~N}$-ter +28.03 Da, $\mathrm{K}+28.03 \mathrm{Da}$ & 47,8 & $-1,876$ & 4 & 3088,6 & K1C10_MOUSE & A2A513_MOUSE Keratin, type I cytoskeletal 10; & $\begin{array}{l}56 \text { kDa cytokeratin;Cytokeratin-10;Keratin, type } \\
\text { I cytoskeletal } 59 \mathrm{kDa} \text {;eratin-10; }\end{array}$ \\
\hline ELQSQLALKQ & SLEASLAETEGR & 0,5 & $\mathrm{~N}$-ter +28.03 Da & 52,5 & 0,310 & 2 & 1289,7 & K1C10_MOUSE & A2A513_MOUSE Keratin, type I cytoskeletal 10; & $\begin{array}{l}56 \text { kDa cytokeratin;Cytokeratin-10;Keratin, type } \\
\text { I cytoskeletal } 59 \mathrm{kDa} \text {;eratin-10; }\end{array}$ \\
\hline GSVRVSSTRG & SLGGGYSSGGFSGGSFSR & 0,9 & N-ter +34.06 Da & 88,0 & $-1,293$ & 2 & 1699,8 & K1C10_MOUSE & A2A513_MOUSE Keratin, type I cytoskeletal 10; & $\begin{array}{l}56 \text { kDa cytokeratin; Cytokeratin-10;keratin, type } \\
\text { I cytoskeletal } 59 \mathrm{kDa} \text {;Keratin-10; }\end{array}$ \\
\hline RLENEIQTYR & SLLEGEGSSSGGGGGR & 3,8 & $\mathrm{~N}$-ter $+28.03 \mathrm{Da}$ & 55,3 & $-2,438$ & 2 & 1433,7 & K1C10_MOUSE & A2A513_MOUSE Keratin, type I cytoskeletal 10; & $\begin{array}{l}56 \text { kDa cytokeratin;Cytokeratin-10;Keratin, type } \\
\text { I cytoskeletal } 59 \mathrm{kDa} \text {;eratin-10; }\end{array}$ \\
\hline KSKELTTEID & SNIEQMSSHKSEITELR & 0,0 & $\mathrm{~N}$-ter $+34.06 \mathrm{Da}, \mathrm{K}+34.06 \mathrm{Da}$ & 72,7 & $-0,340$ & 3 & 2056,1 & K1C10_MOUSE & A2A513_MOUSE Keratin, type I cytoskeletal 10; & $\begin{array}{l}56 \text { kDa cytokeratin;Cytokeratin-10;Keratin, type } \\
\text { I cytoskeletal } 59 \text { kDa;Keratin-10; }\end{array}$ \\
\hline ETEGRYCVQL & SQIOSQISALEEQLQQIR & 0,0 & N-ter +28.03 Da & 71,5 & $-0,376$ & 2 & 2126,1 & K1C10_MOUSE & A2A513_MOUSE Keratin, type I cytoskeletal 10; & $\begin{array}{l}56 \text { kDa cytokeratin; Cytokeratin-10;Keratin, type } \\
\text { I cytoskeletal } 59 \mathrm{kDa} \text {;Keratin-10; }\end{array}$ \\
\hline Rrcvalsala & SQISALEEQLQQIR & 0,1 & $\mathrm{~N}$-ter $+34.06 \mathrm{Da}$ & 36,5 & $-0,358$ & 2 & 1675,9 & K1C10_MOUSE & A2A513_MOUSE Keratin, type I cytoskeletal 10; & $\begin{array}{l}56 \text { kDa cytokeratin;Cytokeratin-10;Keratin, type } \\
\text { I cytoskeletal } 59 \text { kDa;Keratin-10; }\end{array}$ \\
\hline STRGSLGGGY & SSGGFSGGSFSR & 0,4 & N-ter +34.06 Da & 28,4 & $-3,169$ & 2 & 1165,6 & K1C10_MOUSE & A2A513_MOUSE Keratin, type I cytoskeletal 10; & $\begin{array}{l}56 \text { kDa cytokeratin; Cytokeratin-10;Keratin, type } \\
\text { I cytoskeletal } 59 \text { kDa; Keratin-10; }\end{array}$ \\
\hline SSGGAGGHGG & SSGGGYGGGSSSGGQGGSGGFKSSGGGDQSSKGPR & 1,5 & $\mathrm{~N}$-ter +28.03 Da, $\mathrm{K}+28.03 \mathrm{Da}$ & 47,8 & $-5,780$ & 4 & 3075,4 & K1C10_MOUSE & A2A513_MOUSE Keratin, type I cytoskeletal 10; & $\begin{array}{l}56 \text { kDa cytokeratin; Cytokeratin-10;Keratin, type } \\
\text { I cytoskeletal } 59 \text { kDa; Keratin-10; }\end{array}$ \\
\hline SSGGGYGGGS & SSGGQGGSGGFKSSGGGDQSSKGPR & 1,6 & $\mathrm{~N}$-ter $+34.06 \mathrm{Da}, \mathrm{K}+34.06 \mathrm{Da}$ & 23,3 & 6,520 & 4 & 2327,2 & K1C10_MOUSE & A2A513_MOUSE Keratin, type I cytoskeletal 10; & $\begin{array}{l}56 \text { kDa cytokeratin; Cytokeratin-10;Keratin, type } \\
\text { I cytoskeletal } 59 \text { kDa; Keratin-10; }\end{array}$ \\
\hline GSSGGGYGGG & SSSGGAGGHGGSSGGGYGGGSSSGGQGGSGGFKSSS & 3,0 & N-ter +28.03 Da, K+28.03 Da & 21,8 & 1,156 & 5 & 3886,7 & K1C10_MOUSE & A2A513_MOUSE Keratin, type I cytoskeletal 10; & $\begin{array}{l}56 \text { kDa cytokeratin; Cytokeratin-10;Keratin, type } \\
\text { I cytoskeletal } 59 \text { kDa;Keratin-10; }\end{array}$ \\
\hline GSSGGGYGGG & SSSGGQGGSGGFKSSGGGDQSSKGPR & 1,9 & N-ter +28.03 Da, K+28.03 Da & 32,0 & 1,042 & 4 & 2396,1 & K1C10_MOUSE & A2A513_MOUSE Keratin, type I cytoskeletal 10; & $\begin{array}{l}56 \text { kDa cytokeratin; Cytokeratin-10;Keratin, type } \\
\text { I cytoskeletal } 59 \text { kDa;keratin-10; }\end{array}$ \\
\hline KYENEVTLRQ & SVEADINGLR & 0,7 & $\mathrm{~N}$-ter +28.03 Da & 27,1 & 0,000 & 2 & 1100,6 & K1C10_MOUSE & K1C13_MOUSE Keratin, type I cytoskeletal 10; & $\begin{array}{l}56 \text { kDa cytokeratin;Cytokeratin-10;Keratin, type } \\
\text { I cytoskeletal } 59 \mathrm{kDa} \text { keratin-10; }\end{array}$ \\
\hline
\end{tabular}


Supplementary table 11: Dimethylated (naturally unmodified) N-termini identified in the TAlLS experiment comparing wild-type and Adam $17^{4 \mathrm{KCC}}$ epidermis at P3 (replicate 2). This is a comprehensive, non redundant listing. Up to two potential protein IDS per peptide are stated.

\begin{tabular}{|c|c|c|c|c|c|c|c|c|c|c|c|}
\hline Non Prime Site & Prime Site & $\begin{array}{l}\text { Fold change of } \\
\text { XPRESS ratio } \\
\left.\text { (Adam17 } 7^{\alpha K C} / \mathrm{WT}\right)\end{array}$ & Modifications & Hyperscore & $\begin{array}{l}\text { Mass error } \\
\text { in ppm }\end{array}$ & Charge & $\begin{array}{c}\text { Precursor } \\
\text { neutral mass } \\
\text { in Da }\end{array}$ & Uniprot ID 1 & Uniprot ID 2 & Protein Name 1 & Protein Name 2 \\
\hline$\overline{\text { EYGGTKVVLD }}$ & DKDYFLFR & 0,1 & $\mathrm{~N}$-ter $+28.03 \mathrm{Da}, \mathrm{K}+28.03 \mathrm{Da}$ & 16,6 & 0,430 & 3 & 1158,6 & CH10_MOUSE & & 10 kDa heat shock protein, mitochondrial; & 10 kDa chaperonin; Chaperonin 10; \\
\hline QGAELSNEER & NLLSVAYKNVVGAR & $-8,4$ & $\mathrm{~N}$-ter +28.03 Da, $\mathrm{K}+28.03 \mathrm{Da}$ & 19,4 & 5,314 & 3 & 1558,9 & 1433B_MOUSE & 1433F_MOUSE & 14-3-3 protein beta/alpha; & Protein kinase C inhibitor protein 1 ; \\
\hline & DDREDLVYQAKLAEQAER & $-0,3$ & $\mathrm{~N}$-ter +28.03 Da, $\mathrm{K}+28.03 \mathrm{Da}$ & 19,6 & 0,000 & 3 & 2335,2 & 1433E_MOUSE & D6REF3_MOUSE & 14-3-3 protein epsilon; & \\
\hline AFDEAIAELD & TLSEESYKDSTLIMQLLR & 3,5 & $\mathrm{~N}$-ter $+28.03 \mathrm{Da}, \mathrm{K}+28.03 \mathrm{Da}$ & 52,5 & 4,027 & 3 & 2182,2 & 1433E_MOUSE & 1433Z_MOUSE & 14-3-3 protein epsilon; & \\
\hline MER & ASLIOKAKLAEQAER & $-0,6$ & $\mathrm{~N}$-ter $+28.03 \mathrm{Da}, \mathrm{K}+28.03 \mathrm{Da}$ & 17,5 & 0,861 & 4 & 1739,0 & 1433S_MOUSE & & 14-3-3 protein sigma; & Stratifin; \\
\hline VLSSIEQKSN & EEGSEEKGPEVKEYR & $-0,5$ & $\mathrm{~N}$-ter $+28.03 \mathrm{Da}, \mathrm{K}+28.03 \mathrm{Da}$ & 16,8 & $-1,458$ & 3 & 1848,9 & 1433S_MOUSE & & 14-3-3 protein sigma; & Stratifin; \\
\hline KGEELSCEER & NLLSVAYKNVVGGQR & $-6,4$ & $\mathrm{~N}$-ter $+28.03 \mathrm{Da}, \mathrm{K}+28.03 \mathrm{Da}$ & 48,6 & 0,835 & 3 & 1673,0 & 1433s_MOUSE & & 14-3-3 protein sigma; & Stratifin; \\
\hline RYEDMAAFMK & SAVEKGEELSCEER & $-1,1$ & $\begin{array}{l}\text { N-ter }+28.03 \\
+57.02 \mathrm{Da}\end{array}$ & 15,3 & 1,666 & 3 & 1677,8 & 1433S_MOUSE & & 14-3-3 protein sigma; & Stratifin; \\
\hline DKKRIIDSAR & SAYQEAMDISKKEMPPTNPIR & $-9,0$ & $\mathrm{~N}$-ter +28.03 Da, K+28.03 Da & 26,5 & $-3,210$ & 3 & 2489,3 & 1433S_MOUSE & & 14-3-3 protein sigma; & Stratifin; \\
\hline DEAMADLHTL & SEDSYKDSTLIMQLLR & $-0,5$ & $\mathrm{~N}$-ter $+28.03 \mathrm{Da}, \mathrm{K}+28.03 \mathrm{Da}$ & 33,1 & 0,358 & 3 & 1954,0 & 1433S_MOUSE & & 14-3-3 protein sigma; & Stratifin; \\
\hline SIEQKSNEEG & SEEKGPEVKEYR & 0,1 & $\mathrm{~N}$-ter $+34.06 \mathrm{Da}, \mathrm{K}+34.06 \mathrm{Da}$ & 15,5 & $-1,221$ & 4 & 1551,9 & 1433S_MOUSE & & 14-3-3 protein sigma; & Stratifin; \\
\hline ELSCEERNLL & SVAYKNVVGGQR & 0,2 & $\mathrm{~N}$-ter $+28.03 \mathrm{Da}, \mathrm{K}+28.03 \mathrm{Da}$ & 26,8 & 0,225 & 3 & 1332,8 & 1433S_MOUSE & & 14-3-3 protein sigma; & Stratifin; \\
\hline IAELDTLNED & SYKDSTLIMQLLR & $-0,3$ & $\mathrm{~N}$-ter $+28.03 \mathrm{Da}, \mathrm{K}+28.03 \mathrm{Da}$ & 28,6 & 1,969 & 2 & 1622,9 & 1433S_MOUSE & 1433B_MOUSE & 14-3-3 protein sigma; & Stratifin; \\
\hline TFDEAMADLH & TLSEDSYKDSTLIMQLLR & 0,0 & $\mathrm{~N}-\operatorname{ter}+34.06 \mathrm{Da}, \mathrm{K}+34.06 \mathrm{Da}$ & 16,1 & $-2,611$ & 3 & 2180,2 & 14335_MOUSE & & 14-3-3 protein sigma; & Stratifin; \\
\hline IPNASQPESK & $\begin{array}{l}\text { VFYLKMKGDYYR } \\
\text { GYTCOFI }\end{array}$ & $-4,2$ & $\mathrm{~N}$-ter $+28.03 \mathrm{Da}, \mathrm{K}+28.03 \mathrm{Da}$ & 26,7 & $-0,180$ & 4 & $\begin{array}{l}1665,9 \\
1577\end{array}$ & 14335_MOUSE & 1433F_MOUSE & 14-3-3 protein sigma; & Stratifin; \\
\hline RYDDMAACMK & $\begin{array}{l}\text { SVTEQGAELSNEER } \\
\text { AFASECP }\end{array}$ & 0,0 & $\mathrm{~N}$-ter $+28.03 \mathrm{Da}$ & 27,8 & - -7,796 & 2 & 1575,7 & 1433Z_MOUSE & D3YXF4_MOUSE & 14-3-3 protein zeta/delta; & Protein kinase C inhibitor protein 1;EZZ-2; \\
\hline TAFQAASALG & AEFASEACR & 0,1 & $\mathrm{~N}$-ter +34.06 Da, C+57.02 Da & 27,0 & 2,139 & 2 & 1073,5 & SEP15_MOUSE & D3Z3Y8_MOUSE & 15 kDa selenoprotein; & 265 proteasome AAA-ATPase subunit \\
\hline ATVWDEAEQD & GIGEEVLKMSTEEIVQR & $-0,9$ & $\mathrm{~N}$-ter +34.06 Da, K+34.06 Da & 19,4 & 1,258 & 3 & 1985,1 & PRS6A_MOUSE & F6Q2E3_MOUSE & 265 protease regulatory subunit $6 \mathrm{~A} ;$ & $\begin{array}{l}\text { RPTS;Proteasome } 265 \text { subunit ATPase 3;Tat- } \\
\text { binding protein 1; } \\
265 \text { proteasome AAA-ATPase subunit }\end{array}$ \\
\hline SRPQTGLSFL & GPEPEDLEDLYSR & 0,6 & $\mathrm{~N}$-ter +34.06 Da & 23,8 & $-0,193$ & 2 & 1552,8 & PRS6B_MOUSE & & 265 protease regulatory subunit 6B; & $\begin{array}{l}\text { RPT3;CIP21;:MB67-interacting } \\
\text { protein;MIIP2224; ritoteasome 265 subunit } \\
\text { ATPase 4; Tat-binding protein 7; }\end{array}$ \\
\hline GLAPPALWDL & AADKQTLQSEQPLQVAR & 0,2 & N-ter +34.06 Da, K+34.06 Da & 38,0 & $-0,102$ & 3 & 1950,1 & PRS7_MOUSE & Q8BVQ9_MOUSE & 265 protease regulatory subunit 7 ; & $\begin{array}{l}265 \text { proteasome AAA-ATPase subunit } \\
\text { RPT1; } 1 \text { roteasome } 265 \text { subunit ATPase 2;Protein } \\
\text { MSS1; }\end{array}$ \\
\hline M & KDVPAFLQQSOSSGPGQAAVWHR & $-0,1$ & $\mathrm{~N}$-ter $+28.03 \mathrm{Da}, \mathrm{K}+28.03 \mathrm{Da}$ & 24,7 & 6,035 & 4 & 2680,4 & PSD13_MOUSE & E9Q519_MOUSE & 265 proteasome non-ATPase regulatory subunit 13; & $\begin{array}{l}265 \text { proteasome regulatory subunit RPN9; } 26 \mathrm{~S} \\
\text { proteasome regulatory subunit S11;265 } \\
\text { proteasome regulatory subunit p40.5; }\end{array}$ \\
\hline MVL & ESTMVCVDNSEYMR & 0,0 & $\mathrm{~N}$-ter $+28.03 \mathrm{Da}, \mathrm{C}+57.02 \mathrm{Da}$ & 28,9 & 2,000 & 2 & 1747,7 & PSMD4_MOUSE & & 265 proteasome non-ATPase regulatory subunit 4 & $\begin{array}{l}265 \text { proteasome regulatory subunit RPN10;26S } \\
\text { proteasome regulatory subunit } \\
\text { S5A;Multiubiquitin chain-binding protein; } \\
265 \text { proteasome regulatory subunit RPN10;26S }\end{array}$ \\
\hline MV & LESTMVCVDNSEYMR & $-0,2$ & $\mathrm{~N}$-ter $+28.03 \mathrm{Da}, \mathrm{C}+57.02 \mathrm{Da}$ & 15,1 & $-0,430$ & 2 & 1860,8 & PSMD4_MOUSE & & 265 proteasome non-ATPase regulatory subunit 4; & 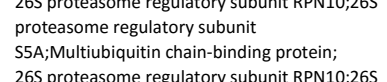 \\
\hline m & VLESTMVCVDNSEYMR & $-0,1$ & $\mathrm{~N}$-ter $+28.03 \mathrm{Da}, \mathrm{C}+57.02 \mathrm{Da}$ & 45,4 & $-0,612$ & 2 & 1959,9 & PSMD4_MOUSE & & 265 proteasome non-ATPase regulatory subunit 4 ; & $\begin{array}{l}\text { proteasome regulatory subunit } \\
\text { S5A;Multiubiquitin chain-binding protein; }\end{array}$ \\
\hline SRLCLAGKRC & LLSAAYVDSHQWEAR & 0,0 & $\mathrm{~N}$-ter +28.03 Da & 17,6 & 1,464 & 3 & 1772,9 & RT27_MOUSE & & 28 S ribosomal protein 527 , mitochondrial; & \\
\hline GGMVPGPVSA & AAPASEAAEEEDVPKQKER & 0,2 & $\mathrm{~N}$-ter $+28.03 \mathrm{Da}, \mathrm{K}+28.03 \mathrm{Da}$ & 18,1 & 2,334 & 4 & 2138,1 & RM12_MOUSE & & 39 S ribosomal protein L12, mitochondrial; & \\
\hline LGRSYGRRKL & SQTQGPPDNPGFVESVDEYQFVER & 0,8 & $\mathrm{~N}$-ter+28.03 Da & 31,0 & $-2,795$ & 3 & 2752,3 & RM49_MOUSE & & 39 S ribosomal protein $L 49$, mitochondrial; & Acetyl-CoA acyltransferase:Beta- \\
\hline AAYLARHVGL & RVGVPTETGALLLNR & 0,2 & N-ter +34.06 Da & 18,6 & 3,457 & 3 & 1617,0 & THIM_MOUSE & & 3-ketoacyl-CoA thiolase, mitochondrial; & $\begin{array}{l}\text { ketothiolase;Mitochondrial 3-oxoacyl-CoA } \\
\text { thiolase; }\end{array}$ \\
\hline NTALQEVLKT & ALHDGLAR & 0,0 & $\mathrm{~N}$-ter +28.03 Da & 23,1 & $-3,415$ & 3 & 992,6 & RS12_MOUSE & Q6ZWZ6_MOUSE & 40S ribosomal protein $\mathrm{S} 12$ & \\
\hline TYRGVDLDQL & LDMSYEQLMQLYSAR & 0,4 & N-ter +34.06 Da & 28,3 & $-2,495$ & 3 & 1880,9 & RS15_MOUSE & & $40 \mathrm{~S}$ ribosomal protein S15; & RIG protein; \\
\hline ASTARHLYLR & GGAGVGSMTKIYGGR & 0,2 & $\mathrm{~N}$-ter $+28.03 \mathrm{Da}, \mathrm{K}+28.03 \mathrm{Da}$ & 31,0 & $-0,7$ & 3 & 1465,8 & RS19_MOUSE & D3Z722_MOUSE & 40S ribosomal protein S19; & \\
\hline SACLSLPQMP & GVTVKDVNQQEFVR & $-0,3$ & $\mathrm{~N}$-ter $+28.03 \mathrm{Da}, \mathrm{K}+28.03 \mathrm{Da}$ & 33,5 & $-0,060$ & 3 & 1673,9 & RS19_MOUSE & S4R223_MOUSE & 40 S ribosomal protein S19; & \\
\hline SLPQMPGVTV & KDVNQQEFVR & 0,0 & $\mathrm{~N}$-ter +34.06 Da, K +34.06 Da & 25,3 & 0,525 & 3 & 1329,8 & RS19_MOUSE & S4R223_MOUSE & 40S ribosomal protein S19; & \\
\hline WVDTVKLAKH & KELAPYDENWFYTR & 0,1 & $\mathrm{~N}$-ter +34.06 Da, K +34.06 Da & 19,0 & 2,261 & 3 & 1899,0 & RS19_MOUSE & S4R223_MOUSE & $40 \mathrm{~S}$ ribosomal protein S19; & \\
\hline CLSLPQMPGV & TVKDVNQQEFVR & $-1,2$ & $\mathrm{~N}$-ter +34.06 Da, K +34.06 Da & 19,9 & 2,609 & 3 & 1529,9 & RS19_MOUSE & S4R223_MOUSE & $40 \mathrm{~S}$ ribosomal protein S19; & \\
\hline ACLSLPQMPG & VTVKDVNQQEEFVR & $-0,1$ & $\mathrm{~N}$-ter $+28.03 \mathrm{Da}, \mathrm{K}+28.03 \mathrm{Da}$ & 24,4 & 2,593 & 3 & 1616,9 & RS19_MOUSE & S4R223_MOUSE & 40 S ribosomal protein S19; & \\
\hline RKCSASNRII & AAKDHASIQMNNVAEVDR & $-0,3$ & $\mathrm{~N}$-ter +34.06 Da, K +34.06 Da & 35,1 & $-7,013$ & 3 & 1922,0 & RS21_MOUSE & & 40 S ribosomal protein $521 ;$ & \\
\hline MQND & AGEFVDLYVPR & 0,5 & $\mathrm{~N}$-ter $+34.06 \mathrm{Da}$ & 24,3 & 1,461 & 2 & 1298,7 & RS21_MOUSE & & $40 \mathrm{~S}$ ribosomal protein $\mathrm{S21}$ & \\
\hline KCSASNRIIA & AKDHASIQMNVAEVDR & 0,3 & $\mathrm{~N}$-ter $+28.03 \mathrm{Da}, \mathrm{K}+28.03 \mathrm{Da}$ & 49,4 & $-0,109$ & 3 & 1838,9 & RS21_MOUSE & & $40 \mathrm{~S}$ ribosomal protein $521 ;$ & \\
\hline MQN & DAGEFVDLYVPR & 0,1 & $\mathrm{~N}$-ter +34.06 Da & 35,1 & 0,565 & 2 & 1413,7 & RS21_MOUSE & & $40 S$ ribosomal protein $521 ;$ & \\
\hline MQNDA & GEFVDLYVPR & 0,1 & N-ter +28.03 Da & 28,8 & $-0,082$ & 2 & 1221,6 & RS21_MOUSE & & $40 S$ ribosomal protein $521 ;$ & \\
\hline YGICGAIRRM & GESDDSILR & 0,2 & N-ter +28.03 Da & 19,5 & 2,548 & & 1018,5 & RS21_MOUSE & & $40 S$ ribosomal protein S21; & \\
\hline
\end{tabular}




\begin{tabular}{|c|c|c|c|c|c|c|c|c|c|c|c|}
\hline Non Prime Site & Prime Site & $\begin{array}{l}\text { Fold change of } \\
\text { XPRESS ratio } \\
\text { (Adam17 } 7^{\text {AKC } / W T)} \\
\end{array}$ & Modifications & Hyperscore & $\begin{array}{c}\text { Mass error } \\
\text { in ppm }\end{array}$ & Charge & $\begin{array}{c}\text { Precursor } \\
\text { neutral mass } \\
\text { in Da }\end{array}$ & Uniprot ID 1 & Uniprot ID 2 & Protein Name 1 & Protein Name 2 \\
\hline $\begin{array}{l}\text { VPRKCSASNR } \\
\text { SGCWDMA }\end{array}$ & IIAAKDHASIQMNNVAEVDR & $-1,7$ & N-ter +28.03 Da, K +28.03 Da & 14,5 & 0,561 & 4 & 2136,1 & RS21_MOUSE & & 40S ribosomal protein S21; & \\
\hline CSASNRIIAA & KDHASIOMNVAEVDR & 0,2 & N-ter $+28.03 \mathrm{Da}, \mathrm{K}+28.03 \mathrm{Da}$ & 41,9 & $-0,113$ & 3 & 1767,9 & RS21_MOUSE & & $40 S$ ribosomal protein S21; & \\
\hline TYGICGAIRR & MGESDDSILR & 0,0 & $\mathrm{~N}$-ter +28.03 Da & 18,1 & 0,347 & 2 & 1149,5 & RS21_MOUSE & & 40 ribosomal protein S21; & \\
\hline KTYGICGAIR & RMGESDDSILR & 0,1 & N-ter +28.03 Da & 22,5 & 0,153 & 3 & 1305,6 & RS21_MOUSE & & $40 S$ ribosomal protein S21; & \\
\hline NRIIAAKDHA & SIQMNVAEVDR & 0,1 & $\mathrm{~N}$-ter +28.03 Da & 40,6 & $-0,387$ & 2 & 1288,6 & RS21_MOUSE & & $40 S$ ribosomal protein S21; & \\
\hline AQTVVLCVGC & STVLCQPTGGKAR & 0,2 & $\begin{array}{l}\text { N-ter +34.06 Da, } \mathrm{K}+34.06 \mathrm{Da}, \mathrm{C} \\
+57.02 \mathrm{Da}\end{array}$ & 24,6 & 1,315 & 3 & 1441,8 & RS27L_MOUSE & RS27_MOUSE & 40 S ribosomal protein S27-like; & \\
\hline QTVVLCVGCS & TVLCQPTGGKAR & 0,0 & $\begin{array}{l}\text { N-ter +28.03 Da, K +28.03 Da, C } \\
+57.02 \mathrm{Da}\end{array}$ & 20,7 & 2,824 & 3 & 1342,7 & RS27L_MOUSE & RS27_MOUSE & 40S ribosomal protein S27-like; & \\
\hline SQGQCTQVRV & EFMDDTSR & $-0,1$ & $\mathrm{~N}$-ter $+28.03 \mathrm{Da}$ & 20,6 & 2,914 & 2 & 1027,4 & RS28_MOUSE & J3QNN8_MOUSE & $40 \mathrm{~S}$ ribosomal protein $\mathrm{S28}$ & \\
\hline IRNVKGPVRE & GDVLTLLESER & 0,1 & $\mathrm{~N}$-ter +28.03 Da & 17,4 & 0,635 & 2 & 1258,7 & RS28_MOUSE & G3UYV7_MOUSE & 40 r ribosomal protein 528; & \\
\hline TGSQGQCTQV & RVEFMDDTSR & $-0,1$ & $\mathrm{~N}$-ter +34.06 Da & 21,8 & $-1,239$ & 3 & 1288,6 & RS28_MOUSE & J3QNN8_MOUSE & 40 S ribosomal protein 528; & \\
\hline QRAKSMKFVD & GLMIHSGDPVNYYVDTAVR & 0,2 & $\mathrm{~N}$-ter +34.06 Da & 38,7 & 2,006 & 3 & 2140,1 & RS3_MOUSE & & $40 \mathrm{~S}$ ribosomal protein $\mathrm{S3} ;$ & \\
\hline IFKAELNEFL & TRELAEDGYSGVEVR & 1,0 & N-ter $+34.06 \mathrm{Da}$ & 20,5 & 1,864 & 3 & 1713,9 & RS3_MOUSE & D3YV43_MOUSE & $40 \mathrm{~S}$ ribosomal protein S3; & \\
\hline IPDSIGKDIE & KACQSIYPLHDVFVR & 3,0 & $\begin{array}{l}\text { N-ter }+34.06 \text { Da, } \mathrm{K}+34.066 \mathrm{Da}, \mathrm{C} \\
+57.02 \mathrm{Da}\end{array}$ & 17,5 & $-4,624$ & 3 & 1900,1 & RS3A_MOUSE & D3Z6C3_MOUSE & $\begin{array}{l}\text { 40S ribosomal protein S3a \{ECO:0000255|HAMAP- } \\
\text { Rule:MF__3122\};; }\end{array}$ & Protein TU-11; \\
\hline IGKDIEKACQ & SIYPLHDVFVR & $-0,1$ & $\mathrm{~N}$-ter +34.06 Da & 32,0 & 0,217 & 3 & 1378,8 & RS3A_MOUSE & D3Z6C3_MOUSE & $\begin{array}{l}\text { 40S ribosomal protein S3a \{ECO:0000255|HAMAP- } \\
\text { Rule:MF_03122\}; }\end{array}$ & Protein TU-11; \\
\hline M & KLNISFPATGCQKLEVVDDER & 0,0 & $\begin{array}{l}\text { N-ter +34.06 Da, K+34.06 Da, C } \\
+57.02 \mathrm{Da}\end{array}$ & 28,0 & 0,946 & 4 & 2534,4 & RS6_MOUSE & & 40 S ribosomal protein S6; & Phosphoprotein NP33; \\
\hline $\begin{array}{l}\text { RSRTLTAVHD } \\
\text { SRPGQCGRAD }\end{array}$ & $\begin{array}{l}\text { AlLDLVFPSEIGGR } \\
\text { GYVLEGKELFYLR }\end{array}$ & $\begin{array}{l}-0,3 \\
-0,3\end{array}$ & $\begin{array}{l}\mathrm{N} \text {-ter +28.03 Da, } \mathrm{K}+28.03 \mathrm{Da} \\
\mathrm{N} \text {-ter +34.06 Da, } \mathrm{K}+34.06 \mathrm{Da}\end{array}$ & $\begin{array}{l}43,1 \\
20,1\end{array}$ & $\begin{array}{l}6,236 \\
4,759\end{array}$ & $\begin{array}{l}3 \\
3\end{array}$ & $\begin{array}{l}1841,1 \\
1783,0\end{array}$ & $\begin{array}{l}\text { RS7_MOUSE } \\
\text { RS8_MOUSE }\end{array}$ & J3QJZ3_MOUSE & $\begin{array}{l}40 S \text { ribosomal protein } 57 ; \\
40 \text { r ribosomal protein } 58 ;\end{array}$ & \\
\hline NTGQRAVLKF & AAATGATPIAGR & $-0,1$ & N-ter+28.03 Da & 31,2 & $-1,013$ & 2 & 1083,6 & RSSA_MOUSE & DЗYTT7_MOUSE & $\begin{array}{l}\text { 40S ribosomal protein SA \{ECO:0000255|HAMAP- } \\
\text { Rule:MF_03016\}; }\end{array}$ & 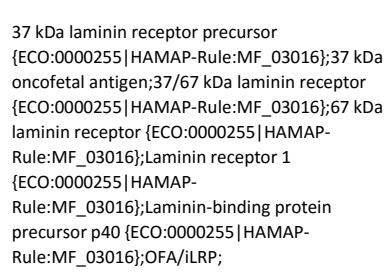 \\
\hline RTWEKLLLAA & RAIVAIENPADVSVISSR & 0,1 & $\mathrm{~N}$-ter +28.03 Da & 28,0 & 7,005 & 3 & 1924,1 & RSSA_MOUSE & DЗYTT7_MOUSE & $\begin{array}{l}\text { 40S ribosomal protein SA \{ECO:0000255|HAMAP- } \\
\text { Rule:MF_03016\}; }\end{array}$ & 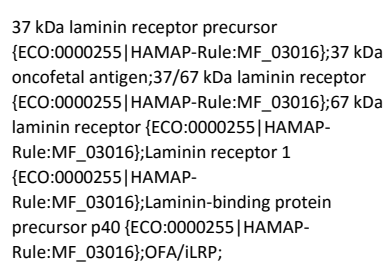 \\
\hline RVTDALNATR & AAVEEGIVLGGGCALLR & $-10,0$ & N-ter +28.03 Da, C +57.02 Da & 27,5 & $-2,392$ & 2 & 1711,9 & CH60_MOUSE & & 60 kDa heat shock protein, mitochondrial; & $\begin{array}{l}60 \mathrm{kDa} \text { chaperonin;Chaperonin } 60 ; \mathrm{HSP}-65 ; \text {; Heat } \\
\text { shock protein } 60 \text {;Mitochondrial matrix protein } \\
\text { P1; } \\
60 \text { kDa chaperonin;Chaperonin } 60 ; \mathrm{HSP}-65 \text {; Heat }\end{array}$ \\
\hline ALAPHLTRAY & AKDVKFGADAR & $-0,2$ & $\mathrm{~N}$-ter $+28.03 \mathrm{Da}, \mathrm{K}+28.03 \mathrm{Da}$ & 27,3 & $-0,474$ & 4 & 1260,7 & CH6O_MOUSE & D3Z2F2_MOUSE & $60 \mathrm{kDa}$ heat shock protein, mitochondrial; & $\begin{array}{l}\text { shock protein } 60 \text {;Mitochondrial matrix protein } \\
\text { P1; } \\
60 \mathrm{kDa} \text { chaperonin; Chaperonin } 60 ; \mathrm{HSP}-65 \text {; Heat }\end{array}$ \\
\hline KGVITVKDGK & TLNDELEIIEGMKFDR & 0,6 & $\mathrm{~N}$-ter $+28.03 \mathrm{Da}, \mathrm{K}+28.03 \mathrm{Da}$ & 17,1 & 9,843 & 3 & 1978,0 & CH60_MOUSE & & $60 \mathrm{kDa}$ heat shock protein, mitochondrial; & $\begin{array}{l}\text { shock protein 60;Mitochondrial matrix protein } \\
\mathrm{P} 1 \text {; }\end{array}$ \\
\hline NGSIYNPEVL & DITEQALHSR & 0,6 & $\mathrm{~N}$-ter $+34.06 \mathrm{Da}$ & 24,1 & 3,235 & 3 & 1202,7 & RLAO_MOUSE & S4R1N1_MOUSE & $60 S$ acidic ribosomal protein $\mathrm{PO}$; & 60S ribosomal protein L10E; \\
\hline SFGLIIQQVFF & DNGSIYNPEVLDITEQALHSR & 1,0 & $\mathrm{~N}$-ter +28.03 Da & 28,9 & 0,541 & 3 & 2398,2 & RLAO_MOUSE & SAR1N1_MOUSE & $60 \mathrm{~S}$ acidic ribosomal protein $\mathrm{PO}$; & 60S ribosomal protein L10E; \\
\hline GLIIQQVFDN & GSIYNPEVLDITEQALHSR & 1,8 & $\mathrm{~N}$-ter $+34.06 \mathrm{Da}$ & 45,4 & $-4,821$ & 3 & 2175,1 & RLAO_MOUSE & SAR1N1_MOUSE & $60 \mathrm{~S}$ acidic ribosomal protein $\mathrm{PO}$; & 60S ribosomal protein L10E; \\
\hline MRYVASYLL & AALGGNSSPSAKDIKKILDSVGIEADDDR & 0,3 & $\mathrm{~N}$-ter $+34.06 \mathrm{Da}, \mathrm{K}+34.06 \mathrm{Da}$ & 29,9 & 3,860 & 5 & 3077,8 & RLA2_MOUSE & & $60 \mathrm{~S}$ acidic ribosomal protein $\mathrm{P} 2 ;$ & \\
\hline MRYVASYLLA & ALGGNSSPSAKDIKKILDSVGIEADDDR & 0,3 & $\mathrm{~N}$-ter $+28.03 \mathrm{Da}, \mathrm{K}+28.03 \mathrm{Da}$ & 33,4 & 1,540 & 4 & 2982,6 & RLA2_MOUSE & & $60 \mathrm{~S}$ acidic ribosomal protein $\mathrm{P2}$ & \\
\hline YVASYLLAAL & GGNSSPSAKDIKKILDSVGIEADDDR & 0,4 & $\mathrm{~N}-\operatorname{ter}+34.06 \mathrm{Da}, \mathrm{K}+34.06 \mathrm{Da}$ & 24,5 & 6,085 & 4 & 2822,6 & RLA2_MOUSE & & 60 a acidic ribosomal protein P2; & \\
\hline KWGFTKFNAD & EFEDMVAEKR & 1,5 & N-ter $+34.06 \mathrm{Da}, \mathrm{K}+34.06 \mathrm{Da}$ & 17,7 & $-3,173$ & 3 & 1320,7 & RL10_MOUSE & 17HLV2_MOUSE & 60 r ribosomal protein L10; & Protein QM homolog; \\
\hline DRKKQKNIKH & SGNITFDEIVNIAR & 1,7 & $\mathrm{~N}$-ter $+34.06 \mathrm{Da}$ & 27,9 & 4,735 & 2 & 1581,9 & RL12_MOUSE & & 60S ribosomal protein L12; & \\
\hline M & AEGQVLVLDGR & $-0,1$ & $\mathrm{~N}$-ter $+28.03 \mathrm{Da}$ & 32,9 & 1,181 & 2 & 1183,7 & RL13A_MOUSE & D3YY61_MOUSE & 60 r ribosomal protein L13a; & $\begin{array}{l}\text { Transplantation antigen P198;Tum-P198 } \\
\text { antigen; }\end{array}$ \\
\hline
\end{tabular}




\begin{tabular}{|c|c|c|c|c|c|c|c|c|c|c|c|}
\hline Non Prime Site & Prime Site & 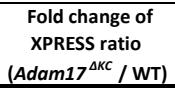 & Modifications & Hyperscore & $\begin{array}{c}\text { Mass error } \\
\text { in ppm }\end{array}$ & Charge & $\begin{array}{c}\text { Precursor } \\
\text { neutral mass } \\
\text { in Da }\end{array}$ & Uniprot ID 1 & Uniprot ID 2 & Protein Name 1 & Protein Name 2 \\
\hline M & GAYKYIQELWR & $-0,1$ & & 52,8 & $-0,809$ & 2 & $1481,8 \quad$ & RL15_MOUSE & B8JKK2_MOUSE & 60S ribosomal protein L15; & \\
\hline MA & KFNPFVTSDR & $-0,1$ & & 34,4 & $-0,638$ & 3 & 1408,8 & RL26_MOUSE & BIARA__MOUSE & 60S ribosomal protein L26; & Silica-induced gene 20 protein; \\
\hline TAGGKYYRVC & $\begin{array}{l}\text { TLAIIDPGDSDIIR } \\
\text { LVIINOKENLR }\end{array}$ & 0,3 & $\mathrm{~N}$-ter $+34.06 \mathrm{Da}$ & 18,7 & $-1,304$ & 2 & 1531,9 & RL30_MOUSE & & 60 S ribosomal protein L30; & \\
\hline $\begin{array}{l}\text { VVRKSIARVL } \\
\text { TVAGGAWTYN }\end{array}$ & $\begin{array}{l}\text { TVINQTQKENLR } \\
\text { TTSAVTVKSAIR }\end{array}$ & $\begin{array}{l}-2,9 \\
-0,2\end{array}$ & & 21,6 & $-1,387$ & 3 & 1510,9 & RL35_MOUSE & & 60S ribosomal protein L35; & \\
\hline & & & & 32,9 & $-0,767$ & 3 & & RL37A_MOUSE & & $60 S$ ribosomal protein L37a; & \\
\hline ARLGHLVHRK & TCTVAFTQVNSEDKGALAKLVEAIR & 0,7 & $\begin{array}{l}\text { N-ter +34.06 Da, K +34.06 Da, C } \\
+57.02 \mathrm{Da}\end{array}$ & 26,6 & $-1,093$ & 4 & 2923,7 & RL7A_MOUSE & D3YVE6_MOUSE & $60 S$ ribosomal protein L7a; & Surfeit locus protein 3; \\
\hline $\begin{array}{l}\text { QKDELLLEGN } \\
\text { M }\end{array}$ & $\begin{array}{l}\text { DIELVSNSAALIQQATVVKNKDIR } \\
\text { KTILSNQTVDIPENVEITLKGR }\end{array}$ & $\begin{array}{l}-0,1 \\
0,1\end{array}$ & $\begin{array}{l}\mathrm{N} \text {-ter }+34.06 \mathrm{Da}, \mathrm{K}+34.06 \mathrm{Da} \\
\mathrm{N} \text {-ter }+34.06 \mathrm{Da}, \mathrm{K}+34.06 \mathrm{Da}\end{array}$ & $\begin{array}{l}18,0 \\
47,2\end{array}$ & $\begin{array}{l}0,146 \\
0,111\end{array}$ & $\begin{array}{l}4 \\
4\end{array}$ & $\begin{array}{l}2728,6 \\
2700,6\end{array}$ & $\begin{array}{l}\text { RL9_MOUSE } \\
\text { RL9_MOUSE }\end{array}$ & $\begin{array}{l}\text { G3UW34_MOUSE } \\
\text { G3UW34_MOUSE }\end{array}$ & $\begin{array}{l}\text { 60S ribosomal protein } 19 ; \\
60 \text { r ribosomal protein } 19 ;\end{array}$ & \\
\hline M & RPLTEEETR & 0,0 & $\mathrm{~N}$-ter $+34.06 \mathrm{Da}$ & 28,4 & 1,156 & 3 & 1294,7 & NIP7_MOUSE & E9Q109_MOUSE & $\begin{array}{l}60 \text { ribosome subunit biogenesis protein NIP7 } \\
\text { homolog. }\end{array}$ & PEachy;kDa93; \\
\hline VTLTLPVLNA & AQSIIFVATGEGKAAVLKR & 0,0 & $\mathrm{~N}$-ter $+28.03 \mathrm{Da}, \mathrm{K}+28.03 \mathrm{Da}$ & 19,0 & $-7,233$ & 4 & 2042,2 & 6PGL_MOUSE & D3Z4X1_MOUSE & 6-phosphogluconolactonase; & \\
\hline NGVFEVVATN & GDTHLGGEDFDQR & $-0,3$ & $\mathrm{~N}$-ter+28.03 Da & 17,4 & $-0,271$ & 3 & 1473,7 & GRP78_MOUSE & & 78 kDa glucose-regulated protein; & $\begin{array}{l}\text { Heat shock } 70 \mathrm{kDa} \text { protein 5:Immunoglobulin } \\
\text { heavy chain-binding protein; }\end{array}$ \\
\hline CPLTLGIETV & GGVMTKLIPR & 0,3 & $\mathrm{~N}$-ter $+28.03 \mathrm{Da}, \mathrm{K}+28.03 \mathrm{Da}$ & 21,4 & 1,239 & 3 & 1126,7 & GRP78_MOUSE & & 78 kDa glucose-regulated protein; & $\begin{array}{l}\text { Heat shock } 70 \mathrm{kDa} \text { protein 5:Immunoglobulin } \\
\text { heavy chain-binding protein; }\end{array}$ \\
\hline AGTIAGLNVM & RIINEPTAAAIAYGLDKR & 1,0 & $\mathrm{~N}$-ter $+28.03 \mathrm{Da}, \mathrm{K}+28.03 \mathrm{Da}$ & 19,2 & 8,960 & 4 & 2027,2 & GRP78_MOUSE & & 78 kDa glucose-regulated protein; & $\begin{array}{l}\text { Heat shock } 70 \mathrm{kDa} \text { protein 5:Immunoglobulin } \\
\text { heavy chain-binding protein; }\end{array}$ \\
\hline TALFTRQDL & CGATTCDTLGMADVGTMCDPKR & 10,0 & $\begin{array}{l}\text { N-ter }+34.06 \mathrm{Da}, \mathrm{K}+34.06 \mathrm{Da}, \mathrm{C} \\
+57.02 \mathrm{Da}\end{array}$ & 15,9 & 5,669 & 3 & 2484,1 & ATS15_MOUSE & & $\begin{array}{l}\text { A disintegrin and metalloproteinase with } \\
\text { thrombospondin motifs 15; }\end{array}$ & \\
\hline QEVRCLERSY & ASKPTLNEVVIVSAIR & 0,0 & N-ter +28.03 Da, K+28.03 Da & 55,8 & 0,057 & 3 & 1752,1 & THIL_MOUSE & & Acetyl-CoA acetyltransferase, mitochondrial; & Acetoacetyl-CoA thiolase; \\
\hline EEGYNDGEVD & DEEDEEEAGEEEGSQKR & 0,5 & $\mathrm{~N}$-ter $+28.03 \mathrm{Da}, \mathrm{K}+28.03 \mathrm{Da}$ & 36,7 & 1,631 & 3 & 2020,8 & AN32A_MOUSE & D3Z7M9_MOUSE & $\begin{array}{l}\text { Acidic leucine-rich nuclear phosphoprotein } 32 \text { family } \\
\text { member } A \text {; }\end{array}$ & $\begin{array}{l}\text { Acidic nuclear phosphoprotein pp32; Leucine- } \\
\text { rich acidic nuclear protein;Potent heat-stable } \\
\text { protein phosphatase } 2 A \text { inhibitor IIPP2A; }\end{array}$ \\
\hline MCD & DEETTALVCDNGSGLVKAGFAGDDAPR & $-0,5$ & $\begin{array}{l}\text { N-ter +28.03 Da, K +28.03 Da, C } \\
+57.02 \mathrm{Da}\end{array}$ & 24,6 & 5,136 & 3 & 2820,3 & ACTC_MOUSE & F6WX90_MOUSE & Actin, alpha cardiac muscle 1; & Alpha-cardiac actin; \\
\hline MCE & EEDSTALVCDNGSGLCKAGFAGDDAPR & 2,8 & $\begin{array}{l}\text { N-ter +34.06 Da, K+34.06 Da, C } \\
+57.02 \mathrm{Da}\end{array}$ & 29,3 & 0,069 & 3 & 2879,3 & ACTA_MOUSE & & Actin, aortic smooth muscle; & Alpha-actin-2; \\
\hline RKDLYANTVL & SGGTTMYPGIADR & 0,1 & $\mathrm{~N}$-ter +28.03 Da & 37,2 & 0,074 & 2 & 1352,6 & ACTA_MOUSE & ACTB_MOUSE & Actin, aortic smooth muscle; & Alpha-actin-2; \\
\hline DRMQKEITAL & APSTMKIKIIAPPER & 0,2 & $\mathrm{~N}$-ter $+28.03 \mathrm{Da}, \mathrm{K}+28.03 \mathrm{Da}$ & 31,3 & $-0,345$ & 4 & 1735,0 & ACTB_MOUSE & ACTA_MOUSE & Actin, cytoplasmic 1; & Beta-actin; \\
\hline YSVWIGGSIL & ASLSTFQQMWISKQEYDESGPSIVHR & 1,2 & $\mathrm{~N}$-ter +34.06 Da, K+34.06 Da & 22,9 & 0,969 & 4 & 3091,6 & ACTB_MOUSE & ACTG_MOUSE & Actin, cytoplasmic 1; & Beta-actin; \\
\hline AGFAGDDAPR & AVFPSIVGRPR & $-3,5$ & $\mathrm{~N}$-ter $+28.03 \mathrm{Da}$ & 33,6 & $-0,407$ & 3 & 1225,7 & ACTB_MOUSE & ACTA_MOUSE & Actin, cytoplasmic 1; & Beta-actin; \\
\hline MFAM & DDDIAALVVDNGSGMCKAGFAGDDAPR & $-9,0$ & $\begin{array}{l}\text { N-ter }+28.03 \mathrm{Da}, \mathrm{K}+28.03 \mathrm{Da}, \mathrm{C} \\
+57.02 \mathrm{Da}\end{array}$ & 23,8 & $-8,472$ & 4 & 2923,3 & ACTB_MOUSE & E9Q1F2_MOUSE & Actin, cytoplasmic 1; & Beta-actin; \\
\hline MFAMD & DDIAALVVDNGSGMCKAGFAGDDAPR & 1,0 & $\begin{array}{l}\text { N-ter +34.06 Da, K +34.06 Da, C } \\
+57.02 \text { Da }\end{array}$ & 31,2 & 6,129 & 3 & 2689,3 & ACTB_MOUSE & E9Q1F2_MOUSE & Actin, cytoplasmic 1; & Beta-actin; \\
\hline QQMWISKQEY & DESGPSIVHR & 0,0 & $\mathrm{~N}$-ter $+28.03 \mathrm{Da}$ & 18,5 & 4,971 & 3 & 1123,6 & ACTB_MOUSE & ACTG_MOUSE & Actin, cytoplasmic 1; & Beta-actin; \\
\hline MFAMDD & DIAALVVDNGSGMCKAGFAGDDAPR & 0,5 & N-ter +34.06 Da, K +34.06 Da, C & 36,2 & $-2,522$ & 3 & 2574,3 & ACTB_MOUSE & E9Q1F2_MOUSE & Actin, cytoplasmic 1; & Beta-actin; \\
\hline AlLRLDLAGR & DLTDYLMKILTER & $-6,3$ & $\begin{array}{l}+57.02 \mathrm{Da} \\
\mathrm{N} \text {-ter }+28.03 \mathrm{Da}, \mathrm{K}+28.03 \mathrm{Da}\end{array}$ & 23,7 & 0,659 & 3 & 1665,9 & ACTB_MOUSE & ACTA_MOUSE & Actin, cytoplasmic 1; & Beta-actin; \\
\hline ASGRTTGIVM & DSGDGVTHTVPIYEGYALPHAILR & 0,6 & $\mathrm{~N}$-ter $+28.03 \mathrm{Da}$ & 21,7 & 0,651 & 4 & 2608,3 & ACTB_MOUSE & ACTBL_MOUSE & Actin, cytoplasmic 1; & Beta-actin; \\
\hline MATAASSSSL & EKSYELPDGQVITIGNER & $-1,2$ & $\mathrm{~N}$-ter +34.06 Da, K+34.06 Da & 19,7 & $-9,678$ & 3 & 2115,1 & ACTB_MOUSE & ACTA_MOUSE & Actin, cytoplasmic 1; & Beta-actin; \\
\hline GRPRHOGVMV & GMGOKDSWVGDEAOSKR & 0,2 & $\mathrm{~N}$-ter $+28.03 \mathrm{Da} \mathrm{K}+28.03 \mathrm{Da}$ & 35,6 & -0.566 & 4 & 1939,0 & ACTB MOUSE & ACTA MOUSE & $\begin{array}{l}\text { Actin, cytoplasmic 1: } \\
\text { int }\end{array}$ & Beta-actin: \\
\hline ASLSTFOOMW & ISKOEYDESGPSIVHR & -0.2 & $\mathrm{~N}$-ter $+34.06 \mathrm{Da} \mathrm{K}+34.06 \mathrm{Da}$ & 35.5 & 0,313 & 3 & 1912,0 & ACTB MOUSE & ACTG MOUSE & Actin, cytoplasmic 1: & Beta-actin: \\
\hline LSTFQQMWIS & KQEYDESGPSIVHR & 0,3 & $\mathrm{~N}$-ter +28.03 Da, K +28.03 Da & 27,7 & $-9,102$ & 3 & $\begin{array}{l}1699,8 \\
169,0\end{array}$ & ACTB_MOUSE & ACTG_MOUSE & Actin, cytoplasmic 1; & Beta-actin; \\
\hline LRVAPEEHPV & LLTEAPLNPKANR & 2,5 & N-ter +34.06 Da, K +34.06 Da & 39,7 & $-0,730$ & 3 & $\begin{array}{l}1503,9 \\
150\end{array}$ & ACTB_MOUSE & ACTA_MOUSE & Actin, cytoplasmic 1; & Beta-actin; \\
\hline RVAPEEHPVL & LTEAPLNPKANR & 0,7 & N-ter +34.06 Da, K +34.06 Da & 33,4 & 0,646 & 3 & 1390,9 & ACTB_MOUSE & ACTA_MOUSE & Actin, cytoplasmic 1; & Beta-actin; \\
\hline TMMYPGIADR & MQKEITALAPSTMKIKIIAPPER & 2,5 & $\mathrm{~N}$-ter $+28.03 \mathrm{Da}, \mathrm{K}+28.03 \mathrm{Da}$ & 24,9 & $-0,224$ & 5 & 2677,6 & ACTB_MOUSE & ACTA_MOUSE & Actin, cytoplasmic 1 & Beta-actin; \\
\hline DDDIAALVVD & NGSGMCKAGFAGDDAPR & $-10,0$ & N-ter +28.03 Da, K+28.03 Da, C & 19,4 & $-5,541$ & 3 & 1765,8 & ACTB_MOUSE & ACTG_MOUSE & Actin, cytoplasmic 1; & Beta-actin; \\
\hline TDYLMKLITE & RGYSFTTAER & 0,3 & $\begin{array}{l}+57.02 \mathrm{Da} \\
\mathrm{N} \text {-ter } 28.03 \mathrm{Da}\end{array}$ & 18,3 & 4,323 & 3 & 1315,7 & АСТB MOUSE & ACTG MOUSE & Actin, cytoplasmic 1; & Beta-actin: \\
\hline SGRTGIVMD & SGDGVTHTVPIYEGYALPHAILR & 0,2 & $\mathrm{~N}$-ter $+28.03 \mathrm{Da}$ & 40,7 & $-4,325$ & 4 & 2493,3 & ACTB_MOUSE & ACTBL_MOUSE & Actin, cytoplasmic 1; & Beta-actin; \\
\hline SLSTFQQMWI & SKOEYDESGPSIVHR & 0,2 & $\mathrm{~N}$-ter $+28.03 \mathrm{Da}, \mathrm{K}+28.03 \mathrm{Da}$ & 19,9 & $-0,168$ & 4 & 1786,9 & ACTB_MOUSE & ACTG_MOUSE & Actin, cytoplasmic 1; & \\
\hline QEMATAASSS & SLEKSYEI & 0,2 & $\mathrm{~N}-\mathrm{ter}+28.03 \mathrm{Da}, \mathrm{K}+28.03 \mathrm{Da}$ & 72,0 & 1,431 & 3 & 2303,2 & АСTB MOUSE & ACTA MOUSE & Actin, cytoplasmic 1 & Beta-actin; \\
\hline WIGGSILASL & STFQQMWISKQEYDESGPSIVHR & 0,3 & N-ter +34.06 Da, & 38,3 & $-1,310$ & 4 & 2820,4 & ACTB MOUSE & ACTG MOUSE & Actin, cytoplasmic 1 & 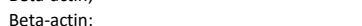 \\
\hline VAPEEHPVLL & TEAPLNPKANR & 0,1 & $\mathrm{~N}-\mathrm{ter}+28.03 \mathrm{Da}, \mathrm{K}+28.03 \mathrm{Da}$ & 42,1 & 1,576 & 3 & 1265,7 & ACTB MOUSE & ACTA MOUSE & Actin, cytoplasmic 1 & Beta-actin; \\
\hline VISLYASGRT & TGIVMDSGDGVTHTVPIYEGYALPHALR & -01 & N-ter $+2803 \mathrm{Da}$ & 358 & -0.899 & 4 & 31096 & ACTB & ACTBL MOUSE & Actit, cytoplasmic 1. & 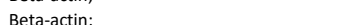 \\
\hline VDIRKDLYAN & TVLSGGTTMYPGIADR & 0,1 & $\mathrm{~N}$-ter +34.06 Da & 16,8 & $-0,418$ & 3 & 1671,9 & АСTB & ACTG_MOUSE & oplasmic 1; & Beta \\
\hline VMDSGDGVTH & TVPYYGGYALPHAILR & $-0,1$ & N-ter +28.03 Da & 38,5 & 2,984 & 3 & 1840,0 & АСТB_MOUSE & ACTBL_MOUSE & Actin, cytoplasmic 1; & Beta-actin; \\
\hline
\end{tabular}




\begin{tabular}{|c|c|c|c|c|c|c|c|c|c|c|c|}
\hline Non Prime Site & Prime Site & $\begin{array}{l}\text { Fold change of } \\
\text { XPRESS ratio } \\
\text { (Adam17 } 7^{\text {AkC } / \text { WT) }}\end{array}$ & Modifications & Hyperscore & $\begin{array}{c}\text { Mass error } \\
\text { in ppm }\end{array}$ & Charge & $\begin{array}{c}\text { Precursor } \\
\text { neutral mass } \\
\text { in Da }\end{array}$ & Uniprot ID 1 & Uniprot ID 2 & Protein Name 1 & Protein Name 2 \\
\hline WHHTFYNELR & VAPEEHPVLLTEAPLNPK & 0,3 & N-ter +34.06 Da, K+34.06 Da & 26,1 & $-2,964$ & 3 & 2021,2 & ACTB_MOUSE & ACTG_MOUSE & Actin, cytoplasmic 1; & Beta-actin; \\
\hline FAMDDDIAAL & VVDNGSGMCKAGFAGDDAPR & $-0,6$ & $\begin{array}{l}\text { N-ter +34.06 Da, K+34.06 Da, C } \\
+57.02 \mathrm{Da}\end{array}$ & 16,5 & $-3,438$ & 3 & 2091,0 & ACTB_MOUSE & E9Q1F2_MOUSE & Actin, cytoplasmic 1; & Beta-actin; \\
\hline MEEEI & AALVIDNGSGMCKAGFAGDDAPR & $-0,1$ & $\begin{array}{l}\text { N-ter +28.03 Da, K+28.03 Da, C } \\
+57.02 \mathrm{Da}\end{array}$ & 32,0 & 5,317 & 3 & 2348,1 & ACTG_MOUSE & G3UYGO_MOUSE & Actin, cytoplasmic 2; & Gamma-actin; \\
\hline MDDDIAALVV & DNGSGMCKAGFAGDDAPR & 0,4 & $\begin{array}{l}\text { N-ter +34.06 Da, K+34.06 Da, C } \\
+57.02 \mathrm{Da}\end{array}$ & 39,0 & 1,055 & 3 & 1892,9 & ACTG_MOUSE & ACTB_MOUSE & Actin, cytoplasmic 2; & Gamma-actin; \\
\hline M & EEEIAALVIDNGSGMCKAGFAGDDAPR & $-10,0$ & $\begin{array}{l}\text { N-ter + 28.03 Da, K +28.03 Da, C } \\
+57.02 \mathrm{Da}\end{array}$ & 17,6 & $-3,262$ & 3 & 2848,3 & ACTG_MOUSE & G3UYGO_MOUSE & Actin, cytoplasmic 2; & Gamma-actin; \\
\hline FEQEMATAAS & SSSLEKSYELPDGQVITIGNER & $-6,5$ & N-ter +34.06 Da, $\mathrm{K}+34.06 \mathrm{Da}$ & 28,6 & $-4,213$ & 3 & 2489,3 & ACTG_MOUSE & ACTA_MOUSE & Actin, cytoplasmic 2; & Gamma-actin; \\
\hline DFEQEMATAA & SSSSLEKSYELPDGQVITIGNER & 0,0 & $\mathrm{~N}-\operatorname{ter}+34.06 \mathrm{Da}, \mathrm{K}+34.06 \mathrm{Da}$ & 40,3 & $-4,846$ & 3 & 2576,4 & ACTG_MOUSE & ACTA_MOUSE & Actin, cytoplasmic 2; & Gamma-actin; \\
\hline VALDFEQEMA & TAASSSSLEKSYELPDGQVITIGNER & $-0,1$ & N-ter +28.03 Da, $\mathrm{K}+28.03 \mathrm{Da}$ & 22,4 & 2,491 & 3 & 2807,4 & ACTG_MOUSE & ACTA_MOUSE & Actin, cytoplasmic 2; & Gamma-actin; \\
\hline MEEEIAAL & VIDNGSGMCKAGFAGDDAPR & 0,6 & $\begin{array}{l}\text { N-ter +34.06 Da, K+34.06 Da, C } \\
+57.02 \mathrm{Da}\end{array}$ & 21,1 & 0,901 & 3 & 2105,0 & ACTG_MOUSE & G3UYGO_MOUSE & Actin, cytoplasmic 2; & Gamma-actin; \\
\hline MC & EEETALIVCDNGSGLCKAGFAGDDAPR & 0,6 & $\begin{array}{l}\text { N-ter +34.06 Da, K+34.06 Da, C } \\
+57.02 \mathrm{Da}\end{array}$ & 25,4 & $-9,449$ & 3 & 2907,3 & ACTH_MOUSE & DЗZ2K__MOUSE & Actin, gamma-enteric smooth muscle; & $\begin{array}{l}\text { Alpha-actin-3;Gamma-2-actin;Smooth muscle } \\
\text { gamma-actin; }\end{array}$ \\
\hline MCE & EETTALVCDNGSGLCKAGFAGDDAPR & 10,0 & $\begin{array}{l}\text { N-ter +34.06 Da, K+34.06 Da, C } \\
\text { +57.02 Da }\end{array}$ & 23,8 & $-5,213$ & 3 & 2778,3 & ACTH_MOUSE & DзZ2K3_MOUSE & Actin, gamma-enteric smooth muscle; & $\begin{array}{l}\text { Alpha-actin-3;Gamma-2-actin;Smooth muscle } \\
\text { gamma-actin; }\end{array}$ \\
\hline MCEEETTA & LVCDNGSGLCKAGFAGDDAPR & $-4,2$ & $\begin{array}{l}\text { N-ter +28.03 Da, K+28.03 Da, C } \\
+57.02 \mathrm{Da}\end{array}$ & 21,1 & $-1,519$ & 3 & 2235,0 & ACTH_MOUSE & ACTA_MOUSE & Actin, gamma-enteric smooth muscle; & $\begin{array}{l}\text { Alpha-actin-3;Gamma-2-actin;Smooth muscle } \\
\text { gamma-actin; }\end{array}$ \\
\hline LAPEVFWEIF & GMSIQEFDKLPLWR & 0,6 & $\mathrm{~N}$-ter $+34.06 \mathrm{Da}, \mathrm{K}+34.06 \mathrm{Da}$ & 18,0 & 5,866 & 3 & 1787,0 & ABLM1_MOUSE & E9QK41_MOUSE & Actin-binding LIM protein 1; & Actin-binding LIM protein family member 1 ; \\
\hline $\begin{array}{l}\text { M } \\
\text { TDGSKWIKQY }\end{array}$ & $\begin{array}{l}\text { ILLEVNNR } \\
\text { TGVNAISKKEFSIDVGYER }\end{array}$ & $\begin{array}{l}0,0 \\
0,5\end{array}$ & $\begin{array}{l}\text { N-ter }+28.03 \mathrm{Da} \\
\mathrm{N} \text {-ter }+34.06 \mathrm{Da}, \mathrm{K}+34.06 \mathrm{Da}\end{array}$ & $\begin{array}{l}24,2 \\
15,5\end{array}$ & $\begin{array}{l}-0,177 \\
7,303\end{array}$ & $\begin{array}{l}2 \\
4\end{array}$ & $\begin{array}{l}1128,6 \\
2214,3\end{array}$ & $\begin{array}{l}\text { ARPC2_MOUSE } \\
\text { ARP3_MOUSE }\end{array}$ & & $\begin{array}{l}\text { Actin-related protein } 2 / 3 \text { complex subunit 2; } \\
\text { Actin-related protein } 3 ;\end{array}$ & $\begin{array}{l}\text { Arp } 2 / 3 \text { complex } 34 \text { kDa subunit; } \\
\text { Actin-like protein } 3 \text {; }\end{array}$ \\
\hline GTVTHLCRQY & SDAPPLTLDGIKDR & 0,0 & N-ter +34.06 Da, K +34.06 Da & 50,2 & 0,000 & 3 & 1564,9 & ACPM_MOUSE & F8WJ64_MOUSE & Acyl carrier protein, mitochondrial; & $\begin{array}{l}\text { Cl-SDAP;NADH-ubiquinone oxidoreductase } 9.6 \\
\text { kDa subunit; }\end{array}$ \\
\hline FAKPPVGPLR & FAPPEAPEPWSGVR & 0,9 & N-ter +28.03 Da & 21,8 & 1,211 & 2 & 1566,8 & EST2C_MOUSE & EST2E_MOUSE & Acylcarnitine hydrolase; & $\begin{array}{l}\text { Carboxylesterase 2;Peroxisome proliferator- } \\
\text { inducible acylcarnitine hydrolase; }\end{array}$ \\
\hline SPCLLPRVHL & ASAFGSSTESLVAR & 0,0 & $\mathrm{~N}$-ter +28.03 Da & 59,0 & $-0,708$ & 2 & 1409,7 & ACO15_MOUSE & & Acyl-coenzyme A thioesterase THEM5; & $\begin{array}{l}\text { Acyl-coenzyme A thioesterase 15; Thioesterase } \\
\text { superfamily member 5; }\end{array}$ \\
\hline LPRVHLASAF & GSSTESLVAR & 0,2 & $\mathrm{~N}$-ter +28.03 Da & 25,5 & 1,062 & 2 & 1033,5 & ACO15_MOUSE & & Acyl-coenzyme A thioesterase THEM5; & $\begin{array}{l}\text { Acyl-coenzyme A thioesterase } 15 \text {; Thioesterase } \\
\text { superfamily member } 5 \text {; }\end{array}$ \\
\hline PCLLPRVHLA & SAFGSSTESLVAR & $-10,0$ & $\mathrm{~N}$-ter +28.03 Da & 42,3 & $-9,920$ & 2 & 1338,7 & ACO15_MOUSE & & Acyl-coenzyme A thioesterase THEM5; & $\begin{array}{l}\text { Acyl-coenzyme A thioesterase } 15 \text {; Thioesterase } \\
\text { superfamily member } 5 \text {; } \\
\text { ATP pyrophosphate-lyase } 9 \text {;Adenylate cyclase }\end{array}$ \\
\hline PHQQLLHHHS & TEVSCDSSGDSNSVR & $-4,9$ & N-ter $+28.03 \mathrm{Da}, \mathrm{C}+57.02 \mathrm{Da}$ & 14,3 & $-1,410$ & 4 & 1626,7 & ADCY9_MOUSE & & Adenylate cyclase type 9; & $\begin{array}{l}\text { type IX;AdenylyI cyclase 9;AdenylyI cyclase type } \\
10 ; \\
\text { ATP-AMP transphosphorylase } 2 \\
\text { \{ECO:000255|HAMAP- }\end{array}$ \\
\hline M & APNVLASEPEIPKGIR & $-0,1$ & N-ter +28.03 Da, K+28.03 Da & 61,3 & $-0,057$ & 3 & 1746,0 & KAD2_MOUSE & & $\begin{array}{l}\text { Adenylate kinase 2, mitochondrial } \\
\text { \{ECO:0000255|HAMAP-Rule:MF_03168\}; }\end{array}$ & 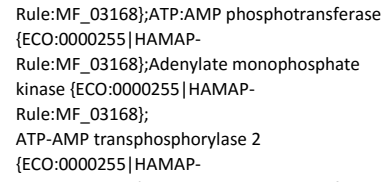 \\
\hline & NVLASEPEIPKGIR & 0,0 & $\mathrm{~N}$-ter $+28.03 \mathrm{Da}, \mathrm{K}+28.03 \mathrm{Da}$ & 27,3 & 1,455 & 3 & 1577,9 & KAD2_MOUSE & F7BP55_MOUSE & $\begin{array}{l}\text { Adenylate kinase 2, mitochondrial } \\
\text { \{ECO:0000255|HAMAP-Rule:MF_03168\}; }\end{array}$ & $\begin{array}{l}\text { Rule:MF_03168\};ATP:AMP phosphotransferase } \\
\text { \{ECO:0000255|HAMAP- } \\
\text { Rule:MF_03168\};Adenylate monophosphate } \\
\text { kinase \{ECO:0000255|HAMAP- } \\
\text { Rule:MF__36168\}; } \\
\text { ATP-AMP transhosphorylase 2 } \\
\text { \{ECO:0000255|HAMAP- }\end{array}$ \\
\hline NVLA & SEPEIPKGIR & 0,1 & N-ter $+34.06 \mathrm{Da}, \mathrm{K}+34.06 \mathrm{Da}$ & 34,6 & $-1,422$ & 3 & 1192,7 & KAD2_MOUSE & F7BP55_MOUSE & $\begin{array}{l}\text { Adenylate kinase 2, mitochondrial } \\
\text { \{ECO:0000255|HAMAP-Rule:MF_03168\}; }\end{array}$ & $\begin{array}{l}\text { Rule:MF_03168\};ATP:AMP phosphotransferase } \\
\text { \{ECO:0000255|HAMAP- } \\
\text { Rule:MF_03168\};Adenylate monophosphate } \\
\text { kinase \{ECO:0000255|HAMAP- } \\
\text { Rule:MF_03168\}; }\end{array}$ \\
\hline GLPSG & GPPPPPPGPPPPPIS & $-0,2$ & $\mathrm{~N}$-ter +28.03 Da & 36,0 & $-5,844$ & 3 & 2478,2 & CAP1_MOUSE & & & \\
\hline SGLPSGPSVG & SGPPPPPPGPPPPPISTSSGSDDSASR & 0,0 & N-ter +28.03 Da & 51,0 & 5,257 & 3 & 2565,3 & CAP1_MOUSE & & Adenylyy cyclase-associated protein 1; & \\
\hline
\end{tabular}




\begin{tabular}{|c|c|c|c|c|c|c|c|c|c|c|c|}
\hline Non Prime Site & Prime Site & 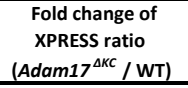 & Modifications & Hyperscore & $\begin{array}{c}\text { Mass error } \\
\text { in ppm }\end{array}$ & Charge & $\begin{array}{c}\text { Precursor } \\
\text { neutral mass } \\
\text { in Da }\end{array}$ & Uniprot ID 1 & Uniprot ID 2 & Protein Name 1 & Protein Name 2 \\
\hline MTDAAVSF & AKDFLAGGVAAAISKTAVAPIER & 0,6 & N-ter +34.06 Da, K +34.06 Da & 34,3 & 6,143 & 3 & 2357,5 & ADT2_MOUSE & & ADP/ATP translocase 2; & $\begin{array}{l}\text { ADP,ATP carrier protein 2;Adenine nucleotide } \\
\text { translocator 2;Solute carrier family } 25 \text { member } \\
5 \text {; }\end{array}$ \\
\hline RTFGTTGERR & AGEEAADSPELPR & 0,3 & $\mathrm{~N}$-ter+28.03 Da & 26,9 & $-7,077$ & 2 & 1368,6 & ADXL_MOUSE & & Adrenodoxin-like protein, mitochondrial; & $\begin{array}{l}\text { Ferredoxin-1-like protein; } \\
\text { Pare }\end{array}$ \\
\hline QKLLSESPFS & CDELTESDNKR & $-2,1$ & $\begin{array}{l}\text { N-ter +28.03 Da, } \mathrm{K}+28.03 \mathrm{Da}, \mathrm{C} \\
+57.02 \mathrm{Da}\end{array}$ & 16,0 & 1,474 & 3 & 1421,7 & AKAP4_MOUSE & & A-kinase anchor protein 4; & $\begin{array}{l}\text { A-kinase anchor protein } 82 \text { kDa;FSC1;Major } \\
\text { sperm fibrous sheath protein; Protein kinase A- } \\
\text { anchoring protein 4;p82; }\end{array}$ \\
\hline INRREKPLAL & YAFSNNNQVVNQMLER & 0,7 & $\mathrm{~N}$-ter+28.03 Da & 33,4 & 0,511 & 3 & 1953,9 & E9Q3E1_MOUSE & & $\begin{array}{l}\text { Aldehyde dehydrogenase } \\
\text { \{ECO:0000256|PIRNR:PIRNR036492\}; }\end{array}$ & \\
\hline PALQnatTrF & YGDNPQTSPNLGR & 1,3 & $\mathrm{~N}$-ter+28.03 Da & 28,1 & $-2,003$ & 2 & 1445,7 & E9Q3E1_MOUSE & AL3B1_MOUSE & $\begin{array}{l}\text { Aldehyde dehydrogenase } \\
\text { \{ECO:0000256|PIRNR:PIRNR036492\}; }\end{array}$ & \\
\hline RRGPRLSRLL & SAAATSAVPAPNHQPEVFCNQIFINNEWHDAVSR & 0,4 & N-ter +28.03 Da, C +57.02 Da & 50,2 & 0,053 & 4 & 3804,8 & ALDH2_MOUSE & D3YYF3_MOUSE & Aldehyde dehydrogenase, mitochondrial; & AHD-M1;ALDH class 2;ALDH-E2;ALDHI; \\
\hline QMKGSGKCCM & DPDEKCLESR & 2,1 & $\begin{array}{l}\text { N-ter +34.06 Da, K+34.06 Da, C } \\
+57.02 \mathrm{Da}\end{array}$ & 18,6 & $-3,792$ & 3 & 1315,7 & Q3TYQ9_MOUSE & & Aldehyde oxidase 4; & $\begin{array}{l}\text { Aldehyde oxidase homolog 2;Azaheterocycle } \\
\text { hydroxylase 4; Retinal oxidase; }\end{array}$ \\
\hline $\begin{array}{l}\text { NAVGPVPTAN } \\
\text { VPTANAALPA }\end{array}$ & $\begin{array}{l}\text { AALPADPPASVVVGPVVVPR } \\
\text { DPPASVVVGPVVVPR }\end{array}$ & $\begin{array}{l}0,0 \\
0,5\end{array}$ & N-ter +34.06 Da & 20,8 & $\begin{array}{l}-2,208 \\
1,912\end{array}$ & $\begin{array}{l}3 \\
2\end{array}$ & $\begin{array}{l}1944,2 \\
1514,9\end{array}$ & $\begin{array}{l}\text { FETUA_MOUSE } \\
\text { FETUA MOUSE }\end{array}$ & & $\begin{array}{l}\text { Alpha-2-HS-glycoprotein; } \\
\text { Alpha-2-HS--glycoprotein: }\end{array}$ & Countertrypin;Fetuin-A; \\
\hline VPTANAALPA & $\begin{array}{l}\text { DPPASWVVGPVVVPR } \\
\text { SPKYGOPGAG }\end{array}$ & 0,5 & $\mathrm{~N}$-ter $+28.03 \mathrm{Da}$ & 25,4 & 1,912 & 2 & 1514,9 & FETUA_MOUSE & & Alpha-2-HS-glycoprotein; & Countertrypin;Fetuin-A; \\
\hline VESASGETLH & SPKVGQPGAAGPVSPMCPGR & 2,0 & $\begin{array}{l}\text { N-ter +28.03 Da, K +28.03 Da, C } \\
\text { +57.02 Da }\end{array}$ & 31,8 & $-5,179$ & 3 & 2005,0 & FETUA_MOUSE & & Alpha-2-HS-glycoprotein; & Countertrypin;Fetuin-A; \\
\hline KQDGQFRVMH & TQCHSTPDSAEDVR & 0,0 & N-ter +28.03 Da, C+57.02 Da & 17,5 & 3,430 & 3 & 1629,7 & FETUA_MOUSE & & Alpha-2-HS-glycoprotein; & Countertrypin;Fetuin-A; \\
\hline NEHLMEDYER & LASDLLEWIR & $-7,6$ & $\mathrm{~N}$-ter +28.03 Da & 29,7 & $-0,402$ & 2 & 1242,7 & ACTN1_MOUSE & ACTN4_MOUSE & Alpha-actinin-1; & $\begin{array}{l}\text { Alpha-actinin cytoskeletal isoform;F-actin cross- } \\
\text { linking protein;Non-muscle alpha-actinin-1; }\end{array}$ \\
\hline QEALIFDNKH & TNYTMEHIR & 0,1 & N-ter +28.03 Da & 21,9 & $-0,084$ & 3 & 1191,6 & ACTN2_MOUSE & ACTN4_MOUSE & Alpha-actinin-2; & $\begin{array}{l}\text { Alpha-actinin skeletal muscle isoform 2;F-actin } \\
\text { cross-linking protein; }\end{array}$ \\
\hline TAANRICKVL & AVNQENEHLMEDYER & 0,8 & N-ter +34.06 Da & 33,4 & $-2,823$ & 3 & 1909,9 & ACTN4_MOUSE & E9Q2W9_MOUSE & Alpha-actinin-4; & $\begin{array}{l}\text { F-actin cross-linking protein;Non-muscle alpha- } \\
\text { actinin 4; }\end{array}$ \\
\hline GPEEFKACLI & SLGYDVENDR & 0,9 & N-ter +28.03 Da & 22,0 & 2,340 & 2 & 1194,6 & ACTN4_MOUSE & & Alpha-actinin-4; & $\begin{array}{l}\text { F-actin cross-linking protein;Non-muscle alpha- } \\
\text { actinin 4; }\end{array}$ \\
\hline DLYTAKGLFR & AAVPSGASTGIYEALELR & $-3,8$ & N-ter +28.03 Da & 38,0 & $-1,253$ & 3 & 1832,0 & ENOA_MOUSE & ENOB_MOUSE & Alpha-enolase; & $\begin{array}{l}\text { 2-phospho-D-glycerate hydro-lyase;Enolase } \\
\text { 1;Non-neural enolase; }\end{array}$ \\
\hline IOACKLAQEN & GWGVMVSHR & $-0,1$ & N-ter +28.03 Da & 15,5 & 0,378 & 3 & 1055,5 & ENOA_MOUSE & ENOB_MOUSE & Alpha-enolase; & $\begin{array}{l}\text { 2-phospho-D-glycerate hydro-lyase;Enolase } \\
\text { 1;Non-neural enolase; }\end{array}$ \\
\hline GVISQAMVMC & ASSPEKVEILAPPNGSVPGDR & $-0,2$ & $\mathrm{~N}$-ter +28.03 Da, K+28.03 Da & 21,4 & 1,974 & 3 & 2175,2 & AIMP1_MOUSE & Q3UZG4_MOUSE & $\begin{array}{l}\text { Aminoacyl tRNA synthase complex-interacting } \\
\text { multifunctional protein } 1 \text {; }\end{array}$ & $\begin{array}{l}\text { Multisynthase complex auxiliary component } \\
\text { p43; }\end{array}$ \\
\hline KKGEKKEKQQ & SAAASTDSKPIDASR & 2,0 & $\mathrm{~N}$-ter +28.03 Da, K+28.03 Da & 19,3 & $-4,170$ & 3 & 1531,8 & AIMP1_MOUSE & Q3UZG4_MOUSE & $\begin{array}{l}\text { Aminoacyl tRNA synthase complex-interacting } \\
\text { multifunctional protein } 1 \text {; }\end{array}$ & $\begin{array}{l}\text { Multisynthase complex auxiliary component } \\
\text { p43; }\end{array}$ \\
\hline M & GKEQELLEAAR & 0,1 & $\mathrm{~N}$-ter +28.03 Da, K+28.03 Da & 27,9 & 3,303 & 3 & 1298,7 & ANS1A_MOUSE & Q3UHP6_MOUSE & $\begin{array}{l}\text { Ankyrin repeat and SAM domain-containing protein } \\
1 \mathrm{~A} ;\end{array}$ & Odin; \\
\hline MTEEetARLR & AGALMARPNSPQSSGSGR & $-0,5$ & N-ter +28.03 Da & 23,2 & $-0,958$ & 3 & 1770,9 & ANR63_MOUSE & & $\begin{array}{l}\text { Ankyrin repeat domain-containing protein } 63 \\
\{\text { ECOO:0000250|UniProtKB:C9JTQO }\} \text {; }\end{array}$ & \\
\hline VPSKEQHLTF & TREFDSDSLR & 0,7 & N-ter +34.06 Da & 18,4 & $-6,975$ & 3 & 1258,6 & ANK3_MOUSE & G3X971_MOUSE & Ankyrin-3; & $\begin{array}{l}\text { Ankyrin-G; } \\
\text { Annexin I;Annexin-1;Calpactin II;Calpactin- }\end{array}$ \\
\hline HLEEVVLAML & KTPAQFDADELR & 0,8 & N-ter +34.06 Da, K+34.06 Da & 25,5 & 8,557 & 3 & 1457,8 & ANXA1_MOUSE & & Annexin $\mathrm{A} 1$; & $\begin{array}{l}\text { 2; Chromobindin-9;Lipocortin l;:Phospholipase } \\
\text { A2 inhibitory protin;;335; } \\
\text { Annexin I:Annexin-1;Calpactin Il:Calpactin- }\end{array}$ \\
\hline ELRGAMKGLG & TDEDTLELLTTR & 0,5 & N-ter +34.06 Da & 29,5 & 2,701 & 2 & 1552,9 & ANXA1_MOUSE & & Annexin A1; & $\begin{array}{l}\text { 2; Chromobindin-9;ipocortin l;:Phospholipase } \\
\text { A2 inhibitory protin;;335; } \\
\text { Annexin I:Annexin-1;Calpactin Il;Calpactin- }\end{array}$ \\
\hline LYEAGERRKG & TDVNVFTTLLTSR & 0,7 & N-ter +34.06 Da & 29,0 & 2,797 & 2 & 1499,9 & ANXA1_MOUSE & & Annexin A1; & $\begin{array}{l}\text { 2; Chromobindin-9;lipocortin l;:Phospholipase } \\
\text { A2 inhibitory protein;p35; }\end{array}$ \\
\hline LCKLSLEGDH & STPPSAYGSVKPYTNFDAER & 0,2 & $\mathrm{~N}$-ter +28.03 Da, K+28.03 Da & 22,3 & $-1,247$ & 3 & 2242,1 & ANXA2_MOUSE & BOV2N7_MOUSE & Annexin A2; & $\begin{array}{l}\text { Annexin II;Annexin-2;Calpactin I heavy } \\
\text { chain;Calpactin-1 heavy chain; Chromobindin- } \\
\text { 8; ilipococtin II:Placental anticoagulant protein } \\
\text { IV;Protein I; } 336 \text {; }\end{array}$ \\
\hline DALNIETAVK & TKGVDEVTIVNILTNR & 0,4 & $\mathrm{~N}$-ter $+28.03 \mathrm{Da}, \mathrm{K}+28.03 \mathrm{Da}$ & 21,1 & $-4,809$ & 3 & 1827,0 & ANXA2_MOUSE & BOV2N7_MOUSE & Annexin A2; & $\begin{array}{l}\text { Annexin II;Annexin-2;Calpactin I heavy } \\
\text { chain;Calpactin-1 heavy chain;Chromobindin- } \\
\text { 8;Lipocortin II;Placental anticoagulant protein } \\
\text { IV;Protein I; } 36 \text {; }\end{array}$ \\
\hline
\end{tabular}




\begin{tabular}{|c|c|c|c|c|c|c|c|c|c|c|c|}
\hline Non Prime Site & Prime Site & $\begin{array}{c}\text { Fold change of } \\
\text { XPRESS ratio } \\
\text { (Adam17 } 7^{\text {AKc }} / \text { WT) } \\
\end{array}$ & Modifications & Hyperscore & $\begin{array}{l}\text { Mass error } \\
\text { in ppm }\end{array}$ & Charge & $\begin{array}{c}\text { Precursor } \\
\text { neutral mass } \\
\text { in Da } \\
\end{array}$ & Uniprot ID 1 & Uniprot ID 2 & Protein Name 1 & Protein Name 2 \\
\hline DSLEIICSR & TNQELQEINR & $-4,4$ & $\mathrm{~N}$-ter +28.03 Da & 28,8 & 0,393 & 2 & 1271,7 & ANXA2_MOUSE & BOV2N5_MOUSE & Annexin A2; & $\begin{array}{l}\text { Annexin II;Annexin-2;Calpactin I heavy } \\
\text { chain;Calpactin-1 heavy chain;Chromobindin- } \\
\text { 8;Lipocortin II;Placental anticoagulant protein } \\
\text { IV;Protein I;336; }\end{array}$ \\
\hline CKLSLEGDHS & TPPSAYGSVKPYTNFDAER & 0,3 & N-ter +34.06 Da, K +34.06 Da & 33,8 & $-1,336$ & 3 & 2167,1 & ANXA2_MOUSE & BOV2N7_MOUSE & Annexin A2; & $\begin{array}{l}\text { Annexin II;Annexin-2;Calpactin I heavy } \\
\text { chain;Calpactin-1 heavy chain;Chromobindin- } \\
\text { 8;Lipocortin II;Placental anticoagulant protein } \\
\text { IV;Protein I;336; }\end{array}$ \\
\hline GRADAEVLRK & AMKGLGTDEDSILNLLTSR & 1,7 & N-ter +34.06 Da, K+34.06 Da & 21,1 & 2,519 & 3 & 2101,2 & ANXA5_MOUSE & & Annexin A5; & $\begin{array}{l}\text { Anchorin Cll;Annexin V;Annexin-5;Calphobindin } \\
\text { l;;Endonexin II;ipocortin V;Placental } \\
\text { anticoagulant protein 4;Placental anticoagulant } \\
\text { protein l;Thromboplastin inhibitor; Vascular } \\
\text { anticoagulant-alpha; }\end{array}$ \\
\hline GALGCAICHG & NPVDDICIAKPR & 0,6 & $\begin{array}{l}\text { N-ter }+28.03 \mathrm{Da}, \mathrm{K}+28.03 \mathrm{Da}, \mathrm{C} \\
+57.02 \mathrm{Da}\end{array}$ & 30,7 & $-0,206$ & 3 & 1452,8 & ANT3_MOUSE & & Antithrombin-III; & Serpin C1; \\
\hline M & IQAILVFNNHGKPR & 0,3 & N-ter +28.03 Da, K+28.03 Da & 27,6 & $-0,723$ & 4 & 1793,0 & AP3S2_MOUSE & & AP-3 complex subunit sigma-2; & $\begin{array}{l}\text { AP-3 complex subunit sigma-3B;Adaptor- } \\
\text { related protein complex } 3 \text { subunit sigma- } \\
2 \text {;;igma-3B-adaptin;Sigma-adaptin } 3 \text { b; }\end{array}$ \\
\hline GSQAWHVWQQ & DEPQSQWDKVKDFANVYVDAVKDSGR & 0,5 & N-ter +34.06 Da, K+34.06 Da & 30,7 & 1,244 & 4 & 3131,7 & APOA1_MOUSE & & Apolipoprotein A-l; & Apolipoprotein A1; \\
\hline PLTRDFWDNL & EKETDWVR & 0,6 & $\mathrm{~N}$-ter +34.06 Da, $\mathrm{K}+34.06 \mathrm{Da}$ & 18,7 & $-0,177$ & 3 & 1129,6 & APOA1_MOUSE & & Apolipoprotein A-l; & Apolipoprotein A1; \\
\hline SSSLGQQLNL & NLLENWDTLGSTVSQLQER & 0,6 & $\mathrm{~N}$-ter +34.06 Da & 24,7 & $-3,126$ & 3 & 2236,2 & APOA1_MOUSE & & Apolipoprotein A-l; & Apolipoprotein A1; \\
\hline ARQKLQELQG & $\begin{array}{l}\text { RLSPVAEEFR } \\
\text { R CUCh }\end{array}$ & 0,1 & $\mathrm{~N}$-ter +34.06 Da & 18,7 & $-4,921$ & 3 & 1236,7 & APOA1_MOUSE & & Apolipoprotein A-l; & Apolipoprotein A1; \\
\hline TLEGPAPAQA & APDLSGTLESIPDKLKEFGNTLEDKAR & 0,6 & N-ter +28.03 Da, K+28.03 Da & 27,2 & $-0,163$ & 5 & 305,6 & APOC1_MOUSE & & Apolipoprotein C-1; & Apolipoprotein C1; \\
\hline EGPAPAQAAP & DLSGTLESIPDKLKEFGNTLEDKAR & 0,6 & N-ter +28.03 Da, $\mathrm{K}+28.03 \mathrm{Da}$ & 23,7 & 5,602 & 4 & 2887,6 & APOC1_MOUSE & & Apolipoprotein C-l; & Apolipoprotein C1; \\
\hline LQELAEQLQT & TKEESGAVAAAASVPAQSTAR & $-0,3$ & N-ter +28.03 Da, K+28.03 Da & 16,6 & 1,214 & 3 & 2057,1 & ASC_MOUSE & & $\begin{array}{l}\text { Apoptosis-associated speck-like protein containing a } \\
\text { CARD; }\end{array}$ & PYD and CARD domain-containing protein; \\
\hline M & KMTVDFEECLKDSPR & 0,2 & $\begin{array}{l}\text { N-ter +34.06 Da, K+34.06 Da, C } \\
+57.02 \mathrm{Da}\end{array}$ & 27,8 & $-1,991$ & 3 & 1956,1 & ACAP2_MOUSE & & $\begin{array}{l}\text { Arf-GAP with coiled-coil, ANK repeat and PH domain- } \\
\text { containing protein 2; }\end{array}$ & Centaurin-beta-2; \\
\hline GQIPCVLTRS & VHSVAIVGAPFSR & 0,7 & $\mathrm{~N}$-ter+28.03 Da & 21,8 & $-0,365$ & 3 & 1366,8 & ARGI2_MOUSE & & Arginase-2, mitochondrial; & $\begin{array}{l}\text { Kidney-type arginase; Non-hepatic } \\
\text { arginase;Type II arginase; }\end{array}$ \\
\hline ESGWTDTESD & SDSEPDVPQR & 10,0 & $\mathrm{~N}$-ter +34.06 Da & 15,2 & 5,405 & 3 & 1162,6 & ARMX2_MOUSE & & Armadillo repeat-containing X-linked protein 2; & \\
\hline LPIGGLAEFC & KASAELALGENNEVLKSGR & $-1,4$ & $\mathrm{~N}$-ter +34.06 Da, $\mathrm{K}+34.06 \mathrm{Da}$ & 23,2 & 3,347 & 4 & 2087,3 & AATM_MOUSE & & Aspartate aminotransferase, mitochondrial; & $\begin{array}{l}\text { Fatty acid-binding protein;Glutamate } \\
\text { oxaloacetate transaminase 2;Kynurenine } \\
\text { aminotransferase 4; 4Kynurenine } \\
\text { aminotransferase IV;Kynurenine---oxoglutarate } \\
\text { transaminase 4;Kynurenine--oxoglutarate } \\
\text { transaminase IV;Plasma membrane-associated } \\
\text { fatty acid-binding protein;Transaminase A; }\end{array}$ \\
\hline SULRRPPGR & EAYPGDVFYLHSR & $-3,6$ & $\mathrm{~N}$-ter $+28.03 \mathrm{Da}$ & 16,9 & 2,778 & 3 & 1580,8 & ATPA_MOUSE & D3Z6F5_MOUSE & ATP synthase subunit alpha, mitochondrial; & \\
\hline NLHASNTRLQ & KTGTAEMSSILEER & $-0,2$ & $\mathrm{~N}-\operatorname{ter}+34.06 \mathrm{Da}, \mathrm{K}+34.06 \mathrm{Da}$ & 24,5 & 6,474 & 3 & 1618,9 & ATPA_MOUSE & D6RJ16_MOUSE & ATP synthase subunit alpha, mitochondrial; & \\
\hline RNLHASNTRL & QKTGTAEMSSILEER & 0,2 & $\mathrm{~N}$-ter +28.03 Da, $\mathrm{K}+28.03 \mathrm{Da}$ & 43,8 & $-1,036$ & 3 & 1734,9 & ATPA_MOUSE & D6RJ16_MOUSE & ATP synthase subunit alpha, mitochondrial; & \\
\hline PAGVHPARDY & AAQASAAPKAGTATGR & 0,0 & $\mathrm{~N}$-ter $+28.03 \mathrm{Da}, \mathrm{K}+28.03 \mathrm{Da}$ & 38,6 & $-0,135$ & 3 & 1483,8 & ATPB_MOUSE & & ATP synthase subunit beta, mitochondrial; & \\
\hline SVQAIYVPAD & DLTDPAPATTFAHLDATTVLSR & 0,6 & $\mathrm{~N}$-ter $+34.06 \mathrm{Da}$ & 34,6 & $-4,470$ & 3 & 2346,2 & ATPB_MOUSE & & ATP synthase subunit beta, mitochondrial; & \\
\hline GSEVSALLGR & IPSAVGYQPTLATDMGTMQER & $-2,1$ & $\mathrm{~N}$-ter +28.03 Da & 27,5 & 4,137 & 3 & 2293,1 & ATPB_MOUSE & & ATP synthase subunit beta, mitochondrial: & \\
\hline QAIYYPADDL & TDPAPATTFAHLDATTVLSR & 0,1 & $\mathrm{~N}$-ter $+28.03 \mathrm{Da}$ & 26,9 & $-0,047$ & 3 & 2112,1 & ATPB MOUSE & & ATP synthase subunit beta, mitochondrial: & \\
\hline APAGVHPARD & YAAQASAAPKAGTATGR & 0,0 & $\mathrm{~N}$-ter $+28.03 \mathrm{Da}, \mathrm{K}+28.03 \mathrm{Da}$ & 21,7 & 1,394 & 3 & 1646,9 & ATPB_MOUSE & & ATP synthase subunit beta, mitochondrial; & \\
\hline SWNETFHARL & ASLSEKPPAIDWAYYR & 0,3 & N-ter +34.06 Da, K +34.06 Da & 27,8 & $-4,956$ & 3 & 1934,1 & ATP5H_MOUSE & G3X9L6_MOUSE & ATP synthase subunit d, mitochondrial; & \\
\hline ETFHARLASL & SEKPPAIDWAYYR & 0,3 & $\mathrm{~N}-\operatorname{ter}+34.06 \mathrm{Da}, \mathrm{K}+34.06 \mathrm{Da}$ & 27,0 & $-2,041$ & 3 & 1662,9 & ATP5H_MOUSE & G3X916 MOUSE & ATP synthase subunit d, mitochondrial; & \\
\hline VNADSSVQLL & AEEAVTLDMLDLGAAR & $-4,0$ & $\mathrm{~N}$-ter $+28.03 \mathrm{Da}$ & 20,9 & 2,524 & 2 & 1701,9 & ATPD_MOUSE & & ATP synthase subunit delta, mitochondrial; & F-ATPase delta subunit; \\
\hline SPLIKFGRYS & ALIIGM & 0,6 & $\mathrm{~N}$-ter $+34.06 \mathrm{Da}, \mathrm{K}+34.06 \mathrm{Da}$ & 20,0 & $-0,900$ & 3 & 1330,8 & ATP5I MOUSE & Q8BTB6 MOUSE & ATP synthase subunit e, mitochondrial; & \\
\hline M & VPPVQVSPLIKFGR & 0,0 & $\mathrm{~N}-\operatorname{ter}+34.06 \mathrm{Da}, \mathrm{K}+34.06 \mathrm{Da}$ & 25,7 & 1,43 & 3 & 1604,1 & ATP5I MOUSE & Q8BTB6 MOUSE & ATP synthase subunit e, mitochondrial; & \\
\hline M & ATLKDITR & 0,0 & $\mathrm{~N}-\operatorname{ter}+28.03 \mathrm{Da}, \mathrm{K}+28.03 \mathrm{Da}$ & 21,3 & 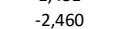 & 3 & 972,6 & ATPG_MOUSE & Q8C208 MOUSE & ATP synthase subunit gamma, mitochondrial; & F-ATPase gamma subunit; \\
\hline RSESTSVVRP & FAKLVRPPVQVYGIEGR & 0,0 & N-ter +34.06 Da, K & 34,9 & $-7,899$ & 4 & 1996,2 & ATPO MOUSE & F6XVM5 ${ }^{-}$MOUSE & ATP synthase subunit 0 , mitochondrial; & Oligomycin sensitivity conferral protein; \\
\hline RNIGVTAVAF & NKELDPVQKLFVDKIR & $-0,1$ & $\mathrm{~N}-\operatorname{ter}+34.06 \mathrm{Da}, \mathrm{K}+34.06 \mathrm{Da}$ & 20,7 & 6,34 - & 4 & 2077,4 & ATP5J MOUSE & E9QADG MOUSE & ATP synthase-coupling factor 6 , mitochondrial; & \\
\hline QTRGFVSDSS & DSMDTGAGSIR & 0,0 & $\mathrm{~N}$-ter $+34.06 \mathrm{Da}$ & 34,5 & $-0,1$ & 2 & 1142,6 & ATIF1 MOUSE & & ATPase inhibitor, mitochondrial; & Inhibitor of $F(1)$ F(o)-ATPase: \\
\hline KVLQTRGFVS & DSSDSMDTGAGSIR & $-0,1$ & $\mathrm{~N}$-ter $+34.06 \mathrm{Da}$ & & 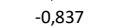 & 2 & 1431,6 & ATIFF MOUSE & & ATPase inhibitor, mitochondrial; & $\begin{array}{l}\text { Inhibitor of F(1) E(I)-ATPase: } \\
\text { (a) }\end{array}$ \\
\hline LQTRGFVSDS & SDSMDTGAGSIR & 0,4 & $\mathrm{~N}$-ter $+28.03 \mathrm{Da}$ & 1 & $-0,0$ & 2 & 1223,6 & ATIF1_MOUSE & & ATPase inhibitor, mitochondrial; & Inhibitor of $\mathrm{F}$ \\
\hline MKVLQTRGFV & SDSSDSMDTGAGSIR & 0,0 & $\mathrm{~N}$-ter $+34.06 \mathrm{Da}$ & & -0, & 2 & 1518,7 & ATIF1_MOUSE & & ATPase inhibitor, mitochondrial; & Inhibitor of $F(1) F(0)-A T P a s e ;$ \\
\hline TRGFVSDSSD & SMDTGAGSIR & 0,3 & N-ter +34.06 Da & 22,7 & 0,874 & 2 & 1027,5 & ATIF1_MOUSE & & ATPase inhibitor, mitochondrial; & Inhibitor of $F(1) F(0)-A T P a s e ;$ \\
\hline
\end{tabular}




\begin{tabular}{|c|c|c|c|c|c|c|c|c|c|c|c|}
\hline Non Prime Site & Prime Site & $\begin{array}{l}\text { Fold change of } \\
\text { XPRESS ratio } \\
\text { (Adam17 } 7^{\text {AKc } / W T)} \\
\end{array}$ & Modifications & Hyperscore & $\begin{array}{c}\text { Mass error } \\
\text { in ppm }\end{array}$ & Charge & $\begin{array}{c}\text { Precursor } \\
\text { neutral mass } \\
\text { in Da }\end{array}$ & Uniprot ID 1 & Uniprot ID 2 & Protein Name 1 & Protein Name 2 \\
\hline $\begin{array}{l}\text { VLQTRGFVSD } \\
\text { GMKVLQTRGF }\end{array}$ & $\begin{array}{l}\text { SSDSMDTGAGSIR } \\
\text { VSDSSDSMDTGAGSIR }\end{array}$ & $\begin{array}{l}0,1 \\
-0,1\end{array}$ & $\begin{array}{l}\text { N-ter +34.06 Da } \\
\text { N-ter +28.03 Da }\end{array}$ & $\begin{array}{l}49,4 \\
65,6\end{array}$ & $\begin{array}{c}0,379 \\
-0,062\end{array}$ & $\begin{array}{l}2 \\
2\end{array}$ & $\begin{array}{l}1316,6 \\
1611,7\end{array}$ & $\begin{array}{l}\text { ATIF1_MOUSE } \\
\text { ATIF1_MOUSE }\end{array}$ & & $\begin{array}{l}\text { ATPase inhibitor, mitochondrial; } \\
\text { ATPase inhibitor, mitochondrial; }\end{array}$ & $\begin{array}{l}\text { Inhibitor of } F(1) F(0)-A T P a s e ; \\
\text { Inhibitor of } F(1) F(0)-A T \text { Pase; }\end{array}$ \\
\hline GAHNTIICAR & AGKDLVSSLTSGLLTIGDR & $-0,1$ & $\mathrm{~N}$-ter +28.03 Da, $\mathrm{K}+28.03 \mathrm{Da}$ & 22,6 & 1,530 & 3 & 1958,1 & ACLY_MOUSE & Q3V117_MOUSE & ATP-citrate synthase; & $\begin{array}{l}\text { ATP-citrate (pro-S--)-yase; Citrate cleavage } \\
\text { enzyme; }\end{array}$ \\
\hline LGLDQQFAGL & DLNSSDNQSGGSTASKGR & 0,0 & $\mathrm{~N}$-ter +28.03 Da, K+28.03 Da & 46,5 & $-0,761$ & 3 & 1835,9 & DDX3X_MOUSE & & ATP-dependent RNA helicase DDX3X; & $\begin{array}{l}\text { helicase DEADD; DEAD box protein } 3, X- \\
\text { chromosomal;Embryonic RNA helicase; }\end{array}$ \\
\hline HQSWSRDGGP & RGSGQGSSGGGGGGSR & 2,1 & N-ter+34.06 Da & 20,1 & $-3,907$ & 3 & 1353,7 & DHX36_MOUSE & & ATP-dependent RNA helicase DHX36; & $\begin{array}{l}\text { DEAH box protein 36;MLE-like protein 1;RNA } \\
\text { helicase associated with AU-rich element ARE; }\end{array}$ \\
\hline M & KALDEPPYLTVGTDVSAKYR & 0,1 & $\mathrm{~N}$-ter +28.03 Da, $\mathrm{K}+28.03 \mathrm{Da}$ & 27,3 & $-0,901$ & 4 & 2437,3 & ARI4B_MOUSE & & AT-rich interactive domain-containing protein $4 \mathrm{~B}$; & $\begin{array}{l}180 \text { KDa Sin3-associated polypeptide; Histone } \\
\text { deacetylase complex subunit SAP180; }\end{array}$ \\
\hline MGEKPVGSLA & GIGDVLSKR & 0,6 & $\mathrm{~N}$-ter +34.06 Da, $\mathrm{K}+34.06 \mathrm{Da}$ & 19,6 & $-3,449$ & 3 & 1011,7 & BAF_MOUSE & & Barrier-to-autointegration factor; & $\begin{array}{l}\text { Breakpoint cluster region protein 1;LAP2- } \\
\text { binding protein 1; }\end{array}$ \\
\hline RGFDKAYVVL & GQFLVLKKDEDLFR & 0,7 & N-ter +34.06 Da, K+34.06 Da & 35,3 & $-1,159$ & 3 & 1809,1 & BAF_MOUSE & & Barrier-to-autointegration factor; & $\begin{array}{l}\text { Breakpoint cluster region protein 1;LAP2- } \\
\text { binding protein 1; }\end{array}$ \\
\hline DKAYVVLGQF & LVLKKDEDLFR & 0,5 & N-ter +34.06 Da, K+34.06 Da & 18,0 & 0,675 & 4 & 1477,0 & BAF_MOUSE & & Barrier-to-autointegration factor; & $\begin{array}{l}\text { Breakpoint cluster region protein 1;LAP2- } \\
\text { binding protein 1; }\end{array}$ \\
\hline KAYVVLGQFL & VLKKDEDLFR & 0,7 & N-ter $+28.03 \mathrm{Da}, \mathrm{K}+28.03 \mathrm{Da}$ & 14,9 & 0,371 & 3 & 1345,8 & BAF_MOUSE & & Barrier-to-autointegration factor; & $\begin{array}{l}\text { Breakpoint cluster region protein 1; LAP2- } \\
\text { binding protein 1; }\end{array}$ \\
\hline LISGYSGAQA & ELHVSVPPR & 0,1 & N-ter +28.03 Da & 24,5 & $-0,094$ & 3 & 1060,6 & BCAM_MOUSE & DЗYTK7_MOUSE & Basal cell achesion molecule; & $\begin{array}{l}\text { B-CAM cell surface glycoprotein;Lutheran } \\
\text { antigen; }\end{array}$ \\
\hline TLHVavSGTS & TAPVASIHPPQLTVQPGQQAEFR & 0,7 & $\mathrm{~N}$-ter +28.03 Da & 20,0 & 5,795 & 3 & 2499,3 & PGBM_MOUSE & B1BOC7_MOUSE & $\begin{array}{l}\text { Basement membrane-specific heparan sulfate } \\
\text { proteoglycan core protein; }\end{array}$ & \\
\hline GEKPYKCETC & GARFVQVAHLR & 0,1 & N-ter $+28.03 \mathrm{Da}$ & 17,3 & $-6,855$ & 3 & 1280,7 & BCL6_MOUSE & & $\begin{array}{l}\text { B-cell lymphoma } 6 \text { protein homolog; } \\
\text { Pat }\end{array}$ & \\
\hline MVDDELTA & LVVDNGSGMCKAGFGGDDAPR & $-6,3$ & $\begin{array}{l}\text { N-ter +28.03 Da, K +28.03 Da, C } \\
+57.02 \mathrm{Da}\end{array}$ & 20,6 & $-0,734$ & 3 & 2178,0 & ACTBL_MOUSE & & Beta-actin-like protein 2; & Kappa-actin; \\
\hline MVDDELTAL & VVDNGSGMCKAGFGGDDAPR & $-5,2$ & $\begin{array}{l}\text { N-ter +28.03 Da, } \mathrm{K}+28.03 \mathrm{Da}, \mathrm{C} \\
+57.02 \mathrm{Da}\end{array}$ & 20,6 & 0,097 & 3 & 2064,9 & ACTBL_MOUSE & & Beta-actin-like protein 2; & Kappa-actin; \\
\hline M & APSQLALFSVSDKTGLVEFAR & $-0,2$ & N-ter +28.03 Da, K+28.03 Da & 46,4 & $-0,218$ & 3 & 2291,3 & PUR9_MOUSE & & Bifunctional purine biosynthesis protein PURH; & \\
\hline MAP & SQLALFSVSDKTGLVEFAR & $-6,8$ & $\mathrm{~N}-\operatorname{ter}+28.03 \mathrm{Da}, \mathrm{K}+28.03 \mathrm{Da}$ & 25,7 & 2,399 & 3 & 2123,2 & PUR9_MOUSE & & Bifunctional purine biosynthesis protein PURH; & \\
\hline LAFGESLMTH & AMTFTAVSEKDNQEGTFVKWR & 0,3 & N-ter $+34.06 \mathrm{Da}, \mathrm{K}+34.06 \mathrm{Da}$ & 19,2 & 2,000 & 4 & 2546,4 & BLMH_MOUSE & & Bleomycin hydrolase; & \\
\hline $\begin{array}{l}\text { SLMTHAMTFT } \\
\text { LITA }\end{array}$ & AVSEKDNQEGTFVKWR & 0,4 & $\mathrm{~N}-\operatorname{ter}+34.06 \mathrm{Da}, \mathrm{K}+34.06$ Da & 19,1 & 1,101 & 4 & 1995,1 & BLMH_MOUSE & & Bleomycin hydrolase; & \\
\hline EEFESSQSYL & $\begin{array}{l}\text { FFWDKVER } \\
\text { F }\end{array}$ & 0,0 & $\mathrm{~N}-\operatorname{ter}+28.03 \mathrm{Da}, \mathrm{K}+28.03 \mathrm{Da}$ & 24,2 & $-0,422$ & 3 & 1181,6 & BLMH_MOUSE & E9PYUo_MOUSE & Bleomycin hydrolase; & \\
\hline FWDKVERCYF & $\begin{array}{l}\text { FLNAFVDTAQKKEPEDGR } \\
\text {. }\end{array}$ & 0,5 & $\mathrm{~N}-\operatorname{ter}+34.06 \mathrm{Da}, \mathrm{K}+34.06 \mathrm{Da}$ & 15,6 & 0,876 & 4 & 2166,2 & BLMH_MOUSE & E9PYUO_MOUSE & Bleomycin hydrolase; & \\
\hline VERCYFFLNA & FVDTAQKKEPEDGR & 0,0 & $\mathrm{~N}-\operatorname{ter}+28.03 \mathrm{Da}, \mathrm{K}+28.03 \mathrm{Da}$ & 25,1 & 0,000 & 3 & 1702,9 & BLMH_MOUSE & E9PYUO_MOUSE & Bleomycin hydrolase; & \\
\hline EFEFSQSYLF & FWDKVER & 0,3 & $\mathrm{~N}-\operatorname{ter}+28.03 \mathrm{Da}, \mathrm{K}+28.03 \mathrm{Da}$ & 21,3 & $-0,675$ & 3 & 1034,6 & BLMH_MOUSE & E9PYUO_MOUSE & Bleomycin hydrolase; & \\
\hline EIFRVVCICL & GNPPETFTWEYR & 0,2 & $\mathrm{~N}$-ter $+28.03 \mathrm{Da}$ & 50,6 & 0,852 & 2 & 1523,7 & BLMH_MOUSE & E9PY26_MOUSE & Bleomycin hydrolase; & \\
\hline MNVYDHELVF & GVSLKNMNKAER & 0,2 & $\mathrm{~N}-\operatorname{ter}+34.06 \mathrm{Da}, \mathrm{K}+34.06 \mathrm{Da}$ & 24,8 & $-0,895$ & 4 & 1447,9 & BLMH_MOUSE & & Bleomycin hydrolase; & \\
\hline MMEEIFRVVC & ICLGNPPETFTWEYR & 0,5 & N-ter $+34.06 \mathrm{Da}, \mathrm{C}+57.02 \mathrm{Da}$ & 63,2 & 0,104 & 2 & 1915,9 & BLMH_MOUSE & E9PY26_MOUSE & Bleomycin hydrolase; & \\
\hline EEIFRVVCIC & LGNPPETFTWEYR & $-0,1$ & $\mathrm{~N}$-ter $+28.03 \mathrm{Da}$ & 30,6 & 0,610 & 2 & 1636,8 & BLMH_MOUSE & E9PY26_MOUSE & Bleomycin hydrolase; & \\
\hline $\begin{array}{l}\text { DKVERCYFFL } \\
\text { DKF }\end{array}$ & NAFVDTAQKKEPEDGR & 0,3 & $\mathrm{~N}$-ter $+34.06 \mathrm{Da}, \mathrm{K}+34.06 \mathrm{Da}$ & $\begin{array}{l}17,5 \\
17, \quad-1\end{array}$ & 0,681 & 4 & 1906,1 & BLMH_MOUSE & E9PYUO_MOUSE & $\begin{array}{l}\text { Bleomycin hydrolase; } \\
\text { Blose }\end{array}$ & \\
\hline MTFTAVSEKD & NQEGTFVKWR & 0,2 & N-ter +34.06 Da, K+34.06 Da & 23,1 & 0,375 & 3 & 1331,8 & BLMH_MOUSE & & Bleomycin hydrolase; & \\
\hline ESLMTHAMTF & TAVSEKDNQEGTFVKWR & 0,4 & N-ter +34.06 Da, K+34.06 Da & 51,8 & $-0,095$ & 4 & 2096,2 & BLMH_MOUSE & & Bleomycin hydrolase; & \\
\hline FGESLMTHAM & TFTAVSEKDNQEGTFVKWR & 0,3 & N-ter +28.03 Da, K+28.03 Da & 27,1 & 3,433 & 4 & 2326,2 & BLMH_MOUSE & & Bleomycin hydrolase; & \\
\hline SDMNVYDHEL & VFGVSLKNMNKAER & 0,3 & $\mathrm{~N}-\operatorname{ter}+34.06 \mathrm{Da}, \mathrm{K}+34.06 \mathrm{Da}$ & 24,1 & 6,596 & 4 & 1694,1 & BLMH_MOUSE & & Bleomycin hydrolase; & \\
\hline LMTHAMTFTA & VSEKDNQEGTFVKWR & 0,5 & N-ter $+28.03 \mathrm{Da}, \mathrm{K}+28.03 \mathrm{Da}$ & 18,2 & 1,990 & 4 & 1906,0 & BLMH_MOUSE & & Bleomycin hydrolase; & \\
\hline VQGAQHVFaH & VVPQEGKPVTNQKSSGR & 0,4 & $\mathrm{~N}-\operatorname{ter}+34.06 \mathrm{Da}, \mathrm{K}+34.06 \mathrm{Da}$ & 24,5 & 5,845 & 4 & 1912,2 & BLMH_MOUSE & E9PZH4_MOUSE & Bleomycin hydrolase; & \\
\hline CECPVHKNCY & CESFLAYTR & 10,0 & N-ter +34.06 Da, C +57.02 Da & 13,7 & $-2,454$ & 2 & 1179,6 & BMPER_MOUSE & & BMP-binding endothelial regulator protein; & $\begin{array}{l}\text { Bone morphogenetic protein-binding } \\
\text { endothelial cell precursor-derived } \\
\text { regulator:Protein crossveinless-2; }\end{array}$ \\
\hline PLWHCAQRMF & ASQTEGELKVTQVLKEKFPR & 0,2 & $\mathrm{~N}$-ter $+34.06 \mathrm{Da}, \mathrm{K}+34.06 \mathrm{Da}$ & 23,5 & $-6,509$ & 4 & 2423,5 & BOLA3_MOUSE & E9Q705_MOUSE & BolA-like protein 3; & \\
\hline NTINAAEPHV & VTVTMNGVAGR & 1,8 & $\mathrm{~N}$-ter +34.06 Da & 25,2 & $-1,228$ & 2 & 1137,6 & BMPR2_MOUSE & & Bone morphogenetic protein receptor type-2; & $\begin{array}{l}\text { BRK-3;Bone morphogenetic protein receptor } \\
\text { type I; }\end{array}$ \\
\hline EYEQTGNEYN & NEYEVYDNENGEPR & $-0,4$ & N-ter +34.06 Da & 18,0 & $-1,361$ & 3 & 1760,8 & SIAL_MOUSE & & Bone sialoprotein 2; & $\begin{array}{l}\text { Bone sialoprotein II;Cell-binding } \\
\text { sialoprotein;Integrin-binding sialoprotein; } \\
22 \text { kDa neuronal tissue-enriched acidic }\end{array}$ \\
\hline ASEAPSSAAK & APAPAAPAAAEPQAEAPAAAASSEQSVAVK & $-0,2$ & $\mathrm{~N}$-ter $+34.06 \mathrm{Da}, \mathrm{K}+34.06 \mathrm{Da}$ & 21,4 & $-1,741$ & 4 & 2925,5 & BASP1_MOUSE & & Brain acid soluble protein 1 ; & $\begin{array}{l}\text { protein;Neuronal axonal membrane protein } \\
\text { NAP-22; }\end{array}$ \\
\hline in & APAGLNGKLVSAQIR & $-0,1$ & $\mathrm{~N}$-ter $+28.03 \mathrm{Da}, \mathrm{K}+28.03 \mathrm{Da}$ & 48,6 & $-1,261$ & 3 & 1663,0 & C1TC_MOUSE & & C-1-tetrahydrofolate synthase, $\mathrm{C}$ & \\
\hline PRFT & DCELDTEAGR & $-3,6$ & $\mathrm{~N}-\operatorname{ter}+28.03 \mathrm{Da}, \mathrm{C}+57.02 \mathrm{Da}$ & 16,7 & 1,674 & 2 & 1192,5 & CELR3_MOUSE & & Cadherin EGF LAG seven-pass $G$ & \\
\hline
\end{tabular}




\begin{tabular}{|c|c|c|c|c|c|c|c|c|c|c|c|}
\hline Non Prime Site & Prime Site & 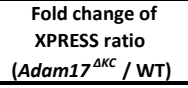 & Modifications & Hyperscore & $\begin{array}{c}\text { Mass error } \\
\text { in ppm }\end{array}$ & charge & $\begin{array}{c}\text { Precursor } \\
\text { neutral mass } \\
\text { in Da }\end{array}$ & Uniprot ID 1 & Uniprot ID 2 & Protein Name 1 & Protein Name 2 \\
\hline VYPGLRRQKR & DWVIPPISCPENEKGEFPKNLVQIKSNR & 0,1 & $\begin{array}{l}\text { N-ter +34.06 Da, K+34.06 Da, C } \\
\text { +57.02 Da }\end{array}$ & 26,3 & 0,670 & 4 & 3429,9 & CADH1_MOUSE & & Cadherin-1; & ARC-1;Epithelial cadherin;Uvomorulin; \\
\hline DKETKVFYSI & TGQGADKPPVGVFIIER & 0,6 & N-ter +34.06 Da, K +34.06 Da & 26,7 & 4,692 & 3 & 1851,1 & CADH1_MOUSE & & Cadherin-1; & ARC-1;Epithelial cadherin;Uvomorulin; \\
\hline LLSQVLLVTS & $\begin{array}{l}\text { ADDLECTPGFQR } \\
\text { G }\end{array}$ & 0,4 & $\mathrm{~N}$-ter $+28.03 \mathrm{Da}, \mathrm{C}+57.02 \mathrm{Da}$ & 24,1 & $-4,591$ & 2 & 1435,6 & CAD13_MOUSE & & Cadherin-13; & Heart cadherin;Truncated cadherin; \\
\hline RTSPVPRQKR & SIVVSPILIPENQR & 0,2 & N-ter +34.06 Da & 34,9 & 2,188 & 2 & 1598,0 & CAD13_MOUSE & & Cadherin-13; & Heart cadherin; Truncated cadherin; \\
\hline SPELLFLGAG & LKLKENGYDGLLVAINPR & 0,1 & $\mathrm{~N}$-ter +28.03 Da, $\mathrm{K}+28.03 \mathrm{Da}$ & 35,2 & $-3,811$ & 3 & 2096,2 & CLCA2_MOUSE & & Calcium-activated chloride channel regulator 2; & Calcium-activated chloride channel family \\
\hline KEAFSLFDKD & GDGTITTKELGTVMR & $-0,2$ & N-ter +34.06 Da, K+34.06 Da & 32,7 & $-4,063$ & 3 & 1645,9 & CALM_MOUSE & Q3UKW2_MOUSE & Calmodulin; & \\
\hline MINEVDADGN & GTIDFPEFLTMMAR & $-0,1$ & $\mathrm{~N}$-ter +28.03 Da & 16,1 & 1,689 & 2 & 1655,8 & CALM_MOUSE & Q3UKW2_MOUSE & Calmodulin; & \\
\hline AFSLFDKDGD & GTITTKELGTVMR & 0,4 & $\mathrm{~N}$-ter +34.06 Da, $\mathrm{K}+34.06 \mathrm{Da}$ & 27,6 & $-0,542$ & 3 & 1473,9 & CALM_MOUSE & Q3UKW2_MOUSE & Calmodulin; & \\
\hline INEVDADGNG & TIDFPEFLTMMAR & 0,0 & $\mathrm{~N}$-ter +34.06 Da & 40,0 & $-0,062$ & 2 & 1604,8 & CALM_MOUSE & Q3UKW2_MOUSE & Calmodulin; & \\
\hline FSLFDKDGDG & TITTKELGTVMR & 0,0 & $\mathrm{~N}$-ter +28.03 Da, $\mathrm{K}+28.03 \mathrm{Da}$ & 20,1 & 0,994 & 3 & 1404,8 & CALM_MOUSE & Q3UKW2_MOUSE & Calmodulin; & \\
\hline QEELEDMIRV & ADVDQDGKVKYEEFVR & 0,1 & $\mathrm{~N}$-ter +34.06 Da, $\mathrm{K}+34.06 \mathrm{Da}$ & 26,7 & 9,585 & 4 & 1999,1 & CALM4_MOUSE & & Calmodulin-4; & Calcium-binding protein Dd112; \\
\hline MSH & GFTKEEVAEFQAAFNR & 1,1 & $\mathrm{~N}$-ter +28.03 Da, $\mathrm{K}+28.03 \mathrm{Da}$ & 25,3 & $-0,316$ & 3 & 1899,0 & CALM4_MOUSE & & Calmodulin-4; & Calcium-binding protein Dd112; \\
\hline MIRVADVDQD & GKVKYEEFVR & 0,0 & $\mathrm{~N}$-ter +28.03 Da, $\mathrm{K}+28.03 \mathrm{Da}$ & 19,9 & 0,448 & 3 & 1337,8 & CALM4_MOUSE & & Calmodulin-4; & Calcium-binding protein Dd112; \\
\hline MSHGFT & KEEVAEFQAAFNR & 0,4 & $\mathrm{~N}$-ter +28.03 Da, $\mathrm{K}+28.03 \mathrm{Da}$ & 19,6 & 3,444 & 3 & 1593,8 & CALM4_MOUSE & & Calmodulin-4; & Calcium-binding protein Dd112; \\
\hline LKESLSKLGE & SLSQEELEDMIR & $-0,1$ & $\mathrm{~N}$-ter +28.03 Da & 44,9 & $-0,744$ & 2 & 1476,7 & CALM4_MOUSE & & Calmodulin-4; & Calcium-binding protein Dd112; \\
\hline ESLSKLGESL & SQEELEDMIR & $-0,1$ & N-ter +28.03 Da & 18,2 & $-0,704$ & 2 & 1276,6 & CALM4_MOUSE & & Calmodulin-4; & Calcium-binding protein Dd112; \\
\hline MSHGF & TKEEVAEFQAAFNR & 0,2 & N-ter +34.06 Da, K +34.06 Da & 39,4 & 0,409 & 3 & 1706,9 & CALM4_MOUSE & & Calmodulin-4; & Calcium-binding protein Dd112; \\
\hline SQEELEDMIR & VADVDQDGKVKYEEFVR & $-6,3$ & N-ter +28.03 Da, K +28.03 Da & 26,9 & $-0,480$ & 4 & 2080,1 & CALM4_MOUSE & & Calmodulin-4; & Calcium-binding protein Dd112; \\
\hline ELEDMIRVAD & VDQDGKVKYEEFVR & 0,4 & N-ter +28.03 Da, $\mathrm{K}+28.03 \mathrm{Da}$ & 24,0 & 0,278 & 4 & 1795,0 & CALM4_MOUSE & & Calmodulin-4; & Calcium-binding protein Dd112; \\
\hline FLTMMARKMK & DTDSEEEIR & 0,2 & $\mathrm{~N}$-ter +34.06 Da & 22,8 & $-0,443$ & 2 & 1126,5 & CALL3_MOUSE & CALM_MOUSE & Calmodulin-like protein 3; & \\
\hline FSLFDKDGDG & SITTQELGTVMR & 0,3 & $\mathrm{~N}$-ter $+28.03 \mathrm{Da}$ & 22,7 & $-0,147$ & 2 & 1362,7 & CALL__MOUSE & & Calmodulin-like protein 3 ; & \\
\hline VNEIDKDGNG & $\begin{array}{l}\text { TVDFPEFLTMMSR } \\
\text { TVET }\end{array}$ & 0,0 & $\mathrm{~N}$-ter $+34.06 \mathrm{Da}$ & 28,0 & $-0,435$ & 2 & 1606,8 & CALI_MOUSE & & Calmodulin-like protein 3; & \\
\hline SEEEIREAFR & VFDKDGNGFVSAAELR & $-3,0$ & $\mathrm{~N}$-ter +28.03 Da, $\mathrm{K}+28.03 \mathrm{Da}$ & 24,8 & $-0,561$ & 3 & 1779,9 & CALL3_MOUSE & & Calmodulin-like protein 3; & \\
\hline AMRILGGVIS & AISEAAAQYNPEPPPPR & $-0,3$ & N-ter +34.06 Da & 25,2 & 4,176 & 3 & 1841,0 & CPNS1_MOUSE & D3rW48_MOUSE & Calpain small subunit 1 ; & $\begin{array}{l}\text { Calcium-activated neutral proteinase small } \\
\text { subuniti;Calcium-dependent protease small } \\
\text { subunutitCalcium-dependent protease small } \\
\text { subunit } 1 ; \text {;Calpain regulatory subunit; }\end{array}$ \\
\hline PEPPPQQQHF & TVVEASESEEVRR & 0,7 & N-ter +34.06 Da & 21,3 & 3,668 & 3 & 1523,8 & CPNS2_MOUSE & & Calpain small subunit 2 ; & Calcium-dependent protease small subunit 2 ; \\
\hline PEPPPQQQHF & TVVEASESEEVR & 0,5 & N-ter +34.06 Da & 36,0 & $-0,073$ & 2 & 1367,7 & CPNS2_MOUSE & & Calpain small subunit 2; & Calcium-dependent protease small subunit 2; \\
\hline DNFKTLFSKL & AGDDMEISVKELQTILNR & 1,4 & N-ter +34.06 Da, K +34.06 Da & 43,2 & 4,995 & 3 & 2099,2 & CAN1_MOUSE & & Calpain-1 catalytic subunit; & $\begin{array}{l}\text { Calcium-activated neutral proteinase 1;Calpain } \\
\text { mu-type;Calpain-1 large subunit;iMirromolar- } \\
\text { calpain; }\end{array}$ \\
\hline RDFIREFTKL & EICNLTPDALKSR & 0,7 & $\begin{array}{l}\text { N-ter +34.06 Da, K+34.06 Da, C } \\
\text { +57.02 Da }\end{array}$ & 16,2 & $-5,987$ & 3 & 1583,9 & CAN1_MOUSE & & Calpain-1 catalytic subunit; & $\begin{array}{l}\text { Calcium-activated neutral proteinase 1;Calpain } \\
\text { mu-type;;Calpain-1 large subunit;:Micromolar- } \\
\text { calpain; }\end{array}$ \\
\hline RIRLPPGEYI & WVPSTFEPNKEGDFLLR & 0,5 & N-ter +34.06 Da, K+34.06 Da & 24,1 & 0,694 & 3 & 2015,1 & CAN1_MOUSE & & Calpain-1 catalytic subunit; & $\begin{array}{l}\text { Calcium-activated neutral proteinase 1;Calpain } \\
\text { mu-type;Calpain-1 large subunit;Micromolar- } \\
\text { calpain; }\end{array}$ \\
\hline GGCRNYPATF & WVNPQFKIR & $-1,7$ & N-ter +34.06 Da, K+34.06 Da & 16,0 & $-3,260$ & 3 & 1254,8 & CAN1_MOUSE & & Calpain-1 catalytic subunit; & $\begin{array}{l}\text { Calcium-activated neutral proteinase 1;Calpain } \\
\text { mu-type;Calpain-1 large subunit;Micromolar- } \\
\text { calpain; }\end{array}$ \\
\hline IKPEHSEKLG & ERDDTIPPEYR & 0,6 & N-ter +28.03 Da & 19,4 & 2,816 & 3 & 1417,7 & ICAL_MOUSE & Q8CE80_MOUSE & Calpastatin; & Calpain inhibitor; \\
\hline RSAPPKLASL & KGVVPEDAVETLAGSLGTR & $-0,3$ & N-ter $+28.03 \mathrm{Da}, \mathrm{K}+28.03 \mathrm{Da}$ & 25,7 & 7,409 & 3 & 1954,1 & ICAL_MOUSE & Q8CE80_MOUSE & Calpastatin; & Calpain inhibitor; \\
\hline VPRASMCSIR & SAPPKLASLKGVVPEDAVETLAGSLGTR & 0,0 & $\mathrm{~N}$-ter $+28.03 \mathrm{Da}, \mathrm{K}+28.03 \mathrm{Da}$ & 38,2 & 1,263 & 4 & 2846,6 & ICAL_MOUSE & Q8CE80_MOUSE & Calpastatin; & Calpain inhibitor; \\
\hline LLGLLLAAA & DPAIYFKEQFLDGDAWTNR & 0,3 & $\mathrm{~N}$-ter $+28.03 \mathrm{Da}, \mathrm{K}+28.03 \mathrm{Da}$ & 49,8 & 1,706 & 3 & 2341,1 & CALR_MOUSE & & Calreticulin; & $\begin{array}{l}\text { CRP55;Calregulin;Endoplasmic reticulum } \\
\text { resident protein 60;HACBP; }\end{array}$ \\
\hline HKEPLQQCNP & DGPLGAAVGAAGAGWGRPGSPPAAPPR & 1,1 & N-ter+34.06 Da & 17,4 & 1,840 & 3 & 2443,3 & CHST2_MOUSE & & Carbohydrate sulfotransferase 2 ; & $\begin{array}{l}\text { Galactose/N-acetylglucosamine/ } / \mathrm{N} \text { - } \\
\text { acety/glucosamine 6-O-sulfotransferase 2;N- } \\
\text { acetyglucosamine 6-O-sulfotransferase } 1 ;\end{array}$ \\
\hline M & KLNFSGLR & 0,1 & $\mathrm{~N}$-ter +28.03 Da, $\mathrm{K}+28.03 \mathrm{Da}$ & 21,0 & 2,937 & 3 & 1120,7 & CBR2_MOUSE & & Carbonyl reductase [NADPH] 2; & $\begin{array}{l}\text { Adipocyte protein P27; Lung carbonyl } \\
\text { reductase;NADPH-dependent carbonyl } \\
\text { reductase 2; }\end{array}$ \\
\hline VTIEDLQA! & DNEDEEMQHNEGIER & 0,7 & $\mathrm{~N}$-ter +34.06 Da & 42,9 & 2,658 & 3 & 1877,8 & CBPA4_MOUSE & & & \\
\hline SVTIEDL & LDNEDEEMQHNEGIER & 0,6 & $\mathrm{~N}$-ter +34.06 Da & 18,1 & $-1,254$ & 3 & 1990,9 & CBPA4_MOUSE & & Carboxypeptidase A4; & \\
\hline NPDGFERARE & GDGGLGDSGPPGTSGR & 10,0 & N-ter +34.06 Da, C+57.02 Da & 16,7 & $-2,753$ & 3 & 1522,7 & CBPD_MOUSE & & Carboxypeptidase D; & Metallocarboxypeptidase D;gp180; \\
\hline
\end{tabular}




\begin{tabular}{|c|c|c|c|c|c|c|c|c|c|c|c|}
\hline Non Prime Site & Prime Site & 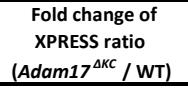 & Modifications & Hyperscore & $\begin{array}{l}\text { Mass error } \\
\text { in ppm }\end{array}$ & Charge & $\begin{array}{c}\begin{array}{c}\text { Precursor } \\
\text { neutral mass } \\
\text { in Da }\end{array} \\
\end{array}$ & Uniprot ID 1 & Uniprot ID 2 & Protein Name 1 & Protein Name 2 \\
\hline PAAGMRRRRR & LQQEDGISFEYHR & 1,3 & N-ter+34.06 Da & 20,5 & $-3,197$ & 3 & 1654,8 & CBPE_MOUSE & & Carboxypeptidase E; & $\begin{array}{l}\text { Carboxypeptidase H;Enkephalin } \\
\text { convertase;Prohormone-processing } \\
\text { carboxypeptidase; }\end{array}$ \\
\hline HWIMDIPFVL & SANLHGGDLVANYPYDETR & 0,3 & $\mathrm{~N}$-ter +28.03 Da & 22,1 & 2,074 & 3 & 2119,0 & CBPE_MOUSE & & Carboxypeptidase E; & $\begin{array}{l}\text { Carboxypeptidase H;Enkephalin } \\
\text { convertase; Prohormone-processing } \\
\text { carboxypeptidase; }\end{array}$ \\
\hline EDVVDSFQVYY & CVEEPQDDQEINELVEEYR & 0,2 & $\mathrm{~N}$-ter +34.06 Da, C +57.02 Da & 18,7 & $-2,262$ & 4 & 2427,1 & CMYAS_MOUSE & E9QபO_MOUSE & Cardiomyopathy-associated protein 5; & $\begin{array}{l}\text { Heart and skeletal muscle-specific and sprouty } \\
\text { domain-containing;:Myospryn; } ; \text { Stretch-response } \\
\text { protein } 553 ; \text { Stretch-responsive fibronectin } \\
\text { protein type 3;TRIM-like protein; }\end{array}$ \\
\hline SFQVYCVEEP & QDDQEINELVEEYR & 0,8 & $\mathrm{~N}$-ter $+28.03 \mathrm{Da}$ & 30,2 & 7,574 & 2 & 1806,8 & CMYA5_MOUSE & E9QU0_MOUSE & Cardiomyopathy-associated protein 5; & $\begin{array}{l}\text { Heart and skeletal muscle-specific and sprouty } \\
\text { domain-containing;Myospryn; Stretch-response } \\
\text { protein 553;Stretch-responsive fibronectin } \\
\text { protein type 3;TRIM-like protein; }\end{array}$ \\
\hline LVLGAPSRPL & SAVSGPAEYLQHSIVPTMHYQDSLPR & 0,0 & $\mathrm{~N}$-ter +34.06 Da & 37,1 & $-5,068$ & 4 & 2916,5 & CPT2_MOUSE & A2A8E7_MOUSE & Carnitine O-palmitoyltransferase 2, mitochondrial; & Carnitine palmitoyltransferase II; \\
\hline ELTEEITRLM & ANTEVMQEGKPR & $-0,9$ & N-ter +28.03 Da, K+28.03 Da & 38,7 & $-0,988$ & 3 & 1414,7 & CASPE_MOUSE & & Caspase-14; & Mini-IEE; \\
\hline RLALTLCVTK & AREGSEVDMEALER & 0,5 & $\mathrm{~N}$-ter +34.06 Da & 36,8 & $-1,167$ & 3 & 1624,8 & CASPE_MOUSE & & Caspase-14; & Mini-ICE; \\
\hline MESEMS & DPQPLQEER & 0,3 & $\mathrm{~N}$-ter $+28.03 \mathrm{Da}$ & 30,2 & 1,666 & 2 & 1138,6 & CASPE_MOUSE & & Caspase-14; & Mini-ICE; \\
\hline ALTLCVTKAR & EGSEVDMEALER & $-6,1$ & N-ter $+28.03 \mathrm{Da}$ & 24,4 & 2,009 & 2 & 1391,6 & CASPE_MOUSE & & Caspase-14; & Mini-ICE; \\
\hline QSIPTYTDTL & HIYSTVEGYLSYR & 0,6 & $\mathrm{~N}$-ter $+28.03 \mathrm{Da}$ & 28,8 & 1,422 & 3 & 1614,8 & CASPE_MOUSE & & Caspase-14; & Mini-ICE; \\
\hline MESE & MSDPQPLQEER & 0,7 & $\mathrm{~N}$-ter $+28.03 \mathrm{Da}$ & 39,3 & 0,442 & 2 & 1356,6 & CASPE_MOUSE & & Caspase-14; & Mini-ICE; \\
\hline LTEEITRLMA & NTEVMQEGKPR & $-0,7$ & $\mathrm{~N}$-ter $+28.03 \mathrm{Da}, \mathrm{K}+28.03 \mathrm{Da}$ & 30,2 & $-6,609$ & 3 & 1343,7 & CASPE_MOUSE & & Caspase-14; & Mini-ICE; \\
\hline LALTLCVTKA & REGSEVDMEALER & 0,5 & $\mathrm{~N}$-ter $+28.03 \mathrm{Da}$ & 34,1 & 1,741 & 3 & 1547,7 & CASPE_MOUSE & & Caspase-14; & Mini-ICE; \\
\hline MESEM & SDPQPLQEER & 0,8 & $\mathrm{~N}-$ ter $+34.06 \mathrm{Da}$ & 39,6 & $-0,649$ & 2 & 1231,6 & CASPE_MOUSE & & Caspase-14; & Mini-ICE; \\
\hline PTYTDTLHIY & STVEGYLSYR & 0,7 & $\mathrm{~N}-$ ter $+34.06 \mathrm{Da}$ & 29,3 & $-2,067$ & 2 & 1207,6 & CASPE_MOUSE & & Caspase-14; & Mini-ICE; \\
\hline VNYFAEVEQM & AFDPSNMPPGIEPSPDKMLQGR & 0,9 & $\mathrm{~N}$-ter $+34.06 \mathrm{Da}, \mathrm{K}+34.06 \mathrm{Da}$ & 47,9 & $-0,448$ & 3 & 2451,3 & CATA_MOUSE & & Catalase; & \\
\hline AGSRGPLLVQ & DVVFTDEMAHFDR & 1,4 & $\mathrm{~N}$-ter +34.06 Da & 15,5 & $-0,680$ & 3 & 1614,8 & CATA_MOUSE & A2AL20_MOUSE & Catalase; & \\
\hline LLVQDVVFTD & EMAHFDR & 2,1 & $\mathrm{~N}-$ ter $+34.06 \mathrm{Da}$ & 15,6 & $-1,912$ & 3 & 938,5 & CATA_MOUSE & A2AL20_MOUSE & Catalase; & \\
\hline SPDKMLQGRL & FAYPDTHR & 2,0 & $\mathrm{~N}-$ ter $+34.06 \mathrm{Da}$ & 16,4 & $-0,959$ & 3 & 1039,5 & CATA_MOUSE & & Catalase; & \\
\hline NYFAEVEQMA & FDPSNMPPGIEPSPDKMLQGR & 1,4 & N-ter +28.03 Da, K +28.03 Da & 16,8 & $-3,247$ & 3 & 2368,2 & CATA_MOUSE & & Catalase; & \\
\hline ERIPERVVHA & KGAGAFGYFEVTHDITR & 1,5 & $\mathrm{~N}$-ter +34.06 Da, K +34.06 Da & 34,7 & 0,000 & 4 & 1936,1 & CATA_MOUSE & A2AL20_MOUSE & Catalase; & \\
\hline PSPDKMLQGR & $\begin{array}{l}\text { LFAYPDTHR } \\
\text { Lation }\end{array}$ & $-0,2$ & $\mathrm{~N}$-ter +28.03 Da & 22,5 & $-3,827$ & 3 & 1146,6 & CATA_MOUSE & & Catalase; & \\
\hline QCAVDVKRFN & SANEDNVTQVR & 0,4 & N-ter +28.03 Da & 27,4 & 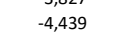 & 2 & 1259,6 & CATA_MOUSE & & Catalase; & \\
\hline GKRTPIAVRF & STVTGESGSADTVR & 1,0 & N-ter +34.06 Da & 49,8 & $-1,712$ & 2 & 1399,7 & CATA_MOUSE & & Catalase; & \\
\hline GPLVQDVVF & TDEMAHFDR & 1,6 & N-ter +34.06 Da & 26,5 & $-0,346$ & 3 & 1154,5 & CATA_MOUSE & A2AL20_MOUSE & Catalase; & \\
\hline GSRGPLLVQD & VVFTDEMAHFDR & 1,5 & N-ter +34.06 Da & 37,2 & 0,998 & 3 & 1499,7 & CATA_MOUSE & A2AL20_MOUSE & Catalase; & \\
\hline KLPGRVAFGE & DIDLPETFDAR & $-0,4$ & $\mathrm{~N}$-ter $+28.03 \mathrm{Da}$ & 22,0 & $-0,379$ & 2 & 1318,6 & CATB_MOUSE & & Cathepsin B; & Cathepsin B1; \\
\hline DNGFFKLRG & ENHCGIESEIVAGIPR & $-0,9$ & N-ter +28.03 Da, C +57.02 Da & 19,1 & 5,025 & 3 & 1807,9 & CATB_MOUSE & & Cathepsin B; & Cathepsin B1; \\
\hline GFFKILRGEN & HCGIESEIVAGIPR & $-0,6$ & N-ter +34.06 Da, C +57.02 Da & 13,7 & 1,779 & 3 & 1570,8 & CATB_MOUSE & & Cathepsin B; & Cathepsin B1; \\
\hline DIDLPETFDA & REQWSNCPTIGQIR & 0,1 & N-ter +28.03 Da, C +57.02 Da & 16,8 & 0,789 & 3 & 1771,9 & CATB_MOUSE & & Cathepsin B; & Cathepsin B1; \\
\hline IPPCEHHVNG & SRPPCTGEGDTPR & 0,0 & N-ter +28.03 Da, C +57.02 Da & 18,0 & 1,576 & 3 & 1456,7 & CATB_MOUSE & & Cathepsin B; & Cathepsin B1; \\
\hline KNIFSFYLNR & DPEGQPGGELMLGGTDSK & 0,3 & $\mathrm{~N}$-ter +28.03 Da, $\mathrm{K}+28.03 \mathrm{Da}$ & 33,2 & 2,492 & 3 & 1842,9 & CATD_MOUSE & FGYGL6_MOUSE & Cathepsin $\mathrm{D} ;$ & \\
\hline ILGDVFIGSY & YTVFDRDNNR & 0,3 & N-ter +34.06 Da & 16,2 & 0,599 & 3 & 1332,7 & CATD_MOUSE & & Cathepsin $\mathrm{D} ;$ & \\
\hline QWKSTHRRLY & GTNEEEWR & 0,5 & $\mathrm{~N}$-ter +28.03 Da & 15,9 & $-1,620$ & 2 & 1047,5 & CATL1_MOUSE & & Cathepsin Li; & $\begin{array}{l}\text { Cathepsin L;Major excreted protein;;39 } \\
\text { austeine Lroteinase. }\end{array}$ \\
\hline ERLRNRVDQL & TQELDTLR & $-0,6$ & $\mathrm{~N}$-ter $+34.06 \mathrm{Da}$ & 14,5 & $-1,385$ & 2 & 1008,6 & CEBPE_MOUSE & & CCAAT/enhancer-binding protein epsilon; & \\
\hline EFSALVHKEN & TDIQLTVTGPGIPR & 0,4 & $\mathrm{~N}$-ter +28.03 Da & 39,0 & 1,737 & 2 & 1494,8 & CD109_MOUSE & & CD109 antigen; & $\begin{array}{l}\text { GPl-anchored alpha-2 macroglobulin-related } \\
\text { rotein. }\end{array}$ \\
\hline GVLCLCLTGA & VEVQVSEDPVVALVDTDATLR & $-1,0$ & $\mathrm{~N}$-ter $+28.03 \mathrm{Da}$ & 29,9 & $-1,225$ & 3 & 2282,2 & CD276_MOUSE & & CD276 antigen; & $\begin{array}{l}\text { protein; } \\
\text { B7 homolog 3;Costimulatory molecule; }\end{array}$ \\
\hline CEPVMEEKVQ & EQSSLLENGAVHQGDTSWGSSGSGSQSSQGR & $-7,6$ & $\mathrm{~N}$-ter $+28.03 \mathrm{Da}$ & 20,6 & 0,508 & 4 & 3147,4 & BORG2_MOUSE & & Cdc42 effector protein 3; & Binder of Rho GTPases 2; \\
\hline VEELPNOFYR & DPNALHELLR & $-4,4$ & $\mathrm{~N}$-ter $+28.03 \mathrm{Da}$ & 20,5 & 0,745 & 3 & 1204,7 & RAD17_MOUSE & & Cell cycle checkpoint protein RAD17; & \\
\hline MAMNYSAK & DEVDGGPAGPPGGAAKTR & 0,9 & N-ter +34.06 Da, $\mathrm{K}+34.06 \mathrm{Da}$ & 34,3 & 0,581 & 3 & 1718,9 & CC5OA_MOUSE & D3rVV1_MOUSE & Cell cycle control protein 50A; & $\begin{array}{l}\text { P4-ATPase flippase complex beta subunit } \\
\text { TMEM } 30 \text { A Transmembrane protein } 30 \mathrm{~A} \text {. }\end{array}$ \\
\hline RGRGGFTSDR & GFQFVSSSLPDICYR & $-1,5$ & N-ter +28.03 Da, C +57.02 Da & 21,4 & 1,274 & 2 & 1802,9 & CNBP_MOUSE & D3YYF6_MOUSE & Cellular nucleic acid-binding protein; & $\begin{array}{l}\text { TMEM } 30 \text { A; Transmembrane protein 30A; } \\
\text { Zinc finger protein 9; }\end{array}$ \\
\hline SDRGFAFUSS & SLPDICYR & 0,0 & $\mathrm{~N}$-ter +34.06 Da, C +57.02 Da & 16,9 & 0,756 & 2 & 105,6 & CNBP_MOUSE & D3YYF__MOUSE & Cellular nucleic acid-binding protein; & Zinc finger protein 9 ; \\
\hline GRKEITPPDQ & TIKTLFADGQEESIFPDGTIVR & $-4,0$ & $\mathrm{~N}$-ter +28.03 Da, K +28.03 Da & 18,5 & 0,601 & 3 & 2492,3 & CENPJ_MOUSE & & Centromere protein J; & \\
\hline ITSDALIPLP & LNTVQDPASAQEAVGASR & $-10,0$ & $\mathrm{~N}$-ter $+28.03 \mathrm{Da}$ & 21,2 & $-1,574$ & 2 & 1840,9 & CEP78_MOUSE & & Centrosomal protein of $78 \mathrm{kDa}$; & \\
\hline MGIPTFGKSF & TLASSENQLGAPISGEGLPGR & 1,6 & $\mathrm{~N}$-ter +34.06 Da & 43,7 & 2,153 & 3 & 2087,1 & CH3L1_MOUSE & D3Z2P2_MOUSE & Chitinase-3--ike protein 1 ; & $\begin{array}{l}\text { BRP39 protein;Breast regression protein } \\
39 \text { Cartiage ofycorototein } 3 \text {. }\end{array}$ \\
\hline TFVKSALSFL & RTQGFDGLDLDWEFPGGR & 1,7 & N-ter +34.06 Da & 27,8 & $-4,995$ & 3 & 2099,0 & CHIT1_MOUSE & & Chitotriosidase-1; & $\begin{array}{l}\text { Chitinase- } 1 \text {; } \\
\text {; }\end{array}$ \\
\hline
\end{tabular}




\begin{tabular}{|c|c|c|c|c|c|c|c|c|c|c|c|}
\hline Non Prime Site & Prime Site & $\begin{array}{l}\text { Fold change of } \\
\text { XPRESS ratio } \\
\text { (Adam17 } 7^{\text {Akc } / \text { WT) }} \\
\end{array}$ & Modifications & Hyperscore & $\begin{array}{c}\text { Mass error } \\
\text { in ppm }\end{array}$ & Charge & $\begin{array}{c}\begin{array}{c}\text { Precursor } \\
\text { neutral mass } \\
\text { in Da }\end{array} \\
\end{array}$ & Uniprot ID 1 & Uniprot ID 2 & Protein Name 1 & Protein Name 2 \\
\hline GQPCPPCSRA & VASVGKDEYTFVVVGAGSAGCVLASR & $-0,3$ & $\begin{array}{l}\text { N-ter +34.06 Da, K +34.06 Da, C } \\
+57.02 \text { Da }\end{array}$ & 24,8 & 0,150 & 3 & 2666,4 & CHDH_MOUSE & & Choline dehydrogenase, mitochondrial; & \\
\hline SSCLVLAARH & ASASSTNLKDVISNLIPKEOAR & 0,0 & $\mathrm{~N}$-ter +28.03 Da, K+28.03 Da & 43,8 & 6,668 & 4 & 2425,4 & CISY_MOUSE & & Citrate synthase, mitochondrial; & Citrate (Si)-synthase; \\
\hline $\begin{array}{l}\text { CVAAKIYRNL } \\
\text { DGYAAIAQAD }\end{array}$ & $\begin{array}{l}\text { YREGSSIGAIDSR } \\
\text { RLTQEPESIR }\end{array}$ & $\begin{array}{c}0,8 \\
-0,1\end{array}$ & $\begin{array}{l}\mathrm{N} \text {-ter }+34.06 \mathrm{Da} \\
\mathrm{N}-\mathrm{ter}+34.06 \mathrm{Da}\end{array}$ & $\begin{array}{l}21,0 \\
20,6\end{array}$ & $\begin{array}{l}-1,659 \\
-0,158\end{array}$ & $\begin{array}{l}3 \\
3\end{array}$ & $\begin{array}{l}1443,8 \\
1261,7\end{array}$ & $\begin{array}{l}\text { CISY_MOUSE } \\
\text { CLCB_MOUSE }\end{array}$ & F7BHJO_MOUSE & $\begin{array}{l}\text { Citrate synthase, mitochondrial; } \\
\text { Clathrin light chain B; }\end{array}$ & Citrate (Si)-synthase; \\
\hline EMIHQAQQAM & DVQLHSPAFQFPDVDFLR & $-0,8$ & N-ter +28.03 Da & 23,2 & 1,434 & 3 & 2158,1 & CLUS_MOUSE & & Clusterin; & $\begin{array}{l}\text { Apolipoprotein J;Clustrin;Sulfated glycoprotein } \\
2 ;\end{array}$ \\
\hline LIWDNGMVLG & EQEVSDNELQELSTQGSR & $-0,6$ & N-ter +34.06 Da & 33,8 & 0,384 & 3 & 2082,0 & CLUS_MOUSE & E9PXG5_MOUSE & Clusterin; & $\begin{array}{l}\text { Apolipoprotein J;Clustrin;Sulfated glycoprotein } \\
2 \text {; }\end{array}$ \\
\hline NAVQGVKHIK & TLEKTNAER & $-1,0$ & N-ter +34.06 Da, K+34.06 Da & 21,0 & 4,981 & 3 & 1241,8 & CLUS_MOUSE & E9PXG5_MOUSE & Clusterin; & $\begin{array}{l}\text { Apolipoprotein } J \text {;Clustrin;Sulfated glycoprotein } \\
2 \text {; }\end{array}$ \\
\hline GEDKYYLRVS & TVTTHSSDSEVPSR & 0,1 & $\mathrm{~N}$-ter +34.06 Da & 35,9 & 2,470 & 3 & 1535,8 & CLUS_MOUSE & & Clusterin; & $\begin{array}{l}\text { Apolipoprotein J;Clustrin;Sulfated glycoprotein } \\
2 \text {; }\end{array}$ \\
\hline M & VLLAAAVCTKAGKAIVSR & 0,5 & $\begin{array}{l}\text { N-ter +34.06 Da, K+34.06 Da, C } \\
+57.02 \text { Da }\end{array}$ & 64,9 & $-1,035$ & 3 & 1929,3 & COPD_MOUSE & & Coatomer subunit delta; & Archain;Delta-coat protein; \\
\hline MRAAPRR & APAAQPPAAAAPSAVGSPAAAPR & $-0,5$ & $\mathrm{~N}$-ter $+28.03 \mathrm{Da}$ & 50,2 & $-1,233$ & 3 & 2024,1 & CHCH2_MOUSE & B2RPU8_MOUSE & $\begin{array}{l}\text { Coiled-coil-helix-coiled-coil-helix domain-containing } \\
\text { protein 2, mitochondrial; }\end{array}$ & \\
\hline MRAAPR & RAPAAQPPAAAAPSAVGSPAAAPR & $-0,4$ & $\mathrm{~N}$-ter $+28.03 \mathrm{Da}$ & 40,1 & $-0,962$ & 3 & 2180,2 & CHCH2_MOUSE & B2RPU8_MOUSE & $\begin{array}{l}\text { Coiled-coil-helix-coiled-coil-helix domain-containing } \\
\text { protein 2, mitochondrial; }\end{array}$ & \\
\hline QALEQVFSKY & GQISEVVVVKDR & 1,0 & $\mathrm{~N}-\operatorname{ter}+28.03 \mathrm{Da}, \mathrm{K}+28.03 \mathrm{Da}$ & 21,2 & 6,634 & 3 & 1383,8 & CIRBP_MOUSE & K4D165_MOUSE & Cold-inducible RNA-binding protein; & $\begin{array}{l}\text { A18 hnRNP;Glycine-rich RNA-binding protein } \\
\text { CIRP; }\end{array}$ \\
\hline NFASQMSYGY & DEKSAGVSVPGPMGPSGPR & 0,2 & $\mathrm{~N}$-ter $+28.03 \mathrm{Da}, \mathrm{K}+28.03 \mathrm{Da}$ & 20,4 & 6,107 & 3 & 1880,0 & CO1A1_MOUSE & & Collagen alpha-1(I) chain; & Alpha-1 type I collagen; \\
\hline GGNFASQMSY & GYDEKSAGVSVPGPMGPSGPR & $-0,3$ & $\mathrm{~N}-\operatorname{ter}+34.06 \mathrm{Da}, \mathrm{K}+34.06 \mathrm{Da}$ & 20,2 & 1,513 & 3 & 2112,1 & COIA1_MOUSE & & Collagen alpha-1(I) chain; & Alpha-1 type I collagen; \\
\hline GLTGNFAAQY & SDKGVSSGPGPMGLMGPR & 0,1 & $\mathrm{~N}-\operatorname{ter}+28.03 \mathrm{Da}, \mathrm{K}+28.03 \mathrm{Da}$ & 33,9 & $-1,958$ & 3 & 1784,9 & CO1A2_MOUSE & EOCXI2_MOUSE & Collagen alpha-2(l) chain; & Alpha-2 type I collagen; \\
\hline PSGPVGKDGR & SGQPGPVGPAGVR & $-0,3$ & $\mathrm{~N}$-ter $+34.06 \mathrm{Da}$ & 28,9 & 1,154 & 2 & 1211,7 & CO1A2_MOUSE & & Collagen alpha-2(I) chain; & Alpha-2 type I collagen; \\
\hline PGLTGNFAAQ & YSDKGUSSGPGPMGLMGPR & 0,1 & $\mathrm{~N}-\operatorname{ter}+28.03 \mathrm{Da}, \mathrm{K}+28.03 \mathrm{Da}$ & 30,6 & $-2,512$ & 3 & 1948,0 & CO1A2_MOUSE & EOCXI2_MOUSE & Collagen alpha-2(I) chain; & Alpha-2 type I collagen; \\
\hline HRPDQGSLAN & MPNLDAVALKAAMAQFDR & 10,0 & $\mathrm{~N}-\operatorname{ter}+34.06 \mathrm{Da}, \mathrm{K}+34.06 \mathrm{Da}$ & 17,0 & $-1,772$ & 3 & 2029,1 & COG6_MOUSE & & Conserved oligomeric Golgi complex subunit 6; & Component of oligomeric Golgi complex 6; \\
\hline IRGSPGAPSF & AAGPPVSEGKYFSSNPIIPSR & $-0,6$ & $\mathrm{~N}-\operatorname{ter}+34.06 \mathrm{Da}, \mathrm{K}+34.06 \mathrm{Da}$ & 18,9 & $-0,223$ & 3 & 2241,2 & CDSN_MOUSE & & Corneodesmosin; & \\
\hline RGSPGAPSFA & AGPPVSEGKYFSSNPIIPSR & $-0,1$ & $\mathrm{~N}-\operatorname{ter}+28.03 \mathrm{Da}, \mathrm{K}+28.03 \mathrm{Da}$ & 42,1 & $-0,185$ & 3 & 2158,1 & CDSN_MOUSE & & Corneodesmosin; & \\
\hline QVKPLGPQLM & DPKVSLPQGEPQGEPLEK & 0,2 & $\mathrm{~N}-\operatorname{ter}+28.03 \mathrm{Da}, \mathrm{K}+28.03 \mathrm{Da}$ & 22,7 & 1,650 & 3 & 2118,1 & CDSN_MOUSE & & Corneodesmosin; & \\
\hline SQSGSSGSSR & DRPGSGSALPTGDK & $-0,3$ & $\mathrm{~N}-\operatorname{ter}+28.03 \mathrm{Da}, \mathrm{K}+28.03 \mathrm{Da}$ & 26,4 & $-0,283$ & 3 & 1412,7 & CDSN_MOUSE & & Corneodesmosin; & \\
\hline SGGKIYPVGY & FTKDNPIR & 0,0 & $\mathrm{~N}-\operatorname{ter}+28.03 \mathrm{Da}, \mathrm{K}+28.03 \mathrm{Da}$ & 19,3 & $-0,572$ & 3 & 1045,6 & CDSN_MOUSE & & Corneodesmosin; & \\
\hline TKDNPIRGSP & GAPSFAAGPPVSEGKYFSSNPIIPSR & 0,6 & $\mathrm{~N}-\operatorname{ter}+34.06 \mathrm{Da}, \mathrm{K}+34.06 \mathrm{Da}$ & 25,1 & 4,402 & 3 & 2700,5 & CDSN_MOUSE & & Corneodesmosin; & \\
\hline GSPGAPSFAA & GPPVSEGKYFSSNPIIPSR & 0,2 & $\mathrm{~N}$-ter $+28.03 \mathrm{Da}, \mathrm{K}+28.03 \mathrm{Da}$ & 26,2 & 0,096 & 3 & 2087,1 & CDSN_MOUSE & & Corneodesmosin; & \\
\hline TYSGGKIYPV & GYFTKDNPIR & $-1,6$ & $\mathrm{~N}-\operatorname{ter}+28.03 \mathrm{Da}, \mathrm{K}+28.03 \mathrm{Da}$ & 18,2 & 2,128 & 3 & 1265,7 & CDSN_MOUSE & & Corneodesmosin; & \\
\hline AGLILPGILA & KSIGTLSDPCKDPTR & $-0,3$ & $\begin{array}{l}\text { N-ter +28.03 Da, K +28.03 Da, C } \\
+57.02 \mathrm{Da}\end{array}$ & 21,4 & 0,227 & 3 & 1757,9 & CDSN_MOUSE & & Corneodesmosin; & \\
\hline TQVKPLGPQL & MDPKVSLPQGEPQGEPLEK & 0,2 & $\mathrm{~N}$-ter +34.06 Da, $\mathrm{K}+34.06 \mathrm{Da}$ & 20,0 & 0,441 & 3 & 2267,3 & CDSN_MOUSE & & Corneodesmosin; & \\
\hline SSGYPVGVAF & QPVGSGGVQPCGTGSVSSKGPCSGTR & 0,0 & N-ter +34.06 Da, K+34.06 Da, C & 13,9 & 1,049 & 3 & 2571,3 & CDSN_MOUSE & & Corneodesmosin; & \\
\hline NSYLVPGMTY & SGGKIYPVGYFTKDNPIR & $-0,2$ & $\begin{array}{l}+57.02 \mathrm{Da} \\
\mathrm{N}+\mathrm{er}+34.06 \mathrm{Da}, \mathrm{K}+34.06 \mathrm{Da}\end{array}$ & 39,1 & 0,189 & 4 & 2113,2 & CDSN MOUSE & & Corneodesmosin; & \\
\hline GLLPGILAK & SIGTLSDPCKDPTR & $-0,1$ & N-ter $+34.06 \mathrm{Da}, \mathrm{K}+34.06 \mathrm{Da}, \mathrm{C}$ & 29,1 & 1,794 & 3 & 1613,9 & CDSN_MOUSE & & Corneodesmosin; & \\
\hline GPPVSEGKYF & SSNPIIISR & 0,1 & $\begin{array}{l}+57.02 \text { a } \\
\text { N-ter }+34.06 \text { Da }\end{array}$ & 16,4 & $-0,497$ & 2 & 1003,6 & CDSN_MOUSE & & Corneodesmosin; & \\
\hline GSQSGRWVSS & SSQWVSSSSQSGSSGSSR & 10,0 & $\mathrm{~N}$-ter +34.06 Da & 17,7 & $-3,893$ & 3 & 1820,8 & CDSN_MOUSE & & Corneodesmosin; & \\
\hline SGSQSGRWVS & SSSQWVSSSSOSGSSGSSR & 0,2 & $\mathrm{~N}$-ter $+28.03 \mathrm{Da}$ & 36,0 & 5,200 & 2 & 1901,9 & CDSN_MOUSE & & Corneodesmosin; & \\
\hline LENLRLQKRG & TGGVDTAATGSVFDISNLDR & 0,3 & $\mathrm{~N}$-ter +34.06 Da & 20,1 & 5,167 & 3 & 2029,0 & KCRU_MOUSE & & Creatine kinase U-type, mitochondrial; & $\begin{array}{l}\text { Acidic-type mitochondrial creatine } \\
\text { kinase;Ubiquitous mitochondrial creatine } \\
\text { kinase; }\end{array}$ \\
\hline NGYDPRTMKH & TTDLDASKIR & 0,2 & N-ter $+28.03 \mathrm{Da}, \mathrm{K}+28.03 \mathrm{Da}$ & 32,8 & $-1,783$ & 3 & 1174,7 & KCRU_MOUSE & BOROFO_MOUSE & Creatine kinase U-type, mitochondrial; & $\begin{array}{l}\text { Acidic-type mitochondrial creatine } \\
\text { kinase;Ubiquitous mitochondrial creatine } \\
\text { kinase; }\end{array}$ \\
\hline M & LPKPGVYYFPWEVSDGHVPEGSTLR & 0,1 & $\mathrm{~N}$-ter $+34.06 \mathrm{Da}, \mathrm{K}+34.06 \mathrm{Da}$ & 34,0 & $-1,781$ & 4 & 3028,6 & TEN1L_MOUSE & & CST complex subunit TEN1; & $\begin{array}{l}\text { Protein telomeric pathways with STN1 } \\
\text { homolog; Telomere length regulation protein } \\
\text { TEN1 homolog; }\end{array}$ \\
\hline GFKIRYAAPY & CSLTSTLR & $-7,6$ & N-ter $+28.03 \mathrm{Da}, \mathrm{C}+57.02 \mathrm{Da}$ & 11,2 & 2,587 & 2 & 964,5 & CSMD1_MOUSE & & CUB and sushi domain-containing protein $1 ;$ & CUB and sushi multiple domains protein $1 ;$ \\
\hline & TSLFRR & $-0,2$ & $\mathrm{~N}$-ter +34.06 Da & 20,0 & $-0,105$ & 3 & 1902,0 & CUED1_MOUSE & F2Z3X3_MOUSE & CUE domain-containing protein 1 ; & \\
\hline LQDLGAMKSV & CPGTSGFSSPNPSAASAAAQEVR & $-4,6$ & $\mathrm{~N}$-ter $+28.03 \mathrm{Da}, \mathrm{C}+57.02 \mathrm{Da}$ & 16,1 & $-0,614$ & 3 & 2276,1 & CUL4B_MOUSE & & Cullin-4B \{ECO:0000312|EMBL:CAM17145.1\}; & \\
\hline LLNLLCNHH & TLGDQIITQELR & $-10,0$ & $\mathrm{~N}$-ter $+28.03 \mathrm{Da}$ & 17,0 & 1,625 & 2 & 1413,8 & CUL9_MOUSE & S4R1Y1_MOUSE & Cullin-9; & p53-associated parkin-like cytoplasmic protein; \\
\hline MQYQQRQNQR & RFSMEDLNKR & 1,1 & $\mathrm{~N}$-ter $+28.03 \mathrm{Da}, \mathrm{K}+28.03 \mathrm{Da}$ & 17,4 & 5,319 & 3 & 1350,7 & CDK18_MOUSE & & Cyclin-dependent kinase 18; & $\begin{array}{l}\text { Cell division protein kinase 18;PCTAIRE-motif } \\
\text { protein kinase 3;Serine/threonine-protein } \\
\text { kinase PCTAIRE-3; }\end{array}$ \\
\hline
\end{tabular}




\begin{tabular}{|c|c|c|c|c|c|c|c|c|c|c|c|}
\hline Non Prime Site & Prime Site & $\begin{array}{l}\text { Fold change of } \\
\text { XPRESS ratio } \\
\text { (Adam17 } 7^{\text {AKC } / W T)} \\
\end{array}$ & Modifications & Hyperscore & $\begin{array}{l}\text { Mass error } \\
\text { in ppm }\end{array}$ & Charge & $\begin{array}{c}\text { Precursor } \\
\text { neutral mass } \\
\text { in Da }\end{array}$ & Uniprot ID 1 & Uniprot ID 2 & Protein Name 1 & Protein Name 2 \\
\hline $\begin{array}{l}\text { IGGGLTEARP } \\
\text { PGI }\end{array}$ & $\begin{array}{l}\text { ATAEVQEIADR } \\
\end{array}$ & 0,0 & N-ter +28.03 Da & 23,3 & 0,487 & 2 & 1229,6 & CYTA_MOUSE & EOCZ96_MOUSE & Cystatin-A; & Cystatin-A1;Stefin-A; \\
\hline MIP & GGLTEARPATAEVQEIADR & 0,8 & N-ter +28.03 Da & 16,0 & $-2,781$ & 3 & 2011,0 & CYTA_MOUSE & EOCZ96_MOUSE & Cystatin-A; & Cystatin-A1;Stefin-A; \\
\hline M & IPGGLTEARPATAEVQEIADR & $-0,3$ & $\mathrm{~N}$-ter +34.06 Da & 30,2 & 0,508 & 3 & 2358,2 & CYTA_MOUSE & EOCZ96_MOUSE & Cystatin-A; & Cystatin-A1;Stefin-A; \\
\hline M & IPGGLTEAR & 0,2 & $\mathrm{~N}$-ter +34.06 Da & 25,8 & $-0,463$ & 2 & 1077,6 & CYTA_MOUSE & EOCZ96_MOUSE & Cystatin-A; & Cystatin-A1;Stefin-A; \\
\hline MIPGGLTEA & RPATAEVQEIADR & 0,4 & $\mathrm{~N}$-ter +28.03 Da & 34,4 & $-1,010$ & 3 & 1482,8 & CYTA_MOUSE & EOCZ96_MOUSE & Cystatin-A; & Cystatin-A1;Stefin-A; \\
\hline PGGLTEARPA & TAEVQEIADR & 0,8 & $\mathrm{~N}$-ter +28.03 Da & 28,2 & 0,345 & 2 & 1158,6 & CYTA_MOUSE & EOCZ96_MOUSE & Cystatin-A; & Cystatin-A1;Stefin-A; \\
\hline VNIGGAGSYI & YEKPQTEAPQVTGGIEVPVVR & $-0,2$ & $\mathrm{~N}$-ter +28.03 Da, K +28.03 Da & 21,2 & $-1,002$ & 3 & 2392,3 & CRIP2_MOUSE & & Cysteine-rich protein 2; & $\begin{array}{l}\text { Heart LIM protein; } \\
\text { Complex III subunit 1;Core protein l;Ubiquinol- }\end{array}$ \\
\hline LPALRGTATF & AQALQSVPETQVIILDNGLR & 0,9 & $\mathrm{~N}$-ter +34.06 Da & 24,8 & $-3,908$ & 3 & 2172,2 & QCR1_MOUSE & & Cytochrome b-c1 complex subunit 1, mitochondrial; & $\begin{array}{l}\text { cytochrome-c reductase complex core protein } \\
1 ; \\
\text { Complex III subunit 1;Core protein l; Ubiquinol- }\end{array}$ \\
\hline ALLRLPALRG & TATFAQALQSVPETQVIILDNGLR & 0,1 & N-ter +28.03 Da & 60,8 & $-0,927$ & 3 & 2586,4 & QCR1_MOUSE & & Cytochrome b-c1 complex subunit 1, mitochondrial; & $\begin{array}{l}\text { cytochrome-c reductase complex core protein } \\
1 ;\end{array}$ \\
\hline LRLPALRGTA & TFAQALQSVPETQVSILDNGLR & 1,5 & $\mathrm{~N}$-ter $+28.03 \mathrm{Da}$ & 37,4 & 3,020 & 3 & 2414,3 & QCR1_MOUSE & & Cytochrome b-c1 complex subunit 1, mitochondrial; & $\begin{array}{l}\text { Complex III subunit 1; Core protein I; } ; \text { biquinol- } \\
\text { cytochrome-c reductase complex core protein } \\
1\end{array}$ \\
\hline NLGTSHLLRL & ASSLTTKGASSFKITR & 1,1 & N-ter +34.06 Da, K+34.06 Da & 23,8 & $-0,114$ & 4 & 1756,1 & QCR2_MOUSE & & Cytochrome b-c1 complex subunit 2, mitochondrial; & $\begin{array}{l}\text { Complex III subunit 2;Core protein II;Ubiquinol- } \\
\text { cytochrome-c reductase complex core protein } \\
2 \text {; } \\
\text { Complex III subunit 6;Complex III subunit } \\
\text { VIII;Cytochrome c1 non-heme } 11 \mathrm{kDa}\end{array}$ \\
\hline DERKMLTGSG & DPKEEEEEELVDPLTTVR & 0,0 & N-ter +28.03 Da, K+28.03 Da & 19,0 & $-3,065$ & 3 & 2183,1 & QCRG_MOUSE & & Cytochrome b-c1 complex subunit 6, mitochondrial; & $\begin{array}{l}\text { protein; Mitochondrial hinge protein;Ubiquinol- } \\
\text { cytochrome c reductase complex } 11 \mathrm{kDa} \\
\text { protein; } \\
\text { Complex III subunit 6;Complex III subunit } \\
\text { VIII;Cytochrome c1 non-heme } 11 \mathrm{kDa}\end{array}$ \\
\hline EDERKMLTGS & GDPKEEEEEELVDPLTTVR & 0,2 & $\mathrm{~N}$-ter +34.06 Da, K+34.06 Da & 48,6 & 0,576 & 3 & 2252,2 & QCRG_MOUSE & & Cytochrome b-c1 complex subunit 6, mitochondrial; & $\begin{array}{l}\text { protein; Mitochondrial hinge protein;Ubiquinol- } \\
\text { cytochrome c reductase complex } 11 \mathrm{kDa} \\
\text { protein; } \\
\text { Complex III subunit 6;Complex III subunit } \\
\text { VIII;Cytochrome c1 non-heme } 11 \mathrm{kDa}\end{array}$ \\
\hline ELCDNRVSSR & SQTEEDCTEELFDFLHAR & $-1,1$ & N-ter +28.03 Da, C +57.02 Da & 38,4 & 5,538 & 3 & 2254,0 & QCRG_MOUSE & & Cytochrome b-c1 complex subunit 6, mitochondrial; & $\begin{array}{l}\text { protein; Mitochondrial hinge protein; Ubiquinol- } \\
\text { cytochrome c reductase complex } 11 \mathrm{kDa} \\
\text { protein; } \\
\text { Complex III subunit 8;Complex III subunit } \\
\text { VIII;Ubiquinol-cytochrome c reductase complex }\end{array}$ \\
\hline M & GREFGNLAR & 0,8 & $\mathrm{~N}$-ter $+28.03 \mathrm{Da}$ & 21,7 & $-4,097$ & 3 & 1046,6 & QCR8_MOUSE & & Cytochrome b-c1 complex subunit 8; & $\begin{array}{l}9.5 \mathrm{kDa} \text { protein;Ubiquinol-cytochrome } \mathrm{C} \\
\text { reductase complex ubiquinone-binding protein } \\
\text { QP-C; } \\
\text { Complex III subunit 8;Complex III subunit } \\
\text { VIII;Ubiquinol-cytochrome c reductase complex }\end{array}$ \\
\hline MLAFYVUYLI & YTWGNQEFEQSKR & 0,4 & N-ter +34.06 Da, K+34.06 Da & 21,4 & 0,574 & 3 & 1739,9 & QCR8_MOUSE & 17HPX6_MOUSE & Cytochrome b-c1 complex subunit 8; & $\begin{array}{l}9.5 \mathrm{kDa} \text { protein;Ubiquinol-cytochrome c } \\
\text { reductase complex ubiquinone-binding protein } \\
\text { QP-C; } \\
\text { Complex III subunit 9; Complex III subunit }\end{array}$ \\
\hline LFRRTSTFAL & TIAVGALFFER & 0,7 & N-ter +28.03 Da & 24,8 & 0,399 & 2 & 1250,7 & QCR9_MOUSE & & Cytochrome b-c1 complex subunit 9; & $\begin{array}{l}\text { x;Cytochrome c1 non-heme } 7 \mathrm{kDa} \\
\text { protein;Ubiquinol-cytochrome c reductase } \\
\text { complex } 7.2 \text { kDa protein; }\end{array}$ \\
\hline MLSVAA & RSGPFAPVLSATSR & 0,2 & $\mathrm{~N}$-ter+28.03 Da & 34,3 & $-1,762$ & 3 & 1472,8 & UCRI_MOUSE & & $\begin{array}{l}\text { Cytochrome b-c1 complex subunit Rieske, } \\
\text { mitochondrial; }\end{array}$ & $\begin{array}{l}\text { Complex III subunit 5;Cytochrome b-c1 complex } \\
\text { subuniti 5;Reieke iron-sulfur protein,Ubiquinol- } \\
\text { cytochrome c reductase iron-sulfur subunit; }\end{array}$ \\
\hline GLNVPASVRF & SHTDVKVPDFSDYR & 0,1 & N-ter +34.06 Da, K +34.06 Da & 18,5 & $-0,749$ & 3 & 1732,9 & UCRI_MOUSE & & $\begin{array}{l}\text { Cytochrome b-c1 complex subunit Rieske, } \\
\text { mitochondrial; }\end{array}$ & $\begin{array}{l}\text { Complex III subunit 5;Cytochrome b-c1 complex } \\
\text { subunit 5;Rieske iron-sulfur protein;Ubiquinol- } \\
\text { cytochrome c reductase iron-sulfur subunit; }\end{array}$ \\
\hline RAISTSVCLR & AHGSVVKSEDYAFPTYADR & 0,2 & $\mathrm{~N}$-ter +34.06 Da, K +34.06 Da & 16,1 & 6,044 & 4 & 2180,1 & COX41_MOUSE & & $\begin{array}{l}\text { Cytochrome c oxidase subunit } 4 \text { isoform } 1 \text {, } \\
\text { mitochondrial; }\end{array}$ & $\begin{array}{l}\text { Cytochrome c oxidase polypeptide } \\
\text { IV;Cytochrome c oxidase subunit IV isoform 1; }\end{array}$ \\
\hline ISTSVCLRAH & GSVVKSEDYAFPTYADR & 0,1 & N-ter +28.03 Da, K+28.03 Da & 25,9 & 5,094 & 3 & 1960,0 & COX41_MOUSE & & $\begin{array}{l}\text { Cytochrome coxidase subunit } 4 \text { isoform } 1 \text {, } \\
\text { mitochondrial; }\end{array}$ & $\begin{array}{l}\text { Cytochrome c oxidase polypeptide } \\
\text { IV;Cytochrome c oxidase subunit IV isoform 1; }\end{array}$ \\
\hline
\end{tabular}




\begin{tabular}{|c|c|c|c|c|c|c|c|c|c|c|c|}
\hline Non Prime Site & Prime Site & $\begin{array}{c}\text { Fold change of } \\
\text { XPRESS ratio } \\
\text { (Adam17 } 7^{\text {AKc }} / \text { WT) } \\
\end{array}$ & Modifications & Hyperscore & $\begin{array}{l}\text { Mass error } \\
\text { in ppm }\end{array}$ & Charge & $\begin{array}{c}\text { Precursor } \\
\text { neutral mass } \\
\text { in Da } \\
\end{array}$ & Uniprot ID 1 & Uniprot ID 2 & Protein Name 1 & Protein Name 2 \\
\hline STSVCLRAHG & SWVKSEDYAFPTYADR & 2,1 & N-ter +34.06 Da, K+34.06 Da & 17,2 & $-1,147$ & 3 & 1915,0 & COX41_MOUSE & & $\begin{array}{l}\text { Cytochrome c oxidase subunit } 4 \text { isoform } 1 \text {, } \\
\text { mitochondrial; }\end{array}$ & $\begin{array}{l}\text { Cytochrome c oxidase polypeptide } \\
\text { IV;Cytochrome coxidase subunit IV isoform 1; }\end{array}$ \\
\hline QELRPTLNEL & GISTPEELGLDK & 0,3 & $\mathrm{~N}$-ter $+34.06 \mathrm{Da}, \mathrm{K}+34.06 \mathrm{Da}$ & 35,2 & $-0,491$ & 2 & 1424,8 & COX5A_MOUSE & & Cytochrome c oxidase subunit 5A, mitochondrial; & Cytochrome c oxidase polypeptide Va; \\
\hline AVCSIRCYSH & GSHETDEEFDAR & $-0,1$ & N-ter +28.03 Da & 17,7 & $-8,787$ & 3 & 1419,6 & COX5A_MOUSE & & Cytochrome c oxidase subunit 5A, mitochondrial; & Cytochrome c oxidase polypeptide Va; \\
\hline EIYPYVIQEL & RPTLNELGISTPEELGLDK & 0,4 & $\mathrm{~N}-\operatorname{ter}+34.06 \mathrm{Da}, \mathrm{K}+34.06 \mathrm{Da}$ & 21,4 & 0,267 & 3 & 2248,3 & COX5A_MOUSE & & Cytochrome c oxidase subunit 5A, mitochondrial; & Cytochrome c oxidase polypeptide Va; \\
\hline WELRKGMNTL & VGYDLVPEPRIIIDAALR & $-0,8$ & $\mathrm{~N}-\operatorname{ter}+34.06 \mathrm{Da}, \mathrm{K}+34.06 \mathrm{Da}$ & 28,4 & 1,702 & 3 & 1936,2 & COX5A_MOUSE & & Cytochrome c oxidase subunit $5 \mathrm{~A}$, mitochondrial; & Cytochrome c oxidase polypeptide Va; \\
\hline PRGAAVTRSM & ASGGGVPTDEEQATGLER & 0,0 & N-ter +28.03 Da & 48,8 & $-0,388$ & 2 & 1800,9 & COX5B_MOUSE & Q9D881_MOUSE & Cytochrome c oxidase subunit $5 \mathrm{~B}$, mitochondrial; & Cytochrome c oxidase polypeptide Vb; \\
\hline GPRGAAVTRS & MASGGGVPTDEEQATGLER & 0,1 & N-ter +28.03 Da & 44,8 & $-0,879$ & 3 & 1931,9 & COX5B_MOUSE & Q9D881_MOUSE & Cytochrome c oxidase subunit 5B, mitochondrial; & Cytochrome c oxidase polypeptide Vb; \\
\hline RGAAVTRSMA & SGGGVPTDEEQATGLER & 0,0 & N-ter+28.03 Da & 26,7 & $-2,598$ & 2 & 1729,8 & COX5B_MOUSE & Q9D881_MousE & Cytochrome c oxidase subunit 5B, mitochondrial; & Cytochrome c oxidase polypeptide vb; \\
\hline HGPRGAAVTR & SMASGGGVPTDEEQATGLER & $-4,3$ & N-ter +28.03 Da & 20,4 & $-1,830$ & 3 & 2018,9 & COX5B_MOUSE & Q9D881_MousE & Cytochrome c oxidase subunit 5B, mitochondrial; & Cytochrome c oxidase polypeptide vb; \\
\hline ALPGVGVSML & NVFLKSR & 0,8 & $\mathrm{~N}-\operatorname{ter}+28.03 \mathrm{Da}, \mathrm{K}+28.03 \mathrm{Da}$ & 21,1 & 0,217 & 3 & 918,6 & CX6A1_MOUSE & Q9DCW5_MOUSE & Cytochrome c oxidase subunit 6A1, mitochondrial; & Cytochrome c oxidase polypeptide Vla-liver; \\
\hline YFVALPGVGV & SMLNVFLKSR & 0,7 & $\mathrm{~N}$-ter $+34.06 \mathrm{Da}, \mathrm{K}+34.06 \mathrm{Da}$ & 33,6 & $-5,930$ & 3 & 1261,8 & CX6A1_MOUSE & Q9DCW5_MOUSE & Cytochrome c oxidase subunit 6A1, mitochondrial; & Cytochrome c oxidase polypeptide Vla-liver; \\
\hline WQNYLDFHRC & EKAMTAKGGDVSVCEWYR & $-1,1$ & $\begin{array}{l}\text { N-ter +34.06 Da, K+34.06 Da, C } \\
+57.02 \mathrm{Da}\end{array}$ & 16,5 & $-1,232$ & 3 & 2188,2 & CX6B1_MOUSE & & Cytochrome c oxidase subunit 6B1; & Cytochrome c oxidase subunit VIb isoform 1; \\
\hline FHRCEKAMTA & KGGDVSVCEWYR & 0,0 & $\begin{array}{l}\text { N-ter +34.06 Da, K +34.06 Da, C } \\
+57.02 \mathrm{Da}\end{array}$ & 20,7 & $-6,357$ & 3 & 1522,8 & CX6B1_MOUSE & & Cytochrome c oxidase subunit 6B1; & Cytochrome c oxidase subunit Vib isoform 1; \\
\hline M & SSGALLPKPQMR & $-0,1$ & N-ter +28.03 Da, $\mathrm{K}+28.03 \mathrm{Da}$ & 26,9 & $-0,745$ & 3 & 1339,8 & COX6C_MOUSE & D3Z6E1_MOUSE & Cytochrome c oxidase subunit $6 \mathrm{C}$; & Cytochrome c oxidase polypeptide VIC; \\
\hline ENKVPEKQKL & FQEDNGMPVHLKGGASDALLYR & 0,2 & $\mathrm{~N}$-ter $+34.06 \mathrm{Da}, \mathrm{K}+34.06 \mathrm{Da}$ & 30,7 & $-6,347$ & 4 & 2485,3 & CX7A2_MOUSE & & Cytochrome c oxidase subunit 7A2, mitochondrial; & Cytochrome c oxidase subunit VIla-liver/heart; \\
\hline AVALHSAVSA & SDLELHPPSYPWSHR & 0,2 & $\mathrm{~N}$-ter +28.03 Da & 21,4 & $-4,160$ & 3 & 1847,9 & CY1_MOUSE & & Cytochrome c1, heme protein, mitochondrial; & $\begin{array}{l}\text { Complex III subunit 4;Complex III subunit } \\
\text { IV;Cytochrome b-c1 complex subunit } \\
\text { 4;Ubiquinol-cytochrome-c reductase complex } \\
\text { cytochrome c1 subunit; }\end{array}$ \\
\hline EVEADITLAG & FHLPKGTMVLTNLTALHR & $-10,0$ & $\mathrm{~N}-\operatorname{ter}+28.03 \mathrm{Da}, \mathrm{K}+28.03 \mathrm{Da}$ & 22,8 & $-1,803$ & 4 & 2104,2 & CP2J6_MOUSE & G3UXTO_MOUSE & Cytochrome P450 2J6; & Arachidonic acid epoxygenase;CYPIIJ6; \\
\hline RLDLWNLNND & TEVPTASISVEGNPALNR & 0,6 & $\mathrm{~N}$-ter $+34.06 \mathrm{Da}$ & 19,6 & 8,725 & 3 & 1888,0 & DC112_MOUSE & Q3TPJ8_MOUSE & Cytoplasmic dynein 1 intermediate chain 2 ; & $\begin{array}{l}\text { Cytoplasmic dynein intermediate chain } \\
\text { 2. }\end{array}$ \\
\hline WTRERLHDIE & DDPILPEIPR & 10,0 & $\mathrm{~N}$-ter +34.06 Da & 15,0 & 2,667 & 2 & 1197,7 & PA24E_MOUSE & & Cytosolic phospholipase A2 epsilon; & Phospholipase A2 group IVE; \\
\hline SDDDPVLIPG & ARYRTGPGDR & $-0,2$ & N-ter +34.06 Da & 15,9 & 3,545 & 3 & 1181,7 & DCAFG_MOUSE & & DDB1- and CUL4-associated factor 6; & $\begin{array}{l}\text { IQ motif and WD repeat-containing protein } \\
\text { 1;Nuclear receptor interaction protein; }\end{array}$ \\
\hline TEPCAHLLVS & SIGVVGTAEQNR & 1,9 & N-ter +34.06 Da & 21,4 & 5,214 & 2 & 1263,7 & DOPD_MOUSE & G3UZN1_MOUSE & D-dopachrome decarboxylase; & D-dopachrome tautomerase; \\
\hline M & VAPMKGQVCVVTGASR & 0,1 & $\begin{array}{l}\text { N-ter + 34.06 Da, K +34.06 Da, C } \\
\text { +57.02 Da }\end{array}$ & 41,5 & $-0,173$ & 3 & 1727,0 & DHRS1_MOUSE & & Dehydrogenase/reductase SDR family member 1; & \\
\hline M & VTEQEEEAIGKTLVDPKQPLQAR & $-0,7$ & $\mathrm{~N}$-ter +28.03 Da, K+28.03 Da & 29,6 & 0,036 & 4 & 2777,5 & DOHH_MOUSE & DЗYZMO_MOUSE & $\begin{array}{l}\text { Deoxyhypusine hydroxylase \{ECO:0000255|HAMAP- } \\
\text { Rule:MF_03101\}; }\end{array}$ & $\begin{array}{l}\text { Deoxyhypusine dioxygenase } \\
\text { \{ECO:O000255|HAMAP- } \\
\text { Rule:MF_03101\};Deoxyhypusine } \\
\text { monooxygenase \{ECO:0000255|HAMAP- } \\
\text { Rule:MF_03101\}; }\end{array}$ \\
\hline GSDLSSLQKR & AGGADQFSKPEAR & 0,1 & $\mathrm{~N}$-ter $+28.03 \mathrm{Da}, \mathrm{K}+28.03 \mathrm{Da}$ & 45,1 & 0,072 & 3 & 1388,7 & DMKN_MOUSE & E9Q2P1_MOUSE & Dermokine; & Epidermis-specific secreted protein SK30/אK89; \\
\hline KEAASSGIQN & ALGQGHGEEGGSTLMGSR & $-0,1$ & N-ter +34.06 Da & 39,8 & 1,798 & 3 & 1776,9 & DMKN_MOUSE & E9Q2P1_MOUSE & Dermokine; & Epidermis-specific secreted protein SK30/SK89; \\
\hline GSRDIETSNF & DEGYSVSR & 1,1 & $\mathrm{~N}$-ter+28.03 Da & 14,9 & $-0,319$ & 2 & 939,4 & DMKN_MOUSE & E9Q2P1_MOUSE & Dermokine; & Epidermis-specific secreted protein SK30/SK89; \\
\hline NSGNSGSGSR & DIETSNFDEGYSVSR & 0,2 & $\mathrm{~N}$-ter+28.03 Da & 40,7 & $-1,030$ & 2 & 1745,8 & DMKN_MOUSE & E9Q2P1_MOUSE & Dermokine; & Epidermis-specific secreted protein SK30/SK89; \\
\hline SSLQKRAGGA & DQFSKPEAR & 0,1 & $\mathrm{~N}-\operatorname{ter}+28.03 \mathrm{Da}, \mathrm{K}+28.03 \mathrm{Da}$ & 33,0 & $-0,176$ & 2 & 1132,6 & DMKN_MOUSE & E9Q2P1_MOUSE & Dermokine; & Epidermis-specific secreted protein SK30/SK89; \\
\hline VGQGGNGGPL & DYETNAQGAVAQPGYGTVR & $-0,5$ & $\mathrm{~N}$-ter +34.06 Da & 19,3 & $-1,968$ & 3 & 2030,0 & DMKN_MOUSE & & Dermokine; & Epidermis-specific secreted protein SK30/SK89; \\
\hline DLSSLQKRAG & GADQFSKPEAR & 0,2 & N-ter +34.06 Da, K+34.06 Da & 36,1 & $-9,798$ & 3 & 1272,7 & DMKN_MOUSE & E9Q2P1_MOUSE & Dermokine; & Epidermis-specific secreted protein SK30/SK89; \\
\hline
\end{tabular}




\begin{tabular}{|c|c|c|c|c|c|c|c|c|c|c|c|}
\hline Non Prime Site & Prime Site & 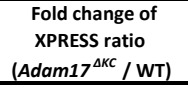 & Modifications & Hyperscore & $\begin{array}{c}\text { Mass error } \\
\text { in ppm }\end{array}$ & charge & $\begin{array}{c}\text { Precursor } \\
\text { neutral mass } \\
\text { in Da }\end{array}$ & Uniprot ID 1 & Uniprot ID 2 & Protein Name 1 & Protein Name 2 \\
\hline SDLSSLQKRA & GGADQFSKPEAR & 0,1 & N-ter +28.03 Da, K+28.03 Da & 20,2 & 0,076 & 3 & 1317,7 & DMKN_MOUSE & E9Q2P1_MOUSE & Dermokine; & Epidermis-specific secreted protein SK30/\$K89; \\
\hline GSGSRGTGSR & GGSGGSGGSGGGNKPECNNPGNDVR & 0,1 & $\begin{array}{l}\text { N-ter +28.03 Da, K+28.03 Da, C } \\
+57.02 \mathrm{Da}\end{array}$ & 66,5 & 0,000 & 3 & 2343,0 & DMKN_MOUSE & E9Q2P1_MOUSE & Dermokine; & Epidermis-specific secreted protein SK30/SK89; \\
\hline SSGIQNALGQ & GHGEEGGSTLMGSR & 0,1 & N-ter +34.06 Da & 17,0 & 1,205 & 3 & 1407,7 & DMKN_MOUSE & E9Q2P1_MOUSE & Dermokine; & Epidermis-specific secreted protein SK30/אK89; \\
\hline VAQPGYGTVR & GNNQNSGCTNPPPSGSHESFSNSGGSSNDGSR & 0,2 & N-ter +28.03 Da, C +57.02 Da & 62,4 & 0,558 & 3 & 3220,3 & DMKN_MOUSE & E9Q2P1_MOUSE & Dermokine; & Epidermis-specific secreted protein SK30/SK89; \\
\hline SGSRGTGSRG & GSGGSGGSGGGNKPECNNPGNDVR & 0,3 & $\begin{array}{l}\text { N-ter +34.06 Da, K+34.06 Da, C } \\
+57.02 \mathrm{Da}\end{array}$ & 32,5 & $-0,087$ & 3 & 2298,1 & DMKN_MOUSE & E9Q2P1_MOUSE & Dermokine; & Epidermis-specific secreted protein SK30/SK89; \\
\hline SVGQGGNGGP & LDYETNAQGAVAQPGYGTVR & $-0,4$ & $\mathrm{~N}$-ter +34.06 Da & 25,3 & 5,359 & 3 & 2143,1 & DMKN_MOUSE & & Dermokine; & Epidermis-specific secreted protein SK30/SK89; \\
\hline RGGGQGNSDN & $\begin{array}{l}\text { NGSSSSSSGSNSGNSNSGNSGNSNSGNSGNSGSGS } \\
\mathrm{R}\end{array}$ & 0,1 & $\mathrm{~N}$-ter +28.03 Da & 22,2 & 7,193 & 4 & 3221,3 & DMKN_MOUSE & E9Q2P1_MOUSE & Dermokine; & Epidermis-specific secreted protein SK30/SK89; \\
\hline RGNNQNSGCT & NPPPSGSHESFSNSGGSSNDGSR & $-0,1$ & N-ter +28.03 Da & 55,9 & 0,000 & 3 & 2288,0 & DMKN_MOUSE & E9Q2P1_MOUSE & Dermokine; & Epidermis-specific secreted protein $\mathrm{KK} 30 / 5 K 89 ;$ \\
\hline ENLKSKTRFI & NWDAINKGHAPSPSTR & 0,3 & $\mathrm{~N}$-ter +28.03 Da, $\mathrm{K}+28.03 \mathrm{Da}$ & 15,4 & 0,276 & 3 & 1805,9 & DMKN_MOUSE & E9Q2P1_MOUSE & Dermokine; & Epidermis-specific secreted protein SK30/S \\
\hline PFFNWKQIEG & SDLSSLQKR & 0,1 & $\mathrm{~N}$-ter +28.03 Da, $\mathrm{K}+28.03 \mathrm{Da}$ & 21,9 & $-1,283$ & 3 & 1088,6 & DMKN_MOUSE & E9Q2P1_MOUSE & Dermokine; & Epidermis-specific secreted protein $5 K 30 / \varsigma K 89 ;$ \\
\hline GTGSRGGSGG & SGGSGGGNKPECNNPGNDVR & 4,3 & $\begin{array}{l}\text { N-ter +34.06 Da, K+34.06 Da, C } \\
+57.02 \mathrm{Da}\end{array}$ & 29,6 & 3,084 & 3 & 2040,0 & DMKN_MOUSE & E9Q2P1_MOUSE & Dermokine; & Epidermis-specific secreted protein SK30/SK89; \\
\hline GSRGTGSRGG & SGGSGGSGGGNKPECNNPGNDVR & 0,4 & $\begin{array}{l}\text { N-ter +34.06 Da, K+34.06 Da, C } \\
+57.02 \mathrm{Da}\end{array}$ & 28,3 & $-3,877$ & 3 & 2241,0 & DMKN_MOUSE & E9Q2P1_MOUSE & Dermokine; & Epidermis-specific secreted protein SK30/SK89; \\
\hline WASGGNYGTN & SLGGSVGQGGNGGPLDYETNAQGAVAQPGYGTVR & 0,1 & N-ter +28.03 Da & 36,2 & $-0,582$ & 3 & 3261,6 & DMKN_MOUSE & & Dermokine; & Epidermis-specific secreted protein SK30/SK89; \\
\hline FEHRLGEAAR & SLGNAGNEIGR & 0,0 & $\mathrm{~N}-\operatorname{ter}+28.03 \mathrm{Da}$ & 33,7 & 1,881 & 2 & 1114,6 & DMKN_MOUSE & E9Q2P1_MOUSE & Dermokine; & Epidermis-specific secreted protein SK30/SK89; \\
\hline SGSGSRDIET & SNFDEGYSVSR & 0,2 & N-ter+34.06 Da & 18,1 & $-1,312$ & 2 & 1293,6 & DMKN_MOUSE & E9Q2P1_MOUSE & Dermokine; & Epidermis-specific secreted protein SK30/SK89; \\
\hline SGNSGNSGSG & SRDIETSNFDEGYSVSR & 0,5 & N-ter+34.06 Da & 15,4 & 4,204 & 3 & 1995,0 & DMKN_MOUSE & E9Q2P1_MOUSE & Dermokine; & Epidermis-specific secreted protein SK30/SK89; \\
\hline GNYGTNSLGG & SVGQGGNGGPLDYETNAQGAVAQPGYGTVR & 0,0 & N-ter+28.03 Da & 23,9 & 0,475 & 3 & 2947,4 & DMKN_MOUSE & & Dermokine; & Epidermis-specific secreted protein SK30/SK89; \\
\hline VRGNNQNSGC & TNPPPSGSHESFSNSGGSSNDGSR & $-0,1$ & $\mathrm{~N}$-ter $+28.03 \mathrm{Da}$ & 49,7 & 0,167 & 3 & 2389,0 & DMKN_MOUSE & E9Q2P1_MOUSE & Dermokine; & Epidermis-specific secreted protein SK30/אK89; \\
\hline NSGSGSRDIE & TSNFDEGYSVSR & $-0,1$ & N-ter +28.03 Da & 29,2 & 0,719 & 2 & 1388,6 & DMKN_MOUSE & E9Q2P1_MOUSE & Dermokine; & Epidermis-specific secreted protein SK30/SK89; \\
\hline $\begin{array}{l}\text { PAGQELLGYK } \\
\text { DGLPAGQELL }\end{array}$ & $\begin{array}{l}\text { AVDPETSSGEGLR } \\
\text { GYKAVDPETSSGEGLR }\end{array}$ & $\begin{array}{c}0,1 \\
-0,1\end{array}$ & $\begin{array}{l}\text { N-ter +28.03 Da } \\
\text { N-ter +34.06 Da, K+34.06 Da }\end{array}$ & $\begin{array}{l}29,6 \\
39,7\end{array}$ & $\begin{array}{c}-1,782 \\
0,576\end{array}$ & $\begin{array}{l}2 \\
3\end{array}$ & $\begin{array}{l}1344,7 \\
1732,9\end{array}$ & $\begin{array}{l}\text { DSC1_MOUSE } \\
\text { DSC1 MOUSE }\end{array}$ & $\begin{array}{l}\text { Q32ME9_MOUSE } \\
\text { Q32ME9 MOUSE }\end{array}$ & $\begin{array}{l}\text { Desmocollin-1; } \\
\text { Desmocollin-1; }\end{array}$ & \\
\hline VDKEPYNLFY & IEKDTGDIYCTR & 0,2 & N-ter +34.06 Da, K+34.06 Da, C & 22,3 & $-1,428$ & 3 & 1537,8 & DSC1_MOUSE & Q32ME9_MOUSE & Desmocollin-1; & \\
\hline LPAGQELLGY & KAVDPETSSGEGLR & $-2,1$ & N-ter +34.06 Da, $\mathrm{K}+34.06 \mathrm{Da}$ & 34,3 & 3,826 & 3 & 1512,9 & DSC1_MOUSE & Q32ME9_MOUSE & Desmocollin-1; & \\
\hline VGKVNLEECL & KSPSLILSSDPAFR & 0,1 & $\mathrm{~N}$-ter +28.03 Da, $\mathrm{K}+28.03 \mathrm{Da}$ & 16,3 & $-0,254$ & 3 & 1572,9 & DSC1_MOUSE & Q32ME9_MOUSE & Desmocollin-1; & \\
\hline TSSGEGLRYE & MVGDEDNWFEINKITGDLR & 0,1 & $\mathrm{~N}$-ter +34.06 Da, $\mathrm{K}+34.06 \mathrm{Da}$ & 40,0 & 1,550 & 3 & 2319,2 & DSC1_MOUSE & Q32ME9_MOUSE & Desmocollin-1; & \\
\hline SEKRGFSILL & SDGQGQEQKKLEVVLSAR & 0,8 & N-ter +34.06 Da, K +34.06 Da & 31,7 & $-5,057$ & 3 & 2073,2 & DSC1_MOUSE & Q32ME9_MOUSE & Desmocollin-1; & \\
\hline NSPYFTQTSY & TTEVEENRIDVELLR & $-0,4$ & $\mathrm{~N}$-ter $+28.03 \mathrm{Da}$ & 27,6 & $-1,138$ & 3 & 1843,0 & DSC1_MOUSE & Q32ME9_MOUSE & Desmocollin-1; & \\
\hline GVDKEPYNLF & YIEKDTGDIYCTR & $-0,3$ & $\begin{array}{l}\text { N-ter +34.06 Da, K+34.06 Da, C } \\
+57.02 \mathrm{Da}\end{array}$ & 26,5 & $-7,923$ & 3 & 1700,9 & DSC1_MOUSE & Q32ME9_MOUSE & Desmocollin-1; & \\
\hline GLPAgqeLLG & YKAVDPETSSGEGLR & 0,1 & N-ter $+34.06 \mathrm{Da}, \mathrm{K}+34.06 \mathrm{Da}$ & 31,5 & 3,395 & 3 & 1675,9 & DSC1_MOUSE & Q32ME9_MOUSE & Desmocollin-1; & \\
\hline NDNAPTFRQN & TYETAVEENTYNVEILR & 0,2 & $\mathrm{~N}$-ter +28.03 Da & 19,8 & $-6,509$ & 3 & 2071,0 & DSC3_MOUSE & G5E8S6_MOUSE & Desmocollin-3; & \\
\hline ANVIVTERVI & APGSSLPTSLTIPNPQETSNVVVTER & 0,5 & N-ter +28.03 Da & 62,0 & 3,414 & 3 & 2721,4 & DSG1A_MOUSE & & Desmoglein-1-alpha; & DG1;DG|;Desmosomal glycoprotein I; \\
\hline RNPIAKIHSD & CAANQPVTYR & 0,4 & N-ter +34.06 Da, C +57.02 Da & 13,9 & $-6,916$ & 2 & 1212,6 & DSG1A_MOUSE & DSG1B_MOUSE & Desmoglein-1-alpha; & DG1;DGl;Desmosomal glycoprotein I; \\
\hline NIPYLEQSSY & DITIEENALHSQLVQIR & $-0,3$ & N-ter +34.06 Da & 39,4 & $-1,836$ & 3 & 2012,1 & DSG1A_MOUSE & & Desmoglein-1-alpha; & DG1;DGI;Desmosomal glycoprotein I; \\
\hline TWQTVRRQKR & EWIKFAAACR & $-0,1$ & $\begin{array}{l}\text { N-ter +28.03 Da, K+28.03 Da, C } \\
+57.02 \mathrm{Da}\end{array}$ & 59,5 & 1,910 & 2 & 1306,7 & DSG1A_MOUSE & DSG1B_MOUSE & Desmoglein-1-alpha; & DG1;DG|;Desmosomal glycoprotein I; \\
\hline VTMEQYQRLN & GEYKGTVLIDDSLQR & 0,5 & N-ter +34.06 Da, K +34.06 Da & 31,1 & 6,213 & 3 & 1848,0 & DSG1A_MOUSE & DSG1B_MOUSE & Desmoglein-1-alpha; & DG1:DG|:Desmosomal glycoprotein I: \\
\hline VTERVIQPTS & GMIGNL & 0,6 & $\mathrm{~N}$-ter $+28.03 \mathrm{Da}$ & 36,8 & 2,655 & 3 & 2483,3 & DSG1A_MOUSE & & Desmoglein-1-alpha; & DG1;DG;;Desmosomal gly \\
\hline RVIQPTSGMI & GNLSMTPELSSAHNVIITER & 3,3 & N-ter +34.06 Da & 40,5 & 4,792 & 3 & 2188,2 & DSG1A_MOUSE & & Desmoglein-1-alpha; & DG1;DG|;Desmosomal gly \\
\hline SNWWVTERVV & GPISGADLHGMLEIPDLR & 0,5 & N-ter +28.03 Da & 56,3 & 0,469 & 3 & 1918,0 & DSG1A_MOUSE & & Desmoglein-1-alpha; & DG1;DG;:Desmosomal glycoprotein I; \\
\hline ISENAYHSGP & GVQHPVPIPDPLGYGNVTVR & 2,8 & N-ter +34.06 Da & 21,0 & 9,386 & 4 & 2148,2 & DSG1A_MOUSE & & Desmoglein-1-alpha; & DG1;DG|;Desmosomal gly \\
\hline VVGPVPGADL & HGMLEIPDLR & 0,1 & N-ter +34.06 Da & 18,1 & 0,822 & 3 & 1213,7 & DSG1A_MOUSE & DSG1B_MOUSE & Desmoglein-1-alpha; & DG1;DGl;Desmosomal glycoprotein I; \\
\hline PTSGMIGNIS & MTPELSSAHNVIVTER & 0,2 & N-ter +28.03 Da & 57,6 & 0,772 & 3 & 1810,9 & DSG1A_MOUSE & & Desmoglein-1-alpha; & DG1;DG|;Desmosomal glycoprotein I; \\
\hline
\end{tabular}




\begin{tabular}{|c|c|c|c|c|c|c|c|c|c|c|c|}
\hline Non Prime Site & Prime Site & $\begin{array}{l}\text { Fold change of } \\
\text { XPRESS ratio } \\
\text { (Adam17 } 7 \text { «KC } / \text { WT) }\end{array}$ & Modifications & Hyperscore & $\begin{array}{c}\text { Mass error } \\
\text { in ppm }\end{array}$ & charge & $\begin{array}{l}\text { Precursor } \\
\text { neutral mass } \\
\text { in Da }\end{array}$ & Uniprot ID 1 & Uniprot ID 2 & Protein Name 1 & Protein Name 2 \\
\hline 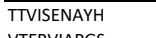 & SGPGVQHPVPIPDPLGYGNVTVR & 0,4 & N-ter+28.03 Da & 49,8 & 1,257 & 3 & 2383,3 & DSG1A_MOUSE & & Desmoglein-1-alpha; & DG1;DGl;Desmosomal glycoprotein I; \\
\hline VTERVIAPGS & SLPTSLTIPNPQETSNVVVTER & 0,5 & $\mathrm{~N}$-ter +28.03 Da & 44,9 & 0,539 & 3 & 2409,3 & DSG1A_MOUSE & & Desmoglein-1-alpha; & DG1;DGl;Desmosomal glycoprotein I; \\
\hline QPTSGMIGNL & SMTPELSSAHNVIVTER & 0,2 & $\mathrm{~N}$-ter $+34.06 \mathrm{Da}$ & 61,5 & 0,420 & 3 & 1904,0 & DSG1A_MOUSE & & Desmoglein-1-alpha; & DG1;DGl;Desmosomal glycoprotein I; \\
\hline IVTERVIAPG & SSLPTSLTIPNPQETSNVVVTER & 0,7 & $\mathrm{~N}$-ter $+34.06 \mathrm{Da}$ & 46,6 & $-0,479$ & 3 & 2502,3 & DSG1A_MOUSE & & Desmoglein-1-alpha; & DG1;DGl;Desmosomal glycoprotein I; \\
\hline LVSTTMGAAG & TGLNMGGTATIGHMR & 2,5 & $\mathrm{~N}$-ter $+34.06 \mathrm{Da}$ & 22,5 & 0,708 & 3 & 1549,8 & DSG1A_MOUSE & DSG1B_MOUSE & Desmoglein-1-alpha; & DG1;DGl;Desmosomal glycoprotein I; \\
\hline APGSSLPTSL & TIPNPQETSNVVVTER & 0,2 & $\mathrm{~N}$-ter $+34.06 \mathrm{Da}$ & 28,8 & $-5,113$ & 2 & 1817,0 & DSG1A_MOUSE & & Desmoglein-1-alpha; & DG1;DGl;Desmosomal glycoprotein I; \\
\hline GIGSSGLVST & TMGASGTGLNMGGTATIGHMR & 0,7 & $\mathrm{~N}$-ter $+28.03 \mathrm{Da}$ & 36,2 & $-1,170$ & 3 & 2048,0 & DSG1A_MOUSE & & Desmoglein-1-alpha; & DG1;DGl;Desmosomal glycoprotein I; \\
\hline TSGMIGNLSM & TPELSSAHNVIVTER & 0,3 & $\mathrm{~N}$-ter $+34.06 \mathrm{Da}$ & 39,8 & 1,007 & 3 & 1685,9 & DSG1A_MOUSE & & Desmoglein-1-alpha; & DG1;DGl;Desmosomal glycoprotein I; \\
\hline YGNITVRESY & TTSGTLKPSVHFHDNQQASNVVVTER & 10,0 & $\mathrm{~N}$-ter +34.06 Da, K +34.06 Da & 18,1 & 7,935 & 4 & 2919,6 & DSG1A_MOUSE & DSG1C_MOUSE & Desmoglein-1-alpha; & DG1;DGl;Desmosomal glycoprotein I; \\
\hline GGANVIVTER & VIAPGSSLPTSLTIPNPQETSNVVVTER & $-1,4$ & $\mathrm{~N}$-ter $+28.03 \mathrm{Da}$ & 27,7 & 0,443 & 3 & 2933,6 & DSG1A_MOUSE & & Desmoglein-1-alpha; & DG1;DGl;Desmosomal glycoprotein I; \\
\hline FELMDGVKTS & AAPEICQEYSGTLR & 0,6 & $\mathrm{~N}$-ter +34.06 Da, C +57.02 Da & 25,9 & $-2,332$ & 2 & 1627,8 & DSG1B_MOUSE & DSG1A_MOUSE & Desmoglein-1-beta; & Desmoglein-5; \\
\hline ICVPQMPPGN & ANVIEYIDNSGVYTNEYCGR & 0,6 & N-ter +34.06 Da, C +57.02 Da & 25,7 & $-1,348$ & 3 & 2370,1 & DSG1B_MOUSE & DSG1A_MOUSE & Desmoglein-1-beta; & Desmoglein-5; \\
\hline $\begin{array}{l}\text { VTERVIQPTS } \\
\text { V }\end{array}$ & GMIGNLSMTPELSSAQNVIVTER & 0,7 & $\mathrm{~N}$-ter $+28.03 \mathrm{Da}$ & 28,6 & 2,785 & 3 & 2474,3 & DSG1B_MOUSE & & Desmoglein-1-beta; & Desmoglein-5; \\
\hline PTSGMIGNLS & MTPELSSAQNVIVTER & $-0,3$ & $\mathrm{~N}-$ ter $+28.03 \mathrm{Da}$ & 17,7 & 0,277 & 3 & 1801,9 & DSG1B_MOUSE & & Desmoglein-1-beta; & Desmoglein-5; \\
\hline QPTSGMIGNL & SMTPELSSAQNVIVTER & $-0,1$ & $\mathrm{~N}-$ ter $+28.03 \mathrm{Da}$ & 16,7 & 0,740 & 3 & 1889,0 & DSG1B_MOUSE & & Desmoglein-1-beta; & Desmoglein-5; \\
\hline PLGYGNITVR & ESYTTSGTLKPSVHFHDNQQASNVVVTER & $-10,0$ & $\mathrm{~N}$-ter +28.03 Da, K +28.03 Da & 21,4 & $-3,586$ & 4 & 3286,6 & DSG1C_MOUSE & DSG1A_MOUSE & Desmoglein-1-gamma; & Desmoglein-6; \\
\hline SGVYTNEYCG & REMQDLGGGER & $-0,1$ & N-ter $+28.03 \mathrm{Da}$ & 22,4 & $-1,565$ & 3 & 1274,6 & DSG1C_MOUSE & DSG1A_MOUSE & Desmoglein-1-gamma; & Desmoglein-6; \\
\hline LQLAKRRYKR & EWVKFAKPCR & $-0,1$ & $\begin{array}{l}\text { N-ter }+28.03 \mathrm{Da}, \mathrm{K}+28.03 \mathrm{Da}, \mathrm{C} \\
+57.02 \mathrm{Da}\end{array}$ & 23,7 & 1,066 & 3 & 1403,8 & DSG3_MOUSE & Q3UFC6_MOUSE & Desmoglein-3; & 130 kDa pemphigus vulgaris antigen homolog; \\
\hline LSELLSDPSD & DTKGFEDNTEENLTYLQLKER & 1,4 & $\begin{array}{l}+57.02 \mathrm{Da} \\
\text { N-ter +34.06 Da, } \mathrm{K}+34.06 \mathrm{Da}\end{array}$ & 18,0 & 1,375 & 4 & 2759,5 & DESP_MOUSE & E9PZWO MOUSE & Desmoplakin; & \\
\hline $\begin{array}{l}\text { VRPGTALELL } \\
\text { VRP }\end{array}$ & EAQAATGFIVDPVSNLR & $\begin{array}{l}1,4 \\
1,6\end{array}$ & N-ter +34.06 Da & $\begin{array}{l}20,0 \\
29,4\end{array}$ & 1,151 & 3 & $\begin{array}{l}1821,0 \\
182,0\end{array}$ & DESP_MOUSE & E9PZWO_MOUSE & Desmoplakin; & \\
\hline IEKGHGIRLL & EAQIATGGIIDPKESHR & 0,8 & $\mathrm{~N}$-ter +34.06 Da, K+34.06 Da & 22,8 & 0,740 & 4 & $\begin{array}{l}1821,0 \\
1889,1\end{array}$ & DESP_MOUSE & E9PZWO_MOUSE & Desmoplakin; & \\
\hline SGILRPSTVN & ELESGQISYDEVGER & 1,2 & $\mathrm{~N}$-ter $+28.03 \mathrm{Da}$ & 61,4 & $-0,402$ & 2 & 1737,8 & DESP_MOUSE & E9PZWO_MOUSE & Desmoplakin; & \\
\hline NLDKCYGQIK & ELNEKITR & $-2,7$ & $\mathrm{~N}$-ter +28.03 Da, K +28.03 Da & 13,8 & $-1,038$ & 2 & 1057,6 & DESP_MOUSE & E9PZWO_MOUSE & Desmoplakin; & \\
\hline $\begin{array}{l}\text { GLFQKLENIN } \\
\text { GLF }\end{array}$ & GVSDGYLNSLCSVR & $\begin{array}{l}-2,1 \\
0,7\end{array}$ & N-ter +28.03 Da, C+57.02 Da & $\begin{array}{l}13,8 \\
37,8\end{array}$ & $\begin{array}{l}-1,008 \\
6,299\end{array}$ & 2 & $\begin{array}{l}1053,0 \\
1553,8\end{array}$ & DESP_MOUSE & E9PZWO_MOUSE & $\begin{array}{l}\text { Desmoplakin; } \\
\text { Desmoping }\end{array}$ & \\
\hline YENELAKVRN & $\begin{array}{l}\text { HYNEEMSNLR } \\
\text { HoN }\end{array}$ & 0,6 & N-ter +34.06 Da & $\begin{array}{l}30,4 \\
20,4\end{array}$ & $\begin{array}{l}0,599 \\
-5,193\end{array}$ & 3 & $\begin{array}{l}1353,6 \\
1325,6\end{array}$ & DESP_MOUSE & & Desmoplakin; & \\
\hline KSLVSWHYCM & IDIEKIR & 1,0 & $\mathrm{~N}$-ter $+28.03 \mathrm{Da}, \mathrm{K}+28.03 \mathrm{Da}$ & 19,2 & $-3,388$ & 3 & 941,6 & DESP_MOUSE & ANR17_MOUSE & Desmoplakin; & \\
\hline ARLTEEETVC & LDLDKVEAYR & 3,0 & $\mathrm{~N}-\operatorname{ter}+34.06 \mathrm{Da}, \mathrm{K}+34.06 \mathrm{Da}$ & 21,7 & 6,812 & 3 & 1288,8 & DESP_MOUSE & E9PZWO_MOUSE & Desmoplakin; & \\
\hline LVRPGTALEL & LEAQAATGFIVDPVSNLR & 1,3 & $\mathrm{~N}$-ter $+34.06 \mathrm{Da}$ & 36,0 & $-0,878$ & 3 & 1934,1 & DESP_MOUSE & E9PZWO_MOUSE & Desmoplakin; & \\
\hline LEKGHGIRL & LEAQIATGGIIDPKESHR & 0,8 & $\mathrm{~N}$-ter $+34.06 \mathrm{Da}, \mathrm{K}+34.06 \mathrm{Da}$ & 14,6 & $-0,299$ & 4 & 2002,2 & DESP_MOUSE & E9PZWO_MOUSE & Desmoplakin; & \\
\hline FIVDPVSNLR & LPVEEAYKR & $-1,6$ & $\mathrm{~N}-\operatorname{ter}+28.03 \mathrm{Da}, \mathrm{K}+28.03 \mathrm{Da}$ & 15,6 & $-1,720$ & 3 & 1159,7 & DESP_MOUSE & E9PZWO_MOUSE & Desmoplakin; & \\
\hline EYENELAKVR & NHYNEEMSNLR & $-5,0$ & $\mathrm{~N}-$ ter $+28.03 \mathrm{Da}$ & 19,4 & 2,158 & 3 & 1433,6 & DESP_MOUSE & & Desmoplakin; & \\
\hline ASFERMDHLR & QLQNIIQATSR & $-4,7$ & $\mathrm{~N}-$ ter $+28.03 \mathrm{Da}$ & 20,4 & $-4,305$ & 2 & 1298,7 & DESP_MOUSE & E9PZWO_MOUSE & Desmoplakin; & \\
\hline PIKRTMVQSP & SGVILQEAADIHAR & 2,7 & $\mathrm{~N}-$ ter $+34.06 \mathrm{Da}$ & 13,7 & 0,330 & 3 & 1512,9 & DESP_MOUSE & E9PZWO_MOUSE & Desmoplakin; & \\
\hline GAGGYTCQSG & SGWDEFTKR & 0,4 & $\mathrm{~N}$-ter $+28.03 \mathrm{Da}, \mathrm{K}+28.03 \mathrm{Da}$ & 23,7 & $-1,605$ & 3 & 1180,6 & DESP_MOUSE & E9PZWO_MOUSE & Desmoplakin; & \\
\hline ERCRRKLEDS & SRETQSQLESER & 1,0 & $\mathrm{~N}$-ter $+28.03 \mathrm{Da}$ & 17,2 & 7,096 & 3 & 1476,7 & DESP_MOUSE & E9PZWO_MOUSE & Desmoplakin; & \\
\hline AYFQFFEEAQ & STEAYLKGLLDSIR & 0,9 & $\mathrm{~N}$-ter +28.03 Da, $\mathrm{K}+28.03 \mathrm{Da}$ & 17,6 & $-3,295$ & 3 & 1635,9 & DESP_MOUSE & E9PZWO_MOUSE & Desmoplakin; & \\
\hline VTKTEITHLG & TCQDVNHNKVIETNR & 0,3 & $\begin{array}{l}\text { N-ter +28.03 Da, K+28.03 Da, C } \\
+57.02 \mathrm{Da}\end{array}$ & 25,4 & $-0,742$ & 4 & 1882,9 & DESP_MOUSE & E9PZWO_MOUSE & Desmoplakin; & \\
\hline DMLKVYEARL & TEEETVCLDLDKVEAYR & 1,1 & $\mathrm{~N}$-ter +28.03 Da, K +28.03 Da, C & 24,4 & 1,363 & 3 & 2125,0 & DESP MOUSE & E9PZWO MOUSE & Desmoplakin; & \\
\hline RPYGSHEETO & TEEEIVCLDLDKVEAYR & $\begin{array}{l}1,1 \\
0,9\end{array}$ & $\begin{array}{l}+57.02 \mathrm{Da} \\
\text { N-ter +28.03 Da, } \mathrm{K}+28.03 \mathrm{Da}\end{array}$ & $\begin{array}{l}24,4 \\
31,1\end{array}$ & $\begin{array}{l}1,363 \\
0,272\end{array}$ & 3 & $\begin{array}{l}2125,0 \\
2200,1\end{array}$ & DESP_MOUSE & E9PZWO MOUSE & $\begin{array}{l}\text { Desmoplakin; } \\
\text { Desmoplakin; }\end{array}$ & \\
\hline ALELEAQAA & TGFIVDPVSNLR & 0,7 & N-ter +28.03 Da & $\begin{array}{l}18,7 \\
18,7\end{array}$ & 0,965 & 2 & $\begin{array}{l}2204,1 \\
1344\end{array}$ & DESP_MOUSE & E9PZWO_MOUSE & Desmoplakin; & \\
\hline GQRELFFOFL & TGGLVDPEVHGR & $-0,3$ & $\mathrm{~N}$-ter +34.06 Da & 21,6 & 6,914 & 3 & 1269,7 & DESP_MOUSE & E9PZWO_MOUSE & Desmoplakin; & \\
\hline SEILSDPSDD & TKGFFDPNTEENLTYLQLKER & 0,2 & $\mathrm{~N}-\operatorname{ter}+28.03 \mathrm{Da}, \mathrm{K}+28.03 \mathrm{Da}$ & 17,7 & 2,357 & 4 & 2626,4 & DESP_MOUSE & E9PZWO_MOUSE & Desmoplakin; & \\
\hline KSLEDLKLKN & TKIEVLEEELR & 0,1 & $\mathrm{~N}$-ter +34.06 Da, $\mathrm{K}+34.06 \mathrm{Da}$ & 23,3 & $-4,5$ & 2 & 1425,9 & DESP_MOUSE & E9PZWO_MOUSE & Desmoplakin; & \\
\hline $\begin{array}{l}\text { RSQLQLSNNR } \\
\text { RS }\end{array}$ & TLELQGLINDLQR & $-10,0$ & $\mathrm{~N}$-ter +28.03 Da & 46,6 & 1,881 & 2 & 1539,9 & DESP_MOUSE & & Desmoplakin; & \\
\hline ETLLIPIIKR & $\begin{array}{l}\text { TMVQSPSGVILQEAADIHAR } \\
\text { ThE }\end{array}$ & $-0,3$ & $\mathrm{~N}$-ter $+28.03 \mathrm{Da}$ & 58,0 & 1,997 & 3 & 2150,1 & DESP_MOUSE & E9PZWO_MOUSE & Desmoplakin; & \\
\hline ELLYDWSDKN & TNIAQKQEAFSIR & $-0,1$ & N-ter +34.06 Da, K+34.06 Da & 16,3 & 7,297 & 3 & 1572,9 & DESP_MOUSE & E9PZWO_MOUSE & Desmoplakin; & \\
\hline VYEARLTEEE & TVCLDLDKVEAYR & 0,4 & $\begin{array}{l}\text { N-ter +28.03 Da, K+28.03 Da, C } \\
+57.02 \mathrm{Da}\end{array}$ & 41,2 & 1,159 & 3 & 1636,9 & DESP_MOUSE & EgPZWO_MOUSE & Desmoplakin; & \\
\hline AECSQFKAKL & VSLEELKR & 1,5 & $\begin{array}{l}+27.02 \mathrm{Da} \\
\mathrm{N} \text {-ter +34.06 Da, } \mathrm{K}+34.06 \mathrm{Da}\end{array}$ & 17,0 & $-8,432$ & 3 & 1040,7 & DESP_MOUSE & E9PZW0_MOUSE & Desmoplakin; & \\
\hline LAFVSSSALP & IHDVDSQQNTSGFLGLQR & $-0,7$ & $\mathrm{~N}$-ter +34.06 Da & 18,3 & $-1,024$ & 3 & 2048,1 & DKKL1_MOUSE & & Dickkopf-like protein 1 ; & Protein soggy-1; \\
\hline GGSKKGKSLG & QAYPCSSDKECEVGR & $-0,2$ & $\begin{array}{l}\text { N-ter +28.03 Da, K +28.03 Da, C } \\
+57.02 \text { Da }\end{array}$ & 12,2 & 1,138 & 4 & 1840,8 & DKK2_MOUSE & & Dickkopf-related protein 2; & \\
\hline LLGSPSRRSY & SLPPHQKVPLPSLSPTMQAGTIAR & 0,2 & N-ter +34.06 Da, K+34.06 Da & 39,4 & 0,693 & 4 & 2593,5 & ODP2_MOUSE & & $\begin{array}{l}\text { Dihydrolipoyllysine-residue acetyltransferase } \\
\text { component of pyruvate dehydrogenase complex, } \\
\text { mitochondrial; }\end{array}$ & $\begin{array}{l}\text { Dihydrolipoamide acetyltransferase component } \\
\text { of pyruvate dehydrogenase complex; Pyruvate } \\
\text { dehydrogenase complex component E2; }\end{array}$ \\
\hline QLLGSPSRRS & YSLPPHQKVPLPSLSPTMQAGTIAR & $-2,7$ & $\mathrm{~N}$-ter $+28.03 \mathrm{Da}, \mathrm{K}+28.03 \mathrm{Da}$ & 22,9 & $-5,021$ & 4 & 2744,5 & ODP2_MOUSE & & $\begin{array}{l}\text { Dihydrolipoyllysine-residue acetyltransferase } \\
\text { component of pyruvate dehydrogenase complex, } \\
\text { mitochondrial; }\end{array}$ & $\begin{array}{l}\text { Dihydrolipoamide acetyltransferase component } \\
\text { of pyruvate dehydrogenase complex; Pyruvate } \\
\text { dehydrogenase complex component } E 2 ;\end{array}$ \\
\hline
\end{tabular}




\begin{tabular}{|c|c|c|c|c|c|c|c|c|c|c|c|}
\hline Non Prime Site & Prime Site & $\begin{array}{c}\text { Fold change of } \\
\text { XPRESS ratio } \\
\left.\text { (Adam17 } 7^{\text {AKc }} / \mathrm{WT}\right) \\
\end{array}$ & Modifications & Hyperscore & $\begin{array}{l}\text { Mass error } \\
\text { in ppm }\end{array}$ & charge & $\begin{array}{c}\text { Precursor } \\
\text { neutral mass } \\
\text { in Da } \\
\end{array}$ & Uniprot ID 1 & Uniprot ID 2 & Protein Name 1 & Protein Name 2 \\
\hline MSAFVKASAF & ALQEQPVVNAVIDDATKEVVYR & 1,0 & N-ter +34.06 Da, K +34.06 Da & 32,9 & 0,198 & 3 & 2524,4 & ODO2_MOUSE & & $\begin{array}{l}\text { Dihydrolipoyllysine-residue succinyltransferase } \\
\text { component of 2-oxoglutarate dehydrogenase } \\
\text { complex, mitochondrial; }\end{array}$ & $\begin{array}{l}\text { 2-oxoglutarate dehydrogenase complex } \\
\text { component E2;ihydrolipoamide } \\
\text { succinyltransferase component of } 2- \\
\text { oxoglutaratate dehydrogenase complex;E2K; }\end{array}$ \\
\hline KPVSAIKPTA & APPLAEAGAAKGLR & 1,4 & N-ter +34.06 Da, K +34.06 Da & 23,8 & 7,544 & 3 & 1388,9 & ODO2_MOUSE & & $\begin{array}{l}\text { Dihydrolipoyllysine-residue succinyltransferase } \\
\text { component of 2-oxoglutarate dehydrogenase } \\
\text { complex, mitochondrial; }\end{array}$ & $\begin{array}{l}\text { 2-oxoglutarate dehydrogenase complex } \\
\text { component E2;Dihydrolipoamide } \\
\text { succinyltransferase component of } 2 \text { - } \\
\text { oxoglutarate dehydrogenase complex; E2K; }\end{array}$ \\
\hline FFQTTAVCKN & DVITVQTPAFAESVTEGDVR & 0,1 & $\mathrm{~N}$-ter+28.03 Da & 35,5 & $-1,155$ & 3 & 2161,1 & ODO2_MOUSE & & $\begin{array}{l}\text { Dihydrolipoyllysine-residue succinyltransferase } \\
\text { component of 2-oxogglutarate dehydrogenase } \\
\text { complex, mitochondrial; }\end{array}$ & $\begin{array}{l}\text { 2-oxoglutarate dehydrogenase complex } \\
\text { component E2;0ihydrolipoamide } \\
\text { succinyltransferase component of } 2 \text { - } \\
\text { oxoglutaratate dehydrogenase complex;E2K; }\end{array}$ \\
\hline RFFQTTAVCK & NDVITVQTPAFAESVTEGDVR & 0,1 & N-ter +28.03 Da & 60,3 & 0,527 & 3 & 2275,1 & ODO2_MOUSE & & $\begin{array}{l}\text { Dihydrolipoyllysine-residue succinyltransferase } \\
\text { component of 2-oxoglutarate dehydrogenase } \\
\text { complex, mitochondrial; }\end{array}$ & $\begin{array}{l}\text { 2-oxoglutarate dehydrogenase complex } \\
\text { component E2;Dihydrolipoamide } \\
\text { succinyltransferase component of } 2 \text { - } \\
\text { oxoglutarate dehydrogenase complex; E2K; }\end{array}$ \\
\hline PANGIIEALL & VPDGGKVEGGTPLFTLR & 1,4 & N-ter +34.06 Da, K+34.06 Da & 19,1 & $-2,592$ & 3 & 1810,1 & ODO2_MOUSE & & $\begin{array}{l}\text { Dihydrolipoyllysine-residue succinyltransferase } \\
\text { component of 2-oxoglutarate dehydrogenase } \\
\text { complex, mitochondrial; }\end{array}$ & $\begin{array}{l}\text { 2-oxoglutarate dehydrogenase complex } \\
\text { component E2;Dihydrolipoamide } \\
\text { succinyltransferase component of 2- } \\
\text { oxoglutarate dehydrogenase complex;E2K; }\end{array}$ \\
\hline KTVTPASSAK & TSPAKQQAPPVR & 1,0 & N-ter +34.06 Da, K+34.06 Da & 27,2 & $-0,148$ & 3 & 134,8 & DPYL2_MOUSE & & Dihydropyrimidinase-related protein 2; & Unc-33-like phosphoprotein 2; \\
\hline GAGVSGLISL & KCCVDEGLEPTCFER & 1,1 & $\begin{array}{l}\text { N-ter +34.06 Da, K +34.06 Da, C } \\
\text { +57.02 Da }\end{array}$ & 28,1 & 2,792 & 3 & 1966,9 & FMO2_MOUSE & D3YVR1_MOUSE & Dimethylaniline monooxygenase [N-oxide-forming] 2; & $\begin{array}{l}\text { Dimethylaniline oxidase 2;Pulmonary flavin- } \\
\text { containing monooxygenase } 2 \text {; }\end{array}$ \\
\hline GLVPQNKYLM & KEPVLNDDLPSR & 0,9 & $\mathrm{~N}$-ter +28.03 Da, K+28.03 Da & 14,2 & 3,540 & 3 & 1437,8 & FMO2_MOUSE & & Dimethylaniline monooxygenase [N-oxide-forming] 2; & $\begin{array}{l}\text {; imethylaniline oxidase 2;Pulmonary flavin- } \\
\text { containing monooxygenase 2; }\end{array}$ \\
\hline YFGPCNSYQY & RLVGPGQWEGAR & 0,5 & $\mathrm{~N}$-ter $+34.06 \mathrm{Da}$ & 20,9 & 3,451 & 3 & 1358,8 & FMO2_MOUSE & & Dimethylaniline monooxygenase [N-oxide-forming] 2; & $\begin{array}{l}\text { Dimethylaniline oxidase 2;Pulmonary flavin- } \\
\text { containing monooxygenase 2; }\end{array}$ \\
\hline FKYEVRGHTA & ISYCHETMTGWVHDVLGR & 0,2 & $\mathrm{~N}$-ter +34.06 Da, C +57.02 Da & 18,5 & $-0,319$ & 4 & 2194,1 & CATC_MOUSE & D3Z220_MOUSE & Dipeptidyl peptidase 1 ; & $\begin{array}{l}\text { Cathepsin C;Cathepsin J;Dipeptidyl peptidase } \\
\text { l;Dipeptidyl transferase; }\end{array}$ \\
\hline PMTDEIQQQI & LNLPESWDWR & 0,4 & $\mathrm{~N}$-ter +34.06 Da & 22,6 & $-0,518$ & 2 & 1348,7 & CATC_MOUSE & D3Z220_MOUSE & Dipeptidyl peptidase 1 ; & $\begin{array}{l}\text { Cathepsin C;Cathepsin J;Dipeptidyl peptidase } \\
\text { l;Dipeptidyl transferase; }\end{array}$ \\
\hline MTDEIQQQIL & NLPESWDWR & 0,1 & $\mathrm{~N}$-ter +34.06 Da & 11,9 & 0,646 & 2 & 1235,6 & CATC_MOUSE & D3Z220_MOUSE & Dipeptidyl peptidase 1 ; & $\begin{array}{l}\text { Cathepsin C;Cathepsin J;Dipeptidyl peptidase } \\
\text { I;Dipeptidyl transferas;; }\end{array}$ \\
\hline ILNLPESWDW & RNVQGVNYVSPVR & $-0,2$ & $\mathrm{~N}$-ter +28.03 Da & 27,9 & $-2,635$ & 3 & 1514,8 & CATC_MOUSE & D3Z220_MOUSE & Dipeptidyl peptidase 1; & $\begin{array}{l}\text { Cathepsin C;Cathepsin J;Dipeptidyl peptidase } \\
\text { l;Dipeptidyl transferase; }\end{array}$ \\
\hline KYEVRGHTAI & SYCHETMTGWVHDVLGR & $-0,2$ & $\mathrm{~N}$-ter +28.03 Da, C +57.02 Da & 28,8 & $-3,609$ & 3 & 2074,9 & CATC_MOUSE & D3Z220_MOUSE & Dipeptidyl peptidase 1; & $\begin{array}{l}\text { Cathepsin C;Cathepsin J;Dipeptidyl peptidase } \\
\text { l;Dipeptidyl transferas;; }\end{array}$ \\
\hline NADDIVATGL & AVESIKTVYR & 0,1 & N-ter +34.06 Da, $\mathrm{K}+34.06 \mathrm{Da}$ & 16,0 & $-7,040$ & 3 & 1232,8 & DIP2B_MOUSE & B2RQC7_MOUSE & Disco-interacting protein 2 homolog B; & \\
\hline VQPVITWEIA & SLPSTAVTSETLPGSLSPPVEKYR & $-0,1$ & $\mathrm{~N}$-ter +28.03 Da, K+28.03 Da & 20,3 & 7,186 & 3 & 2571,4 & DLG1_MOUSE & H7BWY4_MOUSE & Disks large homolog 1; & $\begin{array}{l}\text { Embryo-dlg/synapse-associated protein } \\
\text { 97:Synapse-associated protein 97; }\end{array}$ \\
\hline VENHEFLVKP & SFDPNLSELR & 0,9 & N-ter +28.03 Da & 23,3 & 2,155 & 2 & 1204,6 & MSH2_MOUSE & G3UWZ8_MOUSE & DNA mismatch repair protein Msh2; & Muts protein homolog 2; \\
\hline TSFHTSASLA & KDDYYQILGVPR & 0,1 & $\mathrm{~N}$-ter +34.06 Da, K+34.06 Da & 22,0 & 1,366 & 3 & 1533,9 & DNJA3_MOUSE & & DnaJ homolog subfamily A member 3, mitochondrial; & $\begin{array}{l}\text { Dnaj protein Tid-1; Tumorous imaginal discs } \\
\text { protein Tid56 homolog: }\end{array}$ \\
\hline$M$ & GKDYYQTLGLAR & 0,1 & N-ter +34.06 Da, K+34.06 Da & 24,9 & $-0,206$ & 3 & 1451,8 & DNIB1_MOUSE & & DnaJ homolog subfamily B member 1; & Heat shock 40 kDa protein 1 ; \\
\hline $\begin{array}{l}\text { M } \\
\text { GALAPTPGSA }\end{array}$ & $\begin{array}{l}\text { VDYYEVLGVQR } \\
\text { SSEAPPLVNEDVKR }\end{array}$ & $\begin{array}{l}0,2 \\
0,1\end{array}$ & $\begin{array}{l}\mathrm{N} \text {-ter }+28.03 \mathrm{Da} \\
\mathrm{N} \text {-ter +28.03 Da, K+28.03 Da }\end{array}$ & $\begin{array}{l}25,6 \\
55,8\end{array}$ & $\begin{array}{l}-2,555 \\
3,190\end{array}$ & $\begin{array}{l}2 \\
3\end{array}$ & $\begin{array}{l}1367,7 \\
1595,9\end{array}$ & $\begin{array}{l}\text { DNIBG_MOUSE } \\
\text { RPN1_MOUSE }\end{array}$ & DNJB7_MOUSE & $\begin{array}{l}\text { Dnal homolog subfamily B member } 6 \text {; } \\
\text { Dolichyl-diphosphooligosaccharide--protein } \\
\text { glycosyltransferase subunit } 1 \text {; }\end{array}$ & $\begin{array}{l}\text { Heat shock protein J2;MRJ;mDj4; } \\
\text { Dolichyl-diphosphooligosaccharide--protein } \\
\text { glycosyltransferase } 67 \text { kDa subunit;;Ribophorin } \\
\text { I:Ribophorin-1: }\end{array}$ \\
\hline ALITASVOA & LTPTHYLTKQDVER & 0,1 & N-ter +34.06 Da, K +34.06 Da & 37,0 & 7,058 & 3 & 1768,0 & RPN2_MOUSE & A2ACG7_MOUSE & $\begin{array}{l}\text { Dolichyl-diphosphooligosaccharide--protein } \\
\text { glycosyltransferase subunit 2; }\end{array}$ & $\begin{array}{l}\text {;;Ribophorin-1; } \\
\text { Dolichyl-diphoshooligosaccharide--protein } \\
\text { glycosyltransferase } 63 \text { kDa subunit;:Ribophorin } \\
\text { I;;Ribophorin-2; }\end{array}$ \\
\hline LITTASVQAL & TPTHYLTKQDVER & 0,3 & $\mathrm{~N}$-ter +28.03 Da, K+28.03 Da & 27,7 & $-0,061$ & 3 & 1642,9 & RPN2_MOUSE & A2ACG7_MOUSE & $\begin{array}{l}\text { Dolichyl-diphosphooligosaccharide--protein } \\
\text { glycosyltransferase subunit 2; }\end{array}$ & $\begin{array}{l}\text { Dolichyl-diphosphooligosaccharide--protein } \\
\text { glycosyltransferase } 63 \text { kDa subunit;Ribophorin } \\
\text { II;Ribophorin-2; }\end{array}$ \\
\hline IFKOKERAMS & TTSVTSSQPGKLR & $-0,1$ & $\mathrm{~N}$-ter +28.03 Da, $\mathrm{K}+28.03 \mathrm{Da}$ & 24,0 & $-4,085$ & 3 & 1416,8 & DBNL_MOUSE & & Drebrin-like protein; & $\begin{array}{l}\text { Actin-binding protein 1;SH3 domain-containing } \\
\text { protein 7; }\end{array}$ \\
\hline ESRRTQWKRP & SPDDDLTDEDNDDMQLQAQR & $-4,3$ & $\mathrm{~N}$-ter +28.03 Da & 11,8 & $-0,213$ & 4 & 2348,0 & NEDD4_MOUSE & & E3 ubiquitin-protein ligase NEDD4; & $\begin{array}{l}\text { Neural precursor cell expressed } \\
\text { developmentally down-resulated protein 4; }\end{array}$ \\
\hline RAGESSDDVT & NSDSIIDWLNSVR & $-10,0$ & $\mathrm{~N}$-ter +28.03 Da & 19,3 & 4,006 & 2 & 1545,8 & RNF12_MOUSE & & E3 ubiquitin-protein ligase RLIM; & $\begin{array}{l}\text { LIM domain-interacting RING finger } \\
\text { protein;:RING finger LM domain-binding } \\
\text { protein;;IING finger protein 12; }\end{array}$ \\
\hline
\end{tabular}




\begin{tabular}{|c|c|c|c|c|c|c|c|c|c|c|c|}
\hline Non Prime Site & Prime Site & 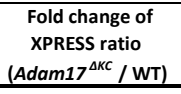 & Modifications & Hyperscore & $\begin{array}{l}\text { Mass error } \\
\text { in ppm }\end{array}$ & Charge & $\begin{array}{c}\text { Precursor } \\
\text { neutral mass } \\
\text { in Da } \\
\end{array}$ & Uniprot ID 1 & Uniprot ID 2 & Protein Name 1 & Protein Name 2 \\
\hline LPEHVQLLQ & VDSEDNESQGQVEGR & 4,0 & N-ter +34.06 Da & 18,2 & 0,475 & 3 & 1681,8 & RN123_MOUSE & Q05CH9_MOUSE & E3 ubiquitin-protein ligase RNF123; & $\begin{array}{l}\text { Kip1 ubiquitination-promoting complex protein } \\
\text { 1; IING finger protein 123; }\end{array}$ \\
\hline TRWADLSKNF & IETDNEGNGILR & $-3,8$ & $\mathrm{~N}$-ter $+28.03 \mathrm{Da}$ & 16,1 & 0,883 & 2 & 1357,7 & EFCB6_MOUSE & & $\begin{array}{l}\text { EF-hand calcium-binding domain-containing protein } \\
6 \text {; }\end{array}$ & DJ-1-binding protein; \\
\hline FSRRLLKDLE & KMFKTYDAGR & $-4,5$ & $\mathrm{~N}$-ter +28.03 Da, K+28.03 Da & 17,2 & 2,456 & 3 & 1299,7 & EFHD1_MOUSE & & EF-hand domain-containing protein D1; & $\begin{array}{l}\text { EF-hand domain-containing protein 1;Swiprosin- } \\
2 \text {; }\end{array}$ \\
\hline LRRAASLLRF & QSTLVIAEHANDSLAPITLNTITAAGR & 0,2 & $\mathrm{~N}$-ter +28.03 Da & 34,0 & $-1,923$ & 3 & 2804,5 & ETFA_MOUSE & & $\begin{array}{l}\text { Electron transfer flavoprotein subunit alpha, } \\
\text { mitochondrial; }\end{array}$ & \\
\hline KCLPLCAPRC & SSTSAVPQITTHYTVHPR & 0,2 & $\mathrm{~N}$-ter +34.06 Da & 45,8 & $-0,248$ & 4 & 2015,1 & ETFD_MOUSE & & $\begin{array}{l}\text { Electron transfer flavoprotein-ubiquinone } \\
\text { oxidoreductase, mitochondrial; }\end{array}$ & $\begin{array}{l}\text { Electron-transferring-flavoprotein } \\
\text { dehydrogenase; }\end{array}$ \\
\hline DGPKFLKSGD & AAIVDMVPGKPMCVESFSDYPPLGR & $-0,4$ & $\begin{array}{l}\text { N-ter +34.06 Da, K+34.06 Da, C } \\
\text { +57.02 Da }\end{array}$ & 31,1 & $-0,036$ & 3 & 2803,4 & EF1A1_MOUSE & & Elongation factor 1-alpha 1; & $\begin{array}{l}\text { Elongation factor Tu; Eukaryotic elongation } \\
\text { factor } 1 \mathrm{~A}-1 \text {; }\end{array}$ \\
\hline SGTTLLEALD & CILPPTRPTDKPLR & $-0,8$ & $\begin{array}{l}\text { N-ter +28.03 Da, K+28.03 Da, C } \\
+57.02 \mathrm{Da}\end{array}$ & 15,1 & $-9,170$ & 4 & 1719,0 & EF1A1_MOUSE & D3YZ68_MOUSE & Elongation factor 1-alpha 1; & $\begin{array}{l}\text { Elongation factor Tu; Eukaryotic elongation } \\
\text { factor } 1 \mathrm{~A}-1 \text {; }\end{array}$ \\
\hline KQLIVGVNKM & DSTEPPYSQKR & $-1,0$ & $\mathrm{~N}$-ter $+28.03 \mathrm{Da}, \mathrm{K}+28.03 \mathrm{Da}$ & 23,4 & $-0,366$ & 3 & 1362,7 & EF1A1_MOUSE & D3YZ68_MOUSE & Elongation factor 1-alpha 1; & $\begin{array}{l}\text { Elongation factor Tu; Eukaryotic elongation } \\
\text { factor } 1 \mathrm{~A}-1 ;\end{array}$ \\
\hline VAFVPISGWN & GDNMLEPSANMPWFKGWKVTR & $-0,1$ & $\mathrm{~N}$-ter $+28.03 \mathrm{Da}, \mathrm{K}+28.03 \mathrm{Da}$ & 27,1 & 5,566 & 4 & 2547,3 & EF1A1_MOUSE & D3YZ68_MOUSE & Elongation factor 1-alpha 1; & $\begin{array}{l}\text { Elongation factor Tu; Eukaryotic elongation } \\
\text { factor } 1 \mathrm{~A}-1 ;\end{array}$ \\
\hline DRRSGKKLED & GPKFLKSGDAAIVDMVPGKPMCVESFSDYPPLGR & $-0,2$ & $\begin{array}{l}\text { N-ter +28.03 Da, K +28.03 Da, C } \\
+57.02 \mathrm{Da}\end{array}$ & 33,2 & 2,909 & 5 & 3777,0 & EF1A1_MOUSE & & Elongation factor 1-alpha 1; & $\begin{array}{l}\text { Elongation factor Tu; Eukaryotic elongation } \\
\text { factor } 1 \mathrm{~A}-1 ;\end{array}$ \\
\hline FKGWKVTRKD & GSASGTTLLEALDCILPPTRPTDKPLR & 0,2 & $\begin{array}{l}\text { N-ter }+34.06 \mathrm{Da}, \mathrm{K}+34.06 \mathrm{Da}, \mathrm{C} \\
+57.02 \mathrm{Da}\end{array}$ & 23,3 & 0,678 & 4 & 2946,7 & EF1A1_MOUSE & D3YZ68_MOUSE & Elongation factor 1-alpha 1; & $\begin{array}{l}\text { Elongation factor Tu; Eukaryotic elongation } \\
\text { factor } 1 \mathrm{~A}-1 \text {; }\end{array}$ \\
\hline PLRLPLQDVY & KIGGIGTVPVGR & 0,4 & $\mathrm{~N}$-ter $+28.03 \mathrm{Da}, \mathrm{K}+28.03 \mathrm{Da}$ & 20,1 & $-4,951$ & 3 & 1208,8 & EF1A1_MOUSE & EF1A2_MOUSE & Elongation factor 1-alpha 1; & $\begin{array}{l}\text { Elongation factor Tu; Eukaryotic elongation } \\
\text { factor } 1 \mathrm{~A}-1 ;\end{array}$ \\
\hline GVKRLIVGVN & KMDSTEPPYSQKR & $-0,8$ & $\mathrm{~N}$-ter $+28.03 \mathrm{Da}, \mathrm{K}+28.03 \mathrm{Da}$ & 19,7 & $-0,242$ & 4 & 1649,8 & EFIA1_MOUSE & D3YZ68_MOUSE & Elongation factor 1-alpha 1; & $\begin{array}{l}\text { Elongation factor Tu; Eukaryotic elongation } \\
\text { factor } 1 \mathrm{~A}-1 ;\end{array}$ \\
\hline RPTDKPLRLP & LQDVYKIGGIGTVPVGR & 1,9 & $\mathrm{~N}$-ter $+34.06 \mathrm{Da}, \mathrm{K}+34.06 \mathrm{Da}$ & 20,7 & 1,249 & 3 & 1839,1 & EF1A1_MOUSE & EF1A2_MOUSE & Elongation factor 1-alpha 1; & $\begin{array}{l}\text { Elongation factor Tu; Eukaryotic elongation } \\
\text { factor } 1 \mathrm{~A}-1 ;\end{array}$ \\
\hline LKSGDAAIVD & MVPGKPMCVESFSDYPPLGR & 0,0 & $\begin{array}{l}\text { N-ter +34.06 Da, K+34.06 Da, C } \\
+57.02 \text { Da }\end{array}$ & 25,0 & $-0,043$ & 3 & 2334,2 & EF1A1_MOUSE & & Elongation factor 1-alpha 1; & $\begin{array}{l}\text { Elongation factor Tu; Eukaryotic elongation } \\
\text { factor } 1 \mathrm{~A} 1 \mathrm{1} \text {; }\end{array}$ \\
\hline KGWKVTRKDG & SASGTTLLEALDCILPPTRPTDKPLR & 0,0 & $\begin{array}{l}\text { N-ter +34.06 Da, K +34.06 Da, C } \\
+57.02 \mathrm{Da}\end{array}$ & 19,7 & 0,864 & 4 & 2889,6 & EF1A1_MOUSE & D3YZ68_MOUSE & Elongation factor 1-alpha 1; & $\begin{array}{l}\text { Elongation factor Tu; Eukaryotic elongation } \\
\text { factor } 1 \mathrm{~A}-1 \text {; }\end{array}$ \\
\hline PGKPMCVESF & SDYPPLGR & 0,0 & N-ter +28.03 Da & 17,1 & $-0,321$ & 2 & 931,5 & EF1A1_MOUSE & & Elongation factor 1-alpha 1; & $\begin{array}{l}\text { Elongation factor Tu; Eukaryotic elongation } \\
\text { factor } 1 \mathrm{~A}-1 \text {; }\end{array}$ \\
\hline IDISLWKFET & SKYYVTIIDAPGHR & $-0,1$ & $\mathrm{~N}$-ter +34.06 Da, K +34.06 Da & 14,2 & $-1,478$ & 4 & 1687,0 & EF1A1_MOUSE & D3YZ68_MOUSE & Elongation factor 1-alpha 1; & $\begin{array}{l}\text { Elongation factor Tu; Eukaryotic elongation } \\
\text { factor } 1 \mathrm{~A}-1 \text {; }\end{array}$ \\
\hline VTRKDGSASG & TTLEALDCILPPTRPTDKPLR & 0,1 & $\begin{array}{l}\text { N-ter }+34.06 \mathrm{Da}, \mathrm{K}+34.06 \mathrm{Da}, \mathrm{C} \\
+57.02 \mathrm{Da}\end{array}$ & 40,1 & $-5,325$ & 4 & 2587,5 & EF1A1_MOUSE & D3YZ68_MOUSE & Elongation factor 1-alpha 1; & $\begin{array}{l}\text { Elongation factor Tu; Eukaryotic elongation } \\
\text { factor } 1 \mathrm{~A}-1 \text {; }\end{array}$ \\
\hline DDDIDLFGSD & DEEESEEAKKLR & $-0,9$ & $\mathrm{~N}$-ter $+28.03 \mathrm{Da}, \mathrm{K}+28.03 \mathrm{Da}$ & 21,6 & $-0,517$ & 3 & 1545,8 & EF1B_MOUSE & & Elongation factor 1-beta; & \\
\hline $\begin{array}{l}\text { QADVAVFEAV } \\
\text { VGDMIEFOOA }\end{array}$ & 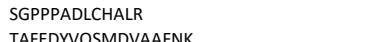 & $-0,3$ & $\mathrm{~N}$-ter +28.03 Da, C +57.02 Da & $\begin{array}{l}58,4 \\
19,1\end{array}$ & -0,634 & 3 & 1417,7 & $\begin{array}{l}\text { EF1B_MOUSE } \\
\text { EFB MOUSE }\end{array}$ & G3UX43_MOUSE & $\begin{array}{l}\text { Elongation factor 1-beta; } \\
\text { Elpogation fator }\end{array}$ & \\
\hline $\begin{array}{l}\text { VGTDMLEEQI } \\
\text { SQADVAVFEA }\end{array}$ & $\begin{array}{l}\text { TAFEDYVQSMDVAAFNK } \\
\text { VSGPPPADLCHALR }\end{array}$ & $\begin{array}{c}6,8 \\
-0,4\end{array}$ & $\begin{array}{l}\text { N-ter +28.03 Da, K+28.03 Da } \\
\text { N-ter +28.03 Da, C +57.02 Da }\end{array}$ & $\begin{array}{l}19,1 \\
45,6\end{array}$ & $\begin{array}{l}4,319 \\
0,790\end{array}$ & $\begin{array}{l}3 \\
3\end{array}$ & $\begin{array}{l}2104,0 \\
1516,8\end{array}$ & $\begin{array}{l}\text { EF1B_MOUSE } \\
\text { EF1B MOUSE }\end{array}$ & G3UX43 MOUSE & $\begin{array}{l}\text { Elongation factor 1-beta; } \\
\text { Elongation factor 1-beta; }\end{array}$ & \\
\hline & $\begin{array}{l}\text { DETDMAQLETCVR } \\
\text { DETPA }\end{array}$ & $-0,2$ & $\begin{array}{l}\text { N-ter +28.03 Da, C +57.02 Da } \\
\text { N-ter +28.03 Da, C +57.02 Da }\end{array}$ & 23,0 & $-7,077$ & 2 & $\begin{array}{l}1594,7 \\
159,7\end{array}$ & EF1D_MOUSE & Q91VK2_MOUSE & $\begin{array}{l}\text { Elongagition factoro 1-beta; } \\
\text { Elongation factor 1-delta; }\end{array}$ & \\
\hline IQLDGLVWGA & $\begin{array}{l}\text { SKLVPVGYGIR } \\
\text { SKI }\end{array}$ & -0,1 & $\mathrm{N}$-ter +34.06 Da, K +34.06 Da & 19,4 & 7,070 & 3 & 1255,8 & EF1D_MOUSE & Q91VK2_MOUSE & Elongation factor 1-delta; & \\
\hline TENAKEEVKR & ILGLLDTHLKTR & $-10,0$ & N-ter +28.03 Da, K+28.03 Da & 13,8 & $-0,973$ & 4 & 1434,9 & EF1G_MOUSE & & Elongation factor 1-gamma; & eEF-1B gamma; \\
\hline KDSVVAGFQW & ATKEGALCEENMR & 0,3 & $\begin{array}{l}\text { N-ter }+28.03 \mathrm{Da}, \mathrm{K}+28.03 \mathrm{Da}, \mathrm{C} \\
+5502 \mathrm{Da}\end{array}$ & 27,2 & $-2,681$ & 3 & 1563,7 & EF2_MOUSE & & Elongation factor 2; & \\
\hline DGSGFLINLI & DSPGHVDFSSEVTAAL & 0,9 & $\mathrm{~N}$-ter +34.06 Da & 38,0 & 3,344 & 3 & 1820,9 & EF2_MOUSE & & Elongation factor 2; & \\
\hline KDGSGFLINL & IDSPGHVD & 1,1 & $\mathrm{~N}$-ter +28.03 Da & 43,4 & $-3,2$ & 3 & 1928,0 & EF2_MOUSE & & & \\
\hline RLMEPIYLVE & IQCPEQVVGGIYGVLNR & 0,2 & $\mathrm{~N}-\operatorname{ter}+34.06 \mathrm{Da}, \mathrm{C}+57.02 \mathrm{Da}$ & 25,3 & 0,4 & 3 & 1935,1 & EF2_MOUSE & & & \\
\hline & NFTVDC & 0,0 & $\mathrm{~N}$-ter $+34.06 \mathrm{Da}$ & 21,6 & 0,7 & 2 & 1025,6 & EF2_MOUSE & & & \\
\hline LMEPIYLVEI & QCPEQ & $-0,3$ & $\mathrm{~N}$-ter $+28.03 \mathrm{Da}, \mathrm{C}+57.02 \mathrm{Da}$ & 16,4 & 4,1 & 3 & 1815,9 & EF2_MOUSE & & tor 2; & \\
\hline & IVAETR & 1,1 & $\mathrm{~N}$-ter +28.03 Da & 17,3 & 1,2 & 3 & 2440,2 & EF2_MOUSE & & i 2; & \\
\hline & & $-0,5$ & N-ter & 23,4 & & 3 & 1699,9 & EF2_MOUSE & & & \\
\hline QPRLMEPIYL & VEILCPEQVVGGIYGVLNR & 0,4 & $\mathrm{~N}$-ter $+28.03 \mathrm{Da}, \mathrm{C}+57.02 \mathrm{Da}$ & 64,9 & 0,232 & 3 & 2157,1 & EF2_MOUSE & & Elongation factor $2 ;$ & \\
\hline M & VLSGVDKMIR & 0,0 & $\mathrm{~N}$-ter +34.06 Da, K+34.06 Da & 24,1 & 0,758 & 3 & 1184,8 & ETUD1_MOUSE & D3Z1V3_MOUSE & $\begin{array}{l}\text { Elongation factor Tu GTP-binding domain-containing } \\
\text { protein } 1 \text { : }\end{array}$ & \\
\hline & VSSVLE & 0,6 & N-ter +28 & 21 & -3, & 3 & 1916,9 & ELP2_MOUSE & & Elongator complex protein 2 ; & STAT3-interacting protein 1; \\
\hline LLALAGLLQA & RLLPQQAGFGECDR & 0,0 & N-ter +28.03 Da, C +57.02 Da & 33,4 & 0,335 & 3 & 1786,9 & ENDD1_MOUSE & & Endonuclease domain-containing 1 protein; & \\
\hline
\end{tabular}




\begin{tabular}{|c|c|c|c|c|c|c|c|c|c|c|c|}
\hline Non Prime Site & Prime Site & 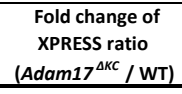 & Modifications & Hyperscore & $\begin{array}{l}\text { Mass error } \\
\text { in ppm }\end{array}$ & Charge & $\begin{array}{c}\text { Precursor } \\
\text { neutral mass } \\
\text { in Da } \\
\end{array}$ & Uniprot ID 1 & Uniprot ID 2 & Protein Name 1 & Protein Name 2 \\
\hline VLLTFGFVRA & DDEVDVDGTVEEDLGKSR & $-0,1$ & $\mathrm{~N}$-ter +34.06 Da, K+34.06 Da & 81,9 & 5,127 & 3 & 2045,0 & ENPL_MOUSE & & Endoplasmin; & $\begin{array}{l}94 \text { kDa glucose-regulated protein;Endoplasmic } \\
\text { reticulum resident protein 99; Heat shock } \\
\text { protein } 90 \text { kDa beta member 1;Polymorphic } \\
\text { tumor rejection antigen 1;Tumor rejection } \\
\text { antigen gp96; }\end{array}$ \\
\hline LTFGFVRADD & EVDVDGTVEEDLGKSR & 0,0 & N-ter +28.03 Da, K+28.03 Da & 18,9 & $-1,108$ & 3 & 1802,9 & ENPL_MOUSE & & Endoplasmin; & $\begin{array}{l}94 \text { kDa glucose-regulated protein;Endoplasmic } \\
\text { reticulum resident protein } 99 ; \text { Heat shock } \\
\text { protein } 90 \text { kDa beta member 1;Polymorphic } \\
\text { tumor rejection antigen 1;Tumor rejection } \\
\text { antigen gp96; }\end{array}$ \\
\hline WESSVVSRLL & TPTHSFLAR & 0,3 & N-ter +28.03 Da & 17,0 & $-1,793$ & 3 & 1056,6 & MAP7_MOUSE & D3YWN7_MOUSE & Ensconsin; & $\begin{array}{l}\text { Epithelial microtubule-associated protein of } \\
115 \text { kDa;Microtubule-associated protein 7; }\end{array}$ \\
\hline YELQADTYRC & SLEPALAVSAPKR & 1,8 & N-ter +34.06 Da, K+34.06 Da & 15,1 & $-3,194$ & 3 & 1405,9 & EVPL_MOUSE & & Envoplakin; & $\begin{array}{l}210 \mathrm{kDa} \text { cornified envelope precursor } \\
\text { protein;p210; }\end{array}$ \\
\hline QKPKVQLQER & VSEIFQVLPETEQEIR & $-3,2$ & $\mathrm{~N}$-ter +28.03 Da & 22,0 & 8,988 & 3 & 1944,0 & EVPL_MOUSE & & Envoplakin; & $\begin{array}{l}210 \mathrm{kDa} \text { cornified envelope precursor } \\
\text { protein;p210; }\end{array}$ \\
\hline LTTYYNEGH & CVLEKVAATQVDGSAKYKVTR & 5,8 & $\begin{array}{l}\text { N-ter }+34.06 \mathrm{Da}, \mathrm{K}+34.06 \mathrm{Da}, \mathrm{C} \\
+57.02 \mathrm{Da}\end{array}$ & 15,1 & 0,609 & 4 & 2458,5 & LCN5_MOUSE & & Epididymal-specific lipocalin-5; & $\begin{array}{l}\text { Epididyyal retinoic acid-binding } \\
\text { protein;:Epididymal secretory protein 10; }\end{array}$ \\
\hline DASLKQQDTG & ARGSGTSPDEGDAQDSSESAR & 0,7 & $\mathrm{~N}$-ter +34.06 Da & 44,2 & 0,473 & 3 & 2112,9 & EPIPL_MOUSE & EPIPL_MOUSE & Epiplakin; & \\
\hline VPKIKKNQLG & ASTPSRPQAEAALPTTAR & 0,1 & $\mathrm{~N}$-ter +34.06 Da & 30,3 & 1,182 & 3 & 1858,0 & EPIPL_MOUSE & & Epiplakin; & \\
\hline VYAKLLSAEH & AVTGYTDPYSGEQISLFQAMQR & $-1,7$ & $\mathrm{~N}$-ter $+28.03 \mathrm{Da}$ & 40,6 & 3,250 & 3 & 2489,2 & EPIPL_MOUSE & & Epiplakin; & \\
\hline TYMKLLSAER & AVTGYTDPYTGEQISLFRAMQR & $-3,5$ & $\mathrm{~N}$-ter $+28.03 \mathrm{Da}$ & 21,4 & 0,998 & 3 & 2503,2 & EPIPL_MOUSE & & Epiplakin; & \\
\hline ETQESKPKPR & DASLKQQDTGAR & $-1,1$ & $\mathrm{~N}$-ter +28.03 Da, K+28.03 Da & 24,2 & $-1,410$ & 3 & 1344,7 & EPIPL_MOUSE & EPIPL_MOUSE & Epiplakin; & \\
\hline IVKNHGIRLL & EAQIATGGVIDPVHSHR & 1,4 & N-ter +34.06 Da & 22,6 & 6,857 & 3 & 1820,0 & EPIPL_MOUSE & EPIPL_MOUSE & Epiplakin; & \\
\hline PVNNRRLSVE & EAVAAGVVGGEIQEKLLSAER & 3,5 & $\mathrm{~N}$-ter +34.06 Da, $\mathrm{K}+34.06 \mathrm{Da}$ & 20,9 & $-7,513$ & 3 & 2193,3 & EPIPL_MOUSE & EPIPL_MOUSE & Epiplakin; & \\
\hline ELAQGTKTIH & EVTEMDSVKR & 1,2 & $\mathrm{~N}$-ter +28.03 Da, $\mathrm{K}+28.03 \mathrm{Da}$ & 36,5 & $-0,240$ & 3 & 1248,6 & EPIPL_MOUSE & EPIPL_MOUSE & Epiplakin; & \\
\hline EDMNSILADP & GDDTKGFFDPNTHENLTYLQLLR & 2,3 & $\mathrm{~N}$-ter +34.06 Da, $\mathrm{K}+34.06 \mathrm{Da}$ & 19,0 & 5,858 & 4 & 2761,5 & EPIPL_MOUSE & EPIPL_MOUSE & Epiplakin; & \\
\hline MEVKRGHLRG & HEVPVWDILTSNYVSR & $-5,0$ & $\mathrm{~N}$-ter +28.03 Da & 16,1 & $-2,931$ & 3 & 1942,0 & EPIPL_MOUSE & 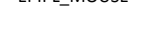 & Epiplakin; & \\
\hline VLRPGTALVL & LEAQAATGFIIDPVNNR & 0,2 & $\mathrm{~N}$-ter +34.06 Da & 42,7 & 3,164 & 3 & 1862,0 & EPIPL_MOUSE & EPIPL_MOUSE & Epiplakin; & \\
\hline DIVKNHGIR & LLEAQIATGGVIDPVHSHR & $-3,0$ & $\mathrm{~N}$-ter +28.03 Da & 42,6 & 9,393 & 4 & 2040,1 & EPIPL_MOUSE & EPIPL_MOUSE & Epiplakin; & \\
\hline HEVTEMDSVK & $\begin{array}{l}\text { RYLGSSCIAGVLVPVQGEPGR } \\
\text { RYST }\end{array}$ & 0,9 & $\mathrm{~N}$-ter +34.06 Da, C+57.02 Da & 16,0 & $-0,477$ & 3 & 2305,2 & EPIPL_MOUSE & EPIPL_MOUSE & Epiplakin; & \\
\hline TLPMLKRRLT & TIIEEAEETQESKPKPR & 0,7 & $\mathrm{~N}$-ter +34.06 Da, K +34.06 Da & 27,8 & 4,689 & 4 & 2086,2 & EPIPL_MOUSE & EPIPL_MOUSE & Epiplakin; & \\
\hline NSILADPGDD & $\begin{array}{l}\text { TKGFFDPNTHENLTYLQLLR } \\
\text { ThIT }\end{array}$ & 0,3 & $\mathrm{~N}$-ter +28.03 Da, K+28.03 Da & 22,5 & $-2,149$ & 4 & 2462,3 & EPIPL_MOUSE & EPIPL_MOUSE & Epiplakin; & \\
\hline QQDTGARGSG & TSPDEGDAQDSSESAR & 0,8 & $\mathrm{~N}$-ter $+34.06 \mathrm{Da}$ & 34,2 & 0,296 & 2 & 1684,7 & EPIPL_MOUSE & EPIPL_MOUSE & Epiplakin; & \\
\hline QSISVWELLF & YREVPESLR & 0,7 & $\mathrm{~N}$-ter $+34.06 \mathrm{Da}$ & 19,7 & $-0,844$ & 3 & 1181,7 & EPIPL_MOUSE & & Epiplakin; & \\
\hline LLLSGDAHS & SEVPGAAAEGPGGSGVGLGDR & 0,7 & $\mathrm{~N}$-ter +34.06 Da & 24,3 & $-0,640$ & 3 & 1872,9 & EMC7_MOUSE & & ER membrane protein complex subunit 7 ; & \\
\hline FSPTGSPLSS & AAYSSPKFHGSLPLAPCEAR & 10,0 & $\begin{array}{l}\text { N-ter +34.06 Da, K+34.06 Da, C } \\
+57.02 \mathrm{Da}\end{array}$ & 23,6 & 8,295 & 4 & 2226,2 & GATA1_MOUSE & & Erythroid transcription factor; & $\begin{array}{l}\text { Ervf1;GATA-binding factor 1;NF-E1 DNA-binding } \\
\text { protein; }\end{array}$ \\
\hline M & AGGEGGVTLGQPHLSR & $-0,2$ & N-ter $+34.06 \mathrm{Da}$ & 52,3 & 6,044 & 3 & 1568,9 & IF2G_MOUSE & A2AAW9_MOUSE & $\begin{array}{l}\text { Eukaryotic translation initiation factor } 2 \text { subunit } 3, \mathrm{X} \text { - } \\
\text { linked; }\end{array}$ & $\begin{array}{l}\text { Eukaryotic translation initiation factor } 2 \text { subunit } \\
\text { gamma, } X \text {-linked; }\end{array}$ \\
\hline VGQTEDAEED & AEAGPEPEVR & 0,6 & N-ter +28.03 Da & 21,5 & 1,015 & 2 & 1081,5 & ElF3B_MOUSE & & $\begin{array}{l}\text { Eukaryotic translation initiation factor } 3 \text { subunit B } \\
\text { \{ECO:0000255|HAMAP-Rule:MF_03001\}; }\end{array}$ & 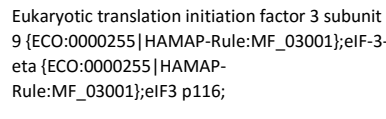 \\
\hline MQD & AENVAVPEAAEER & $-0,9$ & N-ter+28.03 Da & 17,3 & $-6,579$ & 2 & 1411,7 & EIF3B_MOUSE & & $\begin{array}{l}\text { Eukaryotic translation initiation factor } 3 \text { subunit B } \\
\text { \{ECO:0000255|HAMAP-Rule:MF_03001\}; }\end{array}$ & $\begin{array}{l}\text { Eukaryotic translation initiation factor } 3 \text { subunit } \\
9\{\text { \{CO:0000255|HAMAP-Rule:MF_03001\}; } 1 \text {;F-3. } \\
\text { eta \{ECO:0000255|HAMAP- } \\
\text { Rule:MF_03001\};:IF3 p116; }\end{array}$ \\
\hline MQDAENV & AVPEAAEER & $-0,2$ & N-ter +34.06 Da & 14,1 & 1,888 & 2 & 1004,5 & EIF3B_MOUSE & & $\begin{array}{l}\text { Eukaryotic translation initiation factor } 3 \text { subunit B } \\
\text { \{ECO:0000255|HAMAP-Rule:MF_03001\}; }\end{array}$ & $\begin{array}{l}\text { Eukaryotic translation initiation factor } 3 \text { subunit } \\
9\{\text { \{CO:0000255|HAMAP-Rule:MF_03001\}; elF-3. } \\
\text { eta \{ECO:0000255|HAMAP- } \\
\text { Rule:MF_03001\};:IF3 p116; }\end{array}$ \\
\hline EVRAKPAAQS & EEETATSPAASPTPQSAER & 0,6 & $\mathrm{~N}$-ter $+28.03 \mathrm{Da}$ & 23,5 & 0,000 & 3 & 1985,9 & EIF3B_MOUSE & & $\begin{array}{l}\text { Eukaryotic translation initiation factor } 3 \text { subunit B } \\
\text { \{ECO:0000255|HAMAP-Rule:MF_03001\}; }\end{array}$ & 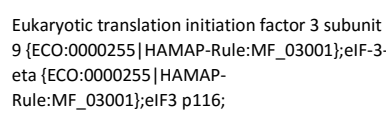 \\
\hline
\end{tabular}




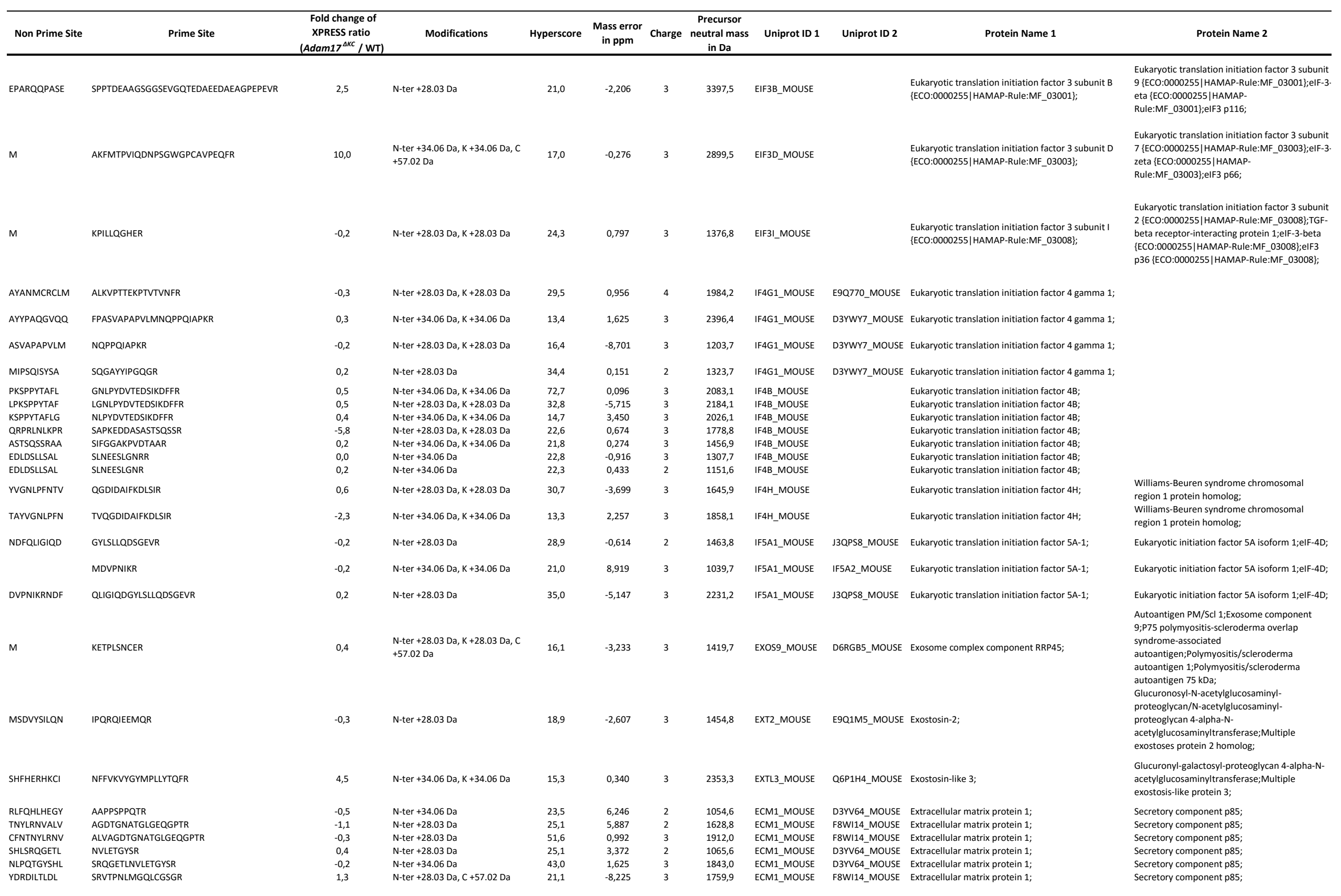




\begin{tabular}{|c|c|c|c|c|c|c|c|c|c|c|c|}
\hline Non Prime Site & Prime Site & 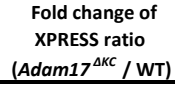 & Modifications & Hyperscore & $\begin{array}{l}\text { Mass error } \\
\text { in ppm }\end{array}$ & Charge & $\begin{array}{c}\text { Precursor } \\
\text { neutral mass } \\
\text { in Da }\end{array}$ & Uniprot ID 1 & Uniprot ID 2 & Protein Name 1 & Protein Name 2 \\
\hline HTCTWKAWEG & $\begin{array}{l}\text { TLDGYCER } \\
\end{array}$ & 0,1 & N-ter $+28.03 \mathrm{Da}, \mathrm{C}+57.02 \mathrm{Da}$ & 14,5 & $-1,055$ & 2 & 1040,5 & ECM1_MOUSE & F8W114_MOUSE & Extracellular matrix protein 1; & Secretory component p85; \\
\hline $\begin{array}{l}\text { NTRYLRNVAL } \\
\text { ARDENKRTHN }\end{array}$ & $\begin{array}{l}\text { VAGDNGNATGLGEQGPTR } \\
\text { DIIHENMR }\end{array}$ & $\begin{array}{l}-0,1 \\
0,5\end{array}$ & N-ter +34.06 Da & 37,1 & 4,606 & 3 & 1733,9 & ECM1_MOUSE & F8WI14_MOUSE & Extracellular matrix protein 1 ; & Secretory component p85; \\
\hline ARDENKRTHN & DIIHNENMR & 0,5 & $\mathrm{~N}$-ter +34.06 Da & 20,1 & 0,085 & 3 & 1174,6 & EZRI_MOUSE & & & Cytovillin;Villin-2;p81; \\
\hline ERAGVKMVMI & QDGPQNTGADKPLR & 0,2 & N-ter $+28.03 \mathrm{Da}, \mathrm{K}+28.03 \mathrm{Da}$ & 35,3 & $-8,040$ & 3 & 1551,8 & FUBP1_MOUSE & Q3TUE1_MOUSE & Far upstream element-binding protein $1 ;$ & \\
\hline ADPNMKLFTI & RGTPQQIDYAR & 1,8 & $\mathrm{~N}$-ter +34.06 Da & 20,7 & $-2,014$ & 3 & 1337,7 & FUBP1_MOUSE & Q3TUE1_MOUSE & Far upstream element-binding protein $1 ;$ & \\
\hline GGPPPGPPPP & AGGGGGAAGAGGGPPPGPPGAGDR & 0,2 & N-ter+28.03 Da & 51,9 & 5,691 & 3 & 1841,9 & FUBP2_MOUSE & & Far upstream element-binding protein 2; & KH type-splicing regulatory protein; \\
\hline MSDYNTG & GPPPGPPPPAGGGGGAAGAGGGPPPGPPGAGDR & $-1,2$ & N-ter+28.03 Da & 22,1 & $-8,149$ & 3 & 2635,3 & FUBP2_MOUSE & & Far upstream element-binding protein 2; & KH type-splicing regulatory protein; \\
\hline $\begin{array}{l}\text { ERAGVKMILI } \\
\text { RIINDLLSL }\end{array}$ & QDGSQNTNVDKPLR & 0,4 & $\mathrm{~N}-\operatorname{ter}+34.06 \mathrm{Da}, \mathrm{K}+34.06 \mathrm{Da}$ & 30,4 & 1,096 & 3 & 1638,9 & FUBP2_MOUSE & & Far upstream element-binding protein 2; & KH type-splicing regulatory protein; \\
\hline RIINDLLQSL & RSGPPGPPGAPGMPPGGR & 0,8 & $\mathrm{~N}$-ter +28.03 Da & 21,8 & $-2,393$ & 3 & 1668,9 & FUBP2_MOUSE & & Far upstream element-binding protein 2; & KH type-splicing regulatory protein; \\
\hline MSDYN & $\begin{array}{l}\text { TRGPPPGPPPAGGGGGAGAGGGPPPGPGAGD } \\
\mathrm{R}\end{array}$ & 0,1 & N-ter +34.06 Da & 28,5 & 2,748 & 3 & 2799,4 & FUBP2_MOUSE & & Far upstream element-binding protein 2; & KH type-splicing regulatory protein; \\
\hline M & APAPPPAASFTPAEVQR & $-1,2$ & N-ter +28.03 Da & 27,0 & 4,433 & 3 & 1733,9 & FA2H_MOUSE & & Fatty acid 2-hydroxylase; & Fatty acid alpha-hydroxylase; \\
\hline STVKTTVFSC & NLGEKFDETTADGR & 1,8 & $\mathrm{~N}$-ter $+28.03 \mathrm{Da}, \mathrm{K}+28.03 \mathrm{Da}$ & 27,4 & $-1,118$ & 3 & 1607,8 & FABP5_MOUSE & & Fatty acid-binding protein, epidermal; & $\begin{array}{l}\text { Epidermal-type fatty acid-binding protein;Fatty } \\
\text { acid-binding protein 5;Keratinocyte lipid- } \\
\text { binding protein;Psoriasis-associated fatty acid- } \\
\text { binding protein homolog; }\end{array}$ \\
\hline TESTVKTTVF & SCNLGEKFDETTADGR & 0,0 & $\begin{array}{l}\text { N-ter +28.03 Da, K+28.03 Da, C } \\
+57.02 \mathrm{Da}\end{array}$ & 21,8 & $-1,346$ & 3 & 1854,8 & FABP5_MOUSE & & Fatty acid-binding protein, epidermal; & $\begin{array}{l}\text { Epidermal-type fatty acid-binding protein;Fatty } \\
\text { acid-binding protein 5;Keratinocyte lipid- } \\
\text { binding protein;Psoriasis-associated fatty acid- } \\
\text { binding protein homolog; }\end{array}$ \\
\hline DGNNITVKTE & STVKTTVFSCNLGEKFDETTADGR & 0,2 & $\begin{array}{l}\text { N-ter +34.06 Da, K +34.06 Da, C } \\
+57.02 \mathrm{Da}\end{array}$ & 23,5 & 2,420 & 4 & 2764,5 & FABP5_MOUSE & & Fatty acid-binding protein, epidermal; & $\begin{array}{l}\text { Epidermal-type fatty acid-binding protein;Fatty } \\
\text { acid-binding protein ; FKeratinocyte lipid- } \\
\text { binding proteiniPsoriasis-associated fatty acid- } \\
\text { binding protein homolog; }\end{array}$ \\
\hline GNNITVKTES & TVKTTVFSCNLGEKFDETTADGR & 0,1 & $\begin{array}{l}\text { N-ter +34.06 Da, K +34.06 Da, C } \\
\text { +57.02 Da }\end{array}$ & 25,4 & 1,193 & 4 & 2677,4 & FABP5_MOUSE & & Fatty acid-binding protein, epidermal; & $\begin{array}{l}\text { Epidermal-type fatty acid-binding protein;Fatty } \\
\text { acid-binding protein 5;Keratinocyte lipid- } \\
\text { binding protein;Psoriasis-associated fatty acid- } \\
\text { bidding protein homolog; }\end{array}$ \\
\hline LPRRISWQLL & FLERPLYR & 1,1 & $\mathrm{~N}$-ter+34.06 Da & 14,5 & 0,620 & 3 & 1126,7 & FBX50_MOUSE & & F-box only protein 50; & $\begin{array}{l}\text { NCC receptor protein 1;Non-specific cytotoxic } \\
\text { cell receptor protein } 1 \text { homolog; }\end{array}$ \\
\hline PPRISWQLLF & LERPLYR & $-0,2$ & N-ter +34.06 Da & 16,1 & $-4,987$ & 3 & 979,6 & FBX50_MOUSE & & F-box only protein 50; & $\begin{array}{l}\text { NCC receptor protein 1;Non-specific cytotoxic } \\
\text { cell receptor protein } 1 \text { homolog; }\end{array}$ \\
\hline LERPLYRNLL & SSPNPEGINIYQPAPPTGPTR & 0,5 & $\mathrm{~N}$-ter +34.06 Da & 21,0 & 3,813 & 3 & 2226,2 & FBX50_MOUSE & & F-box only protein 50; & $\begin{array}{l}\text { NCC receptor protein 1;Non-specific cytotoxic } \\
\text { cell receptor protein } 1 \text { homolog; }\end{array}$ \\
\hline M & GEIEQKPTPASR & $-0,8$ & $\mathrm{~N}-\operatorname{ter}+28.03 \mathrm{Da}, \mathrm{K}+28.03 \mathrm{Da}$ & 16,9 & $-2,262$ & 3 & 1367,7 & FARP1_MOUSE & E9Q805_MOUSE & $\begin{array}{l}\text { FERM, RhoGEF and pleckstrin domain-containing } \\
\text { protein } 1 ;\end{array}$ & \\
\hline SPGRPPSFTQ & DCSHHSPGLEAEIR & 1,7 & $\mathrm{~N}-\operatorname{ter}+34.06 \mathrm{Da}, \mathrm{C}+57.02 \mathrm{Da}$ & 17,4 & $-6,996$ & 3 & 1640,8 & FARP2_MOUSE & & $\begin{array}{l}\text { FERM, RhoGEF and pleckstrin domain-containing } \\
\text { protein 2; }\end{array}$ & FERM domain including RhoGEF; \\
\hline ILSVASTVWT & TDTEDKGEFLSEGGGVR & 0,7 & N-ter $+28.03 \mathrm{Da}, \mathrm{K}+28.03 \mathrm{Da}$ & 39,6 & $-3,882$ & 3 & 1851,9 & Q99K47_MOUSE & E9PV24_MOUSE & Fibrinogen alpha chain; & \\
\hline LSLLAARANA & DISMEACCTDGNQMANQHR & 0,2 & N-ter $+34.06 \mathrm{Da}, \mathrm{C}+57.02 \mathrm{Da}$ & 26,6 & 0,132 & 3 & 2270,9 & FBLN1_MOUSE & & Fibulin-1; & Basement-membrane protein 90; \\
\hline RGQSSSANRR & AGSSSGSGVQGASAGGLAADASRR & $-1,3$ & N-ter +28.03 Da & 30,5 & 0,997 & 3 & 2104,0 & FILA_MOUSE & J3QMA3_MOUSE & Filaggrin; & \\
\hline RPRQPSASQS & SDSQVHSGVQVEAQR & 0,1 & $\mathrm{~N}$-ter $+28.03 \mathrm{Da}$ & 43,4 & 4,044 & 3 & 1653,8 & FILA_MOUSE & FGTLO2_MOUSE & Filaggrin; & \\
\hline RRDRPRQPSA & SOSSDSQVHSGVQVEAQR & 0,0 & N-ter +28.03 Da & 53,1 & 0,817 & 3 & 1955,9 & FILA_MOUSE & F6TLO2_MOUSE & Filaggrin; & \\
\hline RQPSASQSSD & SQVHSGVQVEAQR & $-0,1$ & N-ter +28.03 Da & 33,2 & 0,825 & 3 & 1451,8 & FILA_MOUSE & F6TLO2_MOUSE & Filaggrin; & \\
\hline QSSSANRRAG & SSSGSGVOGASAGGLAADASRR & 0,4 & $\mathrm{~N}$-ter +28.03 Da & 35,1 & $-4,699$ & 3 & 1976,0 & FILA_MOUSE & J3QMA3_MOUSE & Filaggrin; & \\
\hline QPVRPQGPSQ & DSSSQPQASQGQPSQSGSGR & 0,8 & N-ter +34.06 Da & 21,8 & 0,944 & 3 & 2008,9 & FILA2_MOUSE & & Filaggrin-2; & Intermediate filament-associated protein; \\
\hline SPVHPESSEG & EEHSVUPQR & 0,2 & N-ter +34.06 Da & 30,5 & 1,255 & 2 & 1113,6 & FILA2_MOUSE & FILA2_MOUSE & Filaggrin-2; & Intermediate filament-associated protein; \\
\hline RRSPVHPESS & EGEEHSVIPQR & $-1,5$ & N-ter +34.06 Da & 34,3 & 0,152 & 2 & 1313,7 & FILA2_MOUSE & & Filaggrin-2; & Intermediate filament-associated protein; \\
\hline RRSPVHPESS & EGEEHSVYPQR & 0,5 & N-ter +34.06 Da & 60,0 & 0,99 & 2 & 1299,7 & FILA2_MOUSE & FILA2_MOUSE & Filaggrin-2; & Intermediate filament-associated protein; \\
\hline RSPVHPESSE & GEEHSVIPQR & 1,3 & N-ter +28.03 & 26 & 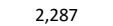 & 2 & 1178,6 & FILA2_MOUSE & & Filaggrin-2; & Intermediate filament-associated protein; \\
\hline YTQPGTICKY & NQFLDG & 0,2 & N-ter +34.06 Da & 14 & $-1,24$ & 2 & 882,5 & FILA2_MOUSE & E9QPZ3_MOUSE & Filaggrin-2; & Intermediate filament-associated protein; \\
\hline GQPVRPQGPS & QASOGQPSOSGSGR & 5,3 & N-ter +34.06 & 15 & $-6,44$ & 4 & 2137,0 & FILA2_MOUSE & & Filaggrin-2; & Intermediate filament-associated protein; \\
\hline ESVHGQPVRP & SSOSGSGR & 0,1 & N-ter +34.06 & & $-0,5$ & 3 & 2506,2 & FILA2_MOUSE & & Filaggrin-2; & Intermediate filament-associated protein; \\
\hline QSGSRRSPRS & QPVHPESSEGEEHSVYPQR & $-2,3$ & N-ter +28.03 & & 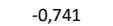 & 3 & 2155,0 & FILA2_MOUSE & & Filaggrin-2; & Intermediate filament-asso \\
\hline PRRSPVHPES & SEGEEH & 1,1 & N-ter +34.06 & & $-0,8>0$ & 3 & 1400,7 & FILA2_MOUSE & & Filaggrin-2; & Intermediate filament-asso \\
\hline RPQGPSQDSS & SQSGSGR & 1,1 & N-ter +34.06 Da & & 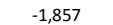 & 3 & 1719,8 & FILA2_MOUSE & & Filaggrin-2; & Intermediate filament-a \\
\hline SPRRSPVHPE & SSEGEEHSVIP & 1,3 & $\mathrm{~N}$-ter $+34.06 \mathrm{Da}$ & & $-0,2$ & 2 & 1487,7 & FILA2_MOUSE & & & Intermediate filament-associated protein; \\
\hline VRPQGPSQDS & SSQPQASQGQPSQSGSGR & 1,0 & N-ter +34.06 Da & 24,1 & 5,801 & 3 & 1806,9 & FILA2_MOUSE & & Filaggrin-2; & Intermediate filament-associated protein; \\
\hline
\end{tabular}




\begin{tabular}{|c|c|c|c|c|c|c|c|c|c|c|c|}
\hline Non Prime Site & Prime Site & $\begin{array}{l}\text { Fold change of } \\
\text { XPRESS ratio } \\
\text { (Adam17 } 7^{\text {AkC } / \text { WT) }}\end{array}$ & Modifications & Hyperscore & $\begin{array}{c}\text { Mass error } \\
\text { in ppm }\end{array}$ & Charge & $\begin{array}{c}\text { Precursor } \\
\text { neutral mass } \\
\text { in Da }\end{array}$ & Uniprot ID 1 & Uniprot ID 2 & Protein Name 1 & Protein Name 2 \\
\hline PVRPQGPSQD & SSSQPQASQGQPSQSGSGR & 1,4 & N-ter+34.06 Da & 31,2 & 4,692 & 3 & 1893,9 & FILA2_MOUSE & & Filaggrin-2; & Intermediate filament-associated protein; \\
\hline QGQAGHQQRE & SVHGQPVRPQGPSQDSSSQPQASQGQPSQSGSGR & 1,8 & N-ter +34.06 Da & 57,2 & $-0,548$ & 4 & 3463,7 & FLLA2_MOUSE & & Filaggrin-2; & Intermediate filament-associated protein; \\
\hline VSPSGAAVPC & KVEPGLGADNSVVR & $-2,0$ & $\mathrm{~N}-\operatorname{ter}+34.06 \mathrm{Da}, \mathrm{K}+34.06 \mathrm{Da}$ & 17,4 & $-2,383$ & 3 & 1507,9 & FLNA_MOUSE & B7FAU9_MOUSE & Filamin-A; & $\begin{array}{l}\text { Actin-binding protein 280;Alpha- } \\
\text { filamin;Endothelial actin-binding } \\
\text { protein;Filamin-1;Non-muscle filamin; } \\
\text { Actin-binding protein 280;Alpha- }\end{array}$ \\
\hline PGDYSILVKY & NDQHIPGSPFTAR & 3,3 & N-ter +28.03 Da & 24,7 & 0,340 & 3 & 1466,7 & FLNA_MOUSE & B7FAU9_MOUSE & Filamin-A; & $\begin{array}{l}\text { filamin;Endothelial actin-binding } \\
\text { protein;Filamin-1;Non-muscle filamin; }\end{array}$ \\
\hline AIKHTIAVVW & GGVNIPHSPYR & 0,0 & N-ter $+34.06 \mathrm{Da}$ & 15,8 & 3,975 & 3 & 1229,7 & FLNB_MOUSE & & Filamin-B; & $\begin{array}{l}\text { ABP-280-like protein;Actin-binding-like } \\
\text { protein;Beta-filamin; }\end{array}$ \\
\hline SDDARCLTVL & SLQESGLKVNQPASFAIR & 0,1 & $\mathrm{~N}$-ter $+34.06 \mathrm{Da}, \mathrm{K}+34.06 \mathrm{Da}$ & 19,7 & $-0,645$ & 3 & 2012,2 & FLNB_MOUSE & & Filamin-B; & $\begin{array}{l}\text { ABP-280-like protein;Actin-binding-like } \\
\text { protein;Beta-filamin; } \\
\text { Biliverdin reductanse B-Biliverdin-IX beta- }\end{array}$ \\
\hline m & TVKKIAIFGATGR & 0,4 & $\mathrm{~N}$-ter $+34.06 \mathrm{Da}, \mathrm{K}+34.06 \mathrm{Da}$ & 18,9 & 1,909 & 4 & 1463,0 & BLVRB_MOUSE & E9PZC3_MOUSE & Flavin reductase (NADPH); & $\begin{array}{l}\text { reductase;NADPH-dependent } \\
\text { diaphorase;NADPH-flavin reductase; }\end{array}$ \\
\hline TPKSPPKARR & QQMDLISELKR & $-10,0$ & $\mathrm{~N}$-ter $+28.03 \mathrm{Da}, \mathrm{K}+28.03 \mathrm{Da}$ & 20,0 & $-2,116$ & 2 & 1415,8 & FMNL_MOUSE & A2AB60_MOUSE & Formin-like protein 1 & Formin-related protein; \\
\hline SVCVVHLRNL & GTLNPPSSLDETAYER & 0,2 & $\mathrm{~N}$-ter $+28.03 \mathrm{Da}$ & 21,4 & $-1,224$ & 2 & 1794,8 & FRDA_MOUSE & E9Q2P9_MOUSE & Frataxin, mitochondrial; & \\
\hline LLCGEEQGAD & AALHFNPR & 0,1 & $\mathrm{~N}$-ter $+28.03 \mathrm{Da}$ & 22,1 & $-0,105$ & 3 & 952,5 & Q9CRB1_MOUSE & LEG7_MOUSE & Galectin \{ECO:0000256|RuleBase:RU102079\}; & \\
\hline DAALHFNPRL & DTSEVVFNTKEQGKWGR & $-0,1$ & N-ter $+34.06 \mathrm{Da}, \mathrm{K}+34.06 \mathrm{Da}$ & 50,4 & 0,576 & 3 & 2082,2 & Q9CRB1_MOUSE & D3Z141_MOUSE & Galectin \{ECO:0000256|RuleBase:RU102079\}; & \\
\hline LHFNPRLDTS & EVVFNTKEQGKWGR & 0,2 & $\mathrm{~N}$-ter $+28.03 \mathrm{Da}, \mathrm{K}+28.03 \mathrm{Da}$ & 27,8 & 0,397 & 4 & 1761,0 & Q9CRB1_MOUSE & D3Z141_MOUSE & Galectin \{ECO:0000256|RuleBase:RU102079\}; & \\
\hline RGMVPDQAGR & FHVNLLCGEEQGADAALHFNPR & $-10,0$ & N-ter $+28.03 \mathrm{Da}, \mathrm{C}+57.02 \mathrm{Da}$ & 53,3 & $-1,663$ & 3 & 2522,2 & Q9CRB1_MOUSE & LEG7_MOUSE & Galectin \{ECO:0000256|RuleBase:RU102079\}; & \\
\hline VRVGTVMRIR & GMVPDQAGR & 0,0 & $\mathrm{~N}$-ter $+28.03 \mathrm{Da}$ & 22,7 & $-0,313$ & 2 & 957,5 & Q9CRB1_MOUSE & D3Z141_MOUSE & Galectin \{ECO:0000256|RuleBase:RU102079\}; & \\
\hline QGVRVGTVMR & IRGMVPDQAGR & $-0,8$ & $\mathrm{~N}$-ter $+28.03 \mathrm{Da}$ & 25,6 & $-0,976$ & 3 & 1226,7 & Q9CRB1_MOUSE & D3Z141_MOUSE & Galectin \{ECO:0000256|RuleBase:RU102079\}; & \\
\hline MSATQH & KTSLPQGVR & 0,2 & $\mathrm{~N}-\operatorname{ter}+34.06 \mathrm{Da}, \mathrm{K}+34.06 \mathrm{Da}$ & 19,5 & $-0,853$ & 3 & 1052,7 & Q9CRB1_MOUSE & LEG7_MOUSE & Galectin \{ECO:0000256|RuleBase:RU102079\}; & \\
\hline DQAGRFHVNL & LCGEEQGADAALHFNPR & 0,0 & N-ter $+28.03 \mathrm{Da}, \mathrm{C}+57.02 \mathrm{Da}$ & 81,5 & 1,097 & 3 & 1911,9 & Q9CRB1_MOUSE & LEG7_MOUSE & Galectin \{ECO:0000256|RuleBase:RU102079\}; & \\
\hline ADAALHFNPR & LDTSEVVFNTKEQGKWGR & $-7,4$ & N-ter $+28.03 \mathrm{Da}, \mathrm{K}+28.03 \mathrm{Da}$ & 28,7 & $-4,447$ & 4 & 2177,1 & Q9CRB1_MOUSE & D3Z141_MOUSE & Galectin \{ECO:0000256|RuleBase:RU102079\}; & \\
\hline VPDQAGRFHV & NLLCGEEQGADAALHFNPR & 0,2 & N-ter $+28.03 \mathrm{Da}, \mathrm{C}+57.02 \mathrm{Da}$ & 55,6 & 1,214 & 3 & 2139,0 & Q9CRB1_MOUSE & LEG7_MOUSE & Galectin \{ECO:0000256|RuleBase:RU102079\}; & \\
\hline GVRVGTVMRI & RGMVPDQAGR & 0,1 & $\mathrm{~N}$-ter $+28.03 \mathrm{Da}$ & 27,3 & $-0,448$ & 3 & 1113,6 & Q9CRB1_MOUSE & D3Z141_MOUSE & Galectin \{ECO:0000256|RuleBase:RU102079\}; & \\
\hline KEQGKWGREE & RGTGIPFER & 0,0 & $\mathrm{~N}$-ter $+28.03 \mathrm{Da}$ & 18,7 & $-1,129$ & 3 & 1059,6 & Q9CRB1_MOUSE & D3Z141_MOUSE & Galectin \{ECO:0000256|RuleBase:RU102079\}; & \\
\hline ALHFNPRLDT & $\begin{array}{l}\text { SEVVFNTKEQGKWGR } \\
\text { SENT }\end{array}$ & 0,2 & $\mathrm{~N}-\operatorname{ter}+28.03 \mathrm{Da}, \mathrm{K}+28.03 \mathrm{Da}$ & 33,6 & $-6,213$ & 3 & 1848,0 & Q9CRB1_MOUSE & D3Z141_MOUSE & Galectin $\{E C O: 0000256 \mid$ RuleBase:RU102079\}; & \\
\hline MSA & TQHKTSLPQGVR & 0,2 & $\mathrm{~N}-\operatorname{ter}+28.03 \mathrm{Da}, \mathrm{K}+28.03 \mathrm{Da}$ & 17,8 & $-1,135$ & 3 & 1406,8 & Q9CRB1_MOUSE & D3Z141_MOUSE & Galectin $\{E C O: 0000256 \mid$ RuleBase:RU102079\}; & \\
\hline AALHFNPRLD & TSEVVFNTKEQGKWGR & 0,1 & $\mathrm{~N}-\operatorname{ter}+28.03 \mathrm{Da}, \mathrm{K}+28.03 \mathrm{Da}$ & 41,9 & 0,461 & 3 & 1949,0 & Q9CRB1_MOUSE & D3Z141_MOUSE & Galectin \{ECO:0000256|RuleBase:RU102079\}; & \\
\hline FNPRLDTSEV & VFNTKEQGKWGR & $-0,1$ & $\mathrm{~N}$-ter $+28.03 \mathrm{Da}, \mathrm{K}+28.03 \mathrm{Da}$ & 26,0 & $-0,195$ & 4 & 1532,9 & Q9CRB1_MOUSE & D3Z141_MOUSE & Galectin $\{E C O: 0000256 \mid$ RuleBase:RU102079\}; & \\
\hline SDAKSFVLNL & GKDSNNLCLHFNPR & 0,4 & $\begin{array}{l}\text { N-ter +34.06 Da, K+34.06 Da, C } \\
\text { +57.02 Da }\end{array}$ & 39,8 & 0,057 & 4 & 1738,9 & LEG1_MOUSE & & Galectin-1; & $\begin{array}{l}14 \text { kDa lectin;Beta-galactoside-binding lectin L- } \\
14-\text {-1;Galaptin;Lactose-binding lectin } 1 ; \text {;ectin } \\
\text { galactoside-binding soluble 1;-L-Lac lectin 1; }\end{array}$ \\
\hline VLNLGKDSNN & LCLHFNPR & 0,0 & N-ter $+34.06 \mathrm{Da}, \mathrm{C}+57.02 \mathrm{Da}$ & 17,0 & $-0,549$ & 3 & 1089,6 & LEG1_MOUSE & & Galectin-1; & $\begin{array}{l}14 \text { kDa lectin;Beta-galactoside-binding lectin L- } \\
14-\text { - Galaptin;Lactose-binding lectin } 1 ; \text {;ectin } \\
\text { galactoside-binding soluble 1;-L-Lac lectin 1; }\end{array}$ \\
\hline VASDAKSFVL & NLGKDSNNLCLHFNPR & 0,0 & $\begin{array}{l}\text { N-ter }+28.03 \mathrm{Da}, \mathrm{K}+28.03 \mathrm{Da}, \mathrm{C} \\
+57.02 \mathrm{Da}\end{array}$ & 28,0 & 6,898 & 3 & 1954,0 & LEG1_MOUSE & & Galectin-1; & 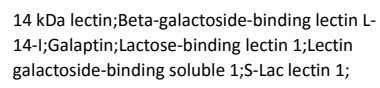 \\
\hline MACGLVASNL & NLKPGECLKVR & 0,5 & $\begin{array}{l}\text { N-ter +34.06 Da, K+34.06 Da, C } \\
+57.02 \mathrm{Da}\end{array}$ & 29,5 & 0,423 & 3 & 1414,9 & LEG1_MOUSE & & Galectin-1; & $\begin{array}{l}14 \text { kDa lectin;Beta-galactoside-binding lectin L- } \\
14-\text { - Galaptin;:Lactose-binding lectin } 1 ; \text {;ectin } \\
\text { galactoside-binding soluble 1;s-Lac lectin } 1 ;\end{array}$ \\
\hline MACGLVAS & NLNLKPGECLKVR & 0,5 & $\begin{array}{l}\text { N-ter +34.06 Da, K+34.06 Da, C } \\
+57.02 \mathrm{Da}\end{array}$ & 16,8 & 3,402 & 4 & 1642,1 & LEG1_MOUSE & & Galectin-1; & $\begin{array}{l}14 \text { kDa lectin;Beta-galactoside-binding lectin L- } \\
14-\text { - Galaptin;Lactose-binding lectin } 1 ; \text {;ectin } \\
\text { galactoside-binding soluble 1;5-Lac lectin 1; }\end{array}$ \\
\hline MACGLVA & SNLNLKPGECLKVR & 0,4 & $\begin{array}{l}\text { N-ter +28.03 Da, K+28.03 Da, C } \\
+57.02 \mathrm{Da}\end{array}$ & 24,5 & $-0,175$ & 4 & 1711,0 & LEG1_MOUSE & & Galectin-1; & $\begin{array}{l}14 \text { kDa lectin;Beta-galactoside-binding lectin L- } \\
14-\text { - Galaptin;Lactose-binding lectin } 1 ; \text {;ectin } \\
\text { galactoside-binding soluble 1;5-Lac lectin 1; }\end{array}$ \\
\hline RIVLDFRRGN & DVAFHFNPR & 0,1 & N-ter +34.06 Da & 21,9 & $-0,878$ & 3 & 1135,6 & LEG3_MOUSE & LEG4_MOUSE & Galectin-3; & $\begin{array}{l}35 \text { kDa lectin;Carbohydrate-binding protein } \\
35 ; G \text { Galactose-specific lectin 3;/IE-binding } \\
\text { protein;:--34 galactoside-binding lectin;Laminin } \\
\text { binding protein;Lectin L-29;Mac-2 antigen; }\end{array}$ \\
\hline
\end{tabular}




\begin{tabular}{|c|c|c|c|c|c|c|c|c|c|c|c|}
\hline Non Prime Site & Prime Site & $\begin{array}{l}\text { Fold change of } \\
\text { XPRESS ratio } \\
\text { (Adam17 } 1 \text { ¿Kc / WT) } \\
\end{array}$ & Modifications & Hyperscore & $\begin{array}{l}\text { Mass error } \\
\text { in ppm }\end{array}$ & Charge & $\begin{array}{c}\text { Precursor } \\
\text { neutral mass } \\
\text { in Da } \\
\end{array}$ & Uniprot ID 1 & Uniprot ID 2 & Protein Name 1 & Protein Name 2 \\
\hline LPLPGGVMPR & MLITIMGTVKPNANR & $-5,7$ & N-ter +28.03 Da, K+28.03 Da & 28,6 & 2,097 & 3 & 1714,0 & LEG3_MOUSE & Q8C253_MOUSE & Galectin-3; & $\begin{array}{l}35 \text { kDa lectin;Carbohydrate-binding protein } \\
35 ; G \text { Galactose-specific lectin 3;/IE-binding } \\
\text { protein;-L-30 galactoside-binding lectinitaminin- } \\
\text { binding protein;Lectin L-29;Mac-2 antigen; }\end{array}$ \\
\hline PGGVMPRMLI & TIMGTVKPNANR & $-0,6$ & N-ter +28.03 Da, K+28.03 Da & 24,2 & 2,206 & 3 & 1356,8 & LEG3_MOUSE & Q8C253_MOUSE & Galectin-3; & $\begin{array}{l}35 \text { kDa lectin;Carbohydrate-binding protein } \\
35 ; G \text { Galactose-specific lectin 3;IIE-binding } \\
\text { protein;-L-34 galactoside-binding lectinitLaminin- } \\
\text { binding protein;lectin L-29;Mac-2 antigen; }\end{array}$ \\
\hline IVLDFRRGND & VAFHFNPR & 0,3 & $\mathrm{~N}$-ter +28.03 Da & 18,5 & 0,098 & 3 & 1014,5 & LEG3_MOUSE & LEG4_MOUSE & Galectin-3; & $\begin{array}{l}35 \mathrm{kDa} \text { lectin;Carbohydrate-binding protein } \\
35 \text {;Galactose-specific lectin 3;/IE-binding } \\
\text { protein;;--34 galactoside-binding lectin; Laminin- } \\
\text { binding protein;Lectin L-29;Mac-2 antigen; }\end{array}$ \\
\hline QAGRFHVNLL & CGEEQGADAALHFNPR & 0,0 & N-ter +34.06 Da, C +57.02 Da & 72,5 & $-0,664$ & 3 & 1804,8 & LEG7_MOUSE & Q9CRB1_MOUSE & Galectin-7; & \\
\hline GRFHVNLLCG & EEQGADAALHFNPR & 0,3 & $\mathrm{~N}$-ter +28.03 Da & 48,9 & 1,830 & 3 & 1581,8 & LEG7_MOUSE & Q9CRB1_MOUSE & Galectin-7; & \\
\hline RFHVNLLCGE & EQGADAALHFNPR & 0,6 & N-ter +28.03 Da & 15,5 & $-1,511$ & 3 & 1452,7 & LEG7_MOUSE & Q9CRB1_MOUSE & Galectin-7; & \\
\hline HVNLLCGEEQ & GADAALHFNPR & 0,2 & N-ter +34.06 Da & 18,8 & $-1,660$ & 3 & 1201,6 & LEG7_MOUSE & Q9CRB1_MOUSE & Galectin-7; & \\
\hline AGRFHVNLLC & GEEQGADAALHFNPR & 0,2 & $\mathrm{~N}$-ter $+28.03 \mathrm{Da}$ & 57,9 & 1,218 & 3 & 1638,8 & LEG7_MOUSE & Q9CRB1_MOUSE & Galectin-7; & \\
\hline PDQAGRFHVN & LLCGEEQGADAALHFNPR & 0,1 & $\mathrm{~N}$-ter +28.03 Da, C+57.02 Da & 64,0 & 0,395 & 3 & 2025,0 & LEG7_MOUSE & Q9CRB1_MOUSE & Galectin-7; & \\
\hline MVPDQAGRFH & VNLLCGEEQGADAALHFNPR & $-0,1$ & $\mathrm{~N}$-ter +28.03 Da, C +57.02 Da & 67,8 & 0,535 & 3 & 2238,1 & LEG7_MOUSE & Q9CRB1_MOUSE & Galectin-7; & \\
\hline LGNYYIAASY & VKYIESAGAR & 1,0 & $\mathrm{~N}$-ter +28.03 Da, K +28.03 Da & 23,9 & $-6,599$ & 3 & 1148,7 & GGH_MOUSE & & Gamma-glutamyl hydrolase; & $\begin{array}{l}\text { Conjugase;FGPH;Folylpolyglutamate } \\
\text { hydrolase;GH;Gamma-Glu-x carboxypeptidase; }\end{array}$ \\
\hline FGKMAKLGNY & YIAASYVKYIESAGAR & 0,5 & N-ter +34.06 Da, K +34.06 Da & 43,0 & 1,747 & 3 & 1829,0 & GGH_MOUSE & & Gamma-glutamyl hydrolase; & $\begin{array}{l}\text { Conjugase;FGPH;Folyl|polyglutamate } \\
\text { hydrolase;GH;Gamma-Glu-x carboxypeptidase; }\end{array}$ \\
\hline HAPNAVKTSF & YLAEFLVSEAR & 0,7 & $\mathrm{~N}$-ter+28.03 Da & 30,7 & 0,678 & 2 & 1324,7 & GGH_MOUSE & & Gamma-glutamyl hydrolase; & $\begin{array}{l}\text { Conjugase;FGPH;Folylpolyylutamate } \\
\text { hydrolase;GH;Gamma-Glu-x carboxypeptidase; }\end{array}$ \\
\hline KLGNYYIAAS & YVKYIESAGAR & 1,2 & N-ter +28.03 Da, K+28.03 Da & 24,4 & $-0,913$ & 3 & 1311,7 & GGH_MOUSE & & Gamma-glutamyl hydrolase; & $\begin{array}{l}\text { Conjugase;FGPH;Folylyolyglutamate } \\
\text { hydrolase;GH;Gamma-Glu-x carboxypeptidase; }\end{array}$ \\
\hline TCKAHDVCLL & GPRPLPPSPPVR & 0,4 & N-ter +28.03 Da & 31,9 & $-0,154$ & 3 & 1296,8 & GILT_MOUSE & & $\begin{array}{l}\text { Gamma-interferon-inducible lysosomal thiol } \\
\text { reductase; }\end{array}$ & $\begin{array}{l}\text { Gamma-interferon-inducible protein IP- } \\
\text { 30;Lysosomal thiol reductase IP30; }\end{array}$ \\
\hline YGNAQERNVS & GTWEFTCQHGELECR & 1,3 & N-ter +34.06 Da, C +57.02 Da & 20,5 & 8,479 & 3 & 1942,9 & GILT_MOUSE & & $\begin{array}{l}\text { Gamma-interferon-inducible Iysosomal thiol } \\
\text { reductase; }\end{array}$ & $\begin{array}{l}\text { Gamma-interferon-inducible protein IP- } \\
\text { 30; Lysosomal thiol reductase IP30; }\end{array}$ \\
\hline KAHDVCLLGP & RPLPPSPPVR & 0,9 & N-ter+34.06 Da & 28,1 & $-2,171$ & 3 & 1148,7 & GILT_MOUSE & & $\begin{array}{l}\text { Gamma-interferon-inducible Iysosomal thiol } \\
\text { reductase; }\end{array}$ & $\begin{array}{l}\text { Gamma-interferon-inducible protein IP- } \\
\text { 30; Lysosomal thiol reductase IP30; }\end{array}$ \\
\hline GNAQERNVSG & TWEFTCQHGELECR & 0,8 & N-ter +28.03 Da, C +57.02 Da & 18,1 & 5,099 & 3 & 1879,8 & GILT_MOUSE & & $\begin{array}{l}\text { Gamma-interferon-inducible Iysosomal thiol } \\
\text { reductase; }\end{array}$ & $\begin{array}{l}\text { Gamma-interferon-inducible protein IP- } \\
\text { 30; Lysosomal thiol reductase IP30; }\end{array}$ \\
\hline $\begin{array}{l}\text { ERGENLYVVM } \\
\text { VKGTAGSPS }\end{array}$ & EVVETLQEVTLER & 0,5 & $\mathrm{~N}$-ter +34.06 Da & 22,4 & $\begin{array}{r}-4,747 \\
2,364\end{array}$ & 2 & 1577,9 & GSDMA_MOUSE & & Gasdermin-A; & Gasdermin-1;Gasdermin-A1; \\
\hline VKGTAGLSRS & STLEVQTLSVAPTALENLHKER & 0,5 & N-ter +28.03 Da, K+28.03 Da & 20,1 & 2,364 & 4 & 2491,4 & GSDMA_MOUSE & & Gasdermin-A; & Gasdermin-1;Gasdermin-A1; \\
\hline GPRPRGPRYA & EGTFISDYSIAMDKIR & $-5,2$ & N-ter +28.03 Da, K+28.03 Da & 19,4 & 9,716 & 3 & 1901,0 & GIP_MOUSE & & Gastric inhibitory polypeptide; & Glucose-dependent insulinotropic polypeptide; \\
\hline $\begin{array}{l}\text { GTQCPNLEIS } \\
\text { VOVEEGSEDD }\end{array}$ & $\begin{array}{l}\text { SATSPASLQR } \\
\text { START }\end{array}$ & 2,4 & $\mathrm{~N}$-ter +34.06 Da & 18,0 & $-3,040$ & 2 & 1050,6 & ZGLP1_MOUSE & & GATA-type zinc finger protein $1 ;$ & GATA-like protein 1; \\
\hline $\begin{array}{l}\text { VQVEEGSEPD } \\
\text { TPSAAYLWVG }\end{array}$ & $\begin{array}{l}\text { AFWEAAGGGTAAR } \\
\text { AGAEAEKTAQELLVVR }\end{array}$ & $\begin{array}{l}-0,6 \\
0,6\end{array}$ & 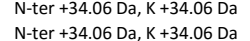 & $\begin{array}{l}24,3 \\
16,6\end{array}$ & $\begin{array}{l}3,422 \\
0,289\end{array}$ & $\begin{array}{l}3 \\
4\end{array}$ & $\begin{array}{l}1536,9 \\
2072,3\end{array}$ & $\begin{array}{l}\text { GELS_MOUSE } \\
\text { GES MOUSE }\end{array}$ & & $\begin{array}{l}\text { Gelsolin; } \\
\text { Gelsolin; }\end{array}$ & $\begin{array}{l}\text { Actin-depolymerizing factor; Brevin; } \\
\text { Actin-depolymerizing factor:Brevin; }\end{array}$ \\
\hline AMLQVLGPKP & ALPEGTEDTAKEDAANR & 0,7 & $\mathrm{~N}$-ter +28.03 Da, K +28.03 Da & 22,8 & 6,230 & 3 & 1842,9 & GELS_MOUSE & A6PWS5_MOUSE & Gelsolin; & Actin-depolymerizing factor; Brevin; \\
\hline FAQGALRSED & CFLLDHGR & $-0,8$ & $\mathrm{~N}$-ter $+28.03 \mathrm{Da}, \mathrm{C}+57.02 \mathrm{Da}$ & 16,2 & 0,286 & 3 & 1044,5 & GELS_MOUSE & & Gelsolin; & Actin-depolymerizing factor;Brevin; \\
\hline AGSMSVSLVA & DENPFAQGALR & 0,7 & N-ter $+28.03 \mathrm{Da}$ & 27,1 & 1,765 & 2 & 1244,6 & GELS_MOUSE & & Gelsolin; & Actin-depolymerizing factor;Brevin; \\
\hline KNWRDPDQTD & GPGLGYLSSHIANVER & $-0,3$ & $\mathrm{~N}$-ter $+28.03 \mathrm{Da}$ & 33,3 & 4,765 & 3 & 1696,9 & GELS_MOUSE & & Gelsolin; & Actin-depolymerizing factor:Brevin: \\
\hline KLYKVSNGAG & SMSVSLVADENPFAQGALR & 0,5 & N-ter +34.06 Da & 24,7 & $-1,726$ & 3 & 2025,0 & GELS_MOUSE & & Gelsolin; & Actin-depolymerizing factor;Brevin; \\
\hline YKVSNGAGSM & SVSLVADENPFAQGALR & 0,5 & $\mathrm{~N}$-ter $+34.06 \mathrm{Da}$ & 16,3 & $-0,111$ & 2 & 1807,0 & GELS_MOUSE & & Gelsolin; & Actin-depolymerizing factor;Brevin; \\
\hline M & KLLPGVGVFGTGSSAR & $-0,3$ & $\mathrm{~N}$-ter $+28.03 \mathrm{Da}, \mathrm{K}+28.03 \mathrm{Da}$ & 13,4 & $-1,614$ & 3 & 1732,0 & GFOD2_MOUSE & D3Z3T3_MOUSE & $\begin{array}{l}\text { Glucose-fructose oxidoreductase domain-containing } \\
\text { protein 2: }\end{array}$ & \\
\hline HVTGWTDWNL & ALNPEGGPNWVR & 0,4 & $\mathrm{~N}$-ter +34.06 Da & 16,9 & $-0,074$ & 2 & 1342,7 & GLCM_MOUSE & & Glucosylceramidase; & $\begin{array}{l}\text { Acid beta-glucosidase;Beta- } \\
\text { glucocerebrosidase; } \mathrm{D}-\text {-lucosyl-N- } \\
\text { acylsphingosine glucohydrolase; }\end{array}$ \\
\hline PFKQGLLEFV & DITATNNTSAIQDYLQQLTGA & $-0,9$ & $\mathrm{~N}$-ter $+34.06 \mathrm{Da}$ & 21,2 & 0,988 & 3 & 2427,3 & GLRX1_MOUSE & & Glutaredoxin-1; & Thioltransferase-1; \\
\hline LPFKQGLLEF & VDITATNNTSAIQDYLQQLLTGAR & 0,5 & N-ter +28.03 Da & 38,0 & $-1,783$ & 3 & 2520,3 & GLRX1_MOUSE & & Glutaredoxin-1; & Thioltransferase- 1 ; \\
\hline
\end{tabular}




\begin{tabular}{|c|c|c|c|c|c|c|c|c|c|c|c|}
\hline Non Prime Site & Prime Site & $\begin{array}{c}\text { Fold change of } \\
\text { XPRESS ratio } \\
\text { (Adam17 } 7^{\text {KKc } / W T)}\end{array}$ & Modifications & Hyperscore & $\begin{array}{c}\text { Mass error } \\
\text { in ppm }\end{array}$ & Charge & $\begin{array}{c}\text { Precursor } \\
\text { neutral mass } \\
\text { in Da } \\
\end{array}$ & is Uniprot ID 1 & Uniprot ID 2 & Protein Name 1 & Protein Name 2 \\
\hline AAQSTVYAFS & ARPLTGGEPVSLGSLR & 0,9 & N-ter +34.06 Da & 20,9 & $-0,061$ & 3 & 1643,0 & GPX1_MOUSE & & Glutathione peroxidase 1; & $\begin{array}{l}\text { Celluluar glutathione peroxidase; Selenium- } \\
\text { dependent glutathione peroxidase } 1 \text {; }\end{array}$ \\
\hline VCRNDIAWNF & EKFLVGPDGVPVR & $-4,1$ & N-ter $+34.06 \mathrm{Da}, \mathrm{K}+34.06 \mathrm{Da}$ & 15,4 & $-1,214$ & 3 & 1479,9 & GPX1_MOUSE & & Glutathione peroxidase 1; & $\begin{array}{l}\text { Celluluar glutathione peroxidase; Selenium- } \\
\text { dependent glutathione peroxidase } 1 \text {; }\end{array}$ \\
\hline AAAQSTVYAF & SARPLTGGEPVSLGSLR & 0,5 & N-ter +34.06 Da & 38,7 & $-0,173$ & 3 & 1730,0 & GPX1_MOUSE & & Glutathione peroxidase $1 ;$ & $\begin{array}{l}\text { Cellular glutathione peroxidase:Selenium- } \\
\text { dependent glutathione peroxidase 1; }\end{array}$ \\
\hline TPSDDPTALM & TDPKYIIWSPVCR & 0,9 & $\begin{array}{l}\text { N-ter }+34.06 \mathrm{Da}, \mathrm{K}+34.06 \mathrm{Da}, \mathrm{C} \\
+57.02 \mathrm{Da}\end{array}$ & 15,9 & 7,331 & 3 & 1702,0 & GPX1_MOUSE & & Glutathione peroxidase 1 ; & $\begin{array}{l}\text { Cellular glutathione peroxidase;Selenium- } \\
\text { dependent glutathione peroxidase } 1 \text {; }\end{array}$ \\
\hline LLWAAACAQS & EQDFYDFKAVNIR & $-1,9$ & N-ter +34.06 Da, K +34.06 Da & 17,9 & $-0,700$ & 3 & 1711,9 & GPX7_MOUSE & & Glutathione peroxidase 7; & \\
\hline SMRFCPFAQR & TLMVLKAKGIR & 1,4 & $\mathrm{~N}-\operatorname{ter}+34.06 \mathrm{Da}, \mathrm{K}+34.06 \mathrm{Da}$ & 12,5 & 9,139 & 4 & 1331,0 & GSTO1_MOUSE & & Glutathione S-transferase omega-1; & $\begin{array}{l}\text { Glutathione S-transferase omega 1- } \\
\text { 1;Glutathione-dependent dehydroascorbate } \\
\text { reductase;Monomethylarsonic acid reductase;-S- } \\
\text { (Phenacyl)glutathione reductase;p28; }\end{array}$ \\
\hline SEERASYILM & EKIEPEPFR & 1,3 & N-ter $+28.03 \mathrm{Da}, \mathrm{K}+28.03 \mathrm{Da}$ & 15,9 & 0,083 & 3 & 1199,7 & GSHB_MOUSE & Q3UEE2_MOUSE & Glutathione synthetase; & Glutathione synthase; \\
\hline KAENGKLVIN & GKPITIFQER & 0,2 & N-ter +34.06 Da, K+34.06 Da & 19,4 & $-1,033$ & 3 & 1255,8 & S4R257_MOUSE & G3P_MOUSE & $\begin{array}{l}\text { Glyceraldehyde-3-phosphate dehydrogenase } \\
\text { \{ECO:0000256|RuleBase:RU361160\}; }\end{array}$ & \\
\hline PELNGKLTGM & AFRVPTPNVSVVDLTCR & 0,7 & $\mathrm{~N}-\operatorname{ter}+34.06 \mathrm{Da}, \mathrm{C}+57.02 \mathrm{Da}$ & 26,4 & $-0,864$ & 3 & 1964,1 & G3PT_MOUSE & G3P_MOUSE & $\begin{array}{l}\text { Glyceraldehyde-3-phosphate dehydrogenase, testis- } \\
\text { specific; }\end{array}$ & $\begin{array}{l}\text { Spermatogenic cell-specific glyceraldehyde 3- } \\
\text { phosphate dehydrogenase 2;Spermatogenic } \\
\text { glyceraldehyde-3-phosphate dehydrogenase; }\end{array}$ \\
\hline ELNGKLTGMA & FRVPTPNVSVVDLTCR & $-0,2$ & N-ter $+34.06 \mathrm{Da}, \mathrm{C}+57.02 \mathrm{Da}$ & 47,8 & 0,580 & 3 & 1893,0 & G3PT_MOUSE & G3P_MOUSE & $\begin{array}{l}\text { Glyceraldehyde-3-phosphate dehydrogenase, testis- } \\
\text { specific; }\end{array}$ & $\begin{array}{l}\text { Spermatogenic cell-specific glyceraldehyde 3- } \\
\text { phosphate dehydrogenase 2; } 5 \text {-Spermatogenic } \\
\text { glyceraldehyde-3-phosphate dehydrogenase; }\end{array}$ \\
\hline IVEGLMTTVH & AITATQKTVDGPSGKLWR & 1,0 & $\mathrm{~N}$-ter $+34.06 \mathrm{Da}, \mathrm{K}+34.06 \mathrm{Da}$ & 35,3 & 1,868 & 4 & 2030,2 & G3P_MOUSE & D3YY15_MOUSE & Glyceraldehyde-3-phosphate dehydrogenase; & Peptidyl-cysteine S-nitrosylase GAPDH; \\
\hline GLMTTVHAIT & ATQKTVDGPSGKLWR & 0,6 & $\mathrm{~N}$-ter $+34.06 \mathrm{Da}, \mathrm{K}+34.06 \mathrm{Da}$ & 13,9 & 0,687 & 3 & 1745,1 & G3P_MOUSE & D3YYI5_MOUSE & Glyceraldehyde-3-phosphate dehydrogenase; & Peptidyl-cysteine S-nitrosylase GAPDH; \\
\hline DNFVKLISWY & DNEYGYSNR & $-0,2$ & $\mathrm{~N}$-ter +28.03 Da & 24,1 & 0,698 & 2 & 1144,5 & G3P_MOUSE & G3PT_MOUSE & Glyceraldehyde-3-phosphate dehydrogenase; & Peptidyl-cysteine S-nitrosylase GAPDH; \\
\hline AITATQKTVD & GPSGKLWR & 0,3 & $\mathrm{~N}-\operatorname{ter}+34.06 \mathrm{Da}, \mathrm{K}+34.06 \mathrm{Da}$ & 22,0 & $-0,412$ & 3 & 967,6 & G3P_MOUSE & D3YYI5_MOUSE & Glyceraldehyde-3-phosphate dehydrogenase; & Peptidyl-cysteine S-nitrosylase GAPDH; \\
\hline IALNDNFVKL & ISWYDNEYGYSNR & 0,2 & $\mathrm{~N}$-ter $+28.03 \mathrm{Da}$ & 31,9 & 1,886 & 3 & 1693,7 & G3P_MOUSE & D3YYIS_MOUSE & Glyceraldehyde-3-phosphate dehydrogenase; & Peptidyl-cysteine S-nitrosylase GAPDH; \\
\hline AGIALNDNFV & KLISWYDNEYGYSNR & 0,2 & N-ter $+28.03 \mathrm{Da}, \mathrm{K}+28.03 \mathrm{Da}$ & 21,5 & $-4,985$ & 3 & 1962,9 & G3P_MOUSE & D3YYI5_MOUSE & Glyceraldehyde-3-phosphate dehydrogenase; & Peptidyl-cysteine S-nitrosylase GAPDH; \\
\hline MV & $\begin{array}{l}\text { KVGVNGFGR } \\
\text { KVIT }\end{array}$ & 0,5 & $\mathrm{~N}-\operatorname{ter}+34.06 \mathrm{Da}, \mathrm{K}+34.06$ Da & 16,1 & $-0,100$ & 3 & 1000,7 & G3P_MOUSE & D3YYIS_MOUSE & Glyceraldehyde-3-phosphate dehydrogenase; & Peptidyl-cysteine S-nitrosylase GAPDH; \\
\hline LNGKLTGMAF & $\begin{array}{l}\text { RVPTPNVSVVDLTCR } \\
\text { RUN }\end{array}$ & 0,9 & $\mathrm{~N}-\operatorname{ter}+34.06 \mathrm{Da}, \mathrm{C}+57.02 \mathrm{Da}$ & 35,3 & $-0,858$ & 3 & 1746,0 & G3P_MOUSE & G3PT_MOUSE & Glyceraldehyde-3-phosphate dehydrogenase; & Peptidyl-cysteine S-nitrosylase GAPDH; \\
\hline ALNDNFVKLI & SWYDNEYGYSNR & 0,0 & $\mathrm{~N}-\operatorname{ter}+28.03 \mathrm{Da}$ & 49,5 & 0,442 & 2 & 1580,7 & G3P_MOUSE & D3YYIS_MOUSE & Glyceraldehyde-3-phosphate dehydrogenase; & Peptidyl-cysteine S-nitrosylase GAPDH; \\
\hline LMTTVHAITA & TQKTVDGPSGKLWR & 0,5 & $\mathrm{~N}-\operatorname{ter}+34.06 \mathrm{Da}, \mathrm{K}+34.06 \mathrm{Da}$ & 25,9 & 0,358 & 4 & 1674,0 & G3P_MOUSE & D3YYIS_MOUSE & Glyceraldehyde-3-phosphate dehydrogenase; & Peptidyl-cysteine S-nitrosylase GAPDH; \\
\hline TVHAITATQK & TVDGPSGKLWR & 0,6 & $\mathrm{~N}-\operatorname{ter}+34.06 \mathrm{Da}, \mathrm{K}+34.06 \mathrm{Da}$ & 28,7 & 0,233 & 3 & 1282,8 & G3P_MOUSE & D3YYIS_MOUSE & Glyceraldehyde-3-phosphate dehydrogenase; & Peptidyl-cysteine S-nitrosylase GAPDH; \\
\hline GAGIALNDNF & $\begin{array}{l}\text { VKLISWYDNEYGYSNR } \\
\text { VIS }\end{array}$ & 0,1 & $\mathrm{~N}$-ter $+34.06 \mathrm{Da}, \mathrm{K}+34.06 \mathrm{Da}$ & $\begin{array}{l}20,1 \\
43,1\end{array}$ & $\begin{array}{l}-0,048 \\
-0,230\end{array}$ & 3 & 2074,1 & G3P_MOUSE & D3YYIS_MOUSE & Glyceraldehyde-3-phosphate dehydrogenase; & Peptidyl-cysteine S-nitrosylase GAPDH; \\
\hline M & $\begin{array}{l}\text { VKVGVNGFGR } \\
\text { VKIM }\end{array}$ & 0,3 & $\mathrm{~N}$-ter $+28.03 \mathrm{Da}, \mathrm{K}+28.03 \mathrm{Da}$ & 34,8 & $\begin{array}{l}3,301 \\
3,301\end{array}$ & 3 & 1087,7 & G3P_MOUSE & D3YYIS_MOUSE & Glyceraldehyde-3-phosphate dehydrogenase; & Peptidyl-cysteine S-nitrosylase GAPDH; \\
\hline LNDNFVKLIS & WYDNEYGYSNR & 0,2 & $\mathrm{~N}$-ter +34.06 Da & 35,3 & 4,528 & 2 & 1499,7 & G3P_MOUSE & G3PT_M̄MOUSE & Glyceraldehyde-3-phosphate dehydrogenase; & Peptidyl-cysteine S-nitrosylase GAPDH; \\
\hline NDNFVKLISW & YDNEYGYSNR & 0,1 & $\mathrm{~N}$-ter $+28.03 \mathrm{Da}$ & 15,5 & 1,985 & 2 & 1307,5 & G3P_MOUSE & G3PT_MOUSE & $\begin{array}{l}\text { Glyceraldehyde-3-phosphate dehydrogenase; }\end{array}$ & Peptidyl-cysteine S-nitrosylase GAPDH; \\
\hline \multirow[t]{2}{*}{ M } & KVFVTGPLPAEGR & $-3,3$ & $\mathrm{~N}-\operatorname{ter}+34.06 \mathrm{Da}, \mathrm{K}+34.06 \mathrm{Da}$ & 22,7 & $-0,139$ & 3 & 1437,9 & GRHPR_MOUSE & DGREG4_MOUSE & Glyoxylate reductase/hydroxypyruvate reductase; & \\
\hline & MKVFVTGPLPAEGR & 0,5 & N-ter +34.06 Da, K+34.06 Da & 23,8 & 0,382 & 3 & 1568,9 & GRHPR_MOUSE & DGREG4_MOUSE & Glyoxylate reductase/hydroxypyruvate reductase; & \\
\hline VQNEETNEIQ & AVGEEHQQASIQGQAVADGTR & $-1,3$ & $\mathrm{~N}$-ter $+28.03 \mathrm{Da}$ & 22,2 & $-1,833$ & 3 & 2179,1 & GOLM1_MOUSE & & Golgi membrane protein 1; & $\begin{array}{l}\text { Golgi membrane protein GP73;Golgi } \\
\text { phosphoprotein 2; }\end{array}$ \\
\hline TSQLTAFPLG & APPPWPIPQDSSGPELGSR & 0,5 & $\mathrm{~N}$-ter $+28.03 \mathrm{Da}$ & 19,8 & 3,271 & 3 & 2015,0 & GORS1_MOUSE & & Golgi reassembly-stacking protein 1; & $\begin{array}{l}\text { Golgi peripheral membrane protein p65;Golgi } \\
\text { reassembly-stacking protein of } 65 \mathrm{kDa} \text {; }\end{array}$ \\
\hline $\begin{array}{l}\text { KLDKKQMELE } \\
\text { M }\end{array}$ & $\begin{array}{l}\text { SVSSELLEALR } \\
\text { RPOOAPVSGKVFIOR }\end{array}$ & $\begin{array}{l}1,0 \\
0,2\end{array}$ & $\begin{array}{l}\text { N-ter +34.06 Da } \\
\text { N-ter +34.06 Da K }+34.06 \text { Da }\end{array}$ & $\begin{array}{l}14,9 \\
16,9\end{array}$ & $\begin{array}{r}2,721 \\
-2,666\end{array}$ & $\begin{array}{l}2 \\
4\end{array}$ & $\begin{array}{l}1210,7 \\
1909,1\end{array}$ & $\begin{array}{l}\text { GOGA4_MOUSE } \\
\text { GOGA7 MOUSE }\end{array}$ & & $\begin{array}{l}\text { Golgin subfamily A member } 4 \text {; } \\
\text { Golgin subfamily A member } 7\end{array}$ & tGolgin-1; \\
\hline M & $\begin{array}{l}\text { PPQDAPVSGKVHIQR } \\
\text { AAAGAATDLEVVR }\end{array}$ & $\begin{array}{l}0,2 \\
0,1\end{array}$ & $\begin{array}{l}\mathrm{N}-\operatorname{ter}+34.06 \mathrm{Da}, \mathrm{K}+34.06 \mathrm{Da} \\
\mathrm{N} \text {-ter }+34.06 \mathrm{Da}\end{array}$ & $\begin{array}{l}16,9 \\
25,4\end{array}$ & $\begin{array}{l}-2,666 \\
-0,074\end{array}$ & $\begin{array}{l}4 \\
2\end{array}$ & $\begin{array}{l}1909,1 \\
1347,8\end{array}$ & $\begin{array}{l}\text { GOGA7_MOUSE } \\
\text { PIGS_MOUSE }\end{array}$ & & $\begin{array}{l}\text { Golgin subfamily A member 7; } \\
\text { GPI transamidase component PIG-S; }\end{array}$ & $\begin{array}{l}\text { Phosphatidylinositol-glycan biosynthesis class S } \\
\text { protein; }\end{array}$ \\
\hline LGLFSISAVE & NSATSIDVEESNR & 10,0 & N-ter +34.06 Da & 17,6 & 1,166 & 3 & 1454,7 & GPR98_MOUSE & B8JJEO_MOUSE & G-protein coupled receptor 98; & $\begin{array}{l}\text { Monogenic audiogenic seizure susceptibility } \\
\text { protein 1;Neurepin;Very large G-protein } \\
\text { coupled receptor 1; }\end{array}$ \\
\hline LTKLPGYPVK & EVKCDMEVSCPEGYTCCR & 0,5 & $\begin{array}{l}\text { N-ter +34.06 Da, K +34.06 Da, C } \\
\text { +57.02 Da }\end{array}$ & 52,6 & $-0,298$ & 3 & 2347,0 & GRN_MOUSE & Q3U9N4_MOUSE & E Granulins; & PC cell-derived growth factor;Proepithelin; \\
\hline LLTKLPGYPV & KEVKCDMEVSCPEGYTCCR & 1,4 & $\begin{array}{l}\text { N-ter +34.06 Da, K +34.06 Da, C } \\
\text { +57.02 Da }\end{array}$ & 15,9 & 3,064 & 4 & 2509,2 & GRN_MOUSE & Q3U9N4_MOUSE & E Granulins; & PC cell-derived growth factor;Proepithelin; \\
\hline
\end{tabular}




\begin{tabular}{|c|c|c|c|c|c|c|c|c|c|c|c|}
\hline Non Prime Site & Prime Site & $\begin{array}{c}\text { Fold change of } \\
\text { XPRESS ratio } \\
\left.\text { (Adam1 }{ }^{\Delta K c} / \mathrm{WT}\right) \\
\end{array}$ & Modifications & Hyperscore & $\begin{array}{c}\text { Mass error } \\
\text { in ppm }\end{array}$ & charge & $\begin{array}{c}\text { Precursor } \\
\text { neutral mass } \\
\text { in Da }\end{array}$ & Uniprot ID 1 & Uniprot ID 2 & Protein Name 1 & Protein Name 2 \\
\hline$\overline{\text { AGSVVTTAHS }}$ & $\begin{array}{l}\text { SLLNLKSMVEAITHR } \\
\text { S }\end{array}$ & 0,2 & N-ter +28.03 Da, K+28.03 Da & 27,2 & 0,057 & 3 & 1767,0 & $\begin{array}{l}\text { PA2GF_MOUSE } \\
\end{array}$ & & Group IIF secretory phospholipase A2; & Phosphatidylcholine 2-acylhydrolase $2 F$; \\
\hline м & TKVKAAPEESEAQAEGCSEER & 0,1 & $\begin{array}{l}\text { N-ter +28.03 Da, K+28.03 Da, C } \\
+57.02 \mathrm{Da}\end{array}$ & 27,5 & $-5,349$ & 4 & 2389,1 & NHP2_MOUSE & & H/ACA ribonucleoprotein complex subunit 2; & $\begin{array}{l}\text { Nucleolar protein family A member 2;snoRNP } \\
\text { protein NHP2; }\end{array}$ \\
\hline M & FLQYYLNEQGDR & $-0,2$ & $\mathrm{~N}$-ter +28.03 Da & 35,7 & $-1,641$ & 2 & 1703,8 & NOP10_MOUSE & & H/ACA ribonucleoprotein complex subunit 3; & $\begin{array}{l}\text { Nucleolar protein 10;Nucleolar protein family A } \\
\text { member 3;snoRNP protein NOP10; }\end{array}$ \\
\hline LIGDAAKNQV & ALNPQNTVFDAKR & $-0,2$ & N-ter +34.06 Da, K+34.06 Da & 20,9 & 1,101 & 3 & 1540,9 & HS71A_MOUSE & HS71B_MOUSE & Heat shock $70 \mathrm{kDa}$ protein $1 \mathrm{~A}$; & Heat shock 70 kDa protein 3;Hsp68; \\
\hline IDLGTTYSCV & GVFOHGKVEIIANDQGNR & 0,2 & $\mathrm{~N}$-ter +28.03 Da, $\mathrm{K}+28.03 \mathrm{Da}$ & 46,5 & $-3,823$ & 3 & 2037,1 & HS71A_MOUSE & HS71B_MOUSE & Heat shock 70 kDa protein 1A; & Heat shock 70 kDa protein 3;Hsp68; \\
\hline QTQTFTYSD & NQPGVLIQVYEGER & 0,2 & $\mathrm{~N}$-ter +34.06 Da & 16,4 & 1,404 & 3 & 1634,9 & HS71A_MOUSE & HS71B_MOUSE & Heat shock 70 kDa protein $1 \mathrm{~A}$; & Heat shock 70 kDa protein 3;Hsp68; \\
\hline AGVIAGLNVL & RIINEPTAAAIAYGLDR & 0,5 & $\mathrm{~N}-\operatorname{ter}+28.03 \mathrm{Da}$ & 31,0 & $-4,642$ & 3 & 1871,0 & HS71A_MOUSE & HS71B_MOUSE & Heat shock $70 \mathrm{kDa}$ protein $1 \mathrm{~A}$; & Heat shock 70 kDa protein 3;Hsp68; \\
\hline VEIIANDQGN & RTTPSYVAFTDTER & 0,1 & $\mathrm{~N}$-ter $+28.03 \mathrm{Da}$ & 27,4 & 0,836 & 3 & 1670,8 & HS71A_MOUSE & HS71B_MOUSE & Heat shock 70 kDa protein $1 \mathrm{~A}$ & Heat shock 70 kDa protein 3;Hsp 68; \\
\hline AVGIDLGTTY & SCVGVFaHGKVEIIANDQGNR & $-0,1$ & $\begin{array}{l}\text { N-ter +28.03 Da, K+28.03 Da, C } \\
+57.02 \mathrm{Da}\end{array}$ & 39,8 & $-0,838$ & 4 & 2383,2 & HS71A_MOUSE & HS71B_MOUSE & Heat shock 70 kDa protein $1 \mathrm{~A}$; & Heat shock 70 kDa protein 3;Hsp68; \\
\hline ткQТатFтTY & SDNQPGVLIQVYYGER & 0,4 & $\mathrm{~N}$-ter +34.06 Da & 49,2 & 0,435 & 2 & 1836,9 & HS71A_MOUSE & HS71B_MOUSE & Heat shock $70 \mathrm{kDa}$ protein $1 \mathrm{~A}$; & Heat shock 70 kDa protein 3;Hsp68; \\
\hline IEDGIFEVKS & TAGDTHLGGEDFDNR & 0,0 & $\mathrm{~N}$-ter +28.03 Da & 19,3 & $-5,995$ & 3 & 1631,7 & HS71A_MOUSE & HS71B_MOUSE & Heat shock $70 \mathrm{kDa}$ protein $1 \mathrm{~A}$; & Heat shock 70 kDa protein 3;Hsp68; \\
\hline NTTIPTKQTQ & TFTTYSDNQPGVLIQVYYGER & 0,2 & $\mathrm{~N}$-ter +34.06 Da & 20,5 & 4,076 & 3 & 2450,2 & HS71A_MOUSE & HS71B_MOUSE & Heat shock 70 kDa protein $1 \mathrm{~A}$; & Heat shock 70 kDa protein 3;Hsp68; \\
\hline IFEVKSTAGD & THLGGEDFDNR & 0,0 & $\mathrm{~N}$-ter +28.03 Da & 29,9 & $-5,114$ & 3 & 1287,6 & HS71A_MOUSE & HS71B_MOUSE & Heat shock 70 kDa protein $1 \mathrm{~A}$; & Heat shock 70 kDa protein 3;Hsp68; \\
\hline VMTVLIKRNT & TIPTKQTQTFTTYSDNQPGVLIQVYEGER & 1,7 & N-ter +34.06 Da, K +34.06 Da & 22,1 & $-0,709$ & 4 & 3381,8 & HS71A_MOUSE & HS71B_MOUSE & Heat shock 70 kDa protein $1 \mathrm{~A}^{\prime}$ & Heat shock 70 kDa protein 3;Hsp68; \\
\hline AYFNDSQRQA & TKDAGVIAGLNVLR & 0,1 & N-ter +34.06 Da, K +34.06 Da & 15,7 & $-2,138$ & 3 & 1494,0 & HS71A_MOUSE & HS71B_MOUSE & Heat shock 70 kDa protein $1 \mathrm{~A}^{\prime}$ & Heat shock 70 kDa protein 3;Hsp68; \\
\hline KRNTTIPTKQ & TQTFTTYSDNQPGVLIQVYEGER & 0,1 & $\mathrm{~N}$-ter +34.06 Da & 44,5 & 5,033 & 3 & 2679,3 & HS71A_MOUSE & HS71B_MOUSE & Heat shock 70 kDa protein $1 \mathrm{~A}^{\prime}$ & Heat shock 70 kDa protein 3;Hsp68; \\
\hline TІРТКQTQTF & TTYSDNQPGVLIQVYEGER & 1,4 & $\mathrm{~N}$-ter $+28.03 \mathrm{Da}$ & 53,6 & 0,409 & 3 & 2196,1 & HS71A_MOUSE & HS71B_MOUSE & Heat shock 70 kDa protein $1 \mathrm{~A} ;$ & Heat shock 70 kDa protein 3;Hsp68; \\
\hline IPTKQTQTFT & TYSDNQPGVLIQVYEGER & 0,5 & $\mathrm{~N}$-ter +28.03 Da & 41,1 & 1,954 & 3 & 2095,0 & HS71A_MOUSE & HS71B_MOUSE & Heat shock 70 kDa protein $1 \mathrm{~A}$; & Heat shock 70 kDa protein 3;Hsp68; \\
\hline GIDLGTTYSC & 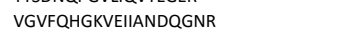 & 0,0 & $\mathrm{~N}-\operatorname{ter}+28.03 \mathrm{Da}, \mathrm{K}+28.03 \mathrm{Da}$ & 19,5 & $-6,448$ & 4 & 2136,1 & HS71A_MOUSE & HS71B_MOUSE & Heat shock 70 kDa protein 1A; & Heat shock 70 kDa protein 3;Hsp68; \\
\hline РтКQTQTFT & YSDNQPGVLIQVYEGER & 0,5 & $\mathrm{~N}-\operatorname{ter}+34.06 \mathrm{Da}$ & 30,8 & 5,741 & 3 & 2000,0 & HS71A_MOUSE & HS71B_MOUSE & Heat shock 70 kDa protein $1 \mathrm{~A}$; & Heat shock 70 kDa protein 3;Hsp68; \\
\hline DVTPLSLGIE & TAGGVMTVLIKR & $-0,1$ & N-ter +34.06 Da, K +34.06 Da & 25,4 & $-0,912$ & 3 & 1312,9 & HS71L_MOUSE & HSP7C_MOUSE & Heat shock 70 kDa protein 1-like; & $\begin{array}{l}\text { Heat shock } 70 \text { kDa-like protein 1;Spermatid- } \\
\text { specific heat shock protein } 70 \text {; }\end{array}$ \\
\hline VMTVLIKRNS & TIPTKQTQIFTTYSDNQPGVLIQVYEGER & $-3,1$ & $\mathrm{~N}$-ter +28.03 Da, K+28.03 Da & 21,7 & 7,443 & 4 & 3381,8 & HS71L_MOUSE & & Heat shock 70 kDa protein 1-like; & $\begin{array}{l}\text { Heat shock } 70 \text { kDa-like protein 1;5permatid- } \\
\text { specific heat shock protein 70; }\end{array}$ \\
\hline EIAEAYLGKA & TKDAGTIAGLNVLR & 0,2 & $\mathrm{~N}$-ter +28.03 Da, K+28.03 Da & 21,4 & $-2,959$ & 3 & 1483,9 & HSP7C_MOUSE & Q504P4_MOUSE & Heat shock cognate 71 kDa protein; & Heat shock 70 kDa protein 8; \\
\hline EELNADLFRG & TLDPVEKALR & $-0,3$ & $\mathrm{~N}$-ter $+28.03 \mathrm{Da}, \mathrm{K}+28.03 \mathrm{Da}$ & 32,2 & 1,500 & 3 & 1196,7 & HSP7C_MOUSE & Q504P4_MOUSE & Heat shock cognate 71 kDa protein; & Heat shock 70 kDa protein 8; \\
\hline GYVRPLPAAT & AEGPAAVTLAAPAFSR & 0,0 & $\mathrm{~N}$-ter +28.03 Da & 46,0 & $-0,128$ & 2 & 1555,8 & HSPB1_MOUSE & D3YZO6_MOUSE & Heat shock protein beta-1; & $\begin{array}{l}\text { Growth-related } 25 \text { kDa protein;Heat shock } 25 \\
\text { KDa protein;Heat shock } 27 \text { kDa protein;;25; }\end{array}$ \\
\hline DWYPAHSRLF & DQAFGVPR & 0,2 & $\mathrm{~N}$-ter +34.06 Da & 15,3 & 0,541 & 2 & 922,5 & HSPB1_MOUSE & D3YZO6_MOUSE & Heat shock protein beta-1; & $\begin{array}{l}\text { Growth-related } 25 \text { kDa protein;Heat shock } 25 \\
\text { kDa protein;Heat shock } 27 \text { kDa protein;p25; }\end{array}$ \\
\hline YVRPLPAATA & EGPAAVTLAAPAFSR & 2,1 & $\mathrm{~N}$-ter+28.03 Da & 24,8 & 0,067 & 2 & 1484,8 & HSPB1_MOUSE & D3YZO6_MOUSE & Heat shock protein beta-1; & $\begin{array}{l}\text { Growth-related } 25 \text { kDa protein; Heat shock } 25 \\
\text { kDa protein;Heat shock } 27 \text { kDa protein;p25; }\end{array}$ \\
\hline RDWYPAHSRL & FDQAFGVPR & 0,2 & $\mathrm{~N}$-ter +28.03 Da & 18,7 & $-0,094$ & 2 & 1063,6 & HSPB1_MOUSE & D3YZO6_MOUSE & Heat shock protein beta-1; & $\begin{array}{l}\text { Growth-related } 25 \text { kDa protein;Heat shock } 25 \\
\text { kDa protein;Heat shock } 27 \text { kDa protein; } 225 ;\end{array}$ \\
\hline DEWSQWFSAA & GWPGYVRPLPAATAEGPAAVTLAAPAFSR & 0,3 & N-ter $+28.03 \mathrm{Da}$ & 29,7 & $-9,403$ & 3 & 2921,5 & HSPB1_MOUSE & D3YZO6_MOUSE & Heat shock protein beta-1; & $\begin{array}{l}\text { Growth-related } 25 \text { kDa protein;Heat shock } 25 \\
\text { kDa protein;Heat shock } 27 \text { kDa protein;p25; }\end{array}$ \\
\hline LAAPAFSRAL & NRQLSSGVSEIR & 0,1 & N-ter +28.03 Da & 35,3 & $-0,727$ & 3 & 1372,7 & HSPB1_MOUSE & D3YZO6_MOUSE & Heat shock protein beta-1; & $\begin{array}{l}\text { Growth-related } 25 \text { kDa protein;Heat shock } 25 \\
\text { kDa protein;Heat shock } 27 \text { kDa protein;p25; }\end{array}$ \\
\hline APAFSRALNR & QLSSGVSEIR & 0,2 & N-ter +34.06 Da & 26,4 & $-0,180$ & 2 & 1108,6 & HSPB1_MOUSE & D3YZO6_MOUSE & Heat shock protein beta-1; & $\begin{array}{l}\text { Growth-related } 25 \text { kDa protein;Heat shock } 25 \\
\text { kDa protein;Heat shock } 27 \text { kDa protein;p25; }\end{array}$ \\
\hline PFRDWYPAHS & RLFDQAFGVPR & 0,1 & N-ter +34.06 Da & 31,1 & $-3,205$ & 3 & 1338,8 & HSPB1_MOUSE & D3YZO6_MOUSE & Heat shock protein beta-1; & $\begin{array}{l}\text { Growth-related } 25 \text { kDa protein;Heat shock } 25 \\
\text { kDa protein;Heat shock } 27 \text { kDa protein;p25; }\end{array}$ \\
\hline TERRVPFSLL & RSPSWEPFR & 0,2 & N-ter +34.06 Da & 23,8 & 1,670 & 3 & 1194,6 & HSPB1_MOUSE & D3YZO6_MOUSE & Heat shock protein beta-1; & $\begin{array}{l}\text { Growth-related } 25 \text { kDa protein;Heat shock } 25 \\
\text { kDa protein;Heat shock } 27 \text { kDa protein;p25; }\end{array}$ \\
\hline RLPDEWSQWF & SAAGWPGYVRPLPAATAEGPAAVTLAAPAFSR & 0,3 & N-ter +34.06 Da & 34,5 & $-8,070$ & 3 & 3156,7 & HSPB1_MOUSE & D3YZO6_MOUSE & Heat shock protein beta-1; & $\begin{array}{l}\text { Growth-related } 25 \text { kDa protein;Heat shock } 25 \\
\text { KDa protein;Heat shock } 27 \text { kDa protein;p25; }\end{array}$ \\
\hline
\end{tabular}




\begin{tabular}{|c|c|c|c|c|c|c|c|c|c|c|c|}
\hline Non Prime Site & Prime Site & 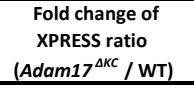 & Modifications & Hyperscore & $\begin{array}{c}\text { Mass error } \\
\text { in ppm }\end{array}$ & Charge & $\begin{array}{c}\text { Precursor } \\
\text { neutral mass } \\
\text { in Da }\end{array}$ & Uniprot ID 1 & Uniprot ID 2 & Protein Name 1 & Protein Name 2 \\
\hline PGYVRPLPAA & TAEGPAAVTLAAPAFSR & 0,1 & $\mathrm{~N}$-ter +28.03 Da & 35,7 & 0,362 & 2 & 1656,9 & HSPB1_MOUSE & D3YZ06_MOUSE & Heat shock protein beta-1; & $\begin{array}{l}\text { Growth-related } 25 \text { kDa protein;Heat shock } 25 \\
\text { kDa protein;Heat shock } 27 \text { kDa protein;p25; }\end{array}$ \\
\hline IKLGLGIDED & DPTVDDTSAAVTEEMPPLEGDDDTSR & $-0,7$ & $\mathrm{~N}$-ter +28.03 Da & 30,2 & $-5,191$ & 3 & 2790,2 & HS90A_MOUSE & & Heat shock protein HSP 90-alpha; & $\begin{array}{l}\text { Heat shock } 86 \text { kDa;Tumor-specific } \\
\text { transplantation } 8 \text { kDa antigen; }\end{array}$ \\
\hline SEDLPINISR & EMLQQSKILKVIR & $-5,4$ & $\mathrm{~N}$-ter +28.03 Da, K+28.03 Da & 22,0 & 1,674 & 4 & 1669,0 & HS9OA_MOUSE & HS9OB_MOUSE & Heat shock protein HSP 90-alpha; & $\begin{array}{l}\text { Heat shock } 86 \text { kDa;Tumor-specific } \\
\text { transplantation } 86 \text { kDa antigen; }\end{array}$ \\
\hline KYIDQEELINK & TKPIWTR & 0,2 & $\mathrm{~N}$-ter +34.06 Da, K +34.06 Da & 16,9 & 0,618 & 3 & 968,7 & HS9OA_MOUSE & HS9OB_MOUSE & Heat shock protein HSP 90-alpha; & $\begin{array}{l}\text { Heat shock } 86 \text { kDa;Tumor-specific } \\
\text { transplantation } 86 \text { kDa antigen; }\end{array}$ \\
\hline QLMSLINTFF & YSNKEIFLR & 0,1 & $\mathrm{~N}$-ter +34.06 Da, K+34.06 Da & 17,1 & $-1,694$ & 3 & 1236,8 & HS9OA_MOUSE & HS9OB_MOUSE & Heat shock protein HSP 90-alpha; & $\begin{array}{l}\text { Heat shock } 86 \text { kDa; Tumor-specific } \\
\text { transplantation } 86 \text { kDa antigen; }\end{array}$ \\
\hline IKLGLGIDED & EVTAEEPSAAVPDEIPPLEGDEDASR & 0,0 & N-ter +34.06 Da & 17,8 & 3,262 & 3 & 2756,3 & HS9OB_MOUSE & & Heat shock protein HSP 90-beta; & $\begin{array}{l}\text { Heat shock } 84 \text { kDa; Tumor-specific } \\
\text { transplantation } 84 \text { kDa antigen; }\end{array}$ \\
\hline LYVRRVFIMD & SCDELPEYYLNFIR & $-0,3$ & N-ter +28.03 Da, C +57.02 Da & 15,9 & 0,334 & 2 & 1795,9 & HS9OB_MOUSE & E9Q3D6_MOUSE & Heat shock protein HSP 90-beta; & $\begin{array}{l}\text { Heat shock } 84 \text { kDa; Tumor-specific } \\
\text { transplantation } 84 \text { kDa antigen; }\end{array}$ \\
\hline RRVKEVVKKH & SQFIGYPITLYLEKER & $-0,9$ & N-ter +34.06 Da, K+34.06 Da & 23,5 & 2,910 & 3 & 2024,2 & HS90B_MOUSE & & Heat shock protein HSP 90-beta; & $\begin{array}{l}\text { Heat shock } 84 \text { kDa;Tumor-specific } \\
\text { transplantation } 84 \text { kDa antigen; }\end{array}$ \\
\hline RMKETQKSIY & YITGESKEQVANSAFVER & 0,7 & $\mathrm{~N}$-ter $+28.03 \mathrm{Da}, \mathrm{K}+28.03 \mathrm{Da}$ & 22,5 & $-0,384$ & 3 & 2083,1 & HS90B_MOUSE & E9Q00__MOUSE & Heat shock protein HSP 90-beta; & $\begin{array}{l}\text { Heat shock } 84 \text { kDa;Tumor-specific } \\
\text { transplantation } 84 \text { kDa antigen; }\end{array}$ \\
\hline TDFQANLAQM & EEKPVPAAPVPSPVAPAPVPSR & 0,3 & $\mathrm{~N}$-ter $+28.03 \mathrm{Da}, \mathrm{K}+28.03 \mathrm{Da}$ & 34,7 & 6,888 & 3 & 2247,3 & HN1_MOUSE & & Hematological and neurological expressed 1 protein; & \\
\hline LRPPGGGSNF & SLGFDEPAEQPVR & 0,2 & $\mathrm{~N}$-ter +34.06 Da & 28,2 & $-0,203$ & 2 & 1477,8 & HN1_MOUSE & & Hematological and neurological expressed 1 protein; & \\
\hline $\begin{array}{l}\text { SGEDKSNIKA } \\
\text { M }\end{array}$ & $\begin{array}{l}\text { AWGKIGGHGAEYGAEALER } \\
\text { VLSGEDKSNIKAAWGKIGGHGAEYGAEALER }\end{array}$ & $\begin{array}{c}-0,2 \\
0,1\end{array}$ & $\begin{array}{l}\text { N-ter }+34.06 \mathrm{Da}, \mathrm{K}+34.06 \mathrm{Da} \\
\mathrm{N} \text {-ter }+34.06 \mathrm{Da} \mathrm{K}+34.06 \mathrm{Da}\end{array}$ & $\begin{array}{l}32,1 \\
274\end{array}$ & $\begin{array}{l}-4,064 \\
-0,328\end{array}$ & $\begin{array}{l}3 \\
5\end{array}$ & $\begin{array}{l}2039,1 \\
33989\end{array}$ & $\begin{array}{l}\text { HBA_MOUSE } \\
\text { HBA MOUSE }\end{array}$ & $\begin{array}{l}\text { Q91VB8_MOUSE } \\
\text { Q91VB8 MOUSE }\end{array}$ & Hemoglobin subunit alpha; & Alpha-globin;Hemoglobin alpha chain; \\
\hline м & VHLTDAEKAAVSCLWGKVNSDEVGGEALGR & 0,0 & $\begin{array}{l}\text { N-ter +28.03 Da, } \mathrm{K}+28.03 \mathrm{Da}, \mathrm{C} \\
+57.02 \mathrm{Da}\end{array}$ & 40,5 & 2,119 & 4 & $\begin{array}{l}3348,9 \\
3251,7\end{array}$ & HBB1_MOUSE & QTivo__Nivose & $\begin{array}{l}\text { Hemogiobin subunit alpha; } \\
\text { Hemoglobin subunit beta-1; }\end{array}$ & $\begin{array}{l}\text { Beta-1-globin;Hemoglobin beta-1 } \\
\text { chain;Hemoglobin beta-major chain; }\end{array}$ \\
\hline DAEKAAVSCL & WGKVNSDEVGGEALGR & 0,0 & $\mathrm{~N}$-ter +28.03 Da, K +28.03 Da & 27,0 & 3,407 & 3 & 1728,9 & HBB1_MOUSE & & Hemoglobin subunit beta-1; & $\begin{array}{l}\text { Beta-1-globin;Hemoglobin beta-1 } \\
\text { chain;Hemoglobin beta-major chain; }\end{array}$ \\
\hline M & VHLTDAEKSAVSCLWAKVNPDEVGGEALGR & $-0,2$ & $\begin{array}{l}\text { N-ter }+28.03 \mathrm{Da}, \mathrm{K}+28.03 \mathrm{Da}, \mathrm{C} \\
+57.02 \mathrm{Da}\end{array}$ & 42,6 & 0,334 & 4 & 3291,7 & HBB2_MOUSE & & Hemoglobin subunit beta-2; & $\begin{array}{l}\text { Beta-2-globin;Hemoglobin beta-2 } \\
\text { chain;Hemoglobin beta-minor chain; }\end{array}$ \\
\hline M & VNFTAEEKTLINGLWSKVNVEEVGGEALGR & $-2,3$ & $\mathrm{~N}$-ter $+28.03 \mathrm{Da}, \mathrm{K}+28.03 \mathrm{Da}$ & 27,3 & $-5,705$ & 5 & 3342,8 & HBE_MOUSE & Q9CR49_MOUSE & Hemoglobin subunit epsilon-Y2; & Epsilon-Y2-globin;Hemoglobin epsilon-Y2 chain; \\
\hline KDLKDYFTKF & GEVVDCTIKMDPNTGR & 0,5 & $\begin{array}{l}\text { N-ter +34.06 Da, K+34.06 Da, C } \\
\text { +57.02 Da }\end{array}$ & 26,7 & 0,000 & 3 & 1859,0 & ROAA_MOUSE & Q80XR6_MOUSE & Heterogeneous nuclear ribonucleoprotein $\mathrm{A} / \mathrm{B}$; & CArG-binding factor-A; \\
\hline SGLRGHFEAF & GTLTDCVVVVNPQTKR & $-0,5$ & $\begin{array}{l}\text { N-ter +28.03 Da, K+28.03 Da, C } \\
+57.02 \mathrm{Da}\end{array}$ & 17,6 & 2,656 & 3 & 1842,0 & ROAO_MOUSE & & Heterogeneous nuclear ribonucleoprotein $\mathrm{A} 0$; & \\
\hline YNLRDYFEKY & GKIETIEVI & 0,2 & $\mathrm{~N}$-ter +34.06 Da, K+34.06 Da & 24,1 & $-0,671$ & 3 & 1486,8 & ROA3_MOUSE & B2RXM2_MOUSE & Heterogeneous nuclear ribonucle & \\
\hline $\mathrm{DFC}$ & & 0,2 & $\mathrm{~N}$-ter $+28.03 \mathrm{Da}, \mathrm{K}+28.03 \mathrm{Da}$ & 26,4 & $-0,7$ & 3 & 1469,7 & ROA3_MOUSE & B2RXM2_MOUSE & Heterogeneous nuclear ribon & \\
\hline GNYSGQQQSN & YGPMK & 0,3 & N-ter +34.06 Da, K & 17,4 & 8,179 & 3 & 1280,7 & ROA3_MOUSE & B2RXM2_MOUSE & Heterogeneous nuclear ribonucleoprotein A3; & \\
\hline & MLGPE & 0,1 & N-ter +28.03 & 32,2 & 0,000 & 3 & 1659,9 & HNRPF_MOUSE & J3QMQS_MOUSE & Heterogeneous nuclear ribonucle & \\
\hline GGQRIKQIRH & ESGASIKIDEPLEGS & 0,0 & $\mathrm{~N}$-ter +34.06 Da, K+34.06 Da & 17,9 & $-5,245$ & 3 & 1999,0 & HNRPK_MOUSE & B2M1R6_MOUSE & Heterogeneous nuclear ribonucleoprotein K; & \\
\hline LGGPIITTQV & TIPKDLAGSIIGKGGQR & 0,2 & $\mathrm{~N}$-ter $+28.03 \mathrm{Da}, \mathrm{K}+28.03 \mathrm{Da}$ & 17,1 & $-0,890$ & 3 & 1794,1 & HNRPK_MOUSE & B2M1R6_MOUSE & Heterogeneous nuclear ribonucleoprotein $\mathrm{K}_{\text {; }}$ & \\
\hline HHLRDYFEEY & GKIDTIEITDR & 0,7 & $\mathrm{~N}$-ter +34.06 Da, K+34.06 Da & 20,5 & $-3,186$ & 3 & 1440,9 & ROA2_MOUSE & & Heterogeneous nuclear ribonucleoproteins $\mathrm{A} 2 / \mathrm{B} 1$ & \\
\hline MSTNTDLSL & SSYDEGQGSKFIR & 0,9 & $\mathrm{~N}$-ter $+28.03 \mathrm{Da}, \mathrm{K}+28.03 \mathrm{Da}$ & 17,6 & $-2,873$ & 3 & 1528,8 & HIGIA_MOUSE & J3QPX3_MOUSE & HIG1 domain family member $1 \mathrm{~A}$, mitochondrial; & Hypoxia-inducible gene 1 protein; \\
\hline QEAGEEGTAP & SANGDTKVEEAQR & $-6,8$ & $\mathrm{~N}$-ter $+28.03 \mathrm{Da}, \mathrm{K}+28.03 \mathrm{Da}$ & 20,6 & 1,026 & 2 & 1459,7 & HMGN3_MOUSE & & $\begin{array}{l}\text { High mobility group nucleosome-binding domain- } \\
\text { containing protein 3; }\end{array}$ & \\
\hline EGVFLYSKYR & EPEKYIALDGDSLSTE & 0,7 & N-ter +34.06 Da, K+34.06 Da & 47,0 & 2,691 & 4 & 2820,6 & HUTH_MOUSE & F8WH73_MOUSE & Histidine ammonia-lyase; & \\
\hline PEKYIALDGD & SLSTEDLVNLGKGR & 1,8 & $\mathrm{~N}$-ter $+34.06 \mathrm{Da}, \mathrm{K}+34.06 \mathrm{Da}$ & 16,2 & 4,619 & 3 & 1555,9 & HUTH_MOUSE & F8WH73_MOUSE & Histidine ammonia-lyase; & \\
\hline MADEIAKAQV & AQPGGDTIFGKIIIR & 0,4 & $\mathrm{~N}$-ter $+34.06 \mathrm{Da}, \mathrm{K}+34.06 \mathrm{Da}$ & 19,9 & 0,389 & 3 & 1539,9 & HINT1_MOUSE & BOR1E3_MOUSE & Histidine triad nucleotide-binding protein 1; & $\begin{array}{l}\text { Adenosine 5'-monophosphoramidase;Protein } \\
\text { kinase C inhibitor 1;Protein kinase C-interacting } \\
\text { protein 1; }\end{array}$ \\
\hline PRHLQL & DEELNKL & $-0,1$ & $\mathrm{~N}$-ter +28.03 Da, K+28.03 Da & 30 & 1,7 & 3 & 1241 & H2A1F_MOUSE & H2A1H_MOUSE & Histone $\mathrm{H} 2 \mathrm{~A}$ & \\
\hline & & 0,6 & N-te & & & 2 & 176 & H2A1 & USE & & \\
\hline & LEYLT & 0, & $\mathrm{~N}-\mathrm{t}$ & 26 & & 3 & $188 \mathrm{C}$ & H2A1 & USE & & \\
\hline & & 0, & & & & 2 & 136 & HZA1 & & & \\
\hline & VLE & 0, & $\mathrm{~N}-\mathrm{-}$ & 53 & & 3 & 19 & H2A1 & & & \\
\hline & & 0,9 & & & & 2 & 1637,9 & & & & \\
\hline GISSKAMGIM & NSFVNDIFER & 0,5 & a & 17,9 & $\begin{array}{l}-0,551 \\
-0,10\end{array}$ & 2 & 1267,6 & H2B1B_MOUSE & H2B1C_MOUSE & Histone $H 2 B$ type 1-B; & h2B-143; \\
\hline LQEASEAYLV & GLFEDTNLCAIHAKR & 0,2 & N-ter $+28.03 \mathrm{Da}$, & 25,6 & $-2,605$ & 4 & 1799,9 & H31_MOUSE & H32_MOUSE & Histone H3.1; & \\
\hline
\end{tabular}




\begin{tabular}{|c|c|c|c|c|c|c|c|c|c|c|c|}
\hline Non Prime Site & Prime Site & 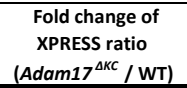 & Modifications & Hyperscore & $\begin{array}{l}\text { Mass error } \\
\text { in ppm }\end{array}$ & charge & $\begin{array}{c}\text { Precursor } \\
\text { neutral mass } \\
\text { in Da }\end{array}$ & Uniprot ID 1 & Uniprot ID 2 & Protein Name 1 & Protein Name 2 \\
\hline $\begin{array}{l}\text { NLCAIHAKRV } \\
\end{array}$ & TIMPKDIQLAR & 0,0 & $\mathrm{~N}$-ter +34.06 Da, K+34.06 Da & 15,0 & 6,785 & 3 & 1352,9 & $\overline{\text { H31_MOUSE }}$ & $\begin{array}{l}\text { H32_MOUSE } \\
\end{array}$ & Histone H3.1; & \\
\hline QSAAIGALQE & ASEAYLVGLFEDTNLCAIHAKR & 0,4 & $\begin{array}{l}\text { N-ter }+28.03 \text { Da, K+ } 28.03 \text { Da, C } \\
+57.02 \text { Da }\end{array}$ & 35,9 & 0,867 & 4 & 2533,3 & H32_MOUSE & H33_MOUSE & Histone H3.2; & \\
\hline SAAIGALQEA & SEAYLVGLFEDTNLCAIHAKR & $-0,1$ & $\begin{array}{l}\text { N-ter +28.03 Da, K+28.03 Da, C } \\
+57.02 \mathrm{Da}\end{array}$ & 26,2 & $-4,785$ & 4 & 2462,3 & H32_MOUSE & H33_MOUSE & Histone H3.2; & \\
\hline RLARRGGVKR & ISGLIYEETR & $-2,1$ & $\mathrm{~N}$-ter $+28.03 \mathrm{Da}$ & 21,6 & $-0,909$ & 2 & 1207,6 & H4_MOUSE & & Histone $\mathrm{H} 4$; & \\
\hline RRLARRGGVK & RISGLIYEETR & $-2,1$ & $\mathrm{~N}$-ter $+28.03 \mathrm{Da}$ & 23,1 & 1,317 & 3 & 1363,8 & H4_MOUSE & & Histone $\mathrm{H} 4$; & \\
\hline EGDSDRDTGN & DWASSSSEANSR & 6,8 & N-ter +34.06 Da & 15,6 & $-5,332$ & 2 & 1329,6 & EZH1_MOUSE & & Histone-lysine N-methyltransferase EZH1; & ENX-2; Enhancer of zeste homolog $1 ;$ \\
\hline TLKVKEEEIE & RLKQEIDNAR & 0,5 & $\mathrm{~N}$-ter +28.03 Da, K+28.03 Da & 17,3 & 0,923 & 3 & 1297,8 & HOME1_MOUSE & E9Q018_MOUSE & Homer protein homolog 1 & $\begin{array}{l}\text { VASP/Ena-related gene up-re } \\
\text { seizure and LTP } 1 \text {; }\end{array}$ \\
\hline STaWSHGSGS & EQSSGLGHYGSTSGQTASSTR & 0,0 & N-ter $+28.03 \mathrm{Da}$ & 28,3 & 0,282 & 3 & 2125,0 & HORN_MOUSE & HORN_MOUSE & Hornerin; & \\
\hline QNYNSTRQGC & GQGKSSGSEQYGASSGQSSGCSSGQSTR & 1,0 & $\begin{array}{l}\text { N-ter +28.03 Da, K+28.03 Da, C } \\
\text { +57.02 Da }\end{array}$ & 23,1 & 4,517 & 3 & 2764,2 & HORN_MOUSE & E9QNP3_MOUSE & Hornerin; & \\
\hline SSSTQWSHGS & GSEQSSGLGHYGSTSGQTASSTR & 0,3 & N-ter +28.03 Da & 52,6 & 0,528 & 3 & 2269,0 & HORN_MOUSE & HORN_MOUSE & Hornerin; & \\
\hline SQSSSTQWSH & GSGSEQSSGLGHYGSTSGQTASSTR & 0,1 & N-ter+28.03 Da & 25,9 & 0,869 & 3 & 2413,1 & HORN_MOUSE & HORN_MOUSE & Hornerin; & \\
\hline RSRSTSRESS & GSQEYSSGSSEEPGFTHGSGR & 0,1 & $\mathrm{~N}-\mathrm{ter}+28.03 \mathrm{Da}$ & 27,4 & $-5,753$ & 3 & 2169,9 & HORN_MOUSE & E9QNP3_MOUSE & Hornerin; & \\
\hline TQWSHGSGSE & QSSGLGHYGSTSGQTASSTR & 0,2 & $\mathrm{~N}$-ter $+34.06 \mathrm{Da}$ & 48,3 & 0,349 & 3 & 2002,0 & HORN_MOUSE & HORN_MOUSE & Hornerin; & \\
\hline TQWSHGSGSE & QSSGLGQYGSPSGQTSSSTRQGSGQGQASGSGR & 1,1 & N-ter+34.06 Da & 33,0 & $-4,897$ & 3 & 3162,5 & HORN_MOUSE & E9QNP3_MOUSE & Hornerin; & \\
\hline SSTQWSHGSG & SEQSSGLGHYGSTSGQTASSTR & 0,4 & $\mathrm{~N}$-ter $+28.03 \mathrm{Da}$ & 67,8 & 1,716 & 3 & 2212,0 & HORN_MOUSE & HORN_MOUSE & Hornerin; & \\
\hline RGHGSRSGRS & SGLGQYGSPSGQTSSSTRQGSGQGQASGSGR & 0,5 & $\mathrm{~N}$-ter +34.06 Da & 25,3 & 1,695 & 3 & 2947,4 & HORN_MOUSE & HORN_MOUSE & Hornerin; & \\
\hline QSSSTQWSHG & SGSEQSSGLGHYGSTSGQTASSTR & 0,5 & $\mathrm{~N}$-ter +34.06 Da & 77,6 & $-0,381$ & 3 & 2362,1 & HORN_MOUSE & HORN_MOUSE & Hornerin; & \\
\hline RRSRSTSRES & SGSQEYSSGSSEEPGFTHGSGR & 0,2 & $\mathrm{~N}$-ter +34.06 Da & 63,6 & $-6,840$ & 3 & 2263,0 & HORN_MOUSE & E9QNP3_MOUSE & Hornerin; & \\
\hline SRSTSRESSG & SQEYSSGSSEEPGFTHGSGR & 0,1 & $\mathrm{~N}$-ter $+34.06 \mathrm{Da}$ & 49,2 & 3,535 & 3 & 2118,9 & HORN_MOUSE & E9QNP3_MOUSE & Hornerin; & \\
\hline QRGHGSRSGR & SSGLGQYGSPSGQTSSSTRQGSGQGQASGSGR & 0,2 & N-ter +34.06 Da & 30,2 & 0,955 & 3 & 3034,4 & HORN_MOUSE & HORN_MOUSE & Hornerin; & \\
\hline KKTHVSGSGH & SSSTGKYTSTSGQNYNSTR & 0,3 & $\mathrm{~N}$-ter +34.06 Da, K+34.06 Da & 51,8 & $-5,487$ & 3 & 2093,0 & HORN_MOUSE & E9QNP3_MOUSE & Hornerin; & \\
\hline M & LPNTGKLAGCTVFITGASR & 0,4 & $\begin{array}{l}\text { N-ter +28.03 Da, K +28.03 Da, C } \\
+57.02 \mathrm{Da}\end{array}$ & 30,2 & 4,182 & 3 & 2149,2 & HSDL2_MOUSE & & Hydroxysteroid dehydrogenase-like protein 2; & \\
\hline ERTISKIKGL & VRAPQVYILPPPAEQLSR & 1,9 & $\mathrm{~N}$-ter $+34.06 \mathrm{Da}$ & 32,3 & 6,038 & 3 & 2067,2 & IGG2B_MOUSE & & Ig gamma-2B chain C region; & \\
\hline D & VVMTQTPLSLPVSLGDQASISCR & 1,6 & $\mathrm{~N}$-ter + 34.06 Da, C+57.02 Da & 31,6 & $-3,141$ & 3 & 2607,3 & KV2A7_MOUSE & & Ig kappa chain V-II region 26-10; & \\
\hline & NIVLTQSPASLAVSLGQR & 0,8 & $\mathrm{~N}$-ter $+28.03 \mathrm{Da}$ & 37,7 & $-0,319$ & 3 & 1881,1 & KV3A9_MOUSE & KVЗAA_MOUSE & Ig kappa chain V-III region MOPC 63; & \\
\hline $\mathrm{D}$ & IVLTQSPASLAVSLGQR & 0,4 & $\mathrm{~N}$-ter +34.06 Da & 45,1 & 1,904 & 3 & 1888,1 & KV3A1_MOUSE & KV3A2_MOUSE & Ig kappa chain V-III region PC 2880/PC 1229; & \\
\hline D & IQMTOTTSSLSASLGDR & 0,2 & N-ter +28.03 Da & 26,0 & $-9,532$ & 3 & 1937,9 & KVSAA_MOUSE & KVSAB_MOUSE & Ig kappa chain V-V region MOPC 173; & \\
\hline LLWFPGARC & DIQMTQSPSSISASLGER & 0,3 & N-ter +34.06 Da & 28,3 & 7,463 & 3 & 1940,0 & KVSA7_MOUSE & KVSA8_MOUSE & Ig kappa chain V-V region MOPC 41; & \\
\hline SEGHGSELDR & YGQGQCHDPQLGAGAGQR & $-5,2$ & $\mathrm{~N}$-ter $+28.03 \mathrm{Da}, \mathrm{C}+57.02 \mathrm{Da}$ & 16,7 & 1,192 & 3 & 1926,9 & IGFN1_MOUSE & & $\begin{array}{l}\text { Immunoglobulin-like and fibronectin type III domain- } \\
\text { containing protein 1; }\end{array}$ & \\
\hline LLPLAGAQA & AIVFIKEPSSQDALQGR & $-0,2$ & $\mathrm{~N}$-ter +34.06 Da, K+34.06 Da & 51,0 & 1,037 & 3 & 1926,1 & PTK7_MOUSE & & Inactive tyrosine-protein kinase 7; & $\begin{array}{l}\text { Protein chuzhoi;:Protein-tyrosine kinase } \\
\text { 7; Pseudo tyrosine kinase receptor 7;Tyrosine- } \\
\text { protein kinase-like } 7 \text {; } \\
\text { Non-canonical purine NTP pyrophosphatase } \\
\text { \{ECO:0000255|HAMAP-Rule:MF_03148\};Non- } \\
\text { standard purine NTP pyrophosphatase }\end{array}$ \\
\hline DKSAYALCTF & ALSTGDPSQPVLLFR & 1,8 & N-ter +34.06 Da & 19,1 & $-0,122$ & 2 & 1633,9 & ITPA_MOUSE & & $\begin{array}{l}\text { Inosine triphosphate pyrophosphatase } \\
\text { \{ECO:0000255|HAMAP-Rule:MF_03148\}; }\end{array}$ & $\begin{array}{l}\text { Rule:MF_03148\};:Nucleoside-triphosphate } \\
\text { diphosphatase ECOO0000255|HAMAP- } \\
\text { Rule:MF_03148\};:Nucleoside-triphosphate } \\
\text { pyrophosphatase \{ECO:0000255|HAMAP- } \\
\text { Rule:MF_03148\}; }\end{array}$ \\
\hline DDLTNLVVKL & FSEVENKNVPLPEFPEHPFQEEHLR & 1,3 & $\mathrm{~N}$-ter $+34.06 \mathrm{Da}, \mathrm{K}+34.06 \mathrm{Da}$ & 24,4 & 2,948 & 5 & 3115,6 & IDE_MOUSE & FGRPJg_MOUSE & Insulin-degrading enzyme; & 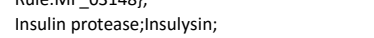 \\
\hline STMSNPAIQR & IEDQIVKSPEDK & $-0,3$ & $\mathrm{~N}$-ter +34.06 Da, K+34.06 Da & 20,8 & 0,066 & 3 & 1501,9 & IDE_MOUSE & FGRPJ9_MOUSE & Insulin-degrading enzyme; & Insulin protease;Insulysin; \\
\hline RANGIQGLRF & "IOSEKPPHYLESR & 1,4 & $\mathrm{~N}-\operatorname{ter}+34.06 \mathrm{Da}, \mathrm{K}+34.06 \mathrm{Da}$ & 22,5 & 1,753 & 4 & 1764,0 & IDE MOUSE & FGRPJ9 MOUSE & Insulin-degrading enzyme; & Insulin protease:Insulysin: \\
\hline VVGEFPSQND & INLSEAPPLPQPEVIHNMTEFKR & 3,5 & $\mathrm{~N}$-ter $+34.06 \mathrm{Da}, \mathrm{K}+34.06 \mathrm{Da}$ & 17,5 & 9,226 & 4 & 2727,5 & IDE_MOUSE & FGRPJ9_MOUSE & Insulin-degrading enzyme; & Insulin protease:IInsulysin; \\
\hline ANGIQGLRFI & IQSEKPPHYLESR & 1,4 & $\mathrm{~N}$-ter $+34.06 \mathrm{Da}, \mathrm{K}+34.06 \mathrm{Da}$ & 17,7 & $-3,686$ & 4 & 1650,9 & IDE_MOUSE & FGRPJ9_MOUSE & Insulin-degrading enzyme; & Insulin protease;Insulysin; \\
\hline HVEDIILHMF & QYIQKLR & 0,6 & $\mathrm{~N}-\mathrm{ter}+34.06 \mathrm{Da}, \mathrm{K}+34.06 \mathrm{Da}$ & 18,5 & 6,18 & 3 & 1015,7 & IDE_MOUSE & FGRPJ9_MOUSE & Insulin-degrading enzyme; & Insulin protease;Insulysin; \\
\hline PLLPSQLVRY & REVQLPDR & 0,1 & $\mathrm{~N}$-ter $+34.06 \mathrm{Da}$ & 19,5 & 3,910 & 3 & 1045,6 & IDE_MOUSE & FGRPJ9_MOUSE & Insulin-degrading enzyme; & Insulin protease;Insulysin; \\
\hline GTGNKYTLET & RPNQEGIDVR & 0,3 & $\mathrm{~N}$-ter $+34.06 \mathrm{Da}$ & 19,1 & 0,16 & 3 & 1216,7 & IDE_MOUSE & FGRPJ9_MOUSE & Insulin-degrading enzyme; & Insulin protease;Insulysin; \\
\hline IISEPCFNTL & RTKEQLGYIVFSGPR & 1,6 & $\mathrm{~N}$-ter $+34.06 \mathrm{Da}, \mathrm{K}+34.06 \mathrm{Da}$ & 15,6 & 1,97 & 4 & 1818,1 & IDE_MOUSE & FGRPJ9_MOUSE & Insulin-degrading enzyme; & Insulin protease;Insulysin; \\
\hline FGTGNKYTLE & TRPNQEGIDVR & $-1,0$ & N-ter +34.06 Da & 21,7 & 4,92 & 3 & 1317,7 & IDE_MOUSE & FGRPJ9_MOUSE & Insulin-degrading enzyme; & Insulin protease:IInsulysin: \\
\hline KQAALGVMQM & VEDTLEHAHTKPLLPSQLVR & 1,5 & $\mathrm{~N}$-ter $+34.06 \mathrm{Da}, \mathrm{K}+34.06 \mathrm{Da}$ & 30,8 & 0,04 & 4 & 2463,5 & IDE_MOUSE & FGRPJ9_MOUSE & Insulin-degrading enzyme; & Insulin protease;Insulysin; \\
\hline NYDRDNIEVA & YLKTLTKDDIIR & 1,7 & $\mathrm{~N}-\mathrm{ter}+34.06 \mathrm{Da}, \mathrm{K}+34.06 \mathrm{Da}$ & 26,5 & 1,074 & 3 & 1580,0 & IDE_MOUSE & FGRPJ9_MOUSE & Insulin-degrading enzyme; & Insulin protease;Insulysin; \\
\hline TLTKDDIIRF & YQEMLAVDAPR & 1,1 & $\mathrm{~N}$-ter $+28.03 \mathrm{Da}$ & 21,6 & $-0,681$ & 2 & 1319,7 & IDE_MOUSE & FGRPJ9_MOUSE & Insulin-degrading enzyme; & Insulin protease; Insulysin; \\
\hline RETRGIQKR & EASNCFTIR & 0,9 & $\mathrm{~N}$-ter $+34.06 \mathrm{Da}, \mathrm{C}+57.02 \mathrm{Da}$ & 29,6 & 1,589 & 2 & 1130,6 & ITM2B_MOUSE & & Integral membrane protein $2 \mathrm{~B}$; & $\begin{array}{l}\text { Immature BR12;Protein E25B; Transmembrane } \\
\text { protein BRI: }\end{array}$ \\
\hline
\end{tabular}




\begin{tabular}{|c|c|c|c|c|c|c|c|c|c|c|c|}
\hline Non Prime Site & Prime Site & $\begin{array}{c}\text { Fold change of } \\
\text { XPRESS ratio } \\
\text { (Adam17 } 7^{\text {Acc } / \text { WT) }} \\
\end{array}$ & Modifications & Hyperscore & $\begin{array}{c}\text { Mass error } \\
\text { in ppm }\end{array}$ & Charge & $\begin{array}{c}\text { Precursor } \\
\text { neutral mass } \\
\text { in Da } \\
\end{array}$ & Uniprot ID 1 & Uniprot ID 2 & Protein Name 1 & Protein Name 2 \\
\hline PDDVYYCGLK & YIKDDVILNEPSADAPAAR & $-0,4$ & $\mathrm{~N}$-ter $+28.03 \mathrm{Da}, \mathrm{K}+28.03 \mathrm{Da}$ & 24,6 & 5,907 & 3 & 2113,1 & ITM2B_MOUSE & & Integral membrane protein 2B; & $\begin{array}{l}\text { Immature BRI2; Protein E25B; Transmembrane } \\
\text { protein BRI: }\end{array}$ \\
\hline NSYNSPGGGG & GSDYSYDSKFNYSGSGGR & $-0,7$ & $\mathrm{~N}$-ter $+28.03 \mathrm{Da}, \mathrm{K}+28.03 \mathrm{Da}$ & 17,8 & $-9,871$ & 4 & 2001,9 & ILF3_MOUSE & & Interleukin enhancer-binding factor 3; & \\
\hline RRLSFSETFT & EDDLQSITHDLEETIQPR & 1,1 & $\mathrm{~N}$-ter $+34.06 \mathrm{Da}$ & 17,3 & $-0,506$ & 3 & 2172,1 & IL1A_MOUSE & & Interleukin-1 alpha; & \\
\hline ILKKRRLSFS & ETFTEDDLQSITHDLEETIQPR & 0,8 & $\mathrm{~N}$-ter $+28.03 \mathrm{Da}$ & 21,7 & $-4,722$ & 3 & 2644,2 & IL1A_MOUSE & & Interleukin-1 alpha; & \\
\hline KRRLSFSETF & TEDDLQSITHDLEETIQPR & 0,9 & $\mathrm{~N}$-ter $+34.06 \mathrm{Da}$ & 51,5 & 6,370 & 3 & 2273,1 & ILIA_MOUSE & & Interleukin-1 alpha; & \\
\hline SIDELDKMTG & TDSNCPNNEPNFFR & $-0,1$ & $\mathrm{~N}-\operatorname{ter}+34.06 \mathrm{Da}, \mathrm{C}+57.02 \mathrm{Da}$ & 11,6 & 1,659 & 3 & 1744,8 & IL7_MOUSE & & Interleukin-7; & \\
\hline PQEPQGLLCL & EQQQQQEPQMQEQHLR & $-0,4$ & N-ter +28.03 Da & 31,1 & 1,193 & 3 & 2092,0 & INVO_MOUSE & G3X9D9_MOUSE & Involucrin; & \\
\hline LLPYVELDL & HSYDLGIENR & 1,9 & $\mathrm{~N}$-ter +34.06 Da & 19,4 & $-0,323$ & 3 & 1236,6 & IDHC_MOUSE & D3YVY3_MOUSE & Isocitrate dehydrogenase [NADP] cytoplasmic; & $\begin{array}{l}\text { Cytosolic NADP-isocitrate } \\
\text { dehydrogenase;IDP;NADP(+)-specific } \\
\text { ICDH;Oxalosuccinate decarboxylase; }\end{array}$ \\
\hline VEALLHALLR & AGDKDDITEPAVCALR & $-3,0$ & $\begin{array}{l}\text { N-ter +28.03 Da, K+28.03 Da, C } \\
+57.02 \mathrm{Da}\end{array}$ & 15,4 & $-1,901$ & 3 & 1785,9 & PLAK_MOUSE & & Junction plakoglobin; & Desmoplakin III;Desmoplakin-3; \\
\hline PLMELLHSRN & EGTATYAAAVLFR & $-0,3$ & $\begin{array}{l}\text { N-ter }+34.06 \mathrm{Da} \\
\text { Ner }\end{array}$ & 19,5 & $-2,207$ & 2 & 1402,8 & PLAK_MOUSE & & Junction plakoglobin; & Desmoplakin III;Desmoplakin-3; \\
\hline $\begin{array}{l}\text { LVKATIGLIR } \\
\text { AADAIDAEGA }\end{array}$ & $\begin{array}{l}\text { NLALCPANHAPLQEAAVIPR } \\
\text { SAPLMELHSR }\end{array}$ & $\begin{array}{r}-4,9 \\
0,6\end{array}$ & $\begin{array}{l}\text { N-ter }+28.03 \mathrm{Da}, \mathrm{C}+57.02 \mathrm{Da} \\
\mathrm{N} \text {-ter }+34.06 \mathrm{Da}\end{array}$ & $\begin{array}{l}34,2 \\
38,9\end{array}$ & $\begin{array}{r}1,693 \\
-0,155\end{array}$ & $\begin{array}{l}3 \\
3\end{array}$ & $\begin{array}{l}2182,2 \\
1286,7\end{array}$ & $\begin{array}{l}\text { PLAK_MOUSE } \\
\text { PLAK_MOUSE }\end{array}$ & & $\begin{array}{l}\text { Junction plakoglobin; } \\
\text { Junction plakoglobin; }\end{array}$ & $\begin{array}{l}\text { Desmoplakin III;Desmoplakin-3; } \\
\text { Desmoplakin III;Desmoplakin-3; }\end{array}$ \\
\hline ASAPLMELLH & $\begin{array}{l}\text { SRNEGTATYAAAVLFR } \\
\text { SRET }\end{array}$ & 0,5 & $\mathrm{~N}$-ter $+28.03 \mathrm{Da}$ & 30,9 & $-1,025$ & 3 & 1753,9 & PLAK_MOUSE & & $\begin{array}{l}\text { Junction plakoglobin; } \\
\text { J }\end{array}$ & Desmoplakin III;Desmoplakin-3; \\
\hline MELLHSRNEG & $\begin{array}{l}\text { TATYAAAVLFR } \\
\text { ThIn }\end{array}$ & 0,6 & N-ter $+28.03 \mathrm{Da}$ & 16,7 & 2,227 & 2 & 1210,7 & PLAK_MOUSE & & 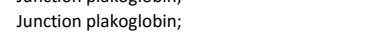 & Desmoplakin III;Desmoplakin-3; \\
\hline YDSGIHSGVN & TCVPSVSSKGIMDEDDACGR & 0,4 & $\begin{array}{l}\text { N-ter +28.03 Da, } \mathrm{K}+28.03 \mathrm{Da}, \mathrm{C} \\
+57.02 \mathrm{Da}\end{array}$ & 17,7 & 2,632 & 3 & 2239,0 & PLAK_MOUSE & & Junction plakoglobin; & Desmoplakin III;Desmoplakin-3; \\
\hline $\begin{array}{l}\text { SGEDSSLLLA } \\
\text { LKKTTYTQG }\end{array}$ & $\begin{array}{l}\text { TQVEGQTTNLQR } \\
\text { VPQNQGDLEYQMSTTAR }\end{array}$ & $\begin{array}{l}0,7 \\
-0,6\end{array}$ & $\begin{array}{l}\text { N-ter }+28.03 \mathrm{Da} \\
\mathrm{N} \text {-ter }+28.03 \mathrm{Da}\end{array}$ & $\begin{array}{l}27,6 \\
24,7\end{array}$ & $\begin{array}{l}-0,641 \\
-4,268\end{array}$ & $\begin{array}{l}2 \\
3\end{array}$ & $\begin{array}{l}1401,7 \\
1964,9\end{array}$ & $\begin{array}{l}\text { PLAK_MOUSE } \\
\text { PLAK_MOUSE }\end{array}$ & & $\begin{array}{l}\text { Junction plakoglobin; } \\
\text { Junction plakoglobin; }\end{array}$ & $\begin{array}{l}\text { Desmoplakin III;Desmoplakin-3; } \\
\text { Desmoplakin III;Desmoplakin-3; }\end{array}$ \\
\hline SGIHSGVNTC & VPSVSSKGIMDEDDACGR & 0,6 & $\begin{array}{l}\text { N-ter +28.03 Da, } \mathrm{K}+28.03 \mathrm{Da}, \mathrm{C} \\
+57.02 \mathrm{Da}\end{array}$ & 18,1 & $-2,928$ & 3 & 1977,9 & PLAK_MOUSE & & Junction plakoglobin; & Desmoplakin III;Desmoplakin-3; \\
\hline SMILGSLVQG & KGSVYTAQSDVQVPENESIKLTCTYSGFSSPR & 0,0 & $\begin{array}{l}\text { N-ter +28.03 Da, K+28.03 Da, C } \\
+57.02 \mathrm{Da}\end{array}$ & 73,7 & 1,601 & 4 & 3618,8 & JAM1_MOUSE & & Junctional adhesion molecule A; & Junctional adhesion molecule 1; \\
\hline DVTFPSDLMC & SDVKLISSR & 0,1 & $\mathrm{~N}$-ter +28.03 Da, K+28.03 Da & 19,0 & 0,000 & 3 & 1059,6 & KLK7_MOUSE & & Kallikrein-7; & $\begin{array}{l}\text { Serine protease 6;Stratum corneum } \\
\text { chymotryptic enzyme; Thymopsin; }\end{array}$ \\
\hline ATKSFRHPGY & STKTHVNDIMLVR & 0,0 & $\mathrm{~N}$-ter $+28.03 \mathrm{Da}, \mathrm{K}+28.03 \mathrm{Da}$ & 20,4 & 2,099 & 3 & 1568,9 & KLK7_MOUSE & & Kallikrein-7; & $\begin{array}{l}\text { Serine protease 6;Stratum corneum } \\
\text { chymotryptic enzyme; Thymopsin; }\end{array}$ \\
\hline TSCTVSGWGT & TTSPDVTFPSDLMCSDVKLISSR & 0,5 & $\begin{array}{l}\text { N-ter +28.03 Da, } \mathrm{K}+28.03 \mathrm{Da}, \mathrm{C} \\
+57.02 \mathrm{Da}\end{array}$ & 15,6 & 4,016 & 3 & 2611,3 & KLK7_MOUSE & & Kallikrein-7; & $\begin{array}{l}\text { Serine protease 6;Stratum corneum } \\
\text { chymotryptic enzyme; Thymopsin; }\end{array}$ \\
\hline GTSCTVSGWG & TTTSPDVTFPSDLMCSDVKLISSR & 0,6 & $\begin{array}{l}\text { N-ter +34.06 Da, K +34.06 Da, C } \\
+57.02 \mathrm{Da}\end{array}$ & 41,1 & $-0,330$ & 3 & 2724,4 & KLK7_MOUSE & & Kallikrein-7; & $\begin{array}{l}\text { Serine protease 6;Stratum corneum } \\
\text { chymotryptic enzyme-Thymoosin: }\end{array}$ \\
\hline NTLRCQLGDR & LNVEVDAAPTVDLNR & $-10,0$ & $\mathrm{~N}$-ter +28.03 Da & 19,5 & 0,604 & 2 & 1652,9 & K1H1_MOUSE & KRT34_MOUSE & Keratin, type I cuticular Ha1; & HKA-1;Hair keratin, type I Ha1;Keratin-31; \\
\hline EIELQAQQSM & RDALDSTLAETEGR & 0,4 & N-ter +34.06 Da & 17,9 & 7,326 & 3 & 1566,8 & KRT35_MOUSE & & $\begin{array}{l}\text { Keratin, type I cuticular Ha5 } \\
\{\text { ECO:0000250|UniiProtKB:Q92764); }\end{array}$ & 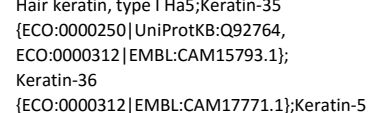 \\
\hline RVPYIPSTTC & TPAGPCTPAGPCTPAPQVSTQIR & 1,0 & $\mathrm{~N}-\operatorname{ter}+28.03 \mathrm{Da}, \mathrm{C}+57.02 \mathrm{Da}$ & 19,6 & 8,562 & 3 & 2391,2 & KRT36_MOUSE & & $\begin{array}{l}\text { Keratin, type I cuticular Ha6 } \\
\{\text { \{CO:0000250|UniprotKB:076013\}; }\end{array}$ & 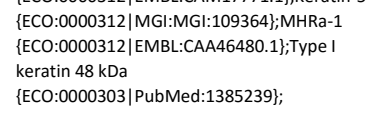 \\
\hline Q.Lalasals & ALEEQLQQIR & 1,5 & N-ter +28.03 Da & 41,2 & $-2,944$ & 2 & 1254,7 & K1C10_MOUSE & A2A513_MOUSE & Keratin, type I cytoskeletal 10; & $\begin{array}{l}56 \text { kDa cytokeratin;Cytokeratin-10;Keratin, type } \\
\text { I cytoskeletal } 59 \mathrm{kD} \text {; Keratin-10; }\end{array}$ \\
\hline GQILTLTDDN & ANVLLQIDNAR & 0,8 & $\mathrm{~N}$-ter $+28.03 \mathrm{Da}$ & 45,6 & 0,478 & 2 & 1253,7 & K1C10_MOUSE & K1C14_MOUSE & Keratin, type I cytoskeletal 10; & $\begin{array}{l}56 \text { kDa cytokeratin;Cytokeratin-10;Keratin, type } \\
\text { I cytoskeletal } 59 \mathrm{kDa} \text {;Keratin-10; }\end{array}$ \\
\hline LKGQILTLT & DNANVLLQIDNAR & 0,8 & $\mathrm{~N}$-ter $+28.03 \mathrm{Da}$ & 54,8 & 0,606 & 2 & 1482,8 & K1C10_MOUSE & K1C14_MOUSE & Keratin, type I cytoskeletal 10; & $\begin{array}{l}56 \text { kDa cytokeratin; Cytokeratin-10;Keratin, type } \\
\text { I cytoskeletal } 59 \text { kDa;Keratin-10; }\end{array}$ \\
\hline NEKVTMQNLN & DRLASYMDKVR & 0,4 & $\mathrm{~N}$-ter $+28.03 \mathrm{Da}, \mathrm{K}+28.03 \mathrm{Da}$ & 22,9 & $-0,425$ & 4 & 1408,8 & K1C10_MOUSE & A2A513_MOUSE & Keratin, type I cytoskeletal 10; & $\begin{array}{l}56 \text { kDa cytokeratin;Cytokeratin-10;Keratin, type } \\
\text { I cytoskeletal } 59 \text { kDa;Keratin-10; }\end{array}$ \\
\hline ENEVTLRQSV & EADINGLRR & 1,5 & N-ter +34.06 Da & 19,7 & $-0,741$ & 3 & 1076,6 & K1C10_MOUSE & K1C12_MOUSE & Keratin, type I cytoskeletal 10; & $\begin{array}{l}56 \text { kDa cytokeratin; Cytokeratin-10;Keratin, type } \\
\text { cytoskeletal } 59 \text { kDa;Keratin-10; }\end{array}$ \\
\hline QSQLALKQSL & EASLAETEGR & 1,5 & $\mathrm{~N}$-ter +34.06 Da & 35,5 & $-0,638$ & 2 & 1095,6 & K1C10_MOUSE & A2A513_MOUSE & Keratin, type I cytoskeletal 10; & $\begin{array}{l}56 \text { kDa cytokeratin;Cytokeratin-10;Keratin, type } \\
\text { cytoskeletal } 59 \text { kDa;Keratin-10; }\end{array}$ \\
\hline SQIOSQISAL & EEQLQQIR & 2,2 & $\mathrm{~N}$-ter +34.06 Da & 25,6 & $-1,205$ & 2 & 1076,6 & K1C10_MOUSE & K1C26_MOUSE & Keratin, type I cytoskeletal 10; & $\begin{array}{l}56 \text { kDa cytokeratin;Cytokeratin-10;Keratin, type } \\
\text { I cytoskeletal } 59 \text { kDa;Keratin-10; }\end{array}$ \\
\hline FNQKSKELT & EIDSNIEQMSSHKSEITELR & 1,4 & N-ter $+34.06 \mathrm{Da}, \mathrm{K}+34.06 \mathrm{Da}$ & 39,0 & 0,579 & 4 & 2413,2 & K1C10_MOUSE & A2A513_MOUSE & Keratin, type I cytoskeletal 10; & $\begin{array}{l}56 \text { kDa cytokeratin;Cytokeratin-10;Keratin, type } \\
\text { I cytoskeletal } 59 \text { kDa;Keratin-10; }\end{array}$ \\
\hline DELTLSKSDL & EMQIESLNEELAYLKKNHEEEMR & 1,9 & N-ter $+34.06 \mathrm{Da}, \mathrm{K}+34.06 \mathrm{Da}$ & 49,4 & $-3,975$ & 4 & 2964,5 & K1C10_MOUSE & A2A513_MOUSE & Keratin, type I cytoskeletal 10; & $\begin{array}{l}56 \text { kDa cytokeratin;Cytokeratin-10;Keratin, type } \\
\text { I yytoskeletal } 59 \mathrm{kDa} \text { keratin-10; }\end{array}$ \\
\hline
\end{tabular}




\begin{tabular}{|c|c|c|c|c|c|c|c|c|c|c|c|}
\hline Non Prime Site & Prime Site & 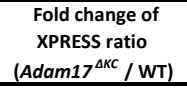 & Modifications & Hyperscore & $\begin{array}{l}\text { Mass error } \\
\text { in ppm }\end{array}$ & charge & $\begin{array}{c}\text { Precursor } \\
\text { neutral mass } \\
\text { in Da }\end{array}$ & Uniprot ID 1 & Uniprot ID 2 & Protein Name 1 & Protein Name 2 \\
\hline QQLLDIKTRL & ENEIOTYR & 1,5 & N-ter +34.06 Da & 26,1 & 0,092 & 2 & 1085,6 & K1C10_MOUSE & A2A513_MOUSE & Keratin, type I cytoskeletal 10; & $\begin{array}{l}56 \text { kDa cytokeratin;Cytokeratin-10;Keratin, type } \\
\text { I cytoskeletal } 59 \mathrm{kDa} \text {;Keratin-10; }\end{array}$ \\
\hline EMRDLQNVST & GDVNVEMNAAPGVDLTQLLNNMR & 1,7 & N-ter +28.03 Da & 17,7 & 0,840 & 3 & 2498,2 & K1C10_MOUSE & A2A513_MOUSE & Keratin, type I cytoskeletal 10; & $\begin{array}{l}56 \text { kDa cytokeratin; Cytokeratin-10;Keratin, type } \\
\text { I cytoskeletal } 59 \mathrm{kDa} \text {; Keratin-10; }\end{array}$ \\
\hline RGSLGGGYSS & GGFSGGSFSR & 0,4 & $\mathrm{~N}$-ter+28.03 Da & 18,6 & $-1,620$ & 2 & 985,5 & K1C10_MOUSE & A2A513_MOUSE & Keratin, type I cytoskeletal 10; & $\begin{array}{l}56 \text { kDa cytokeratin; Cytokeratin-10;Keratin, type } \\
\text { I cytoskeletal } 59 \mathrm{kDa} \text {; Keratin-10; }\end{array}$ \\
\hline GGAGGHGGSS & GGGYGGGSSSGGQGGSGGFKSSGGGDQSSKGPR & 0,3 & $\mathrm{~N}$-ter +34.06 Da, K +34.06 Da & 21,1 & $-2,976$ & 4 & 2919,4 & K1C10_MOUSE & A2A513_MOUSE & Keratin, type I cytoskeletal 10; & $\begin{array}{l}56 \text { kDa cytokeratin; Cytokeratin-10;Keratin, type } \\
\text { I cytoskeletal } 59 \mathrm{kDa} \text {; Keratin-10; }\end{array}$ \\
\hline VRVSSTRGSL & GGGYSSGGFSGGSFSR & 10,0 & N-ter +34.06 Da & 26,5 & $-0,733$ & 2 & 1499,7 & K1C10_MOUSE & A2A513_MOUSE & Keratin, type I cytoskeletal 10; & $\begin{array}{l}56 \text { kDa cytokeratin;Cytokeratin-10; Keratin, type } \\
\text { I cytoskeletal } 59 \mathrm{kDa} \text {;Keratin-10; }\end{array}$ \\
\hline GSSSGGAGGH & $\begin{array}{l}\text { GGSSGGGYGGGSSSGGQGGSGGFKSSGGGDQSSK } \\
\text { GPR }\end{array}$ & 0,4 & $\mathrm{~N}$-ter $+28.03 \mathrm{Da}, \mathrm{K}+28.03 \mathrm{Da}$ & 35,4 & $-6,200$ & 4 & 3189,4 & K1C10_MOUSE & A2A513_MOUSE & Keratin, type I cytoskeletal 10; & $\begin{array}{l}56 \text { kDa cytokeratin; Cytokeratin-10;Keratin, type } \\
\text { I cytoskeletal } 59 \mathrm{kDa} \text {; } k \text { eratin-10; }\end{array}$ \\
\hline HGGSSGGGYG & GGSSSGGQGGSGGFKSSGGGDQSSKGPR & 0,5 & $\mathrm{~N}$-ter $+34.06 \mathrm{Da}, \mathrm{K}+34.06 \mathrm{Da}$ & 24,5 & $-7,819$ & 4 & 2528,3 & K1C10_MOUSE & A2A513_MOUSE & Keratin, type I cytoskeletal 10; & $\begin{array}{l}56 \text { kDa cytokeratin; Cytokeratin-10;Keratin, type } \\
\text { I cytoskeletal } 59 \mathrm{kDa} \text {; Keratin-10; }\end{array}$ \\
\hline GAGGHGGSSG & GGYGGGSSSGGQGGSGGFKSSGGGDQSSKGPR & 0,3 & $\mathrm{~N}$-ter $+28.03 \mathrm{Da}, \mathrm{K}+28.03 \mathrm{Da}$ & 39,2 & $-4,143$ & 4 & 2844,3 & K1C10_MOUSE & A2A513_MOUSE & Keratin, type I cytoskeletal 10; & $\begin{array}{l}56 \text { kDa cytokeratin; Cytokeratin-10;Keratin, type } \\
\text { I cytoskeletal } 59 \mathrm{kDa} \text {; } k \text { eratin-10; }\end{array}$ \\
\hline RVSSTRGSLG & GGYSSGGFSGGSFSR & 0,7 & N-ter +28.03 Da & 57,7 & $-0,834$ & 2 & 1436,6 & K1C10_MOUSE & A2A513_MOUSE & Keratin, type I cytoskeletal 10; & $\begin{array}{l}56 \text { kDa cytokeratin; Cytokeratin-10;Keratin, type } \\
\text { I cytoskeletal } 59 \mathrm{kDa} \text {;Keratin-10; }\end{array}$ \\
\hline GGSVRVSSTR & GSLGGGYSSGGFSGGSFSR & 1,7 & N-ter +34.06 Da & 79,6 & 0,398 & 2 & 1756,8 & K1C10_MOUSE & A2A513_MOUSE & Keratin, type I cytoskeletal 10; & $\begin{array}{l}56 \text { kDa cytokeratin; Cytokeratin-10;Keratin, type } \\
\text { I cytoskeletal } 59 \mathrm{kDa} \text {;Keratin-10; }\end{array}$ \\
\hline GGSSGGGYGG & GSSSGGQGGSGGFKSSGGGDQSSKGPR & 0,4 & $\mathrm{~N}$-ter $+28.03 \mathrm{Da}, \mathrm{K}+28.03 \mathrm{Da}$ & 37,5 & 7,000 & 4 & 2453,2 & K1C10_MOUSE & A2A513_MOUSE & Keratin, type I cytoskeletal 10 ; & $\begin{array}{l}56 \text { kDa cytokeratin; Cytokeratin-10;Keratin, type } \\
\text { I cytoskeletal } 59 \mathrm{kDa} \text {;Keratin-10; }\end{array}$ \\
\hline DVNVEMNAAP & GVDLTQLLNNMR & 2,5 & $\mathrm{~N}$-ter $+28.03 \mathrm{Da}$ & 47,7 & 0,570 & 2 & 1400,8 & K1C10_MOUSE & A2A513_MOUSE & Keratin, type I cytoskeletal 10; & $\begin{array}{l}56 \text { kDa cytokeratin;Cytokeratin-10;Keratin, type } \\
\text { I cytoskeletal } 59 \mathrm{kDa} \text {-Keratin-10; }\end{array}$ \\
\hline AGGHGGSSGG & GYGGGSSSGGQGGSGGFKSSGGGDQSSKGPR & 0,4 & $\mathrm{~N}$-ter $+34.06 \mathrm{Da}, \mathrm{K}+34.06 \mathrm{Da}$ & 17,4 & 0,534 & 4 & 2805,4 & K1C10_MOUSE & A2A513_MOUSE & Keratin, type I cytoskeletal 10; & $\begin{array}{l}56 \text { kDa cytokeratin;Cytokeratin-10;Keratin, type } \\
\text { I cytoskeletal } 59 \mathrm{kDa} \text {-Keratin-10; }\end{array}$ \\
\hline vsSTRGSLGG & GYSSGGFSGGSFSR & 0,8 & $\mathrm{~N}$-ter +34.06 Da & 30,6 & 0,072 & 2 & 1385,6 & K1C10_MOUSE & A2A513_MOUSE & Keratin, type I cytoskeletal 10; & $\begin{array}{l}56 \text { kDa cytokeratin;Cytokeratin-10;Keratin, type } \\
\text { I cytoskeletal } 59 \mathrm{kDa} \text { keratin-10; }\end{array}$ \\
\hline NQKSKELTTE & IDSNIEQMSSHKSEITELR & 1,6 & $\mathrm{~N}$-ter $+34.06 \mathrm{Da}, \mathrm{K}+34.06 \mathrm{Da}$ & 19,8 & 2,273 & 4 & 2284,2 & K1C10_MOUSE & A2A513_MOUSE & Keratin, type I cytoskeletal 10; & $\begin{array}{l}56 \text { kDa cytokeratin;Cytokeratin-10;Keratin, type } \\
\text { I cytoskeletal } 59 \mathrm{kDa} \text {-Keratin-10; }\end{array}$ \\
\hline KELTTEIDSN & IEQMSSHKSEITELR & 0,7 & $\mathrm{~N}$-ter $+34.06 \mathrm{Da}, \mathrm{K}+34.06 \mathrm{Da}$ & 33,6 & $-2,205$ & 4 & 1855,0 & K1C10_MOUSE & A2A513_MOUSE & Keratin, type I cytoskeletal 10; & $\begin{array}{l}56 \text { kDa cytokeratin;Cytokeratin-10;Keratin, type } \\
\text { | cytoskeletal } 59 \text { kDa;Keratin-10; }\end{array}$ \\
\hline YKTIEDLKGQ & ILTLTTDNANVLLQIDNAR & 3,5 & $\mathrm{~N}$-ter +34.06 Da & 44,3 & $-0,141$ & 3 & 2131,2 & K1C10_MOUSE & A2A513_MOUSE & Keratin, type I cytoskeletal 10; & $\begin{array}{l}56 \text { kDa cytokeratin;Cytokeratin-10;Keratin, type } \\
\text { | cytoskeletal } 59 \mathrm{kDa} \text { keratin-10; }\end{array}$ \\
\hline ARLAADDFRL & KYENEVTLR & 0,9 & $\mathrm{~N}$-ter $+34.06 \mathrm{Da}, \mathrm{K}+34.06 \mathrm{Da}$ & 22,3 & $-0,327$ & 3 & 1218,7 & K1C10_MOUSE & A2A513_MOUSE & Keratin, type I cytoskeletal 10; & $\begin{array}{l}56 \text { kDa cytokeratin;Cytokeratin-10;Keratin, type } \\
\text { I cytoskeletal } 59 \text { kDa;Keratin-10; }\end{array}$ \\
\hline YQQLLDIKTR & LENEIQTYR & $-5,2$ & N-ter +28.03 Da & 33,5 & 1,758 & 2 & 1192,6 & K1C10_MOUSE & A2A513_MOUSE & Keratin, type I cytoskeletal 10; & $\begin{array}{l}56 \text { kDa cytokeratin;Cytokeratin-10;Keratin, type } \\
\text { I cytoskeletal } 59 \mathrm{kDa} \text {;Keratin-10; }\end{array}$ \\
\hline LENEIQTYRS & LLEGEGSSSGGGGGR & 0,7 & N-ter +28.03 Da & 43,7 & $-1,112$ & 2 & 1346,6 & K1C10_MOUSE & A2A513_MOUSE & Keratin, type I cytoskeletal 10; & $\begin{array}{l}56 \text { kDa cytokeratin;Cytokeratin-10;Keratin, type } \\
\text { I cytoskeletal } 59 \text { kDa;Keratin-10; }\end{array}$ \\
\hline AETEGRYCVQ & LSQIQSQISALEEQLQQIR & 2,8 & $\mathrm{~N}$-ter +34.06 Da & 51,9 & $-5,115$ & 3 & 2245,2 & K1C10_MOUSE & A2A513_MOUSE & Keratin, type I cytoskeletal 10; & $\begin{array}{l}56 \text { kDa cytokeratin; Cytokeratin-10;Keratin, type } \\
\text { I cytoskeletal } 59 \text { kDa;Keratin-10; }\end{array}$ \\
\hline KTIEDLKGQI & LTLTTDNANVLLQIDNAR & 3,8 & N-ter +34.06 Da & 61,9 & 0,346 & 3 & 2018,1 & K1C10_MOUSE & A2A513_MOUSE & Keratin, type I cytoskeletal 10; & $\begin{array}{l}56 \text { kDa cytokeratin;Cytokeratin-10;Keratin, type } \\
\text { I cytoskeletal } 59 \mathrm{kDa} \text {;Keratin-10; }\end{array}$ \\
\hline VEMNAAPGVD & LTQLLNNMR & 1,5 & N-ter +28.03 Da & 18,0 & $-0,619$ & 2 & 1129,6 & K1C10_MOUSE & A2A513_MOUSE & Keratin, type I cytoskeletal 10; & $\begin{array}{l}56 \text { kDa cytokeratin;Cytokeratin-10;Keratin, type } \\
\text { I cytoskeletal } 59 \mathrm{kDa} \text {;Keratin-10; }\end{array}$ \\
\hline VSTGDVNVEM & NAAPGVDLTQLLNNMR & 0,4 & $\mathrm{~N}$-ter +28.03 Da & 65,7 & $-1,025$ & 2 & 1753,9 & K1C10_MOUSE & A2A513_MOUSE & Keratin, type I cytoskeletal 10; & $\begin{array}{l}56 \text { kDa cytokeratin;Cytokeratin-10;Keratin, type } \\
\text { I cytoskeletal } 59 \mathrm{kDa} \text {; Keratin-10; }\end{array}$ \\
\hline кGQILTLTD & NANVLLQIDNAR & 0,3 & $\mathrm{~N}$-ter +28.03 Da & 52,0 & $-1,606$ & 2 & 1367,8 & K1C10_MOUSE & K1C14_MOUSE & Keratin, type I cytoskeletal 10; & $\begin{array}{l}56 \text { kDa cytokeratin; Cytokeratin-10;Keratin, type } \\
\text { I cytoskeletal } 59 \mathrm{kDa} \text {; Keratin-10; }\end{array}$ \\
\hline QILTLTDNA & NVLLQIDNAR & 0,6 & $\mathrm{~N}$-ter $+28.03 \mathrm{Da}$ & 26,7 & 0,591 & 2 & 1182,7 & K1C10_MOUSE & K1C14_MOUSE & Keratin, type I cytoskeletal 10; & $\begin{array}{l}56 \text { kDa cytokeratin; Cytokeratin-10;Keratin, type } \\
\text { I cytoskeletal } 59 \mathrm{kDa} \text {; Keratin-10; }\end{array}$ \\
\hline Ycvalsolas & QISALEEQLQQIR & 1,1 & $\mathrm{~N}$-ter $+28.03 \mathrm{Da}$ & 53,5 & $-1,830$ & 2 & 1582,9 & K1C10_MOUSE & A2A513_MOUSE & Keratin, type I cytoskeletal 10; & $\begin{array}{l}56 \text { kDa cytokeratin;Cytokeratin-10;Keratin, type } \\
\text { I cytoskeletal } 59 \text { kDa;Keratin-10; }\end{array}$ \\
\hline LAETEGRYCV & QLSQIQSQISALEEQLQQIR & 1,5 & $\mathrm{~N}$-ter +34.06 Da & 61,3 & 0,084 & 3 & 2373,3 & K1C10_MOUSE & A2A513_MOUSE & Keratin, type I cytoskeletal 10; & $\begin{array}{l}56 \text { kDa cytokeratin;Cytokeratin-10;Keratin, type } \\
\text { I cytoskeletal } 59 \text { kDa;Keratin-10; }\end{array}$ \\
\hline LTEIDSNIE & QMSSHKSEITELR & 3,5 & $\mathrm{~N}$-ter +34.06 Da, K+34.06 Da & 19,3 & 6,127 & 3 & 1612,9 & K1C10_MOUSE & A2A513_MOUSE & Keratin, type I cytoskeletal 10; & $\begin{array}{l}56 \text { kDa cytokeratin;Cytokeratin-10;Keratin, type } \\
\text { I cytoskeletal } 59 \mathrm{kDa} \text {;Keratin-10; }\end{array}$ \\
\hline LKYENEVTLR & QSVEADINGLR & $-0,9$ & $\mathrm{~N}$-ter $+28.03 \mathrm{Da}$ & 36,4 & $-0,650$ & 2 & 1228,6 & K1C10_MOUSE & K1C13_MOUSE & Keratin, type I cytoskeletal 10; & $\begin{array}{l}56 \text { kDa cytokeratin;Cytokeratin-10;Keratin, type } \\
\text { I cytoskeletal } 59 \text { kDa;Keratin-10; }\end{array}$ \\
\hline SALEEQLQQI & RAETECQNAEYQQLLDIKTR & 1,8 & $\begin{array}{l}\text { N-ter +28.03 Da, K+28.03 Da, C } \\
+57.02 \mathrm{Da}\end{array}$ & 24,0 & $-1,109$ & 4 & 2521,3 & K1C10_MOUSE & A2A513_MOUSE & Keratin, type I cytoskeletal 10; & $\begin{array}{l}56 \text { kDa cytokeratin; Cytokeratin-10;Keratin, type } \\
\text { I cytoskeletal } 59 \mathrm{kDa} \text {; Keratin-10; }\end{array}$ \\
\hline
\end{tabular}




\begin{tabular}{|c|c|c|c|c|c|c|c|c|c|c|c|}
\hline Non Prime Site & Prime Site & 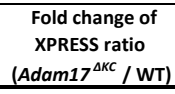 & Modifications & Hyperscore & $\begin{array}{c}\text { Mass error } \\
\text { in ppm }\end{array}$ & Charge & $\begin{array}{c}\text { Precursor } \\
\text { neutral mass } \\
\text { in Da } \\
\end{array}$ & Uniprot ID 1 & Uniprot ID 2 & Protein Name 1 & Protein Name 2 \\
\hline GGGSVRVSST & RGSLGGGYSSGGFSGGSFSR & 0,6 & N-ter+34.06 Da & 65,0 & $-0,679$ & 3 & 1912,9 & K1C10_MOUSE & A2A513_MOUSE & Keratin, type I cytoskeletal 10; & $\begin{array}{l}56 \text { kDa cytokeratin;Cytokeratin-10;Keratin, type } \\
\text { I cytoskeletal } 59 \text { kDa; Keratin-10; }\end{array}$ \\
\hline ANVLLaIdNA & RLAADDFR & 0,3 & $\mathrm{~N}$-ter +28.03 Da & 22,1 & $-0,201$ & 3 & 990,5 & K1C10_MOUSE & K1C13_MOUSE & Keratin, type I cytoskeletal 10; & $\begin{array}{l}56 \text { kDa cytokeratin;Cytokeratin-10;Keratin, type } \\
\text { I cytoskeletal } 59 \text { kDa;Keratin-10; }\end{array}$ \\
\hline EYQQLLIKT & RLENEIQTYR & 1,3 & N-ter +34.06 Da & 23,6 & $-0,442$ & 3 & 1354,7 & K1C10_MOUSE & A2A513_MOUSE & Keratin, type I cytoskeletal 10; & $\begin{array}{l}56 \text { kDa cytokeratin; Cytokeratin-10;Keratin, type } \\
\text { cytoskeletal } 59 \text { kDa;Keratin-10; }\end{array}$ \\
\hline RLKYENEVTL & RQSVEADINGLR & 0,3 & N-ter +28.03 Da & 40,5 & 1,082 & 2 & 1384,7 & K1C10_MOUSE & K1C13_MOUSE & Keratin, type I cytoskeletal 10; & $\begin{array}{l}56 \text { kDa cytokeratin; Cytokeratin-10;Keratin, type } \\
\text { cytoskeletal } 59 \text { kDa;Keratin-10; }\end{array}$ \\
\hline TRLENEIQTY & RSLLEGEGSSSGGGGGR & 0,6 & N-ter +28.03 Da & 51,7 & $-5,023$ & 3 & 1589,8 & K1C10_MOUSE & A2A513_MOUSE & Keratin, type I cytoskeletal 10; & $\begin{array}{l}56 \text { kDa cytokeratin;Cytokeratin-10;Keratin, type } \\
\text { I cytoskeletal } 59 \text { kDa;Keratin-10; }\end{array}$ \\
\hline valsalosal & SALEEQLQQIR & 5,8 & N-ter +34.06 Da & 45,9 & $-9,631$ & 2 & 1347,7 & K1C10_MOUSE & A2A513_MOUSE & Keratin, type I cytoskeletal 10; & $\begin{array}{l}56 \text { kDa cytokeratin;Cytokeratin-10;Keratin, type } \\
\text { I cytoskeletal } 59 \text { kDa;Keratin-10; }\end{array}$ \\
\hline RVLDELTISK & SDLEMQIESLNEELAYLKKNHEEEMR & 0,2 & N-ter +34.06 Da, K+34.06 Da & 36,5 & 0,822 & 5 & 3279,7 & K1C10_MOUSE & A2A513_MOUSE & Keratin, type I cytoskeletal 10; & $\begin{array}{l}56 \text { kDa cytokeratin;Cytokeratin-10;Keratin, type } \\
\text { cytoskeletal } 59 \text { kDa;Keratin-10; }\end{array}$ \\
\hline TRGSLGGGYS & SGGFSGGSFSR & 0,8 & N-ter +28.03 Da & 24,8 & 1,861 & 2 & 1072,5 & K1C10_MOUSE & A2A513_MOUSE & Keratin, type I cytoskeletal 10; & $\begin{array}{l}56 \text { kDa cytokeratin;Cytokeratin-10;Keratin, type } \\
\text { cytoskeletal } 59 \text { kDa;Keratin-10; }\end{array}$ \\
\hline SGGAGGHGGS & SGGGYGGGSSSGGQGGSGGFKSSGGGDOSSKGPR & 0,4 & N-ter +34.06 Da, K+34.06 Da & 29,6 & 0,000 & 4 & 3006,5 & K1C10_MOUSE & A2A513_MOUSE & Keratin, type I cytoskeletal 10; & $\begin{array}{l}56 \text { KDa cytokeratin; Cytokeratin-10;Keratin, type } \\
\text { cytoskeletal } 59 \text { kDa;Keratin-10; }\end{array}$ \\
\hline SGGGYGGGSS & SGGQGGSGGFKSSGGGDQSSKGPR & 0,3 & N-ter +28.03 Da, K+28.03 Da & 27,1 & 0,674 & 4 & 2222,1 & K1C10_MOUSE & A2A513_MOUSE & Keratin, type I cytoskeletal 10; & $\begin{array}{l}56 \text { kDa cytokeratin;Cytokeratin-10;Keratin, type } \\
\text { I cytoskeletal } 59 \mathrm{kDa} \text { KReratin-10; }\end{array}$ \\
\hline LRRVLDELTL & SKSDLEMQIIESLNEELAYYKKNHEEEMR & $-7,4$ & N-ter +28.03 Da, K+28.03 Da & 25,6 & $-10,001$ & 5 & 3504,7 & K1C10_MOUSE & A2A513_MOUSE & Keratin, type I cytoskeletal 10; & $\begin{array}{l}56 \text { kDa cytokeratin;Cytokeratin-10;Keratin, type } \\
\text { I cytoskeletal } 59 \mathrm{kDa} \text { KKeratin-10; }\end{array}$ \\
\hline ELQSQLALKa & SLEASLAETEGR & 0,5 & $\mathrm{~N}$-ter+34.06 Da & 49,2 & $-0,077$ & 2 & 1295,7 & K1C10_MOUSE & A2A513_MOUSE & Keratin, type I cytoskeletal 10; & $\begin{array}{l}56 \text { kDa cytokeratin;Cytokeratin-10;Keratin, type } \\
\text { c cytoskeletal } 59 \mathrm{kDa} \text { KKeratin-10; }\end{array}$ \\
\hline GSVRVSSTRG & SLGGGYSSGGFSGGSFSR & 0,9 & N-ter +34.06 Da & 84,4 & $-2,527$ & 2 & 1699,8 & K1C10_MOUSE & A2A513_MOUSE & Keratin, type I cytoskeletal 10; & $\begin{array}{l}56 \text { kDa cytokeratin;Cytokeratin-10; Keratin, type } \\
\text { cytoskeletal } 59 \mathrm{kDa} \text { KKeratin-10; }\end{array}$ \\
\hline RLENEIOTYR & SLLEGEGSSSGGGGGR & $-6,8$ & N-ter +28.03 Da & 61,0 & 0,070 & 2 & 1433,7 & K1C10_MOUSE & A2A513_MOUSE & Keratin, type I cytoskeletal 10; & $\begin{array}{l}56 \text { kDa cytokeratin;Cytokeratin-10;Keratin, type } \\
\text { c cytoskeletal } 59 \mathrm{kDa} \text { KKeratin-10; }\end{array}$ \\
\hline KSKELTTEID & SNIEQMSSHKSEITELR & 0,9 & N-ter +34.06 Da, K+34.06 Da & 70,8 & $-0,534$ & 3 & 2056,1 & K1C10_MOUSE & A2A513_MOUSE & Keratin, type I cytoskeletal 10; & $\begin{array}{l}56 \text { kDa cytokeratin;Cytokeratin-10;Keratin, type } \\
\text { c cytoskeletal } 59 \mathrm{kDa} \text { KKeratin-10; }\end{array}$ \\
\hline ETEGRYCVQL & SQIQSQISALEEQLQQIR & 0,2 & N-ter +28.03 Da & 79,9 & 1,081 & 2 & 2126,1 & K1C10_MOUSE & A2A513_MOUSE & Keratin, type I cytoskeletal 10; & $\begin{array}{l}56 \text { kDa cytokeratin;Cytokeratin-10; Keratin, type } \\
\text { cytoskeletal } 59 \mathrm{kDa} \text { KReratin-10; }\end{array}$ \\
\hline STRGSLGGGY & SSGGFSGGSFSR & 0,6 & $\mathrm{~N}$-ter +34.06 Da & 31,0 & $-2,056$ & 2 & 1165,6 & K1C10_MOUSE & A2A513_MOUSE & Keratin, type I cytoskeletal 10; & $\begin{array}{l}56 \text { kDa cytokeratin;Cytokeratin-10; Keratin, type } \\
\text { I cytoskeletal } 59 \text { kDa;Keratin-10; }\end{array}$ \\
\hline SSGGAGGHGG & SSGGGYGGGSSSGGQGGSGGFKSSGGGDQSSKGPR & 0,5 & $\mathrm{~N}$-ter +34.06 Da, $\mathrm{K}+34.06 \mathrm{Da}$ & 44,1 & $-1,033$ & 4 & 3093,5 & K1C10_MOUSE & A2A513_MOUSE & Keratin, type I cytoskeletal 10; & $\begin{array}{l}56 \text { KDa cytokeratin;Cytokeratin-10;Keratin, type } \\
\text { I cytoskeletal } 59 \text { kDa; Keratin-10; }\end{array}$ \\
\hline SSGGGYGGGS & SSGGQGGSGGFKSSGGGDQSSKGPR & 0,4 & $\mathrm{~N}$-ter +34.06 Da, $\mathrm{K}+34.06 \mathrm{Da}$ & 25,2 & $-2,745$ & 4 & 2327,2 & K1C10_MOUSE & A2A513_MOUSE & Keratin, type I cytoskeletal 10; & $\begin{array}{l}56 \text { KDa cytokeratin;Cytokeratin-10;Keratin, type } \\
\text { I cytoskeletal } 59 \text { kDa; Keratin-10; }\end{array}$ \\
\hline GSSGGGYGGG & SSSGGQGGSGGFKSSGGGDQSSKGPR & 0,4 & $\mathrm{~N}$-ter +28.03 Da, $\mathrm{K}+28.03 \mathrm{Da}$ & 26,3 & 0,292 & 3 & 2396,1 & K1C10_MOUSE & A2A513_MOUSE & Keratin, type I cytoskeletal 10; & $\begin{array}{l}56 \text { KDa cytokeratin;Cytokeratin-10;Keratin, type } \\
\text { I cytoskeletal } 59 \text { kDa; Keratin-10; }\end{array}$ \\
\hline MSVLY & SSSSKOFSSSR & 0,9 & $\mathrm{~N}$-ter +34.06 Da, $\mathrm{K}+34.06 \mathrm{Da}$ & 15,2 & $-0,636$ & 3 & 1254,7 & K1C10_MOUSE & A2A513_MOUSE & Keratin, type I cytoskeletal 10; & $\begin{array}{l}56 \text { kDa cytokeratin; Cytokeratin-10;Keratin, type } \\
\text { I cytoskeletal } 59 \text { kDa; Keratin-10; }\end{array}$ \\
\hline KYENEVTLRQ & SVEADINGLR & 0,7 & $\mathrm{~N}$-ter +28.03 Da & 29,8 & 0,998 & 2 & 1100,6 & K1C10_MOUSE & K1C13_MOUSE & Keratin, type I cytoskeletal 10; & $\begin{array}{l}56 \text { kDa cytokeratin;Cytokeratin-10;Keratin, type } \\
\text { I cytoskeletal } 59 \text { kDa; Keratin-10; }\end{array}$ \\
\hline TMQNLNDRLA & SYMDKVR & 0,3 & N-ter +34.06 Da, K +34.06 Da & 21,7 & $-1,549$ & 3 & 965,6 & K1C10_MOUSE & A2A513_MOUSE & Keratin, type I cytoskeletal 10; & $\begin{array}{l}56 \text { kDa cytokeratin;Cytokeratin-10;Keratin, type } \\
\text { I cytoskeletal } 59 \text { kDa; Keratin-10; }\end{array}$ \\
\hline DLKGQLITLT & TDNANVLLQIDNAR & 0,9 & $\mathrm{~N}$-ter +28.03 Da & 54,4 & $-0,189$ & 2 & 1583,8 & K1C10_MOUSE & A2A513_MOUSE & Keratin, type I cytoskeletal 10; & $\begin{array}{l}56 \text { kDa cytokeratin;Cytokeratin-10;Keratin, type } \\
\text { I cytoskeletal } 59 \text { kDa;keratin-10; }\end{array}$ \\
\hline WFNQKSKELT & TEIDSNIEQMSSHKSEITELR & 1,0 & N-ter +34.06 Da, K +34.06 Da & 68,7 & $-1,589$ & 3 & 2514,3 & K1C10_MOUSE & A2A513_MOUSE & Keratin, type I cytoskeletal 10; & $\begin{array}{l}56 \text { kDa cytokeratin; Cytokeratin-10;Keratin, type } \\
\text { I cytoskeletal } 59 \text { kDa;Keratin-10; }\end{array}$ \\
\hline EEMRDLanvS & TGDVNVEMNAAPGVDLTQLLNNMR & 1,0 & N-ter +34.06 Da & 60,5 & 1,035 & 3 & 2605,3 & K1C10_MOUSE & A2A513_MOUSE & Keratin, type I cytoskeletal 10; & $\begin{array}{l}56 \text { kDa cytokeratin;Cytokeratin-10;Keratin, type } \\
\text { I cytoskeletal } 59 \text { kDa;Keratin-10; }\end{array}$ \\
\hline TIEDLKGQIL & TLITDNANVLLOIDNAR & 0,4 & $\mathrm{~N}$-ter +28.03 Da & 82,5 & 0,736 & 2 & 1899,0 & K1C10_MOUSE & A2A513_MOUSE & Keratin, type I cytoskeletal 10; & $\begin{array}{l}56 \text { kDa cytokeratin; Cytokeratin-10;Keratin, type } \\
\text { cytoskeletal } 59 \text { kDa;Keratin-10; }\end{array}$ \\
\hline AEYQQLLDIK & TRLENEIQTYR & 2,3 & N-ter +34.06 Da & 25,0 & $-0,411$ & 3 & 1455,8 & K1C10_MOUSE & A2A513_MOUSE & Keratin, type I cytoskeletal 10; & $\begin{array}{l}56 \text { kDa cytokeratin;Cytokeratin-10;Keratin, type } \\
\text { I cytoskeletal } 59 \mathrm{kDa} \text { KKeratin-10; }\end{array}$ \\
\hline EDLKGQILTL & TTDNANVLLQIDNAR & 0,3 & $\mathrm{~N}$-ter +28.03 Da & 74,1 & 2,431 & 2 & 1684,9 & K1C10_MOUSE & A2A513_MOUSE & Keratin, type I cytoskeletal 10; & $\begin{array}{l}56 \text { kDa cytokeratin;Cytokeratin-10;Keratin, type } \\
\text { I cytoskeletal } 59 \mathrm{kDa} \text { kReratin-10; }\end{array}$ \\
\hline EWFNOKSKEL & TTEIDSNIEQMSSHKSEITELR & 1,1 & N-ter +34.06 Da, K+34.06 Da & 63,3 & $-0,115$ & 4 & 2615,3 & K1C10_MOUSE & A2A513_MOUSE & Keratin, type I cytoskeletal 10; & $\begin{array}{l}56 \text { KDa cytokeratin;Cytokeratin-10;Keratin, type } \\
\text { I cytoskeletal } 59 \text { kDa; Keratin-10; }\end{array}$ \\
\hline VNVEMNAAPG & VDLTQLLNNMR & 1,1 & $\mathrm{~N}$-ter +28.03 Da & 25,0 & $-0,223$ & 2 & 1343,7 & K1C10_MOUSE & A2A513_MOUSE & Keratin, type I cytoskeletal 10; & $\begin{array}{l}56 \text { kDa cytokeratin;Cytokeratin-10;Keratin, type } \\
\text { I cytoskeletal } 59 \mathrm{kDa} \text { KKeratin-10; }\end{array}$ \\
\hline
\end{tabular}




\begin{tabular}{|c|c|c|c|c|c|c|c|c|c|c|c|}
\hline Non Prime Site & Prime Site & 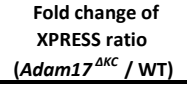 & Modifications & Hyperscore & $\begin{array}{l}\text { Mass error } \\
\text { in ppm }\end{array}$ & Charge & $\begin{array}{c}\text { Precursor } \\
\text { neutral mass } \\
\text { in Da } \\
\end{array}$ & Uniprot ID 1 & Uniprot ID 2 & Protein Name 1 & Protein Name 2 \\
\hline LQNVSTGDVN & VEMNAAPGVDLTQLLNNMR & 0,7 & $\mathrm{~N}$-ter $+34.06 \mathrm{Da}$ & 65,8 & 1,226 & 2 & 2119,1 & K1C10_MOUSE & A2A513_MOUSE & Keratin, type I cytoskeletal 10; & $\begin{array}{l}56 \text { kDa cytokeratin;Cytokeratin-10;Keratin, type } \\
\text { I cytoskeletal } 59 \mathrm{kDa} \text {;Keratin-10; }\end{array}$ \\
\hline RDLQNVSTGD & VNVEMNAAPGVDLTQLLNNMR & 0,9 & $\mathrm{~N}$-ter +34.06 Da & 56,3 & $-0,257$ & 3 & 2332,2 & K1C10_MOUSE & A2A513_MOUSE & Keratin, type I cytoskeletal 10; & $\begin{array}{l}56 \text { kDa cytokeratin;Cytokeratin-10;Keratin, type } \\
\text { I cytoskeletal } 59 \mathrm{kDa} \text {;Keratin-10; }\end{array}$ \\
\hline SLAETEGRYC & VQLSQIQSQISALEEQLQQIR & 2,5 & N-ter +34.06 Da & 67,9 & $-0,242$ & 3 & 2472,4 & K1C10_MOUSE & A2A513_MOUSE & Keratin, type I cytoskeletal 10; & $\begin{array}{l}56 \text { kDa cytokeratin;Cytokeratin-10;Keratin, type } \\
\text { I cytoskeletal } 59 \text { kDa;Keratin-10; }\end{array}$ \\
\hline HEEEMRDLQN & VSTGDVNVEMNAAPGVDLTQLLNNMR & 0,9 & $\mathrm{~N}$-ter $+34.06 \mathrm{Da}$ & 64,0 & $-0,716$ & 3 & 2791,4 & K1C10_MOUSE & A2A513_MOUSE & Keratin, type I cytoskeletal 10; & $\begin{array}{l}56 \text { kDa cytokeratin;Cytokeratin-10;Keratin, type } \\
\text { I cytoskeletal } 59 \text { kDa;Keratin-10; }\end{array}$ \\
\hline EASLAETEGR & YCVQLSQIQSQISALEEQLQQIR & $-2,0$ & N-ter +28.03 Da, C +57.02 Da & 16,9 & 2,220 & 3 & 2789,4 & K1C10_MOUSE & A2A513_MOUSE & Keratin, type I cytoskeletal 10; & $\begin{array}{l}56 \text { kDa cytokeratin;Cytokeratin-10;Keratin, type } \\
\text { I cytoskeletal } 59 \mathrm{kDa} \text {; Keratin-10; }\end{array}$ \\
\hline GGHGGSSGGG & YGGGSSSGGQGGSGGFKSSGGGDQSSKGPR & 0,3 & $\mathrm{~N}$-ter $+28.03 \mathrm{Da}, \mathrm{K}+28.03 \mathrm{Da}$ & 17,9 & $-3,255$ & 4 & 2730,3 & K1C10_MOUSE & A2A513_MOUSE & Keratin, type I cytoskeletal 10; & $\begin{array}{l}56 \text { kDa cytokeratin;Cytokeratin-10;Keratin, type } \\
\text { I cytoskeletal } 59 \mathrm{kDa} \text {; } \mathrm{Keratin}-10 \text {; }\end{array}$ \\
\hline KTRLENEIQT & YRSLLEGEGSSSGGGGGR & 3,5 & $\mathrm{~N}$-ter +34.06 Da & 45,2 & 3,916 & 3 & 1758,9 & K1C10_MOUSE & A2A513_MOUSE & Keratin, type I cytoskeletal 10; & $\begin{array}{l}56 \text { kDa cytokeratin;Cytokeratin-10;Keratin, type } \\
\text { I cytoskeletal } 59 \text { kDa;Keratin-10; }\end{array}$ \\
\hline MSVL & YSSSSKQFSSSRSGGGGGGGSVR & 0,6 & $\mathrm{~N}$-ter $+34.06 \mathrm{Da}, \mathrm{K}+34.06 \mathrm{Da}$ & 16,2 & $-0,311$ & 4 & 2246,1 & K1C10_MOUSE & A2A513_MOUSE & Keratin, type I cytoskeletal 10; & $\begin{array}{l}56 \text { kDa cytokeratin;Cytokeratin-10;Keratin, type } \\
\text { I cytoskeletal } 59 \text { kDa;Keratin-10; }\end{array}$ \\
\hline MKYENELALR & QTVEADINGLRR & $-8,0$ & N-ter +28.03 Da & 26,7 & 0,785 & 3 & 1398,8 & K1C12_MOUSE & & Keratin, type I cytoskeletal 12; & Cytokeratin-12;Keratin-12; \\
\hline MKYENELALR & QTVEADINGLR & $-8,0$ & $\mathrm{~N}$-ter +28.03 Da & 40,9 & $-0,241$ & 2 & 1242,7 & K1C12_MOUSE & & Keratin, type I cytoskeletal 12; & Cytokeratin-12;Keratin-12; \\
\hline RMKYENELAL & RQTVEADINGLR & $-5,6$ & $\mathrm{~N}$-ter+28.03 Da & 32,5 & $-0,071$ & 3 & 1398,8 & K1C12_MOUSE & & Keratin, type I cytoskeletal 12; & Cytokeratin-12;Keratin-12; \\
\hline TIEDLKSKIL & AATVDNANVLLQIDNAR & 0,6 & $\mathrm{~N}$-ter+34.06 Da & 43,5 & $-1,854$ & 3 & 1831,0 & K1C14_MOUSE & & Keratin, type I cytoskeletal 14; & Cytokeratin-14;Keratin-14; \\
\hline EKMAEKNRKD & AEEWFFSKTEELNR & 0,2 & $\mathrm{~N}$-ter +28.03 Da, K+28.03 Da & 45,2 & $-0,325$ & 3 & 1840,9 & K1C14_MOUSE & & Keratin, type I cytoskeletal 14; & Cytokeratin-14;Keratin-14; \\
\hline TYRRLLEGED & AHLSSSQFSSSSQFSSGSQSSR & 0,0 & $\mathrm{~N}$-ter +28.03 Da & 18,7 & $-0,477$ & 3 & 2303,0 & K1C14_MOUSE & & Keratin, type I cytoskeletal 14; & Cytokeratin-14;Keratin-14; \\
\hline YLDKVRALEQ & ANTELEVKIR & 0,1 & $\mathrm{~N}$-ter +34.06 Da, K +34.06 Da & 37,7 & $-0,966$ & 3 & 1239,8 & K1C14_MOUSE & K1C15_MOUSE & Keratin, type I cytoskeletal 14; & Cytokeratin-14;Keratin-14; \\
\hline ETKGRYCMQL & AQIQEMIGSVEEQLAQLR & 0,1 & $\mathrm{~N}$-ter +34.06 Da & 47,6 & 0,192 & 3 & 2076,1 & K1C14_MOUSE & & Keratin, type I cytoskeletal 14; & Cytokeratin-14;Keratin-14; \\
\hline VTMQNLNDRL & ATYLDKVR & $-0,1$ & $\mathrm{~N}$-ter +34.06 Da, K +34.06 Da & 24,2 & $-0,869$ & 3 & 1032,7 & K1C14_MOUSE & K1C16_MOUSE & Keratin, type I cytoskeletal 14; & Cytokeratin-14;keratin-14; \\
\hline QVGGDVNVEM & DAAPGVDLSR & 0,4 & $\mathrm{~N}$-ter +28.03 Da & 30,2 & $-0,486$ & 2 & 1027,5 & K1C14_MOUSE & K1C16_MOUSE & Keratin, type I cytoskeletal 14; & Cytokeratin-14;Keratin-14; \\
\hline DLSRILNEMR & DQYEKMAEKNR & $-3,6$ & $\mathrm{~N}$-ter +28.03 Da, K+28.03 Da & 16,4 & 0,334 & 3 & 1494,8 & K1C14_MOUSE & K1C17_MOUSE & Keratin, type I cytoskeletal 14 & Cytokeratin-14;keratin-14; \\
\hline SYLDKVRALE & EANTELEVKIR & 0,1 & $\mathrm{~N}$-ter +34.06 Da, K+34.06 Da & 23,9 & $-5,176$ & 3 & 1368,8 & K1C14_MOUSE & K1C17_MOUSE & Keratin, type I cytoskeletal 14; & Cytokeratin-14;Keratin-14; \\
\hline ELNREVATNS & ELVQSGKSEISELR & 0,6 & $\mathrm{~N}$-ter +34.06 Da, K+34.06 Da & 21,6 & 6,383 & 3 & 1642,0 & K1C14_MOUSE & K1C17_MOUSE & Keratin, type I cytoskeletal 14; & Cytokeratin-14;Keratin-14; \\
\hline RGQVGGDVNV & EMDAAPGVDLSR & $-0,1$ & $\mathrm{~N}$-ter +28.03 Da & 15,2 & $-6,126$ & 2 & 1287,6 & K1C14_MOUSE & K1C16_MOUSE & Keratin, type I cytoskeletal 14; & Cytokeratin-14;keratin-14; \\
\hline VEEQLAQLRC & EMEQQNQEYKILLDVKTR & 0,3 & $\mathrm{~N}$-ter +28.03 Da, K+28.03 Da & 34,5 & $-3,996$ & 4 & 2348,2 & K1C14_MOUSE & K1C17_MOUSE & Keratin, type I cytoskeletal 14; & Cytokeratin-14;Keratin-14; \\
\hline EQLAQLRCEM & EQQNQEYKILLDVKTR & 1,0 & $\mathrm{~N}$-ter $+28.03 \mathrm{Da}, \mathrm{K}+28.03 \mathrm{Da}$ & 17,8 & $-0,048$ & 3 & 2088,2 & K1C14_MOUSE & K1C17_MOUSE & Keratin, type I cytoskeletal 14 & Cytokeratin-14;Keratin-14; \\
\hline FFSKTELLNR & EVATNSELVQSGKSEISELRR & 1,7 & $\mathrm{~N}$-ter $+28.03 \mathrm{Da}, \mathrm{K}+28.03 \mathrm{Da}$ & 35,5 & $-6,189$ & 4 & 2387,3 & K1C14_MOUSE & K1C17_MOUSE & Keratin, type I cytoskeletal 14 & Cytokeratin-14;Keratin-14; \\
\hline MAEKNRKDAE & EWFFSKTEELNR & 2,9 & $\mathrm{~N}$-ter +34.06 Da, $\mathrm{K}+34.06 \mathrm{Da}$ & 16,0 & 2,597 & 3 & 1652,9 & K1C14_MOUSE & & Keratin, type I cytoskeletal 14 & Cytokeratin-14;Keratin-14; \\
\hline EMNALRGQVG & GDVNVEMDAAPGVDLSR & $-0,4$ & $\mathrm{~N}$-ter +28.03 Da & 24,7 & $-0,451$ & 2 & 1771,8 & K1C14_MOUSE & K1C16_MOUSE & Keratin, type I cytoskeletal 14; & Cytokeratin-14;Keratin-14; \\
\hline IRTKVMDVHD & GKVVSTHEQVLR & 0,2 & N-ter +34.06 Da, K +34.06 Da & 26,0 & $-0,211$ & 4 & 1419,9 & K1C14_MOUSE & & Keratin, type I cytoskeletal 14; & Cytokeratin-14;Keratin-14; \\
\hline GGGLGGGIGD & GLLVGSEKVTMQNLNDR & 0,1 & $\mathrm{~N}$-ter +34.06 Da, K+34.06 Da & 52,6 & $-0,463$ & 3 & 1941,1 & K1C14_MOUSE & K1C16_MOUSE & Keratin, type I cytoskeletal 14; & Cytokeratin-14;Keratin-14; \\
\hline NHEEEMNALR & GQVGGDVNVEMDAAPGVDLSR & $-1,7$ & $\mathrm{~N}$-ter +28.03 Da & 25,0 & 7,798 & 3 & 2113,0 & K1C14_MOUSE & K1C42_MOUSE & Keratin, type I cytoskeletal 14; & Cytokeratin-14;Keratin-14; \\
\hline GGGIGDGLLV & GSEKVTMQNLNDR & 0,1 & N-ter +34.06 Da, K +34.06 Da & 24,3 & $-2,241$ & 3 & 1558,8 & K1C14_MOUSE & K1C16_MOUSE & Keratin, type I cytoskeletal 14; & Cytokeratin-14;Keratin-14; \\
\hline KEELAYLKKN & HEEEMASMR & $-0,6$ & $\mathrm{~N}$-ter +28.03 Da & 21,0 & 6,960 & 3 & 1146,5 & K1C14_MOUSE & & Keratin, type I cytoskeletal 14; & Cytokeratin-14;Keratin-14; \\
\hline EIELQSQLSM & KASLENNLEETKGR & 0,3 & N-ter +34.06 Da, K +34.06 Da & 19,8 & $-7,974$ & 3 & 1690,0 & K1C14_MOUSE & & Keratin, type I cytoskeletal 14; & Cytokeratin-14;Keratin-14; \\
\hline KTIEDLKSKI & LAATVDNANVLLQIDNAR & 3,5 & $\mathrm{~N}$-ter +34.06 Da & 44,4 & 6,420 & 3 & 1944,1 & K1C14_MOUSE & & Keratin, type I cytoskeletal 14; & Cytokeratin-14;Keratin-14; \\
\hline KVTMQNLNDR & LATYLDKVR & $-4,1$ & N-ter +28.03 Da, K+28.03 Da & 30,3 & $-1,496$ & 3 & 1133,7 & K1C14_MOUSE & K1C16_MOUSE & Keratin, type I cytoskeletal 14; & Cytokeratin-14;Keratin-14; \\
\hline YNMLLDIKTR & LEQEIATYR & $-4,6$ & $\mathrm{~N}$-ter +28.03 Da & 33,0 & 0,955 & 2 & 1149,6 & K1C14_MOUSE & K1C13_MOUSE & Keratin, type I cytoskeletal 14; & Cytokeratin-14;Keratin-14; \\
\hline QNEEIELOSQ & LSMKASLENNLEETKGR & $-1,2$ & N-ter +34.06 Da, K +34.06 Da & 17,9 & 1,432 & 4 & 2021,2 & K1C14_MOUSE & & Keratin, type I cytoskeletal 14; & Cytokeratin-14;Keratin-14; \\
\hline TKYETELNLR & MSVEADINGLRR & $-3,7$ & $\mathrm{~N}$-ter +28.03 Da & 22,4 & $-1,222$ & 3 & 1387,7 & K1C14 MOUSE & K1C17_MOUSE & Keratin, type I cytoskeletal 14 & Cytokeratin-14;Keratin-14; \\
\hline LDKVRALEQA & NTELEVKIR & $-0,1$ & N-ter +34.06 Da, K +34.06 Da & 26,0 & 3,158 & 3 & 1168,8 & K1C14 MOUSE & K1C15 MOUSE & Keratin, type I cytoskeletal $14 ;$ & Cytokeratin-14;Keratin-14; \\
\hline QLRCEMEQQN & QEYKILLDVKTR & 0,1 & N-ter +34.06 Da, K +34.06 Da & 37,7 & $-1,304$ & 3 & 1607,1 & K1C14 MOUSE & K1C17 MOUSE & Keratin, type I cytoskeletal $14 ;$ & Cytokeratin-14;Keratin-14; \\
\hline DRLASYLDKV & RALEEANTELEVKIR & 0,1 & N-ter +34.06 Da, K +34.06 Da & 59,0 & $-6,246$ & 3 & 1838,1 & K1C14_MOUSE & $\mathrm{K}^{\mathrm{K} 1 \mathrm{C1} 7^{-} \text {MOUSE }}$ & Keratin, type I cytoskeletal $14 ;$ & Cytokeratin-14;Keratin-14; \\
\hline WFFSKTEELN & REVATNSELVQSGKSEISELR & $-0,2$ & N-ter +34.06 Da, K +34.06 Da & 30,3 & 0,499 & 4 & 2399,3 & K1C14_MOUSE & $\mathrm{K}^{\mathrm{K} 1 \mathrm{C1} 7^{-} \text {MOUSE }}$ & Keratin, type I cytoskeletal $14 ;$ & Cytokeratin-14;Keratin-14; \\
\hline RTKYETELINL & RMSVEADINGLR & 0,2 & $\mathrm{~N}$-ter +34.06 Da & 33,7 & $-0,143$ & 3 & 1393,8 & K1C14_MOUSE & K1C17_MOUSE & Keratin, type I cytoskeletal $14 ;$ & Cytokeratin-14;Keratin-14; \\
\hline GGIGDGLLVG & SEKVTMQNLNDR & 0,3 & N-ter +28.03 Da, K +28.03 Da & 27,7 & $-0,469$ & 3 & 1489,8 & K1C14_MOUSE & K1C16 MOUSE & Keratin, type I cytoskeletal 14; & Cytokeratin-14;Keratin-14; \\
\hline EELNREVATN & SELVQSGKSEISELRR & 1,6 & $\mathrm{~N}$-ter +28.03 Da, K +28.03 Da & 24,3 & 0,852 & 4 & 1873,0 & K1C14_MOUSE & K1C17_MOUSE & Keratin, type I cytoskeletal 14 & Cytokeratin-14;Keratin-14; \\
\hline GGYGGSFSSS & SFGGGLGSGFC & 0,2 & $\mathrm{~N}$-ter +28.03 Da & 26,2 & $-2,026$ & 2 & 1182,6 & K1C14_MOUSE & K1C16_MOUSE & Keratin, type I cytoskeletal 14; & Cytokeratin-14;Keratin-14; \\
\hline VCGIGGGYGG & SFSSSSFGGGLGSGFGGR & 0,2 & $\mathrm{~N}$-ter $+34.06 \mathrm{Da}$ & 27,9 & $-2,491$ & 2 & 1683,8 & K1C14_MOUSE & K1C16_MOUSE & Keratin, type I cytoskeletal 14 & Cytokeratin-14;Keratin-14; \\
\hline
\end{tabular}




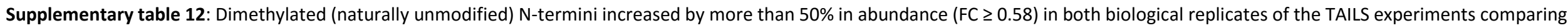
wild-type and Adam17 ${ }^{\Delta K C}$ epidermis at P3.

\begin{tabular}{|c|c|c|c|c|c|}
\hline Uniprot ID & Protein Name & $\begin{array}{c}\text { Fold change of } \\
\text { XPRESS ratio } \\
\left.\text { (Adam17 } 17^{\Delta K C} / \mathrm{WT}\right) \\
\text { (replicate 1) }\end{array}$ & $\begin{array}{c}\text { Fold change of } \\
\text { XPRESS ratio } \\
\text { (Adam } 17^{\Delta K C} / \mathrm{WT} \text { ) } \\
\text { (replicate 2) }\end{array}$ & Non Prime Site & Prime Site \\
\hline ACTB_MOUSE & Actin, cytoplasmic 1 & 1,6 & 2,5 & LRVAPEEHPV & LLTEAPLNPKANR \\
\hline Q3TYQ9_MOUSE & Aldehyde oxidase 4 & 1,0 & 2,1 & QMKGSGKCCM & DPDEKCLESR \\
\hline ATP5I_MOUSE & ATP synthase subunit e, mitochondrial & 7,6 & 0,6 & SPLIKFGRYS & ALIIGMAYGAKR \\
\hline CAN1_MOUSE & Calpain-1 catalytic subunit & 1,1 & 1,4 & DNFKTLFSKL & AGDDMEISVKELQTILNR \\
\hline CH3L1_MOUSE & Chitinase-3-like protein 1 & 1,2 & 1,6 & MGIPTFGKSF & TLASSENQLGAPISGEGLPGR \\
\hline DESP_MOUSE & Desmoplakin & 1,8 & 1,1 & DMLKVYEARL & TEEETVCLDLDKVEAYR \\
\hline EPIPL_MOUSE & Epiplakin & 0,7 & 1,2 & ELAQGTKTIH & EVTEMDSVKR \\
\hline EPIPL_MOUSE & Epiplakin & 2,5 & 0,8 & QQDTGARGSG & TSPDEGDAQDSSESAR \\
\hline Q99K47_MOUSE & Fibrinogen alpha chain & 0,9 & 0,7 & ILSVASTVWT & TDTEDKGEFLSEGGGVR \\
\hline E9Q019_MOUSE & Filaggrin & 1,0 & 0,9 & SANRRAGSSS & GSGVQGTSAGGLAADASR \\
\hline E9Q019_MOUSE & Filaggrin & 1,4 & 0,6 & ESQASDSEGH & SDFSEGQAVGAHR \\
\hline E9Q019_MOUSE & Filaggrin & 1,4 & 0,8 & GRRGVSESQA & SDSEGHSDFSEGQAVGAHR \\
\hline E9Q019_MOUSE & Filaggrin & 1,3 & 0,8 & DRQGRRGVSE & SQASDSEGHSDFSEGQAVGAHR \\
\hline J3QMA3_MOUSE & Filaggrin & 0,9 & 0,6 & DRPRQPSPSQ & SSDSHVHSGVQVEGR \\
\hline E9Q019_MOUSE & Filaggrin & 1,1 & 0,8 & SSSANRRAGS & SSGSGVQGTSAGGLAADASR \\
\hline FILA2_MOUSE & Filaggrin-2 & 1,3 & 1,3 & RSPVHPESSE & GEEHSVIPQR \\
\hline E9QPZ3_MOUSE & Filaggrin-2 & 0,6 & 6,8 & RPEVPTQDSS & RQPQADQGQPSQSGSGR \\
\hline E9QPZ3_MOUSE & Filaggrin-2 & 1,0 & 0,9 & GQSNGFGENE & SGPDQEGYQQR \\
\hline E9QPZ3_MOUSE & Filaggrin-2 & 1,0 & 0,8 & QSSSGRSPRR & SPVHPESSEGEEHSVVPQR \\
\hline E9QPZ3_MOUSE & Filaggrin-2 & 1,3 & 1,3 & GRQQRMGSSH & SSCCGPYGSGATQSSGCGQQR \\
\hline E9QPZ3_MOUSE & Filaggrin-2 & 0,9 & 1,5 & GNSTGFGEHG & SSSHPLPSSGQNESSSGQSSR \\
\hline GELS_MOUSE & Gelsolin & 1,0 & 0,7 & AGSMSVSLVA & DENPFAQGALR \\
\hline G3P_MOUSE & Glyceraldehyde-3-phosphate dehydrogenase & 0,7 & 0,6 & TVHAITATQK & TVDGPSGKLWR \\
\hline HS71A_MOUSE & Heat shock $70 \mathrm{kDa}$ protein $1 \mathrm{~A}$ & 1,3 & 1,7 & VMTVLIKRNT & TIPTKQTQTFTTYSDNQPGVLIQVYEGER \\
\hline ROA2_MOUSE & $\begin{array}{l}\text { Heterogeneous nuclear ribonucleoproteins } \\
\text { A2/B1 }\end{array}$ & 2,2 & 0,7 & HHLRDYFEEY & GKIDTIEIITDR \\
\hline E9QNP3_MOUSE & Hornerin & 0,7 & 0,9 & GRSTSRESSC & SQHDVSGSGESSSFSQHR \\
\hline E9QNP3_MOUSE & Hornerin & 1,0 & 0,9 & CFGQTEGSFQ & SSNCGQQGTTSYQSSGFDQQR \\
\hline E9QNP3_MOUSE & Hornerin & 0,6 & 0,7 & HGVGCSHGFN & TGQYGSGSYPSSNSR \\
\hline E9QNP3_MOUSE & Hornerin & 0,8 & 1,0 & QSSNCGQQGT & TSYQSSGFDQQR \\
\hline E9QNP3_MOUSE & Hornerin & 1,9 & 0,7 & FQSSNCGQQG & TTSYQSSGFDQQR \\
\hline
\end{tabular}




\begin{tabular}{|c|c|c|c|c|c|}
\hline Uniprot ID & Protein Name & $\begin{array}{c}\text { Fold change of } \\
\text { XPRESS ratio } \\
\left.\text { (Adam17 } 17^{\Delta K C} / \mathrm{WT}\right) \\
\text { (replicate } 1) \\
\end{array}$ & $\begin{array}{c}\text { Fold change of } \\
\text { XPRESS ratio } \\
\left.\text { (Adam17 } 17^{\Delta K C} / \mathrm{WT}\right) \\
\text { (replicate 2) }\end{array}$ & Non Prime Site & Prime Site \\
\hline F6RPJ9_MOUSE & Insulin-degrading enzyme & 0,7 & 1,1 & RDNIEVAYLK & TLTKDDIIR \\
\hline A2A513_MOUSE & Keratin, type I cytoskeletal 10 & 1,4 & 0,8 & GGGGFGGDGG & SLLSGNEKVTMQNLNDR \\
\hline A2A513_MOUSE & Keratin, type I cytoskeletal 10 & 1,2 & 0,9 & TVQGLEIELQ & SQLALKQSLEASLAETEGR \\
\hline A2A513_MOUSE & Keratin, type I cytoskeletal 10 & 0,8 & 1,8 & GGGGGSVRVS & STRGSLGGGYSSGGFSGGSFSR \\
\hline A2A513_MOUSE & Keratin, type I cytoskeletal 10 & 0,9 & 0,7 & KYENEVTLRQ & SVEADINGLRR \\
\hline A2A513_MOUSE & Keratin, type I cytoskeletal 10 & 1,5 & 1,1 & SFGGGSFGGG & $\begin{array}{l}\text { SYGGGFGGGGFGGDGGSLLSGNEKVTMQN } \\
\text { LNDR }\end{array}$ \\
\hline A2A513_MOUSE & Keratin, type I cytoskeletal 10 & 1,5 & 1,0 & EEQLQQIRAE & TECQNAEYQQLLDIKTR \\
\hline A2A513_MOUSE & Keratin, type I cytoskeletal 10 & 1,3 & 0,9 & GGGGSVRVSS & TRGSLGGGYSSGGFSGGSFSR \\
\hline K1C10_MOUSE & Keratin, type I cytoskeletal 10 & 0,6 & 1,7 & GGSVRVSSTR & GSLGGGYSSGGFSGGSFSR \\
\hline K1C10_MOUSE & Keratin, type I cytoskeletal 10 & 1,1 & 0,8 & TRGSLGGGYS & SGGFSGGSFSR \\
\hline K1C10_MOUSE & Keratin, type I cytoskeletal 10 & 0,9 & 0,9 & GSVRVSSTRG & SLGGGYSSGGFSGGSFSR \\
\hline K1C10_MOUSE & Keratin, type I cytoskeletal 10 & 0,7 & 0,7 & KYENEVTLRQ & SVEADINGLR \\
\hline K1C10_MOUSE & Keratin, type I cytoskeletal 10 & 0,7 & 0,9 & DLKGQILTLT & TDNANVLLQIDNAR \\
\hline K1C10_MOUSE & Keratin, type I cytoskeletal 10 & 2,0 & 1,0 & WFNQKSKELT & TEIDSNIEQMSSHKSEITELR \\
\hline K1C10_MOUSE & Keratin, type I cytoskeletal 10 & 2,9 & 1,0 & EEMRDLQNVS & TGDVNVEMNAAPGVDLTQLLNNMR \\
\hline K1C10_MOUSE & Keratin, type I cytoskeletal 10 & 1,2 & 1,1 & EWFNQKSKEL & TTEIDSNIEQMSSHKSEITELR \\
\hline K1C10_MOUSE & Keratin, type I cytoskeletal 10 & 0,8 & 0,6 & MSVL & YSSSSKQFSSSRSGGGGGGGSVR \\
\hline K2C1_MOUSE & Keratin, type II cytoskeletal 1 & 1,0 & 4,8 & QRLRSEIDGC & KKQISQIQQNINDAEQR \\
\hline K2C1_MOUSE & Keratin, type II cytoskeletal 1 & 0,7 & 1,5 & MEMSQMQTQI & SETNVVLSMDNNR \\
\hline K2C1_MOUSE & Keratin, type II cytoskeletal 1 & 1,6 & 1,1 & GSFGGGGFGG & SGFGGGLGGGGGFGSGGGFGGGR \\
\hline K2C1_MOUSE & Keratin, type II cytoskeletal 1 & 1,4 & 1,1 & SGGGFCGSSG & SGFGSKSLMNLGGGR \\
\hline K2C1_MOUSE & Keratin, type II cytoskeletal 1 & 1,2 & 0,7 & GSGGGSYGGS & $\begin{array}{l}\text { SGGGGSGGSYGGGSGGGSYGGGSGGGSSG } \\
\text { SHR }\end{array}$ \\
\hline K2C1_MOUSE & Keratin, type II cytoskeletal 1 & 2,6 & 1,3 & YGGGGSYGGG & $\begin{array}{l}\text { SGGGSYGGSSGGGGSGGSYGGGSGGGSYG } \\
\text { GGSGGGSSGSHR }\end{array}$ \\
\hline K2C1_MOUSE & Keratin, type II cytoskeletal 1 & 1,2 & 1,1 & RFSGGGFCGS & SGSGFGSKSLMNLGGGR \\
\hline K2C1_MOUSE & Keratin, type II cytoskeletal 1 & 1,4 & 0,9 & FCGSSGSGFG & SKSLMNLGGGR \\
\hline K2C1_MOUSE & Keratin, type II cytoskeletal 1 & 0,7 & 0,7 & GGIQEVTINQ & SLLQPLNVEVDPQIQKVKSQER \\
\hline K2C1_MOUSE & Keratin, type II cytoskeletal 1 & 0,8 & 1,0 & VKSQEREQIK & SLNDKFASFIDKVR \\
\hline K2C1_MOUSE & Keratin, type II cytoskeletal 1 & 1,4 & 0,9 & RKVDSLKSDQ & SRMDSELKNMQDLVEEYR \\
\hline K2C1_MOUSE & Keratin, type II cytoskeletal 1 & 1,4 & 0,8 & GGSGGGSYGG & $\begin{array}{l}\text { SSGGGGSGGSYGGGSGGGSYGGGSGGGSS } \\
\text { GSHR }\end{array}$ \\
\hline K2C1_MOUSE & Keratin, type II cytoskeletal 1 & 1,7 & 0,8 & GSSGGGGSGG & SYGGGSGGGSYGGGSGGGSSGSHR \\
\hline
\end{tabular}




\begin{tabular}{|c|c|c|c|c|c|}
\hline Uniprot ID & Protein Name & $\begin{array}{c}\text { Fold change of } \\
\text { XPRESS ratio } \\
\left.\text { (Adam17 } 17^{\Delta K C} / \mathrm{WT}\right) \\
\text { (replicate 1) }\end{array}$ & $\begin{array}{c}\text { Fold change of } \\
\text { XPRESS ratio } \\
\left.\text { (Adam17 } 17^{\Delta K C} / \mathrm{WT}\right) \\
\text { (replicate 2) }\end{array}$ & Non Prime Site & Prime Site \\
\hline K2C1_MOUSE & Keratin, type II cytoskeletal 1 & 2,6 & 0,8 & GSYGGGSGGG & $\begin{array}{l}\text { SYGGSSGGGGSGGSYGGGSGGGSYGGGSG } \\
\text { GGSSGSHR }\end{array}$ \\
\hline K2C1_MOUSE & Keratin, type II cytoskeletal 1 & 1,3 & 0,8 & AGQRGDDLRT & TKMEISELNR \\
\hline K2C1_MOUSE & Keratin, type II cytoskeletal 1 & 1,4 & 1,3 & FLEQQNQVLQ & TKWELLQQVDTTTR \\
\hline K2C1_MOUSE & Keratin, type II cytoskeletal 1 & 3,1 & 0,8 & NMQDLVEEYR & TKYEDEINKR \\
\hline K2C1_MOUSE & Keratin, type II cytoskeletal 1 & 1,9 & 1,2 & MSQMQTQISE & TNVVLSMDNNR \\
\hline K2C1_MOUSE & Keratin, type II cytoskeletal 1 & 1,7 & 1,1 & LYQMEMSQMQ & TQISETNVVLSMDNNR \\
\hline K2C1_MOUSE & Keratin, type II cytoskeletal 1 & 1,3 & 1,2 & WELLQQVDTT & TRTQNLDPFFENYISILR \\
\hline K2C1_MOUSE & Keratin, type II cytoskeletal 1 & 2,0 & 1,1 & TKLALDMEIA & TYKKLLEGEEIR \\
\hline K2C1_MOUSE & Keratin, type II cytoskeletal 1 & 1,3 & 1,8 & AEAETFYQSK & YEELQITAGKHGDSVR \\
\hline K2C5_MOUSE & $\begin{array}{l}\text { Keratin, type II cytoskeletal } 5 \\
\text { Platelet-activating factor acetylhydrolase IB }\end{array}$ & 1,0 & 1,6 & LNLDSIIAEV & KAQYEDIANR \\
\hline LIS1_MOUSE & $\begin{array}{l}\text { subunit alpha }\{\text { ECO:0000255|HAMAP- } \\
\text { Rule:MF_03141\} }\end{array}$ & 0,6 & 1,1 & SVMVSASEDA & TIKVWDYETGDFER \\
\hline ENDOU_MOUSE & Poly(U)-specific endoribonuclease & 0,8 & 2,5 & DHEGFSHSSD & AVTKEELESISETIYR \\
\hline TCTP_MOUSE & Translationally-controlled tumor protein & 0,7 & 0,6 & ELFSDIYKIR & EIADGLCLEVEGKMVSR \\
\hline
\end{tabular}




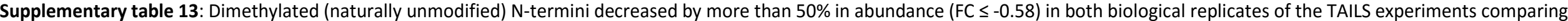
wild-type and Adam $17^{\triangle K C}$ epidermis at P3.

\begin{tabular}{|c|c|c|c|c|c|}
\hline Uniprot ID & Protein Name & $\begin{array}{c}\text { Fold change of } \\
\text { XPRESS ratio } \\
\text { (Adam17 }^{\Delta K C} / \mathrm{WT} \text { ) } \\
\text { (replicate 1) }\end{array}$ & $\begin{array}{c}\text { Fold change of } \\
\text { XPRESS ratio } \\
\left.\text { (Adam } 17^{\Delta K C} / \mathrm{WT}\right) \\
\text { (replicate 2) }\end{array}$ & Non Prime Site & Prime Site \\
\hline K1C25_MOUSE & Keratin, type I cytoskeletal 25 & $-6,4$ & $-1,8$ & AGGNVNVEMN & AAPGVDLTVLLNNMR \\
\hline ATPD_MOUSE & ATP synthase subunit delta, mitochondrial & $-0,7$ & $-4,0$ & VNADSSVQLL & AEEAVTLDMLDLGAAR \\
\hline EIF3B_MOUSE & $\begin{array}{l}\text { Eukaryotic translation initiation factor } 3 \text { subunit } \\
\text { B \{ECO:0000255|HAMAP-Rule:MF_03001\} }\end{array}$ & $-1,2$ & $-0,9$ & MQD & AENVAVPEAAEER \\
\hline SBSN_MOUSE & Suprabasin & $-0,7$ & $-0,9$ & HHAFGQGRDM & AETFDQGAHHAFGQGGR \\
\hline PLAK_MOUSE & Junction plakoglobin & $-1,6$ & $-3,0$ & VEALIHAILR & AGDKDDITEPAVCALR \\
\hline K2C71_MOUSE & Keratin, type II cytoskeletal 71 & $-4,0$ & $-2,6$ & YQVTVNKSLL & APLNVELDPEIQKVR \\
\hline E9Q019_MOUSE & Filaggrin & $-0,8$ & $-0,7$ & PRQPSPSQSS & DSQVHSGVQVEGR \\
\hline TCTP_MOUSE & Translationally-controlled tumor protein & $-0,6$ & $-0,9$ & ILANFNNYQF & FIGENMNPDGMVALLDYR \\
\hline E9QPZ3_MOUSE & Filaggrin-2 & $-1,0$ & $-0,6$ & SCFDDSQYIL & FQKHLESPSFGNQSGFSPNER \\
\hline K2C74_MOUSE & $\begin{array}{l}\text { Keratin, type II cytoskeletal } 74 \\
\{\text { ECO:0000250|UniProtKB:Q7RT57\} }\end{array}$ & $-2,4$ & $-1,1$ & KSLLAPLNVE & LDPEIQKVR \\
\hline NDKB_MOUSE & Nucleoside diphosphate kinase B & $-0,6$ & $-1,4$ & LNVVKTGRVM & LGETNPADSKPGTIR \\
\hline K1C25_MOUSE & Keratin, type I cytoskeletal 25 & $-0,8$ & $-0,8$ & DDAGATTSAR & NELTEMKR \\
\hline K2C5_MOUSE & Keratin, type II cytoskeletal 5 & $-6,4$ & $-1,0$ & EEKEQIKTLN & NKFASFIDKVR \\
\hline J3QMĀ3_MOUSE & Filaggrin & $-10,0$ & $-3,0$ & RSSNRRDRPR & QPSPSQSSDSHVHSGVQVEGR \\
\hline H4_MOUSE & Histone $\mathrm{H} 4$ & $-3,8$ & $-2,1$ & RRLARRGGVK & RISGLIYEETR \\
\hline K1C12_MOUSE & Keratin, type I cytoskeletal 12 & $-1,0$ & $-5,6$ & RMKYENELAL & RQTVEADINGLR \\
\hline 1433S_MOUSE & $14-3-3$ protein sigma & $-2,0$ & $-1,1$ & RYEDMAAFMK & SAVEKGEELSCEER \\
\hline KRA31_MOUSE & Keratin-associated protein 3-1 & $-5,3$ & $-4,3$ & MACCVALCC & SVPTGPATTICSSDKSCR \\
\hline KR195_MOUSE & Keratin-associated protein $19-5$ & $-10,0$ & $-10,0$ & M & SYYGSYYGGLGSGIR \\
\hline K1C17_MOUSE & Keratin, type I cytoskeletal 17 & $-2,5$ & $-1,9$ & EDLKNKILVA & TVDNASILLQIDNAR \\
\hline CALL3_MOUSE & Calmodulin-like protein 3 & $-0,7$ & $-3,0$ & SEEEIREAFR & VFDKDGNGFVSAAELR \\
\hline
\end{tabular}


Supplementary table 14: Acetylated (naturally modified) N-termini identified in the TAlLS experiment comparing wild-type and Adam17 $7^{\text {AKC }}$ epidermis at P3 (replicate 1). This is a comprehensive, non redundant listing. Up to two potential protein IDS per peptide are stated.

\begin{tabular}{|c|c|c|c|c|c|c|c|c|c|c|c|}
\hline Non Prime Site & Prime Site & $\begin{array}{l}\text { Fold change of } \\
\text { XPRESS ratio } \\
\text { (Adam17 } 7^{\text {NKC } / W T)} \\
\end{array}$ & Modifications & Hyperscore & $\begin{array}{l}\text { Mass error } \\
\text { in ppm }\end{array}$ & Charge & $\begin{array}{c}\text { Precursor } \\
\text { neutral mass } \\
\text { in Da }\end{array}$ & Uniprot ID 1 & Uniprot ID 2 & Protein Name 1 & Protein Name 2 \\
\hline$\overline{\mathrm{MT}}$ & MDKSELVQKAKLAEQAER & 0,1 & N-ter + 42.01 Da, K +34.06 Da & 36,7 & 0,090 & 1 & 2217,3 & 1433B_MOUSE & A2A5N1_MOUSE & 14-3-3 protein beta/alpha; & Protein kinase C inhibitor protein $1 ;$ \\
\hline M & TMDKSELVQKAKLAEQAER & n.d. & $\mathrm{N}$-ter + 42.01 Da, K +28.03 Da & 47,3 & 0,651 & 1 & 2300,2 & 1433B_MOUSE & A2A5N1_MOUSE & 14-3-3 protein beta/alpha; & Protein kinase C inhibitor protein 1 \\
\hline M & DDREDLVYQAKLAEQAER & n.d. & N-ter + 42.01 Da, K +34.06 Da & 81,4 & $-2,078$ & 1 & 2355,2 & 1433E_MOUSE & D6REF3_MOUSE & 14-3-3 protein epsilon; & \\
\hline M & DDREDLVYQAK & n.d. & N-ter + 42.01 Da, K +34.06 Da & 59,1 & $-1,282$ & 1 & 1557,8 & 1433E_MOUSE & D6REF3_MOUSE & 14-3-3 protein epsilon; & \\
\hline M & GDREQLLQR & n.d. & $\mathrm{N}$-ter + 42.01 Da & 40,2 & $-1,296$ & 1 & 1155,6 & 1433F_MOUSE & & 14-3-3 protein eta; & \\
\hline M & VDREQLVQKAR & n.d. & N-ter + 42.01 Da, K +34.06 Da & 46,9 & $-2,183$ & 1 & 1416,8 & 1433G_MOUSE & & 14-3-3 protein gamma; & \\
\hline MER & ASLIOKAKLAEQAER & n.d. & N-ter + 42.01 Da, K +34.06 Da & 26,1 & $-8,767$ & 1 & 1765,1 & 14335_MOUSE & & 14-3-3 protein sigma; & Stratifin; \\
\hline M & ERASLIOKAKLAEQAER & n.d. & $\mathrm{N}$-ter + 42.01 Da, K +34.06 Da & 38,7 & 0,092 & 1 & 2181,3 & 14335_MOUSE & & 14-3-3 protein sigma; & Stratifin; \\
\hline PARRSPSLAM & EKTELIQKAKLAEQAER & n.d. & $\mathrm{N}$-ter + 42.01 Da, K +34.06 Da & 65,4 & $-3,625$ & 1 & 2259,3 & 1433T_MOUSE & F6VW30_MOUSE & 14-3-3 protein theta; & 14-3-3 protein tau; \\
\hline PARRSPSLAM & EKTELIQKAK & n.d. & N-ter + 42.01 Da, K +34.06 Da & 46,8 & 0,956 & 1 & 1461,9 & 1433T_MOUSE & & 14-3-3 protein theta; & 14-3-3 protein tau; \\
\hline PARRSPSLAM & ЕКTELIQK & n.d. & N-ter + 42.01 Da, K +34.06 Da & 41,1 & $-1,219$ & 1 & 1228,7 & 1433T_MOUSE & & 14-3-3 protein theta; & 14-3-3 protein tau; \\
\hline M & DKNELVQKAKLAEQAER & n.d. & $\mathrm{N}$-ter + 42.01 Da, K +34.06 Da & 73,9 & $-1,157$ & 1 & 2244,3 & 1433Z_MOUSE & D3YXF4_MOUSE & 14-3-3 protein zeta/delta; & Protein kinase C inhibitor protein 1;SEZ-2; \\
\hline M & DKNELVQKAK & n.d. & $\mathrm{N}$-ter + 42.01 Da, K +34.06 Da & 51,8 & 0,276 & 1 & 1446,9 & 1433Z_MOUSE & D3YXF4_MOUSE & 14-3-3 protein zeta/delta; & Protein kinase C inhibitor protein 1;SEZ-2; \\
\hline M & DKNELVaK & n.d. & $\mathrm{N}$-ter + 42.01 Da, K+34.06 Da & 40,3 & 3,044 & 1 & 1213,7 & 1433Z_MOUSE & D3YXF4_MOUSE & 14-3-3 protein zeta/delta; & $\begin{array}{l}\text { Protein kinase C inhibitor protein 1; } ; \text { EZ-2; } \\
\text { FVVE finger-containing phosphoinositide }\end{array}$ \\
\hline M & ATDDKSSPTLDSANDLPR & n.d. & $\mathrm{N}$-ter + 42.01 Da, K+34.06 Da & 28,6 & $-1,262$ & 1 & 1978,0 & FYV1_MOUSE & D3Z5N5_MOUSE & 1-phosphatidylinositol 3-phosphate 5-kinase; & $\begin{array}{l}\text { kinase;PIKfyve;Phosphatidylinositol 3- } \\
\text { phosphate 5-kinase type III;p235; } \\
\text { 26S proteasome AAA-ATPase subunit }\end{array}$ \\
\hline M & ALDGPEQMELEEGKAGSGLR & 0,4 & N-ter + 42.01 Da, K+34.06 Da & 64,5 & $-0,324$ & 1 & 2162,1 & PRS8_MOUSE & Q8к1K2_MOUSE & 265 protease regulatory subunit 8 & $\begin{array}{l}\text { RPT6;Proteasome e 65 subunit ATPase } \\
\text { 5;Proteasome subunit } 455 \text {;p45/SUG; } \\
\text { 26S proteasome AAA-ATPase subunit }\end{array}$ \\
\hline MALDGPEQ & MELEEGKAGSGLR & n.d. & N-ter + 42.01 Da, K+28.03 Da & 38,4 & 4,559 & 1 & 1445,7 & PRS8_MOUSE & Q8K1K2_MOUSE & 265 protease regulatory subunit 8 & $\begin{array}{l}\text { RPT6;Proteasome } 265 \text { subunit ATPase } \\
\text { 5;Proteasome subunit p45;p45/SUG; }\end{array}$ \\
\hline M & SGEDVPHR & n.d. & N-ter $+42.01 \mathrm{Da}$ & 21,8 & $-0,426$ & 1 & 937,4 & PSMD9_MOUSE & & $26 \mathrm{~S}$ proteasome non-ATPase regulatory subunit $9 ;$ & 265 proteasome regulatory subunit p27; \\
\hline m & ASSHTVLMR & n.d. & N-ter $+42.01 \mathrm{Da}$ & 22,0 & 0,479 & 1 & 1042,5 & BPNT1_MOUSE & D3Z5X0_MOUSE & $3^{\prime}\left(2^{\prime}\right), 5^{\prime}$-bisphosphate nucleotidase 1; & $\begin{array}{l}\text { Bisphosphate 3'-nucleotidase 1;PAP-inositol 1,4 } \\
\text { phosphatase: }\end{array}$ \\
\hline SRGSKSVARR & VLQALEGLKMVEKDQDGGR & n.d. & $\mathrm{N}$-ter + 42.01 Da, K +34.06 Da & 32,7 & 1,001 & 1 & 2195,2 & RS19_MOUSE & D3Z722_MOUSE & 40S ribosomal protein S19; & \\
\hline & AFKDTGKTPVEPEVAIHR & n.d. & N-ter + 42.01 Da, $\mathrm{K}+34.06 \mathrm{Da}$ & 64,4 & 0,000 & 1 & 2104,2 & RS20_MOUSE & & 40S ribosomal protein S20; & \\
\hline M & DTSRVQPIKLAR & n.d. & $\mathrm{N}$-ter + 42.01 Da, K +34.06 Da & 27,5 & 1,004 & 1 & 1589,9 & RS28_MOUSE & J3QNN8_MOUSE & 40S ribosomal protein S28; & \\
\hline M & DPEPTEHSTDGVSVPR & n.d. & $\mathrm{N}$-ter + 42.01 Da & 45,2 & $-1,845$ & 1 & 1894,8 & G3UWA6_MOUSE & & 4F2 cell-surface antigen heavy chain; & Solute carrier family 3 member 2 ; \\
\hline M & TLKSSEGEGGNSMR & n.d. & $\mathrm{N}$-ter + 42.01 Da, K+34.06 Da & 34,3 & 2,549 & 1 & 1527,8 & EM55_M̄MOUSE & B7ZCMO_MOUSE & 55 kDa erythrocyte membrane protein; & Membrane protein, palmitoylated 1 ; \\
\hline M & ATESSPLTTHVLDTASGLPAQGLCLR & n.d. & N-ter + 42.01 Da, C+57.02 Da & 78,5 & 1,059 & 1 & 2736,4 & HIUH_MOUSE & & 5-hydroxyisourate hydrolase; & Transthyretin-related protein; \\
\hline M & AQDQGEKENPMR & n.d. & $\mathrm{N}$-ter + 42.01 Da, K +34.06 Da & 68,0 & $-1,419$ & 1 & 1477,7 & RL11_MOUSE & D3Z3K1_MOUSE & 60S ribosomal protein L11; & \\
\hline M & SAHLQWMVVR & n.d. & N-ter + 42.01 Da & 31,9 & 1,024 & 1 & 1267,7 & RL28_MOUSE & & 60S ribosomal protein L28; & \\
\hline M & EAVPEKK & n.d. & N-ter + 42.01 Da, K +34.06 Da & 24,9 & $-0,767$ & 1 & 1040,6 & RL7_MOUSE & & 60 S ribosomal protein L7; & \\
\hline M & AEEVSSLMKATVLMR & n.d. & $\mathrm{N}$-ter + 42.01 Da, K+34.06 Da & 38,7 & 4,363 & 1 & 1740,0 & 5NT3B_MOUSE & & $\begin{array}{l}\text { 7-methylguanosine phosphate-specific 5'- } \\
\text { nucleotidase; }\end{array}$ & $\begin{array}{l}\text { Cytosolic 5'-nucleotidase 3B;Cytosolic 5'- } \\
\text { nucleotidase III-like protein; ;N(7)- } \\
\text { methylguanylate 5'-phosphatase; } \\
\text { Abelson interactor 1;Ablphilin-1;Eps8 SH3 }\end{array}$ \\
\hline M & AELQMLLEEEIPSGKR & n.d. & $\mathrm{N}$-ter + 42.01 Da, K +34.06 Da & 34,3 & $-0,833$ & 1 & 1918,0 & AB11_MOUSE & J3QNK8_MOUSE & Abl interactor 1; & $\begin{array}{l}\text { domain-binding protein;Spectrin SH3 domain- } \\
\text { binding protein } 1 ; \mathrm{e} 3 \mathrm{~B} 1 \text {; }\end{array}$ \\
\hline VFPSIVGRPR & HQGVMVGMGQKDSYVGDEAQSKR & $-0,5$ & $\mathrm{~N}$-ter + 42.01 Da, K +28.03 Da & 22,6 & 3,067 & 1 & 2604,3 & ACTA_MOUSE & ACTB_MOUSE & Actin, aortic smooth muscle; & Alpha-actin-2; \\
\hline MFAM & DDDIAALVVDNGSGMCKAGFAGDDAPR & n.d. & $\begin{array}{l}\text { N-ter + 42.01 Da, } \mathrm{K}+34.06 \mathrm{Da}, \\
\mathrm{C}+57.02 \mathrm{Da}\end{array}$ & 59,4 & 0,675 & 1 & 2812,3 & ACTB_MOUSE & E9Q1F2_MOUSE & Actin, cytoplasmic 1; & Beta-actin; \\
\hline M & EEEIAALVIDNGSGMCKAGFAGDDAPR & n.d. & $\begin{array}{l}\text { N-ter + 42.01 Da, K+34.06 Da, } \\
\text { C+57.02 Da }\end{array}$ & 67,2 & 0,313 & 1 & 2868,4 & ACTG_MOUSE & G3UYGO_MOUSE & Actin, cytoplasmic 2; & Gamma-actin; \\
\hline MC & EEETTALVCDNGSGLCKAGFAGDDAPR & 4,8 & $\begin{array}{l}\text { N-ter + 42.01 Da, K+28.03 Da, } \\
\text { C +57.02 Da }\end{array}$ & 20,6 & 3,330 & 1 & 2909,3 & ACTH_MOUSE & D3Z2K3_MOUSE & Actin, gamma-enteric smooth muscle; & $\begin{array}{l}\text { Alpha-actin-3;Gamma-2-actin;Smooth muscle } \\
\text { gamma-actin; }\end{array}$ \\
\hline ETTALVCDNG & SGLCKAGFAGDDAPR & 10,6 & $\begin{array}{l}\text { N-ter + 42.01 Da, } \mathrm{K}+28.03 \mathrm{Da}, \\
\mathrm{C}+57.02 \mathrm{Da}\end{array}$ & 20,8 & $-0,628$ & 1 & 1590,8 & ACTH_MOUSE & ACTA_MOUSE & Actin, gamma-enteric smooth muscle; & $\begin{array}{l}\text { Alpha-actin-3;Gamma-2-actin;SSmooth muscle } \\
\text { gamma-actin; }\end{array}$ \\
\hline $\begin{array}{l}M \\
M\end{array}$ & $\begin{array}{l}\text { SLHQFLLEPITCHAWNR } \\
\text { TATLRPYSAVRR }\end{array}$ & $\begin{array}{l}\text { n.d. } \\
\text { n.d. }\end{array}$ & $\begin{array}{l}\text { N-ter + 42.01 Da, C+57.02 Da } \\
\text { N-ter + 42.01 Da }\end{array}$ & $\begin{array}{l}40,6 \\
43,3\end{array}$ & $\begin{aligned} 1,154 \\
-0,935\end{aligned}$ & $\begin{array}{l}1 \\
1\end{array}$ & $\begin{array}{l}2163,1 \\
1388,8\end{array}$ & $\begin{array}{l}\text { ARC1A_MOUSE } \\
\text { ARPC_MOUSE }\end{array}$ & D3YVI5_MOUSE & $\begin{array}{l}\text { Actin-related protein } 2 / 3 \text { complex subunit } 1 \mathrm{~A} ; \\
\text { Actin-related protein } 2 / 3 \text { complex subunit } 4 ;\end{array}$ & $\begin{array}{l}\text { SOP2-like protein;Sid 329; } \\
\text { Arp2/3 complex } 20 \text { kDa subunit; }\end{array}$ \\
\hline M & DSVEEPQKKVFKAR & n.d. & $\mathrm{N}$-ter + 42.01 Da, K+34.06 Da & 18,6 & 0,103 & 1 & 1935,1 & MCAF1_MOUSE & & Activating transcription factor 7 -interacting protein 1; & 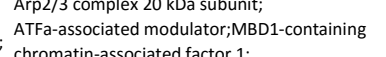 \\
\hline $\begin{array}{l}M \\
M\end{array}$ & $\begin{array}{l}\text { SQAEFDKAAEEVKR } \\
\text { SEPELKLVAR }\end{array}$ & $\begin{array}{l}\text { n.d. } \\
\text { n.d. }\end{array}$ & $\begin{array}{l}\mathrm{N} \text {-ter + 42.01 Da, K+34.06 Da } \\
\mathrm{N} \text {-ter + 42.01 Da, K+34.06 Da }\end{array}$ & $\begin{array}{l}80,4 \\
43,5\end{array}$ & $\begin{array}{l}-2,385 \\
-0,246\end{array}$ & $\begin{array}{l}1 \\
1\end{array}$ & $\begin{array}{l}1716,9 \\
1216,7\end{array}$ & $\begin{array}{l}\text { ACBP_MOUSE } \\
\text { APT_MOUSE }\end{array}$ & MoQWU8_MOUSE & $\begin{array}{l}\text { EAcyl-CoA-binding protein; } \\
\text { Adenine phosphoribosyltransferase; }\end{array}$ & $\begin{array}{l}\text { chromatin-associated factor } 1 ; \\
\text { Diazepam-binding inhibitor; Endozepine; }\end{array}$ \\
\hline m & SDKLPYKVADIGLAAWGR & n.d. & N-ter + 42.01 Da, K+34.06 Da & 54,8 & 0,531 & 1 & 2069,2 & SAHH_MOUSE & A2ALTS_MOUSE & Adenosylhomocysteinase; & $\begin{array}{l}\text { CUBP; Liver copper-binding protein;S-adenosyl- } \\
\text { L-homocysteine hydrolase. }\end{array}$ \\
\hline
\end{tabular}




\begin{tabular}{|c|c|c|c|c|c|c|c|c|c|c|c|}
\hline Non Prime Site & Prime Site & $\begin{array}{c}\text { Fold change of } \\
\text { XPRESS ratio } \\
\left.\text { (Adam17 }^{\Delta K c} / \mathrm{WT}\right) \\
\end{array}$ & Modifications & Hyperscore & $\begin{array}{l}\text { Mass error } \\
\text { in ppm }\end{array}$ & charge & $\begin{array}{c}\text { Precursor } \\
\text { neutral mass } \\
\text { in Da }\end{array}$ & Uniprot ID 1 & Uniprot ID 2 & Protein Name 1 & Protein Name 2 \\
\hline LFEEAEKNVQ & KGKGLDGWEKR & n.d. & $\mathrm{N}$-ter + 42.01 Da, K+34.06 Da & 16,9 & $-5,564$ & 1 & 1416,9 & PURA2_MOUSE & & $\begin{array}{l}\text { Adenylosuccinate synthetase isozyme } 2 \\
\text { \{ECO:0000255|HAMAP-Rule:MF_03127\}; }\end{array}$ & $\begin{array}{l}\text { Adenylosuccinate synthetase, acidic isozyme } \\
\text { \{ECO:0000255|HAMAP- } \\
\text { Rule:MF_03127\};Adenylosuccinate synthetase, } \\
\text { liver isozyme \{ECC:0000255|HAMAP- } \\
\text { Rule:MF_03127):|MPP--aspartate lipase 2 } \\
\text { \{ECO:0000255|HAMAP-Rule:MF_03127\}; }\end{array}$ \\
\hline M & AASPSKTEIQTIFKR & n.d. & $\mathrm{N}$-ter + 42.01 Da, K +34.06 Da & 38,1 & $-0,894$ & 1 & 1786,1 & ARFG2_MOUSE & & ADP-ribosylation factor GTPase-activating protein 2; & $\begin{array}{l}\text { GTPase-activating protein ZNF289;Zinc finger } \\
\text { protein 289; }\end{array}$ \\
\hline M & GDPSKQDILAIFKR & n.d. & $\mathrm{N}$-ter + 42.01 Da, K+34.06 Da & 17,5 & 1,765 & 1 & 1697,0 & ARFG3_MOUSE & & ADP-ribosylation factor GTPase-activating protein $3 ;$ & \\
\hline M & AQELQHPEFAR & n.d. & $\mathrm{N}$-ter $+42.01 \mathrm{Da}$ & 38,4 & 1,461 & 1 & 1366,7 & ADSV_MOUSE & & Adseverin; & Gelsolin-like protein;Scinderin; \\
\hline M & SAAETGSEPSQGAGPSEATLHSLR & n.d. & $\mathrm{N}$-ter $+42.01 \mathrm{Da}$ & 76,1 & 1,007 & 1 & 2381,1 & E9Q3E1_MOUSE & & $\begin{array}{l}\text { Aldehyde dehydrogenase } \\
\text { \{ECO:0000256|PIRNR:PIRNR036492\}; }\end{array}$ & \\
\hline M & AGVDQHEGTIQVQGQNLFFR & n.d. & $\mathrm{N}$-ter $+42.01 \mathrm{Da}$ & 51,8 & $-0,393$ & 1 & 2285,1 & ABHEB_MOUSE & & Alpha/beta hydrolase domain-containing protein $14 \mathrm{~B}$; & ; CCG1-interacting factor B; \\
\hline YTEQTQHILN & SMVSKLQEDPR & n.d. & $\mathrm{N}$-ter + 42.01 Da, K+34.06 Da & 24,1 & $-5,561$ & 1 & 1364,7 & MA2A2_MOUSE & & Alpha-mannosidase $2 x ;$ & $\begin{array}{l}\text { Alpha-mannosidase IIx;Mannosidase alpha } \\
\text { class } 2 \mathrm{~A} \text { member 2;Mannosyl-oligosaccharide } \\
\text { 1,3-1,6-alpha-mannosidase; }\end{array}$ \\
\hline M & DNSGKQAEAMALLAEAER & n.d. & $\mathrm{N}$-ter + 42.01 Da, K+34.06 Da & 75,4 & $-1,089$ & 1 & 2110,0 & SNAA_MOUSE & & Alpha-soluble NSF attachment protein; & $\begin{array}{l}\text { N-ethylmaleimide-sensitive factor attachment } \\
\text { protein alpha; }\end{array}$ \\
\hline M & ADKMDMSLDDIIKLNR & 0,1 & $\mathrm{~N}$-ter + $42.01 \mathrm{Da}, \mathrm{K}+28.03 \mathrm{Da}$ & 69,9 & 0,303 & 1 & 1975,0 & ALRF2_MOUSE & THOC4_MOUSE & Aly/REF export factor 2; & Alyref;RNA and export factor-binding protein 2 ; \\
\hline MFLCRFWGKM & ATNDAVLKR & n.d. & N-ter + 42.01 Da, K+34.06 Da & 36,8 & 0,751 & 1 & 1062,6 & AIMP1_MOUSE & Q3UZG4_MOUSE & $\begin{array}{l}\text { Aminoacyl tRNA synthase complex-interacting } \\
\text { multifunctional protein } 1 \text {; }\end{array}$ & $\begin{array}{l}\text { Multisynthase complex auxiliary component } \\
\text { p43; }\end{array}$ \\
\hline M & TTKDPESEHPSVTLFR & n.d. & $\mathrm{N}$-ter + 42.01 Da, K+34.06 Da & 45,4 & 0,885 & 1 & 1919,0 & ACY1_MOUSE & & Aminoacylase-1; & N-acyl-L-amino-acid amidohydrolase; \\
\hline м & AQETNHSQAPMLCSTGCGFYGNPR & n.d. & N-ter + 42.01 Da, C+57.02 Da & 34,0 & $-0,440$ & 1 & 2724,2 & ZFAN6_MOUSE & & AN1-type zinc finger protein 6 ; & $\begin{array}{l}\text { Associated with PRK1 protein;Zinc finger A20 } \\
\text { domain-containing protein 3; }\end{array}$ \\
\hline SESVAPSQVD & STSVDKAVSETEDLQGPR & n.d. & N-ter + 42.01 Da, K+34.06 Da & 29,1 & 8,262 & 1 & 1994,0 & ZFAN6_MOUSE & & AN1-type zinc finger protein 6; & $\begin{array}{l}\text { Associated with PRK1 protein;Zinc finger A20 } \\
\text { domain-containing protein 3; } \\
\text { Annexin I;Annexin-1;Calpactin I;;Calpactin- }\end{array}$ \\
\hline M & AMVSEFLKQAR & n.d. & N-ter + 42.01 Da, K+34.06 Da & 61,1 & $-3,980$ & 1 & 1354,7 & ANXA1_MOUSE & & Annexin A1; & $\begin{array}{l}\text { 2; Chromobindin-9;ipipocortin l;Phospholipase } \\
\text { A2 inhibitory protein;;35; }\end{array}$ \\
\hline DSLEIIISR & TNQELQEINR & n.d. & $\mathrm{N}$-ter + $42.01 \mathrm{Da}$ & 21,3 & $-0,932$ & 1 & 1285,6 & ANXA2_MOUSE & BOV2N5_MOUSE & Annexin A2; & $\begin{array}{l}\text { Annexin II;Annexin-2;Calpactin I heavy } \\
\text { chain;Calpactin-1 heavy chain; Chromobindin- } \\
\text { 8;Lipocortin II;Placental anticoagulant protein } \\
\text { IV;Protein I;p36; }\end{array}$ \\
\hline M & EAKGGTVKAASGFNATEDAQTLR & 0,2 & N-ter + 42.01 Da, K+28.03 Da & 71,8 & 0,666 & 1 & 2550,3 & ANXA4_MOUSE & SAR1F2_MOUSE & Annexin A4; & $\begin{array}{l}\text { Annexin IV;Annexin-4; } \\
67 \text { kDa calelectrin;Annexin Vi;Annexin- }\end{array}$ \\
\hline M & AKIAQGAMYR & n.d. & $\mathrm{N}$-ter + 42.01 Da, K+34.06 Da & 44,5 & $-0,675$ & 1 & 1183,7 & ANXA6_MOUSE & F8WIT2_MOUSE & Annexin A6; & $\begin{array}{l}\text { 6;Calphobindin-II;Chromobindin-20;Lipocortin } \\
\text { VI;Protein III;66;;p70; }\end{array}$ \\
\hline GTDPSTSRKM & AELEEVTLDGKPLQALR & n.d. & $\mathrm{N}$-ter + 42.01 Da, K+34.06 Da & 69,3 & 0,459 & 1 & 1957,1 & ACINU_MOUSE & F6RJ39_MOUSE & $\begin{array}{l}\text { Apoptotic chromatin condensation inducer in the } \\
\text { nucleus; }\end{array}$ & \\
\hline CEPVYGPLTM & SLKPQPQPPAPATGR & $-0,2$ & $\mathrm{~N}$-ter + 42.01 Da, K+28.03 Da & 49,0 & 0,495 & 1 & 1613,9 & ATX2_MOUSE & E9QM77_MOUSE & Ataxin-2; & Spinocerebellar ataxia type 2 protein homolog; \\
\hline M & LKPQPPQQTSQPQQPPPTQQAVAR & n.d. & N-ter + 42.01 Da, K+34.06 Da & 64,8 & 0,210 & 1 & 2856,5 & ATX2L_MOUSE & & Ataxin-2-like protein; & \\
\hline M & SWLFGIKGPKGEGTGPPLPLPPAQPGAEGGGDR & n.d. & $\mathrm{N}$-ter + 42.01 Da, K+34.06 Da & 20,5 & 0,298 & 1 & 3349,8 & ATAD3_MOUSE & H3BKI6_MOUSE & ATPase family AAA domain-containing protein $3 ;$ & AAA-ATPase TOB3; \\
\hline $\begin{array}{l}\text { KVLQTRGFVS } \\
\text { MKVLQTRGFV }\end{array}$ & $\begin{array}{l}\text { DSSDSMDTGAGSIR } \\
\text { SDSSDSMDTGAGSIR }\end{array}$ & $\begin{array}{l}\text { n.d. } \\
\text { n.d. }\end{array}$ & $\begin{array}{l}\text { N-ter + 42.01 Da } \\
\text { N-ter + 42.01 Da }\end{array}$ & $\begin{array}{l}20,6 \\
32,7\end{array}$ & $\begin{array}{r}-1,526 \\
6,215\end{array}$ & $\begin{array}{l}1 \\
1\end{array}$ & $\begin{array}{l}1439,6 \\
1526,6\end{array}$ & $\begin{array}{l}\text { ATIF1_MOUSE } \\
\text { ATIF1_MOUSE }\end{array}$ & & $\begin{array}{l}\text { ATPase inhibitor, mitochondrial; } \\
\text { ATPase inhibitor, mitochondrial; }\end{array}$ & $\begin{array}{l}\text { Inhibitor of } \mathrm{F}(1) \mathrm{F}(0) \text {-ATPase; } \\
\text { Inhibitor of } \mathrm{F}(1) \mathrm{F}(0) \text {-ATPase; }\end{array}$ \\
\hline M & SAKAISEQTGKELYYKYICTTSAIQNR & n.d. & $\begin{array}{l}\text { N-ter + 42.01 Da, K+34.06 Da, } \\
\text { C+57.02 Da }\end{array}$ & 41,7 & 0,186 & 1 & 3216,8 & ACLY_MOUSE & Q3V117_MOUSE & ATP-citrate synthase; & $\begin{array}{l}\text { ATP-citrate (pro-S--)-yase; Citrate cleavage } \\
\text { enzyme; }\end{array}$ \\
\hline M & ATVDLEKLR & n.d. & N-ter + 42.01 Da, K+34.06 Da & 21,8 & $-0,535$ & 1 & 1119,7 & KGPL_MOUSE & & $\begin{array}{l}\text { ATP-dependent 6-phosphofructokinase, liver type } \\
\text { \{ECO:0000255|HAMAP-Rule:MF_03184\}; }\end{array}$ & $\begin{array}{l}\text { 6-phosphoffuctokinase type B;Phosphoffucto-1- } \\
\text { kinase isozyme B;Phosphohexokinase } \\
\text { \{ECO:0000255|HAMAP-Rule:MF_03184\}; }\end{array}$ \\
\hline M & GDIKNFLYAWCGKR & n.d. & $\begin{array}{l}\mathrm{N} \text {-ter }+42.01 \mathrm{Da}, \mathrm{K}+34.06 \mathrm{Da}, \\
\mathrm{C}+57.02 \mathrm{Da}\end{array}$ & 23,1 & $-0,163$ & 1 & 1837,0 & DHX9_MOUSE & EganN1_MOUSE & ATP-dependent RNA helicase A; & DEAH box protein 9;Nuclear DNA helicase Il; \\
\hline M & NWNKGGPGTKR & n.d. & $\mathrm{N}$-ter + 42.01 Da, K+34.06 Da & 31,1 & $-2,812$ & 1 & 145,8 & DDX42_MOUSE & & ATP-dependent RNA helicase DDX42; & DEAD box protein 42 ; \\
\hline
\end{tabular}




\begin{tabular}{|c|c|c|c|c|c|c|c|c|c|c|c|}
\hline Non Prime Site & Prime Site & $\begin{array}{l}\text { Fold change of } \\
\text { XPRESS ratio } \\
\left.\text { (Adam17 } 17^{\Delta K C} / \mathrm{WT}\right) \\
\end{array}$ & Modifications & Hyperscore & $\begin{array}{l}\text { Mass error } \\
\text { in ppm }\end{array}$ & Charge & $\begin{array}{c}\text { Precursor } \\
\text { neutral mass } \\
\text { in Da } \\
\end{array}$ & Uniprot ID 1 & Uniprot ID 2 & Protein Name 1 & Protein Name 2 \\
\hline M & SYDYHQSWSR & n.d. & $\mathrm{N}$-ter $+42.01 \mathrm{Da}$ & 16,8 & $-0,729$ & 1 & 1369,6 & DHX36_MOUSE & & ATP-dependent RNA helicase DHX36; & $\begin{array}{l}\text { DEAH box protein } 36 ; \text { MLE-like protein 1;RNA } \\
\text { helicase associated with AU-rich element ARE; }\end{array}$ \\
\hline M & AAQVAPAAASSLGNPPPPPSELKKAEQQQR & n.d. & $\mathrm{N}$-ter + 42.01 Da, K+34.06 Da & 49,2 & $-0,095$ & 1 & 3147,7 & ARIIA_MOUSE & E9QAQ7_MOUSE & AT-rich interactive domain-containing protein $1 \mathrm{~A}$; & $\begin{array}{l}\text { BRG1-associated factor 250;BRG1-associated } \\
\text { factor 250a;Osa homolog 1; ;SWI-like } \\
\text { protein;;SWI/SNF complex protein } \\
\text { p270; SWI/SNF-related, matrix-associated, actin } \\
\text { dependent regulator of chromatin subfamily F } \\
\text { member 1; }\end{array}$ \\
\hline m & TDDKDVLR & n.d. & $\mathrm{N}$-ter + 42.01 Da, K+34.06 Da & 21,3 & 0,193 & 1 & 1036,6 & ATG5_MOUSE & & Autophagy protein 5; & APG5-like; \\
\hline AEEVTQTDNM & AKTEEMVQTEEMETPR & 0,3 & $\mathrm{~N}$-ter + 42.01 Da, $\mathrm{K}+28.03 \mathrm{Da}$ & 68,6 & $-0,202$ & 1 & 1977,9 & BAG1_MOUSE & F6TCF9_MOUSE & BAG family molecular chaperone regulator 1 & Bcl-2-associated athanogene 1; \\
\hline M & AQAKISAKAHEGR & 0,0 & $\mathrm{~N}$-ter + 42.01 Da, K+28.03 Da & 26,1 & 1,703 & 1 & 1463,8 & BAG2_MOUSE & & BAG family molecular chaperone regulator 2 & Bcl-2-associated athanogene 2; \\
\hline M & DMGNQHPSISR & n.d. & $\mathrm{N}$-ter $+42.01 \mathrm{Da}$ & 27,1 & 7,064 & 1 & 1413,6 & BAG5_MOUSE & EOCYW3_MOUSE & BAG family molecular chaperone regulator 5 ; & Bcl-2-associated athanogene 5; \\
\hline M & NNQKQQKPTLSGQR & $-1,3$ & $\mathrm{~N}$-ter $+42.01 \mathrm{Da}, \mathrm{K}+28.03 \mathrm{Da}$ & 32,3 & $-2,691$ & 1 & 1855,0 & BZW1_MOUSE & & $\begin{array}{l}\text { Basic leucine zipper and W2 domain-containing } \\
\text { protein 1; }\end{array}$ & \\
\hline M & NKHQKPVLTGQR & n.d. & $\mathrm{N}$-ter + 42.01 Da, K+34.06 Da & 26,5 & $-0,242$ & 1 & 1646,0 & BZW2_MOUSE & & $\begin{array}{l}\text { Basic leucine zipper and W2 domain-containing } \\
\text { protein 2; }\end{array}$ & \\
\hline M & DKLNKITVPASQKLR & n.d. & $\mathrm{N}$-ter + 42.01 Da, K+34.06 Da & 21,4 & $-2,564$ & 1 & 1985,3 & BLNK_MOUSE & D3YWR2_MOUSE & E-cell linker protein; & 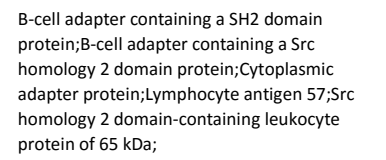 \\
\hline M & GTPKQPSLAPAHALGLR & n.d. & $\mathrm{N}$-ter + 42.01 Da, K+34.06 Da & 24,0 & 2,288 & 1 & 1789,1 & BAD_MOUSE & D3YZR8_MOUSE & Bc12-associated agonist of cell death; & $\begin{array}{l}\text { Bcl-2-binding component } 6 ; \mathrm{BCl}-\mathrm{xL} / \mathrm{BCCl}-2- \\
\text { associated death promoter; } \mathrm{BCl} \text { antagonist of } \\
\text { cell death; }\end{array}$ \\
\hline M & AKQPSDVSSECDR & n.d. & $\begin{array}{l}\text { N-ter + 42.01 Da, K+34.06 Da, } \\
\mathrm{C}+57.02 \mathrm{Da}\end{array}$ & 17,1 & 2,957 & 1 & 1553,7 & B2L11_MOUSE & Q6PEB3_MOUSE & Bcl-2-like protein 11; & Bc12-interacting mediator of cell death; \\
\hline M & AMQKIFAR & n.d. & N-ter + 42.01 Da, K+34.06 Da & 21,6 & 0,096 & 1 & 1039,6 & ENOB_MOUSE & Q55X59_MOUSE & Beta-enolase; & $\begin{array}{l}\text { 2-phospho-D-Dlycerate hydro-lyase;Enolase } \\
\text { 3;Muscle-specific enolase;;keletal muscle } \\
\text { enolase; }\end{array}$ \\
\hline M & NNAGLNSEKVSALIQK & n.d. & N-ter + 42.01 Da, K+34.06 Da & 81,1 & 0,052 & 1 & 1926,1 & BLMH_MOUSE & E9Q6V3_MOUSE & Bleomycin hydrolase; & \\
\hline GMLQNRVLLW & KEQSEASR & 3,9 & $\mathrm{~N}$-ter + 42.01 Da, K+28.03 Da & 19,4 & $-0,796$ & 1 & 1003,5 & B12L2_MOUSE & E9QAX1_MOUSE & $\begin{array}{l}\text { Brain-specific angiogenesis inhibitor 1-associated } \\
\text { protein 2-like protein } 2 \text {; }\end{array}$ & $\begin{array}{l}\text { Planar intestinal- and kidney-specific BAR } \\
\text { domain protein; }\end{array}$ \\
\hline M & ATGTGKHKLLSTGPTEPWSIR & $-0,6$ & N-ter + 42.01 Da, K+28.03 Da & 20,0 & $-8,040$ & 1 & 2334,3 & BRD8_MOUSE & D3YZC7_MOUSE & Bromodomain-containing protein 8 & \\
\hline M & AAAKVALTKR & n.d. & $\mathrm{N}$-ter + 42.01 Da, K+34.06 Da & 32,4 & $-3,068$ & 1 & 1137,8 & CMC2_MOUSE & & $\begin{array}{l}\text { Calcium-binding mitochondrial carrier protein } \\
\text { Aralar2; }\end{array}$ & $\begin{array}{l}\text { Citrin;Mitochondrial aspartate glutamate } \\
\text { carrier 2; } \text {;olute carrier family } 25 \text { member 13; }\end{array}$ \\
\hline M & SSEPPPPPLQPPTHQTSVGLLDTPR & n.d. & $\mathrm{N}$-ter $+42.01 \mathrm{Da}$ & 42,3 & $-0,371$ & 1 & 2689,4 & CHSP1_MOUSE & J3QPI4_MOUSE & Calcium-regulated heat stable protein 1 ; & $\begin{array}{l}\text { Calcium-regulated heat-stable protein of } 24 \\
\text { kDa; }\end{array}$ \\
\hline M & SHGFTKEEVAEFQAAFNR & n.d. & N-ter + 42.01 Da, K+34.06 Da & 102,1 & 0,140 & 1 & 2143,1 & CALM4_MOUSE & & Calmodulin-4; & Calcium-binding protein Dd112; \\
\hline M & FLAKAILEGADR & n.d. & $\mathrm{N}$-ter + 42.01 Da, $\mathrm{K}+34.06 \mathrm{Da}$ & 25,2 & 0,794 & 1 & 1509,9 & CPNS2_MOUSE & & Calpain small subunit 2 ; & Calcium-dependent protease small subunit 2 ; \\
\hline M & AGIAIKLAKDR & n.d. & N-ter + 42.01 Da, K+34.06 Da & 18,0 & $-1,026$ & 1 & 1264,9 & CAN2_MOUSE & & Calpain-2 catalytic subunit; & $\begin{array}{l}80 \mathrm{kDa} \text { M-calpain subunit;CCalcium-activated } \\
\text { neutral proteinase 2;Calpain M-type;Calpain-2 } \\
\text { large subunit;Millimolar-calpain; }\end{array}$ \\
\hline NKPIIFVSDR & ANSNKELGVDQEAEEGKDKAGPEK & 0,2 & N-ter + 42.01 Da, K+28.03 Da & 16,6 & 5,886 & 1 & 2696,4 & CAN3_MOUSE & & Calpain-3; & $\begin{array}{l}\text { Calcium-activated neutral proteinase 3;Calpain } \\
\text { L3;CCalpain p94;Muscle-specific calcium- } \\
\text { activated neutral protease 3; }\end{array}$ \\
\hline M & SQPGPKPAASPRPSR & n.d. & N-ter + 42.01 Da, K+34.06 Da & 20,5 & $-0,062$ & 1 & 1607,9 & ICAL_MOUSE & & Calpastatin; & Calpain inhibitor; \\
\hline M & SSTQFNKGPSYGLSAEVKNR & n.d. & N-ter + 42.01 Da, K+34.06 Da & 83,2 & 0,044 & 1 & 2279,2 & CNN2_MOUSE & D3Z7R6_MOUSE & Calponin-2; & Calponin H2, smooth muscle; Neutral calponin; \\
\hline M & SSSRPVALVTGANKGIGFAITR & n.d. & N-ter + 42.01 Da, K+34.06 Da & 64,9 & 0,789 & 1 & 2277,3 & CBR1_MOUSE & & Carbonyl reductase [NADPH] 1; & $\begin{array}{l}\text { 15-hydroxyprostaglandin dehydrogenase } \\
\text { [NADP(+)];NADPH-dependent carbonyl } \\
\text { reductase 1;Prostaglandin 9- } \\
\text { ketoreductase; Prostaglandin-E(2) 9-reductase; }\end{array}$ \\
\hline LKFSTGGGKK & RPAIWLNAGIHAR & n.d. & $\mathrm{N}$-ter $+42.01 \mathrm{Da}$ & 17,4 & 3,621 & 1 & 1515,9 & CBPA4_MOUSE & & Carboxypeptidase A4; & \\
\hline M & TETDGFYKSR & n.d. & N-ter + 42.01 Da, K+34.06 Da & 30,9 & $-4,295$ & 1 & 1278,6 & CASPG_MOUSE & & Caspase-6; & Apoptotic protease Mch-2; \\
\hline M & SDSRDPASDQMKQWKEQR & 0,2 & $\mathrm{~N}$-ter + 42.01 Da, $\mathrm{K}+28.03 \mathrm{Da}$ & 28,9 & 4,230 & 1 & 2289,1 & CATA_MOUSE & A2AL20_MOUSE & Catalase; & \\
\hline M & SGGKYVDSEGHLYTVPIR & 0,8 & $\mathrm{~N}$-ter + 42.01 Da, K+28.03 Da & 38,4 & $-2,976$ & 1 & 2047,0 & CAV1_MOUSE & H3BLK5_MOUSE & Caveolin-1; & \\
\hline APAGPGGAVM & SAGAHGPPPGYGCAAAGYLDGR & n.d. & $\mathrm{N}$-ter + 42.01 Da, C+57.02 Da & 24,8 & $-0,186$ & 1 & 2143,0 & CEBPA_MOUSE & & CCAAT/enhancer-binding protein alpha; & \\
\hline
\end{tabular}




\begin{tabular}{|c|c|c|c|c|c|c|c|c|c|c|c|}
\hline Non Prime Site & Prime Site & 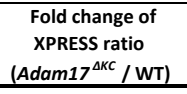 & Modifications & Hyperscore & $\begin{array}{l}\text { Mass error } \\
\text { in ppm }\end{array}$ & Charge & $\begin{array}{c}\text { Precursor } \\
\text { neutral mass } \\
\text { in Da } \\
\end{array}$ & Uniprot ID 1 & Uniprot ID 2 & Protein Name 1 & Protein Name 2 \\
\hline & LKAIHFR & n.d. & $\mathrm{N}$-ter + 42.01 Da, K+34.06 Da & 11,9 & 1,870 & 1 & 959,6 & RAD1_MOUSE & E9PXKO_MOUSE & Cell cycle checkpoint protein RAD1; & $\begin{array}{l}\text { DNA repair exonuclease rad1 homolog;Rad1- } \\
\text { like DNA damage checkpoint protein; }\end{array}$ \\
\hline M & AMNYSAKDEVDGGPAGPPGGAAKTR & n.d. & N-ter + 42.01 Da, K+34.06 Da & 87,3 & 0,830 & 1 & 2526,3 & CC50A_MOUSE & D3YVV1_MOUSE & Cell cycle control protein $50 \mathrm{~A}$ & $\begin{array}{l}\text { P4-ATPase flippase complex beta subunit } \\
\text { TMEM30A; Transmembrane protein 30A; }\end{array}$ \\
\hline MA & MNYSAKDEVDGGPAGPPGGAAKTR & n.d. & N-ter + 42.01 Da, K+34.06 Da & 48,7 & $-2,441$ & 1 & 2455,2 & CC5OA_MOUSE & D3rVV1_MOUSE & Cell cycle control protein 50A; & $\begin{array}{l}\text { P4-ATPase flippase complex beta subunit } \\
\text { TMEM30A;Transmembrane protein 30A; }\end{array}$ \\
\hline M & HSLATAAPVPTALAQVDR & n.d. & $\mathrm{N}$-ter $+42.01 \mathrm{Da}$ & 52,0 & $-0,050$ & 1 & 1990,0 & RCD1_MOUSE & & Cell differentiation protein RCD1 homolog; & $\begin{array}{l}\text { CCR4-NOT transcription complex subunit 9; EPO- } \\
\text { induced protein FL10; }\end{array}$ \\
\hline M & SSNECFKCGR & n.d. & $\begin{array}{l}\text { N-ter + 42.01 Da, K+34.06 Da, } \\
\text { C +57.02 Da }\end{array}$ & 44,1 & $-1,362$ & 1 & 1319,6 & CNBP_MOUSE & D3YYF6_MOUSE & Cellular nucleic acid-binding protein; & Zinc finger protein 9 \\
\hline M & ASNFKKTTMASSAQR & $-0,1$ & $\mathrm{~N}$-ter + $42.01 \mathrm{Da}, \mathrm{K}+28.03 \mathrm{Da}$ & 37,7 & 4,746 & 1 & 1724,9 & CETN2_MOUSE & & Centrin-2; & Caltractin isoform 1; \\
\hline M & ASFVTEVLAHSGSLEKEDLGTR & n.d. & N-ter + 42.01 Da, K+34.06 Da & 33,2 & 0,949 & 1 & 2421,3 & ZW10_MOUSE & & Centromere/kinetochore protein zw10 homolog; & \\
\hline M & SVFGKLFGAGGGKAGKGGPTPQEAIQR & 0,0 & $\mathrm{~N}$-ter + 42.01 Da, K+28.03 Da & 41,4 & 1,931 & 1 & 2740,5 & CHM4B_MOUSE & & Charged multivesicular body protein $4 \mathrm{~b}$; & Chromatin-modifying protein $4 \mathrm{~b}$; \\
\hline M & AEEQPQVELFVKAGSDGAKIGNCPFSQR & n.d. & $\begin{array}{l}\text { N-ter + 42.01 Da, K+34.06 Da, } \\
\mathrm{C}+57.02 \mathrm{Da}\end{array}$ & 39,1 & $-0,977$ & 1 & 3171,6 & CLIC1_MOUSE & & Chloride intracellular channel protein 1; & Nuclear chloride ion channel 27; \\
\hline M & AETTKLQLFVKASEDGESVGHCPSCQR & n.d. & $\begin{array}{l}\text { N-ter + 42.01 Da, K+34.06 Da, } \\
\mathrm{C}+57.02 \mathrm{Da}\end{array}$ & 25,3 & $-0,191$ & 1 & 3143,6 & CLIC3_MOUSE & & Chloride intracellular channel protein 3; & \\
\hline CCGERESRTP & EELTILGETQEEEDELIPR & n.d. & $\mathrm{N}$-ter $+42.01 \mathrm{Da}$ & 17,8 & $-3,498$ & 1 & 2284,1 & CLCN6_MOUSE & A2A7F6_MOUSE & Chloride transport protein $6 ;$ & $\begin{array}{l}\text { Chloride channel protein 6; } \\
\text { CCT-alpha; CTP:phosphocholine }\end{array}$ \\
\hline M & DAQSSAKVNSR & n.d. & $\mathrm{N}$-ter + 42.01 Da, K+34.06 Da & 40,1 & $-0,146$ & 1 & 1368,7 & PCY1A_MOUSE & D3Z3T5_MOUSE & Choline-phosphate cytidylyltransferase A; & $\begin{array}{l}\text { cytidylyltransferase A;Phosphorylcholine } \\
\text { transferase A; }\end{array}$ \\
\hline $\begin{array}{l}\text { M } \\
\text { RKWREEQKKR }\end{array}$ & $\begin{array}{l}\text { AMHNKTAPPQIPDTR } \\
\text { LQELDAASKVTEQEWR }\end{array}$ & $\begin{array}{l}\text { n.d. } \\
\text { n.d. }\end{array}$ & $\begin{array}{l}\text { N-ter + 42.01 Da, } \mathrm{K}+34.06 \mathrm{Da} \\
\text { N-ter + 42.01 Da, K+34.06 Da }\end{array}$ & $\begin{array}{l}20,7 \\
27,1\end{array}$ & $\begin{array}{l}-1,311 \\
2,928\end{array}$ & $\begin{array}{l}1 \\
1\end{array}$ & $\begin{array}{l}1751,9 \\
1978,0\end{array}$ & $\begin{array}{l}\text { EAFG_MOUSE } \\
\text { CLCB MOUSE }\end{array}$ & F7BHJO MOUSE & $\begin{array}{l}\text { Chromatin modification-related protein MEAF6; } \\
\text { Clathrin light chain B; }\end{array}$ & Esa1-associated factor 6 homolog; \\
\hline M & SAIPAEESDQLLIRPLGAGQEVGR & n.d. & $\mathrm{N}$-ter $+42.01 \mathrm{Da}$ & 34,9 & 3,333 & 1 & 2547,3 & CPSF3_MOUSE & & $\begin{array}{l}\text { cleavage and polyadenylation specificity factor } \\
\text { subunit } 3 \text {; }\end{array}$ & $\begin{array}{l}\text { Cleavage and polyadenylation specificity factor } \\
73 \text { kDa subunit; }\end{array}$ \\
\hline M & SSLAVRDPAMDR & n.d. & $\mathrm{N}$-ter + 42.01 Da & 19,2 & $-1,617$ & 1 & 1358,7 & CSTFT_MOUSE & & Cleavage stimulation factor subunit 2 tau variant; & 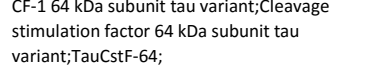 \\
\hline M & AGLPVRDPAVDR & n.d. & $\mathrm{N}$-ter $+42.01 \mathrm{Da}$ & 26,6 & 0,535 & 1 & 1306,7 & CSTF2_MOUSE & A2AEK1_MOUSE & Cleavage stimulation factor subunit 2 ; & $\begin{array}{l}\text { CF-1 } 64 \text { kDa subunit;Cleavage stimulation } \\
\text { factor } 64 \text { kDa subunit;; }\end{array}$ \\
\hline M & ATKIDKEACR & 0,3 & $\begin{array}{l}\text { N-ter + 42.01 Da, K+28.03 Da, } \\
\mathrm{C}+57.02 \mathrm{Da}\end{array}$ & 24,4 & 3,177 & 1 & 1288,7 & COTL1_MOUSE & & Coactosin-like protein; & \\
\hline M & ASGVAVSDGVIKVFNDMKVR & n.d. & $\mathrm{N}$-ter $+42.01 \mathrm{Da}, \mathrm{K}+34.06 \mathrm{Da}$ & 58,9 & $-2,132$ & 1 & 2201,3 & COF1_MOUSE & F8WGL3_MOUSE & Cofilin-1; & Cofilin, non-muscle isoform; \\
\hline & NDMKVR & 0,0 & $\mathrm{~N}$-ter + 42.01 Da, K+28.03 Da & 50,4 & $-0,259$ & 1 & 2318,2 & COF2_MOUSE & & Cofilin-2; & Cofilin, muscle isoform; \\
\hline MQSELVPVSM & SETEHIASISSDATTEKTSELR & n.d. & $\mathrm{N}$-ter + 42.01 Da, K+34.06 Da & 29,5 & 0,486 & 1 & 2467,2 & CJ118_MOUSE & & Coiled-coil domain-containing protein 186; & Oocyte-testis gene 1 protein; \\
\hline M & ADVSVDQSKLPGVKEVCR & $-0,2$ & $\begin{array}{l}\text { N-ter + 42.01 Da, K+28.03 Da, } \\
\mathrm{C}+57.02 \mathrm{Da}\end{array}$ & 30,2 & 6,947 & 1 & 2084,1 & CCD50_MOUSE & & Coiled-coil domain-containing protein 50; & Protein Ymer; \\
\hline LQARTINPPA & TSLPKTAVSVI & 3,1 & $\mathrm{~N}$-ter + 42.01 Da, K+28.03 Da & 24,0 & $-7,762$ & 1 & 1452,8 & CCD71_MOUSE & & Coiled-coil domain-containing & \\
\hline ERGP & GLPGAIGLPGDPGPASYGKNGR & 4,7 & N-ter + 42.01 Da, K +28.03 Da & 21,0 & 2,967 & 1 & 2120,1 & COSA1_MOUSE & G3X995_MOUSE & Collagen alpha-1(IX) chain; & \\
\hline M & AALTAEHFVALQSLLKASSKDVVR & $-0,1$ & $\mathrm{~N}$-ter + 42.01 Da, K+28.03 Da & 18,9 & $-4,067$ & 1 & 2651,5 & COMD9_MOUSE & & COMM domain-containing protein 9 ; & \\
\hline M & AAATASSALKR & n.d. & $\mathrm{N}$-ter + 42.01 Da, K+34.06 Da & 23,8 & $-1,602$ & 1 & 1121,7 & COG1_MOUSE & Q810S7_MOUSE & Conserved oligomeric Golgi complex subunit 1; & $\begin{array}{l}\text { Component of oligomeric Golgi complex 1; Low } \\
\text { densityl lipoprotein receptor defect B- } \\
\text { complementing protein; }\end{array}$ \\
\hline M & NVEHEVNLLVEEIHR & n.d. & $\mathrm{N}$-ter + 42.01 Da & 51,9 & $-0,349$ & 1 & 2002,0 & ABRAL_MOUSE & E9QMV2_MOUSE & Costars family protein ABRACL; & ABRA C-terminal-like protein; \\
\hline M & ALDPAEQHLR & n.d. & $\mathrm{N}-\operatorname{ter}+42.01 \mathrm{Da}$ & 32,2 & $-0,084$ & 1 & 1190,6 & COXM1_MOUSE & & COX assembly mitochondrial protein homolog; & \\
\hline LENLRLQKRG & TGGVDTAATGSVFDISNLDR & n.d. & $\mathrm{N}$-ter + 42.01 Da & 21,3 & 8,578 & 1 & 2037,0 & KCRU_MOUSE & & Creatine kinase U-type, mitochondrial; & $\begin{array}{l}\text { Acidic-type mitochondrial creatine } \\
\text { kinase; } b \text { biquitous mitochondrial creatine } \\
\text { kinase; }\end{array}$ \\
\hline M & ASASYHISNLLEKMTSSDKDFR & n.d. & $\mathrm{N}$-ter + 42.01 Da, K+34.06 Da & 25,8 & $-4,898$ & 1 & 2609,3 & CAND1_MOUSE & & Cullin-associated NEDD8-dissociated protein 1; & $\begin{array}{l}\text { Cullin-associated and neddylation-dissociated } \\
\text { protein 1;p120 CAND1; }\end{array}$ \\
\hline M & IPGGLTEARPATAEVQEIADR & n.d. & $\mathrm{N}$-ter + 42.01 Da & 52,7 & 0,380 & 1 & 2366,2 & CYTA_MOUSE & EOCZ96_MOUSE & Cystatin-A; & Cystatin-A1;Stefin-A; \\
\hline M & ATPEASGSGEKVEGSEPSVTYYR & n.d. & $\mathrm{N}$-ter + $42.01 \mathrm{Da}, \mathrm{K}+34.06 \mathrm{Da}$ & 51,6 & $-0,081$ & 1 & 2476,2 & CYBSB_MOUSE & & Cytochrome b5 type B; & $\begin{array}{l}\text { Cytochrome b5 outer mitochondrial membrane } \\
\text { isoform; } \\
\text { Complex III subunit 6;Complex III subunit } \\
\text { VIIIlCytochrome c1 non-heme } 11 \mathrm{kDa}\end{array}$ \\
\hline EDERKMLTGS & GDPKEEEEEELVDPLTTVR & 0,6 & N-ter + 42.01 Da, K+28.03 Da & 39,1 & 1,108 & 1 & 2254,1 & QCR6_MOUSE & & Cytochrome b-c1 complex subunit 6, mitochondrial; & $\begin{array}{l}\text { protein;Mitochondrial hinge protein; Ubiquinol- } \\
\text { cytochrome c reductase complex } 11 \mathrm{kDa} \\
\text { protein; }\end{array}$ \\
\hline M & SSGALPKPQMR & n.d. & N-ter + $42.01 \mathrm{Da}, \mathrm{K}+34.06 \mathrm{Da}$ & 50,5 & $-0,367$ & 1 & 1359,8 & COX6C_MOUSE & D3Z6E1_MOU & Cytochrome c oxidase subunit $6 \mathrm{C} ;$ & Cytochrome c oxidase polypeptide VIC; \\
\hline & EKVPGDMEIER & 0,4 & N-ter + 42.01 Da, K+28.03 Da & 24,5 & $-0,199$ & 1 & 1502,7 & CYGB_MOUSE & & Cytoglobin; & Histoglobin; \\
\hline
\end{tabular}




\begin{tabular}{|c|c|c|c|c|c|c|c|c|c|c|c|}
\hline Non Prime Site & Prime Site & $\begin{array}{l}\text { Fold change of } \\
\text { XPRESS ratio } \\
\left.\text { (Adam1 } 1 \mathrm{D}^{\wedge \mathrm{KC}} / \mathrm{WT}\right)\end{array}$ & Modifications & Hyperscore & $\begin{array}{l}\text { Mass error } \\
\text { in ppm }\end{array}$ & Charge & $\begin{array}{l}\text { Precursor } \\
\text { neutral mass } \\
\text { in Da }\end{array}$ & Uniprot ID 1 & Uniprot ID 2 & Protein Name 1 & Protein Name 2 \\
\hline M & KGAMEPEPEVLLQEAR & n.d. & N-ter + 42.01 Da, K+34.06 Da & 31,2 & 9,721 & 1 & 2003,1 & CRLF__MOUSE & J3QMV5_MOUSE & Cytokine receptor-like factor 3; & $\begin{array}{l}\text { Cytokine receptor-like molecule 9;Cytokine } \\
\text { receptor-related factor 4; }\end{array}$ \\
\hline M & SEPGGGEDGSAGLEVSAVQNVADVAVLQKHLR & 0,0 & $\mathrm{~N}-\operatorname{ter}+42.01 \mathrm{Da}, \mathrm{K}+28.03 \mathrm{Da}$ & 31,7 & 1,134 & 1 & 3258,7 & DYHC1_MOUSE & & Cytoplasmic dynein 1 heary chain $1 ;$ & $\begin{array}{l}\text { Cytoplasmic dynein heavy chain 1; Dynein heavy } \\
\text { chain, cytosolic; }\end{array}$ \\
\hline GRMLSREANM & SDKSDLKAELER & n.d. & $\mathrm{N}-\operatorname{ter}+42.01 \mathrm{Da}, \mathrm{K}+34.06 \mathrm{Da}$ & 27,4 & 2,131 & 1 & 1499,9 & DC111_MOUSE & DC112_MOUSE & Cytoplasmic dynein 1 intermediate chain 1 ; & $\begin{array}{l}\text { Cytoplasmic dynein intermediate chain } \\
\text { 1;Dynein intermediate chain 1, cytosolic; }\end{array}$ \\
\hline M & EEAPHGCPGADSAQAGR & n.d. & $\mathrm{N}$-ter + 42.01 Da, C+57.02 Da & 55,4 & $-0,637$ & 1 & 1881,8 & NUBP1_MOUSE & & $\begin{array}{l}\text { Cytosolic Fe-S cluster assembly factor NUBP1 } \\
\text { \{ECO:0000255|HAMAP-Rule:MF_03038\}; }\end{array}$ & $\begin{array}{l}\text { Nucleotide-binding protein } 1 \\
\text { \{ECO:0000255|HAMAP-Rule:MF_03038\}; }\end{array}$ \\
\hline M & SALKAVFQYIDENQDR & 0,0 & N-ter + 42.01 Da, K+28.03 Da & 85,1 & $-0,051$ & 1 & 1966,0 & CNDP2_MOUSE & & Cytosolic non-specific dipeptidase; & $\begin{array}{l}\text { CNDP dipeptidase 2;Glutamate } \\
\text { carboxypeptidase-like protein 1; }\end{array}$ \\
\hline M & DFEDDYVHSTCR & n.d. & $\mathrm{N}$-ter + 42.01 Da, C+57.02 Da & 18,4 & $-0,466$ & 1 & 1715,7 & ZN326_MOUSE & D6RG92_MOUSE & DBIRD complex subunit ZNF326; & $\begin{array}{l}\text { interacting with mRNPs;zinc finger protein- } \\
\text { associated with nuclear matrix of } 75 \mathrm{kDa} \text {; }\end{array}$ \\
\hline M & SSPPEGKLETKAGHPPAVKAGGMR & n.d. & N-ter + 42.01 Da, K+34.06 Da & 15,9 & 1,255 & 1 & 2545,5 & DAP1_MOUSE & & Death-associated protein $1 ;$ & \\
\hline M & ANEVQVLPSPLKGR & 0,7 & N-ter + 42.01 Da, K+28.03 Da & 53,8 & $-0,570$ & 1 & 1576,9 & DAPL1_MOUSE & & Death-associated protein-like 1; & $\begin{array}{l}\text { Early epithelial differentiation-associated } \\
\text { protein }\end{array}$ \\
\hline LSALSMPNLF & GQIPMGAHTSPLQPLGPPAVAPSR & n.d. & $\mathrm{N}$-ter + $42.01 \mathrm{Da}$ & 16,8 & $-2,352$ & 1 & 2420,3 & DEN1A_MOUSE & & DENN domain-containing protein $1 \mathrm{~A}$; & Connecdenn 1; \\
\hline SQPRVSEVAM & QSAPLEQPAKRPR & n.d. & N-ter + 42.01 Da, K+34.06 Da & 22,5 & $-0,652$ & 1 & 1683,9 & SAMH1_MOUSE & F8WJEO_MOUSE & $\begin{array}{l}\text { Deoxynucleoside triphosphate triphosphohydrolase } \\
\text { SAMHD1; }\end{array}$ & $\begin{array}{l}\text { Interferon-gamma-inducible protein } \\
\text { Mg11;SAM domain and HD domain-containing } \\
\text { protein 1; }\end{array}$ \\
\hline YAFEVPEGSR & LGTVVGTVCATDKDEPDTMHTR & n.d. & $\begin{array}{l}\text { N-ter + } 42.01 \mathrm{Da}, \mathrm{K}+34.06 \mathrm{Da}, \\
\mathrm{C}+57.02 \mathrm{a}\end{array}$ & 21,3 & 6,526 & 1 & 2478,2 & DSC3_MOUSE & G5E8S6_MOUSE & Desmocollin-3; & \\
\hline M & ADFLKGLPVYNKSNFSR & $-0,2$ & N-ter + 42.01 Da, K+28.03 Da & 35,3 & $-0,730$ & 1 & 2053,1 & DDA1_MOUSE & D3YXY5_MOUSE & DET1- and DDB1-associated protein 1; & \\
\hline M & SGTLAKIAEIEAEMAR & $-0,1$ & $\mathrm{~N}$-ter + 42.01 Da, K+28.03 Da & 32,5 & 0,965 & 1 & 1758,9 & DRG1_MOUSE & & Developmentally-regulated GTP-binding protein 1; & $\begin{array}{l}\text { Neural precursor cell expressed } \\
\text { developmentally down-regulated protein 3; }\end{array}$ \\
\hline TDTPWLCQPL & EPGEEESLQQNVMLDLTKR & 0,2 & N-ter + 42.01 Da, K+28.03 Da & 17,3 & $-5,900$ & 1 & 2285,1 & E9PUQ8_MOUSE & & $\begin{array}{l}\text { Diacylglycerol kinase } \\
\{\text { ECO.000025/RuleBase }\end{array}$ & \\
\hline M & SYQGKKNIPR & 0,4 & N-ter + 42.01 Da, K+28.03 Da & 29,4 & $-3,799$ & 1 & 1287,7 & DPYL2_MOUSE & DPYL3_MOUSE & 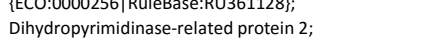 & Unc-33-like phosphoprotein 2; \\
\hline M & MKLKSNQTR & 0,0 & $\mathrm{~N}$-ter + 42.01 Da, $\mathrm{K}+28.03 \mathrm{Da}$ & 17,3 & $-1,496$ & 1 & 1333,7 & NUDT3_MOUSE & 11E4X7_MOUSE & $\begin{array}{l}\text { Diphosphoinositol polyphosphate phosphohydrolase } \\
1 ;\end{array}$ & $\begin{array}{l}\text { Diadenosine 5',5"'-P1,P6-hexaphosphate } \\
\text { hydrolase 1;Nucleoside diphosphate-linked } \\
\text { moiety X motif } 3 \text {; }\end{array}$ \\
\hline M & NHPDYKLNLR & n.d. & $\mathrm{N}$-ter + 42.01 Da, K+34.06 Da & 21,9 & $-5,072$ & 1 & 1475,8 & DI3L2_MOUSE & & $\begin{array}{l}\text { DIS3-like exonuclease } 2 \text { \{ECO:0000255 |HAMAP- } \\
\text { Rule:MF_03045\}; }\end{array}$ & \\
\hline M & DPGKDEESRPQPSGPPTR & 0,1 & N-ter + 42.01 Da, K +28.03 Da & 15,6 & 5,341 & 1 & 2150,0 & ERCC1_MOUSE & E9PUMO_MOUSE & DNA excision repair protein ERCC-1; & \\
\hline M & AVQPKETLQLEGAAEAGFVR & 0,0 & $\mathrm{~N}$-ter + 42.01 Da, $\mathrm{K}+28.03 \mathrm{Da}$ & 49,4 & $-9,377$ & 1 & 2183,1 & MSH2_MOUSE & & DNA mismatch repair protein Msh2; & $\begin{array}{l}\text { MutS protein homolog 2; } \\
\text { DNA polymerase II subunit 3;DNA polymerase }\end{array}$ \\
\hline M & AERPEDLNLPNAVITR & n.d. & $\mathrm{N}$-ter + 42.01 Da & 55,6 & $-0,648$ & 1 & 1849,0 & DPOE3_MOUSE & DGRDT4_MOUSE & DNA polymerase epsilon subunit 3; & $\begin{array}{l}\text { epsilon subunit p17;NF-YB-like protein;YB-like } \\
\text { protein 1; }\end{array}$ \\
\hline M & ATAEEKQTSPEPVAADEPAQLPAAVR & n.d. & $\mathrm{N}$-ter + 42.01 Da, K+34.06 Da & 40,5 & $-5,990$ & 1 & 2751,4 & XPA_MOUSE & Q8K2X7_MOUSE & $\begin{array}{l}\text { DNA repair protein complementing XP-A cells } \\
\text { homolog; }\end{array}$ & $\begin{array}{l}\text { Xeroderma pigmentosum group A- } \\
\text { complementing protein homolog; }\end{array}$ \\
\hline $\begin{array}{l}\mathrm{M} \\
\mathrm{M}\end{array}$ & $\begin{array}{l}\text { DLAAAAEPGAGSQHPEVR } \\
\text { SGDHLHNDSOIEADFR }\end{array}$ & $\begin{array}{l}\text { n.d. } \\
\text { n.d. }\end{array}$ & $\begin{array}{l}\text { N-ter + 42.01 Da } \\
\text { N-ter + 42.01 Da }\end{array}$ & $\begin{array}{l}50,7 \\
44,1\end{array}$ & $\begin{array}{l}-1,743 \\
-0,743\end{array}$ & $\begin{array}{l}1 \\
1\end{array}$ & $\begin{array}{l}1947,9 \\
1881,8\end{array}$ & $\begin{array}{l}\text { MCM6_MOUSE } \\
\text { TOP1 MOUSE }\end{array}$ & & $\begin{array}{l}\text { DNA replication licensing factor MCM6; } \\
\text { DNA topoisomerase } 1 \text {. }\end{array}$ & Mis5 homolog; \\
\hline M & AEVKVKVQPPDADPVEIENR & $-0,1$ & N-ter + 42.01 Da, K+28.03 Da & 54,4 & 4,114 & 1 & 2330,3 & RPC6_MOUSE & Q9CZ23_MOUSE & DNA-directed RNA polymerase III subunit RPC6; & DNA-directed RNA polymerase III subunit F; \\
\hline M & ANVADTKLYOLLGVPPGASENELKKAYR & $-0,3$ & $\mathrm{~N}$-ter $+42.01 \mathrm{Da}, \mathrm{K}+28.03$ & 64,2 & 0,285 & 1 & 3157,7 & DNJA2_MOUSE & & DnaJ homolog subfamily A mem! & $\mathrm{mDj} 3 ;$ \\
\hline M & ESNKDEA & $\begin{array}{ll} & \\
-0,3 \\
\text { n.d. }\end{array}$ & $\mathrm{N}-\mathrm{ter}+42.0$ & 39 & $\begin{array}{cc}-4,2 \\
-4,\end{array}$ & 1 & 1283,6 & DJB12_MOUSE & Q8C4C9_MOUSE & DnaJ homolog subfamily B member 12; & mDj10; \\
\hline M & AASGESGASGGGGSTEEAFMTFYSEVKOIEKR & $-0,1$ & $\mathrm{~N}$-ter + 42.01 Da, $\mathrm{K}+28.03 \mathrm{Da}$ & 27,4 & 1,128 & 1 & 3365,6 & DNJC8_MOUSE & D3Z415_MOUSE & DnaJ homolog subfamily C member 8; & \\
\hline M & AGVSFSGHR & n.d. & $\mathrm{N}$-ter $+42.01 \mathrm{Da}$ & 24,6 & $-0,417$ & 1 & 958,5 & DREB_MOUSE & & Drebrin; & Developmentally-regulated brain protein; \\
\hline M & ESPAASPPASLPQTKGK & n.d. & $\mathrm{N}$-ter + 42.01 Da, $\mathrm{K}+34.06 \mathrm{Da}$ & 27,2 & 3,772 & 1 & 1906,1 & MP2K3_MOUSE & & $\begin{array}{l}\text { Dual specificity mitogen-activated protein kinase } \\
\text { kinase 3; }\end{array}$ & MAPK/ERK kinase 3; \\
\hline M & STEASARPLR & n.d. & $\mathrm{N}$-ter + 42.01 Da & 23,5 & $-0,177$ & 1 & 1128,6 & DCTN1_MOUSE & D3Z2M9_MOUSE & Dynactin subunit 1; & $\begin{array}{l}150 \text { KDa dynein-associated polypeptide;DAP- } \\
\text { 150;p150-glued; }\end{array}$ \\
\hline M & ADPKYADLPGIAR & n.d. & $\mathrm{N}$-ter + 42.01 Da, K+34.06 Da & 40,3 & $-0,410$ & 1 & 1461,8 & DCTN2_MOUSE & & Dynactin subunit 2; & $\begin{array}{l}50 \text { kDa dynein-associated polypeptide; Dynactin } \\
\text { complex } 50 \text { kDa subuunit;Growth cone } \\
\text { membrane protein 23-48K; ;550 dynamitin; }\end{array}$ \\
\hline M & AEKTQKSVKIAPGAVVCVESEIR & n.d. & $\begin{array}{l}\text { N-ter + } 42.01 \mathrm{Da}, \mathrm{K}+34.06 \mathrm{Da}, \\
\mathrm{C}+57.02 \mathrm{Da}\end{array}$ & 37,2 & $-8,237$ & 1 & 2642,5 & DCTN6_MOUSE & D3Z6A1_MOUSE & Dynactin subunit 6 ; & Dynactin subunit p27;Protein WS-3; \\
\hline MAFPPPLWE & AEVEETLKR & 0,0 & $\mathrm{~N}$-ter $+42.01 \mathrm{Da}, \mathrm{K}+28.03 \mathrm{Da}$ & 47,5 & 0,087 & 1 & 1143,6 & DLRB1_MOUSE & A2AVR9_MOUSE & 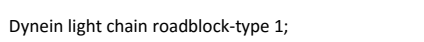 & (I) \\
\hline & AAAMDVDTPSGTNSGAGKKR & n.d. & $\mathrm{N}$-ter + 42.01 Da, K+34.06 Da & 25,9 & $-1,906$ & 1 & 2043,1 & RBX1_MOUSE & & E3 ubiquitin-protein ligase RBX1; & RING finger protein $75 ;$;ING-box protein 1 \\
\hline
\end{tabular}




\begin{tabular}{|c|c|c|c|c|c|c|c|c|c|c|c|}
\hline Non Prime Site & Prime Site & 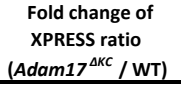 & Modifications & Hyperscore & $\begin{array}{c}\text { Mass error } \\
\text { in ppm }\end{array}$ & Charge & $\begin{array}{c}\text { Precursor } \\
\text { neutral mass } \\
\text { in Da }\end{array}$ & Uniprot ID 1 & Uniprot ID 2 & Protein Name 1 & Protein Name 2 \\
\hline M & ENSDSNDKGSDQSAAQR & n.d. & $\mathrm{N}$-ter + 42.01 Da, K+34.06 Da & 23,4 & $-1,586$ & 1 & 2014,9 & RNF12_MOUSE & & E3 ubiquitin-protein ligase RLIM; & $\begin{array}{l}\text { LIM domain-interacting RING finger } \\
\text { protein;RIING finger LIM domain-binding } \\
\text { protein;RING finger protein 12; }\end{array}$ \\
\hline M & ASYFDEHDCEPLNPER & n.d. & N-ter + 42.01 Da, C+57.02 Da & 17,7 & 2,670 & 1 & 2019,8 & RN181_MOUSE & D3YU1__MOUSE & E3 ubiquitin-protein ligase RNF181; & $\begin{array}{l}\text { RING finger protein 181; } \\
\text { ADP-ribosylation factor domain-containing }\end{array}$ \\
\hline GKHQGHKHSV & LEPEANQIR & n.d. & $\mathrm{N}$-ter + 42.01 Da & 17,4 & $-1,618$ & 1 & 1110,6 & TRI23_MOUSE & & E3 ubiquitin-protein ligase TRIM23; & $\begin{array}{l}\text { protein 1;GTP-binding protein ARD-1; Tripartite } \\
\text { motif-containing protein 23; }\end{array}$ \\
\hline M & SNRPNNNPGGSLR & n.d. & $\mathrm{N}$-ter $+42.01 \mathrm{Da}$ & 26,0 & $-0,281$ & 1 & 1423,7 & TRIPC_MOUSE & & E3 ubiquitin-protein ligase TRIP12; & Thyroid receptor-interacting protein 12; \\
\hline VAREKAIDYT & TKIYAVSIR & $-0,3$ & N-ter + 42.01 Da, K+28.03 Da & 14,8 & 2,316 & 1 & 1119,7 & EMAL6_MOUSE & & Echinoderm microtubule-associated protein-like 6; & $\begin{array}{l}\text { Echinoderm microtubule-associated protein- } \\
\text { like 5-like; }\end{array}$ \\
\hline M & SSEELACKLQR & n.d. & $\begin{array}{l}\text { N-ter + 42.01 Da, K+34.06 Da, } \\
\text { C +57.02 Da }\end{array}$ & 56,1 & $-2,075$ & 1 & 1395,7 & EFHD1_MOUSE & & EF-hand domain-containing protein D1; & $\begin{array}{l}\text { EF-hand domain-containing protein 1;Swiprosin- } \\
2 \text {; }\end{array}$ \\
\hline $\begin{array}{l}M \\
M\end{array}$ & $\begin{array}{l}\text { ATDELASKLSR } \\
\text { FSWVSKDAR }\end{array}$ & $\begin{array}{l}0,1 \\
\text { n.d. }\end{array}$ & $\begin{array}{l}\mathrm{N} \text {-ter + 42.01 Da, } \mathrm{K}+28.03 \mathrm{Da} \\
\mathrm{N} \text {-ter + 42.01 Da, K+34.06 Da }\end{array}$ & $\begin{array}{l}58,8 \\
27,0\end{array}$ & $\begin{array}{l}-0,872 \\
-0,690\end{array}$ & $\begin{array}{l}1 \\
1\end{array}$ & $\begin{array}{l}1259,7 \\
1301,7\end{array}$ & $\begin{array}{l}\text { EFHD2_MOUSE } \\
\text { EHD1_MOUSE }\end{array}$ & Q8C845_MOUSE & $\begin{array}{l}\text { EF-hand domain-containing protein D2; } \\
\text { EH domain-containing protein 1; }\end{array}$ & $\begin{array}{l}\text { Swiprosin-1; } \\
\text { PAST homolog 1; }\end{array}$ \\
\hline M & QTAHTGLSHTADGADGQTSR & n.d. & $\mathrm{N}$-ter $+42.01 \mathrm{Da}$ & 18,3 & 0,503 & 1 & 2183,0 & LAGE3_MOUSE & J3JS94_MOUSE & EKC/KEOPS complex subunit Lage3; & $\begin{array}{l}\text { ITBA2 protein homolog; Lantigen family } \\
\text { member 3; }\end{array}$ \\
\hline MSNGYEDHM & AEDCRDDIGR & n.d. & N-ter + 42.01 Da, C +57.02 Da & 26,0 & $-1,761$ & 1 & 1247,5 & ELAV1_MOUSE & & ELAV-like protein 1; & Elav-like generic protein;Hu-antigen R;MelG; \\
\hline PTRPTDKPLR & LPLQDVYKIGGIGTVPVGR & $-0,1$ & N-ter + 42.01 Da, K+28.03 Da & 55,0 & 9,493 & 1 & 2051,2 & EF1A1_MOUSE & EF1A2_MOUSE & Elongation factor 1-alpha 1; & $\begin{array}{l}\text { Elongation factor Tu; Eukaryotic elongation } \\
\text { factor } 1 \mathrm{~A}-1 \text {; }\end{array}$ \\
\hline GGDHSELIVR & ITSLEVENQNLR & n.d. & $\mathrm{N}$-ter + $42.01 \mathrm{Da}$ & 24,2 & 8,706 & 1 & 145,8 & EF1D_MOUSE & Q91VK2_MOUSE & Elongation factor 1-delta; & \\
\hline MNI & MDFNVKKLAADAGTFLSR & n.d. & N-ter + 42.01 Da, K+34.06 Da & 24,1 & 2,958 & 1 & 2093,2 & SHLB1_MOUSE & & Endophilin-B1; & SH3 domain-containing GRB2-like protein B1; \\
\hline $\begin{array}{l}\text { M } \\
\text { RSPGLELPVM }\end{array}$ & $\begin{array}{l}\text { ASCASIDIEDATQHLR } \\
\text { SHTLLIVQPTKRPEGR }\end{array}$ & $\begin{array}{l}\text { n.d. } \\
\text { n.d. }\end{array}$ & $\begin{array}{l}\text { N-ter + 42.01 Da, C+57.02 Da } \\
\text { N-ter + 42.01 Da, K+34.06 Da }\end{array}$ & $\begin{array}{l}17,4 \\
33,8\end{array}$ & $\begin{array}{c}-0,710 \\
0,471\end{array}$ & $\begin{array}{l}1 \\
1\end{array}$ & $\begin{array}{l}1827,8 \\
1907,1\end{array}$ & $\begin{array}{l}\text { EDC4_MOUSE } \\
\text { ERH_MOUSE }\end{array}$ & $\begin{array}{l}\text { G5E896_MOUSE } \\
\text { G3UW85_MOUSE }\end{array}$ & $\begin{array}{l}\text { Enhancer of mRNA-decapping protein } 4 ; \\
\text { Enhancer of rudimentary homolog; }\end{array}$ & \\
\hline HAVGDQLDLR & EKIVQDASLTYQQLR & 2,5 & $\mathrm{~N}$-ter + 42.01 Da, K+28.03 Da & 18,9 & 1,770 & 1 & 1861,0 & EVPL_MOUSE & & Envoplakin; & $\begin{array}{l}210 \mathrm{kDa} \text { cornified envelope precursor } \\
\text { protein;p210; }\end{array}$ \\
\hline M & STTTGPEAAPKPSAKSIYEQR & 0,1 & $\mathrm{~N}$-ter + 42.01 Da, K+28.03 Da & 28,0 & 1,466 & 1 & 2316,2 & ES8L1_MOUSE & E9Q4X5_MOUSE & $\begin{array}{l}\text { Epidermal growth factor receptor kinase substrate 8- } \\
\text { like protein } 1 \text {; }\end{array}$ & $\begin{array}{l}\text { Epidermal growth factor receptor pathway } \\
\text { substrate 8-related protein } 1 \text {; }\end{array}$ \\
\hline M & AAAAQLSLTQLLSSGNPVYEKYYR & n.d. & N-ter + 42.01 Da, K+34.06 Da & 39,1 & 1,112 & 1 & 2605,4 & EPS15_MOUSE & Q.5JC28_MOUSE & Epidermal growth factor receptor substrate 15; & Protein AF-1p; \\
\hline EDLEMFNAAY & QSIQSYLR & n.d. & $\mathrm{N}$-ter $+42.01 \mathrm{Da}$ & 9,8 & $-9,542$ & 1 & 1035,5 & EDEM1_MOUSE & & $\begin{array}{l}\text { ER degradation-enhancing alpha-mannosidase-like } \\
\text { protein 1; }\end{array}$ & \\
\hline MM & GDPKEAGAEASPSGAAAR & n.d. & N-ter + 42.01 Da, K+34.06 Da & 23,4 & 1,222 & 1 & 1716,9 & EDRF1_MOUSE & E9Q9F9_MOUSE & Erythroid differentiation-related factor 1 ; & \\
\hline M & SSKQEIMDDQR & 1,4 & $\mathrm{~N}$-ter + 42.01 Da, K+28.03 Da & 27,3 & $-2,415$ & 1 & 1405,7 & ESF1_MOUSE & & ESF1 homolog; & ABT1-associated protein; \\
\hline M & AKCLLTSSLSVR & n.d. & $\begin{array}{l}\text { N-ter + 42.01 Da, K+34.06 Da, } \\
\mathrm{C}+57.02 \mathrm{Da}\end{array}$ & 25,9 & $-5,312$ & 1 & 1409,8 & ECHD1_MOUSE & EOCXS3_MOUSE & Ethylmalonyl-CoA decarboxylase; & $\begin{array}{l}\text { Enoyl-CoA hydratase domain-containing } \\
\text { protein 1;Methylmalonyl-CoA decarboxylase; }\end{array}$ \\
\hline M & ADSVKTFLQDLGR & n.d. & $\mathrm{N}$-ter + 42.01 Da, K+34.06 Da & 20,4 & $-2,096$ & 1 & 1524,8 & EI24_MOUSE & & Etoposide-induced protein 2.4; & p53-induced gene 8 protein; \\
\hline GIDAVKEALR & AGLNCSTETMPIIINLIAPPR & 5,6 & $\begin{array}{l}\text { N-ter + 42.01 Da, K+34.06 Da, } \\
\mathrm{C}+57.02 \mathrm{Da}\end{array}$ & 26,9 & 6,949 & 1 & 2371,3 & IF2A_MOUSE & & Eukaryotic translation initiation factor 2 subunit 1 ; & $\begin{array}{l}\text { Eukaryotic translation initiation factor } 2 \text { subunit } \\
\text { alpha; }\end{array}$ \\
\hline KEALRAGLNC & STETMPIKINLIAPPR & n.d. & N-ter + 42.01 Da, K+28.03 Da & 24,1 & $-8,365$ & 1 & 1850,0 & IF2A_MOUSE & & Eukaryotic translation initiation factor 2 subunit 1; & $\begin{array}{l}\text { Eukaryotic translation initiation factor } 2 \text { subunit } \\
\text { alpha; }\end{array}$ \\
\hline M & AGGEGGVTLGQPHLSR & n.d. & N-ter + 42.01 Da & 57,0 & 0,570 & 1 & 1576,8 & IF2G_MOUSE & AZAAW9_MOUSE & $\begin{array}{l}\text { Eukaryotic translation initiation factor } 2 \text { subunit } 3, \mathrm{X} \text { - } \\
\text { linked; }\end{array}$ & $\begin{array}{l}\text { Eukaryotic translation initiation factor } 2 \text { subunit } \\
\text { gamma, } X \text {-linked; }\end{array}$ \\
\hline M & AGGEAGVTLGQPHLSR & n.d. & $\mathrm{N}$-ter + 42.01 Da & 60,8 & $-0,126$ & 1 & 1590,8 & IF2H_MOUSE & & $\begin{array}{l}\text { Eukaryotic translation initiation factor } 2 \text { subunit } 3, Y \text { - } \\
\text { linked; }\end{array}$ & $\begin{array}{l}\text { Eukaryotic translation initiation factor } 2 \text { subunit } \\
\text { gamma, Y-linked; }\end{array}$ \\
\hline PASVAPAPVL & MNQPPQIAPKR & n.d. & $\mathrm{N}$-ter + 42.01 Da, K+34.06 Da & 30,5 & $-0,590$ & 1 & 1354,8 & IF4G1_MOUSE & D3rWY7_MOUSE & Eukaryotic translation initiation factor 4 gamma 1 ; & \\
\hline M & NKAPQPTGPPPAR & n.d. & $\mathrm{N}$-ter + 42.01 Da, K+34.06 Da & 47,8 & $-0,780$ & 1 & 1536,8 & IF4G1_MOUSE & D6RCH1_MOUSE & Eukaryotic translation initiation factor 4 gamma 1 ; & \\
\hline M & TAIKHALQR & 0,1 & $\mathrm{~N}$-ter + 42.01 Da, K+28.03 Da & 18,3 & 0,090 & 1 & 1106,7 & EXOC1_MOUSE & Q6P1Y9_MOUSE & Exocyst complex component 1 & Exocyst complex component Sec3; \\
\hline MERAKM & AEESLETAAEHER & n.d. & $\mathrm{N}$-ter + 42.01 Da & 24,5 & $-2,773$ & 1 & 1512,7 & EXC6B_MOUSE & EOCXR2_MOUSE & Exocyst complex component $6 \mathrm{~B} ;$ & $\begin{array}{l}\text { Exocyst complex component Sec15B;SEC15-like } \\
\text { protein 2; }\end{array}$ \\
\hline M & AAGFKTVEPLEYYR & n.d. & $\mathrm{N}$-ter + 42.01 Da, K+34.06 Da & 55,8 & 2,557 & 1 & 1718,9 & EXOS8_MOUSE & D3YYN3_MOUSE & Exosome complex component RRP43; & $\begin{array}{l}\text { Exosome component 8; Ribosomal RNA- } \\
\text { processing protein 43; }\end{array}$ \\
\hline M & ASVALSEAEKVYIVHGVQEDLR & n.d. & N-ter + 42.01 Da, K+34.06 Da & 44,7 & $-4,215$ & 1 & 2488,3 & EXOS7_MOUSE & & Exosome complex exonuclease RRP42; & $\begin{array}{l}\text { Exosome component 7;Ribosomal RNA- } \\
\text { processing protein 42; }\end{array}$ \\
\hline KAAKSKNLQI & KTDTAKSLADSLDR & n.d. & $\mathrm{N}$-ter + 42.01 Da, K+34.06 Da & 27,0 & $-2,696$ & 1 & 1629,9 & RRP44_MOUSE & & Exosome complex exonuclease RRP44; & $\begin{array}{l}\text { Protein DIS3 homolog; Ribosomal RNA- } \\
\text { processing protein 44; }\end{array}$ \\
\hline MPAIM & TMLADHAAR & n.d. & $\mathrm{N}$-ter $+42.01 \mathrm{Da}$ & 31,7 & 0,972 & 1 & 1026,5 & XPO1_MOUSE & A2AKT6_MOUSE & Exportin-1; & $\begin{array}{l}\text { Chromosome region maintenance } 1 \text { protein } \\
\text { homolog; }\end{array}$ \\
\hline M & EHSPEEGASPEPSGQPPATDSTR & n.d. & $\mathrm{N}$-ter $+42.01 \mathrm{Da}$ & 50,1 & 6,107 & 1 & 2535,1 & ESYT1_MOUSE & & Extended synaptotagmin-1; & $\begin{array}{l}\text { Membrane-bound C2 domain-containing } \\
\text { protein; }\end{array}$ \\
\hline
\end{tabular}




\begin{tabular}{|c|c|c|c|c|c|c|c|c|c|c|c|}
\hline Non Prime Site & Prime Site & 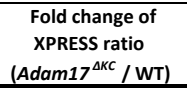 & Modifications & Hyperscore & $\begin{array}{c}\text { Mass error } \\
\text { in ppm }\end{array}$ & Charge & $\begin{array}{c}\text { Precursor } \\
\text { neutral mass } \\
\text { in Da } \\
\end{array}$ & Uniprot ID 1 & Uniprot ID 2 & Protein Name 1 & Protein Name 2 \\
\hline M & AVTLDKDAYYR & n.d. & N-ter + 42.01 Da, K+34.06 Da & 27,0 & $-7,401$ & 1 & 1389,7 & SP16H_MOUSE & G3X956_MOUSE & FACT complex subunit SPT16; & 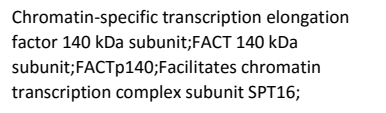 \\
\hline M & AETLEFNDIFQEVKGSMNDGR & n.d. & N-ter + 42.01 Da, K+34.06 Da & 52,5 & $-0,121$ & 1 & 2475,2 & SSRP1_MOUSE & A2AW05_MOUSE F & FACT complex subunit SSRP1; & $\begin{array}{l}\text { Facilitates chromatin transcription complex } \\
\text { subunit SSRP } 1 ; \text { Recombination signal sequence } \\
\text { recognition protein 1;Structure-specific } \\
\text { recognition protein 1;T160; }\end{array}$ \\
\hline M & ADLEEQLSDEEKVR & n.d. & $\mathrm{N}$-ter + 42.01 Da, K+34.06 Da & 75,0 & $-1,266$ & 1 & 1735,9 & CAZAZ_MOUSE & DGRCW7_MOUSE & F-actin-capping protein subunit alpha-2; & Capz alpha-2; \\
\hline M & ADYSTVPPPSSGSAGGGGGGVVNDAFKDALQR & n.d. & $\mathrm{N}$-ter + 42.01 Da, K +34.06 Da & 39,3 & $-1,317$ & 1 & 3109,5 & FUBP1_MOUSE & Q3TUE1_MOUSE $\mathrm{F}$ & Far upstream element-binding protein $1 ;$ & \\
\hline M & ASLKDLEGKWR & $-0,4$ & $\mathrm{~N}$-ter + 42.01 Da, K+28.03 Da & 59,8 & $-2,711$ & 1 & 1399,8 & FABP5_MOUSE & & Fatty acid-binding protein, epidermal; & $\begin{array}{l}\text { Epidermal-type fatty acid-binding protein;Fatty } \\
\text { acid-binding protein 5;Keratinocyte lipid- } \\
\text { binding protein;Psoriasis-associated fatty acid- } \\
\text { binding protein homolog; }\end{array}$ \\
\hline WDGKESTITR & KLKDGKMIVECVMNNATCTR & $-0,1$ & $\begin{array}{l}\text { N-ter + 42.01 Da, K+34.06 Da, } \\
\mathrm{C}+57.02 \mathrm{Da}\end{array}$ & 36,8 & 5,767 & 1 & 2511,4 & FABP5_MOUSE & & Fatty acid-binding protein, epidermal; & $\begin{array}{l}\text { Epidermal-type fatty acid-binding protein;Fatty } \\
\text { acid-binding protein 5;Keratinocyte lipid- } \\
\text { binding protein;Psoriasis-associated fatty acid- } \\
\text { binding protein homolog; }\end{array}$ \\
\hline MA & SLKDLEGKWR & n.d. & N-ter + 42.01 Da, K+28.03 Da & 28,7 & $-5,557$ & 1 & 1328,7 & FABP5_MOUSE & & Fatty acid-binding protein, epidermal; & $\begin{array}{l}\text { Epidermal-type fatty acid-binding protein;Fatty } \\
\text { acid-binding protein 5;Keratinocyte lipid- } \\
\text { binding protein;Psoriasis-associated fatty acid- } \\
\text { binding protein homolog; }\end{array}$ \\
\hline M & EKTQDRDTLSGR & 0,3 & N-ter + 42.01 Da, K+28.03 Da & 40,5 & $-1,119$ & 1 & 1605,8 & FBX50_MOUSE & & F-box only protein 50; & $\begin{array}{l}\text { NCC receptor protein 1;Non-specific cytotoxic } \\
\text { cell receptor protein } 1 \text { homolog; }\end{array}$ \\
\hline PEVDDEGFTV & RPDISQNNGAEPPR & n.d. & $\mathrm{N}$-ter $+42.01 \mathrm{Da}$ & 19,2 & 4,016 & 1 & 1591,8 & FCHO1_MOUSE & & FCH domain only protein $1 ;$ & \\
\hline M & SCTIEKALADAKALVER & n.d. & $\begin{array}{l}\text { N-ter + 42.01 Da, K+34.06 Da, } \\
\mathrm{C}+57.02 \mathrm{Da}\end{array}$ & 43,0 & $-6,290$ & 1 & 1984,1 & FGOP2_MOUSE & F8WGG1_MOUSE F & FGFR1 oncogene partner 2 homolog; & \\
\hline $\begin{array}{l}\text { RSSNRRDRPR } \\
\text { RGVSESOASD }\end{array}$ & QLSPSOSSDSQVHSGVQVEGR & n.d. & $\mathrm{N}$-ter $+42.01 \mathrm{Da}$ & 35,8 & 2,482 & 1 & 2253,1 & FILA_MOUSE & & Filaggrin; & \\
\hline $\begin{array}{l}\text { RGVSESQASD } \\
\text { GGLAADASRR }\end{array}$ & & $\begin{array}{l}\text { n.d. } \\
\text { n.d. }\end{array}$ & $\begin{array}{l}\mathrm{N} \text {-ter + 42.01 Da } \\
\mathrm{N} \text {-ter + 42.01 Da }\end{array}$ & $\begin{array}{l}33,2 \\
38,3\end{array}$ & $\begin{array}{l}3,527 \\
9,251\end{array}$ & $\begin{array}{l}1 \\
1\end{array}$ & $\begin{array}{l}1811,8 \\
2255,1\end{array}$ & $\begin{array}{l}\text { FLIA_MOUSE } \\
\text { FLLA MOUSE }\end{array}$ & $\begin{array}{l}\text { J3QM500MOUSE F } \\
\text { JOM50 MOUSE }\end{array}$ & $\begin{array}{l}\text { Filaggrin; } \\
\text { Filaggrin; }\end{array}$ & \\
\hline $\begin{array}{l}\text { GGLAADDSRR } \\
\text { RRSPVHPESS }\end{array}$ & $\begin{array}{l}\text { SGARQGQASAQGRAGSQGQAQGR } \\
\text { EGEEHSVVPQR }\end{array}$ & $\begin{array}{l}\text { n.d. } \\
\text { n.d. }\end{array}$ & $\begin{array}{l}\text { N-ter }+42.01 \mathrm{Da} \\
\mathrm{N} \text {-et }+42.01 \mathrm{Da}\end{array}$ & 22,6 & $\begin{array}{l}7,521 \\
5,494\end{array}$ & 1 & $\begin{array}{l}2253,1 \\
1307,6\end{array}$ & $\begin{array}{l}\text { FLA_MOUSE } \\
\text { FILA2 MOUSE }\end{array}$ & J3OM50_MOUSE & Filaggrin; & \\
\hline $\begin{array}{l}\text { RSRPVHPESS } \\
\text { ESVHGQPVRP }\end{array}$ & $\begin{array}{l}\text { EGEEHSVVPQR } \\
\text { QGPSQDSSSQPQASQGQPSQSGSGR }\end{array}$ & $\begin{array}{l}\text { n.d. } \\
\text { n.d. }\end{array}$ & $\begin{array}{l}\text { N-ter }+42.01 \text { Da } \\
\text { N-ter + 42.01 Da }\end{array}$ & 20,1 & $-2,462$ & 1 & $\begin{array}{l}1301,6 \\
2514,1\end{array}$ & $\begin{array}{l}\text { FLAA2_MOUSE } \\
\text { FILA2 MOUSE }\end{array}$ & FILA2_MOUSE & $\begin{array}{l}\text { Filaggrin-2; } \\
\text { Filagarin-2 }\end{array}$ & 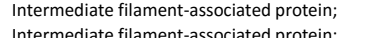 \\
\hline $\begin{array}{l}\text { ESVHGQPVRP } \\
\text { QSGSRRSPRS }\end{array}$ & $\begin{array}{l}\text { QGPSQDSSSQPQASQGQPSQSGSGR } \\
\text { QPVHPESSEGEEHSVYPQR }\end{array}$ & n.d. & $\mathrm{N}$-ter $+42.01 \mathrm{Da}$ & 19,1 & $-0,414$ & 1 & 2169,0 & $\begin{array}{l}\text { FLA_2 MOUSE } \\
\text { FILA2MOUSE }\end{array}$ & & $\begin{array}{l}\text { Filagginin-2; } \\
\text { Filaggrin-2; }\end{array}$ & $\begin{array}{l}\text { Intermediate filament-associated protein; } \\
\text { Intermediate filament-associated protein; }\end{array}$ \\
\hline QRESVHGQPV & RPQGPSQDSSSQPQASQGQPSQSGSGR & n.d. & $\mathrm{N}$-ter + 42.01 Da & 20,0 & $-0,036$ & 1 & 2767,3 & FILA2_MOUSE & & Filaggrin-2; & Intermediate filament-associated protein; \\
\hline RPQGPSQDSS & RQPQAGQGQPSQSGSC & n.d. & $\mathrm{N}$-ter + 42.01 Da & 34,7 & 9,549 & 1 & 1766,9 & FILA2_MOUSE & FILA2_MOUSE & Filaggrin-2; & Intermediate filament-associated protein; \\
\hline SQSSGYSQHR & VGSGQSCHYGQHGSSSGQSSSSGR & n.d. & N-ter + 42.01 Da, C+57.02 Da & 30,0 & 1,072 & 1 & 2422,0 & FILA2_MOUSE & E9QPZ3__MOUSE F & Filaggrin-2; & Intermediate filament-associated protein; \\
\hline EFHQNQDKIM & AKLTNEDSQNR & $-0,1$ & N-ter + 42.01 Da, K+28.03 Da & 43,9 & $-0,743$ & 1 & 1344,7 & FILIL_MOUSE & EOCYH7_MOUSE & Filamin A-interacting protein 1-like; & $\begin{array}{l}\text { Protein down-regulated in ovarian cancer } 1 \\
\text { homolog; }\end{array}$ \\
\hline AILPDFEQLS & YESEQGTSDSEGSGR & n.d. & $\mathrm{N}$-ter + 42.01 Da & 14,4 & 4,713 & 1 & 1629,7 & GEN_MOUSE & & Flap endonuclease GEN homolog 1; & \\
\hline M & SEKFDCHYCR & 0,3 & $\begin{array}{l}\text { N-ter + } 42.01 \mathrm{Da}, \mathrm{K}+28.03 \mathrm{Da}, \\
\mathrm{C}+57.02 \mathrm{Da}\end{array}$ & 19,3 & $-0,814$ & 1 & 1470,6 & FHL1_MOUSE & A2AEX8_MOUSE & Four and a half LIM domains protein 1; & $\begin{array}{l}\text { KyoT;RBP-associated molecule 14-1;;keletal } \\
\text { muscle LIM-protein } 1 ;\end{array}$ \\
\hline M & SEAFDCAKCNESLYGR & n.d. & $\begin{array}{l}\text { N-ter + } 42.01 \text { Da, } \mathrm{K}+34.06 \mathrm{Da}, \\
\mathrm{C}+57.02 \mathrm{Da}\end{array}$ & 33,8 & $-1,360$ & 1 & 1981,9 & FHL__MOUSE & F2Z455_MOUSE & Four and a half LIM domains protein 3; & Skeletal muscle LIM-protein 2; \\
\hline M & ANHAPFETDISTLTR & n.d. & $\mathrm{N}$-ter + 42.01 Da & 41,6 & $-1,399$ & 1 & 1713,8 & F16P1_MOUSE & E9Q0T7_MOUSE F & Fructose-1,6-bisphosphatase 1; & $\begin{array}{l}\text { D-fructose-1,6-bisphosphate 1- } \\
\text { phosphohydrolase 1;Fructose-1,6- } \\
\text { bisphosphatase isozyme 3;liver FBPase; }\end{array}$ \\
\hline M & SLVDLGKKLLEAAR & $-0,1$ & $\mathrm{~N}$-ter + 42.01 Da, K +28.03 Da & 43,3 & 0,496 & 1 & 1610,0 & GABP1_MOUSE & Q3UT69_MOUSE & GA-binding protein subunit beta- 1 ; & GABP subunit beta-2; \\
\hline & ATQHKTSLPQGVR & 0,2 & N-ter + 42.01 Da, K +28.03 Da & 36 & $-2,1$ & 1 & 1491,8 & Q9CRB1_MOUSE & D3Z141_MOUSE & Galectin \{ECO:0000256|RuleBase:RU102079\}; & \\
\hline QAGRFHVNLL & CGEEQGADAALHFNPR & 0,3 & $\mathrm{~N}$-ter + 42.01 Da, C+57.02 Da & 24 & 1,55 & 1 & 1812,8 & Q9CRB1_MOUSE & LEG7_MOUSE & Galectin \{ECO:0000256|RuleBase:RU102079\}; & \\
\hline RGMVPDQAGR & FHVNLLCGEEQGADAALHFNPR & n.d. & $\mathrm{N}$-ter + 42.01 Da, C+57.02 Da & 32,3 & 6,45 & 1 & 2536,2 & Q9CRB1_MOUSE & LEG7_MOUSE & Galectin \{ECO:0000256|RuleBase:RU102079\}; & \\
\hline $\begin{array}{l}\text { ADAALHFNPR } \\
M\end{array}$ & $\begin{array}{l}\text { LDTSEVWFNTKEQGKWGR } \\
\text { SATOHKSLPOGR }\end{array}$ & $\begin{array}{l}\text { n.d. } \\
\text { n.d. }\end{array}$ & $\begin{array}{l}\mathrm{N} \text {-ter + 42.01 Da, } \mathrm{K}+34.06 \mathrm{Da} \\
\mathrm{N} \text {-er + } 24.01 \mathrm{Da} \mathrm{K}+28.03 \mathrm{Da}\end{array}$ & 36,9 & $\begin{array}{r}8,699 \\
-3,859\end{array}$ & 1 & $\begin{array}{l}2203,2 \\
1587,8\end{array}$ & $\begin{array}{l}\text { Q9CRB1_MOUSE } \\
\text { O99RB1 MOUSE }\end{array}$ & D3Z141_MOUSE & 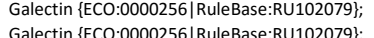 & \\
\hline M & ACGLVASNLNLKPGECLKVR & n.d. & $\begin{array}{l}\text { N-ter + 42.01 Da, K+34.06 Da, } \\
\text { C+57.02 Da }\end{array}$ & 61,8 & $-1,168$ & 1 & 2308,3 & LEG1_MOUSE & & 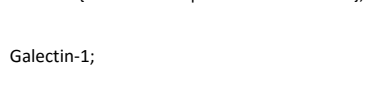 & $\begin{array}{l}14 \text { kDa lectin;Beta-galactoside-binding lectin L- } \\
14-\text { - ;Galaptin;Lactose-binding lectin 1;Lectin } \\
\text { galactoside-binding soluble 1;5-Lac lectin 1; }\end{array}$ \\
\hline PDQAGRFHVN & LLCGEEQGADAALHFNPR & n.d. & N-ter + 42.01 Da, C+57.02 Da & 21,4 & 2,302 & 1 & 2039,0 & LEG7_MOUSE & Q9CRB1_MOUSE & Galectin-7; & \\
\hline & SSDTSPAVVITTPPPPSMPHKER & n.d. & $\mathrm{N}$-ter + 42.01 Da, K+34.06 Da & 27,5 & 0,084 & 1 & 2393,2 & ADDG_MOUSE & & Gamma-adducin; & Adducin-like protein 70; \\
\hline
\end{tabular}




\begin{tabular}{|c|c|c|c|c|c|c|c|c|c|c|c|}
\hline Non Prime Site & Prime Site & $\begin{array}{c}\text { Fold change of } \\
\text { XPRESS ratio } \\
\text { (Adam17 } 1 \text { ¿Kc / WT) } \\
\end{array}$ & Modifications & Hyperscore & $\begin{array}{c}\text { Mass error } \\
\text { in ppm }\end{array}$ & charge & $\begin{array}{c}\text { Precursor } \\
\text { neutral mass } \\
\text { in Da } \\
\end{array}$ & Uniprot ID 1 & Uniprot ID 2 & Protein Name 1 & Protein Name 2 \\
\hline RSLPYLPADI & GNAEGEPVVPER & n.d. & $\mathrm{N}$-ter + 42.01 Da & 15,1 & 1,233 & 1 & 1294,6 & GDAP2_MOUSE & & $\begin{array}{l}\text { Ganglioside-induced differentiation-associated } \\
\text { protein 2; }\end{array}$ & \\
\hline M & ADPDVLTEVPAALKR & n.d. & $\mathrm{N}$-ter + 42.01 Da, K +34.06 Da & 46,6 & $-0,778$ & 1 & 1670,0 & T2EA_MOUSE & & General transcription factor IIE subunit 1; & $\begin{array}{l}\text { General transcription factor IIE } 56 \mathrm{kDa} \\
\text { subunit;:Transcription initiation factor IIE } \\
\text { subunit alpha; }\end{array}$ \\
\hline $\begin{array}{l}M \\
M\end{array}$ & $\begin{array}{l}\text { ATEGMILTNHDHQIR } \\
\text { AEGSAVSDPQHAAR }\end{array}$ & $\begin{array}{l}\text { n.d. } \\
\text { n.d. }\end{array}$ & $\begin{array}{l}\mathrm{N} \text {-ter }+42.01 \mathrm{Da} \\
\mathrm{N} \text {-ter }+42.01 \mathrm{Da}\end{array}$ & $\begin{array}{l}41,6 \\
40,2\end{array}$ & $\begin{array}{l}-1,629 \\
-1,877\end{array}$ & $\begin{array}{l}1 \\
1\end{array}$ & $\begin{array}{l}1776,9 \\
1436,7\end{array}$ & $\begin{array}{l}\text { GEPH_MOUSE } \\
\text { GAN_MOUSE }\end{array}$ & $\begin{array}{l}\text { AOJNY3_MOUSE } \\
\text { FGTZU3_MOUSE }\end{array}$ & $\begin{array}{l}\text { Gephyrin; } \\
\text { Gigaxonin \{ECO:0000312|MGI:MGI:1890619\}; }\end{array}$ & \\
\hline M & SESLVVCDVAEDLVEKLR & n.d. & $\begin{array}{l}\text { N-ter + 42.01 Da, K+34.06 Da, } \\
\text { C +57.02 Da }\end{array}$ & $\begin{array}{l}40,2 \\
64,9\end{array}$ & $-0,842$ & 1 & $\begin{array}{l}1430, \prime \\
2136,1\end{array}$ & GMFB_MOUSE & FotZUS_NIUUSE & Glia maturation factor beta; & \\
\hline M & SDSLVVCEVDPELKETLR & $-0,5$ & $\begin{array}{l}\text { N-ter + } 42.01 \mathrm{Da}, \mathrm{K}+28.03 \mathrm{Da}, \\
\mathrm{C}+57.02 \mathrm{Da}\end{array}$ & 21,8 & 0,879 & 1 & 2158,1 & GMFG_MOUSE & & Glia maturation factor gamma; & \\
\hline M & STASSSSSQTPHSAPQR & n.d. & $\mathrm{N}$-ter $+42.01 \mathrm{Da}$ & 20,6 & 4,207 & 1 & 1756,8 & GLCI1_MOUSE & E9QKK4_MOUSE & Glucocorticoid-induced transcript 1 protein; & $\begin{array}{l}\text { Glucocorticoid-induced gene } 18 \\
\text { protein;Testhymin;Thymocyte/spermatocyte } \\
\text { selection protein 1; }\end{array}$ \\
\hline M & AGKPVLHHFNAR & 0,1 & N-ter + 42.01 Da, K+28.03 Da & 25,3 & 0,282 & 1 & 1415,8 & E9Q6L7_MOUSE & & $\begin{array}{l}\text { Glutathione S-transferase } \\
\text { \{ECO:0000256|RuleBase:RU004454\}; }\end{array}$ & \\
\hline M & AGKPVLHYFNAR & n.d. & $\mathrm{N}$-ter + 42.01 Da, K+34.06 Da & 48,6 & $-1,104$ & 1 & 1447,8 & GSTA1_MOUSE & GSTA2_MOUSE & Glutathione S-transferase A1; & $\begin{array}{l}\text { GST class-alpha member 1;Glutathione S- } \\
\text { transferase Ya;Glutathione S-transferase Ya1; }\end{array}$ \\
\hline M & AAKPKLYYFNGR & n.d. & $\mathrm{N}$-ter + 42.01 Da, K+34.06 Da & 26,8 & $-0,390$ & 1 & 1536,9 & GSTA4_MOUSE & & Glutathione S-transferase A4; & $\begin{array}{l}\text { GST A4-4;GST class-alpha member } \\
\text { 4;Glutathione S-transferase 5.7; }\end{array}$ \\
\hline M & SSKSMVLGYWDIR & n.d. & $\mathrm{N}$-ter + 42.01 Da, K +34.06 Da & 27,2 & 0,062 & 1 & 1616,9 & GSTM5_MOUSE & E9Q5L9_MOUSE & Glutathione S-transferase Mu 5; & Fibrous sheath component 2;GST class-mu 5; \\
\hline M & SGESSRSLGKGSAPPGPVPEGQIR & 0,9 & N-ter + 42.01 Da, K+28.03 Da & 35,5 & $-3,220$ & 1 & 2419,2 & GSTO1_MOUSE & & Glutathione S-transferase omega- $1 ;$ & $\begin{array}{l}\text { Glutathione S-transferase omega 1- } \\
\text { 1;Glutathione-dependent dehydroascorbate } \\
\text { reductase;Monomethylarsonic acid reductase;-S- } \\
\text { (Phenacy)|glutathione reductase;p28; }\end{array}$ \\
\hline M & VKVGVNGFGR & n.d. & $\mathrm{N}$-ter + 42.01 Da, K+34.06 Da & 22,3 & $-1,892$ & 1 & 1107,7 & G3P_MOUSE & D3YYI5_MOUSE & Glyceraldehyde-3-phosphate dehydrogenase; & Peptidyl-cysteine S-nitrosylase GAPDH; \\
\hline M & AAAKKAVLGPLVGAVDQGTSSTR & n.d. & $\mathrm{N}$-ter + 42.01 Da, K+34.06 Da & 37,5 & $-1,472$ & 1 & 2306,4 & GLPK_MOUSE & B1ASZ3_MOUSE & Glycerol kinase; & ATP:Ilycerol 3-phosphotransferase; \\
\hline \multirow{3}{*}{ M } & AKPLTDQEKR & 0,4 & $\mathrm{~N}$-ter + 42.01 Da, K+28.03 Da & 48,6 & $-0,467$ & 1 & 1282,7 & PYGL_MOUSE & & Glycogen phosphorylase, live & \\
\hline & ALLAEHLLKPLPADR & n.d. & N-ter + 42.01 Da, K+34.06 Da & 56,2 & $-0,577$ & 1 & 1732,1 & GLTP_MOUSE & D3Z1H8_MOUSE & Glycolipid transfer protein; & \\
\hline & MKVFVTGPLPAEGR & 0,2 & N-ter + 42.01 Da, K+28.03 Da & 25,0 & 1,526 & 1 & 1570,9 & GRHPR_MOUSE & DGREG4_MOUSE & Glyoxylate reductase/hydroxypyruvate reductase; & \\
\hline M & EPLYQQTNKQVQEIOSHMGR & n.d. & N-ter + 42.01 Da, K+34.06 Da & 48,7 & 1,067 & 1 & 2620,3 & GOSR2_MOUSE & A2A910_MOUSE & Golgi SNAP receptor complex member 2; & 27 kDa Golgi SNARE protein;Membrin; \\
\hline $\begin{array}{l}\text { M } \\
\text { MEKN }-1\end{array}$ & $\begin{array}{l}\text { RPQQAPVSGKVFIQR } \\
\text { TIKSENKGISTPR }\end{array}$ & n.d. & N-ter + 42.01 Da, K+34.06 Da & $\begin{array}{l}42,1 \\
174\end{array}$ & $\begin{array}{c}0,833 \\
-1652\end{array}$ & 1 & $\begin{array}{l}1917,1 \\
20552\end{array}$ & GOGA7_MOUSE & & Golgin subfamily A member 7; & \\
\hline $\begin{array}{l}\text { MEKM } \\
\text { QLGPAAAGRL }\end{array}$ & $\begin{array}{l}\text { TTLKSSENKGLITSTPIR } \\
\text { AGPIPARPSAAAAAAASYSALR }\end{array}$ & $\begin{array}{l}\text { n.d. } \\
\text { n.d. }\end{array}$ & $\begin{array}{l}\text { N-ter + 42.01 Da, K +34.06 Da } \\
\text { N-ter + 42.01 Da }\end{array}$ & $\begin{array}{l}17,4 \\
34,6\end{array}$ & $\begin{array}{l}-1,652 \\
2,879\end{array}$ & $\begin{array}{l}1 \\
1\end{array}$ & $\begin{array}{l}2055,2 \\
2081,1\end{array}$ & $\begin{array}{l}\text { GO45_MOUSE } \\
\text { GRSF1_MOUSE }\end{array}$ & & $\begin{array}{l}\text { Golgin-45; } \\
\text { G-rich sequence factor 1; }\end{array}$ & Basic leucine zipper nuclear factor $1 ;$ \\
\hline M & EDSAPDAVAAAPSGTPKSKLETLPR & n.d. & $\mathrm{N}$-ter + 42.01 Da, $\mathrm{K}+34.06 \mathrm{Da}$ & 18,7 & $-2,253$ & 1 & 2748,5 & GCC2_MOUSE & B2RSU7_MOUSE & GRIP and coiled-coil domain-containing protein 2; & 185 kDa Golgi coiled-coil protein; \\
\hline M & SSKTASTNSIAQAR & n.d. & N-ter + 42.01 Da, K+34.06 Da & 72,2 & $-0,200$ & 1 & 1496,8 & GBG12_MOUSE & & $\begin{array}{l}\text { Guanine nucleotide-binding protein } G(I) / G(S) / G(0) \\
\text { subunit gamma-12; }\end{array}$ & \\
\hline M & SGSSSVAAMKKVVQQLR & $-0,2$ & $\mathrm{~N}$-ter + 42.01 Da, K+28.03 Da & 35,1 & 6,663 & 1 & 1873,1 & GBG5_MOUSE & & $\begin{array}{l}\text { Guanine nucleotide-binding protein } G(I) / G(S) / G(0) \\
\text { subunit gamma-5; }\end{array}$ & \\
\hline M & TKVKAAPEESEAQAEGCSEER & n.d. & $\begin{array}{l}\text { N-ter + 42.01 Da, K+34.06 Da, } \\
\mathrm{C}+57.02 \mathrm{Da}\end{array}$ & 44,1 & 0,744 & 1 & 2415,2 & NHP2_MOUSE & & H/ACA ribonucleoprotein complex subunit 2; & $\begin{array}{l}\text { Nucleolar protein family A member 2;snoRNP } \\
\text { protein NHP2; }\end{array}$ \\
\hline M & ADKEAGGGDAGPR & n.d. & N-ter + 42.01 Da, K+34.06 Da & 46,4 & $-3,366$ & 1 & 1275,6 & HS12A_MOUSE & & Heat shock 70 kDa protein 12A; & \\
\hline SYVAFTDTER & LIGDAAKNQVAMNPQNTVFDAKR & n.d. & N-ter + 42.01 Da, K+34.06 Da & 22,4 & $-4,513$ & 1 & 2610,4 & HS71L_MOUSE & & Heat shock 70 kDa protein 1-like; & $\begin{array}{l}\text { Heat shock } 70 \text { kDa-like protein } 1 \text {; Spermatid- } \\
\text { specific heat shock protein 70; }\end{array}$ \\
\hline EIAEAYLGKA & TKDAGTIAGLNVLR & 1,8 & N-ter + 42.01 Da, K+28.03 Da & 17,7 & $-2,465$ & 1 & 1497,9 & HSP7C_MOUSE & Q504P4_MOUSE & Heat shock cognate 71 kDa protein; & Heat shock 70 kDa protein 8 \\
\hline NRRRLSELLR & YHTSQSGDEMTSLSEYVSR & n.d. & $\mathrm{N}$-ter $+42.01 \mathrm{Da}$ & 29,3 & 8,780 & 1 & 2218,0 & HS90B_MOUSE & E9Q0C3_MOUSE & Heat shock protein HSP 90-beta; & $\begin{array}{l}\text { Heat thock } 8 \text { 4DDa; Tumor-specific } \\
\text { transplantation } 84 \text { kDa antigen; }\end{array}$ \\
\hline M & TTTTFKGVDPNSR & $-0,1$ & N-ter + 42.01 Da, K+28.03 Da & 28,5 & 0,689 & 1 & 1593,8 & HN1_MOUSE & & Hematological and neurological expressed 1 protein; & \\
\hline GISSSKPNRM & ASNIFGPTEEPKNIPKR & 0,5 & $\mathrm{~N}$-ter + 42.01 Da, K+28.03 Da & 29,7 & 1,752 & 1 & 1995,1 & HN1L_MOUSE & & $\begin{array}{l}\text { Hematological and neurological expressed 1-like } \\
\text { protein; }\end{array}$ & \\
\hline M & FQGADSQAGKSGSR & n.d. & N-ter + 42.01 Da, K+34.06 Da & 46,9 & $-0,499$ & 1 & 1601,8 & HN1L_MOUSE & & $\begin{array}{l}\text { Hematological and neurological expressed 1-like } \\
\text { protein: }\end{array}$ & \\
\hline LSGEDKSNIK & AAWGKIGGHGAEYGAEALER & 5,5 & N-ter + $42.01 \mathrm{Da}, \mathrm{K}+28.03 \mathrm{Da}$ & 25,2 & $-0,520$ & 1 & 2112,0 & HBA_MOUSE & Q91VB8_MOUSE & Hemoglobin subunit alpha; & Alpha-globin;Hemoglobin alpha chain; \\
\hline M & ENSQLCKLFIGGLNVQTSESGLR & $-0,1$ & $\begin{array}{l}\text { N-ter + 42.01 Da, K+28.03 Da, } \\
\text { C +57.02 Da }\end{array}$ & 46,1 & 1,707 & 1 & 2750,4 & ROAO_MOUSE & & Heterogeneous nuclear ribonucleoprotein A0; & \\
\hline
\end{tabular}




\begin{tabular}{|c|c|c|c|c|c|c|c|c|c|c|c|}
\hline Non Prime Site & Prime Site & 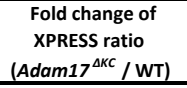 & Modifications & Hyperscore & $\begin{array}{l}\text { Mass error } \\
\text { in ppm }\end{array}$ & Charge & $\begin{array}{c}\text { Precursor } \\
\text { neutral mass } \\
\text { in Da }\end{array}$ & Uniprot ID 1 & Uniprot ID 2 & Protein Name 1 & Protein Name 2 \\
\hline M & SKSESPKEPEQLR & 0,1 & $\mathrm{~N}$-ter + 42.01 Da, K+28.03 Da & 63,5 & $-2,603$ & 1 & 1611,8 & ROA1_MOUSE & Q5EBP8_MOUSE & Heterogeneous nuclear ribonucleoprotein $\mathrm{A} 1$; & $\begin{array}{l}\text { HDP-1; Helix-destabilizing protein;Single-strand- } \\
\text { binding protein;Topoisomerase-inhibitor } \\
\text { suppressed;hnRNP core protein A1; }\end{array}$ \\
\hline M & EVKPPPGRPQPDSGR & n.d. & $\mathrm{N}$-ter + 42.01 Da, K+34.06 Da & 33,7 & 0,164 & 1 & 1823,0 & ROA3_MOUSE & A2AL12_MOUSE & Heterogeneous nuclear ribonucleoprotein A3; & \\
\hline $\begin{array}{l}M \\
M\end{array}$ & MLGPEGGEGYVVKLR & 0,0 & $\mathrm{~N}$-ter + 42.01 Da, K+28.03 Da & 67,8 & $-0,477$ & 1 & 1673,9 & HNRPF_MOUSE & J3QMQ5_MOUSE & Heterogeneous nuclear ribonuc & \\
\hline M & & & Neter $+42.01 \mathrm{da}, \mathrm{K}+34.00 \mathrm{dd}$ & 08,3 & ${ }^{-1,040}$ & & $10<3,9$ & HNRH1_MOUSE & Q8C2QQ_MMOUSE & Heterogeneous nuclear ribonucleoprotelin $\mathrm{H}$; & \\
\hline & EKILETVPLER & 0,0 & N-ter + 42.01 Da, K+28.03 Da & 56,8 & $-1,319$ & 1 & 1514,8 & ROA2_MOUSE & & Heterogeneous nuclear ribonucleoproteins $A 2 / B 1 ;$ & \\
\hline $\begin{array}{l}M \\
M\end{array}$ & $\begin{array}{l}\text { STNTDLSLSSYDEGQGSKFIR } \\
\text { SESGSKSSQPLASKOEKDGTEKR }\end{array}$ & $\begin{array}{r}0,1 \\
-0,2\end{array}$ & $\begin{array}{l}\mathrm{N} \text {-ter + 42.01 Da, } \mathrm{K}+28.03 \mathrm{Da} \\
\mathrm{N} \text {-ter + 42.01 Da, K+28.03 Da }\end{array}$ & $\begin{array}{l}51,7 \\
29,4\end{array}$ & $\begin{array}{l}0,210 \\
-0,114\end{array}$ & $\begin{array}{l}1 \\
1\end{array}$ & $\begin{array}{l}2374,1 \\
2617,4\end{array}$ & $\begin{array}{l}\text { HIG1A_MOUSE } \\
\text { HMGA1_MOUSE }\end{array}$ & J3QPX__MOUSE & $\begin{array}{l}\text { HIG1 domain family member } 1 \mathrm{AA} \text {, mitochondrial; } \\
\text { High mobility group protein HMG-1/HMG-Y; }\end{array}$ & $\begin{array}{l}\text { Hypoxia-inducible gene } 1 \text { protein; } \\
\text { High mobility group AT-hook protein 1; }\end{array}$ \\
\hline M & ADEIAKAQVAQPGGDTIFGKIIR & n.d. & $\mathrm{N}$-ter + 42.01 Da, K+34.06 Da & 76,7 & $-1,434$ & 1 & 2507,4 & HINT1_MOUSE & BOR1E3_MOUSE & Histidine triad nucleotide-binding protein 1 ; & $\begin{array}{l}\text { Adenosine 5'-monophosphoramidase; Protein } \\
\text { kinase Cinhibitor 1;; Protein kinase C-interacting } \\
\text { protein 1; }\end{array}$ \\
\hline M & SETAPAAPAAPAPAEKTPVKKKAR & n.d. & N-ter + 42.01 Da, K+34.06 Da & 30,2 & 1,012 & 1 & 2564,6 & H14_MOUSE & & Histone H1.4; & H1 VAR.2;H1e; \\
\hline M & ADKEAAFDDAVEER & n.d. & $\mathrm{N}$-ter + 42.01 Da, K+34.06 Da & 77,1 & $-0,548$ & 1 & 1640,8 & RBBP4_MOUSE & & Histone-binding protein RBBP4; & $\begin{array}{l}\text { Chromatin assembly factor } 1 \text { subunit } \\
\text { C;Chromatin assembly factor I p48 } \\
\text { subunit;;Nucleosome-remodeling factor subunit } \\
\text { RBAP48;Retinoblastoma-binding protein } \\
\text { 4;Retinoblastoma-binding protein p48; }\end{array}$ \\
\hline M & ASKEMFEDTVEER & n.d. & $\mathrm{N}$-ter + 42.01 Da, K+34.06 Da & 72,3 & 0,182 & 1 & 1645,8 & RBBP7_MOUSE & A2AFJ1_MOUSE & Histone-binding protein RBBP7; & $\begin{array}{l}\text { Histone acetyltransferase type B subunit } \\
\text { 2;Nucleosome-remodeling factor subunit } \\
\text { RBAP46;Retinoblastoma-binding protein } \\
\text { 7;Retinoblastoma-binding protein p46; }\end{array}$ \\
\hline $\begin{array}{l}\text { GQGQASGSGR } \\
\text { TSRESSTSQR }\end{array}$ & $\begin{array}{l}\text { CGASSGQTSGGGSGQSTR } \\
\text { FGSGSGGSSGFSQGR }\end{array}$ & $\begin{array}{l}0,4 \\
\text { n.d. }\end{array}$ & $\begin{array}{l}\mathrm{N}-\text { ter + } 42.01 \mathrm{Da}, \mathrm{C}+57.02 \mathrm{Da} \\
\mathrm{N} \text {-ter + 42.01 Da }\end{array}$ & $\begin{array}{l}17,8 \\
18,8\end{array}$ & $\begin{array}{l}-1,565 \\
-1,129\end{array}$ & $\begin{array}{l}1 \\
1\end{array}$ & $\begin{array}{l}1785,7 \\
1415,6\end{array}$ & $\begin{array}{l}\text { HORN_MOUSE } \\
\text { HORN_MOUSE }\end{array}$ & $\begin{array}{l}\text { HORN_MOUSE } \\
\text { HORN_MOUSE }\end{array}$ & $\begin{array}{l}\text { Hornerin; } \\
\text { Hornerin; }\end{array}$ & \\
\hline SGQNYNSTRQ & GCGQGKSSGSEQYGASSGQSSGCSSGQSTR & n.d. & $\begin{array}{l}\mathrm{N} \text {-ter }+42.01 \mathrm{Da}, \mathrm{K}+28.03 \mathrm{Da}, \\
\mathrm{C}+57.02 \mathrm{Da}\end{array}$ & 44,4 & $-6,504$ & 1 & 2995,2 & HORN_MOUSE & E9QNP3_MOUSE & Hornerin; & \\
\hline TSGQNYNSTR & QGCGQGKSSGSEQYGASSGQSSGCSSGQSTR & n.d. & $\begin{array}{l}\text { N-ter + 42.01 Da, K+34.06 Da, } \\
\mathrm{C}+57.02 \mathrm{Da}\end{array}$ & 47,3 & 6,545 & 1 & 3129,3 & HORN_MOUSE & E9QNP3_MOUSE & Hornerin; & \\
\hline $\begin{array}{l}\text { SQRYGSGSGE } \\
\text { ORGHGSRSGR }\end{array}$ & $\begin{array}{l}\text { SSGFSQGGSGQGR } \\
\text { SSGLGOYGSSGGOSS }\end{array}$ & n.d. & $\mathrm{N}$-ter + 42.01 Da & 20,9 & $\begin{array}{l}1,435 \\
1,806\end{array}$ & 1 & $\begin{array}{l}1252,6 \\
3042,4\end{array}$ & HORN_MOUSE & $\begin{array}{l}\text { HORN_MOUSE } \\
\text { HORN_MOUSE }\end{array}$ & Hornerin; & \\
\hline $\begin{array}{l}\text { ORGHGSSSGR } \\
\text { EQSSGLGHYG }\end{array}$ & $\begin{array}{l}\text { SSGLGQYGSSSGQTSSSTRQGSGQGQASGSGR } \\
\text { STSGQTASTRGGSGQGAASGSGR }\end{array}$ & $\begin{array}{l}\text { n.d. } \\
\text { n.d. }\end{array}$ & $\begin{array}{l}\mathrm{N} \text {-ter }+42.01 \mathrm{Da} \\
\mathrm{N} \text {-ter }+42.01 \mathrm{Da}\end{array}$ & $\begin{array}{l}28,8 \\
32,7\end{array}$ & $\begin{array}{r}1,806 \\
-0,044\end{array}$ & $\begin{array}{l}1 \\
1\end{array}$ & $\begin{array}{l}3028,4 \\
2281,0\end{array}$ & $\begin{array}{l}\text { HOR_MOUSE } \\
\text { HORN_MOSE }\end{array}$ & $\begin{array}{l}\text { HORN_MOUSE } \\
\text { HORN_MOUSE }\end{array}$ & $\begin{array}{l}\text { Hoonnerin; } \\
\text { Hornerin; }\end{array}$ & \\
\hline SGCGSGQSTR & YGEQGSGSRNSSTQSR & n.d. & $\mathrm{N}$-ter $+42.01 \mathrm{Da}$ & 21,7 & 1,950 & 1 & 1741,8 & HORN_MOUSE & HORN_MOUSE & Hornerin; & \\
\hline MRRRGEIEM & ATEGDVELELETETSGPERPPEKPR & n.d. & $\mathrm{N}$-ter + 42.01 Da, K+34.06 Da & 43,1 & 1,020 & 1 & 2841,4 & HYPK_MOUSE & & Huntingtin-interacting protein $\mathrm{K}$; & Huntingtin yeast partner $\mathrm{K}$; \\
\hline ESIERKVSVR & EHATLEETTR & n.d. & $\mathrm{N}$-ter $+42.01 \mathrm{Da}$ & 21,2 & 8,865 & 1 & 1227,6 & HYDIN_MOUSE & & Hydrocephalus-inducing protein; & Protein $\mathrm{Hy}-3$; \\
\hline M & LPNTGKLAGCTVFITGASR & 0,2 & $\begin{array}{l}\text { N-ter + 42.01 Da, K+28.03 Da, } \\
\mathrm{C}+57.02 \mathrm{Da}\end{array}$ & 40,8 & 4,524 & 1 & 2163,1 & HSDL2_MOUSE & & Hydroxysteroid dehydrogenase-like protein 2; & \\
\hline M & ADNEKLDNQR & n.d. & $\mathrm{N}$-ter + 42.01 Da, K+34.06 Da & 48,1 & $-1,641$ & 1 & 1277,6 & IMA3_MOUSE & & Importin subunit alpha-3; & $\begin{array}{l}\text { Importin alpha Q1; Karyopherin subunit alpha- } \\
4 ;\end{array}$ \\
\hline m & AENPGLENHR & n.d. & N-ter +42.01 Da & 33,1 & $-2,289$ & 1 & 1177,6 & IMA4_MOUSE & & Importin subunit alpha-4; & $\begin{array}{l}\text { Importin alpha Q2; Karyopherin subunit alpha- } \\
3 ;\end{array}$ \\
\hline M & ETMASPGKDNYR & 0,0 & $\mathrm{~N}$-ter + 42.01 Da, K+28.03 Da & 22,8 & 5,539 & 1 & 1568,7 & IMA7_MOUSE & & Importin subunit alpha-7; & Importin alpha-S2;Karyopherin subunit alpha-6; \\
\hline M & MNKLYIGNLSPAVTADDLR & 0,3 & $\mathrm{~N}$-ter + 42.01 Da, K+28.03 Da & 21,8 & 1,341 & 1 & 2160,1 & IF2B2_MOUSE & & Insulin-like growth factor 2 mRNA-binding protein 2; & $\begin{array}{l}\text { IGF-II mRNA-binding protein 2;VICKZ family } \\
\text { member 2; }\end{array}$ \\
\hline M & ELQKGKGTVAAAASGAAGGGGGGAGAGAPGGGR & n.d. & $\mathrm{N}$-ter + $42.01 \mathrm{Da}, \mathrm{K}+34.06 \mathrm{Da}$ & 37,6 & 2,896 & 1 & 2863,5 & INT3_MOUSE & & Integrator complex subunit 3; & $\begin{array}{l}\text { SOSS complex subunit A; Sensor of single-strand } \\
\text { DNA complex subunit A; }\end{array}$ \\
\hline SRSIGDGQYK & RCGVTSVPDIR & n.d. & $\mathrm{N}$-ter + 42.01 Da, C+57.02 Da & 16,4 & 1,534 & 1 & 1300,7 & ILKAP_MOUSE & & $\begin{array}{l}\text { Integrin-linked kinase-associated serine/threonine } \\
\text { phosphatase } 2 C_{;}\end{array}$ & \\
\hline PSPIAALKNV & AEALGHSPKDPGGGGGSVR & n.d. & N-ter + 42.01 Da, K+34.06 Da & 24,7 & 7,389 & 1 & 1824,0 & 12BP1_MOUSE & & Interferon regulatory factor 2-binding protein 1 ; & Probable E3 ubiquitin-protein ligase IRF2BP1; \\
\hline $\begin{array}{l}\text { M } \\
\text { QLIKKPLVAS } \\
\text { M }\end{array}$ & $\begin{array}{l}\text { AKDILGEAGLHFDELNKLR } \\
\text { EFVGHLAR } \\
\text { LGSGFKAER }\end{array}$ & $\begin{array}{l}-0,5 \\
\text { n.d. } \\
\text { n.d. }\end{array}$ & $\begin{array}{l}\text { N-ter + 42.01 Da, K+28.03 Da } \\
\text { N-ter + 42.01 Da } \\
\text { N-ter + 42.01 Da, K+34.06 Da }\end{array}$ & $\begin{array}{l}36,4 \\
24,4 \\
40,0\end{array}$ & $\begin{array}{l}0,000 \\
-9,470 \\
-1,023\end{array}$ & $\begin{array}{l}1 \\
1 \\
1\end{array}$ & $\begin{array}{l}2236,2 \\
969,5 \\
1170,6\end{array}$ & $\begin{array}{l}\text { IFT20_MOUSE } \\
\text { IQCAL_MOUSE } \\
\text { IST1_MOUSE }\end{array}$ & & $\begin{array}{l}\text { Intraflagelllar transport protein } 20 \text { homolog; } \\
\text { IQ and AAA domain-containing protein 1-like; } \\
\text { IST1 homolog; }\end{array}$ & Protein IQCA1P1; \\
\hline LVKATIGLR & NLALCPANHAPLQEAAVIPR & n.d. & $\mathrm{N}$-ter $+42.01 \mathrm{Da}, \mathrm{C}+57.02 \mathrm{Da}$ & 17,0 & 4,775 & 1 & 2196,2 & PLAK_MOUSE & & Junction plakoglobin; & Desmoplakin III;Desmoplakin-3; \\
\hline FWMYDHSTNR & WLSKPSLLR & n.d. & N-ter + 42.01 Da, K+34.06 Da & 17,3 & $-8,915$ & 1 & 1174,7 & KBTB8_MOUSE & & Kelch repeat and BTB domain-containing protein 8; & \\
\hline $\begin{array}{l}\text { M } \\
\text { DGSCNLASVE }\end{array}$ & $\begin{array}{l}\text { QPEEKLSGAPR } \\
\text { YYNPVTDKWTLPTNMSTGR }\end{array}$ & $\begin{array}{l}1,1 \\
\text { n.d. }\end{array}$ & $\begin{array}{l}\text { N-ter + 42.01 Da, K+28.03 Da } \\
\text { N-ter + 42.01 Da, K+34.06 Da }\end{array}$ & $\begin{array}{l}26,1 \\
25,8\end{array}$ & $\begin{array}{l}-0,434 \\
-2,217\end{array}$ & $\begin{array}{l}1 \\
1\end{array}$ & $\begin{array}{l}1379,7 \\
2432,2\end{array}$ & $\begin{array}{l}\text { KEAP1_MOUSE } \\
\text { KLHL3_MOUSE }\end{array}$ & J3КMI2 MOUSE & $\begin{array}{l}\text { Kelch-like ECH-associated protein 1; } \\
\text { Kelch-like protein 3; }\end{array}$ & Cytosolic inhibitor of Nrf2; \\
\hline
\end{tabular}




\begin{tabular}{|c|c|c|c|c|c|c|c|c|c|c|c|}
\hline Non Prime Site & Prime Site & 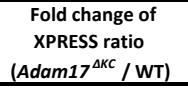 & Modifications & Hyperscore & $\begin{array}{c}\text { Mass error } \\
\text { in ppm }\end{array}$ & Charge & $\begin{array}{c}\text { Precursor } \\
\text { neutral mass } \\
\text { in Da }\end{array}$ & Uniprot ID 1 & Uniprot ID 2 & Protein Name 1 & Protein Name 2 \\
\hline STGDVNVEMN & AAPGVDLTQLLNNMR & 0,3 & N-ter + 42.01 Da & 28,2 & 8,274 & 1 & 1653,9 & K1C10_MOUSE & A2A513_MOUSE & Keratin, type I cytoskeletal 10; & $\begin{array}{l}56 \text { kDa cytokeratin;Cytokeratin-10;Keratin, type } \\
\text { I cytoskeletal } 59 \text { kDa;keratin-10; }\end{array}$ \\
\hline EIELOSQLAL & KaSLEASLAETEGR & 0,3 & N-ter $+42.01 \mathrm{Da}, \mathrm{K}+34.06 \mathrm{Da}$ & 23,0 & 9,337 & 1 & 1593,9 & K1C10_MOUSE & A2A513_MOUSE & Keratin, type I cytoskeletal 10; & $\begin{array}{l}56 \text { kDa cytokeratin;Cytokeratin-10;Keratin, type } \\
\text { I cytoskeletal } 59 \mathrm{kDa} \text {;eratin-10; }\end{array}$ \\
\hline KVTMQNLNDR & LASYMDKVR & 0,9 & N-ter + 42.01 Da, K +28.03 Da & 30,8 & $-1,646$ & 1 & 1151,6 & K1C10_MOUSE & A2A513_MOUSE & Keratin, type I cytoskeletal 10; & $\begin{array}{l}56 \text { kDa cytokeratin;Cytokeratin-10;Keratin, type } \\
\text { I cytoskeletal } 59 \text { kDa;Keratin-10; }\end{array}$ \\
\hline YQQLLIIKTR & LENEIOTYR & n.d. & $\mathrm{N}$-ter $+42.01 \mathrm{Da}$ & 24,1 & $-2,317$ & 1 & 1206,6 & K1C10_MOUSE & A2A513_MOUSE & Keratin, type I cytoskeletal 10; & $\begin{array}{l}56 \text { kDa cytokeratin;Cytokeratin-10;Keratin, type } \\
\text { I cytoskeletal } 59 \text { kDa;Keratin-10; }\end{array}$ \\
\hline NARLAADDFR & LKYENEVTLR & n.d. & N-ter + 42.01 Da, K+28.03 Da & 21,1 & $-0,524$ & 1 & 1333,7 & K1C10_MOUSE & A2A513_MOUSE & Keratin, type I cytoskeletal 10; & $\begin{array}{l}56 \text { kDa cytokeratin;Cytokeratin-10;Keratin, type } \\
\text { I cytoskeletal } 59 \mathrm{kDa} \text {;Keratin-10; }\end{array}$ \\
\hline RRTVQGLEIE & LQSQLALKQSLEASLAETEGR & n.d. & N-ter + 42.01 Da, K+28.03 Da & 25,6 & 7,892 & 1 & 2341,3 & K1C10_MOUSE & A2A513_MOUSE & Keratin, type I cytoskeletal 10; & $\begin{array}{l}56 \mathrm{kDa} \text { cytokeratin;Cytokeratin-10;Keratin, type } \\
\text { I cytoskeletal } 59 \mathrm{kDa} \text {;Keratin-10; }\end{array}$ \\
\hline DLTQLLNNMR & NQYEQLAEKNR & n.d. & N-ter + 42.01 Da, K+34.06 Da & 29,0 & 3,264 & 1 & 1467,8 & K1C10_MOUSE & A2A513_MOUSE & Keratin, type I cytoskeletal 10; & $\begin{array}{l}56 \mathrm{kDa} \text { cytokeratin;Cytokeratin-10;Keratin, type } \\
\text { I cytoskeletal } 59 \mathrm{kDa} \text {;Keratin-10; }\end{array}$ \\
\hline RLENEIQTYR & SLLEGEGSSSGGGGGR & n.d. & $\mathrm{N}$-ter $+42.01 \mathrm{Da}$ & 45,8 & $-6,139$ & 1 & 1447,7 & K1C10_MOUSE & A2A513_MOUSE & Keratin, type I cytoskeletal 10; & $\begin{array}{l}56 \text { kDa cytokeratin;Cytokeratin-10;Keratin, type } \\
\text { I cytoskeletal } 59 \mathrm{kDa} \text {;Keratin-10; }\end{array}$ \\
\hline MSVLYS & SSSKQFSSSRSGGGGGGGSVR & n.d. & $\mathrm{N}$-ter + 42.01 Da, K+34.06 Da & 20,9 & $-2,740$ & 1 & 2004,0 & K1C10_MOUSE & A2A513_MOUSE $\mathrm{K}$ & Keratin, type I cytoskeletal 10; & $\begin{array}{l}56 \text { kDa cytokeratin;Cytokeratin-10;Keratin, type } \\
\text { I cytoskeletal } 59 \text { kDa;Keratin-10; }\end{array}$ \\
\hline M & SVLYSSSSKQFSSSR & n.d. & N-ter $+42.01 \mathrm{Da}, \mathrm{K}+34.06 \mathrm{Da}$ & 84,5 & $-0,463$ & 1 & 1724,9 & K1C10_MOUSE & A2A513_MOUSE & Keratin, type I cytoskeletal 10; & $\begin{array}{l}56 \text { kDa cytokeratin;Cytokeratin-10;Keratin, type } \\
\text { I cytoskeletal } 59 \text { kDa;Keratin-10; }\end{array}$ \\
\hline AQLSELRSEM & ECQNQEYKMLLDIKTR & $-0,3$ & $\begin{array}{l}\text { N-ter + 42.01 Da, K+28.03 Da, } \\
\mathrm{C}+57.02 \mathrm{Da}\end{array}$ & 24,7 & $-7,423$ & 1 & 2166,1 & K1C13_MOUSE & & Keratin, type I cytoskeletal 13; & 47 kDa cytokeratin;Cytokeratin-13;Keratin-13; \\
\hline EAQLSELRSE & MECQNQEYKMLLDIKTR & 1,7 & $\begin{array}{l}\text { N-ter + 42.01 Da, K+28.03 Da, } \\
\mathrm{C}+57.02 \mathrm{Da}\end{array}$ & 24,4 & $-5,869$ & 1 & 2297,1 & K1C13_MOUSE & & Keratin, type I cytoskeletal 13; & 47 kDa cytokeratin;Cytokeratin-13;Keratin-13; \\
\hline KILEATTDNN & RIILEIDNAR & n.d. & $\mathrm{N}$-ter $+42.01 \mathrm{Da}$ & 16,3 & $-0,080$ & 1 & 1253,7 & K1C13_MOUSE & & Keratin, type I cytoskeletal 13; & 47 kDa cytokeratin;Cytokeratin-13;Keratin-13; \\
\hline $\begin{array}{l}\text { DLTRMLAEMR } \\
\text { OYEKMAEKNR }\end{array}$ & $\begin{array}{l}\text { EQYEAIAEKNR } \\
\text { KDARETS }\end{array}$ & n.d. & $\mathrm{N}$-ter + 42.01 Da, K +34.06 Da & 20,5 & $\begin{array}{r}-2,380 \\
8,207\end{array}$ & 1 & 1425,7 & K1C15_MOUSE & K1C13_MOUSE & Keratin, type I cytoskeletal 15; & Cytokeratin-15; Keratin-15; \\
\hline $\begin{array}{l}\text { QYYERAAEKNR } \\
\text { KNKILVATVD }\end{array}$ & $\begin{array}{l}\text { KDAEDWFFSKAEELNR } \\
\text { NASILLOIDNR }\end{array}$ & $\begin{array}{l}\text { n.d. } \\
\text { n.d. }\end{array}$ & $\begin{array}{l}\text { N-ter + 42.01 Da, K+34.06 Da } \\
\text { N-ter + 42.01 Da }\end{array}$ & $\begin{array}{l}21,0 \\
24,3\end{array}$ & $\begin{array}{l}8,227 \\
-0,803\end{array}$ & $\begin{array}{l}1 \\
1\end{array}$ & $\begin{array}{l}2124,1 \\
136,7\end{array}$ & $\begin{array}{l}\text { K1C17_MOUSE } \\
\text { K1C17 MOUSE }\end{array}$ & & $\begin{array}{l}\text { Keratin, type I cytoskeletal 17; } \\
\text { Keratin, type I cytoskeletal 17; }\end{array}$ & $\begin{array}{l}\text { Cytokeratin-17;Keratin-17; } \\
\text { Cytokeratin-17;Keratin-17; }\end{array}$ \\
\hline EDLKNKILVA & TVDNASILLQIDNAR & n.d. & N-ter + 42.01 Da & 24,7 & $-0,830$ & 1 & 1683,9 & K1C17_MOUSE & & 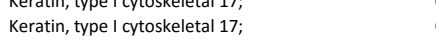 & 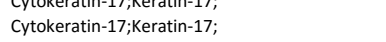 \\
\hline TKSAERDAE & TTLTELRR & n.d. & $\mathrm{N}$-ter + 42.01 Da & 22,7 & $-0,290$ & 1 & 1030,6 & K1C18_MOUSE & & Keratin, type I cytoskeletal 18; & Cytokeratin endo B;Cytokeratin-18;Keratin-18; \\
\hline RTKFETEHAL & RLSVEADINGLR & n.d. & $\mathrm{N}$-ter $+42.01 \mathrm{Da}$ & 20,6 & $-6,490$ & 1 & 1383,7 & K1C19_MOUSE & & Keratin, type I cytoskeletal 19; & Cytokeratin-19;Keratin-19; \\
\hline & NSSHSFNQTYSASVHSLGSTR & n.d. & $\mathrm{N}$-ter + 42.01 Da & 34,4 & $-0,123$ & 1 & 2439,1 & K1C23_MOUSE & & Keratin, type I cytoskeletal 23; & Cytokeratin-23; Keratin-23; \\
\hline KKDVDCAYLR & KSDLEANAEALTQETDFLR & n.d. & $\mathrm{N}$-ter + 42.01 Da, K+34.06 Da & 19,1 & 2,243 & 1 & 2226,1 & KRT81_MOUSE & KRT83_MOUSE & $\begin{array}{l}\text { Keratin, type II cuticular Hb1 } \\
\text { \{ECO:0000250|UniProtkB:Q14533\}; }\end{array}$ & $\begin{array}{l}\text { Keratin-81 } \\
\{\text { ECO:0000303 | PubMed:12399393\}; Type II hair } \\
\text { keratin Hb1; }\end{array}$ \\
\hline VDSLKSDQSR & MDSELKNMQDLVEEYR & 0,4 & $\mathrm{~N}$-ter + 42.01 Da, K+34.06 Da & 21,6 & $-0,722$ & 1 & 2075,0 & K2C1_MOUSE & & Keratin, type II cytoskeletal 1; & $\begin{array}{l}67 \text { kDa cytokeratin;Cytokeratin-1; Keratin- } \\
\text { 1; Type-Il keratin Kb1; }\end{array}$ \\
\hline KKLLEGEEIR & MSGECTPNVSVSVSTSHTSMSGSSSR & 0,7 & N-ter + 42.01 Da, C+57.02 Da & 25,6 & 5,734 & 1 & 2700,2 & K2C1_MOUSE & & Keratin, type II cytoskeletal 1; & $\begin{array}{l}\text { 67 kDa cytokeratin;Cytokeratin-1;keratin- } \\
\text { 1; ;ype-II keratin Kb1; }\end{array}$ \\
\hline KAQYDSICQR & SKAEAETFYQSKYEELQITAGKHGDSVR & n.d. & $\mathrm{N}-\operatorname{ter}+42.01 \mathrm{Da}, \mathrm{K}+28.03 \mathrm{Da}$ & 22,4 & 3,603 & 1 & 3297,7 & K2C1_MOUSE & & Keratin, type II cytoskeletal 1; & $\begin{array}{l}\text { 67 kDa cytokeratin;Cytokeratin-1; Keratin- } \\
\text { 1; ;ype-II keratin Kb1; }\end{array}$ \\
\hline SGECTPNVSV & SVSTSHTSMSGSSSR & n.d. & $\mathrm{N}$-ter $+42.01 \mathrm{Da}$ & 37,9 & 1,233 & 1 & 1538,7 & K2C1_MOUSE & & Keratin, type II cytoskeletal 1; & $\begin{array}{l}\text { 67 kDa cytokeratin;Cytokeratin-1; Keratin- } \\
\text { 1; Type-II keratin Kb1; }\end{array}$ \\
\hline NMQDLVEEYR & TKYEDEINKR & n.d. & $\mathrm{N}$-ter + 42.01 Da, K+28.03 Da & 21,4 & 0,143 & 1 & 1392,7 & K2C1_MOUSE & & Keratin, type II cytoskeletal 1; & $\begin{array}{l}67 \text { KDa cytokeratin;Cytokeratin-1;Keratin- } \\
\text { 1;Type-II keratin Kb1; }\end{array}$ \\
\hline TAGKHGDDLK & NSKMEISELNR & $-0,3$ & $\mathrm{~N}$-ter + 42.01 Da, K+28.03 Da & 20,7 & $-1,725$ & 1 & 1389,7 & K2C1B_MOUSE & & Keratin, type II cytoskeletal 1b; & $\begin{array}{l}\text { Cytokeratin-1B;Embryonic type II keratin- } \\
\text { 1;Keratin-77;Type-II keratin Kb39; }\end{array}$ \\
\hline AGKHGDDLKN & SKMEISELNR & 3,7 & N-ter + 42.01 Da, K+28.03 Da & 22,7 & $-7,122$ & 1 & 1275,6 & K2C1B_MOUSE & & Keratin, type II cytoskeletal 1b; & $\begin{array}{l}\text { Cytokeratin-1B;Embryonic type II keratin- } \\
\text { 1; keratin-77; ;ype-II keratin Kb39; }\end{array}$ \\
\hline M & SRQSSVSFR & n.d. & $\mathrm{N}$-ter + 42.01 Da & 33,4 & $-1,368$ & 1 & 1094,6 & K2C5_MOUSE & & Keratin, type II cytoskeletal 5; & Cytokeratin-5; Keratin-5;Type-II keratin Kb5; \\
\hline NMQDTVEDYK & SKYEDEINKR & n.d. & N-ter + 42.01 Da, K+34.06 Da & 28,9 & $-2,655$ & 1 & 1390,8 & K2C6A_MOUSE & K2C6B_MOUSE & Keratin, type II cytoskeletal 6A; & Cytokeratin-6A;Keratin-6-alpha;Keratin-6A; \\
\hline QINFYRMIYE & AELSQMQNQVSDTSVVISMDNNR & n.d. & $\mathrm{N}$-ter $+42.01 \mathrm{Da}$ & 17,8 & $-1,034$ & 1 & 2607,2 & K2C75_MOUSE & & Keratin, type II cytoskeletal 75; & $\begin{array}{l}\text { Cytokeratin-75;Keratin-6 hair follicle; Keratin- } \\
\text { 75;Type II keratin-KGhf;TYpe-II keratin Kb18; }\end{array}$ \\
\hline IQLEEDITKK & MDKDGEALKAAQAELKEAR & $-0,6$ & $\mathrm{~N}$-ter + 42.01 Da, K+28.03 Da & 24,0 & 0,545 & 1 & 2199,2 & KT222_MOUSE & & Keratin-like protein KRT222; & Keratin-222; \\
\hline M & ADPAECNIKVMCR & 0,1 & $\begin{array}{l}\text { N-ter + } 42.01 \text { Da, } \mathrm{K}+28.03 \mathrm{Da}, \\
\mathrm{C}+57.02 \mathrm{Da}\end{array}$ & 53,7 & $-0,184$ & 1 & 1632,7 & KINH_MOUSE & E9QAK5_MOUSE I & Kinesin-1 heavy chain; & $\begin{array}{l}\text { Conventional kinesin heavy chain;Ubiquitous } \\
\text { kinesin heavy chain; } \\
\text { HIV-1 Ree-binding protein } 2 \text { homolo. KRR-R }\end{array}$ \\
\hline M & ATSAEAPAKEAQKR & n.d. & $\mathrm{N}$-ter + 42.01 Da, K+34.06 Da & 19,0 & 4,841 & 1 & 1566,9 & KRR1_MOUSE & G3UZAO_MOUSE & KRR1 small subunit processome cor & $\begin{array}{l}\text {; HIV-1 Rev-binding protein } 2 \text { homolog;KRR-R } \\
\text { motif-containing protein 1; }\end{array}$ \\
\hline
\end{tabular}




\begin{tabular}{|c|c|c|c|c|c|c|c|c|c|c|c|}
\hline Non Prime Site & Prime Site & $\begin{array}{l}\text { Fold change of } \\
\text { XPRESS ratio } \\
\text { (Adam17 } \text { AKC } / \text { WT) }^{\text {(A) }}\end{array}$ & Modifications & Hyperscore & $\begin{array}{l}\text { Mass error } \\
\text { in ppm }\end{array}$ & Charge & $\begin{array}{c}\text { Precursor } \\
\text { neutral mass } \\
\text { in Da }\end{array}$ & Uniprot ID 1 & Uniprot ID 2 & Protein Name 1 & Protein Name 2 \\
\hline $\bar{M}$ & $\begin{array}{l}\text { ASLPPHAGPATPLSPTR } \\
\end{array}$ & n.d. & 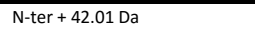 & 62,7 & $-0,117$ & 1 & 1710,9 & LMNB2_MOUSE & & $\begin{array}{l}\text { Lamin-B2; } \\
\end{array}$ & \\
\hline M & ATQVEPLLPAGAPLLQAEEHGLAR & n.d. & $\mathrm{N}$-ter $+42.01 \mathrm{Da}$ & 58,7 & $-0,634$ & 1 & 2522,4 & LARP1_MOUSE & J3QNB1_MOUSE & La-related protein 1; & La ribonucleoprotein domain family member 1 ; \\
\hline MT & SDQDAKVVAEPQAQR & n.d. & $\mathrm{N}$-ter + 42.01 Da, K +34.06 Da & 22,7 & 1,105 & 1 & 1716,9 & LAR4B_MOUSE & & La-related protein 4B; & $\begin{array}{l}\text { La ribonucleoprotein domain family member } \\
\text { 4B;la ribonucleoprotein domain family } \\
\text { member 5;Larelated protein } 5 \\
\text { La ribonucleoprotein domain family member }\end{array}$ \\
\hline M & TSDQDAKVVAEPQAQR & n.d. & $\mathrm{N}$-ter + 42.01 Da, K +34.06 Da & 41,7 & 7,428 & 1 & 1949,0 & LAR4B_MOUSE & & La-related protein $4 B$; & $\begin{array}{l}\text { 4B;:La ribonucleoprotein domain family } \\
\text { member 5;la-related protein 5; }\end{array}$ \\
\hline M & ETENQKTMEESTKR & n.d. & N-ter + 42.01 Da, K+34.06 Da & 28,6 & 0,768 & 1 & 1951,0 & LARP7_MOUSE & & La-related protein $7 ;$ & La ribonucleoprotein domain family member 7 ; \\
\hline M & EIPPTHYAASR & n.d. & $\mathrm{N}$-ter + 42.01 Da & 44,4 & 0,918 & 1 & 1413,7 & LXN_MOUSE & & Latexin; & $\begin{array}{l}\text { Endogenous carboxypeptidase inhibitor; Tissue } \\
\text { carboxypeptidase inhibitor; }\end{array}$ \\
\hline M & AELTNYKDAASNR & n.d. & N-ter + 42.01 Da, K+34.06 Da & 51,9 & 0,588 & 1 & 1527,8 & LUZP1_MOUSE & E9Q9L__MOUSE & Leucine zipper prot & Leucine zipper motif-containing protein; \\
\hline M & AIVHTLPVPLEPAR & n.d. & $\mathrm{N}$-ter $+42.01 \mathrm{Da}$ & 36,6 & $-0,257$ & 1 & 1553,9 & LZTS2_MOUSE & & $\begin{array}{l}\text { Leucine zipper putative tumor suppressor } 2 \\
\text { \{ECO:0000255|HAMAP-Rule:MF_03026\}; }\end{array}$ & $\begin{array}{l}\text { Protein LAPSER1 \{ECO:0000255|HAMAP- } \\
\text { Rule:MF__3026\}; }\end{array}$ \\
\hline M & AELGLNEHHQNEVINYMR & n.d. & $\mathrm{N}$-ter + 42.01 Da & 52,3 & $-1,854$ & 1 & 2208,0 & LZTL1_MOUSE & E9Q9P1_MOUSE & Leucine zipper transcription factor-like protein 1 ; & \\
\hline M & TSPEGAQNKEIDCLSPEAQR & n.d. & $\begin{array}{l}\text { N-ter + 42.01 Da, } \mathrm{K}+34.06 \mathrm{Da}, \\
\mathrm{C}+57.02 \mathrm{Da}\end{array}$ & 37,1 & 0,173 & 1 & 2305,1 & LRRF1_MOUSE & E9Q9T1_MOUSE & Leucine-rich repeat flightless-interacting protein 1 ; & FLL-LRR-associated protein 1;H186 FLAP; \\
\hline M & FQAAGAAQATPSHEAKGSSGSSTVQR & n.d. & $\mathrm{N}$-ter + 42.01 Da, K+34.06 Da & 20,8 & $-0,876$ & 1 & 2737,3 & LIMD2_MOUSE & & LIM domain-containing protein 2; & \\
\hline $\begin{array}{l}\text { M } \\
\text { ISLAPOFSSS }\end{array}$ & SHPSWLPPKSTGEPLGHVPAR & 0,1 & N-ter + 42.01 Da, K+28.03 Da & 38,9 & $-0,990$ & 1 & 2319,2 & $\begin{array}{l}\text { LPP_MOUSE } \\
\text { LPMC9MOUSE }\end{array}$ & & Lipoma-preferred partner homolog; & \\
\hline M & $\begin{array}{l}\text { GQQQAASPPASTASTR } \\
\text { EVHELFR }\end{array}$ & $\begin{array}{l}\text { n.d. } \\
\text { n.d. }\end{array}$ & $\begin{array}{l}\mathrm{N} \text {-ter + 42.01 Da } \\
\mathrm{N}-\mathrm{ter}+42.01 \mathrm{Da}\end{array}$ & $\begin{array}{l}21,8 \\
20,4\end{array}$ & $\begin{array}{l}7,109 \\
-0,272\end{array}$ & $\begin{array}{l}1 \\
1\end{array}$ & $\begin{array}{l}1642,8 \\
1101,5\end{array}$ & $\begin{array}{l}\text { ARMC9_MOUSE } \\
\text { ACSLI_MOUSE }\end{array}$ & D3Z041_MOUSE & $\begin{array}{l}\text { LisH domain-containing protein ARMC9; } \\
\text { Long-chain-fatty-acid--CoA ligase 1; }\end{array}$ & $\begin{array}{l}\text { Long-chain acyl-CoA synthetase } 1 ; \\
\text { Jmjc domain-containing histone demethylation }\end{array}$ \\
\hline M & ADAAASPVGKR & 0,0 & $\mathrm{~N}$-ter + 42.01 Da, K+28.03 Da & 18,5 & $-0,180$ & 1 & 1111,6 & KDM3B_MOUSE & B9EKS2_MOUSE & Lysine-specific demethylase 3B; & $\begin{array}{l}\text { protein 2B;Jumonji domain-containing protein } \\
\text { 1B; }\end{array}$ \\
\hline M & ATLQESEVKVDGEQKLSKNELKR & 0,2 & $\mathrm{~N}$-ter + 42.01 Da, K+28.03 Da & 17,2 & 6,780 & 1 & 2782,6 & SYK_MOUSE & & Lysine--tRNA ligase; & Lysyl-tRNA synthetase; \\
\hline M & ADTAPQLKR & 0,2 & $\mathrm{~N}$-ter + 42.01 Da, K+28.03 Da & 30,0 & 0,841 & 1 & 1068,6 & DCPS_MOUSE & Q3TBW9_MOUSE & m7GpppX diphosphatase; & $\begin{array}{l}\text { DCS-1;Decapping scavenger enzyme; Hint- } \\
\text { related 7meGMP-directed hydrolase;Histidine } \\
\text { triad nucleotide-binding protein 5;Histidine } \\
\text { triad protein member 5;;cavenger mRNA- } \\
\text { decapping enzyme DcpS; }\end{array}$ \\
\hline M & YTPIPQSGSPFPASVQDPGLHIWR & n.d. & $\mathrm{N}$-ter + 42.01 Da & 32,0 & 0,460 & 1 & 2822,4 & CAPG_MOUSE & Q99LB4_MOUSE & Macrophage-capping protein; & $\begin{array}{l}\text { Actin regulatory protein CAP-G;Actin-capping } \\
\text { protein GCAP39;Myc basic motif homolog 1; }\end{array}$ \\
\hline M & AADDKVAILTDDEEEQKR & n.d. & N-ter + 42.01 Da, K+34.06 Da & 36,8 & $-4,865$ & 1 & 2155,1 & MFSD6_MOUSE & D3Z183_MOUSE & $\begin{array}{l}\text { Major facilitator superfamily domain-containing } \\
\text { protein 6; }\end{array}$ & Macrophage MHC class I receptor 2; \\
\hline $\begin{array}{l}M \\
M\end{array}$ & $\begin{array}{l}\text { DGETAGEKGSLVPPPGALGGSALGGAPAPGVR } \\
\text { SKSFOOSSLGR }\end{array}$ & $\begin{array}{c}\text { n.d. } \\
0,1\end{array}$ & $\mathrm{~N}$-ter + 42.01 Da, $\mathrm{K}+34.06 \mathrm{Da}$ & $\begin{array}{l}23,5 \\
31,0\end{array}$ & $\begin{array}{r}0,492 \\
-1,081\end{array}$ & $\begin{array}{l}1 \\
1\end{array}$ & $\begin{array}{l}3048,6 \\
1293,7\end{array}$ & $\begin{array}{l}\text { MAPK3_MOUSE } \\
\text { MATR3 MOUSE }\end{array}$ & & MAP kinase-activated protein kinase 3; & \\
\hline M & $\begin{array}{l}\text { SKSFQQSSLGR } \\
\text { AAGDGDVKLSTLGSGGESGGDGSPGGAGATAAR }\end{array}$ & n.d. & $\begin{array}{l}\text { N-ter + 42.01 Da, K+28.03 Da } \\
\text { N-ter + 42.01 Da, K +34.06 Da }\end{array}$ & 71,0 & $\begin{array}{l}-1,001 \\
-0,174\end{array}$ & 1 & 2878,4 & $\begin{array}{l}\text { MAIR__MOUSE } \\
\text { PGRC2_MOUSE }\end{array}$ & & $\begin{array}{l}\text { Matrin-3; } \\
\text { Membrane-associated progesterone receptor }\end{array}$ & \\
\hline M & ASGSAGKPTGEAASPAPGSAVGGASSQPR & n.d. & N-ter + 42.01 Da, K+34.06 Da & 19,7 & $-0,618$ & 1 & 2585,3 & MESD1_MOUSE & & $\begin{array}{l}\text { component } 2 \\
\text { Mesoderm development candidate 1; }\end{array}$ & \\
\hline M & SAFNLLHLVTKSQPVAPR & n.d. & $\mathrm{N}$-ter + 42.01 Da, K+34.06 Da & 43,9 & $-5,593$ & 1 & 2053,2 & MSRB__MOUSE & D3YUC9_MOUSE & Methionine-R-sulfoxide reductase $B 3$, mitochondrial; & \\
\hline MLSPAM & SFLLSFPPPGSADGLR & n.d. & $\mathrm{N}$-ter + 42.01 Da, K+34.06 Da & 24,2 & 0,228 & 1 & 1751,0 & ICLN_MOUSE & Q923F1_MOUSE & Methylosome subunit plCIn; & $\begin{array}{l}\text { Chloride channel, nucleotide sensitive } \\
\text { 1A;Chloride conductance regulatory protein } \\
\text { ICln; } \text {;Chloride ion current inducer protein; }\end{array}$ \\
\hline M & SGCQAQGDCCSRPCGAQDKEHPR & n.d. & $\begin{array}{l}\text { N-ter + 42.01 Da, K+34.06 Da, } \\
\mathrm{C}+57.02 \mathrm{Da}\end{array}$ & 19,6 & $-4,671$ & 1 & 2736,1 & MTNB_MOUSE & & $\begin{array}{l}\text { Methythioribulose-1-phosphate dehydratase } \\
\text { \{ECO:0000255|HAMAP-Rule:MF_03116\}; }\end{array}$ & $\begin{array}{l}\text { APAF1-interacting protein } \\
\text { \{ECO:0000255|HAMAP- } \\
\text { Rule:MF_03116\};:Monocyte/macrophage } \\
\text { protein 19; }\end{array}$ \\
\hline M & SVPSALMKQPPIQSTAGAVPVR & 0,1 & N-ter + 42.01 Da, K+28.03 Da & 41,7 & $-4,033$ & 1 & 2303,3 & MFAP1_MOUSE & & Microfibrillar-associated protein 1 ; & \\
\hline $\begin{array}{l}\text { M } \\
\text { LRSNSTGARH }\end{array}$ & $\begin{array}{l}\text { ADLSLVDALTEPPPEIEGEIKR } \\
\text { STPRPLDAGR }\end{array}$ & $\begin{array}{l}0,1 \\
\text { n.d. }\end{array}$ & $\begin{array}{l}\text { N-ter + 42.01 Da, K+28.03 Da } \\
\text { N-ter + 42.01 Da }\end{array}$ & $\begin{array}{l}55,5 \\
24,2\end{array}$ & $\begin{array}{r}-1,258 \\
8,359\end{array}$ & $\begin{array}{l}1 \\
1\end{array}$ & $\begin{array}{l}2461,3 \\
1110,6\end{array}$ & $\begin{array}{l}\text { MAP4_MOUSE } \\
\text { MAST3 MOUSE }\end{array}$ & $\begin{array}{l}\text { Q78TF3_MOUSE } \\
\text { E9Q855 MOUSE }\end{array}$ & $\begin{array}{l}\text { Microtubule-associated protein } 4 \text {; } \\
\text { Microtubule-associated serine/threonine-protein }\end{array}$ & \\
\hline EHGAAWQPRI & MEEKALEVYDLIR & n.d. & N-ter + 42.01 Da, K+34.06 Da & 25,3 & $-0,712$ & 1 & 1683,9 & FA96A_MOUSE & & $\begin{array}{l}\text { kinase 3; } \\
\text { MIP18 family protein FAM96A; }\end{array}$ & \\
\hline & AKSSLGQDSDSTAAVAVLKR & 0,1 & $\mathrm{~N}$-ter + $42.01 \mathrm{Da}, \mathrm{K}+28.03 \mathrm{Da}$ & 28,1 & 4,705 & 1 & 2101,2 & MITD1_MOUSE & & MIT domain-containing protein $1 ;$ & \\
\hline M & EAVLNELVSVEDLKNFER & n.d. & N-ter + 42.01 Da, K+34.06 Da & 43,3 & 3,242 & 1 & 2310,2 & FIS1_MOUSE & & Mitochondrial fission 1 protein; & $\begin{array}{l}11 ; \\
11 ;\end{array}$ \\
\hline
\end{tabular}




\begin{tabular}{|c|c|c|c|c|c|c|c|c|c|c|c|}
\hline Non Prime Site & Prime Site & 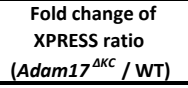 & Modifications & Hyperscore & $\begin{array}{c}\text { Mass error } \\
\text { in ppm }\end{array}$ & Charge & $\begin{array}{c}\text { Precursor } \\
\text { neutral mass } \\
\text { in Da }\end{array}$ & Uniprot ID 1 & Uniprot ID 2 & Protein Name 1 & Protein Name 2 \\
\hline $\bar{M}$ & EANVTIPIWQNKPHGAAR & n.d. & N-ter + 42.01 Da, K+34.06 Da & 22,2 & $-4,749$ & 1 & 2208,2 & MFR1L_MOUSE & $\begin{array}{l}\text { D3YW54_MOUSE } \\
\end{array}$ & Mitochondrial fission regulator 1-like; & \\
\hline M & SQERPTFYR & n.d. & $\mathrm{N}$-ter + 42.01 Da & 26,9 & $-1,304$ & 1 & 1224,6 & MK14_MOUSE & B2KF35_MOUSE & Mitogen-activated protein kinase 14; & $\begin{array}{l}\text { CRK1;Mitogen-activated protein kinase p38 } \\
\text { alpha; }\end{array}$ \\
\hline M & EAPLRPAADILR & n.d. & $\mathrm{N}$-ter + 42.01 Da & 21,8 & 1,738 & 1 & 1493,8 & M4K5_MOUSE & E9PX30_MOUSE & $\begin{array}{l}\text { Mitogen-activated protein kinase kinase kinase kinase } \\
\text { 5; }\end{array}$ & MAPK/ERK kinase kinase kinase 5; \\
\hline M & SSILPFTPPVVKR & n.d. & N-ter + 42.01 Da, K+34.06 Da & 26,0 & 2,899 & 1 & 1515,9 & SMAD2_MOUSE & E9Q3MO_MOUSE & Mothers against decapentaplegic homolog 2; & Mad-related protein 2;SMAD family member 2; \\
\hline MTSM & ASLFSFTSPAVKR & n.d. & N-ter + 42.01 Da, K+34.06 Da & 15,7 & 3,092 & 1 & 1485,9 & SMAD5_MOUSE & & Mothers against decapentaplegic homolog 5 ; & Dwarfin-c;SMAD family member 5 ; \\
\hline M & AENNAQNKAKLISETR & 0,2 & $\mathrm{~N}$-ter + 42.01 Da, K+28.03 Da & 47,9 & $-3,498$ & 1 & 1884,0 & MSPD2_MOUSE & B1AU74_MOUSE & Motile sperm domain-containing protein 2; & RG7MT1;mRNA (guanine-N(7)-)- \\
\hline M & EGSAKASVASDPESPPGGNEPAAASGQR & $-0,3$ & $\mathrm{~N}$-ter + $42.01 \mathrm{Da}, \mathrm{K}+28.03 \mathrm{Da}$ & 27,8 & $-0,035$ & 1 & 2824,3 & MCES_MOUSE & D3Z4K1_MOUSE & mRNA cap guanine-N7 methyltransferase; & $\begin{array}{l}\text { methyltransferase;mRNA cap } \\
\text { methyltransferase; }\end{array}$ \\
\hline M & AAAAAGGLPGKGHDISLALLR & n.d. & N-ter + 42.01 Da, K+34.06 Da & 40,5 & $-2,105$ & 1 & 1992,1 & DCP1B_MOUSE & B9EIXO_MOUSE & mRNA-decapping enzyme 1B; & \\
\hline GRPPKTRRRA & EEDGEHER & n.d. & N-ter + 42.01 Da & 14,4 & 8,817 & 1 & 1041,4 & MINT_MOUSE & AZADBO_MOUSE & Msx2-interacting protein; & $\begin{array}{l}\text { SMART/HDAC1-associated repressor } \\
\text { protein;;PEN homolog; }\end{array}$ \\
\hline AEDGGPGPSP & RSFHAAAYVPAGR & n.d. & N-ter + $42.01 \mathrm{Da}$ & 21,2 & $-6,428$ & 1 & 1443,7 & MEGF8_MOUSE & & $\begin{array}{l}\text { Multiple epidermal growth factor-like domains } \\
\text { protein 8; }\end{array}$ & Epidermal growth factor-like protein 4; \\
\hline $\begin{array}{l}\text { M } \\
\text { VRQFGQQDWK }\end{array}$ & AEMKSPTKAEPATPAEAAQSDR & n.d. & $\mathrm{N}$-ter + 42.01 Da, $\mathrm{K}+34.06 \mathrm{Da}$ & 24,5 & 1,459 & 1 & 2395,2 & MBB1A_MOUSE & & Myb-binding protein 1A; & Myb-binding protein of $160 \mathrm{kDa}$; \\
\hline $\begin{array}{l}\text { VROFGQQDWK } \\
\text { M }\end{array}$ & $\begin{array}{l}\text { FLASHFPNR } \\
\text { ADADKSEAAAGDDGSQQQPAEPR }\end{array}$ & $\begin{array}{l}\text { n.d. } \\
\text { n.d. }\end{array}$ & $\begin{array}{l}\text { N-ter + 42.01 Da } \\
\text { N-ter + 42.01 Da, K +34.06 Da }\end{array}$ & $\begin{array}{ll}15,1 \\
63,9\end{array}$ & $\begin{array}{l}-1,413 \\
1045\end{array}$ & 1 & $\begin{array}{l}1129,6 \\
23891\end{array}$ & $\begin{array}{l}\text { MYBB_MOUSE } \\
\text { MYEF2_MOUSE }\end{array}$ & A2ATP5 MOUSE & $\begin{array}{l}\text { Myb-related protein B; } \\
\text { Myelin expression factor 2; }\end{array}$ & Myb-like protein 2; \\
\hline DDCQVLLSCK & 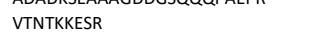 & 10,6 & $\mathrm{~N}$-ter + 42.01 Da, K+28.03 Da & $\begin{array}{l}0,3 \\
14,3\end{array}$ & $\begin{array}{l}-9,728 \\
-9,045\end{array}$ & 1 & 1159,7 & MYOM3_MOUSE & 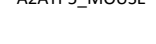 & Myomesin-3; & Myomesin family member 3 ; \\
\hline QGNTELINDR & LKKANLQIDQINTDLNLER & $-0,7$ & $\mathrm{~N}$-ter + 42.01 Da, K+28.03 Da & 20,9 & 4,916 & 1 & 2336,3 & MYH9_MOUSE & & Myosin-9; & $\begin{array}{l}\text { Cellular myosin heavy chain, type A;Myosin } \\
\text { heavy chain 9;Myosin heavy chain, non-muscle } \\
\text { Ila;Non-muscle myosin heavy chain A;Non- } \\
\text { muscle myosin heavy chain Ila; }\end{array}$ \\
\hline NLPLFAFYNE & EKFNVDGWTVYNPVEEYR & 10,6 & $\mathrm{~N}$-ter + 42.01 Da, K +28.03 Da & 18,2 & $-0,043$ & 1 & 2314,1 & MTM1_MOUSE & B1AW21_MOUSE & Myotubularin; & $\begin{array}{l}\text { Phosphatidylinositol-3,5-isphosphate 3- } \\
\text { phosphatase; } 9 \text { hosphatidylinositol-3-phosphate } \\
\text { phosphatase; }\end{array}$ \\
\hline EEKFNVDGWT & UYNPVEEYR & n.d. & $\mathrm{N}$-ter $+42.01 \mathrm{Da}$ & 17,6 & 1,816 & 1 & 1209,6 & MTM1_MOUSE & B1AW21_MOUSE & Myotubularin; & $\begin{array}{l}\text { Phosphatidylinositol-3,5-5isphosphate 3- } \\
\text { phosphatase; } \\
\text { phosposphatidylinositol-3-phosphate }\end{array}$ \\
\hline M & AGLGHPSAFGR & n.d. & $\mathrm{N}$-ter $+42.01 \mathrm{Da}$ & 37,0 & $-0,270$ & 1 & 1110,6 & DDAH1_MOUSE & & $\begin{array}{l}N(G), N(G) \text {-dimethylarginine dimethylaminohydrolase } \\
1 ;\end{array}$ & DDAHI;Dimethylargininase-1; \\
\hline M & AFPKKKLR & n.d. & N-ter + 42.01 Da, K+34.06 Da & 35,9 & $-1,059$ & 1 & 1130,8 & NPL_MOUSE & & $\mathrm{N}$-acetylneuraminate lyase; & $\begin{array}{l}\text { N-acetylneuraminate pyruvate-Iyase;N- } \\
\text { acetylneuraminic acid aldolase;Sialate } \\
\text { lyase;Sialate-pyruvate Iyase;Sialic acid } \\
\text { aldolase;Sialic acid lyase; }\end{array}$ \\
\hline M & ELVEVLKR & 0,0 & N-ter + 42.01 Da, K+28.03 Da & 29,2 & 1,263 & 1 & 1185,7 & NDUAC_MOUSE & & $\begin{array}{l}\text { NADH dehydrogenase [ubiquinone] } 1 \text { alpha } \\
\text { subcomplex subunit 12; }\end{array}$ & $\begin{array}{l}\text { Complex I-B17.2;NADH-ubiquinone } \\
\text { oxidoreductase subunit B17.2; }\end{array}$ \\
\hline M & AASKVKQDMPPPGGYGPIDYKR & 0,0 & $\mathrm{~N}$-ter + 42.01 Da, K+28.03 Da & 25,2 & 2,875 & 1 & 2500,3 & NDUAD_MOUSE & & $\begin{array}{l}\text { NADH dehydrogenase [ubiquinone] } 1 \text { alpha } \\
\text { subcomplex subunit 13; }\end{array}$ & $\begin{array}{l}\text { Cell death regulatory protein GRIM-19;Complex } \\
\text { I-B16.6;Gene associated with retinoic and } \\
\text { interferon-induced mortality } 19 \text { protein;NADH- } \\
\text { ubiquinone oxidoreductase B16.6 subunit; }\end{array}$ \\
\hline M & AGLLKKTTGLVGLAVCDTPHER & 0,2 & $\begin{array}{l}\text { N-ter + 42.01 Da, K+28.03 Da, } \\
\text { C+57.02 Da }\end{array}$ & 49,4 & $-1,026$ & 1 & 2433,3 & NDUA5_MOUSE & & $\begin{array}{l}\text { NADH dehydrogenase [ubiquinone] } 1 \text { alpha } \\
\text { subcomplex subunit } 5 \text {; }\end{array}$ & $\begin{array}{l}\text { Complex I subunit B13;Complex I-13kD-B;NADH- } \\
\text { ubiquinone oxidoreductase } 13 \text { kDa-B subunit; }\end{array}$ \\
\hline M & SGSKYKPAPLATLPSTLDPAEYDVSPETR & n.d. & N-ter + 42.01 Da, K+34.06 Da & 38,9 & $-0,624$ & 1 & 3199,7 & NDUB4_MOUSE & & $\begin{array}{l}\text { NADH dehydrogenase [ubiquinone] } 1 \text { beta } \\
\text { subcomplex subunit } 4 \text {; }\end{array}$ & $\begin{array}{l}\text { Complex I-B15;NADH-ubiquinone } \\
\text { oxidoreductase B15 subunit; }\end{array}$ \\
\hline M & SGYTPDEKLR & n.d. & N-ter + 42.01 Da, K+34.06 Da & 36,3 & 4,024 & 1 & 1240,7 & NDUB6_MOUSE & A2AP32_MOUSE & $\begin{array}{l}\text { NADH dehydrogenase [ubiquinone] } 1 \text { beta } \\
\text { subcomplex subunit } 6 \text {; }\end{array}$ & $\begin{array}{l}\text { Complex I-B17;NADH-ubiquinone } \\
\text { oxidoreductase B17 subunit; }\end{array}$ \\
\hline M & LTLASKLKR & n.d. & $\mathrm{N}$-ter + 42.01 Da, K +34.06 Da & 27,8 & $-0,079$ & 1 & 1269,9 & UB2FA_MOUSE & & NEDD8-conjugating enzyme UBE2F; & $\begin{array}{l}\text { NEDD8 carrier protein UBE2F;NEDD8 protein } \\
\text { ligase UBE2F;NEDD8-conjugating enzyme } \\
\text { 2; ;Ubiquitin-conjugating enzyme E2 F; }\end{array}$ \\
\hline GQWGTICDDG & WTDKHAAVICR & 4,0 & $\begin{array}{l}\text { N-ter + } 42.01 \mathrm{Da}, \mathrm{K}+28.03 \mathrm{Da} \\
\mathrm{C}+57.02 \mathrm{Da}\end{array}$ & 18,5 & 6,369 & 1 & 1425,7 & NETR_MOUSE & & Neurotrypsin; & $\begin{array}{l}\text { Brain-specific serine protease } \\
\text { 3;Motopsin;Serine protease 12; }\end{array}$ \\
\hline M & QSNKAFNLEKQNHTPR & n.d. & N-ter + 42.01 Da, K+34.06 Da & 38,5 & 0,278 & 1 & 2152,2 & NONO_MOUSE & B1AXTO_MOUSE & $\begin{array}{l}\text { Non-POU domain-containing octamer-binding } \\
\text { protein; }\end{array}$ & \\
\hline M & ASVDFKTYVDQACR & n.d. & $\begin{array}{l}\mathrm{N} \text {-ter + 42.01 Da, K+34.06 Da, } \\
\mathrm{C}+57.02 \mathrm{Da}\end{array}$ & 17,5 & $-0,518$ & 1 & 1734,9 & NXT1_MOUSE & & NTF2-related export protein 1 & \\
\hline LFLVIFQRFI & MILTEHLVR & n.d. & N-ter + 42.01 Da & 18,8 & 3,638 & 1 & 1152,6 & NCBP1_MOUSE & & Nuclear cap-binding protein subunit 1; & 80 kDa nuclear cap-binding protein; \\
\hline
\end{tabular}




\begin{tabular}{|c|c|c|c|c|c|c|c|c|c|c|c|}
\hline Non Prime Site & Prime Site & 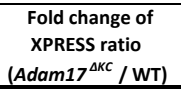 & Modifications & Hyperscore & $\begin{array}{l}\text { Mass error } \\
\text { in ppm }\end{array}$ & Charge & $\begin{array}{c}\text { Precursor } \\
\text { neutral mass } \\
\text { in Da } \\
\end{array}$ & Uniprot ID 1 & Uniprot ID 2 & Protein Name 1 & Protein Name 2 \\
\hline GQDSKSPLGL & YCEQNPVESSVCQSNSR & n.d. & N-ter + 42.01 Da, C+57.02 Da & 15,4 & $-1,101$ & 1 & 2084,9 & NCOA3_MOUSE & A2A468_MOUSE & Nuclear receptor coactivator 3 ; & $\begin{array}{l}\text { Amplified in breast cancer-1 protein } \\
\text { homolog;CGPP-interacting proteininReceptor- } \\
\text { associated coactivator 3;Steroid receptor } \\
\text { coactivator protein 3;Thyroid hormone } \\
\text { receptor activator molecule } 1 \text {; }\end{array}$ \\
\hline MEV & MEGPLNLAHQQSR & n.d. & $\mathrm{N}$-ter + 42.01 Da & 26,8 & 6,038 & 1 & 1521,8 & NRBF2_MOUSE & EOCZ57_MOUSE & Nuclear receptor-binding factor 2; & \\
\hline M & ADEGKSYNEHDDR & n.d. & $\mathrm{N}$-ter + 42.01 Da, K+34.06 Da & 32,1 & 3,532 & 1 & 1610,7 & NXF1_MOUSE & & Nuclear RNA export factor 1; & $\begin{array}{l}\text { Tip-associated protein;Tip-associating } \\
\text { protein;mRNA export factor TAP; } \\
\text { Nuclear-interacting partner of anaplastic }\end{array}$ \\
\hline M & AATSEGPLFAASIEKTWGSVVR & n.d. & N-ter + 42.01 Da, K+34.06 Da & 41,8 & $-1,698$ & 1 & 2352,3 & NIPA_MOUSE & D3ZOB2_MOUSE & Nuclear-interacting partner of ALK; & $\begin{array}{l}\text { lymphoma kinase;Zinc finger C3HC-type } \\
\text { protein 1; }\end{array}$ \\
\hline M & ADPEKQGPAESR & n.d. & N-ter + 42.01 Da, K+34.06 Da & 20,9 & 0,514 & 1 & 1359,7 & NP115_MOUSE & & Nucleosome assembly protein 1-like 5; & \\
\hline QNLIVTQTAL & NWIPSGAPCGSQEGPPPPR & n.d. & $\mathrm{N}$-ter $+42.01 \mathrm{Da}, \mathrm{C}+57.02 \mathrm{Da}$ & 17,1 & 9,033 & 1 & 2045,0 & NUTM1_MOUSE & & NUT family member 1; & Nuclear protein in testis; \\
\hline M & NVGVAHSEVNPNTR & n.d. & $\mathrm{N}$-ter $+42.01 \mathrm{Da}$ & 31,0 & 0,240 & 1 & 1665,8 & ORML1_MOUSE & ORML2_MOUSE & ORM1-like protein 1; & 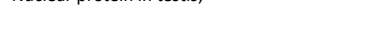 \\
\hline M & SKPPPKPVKPGQVKVFR & n.d. & $\mathrm{N}$-ter $+42.01 \mathrm{Da}, \mathrm{K}+34.06 \mathrm{Da}$ & 30,0 & $-0,338$ & 1 & 2066,4 & OSTF1_MOUSE & 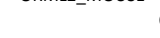 & Osteoclast-stimulating factor 1 ; & SH3 domain protein 3; \\
\hline DIHFTTFDGR & RYTFPATCQYILAKSR & n.d. & $\begin{array}{l}\text { N-ter + 42.01 Da, K+34.06 Da, } \\
\mathrm{C}+57.02 . \mathrm{Da}\end{array}$ & 23,9 & 5,404 & 1 & 2050,1 & OTOG_MOUSE & G3UWC8_MOUSE & Otogelin; & \\
\hline M & ADKEALPKLR & n.d. & $\mathrm{N}$-ter + 42.01 Da, K+34.06 Da & 17,9 & $-4,234$ & 1 & 1249,8 & HTAL__MOUSE & & Oxidoreductase HTATP2; & \\
\hline M & EEAELVKGR & 0,1 & $\mathrm{~N}$-ter + 42.01 Da, K+28.03 Da & 47,5 & $-2,272$ & 1 & 1230,6 & PALMD_MOUSE & Q3UVT7_MOUSE & $\begin{array}{l}\text { Palmdelphin; } \\
\text { Pal }\end{array}$ & \\
\hline M & AEAELHKER & 0,3 & $\mathrm{~N}$-ter + 42.01 Da, K+28.03 Da & 26,9 & $-4,937$ & 1 & 1151,6 & PALM2_MOUSE & G3X9P1_MOUSE & Paralemmin-2; & \\
\hline M & EKPAASTEPQGSRPALGR & 0,2 & $\mathrm{~N}$-ter + 42.01 Da, K+34.06 Da & 39,4 & 1,067 & 1 & 2058,1 & PCTL_MOUSE & E9PVPO_MOUSE & PCTP-like protein; & $\begin{array}{l}\text { 10;Serologically defineding polon cancer antigen } \\
28 \text { homolog;StAR-related lipid transfer protein } \\
10 ; \\
\text { START domain-containing protein }\end{array}$ \\
\hline M & EKPAASTEPQGSR & n.d. & $\mathrm{N}$-ter + 42.01 Da, K+28.03 Da & 40,2 & 0,385 & 1 & 1557,8 & PCTL_MOUSE & G3UY59_MOUSE & PCTP-like protein; & $\begin{array}{l}\text { 10;Serologically defined colon cancer antigen } \\
28 \text { homolog;StAR-related lipid transfer protein } \\
\text { 10; }\end{array}$ \\
\hline M & THSVTLR & n.d. & $\mathrm{N}$-ter $+42.01 \mathrm{Da}$ & 18,6 & $-0,467$ & 1 & 854,5 & PDL4__MOUSE & Q.5SWV3_MOUSE & PDZ and LIM domain protein 4; & LIM protein RLL;Reversion-induced LIM protein; \\
\hline M & ADEEKLPPGWEKR & 0,0 & $\mathrm{~N}$-ter + 42.01 Da, K+28.03 Da & 51,5 & 0,121 & 1 & 1651,9 & PIN1_MOUSE & & $\begin{array}{l}\text { Peptidyl-prolyl cis-trans isomerase NIMA-interacting } \\
1 ;\end{array}$ & Peptidyl-prolyl cis-trans isomerase Pin1; \\
\hline M & SMNKGPTLLDGDLPEQENVLQR & n.d. & $\mathrm{N}$-ter + 42.01 Da, K+34.06 Da & 36,7 & 4,936 & 1 & 2529,3 & PLIN1_MOUSE & & Perilipin-1; & Lipid droplet-associated protein;Perilipin A; \\
\hline M & ENQLTKSVEER & n.d. & $\mathrm{N}$-ter + 42.01 Da, K+34.06 Da & 23,9 & $-0,519$ & 1 & 1538,8 & OCTC_MOUSE & & Peroxisomal carnitine 0-octanoyltransferase; & \\
\hline M & SRPCGLPEPVR & n.d. & N-ter + 42.01 Da, C+57.02 Da & 16,1 & $-0,305$ & 1 & 1308,7 & NUDT7_MOUSE & & Peroxisomal coenzyme A diphosphatase NUDT7; & $\begin{array}{l}\text { Nucleoside diphosphate-linked moiety } \mathrm{X} \text { motif } \\
7 \text {; }\end{array}$ \\
\hline IPITVLapAL & KOYSQTGDLGGLR & 3,7 & $\mathrm{~N}$-ter + 42.01 Da, K +28.03 Da & 15,3 & 2,141 & 1 & 1491,8 & TYSD1_MOUSE & & Peroxisomal leader peptide-processing protease; & Trypsin domain-containing protein 1 \\
\hline M & ADGKAGEEKPEKPQR & $-0,4$ & $\mathrm{~N}$-ter + $42.01 \mathrm{Da}, \mathrm{K}+28.03 \mathrm{Da}$ & 35,8 & 0,057 & 1 & 1764,9 & PCNP_MOUSE & Q3TM89_MOUSE & PEST proteolytic signal-containing nuclear protein; & \\
\hline NQKVVIDHRG & VPKNIHFNPR & n.d. & $\mathrm{N}$-ter + 42.01 Da, K+34.06 Da & 21,7 & $-5,005$ & 1 & 1296,8 & PG2IP_MOUSE & & PGAP2-interacting protein; & $\begin{array}{l}\text { Cell wall biogenesis protein } 43 \mathrm{C} \text {-terminal } \\
\text { homolog: }\end{array}$ \\
\hline EVPKKIQDQS & FGESHIPSR & n.d. & $\mathrm{N}$-ter + 42.01 Da & 17,2 & $-8,858$ & 1 & 1070,5 & PHAR4_MOUSE & & Phosphatase and actin regulator 4; & $\begin{array}{l}\text { Protein Humpty dumpty; } \\
\text { CDP-diacylglycerol synthase:Mitochondrial }\end{array}$ \\
\hline M & ALQALHSSGVGLR & n.d. & $\mathrm{N}$-ter $+42.01 \mathrm{Da}$ & 20,6 & 2,589 & 1 & 1349,8 & TAM41_MOUSE & G5E881_MOUSE & Phosphatidate cytidyly|transferase, mitochondrial; & $\begin{array}{l}\text { translocator assembly and maintenance protein } \\
41 \text { homolog; }\end{array}$ \\
\hline м & AADISQWAGPLCLQEVDEPPQHALR & n.d. & $\mathrm{N}$-ter + 42.01 Da, C+57.02 Da & 57,1 & 2,355 & 1 & 2842,4 & PEBP1_MOUSE & D3Z1V4_MOUSE & Phosphatidylethanolamine-binding protein 1 ; & HCNPpp; \\
\hline MA & ADISQWAGPLCLQEVDEPPQHALR & n.d. & $\mathrm{N}$-ter $+42.01 \mathrm{Da}, \mathrm{C}+57.02 \mathrm{Da}$ & 18,1 & 5,226 & 1 & 2771,4 & PEBP1_MOUSE & D3Z1V4_MOUSE & Phosphatidylethanolamine-binding protein 1 ; & HCNPPp; \\
\hline M & TAIIKEIVSR & n.d. & $\mathrm{N}$-ter + 42.01 Da, K+34.06 Da & 28,0 & $-1,243$ & 1 & 1204,8 & PTEN_MOUSE & & $\begin{array}{l}\text { hhosphatidylinosito 3,4,5-trisphosphate 3- } \\
\text { phosphatase and dual-specificity protein phosphatase } \\
\text { PTEN; }\end{array}$ & $\begin{array}{l}\text { Mutated in multiple advanced cancers } \\
\text { 1;Phosphatase and tensin homolog; }\end{array}$ \\
\hline M & DLEAVCKR & n.d. & $\begin{array}{l}\text { N-ter + 42.01 Da, K+34.06 Da, } \\
\text { C+57.02 Da }\end{array}$ & 28,7 & $-2,086$ & 1 & 1196,6 & AGM1_MOUSE & Q8BWW3_MOUSE & E Phosphoacety|glucosamine mutase \{ECO:0000305\}; & 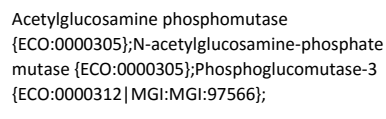 \\
\hline M & AAYKLVLIR & n.d. & $\mathrm{N}$-ter + 42.01 Da, K+34.06 Da & 34,3 & $-0,801$ & 1 & 1121,7 & PGAM1_MOUSE & & Phosphoglycerate mutase 1; & $\begin{array}{l}\text { BPG-dependent PGAM 1;Phosphoglycerate } \\
\text { mutase isozzye B; }\end{array}$ \\
\hline M & EPKAPCPAAVPSEER & n.d. & $\begin{array}{l}\text { N-ter + 42.01 Da, K+34.06 Da, } \\
\mathrm{C}+57.02 \mathrm{Da}\end{array}$ & 32,5 & 1,571 & 1 & 1843,9 & COAC_MOUSE & & Phosphopantothenoylcysteine decarboxylase; & $\mathrm{CoaC}$ \\
\hline M & ASSKKVTLSVISR & 0,5 & N-ter + 42.01 Da, K+28.03 Da & 40,6 & 0,610 & 1 & 1472,9 & PIR_MOUSE & A2AIH8_MOUSE & Pirin; & Probable quercetin 2,3-dioxygenase PIR; \\
\hline
\end{tabular}




\begin{tabular}{|c|c|c|c|c|c|c|c|c|c|c|c|}
\hline Non Prime Site & Prime Site & 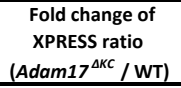 & Modifications & Hyperscore & $\begin{array}{l}\text { Mass error } \\
\text { in ppm }\end{array}$ & Charge & $\begin{array}{c}\begin{array}{c}\text { Precursor } \\
\text { neutral mass } \\
\text { in Da }\end{array} \\
\end{array}$ & Uniprot ID 1 & Uniprot ID 2 & Protein Name 1 & Protein Name 2 \\
\hline M & SQGDSNPAAIPHAAEDIQGDDR & n.d. & N-ter + 42.01 Da & 67,7 & 0,390 & 1 & 2305,0 & PA1B2_MOUSE & & $\begin{array}{l}\text { Platelet-activating factor acetylhydrolase IB subunit } \\
\text { beta; }\end{array}$ & 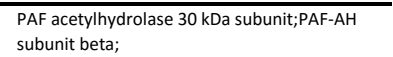 \\
\hline M & SGEGENPASKPTPVQDVQGDGR & n.d. & N-ter + 42.01 Da, K +34.06 Da & 45,1 & 1,389 & 1 & 2300,1 & PA1B3_MOUSE & Q8CA83_MOUSE & $\begin{array}{l}\text { Platelet-activating factor acetylhydrolase IB subunit } \\
\text { gamma; }\end{array}$ & $\begin{array}{l}\text { PAF acetylhydrolase } 29 \text { kDa subunit;;PAF-AH } \\
\text { subunit gamma; }\end{array}$ \\
\hline M & EEESIKEGSEKPR & n.d. & $\mathrm{N}$-ter + 42.01 Da, K+34.06 Da & 22,4 & $-0,454$ & 1 & 1757,9 & PKHO2_MOUSE & & $\begin{array}{l}\text { Pleckstrin homology domain-containing family } 0 \\
\text { member 2; }\end{array}$ & $\begin{array}{l}\text { Pleckstrin homology domain-containing family } \\
\text { Q member 1; }\end{array}$ \\
\hline M & TAAATVLKEGVLEKR & n.d. & N-ter + 42.01 Da, K+34.06 Da & 22,9 & 6,772 & 1 & 1695,1 & PHLA3_MOUSE & & Pleckstrin homology-like domain family A member 3; & ; TDAG51/II homolog 1; \\
\hline M & VEEVQKHSVHTLVFR & 0,4 & N-ter + 42.01 Da, K+28.03 Da & 21,2 & $-0,372$ & 1 & 1877,0 & PLRG1_MOUSE & D3Z4V1_MOUSE & Pleiotropic regulator 1; & \\
\hline MAA & AAAAAAAAGAAGGRGSGPGRR & n.d. & $\mathrm{N}$-ter + 42.01 Da & 45,3 & $-6,392$ & 1 & 1764,9 & PABP2_MOUSE & G3UY42_MOUSE & Polyadenylate-binding protein 2 & $\begin{array}{l}\text { Nuclear poly(A)-binding protein 1;Poly(A)- } \\
\text { binding protein II;Polyadenylate-binding } \\
\text { nuclear protein 1; }\end{array}$ \\
\hline M & DGIVPDIAVGTKR & n.d. & N-ter + 42.01 Da, K+34.06 Da & 45,0 & 0,581 & 1 & 1546,9 & PTBP1_MOUSE & Q92217_MOUSE & Polypyrimidine tract-binding protein 1 & Heterogeneous nuclear ribonucleoprotein I; \\
\hline M & DGIVTEVAVGVKR & n.d. & $\mathrm{N}$-ter + 42.01 Da, K+34.06 Da & 20,9 & 1,677 & 1 & 1548,9 & PTBP2_MOUSE & & Polypyrimidine tract-binding protein 2; & $\begin{array}{l}\text { Brain-enriched polypyrimidine tract-binding } \\
\text { protein;Neural polypyrimidine tract-binding } \\
\text { protein;RRM-type RNA-binding protein brPTB; }\end{array}$ \\
\hline YGLVPELVGD & CCLEEYR & n.d. & $\mathrm{N}$-ter + 42.01 Da, C +57.02 Da & 12,5 & 8,299 & 1 & 1070,4 & KCND1_MOUSE & & $\begin{array}{l}\text { Potassium voltage-gated channel subfamily D } \\
\text { member 1; }\end{array}$ & $\begin{array}{l}\text { Voltage-gated potassium channel subunit } \\
\text { Kv4.1; }\end{array}$ \\
\hline M & AAAKDGCGLETAAGNGR & 0,1 & $\begin{array}{l}\text { N-ter + 42.01 Da, K+28.03 Da, } \\
\mathrm{C}+57.02 \mathrm{Da}\end{array}$ & 68,5 & 0,947 & 1 & 1687,8 & PFD3_MOUSE & Q3TIR6_MOUSE & Prefoldin subunit 3; & Von Hippel-Lindau-binding protein 1; \\
\hline M & AATMKKAAAEDVNVTFEDQQKINKFAR & n.d. & $\mathrm{N}$-ter + 42.01 Da, K+34.06 Da & 44,9 & $-0,468$ & 1 & 3201,8 & Q3UWL8_MOUSE & E9Q6U4_MOUSE & $\begin{array}{l}\text { Prefoldin subunit } 4 \\
\{\text { ECO:0000256|PIRNR:PIRNR016477\}; }\end{array}$ & \\
\hline M & SLICSISNEVPEHPCVSPVSNHYYER & n.d. & $\mathrm{N}$-ter + 42.01 Da, C +57.02 Da & 27,5 & $-1,343$ & 1 & 3050,4 & PRP19_MOUSE & & Pre-mRNA-processing factor $19\{$ \{CO:0000305\}; & 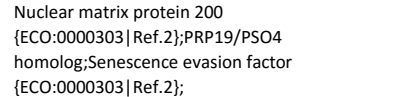 \\
\hline M & DILKSELLR & n.d. & $\mathrm{N}$-ter + 42.01 Da, K +34.06 Da & 19,6 & $-0,155$ & 1 & 1292,8 & PRP18_MOUSE & & Pre-mRNA-splicing factor 18; & $\begin{array}{l}\text { PRP18 homolog; } \\
\text { PR }\end{array}$ \\
\hline M & AAVTEVVVPADGAEARPLAAEELAAQKR & 0,1 & $\mathrm{~N}$-ter + 42.01 Da, K+28.03 Da & 21,1 & $-0,654$ & 1 & 2901,6 & SYF2_MOUSE & & Pre-mRNA-splicing factor SYF2; & CCNDBP1-interactor;mp29;p29; \\
\hline M & TNEEPLPKKVR & n.d. & $\mathrm{N}$-ter + 42.01 Da, K +34.06 Da & 21,1 & $-1,406$ & 1 & 1419,9 & FL2D_MOUSE & EOCYHO_MOUSE & Pre-mRNA-splicing regulator WTAP; & $\begin{array}{l}\text { Female-lethal(2)D homolog;WT1-associated } \\
\text { protein;Wilms tumor 1-associating protein; }\end{array}$ \\
\hline SHLAAVAHKT & LQVKDSPPDVAGAR & 1,0 & $\mathrm{~N}$-ter + 42.01 Da, K+28.03 Da & 15,9 & 1,771 & 1 & 1521,8 & SYRM_MOUSE & & Probable arginine--tRNA ligase, mitochondrial; & Arginyl-tRNA synthetase; \\
\hline GSMEGLEVIT & EFQASSAKADTGR & n.d. & $\mathrm{N}$-ter + 42.01 Da, K+34.06 Da & 17,5 & 6,156 & 1 & 1442,7 & GP101_MOUSE & & Probable G-protein coupled receptor $101 ;$ & \\
\hline M & AQNLKDLAGR & n.d. & $\mathrm{N}$-ter + 42.01 Da, K+34.06 Da & 56,0 & $-0,344$ & 1 & 1160,7 & PHB2_MOUSE & & Prohibitin-2; & $\begin{array}{l}\text { B-cell receptor-associated protein } \\
\text { BAP37;Repressor of estrogen receptor activity; }\end{array}$ \\
\hline M & SGEDEQQEQTIAEDLVVTKYKMGGDIANR & n.d. & $\mathrm{N}$-ter + 42.01 Da, K+34.06 Da & 35,9 & $-0,509$ & 1 & 3333,7 & PA2G4_MOUSE & D3YVH7_MOUSE & Proliferation-associated protein 2G4; & $\begin{array}{l}\text { IRES-specific cellular trans-acting factor } 45 \\
\text { RDa;Mpp1;;Proliferation-associated protein } \\
\text { 1;:Protein } 038 \text {-2G4; }\end{array}$ \\
\hline MSIRLARM & ASGRPEELWEAVVGAAER & n.d. & $\mathrm{N}$-ter + $42.01 \mathrm{Da}$ & 56,5 & $-0,152$ & 1 & 1968,0 & AKTS1_MOUSE & D3Z4W3_MOUSE & Proline-rich AKT1 substrate 1; & \\
\hline M & QPASAKWYDRR & n.d. & $\mathrm{N}$-ter + 42.01 Da, K+34.06 Da & 17,9 & 0,126 & 1 & 1583,8 & TEBP_MOUSE & D3Z7C6_MOUSE & Prostaglandin Esynthase 3; & $\begin{array}{l}\text { Cytosolic prostaglandin E2 synthase;Hsp90 co- } \\
\text { chaperone; ;rogesterone receptor complex } \\
\text { p23;Sid 3177; Telomerase-binding protein p23; }\end{array}$ \\
\hline M & QPASAKWYDR & n.d. & $\mathrm{N}$-ter + 42.01 Da, K +34.06 Da & 41,2 & $-1,539$ & 1 & 1427,7 & TEBP_MOUSE & D3Z7C6_MOUSE & Prostaglandin Esynthase 3; & $\begin{array}{l}\text { Cytosolic prostaglandin E2 synthase;Hsp90 co- } \\
\text { chaperone;Progesterone receptor complex } \\
\text { p23;Sid 3177; Telomerase-binding protein p23; }\end{array}$ \\
\hline M & AATFFGEVVKAPCR & n.d. & $\begin{array}{l}\text { N-ter + 42.01 Da, K+34.06 Da, } \\
\text { C }+57.02 \text { Da }\end{array}$ & 58,1 & $-0,982$ & 1 & 1627,9 & PSMG1_MOUSE & D6RG59_MOUSE & Proteasome assembly chaperone 1 & $\begin{array}{l}\text { Down syndrome critical region protein } 2 \\
\text { homolog: }\end{array}$ \\
\hline M & SAAAAPAAEGEDAPVPPSSEKEPEMPGPR & n.d. & $\mathrm{N}$-ter + 42.01 Da, K+34.06 Da & 43,8 & 1,368 & 1 & 2920,4 & DEK_MOUSE & E9Q8Y1_MOUSE & Protein DEK; & \\
\hline KLKAEGSEIR & LAKVDATEESDLAQQYGVR & n.d. & $\mathrm{N}$-ter + 42.01 Da, K +34.06 Da & 25,2 & 9,442 & 1 & 2168,1 & PDIA1_MOUSE & & Protein disulfide-isomerase; & $\begin{array}{l}\text { Cellular thyroid hormone-binding } \\
\text { protein;:Endoplasmic reticulum resident protein } \\
59 \text {; Prolyl 4-hydroxylase subunit beta;p55; }\end{array}$ \\
\hline M & ESEQMLEGQTQVAENPHSEYGLTDSVER & n.d. & $\mathrm{N}$-ter + 42.01 Da & 37,6 & $-0,539$ & 1 & 3335,5 & DPY30_MOUSE & & Protein dpy-30 homolog; & Dpy-30-like protein; \\
\hline $\begin{array}{l}\text { MESEQ } \\
\text { M }\end{array}$ & $\begin{array}{l}\text { MLEGQTC } \\
\text { DGDSDN }\end{array}$ & n.d. & $\begin{array}{l}\text { N-ter + 42.01 Da } \\
\text { N-ter+ } 4201 \mathrm{Da} \text { K +28.03 Da }\end{array}$ & 19 & & 1 & 2731,2 & DPY30-A & & $\begin{array}{l}\text { Protein dpy-30 homolog; } \\
\text { Pron }\end{array}$ & 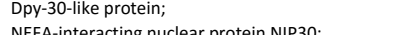 \\
\hline $\begin{array}{l}\mathrm{M} \\
\mathrm{M}\end{array}$ & $\begin{array}{l}\text { DGGDDDNLLIIIKR } \\
\text { LGGLGKLAEGLAHR }\end{array}$ & $\begin{array}{c}-0,1 \\
0,0\end{array}$ & $\begin{array}{l}\text { N-ter + 42.201 Da, K+28.03 Da } \\
\text { N-ter + 42.01 Da K + +88.03 Da }\end{array}$ & $\begin{array}{c}45,1 \\
118,9\end{array}$ & $\begin{array}{l}-0,2,2 \\
-0,2\end{array}$ & $\begin{array}{l}1 \\
1\end{array}$ & $\begin{array}{l}1716,9 \\
1662,9\end{array}$ & $\begin{array}{l}\text { F192A_MOUSE } \\
\text { FM25C MOUSE }\end{array}$ & & $\begin{array}{l}\text { Protein FAM192A; } \\
\text { Protein FAM25. }\end{array}$ & NEFA-interacting nuclear protein NIP30; \\
\hline M & $\begin{array}{l}\text { AQYKGAASEAGR } \\
\text { AQR }\end{array}$ & $-0,3$ & $\begin{array}{l}\text { N-ter + 42.01 Da, } \mathrm{K}+28.03 \mathrm{Da} \\
\mathrm{N} \text {-ter + 42.01 Da, K+28.03 Da }\end{array}$ & 64 & & 1 & 1277,6 & $\begin{array}{l}\text { FASOA_MOUSE } \\
\text { FASL_M }\end{array}$ & & $\begin{array}{l}\text { Protein FAM25C; } \\
\text { Protein FAM50A; }\end{array}$ & Protein XAP-5; \\
\hline APGRWRRPSD & SLSVASSLR & n.d. & $\mathrm{N}$-ter $+42.01 \mathrm{Da}$ & 11,7 & $-6,130$ & 1 & 960,5 & FA83B_MOUSE & & Protein FAM83B; & \\
\hline
\end{tabular}




\begin{tabular}{|c|c|c|c|c|c|c|c|c|c|c|c|}
\hline Non Prime Site & Prime Site & 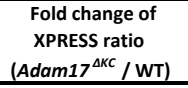 & Modifications & Hyperscore & $\begin{array}{c}\text { Mass error } \\
\text { in ppm }\end{array}$ & Charge & $\begin{array}{c}\text { Precursor } \\
\text { neutral mass } \\
\text { in Da }\end{array}$ & Uniprot ID 1 & Uniprot ID 2 & Protein Name 1 & Protein Name 2 \\
\hline $\begin{array}{l}\text { M } \\
\text { VKNEVNVPCL }\end{array}$ & $\begin{array}{l}\text { AFSQVQCLDDNHVNWR } \\
\text { RNFVESLYDTTLELSSR }\end{array}$ & $\begin{array}{l}\text { n.d. } \\
\text { n.d. }\end{array}$ & $\begin{array}{l}\text { N-ter + 42.01 Da, C +57.02 Da } \\
\text { N-ter + 42.01 Da }\end{array}$ & $\begin{array}{l}30,5 \\
17,7\end{array}$ & $\begin{array}{c}-0,394 \\
1,784\end{array}$ & $\begin{array}{ll}1 \\
1\end{array}$ & $\begin{array}{l}2029,9 \\
2071,0\end{array}$ & $\begin{array}{c}\text { FA83G_MOUSE } \\
\text { FRY_MOUSE }\end{array}$ & & $\begin{array}{l}\text { Protein FAM83G; } \\
\text { Protein furry homolog; }\end{array}$ & \\
\hline RASSSPLEAL & EACLKGIPPGGSSPLQSLAISWSR & n.d. & $\begin{array}{l}\text { N-ter + } 42.01 \mathrm{Da}, \mathrm{K}+34.06 \mathrm{Da}, \\
\mathrm{C}+57.02 \mathrm{Da}\end{array}$ & 20,8 & $\begin{array}{l}-0,077 \\
-0,107\end{array}$ & 1 & 2586,4 & KRBA1_MOUSE & D3Z4B8_MOUSE & Protein KRBA1; & \\
\hline M & AALGEPVRLER & n.d. & $\mathrm{N}$-ter $+42.01 \mathrm{Da}$ & 36,8 & $-1,755$ & 1 & 1251,7 & LIN7C_MOUSE & & Protein lin-7 homolog C; & $\begin{array}{l}\text { Mammalian lin-seven protein 3;Vertebrate lin-7 } \\
\text { homolog 3; }\end{array}$ \\
\hline M & SGGTPYIGSKISLISKAEIR & n.d. & N-ter + 42.01 Da, K+34.06 Da & 34,3 & 0,548 & 1 & 2186,3 & LS14A_MOUSE & & Protein LSM14 homolog A; & Protein FAM61A;RNA-associated protein 55A; \\
\hline M & SGSSGTPYLGSKISLISKAQIR & n.d. & N-ter + 42.01 Da, K+34.06 Da & 32,5 & 0,720 & 1 & 2359,4 & LS14B_MOUSE & & Protein LSM14 homolog B; & Protein FAM61B;RNA-associated protein 55B; \\
\hline M & AHATPPSALEQGGPIR & n.d. & $\mathrm{N}$-ter $+42.01 \mathrm{Da}$ & 22,2 & 0,730 & 1 & 1642,8 & GTL3B_MOUSE & & Protein NATD1; & $\begin{array}{l}\text { Gene trap locus protein F3-2;Gene trap locus } \\
\text { protein F3b;N-acetyltransferase domain-- } \\
\text { containing protein 1; }\end{array}$ \\
\hline M & SALEKSMHLGR & n.d. & $\mathrm{N}$-ter + 42.01 Da, K+34.06 Da & 34,1 & $-0,459$ & 1 & 1303,7 & PPME1_MOUSE & & Protein phosphatase methylesterase 1 ; & \\
\hline & 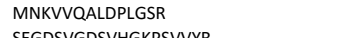 & n.d. & N-ter + 42.01 Da, $\mathrm{K}+34.06 \mathrm{Da}$ & 23,7 & $-1,932$ & 1 & 1602,9 & POF1B_MOUSE & & Protein POF1B; & \\
\hline M & SEGDSVGDSVHGKPSVVYR & n.d. & N-ter + 42.01 Da, K+34.06 Da & 20,9 & 4,773 & 1 & 2050,0 & RER1_MOUSE & & Protein RER1; & \\
\hline M & ANKAPSQMER & n.d. & $\mathrm{N}$-ter + 42.01 Da, K+34.06 Da & 40,2 & $-1,407$ & 1 & 1206,6 & S10A9_MOUSE & & Protein S100-A9; & $\begin{array}{l}\text { Calgranulin-B;:Leukocyte L Lomplex heavy } \\
\text { chain;Migration inhibitory factor-related } \\
\text { protein 14;S100 calcium-binding protein A9; }\end{array}$ \\
\hline M & VSVMNTVDTSHEDMIHDAQMDYYGTR & n.d. & $\mathrm{N}$-ter + $42.01 \mathrm{Da}$ & 29,6 & $-0,294$ & 1 & 3056,3 & SEC13_MOUSE & & Protein SEC13 homolog; & SEC13-like protein 1;SEC13-related protein; \\
\hline m & SESGHSQPGLYGIER & n.d. & $\mathrm{N}$-ter $+42.01 \mathrm{Da}$ & 27,8 & $-0,121$ & 1 & 1657,8 & SMG9_MOUSE & D3Z719_MOUSE & Protein SMG9; & Protein smg-9 homolog; \\
\hline M & STTVPEL & n.d. & $\mathrm{N}$-ter + $42.01 \mathrm{Da}, \mathrm{K}$ & 27,3 & 0,195 & 1 & 1534,9 & SDOS_MOUSE & & Protein syndesmos; & NUDT16-like protein 1; \\
\hline M & NPEKDFA & $-1,5$ & $\mathrm{~N}$-ter + 42.01 Da, $\mathrm{K}+28.03 \mathrm{Da}$ & 27,3 & $-3,868$ & 1 & 1911,0 & VAC14_MOUSE & & Protein VAC14 homolog; & \\
\hline M & HATGLAAPAGTPR & n.d. & $\mathrm{N}$-ter $+42.01 \mathrm{Da}$ & 27,8 & 0,646 & 1 & 1391,7 & YIF1B_MOUSE & D3Z5F9_MOUSE & Protein YIFIB; & $\begin{array}{l}\text { YIP1-interacting factor homolog B; } \\
\text { Peptidylarginine deiminase IV;Protein-ci }\end{array}$ \\
\hline M & AQGAVIHVAPEQPTHAVCVVGTATPLDVR & n.d. & $\mathrm{N}$-ter + 42.01 Da, C+57.02 Da & 27,3 & 0,658 & 1 & 3034,6 & PADI4_MOUSE & & Protein-arginine deiminase type-4; & $\begin{array}{l}\text { Peptidyarginine delminase IV; Protein-ar } \\
\text { deiminase type IV; }\end{array}$ \\
\hline M & SALQIQNVNWQVPMNRR & n.d. & $\mathrm{N}$-ter $+42.01 \mathrm{Da}$ & 20,3 & 1,764 & 1 & 2095,1 & TGM3_MOUSE & & Protein-glutamine gamma-glutamyltransferase E; & Transglutaminase E;Transglutaminase-3; \\
\hline LYLRVCFAM & AWKSGGASHSELIHNLR & n.d. & N-ter + 42.01 Da, K+34.06 Da & 52,1 & $-9,166$ & 1 & 1938,0 & PIMT_MOUSE & F7D432_MOUSE & $\begin{array}{l}\text { Protein-L-isoaspartate(D-aspartate) O- } \\
\text { methyltransferase; }\end{array}$ & $\begin{array}{l}\text { L-isoaspartyl protein carboxyl } \\
\text { methyltransferase; rotein L-isoasparty//D- } \\
\text { aspartyl methyltransferase;Protein-beta- } \\
\text { aspartate methyltransferase; }\end{array}$ \\
\hline M & SDAAVDTSSEITTKDLKEKKEVVEEAENGR & 0,2 & N-ter + 42.01 Da, K+28.03 Da & 32,7 & $-1,193$ & 1 & 3431,8 & PTMA_MOUSE & & Prothymosin alpha; & $\begin{array}{l}\text { CD62 antigen-like family member } P \text {; Granule } \\
\text { membrane protein } 140 \text {;Leukocyte-endothelial }\end{array}$ \\
\hline CSNPWGNFSY & GSTCTFQCPEGQSLNGSVR & n.d. & N-ter + 42.01 Da, C +57.02 Da & 18,4 & $-1,173$ & 1 & 2125,9 & LYAM3_MOUSE & & P-selectin; & $\begin{array}{l}\text { cell adhesion molecule 3;Platelet activation } \\
\text { dependent granule-external membrane } \\
\text { protein; } \\
\text { CD62 antigen-like family member P;Granule } \\
\text { membrane protein 140;Leukocyte-endothelial }\end{array}$ \\
\hline NCSNPWGNFS & YGSTCTFQCPEGQSLNGSVR & n.d. & N-ter + 42.01 Da, C +57.02 Da & 17,2 & $-7,018$ & 1 & 2289,0 & LYAM3_MOUSE & & P-selectin; & $\begin{array}{l}\text { cell adhesion molecule 3;Platelet activation } \\
\text { dependent granule-external membrane } \\
\text { protein; }\end{array}$ \\
\hline QNFQAKPSTP & TSKLGQEEGHDTGSESDSSGR & 0,2 & $\mathrm{~N}$-ter + 42.01 Da, K +28.03 Da & 19,5 & 1,833 & 1 & 2233,0 & PSPC_MOUSE & Q6P8P8_MOUSE & Pulmonary surfactant-associated protein C; & $\begin{array}{l}\text { Pulmonary surfactant-associated proteolipid } \\
\text { SPL(Val):SP5; }\end{array}$ \\
\hline $\begin{array}{l}\mathrm{M} \\
\mathrm{M}\end{array}$ & $\begin{array}{l}\text { NHDFOALALESR } \\
\text { AAAPATLALDPQPQEKQKDASESSELSR }\end{array}$ & $\begin{array}{l}\text { n.d. } \\
0,2\end{array}$ & $\begin{array}{l}\text { N-ter + 42.01 Da } \\
\text { N-ter + 42.01 Da, K+28.03 Da }\end{array}$ & $\begin{array}{l}28,7 \\
35,0\end{array}$ & $\begin{array}{l}-0,191 \\
-0,296\end{array}$ & $\begin{array}{l}1 \\
1\end{array}$ & $\begin{array}{l}1572,7 \\
3035,5\end{array}$ & $\begin{array}{l}\text { PUM2_MOUSE } \\
\text { RABE2_MOUSE }\end{array}$ & E9Q0P1_MOUSE & $\begin{array}{l}\text { Pumilio homolog 2; } \\
\text { Rab GTPase-binding effector protein 2; }\end{array}$ & Rabaptin-5beta; \\
\hline mV & MEKPSPLLVGR & n.d. & $\mathrm{N}$-ter + 42.01 Da, K+34.06 Da & 51,0 & 0,077 & 1 & 1301,8 & G3BP1_MOUSE & G3BP2_MOUSE & Ras GTPase-activating protein-binding protein 1; & ATP-dependent DNA helicase VIII;GAP SH3 \\
\hline $\begin{array}{l}\text { M } \\
\text { YDALVHFGKR }\end{array}$ & $\begin{array}{l}\text { SAAEEVDGLGVVRPHYGSVLDNER } \\
\text { GLNVAATAAVMAASKGQGALSER }\end{array}$ & $\begin{array}{l}\text { n.d. } \\
4,0\end{array}$ & $\begin{array}{l}\text { N-ter + 42.01 Da } \\
\text { N-ter + 42.01 Da, K+28.03 Da }\end{array}$ & $\begin{array}{l}55,5 \\
16,4\end{array}$ & $\begin{array}{r}-0,268 \\
1,693\end{array}$ & $\begin{array}{l}1 \\
1\end{array}$ & $\begin{array}{l}2610,3 \\
2242,2\end{array}$ & $\begin{array}{l}\text { IQGA1_MOUSE } \\
\text { REEP1_MOUSE }\end{array}$ & & $\begin{array}{l}\text { Ras GTPase-activating-like protein IQGAP1; } \\
\text { Receptor expression-enhancing protein 1; }\end{array}$ & domain-binding protein 1;HDH-VIII; \\
\hline M & SSVKLWPTGASAVPLVSR & n.d. & N-ter + 42.01 Da, K+34.06 Da & 21,5 & $-2,588$ & 1 & 1930,1 & RIPK3_MOUSE & G3X8V8_MOUSE & $\begin{array}{l}\text { Receptor-interacting serine/threonine-protein kinase } \\
3 ;\end{array}$ & $\begin{array}{l}\text { RIP-like protein kinase 3;Receptor-interacting } \\
\text { protein 3; }\end{array}$ \\
\hline M & SSIKVECVLR & n.d. & $\begin{array}{l}\text { N-ter + 42.01 Da, K+34.06 Da, } \\
\mathrm{C}+57.02 \mathrm{Da}\end{array}$ & 18,8 & $-1,972$ & 1 & 1265,7 & RGN_MOUSE & & Regucalcin; & $\begin{array}{l}\text { Gluconolactonase;Senescence marker protein } \\
\text { 30; }\end{array}$ \\
\hline M & AAGGGGGSSKASSSSASSAGALESSLDR & n.d. & $\mathrm{N}$-ter + 42.01 Da, K+34.06 Da & 58,8 & $-0,763$ & 1 & 2487,2 & RPRD2_MOUSE & & $\begin{array}{l}\text { Regulation of nuclear pre-mRNA domain-containing } \\
\text { protein 2; }\end{array}$ & \\
\hline M & DSEAFQHAR & n.d. & $\mathrm{N}$-ter + 42.01 Da & 41,8 & $-0,972$ & 1 & 1232,5 & IASPP_MOUSE & D3Z5Q5_MOUSE & RelA-associated inhibitor; & $\begin{array}{l}\text { Inhibitor of ASPP protein;NFkB-interacting } \\
\text { protein 1;PPP1R13B-like protein; }\end{array}$ \\
\hline M & EDIMQLPRAR & n.d. & N-ter + 42.01 Da, K+34.06 Da & 48,4 & $-1,491$ & 1 & 1406,8 & RFA3_MOUSE & & Replication protein A 14 kDa subunit; & Replication factor A protein 3; \\
\hline
\end{tabular}




\begin{tabular}{|c|c|c|c|c|c|c|c|c|c|c|c|}
\hline Non Prime Site & Prime Site & $\begin{array}{c}\text { Fold change of } \\
\text { XPRESS ratio } \\
\left.\text { (Adam17 }^{\Delta K c} / \mathrm{WT}\right) \\
\end{array}$ & Modifications & Hyperscore & $\begin{array}{l}\text { Mass error } \\
\text { in ppm }\end{array}$ & Charge & $\begin{array}{c}\text { Precursor } \\
\text { neutral mass } \\
\text { in Da }\end{array}$ & Uniprot ID 1 & Uniprot ID 2 & Protein Name 1 & Protein Name 2 \\
\hline M & SRPPPTGKMPGAPEAAPGDGAGAGR & 0,0 & $\mathrm{~N}$-ter + 42.01 Da, K+28.03 Da & 51,8 & 3,159 & 1 & 2371,2 & ROCK2_MOUSE & & Rho-associated protein kinase 2; & $\begin{array}{l}\text { Rho-associated, coiled-coil-containing protein } \\
\text { kinase 2; Rho-associated, coiled-coil-containing } \\
\text { protein kinase II;p164 ROCK-2; }\end{array}$ \\
\hline M & AAAPQAPKR & n.d. & $\mathrm{N}$-ter + 42.01 Da, K+34.06 Da & 17,2 & $-1,216$ & 1 & 984,6 & RPP38_MOUSE & A2AJGO_MOUSE & Ribonuclease P protein subunit p38; & \\
\hline M & AGACGKPHMSPASLPGKR & n.d. & $\begin{array}{l}\text { N-ter + 42.01 Da, K+34.06 Da, } \\
\text { C +57.02 Da }\end{array}$ & 20,8 & $-0,052$ & 1 & 1931,1 & BOP1_MOUSE & & $\begin{array}{l}\text { Ribosome biogenesis protein BOP1 } \\
\{E C O: 0000255 \mid \text { HAMAP-Rule:MF_03027\}; }\end{array}$ & $\begin{array}{l}\text { Block of proliferation } 1 \text { protein } \\
\text { \{ECO:0000255|HAMAP-Rule:MF_03027\}; }\end{array}$ \\
\hline M & ASGCKIGPSILNSDLANLGAECLR & n.d. & $\begin{array}{l}\text { N-ter + 42.01 Da, K+34.06 Da, } \\
\mathrm{C}+57.02 \mathrm{Da}\end{array}$ & 33,4 & $-3,161$ & 1 & 2591,3 & RPE_MOUSE & B2KGFO_MOUSE & Ribulose-phosphate 3-epimerase; & Ribulose-5-phosphate-epimerase; \\
\hline M & TSASKAVELQLQVKKHNAEELQDFMR & n.d. & $\mathrm{N}$-ter + 42.01 Da, K+34.06 Da & 37,8 & 6,431 & 1 & 2981,6 & RPAP3_MOUSE & & RNA polymerase II-associated protein 3; & \\
\hline ADVPPELLNN & VGALHFR & n.d. & $\mathrm{N}$-ter + 42.01 Da & 12,7 & $-8,309$ & 1 & 840,5 & CTR9_MOUSE & & RNA polymerase-associated protein CTR9 homolog; & $\begin{array}{l}\text { SH2 domain-binding protein } \\
\text { 1; Tetratricopeptide repeat-containing, SH2- } \\
\text { binding phosphoprotein of } 150 \mathrm{kDa} \text {; }\end{array}$ \\
\hline M & AAALGAGGGAGAGDDDFDQFDKPGAER & n.d. & $\mathrm{N}$-ter + 42.01 Da, K +34.06 Da & 24,5 & 3,022 & 1 & 2611,2 & RBM33_MOUSE & D3Z519_MOUSE & RNA-binding protein 33; & $\begin{array}{l}\text { Proline-rich protein 8;RNA-binding motif } \\
\text { protein 33; }\end{array}$ \\
\hline M & ATVAATTKVPEIR & $-0,1$ & N-ter + 42.01 Da, K+28.03 Da & 69,1 & 0,840 & 1 & 1425,8 & RUVB2_MOUSE & D3YXQ8_MOUSE & RuvB-like 2; & $\begin{array}{l}\text { p47 protein; } \\
\text { p }\end{array}$ \\
\hline M & AAAAGAVVASAASGPAEGKKITELR & 0,0 & $\mathrm{~N}$-ter + 42.01 Da, K+28.03 Da & 95,3 & 0,417 & 1 & 2393,3 & SLTM_MOUSE & & SAFB-like transcription modulator; & $\begin{array}{l}\text { Modulator of estrogen-induced } \\
\text { transcription;SAF-like transcription modulator; }\end{array}$ \\
\hline M & ATKCTKCGPGYSTPLEAMKGPR & n.d. & $\begin{array}{l}\text { N-ter + 42.01 Da, K+34.06 Da, } \\
\mathrm{C}+57.02 \mathrm{Da}\end{array}$ & 32,6 & 0,391 & 1 & 2553,4 & SBP1_MOUSE & D6RHN2_MOUSE & Selenium-binding protein 1 ; & 56 kDa selenium-binding protein; \\
\hline M & ASCDEIKEHPR & n.d. & $\begin{array}{l}\text { N-ter + 42.01 Da, K+34.06 Da, } \\
\mathrm{C}+57.02 \mathrm{Da}\end{array}$ & 23,9 & $-1,972$ & 1 & 1416,7 & SEP10_MOUSE & & Septin-10; & \\
\hline M & AVAVGRPSNEELR & n.d. & $\mathrm{N}$-ter+ $+42.01 \mathrm{Da}$ & 40,4 & $-0,486$ & 1 & 1438,8 & SEP11_MOUSE & & Septin-11; & \\
\hline AGQTGKSRKM & SKQQPTQFINPETPGYVGFANLPNQVHR & n.d. & $\mathrm{N}$-ter + 42.01 Da, K+34.06 Da & 19,0 & $-5,483$ & 1 & 3242,7 & SEPT2_MOUSE & D3Z3CO_MOUSE & Septin-2; & $\begin{array}{l}\text { Neural precursor cell expressed } \\
\text { developmentally down-resulated protein } 5 \text {; }\end{array}$ \\
\hline ERSVNCGTMV & AQPKNLEGYVGFANLPNQVYR & 0,1 & N-ter + 42.01 Da, K+28.03 Da & 55,8 & $-0,775$ & 1 & 2447,3 & SEPT7_MOUSE & E9Q168_MOUSE & Septin-7; & $\begin{array}{l}\text { CDC10 protein homolog; } \\
\text { Protein PR264;Putative myelin regulatory }\end{array}$ \\
\hline M & SYGRPPPDVEGMTSLKVDNLTYR & n.d. & N-ter + 42.01 Da, K+34.06 Da & 72,9 & $-0,898$ & 1 & 2670,4 & SRSF2_MOUSE & & Serine/arginine-rich splicing factor 2; & $\begin{array}{l}\text { factor 1;5plicicing component, } 35 \text { LDa;Splicing } \\
\text { factor SC35;splicing factor, arginine/serine-rich } \\
2 ;\end{array}$ \\
\hline SFEALLADLt & RTLSDNVNLPQGVR & n.d. & $\mathrm{N}$-ter + 42.01 Da & 21,1 & $-1,921$ & 1 & 1609,9 & DCLK1_MOUSE & Q80VB6_MOUSE & Serine/threonine-protein kinase DCLK1; & $\begin{array}{l}\text { Doublecortin-like and CAM kinase-like } \\
\text { 1;Doublecortin-like kinase } 1 ;\end{array}$ \\
\hline M & SNNGVDIQDKPPAPPMR & 0,2 & $\mathrm{~N}$-ter + 42.01 Da, K+28.03 Da & 23,0 & 3,354 & 1 & 1905,0 & PAK1_MOUSE & G5E884_MOUSE & Serine/threonine-protein kinase PAK 1; & $\begin{array}{l}\text { Alpha-PAK;CDC42/RAC effector kinase PAK- } \\
\text { A;p21-activated kinase 1;p65-PAK; }\end{array}$ \\
\hline M & SDNGELEDKPPAPPVR & n.d. & N-ter + 42.01 Da, K+34.06 Da & 56,0 & $-0,501$ & 1 & 1795,9 & PAK2_MOUSE & & Serine/threonine-protein kinase PAK 2; & Gamma-PAK; ;21-activated kinase 2; \\
\hline M & SDSLDNEEKPPAPPLR & n.d. & $\mathrm{N}$-ter + 42.01 Da, K+34.06 Da & 52,5 & $-0,163$ & 1 & 1839,9 & PAK3_MOUSE & A3KGC2_MOUSE & Serine/threonine-protein kinase PAK 3; & $\begin{array}{l}\text { Beta-PAK;CDC42/RAC effector kinase PAK- } \\
\text { B;p21-activated kinase 3; }\end{array}$ \\
\hline M & SEPKAIDPKLSTTDR & 0,1 & $\mathrm{~N}$-ter + 42.01 Da, K+28.03 Da & 21,5 & 0,853 & 1 & 1754,9 & PP2BA_MOUSE & & $\begin{array}{l}\text { Serine/threonine-protein phosphatase } 2 \mathrm{~B} \text { catalytic } \\
\text { subunit alpha isoform; }\end{array}$ & $\begin{array}{l}\text { CAM-PRP catalytic subunit;Calmodulin- } \\
\text { dependent calcineurin A subunit alpha isoform; }\end{array}$ \\
\hline M & SAMEAADVFHR & n.d. & $\mathrm{N}$-ter + 42.01 Da & 32,6 & $-1,880$ & 1 & 1274,6 & CPPED_MOUSE & D3Z7F7_MOUSE & Serine/threonine-protein phosphatase CPPED1; & $\begin{array}{l}\text { Calcineurin-like phosphoesterase domain- } \\
\text { containing protein } 1 \text {; }\end{array}$ \\
\hline M & SDSEKLNLDSIIGR & n.d. & $\mathrm{N}$-ter + 42.01 Da, K+34.06 Da & 52,0 & 0,308 & 1 & 1621,9 & PP1A_MOUSE & & $\begin{array}{l}\text { Serine/threonine-protein phosphatase PP1-alpha } \\
\text { catalytic subunit; }\end{array}$ & \\
\hline M & ADIDKLNIDSIIIQR & $-0,2$ & $\mathrm{~N}$-ter + 42.01 Da, K+28.03 Da & 26,3 & 2,789 & 1 & 1682,9 & PP1G_MOUSE & & $\begin{array}{l}\text { Serine/threonine-protein phosphatase PP1-gamma } \\
\text { catalytic subunit; }\end{array}$ & Protein phosphatase $1 \mathrm{C}$ catalytic subunit; \\
\hline WCTGTVPIGE & YLVNSEDGAHR & n.d. & $\mathrm{N}$-ter + 42.01 Da & 17,9 & $-0,307$ & 1 & 1301,6 & ATM_MOUSE & FGUXV2_MOUSE & Serine-protein kinase ATM; & Ataxia telangiectasia mutated homolog; \\
\hline M & DSLTAANNKFCFDFFR & n.d. & $\begin{array}{l}\text { N-ter + 42.01 Da, K+34.06 Da, } \\
\mathrm{C}+57.02 \mathrm{Da}\end{array}$ & 42,9 & 0,555 & 1 & 2159,0 & SPB12_MOUSE & & Serpin B12; & \\
\hline M & GDSDDEYDRR & n.d. & $\mathrm{N}$-ter + 42.01 Da & 15,0 & 6,218 & 1 & 1268,5 & SRRT_MOUSE & & Serrate RNA effector molecule homolog; & Arsenite-resistance protein 2 \\
\hline M & ADLKKA & $-0,3$ & $\mathrm{~N}$-ter + 42.01 Da, K+28.03 Da & 16,9 & 36 & 1 & 1083,6 & 3BP5L_MOUSE & & SH3 domain-binding protein 5-like; & \\
\hline $\begin{array}{l}M \\
M\end{array}$ & SGEVPPNINIIKEPR & n.d. & $\mathrm{N}$-ter + 42.01 Da, K+34.06 Da & 5,5 & 1,045 & 1 & 1624,9 & SFXN1_MOUSE & & Sideroflexin-1; & \\
\hline & SLWGLISKMSPEKLQR & n.d. & N-ter + 42.01 Da, K+34.06 Da & 16,4 & 0,957 & 1 & 1982,2 & STAT6_MOUSE & D3YU28_MOUSE & Signal transducer and transcription activator 6; & \\
\hline м & FAKGKGSAVPSDGQAR & $-0,3$ & $\mathrm{~N}$-ter + 42.01 Da, K+28.03 Da & 35,1 & 4,206 & 1 & 1803,9 & SSBP3_MOUSE & B1AS34_MOUSE & Single-stranded DNA-binding protein 3; & $\begin{array}{l}\text { Lck-associated signal transsducer;Sequence- } \\
\text { specific single-stranded-DNA-binding protein; }\end{array}$ \\
\hline M & SLLNKPKSEMTPEELQKR & n.d. & $\mathrm{N}$-ter + 42.01 Da, K+34.06 Da & 50,2 & $-0,088$ & 1 & 2271,3 & SMD2_MOUSE & & Small nuclear ribonucleoprotein Sm D2; & snRNP core protein D2; \\
\hline M & TVGKSSKMLQHIDYR & $-0,3$ & $\mathrm{~N}$-ter + 42.01 Da, K+28.03 Da & 15,7 & $-2,843$ & 1 & 1860,0 & RSMB_MOUSE & RSMN_MOUSE & Small nuclear ribonucleoprotein-associated protein & ; Sm protein B; \\
\hline M & EPSPDAEEAHTVR & n.d. & N-ter + 42.01 Da & 29,3 & 0,186 & 1 & 1609,7 & SMTL2_MOUSE & & Smoothelin-like protein $2 ;$ & \\
\hline
\end{tabular}




\begin{tabular}{|c|c|c|c|c|c|c|c|c|c|c|c|}
\hline Non Prime Site & Prime Site & 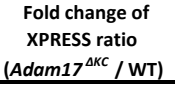 & Modifications & Hyperscore & $\begin{array}{l}\text { Mass error } \\
\text { in ppm }\end{array}$ & Charge & $\begin{array}{c}\text { Precursor } \\
\text { neutral mass } \\
\text { in Da }\end{array}$ & Uniprot ID 1 & Uniprot ID 2 & Protein Name 1 & Protein Name 2 \\
\hline AEQALPLGNG & KAAEEARGSETLGGGGGGAAGTR & 10,6 & $\mathrm{~N}$-ter + 42.01 Da, K +28.03 Da & 22,7 & 0,797 & 1 & 2129,1 & S6A11_MOUSE & & Sodium- and chloride-dependent GABA transporter 3; & $\begin{array}{l}\text { Sodium- and chloride-dependent GABA } \\
\text {; transporter 4;Solute carrier family } 6 \text { member } \\
11 \text {; }\end{array}$ \\
\hline $\begin{array}{l}M \\
M\end{array}$ & $\begin{array}{l}\text { AAPAKGENLSLVVHGPGDIR } \\
\text { SDTAVADTRR }\end{array}$ & $\begin{array}{l}\text { n.d. } \\
\text { n.d. }\end{array}$ & $\begin{array}{l}\text { N-ter + 42.01 Da, K +34.06 Da } \\
\text { N-ter + 42.01 Da }\end{array}$ & $\begin{array}{l}43,2 \\
19,0\end{array}$ & $\begin{array}{r}0,096 \\
-2,027\end{array}$ & $\begin{array}{l}1 \\
1\end{array}$ & $\begin{array}{l}2076,2 \\
1132,6\end{array}$ & $\begin{array}{l}\text { DHSO_MOUSE } \\
\text { SNX12 MOUSE }\end{array}$ & 0 & Sorbitol dehydrogenase; & $\begin{array}{l}\text { L-didtol 2-dehydrogenase; } \\
\text { SDP8 protein; }\end{array}$ \\
\hline M & AANPSGQGFQNKNR & $-0,2$ & $\mathrm{~N}$-ter $+42.01 \mathrm{Da}, \mathrm{K}+28.03 \mathrm{Da}$ & 38,1 & $-0,256$ & 1 & 1557,8 & SOSSC_MOUSE & - & SOSS complex subunit C; & $\begin{array}{l}\text { INTS3- and NABP-interacting protein; Sensor of } \\
\text { single-strand DNA complex subunit } ; \text {; Sensoror of } \\
\text { ssDNA subunit C;Single-stranded DNA-binding } \\
\text { protein-interacting protein 1; }\end{array}$ \\
\hline SFRKRRAQKM & DPSGVKVLETAEDIQER & 0,1 & $\mathrm{~N}$-ter $+42.01 \mathrm{Da}, \mathrm{K}+28.03 \mathrm{Da}$ & 63,6 & $-0,048$ & 1 & 2086,0 & SPTN1_MOUSE & E9Q447_MOUSE & Spectrin alpha chain, non-erythrocytic $1 ;$ & Alpha-II spectrin;Fodrin alpha chain; \\
\hline & ADLLGSILSSMEKPPSLGDQESR & n.d. & $\mathrm{N}$-ter + 42.01 Da, $\mathrm{K}+34.06 \mathrm{Da}$ & 39,4 & $-0,837$ & 1 & 2505,3 & SPAG7_MOUSE & Q5SX56_MOUSE & Sperm-associated antigen 7 ; & \\
\hline GTPLQRIGSM & SSVTSTKEVADSPPPLTR & 0,4 & $\mathrm{~N}$-ter $+42.01 \mathrm{Da}, \mathrm{K}+28.03 \mathrm{Da}$ & 44,8 & 0,772 & 1 & 1941,0 & SSFA2_MOUSE & A2AQD6_MOUSE & Sperm-specific antigen 2 homolog; & Ki-ras-induced actin-interacting protein; \\
\hline M & TTAARPTFEPAR & n.d. & $\mathrm{N}$-ter + 42.01 Da & 39,9 & $-2,058$ & 1 & 1358,7 & CWC15_MOUSE & & Spliceosome-associated protein CWC15 homolog; & Embryonic development factor 1 ; \\
\hline M & ATGANATPLDFPSKKR & n.d. & $\mathrm{N}$-ter + 42.01 Da, K+34.06 Da & 42,7 & $-1,008$ & 1 & 1783,0 & SF01_MOUSE & E9Q4Q2_MOUSE & Splicing factor 1 ; & $\begin{array}{l}\text { CW17; Mammalian branch point-binding } \\
\text { protein;Transcription factor ZFM1;Zinc finger } \\
\text { gene in MEN1 locus;izinc finger protein 162; }\end{array}$ \\
\hline M & DFQHRPGGKTGSGGVASSSESNR & n.d. & $\mathrm{N}$-ter + 42.01 Da, K+34.06 Da & 25,6 & 6,803 & 1 & 2524,2 & SF3A2_MOUSE & G3UVU2_MOUSE & Splicing factor 3 A subunit 2; & SF3a66;Spliceosome-associated protein 62; \\
\hline M & AKIAKTHEDIEAQIR & n.d. & $\mathrm{N}$-ter + 42.01 Da, $\mathrm{K}+34.06 \mathrm{Da}$ & 53,0 & $-0,654$ & 1 & 1832,1 & SF3B1_MOUSE & G5E866_MOUSE & Splicing factor $3 \mathrm{~B}$ subunit 1; & $\begin{array}{l}\text { Pre-mRNA-splicing factor SF3b } 155 \mathrm{kDa} \\
\text { subunit;:Spliceosome-associated protein 155; }\end{array}$ \\
\hline M & AASAIKGLSALR & n.d. & $\mathrm{N}$-ter + 42.01 Da, $\mathrm{K}+34.06 \mathrm{Da}$ & 51,3 & $-0,729$ & 1 & 1232,8 & SLIRP_MOUSE & & $\begin{array}{l}\text { SRA stem-loop-interacting RNA-binding protein, } \\
\text { mitochondrial; }\end{array}$ & \\
\hline M & SDHGDVSLPPQDR & n.d. & $\mathrm{N}$-ter $+42.01 \mathrm{Da}$ & 31,2 & 0,000 & 1 & 1463,7 & STABP_MOUSE & & STAM-binding protein; & $\begin{array}{l}\text { Associated molecule with the SH3 domain of } \\
\text { STAM; }\end{array}$ \\
\hline M & DPSWATQESEAVAEKVLR & n.d. & $\mathrm{N}$-ter + 42.01 Da, K +34.06 Da & 44,1 & $-1,079$ & 1 & 2222,1 & STAR5_MOUSE & D3YUOO_MOUSE & StAR-related lipid transfer protein 5 ; & START domain-containing protein 5; \\
\hline M & ASSDIQVKELEKR & $-0,2$ & $\mathrm{~N}$-ter + 42.01 Da, K+28.03 Da & 63,8 & 0,312 & 1 & 1599,9 & STMN1_MOUSE & D3Z1Z8_MOUSE & Stathmin; & $\begin{array}{l}\text { Leukemia-associated gene protein;Leukemia- } \\
\text { associated phosphoprotein } \\
\text { p18; Metablastin;Oncoprotein } \\
\text { 18; } \\
\text { Pr22osphopprotein p19;Prosolin;Protein } \\
\text { Pri; }\end{array}$ \\
\hline & SLGGVSEASR & n.d. & $\mathrm{N}$-ter + 42.01 Da & 25,6 & $-0,796$ & 1 & 1003,5 & CYT1_MOUSE & Q497JO_MOUSE & Stefin-1; & \\
\hline $\begin{array}{l}\text { CPAGGAEVEM } \\
\text { LSFNPLTSLL }\end{array}$ & $\begin{array}{l}\text { AELIVKPGNKER } \\
\text { QWGGAHNTDQHPYSGSGR }\end{array}$ & $\begin{array}{l}-0,1 \\
\text { n.d. }\end{array}$ & $\begin{array}{l}\text { N-ter + 42.01 Da, K +28.03 Da } \\
\text { N-ter + 42.01 Da }\end{array}$ & $\begin{array}{l}28,5 \\
18,6\end{array}$ & $\begin{array}{l}-1,596 \\
3,700\end{array}$ & $\begin{array}{l}1 \\
1\end{array}$ & $\begin{array}{l}1500,8 \\
1995,9\end{array}$ & $\begin{array}{l}\text { SRA1_MOUSE } \\
\text { SRBP2_MOUSE }\end{array}$ & G3X8R2_MOUSE & $\begin{array}{l}\text { Steroid receptor RNA activator 1; } \\
\text { Sterol regulatory element-binding protein 2; }\end{array}$ & $\begin{array}{l}\text { Steroid receptor RNA activator protein; } \\
\text { Sterol regulatory element-binding transcription }\end{array}$ \\
\hline & VEKEEAGGGGGGGISEEEAAQYDR & 0,1 & $\mathrm{~N}$-ter + 42.01 Da, K+28.03 Da & 22,9 & 7,499 & 1 & 2464,1 & SAE1_MOUSE & & sUMO-activating enzyme subunit 1; & $\begin{array}{l}\text { factor 2; } \\
\text { Ubiquitin-like 1-activating enzyme E1A; }\end{array}$ \\
\hline EG & DKVIHPC & n.d. & $2.01 \mathrm{Da}, \mathrm{K}+34.06 \mathrm{Da}$ & 18,7 & 5,898 & 1 & 2573,3 & SBSN_MOUSE & & Suprabasin; & \\
\hline GAHHAFGQGR & DMAETF & n.d. & $\mathrm{N}$-ter + & 27 & & 1 & 2072,9 & SBSN_MOUSE & E9QPB2_MOUSE & Suprabasin; & \\
\hline$M$ & ASLLLAP & n.d. & N-ter + & 85 & $-0,339$ & 1 & 2063,1 & TCPB_MOUSE & & T-complex protein 1 subunit & CCT-beta; \\
\hline M & LIIKDQDR & n.d. & N-ter + & & -0, & 1 & 2686,4 & TCPE_MOUSE & & T-complex prote & CCT-epsilon; \\
\hline & MGHRP & n.d. & a, K+34.06 Da & 25 & 3,136 & 1 & 1942,1 & TCPG_MOUSE & Q3U013_MOUSE & T-complex protein 1 subunit & CCT-gamma;Matricin;mTRiC-P5; \\
\hline NNRIARACGA & RII & n.d. & & 21 & & 1 & 1295,7 & TCPG_MOUSE & Q3U013_MOUSE & T-comple & CCT-gamma;Matricin;mTRiC-P5; \\
\hline M & & n.d. & $\mathrm{N}-$ & 60 & & 1 & 1577 & TCPZ_MOUSE & IOUSE & & CCT-zeta-1; \\
\hline MA & AVKTLNPKAEVAR & n.d. & $\mathrm{N}$-ter $+42.01 \mathrm{Da}, \mathrm{K}+34.06 \mathrm{Da}$ & 33,2 & 0,133 & 1 & 1506,0 & TCPZ_MOUSE & E9QPA6_MOUSE & T-complex protein 1 subunit zeta; & CCT-zeta-1; \\
\hline M & AEAMDLGKDPNGPTHSSTLFVR & $-0,4$ & $\mathrm{~N}$-ter $+42.01 \mathrm{Da}, \mathrm{K}+28.03 \mathrm{Da}$ & 60,6 & $-6,832$ & 1 & 2412,2 & TE2IP_MOUSE & & $\begin{array}{l}\text { Telomeric repeat-binding factor 2-interacting protein } \\
1_{1}\end{array}$ & Repressor/activator protein 1 homolog; \\
\hline M & ESSEPEPTEDASMDAFLEKFQSQPYR & n.d. & $\mathrm{N}$-ter + 42.01 Da, K +34.06 Da & 18,3 & 0,000 & 1 & 3224,4 & TTC4_MOUSE & A2AVQ9_MOUSE & Tetratricopeptide repeat protein 4; & \\
\hline M & AQGSHQIDFQVLHDLR & n.d. & $\mathrm{N}$-ter + 42.01 Da & 36,3 & $-0,786$ & 1 & 1905,0 & TAB2_MOUSE & D3Z216_MOUSE & $\begin{array}{l}\text { TGF-beta-activated kinase } 1 \text { and MAP3K7-binding } \\
\text { protein 2; }\end{array}$ & $\begin{array}{l}\text { Mitogen-activated protein nikase kinase kinase } \\
\text { 7-interacting protein 2;AK1-binding protein } \\
\text { 2;TGF-beta-activated kinase 1-binding protein } \\
2 ; \\
\text { Mitogen-activated protein kinase kinase kinase }\end{array}$ \\
\hline M & AQNSPQLDIQVLHDLR & n.d. & $\mathrm{N}$-ter + 42.01 Da & 26,4 & $-6,610$ & 1 & 1888,0 & TAB3_MOUSE & F6XIV6_MOUSE & $\begin{array}{l}\text { TGF-beta-activated kinase } 1 \text { and MAP3K7-binding } \\
\text { protein 3; }\end{array}$ & $\begin{array}{l}\text { Mitgoen-activated proteiei kinase kinase kinase } \\
\text { 7-interacting protein 3;AK1-binding protein } \\
\text { 3;TGF-beta-activated kinase 1-binding protein } \\
\text { 3. }\end{array}$ \\
\hline TSEGKM & AA. & n.d. & a & 15 & & 1 & 2291,3 & THOC2_MOUSE & & THO с & \\
\hline $\mathrm{M}$ & EH & n.d. & $\mathrm{N}-$ & & & 1 & 241 & THOCG_MOUSE & D3YUL7_MOUSE & THO complex subunit 6 & WD repeat-containing protein 58; \\
\hline M & ATTAQQSPQPVAGKR & n.d. & - & . & & 1 & 1614,9 & THUM1_MOUSE & & THUMP domain-containing protein 1 ; & \\
\hline M & SDAGGGKKPPVEPQAGPGPGR & n.d. & $\mathrm{N}$-ter + 42.01 Da, K+34.06 Da & 24,0 & $-7,484$ & 1 & 2068,1 & TEF_MOMUSE & & yrotroph embryonic factor; & \\
\hline
\end{tabular}




\begin{tabular}{|c|c|c|c|c|c|c|c|c|c|c|c|}
\hline Non Prime Site & Prime Site & 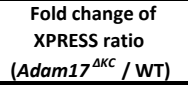 & Modifications & Hyperscore & $\begin{array}{l}\text { Mass error } \\
\text { in ppm }\end{array}$ & charge & $\begin{array}{c}\text { Precursor } \\
\text { neutral mass } \\
\text { in Da }\end{array}$ & Uniprot ID 1 & Uniprot ID 2 & Protein Name 1 & Protein Name 2 \\
\hline M & NSVGEACTDMKR & 0,2 & $\begin{array}{l}\text { N-ter + 42.01 Da, K+28.03 Da, } \\
\text { C+57.02 Da }\end{array}$ & 60,2 & $-0,573$ & 1 & 1567,7 & TRIA1_MOUSE & & TP53-regulated inhibitor of apoptosis 1; & $\begin{array}{l}\text { Protein 15E1.1;WF-1;p53-inducible cell-survival } \\
\text { factor; }\end{array}$ \\
\hline DSIFLHSTPE & APSILAPATPETVCQDR & n.d. & N-ter $+42.01 \mathrm{Da}, \mathrm{C}+57.02 \mathrm{Da}$ & 11,7 & 5,725 & 1 & 1866,9 & TANK_MOUSE & Q8C2D3_MOUSE & $\begin{array}{l}\text { TRAF family member-associated NF-kappa-B } \\
\text { activator; }\end{array}$ & TRAF-interacting protein; \\
\hline MvVsk & MNKDAQMR & n.d. & N-ter + 42.01 Da, K+34.06 Da & 30,1 & $-0,374$ & 1 & 1068,5 & ENY2_MOUSE & & $\begin{array}{l}\text { Transcription and mRNA export factor ENYY2 } \\
\text { \{ECO:0000255|HAMAP-Rule:MF__303046\}; }\end{array}$ & $\begin{array}{l}\text { Enhancer of yellow } 2 \text { transcription factor } \\
\text { homolog \{ECO:0000255|HAMAP- } \\
\text { Rule:MF_03046\}; } \\
\text { Elongin } 15 \text { kDa subunit;:Elongin-C; RNA }\end{array}$ \\
\hline M & DGEEKTYGGCEGPDAMYVK & n.d. & $\begin{array}{l}\text { N-ter + 42.01 Da, K+34.06 Da, } \\
\text { C +57.02 Da }\end{array}$ & 32,0 & $-4,896$ & 1 & 2346,0 & ELOC_MOUSE & & Transcription elongation factor B polypeptide 1 ; & $\begin{array}{l}\text { polymerase II transcription factor SIII subunit } \\
\text { c;sIII p15;stromal membrane-associated } \\
\text { protein SMAP1B homolog; }\end{array}$ \\
\hline M & AAESALQVVEKLLAR & n.d. & $\mathrm{N}$-ter + 42.01 Da, K+34.06 Da & 19,1 & $-1,834$ & 1 & 1688,0 & ELOA1_MOUSE & & Transcription elongation factor B polypeptide 3; & $\begin{array}{l}\text { Elongin } 110 \text { kDa subunit;:Elongin-A;RNA } \\
\text { polymerase II transcription factor SIII subunit } \\
\text { A1;;SIII p110; }\end{array}$ \\
\hline M & ALETVPKDLR & n.d. & $\mathrm{N}$-ter + 42.01 Da, K+34.06 Da & 29,4 & $-1,641$ & 1 & 1216,7 & SPT4A_MOUSE & SPT4B_MOUSE & Transcription elongation factor SPT4-A; & $\begin{array}{l}\text { DRB sensitivity-inducing factor small subunit } \\
\text { 1; Transcription elongation factor SPT } 4 \text { i; }\end{array}$ \\
\hline RESQMKETIM & NQEKLAKLOAQVR & $-0,1$ & $\mathrm{~N}$-ter + 42.01 Da, K+28.03 Da & 47,7 & $-4,098$ & 1 & 1754,0 & BT3L4_MOUSE & BTF3_MOUSE & Transcription factor BTF3 homolog 4; & Basic transcription factor 3-like 4; \\
\hline HVHTVPRALR & MEEDSIHLPTHLR & n.d. & $\mathrm{N}$-ter $+42.01 \mathrm{Da}$ & 17,5 & 0,555 & 1 & 1618,8 & ETV6_MOUSE & E9QAJ7_MOUSE & Transcription factor ETV6; & $\begin{array}{l}\text { ETS translocation variant 6;ETS-related protein } \\
\text { Tel1; }\end{array}$ \\
\hline M & SETPAQSSIKQER & n.d. & N-ter + 42.01 Da, K+34.06 Da & 57,6 & $-0,780$ & 1 & 1535,8 & ETV6_MOUSE & & Transcription factor ETVG; & $\begin{array}{l}\text { ETS translocation variant 6;ETS-related protein } \\
\text { Tel1; }\end{array}$ \\
\hline $\begin{array}{l}\text { MCTK } \\
\text { PGGLLPGAA }\end{array}$ & $\begin{array}{l}\text { MEQPFYHDDSYAAAGYGR } \\
\text { AAAAAAAAAAAASSPVGVGQR }\end{array}$ & $\begin{array}{l}\text { n.d. } \\
\text { n.d. }\end{array}$ & $\begin{array}{l}\text { N-ter + 42.01 Da } \\
\text { N-ter + 42.01 Da }\end{array}$ & $\begin{array}{l}15,5 \\
29,2\end{array}$ & $\begin{array}{l}1,697 \\
-8,133\end{array}$ & $\begin{array}{l}1 \\
1\end{array}$ & $\begin{array}{l}2118,9 \\
1779,9\end{array}$ & $\begin{array}{l}\text { JUNB_MOUSE } \\
\text { SOX_MOUSE }\end{array}$ & A2AM37_MOUSE & $\begin{array}{l}\text { Transcription factor jun-B; } \\
\text { Transcription factor SOX-3; }\end{array}$ & MyD21; \\
\hline m & ANSANTNTVPKLYR & n.d. & N-ter + 42.01 Da, K+34.06 Da & 57,3 & 0,000 & 1 & 1623,9 & TF2AA_MOUSE & & Transcription initiation factor IIA subunit 1; & $\begin{array}{l}\text { General transcription factor IIA subunit 1; } \\
\text { Cell cycle gene } 1 \text { protein; } ; B P \text {-associated factor }\end{array}$ \\
\hline PRRIRMEDIK & KAFPSHSESSIR & 0,9 & N-ter + 42.01 Da, K+28.03 Da & 20,8 & 2,118 & 1 & 1414,7 & TAF1_MOUSE & SAR1B9_MOUSE & Transcription initiation factor TFIID subunit 1; & $\begin{array}{l}250 \text { kDa; Transcription initiation factor TFIID } 250 \\
\text { kDa subunit; }\end{array}$ \\
\hline M & $\begin{array}{l}\text { EPAQQPPPQPAPQGPAPPSVSPAGTPAAPPAPPAG } \\
\text { HQVVHVR }\end{array}$ & n.d. & $\mathrm{N}$-ter + 42.01 Da & 45,1 & $-0,845$ & 1 & 4257,2 & YAP1_MOUSE & G3UYA6_MOUSE & Transcriptional coactivator YAP1; & $\begin{array}{l}\text { Protein yorkie homolog;Yes-associated protein } \\
\text { YAP65 homolog; } \\
\text { ATP-dependent helicase ATRX;HP1 alpha- }\end{array}$ \\
\hline M & MENNKEEGASTSEKSR & 0,3 & $\mathrm{~N}$-ter + 42.01 Da, K +28.03 Da & 34,0 & $-0,896$ & 1 & 1893,9 & ATRX_MOUSE & Q8C2S3_MOUSE & Transcriptional regulator ATRX; & $\begin{array}{l}\text { interacting protein;HP1-BP38 } \\
\text { protein;Heterochromatin protein 2;--linked } \\
\text { nuclear protein; }\end{array}$ \\
\hline M & EGEAVEAIVEESETFIKGKER & n.d. & $\mathrm{N}$-ter + 42.01 Da, K +34.06 Da & 29,6 & $-0,656$ & 1 & 2590,4 & CTCF_MOUSE & & Transcriptional repressor CTCF; & $\begin{array}{l}\text { 11-zinc finger protein;CCCTC-binding } \\
\text { factor; CTCFL paralog; }\end{array}$ \\
\hline M & ANKGPSYGMSR & n.d. & $\mathrm{N}$-ter + 42.01 Da, K+34.06 Da & 43,8 & 0,562 & 1 & 1242,6 & TAGL_MOUSE & & Transgelin; & $\begin{array}{l}\text { Actin-associated protein p27;Smooth muscle } \\
\text { protein 22-alpha; }\end{array}$ \\
\hline M & ANRGPSYGLSR & n.d. & $\mathrm{N}$-ter $+42.01 \mathrm{Da}$ & 43,9 & $-2,867$ & 1 & 1218,6 & TAGL2_MOUSE & TAGL3_MOUSE & Transgelin-2; & SM22-beta; \\
\hline RVVRNNLRVR & LGDVISIQPCPDVKYGKR & 0,1 & $\begin{array}{l}\mathrm{N} \text {-ter + 42.01 Da, } \mathrm{K}+28.03 \mathrm{Da}, \\
\mathrm{C}+57.02 \mathrm{Da}\end{array}$ & 21,1 & 3,310 & 1 & 2142,2 & TERA_MOUSE & & Transitional endoplasmic reticulum ATPase; & $\begin{array}{l}155 \mathrm{Mg}(2+) \text {-ATPase p97 subunit; Valosin- } \\
\text { containing protein; }\end{array}$ \\
\hline KDPDGKVVLS & RQYGSEGR & n.d. & $\mathrm{N}$-ter $+42.01 \mathrm{Da}$ & 16,5 & $-1,306$ & 1 & 993,5 & TMED4_MOUSE & TMED9_MOUSE & Transmembrane emp24 domain-containing protein 4 & $\begin{array}{l}\text { Endoplasmic reticulum stress-response protein } \\
25 \cdot \mathrm{p} 24 \text { family protein alpha-3.p26: }\end{array}$ \\
\hline & EASGSS & n.d. & $\mathrm{N}$-ter $+42.01 \mathrm{Da}$ & 81 & $-0,757$ & 1 & 1847,8 & TMM40_MOUSE & E9QN39_MOUSE & Transmer & 25;p24 family protein alpha-3;p26; \\
\hline & SKS & n.d. & $1.06 \mathrm{Da}$ & & & 1 & 3174,8 & TCHL1_MOUSE & & & \\
\hline M & ENMAEEELLPQEKEEAQVR & n.d. & N-ter + 42.01 Da, K+34.06 Da & 17,0 & 1,894 & 1 & 2478,2 & TRM1L_MOUSE & & TRMT1 & $\begin{array}{l}\text { Methyytransferase-like protein } 1 \\
\text { \{ECO:0000255|HAMAP-Rule:MF_03055\};;RNA }\end{array}$ \\
\hline MM & AGAEAPQPQKR & n.d. & $\mathrm{N}$-ter + 42.01 Da, K +34.06 Da & 25,7 & 1,545 & 1 & 1227,7 & TRMB_MOUSE & D3Z7D2_MOUSE & $\begin{array}{l}\text { tRNA (guanine-N(7)--methyltransferase } \\
\text { \{ECO:0000255|HAMAP-Rule:MF_03055\}; }\end{array}$ & $\begin{array}{l}\text { (guanine(46)-N(7))-methyyltransferase } \\
\text { \{ECO:0000255|HAMAP- } \\
\text { Rule:MF_03055\};:RNA(m7G46)- } \\
\text { methyltransferase \{ECO:0000255|HAMAP- } \\
\text { Rule:MF_03055); }\end{array}$ \\
\hline M & AGNKVPPALASHQPDR & n.d. & $\mathrm{N}$-ter + 42.01 Da, K +34.06 Da & 19,5 & $-4,724$ & 1 & 1732,9 & TRUA_MOUSE & H7BX59_MOUSE & tRNA pseudouridine synthase A, mitochondrial; & $\begin{array}{l}\text { tRNA pseudouridine(38-40) synthase;tRNA } \\
\text { pseudouridylate synthase I;tRNA-uridine } \\
\text { isomerase I; }\end{array}$ \\
\hline $\begin{array}{l}M \\
M\end{array}$ & $\begin{array}{l}\text { DAIKKK } \\
\text { AGLNSLEAVKR }\end{array}$ & $\begin{array}{l}\text { n.d. } \\
\text { n.d. }\end{array}$ & $\begin{array}{l}\text { N-ter + 42.01 Da, } \mathrm{K}+34.06 \mathrm{Da} \\
\text { N-ter + 42.01 Da, K+34.06 Da }\end{array}$ & $\begin{array}{l}16,7 \\
57,6\end{array}$ & $\begin{array}{l}-1,735 \\
-1,701\end{array}$ & $\begin{array}{l}1 \\
1\end{array}$ & $\begin{array}{l}976,7 \\
1232,7\end{array}$ & $\begin{array}{l}\text { TPM1_MOUSE } \\
\text { TPM4_MOUSE }\end{array}$ & TPM2_MOUSE & $\begin{array}{l}\text { Tropomyosin alpha- } 1 \text { chain; } \\
\text { Tropomyosin alpha-4 chain; }\end{array}$ & $\begin{array}{l}\text { Alpha-tropomyosin;Tropomyosin-1; } \\
\text { Tropomyosin-4; }\end{array}$ \\
\hline VAIDNKIEQA & MDLVKSHLMYAVR & n.d. & $\mathrm{N}$-ter + 42.01 Da, K+34.06 Da & 22,0 & $-0,975$ & 1 & 1637,9 & T22D1_MOUSE & H3BLI9_MOUSE & TSC22 domain family protein 1 ; & $\begin{array}{l}\text { Tropomyosin-4; } \\
\text { Regulatory protein TSC-22;TGFB-stimulated } \\
\text { clone } 22 \text { homolog;TSC22-related inducible } \\
\text { leucine zipper 1b;Transforming growth factor } \\
\text { beta-1-induced transcript } 4 \text { protein; }\end{array}$ \\
\hline
\end{tabular}




\begin{tabular}{|c|c|c|c|c|c|c|c|c|c|c|c|}
\hline Non Prime Site & Prime Site & 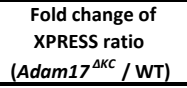 & Modifications & Hyperscore & $\begin{array}{c}\text { Mass error } \\
\text { in ppm }\end{array}$ & Charge & $\begin{array}{c}\text { Precursor } \\
\text { neutral mass } \\
\text { in Da }\end{array}$ & Uniprot ID 1 & Uniprot ID 2 & Protein Name 1 & Protein Name 2 \\
\hline M & SLSHLYR & n.d. & N-ter + 42.01 Da & 16,6 & $-1,960$ & 1 & 916,5 & TFP11_MOUSE & D3YWC8_MOUSE & Tuftelin-interacting protein 11; & $\begin{array}{l}\text { Septin and tuftelin-interacting protein } \\
\text { 1; Tuftelin-interacting protein } 39 ;\end{array}$ \\
\hline GQLDGSAQLD & KADTELTR & n.d. & $\mathrm{N}$-ter + 42.01 Da, K+34.06 Da & 17,8 & $-0,297$ & 1 & 1008,6 & TNR17_MOUSE & & $\begin{array}{l}\text { Tumor necrosis factor receptor superfamily member } \\
{ }_{17} \text {. }\end{array}$ & B-cell maturation protein; \\
\hline M & SHQTGIQASEDVKEIFAR & n.d. & $\mathrm{N}$-ter + 42.01 Da, K+34.06 Da & 65,5 & 0,812 & 1 & 2091,1 & TWF1_MOUSE & & Twinfilin-1; & Protein A6; \\
\hline LRKRIREDRK & ATTAQKVQQMKRQR & 10,6 & $\mathrm{~N}$-ter + 42.01 Da, K+28.03 Da & 30,4 & 1,546 & 1 & 1614,9 & PTN2_MOUSE & D3Z6W2_MOUSE & Tyrosine-protein phosphatase non-receptor type 2 & MPTP; \\
\hline SAGNRDSGAM & GDAPSPEEKLHLITR & n.d. & N-ter + 42.01 Da, K+34.06 Da & 48,7 & $-0,230$ & 1 & 1738,0 & SYYC_MOUSE & A2A7S7_MOUSE & Tyrosine--tRNA ligase, cytoplasmic; & Tyrosyl-tRNA synthetase; \\
\hline M & AANATTNPSQLLPLELVDKCIGSR & n.d. & $\begin{array}{l}\text { N-ter + 42.01 Da, K+34.06 Da, } \\
\mathrm{C}+57.02 \mathrm{Da}\end{array}$ & 70,7 & $-2,419$ & 1 & 2643,4 & LSMS_MOUSE & & U6 snRNA-associated Sm-like protein LSm5; & \\
\hline M & AENGESSGPPRPSR & n.d. & $\mathrm{N}$-ter + 42.01 Da & 23,8 & $-1,078$ & 1 & 1481,7 & UBQL2_MOUSE & & Ubiquilin-2; & $\begin{array}{l}\text { Chap1;DSK2 homolog;Protein linking IAP with } \\
\text { cytoskeleton 2;Ubiquitin-like product } \\
\text { Chap1/Dsk2; }\end{array}$ \\
\hline REEMERMLHV & VDGKVPDTLR & $-0,1$ & $\mathrm{~N}$-ter + 42.01 Da, K+28.03 Da & 18,5 & $-0,854$ & 1 & 1168,6 & F8VPZ3_MOUSE & & $\begin{array}{l}\text { Ubiquitin carboxyl-terminal hydrolase } \\
\text { \{ECO:0000256|RuleBase:RU004435\}; }\end{array}$ & \\
\hline M & FVQEEKIFAGKVLR & n.d. & $\mathrm{N}$-ter + 42.01 Da, K+34.06 Da & 24,0 & 1,730 & 1 & 1904,1 & UBAC1_MOUSE & & Ubiquitin-associated domain-containing protein 1 ; & $\begin{array}{l}\text { E3 ubiquitin-protein ligase subunit KPC2;Kip1 } \\
\text { ubiquitination-promoting complex protein 2; }\end{array}$ \\
\hline SVHTRIAYQS & SASPPDSAPGSVANGHGGGR & n.d. & $\mathrm{N}$-ter + 42.01 Da & 17,2 & 0,275 & 1 & 1818,8 & UBAP2_MOUSE & A2AMY5_MOUSE & Ubiquitin-associated protein 2; & Protein lingerer homolog 1 ; \\
\hline M & AATGSGVKVPR & 0,0 & $\mathrm{~N}$-ter + 42.01 Da, K+28.03 Da & 56,6 & 0,082 & 1 & 1212,7 & UB2V1_MOUSE & D3Z113_MOUSE & Ubiquitin-conjugating enzyme E2 variant 1 ; & CROC-1; \\
\hline M & AVSTGVKVPR & 0,0 & $\mathrm{~N}$-ter + $42.01 \mathrm{Da}, \mathrm{K}+28.03 \mathrm{Da}$ & 52,2 & 0,277 & 1 & 1082,7 & UB2V2_MOUSE & & Ubiquitin-conjugating enzyme E2 variant 2 ; & Ubc-like protein MMS2; \\
\hline M & SKVSFKITLTSDPR & 0,2 & $\mathrm{~N}$-ter + 42.01 Da, K+28.03 Da & 25,2 & 1,370 & 1 & 1676,0 & UFM1_MOUSE & H7BWZ1_MOUSE & Ubiquitin-fold modifier 1 ; & \\
\hline M & SSHVPADMINLR & n.d. & $\mathrm{N}$-ter + 42.01 Da & 16,2 & $-3,472$ & 1 & 1380,7 & UBL3_MOUSE & & Ubiquitin-like protein 3; & $\begin{array}{l}\text { Membrane-anchored ubiquitin-fold } \\
\text { protein:Protein HCG-1: }\end{array}$ \\
\hline $\begin{array}{l}M \\
M\end{array}$ & $\begin{array}{l}\text { QNVINTVKGK } \\
\text { Q }\end{array}$ & n.d. & N-ter + 42.01 Da, K +34.06 Da & 24,8 & $-0,223$ & 1 & 1340,8 & ATG3_MOUSE & & Ubiquitin-like-conjugating enzyme ATG3; & Autophagy-related protein 3; \\
\hline MAHYHDNY & $\begin{array}{l}\text { AAEHDSDSVEPPGSEALLDAVLR } \\
\text { GKNDEVEFVR }\end{array}$ & $\begin{array}{l}\text { n.d. } \\
\text { n.d. }\end{array}$ & $\begin{array}{l}\text { N-ter + 42.01 Da } \\
\text { N-ter + 42.01 Da, K +34.06 Da }\end{array}$ & $\begin{array}{l}26,5 \\
17,5\end{array}$ & $\begin{array}{l}-1,032 \\
8,972\end{array}$ & $\begin{array}{l}1 \\
1\end{array}$ & $\begin{array}{l}2419,2 \\
1267,7\end{array}$ & $\begin{array}{l}\text { CA131_MOUSE } \\
\text { URIC_MOUSE }\end{array}$ & & $\begin{array}{l}\text { Uncharacterized protein C1orf131 homolog; } \\
\text { Uricase: }\end{array}$ & Urate oxidase; \\
\hline M & EGLKDKTLQELEEMQNDPEAIAR & 0,3 & $\mathrm{~N}$-ter $+42.01 \mathrm{Da}, \mathrm{K}+28.03 \mathrm{Da}$ & 38,0 & $-0,692$ & 1 & 2885,4 & VP37C_MOUSE & & Vacuolar protein sorting-associated protein $37 \mathrm{C}$; & ESCRT-I complex subunit VPS37C; \\
\hline $\begin{array}{l}M \\
M\end{array}$ & $\begin{array}{l}\text { SILYVSPHPDAFPSLR } \\
\text { ALPOPYHYR }\end{array}$ & n.d. & $\mathrm{N}$-ter $+42.01 \mathrm{Da}$ & 32,3 & $-0,054$ & 1 & 1840,0 & SYVC_MOUSE & G3UY93_MOUSE & Valine--tRNA ligase; & Protein G7a;Valyl-tRNA synthetase; \\
\hline M & $\begin{array}{l}\text { AAPQDVHVR } \\
\text { AASAASSEHFEKLHEIFR }\end{array}$ & 0,3 & $\begin{array}{l}\text { N-ter + 42.01 Da } \\
\text { N-ter + 42.01 Da, } \mathrm{K}+28.03 \mathrm{Da}\end{array}$ & 27,8 & $\begin{array}{l}0,483 \\
1,998\end{array}$ & 1 & 2009,1 & $\begin{array}{l}\text { SEC20_MOUSE } \\
\text { VT11B_MOUSE }\end{array}$ & $\begin{array}{l}\text { B8JJ4_MOUSE } \\
\text { Q91XH6_MOUSE }\end{array}$ & $\begin{array}{l}\text { Vesilc transport protein SEEC2; } \\
\text { Vesicle transport through interaction with t-SNARES }\end{array}$ & Vesicle transport V-SNARE protein Vti-like \\
\hline WEPTLPPPTL & ANASAYLANTSASPTAAGSAR & n.d. & $\mathrm{N}$-ter + 42.01 Da & 24,5 & $-2,556$ & 1 & 1993,0 & VACHT_MOUSE & & $\begin{array}{l}\text { homolog } 18 ; \\
\text { Vesicular acetylcholine transporter; }\end{array}$ & $\begin{array}{l}\text {;;Vti1-rp1; } \\
\text { Solute carrier family } 18 \text { member 3; }\end{array}$ \\
\hline M & SSVAVLTQESFAEHR & n.d. & $\mathrm{N}$-ter $+42.01 \mathrm{Da}$ & 53,4 & $-0,763$ & 1 & 1701,8 & VIGLN_MOUSE & & Vigilin; & High density lipoprotein-binding protein; \\
\hline M & AVPPTYADLGKSAR & 0,3 & $\mathrm{~N}$-ter + 42.01 Da, K+28.03 Da & 54,7 & $-0,132$ & 1 & 1514,8 & VDAC1_MOUSE & F2Z471_MOUSE & Voltage-dependent anion-selective channel protein & $\begin{array}{l}\text { Outer mitochondrial membrane protein porin } \\
; \text {; 1;lasmalemmal porin;Voltage-dependent } \\
\text { anion-selective channel protein } 5 \text {; }\end{array}$ \\
\hline HKTCYFLGSD & MEAEEDPSPCASSGSGR & n.d. & $\mathrm{N}$-ter + 42.01 Da, C+57.02 Da & 18,2 & $-4,637$ & 1 & 1807,7 & CAC1F_MOUSE & Q7TN13_MOUSE & $\begin{array}{l}\text { Voltage-dependent L-type calcium channel subunit } \\
\text { alpha-1F; }\end{array}$ & $\begin{array}{l}\text { Voltage-gated calcium channel subunit alpha } \\
\text { Cav1.4; }\end{array}$ \\
\hline VHTISLTSAD & RTATEFLR & n.d. & $\mathrm{N}$-ter + 42.01 Da & 11,7 & 0,193 & 1 & 1034,6 & VWA3A_MOUSE & & $\begin{array}{l}\text { von Willebrand factor } A \text { domain-containing protein } \\
3 \mathrm{~A} ;\end{array}$ & \\
\hline M & DFSKLPKIR & 0,2 & $\mathrm{~N}$-ter + $42.01 \mathrm{Da}, \mathrm{K}+28.03 \mathrm{Da}$ & 28,2 & 1,350 & 1 & 1331,8 & VATA_MOUSE & D3YZ23_MOUSE & V-type proton ATPase catalytic subunit A; & $\begin{array}{l}\text { V-ATPase } 69 \text { kDa subunit;Vacuolar proton } \\
\text { pump subunit alpha; }\end{array}$ \\
\hline M & DFSKLPK & n.d. & N-ter + 42.01 Da, K+34.06 Da & 14,2 & $-1,672$ & 1 & 1074,6 & VATA_MOUSE & D3YZ23_MOUSE & V-type proton ATPase catalytic subunit A; & $\begin{array}{l}\text { V-ATPase } 69 \text { kDa subunit; Vacuolar proton } \\
\text { pump subunit alpha: }\end{array}$ \\
\hline M & SEFWLSAPGDKENLQALER & n.d. & N-ter + 42.01 Da, K+34.06 Da & 49,6 & 0,882 & 1 & 2378,2 & VATC2_MOUSE & D3YVW5_MOUSE & V-type proton ATPase subunit C 2; & Vacuolar proton pump subunit C 2; \\
\hline M & ASQSQGIQQLLQAEKR & n.d. & N-ter + 42.01 Da, K+34.06 Da & 70,0 & $-1,074$ & 1 & 1860,0 & VATG1_MOUSE & & V-type proton ATPase subunit $\mathrm{G}$; & $\begin{array}{l}\text { V-ATPase } 13 \mathrm{kDa} \text { subunit 1;Vacuolar proton } \\
\text { pump subunit } \mathrm{G} \text { : }\end{array}$ \\
\hline AHRIGVTAIA & TTSDCKRIISGGGEGEVR & n.d. & $\begin{array}{l}\text { N-ter + 42.01 Da, K +34.06 Da, } \\
\mathrm{C}+57.02 \mathrm{Da}\end{array}$ & 24,3 & 0,050 & 1 & 1997,0 & WDR16_MOUSE & & WD repeat-containing protein 16; & \\
\hline M & ATEEKKPETEAAR & 0,2 & N-ter + 42.01 Da, K+28.03 Da & 38,8 & $-0,577$ & 1 & 1556,8 & WDR5_MOUSE & & WD repeat-containing protein 5 ; & $\begin{array}{l}\text { BMP2-induced 3-kb gene protein;WD repeat- } \\
\text { containing protein BIG-3; }\end{array}$ \\
\hline RISKAHSAPI & NSVLLVDENALVTGDDTGGIR & n.d. & $\mathrm{N}$-ter + $42.01 \mathrm{Da}$ & 17,5 & $-1,862$ & 1 & 2199,1 & WDR55_MOUSE & & WD repeat-containing protein 55; & \\
\hline SVGDGETVEF & DVVEGEKGAEAANVTGPDGVPVEGSR & 8,8 & N-ter + 42.01 Da, K+28.03 Da & 17,6 & 2,336 & 1 & 2608,3 & YBOX__MOUSE & & Y-box-binding protein 3; & $\begin{array}{l}\text { Cold shock domain-containing protein A;DNA- } \\
\text { binding protein A;--box protein 3; }\end{array}$ \\
\hline M & AGGVDGPIGIPFPDHSSDILSGLNEQR & n.d. & $\mathrm{N}$-ter + 42.01 Da & 31,6 & 1,934 & 1 & 2789,4 & ZBT7A_MOUSE & D3YZ18_MOUSE & Zinc finger and BTB domain-containing protein 7A; & $\begin{array}{l}\text { Leukemia//lymphoma-related factor;POZ and } \\
\text { Krueppel erythroid myeloid ontogenic factor; }\end{array}$ \\
\hline GLRSLGPPEA & RSPGSVLPNR & n.d. & $\mathrm{N}$-ter $+42.01 \mathrm{Da}$ & 20,1 & 3,107 & 1 & 1123,6 & ZN541_MOUSE & & Zinc finger protein 541 & Spermatogenic cell HDAC-interacting protein 1 ; \\
\hline FESFSSPFSS & QSASSTLAALSKKVSER & 10,6 & N-ter + 42.01 Da, K+28.03 Da & (2) & 954 & 1 & 1860,0 & ZN827_MOUSE & G5EE & protein 827; & \\
\hline RCMLDMCTGW & KTKEEEELR & n.d. & N-ter + 42.01 Da, K+34.06 Da & 24,3 & 5,653 & 1 & 1270,8 & ZAN_MOUSE & EPPWQ7_MOU & Zonadhesin; & \\
\hline
\end{tabular}




\begin{tabular}{|c|c|c|c|c|c|c|c|c|c|c|c|}
\hline Non Prime Site & Prime Site & 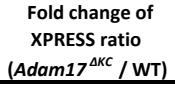 & Modifications & Hyperscore & $\begin{array}{c}\text { Mass error } \\
\text { in ppm }\end{array}$ & Charge & $\begin{array}{c}\begin{array}{c}\text { Precursor } \\
\text { neutral mass } \\
\text { in Da }\end{array} \\
\end{array}$ & Uniprot ID 1 & Uniprot ID 2 & Protein Name 1 & Protein Name 2 \\
\hline M & AAIDLSHGLLSR & $-0,2$ & $\mathrm{~N}$-ter+42.01 Da & 40,7 & $-0,540$ & 1 & 1293,7 & $\begin{array}{l}\text { E9Q2S7_MOUSE } \\
\end{array}$ & & & \\
\hline M & AASAPPPPDKLEGGSGPAPPPAPPSTGR & 0,0 & $\mathrm{~N}$-ter + 42.01 Da, K+34.06 Da & 44,1 & 1,243 & 1 & 2651,4 & Q80V76_MOUSE & & & \\
\hline M & AELVQGQSAPVGMKAEGFVDALHR & 0,1 & $\mathrm{~N}$-ter + 42.01 Da, K+34.06 Da & 52,2 & 0,580 & 1 & 2585,4 & A2AA72_MOUSE & Q3TIX6_MOUSE & & \\
\hline VLHGYEAVKE & AFTDHGEEFAGR & 0,2 & $\mathrm{~N}$-ter + 42.01 Da & 18,5 & 1,377 & 1 & 1377,6 & Q148B1_MOUSE & & & \\
\hline SKKKEKTKAR & AGAEQHSAGAIAWR & 0,3 & $\mathrm{~N}$-ter + 42.01 Da & 20,9 & $-0,818$ & 1 & 1465,7 & G3X9Jo_MOUSE & & & \\
\hline AELQASKGAM & AGHEAEDVLDILR & 0,6 & $\mathrm{~N}$-ter + 42.01 Da & 22,1 & $-0,135$ & 1 & 1478,7 & EOCZ26_MOUSE & E9PYP3_MOUSE & & \\
\hline RQGQASAQGR & AGSQGQAQGRVGSSADR & 5,3 & $\mathrm{~N}$-ter + 42.01 Da & 21,4 & $-1,612$ & 1 & 1672,8 & E9Q019_MOUSE & J3QM50_MOUSE & & \\
\hline RGQSSSANRR & AGSSSGSGVQGASAGGLAADASR & 5,7 & $\mathrm{~N}$-ter + 42.01 Da & 47,0 & 6,870 & 1 & 1961,9 & E9Q019_MOUSE & FILA_MOUSE & & \\
\hline RGQSSSANRR & AGSSSGSGVOGTSAGGLAADASR & 7,4 & $\mathrm{~N}$-ter + 42.01 Da & 37,2 & 1,704 & 1 & 1991,9 & E9Q019_MOUSE & J3QM50_MOUSE & & \\
\hline M & AGTTTIEAVKR & 8,1 & $\mathrm{~N}$-ter + 42.01 Da, K+28.03 Da & 53,5 & 0,493 & 1 & 1215,7 & D3Z2H9_MOUSE & E9Q7Q3_MOUSE & & \\
\hline M & ANSTGKAPPDER & 10,6 & $\mathrm{~N}$-ter + 42.01 Da, $\mathrm{K}+34.06 \mathrm{Da}$ & 18,2 & 1,364 & 1 & 1317,7 & Q9D982_MOUSE & E9Q7E2_MOUSE & & \\
\hline M & ASEAGGIGGGGGGGKIR & n.d. & $\mathrm{N}$-ter + 42.01 Da, $\mathrm{K}+34.06 \mathrm{Da}$ & 21,1 & $-3,722$ & 1 & 1475,8 & E9Q3G__MOUSE & & & \\
\hline M & ASSAHLVTIKR & n.d. & $\mathrm{N}$-ter + 42.01 Da, $\mathrm{K}+34.06 \mathrm{Da}$ & 23,4 & $-3,411$ & 1 & 1257,8 & E9PVX6_MOUSE & & & \\
\hline M & ATPDSLALFTGLGLSENKAR & n.d. & $\mathrm{N}$-ter + 42.01 Da, $\mathrm{K}+34.06 \mathrm{Da}$ & 18,4 & 1,730 & 1 & 2136,2 & Q8BML9_MOUSE & D3Z158_MOUSE & & \\
\hline CQESCIEPIR & CSETCIEPIR & n.d. & N-ter + 42.01 Da, C +57.02 Da & 30,0 & $-0,153$ & 1 & 1305,6 & D3Z724_MOUSE & & & \\
\hline M & DGVVTDLIAVGLKR & n.d. & $\mathrm{N}$-ter + 42.01 Da, $\mathrm{K}+34.06 \mathrm{Da}$ & 38,0 & 0,481 & 1 & 1662,0 & G3UXAG_MOUSE & G8JL74_MOUSE & & \\
\hline M & DYLTTFTGKSGR & n.d. & $\mathrm{N}$-ter + 42.01 Da, $\mathrm{K}+34.06 \mathrm{Da}$ & 45,1 & 2,253 & 1 & 1551,8 & E9Q0A7_MOUSE & & & \\
\hline M & EAATHQVGMAGPR & n.d. & $\mathrm{N}$-ter + 42.01 Da & 38,5 & $-0,467$ & 1 & 1496,7 & E9Q7K1_MOUSE & & & \\
\hline M & EDPGEDTSIHR & n.d. & $\mathrm{N}$-ter + 42.01 Da & 28,0 & 4,407 & 1 & 1427,6 & Q80X98_MOUSE & & & \\
\hline M & EDQSPAEEKGLR & n.d. & $\mathrm{N}$-ter + 42.01 Da, $\mathrm{K}+34.06 \mathrm{Da}$ & 24,8 & 2,234 & 1 & 1564,8 & B1AS67_MOUSE & & & \\
\hline M & EEEQDLPEQPVKKAK & n.d. & $\mathrm{N}$-ter + 42.01 Da, $\mathrm{K}+28.03 \mathrm{Da}$ & 13,1 & $-1,529$ & 1 & 2024,1 & Q6P4T3_MOUSE & A2A953_MOUSE & & \\
\hline SPVHPESSEG & EEHSVVPQR & n.d. & $\mathrm{N}$-ter $+42.01 \mathrm{Da}$ & 18,2 & $-1,335$ & 1 & 1121,6 & E9QPZ3_MOUSE & FILA2_MOUSE & & \\
\hline SGRRRRRRGE & EGHDPKEPEQLR & n.d. & $\mathrm{N}$-ter + 42.01 Da, $\mathrm{K}+34.06 \mathrm{Da}$ & 30,8 & 2,494 & 1 & 1640,8 & B2RXM2_MOUSE & E9Q7H5_MOUSE & & \\
\hline WKCKAEALQQ & EIOTLTKR & n.d. & $\mathrm{N}$-ter $+42.01 \mathrm{Da}, \mathrm{K}+34.06 \mathrm{Da}$ & 13,8 & $-1,220$ & 1 & 1063,6 & Q9ROU9_MOUSE & & & \\
\hline SYKNATEFDF & EKDKYDIR & n.d. & $\mathrm{N}$-ter $+42.01 \mathrm{Da}, \mathrm{K}+34.06 \mathrm{Da}$ & 12,6 & $-1,189$ & 1 & 1175,7 & F7BJB9_MOUSE & & & \\
\hline M & EKEEETTR & n.d. & $\mathrm{N}$-ter $+42.01 \mathrm{Da}, \mathrm{K}+28.03 \mathrm{Da}$ & 45,1 & 1,798 & 1 & 1221,6 & G5E8K8_MOUSE & E9Q616_MOUSE & & \\
\hline M & EMTSTSLKR & n.d. & $\mathrm{N}$-ter $+42.01 \mathrm{Da}, \mathrm{K}+28.03 \mathrm{Da}$ & 45,0 & $-2,072$ & 1 & 1252,6 & Q91VU7_MOUSE & & & \\
\hline M & ENNEKKNIVYGSDVEMEHER & n.d. & $\mathrm{N}$-ter $+42.01 \mathrm{Da}, \mathrm{K}+34.06 \mathrm{Da}$ & 27,4 & 2,177 & 1 & 2660,3 & Q3UOP4_MOUSE & & & \\
\hline RSPVHPESSE & GEEHSVVPQR & n.d. & $\mathrm{N}$-ter $+42.01 \mathrm{Da}$ & 15,1 & 2,118 & 1 & 1178,6 & E9QPZ3_MOUSE & FILA2_MOUSE & & \\
\hline GQSSSANRRA & GSSSGSGVQGTSAGGLAADASR & n.d. & $\mathrm{N}$-ter + 42.01 Da & 41,1 & 0,260 & 1 & 1920,9 & E9Q019_MOUSE & J3QM50_MOUSE & & \\
\hline GSSADRQGRR & GVSESOASDSEGHSDFSEGQAVGAHR & n.d. & $\mathrm{N}$-ter + 42.01 Da & 24,4 & 1,607 & 1 & 2672,1 & J3QM50_MOUSE & J3QMA3_MOUSE & & \\
\hline IASLSYGSGP & $\begin{array}{l}\text { HIVLATTANQPIPR } \\
\end{array}$ & n.d. & $\mathrm{N}$-ter $+42.01 \mathrm{Da}$ & 23,4 & 4,193 & 1 & 1571,9 & F6X714_MOUSE & & & \\
\hline PDKVLLEDAM & KLFDCLPGGTEPEVALHR & n.d. & N-ter + 42.01 Da, K+34.06 Da, & 18,0 & 1,653 & 1 & 2114,1 & FGTNE3_MOUSE & & & \\
\hline & LALAELHHSLVSLLVR & n.d. & $\begin{array}{l}\mathrm{C}+5 \mathrm{~s} .02 \mathrm{Da} \\
\mathrm{N} \text { - }+ \text { + }+42.01 \mathrm{Da}\end{array}$ & 27,5 & $-5,997$ & 1 & 1699,0 & F78YGG_MOUSE & & & \\
\hline RTPTMQECEV & $\begin{array}{l}\text { LGEEIGLPKR } \\
\end{array}$ & n.d. & $\mathrm{N}$-ter + 42.01 Da, K+28.03 Da & 18,7 & $-2,788$ & 1 & 1180,7 & Q2MHN3_MOUSE & & & \\
\hline M & LHSHCOSFR & n.d. & $\mathrm{N}$-ter + 42.01 Da, C+57.02 Da & 23,7 & 0,000 & 1 & 1343,6 & E9PX37_MOUSE & & & \\
\hline LDCLSQVTTF & LKGVTIPNSGADTLGR & n.d. & N-ter + 42.01 Da, K+34.06 Da & 24,1 & 3,819 & 1 & 1674,0 & E9PZP8_MOUSE & & & \\
\hline M & LNMWKVR & n.d. & N-ter + 42.01 Da, K+28.03 Da & 34,7 & 0,000 & 1 & 1146,6 & QSSUH7_MOUSE & Q5SUH6_MOUSE & & \\
\hline TATHAGTARE & LPAEFDKAAEEVKR & n.d. & N-ter + 42.01 Da, K+28.03 Da & 32,2 & $-8,456$ & 1 & 1699,9 & Q4VWZ5_MOUSE & 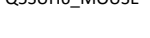 & & \\
\hline M & LSPAMSFLKSFPPPGSADGLR & n.d. & N-ter + 42.01 Da, K+34.06 Da & 51,5 & 0,503 & 1 & 2381,2 & Q923F1_MOUSE & & & \\
\hline SSPRQQGGGM & MMGPGGGGAASLSKAAAGAAAAAGGFQR & n.d. & $\mathrm{N}$-ter + 42.01 Da, $\mathrm{K}+34.06 \mathrm{Da}$ & 52,2 & $-5,872$ & 1 & 2466,2 & E9Q6R4_MOUSE & E9Q4N7_MOUSE & & \\
\hline CRLRGDTSEA & MSCYEKALR & n.d. & $\begin{array}{l}\text { N-ter + 42.01 Da, K+34.06 Da, } \\
\text { C + 57.02 Da }\end{array}$ & 14,3 & $-4,536$ & 1 & 1232,6 & Q3U687_MOUSE & & & \\
\hline M & NTLGKTITCR & n.d. & $\mathrm{N}$-ter + 42.01 Da, K+34.06 Da, & 61,2 & $-0,656$ & 1 & 1369,7 & E904Y1 MOUSE & E90576 MOUSE & & \\
\hline M & QDYNKDDMSYR & n.d. & $\begin{array}{l}\mathrm{C}+57.02 \mathrm{Da} \\
\mathrm{N} \text {-ter }+42.01 \mathrm{Da}, \mathrm{K}+34.06 \mathrm{Da}\end{array}$ & 16,6 & 1,644 & 1 & 1640,7 & O3UPWW3 MOUSE & E9PUSE__VIOUSE & & \\
\hline ADASRRSGAR & QGQASAQGRAGSQGQAQGR & n.d. & $\begin{array}{l}\text { N-ter + +24.01 Da, K+34.06 Da } \\
\text { N-ter + 42.01 Da }\end{array}$ & 37,1 & $-3,604$ & 1 & 1883,9 & E9Q019_MOUSE & $\begin{array}{l}\text { EPPYA__MOUSE } \\
\text { FILA_MOUSE }\end{array}$ & & \\
\hline TLEELDWCLD & QLETIQTYR & n.d. & $\mathrm{N}$-ter + 42.01 Da & 18,3 & 0,670 & 1 & 1192,6 & Q8VBUS_MOUSE & B1AWC9_MOUSE & & \\
\hline PQGPSQDSSR & QPQAGQGQPSQSGSGR & n.d. & $\mathrm{N}$-ter $+42.01 \mathrm{Da}$ & 17,2 & 3,286 & 1 & 1610,8 & E9QPZ3_MOUSE & FILA2_MOUSE & & \\
\hline RSSNRRDRPR & QPSPSOSSDSHVHSGVQVEGR & n.d. & $\mathrm{N}$-ter $+42.01 \mathrm{Da}$ & 17,8 & $-6,003$ & 1 & 2246,0 & J3QMA__MOUSE & & & \\
\hline AISIFHYAKG & QPYIDEVGTFEER & n.d. & $\mathrm{N}$-ter + 42.01 Da, K+28.03 Da & 17,5 & $-4,535$ & 1 & 1650,8 & E9QK82_MOUSE & & & \\
\hline RRGQSSSANR & RAGSSSGSGVQGASAGGLAADASR & n.d. & $\mathrm{N}$-ter $+42.01 \mathrm{Da}$ & 50,1 & 1,320 & 1 & 2118,0 & E9Q019_MOUSE & FILA_MOUSE & & \\
\hline 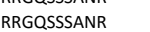 & RAGSSSGSGVQGTSAGGLAADASR & n.d. & $\mathrm{N}-$ ter $+42.01 \mathrm{Da}$ & 32,1 & $-3,626$ & 1 & 2148,0 & E9Q019_MOUSE & J3QM50_MOUSE & & \\
\hline IGSSADRQGR & RGVSESQASDSEGHSDFSEGQAVGAHR & n.d. & $\mathrm{N}$-ter + 42.01 Da & 48,2 & $-2,402$ & 1 & 2828,2 & J3QM50_MOUSE & J3QMA3_MOUSE & & \\
\hline AGGLAADASR & RSGALQGQASAQGRAGSQGQAQGR & n.d. & $\mathrm{N}$-ter $+42.01 \mathrm{Da}$ & 30,4 & 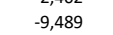 & 1 & 2368,2 & E9Q019_MOUSE & J3QNE6_MOUSE & & \\
\hline M & SAPAGSPHPAAGAR & n.d. & $\mathrm{N}$-ter + 42.01 Da & 47,7 & $-1,628$ & 1 & 1287,6 & Q80ZXO_MOUSE & & & \\
\hline RPRQPSPSQS & SDSQVHSGVQVEGR & n.d. & $\mathrm{N}$-ter + $42.01 \mathrm{Da}$ & 49,3 & $-0,327$ & 1 & 1525,7 & E9Q019_MOUSE & FILA_MOUSE & & \\
\hline PRRSPVHPES & SEGEEHSVVPQR & n.d. & $\mathrm{N}$-ter + 42.01 Da & 25,1 & 0,931 & 1 & 1394,6 & E9QPZ3_MOUSE & FILA2_MOUSE & & \\
\hline GGLAADASRR & SGALQGQASAQGRAGSQGQAQGR & n.d. & $\mathrm{N}$-ter + 42.01 Da & 37,3 & 8,352 & 1 & 2212,1 & E9Q019_MOUSE & J3QNE6_MOUSE & & \\
\hline GGLAADASRR & SGALQGQASAQGR & n.d. & $\mathrm{N}$-ter + 42.01 Da & 34,7 & 0,785 & 1 & 1271,6 & E9Q019 MOUSE & J3QNE6 MOUSE & & \\
\hline QAGFAADGVE & SGTVFSDINLQEKVR & n.d. & $\mathrm{N}$-ter + 42.01 Da, K+34.06 Da & 17,5 & $-3,501$ & 1 & 1768,0 & B7ZC71_MOUSE & & & \\
\hline M & SISSLGSIGKGR & n.d. & $\mathrm{N}$-ter + 42.01 Da, K +34.06 Da & 57,7 & $\begin{array}{l}-0,969 \\
-0,109\end{array}$ & 1 & 1236,7 & G5E8W8_MOUSE & A2B137_MOUSE & & \\
\hline
\end{tabular}




\begin{tabular}{|c|c|c|c|c|c|c|c|c|c|c|c|}
\hline Non Prime Site & Prime Site & 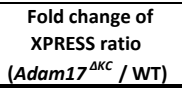 & Modifications & Hyperscore & $\begin{array}{c}\text { Mass error } \\
\text { in ppm }\end{array}$ & Charge & $\begin{array}{c}\text { Precursor } \\
\text { neutral mass } \\
\text { in Da }\end{array}$ & Uniprot ID 1 & Uniprot ID 2 & Protein Name 1 & Protein Name 2 \\
\hline $\begin{array}{l}\text { GKNDIFGEPA } \\
\end{array}$ & SLHARPGKSSADVR & n.d. & $\mathrm{N}$-ter + 42.01 Da, K +28.03 Da & 13,8 & $-5,667$ & 1 & 1549,8 & Q32MEO_MOUSE N & MOQW64_MOUSE & & \\
\hline M & SNIIIFSGSSHQDLSQKIADR & n.d. & N-ter + 42.01 Da, K+34.06 Da & 36,7 & $-1,718$ & 1 & 2440,3 & G3UXL2_MOUSE & & & \\
\hline & SNNCCSEVHSSSSCR & n.d. & N-ter + 42.01 Da, C +57.02 Da & 46,4 & 0,055 & 1 & 1811,7 & Q9D6L__MOUSE & & & \\
\hline RQPSPSOSSD & SQVHSGVQVEGR & n.d. & $\mathrm{N}$-ter + 42.01 Da & 49,4 & $-0,151$ & 1 & 1323,7 & E9Q019_MOUSE & FILA_MOUSE & & \\
\hline VRPEVPTQDS & SRQPQADQGQPSQSGSGR & n.d. & $\mathrm{N}$-ter + 42.01 Da & 22,7 & $-0,261$ & 1 & 1911,9 & E9QPZ3_MOUSE & & & \\
\hline GRPQGPSQDS & SRQPQAGQGQPSQSGSGR & n.d. & $\mathrm{N}$-ter + 42.01 Da & 41,4 & 8,240 & 1 & 1853,9 & E9QPZ3_MOUSE & FILA2_MOUSE & & \\
\hline DRPRQPSPSQ & SSDSQVHHSGVQVEGR & n.d. & $\mathrm{N}$-ter + 42.01 Da & 21,4 & 0,557 & 1 & 1612,8 & E9Q019_MOUSE & FILA_MOUSE & & \\
\hline SPRRSPVHPE & SSEGEEHSVVPQR & n.d. & $\mathrm{N}$-ter + 42.01 Da & 21,4 & $-6,538$ & 1 & 1481,7 & E9QPZ3_MOUSE & FILA2_MOUSE & & \\
\hline PVRPEVPTQD & SSRQPQADQGQPSQSGSGR & n.d. & N-ter + 42.01 Da & 25,1 & $-1,299$ & 1 & 1998,9 & E9QPZ3_MOUSE & & & \\
\hline M & SVAVAPVAVHPDSMLSEAEEPR & n.d. & $\mathrm{N}$-ter + 42.01 Da & 18,9 & 4,497 & 1 & 2332,2 & D3Z325_MOUSE & D3Z0Q1_MOUSE & & \\
\hline PAAVKMYVPP & TLAPLDGR & n.d. & $\mathrm{N}$-ter + 42.01 Da & 12,2 & 0,000 & 1 & 883,5 & J3QM40_MOUSE & & & \\
\hline NMAGSSSVSS & TLTVHVPPR & n.d. & N-ter + 42.01 Da & 14,6 & $-2,162$ & 1 & 1060,6 & D3Z2Q7_MOUSE & D3YXGO_MOUSE & & \\
\hline SAGQRGDDLR & TTKMEISELNR & n.d. & $\mathrm{N}$-ter + 42.01 Da, $\mathrm{K}+34.06 \mathrm{Da}$ & 16,2 & 0,429 & 1 & 1396,8 & E9Q1Z0_MOUSE & & & \\
\hline AISTVLAKTI & TVVMAFKLTTPGRRMR & n.d. & $\mathrm{N}$-ter + 42.01 Da, $\mathrm{K}+28.03 \mathrm{Da}$ & 19,4 & $-0,258$ & 1 & 1933,1 & K7N5U4_MOUSE & G3UW56_MOUSE & & \\
\hline EERSVNCGTM & VAQPKNLEGYVGFANLPNQVYR & n.d. & $\mathrm{N}$-ter + 42.01 Da, $\mathrm{K}+34.06 \mathrm{Da}$ & 42,0 & 5,126 & 1 & 2552,4 & E9Q1G__MOUSE & & & \\
\hline ATLEPICPN & VDLGPVAAAGGAATGR & n.d. & $\mathrm{N}$-ter + 42.01 Da & 17,1 & 2,174 & 1 & 1423,8 & J3KMM_-MOUSE & & & \\
\hline GRNEPCRPVL & VHLQPGQIR & n.d. & $\mathrm{N}$-ter + 42.01 Da & 17,2 & 8,160 & 1 & 1088,6 & A2AQ92_MOUSE & & & \\
\hline M & YGGVSEAKPATPEIQKIADKVR & n.d. & $\mathrm{N}$-ter $+42.01 \mathrm{Da}, \mathrm{K}+34.06 \mathrm{Da}$ & 19,8 & 0,038 & 1 & 2631,5 & L7N257_MOUSE & & & \\
\hline & YGGVSEAKPATPEIQK & n.d. & $\mathrm{N}$-ter $+42.01 \mathrm{Da}, \mathrm{K}+28.03 \mathrm{Da}$ & 37,1 & $-4,355$ & 1 & 1903,0 & L7N257_MOUSE & & & \\
\hline SPRRSQVHPE & YSEGEAHSEVSQR & n.d. & $\mathrm{N}$-ter $+42.01 \mathrm{Da}$ & 21,8 & 6,703 & 1 & 1519,7 & E9QPZ3_MOUSE & FILA2_MOUSE & & \\
\hline
\end{tabular}




\begin{tabular}{|c|c|c|c|c|c|c|c|c|c|c|c|}
\hline Non Prime Site & Prime Site & $\begin{array}{l}\text { Fold change of } \\
\text { XPRESS ratio } \\
\text { (Adam17 } 7^{\text {AkC } / \text { WT) }}\end{array}$ & Modifications & Hyperscore & $\begin{array}{c}\text { Mass error } \\
\text { in ppm }\end{array}$ & Charge & $\begin{array}{l}\text { Precursor } \\
\text { neutral mass } \\
\text { in Da }\end{array}$ & s Uniprot ID 1 & Uniprot ID 2 & Protein Name 1 & Protein Name 2 \\
\hline$\overline{\mathrm{MT}}$ & $\begin{array}{l}\text { MDKSELVQKAKLAEQAER } \\
\end{array}$ & 0,1 & $\mathrm{~N}$-ter + 42.01 Da, K+28.03 Da & 42,1 & $-2,498$ & 3 & 2199,2 & 1433B_MOUSE & A2A5N1_MOUSE & 14-3-3 protein beta/alpha; & Protein kinase Cinhibitor protein 1; \\
\hline M & TMDKSELVQKAKLAEQAER & n.d. & $\mathrm{N}$-ter + 42.01 Da, K +34.06 Da & 54,8 & 0,086 & 3 & 2318,3 & 1433B_MOUSE & A2A5N1_MOUSE & 14-3-3 protein beta/alpha; & Protein kinase C inhibitor protein 1 \\
\hline M & DDREDLVYQAKLAEQAER & 0,0 & $\mathrm{~N}$-ter + $42.01 \mathrm{Da}, \mathrm{K}+28.03 \mathrm{Da}$ & 78,1 & 0,128 & 3 & 2349,1 & 1433E_MOUSE & D6REF3_MOUSE & 14-3-3 protein epsilon; & \\
\hline M & DDREDLVYQAK & 0,2 & $\mathrm{~N}$-ter + 42.01 Da, K +28.03 Da & 44,8 & 0,965 & 2 & 1551,7 & 1433E_MOUSE & D6REF3_MOUSE & 14-3-3 protein epsilon; & \\
\hline M & GDREQLLQR & n.d. & $\mathrm{N}$-ter + 42.01 Da & 40,2 & $-1,037$ & 2 & 1155,6 & 1433F_MOUSE & & 14-3-3 protein eta; & \\
\hline M & VDREQLVQKAR & 0,0 & $\mathrm{~N}$-ter + 42.01 Da, K+28.03 Da & 53,6 & 1,910 & 3 & 1410,8 & 1433G_MOUSE & & 14-3-3 protein gamma; & \\
\hline & ASLIQKAKLAEQAER & n.d. & $\mathrm{N}$-ter + $42.01 \mathrm{Da}, \mathrm{K}+34.06 \mathrm{Da}$ & 13,9 & 9,722 & 4 & 1765,1 & 1433S_MOUSE & & 14-3-3 protein sigma; & Stratifin; \\
\hline DKKRIIIDAR & SAYQEAMDISKKEMPPTNPIR & n.d. & $\mathrm{N}$-ter + 42.01 Da, K +34.06 Da & 30,1 & 8,812 & 4 & 2515,3 & 1433S_MOUSE & & 14-3-3 protein sigma; & Stratifin; \\
\hline PARRSPSLAM & EKTELIOKAKLAEQAER & n.d. & $\mathrm{N}$-ter + 42.01 Da, K +34.06 Da & 57,8 & $-2,387$ & 3 & 2259,3 & 1433T_MOUSE & F6VW30_MOUSE & 14-3-3 protein theta; & 14-3-3 protein tau; \\
\hline PARRSPSLAM & ЕКТЕLQКАК & 0,1 & $\mathrm{~N}$-ter + 42.01 Da, K +28.03 Da & 45,4 & 0,000 & 3 & 1443,8 & 1433T_MOUSE & & 14-3-3 protein theta; & 14-3-3 protein tau; \\
\hline PARRSPSLAM & EKTELOK & n.d. & $\mathrm{N}$-ter + 42.01 Da, K +28.03 Da & 15,9 & 0,492 & 2 & 1216,7 & 1433T_MOUSE & & 14-3-3 protein theta; & 14-3-3 protein tau; \\
\hline M & DKNELVQKAKLAEQAER & n.d. & $\mathrm{N}$-ter $+42.01 \mathrm{Da}, \mathrm{K}+34.06 \mathrm{Da}$ & 67,2 & 0,089 & 3 & 2244,3 & 1433Z_MOUSE & D3YXF4_MOUSE & 14-3-3 protein zeta/delta; & Protein kinase C inhibitor protein 1;SEZ-2; \\
\hline $\begin{array}{l}M \\
M\end{array}$ & $\begin{array}{l}\text { DKNELVQKAK } \\
\text { DKNELVQK }\end{array}$ & $\begin{array}{l}\text { n.d. } \\
0,2\end{array}$ & $\begin{array}{l}\text { N-ter + 42.01 Da, K+34.06 Da } \\
\text { N-ter + 42.01 Da, K+28.03 Da }\end{array}$ & $\begin{array}{l}62,9 \\
27,1\end{array}$ & $\begin{array}{l}-0,828 \\
6,065\end{array}$ & $\begin{array}{l}2 \\
2\end{array}$ & $\begin{array}{l}1446,9 \\
1201,7\end{array}$ & $\begin{array}{l}\text { 1433Z_MOUSE } \\
\text { 1433Z_MOUSE }\end{array}$ & $\begin{array}{l}\text { D3YXF4_MOUSE } \\
\text { D3YXF_ MOUSE }\end{array}$ & $\begin{array}{l}\text { 14-3-3 protein zeta/delta; } \\
\text { 14-3-3 protein zeta/delta; }\end{array}$ & $\begin{array}{l}\text { Protein kinase Cinhibitor protein 1;SEZ-2; } \\
\text { Protein kinase Cinhibitor protein 1:SEZ-2. }\end{array}$ \\
\hline M & AGARPGVHALQLEPPTVVETLR & n.d. & $\mathrm{N}$-ter + 42.01 Da & 20,3 & $-7,303$ & 3 & 2352,3 & PLCB__MOUSE & & $\begin{array}{l}\text { 1-phosphatidylinositol 4,5-5isphosphate } \\
\text { phosphodiesterase beta-3; }\end{array}$ & $\begin{array}{l}\text { Phosphoinositide phospholipase C-beta- } \\
\text { 3; Phospholipase C-beta-3; }\end{array}$ \\
\hline EKGIPEEPLC & QLTPQLGLSLR & n.d. & $\mathrm{N}$-ter + 42.01 Da & 16,2 & 2,520 & 3 & 1266,7 & PLCH2_MOUSE & F7C3AO_MOUSE & $\begin{array}{l}\text { 1-phosphatidylinositol 4,5-bisphosphate } \\
\text { phosphodiesterase eta-2; }\end{array}$ & $\begin{array}{l}\text { Phosphoinositide phospholipase C-eta- } \\
\text { 2;Phosphoinositide phospholipase C-like } \\
\text { 4; Phospholipase C-eta-2; }\end{array}$ \\
\hline YAQTEFSRTV & LYPYCAPYALELDYR & n.d. & $\mathrm{N}$-ter + 42.01 Da, C+57.02 Da & 14,8 & 0,051 & 3 & 1947,9 & PDE12_MOUSE & & 2,5'-phosphodiesterase 12; & $\begin{array}{l}\text { Mitochondrial deadenylase; } \\
\text { 265 proteasome AAA-ATPase subunit }\end{array}$ \\
\hline M & ALDGPEQMELEEGKAGSGLR & 0,1 & $\mathrm{~N}$-ter + $42.01 \mathrm{Da}, \mathrm{K}+28.03 \mathrm{Da}$ & 72,5 & $-0,417$ & 3 & 2156,0 & PRS8_MOUSE & Q8K1K2_MOUSE & 265 protease regulatory subunit $8 ;$ & $\begin{array}{l}\text { RPT6;Proteasome } 265 \text { subunit ATPase } \\
\text {;;Proteasome subunit p45;p } 45 / \text { /SUG; } \\
\text { 26S proteasome AAA-ATPase subunit }\end{array}$ \\
\hline MALDGPEQ & MELEEGKAGSGLR & n.d. & N-ter + 42.01 Da, K+34.06 Da & 17,4 & $-3,577$ & 2 & 1451,8 & PRS8_MOUSE & Q8K1K2_MOUSE & 265 protease regulatory subunit $8 ;$ & $\begin{array}{l}\text { RPTG;Proteasome 265 subunit ATPase } \\
\text { 5;Proteasome subunit p45;p45/SUG; }\end{array}$ \\
\hline M & SGEDVPHR & n.d. & $\mathrm{N}$-ter $+42.01 \mathrm{Da}$ & 20,1 & 0,958 & 2 & 937,4 & PSMD9_MOUSE & & 265 proteasome non-ATPase regulatory subunit $9 ;$ & 265 proteasome regulatory subunit p27; \\
\hline GLIHALGGMA & NANMNCWPLIVIGGSSER & n.d. & $\mathrm{N}$-ter + 42.01 Da, C+57.02 Da & 13,6 & $-7,129$ & 3 & 2059,0 & HACL1_MOUSE & E9PWM4_MOUSE & E 2-hydroxyacyl-CoA lyase 1; & $\begin{array}{l}\text { 2-hydroxyphytanoyl-CoA lyase; Phytanoyl-CoA 2- } \\
\text { hydroxylase 2; }\end{array}$ \\
\hline M & ASSHTVLMR & n.d. & $\mathrm{N}$-ter $+42.01 \mathrm{Da}$ & 25,4 & $-0,766$ & 2 & 1042,5 & BPNT1_MOUSE & D3Z5X0_MOUSE & $3^{\prime}\left(2^{\prime}\right), 5^{\prime}$-bisphosphate nucleotidase 1; & $\begin{array}{l}\text { Bisphosphate 3'-nucleotidase 1;PAP-inositol 1,4- } \\
\text { phosphatase; }\end{array}$ \\
\hline M & TTNTVPLHPYWPR & n.d. & $\mathrm{N}$-ter $+42.01 \mathrm{Da}$ & 20,7 & $-0,246$ & 2 & 1622,8 & EBP_MOUSE & A2AC29_MOUSE & 3-beta-hydroxysteroid-Delta(8),Delta(7)-isomerase; & $\begin{array}{l}\text { Cholestenol Delta-isomerase;Delta(8)-Delta(7) } \\
\text { sterol isomerase;Emopamil-binding protein; }\end{array}$ \\
\hline DGSWVEWYMR & AQPEHIISEGR & n.d. & $\mathrm{N}$-ter + 42.01 Da & 25,2 & $-0,078$ & 2 & 1277,6 & THTM_MOUSE & Q3UW66_MOUSE & E 3-mercaptopyruvate sulfurtransferase; & \\
\hline AEEGIAAGGV & MDVNTALQEVLKTALIHDGLAR & $-0,1$ & $\mathrm{~N}$-ter + 42.01 Da, K+28.03 Da & 53,7 & $-5,443$ & 3 & 2477,3 & RS12_MOUSE & Q6ZWZ6_MOUSE & $=40$ S ribosomal protein S12; & \\
\hline M & AFKDTGKTPVEPEVAIHR & n.d. & $\mathrm{N}$-ter + 42.01 Da, K+34.06 Da & 56,9 & $-1,613$ & 4 & 2104,2 & RS20_MOUSE & & 40S ribosomal protein S20; & \\
\hline ASEGKM & GHQQLYWSHPR & n.d. & $\mathrm{N}$-ter + 42.01 Da & 15,3 & 5,232 & 3 & 1449,7 & RS29_MOUSE & D3YYL7_MOUSE & 40 S ribosomal protein $529 ;$ & \\
\hline $\begin{array}{l}M \\
M\end{array}$ & AVQISKKR & n.d. & $\mathrm{N}$-ter + 42.01 Da, K +34.06 Da & 17,2 & 8,831 & 3 & 1038,7 & RS3_MOUSE & D3YV43_MOUSE & $40 \mathrm{~S}$ ribosomal protein $\mathrm{S} 3 ;$ & \\
\hline M & DPEPTEHSTDGVSVPR & n.d. & $\mathrm{N}$-ter $+42.01 \mathrm{Da}$ & 32,5 & $-1,371$ & 2 & 1894,8 & G3UWA6_MOUSE & & 4F2 cell-surface antigen heavy chain; & Solute carrier family 3 member 2; \\
\hline TATYTVLSSP & AGNLSLTLLR & n.d. & $\mathrm{N}$-ter + 42.01 Da & 28,6 & $-1,302$ & 2 & 1226,7 & HPDL_MOUSE & & 4-hydroxyphenylpyruvate dioxygenase-like protein; & Glyoxalase domain-containing protein $1 ;$ \\
\hline $\begin{array}{l}M \\
M\end{array}$ & $\begin{array}{l}\text { TLKSSEGEGGNSMR } \\
\text { ATESSPLTHVLDTASGLPAQGLCLR }\end{array}$ & $\begin{array}{l}0,4 \\
\text { n.d. }\end{array}$ & $\begin{array}{l}\text { N-ter + 42.01 Da, } \mathrm{K}+28.03 \mathrm{Da} \\
\text { N-ter +42.01 Da, C +57.02 Da }\end{array}$ & $\begin{array}{l}18,7 \\
50,2\end{array}$ & $\begin{array}{l}-2,822 \\
0,584\end{array}$ & $\begin{array}{l}2 \\
3\end{array}$ & $\begin{array}{l}1521,7 \\
2736,4\end{array}$ & $\begin{array}{l}\text { EM55_MOUSE } \\
\text { HIUH MOUSE }\end{array}$ & B7ZCMO_MOUSE & $\begin{array}{l}55 \text { kDa erythrocyte membrane protein; } \\
\text { 5-hydroxyisourate hydrolase: }\end{array}$ & Membrane protein, palmitoylated 1; \\
\hline M & SSKVSRDTLYEAVR & 0,1 & $\mathrm{~N}$-ter + 42.01 Da, K+28.03 Da & 26,7 & 2,139 & 3 & 1679,9 & RLLOA_MOUSE & Q5XJF6_MOUSE & $\begin{array}{l}\text { 5-hydroxylisourate hydrolase; } \\
\text { 60S ribosomal protein L10a; }\end{array}$ & $\begin{array}{l}\text { rransthyretin-related protein; } \\
\text { CSA-19;Neural precursor cell expressed } \\
\text { developmentally down-regulated protein 6; }\end{array}$ \\
\hline M & AQDQGEKENPMR & n.d. & N-ter + 42.01 Da, K+34.06 Da & 59,9 & $-0,338$ & 2 & 1477,7 & RL11_MOUSE & DЗZ3K1_MOUSE & 60 s ribosomal protein L11; & \\
\hline PPRDRKKQKN & IKHSGNITFDEIVNIAR & n.d. & $\mathrm{N}$-ter + 42.01 Da, K +34.06 Da & 25,1 & 5,735 & 3 & 2002,1 & RL12_MOUSE & & 60 S ribosomal protein L12; & \\
\hline M & GKFMKPGKVVLVLAGR & n.d. & N-ter + 42.01 Da, K +34.06 Da & 21,2 & 2,600 & 3 & 1843,2 & RL27_MOUSE & & 60 ribosomal protein L27; & \\
\hline M & SAHLQWMVVR & n.d. & $\mathrm{N}$-ter + 42.01 Da & 34,1 & 0,079 & 2 & 1267,7 & RL28_MOUSE & & $60 S$ ribosomal protein $\mathrm{L28}$; & \\
\hline M & EAVPEKKKK & n.d. & $\mathrm{N}$-ter + 42.01 Da, K +28.03 Da & 14,3 & 0,372 & 3 & 1340,8 & RL7_MOMUSE & & $60 \mathrm{~S}$ ribosomal protein L7; & \\
\hline M & EAVPEKKK & $-0,2$ & $\mathrm{~N}$-ter + 42.01 Da, K+28.03 Da & 19,3 & $-5,136$ & 3 & 1184,7 & RL7_MOUSE & & 605 ribosomal protein L7; & \\
\hline M & EAVPEKK & n.d. & $\mathrm{N}$-ter + 42.01 Da, $\mathrm{K}+34.06 \mathrm{Da}$ & 19,7 & 0,192 & 2 & 1040,6 & RL7_MOUSE & & 60 S ribosomal protein L7; & \\
\hline YVAFTPEGER & LIGDAAKNQLTSNPENTVFDAKR & $-0,7$ & N-ter + 42.01 Da, K +28.03 Da & 30,1 & 3,803 & 4 & 2599,4 & GRP78_MOUSE & & 78 kDa glucose-regulated protein; & $\begin{array}{l}\text { Heat shock } 70 \text { kDa protein 5;Immunoglobulin } \\
\text { heavy chain-binding protein; }\end{array}$ \\
\hline
\end{tabular}




\begin{tabular}{|c|c|c|c|c|c|c|c|c|c|c|c|}
\hline Non Prime Site & Prime Site & $\begin{array}{l}\text { Fold change of } \\
\text { XPRESS ratio } \\
\left.\text { (Adam17 } 17^{\Delta K C} / \mathrm{WT}\right)\end{array}$ & Modifications & Hyperscore & $\begin{array}{l}\text { Mass error } \\
\text { in ppm }\end{array}$ & charge & $\begin{array}{l}\text { Precursor } \\
\text { neutral mass } \\
\text { in Da }\end{array}$ & Uniprot ID 1 & Uniprot ID 2 & Protein Name 1 & Protein Name 2 \\
\hline M & AEEVSSLMKATVLMR & n.d. & $\mathrm{N}$-ter + 42.01 Da, $\mathrm{K}+28.03 \mathrm{Da}$ & 10,1 & $-0,346$ & 2 & 1733,9 & 5NT3B_MOUSE & & $\begin{array}{l}\text { 7-methylguanosine phosphate-specific 5'- } \\
\text { nucleotidase; }\end{array}$ & $\begin{array}{l}\text { Cytosolic 5'-nucleotidase 38;Cytosolic 5'- } \\
\text { nucleotidase III-like protein;N(7)- } \\
\text { methylguanylate 5'-phosphatase; }\end{array}$ \\
\hline HLACKMGKQE & MVRVLLLCNAR & n.d. & $\mathrm{N}$-ter + 42.01 Da, C +57.02 Da & 16,2 & 3,747 & 2 & 1385,8 & PLPL9_MOUSE & & 85/88 kDa calcium-independent phospholipase A2; & $\begin{array}{l}\text { Group VI phospholipase A2;Intracellular } \\
\text { membrane-associated calcium-independent } \\
\text { phospholipase A2 beta; Patatit-like } \\
\text { phospholipase domain-containing protein 9; }\end{array}$ \\
\hline M & AELQMLLEEEIPSGKR & $-0,1$ & $\mathrm{~N}$-ter + 42.01 Da, $\mathrm{K}+28.03 \mathrm{Da}$ & 30,7 & 2,142 & 2 & 1912,0 & ABI1_MOUSE & J3QNK8_MOUSE & Abl interactor 1 & $\begin{array}{l}\text { Abelson interactor 1; ;Abphilin-1; Es } 88 \text { SH3 } \\
\text { domain-binding protein;;Spectrin SH3 domain- } \\
\text { binding protein 1;e3B1; }\end{array}$ \\
\hline $\begin{array}{l}\text { M } \\
\text { SVWIGGSILA }\end{array}$ & $\begin{array}{l}\text { DEPSPLAKTLELNQHSR } \\
\text { SLSTFQQMWITKQEYDEAGPSIVHR }\end{array}$ & $\begin{array}{l}\text { n.d. } \\
\text { n.d. }\end{array}$ & $\begin{array}{l}\text { N-ter + 42.01 Da, K+34.06 Da } \\
\text { N-ter + 42.01 Da, K+34.06 Da }\end{array}$ & $\begin{array}{l}15,7 \\
14,5\end{array}$ & $\begin{array}{r}3,685 \\
-3,894\end{array}$ & $\begin{array}{l}3 \\
4\end{array}$ & $\begin{array}{l}2141,1 \\
3026,5\end{array}$ & $\begin{array}{l}\text { ACACA_MOUSE } \\
\text { ACTS_MOUSE }\end{array}$ & & $\begin{array}{l}\text { Acety-CoA carboxylase 1; } \\
\text { Actin, alpha skeletal muscle; }\end{array}$ & $\begin{array}{l}\text { ACC-alpha;Acetyl-CoA carboxylase 265; } \\
\text { Alpha-actin-1; }\end{array}$ \\
\hline MCEE & EDSTALVCDNGSGLCKAGFAGDDAPR & n.d. & $\begin{array}{l}\text { N-ter + 42.01 Da, K+34.06 Da, } \\
\mathrm{C}+57.02 \mathrm{Da}\end{array}$ & 14,5 & $-0,326$ & 3 & 2758,2 & ACTA_MOUSE & & Actin, aortic smooth muscle; & Alpha-actin-2; \\
\hline MCE & EEDSTALVCDNGSGLCKAGFAGDDAPR & n.d. & $\begin{array}{l}\text { N-ter + 42.01 Da, K+34.06 Da, } \\
\mathrm{C}+57.02 \mathrm{Da}\end{array}$ & 28,2 & 3,182 & 4 & 2887,3 & ACTA_MOUSE & & Actin, aortic smooth muscle; & Alpha-actin-2; \\
\hline TMYYPGIADR & MQKEITALAPSTMKIKIIAPPER & n.d. & N-ter + 42.01 Da, K +28.03 Da & 20,3 & $-4,749$ & 4 & 2691,5 & ACTA_MOUSE & АСTB_MOUSE & Actin, aortic smooth muscle; & Alpha-actin-2; \\
\hline mFAM & DDDIAALVVDNGSGMCKAGFAGDDAPR & 0,1 & $\begin{array}{l}\text { N-ter + 42.01 Da, K+28.03 Da, } \\
\mathrm{C}+57.02 \mathrm{Da}\end{array}$ & 56,2 & $-0,961$ & 3 & 2806,3 & ACTB_MOUSE & E9Q1F__MOUSE & Actin, cytoplasmic 1; & Beta-actin; \\
\hline M & EEEIAALVIDNGSGMCKAGFAGDDAPR & 0,1 & $\begin{array}{l}\text { N-ter + 42.01 Da, K+28.03 Da, } \\
\mathrm{C}+57.02 \mathrm{Da}\end{array}$ & 67,2 & 0,803 & 3 & 2862,3 & ACTG_MOUSE & G3UYGO_MOUSE & Actin, cytoplasmic 2; & Gamma-actin; \\
\hline MC & EEETTALVCDNGSGLCKAGFAGDDAPR & $-3,5$ & $\begin{array}{l}\text { N-ter + 42.01 Da, K+28.03 Da, } \\
\text { C + +57.02 Da }\end{array}$ & 22,1 & 2,404 & 3 & 2909,3 & ACTH_MOUSE & DЗZ2K3_MOUSE & Actin, gamma-enteric smooth muscle; & $\begin{array}{l}\text { Alpha-actin-3;Gamma-2-actin;Smooth muscle } \\
\text { gamma-actin; }\end{array}$ \\
\hline MCE & EETTALVCDNGSGLCKAGFAGDDAPR & $-2,4$ & $\begin{array}{l}\text { N-ter + 42.01 Da, K+28.03 Da, } \\
\mathrm{C}+57.02 \mathrm{Da}\end{array}$ & 24,3 & 7,006 & 3 & 2780,3 & ACTH_MOUSE & D3Z2K3_MOUSE & Actin, gamma-enteric smooth muscle; & $\begin{array}{l}\text { Alpha-actin-3;Gamma-2-actin;Smooth muscle } \\
\text { gamma-actin; }\end{array}$ \\
\hline MCEEET & TALVCDNGSGLCKAGFAGDDAPR & n.d. & $\begin{array}{l}\text { N-ter + 42.01 Da, K+28.03 Da, } \\
\mathrm{C}+57.02 \mathrm{Da}\end{array}$ & 20,5 & 9,282 & 3 & 2421,1 & ACTH_MOUSE & ACTA_MOUSE & Actin, gamma-enteric smooth muscle; & $\begin{array}{l}\text { Alpha-actin-3;Gamma-2-actin;Smooth muscle } \\
\text { gamma-actin; } \\
\text { Intracisternal A particle-promoted }\end{array}$ \\
\hline PRAGMCAVTV & NGLLYSGGR & n.d. & N-ter $+42.01 \mathrm{Da}$ & 20,0 & 3,245 & 2 & 1076,6 & IPP_MOUSE & & Actin-binding protein IPP; & $\begin{array}{l}\text { polypeptide;Murine IAP-promoted placenta- } \\
\text { expressed protein;Protein MIPP; }\end{array}$ \\
\hline M & SLHQFLLEPITCHAWNR & n.d. & $\mathrm{N}$-ter + 42.01 Da, C +57.02 Da & 65,2 & $-0,693$ & 3 & 2163,1 & ARC1A_MOUSE & D3YVI5_MOUSE & Actin-related protein $2 / 3$ complex sub & SOP2-like protein;Sid 329; \\
\hline $\begin{array}{l}M \\
M\end{array}$ & $\begin{array}{l}\text { TATLRPYLSAVR } \\
\text { DVLRPQIVTFDGR }\end{array}$ & n.d. & $\begin{array}{l}\text { N-ter + 42.01 Da } \\
\text { N-ter + 42.01 Da }\end{array}$ & $\begin{array}{l}36,2 \\
265\end{array}$ & $\begin{array}{r}0,719 \\
-0,355\end{array}$ & 2 & $\begin{array}{l}1388,8 \\
16879\end{array}$ & $\begin{array}{l}\text { ARPC4_MOUSE } \\
\text { ASCC1 MOUSE }\end{array}$ & & $\begin{array}{l}\text { Actin-related protein } 2 / 3 \text { complex subunit } 4 \text {; } \\
\text { Activating signal cointegrator } 1 \text { complex subunit } 1 \text {. }\end{array}$ & $\begin{array}{l}\text { Arp2 } 2 / 3 \text { complex } 20 \text { kDa subunit; } \\
\text { ASC-1 complex subunit p50; Trip4 complex }\end{array}$ \\
\hline ESALDDCAPS & GDSQSDEPPSSEDSLPR & n.d. & N-ter +42.01 Da & $\begin{array}{l}26,5 \\
12,2\end{array}$ & $\begin{array}{l}-0,355 \\
3,790\end{array}$ & 3 & $\begin{array}{l}188,9 \\
1843,8\end{array}$ & MCAF1_MOUSE & & Activating transcription factor 7 -interacting protein $1 ;$ & $\begin{array}{l}\text { subunit p50; } \\
\text { ATFa-associated modulator;MBD1-containing } \\
\text { chromatit-associated factor 1. }\end{array}$ \\
\hline M & AKWGEGDPR & n.d. & N-ter + 42.01 Da, K+34.06 Da & 13,2 & 0,275 & 2 & 1090,6 & AHSA1_MOUSE & & $\begin{array}{l}\text { Activator of } 90 \mathrm{kDa} \text { heat shock protein ATPase } \\
\text { homolog 1; }\end{array}$ & chromatin-associated factor 1 ; \\
\hline ICIGCVDGSL & LCFTHSGEQVWR & n.d. & N-ter + 42.01 Da, C +57.02 Da & 15,4 & $-2,432$ & 2 & 1560,7 & ACSF4_MOUSE & & Acyl-CoA synthetase family member 4 ; & $\begin{array}{l}\text { 2-aminoadipic 6-semialdehyde } \\
\text { dehydrogenase; Protein LYS2 homolog;PPutative } \\
\text { aminoadipate-semialdehyde dehydrogenase; }\end{array}$ \\
\hline YCCSKEGHLR & WKFETTAR & n.d. & $\mathrm{N}$-ter + 42.01 Da, K +34.06 Da & 14,6 & 4,209 & 3 & 1113,6 & ACSF4_MOUSE & F7A4F9_MOUSE & Acyl-CoA synthetase family member 4; & $\begin{array}{l}\text { 2-aminoadipic 6-semialdehyde } \\
\text { dehydrogenase; Protein LYS2 homolog;Putative } \\
\text { aminoadipate-semialdehyde dehydrogenase; }\end{array}$ \\
\hline M & SQAEFDKAAEEVKR & 0,0 & N-ter + 42.01 Da, K+28.03 Da & 50,1 & $-1,230$ & 2 & 1704,9 & ACBP_MOUSE & MOQWU8_MOUSE & SE Acyl-CoA-binding protein; & Diazepam-binding inhibitor;Endozepine; \\
\hline M & AAELEYESVLCVKPDVSVYR & n.d. & $\begin{array}{l}\text { N-ter+ +42.01 Da, K+34.06 Da, } \\
\text { C +57.02 Da }\end{array}$ & 32,0 & 1,081 & 3 & 2402,2 & NECP1_MOUSE & & Adaptin ear-binding coat-associated protein 1 & NECAP endocytosis-associated protein 1 ; \\
\hline M & EESEYESVLCVKPEVHVYR & 0,1 & $\begin{array}{l}\text { N-ter + 42.01 Da, } \mathrm{K}+28.03 \mathrm{Da}, \\
\mathrm{C}+57.02 \mathrm{Da}\end{array}$ & 41,6 & 1,252 & 3 & 2552,2 & NECP2_MOUSE & & Adaptin ear-binding coat-associated protein 2; & NECAP endocytosis-associated protein 2; \\
\hline $\begin{array}{l}\text { M } \\
\text { PPPRRASAIP }\end{array}$ & $\begin{array}{l}\text { SEPELKLVAR } \\
\text { RALKREKPAGR }\end{array}$ & $\begin{array}{l}\text { n.d. } \\
\text { n.d. }\end{array}$ & $\begin{array}{l}\text { N-ter + 42.01 Da, } \mathrm{K}+34.06 \mathrm{Da} \\
\mathrm{N} \text {-ter + 42.01 Da, } \mathrm{K}+28.03 \mathrm{Da}\end{array}$ & $\begin{array}{l}17,9 \\
17,5\end{array}$ & $\begin{array}{r}-1,805 \\
2,388\end{array}$ & $\begin{array}{l}2 \\
3\end{array}$ & $\begin{array}{l}1216,7 \\
1378,9\end{array}$ & $\begin{array}{l}\text { APT_MOUSE } \\
\text { APC2_MOUSE }\end{array}$ & G5E832_MOUSE & $\begin{array}{l}\text { Adenine phosphoribosyltransferase; } \\
\text { Adenomatous polyposis coli protein 2; }\end{array}$ & \\
\hline RRVMEETYYH & HIYHTVAIEGNTLTLSEIR & n.d. & N-ter $+42.01 \mathrm{Da}$ & 16,7 & 1,764 & 3 & 2208,2 & FICD_MOUSE & & Adenosine monophosphate-protein transferase FICD; & AMPylator FICD;FIC domain-containing protein; \\
\hline M & SDKLPYKVADIGLAAWGR & 0,1 & $\mathrm{~N}$-ter + 42.01 Da, K+28.03 Da & 58,4 & $-0,049$ & 3 & 2057,1 & SAHH_MOUSE & A2ALT5_MOUSE & Adenosylhomocysteinase; & $\begin{array}{l}\text { CUBP;Liver copper-binding protein;S-adenosyl- } \\
\text { L-homocysteine hydrolase; }\end{array}$ \\
\hline LWQTAVRHIT & EQRFIHGHRGGGGGGVSR & n.d. & $\mathrm{N}$-ter + 42.01 Da & 23,0 & 0,629 & 3 & 1905,0 & ADCY8_MOUSE & & Adenylate cyclase type 8; & $\begin{array}{l}\text { ATP pyrophosphate-lyase 8;Adenylate cyclase } \\
\text { type VIII;Adenylyly cyclase 8;Ca(2+)/calmodulin- } \\
\text { activated adenylyl cyclase; }\end{array}$ \\
\hline
\end{tabular}




\begin{tabular}{|c|c|c|c|c|c|c|c|c|c|c|c|}
\hline Non Prime Site & Prime Site & $\begin{array}{l}\text { Fold change of } \\
\text { XPRESS ratio } \\
\text { (Adam17 } 1 \text { ¿KC / WT) }\end{array}$ & Modifications & Hyperscore & $\begin{array}{l}\text { Mass error } \\
\text { in ppm }\end{array}$ & charge & $\begin{array}{l}\text { Precursor } \\
\text { neutral mass } \\
\text { in Da }\end{array}$ & Uniprot ID 1 & Uniprot ID 2 & Protein Name 1 & Protein Name 2 \\
\hline ATLDCLNGDY & NVEEGHGKER & n.d. & $\mathrm{N}$-ter + 42.01 Da, $\mathrm{K}+34.06 \mathrm{Da}$ & 22,0 & 2,923 & 2 & 1229,6 & ADCY8_MOUSE & & Adenylate cyclase type 8; & $\begin{array}{l}\text { ATP pyrophosphate-lyase 8;Adenylate cyclase } \\
\text { type VIII;Adenylyy cyclase 8;Ca(2+)/calmodulin- } \\
\text { activated adenylylycyclase; }\end{array}$ \\
\hline M & TDAAVSFAKDFLAGGVAAAISKTAVAPIER & 0,2 & $\mathrm{~N}$-ter + 42.01 Da, $\mathrm{K}+28.03 \mathrm{Da}$ & 76,7 & 1,116 & 3 & 3044,7 & ADT2_MOUSE & & ADP/ATP translocase 2; & $\begin{array}{l}\text { ADP,ATP carrier protein 2;Adenine nucleotide } \\
\text { translocator 2; Solute carrier family } 25 \text { member } \\
5 \text {; }\end{array}$ \\
\hline M & AASPSKTEIQTIFKR & n.d. & $\mathrm{N}$-ter + 42.01 Da, K+34.06 Da & 16,8 & 0,783 & 3 & 1786,1 & ARFG2_MOUSE & & ADP-ribosylation factor GTPase-activating protein 2; & $\begin{array}{l}\text { GTPase-activating protein ZNF289;Zinc finger } \\
\text { protein 289; }\end{array}$ \\
\hline $\begin{array}{l}\text { M } \\
\text { LHSYDYWEMA }\end{array}$ & $\begin{array}{l}\text { AQELQHPEFAR } \\
\text { DNLAKENR }\end{array}$ & $\begin{array}{l}\text { n.d. } \\
\text { n.d. }\end{array}$ & $\begin{array}{l}\text { N-ter + 42.01 Da } \\
\text { N-ter + 40.01 Da K +34.06 Da }\end{array}$ & 38,6 & $\begin{array}{r}-3,215 \\
-1929\end{array}$ & 2 & $\begin{array}{l}1366,7 \\
1034,6\end{array}$ & $\begin{array}{l}\text { ADSV_MOUSE } \\
\text { AFF_MOUSE }\end{array}$ & DЗYYI6 MOUSE & $\begin{array}{l}\text { Adseverin; } \\
\text { assuri }\end{array}$ & $\begin{array}{l}\text { Gelsolin-like protein;Scinderin; } \\
\text {. }\end{array}$ \\
\hline $\begin{array}{l}\text { LKSERLHQLVM } \\
\text { LKEM }\end{array}$ & $\begin{array}{l}\text { NKKDKNIAMDVLNYVGR } \\
\text { NIAKE }\end{array}$ & $\begin{array}{l}\text { n.d. } \\
\text { n.d. }\end{array}$ & $\begin{array}{l}\mathrm{N} \text {-ter }+42.201 \mathrm{Da} \mathrm{K}+\mathrm{K}+43.06 \mathrm{Da} \\
\mathrm{N}-\mathrm{ter}+42.01 \mathrm{Da}, \mathrm{K}+28.03 \mathrm{Da}\end{array}$ & $\begin{array}{l}19,8 \\
17,7\end{array}$ & $\begin{array}{l}-1,929 \\
3,229\end{array}$ & $\begin{array}{l}2 \\
3\end{array}$ & $\begin{array}{l}1034,6 \\
2103,2\end{array}$ & $\begin{array}{l}\text { AFF3 MOUSE } \\
\text { ADIPMOUSE }\end{array}$ & D3YYY6_MOUSE & $\begin{array}{l}\text { AFF//FMR2 family member } 3 ; \\
\text { Afadin- and alpha-actinin-binding protein; }\end{array}$ & $\begin{array}{l}\text { Lymphoid nuclear r roteiein related to A A44; } \\
\text { Afadin DIL domain-interacting protein; }\end{array}$ \\
\hline LQMEAQRICL & FVVYSTHADQVR & n.d. & N-ter + 42.01 Da & 12,4 & 3,072 & 2 & 1462,7 & AKAP9_MOUSE & E9QQ10_MOUSE & A-kinase anchor protein 9; & Protein kinase A-anchoring protein 9; \\
\hline M & SAAETGSEPSQGAGPSEATLHSLR & n.d. & $\mathrm{N}$-ter $+42.01 \mathrm{Da}$ & 72,8 & 1,133 & 3 & 2381,1 & E9Q3E1_MOUSE & & $\begin{array}{l}\text { Aldehyde dehydrogenase } \\
\{\text { ECO:0000256|PIRNR:PIRNR036492\}; }\end{array}$ & \\
\hline GVACLREIAK & QRCAPASIR & n.d. & $\mathrm{N}$-ter + 42.01 Da, C +57.02 Da & 14,7 & 3,540 & 2 & 1099,6 & ADAS_MOUSE & A2AL50_MOUSE & $\begin{array}{l}\text { Alkyldihydroxyacetonephosphate synthase, } \\
\text { peroxisomal; }\end{array}$ & AlkyIIIycerone-phosphate synthase; \\
\hline M & AGVDQHEGTIQVQGQNLFFR & n.d. & $\mathrm{N}$-ter $+42.01 \mathrm{Da}$ & 55,4 & 0,962 & 3 & 2285,1 & ABHEB_MOUSE & & Alpha/beta hydrolase domain-containing protein 14B; & ; CCG1-interacting factor B; \\
\hline YTEQTQHILN & SMVSKLQEDPR & n.d. & $\mathrm{N}$-ter + 42.01 Da, K +34.06 Da & 24,1 & $-2,780$ & 2 & 1364,7 & MA2A2_MOUSE & & Alpha-mannosidase $2 \mathrm{x}$; & $\begin{array}{l}\text { Alpha-mannosidase IIx;Mannosidase alpha } \\
\text { class 2A member 2;Mannosyl-oligosaccharide } \\
\text { 1,3-1,6-alpha-mannosidase; }\end{array}$ \\
\hline M & DNSGKOAEAMALLAEAER & n.d. & $\mathrm{N}$-ter + 42.01 Da, $\mathrm{K}+34.06 \mathrm{Da}$ & 79,7 & 1,467 & 3 & 2110,0 & SNAA_MOUSE & & Alpha-soluble NSF attachment protein; & $\begin{array}{l}\text { N-ethylmaleimide-sensitive factor attachment } \\
\text { protein alpha; }\end{array}$ \\
\hline M & ADKMDMSLDDIIKLNR & n.d. & $\mathrm{N}$-ter + 42.01 Da, $\mathrm{K}+34.06 \mathrm{Da}$ & 59,0 & 0,352 & 3 & 1987,1 & ALRF2_MOUSE & THOC4_MOUSE & Aly/REF export factor 2; & Alyref;RNA and export factor-binding protein 2; \\
\hline MFLCRFWGKM & ATNDAVLKR & n.d. & N-ter + 42.01 Da, K+34.06 Da & 45,6 & 0,564 & 2 & 1062,6 & AIMP1_MOUSE & Q3UZG4_MOUSE & $\begin{array}{l}\text { Aminoacyl tRNA synthase complex-interacting } \\
\text { multifunctional protein } 1 \text {; }\end{array}$ & $\begin{array}{l}\text { Multisynthase complex auxiliary component } \\
\text { p43; }\end{array}$ \\
\hline $\begin{array}{l}M \\
M\end{array}$ & $\begin{array}{l}\text { TTKDPESEHPSVTLFR } \\
\text { AELDIGOHCOVOHCR }\end{array}$ & $\begin{array}{l}\text { n.d. } \\
\text { n.d. }\end{array}$ & $\mathrm{N}$-ter + 42.01 Da, K+34.06 Da & $\begin{array}{l}41,8 \\
32,4\end{array}$ & $\begin{array}{r}1,509 \\
-0,264\end{array}$ & $\begin{array}{l}3 \\
3\end{array}$ & $\begin{array}{l}1919,0 \\
18918\end{array}$ & ACY1_MOUSE & 027765 М О४⿻上丨 & Aminoacylase-1; & N-acyl-L-amino-acid amidohydrolase; \\
\hline M & AQETNHSQAPMLCSTGCGFYGNPR & n.d. & $\mathrm{N}$-ter + 42.01 Da, C +57.02 Da & $\begin{array}{l}32,4 \\
65,3\end{array}$ & 0,660 & 3 & 2724,2 & ZFANG_MOUSE & 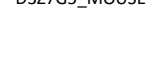 & AN1-type zinc finger protein 6 ; & $\begin{array}{l}\text { Associated with PRK1 protein;Zinc finger A20 } \\
\text { domain-containing protein } 3 \text {; }\end{array}$ \\
\hline M & AKPAGDAAVGSR & n.d. & $\mathrm{N}$-ter + 42.01 Da, K +34.06 Da & 22,8 & 0,935 & 2 & 1174,7 & ANKZ1_MOUSE & J3QM81_MOUSE & $\begin{array}{l}\text { Ankyrin repeat and zinc finger domain-containing } \\
\text { protein 1; }\end{array}$ & \\
\hline M & $\begin{array}{l}\text { EKATVPAAAEGEGSPPAAAAVAAPPAAAAAEVGGG } \\
\text { AR }\end{array}$ & n.d. & N-ter + 42.01 Da, K+34.06 Da & 67,7 & 2,133 & 4 & 3417,8 & ANR17_MOUSE & E9QKG6_MOUSE & Ankyrin repeat domain-containing protein 17 & $\begin{array}{l}\text { Ankyrin repeat domain-containing protein } \\
\text { FOE;Gene trap ankyrin repeat protein; }\end{array}$ \\
\hline M & SALLEQKEQQER & n.d. & $\mathrm{N}$-ter + 42.01 Da, K+34.06 Da & 42,0 & $-3,125$ & 2 & 1533,8 & ANR40_MOUSE & & Ankyrin repeat domain-containing protein 40; & Rnt \\
\hline MMNEDAAQK & SDSGEKFNGSSQR & n.d. & $\mathrm{N}$-ter $+42.01 \mathrm{Da}, \mathrm{K}+28.03 \mathrm{Da}$ & 15,7 & $-0,476$ & 2 & 1467,7 & ANK2_MOUSE & S4R249_MOUSE & Ankyrin-2 \{ECO:0000250|UniProtKB:Q01484\}; & 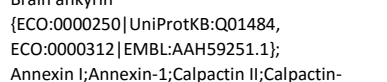 \\
\hline M & AMVSEFLKQAR & 0,2 & N-ter + 42.01 Da, K+28.03 Da & 61,2 & 6,219 & 2 & 1348,7 & ANXA1_MOUSE & & Annexin A1; & $\begin{array}{l}\text { 2;Chromobindin-9;Lipocortin I;Phospholipase } \\
\text { A2 inhibitory protein;p35; }\end{array}$ \\
\hline RELYDAGVKR & KGTDVPKWISIMTER & n.d. & $\mathrm{N}$-ter + 42.01 Da, K+34.06 Da & 14,6 & $-4,324$ & 3 & 1870,1 & ANXA2_MOUSE & BOV2N5_MOUSE & Annexin A2; & $\begin{array}{l}\text { Annexin II;Annexin-2;Calpactin I heavy } \\
\text { chain;CCalpactin-1 heavy chain;Chromobindin- } \\
\text { 8:Lipocortin IIIPlacental anticoagulant protein } \\
\text { IV;Protein I;;36; }\end{array}$ \\
\hline M & STVHELLCKLSLEGDHSTPPSAYGSVKPYTNFDAER & n.d. & $\begin{array}{l}\text { N-ter + 42.01 Da, K +34.06 Da, } \\
\text { C+57.02 Da }\end{array}$ & 45,5 & 2,476 & 5 & 4115,1 & ANXA2_MOUSE & BOV2N7_MOUSE & Annexin A2; & $\begin{array}{l}\text { Annexin II;Annexin-2;Calpactin I heavy } \\
\text { chain;Calpactin-1 heavy chain;Chromobindin- } \\
\text { 8;ipocortin II;Placental anticoagulant protein } \\
\text { IV;Protein I; } 336 \text {; }\end{array}$ \\
\hline M & EAKGGTVKAASGFNATEDAQTLR & 0,1 & N-ter + 42.01 Da, K+28.03 Da & 76,3 & 0,940 & 3 & 2550,3 & ANXA4_MOUSE & S4R1F2_MOUSE & Annexin A4; & Annexin IV;Annexin-4; \\
\hline DGRADAEVLR & KAMKGLGTDEDSILNLLTSR & $-0,3$ & $\mathrm{~N}$-ter + $42.01 \mathrm{Da}, \mathrm{K}+28.03 \mathrm{Da}$ & 17,5 & 1,503 & 3 & 2259,2 & ANXA5_MOUSE & & Annexin A5; & $\begin{array}{l}\text { Anchorin Cll;Annexin V;Annexin-5;Calphobindin } \\
\text { I; Endonexin II;Lipocortin V;Placental } \\
\text { anticoagulant protein 4;Placental anticoagulant } \\
\text { protein I;Thromboplastin inhibitor;:Vascular } \\
\text { anticoagulant-alpha; }\end{array}$ \\
\hline
\end{tabular}




\begin{tabular}{|c|c|c|c|c|c|c|c|c|c|c|c|}
\hline Non Prime Site & Prime Site & $\begin{array}{l}\text { Fold change of } \\
\text { XPRESS ratio } \\
\left.\text { (Adam17 }^{\Delta K C} / \mathrm{WT}\right)\end{array}$ & Modifications & Hyperscore & $\begin{array}{l}\text { Mass error } \\
\text { in ppm }\end{array}$ & charge & $\begin{array}{l}\text { Precursor } \\
\text { neutral mass } \\
\text { in Da }\end{array}$ & Uniprot ID 1 & Uniprot ID 2 & Protein Name 1 & Protein Name 2 \\
\hline M & AKIAQGAMYR & n.d. & $\mathrm{N}$-ter + 42.01 Da, K +34.06 Da & 48,2 & $-1,349$ & 2 & 1183,7 & ANXAG_MOUSE & F8WIT2_MOUSE & Annexin A6; & $\begin{array}{l}67 \text { kDa calelectrin;Annexin VI;Annexin- } \\
\text { 6;Calphobindin-II;Chromobindin-20;Lipocortin } \\
\text { VI;Protein III; } 688 ; p 70 ;\end{array}$ \\
\hline PGRGPGPGPS & AGPGPGPGPSPGSGPAR & n.d. & $\mathrm{N}$-ter $+42.01 \mathrm{Da}$ & 17,5 & $-9,728$ & 3 & 1456,7 & ANTRL_MOUSE & & Anthrax toxin receptor-like; & $\begin{array}{l}\text { AP-mu chain family member mu1B;Adaptor } \\
\text { protein complex AP-1 subunit mu-2;Adaptor- }\end{array}$ \\
\hline LLPGSRLDVS & SASAVFILDVKGKPLISR & n.d. & $\mathrm{N}$-ter + 42.01 Da, K +34.06 Da & 26,7 & 1,689 & 3 & 2010,3 & AP1M2_MOUSE & & AP-1 complex subunit mu-2; & $\begin{array}{l}\text { related protein complex } 1 \text { subunit mu- } \\
\text { 2;Clathrin assembly protein complex } 1 \text { mu-2 } \\
\text { medium chain 2;Golgi adaptor HA1/AP1- } \\
\text { adaptin mu-2 subunit;Mu-adaptin 2;Mu1B- } \\
\text { adaptin; } \\
\text { Apolipoprotein B-100 receptor; }\end{array}$ \\
\hline MAAEIKTEYP & EKEVTLLHSR & n.d. & $\mathrm{N}$-ter + 42.01 Da, K+28.03 Da & 17,1 & $-0,857$ & 3 & 1280,7 & AlFM2_MOUSE & & Apoptosis-inducing factor 2; & $\begin{array}{l}\text { Apoptosis-inducing factor homologous } \\
\text { mitochondrion-associated inducer of } \\
\text { death;Apoptosis--nducing factor-like } \\
\text { mitochondrion-associated inducer of death; }\end{array}$ \\
\hline QQKQRELVLA & VSKVEELTR & n.d. & $\mathrm{N}$-ter + 42.01 Da, K+34.06 Da & 11,6 & $-5,186$ & 2 & 1135,7 & ASPP2_MOUSE & E9QJU8_MOUSE & Apoptosis-stimulating of $\mathrm{p5}$ & Tumor suppressor p53-binding protein 2; \\
\hline GTDPSTSRKM & AELEEVTLDGKPLQALR & 0,0 & $\mathrm{~N}$-ter + 42.01 Da, K+28.03 Da & 55,9 & 1,638 & 2 & 1951,1 & ACINU_MOUSE & F6RJ39_MOUSE & $\begin{array}{l}\text { Apoptotic chromatin conde } \\
\text { nucleus; }\end{array}$ & \\
\hline M & ASESGKLWGGR & 0,3 & $\mathrm{~N}$-ter + 42.01 Da, K+28.03 Da & 42,5 & 4,103 & 2 & 1216,6 & ARLY_MOUSE & EOCY49_MOUSE & Argininosuccinate lyase; & Arginosuccinase; \\
\hline TVVAVLNTIH & EIVSDSLDNAR & n.d. & $\mathrm{N}$-ter $+42.01 \mathrm{Da}$ & 16,1 & 9,432 & 2 & 1259,6 & ARVC_MOUSE & & $\begin{array}{l}\text { Armadillo repeat protein o } \\
\text { syndrome homolog; }\end{array}$ & \\
\hline RCIENIPKKN & DLSLHPNEDRHLVEIIFSPR & n.d. & $\mathrm{N}$-ter + 42.01 Da & 19,0 & $-5,141$ & 3 & 2428,2 & CP19A_MOUSE & & Aromatase; & $\begin{array}{l}\text { CYPXIX;Cytochrome P-450AROM;Cytochrome } \\
\text { P450 19A1;Estrogen synthase; }\end{array}$ \\
\hline AHPDFNGGAW & GPWASEEEEEEEEEEEEGR & n.d. & $\mathrm{N}$-ter $+42.01 \mathrm{Da}$ & 10,9 & $-1,765$ & 3 & 2319,9 & ARSI_MOUSE & & Arylsulfatase I; & \\
\hline MAPRK & $\begin{array}{l}\text { NAKGGGGNSSSSGSGSGSGSGSPS } \\
\mathrm{R}\end{array}$ & n.d. & $\mathrm{N}$-ter + 42.01 Da, K+34.06 Da & 17,0 & $-0,896$ & 4 & 3120,4 & ASPH_MOUSE & Q6P8S1_MOUSE & Asparty//asparaginyl beta-hydroxylase; & $\begin{array}{l}\text { Aspartate beta-hydroxylase;Peptide-aspartate } \\
\text { beta-dioxygenase; }\end{array}$ \\
\hline IPLPVEEQCR & GVLSEPLPDLQLLTGDIR & n.d. & $\mathrm{N}$-ter $+42.01 \mathrm{Da}$ & 15,2 & $-5,303$ & 3 & 1977,1 & ASTN2_MOUSE & E9Q8T4_MOUSE & Astrotactin-2; & \\
\hline CEPVYGPLTM & SLKPQPQPPAPATGR & n.d. & N-ter + 42.01 Da, K+34.06 Da & 27,8 & 0,739 & 3 & 1619,9 & ATX2_MOUSE & E9QM77_MOUSE & Ataxin-2; & Spinocerebellar ataxia type 2 protein homolog; \\
\hline $\begin{array}{l}\text { M } \\
\text { RNLHASNTRL }\end{array}$ & $\begin{array}{l}\text { LKPQPPQQTSQPQQPPPTQQAVAR } \\
\text { QRTGTAEMSSILEER }\end{array}$ & $\begin{array}{l}-0,1 \\
\text { n.d. }\end{array}$ & $\begin{array}{l}\text { N-ter + 42.01 Da, K+28.03 Da } \\
\text { N-ter + 42.01 Da, K+28.03 Da }\end{array}$ & $\begin{array}{l}66,9 \\
17,7\end{array}$ & $\begin{array}{l}-0,140 \\
-2,112\end{array}$ & $\begin{array}{l}3 \\
3\end{array}$ & $\begin{array}{l}2850,5 \\
1748,9\end{array}$ & $\begin{array}{l}\text { ATX2L_MOUSE } \\
\text { ATPA_MOUSE }\end{array}$ & D6RJ16_MOUSE & $\begin{array}{l}\text { Ataxin-2-like protein; } \\
\text { ATP synthase subunit alph }\end{array}$ & \\
\hline LDMLDLGAAR & ANLEKAQSELSGAADEAAR & n.d. & $\mathrm{N}$-ter + 42.01 Da, K+34.06 Da & 24,1 & 2,439 & 3 & 2006,0 & ATPD_MOUSE & & ATP synthase subunit delta, mitochondrial; & F-ATPase delta subunit; \\
\hline M & SWLFGIKGPKGEGTGPLPLPPAQPGAEGGGDR & n.d. & N-ter + 42.01 Da, K+28.03 Da & 20,8 & $-4,728$ & 4 & 3337,7 & ATAD3_MOUSE & H3BKI6_MOUSE & ATPase family AAA domain-containing protein 3 ; & AAA-ATPase TOB3; \\
\hline ANVLICGPNG & CGKSSLFR & n.d. & $\begin{array}{l}\text { N-ter + } 42.01 \mathrm{Da}, \mathrm{K}+34.06 \mathrm{Da}, \\
\mathrm{C}+57.02 \mathrm{Da}\end{array}$ & 11,2 & 5,526 & 2 & 1029,6 & ABCD1_MOUSE & ABCD2_MOUSE & ATP-binding cassette sub-family D member 1 ; & Adrenoleukodystrophy protein; \\
\hline GAHNTIICAR & AGKDLVSSLTSGLLTIGDR & n.d. & N-ter + 42.01 Da, K+34.06 Da & 22,0 & 0,959 & 3 & 1978,1 & ACLY_MOUSE & Q3V117_MOUSE & ATP-citrate synthase; & $\begin{array}{l}\text { ATP-citrate (pro-S--Iyase; Citrate cleavage } \\
\text { enzyme; }\end{array}$ \\
\hline M & SAKAISEQTGKELYYKYICTTSAIQNR & n.d. & $\begin{array}{l}\text { N-ter + 42.01 Da, } \mathrm{K}+34.06 \mathrm{Da} \\
\mathrm{C}+57.02 \mathrm{Da}\end{array}$ & 50,1 & 5,961 & 4 & 3216,8 & ACLY_MOUSE & Q3V117_MOUSE & ATP-citrate synthase; & $\begin{array}{l}\text { ATP-citrate (pro-S--Iyase; Citrate cleavage } \\
\text { enzyme; }\end{array}$ \\
\hline M & ATVDLEKLR & n.d. & N-ter + 42.01 Da, K +34.06 Da & 32,4 & $-0,624$ & 2 & 1119,7 & KGPL_MOUSE & & $\begin{array}{l}\text { ATP-dependent 6-phosphofructokinase, liver type } \\
\text { \{ECO:0000255|HAMAP-Rule:MF_03184\}; }\end{array}$ & $\begin{array}{l}\text { 6-phosphoffructokinase type B;Phosphofructo-1. } \\
\text { kinase isozyme B;Phosphohexokkinase } \\
\text { \{ECO:0000255|HAMAP-Rule:MF_03184\}; }\end{array}$ \\
\hline M & GDIKNFLYAWCGKR & n.d. & $\begin{array}{l}\text { N-ter + 42.01 Da, K+34.06 Da, } \\
\text { C +57.02 Da }\end{array}$ & 23,7 & $-0,163$ & 3 & 1837,0 & DHX9_MOUSE & E9QNN1_MOUSE & ATP-dependent RNA helicase A; & DEAH box protein 9;Nuclear DNA helicase Il; \\
\hline NVCYHKERRK & ILTTEGR & n.d. & $\mathrm{N}-\operatorname{ter}+42.01 \mathrm{Da}$ & 11,5 & $-5,766$ & 2 & 830,5 & DHX9_MOUSE & E9QNN1_MOUSE & ATP-dependent RNA helicase A; & DEAH box protein 9;Nuclear DNA helicase II; \\
\hline M & $\begin{array}{l}\text { SHVAVENALGLDQQFAGLDLNSSDNQSGGSTASKG } \\
\mathrm{R}\end{array}$ & n.d. & $\mathrm{N}$-ter + 42.01 Da, K+34.06 Da & 59,0 & $-2,696$ & 4 & 3705,8 & DDX3X_MOUSE & & ATP-dependent RNA helicase DDX3X; & $\begin{array}{l}\text { D1Pas1-related sequence 2; DEAD box RNA } \\
\text { helicase DEAD3;:DEAD box protein } 3, x- \\
\text { chromosomal;:Embryonic RNA helicase; }\end{array}$ \\
\hline PRRGGKRGRS & NWNKGGPG & n.d. & $+34.06 \mathrm{Da}$ & $\begin{array}{l}23,7 \\
21,4\end{array}$ & $\begin{array}{l}0,755 \\
8,102\end{array}$ & $\begin{array}{l}3 \\
3\end{array}$ & $\begin{array}{l}1454,8 \\
1539,8\end{array}$ & DUSE & & DXX42; & DEAD box protein 42; \\
\hline $\begin{array}{l}\text { PRRGGKRGRS } \\
\text { NEKGEKELEP }\end{array}$ & & n.d. & $\begin{array}{l}\text { N-ter + 42.01 Da } \\
\text { N-ter + 42.01 Da }\end{array}$ & $\begin{array}{l}21,4 \\
17,2\end{array}$ & $\begin{array}{l}8,102 \\
-6,755\end{array}$ & 3 & $\begin{array}{l}1539,8 \\
1299,7\end{array}$ & $\begin{array}{l}\text { DDX54_MOUSE } \\
\text { SUV3 MOUSE }\end{array}$ & & $\begin{array}{l}\text { ATP-dependent RNA helicase DDX54; } \\
\text { ATP-dependent RNA helicase SUPV3L1, }\end{array}$ & DEAD box protein 54; \\
\hline NEKGEKELEP & ITTSQALQIAGR & n.d. & N-ter + 42.01 Da & & & & 1299,1 & SUV3_MOUSE & & mitochondrial; & Suppressor of var1 3-like protein 1; \\
\hline
\end{tabular}




\begin{tabular}{|c|c|c|c|c|c|c|c|c|c|c|c|}
\hline Non Prime Site & Prime Site & $\begin{array}{l}\text { Fold change of } \\
\text { XPRESS ratio } \\
\left.\text { (Adam17 } 17^{\Delta K C} / \mathrm{WT}\right)\end{array}$ & Modifications & Hyperscore & $\begin{array}{l}\text { Mass error } \\
\text { in ppm }\end{array}$ & Charge & $\begin{array}{l}\text { Precursor } \\
\text { neutral mass } \\
\text { in Da }\end{array}$ & Uniprot ID 1 & Uniprot ID 2 & Protein Name 1 & Protein Name 2 \\
\hline SQCWGAAAAA & AAAAAAVSGGAQQR & n.d. & $\mathrm{N}$-ter + 42.01 Da & 19,4 & 1,887 & 2 & 1269,7 & ARI1A_MOUSE & E9QAQ7_MOUSE & AT-rich interactive domain-containing protein $1 \mathrm{~A}$; & $\begin{array}{l}\text { BRG1-associated factor 250;BRG1-associated } \\
\text { factor } 2500 \text {;Osa homolog 1;SWI-like } \\
\text { protein;SWI/SNF complex protein } \\
\text { p270;SWI/SNF-related, matrix-associated, actin } \\
\text { dependent regulator of chromatin subfamily F } \\
\text { member 1; }\end{array}$ \\
\hline RTCGQCLEQP & GCGWCNDPSNTGR & n.d. & $\mathrm{N}$-ter $+42.01 \mathrm{Da}, \mathrm{C}+57.02 \mathrm{Da}$ & 12,0 & $-4,923$ & 2 & 1521,6 & ATRN1_MOUSE & & Attractin-like protein 1 ; & \\
\hline M & TDDKDVLR & n.d. & $\mathrm{N}$-ter + 42.01 Da, $\mathrm{K}+34.06 \mathrm{Da}$ & 24,5 & 0,481 & 2 & 1036,6 & ATG5_MOUSE & & Autophagy protein 5 & APG5-like; \\
\hline ATETTRESNH & GDAAAVHSTVVDLGR & n.d. & $\mathrm{N}$-ter + 42.01 Da & 14,6 & $-3,307$ & 3 & 1508,8 & BIR1E_MOUSE & BIR1G_MOUSE & Baculoviral IAP repeat-containing protein 1e; & Neuronal apoptosis inhibitory protein 5; \\
\hline AEEVTQTDNM & AKTEEMVQTEEMETPR & n.d. & $\mathrm{N}$-ter + 42.01 Da, K+34.06 Da & 44,1 & $-0,554$ & 3 & 1983,9 & BAG1_MOUSE & FGTCF9_MOUSE & BAG family molecular chaperone regulator 1 & BCl-2-associated athanogene $1 ;$ \\
\hline M & AQAKISAKAHEGR & n.d. & $\mathrm{N}$-ter + 42.01 Da, K+34.06 Da & 27,1 & $-0,270$ & 4 & 1475,9 & BAG2_MOUSE & & BAG family molecular chaperone regulator 2 & BCl-2-associated athanogene 2; \\
\hline M & DMGNQHPSISR & n.d. & N-ter+42.01 Da & 31,1 & 0,071 & 2 & 1413,6 & BAG5_MOUSE & EOCYW3_MOUSE & BAG family molecular chaperone regulator 5 ; & Bcl-2-associated athanogene 5; \\
\hline LQTFATQTLG & CIVDRSLSR & n.d. & N-ter + 42.01 Da, C +57.02 Da & 20,8 & $-4,440$ & 2 & 1146,6 & BBS10_MOUSE & & Bardet-Biedl syndrome 10 protein homolog; & \\
\hline RGQTVTFTCV & ATGVPTPIINWR & n.d. & $\mathrm{N}$-ter $+42.01 \mathrm{Da}$ & 15,3 & $-9,132$ & 3 & 1365,7 & PGBM_MOUSE & B1BOC7_MOUSE & $\begin{array}{l}\text { Basement membrane-specific heparan sulfate } \\
\text { protegglycan core protein; }\end{array}$ & \\
\hline M & NNQKQQKPTLLGQR & n.d. & $\mathrm{N}$-ter + 42.01 Da, K +34.06 Da & 28,2 & $-4,973$ & 3 & 1867,0 & BZW1_MOUSE & & $\begin{array}{l}\text { Basic leucine zipper and W2 domain-containing } \\
\text { protein } 1 \text {; }\end{array}$ & \\
\hline HLSEVIKEHE & KMCPLLLCPMNFVQLR & n.d. & $\begin{array}{l}\text { N-ter + 42.01 Da, K+28.03 Da, } \\
\text { C }+57.02 \text { Da }\end{array}$ & 15,9 & 9,130 & 3 & 2089,1 & BATF3_MOUSE & & Basic leucine zipper transcriptional factor ATF-like 3; & \\
\hline M & DKLINKITVPASQKLR & n.d. & $\mathrm{N}$-ter + 42.01 Da, K +34.06 Da & 16,0 & 1,157 & 3 & 1985,3 & BLNK_MOUSE & D3YWR2_MOUSE & B-cell linker protein; & 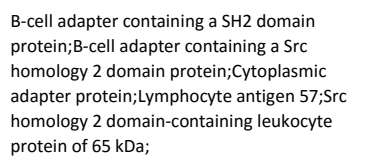 \\
\hline HNGDMEKILL & DAQHESGQSSSR & n.d. & N-ter +42.01 Da & 17,4 & $-7,961$ & 2 & 1329,5 & BNIIL_MOUSE & Q91278_MOUSE & $\begin{array}{l}\text { BCL2/adenovirus E1B } 19 \mathrm{kDa} \text { protein-interacting } \\
\text { protein 3-like; }\end{array}$ & NIP3-like protein $\mathrm{x}$; \\
\hline M & AKQPSDVSSECDR & n.d. & $\begin{array}{l}\text { N-ter + 42.01 Da, } \mathrm{K}+34.06 \mathrm{Da}, \\
\mathrm{C}+57.02 \mathrm{Da}\end{array}$ & 12,5 & 2,957 & 2 & 1553,7 & B2L11_MOUSE & Q6PEB3_MOUSE & Bcl-2-like protein 11; & Bcl2-interacting mediator of cell death; \\
\hline M & EGSKASSSTMQVSFVCQR & 1,0 & $\begin{array}{l}\text { N-ter + 42.01 Da, K+28.03 Da, } \\
\mathrm{C}+57.02 \mathrm{Da}\end{array}$ & 30,5 & $-1,688$ & 3 & 2189,0 & BECN1_MOUSE & E9PX06_MOUSE & Beclin-1; & $\begin{array}{l}\text { Coiled-coil myosin-like BCL2-interacting } \\
\text { protein; }\end{array}$ \\
\hline FWATLRIPG & IPGGISSSSQDVSDLQSKTR & n.d. & $\mathrm{N}$-ter + 42.01 Da, K+28.03 Da & 18,1 & 8,524 & 4 & 2131,1 & GCNT4_MOUSE & & $\begin{array}{l}\text { Beta-1,3-galactosyl-O-glycosyl-glycoprotein beta-1,6- } \\
\text { N-acetylglucosaminyltransferase } 4 \text {; }\end{array}$ & $\begin{array}{l}\text { Core 2-branching enzyme 3;Core2-GIcNAC- } \\
\text { transferase 3; }\end{array}$ \\
\hline Mv & DDELTALVVDNGSGMCKAGFGGDDAPR & n.d. & $\begin{array}{l}\text { N-ter + 42.01 Da, K+28.03 Da, } \\
\mathrm{C}+57.02 \mathrm{Da}\end{array}$ & 14,4 & $-9,333$ & 3 & 2836,2 & ACTBL_MOUSE & & Beta-actin-like protein 2; & Kappa-actin; \\
\hline LVVDNGSGMC & KAGFGGDDAPR & $-0,1$ & $\mathrm{~N}$-ter + 42.01 Da, K +28.03 Da & 20,7 & 0,000 & 2 & 1159,6 & ACTBL_MOUSE & & Beta-actin-like protein 2; & Kappa-actin; \\
\hline M & VDDELTALVVDNGSGMCKAGFGGDDAPR & n.d. & $\begin{array}{l}\text { N-ter + 42.01 Da, K+28.03 Da, } \\
\mathrm{C}+57.02 \mathrm{Da}\end{array}$ & 14,9 & $-1,702$ & 3 & 2935,3 & ACTBL_MOUSE & & Beta-actin-like protein 2; & Kappa-actin; \\
\hline M & AMQKIFAR & $-0,3$ & $\mathrm{~N}$-ter + 42.01 Da, K+28.03 Da & 24,2 & $-2,511$ & 2 & 1033,6 & ENOB_MOUSE & Q55X59_MOUSE & Beta-enolase; & $\begin{array}{l}\text { 2-phospho-D--glycerate hydro-Iyase; Enolase } \\
\text { 3;Muscle-specific enolase;skeletal muscle } \\
\text { enolase; }\end{array}$ \\
\hline MCGVNIIIFa & EAWNMPFAFCTR & n.d. & $\mathrm{N}$-ter + 42.01 Da, C +57.02 Da & 15,4 & $-2,989$ & 2 & 1570,7 & BUP1_MOUSE & & Beta-ureidopropionase; & $\begin{array}{l}\text { Beta-alanine synthase; N-carbamoyl-beta- } \\
\text { alanine amidohydrolase; }\end{array}$ \\
\hline $\begin{array}{l}\text { M } \\
\text { RLLSGEEEEE }\end{array}$ & $\begin{array}{l}\text { NNAGLNSEKVSALIQK } \\
\text { EOSOGTGLEYPERPASR }\end{array}$ & $\begin{array}{l}\text { n.d. } \\
\text { n.d. }\end{array}$ & $\begin{array}{l}\text { N-ter + 42.01 Da, } \mathrm{K}+34.06 \mathrm{Da} \\
\text { N-ter + 42.01 Da }\end{array}$ & $\begin{array}{l}77,3 \\
17,8\end{array}$ & $\begin{array}{l}-0,415 \\
-1,591\end{array}$ & $\begin{array}{l}2 \\
2\end{array}$ & $\begin{array}{l}1926,1 \\
1945,9\end{array}$ & $\begin{array}{l}\text { BLMH_MOUSE } \\
\text { BMP4_MOUSE }\end{array}$ & E9Q6V3_MOUSE & $\begin{array}{l}\text { Bleomycin hydrolase; } \\
\text { Bone morphogenetic protein } 4\end{array}$ & Bone morphogenetic protein $2 \mathrm{~B}$ : \\
\hline GMLQNRVLLW & KEQSEASR & n.d. & $\mathrm{N}$-ter + 42.01 Da, K+28.03 Da & 19,0 & 0,000 & 2 & 1003,5 & B12L2_MOUSE & E9QAX1_MOUSE & $\begin{array}{l}\text { Brain-specific angiogenesis inhibitor 1-associated } \\
\text { protein 2-like protein 2; }\end{array}$ & $\begin{array}{l}\text { Planar intestinal- and kidney-specific BAR } \\
\text { domain protein; }\end{array}$ \\
\hline FLATSSSSSA & SNSSEESKASPGAR & n.d. & N-ter + 42.01 Da, K+34.06 Da & 17,7 & $-6,196$ & 3 & 1481,7 & BRWD1_MOUSE & E9Q2N1_MOUSE & Bromodomain and WD repeat-containing protein $1 ;$ & WD repeat-containing protein 9 ; \\
\hline $\begin{array}{l}\text { M } \\
\text { CAAQRQGFEK } \\
\text { SIIOGOPYFS }\end{array}$ & $\begin{array}{l}\text { AENHCELLPPAPSGLGAGLGGGLCR } \\
\text { KGPVSGLR } \\
\text { VDPKTGVIR }\end{array}$ & $\begin{array}{l}\text { n.d. } \\
\text { n.d. } \\
\text { n.d. }\end{array}$ & $\begin{array}{l}\text { N-ter + 42.01 Da, C +57.02 Da } \\
\text { N-ter + 42.01 Da, K+34.06 Da } \\
\text { N-ter + 42.01 Da K +34.06 Da }\end{array}$ & $\begin{array}{l}57,3 \\
14,9 \\
11,6\end{array}$ & $\begin{array}{l}-0,824 \\
-0,561 \\
-4,404\end{array}$ & $\begin{array}{l}3 \\
2 \\
2\end{array}$ & $\begin{array}{l}2544,2 \\
888,6 \\
1059,7\end{array}$ & $\begin{array}{l}\text { KCTD5_MOUSE } \\
\text { CELR2_MOUSE } \\
\text { CAD22 MOUSE }\end{array}$ & $\begin{array}{l}\text { A2AEE7_MOUSE } \\
\text { O8BBB5 MOUSE }\end{array}$ & $\begin{array}{l}\text { BTB/POZ domain-containing protein KCTD5; } \\
\text { Cadherin EGF LAG seven-pass G-type receptor 2; } \\
\text { Cadherin-22. }\end{array}$ & $\begin{array}{l}\text { Flamingo homolog; } \\
\text { Pituitary and brain cadherin: }\end{array}$ \\
\hline M & SSEPPPPPLQPPTHQTSVGLLDTPR & n.d. & $\mathrm{N}$-ter + 42.01 Da & 50,0 & 0,371 & 3 & 2689,4 & CHSP1_MOUSE & J3QPI4_MOUSE & Calcium-regulated heat stable protein 1 ; & $\begin{array}{l}\text { Pitutatyry and brain caaherin; } \\
\text { Calcium-regulated heat-stable protein of } 24\end{array}$ \\
\hline $\begin{array}{l}\text { SEEEIREAFR } \\
\mathrm{M}\end{array}$ & $\begin{array}{l}\text { VFDKDGNGVISAAELR } \\
\text { SHGFTKEEVAEFQAAFNR }\end{array}$ & $\begin{array}{c}\text { n.d. } \\
0,3\end{array}$ & $\begin{array}{l}\text { N-ter + 42.01 Da, K+34.06 Da } \\
\text { N-ter + 42.01 Da, K+28.03 Da }\end{array}$ & $\begin{array}{l}21,5 \\
106,2\end{array}$ & $\begin{array}{r}5,728 \\
-0,748\end{array}$ & $\begin{array}{l}3 \\
2\end{array}$ & $\begin{array}{l}1830,0 \\
2137,0\end{array}$ & $\begin{array}{l}\text { CALM_MOUSE } \\
\text { CALM4_MOUSE }\end{array}$ & Q3UKW2_MOUSE & $\begin{array}{l}\text { E Calmodulin; } \\
\text { Calmodulin-4; }\end{array}$ & Calcium-binding protein Dd112; \\
\hline M & VEAAPAGSGPLRR & n.d. & N-ter +42.01 Da & 22,7 & 0,907 & 2 & 1321,7 & CAMP3_MOUSE & E9Q5BO_MOUSE & Calmodulin-regulated spectrin-associated protein 3; & Protein Nezha; \\
\hline M & FLAKAILEGADR & 0,3 & $\mathrm{~N}$-ter $+42.01 \mathrm{Da}, \mathrm{K}+28.03 \mathrm{Da}$ & 64,2 & $-0,863$ & 2 & 1503,8 & CPNS2_MOUSE & & Calpain small subunit 2; & Calcium-dependent protease small subunit 2; \\
\hline
\end{tabular}




\begin{tabular}{|c|c|c|c|c|c|c|c|c|c|c|c|}
\hline Non Prime Site & Prime Site & $\begin{array}{l}\text { Fold change of } \\
\text { XPRESS ratio } \\
\text { (Adam17 } 1 \text { ¿Kc / WT) }\end{array}$ & Modifications & Hyperscore & $\begin{array}{l}\text { Mass error } \\
\text { in ppm }\end{array}$ & Charge & $\begin{array}{l}\text { Precursor } \\
\text { neutral mass } \\
\text { in Da }\end{array}$ & Uniprot ID 1 & Uniprot ID 2 & Protein Name 1 & Protein Name 2 \\
\hline M & TEELTPVYCTGVSAQVQKKR & 0,6 & $\begin{array}{l}\text { N-ter + 42.01 Da, K+28.03 Da, } \\
\mathrm{C}+57.02 \mathrm{Da}\end{array}$ & 24,2 & 0,678 & 3 & 2504,3 & CAN1_MOUSE & & Calpain-1 catalytic subunit; & $\begin{array}{l}\text { Calcium-activated neutral proteinase 1;Calpain } \\
\text { mu-type;Calpain-1 large subunit;:Micromolar- } \\
\text { calpain; }\end{array}$ \\
\hline M & SQPGPKPAASPRPSR & n.d. & $\mathrm{N}$-ter + 42.01 Da, $\mathrm{K}+34.06 \mathrm{Da}$ & 20,9 & $-0,186$ & 3 & 1607,9 & ICAL_MOUSE & & Calpastatin; & \\
\hline M & SSTQFNKGPSYGLSAEVKNR & n.d. & N-ter + 42.01 Da, K+34.06 Da & 87,5 & $-0,482$ & 3 & 2279,2 & CNN2_MOUSE & D3Z7R6_MOUSE & Calponin-2; & Calponin $\mathrm{H} 2$, smooth muscle;Neutral calponin; \\
\hline $\begin{array}{l}\text { LGGPPSVCKA } \\
\text { EFASGQWAGI }\end{array}$ & $\begin{array}{l}\text { TLSEETCQQLAR } \\
\text { ELDEPEGKNNGSVGR }\end{array}$ & $\begin{array}{l}\text { n.d. } \\
\text { n.d. }\end{array}$ & $\begin{array}{l}\text { N-ter + 42.01 Da, C +57.02 Da } \\
\text { N-ter + 42.01 Da, K+34.06 Da }\end{array}$ & $\begin{array}{l}20,8 \\
18,2\end{array}$ & $\begin{array}{l}-3,179 \\
-8,637\end{array}$ & $\begin{array}{l}2 \\
3\end{array}$ & $\begin{array}{l}1476,7 \\
1675,8\end{array}$ & $\begin{array}{l}\text { PDEAA_MOUSE } \\
\text { CLIP4_MOUSE }\end{array}$ & & $\begin{array}{l}\text { CAMP-specific 3',5'-cyclic phosphodiesterase 4A; } \\
\text { CAP-Gly domain-containing linker protein 4; }\end{array}$ & Restin-like protein 2; \\
\hline RHEECCTFFH & RYQLETISENIR & n.d. & $\mathrm{N}$-ter + 42.01 Da & 17,4 & $-4,790$ & 3 & 1562,8 & CMTR2_MOUSE & & $\begin{array}{l}\text { Cap-specific mRNA (nucleoside-2'-O-)- } \\
\text { methyltransferase 2; }\end{array}$ & $\begin{array}{l}\text { Cap methyltransferase 2;Cap2 2'O-ribose } \\
\text { methyltransferase 2; Fts methyltransferase } \\
\text { domain-containing protein 1; }\end{array}$ \\
\hline M & EERPSETNSNVDSSAQPSVAQLAGR & n.d. & $\mathrm{N}$-ter $+42.01 \mathrm{Da}$ & 47,0 & $-0,678$ & 3 & 2801,3 & CPZIP_MOUSE & & Capz-interacting protein; & Protein kinase substrate CapZIP; \\
\hline M & SSSRPVALVTGANKGIGFAITR & n.d. & $\mathrm{N}$-ter + 42.01 Da, $\mathrm{K}+34.06 \mathrm{Da}$ & 56,0 & 1,140 & 3 & 2277,3 & CBR1_MOUSE & & Carbonyl reductase [NADPH] 1; & $\begin{array}{l}\text { 15-hydroxyprostaglandin dehydrogenase } \\
\text { [NADP(+)];NADPH-dependent carbonyl } \\
\text { reductase 1; Prostaglandin 9- } \\
\text { ketoreductase;Prostaglandin-E(2) 9-reductase; }\end{array}$ \\
\hline EDVVDSFQVYY & CVEEPQDDQEINELVEEYR & n.d. & $\mathrm{N}$-ter + 42.01 Da, C +57.02 Da & 17,9 & 2,870 & 4 & 2435,1 & CMYAS_MOUSE & E9Q_O_MOUSE & Cardiomyopathy-associated protein 5; & $\begin{array}{l}\text { Heart and skeletal muscle-specific and sprouty } \\
\text { domain-containing;Myospryn;Stretch-response } \\
\text { protein 553;Stretch-responsive fibronectin } \\
\text { protein type 3;TRIM-like protein; }\end{array}$ \\
\hline RTSPDAFVQI & ALQLAHFR & n.d. & $\mathrm{N}$-ter + 42.01 Da & 12,6 & 5,107 & 2 & 996,6 & CPT1B_MOUSE & & Carnitine 0-palmitoyltransferase 1, muscle isoform; & $\begin{array}{l}\text { Carnitine O-palmitoyltransferase I, muscle } \\
\text { isoform; Carnitine palmitoyltransferase } 1 \mathrm{~B} \text {; }\end{array}$ \\
\hline $\begin{array}{l}\text { M } \\
\text { PSGRSGHSTR }\end{array}$ & $\begin{array}{l}\text { ASSSGSKAEFIVGGKYKLVR } \\
\text { GTGSSSSGLMVGPNFR }\end{array}$ & $\begin{array}{l}\text { n.d. } \\
\text { n.d. }\end{array}$ & $\begin{array}{l}\text { N-ter + 42.01 Da, K+34.06 Da } \\
\text { N-ter + 42.01 Da }\end{array}$ & $\begin{array}{l}36,2 \\
12,3\end{array}$ & $\begin{array}{l}0,090 \\
-2,595\end{array}$ & $\begin{array}{l}4 \\
2\end{array}$ & $\begin{array}{l}2227,3 \\
1693,8\end{array}$ & $\begin{array}{l}\text { KC1A_MOUSE } \\
\text { KC1G3_MOUSE }\end{array}$ & E9PWB2_MOUSE & $\begin{array}{l}\text { Casein kinase I isoform alpha; } \\
\text { Casein kinase I isoform gamma-3; }\end{array}$ & \\
\hline M & TETDGFYKSR & 1,4 & $\mathrm{~N}$-ter $+42.01 \mathrm{Da}, \mathrm{K}+28.03 \mathrm{Da}$ & 20,2 & 0,314 & 2 & 1272,6 & CASPG_MOUSE & & Caspase-6; & Apoptotic protease Mch-2; \\
\hline мт & AVHAGNINFKWDPKSLEIR & n.d. & N-ter + 42.01 Da, K+34.06 Da & 16,5 & 2,730 & 3 & 2304,3 & CTNA1_MOUSE & & Catenin alpha-1; & $\begin{array}{l}102 \text { kDa cacherin-associated protein;Alpha E- } \\
\text { catenin;CAP102; }\end{array}$ \\
\hline M & TAVHAGNINFKWDPKSLEIR & 0,0 & $\mathrm{~N}$-ter + 42.01 Da, K+28.03 Da & 36,0 & 2,295 & 3 & 2393,3 & CTNA1_MOUSE & & Catenin alpha-1; & $\begin{array}{l}102 \text { kDa cadherin-associated protein;Alpha E- } \\
\text { catenin;CAP102; }\end{array}$ \\
\hline M & DDSEVESTASLLASVKEQEEAQFEKLITR & n.d. & N-ter+ $42.01 \mathrm{Da}, \mathrm{K}+28.03 \mathrm{Da}$ & 15,1 & 1,944 & 3 & 3238,6 & CTND1_MOUSE & G3X9V2_MOUSE & Catenin delta-1; & $\begin{array}{l}\text { Cadherin-associated Src substrate; } 120 \\
\text { catenin;p120(cas); }\end{array}$ \\
\hline QTNSYRMSAI & IFTCDESEDIGRPQVFSEDR & n.d. & $\mathrm{N}$-ter + 42.01 Da, C +57.02 Da & 12,4 & $-1,105$ & 3 & 2441,1 & MPRI_MOUSE & & Cation-independent mannose-6-phosphate receptor; & $\begin{array}{l}300 \text { kDa mannose 6-phosphate receptor;insulin- } \\
\text { like growth factor } 2 \text { receptor;insulin-like } \\
\text { growth factor II receptor; } 66 \mathrm{P} / \mathrm{IGF2} \text { receptor; }\end{array}$ \\
\hline M & SGGKYVDSEGHLYTVPIR & $-0,1$ & $\mathrm{~N}$-ter + 42.01 Da, K+28.03 Da & 38,4 & $-0,781$ & 3 & 2047,0 & CAV1_MOUSE & H3BLK5_MOUSE & Caveolin-1; & \\
\hline $\begin{array}{l}\text { APAGPGGAVM } \\
\text { FYEVEPRPPM }\end{array}$ & $\begin{array}{l}\text { SAGAHGPPPGYGCAAAGYLDGR } \\
\text { SSHLOSPPHAPSNAAFGFPR }\end{array}$ & $\begin{array}{l}\text { n.d. } \\
\text { n.d. }\end{array}$ & $\begin{array}{l}\text { N-ter + 42.01 Da, C +57.02 Da } \\
\text { N-ter + 42.01 Da }\end{array}$ & $\begin{array}{l}27,8 \\
20,8\end{array}$ & $\begin{array}{l}-7,689 \\
-3,071\end{array}$ & $\begin{array}{l}3 \\
3\end{array}$ & $\begin{array}{l}2142,9 \\
2146,0\end{array}$ & $\begin{array}{l}\text { CEBPA_MOUSE } \\
\text { CEBPA_MOUSE }\end{array}$ & & $\begin{array}{l}\text { CCAAT/enhancer-binding protein alpha; } \\
\text { CCAAT/enhancer-binding protein alpha; }\end{array}$ & \\
\hline M & VDYIVEYDYDAVHDDELTIR & n.d. & $\mathrm{N}$-ter $+42.01 \mathrm{Da}$ & 50,2 & $-0,643$ & 3 & 2484,1 & CD2AP_MOUSE & & CD2-associated protein; & $\begin{array}{l}\text { Mesenchyme-to-epithelium transition protein } \\
\text { with } S H 3 \text { - }\end{array}$ \\
\hline $\begin{array}{l}\text { CEPVMEEKVQ } \\
\text { LGNIERLKLE }\end{array}$ & $\begin{array}{l}\text { EOSSLLENGAVHQGDTSWGSSGGGSQSSQGR } \\
\text { VQKYEAWLAEAESR }\end{array}$ & $\begin{array}{l}\text { n.d. } \\
\text { n.d. }\end{array}$ & $\begin{array}{l}\text { N-ter + 42.01 Da } \\
\text { N-ter + 42.01 Da, } \mathrm{K}+34.06 \mathrm{Da}\end{array}$ & $\begin{array}{l}21,8 \\
20,9\end{array}$ & $\begin{array}{l}-6,066 \\
-9,955\end{array}$ & $\begin{array}{l}4 \\
3\end{array}$ & $\begin{array}{l}3161,4 \\
1754,9\end{array}$ & $\begin{array}{l}\text { BORG2_MOUSE } \\
\text { CIP4_MOUSE }\end{array}$ & & $\begin{array}{l}\text { Cdc42 effector protein 3; } \\
\text { Cdc42-interacting protein 4; }\end{array}$ & $\begin{array}{l}\text { Binder of Rho GTPases 2; } \\
\text { Thyroid receptor-interacting protein 10; }\end{array}$ \\
\hline M & VGGEASAAVEKLVSGVR & n.d. & $\mathrm{N}$-ter + 42.01 Da, K +34.06 Da & 31,6 & 0,871 & 2 & 1835,0 & C2AIL_MOUSE & & CDKN2AIP N-terminal-like protein; & $\begin{array}{l}\text { CDKN2A-interacting protein N-terminal-like } \\
\text { protein; }\end{array}$ \\
\hline M & AMNYSAKDEVDGGPAGPPGGAAKTR & 0,1 & $\mathrm{~N}$-ter $+42.01 \mathrm{Da}, \mathrm{K}+28.03 \mathrm{Da}$ & 43,5 & $-1,549$ & 3 & 2514,2 & CC50A_MOUSE & D3YVV1_MOUSE & Cell cycle control protein 50A; & $\begin{array}{l}\text { P4-ATPase flippase complex beta subunit } \\
\text { TMEM30A; Transmembrane protein 30A; }\end{array}$ \\
\hline M & HSLATAAPVPTALAQVDR & n.d. & $\mathrm{N}$-ter $+42.01 \mathrm{Da}$ & 53,5 & $-1,405$ & 3 & 1990,0 & RCD1_MOUSE & & Cell differentiation protein RCD1 homolog; & $\begin{array}{l}\text { CCR4-NOT transcription complex subunit 9; EPO- } \\
\text { induced protein FL10; }\end{array}$ \\
\hline M & SSNECFKCGR & n.d. & $\begin{array}{l}\text { N-ter + 42.01 Da, K+34.06 Da, } \\
\mathrm{C}+57.02 \mathrm{Da}\end{array}$ & 46,6 & 0,151 & 2 & 1319,6 & CNBP_MOUSE & D3YYF6_MOUSE & Cellular nucleic acid-binding protein; & Zinc finger protein 9 ; \\
\hline M & ASFVTEVLAHSGSLEKEDLGTR & $-0,1$ & $\mathrm{~N}$-ter + 42.01 Da, K+28.03 Da & 29,2 & 1,944 & 3 & 2415,2 & ZW10_MOUSE & & Centromere/kinetochore protein zw10 homolog; & \\
\hline $\begin{array}{l}\text { EELRRHESEK } \\
\text { PHDMLDDELS }\end{array}$ & $\begin{array}{l}\text { ATMLGDVSSLR } \\
\text { SPERHDSDCSMDGR }\end{array}$ & $\begin{array}{l}\text { n.d. } \\
\text { n.d. }\end{array}$ & $\begin{array}{l}\text { N-ter + 42.01 Da } \\
\text { N-ter + 42.01 Da, C +57.02 Da }\end{array}$ & $\begin{array}{l}20,3 \\
14,3\end{array}$ & $\begin{array}{r}0,755 \\
-0,472\end{array}$ & $\begin{array}{l}2 \\
4\end{array}$ & $\begin{array}{l}1190,6 \\
1689,7\end{array}$ & $\begin{array}{l}\text { CP135_MOUSE } \\
\text { A2AUM9_MOUSE }\end{array}$ & & $\begin{array}{l}\text { Centrosomal protein of } 135 \mathrm{kDa} \text {; } \\
\text { Centrosomal protein of } 152 \mathrm{kDa} \text {; }\end{array}$ & Centrosomal protein 4; \\
\hline RIAKNNLELV & NDKMAAQLEETGKR & n.d. & $\mathrm{N}$-ter + 42.01 Da, K+34.06 Da & 19,4 & 4,580 & 3 & 1699,9 & CE290_MOUSE & E9व9M0_MOUSE & Centrosomal protein of $290 \mathrm{kDa}$; & $\begin{array}{l}\text { Bardet-Biedl syndrome } 14 \text { protein } \\
\text { homolog;Nephrocystin-6; }\end{array}$ \\
\hline EGLSQEVRNE & ICYNILVLLLR & n.d. & $\mathrm{N}$-ter $+42.01 \mathrm{Da}, \mathrm{C}+57.02 \mathrm{Da}$ & 14,0 & $-8,988$ & 2 & 1477,8 & ADCK3_MOUSE & F7B1B6_MOUSE & $\begin{array}{l}\text { Chaperone activity of bc1 complex-like, } \\
\text { mitochondrial; }\end{array}$ & aarF domain-containing protein kinase 3; \\
\hline M & SVFGKLFGAGGGKAGKGGPTPQEAIQR & 0,2 & $\mathrm{~N}$-ter $+42.01 \mathrm{Da}, \mathrm{K}+28.03 \mathrm{Da}$ & 36,0 & 2,660 & 4 & 2740,5 & CHM4B_MOUSE & & Charged multivesicular body protein $4 \mathrm{~b}$; & Chromatin-modifying protein 4b; \\
\hline
\end{tabular}




\begin{tabular}{|c|c|c|c|c|c|c|c|c|c|c|c|}
\hline Non Prime Site & Prime Site & $\begin{array}{l}\text { Fold change of } \\
\text { XPRESS ratio } \\
\left.\text { (Adam17 } 17^{\Delta K C} / \mathrm{WT}\right)\end{array}$ & Modifications & Hyperscore & $\begin{array}{c}\text { Mass error } \\
\text { in ppm }\end{array}$ & Charge & $\begin{array}{l}\text { Precursor } \\
\text { neutral mass } \\
\text { in Da }\end{array}$ & Uniprot ID 1 & Uniprot ID 2 & Protein Name 1 & Protein Name 2 \\
\hline M & AEEQPQVELFVKAGSDGAKIGNCPFSQR & 0,1 & $\begin{array}{l}\text { N-ter+42.01 Da, K+28.03 Da, } \\
\mathrm{C}+57.02 \mathrm{Da}\end{array}$ & 43,8 & $-1,486$ & 3 & 3159,6 & CLIC1_MOUSE & & Chloride intracellular channel protein 1; & Nuclear chloride ion channel 27; \\
\hline M & AETTKLQLFVKASEDGESVGHCPSCQR & 0,1 & $\begin{array}{l}\text { N-ter }+42.01 \mathrm{Da}, \mathrm{K}+28.03 \mathrm{Da} \\
\mathrm{C}+57.02 \mathrm{Da}\end{array}$ & 46,5 & $-1,244$ & 4 & 3131,5 & CLIC3_MOUSE & & Chloride intracellular channel protein 3; & \\
\hline M & DAQSSAKVNSR & $-1,7$ & $\mathrm{~N}$-ter + 42.01 Da, K+28.03 Da & 47,2 & $-2,052$ & 2 & 1362,7 & PCY1A_MOUSE & D3Z3T5_MOUSE & Choline-phosphate cytidylyltransferase A; & $\begin{array}{l}\text { CCT-alpha;CTP:phosphocholine } \\
\text { cytidylyltransferase A; } \\
\text { transfersphoryesy;choline }\end{array}$ \\
\hline M & AMHNKTAPPQIPDTR & n.d. & N-ter + 42.01 Da, K+34.06 Da & 30,4 & $-1,311$ & 3 & 1751,9 & EAF6_MOUSE & & Chromatin modification-related protein MEAF6; & Esa1-associated factor 6 homolog; \\
\hline SLDVPGFGTS & FSDKPKQR & n.d. & N-ter + 42.01 Da, K+34.06 Da & 11,3 & 7,074 & 2 & 1114,7 & A3KFM7_MOUSE & & Chromodomain-helicase-DNA-binding protein 6; & ATP-dependent helicase CHDG; \\
\hline YIWNTLNSGR & VVPGYGHAVLR & n.d. & N-ter $+42.01 \mathrm{Da}$ & 12,5 & $-1,322$ & 2 & 1208,7 & CISY_MOUSE & Q80X68_MOUSE & Citrate synthase, mitochondrial; & Citrate (Si)-synthase; \\
\hline KLRVICCKGN & LVKESGTEQHR & 1,0 & N-ter + 42.01 Da, K +28.03 Da & 17,0 & 2,213 & 3 & 1352,7 & CTRO_MOUSE & FGSBRS_MOUSE & Citron Rho-interacting kinase; & $\begin{array}{l}\text { Rho-interacting, serine/threonine-protein } \\
\text { kinase 21; }\end{array}$ \\
\hline CRVLISSLKD & CLHGIEAKSYSVGASGSSSR & n.d. & $\begin{array}{l}\text { N-ter + 42.01 Da, } \mathrm{K}+34.06 \mathrm{Da}, \\
\mathrm{C}+57.02 \mathrm{Da}\end{array}$ & 16,1 & $-7,696$ & 3 & 2128,0 & CPSF7_MOUSE & & $\begin{array}{l}\text { Cleavage and polyadenylation specificity factor } \\
\text { subunit 7; }\end{array}$ & \\
\hline M & SSLAVRDPAMDR & n.d. & $\mathrm{N}$-ter $+42.01 \mathrm{Da}$ & 28,0 & $-0,515$ & 2 & 1358,7 & CSTFT_MOUSE & & Cleavage stimulation factor subunit 2 tau variant; & $\begin{array}{l}\text { CF-1 } 64 \text { kDa subunit tau variant; Cleavage } \\
\text { stimulation factor } 64 \mathrm{kDa} \text { subunit tau } \\
\text { variant; TauCStF-64; }\end{array}$ \\
\hline M & AGLPVRDPAVDR & n.d. & $\mathrm{N}$-ter + 42.01 Da & 32,5 & $-0,688$ & 2 & 1306,7 & CSTF2_MOUSE & A2AEK1_MOUSE & Cleavage stimulation factor subunit 2 ; & $\begin{array}{l}\text { CF-1 } 64 \text { kDa subunit; Cleavage stimulation } \\
\text { factor } 64 \text { kDa subunit; }\end{array}$ \\
\hline YYETVRMAAI & KESWTHNIQR & n.d. & N-ter + 42.01 Da, K+34.06 Da & 14,1 & $-4,361$ & 2 & 1373,7 & SIAT6_MOUSE & Q9CZ48_MOUSE & $\begin{array}{l}\text { CMP-N-acetylneuraminate-beta-1,4-galactoside alpha } \\
\text { 2,3-sialyltransferase; }\end{array}$ & 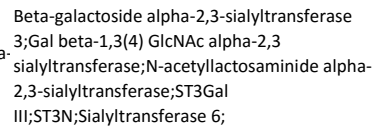 \\
\hline M & ATKIDKEACR & $-0,1$ & $\begin{array}{l}\text { N-ter + 42.01 Da, } \mathrm{K}+28.03 \mathrm{Da} \\
\mathrm{C}+57.02 \mathrm{Da}\end{array}$ & 30,0 & 3,177 & 2 & 1288,7 & COTL1_MOUSE & & Coactosin-like protein; & \\
\hline GHNHYVMCAQ & FHPSEDLVVSASLDQTVR & n.d. & $\mathrm{N}$-ter $+42.01 \mathrm{Da}$ & 11,9 & 6,706 & 2 & 2041,0 & COPA_MOUSE & F8WHL2_MOUSE & Coatomer subunit alpha; & Alpha-coat protein; \\
\hline VTGYLGAGKT & TLLNYITTEQHNR & n.d. & $\mathrm{N}$-ter $+42.01 \mathrm{Da}$ & 16,7 & 2,592 & 3 & 1655,9 & CBWD1_MOUSE & & COBW domain-containing protein $1 ;$ & $\begin{array}{l}\text { Cobalamin synthase } \mathrm{W} \text { domain-containing } \\
\text { protein } 1 \text {; }\end{array}$ \\
\hline M & ASGVAVSDGVIIVFNDMKVR & n.d. & $\mathrm{N}$-ter + 42.01 Da, K +34.06 Da & 55,2 & 0,136 & 3 & 2201,3 & COF1_MOUSE & F8WGL__MOUSE & Cofilin-1; & $\begin{array}{l}\text { Cofilin, non-muscle isoform; } \\
\text { S }\end{array}$ \\
\hline MA & SGVAVSD & n.d. & N-ter + $42.01 \mathrm{Da}, \mathrm{K}+34.06 \mathrm{Da}$ & 29,2 & 1,641 & 3 & 2130,2 & COF1_MOUSE & F8WGL3_MOUSE & Cofilin-1; & Cofilin, non-muscle isoform; \\
\hline M & ASGVTVI & n.d. & $\mathrm{N}$-ter + 42.01 Da, K +34.06 Da & 58,5 & 1,071 & 3 & 2330,3 & COF2_MOUSE & & Cofilin-2; & Cofilin, muscle isoform; \\
\hline $\begin{array}{l}\text { GLPPEAQAPA } \\
\text { MOSELYPVSM }\end{array}$ & LGQALT & n.d. & $\mathrm{N}$-ter + 42.01 Da & 16,7 & $-9,075$ & 4 & 2398,3 & CC142_MOUSE & & Coiled-coil do & \\
\hline MQSELVPVSM & SETEHIASISSDATTEKTSELR & $-0,1$ & $\mathrm{~N}$-ter + 42.01 Da, K+28.03 Da & 23,4 & $-1,704$ & 3 & 2461,2 & CJ118_MOUSE & & Coiled-coil domain-containing protein 186; & Oocyte-testis gene 1 protein; \\
\hline M & ADVSVDQSKLPGVKEVCR & $-0,1$ & $\begin{array}{l}\text { N-ter } 422.01 \text { Da, K+28.03 Da, } \\
\text { C + } 57.02 \text { Da }\end{array}$ & 32,8 & 6,947 & 3 & 2084,1 & CCD50_MOUSE & & Coiled-coil domain-containing protein 50 ; & Protein Ymer; \\
\hline M & AAPSGSVNCEEFAEFQELLKVMR & 0,2 & $\begin{array}{l}\text { N-ter + 42.01 Da, K +28.03 Da, } \\
\mathrm{C}+57.02 \mathrm{Da}\end{array}$ & 17,4 & $-1,378$ & 3 & 2681,3 & CCD58_MOUSE & & Coiled-coil domain-containing protein 58; & \\
\hline KDGIPGFRGD & KGDIGFMGPR & n.d. & N-ter + 42.01 Da, K +34.06 Da & 12,3 & $-8,921$ & 2 & 1152,6 & CO7A1_MOUSE & & Collagen alpha-1(VIII) chain; & Long-chain collagen; \\
\hline LCSLALSLLA & HFRTAELQARVLR & n.d. & $\mathrm{N}$-ter + 42.01 Da & 23,7 & $-0,427$ & 3 & 1637,9 & CODA1_MOUSE & D3Z7B8_MOUSE & Collagen alpha-1(XIII) chain; & \\
\hline LLALALCSLA & LSLLAH & n.d. & N-ter + & 12,4 & 0,700 & 2 & 997,6 & CODA1_MOUSE & D3Z7B__MOUSE & Collagen alpha- & \\
\hline M & AALTAEHFVALQSLLKASSKDVVR & 0,1 & $\mathrm{~N}$-ter + $42.01 \mathrm{Da}, \mathrm{K}+28.03 \mathrm{Da}$ & 22,0 & $-4,067$ & 4 & 2651,5 & COMD9_MOUSE & & COMM domain-containing protein 9 ; & \\
\hline QIFEHMLDVS & KLTDTICGVGNMSANASDQER & n.d. & $\begin{array}{l}\text { N-ter + +22.01 Da, K+28.03 Da, } \\
\text { C+57.02 Da }\end{array}$ & 16,6 & 1,753 & 3 & 2336,1 & CO2_MOUSE & B8JJN2_MOUSE & Complement C2; & C3/C5 convertase; \\
\hline SLESGWDRLR & QLFAKDEQQR & n.d. & $\mathrm{N}$-ter + 42.01 Da, K+28.03 Da & 21,1 & 1,200 & 2 & 1331,7 & TIDC1_MOUSE & & Complex I assembly factor TIMMDC1, mitochondrial; & $\begin{array}{l}\text { Translocase of inner mitochondrial membrane } \\
\text { domain-containing protein } 1 \text {; }\end{array}$ \\
\hline VELFATSOK & FIQETELSQR & n.d. & $\mathrm{N}$-ter $+42.01 \mathrm{Da}$ & 23,5 & $-0,850$ & 2 & 1291,6 & CNDH2_MOUSE & E9PY48_MOUSE & Condensin-2 complex subunit H2; & $\begin{array}{l}\text { Kleisin-beta; Non-SMC condensin II complex } \\
\text { subunit H2; }\end{array}$ \\
\hline NIVEEDTVLY & CYEYEKGRSSSQGR & n.d. & $\begin{array}{l}\text { N-ter + } 42.01 \text { Da, } \mathrm{K}+28.03 \mathrm{Da}, \\
\mathrm{C}+57.02 \mathrm{Da}\end{array}$ & 19,6 & 3,036 & 3 & 1775,8 & CNKR2_MOUSE & A2A178_MOUSE & Connector enhancer of kinase suppressor of ras 2; & CNK homolog protein 2; \\
\hline LRSSVSEGIL & AVDERMSKQEDIR & n.d. & $\mathrm{N}$-ter + 42.01 Da, K+28.03 Da & 11,8 & $-0,970$ & 3 & 1645,8 & COG2_MOUSE & & Conserved oligomeric Golgi complex subunit 2; & $\begin{array}{l}\text { Component of oligomeric Golgi complex 2; Low } \\
\text { density lipoprotein receptor defect C- } \\
\text { complementing protein; }\end{array}$ \\
\hline $\begin{array}{l}\text { AALEDSINNA } \\
\text { EVCMAPEERR }\end{array}$ & $\begin{array}{l}\text { LDVKVLTTPSOSAVR } \\
\text { DSEDRVSK }\end{array}$ & $\begin{array}{l}\text { n.d. } \\
\text { n.d. }\end{array}$ & $\begin{array}{l}\text { N-ter + 42.01 Da, } \mathrm{K}+28.03 \mathrm{Da} \\
\text { N-ter + 42.01 Da, } \mathrm{K}+34.06 \mathrm{Da}\end{array}$ & $\begin{array}{l}17,3 \\
11,7\end{array}$ & $\begin{array}{r}6,130 \\
-4,247\end{array}$ & $\begin{array}{l}3 \\
2\end{array}$ & $\begin{array}{l}1710,0 \\
1010,5\end{array}$ & $\begin{array}{l}\text { COG5_MOUSE } \\
\text { CNST_MOUSE }\end{array}$ & & $\begin{array}{l}\text { Conserved oligomeric Golgi complex subunit 5; } \\
\text { Consortin; }\end{array}$ & Component of oligomeric Golgi complex 5 ; \\
\hline PPLPGARVLV & DAGSALPR & n.d. & $\mathrm{N}$-ter + 42.01 Da & 11,9 & $-8,440$ & 2 & 827,4 & F120C_MOUSE & & $\begin{array}{l}\text { Constitutive coactivator of PPAR-gamma-like protein } \\
2 ;\end{array}$ & Protein FAM120C; \\
\hline DVIHSVCWNS & NGSLLATTCKDKTLR & n.d. & $\begin{array}{l}\text { N-ter + 42.01 Da, K +34.06 Da, } \\
\text { C +57.02 Da }\end{array}$ & 15,5 & $-0,615$ & 3 & 1787,0 & CORO6_MOUSE & B9EIZ7_MOUSE & Coronin-6; & Coronin-like protein E; \\
\hline
\end{tabular}




\begin{tabular}{|c|c|c|c|c|c|c|c|c|c|c|c|}
\hline Non Prime Site & Prime Site & $\begin{array}{l}\text { Fold change of } \\
\text { XPRESS ratio } \\
\text { (Adam17 } 7^{\text {akc } / \text { WT) }}\end{array}$ & Modifications & Hyperscore & $\begin{array}{c}\text { Mass error } \\
\text { in ppm }\end{array}$ & charge & $\begin{array}{l}\text { Precursor } \\
\text { neutral mass } \\
\text { in } \mathrm{Da}\end{array}$ & 5 Uniprot ID 1 & Uniprot ID 2 & Protein Name 1 & Protein Name 2 \\
\hline WNSNGSLLAT & TCKDKTLR & n.d. & N-ter + 42.01 Da, K +34.06 Da, & 20,1 & 5,739 & 2 & 1130,7 & CORO6_MOUSE & B9EIZ7_MOUSE & Coronin-6; & Coronin-like protein $\mathrm{E}$ \\
\hline M & NVEHEVNLLVEEIHR & n.d. & $\mathrm{N}$-ter $+42.01 \mathrm{Da}$ & 52,3 & 0,699 & 2 & 2002,0 & ABRAL_MOUSE & E9QMV2_MOUSE & Costars family protein ABRACL; & ABRA C-terminal-like protein; \\
\hline$\stackrel{M}{M}$ & $\begin{array}{l}\text { ALDPAEQHLR } \\
\text { AENDGPPNR }\end{array}$ & n.d. & N-ter + 42.01 Da & 29,3 & $-1,174$ & 2 & 1190,6 & COXM1_MOUSE & EQYPR5 MOUSE & COX assembly mitochondrial protein hor & \\
\hline M & TSLFRRSSSGSGGGGATGAR & n.d. & N-ter + $42.01 \mathrm{Da}$ & 27,9 & $\begin{array}{l}-3,963 \\
-2,403\end{array}$ & 3 & 2041,0 & CUED1_MOUSE & F2Z3X3_MOUSE & CUE domain-containing protein 1; & \\
\hline M & ASASYHISNLLEKMTSSDKDFR & 0,2 & N-ter + 42.01 Da, K+28.03 Da & 19,0 & 0,192 & 4 & 2597,3 & CAND1_MOUSE & & Cullin-associated NEDD8-dissociated protein $1 ;$ & $\begin{array}{l}\text { Cullin-associated and neddylat } \\
\text { proteien 1-p120 CAND1: }\end{array}$ \\
\hline $\begin{array}{l}\text { SGGQRRGAIM } \\
\text { ASILHCLGRV }\end{array}$ & $\begin{array}{l}\text { SHKOIYYSDKYDDEEFEYR } \\
\text { LNLFEDEEKR }\end{array}$ & $\begin{array}{l}\text { n.d. } \\
\text { n.d. }\end{array}$ & $\begin{array}{l}\mathrm{N} \text {-ter + 42.01 Da, K+34.06 Da } \\
\mathrm{N} \text {-ter + 42.01 Da, K +34.06 Da }\end{array}$ & $\begin{array}{l}20,8 \\
13,4\end{array}$ & $\begin{array}{r}6,164 \\
-6,936\end{array}$ & $\begin{array}{l}4 \\
2\end{array}$ & $\begin{array}{l}2624,3 \\
1367,7\end{array}$ & $\begin{array}{l}\text { CKS1_MOUSE } \\
\text { CCNF_MOUSE }\end{array}$ & Q3UNC9_MOUSE & $\begin{array}{l}\text { Cyclin-dependent kinases regulatory subunit } 1 \text {; } \\
\text { Cyclin-F; }\end{array}$ & Sid 1334; \\
\hline SATKYMNGHS & DVWMGLVSVNSDDLNSR & n.d. & $\mathrm{N}$-ter + $42.01 \mathrm{Da}$ & 12,8 & $-5,633$ & 3 & 1860,9 & CGL_MOUSE & & Cystathionine gamma-Iyase; & $\begin{array}{l}\text { Cysteine-protein sulfhydrase; Gamma- } \\
\text { cystathionase. }\end{array}$ \\
\hline M & IPGGLTEARPATAEVQEIADR & n.d. & $\mathrm{N}$-ter $+42.01 \mathrm{Da}$ & 45,0 & 1,351 & 3 & 2366,2 & CYTA_MOUSE & EOCZ96_MOUSE & Cystatin-A; & Cystatin-A1; ;tefin-A; \\
\hline M & ATPEASGSGEKVEGSEPSVTYYR & n.d. & N-ter + 42.01 Da, K+34.06 Da & 65,2 & 1,089 & 3 & 2476,2 & CYBSB_MOUSE & & Cytochrome b5 type B; & $\begin{array}{l}\text { Cytochrome b5 outer mitochondrial membrane } \\
\text { isoform; } \\
\text { Complex III subunit 6; Complex III subunit }\end{array}$ \\
\hline EDERKMLTGS & GDPKEEEEEELVDPLTTVR & n.d. & $\mathrm{N}$-ter + 42.01 Da, K +34.06 Da & 27,0 & 0,309 & 3 & 2260,1 & QCR6_MOUSE & & Cytochrome b-c1 complex subunit 6 , mitochondrial; & 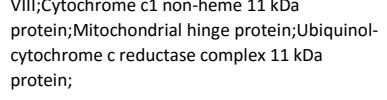 \\
\hline M & AAPGAGDPLNAKNGNAPFAQR & $-0,7$ & N-ter + 42.01 Da, K+28.03 Da & 20,7 & $-1,897$ & 3 & 2106,1 & COA3_MOUSE & E9Q6W2_MOUSE & $\begin{array}{l}\text { Cytochrome coxidase assembly factor } 3 \text { homolog, } \\
\text { mitochondrial; }\end{array}$ & Coiled-coil domain-containing protein 56; \\
\hline M & AAPSMKER & 0,1 & $\mathrm{~N}$-ter + 42.01 Da, K+28.03 Da & 18,1 & $-1,874$ & 2 & 958,5 & COAG_MOUSE & & Cytochrome c oxidase assembly factor 6 homolog; & \\
\hline M & AEDIKTKIKNYKTAPFDSR & n.d. & N-ter + 42.01 Da, K+28.03 Da & 15,1 & 0,378 & 4 & 2378,3 & CX6B1_MOUSE & & Cytochrome c oxidase subunit $6 \mathrm{B1}$; & Cytochrome c oxidase subunit Vlb isoform 1; \\
\hline M & SSGALPKPQI & n.d. & N-ter + 42.01 Da, K+34.06 Da & 46,1 & $-4,186$ & 2 & 1359,8 & COX6C_MOUSE & D3Z6E1_MOUSE & Cytochrome c oxidase subunit $6 \mathrm{C}$; & Cytochrome c oxidase polypeptide VIC; \\
\hline PLKNRLEHLA & TETTSTLR & $n$ & $\mathrm{~N}$-ter + 42.01 Da & 15,7 & 1,555 & 3 & 5,0 & CP237_MOUSE & CY250_MOUSE & Cytochrome P45 & CYPIIC37; \\
\hline $\begin{array}{l}\text { GYEAVKEALV } \\
\mathrm{M}\end{array}$ & $\begin{array}{l}\text { DHGDVFAGR } \\
\text { EKVPGDMEIER }\end{array}$ & $\begin{array}{l}\text { n.d. } \\
\text { n.d. }\end{array}$ & $\begin{array}{l}\mathrm{N} \text {-ter + 42.01 Da } \\
\mathrm{N} \text {-ter + 42.01 Da, } \mathrm{K}+34.06 \mathrm{Da}\end{array}$ & $\begin{array}{l}11,7 \\
13,8\end{array}$ & $\begin{array}{l}-6,788 \\
1,522\end{array}$ & $\begin{array}{l}2 \\
2\end{array}$ & $\begin{array}{l}1014,5 \\
1508,8\end{array}$ & $\begin{array}{l}\text { CP254_MOUSE } \\
\text { CYGB_MOUSE }\end{array}$ & & $\begin{array}{l}\text { Cytochrome P450 2C54; } \\
\text { Cytoglobin; }\end{array}$ & $\begin{array}{l}\text { CYPIIC54; } \\
\text { Histoglobin; }\end{array}$ \\
\hline TVSWPAHSYL & GLDYEVQHR & n.d. & N-ter + 42.01 Da & 15,8 & 9,132 & 3 & $\begin{array}{l}1508,8 \\
1157,6\end{array}$ & $\begin{array}{l}\text { CYGB_MOUSE } \\
\text { CRLF2_MOUSE }\end{array}$ & & $\begin{array}{l}\text { Cytoglobin; } \\
\text { Cytokine receptor-like factor 2; }\end{array}$ & $\begin{array}{l}\text { Histoglobin; } \\
\text { Cytokine receptor-like molecule 2;Thymic } \\
\text { stromal lymphopoietin protein receptor; Type I } \\
\text { cytokine receptor delta 1; }\end{array}$ \\
\hline M & SEPGGGEDGSAGLEVSAVQNVADVAVLOKHLR & 0,2 & $\mathrm{~N}$-ter + 42.01 Da, K+28.03 Da & 35,5 & 0,398 & 4 & 3258,7 & DYHC1_MOUSE & & Cytoplasmic dynein 1 heavy chain 1 ; & $\begin{array}{l}\text { Cytoplasmic dynein heavy chain 1; Dynein heavy } \\
\text { chain, cytosolic; }\end{array}$ \\
\hline GRMLSREANM & SDKSDLKAELER & 0,2 & $\mathrm{~N}$-ter + 42.01 Da, K+28.03 Da & 25,0 & $-0,403$ & 3 & 1487,8 & DC111_MOUSE & DC12_MOUSE & Cytoplasmic dynein 1 intermediate chain 1 ; & $\begin{array}{l}\text { Cytoplasmic dynein intermediate chain } \\
1 \text {;Dynein intermediate chain 1, cytosolic; } \\
\text { Cytoplassmic }\end{array}$ \\
\hline VLEYMSRIDR & VLSFPGGSLLLAGR & n.d. & $\mathrm{N}$-ter + 42.01 Da & 14,8 & 0,140 & 2 & 1427,8 & DYHC2_MOUSE & F6QLS2_MOUSE & Cytoplasmic dynein 2 heavy chain $1 ;$ & $\begin{array}{l}\text { Cytoplasmic dynein } 2 \text { heavy chain; ;ynnein } \\
\text { cytoplasmic heavy chain } 2 \text {; Dynnein heavy chain } \\
11 \text {;Dynein heavy chain isotype } 1 \mathrm{~B} ;\end{array}$ \\
\hline M & EEAPHGCPGADSAQAGR & n.d. & N-ter + 42.01 Da, C+57.02 Da & 55,7 & 0,584 & 2 & 1881,8 & NUBP1_MOUSE & & $\begin{array}{l}\text { Cytosolic Fe-S cluster assembly factor NUBP1 } \\
\text { \{ECO:0000255|HAMAP-Rule:MF_03038\}; }\end{array}$ & $\begin{array}{l}\text { Nuleotide-binding protein } 1 \\
\text { \{CCO:0000255|HAMAP-Rule:MF_03038\}; }\end{array}$ \\
\hline M & SALKAVFQYIDENQDR & 0,1 & $\mathrm{~N}$-ter $+42.01 \mathrm{Da}, \mathrm{K}+28.03 \mathrm{Da}$ & 94,4 & 0,051 & 2 & 1966,0 & CNDP2_MOUSE & & Cytosolic non-specific dipeptidase; & $\begin{array}{l}\text { CNDP dipeptidase 2;Glutamate } \\
\text { carboxypeptidase-like protein 1; }\end{array}$ \\
\hline M & NSAGADEIGKLFVGGLDWSTTQETLR & n.d. & $\mathrm{N}$-ter + 42.01 Da, K +34.06 Da & 15,2 & $-1,076$ & 3 & 2971,5 & DAZP1_MOUSE & Q3UGB5_MOUSE & DAZ-associated protein $1 ;$ & Deleted in azoospermia-associated protein 1; \\
\hline M & SSPPEGKLETKAGHPPAVKAGGMR & 0,9 & $\mathrm{~N}$-ter + 42.01 Da, K+28.03 Da & 16,8 & $-1,580$ & 4 & 2527,4 & DAP1_MOUSE & & Death-associated protein $1 ;$ & \\
\hline M & ANEVQVLPSPLKGR & n.d. & $\mathrm{N}$-ter + 42.01 Da, K+34.06 Da & 46,6 & $-1,893$ & 2 & 1582,9 & DAPL1_MOUSE & & Death-associated protein-like $1 ;$ & $\begin{array}{l}\text { Early epithelial differentiation-associated } \\
\text { protein; }\end{array}$ \\
\hline VTSNLGKMIL & KEEMEKSLPIRR & $-10,0$ & $\mathrm{~N}$-ter + 42.01 Da, K+28.03 Da & 18,6 & 1,858 & 2 & 1612,9 & DEMA_MOUSE & & Dematin; & Dematin actin-binding protein;:Erythrocyte \\
\hline $\begin{array}{l}\text { WQQTLNCKIQ } \\
\text { RMYEENALLR }\end{array}$ & $\begin{array}{l}\text { HITLQLLVGIFR } \\
\text { DHMTVNSLRR }\end{array}$ & $\begin{array}{l}\text { n.d. } \\
\text { n.d. }\end{array}$ & $\begin{array}{l}\text { N-ter + 42.01 Da } \\
\text { N-ter +42.01 Da }\end{array}$ & $\begin{array}{l}11,2 \\
11,3\end{array}$ & $\begin{array}{r}4,336 \\
-2,279\end{array}$ & $\begin{array}{l}2 \\
2\end{array}$ & $\begin{array}{l}1450,9 \\
1226,6\end{array}$ & $\begin{array}{l}\text { DEND3_MOUSE } \\
\text { DENSB_MOUSE }\end{array}$ & G3UYA1_MOUSE & $\begin{array}{l}\text { DENN domain-containing protein 3; } \\
\text { DENN domain-containing protein } 5 B \text {; }\end{array}$ & Rab6IP1-like protein; \\
\hline M & ATDISESSGADCKGDTKNSAKLDADYPLR & 0,2 & $\begin{array}{l}\text { N-ter + 42.01 Da, K+28.03 Da, } \\
\text { C +57.02 Da }\end{array}$ & 18,6 & $-0,062$ & 4 & 3210,5 & DENR_MOUSE & E9Q0G1_MOUSE & Density-regulated protein; & \\
\hline SQPRVSEVAM & QSAPLEQPAKRPR & n.d. & $\mathrm{N}$-ter + 42.01 Da, K +34.06 Da & 29,6 & 0,474 & 3 & 1683,9 & SAMH1_MOUSE & F8WJEO_MOUSE & $\begin{array}{l}\text { Deoxynucleoside triphosphate triphosphohydrolase } \\
\text { SAMHD1; }\end{array}$ & $\begin{array}{l}\text { Interferon-gamma-inducible protein } \\
\text { Mg11;SAM domain and HD domain-containing } \\
\text { protein 1; }\end{array}$ \\
\hline SVATGFPAGQ & THLKTRLEEIR & n.d. & N-ter + 42.01 Da, K+34.06 Da & 21,6 & 2,646 & 3 & 1470,9 & DEOC_MOUSE & & Deoxyribose-phosphate aldolase; & $\begin{array}{l}\text { 2-deooxy-D-ribose 5-phosphate } \\
\text { aldolase;Phosphodeoxyriboaldolase; }\end{array}$ \\
\hline VLNVDYRTVS & NLLTGPR & n.d. & N-ter + 42.01 Da & 13,8 & 6,152 & 2 & 924,6 & DPTOR MOUSE & & DEP domain-containing mTOR-interacting protein; & DEP domain-containing protein $6 ;$ \\
\hline
\end{tabular}




\begin{tabular}{|c|c|c|c|c|c|c|c|c|c|c|c|}
\hline Non Prime Site & Prime Site & $\begin{array}{l}\text { Fold change of } \\
\text { XPRESS ratio } \\
\text { (Adam17 } 1 \text { ¿Kc / WT) }\end{array}$ & Modifications & Hyperscore & $\begin{array}{l}\text { Mass error } \\
\text { in ppm }\end{array}$ & Charge & $\begin{array}{l}\text { Precursor } \\
\text { neutral mass } \\
\text { in Da }\end{array}$ & s Uniprot ID 1 & Uniprot ID 2 & Protein Name 1 & Protein Name 2 \\
\hline 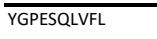 & $\begin{array}{l}\text { DKFILQNGAGNWLAQQIR } \\
\end{array}$ & n.d. & N-ter + 42.01 Da, K+28.03 Da & 17,7 & $-0,187$ & 3 & 2141,1 & DSEL_MOUSE & & Dermatan-sulfate epimerase-like protein; & \\
\hline GSGSRGTGSR & GGSGGSGGSGGGNKPECNNPGNDVR & n.d. & $\begin{array}{l}\text { N-ter + 42.01 Da, K+34.06 Da, } \\
\text { C +57.02 Da }\end{array}$ & 42,5 & 4,438 & 3 & 2363,1 & DMKN_MOUSE & E9Q2P1_MOUSE & Dermokine; & Epidermis-specific secreted protein SK30/SK89; \\
\hline AQPGYGTVRG & NNQNSGCTNPPPSGSHESFSNSGGSSNDGSR & n.d. & $\mathrm{N}$-ter + 42.01 Da, C +57.02 Da & 16,4 & $-6,132$ & 3 & 3177,3 & DMKN_MOUSE & E9Q2P1_MOUSE & Dermokine; & Epidermis-specific secreted protein $\mathrm{SK30/5K89;}$ \\
\hline MWPGVRLRVT & EGWDEDGHHAQDSLHYEGR & n.d. & $\mathrm{N}$-ter $+42.01 \mathrm{Da}$ & 11,5 & 1,796 & 4 & 2278,9 & DHH_MOUSE & & Desert hedgehog protein; & HHG-3; \\
\hline LPAGQELLGY & KAVDPETSSGEGLR & $-2,0$ & $\mathrm{~N}$-ter + 42.01 Da, K +28.03 Da & 24,3 & 6,259 & 3 & 1514,8 & DSC1_MOUSE & Q32ME9_MOUSE & Desmocollin-1; & \\
\hline PTFRQNTYET & AVEENTYNVEILR & n.d. & $\mathrm{N}$-ter $+42.01 \mathrm{Da}$ & 14,3 & $-0,753$ & 3 & 1590,8 & DSC3_MOUSE & G5E856_MOUSE & Desmocollin-3; & \\
\hline $\begin{array}{l}\text { DLMAWGVDAG } \\
\text { M }\end{array}$ & $\begin{array}{l}\text { SVEQHINSHRSIHNTIGDYR } \\
\text { ADFLKGLPVYNKSNFSR }\end{array}$ & $\begin{array}{l}\text { n.d. } \\
\text { n.d. }\end{array}$ & $\begin{array}{l}\mathrm{N} \text {-ter + 42.01 Da } \\
\text { N-ter + 42.01 Da, K +34.06 Da }\end{array}$ & $\begin{array}{l}16,7 \\
40,0\end{array}$ & $\begin{array}{l}3,031 \\
7,011\end{array}$ & $\begin{array}{l}4 \\
3\end{array}$ & $\begin{array}{l}2404,2 \\
2065,2\end{array}$ & $\begin{array}{l}\text { DESP_MOUSE } \\
\text { DDA1 MOUSE }\end{array}$ & $\begin{array}{l}\text { E9PZWO_MOUSE } \\
\text { D3YXY5 MOUSE }\end{array}$ & $\begin{array}{l}\text { Desmoplakin; } \\
\text { DET1-and DDB1-associated protein 1. }\end{array}$ & \\
\hline M & SGTLAKIAEIIEAEMAR & $-0,1$ & $\mathrm{~N}$-ter + 42.01 Da, K +28.03 Da & 85,3 & $-0,341$ & 2 & 1758,9 & DRG1_MOUSE & & Developmentally-regulated GTP-binding protein 1 & $\begin{array}{l}\text { Neural precursor cell expressed } \\
\text { developmentally down-regulated protein 3; }\end{array}$ \\
\hline TDTPWLCQPL & EPGEEESLQQNVMLDLTKR & n.d. & $\mathrm{N}$-ter + 42.01 Da, K+28.03 Da & 16,0 & 7,211 & 3 & 2285,1 & E9PUQ8_MOUSE & & $\begin{array}{l}\text { Diacylglycerol kinase } \\
\text { \{ECO:0000256|RuleBase:RU361128\}; }\end{array}$ & \\
\hline M & AKEKGLISPEDFAQLQKYIEYSTKR & n.d. & $\mathrm{N}$-ter + 42.01 Da, K +34.06 Da & 27,8 & $-1,536$ & 5 & 3119,8 & DGKA_MOUSE & & Diacylglycerol kinase alpha; & $\begin{array}{l}80 \text { kDa diacylglycerol kinase;Diglyceride kinase } \\
\text { alpha; }\end{array}$ \\
\hline $\begin{array}{l}\text { ETEPPKIVQM } \\
\text { LVVISOGKIV }\end{array}$ & $\begin{array}{l}\text { NNYCGIGIDAELSLDFHQAR } \\
\text { LEDGTLHVTEGSGR }\end{array}$ & $\begin{array}{l}\text { n.d. } \\
\text { n.d. }\end{array}$ & $\begin{array}{l}\text { N-ter + 42.01 Da, C +57.02 Da } \\
\text { N-ter + 42.01 Da }\end{array}$ & $\begin{array}{l}14,4 \\
102\end{array}$ & $\begin{array}{l}-0,899 \\
-6144\end{array}$ & $\begin{array}{l}3 \\
2\end{array}$ & $\begin{array}{l}2334,1 \\
15117\end{array}$ & $\begin{array}{l}\text { DGKQ_MOUSE } \\
\text { DPYLI MOUSE }\end{array}$ & & $\begin{array}{l}\text { Diacylglycerol kinase theta; } \\
\text { Dihydropyrimidinase-related protein } 2 .\end{array}$ & Diglyceride kinase theta; \\
\hline M & $\begin{array}{l}\text { LEDGLHVVIEGSGR } \\
\text { SYQGKNIPR }\end{array}$ & $\begin{array}{l}\text { n.d. } \\
-0,1\end{array}$ & $\begin{array}{l}\mathrm{N} \text {-ter }+42.01 \mathrm{Da} \\
\mathrm{N} \text {-ter + 42.01 Da, } \mathrm{K}+28.03 \mathrm{Da}\end{array}$ & $\begin{array}{l}10,2 \\
32,4\end{array}$ & $\begin{array}{l}-6,144 \\
2,404\end{array}$ & $\begin{array}{l}2 \\
2\end{array}$ & $\begin{array}{l}1511,7 \\
1287,7\end{array}$ & $\begin{array}{l}\text { DPYL2_MOUSE } \\
\text { DPYL2_MOUSE }\end{array}$ & DPYL3_MOUSE & $\begin{array}{l}\text { Dihydropyrimidinase-related protein 2; } \\
\text { Dihydropyrimidinase-related protein 2; }\end{array}$ & $\begin{array}{l}\text { Unc-33--ike phosphoprotein 2; } \\
\text { Unc-33-like phosphoprotein 2; }\end{array}$ \\
\hline GKPVPRVAEL & MGQKLPSFGPYLEQR & n.d. & $\mathrm{N}$-ter + 42.01 Da, K+34.06 Da & 16,4 & $-1,258$ & 3 & 1826,0 & DPYD_MOUSE & & Dihydropyrimidine dehydrogenase $[\mathrm{NADP}(+)]$; & $\begin{array}{l}\text { Dihydrothymine dehydrogenase; Dihydrouracil } \\
\text { dehydrogenase; }\end{array}$ \\
\hline AVFSEYVKTM & LRCGYAPVEFFLEGTRSR & n.d. & $\mathrm{N}$-ter + 42.01 Da, C +57.02 Da & 17,8 & $-9,764$ & 3 & 2199,1 & GNPAT_MOUSE & Q05DV2_MOUSE & Dihydroxyacetone phosphate acyltransferase; & $\begin{array}{l}\text { Acyl- } \\
\text { CoA:dihydroxyacetonephosphateacyltransferas } \\
\text { e;Glycerone-phosphate O-acyltransferase; }\end{array}$ \\
\hline M & MKLKSNQTR & 0,2 & $\mathrm{~N}$-ter + 42.01 Da, $\mathrm{K}+28.03 \mathrm{Da}$ & 19,6 & $-0,823$ & 3 & 1333,7 & NUDT3_MOUSE & I1E4X7_MOUSE & $\begin{array}{l}\text { Diphosphoinositol polyphosphate phosphohydrolase } \\
1 \text {; }\end{array}$ & $\begin{array}{l}\text { Diadenosine 5',5"'-P1,PG-hexaphosphate } \\
\text { hydrolase 1;Nucleoside diphosphate-linked } \\
\text { moiety X motif 3; }\end{array}$ \\
\hline KEMTQKLDL & TSDMADYQQPLMIGTGTVAR & n.d. & $\mathrm{N}$-ter $+42.01 \mathrm{Da}$ & 16,6 & 8,413 & 3 & 2196,0 & DCBD1_MOUSE & & Discoidin, CUB and LCCL domain-containing protein 1; & \\
\hline KYGPQGGCAD & HSVFERMR & n.d. & $\mathrm{N}$-ter + 42.01 Da & 20,1 & $-3,893$ & 2 & 1102,5 & ADA10_MOUSE & E9PYF2_MOUSE & $\begin{array}{l}\text { Disintegrin and metalloproteinase domain-containing } \\
\text { protein 10; }\end{array}$ & $\begin{array}{l}\text { Kuzbanian protein homolog;Mammalian } \\
\text { disintegrin-metalloprotease; }\end{array}$ \\
\hline TRGNAFGSCG & RSPGGSYMPCAPR & n.d. & N-ter + 42.01 Da, C +57.02 Da & 16,7 & 3,246 & 2 & 1476,7 & ADA15_MOUSE & & $\begin{array}{l}\text { Disintegrin and metalloproteinase domain-containing } \\
\text { protein 15; }\end{array}$ & $\begin{array}{l}\text { AD56;Metalloprotease RGD disintegrin } \\
\text { protein;Metalloproteinase-like, disintegrin-like, } \\
\text { and cysteine-rich protein 15;Metargidin; }\end{array}$ \\
\hline AKIHDLREQM & MNHSMSSGSGSLR & n.d. & $\mathrm{N}$-ter $+42.01 \mathrm{Da}$ & 14,2 & 0,215 & 2 & 1391,6 & DLG2_MOUSE & D3rWUO_MOUSE & D Disks large homolog 2; & $\begin{array}{l}\text { Channel-associated protein of synapse- } \\
110 \text {;Postsynaptic density protein PSD-93; }\end{array}$ \\
\hline SPEGPLSLSE & GPSSVGPEGGPGGVGAGGGSSTFPR & n.d. & N-ter $+42.01 \mathrm{Da}$ & 13,4 & $-0,414$ & 3 & 2169,0 & DLGP__MOUSE & B1AS06_MOUSE & Disks large-associated protein 3; & $\begin{array}{l}\text { PSD-95/SAP90-binding protein 3;SAP90/PSD-95- } \\
\text { associated protein 3; }\end{array}$ \\
\hline $\begin{array}{l}\text { WVLGCIEDVL } \\
\text { PASHLRTPD }\end{array}$ & $\begin{array}{l}\text { GTQPWKLGFR } \\
\text { NKATADSAR }\end{array}$ & $\begin{array}{l}\text { n.d. } \\
\text { n.d. }\end{array}$ & $\begin{array}{l}\text { N-ter + 42.01 Da, K +34.06 Da } \\
\text { N-ter + 42.01 Da K +34.06 Da }\end{array}$ & $\begin{array}{l}11,4 \\
13,6\end{array}$ & $\begin{array}{l}-0,868 \\
-2,276\end{array}$ & $\begin{array}{l}2 \\
2\end{array}$ & $\begin{array}{l}1264,7 \\
1008,5\end{array}$ & $\begin{array}{l}\text { HELB_MOUSE } \\
\text { HELB MOUSE }\end{array}$ & DGRGT6_MOUSE & $\begin{array}{l}\text { DNA helicase B; } \\
\text { DNA helicase B; }\end{array}$ & \\
\hline GINQLPSVIQ & $\begin{array}{l}\text { ALEKYEGR } \\
\text { ALER }\end{array}$ & $\begin{array}{l}\text { n.a. } \\
\text { n.d. }\end{array}$ & $\mathrm{N}$-ter + 42.01 Da, K +34.06 Da & 10,6 & 2,494 & 2 & 1040,6 & MSH2_MOUSE & & DNA mismatch repair protein Msh2; & Muts protein homolog 2; \\
\hline M & AVQPKETLQLEGAAEAGFVR & n.d. & $\mathrm{N}$-ter + 42.01 Da, K+34.06 Da & 44,4 & 3,330 & 3 & 2189,2 & MSH2_MOUSE & & DNA mismatch repair protein Msh2; & $\begin{array}{l}\text { Muts protein homolog 2; } \\
\text { DNA polymerase II subunit 3;DNA polymerase }\end{array}$ \\
\hline M & AERPEDLNLPNAVITR & n.d. & $\mathrm{N}$-ter $+42.01 \mathrm{Da}$ & 44,0 & $-0,324$ & 2 & 1849,0 & DPOE__MOUSE & DGRDT4_MOUSE & DNA polymerase epsilon subunit 3; & $\begin{array}{l}\text { epsilon subunit p17;NF-YB-like protein;YB-like } \\
\text { protein 1; }\end{array}$ \\
\hline GIGKRMAEKV & MELLESGHLR & n.d. & $\mathrm{N}$-ter + 42.01 Da & 12,7 & $-6,109$ & 2 & 1225,6 & DPOLL_MOUSE & & DNA polymerase lambda; & DNA polymerase kappa; \\
\hline M & ATAEEKQTSPEPVAADEPAQLPAAVR & 0,2 & $\mathrm{~N}$-ter + 42.01 Da, K +28.03 Da & 61,0 & 2,365 & 3 & 2745,4 & XPA_MOUSE & Q8K2X7_MOUSE & $\begin{array}{l}\text { DNA repair protein complementing XP-A cells } \\
\text { homolog; }\end{array}$ & $\begin{array}{l}\text { Xeroderma pigmentosum group A- } \\
\text { complementing protein homolog; }\end{array}$ \\
\hline $\begin{array}{l}\text { GQMQVLQMKNN } \\
\text { M }\end{array}$ & $\begin{array}{l}\text { EHOKLEENIDTIKR } \\
\text { DORS }\end{array}$ & n.d. & N-ter + 42.01 Da, K+34.06 Da & 17,8 & $-3,003$ & 3 & 1862,1 & RAD50_MOUSE & Q.5SV02_MOUSE & DNA repair protein RAD50; & \\
\hline $\begin{array}{l}\mathrm{M} \\
\mathrm{M}\end{array}$ & $\begin{array}{l}\text { DLAAAAEPGAGSOHPEVR } \\
\text { SGDHLHNDSQIEADFR }\end{array}$ & $\begin{array}{l}\text { n.d. } \\
\text { n.d. }\end{array}$ & $\begin{array}{l}\text { N-ter }+42.01 \mathrm{Da} \\
\text { N-ter }+42.01 \mathrm{Da}\end{array}$ & $\begin{array}{l}40,4 \\
44,0\end{array}$ & $\begin{array}{l}1,845 \\
0,478\end{array}$ & $\begin{array}{l}3 \\
3\end{array}$ & $\begin{array}{l}1947,9 \\
1881,8\end{array}$ & $\begin{array}{l}\text { MCM6_MOUSE } \\
\text { TOP1_MOUSE }\end{array}$ & & $\begin{array}{l}\text { DNA replication licensing factor MCM6; } \\
\text { DNA topoisomerase 1; }\end{array}$ & $\begin{array}{l}\text { Mis5 homolog; } \\
\text { DNA topoisomerase I; } \\
\text { DNA-directed RNA polymerase II } 33 \mathrm{kDa}\end{array}$ \\
\hline YSRDCTCEEF & CPECSVEFTLDVR & n.d. & N-ter + 42.01 Da, C +57.02 Da & 14,7 & $-0,846$ & 3 & 1652,7 & RPB3_MOUSE & Q99M46_MOUSE & DNA-directed RNA polymerase II subunit RPB3; & $\begin{array}{l}\text { polypetide;DNA-directed RNA polymerase II } \\
\text { subunit C;RPB31; }\end{array}$ \\
\hline M & AEVKVKVQPPDADPVEIENR & n.d. & N-ter + 42.01 Da, K+34.06 Da & 61,7 & $-0,938$ & 3 & 2342,3 & RPC6_MOUSE & Q9CZ23_MOUSE & DNA-directed RNA polymerase III subunit RPC6; & DNA-directed RNA polymerase III subunit F; \\
\hline M & $\begin{array}{l}\text { ANVADTKLYDILGVPPGASENELKKAYR } \\
\text { ESNKDEAER }\end{array}$ & 0,1 & $\begin{array}{l}\text { N-ter + 42.01 Da, } \mathrm{K}+28.03 \mathrm{Da} \\
\text { N-ter+40.01 Da K+28.03 }\end{array}$ & 64,1 & $\begin{array}{l}-0,127 \\
-0,547\end{array}$ & 4 & $\begin{array}{l}3157,7 \\
12776\end{array}$ & DNJA2_MOUSE & 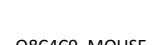 & DnaJ homolog subfamily A member 2; & mDj3; \\
\hline & ESNKDEAER & & N-ter + 42.01 Da, K+28.03 Da & & $-0,547$ & & & DJB12_MOUSE & Q8C4C9_MOUSE & DnaJ homolog subfamily B member 12; & mDj10; \\
\hline
\end{tabular}




\begin{tabular}{|c|c|c|c|c|c|c|c|c|c|c|c|}
\hline Non Prime Site & Prime Site & $\begin{array}{l}\text { Fold change of } \\
\text { XPRESS ratio } \\
\text { (Adam17 } 7^{\text {AKC } / \text { WT) }}\end{array}$ & Modifications & Hyperscore & $\begin{array}{l}\text { Mass error } \\
\text { in ppm }\end{array}$ & Charge & $\begin{array}{l}\text { Precursor } \\
\text { neutral mass } \\
\text { in Da }\end{array}$ & Uniprot ID 1 & Uniprot ID 2 & Protein Name 1 & Protein Name 2 \\
\hline$\overline{\text { AEASSYGESY }}$ & GAAQASSETDLLNR & n.d. & $\mathrm{N}$-ter + 42.01 Da & 15,2 & $-1,288$ & 2 & 1473,7 & DOK4_MOUSE & & Docking protein 4; & Downstream of tyrosine kinase 4; \\
\hline PCLMAENSSS & AQPPPASTPTPAASEGR & n.d. & $\mathrm{N}$-ter $+42.01 \mathrm{Da}$ & 20,5 & $-1,967$ & 2 & 1675,8 & DMRT1_MOUSE & & Doublesex- and mab-3-related transcription factor 1 ; & \\
\hline SNPCLMAENS & SSAQPPPASTPTPAASEGR & n.d. & $\mathrm{N}$-ter $+42.01 \mathrm{Da}$ & 20,9 & $-6,746$ & 3 & 1849,9 & DMRT1_MOUSE & & Doublesex- and mab-3-related transcription factor $1 ;$ & \\
\hline TGKCRWKQCI & CDKCYLTER & n.d. & $\begin{array}{l}\text { N-ter + 42.01 Da, K+34.06 Da, } \\
\text { C+57.02 Da }\end{array}$ & 14,5 & 6,343 & 2 & 1432,7 & DMRTB_MOUSE & & Doublesex- and mab-3-related transcription factor B1; & $\begin{array}{l}\text { Doublesex- and mab-3-related transcription } \\
\text { factor 6; }\end{array}$ \\
\hline RLAQIQQAKK & EKEPEYMLLTER & n.d. & $\mathrm{N}$-ter + 42.01 Da, K+28.03 Da & 15,9 & 0,311 & 2 & 1606,8 & STAU1_MOUSE & Q9DBE7_MOUSE & $\begin{array}{l}\text { Double-stranded RNA-binding protein Staufen } \\
\text { homolog 1; }\end{array}$ & \\
\hline TVTESDSYSA & SLSQDTDKGR & n.d. & $\mathrm{N}$-ter + 42.01 Da, K+34.06 Da & 12,9 & 6,584 & 3 & 1181,6 & DSCL1_MOUSE & E9QPR7_MOUSE & $\begin{array}{l}\text { Down syndrome cell adhesion molecule-like protein } 1 \\
\text { homolog; }\end{array}$ & \\
\hline $\begin{array}{l}\text { M } \\
\text { EEHTTILRPS }\end{array}$ & $\begin{array}{l}\text { AGVSFSGHR } \\
\text { FPGSLSSSSVR }\end{array}$ & $\begin{array}{l}\text { n.d. } \\
\text { n.d. }\end{array}$ & $\begin{array}{l}\text { N-ter + 42.01 Da } \\
\text { N-ter + 42.01 Da }\end{array}$ & $\begin{array}{l}26,1 \\
18,2\end{array}$ & $\begin{array}{l}0,625 \\
6,429\end{array}$ & $\begin{array}{l}2 \\
2\end{array}$ & $\begin{array}{l}958,5 \\
1164,6\end{array}$ & $\begin{array}{l}\text { DREB_MOUSE } \\
\text { CC14A_MOUSE }\end{array}$ & & $\begin{array}{l}\text { Drebrin; } \\
\text { Dual specificity protein phosphatase CDC14A; }\end{array}$ & \multirow{2}{*}{$\begin{array}{l}\text { Developmentally-regulated brain protein; } \\
\text { CDC14 cell division cycle } 14 \text { homolog } A \\
150 \text { kDa dynein-associated polypeptide;DAP- } \\
150 \text {;p150-glued; }\end{array}$} \\
\hline M & STEASARPLR & n.d. & $\mathrm{N}$-ter + 42.01 Da & 23,5 & 0,708 & 2 & 1128,6 & DCTN1_MOUSE & D3Z2M9_MOUSE & Dynactin subunit 1; & \\
\hline M & ADPKYADLPGIAR & n.d. & $\mathrm{N}$-ter + 42.01 Da, K+34.06 Da & 32,1 & 0,683 & 2 & 1461,8 & DCTN2_MOUSE & & Dynactin subunit 2; & $\begin{array}{l}50 \text { kDa dynein-associated polypeptide;Dynactin } \\
\text { complex } 50 \text { KDa subunit; Growth cone } \\
\text { membrane protein 23-48K;p50 dynamitin; }\end{array}$ \\
\hline FSDRIGKTKR & TGYESGDYEMLGEGLGVKETPQQKYQR & $-0,4$ & N-ter + 42.01 Da, K+28.03 Da & 17,5 & 8,911 & 4 & 3160,5 & DCTN2_MOUSE & & Dynactin subunit 2; & $\begin{array}{l}50 \text { kDa dynein-associated polypeptide; Dynactin } \\
\text { complex } 50 \text { KDa subununit;Growth cone } \\
\text { membrane protein } 23-48 K ; \text { p50 dynamitin; }\end{array}$ \\
\hline M & ELGELYYNKSEYIETASGNKVSR & 0,2 & $\mathrm{~N}$-ter + 42.01 Da, K +28.03 Da & 17,3 & 4,062 & 3 & 2828,4 & DCTN5_MOUSE & & Dynactin subunit 5 ; & Dynactin subunit p25; \\
\hline M & AEKTQKSVKIAPGAVVCVESEIR & n.d. & $\begin{array}{l}\text { N-ter + 42.01 Da, K+34.06 Da, } \\
\text { C +57.02 Da }\end{array}$ & 38,5 & 1,700 & 4 & 2642,6 & DCTN6_MOUSE & D3Z6A1_MOUSE & Dynactin subunit $6 ;$ & Dynactin subunit p27; Protein Ws-3; \\
\hline MAFPPPLWE & AEVEETLKR & n.d. & N-ter + 42.01 Da, $\mathrm{K}+34.06 \mathrm{Da}$ & 47,5 & $-1,824$ & 2 & 1149,6 & DLRB1_MOUSE & A2AVR9_MOUSE & Dynein light chain roadblock-type 1 ; & Dynein light chain 2A, cytoplasmic; \\
\hline QGAPCGQKPV & DTECSSSQVHYHSEESMSDASAESGATR & n.d. & N-ter + 42.01 Da, C +57.02 Da & 15,8 & 6,150 & 3 & 3086,2 & DYST_MOUSE & E9Q9x1_MOUSE & Dystonin; & $\begin{array}{l}\text { Bullous pemphigoid antigen 1:Dystonia } \\
\text { musculorum protein;Hemidesmosomal plaque } \\
\text { protein;Microtubule actin cross-linking factor 2; }\end{array}$ \\
\hline M & AAELVEAKNMVMSFR & n.d. & N-ter + 42.01 Da, K +28.03 Da & 18,6 & $-1,528$ & 2 & 1764,9 & PIAS4_MOUSE & D6RGB7_MOUSE & E3 SUMO-protein ligase PIAS4; & $\begin{array}{l}\text { PIASY:Protein inhibitor of activated STAT } \\
\text { protein 4; Protetin inhibitor of activated STAT } \\
\text { protein gamma; }\end{array}$ \\
\hline M & AAAMDVDTPSGTNSGAGKKR & n.d. & N-ter + 42.01 Da, K+34.06 Da & 16,3 & $-0,635$ & 3 & 2043,1 & RBX1_MOUSE & & E3 ubiquitin-protein ligase RBX1; & RING finger protein 75;RING-box protein 1; \\
\hline EIVSASTDSQ & LKLWNVGKPYCLR & n.d. & $\begin{array}{l}\text { N-ter + 42.01 Da, K +28.03 Da, } \\
\text { C +57.02 Da }\end{array}$ & 11,8 & $-4,582$ & 2 & 1744,0 & RFWD2_MOUSE & & E3 ubiquitin-protein ligase RFWD2; & $\begin{array}{l}\text { Constitutive photomorphogenesis protein } 1 \\
\text { homolog;RING finger and WD repeat domain } \\
\text { protein 2; }\end{array}$ \\
\hline GKHQGHKHSV & LEPEANQIR & n.d. & $\mathrm{N}$-ter $+42.01 \mathrm{Da}$ & 17,4 & $-1,708$ & 2 & 1110,6 & TRI23_MOUSE & & E3 ubiquitin-protein ligase TRIM23; & $\begin{array}{l}\text { ADP-ribosylation factor domain-containing } \\
\text { protein 1;GTP-binding protein ARD-1; Tripartite } \\
\text { motif-containing protein 23; }\end{array}$ \\
\hline EQEEAWKLEV & SEKEQAAIWKTQMERR & n.d. & $\mathrm{N}$-ter + 42.01 Da, K+34.06 Da & 16,3 & $-7,370$ & 3 & 2100,1 & TRI68_MOUSE & G3X916_MOUSE & E3 ubiquitin-protein ligase TRIM68; & $\begin{array}{l}\text { RING finger protein } 137 ; \text {;ripartite motif- } \\
\text { containing protein } 68 \text { : }\end{array}$ \\
\hline M & SNRPNNNPGGSLR & n.d. & $\mathrm{N}$-ter $+42.01 \mathrm{Da}$ & 29,6 & $-4,629$ & 2 & 1423,7 & TRIPC_MOUSE & & E3 ubiquitin-protein ligase TRIP12; & Thyroid receptor-interacting protein 12; \\
\hline PEDYSSLINQ & ASNFSCPRSGGDKSR & n.d. & $\begin{array}{l}\text { N-ter + 42.01 Da, K+28.03 Da, } \\
\text { C +57.02 Da }\end{array}$ & 18,8 & 0,236 & 2 & 1694,8 & UBR2_MOUSE & & E3 ubiquitin-protein ligase UBR2; & $\begin{array}{l}\text { N-recognin-2;Ubiquitin-protein ligase E3-alpha- } \\
\text { 2;Ubiquitin-protein ligase E3-alpha-II; }\end{array}$ \\
\hline HESAFQLGLT & EGEINDLLRGIHQVQAPQLR & n.d. & $\mathrm{N}$-ter + 42.01 Da & 18,3 & $-2,918$ & 3 & 2327,2 & ZSWM2_MOUSE & B2FDH1_MOUSE & E3 ubiquitin-protein ligase Zswim2; & $\begin{array}{l}\text { MEKK1-related protein X;ZZ-type zinc finger- } \\
\text { containing protein 2;Zinc finger SWIM domain- } \\
\text { containing protein 2; }\end{array}$ \\
\hline IGKTKEVIFS & MEEGSVKMFLR & 0,9 & $\mathrm{~N}$-ter + 42.01 Da, K +28.03 Da & 65,2 & $-0,572$ & 2 & 1395,7 & EMAL2_MOUSE & E9QK48_MOUSE & Echinoderm microtubule-associated protein-like 2; & \\
\hline M & SSEELACKLQR & $-1,0$ & $\begin{array}{l}\text { N-ter + } 42.01 \mathrm{Da}, \mathrm{K}+28.03 \mathrm{Da}, \\
\mathrm{C}+57.02 \mathrm{Da}\end{array}$ & 39,7 & $-5,174$ & 2 & 1389,7 & EFHD1_MOUSE & & EF-hand domain-containing protein D1; & EF-hand domain-containing protein 1;Swiprosin- \\
\hline $\begin{array}{l}\text { M } \\
\text { RPQQAAARPS }\end{array}$ & $\begin{array}{l}\text { ATDELASKLSR } \\
\text { SAEDTSPAPVSAPAPPVR } \\
\text { FSWYSKDAR }\end{array}$ & $\begin{array}{l}\text { n.d. } \\
\text { n.d. } \\
\text { n.d. }\end{array}$ & $\begin{array}{l}\text { N-ter + 42.01 Da, K +34.06 Da } \\
\text { N-ter + 42.01 Da } \\
\text { N-ter+ 42.01 Da K +34.06 Da }\end{array}$ & $\begin{array}{l}61,6 \\
15,8 \\
183\end{array}$ & $\begin{array}{r}-1,814 \\
1,060 \\
-0,230\end{array}$ & $\begin{array}{l}2 \\
2 \\
2\end{array}$ & $\begin{array}{l}1265,7 \\
1789,9 \\
13017\end{array}$ & $\begin{array}{l}\text { EFHD2_MOUSE } \\
\text { EH111_MOUSE } \\
\text { EHD1MOUSE }\end{array}$ & $\begin{array}{l}\text { Q8C845_MOUSE } \\
\text { E9QP49_MOUSE }\end{array}$ & $\begin{array}{l}\text { EF-hand domain-containing protein D2; } \\
\text { EH domain--inding protein 1-like protein 1; } \\
\text { EH domain-containing protein 1. }\end{array}$ & $\begin{array}{l}\text { Swiprosin-1; } \\
\text { Tangerin; } \\
\text { PAST homolog 1. }\end{array}$ \\
\hline M & QTAHTGLSHTADGADGQTSR & n.d. & $\mathrm{N}$-ter $+42.01 \mathrm{Da}$ & 39,7 & 1,235 & 3 & 2183,0 & LAGE3_MOUSE & J3JS94_MOUSE & EKC/KEOPS complex subunit Lage3; & $\begin{array}{l}\text { ITBA2 protein homolog; Lantigen family } \\
\text { member 3; }\end{array}$ \\
\hline MSNGYEDHM & AEDCRDDIGR & nd & N-ter $+42.01 \mathrm{Da} \mathrm{C}+57.02 \mathrm{Da}$ & 226 & -47222 & 2 & 12475 & ELAV1 MOUSE & & ELAY-like protein 1 . & Elay-like generic protein:Hu-antigen R.MelG. \\
\hline
\end{tabular}




\begin{tabular}{|c|c|c|c|c|c|c|c|c|c|c|c|}
\hline Non Prime Site & Prime Site & $\begin{array}{l}\text { Fold change of } \\
\text { XPRESS ratio } \\
\text { (Adam17 } 7^{\Delta K C} / \mathrm{WT} \text { ) }\end{array}$ & Modifications & Hyperscore & $\begin{array}{l}\text { Mass error } \\
\text { in ppm }\end{array}$ & Charge & $\begin{array}{l}\text { Precursor } \\
\text { neutral mass } \\
\text { in Da }\end{array}$ & Uniprot ID 1 & Uniprot ID 2 & Protein Name 1 & Protein Name 2 \\
\hline M & SNGYEDHMAEDCR & n.d. & N-ter + 42.01 Da, C +57.02 Da & 21,8 & 1,353 & 2 & 1624,6 & ELAV1_MOUSE & & ELAV-like protein $1 ;$ & Elav-like generic protein;Hu-antigen R;MelG; \\
\hline NHEHDQPAHL & TLKDDSIPVNR & n.d. & $\mathrm{N}$-ter + 42.01 Da, K+34.06 Da & 13,0 & 8,609 & 3 & 1332,8 & ETFD_MOUSE & Q6PF96_MOUSE & $\begin{array}{l}\text { Electron transfer flavoprotein-ubiquinone } \\
\text { oxidoreductase, mitochondrial; }\end{array}$ & $\begin{array}{l}\text { Electron-transferring-flavoprotein } \\
\text { dehydrogenase; }\end{array}$ \\
\hline GGDHSELIVR & ITSLEVENQNLR & n.d. & $\mathrm{N}$-ter $+42.01 \mathrm{Da}$ & 14,1 & 8,706 & 2 & 1456,8 & EF1D_MOUSE & Q91VK2_MOUSE & Elongation factor 1-delta; & \\
\hline LDYDYKKRRV & GKIPEEEIR & n.d. & $\mathrm{N}$-ter + 42.01 Da, K+28.03 Da & 15,5 & 0,088 & 2 & 1139,6 & SH3G3_MOUSE & J3QK75_MOUSE & Endophilin-A3; & $\begin{array}{l}\text { Endophilin-3;SH3 domain protein } 2 \mathrm{C} ; \mathrm{SH} 3 \\
\text { domain-containing GRB2-like protein 3;SH3p13; }\end{array}$ \\
\hline MNI & MDFNVKKLAADAGTFLLR & n.d. & N-ter + 42.01 Da, K+34.06 Da & 63,4 & 2,958 & 3 & 2093,2 & SHLB1_MOUSE & & Endophilin-B1; & SH3 domain-containing GRB2-like protein B1; \\
\hline M & DFNMKKLASDAGIFFTR & 0,2 & $\mathrm{~N}$-ter + 42.01 Da, K+28.03 Da & 25,8 & 1,779 & 3 & 2189,1 & SHLB2_MOUSE & A2AWI9_MOUSE & Endophilin-B2; & SH3 domain-containing GRB2-like protein B2; \\
\hline MDFN & MKKLASDAGIFFTR & n.d. & $\mathrm{N}$-ter + 42.01 Da, K+34.06 Da & 13,8 & 0,943 & 3 & 1694,0 & SHLB2_MOUSE & A2AWI9_MOUSE & Endophilin-B2; & SH3 domain-containing GRB2-like protein B2; \\
\hline $\begin{array}{l}\text { M } \\
\text { RSPGLELPVM }\end{array}$ & $\begin{array}{l}\text { ASCASIDIEDATQHLR } \\
\text { SHTILVQPTKRPEGR }\end{array}$ & $\begin{array}{l}\text { n.d. } \\
\text { n.d. }\end{array}$ & $\begin{array}{l}\text { N-ter + 42.01 Da, C +57.02 Da } \\
\text { N-ter +42.01 Da, K+34.06 Da }\end{array}$ & $\begin{array}{l}22,3 \\
37,4\end{array}$ & $\begin{array}{l}1,585 \\
1,047\end{array}$ & $\begin{array}{l}2 \\
3\end{array}$ & $\begin{array}{l}1827,9 \\
1907,1\end{array}$ & $\begin{array}{l}\text { EDC4_MOUSE } \\
\text { ERH_MOUSE }\end{array}$ & $\begin{array}{l}\text { G5E8996_MOUSE } \\
\text { G3UW85_MOUSE }\end{array}$ & $\begin{array}{l}\text { Enhancer of mRNA-decapping protein 4; } \\
\text { Enhancer of rudimentary homolog; }\end{array}$ & \\
\hline RNPPVNSLSL & ECLTEFTISLEKLENDKSIR & n.d. & $\begin{array}{l}\text { N-ter + 42.01 Da, K+28.03 Da, } \\
\text { C +57.02 Da }\end{array}$ & 13,8 & $-1,306$ & 4 & 2522,3 & EC11_MOUSE & & Enoyl-CoA delta isomerase 1, mitochondrial; & $\begin{array}{l}\text { 3,2-trans-enoyl-COA } \\
\text { isomerase;Delta(3),Delta(2)-enoyl-CoA } \\
\text { isomerase;Dodecenoyl-COA isomerase; }\end{array}$ \\
\hline VATDLFCDGE & VNCPDGSDEDTGLCATACDGR & n.d. & N-ter + 42.01 Da, C +57.02 Da & 10,7 & $-3,112$ & 3 & 2310,9 & ENTK_MOUSE & E9Q6Y6_MOUSE & Enteropeptidase; & $\begin{array}{l}\text { Enterokinase;Serine protease } \\
\text { 7;Transmembrane protease serine 15; }\end{array}$ \\
\hline YWDMSGQDVI & KAVEDGFR & n.d. & N-ter + 42.01 Da, K+28.03 Da & 10,5 & 4,429 & 3 & 990,5 & EPHAA_MOUSE & FGWVBO_MOUSE & Ephrin type-A receptor 10; & \\
\hline FEPVNVTTDR & EVPPAVSDIR & n.d. & $\mathrm{N}$-ter + 42.01 Da & 11,9 & $-2,043$ & 2 & 1123,6 & EPHB4_MOUSE & E9PWK7_MOUSE & Ephrin type-B receptor 4; & $\begin{array}{l}\text { Developmental kinase 2; Hepatoma } \\
\text { transmembrane kinas;;Tyrosine kinase MYK-1; }\end{array}$ \\
\hline M & STTTGPEAAPKPSAKSIYEQR & n.d. & $\mathrm{N}$-ter + 42.01 Da, K +34.06 Da & 42,0 & 0,129 & 3 & 2328,3 & ES8L1_MOUSE & E9Q4X5_MOUSE & $\begin{array}{l}\text { Epidermal growth factor receptor kinase substrate 8- } \\
\text { like protein } 1 \text {; }\end{array}$ & $\begin{array}{l}\text { Epidermal growth factor receptor pathway } \\
\text { substrate 8-related protein } 1 \text {; }\end{array}$ \\
\hline M & AAAAQLSLTQLSSGNPVYEKYYR & n.d. & N-ter + 42.01 Da, K+34.06 Da & 60,0 & $-0,843$ & 3 & 2605,4 & EPS15_MOUSE & Q5JC28_MOUSE & Epidermal growth factor receptor substrate 15; & $\begin{array}{l}\text { Protein AF-1p; } \\
\text { Epidermal growth factor receptor pathway }\end{array}$ \\
\hline M & AAPLVPLSQQIPGGNPLYESYYKQVDPAYTGR & n.d. & $\mathrm{N}$-ter + 42.01 Da, $\mathrm{K}+28.03 \mathrm{Da}$ & 13,7 & $-0,533$ & 3 & 3561,8 & EP15R_MOUSE & & Epidermal growth factor receptor substrate 15 -like 1 ; & $\begin{array}{l}\text { substrate 15-related sequence;:Eps15-related } \\
\text { protein; }\end{array}$ \\
\hline TYLQLLRRCV & RDPETGFYMLQLAGKGSSVHHLSEELR & $-2,9$ & N-ter + 42.01 Da, K+28.03 Da & 16,9 & 2,395 & 5 & 3126,6 & EPIPL_MOUSE & EPIPL_MOUSE & Epiplakin; & \\
\hline ESLRQDLLRR & YQAGGLTVHDVTTTLTSLLAR & n.d. & $\mathrm{N}$-ter + 42.01 Da & 24,5 & 3,140 & 3 & 2258,2 & EPIPL_MOUSE & EPIPL_MOUSE & Epiplakin; & \\
\hline $\begin{array}{l}\text { DAHSSEVPGA } \\
\text { M }\end{array}$ & AAEGPGGSGVGLGDR & n.d. & $\mathrm{N}$-ter + 42.01 Da & 19,6 & 0,000 & 2 & 1340,6 & EMC7_MOUSE & & ER membrane protein complex subunit 7; & \\
\hline M & SSKQEIMDDQR & $-1,9$ & $\mathrm{~N}$-ter + 42.01 Da, K +28.03 Da & 32,4 & $-1,421$ & 2 & 1405,7 & ESF1_MOUSE & & ESF1 homolog; & ABT1-associated protein; \\
\hline M & AKCLLTSSISVR & n.d. & $\begin{array}{l}\mathrm{N} \text {-ter + 42.01 Da, K+28.03 Da, } \\
\mathrm{C}+57.02 \mathrm{Da}\end{array}$ & 16,0 & 3,130 & 2 & 1403,8 & ECHD1_MOUSE & EOCXS3_MOUSE & Ethylmalonyl-CoA decarboxylase; & $\begin{array}{l}\text { Enoyl-CoA hydratase domain-containing } \\
\text { protein 1;Methylmalonyl-CoA decarboxylase; }\end{array}$ \\
\hline M & ADSVKTFLQDLGR & 0,5 & $\mathrm{~N}$-ter + 42.01 Da, K+28.03 Da & 27,6 & 0,526 & 2 & 1518,8 & EI24_MOUSE & & Etoposide-induced protein 2.4; & $\begin{array}{l}\text { p53-induced gene } 8 \text { protein; } \\
\text { ATP-dependent RNA helicase DDX48;ATP- }\end{array}$ \\
\hline LISATLPHEI & LEMTNKFMTDPIR & n.d. & $\mathrm{N}$-ter + 42.01 Da, K+34.06 Da & 15,4 & 3,945 & 2 & 1670,9 & IF4A3_MOUSE & A2AFK7_MOUSE & Eukaryotic initiation factor 4A-III; & $\begin{array}{l}\text { dependent RNA helicase elfFA-3;DEAD boo } \\
\text { protein 48;Eukaryotic translation initiation } \\
\text { factor } 4 \text { A isoform 3; }\end{array}$ \\
\hline M & AGGEGGVTLGQPHLSR & n.d. & $\mathrm{N}$-ter $+42.01 \mathrm{Da}$ & 60,4 & 0,000 & 2 & 1576,8 & IF2G_MOUSE & A2AAW9_MOUSE & $\begin{array}{l}\text { Eukaryotic translation initiation factor } 2 \text { subunit } 3, \mathrm{X} \text { - } \\
\text { linked; }\end{array}$ & $\begin{array}{l}\text { Eukaryotic translation initiation factor } 2 \text { subunit } \\
\text { gamma, } X \text {-linked; }\end{array}$ \\
\hline M & AGGEAGVTLGQPHLSR & n.d. & $\mathrm{N}$-ter $+42.01 \mathrm{Da}$ & 61,0 & $-4,646$ & 2 & 1590,8 & IF2H_MOUSE & & $\begin{array}{l}\text { Eukaryotic translation initiation factor } 2 \text { subunit } 3, Y \text { - } \\
\text { linked; }\end{array}$ & $\begin{array}{l}\text { Eukaryotic translation initiation factor } 2 \text { subunit } \\
\text { gamma, Y-linked; }\end{array}$ \\
\hline ARRKEEQEQR & ELLHEIQRR & n.d. & $\mathrm{N}$-ter $+42.01 \mathrm{Da}$ & 16,7 & $-9,703$ & 2 & 1234,7 & E2AK4_MOUSE & A2AUM1_MOUSE & $\begin{array}{l}\text { Eukaryotic translation initiation factor 2-alpha kinase } \\
4 ;\end{array}$ & GCN2-like protein; \\
\hline PASVAPAPVL & MNQPPQIAPKR & n.d. & N-ter + 42.01 Da, K+34.06 Da & 30,2 & 4,643 & 2 & 1354,8 & IF4G1_MOUSE & D3YWY7_MOUSE & Eukaryotic translation initiation factor 4 gamma 1 & \\
\hline M & NKAPQPTGPPPAR & 0,0 & $\mathrm{~N}$-ter + 42.01 Da, K+28.03 Da & 47,9 & $-0,718$ & 2 & 1530,8 & IF4G1_MOUSE & D6RCH1_MOUSE & Eukaryotic translation initiation factor 4 gamma 1; & \\
\hline HWKEYEQSWF & TGPPPAR & n.d. & $\mathrm{N}$-ter $+42.01 \mathrm{Da}$ & 9,9 & 6,501 & 2 & 736,4 & IF4G1_MOUSE & DGRCH1_MOUSE & Eukaryotic translation initiation factor 4 gamma $1 ;$ & \\
\hline M & TAIKHALQR & 0,1 & N-ter + 42.01 Da, K+28.03 Da & 20,6 & 0,631 & 3 & 1106,7 & EXOC1_MOUSE & Q6P1Y9_MOUSE & Exocyst complex component 1 ; & Exocyst complex component Sec3; \\
\hline MERAKM & AEESLETAAEHER & n.d. & $\mathrm{N}$-ter $+42.01 \mathrm{Da}$ & 22,8 & $-2,773$ & 2 & 1512,7 & EXC6B_MOUSE & EOCXR2_MOUSE & Exocyst complex component 6B; & $\begin{array}{l}\text { Exocyst complex component Sec15B;SEC15-like } \\
\text { protein 2; }\end{array}$ \\
\hline M & AAGFKTVEPLEYYR & 0,1 & $\mathrm{~N}$-ter + $42.01 \mathrm{Da}, \mathrm{K}+28.03 \mathrm{Da}$ & 47,8 & 4,432 & 2 & 1712,9 & EXOS8_MOUSE & D3YYN3_MOUSE & Exosome complex component RRP43; & $\begin{array}{l}\text { Exosome component 8; Ribosomal RNA- } \\
\text { processing protein 43; } \\
\text { Exosome component 7-Ribosomal RNA- }\end{array}$ \\
\hline M & ASVALLEAEKVYIVHGVQEDLR & n.d. & $\mathrm{N}$-ter + 42.01 Da, K +34.06 Da & 57,8 & $-4,215$ & 3 & 2488,3 & EXOS7_MOUSE & & Exosome complex exonuclease RRP42; & $\begin{array}{l}\text { Exosome component 7; Ribosomal RNA- } \\
\text { processing protein 42; }\end{array}$ \\
\hline
\end{tabular}




\begin{tabular}{|c|c|c|c|c|c|c|c|c|c|c|c|}
\hline Non Prime Site & Prime Site & $\begin{array}{l}\text { Fold change of } \\
\text { XPRESS ratio } \\
\left.\text { (Adam17 }^{\Delta K C} / \mathrm{WT}\right)\end{array}$ & Modifications & Hyperscore & $\begin{array}{l}\text { Mass error } \\
\text { in ppm }\end{array}$ & Charge & $\begin{array}{l}\text { Precursor } \\
\text { neutral mass } \\
\text { in Da }\end{array}$ & Uniprot ID 1 & Uniprot ID 2 & Protein Name 1 & Protein Name 2 \\
\hline KAAKSKNLQI & KTDTAKSLADSLDR & n.d. & $\mathrm{N}$-ter $+42.01 \mathrm{Da}, \mathrm{K}+34.06 \mathrm{Da}$ & 29,8 & $-3,677$ & 2 & 1629,9 & RRP44_MOUSE & & Exosome complex exonuclease RRP44; & $\begin{array}{l}\text { Protein DIS3 homolog;Ribosomal RNA- } \\
\text { processing protein 44: }\end{array}$ \\
\hline EKQQAMHLCF & ENLMEGIER & n.d. & $\mathrm{N}$-ter $+42.01 \mathrm{Da}$ & 20,6 & $-4,231$ & 3 & 1131,5 & XPO7_MOUSE & E9PUW7_MOUSE & Exportin-7; & Ran-binding protein 16; \\
\hline M & EHSPEEGASPEPSGQPPATDSTR & n.d. & N-ter + 42.01 Da & 57,9 & 0,118 & 3 & 2535,1 & ESYT1_MOUSE & & Extended synaptotagmin-1; & domain-containing \\
\hline YYFQSVHESI & EPTHDVFSFYVSDGSGR & n.d. & $\mathrm{N}$-ter $+42.01 \mathrm{Da}$ & 13,2 & 1,903 & 3 & 1940,9 & FRAS1_MOUSE & & Extracellular matrix protein FRAS1; & \\
\hline M & AVTLDKDAYYR & 0,1 & $\mathrm{~N}$-ter + 42.01 Da, K+28.03 Da & 21,5 & $-1,732$ & 2 & 1383,7 & SP16H_MOUSE & G3X956_MOUSE & FACT complex subunit SPT16; & 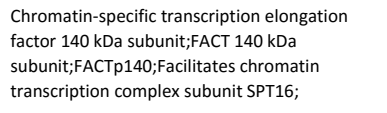 \\
\hline M & AETLEFNDIFQEVKGSMNDGR & n.d. & $\mathrm{N}$-ter + 42.01 Da, K+34.06 Da & 49,0 & 3,147 & 3 & 2475,2 & SSRP1_MOUSE & A2AW05_MOUSE & FACT complex subunit SSRP1; & $\begin{array}{l}\text { Facilitates chromatin transcription complex } \\
\text { subunit SSRP1; Recombination signal sequence } \\
\text { recognition protein 1;Structure-specific } \\
\text { recognition protein 1;T160; }\end{array}$ \\
\hline $\begin{array}{l}\text { M } \\
\text { EPTGAASLRA }\end{array}$ & $\begin{array}{l}\text { ADLEEQLSDEEKVR } \\
\text { KYLPPSTASIR }\end{array}$ & $\begin{array}{l}0,2 \\
\text { n.d. }\end{array}$ & $\begin{array}{l}\text { N-ter + 42.01 Da, } \mathrm{K}+28.03 \mathrm{Da} \\
\text { N-ter + 42.01 Da, K+34.06 Da }\end{array}$ & $\begin{array}{l}71,5 \\
11,9\end{array}$ & $\begin{array}{l}-1,097 \\
-0,016\end{array}$ & $\begin{array}{l}2 \\
2\end{array}$ & $\begin{array}{l}1729,8 \\
1307,8\end{array}$ & $\begin{array}{l}\text { CAZA2_MOUSE } \\
\text { FP100_MOUSE }\end{array}$ & D6RCW7_MOUSE & $\begin{array}{l}\text { F-actin-capping protein subunit alpha-2; } \\
\text { Fanconi anemia-associated protein of } 100 \mathrm{kDa} \text {; }\end{array}$ & CapZ alpha-2; \\
\hline SSDSTLVSNT & KSSPGDTLVKQESAR & n.d. & $\mathrm{N}$-ter + 42.01 Da, K+28.03 Da & 19,0 & $-0,352$ & 3 & 1699,9 & FAN1_MOUSE & & Fanconi-associated nuclease 1; & $\begin{array}{l}\text { FANCD2/FANCl-associated nuclease } \\
\text { 1:Myotubularin-related protein 15; }\end{array}$ \\
\hline $\begin{array}{l}\text { M } \\
\text { PPGPPGAGDR }\end{array}$ & $\begin{array}{l}\text { ADYSTVPPPSSGSAGGGGGGVVNDAFKDALQR } \\
\text { GGGGPGGGGPGGGGASGGPSQPPGGGGPGIR }\end{array}$ & $\begin{array}{l}-0,1 \\
\text { n.d. }\end{array}$ & $\begin{array}{l}\text { N-ter + 42.01 Da, } \mathrm{K}+28.03 \mathrm{Da} \\
\text { N-ter + 42.01 Da }\end{array}$ & $\begin{array}{l}27,2 \\
16,1\end{array}$ & $\begin{array}{l}1,062 \\
-1,097\end{array}$ & $\begin{array}{l}3 \\
3\end{array}$ & $\begin{array}{l}3103,5 \\
2368,1\end{array}$ & $\begin{array}{l}\text { FUBP1_MOUSE } \\
\text { FUBP2_MOUSE }\end{array}$ & Q3TUE1_MOUSE & $\begin{array}{l}\text { Far upstream element-binding protein 1; } \\
\text { Far upstream element-binding protein } 2 ;\end{array}$ & KH type-splicing regulatory protein; \\
\hline ELERAQHRML & LESLQQR & n.d. & N-ter + 42.01 Da & 9,7 & 3,164 & 2 & 914,5 & FBF1_MOUSE & & Fas-binding factor 1 ; & \\
\hline M & ASLKDLEGKWR & n.d. & $\mathrm{N}$-ter + 42.01 Da, K +34.06 Da & 59,7 & $-0,071$ & 2 & 1411,9 & FABP5_MOUSE & & Fatty acid-binding protein, epidermal; & $\begin{array}{l}\text { Epidermal-type fatty acid-binding protein;Fatty } \\
\text { acid-binding protein 5;Keratinocyte lipid- } \\
\text { binding protein;PPoriasis-associated fatty acid- } \\
\text { binding protein homolog; }\end{array}$ \\
\hline MA & SLKDLEGKWR & n.d. & $\mathrm{N}$-ter + 42.01 Da, K +34.06 Da & 16,1 & $-8,558$ & 3 & 1340,8 & FABP5_MOUSE & & Fatty acid-binding protein, epidermal; & $\begin{array}{l}\text { Epidermal-type fatty acid-binding protein;Fatty } \\
\text { acid-binding protein 5;Kreratinocyte lipid- } \\
\text { binding protein;Psoriasis-associated fatty acid- } \\
\text { binding protein homolog; }\end{array}$ \\
\hline PNADFTTSNF & TTHYWNTVSHR & n.d. & $\mathrm{N}$-ter + $42.01 \mathrm{Da}$ & 19,3 & $-3,530$ & 2 & 1442,7 & FACR2_MOUSE & & Fatty acyl-CoA reductase 2; & Male sterility domain-containing protein $1 ;$ \\
\hline RVILLRNYQK & SKVKYEWLSGR & n.d. & $\mathrm{N}$-ter + 42.01 Da, K+34.06 Da & 11,3 & 2,323 & 2 & 1461,9 & FBX48_MOUSE & & F-box only protein 48 & \\
\hline M & EKTQDRDTLSGR & n.d. & $\mathrm{N}$-ter + 42.01 Da, $\mathrm{K}+34.06 \mathrm{Da}$ & 39,6 & $-0,434$ & 3 & 1611,8 & FBX50_MOUSE & & F-box only protein 50; & $\begin{array}{l}\text { NCC receptor protein 1;Non-specific cytotoxic } \\
\text { cell receptor protein } 1 \text { homolog; }\end{array}$ \\
\hline TQDRDTLSGR & MEAEGSLNSEELPPHPQSPPPPPSPR & n.d. & $\mathrm{N}$-ter + 42.01 Da & 33,7 & 2,871 & 3 & 2818,3 & FBX50_MOUSE & & F-box only protein 50; & $\begin{array}{l}\text { NCC receptor protein 1;Non-specific cytotoxic } \\
\text { cell receptor protein } 1 \text { homolog; }\end{array}$ \\
\hline $\begin{array}{l}\text { SAPLPALRL } \\
\text { TVYPLAVENL }\end{array}$ & $\begin{array}{l}\text { DLRWIEDVKDSQLR } \\
\text { SSISAHELHRAVR }\end{array}$ & $\begin{array}{l}\text { n.d. } \\
\text { n.d. }\end{array}$ & $\begin{array}{l}\text { N-ter + 42.01 Da, K+34.06 Da } \\
\text { N-ter + 42.01 Da }\end{array}$ & $\begin{array}{l}14,4 \\
17,0\end{array}$ & $\begin{array}{l}-9,454 \\
1,460\end{array}$ & $\begin{array}{l}3 \\
3\end{array}$ & $\begin{array}{l}1848,0 \\
1503,8\end{array}$ & $\begin{array}{l}\text { FXL19_MOUSE } \\
\text { FRMD8_MOUSE }\end{array}$ & D3Z5B2_MOUSE & $\begin{array}{l}\text { F-box/LRR-repeat protein 19; } \\
\text { FERM domain-containing protein 8; }\end{array}$ & F-box and leucine-rich repeat protein 19; \\
\hline M & GEIEQKPTPASR & n.d. & N-ter + 42.01 Da, K+34.06 Da & 16,1 & 3,742 & 2 & 1387,8 & FARP1_MOUSE & E9Q805_MOUSE & $\begin{array}{l}\text { FERM, RhoGEF and pleckstrin domain-containing } \\
\text { protein } 1 \text {; }\end{array}$ & \\
\hline VSFLYLEPGD & KKLVVLPFPGKEQR & n.d. & $\mathrm{N}$-ter + 42.01 Da, K+28.03 Da & 13,2 & 6,172 & 2 & 1764,1 & FETUB_MOUSE & Q6rJU1_MOUSE & Fetuin-B; & $\begin{array}{l}\text { Fetuin-like protein IRL685; } \\
\text { Forebrain embryonic zinc finger-like protein }\end{array}$ \\
\hline NCGVCCKAEL & GLAPSALPAGR & n.d. & $\mathrm{N}$-ter + 42.01 Da & 15,2 & 2,660 & 2 & 1050,6 & FEZF2_MOUSE & & Fez family zinc finger protein 2 & $\begin{array}{l}\text { 2;Zinc finger protein 312;Zinc finger protein Fez- } \\
\text { like; }\end{array}$ \\
\hline M & SCTIEKALADAKALVER & 0,4 & $\begin{array}{l}\text { N-ter + 42.01 Da, } \mathrm{K}+28.03 \mathrm{Da}, \\
\mathrm{C}+57.02 \mathrm{Da}\end{array}$ & 42,8 & 0,557 & 3 & 1972,1 & FGOP2_MOUSE & F8WGG1_MOUSE & FGFR1 oncogene partner 2 homolog; & \\
\hline CCCEPGRCWS & IGTIPEACPVR & n.d. & N-ter + 42.01 Da, C +57.02 Da & 12,4 & 0,478 & 2 & 1253,6 & FBN2_MOUSE & & Fibrillin-2; & \\
\hline GSLANFIDLK & FKYQDSGKIIR & n.d. & $\mathrm{N}$-ter $+42.01 \mathrm{Da}, \mathrm{K}+34.06 \mathrm{Da}$ & 17,8 & 6,822 & 2 & 1463,9 & PKHL1_MOUSE & E9PUU8_MOUSE & Fibrocystin-L; & $\begin{array}{l}\text { Polycystic kidney and hepatic disease 1-like } \\
\text { protein 1;Protein D86; }\end{array}$ \\
\hline $\begin{array}{l}\text { DVQDNSISVR } \\
\text { ADASRRSGAR }\end{array}$ & $\begin{array}{l}\text { WLPSTSPVTGYR } \\
\text { QGQASAQGRAGSQGQAQGR }\end{array}$ & $\begin{array}{l}\text { n.d. } \\
\text { n.d. }\end{array}$ & $\begin{array}{l}\mathrm{N} \text {-ter }+42.01 \mathrm{Da} \\
\mathrm{N} \text {-ter }+42.01 \mathrm{Da}\end{array}$ & $\begin{array}{l}20,9 \\
38,2\end{array}$ & $\begin{array}{r}-2,844 \\
-3,604\end{array}$ & $\begin{array}{l}2 \\
3\end{array}$ & $\begin{array}{l}1404,7 \\
1883,9\end{array}$ & $\begin{array}{l}\text { FINC_MOUSE } \\
\text { FILA_MOUSE }\end{array}$ & J3QM50_MOUSE & $\begin{array}{l}\text { Fibronectin; } \\
\text { Filaggrin; }\end{array}$ & \\
\hline RSSNRRDRPR & QLSPSQSSDSQVHSGVQVEGR & n.d. & $\mathrm{N}$-ter $+42.01 \mathrm{Da}$ & 24,9 & 2,482 & 3 & 2253,1 & FILA_MOUSE & & Filaggrin; & \\
\hline $\begin{array}{l}\text { RSSNRRDRPR } \\
\text { OPVRPOGPSO }\end{array}$ & $\begin{array}{l}\text { QPSPSOSSDSQVHSGVQVEAQR } \\
\text { DSSSOPOASOGOPSOSGSGR }\end{array}$ & $\begin{array}{l}\text { n.d. } \\
\text { n.d. }\end{array}$ & $\begin{array}{l}\mathrm{N} \text {-ter + 42.01 Da } \\
\mathrm{N} \text {-ter + 42.01 Da }\end{array}$ & $\begin{array}{l}15,3 \\
35,9\end{array}$ & $\begin{array}{r}-3,065 \\
2,228\end{array}$ & 3 & $\begin{array}{l}2379,1 \\
2016,9\end{array}$ & $\begin{array}{l}\text { FILA_MOUSE } \\
\text { FILA2 MOUSE }\end{array}$ & & Filaggrin; & \\
\hline $\begin{array}{l}\text { QPVRPQGPSQ } \\
\text { SPVHPESSEG }\end{array}$ & $\begin{array}{l}\text { DSSSQPQASQGQPSQSGSGR } \\
\text { EEHSVVPQR }\end{array}$ & $\begin{array}{l}\text { n.d. } \\
\text { n.d. }\end{array}$ & $\begin{array}{l}\mathrm{N} \text {-ter + 42.01 Da } \\
\mathrm{N} \text {-ter + 42.01 Da }\end{array}$ & $\begin{array}{l}34,9 \\
14,4\end{array}$ & $\begin{array}{l}2,228 \\
-1,335\end{array}$ & $\begin{array}{l}3 \\
2\end{array}$ & $\begin{array}{l}2016,9 \\
1121,6\end{array}$ & $\begin{array}{l}\text { FILA2_MOUSE } \\
\text { FILA2_MOUSE }\end{array}$ & FILA2 MOUSE & $\begin{array}{l}\text { Filaggrin-2; } \\
\text { Filaggrin-2; }\end{array}$ & $\begin{array}{l}\text { Intermediate filament-associated protein; } \\
\text { Intermediate filament-associated protein; }\end{array}$ \\
\hline RSPVHPESSE & GEEHSVVPQR & n.d. & $\mathrm{N}$-ter + 42.01 Da & 11,7 & $-2,541$ & 2 & 1178,6 & FILA2_MOUSE & FILA2_MOUSE & Filaggrin-2; & Intermediate filament-associated protein; \\
\hline NQYDSSOSSR & HGSYGPQDYDYGQSGYGPSGR & n.d. & N-ter + 42.01 Da & 21,5 & 5,018 & 3 & 2289,0 & FILA2_MOUSE & E9QPZ3_MOUSE & Filaggrin-2; & Intermediate filament-associated protein; \\
\hline GPSQDSSSQP & OASQGQPSQSGSGR & n.d. & $\mathrm{N}$-ter $+42.01 \mathrm{Da}$ & 17,1 & $-0,282$ & 2 & 1415,6 & FILA2_MOUSE & & Filaggrin-2; & Intermediate filament-associated protein; \\
\hline
\end{tabular}




\begin{tabular}{|c|c|c|c|c|c|c|c|c|c|c|c|}
\hline Non Prime Site & Prime Site & $\begin{array}{l}\text { Fold change of } \\
\text { XPRESS ratio } \\
\text { (Adam17 } 1 \text { ¿KC / WT) }\end{array}$ & Modifications & Hyperscore & $\begin{array}{l}\text { Mass error } \\
\text { in ppm }\end{array}$ & charge & $\begin{array}{l}\text { Precursor } \\
\text { neutral mass } \\
\text { in Da }\end{array}$ & Uniprot ID 1 & Uniprot ID 2 & Protein Name 1 & Protein Name 2 \\
\hline GQPVRPQGPS & $\begin{array}{l}\text { QDSSSQPQASQGQPSOSGSGR } \\
\end{array}$ & n.d. & N-ter + 42.01 Da & 25,4 & $\begin{array}{l}-6,751 \\
, 020\end{array}$ & 3 & 2144,9 & $\begin{array}{l}\text { FILA2_MOUSE } \\
\text { LA_MOSF }\end{array}$ & & Filaggrin-2; & Intermediate filament-associated protein; \\
\hline $\begin{array}{l}\text { QSGSRRSPRS } \\
\text { RPPGPSODSS }\end{array}$ & $\begin{array}{l}\text { QPVHPESSEGEEHSVVPQR } \\
\text { RPPOAGOGPSOGSGR }\end{array}$ & n.d. & $\mathrm{N}$-ter + 42.01 Da & 18,0 & 0,828 & 4 & 2169,0 & FILA2_MOUSE & & Filaggrin-2; & Intermediate filament-associated protein; \\
\hline RPQGPSQDSS & RQPQAGQGQPSQSGSGR & n.d. & $\mathrm{N}$-ter + 42.01 Da & 14,4 & 9,323 & 3 & 1766,9 & FILA2_MOUSE & FILA2_MOUSE & Filaggrin-2; & Intermediate filament-associated protein; \\
\hline PRRSPVHPES & SEGEEHSVVPQR & n.d. & $\mathrm{N}$-ter+42.01 Da & 24,9 & 0,143 & 2 & 1394,6 & FILA2_MOUSE & FILA2_MOUSE & Filaggrin-2; & Intermediate filament-associated protein; \\
\hline QQRESVHGQR & SRPQGPFQDSSR & n.d. & $\mathrm{N}$-ter + 42.01 Da & 17,1 & $-0,783$ & 3 & 1402,7 & FILA2_MOUSE & E9QPZ3_MOUSE & Filaggrin-2; & Intermediate filament-associated protein; \\
\hline GRPQGPSQDS & SRQPQAGQGQPSQSGSGR & n.d. & $\mathrm{N}$-ter + 42.01 Da & 34,6 & 7,809 & 3 & 1853,9 & FILA2_MOUSE & FILA2_MMOUSE & Filaggrin-2; & Intermediate filament-associated protein; \\
\hline SPRRSPVHPE & SSEGEEHSVVPQR & n.d. & $\mathrm{N}$-ter + 42.01 Da & 37,6 & 9,908 & 2 & 1481,7 & FILA2_MOUSE & E9QPZZ_MOUSE & Filaggrin-2; & Intermediate filament-associated protein; \\
\hline VRPQGPSQDS & SSQPQASQGQPSQSGSGR & n.d. & $\mathrm{N}$-ter + 42.01 Da & 24,1 & $-2,089$ & 4 & 1814,8 & FILA2_MOUSE & & Filaggrin-2; & Intermediate filament-associated protein; \\
\hline SPRRSQVHPE & $\begin{array}{l}\text { YSEGEAHSEVSQR } \\
\text { Y }\end{array}$ & n.d. & N-ter + $42.01 \mathrm{Da}$ & 15,0 & 7,032 & 2 & 1519,7 & FLAA2_MOUSE & E9QPZ3_MOUSE & Filaggrin-2; & Intermediate filament-associated protein; \\
\hline EFHQNQDKIM & AKLTNEDSQNR & $-0,4$ & $\mathrm{~N}$-ter + 42.01 Da, K+28.03 Da & 40,6 & $-3,119$ & 2 & 1344,7 & FIL1L_MOUSE & EOCYH7_MOUSE & Filamin A-interacting protein 1-like; & $\begin{array}{l}\text { Protein down-regulated in ovarian cancer } 1 \\
\text { homolog; } \\
\text { Actin-binding protein 280;Alpha- }\end{array}$ \\
\hline LEARGDSTYR & CSYQPTMEGVHTVHVTFAGVPIPR & n.d. & $\mathrm{N}$-ter + 42.01 Da, C+57.02 Da & 13,7 & 5,571 & 4 & 2724,3 & FLNA_MOUSE & B7FAU9_MOUSE & Filamin-A; & $\begin{array}{l}\text { filamin;Endothelial actin-binding } \\
\text { protein;Filamin-1;Non-muscle filamin; }\end{array}$ \\
\hline $\begin{array}{l}\text { AlLPDFEQLS } \\
\text { DLEEESESWD }\end{array}$ & $\begin{array}{l}\text { YESEQGTSDSEGSGR } \\
\text { NSEAEEEEKAPVTPEGAEGR }\end{array}$ & n.d. & N-ter + 42.01 Da & 14,3 & 2,999 & 4 & 1629,6 & $\begin{array}{l}\text { GEN_MOUSE } \\
\text { FICN MOUSE }\end{array}$ & & Flap endonuclease GEN homolog 1; & \\
\hline DLEEESESWD & NSEAEEEEKAPVTPEGAEGR & n.d. & $\mathrm{N}$-ter + 42.01 Da, K+28.03 Da & 18,3 & 9,314 & 3 & 2198,0 & FLCN_MOUSE & & Folliculin; & \\
\hline MEKNVQKILL & DAKPDLTLAR & n.d. & N-ter + 42.01 Da, K+28.03 Da & 12,8 & $-6,212$ & 2 & 1269,7 & FHAD1_MOUSE & & Forkhead-associated domain-containing protein 1 ; & \\
\hline TLAERNTEIE & SLKNEGENLKR & n.d. & N-ter + 42.01 Da, K+34.06 Da & 17,4 & 4,786 & 3 & 1396,8 & FHAD1_MOUSE & & Forkhead-associated domain-containing protein 1 ; & \\
\hline M & SEKFDCHYCR & 0,3 & $\begin{array}{l}\text { N-ter + 42.01 Da, K+28.03 Da, } \\
\text { C }+57.02 \text { Da }\end{array}$ & 19,5 & 0,543 & 3 & 1470,6 & FHL1_MOUSE & A2AEX8_MOUSE & Four and a half LMM domains protein 1; & $\begin{array}{l}\text { KyoT;RBP-associated molecule 14-1; } \text {;keletal } \\
\text { muscle LM-protein 1; }\end{array}$ \\
\hline KVTRADTRNR & DDTLALSMYQGPPSADQGNNMADAPR & n.d. & $\mathrm{N}$-ter + 42.01 Da & 11,8 & 1,295 & 4 & 2776,2 & FMR1N_MOUSE & B7ZNB2_MOUSE & Fragile X mental retardation 1 neighbor protein; & $\begin{array}{l}\text { Protein mNY-SAR-35; } \\
\text { D-fructose-1,6-bisphosphate 1- }\end{array}$ \\
\hline M & ANHAPFETDISTLTR & n.d. & $\mathrm{N}$-ter $+42.01 \mathrm{Da}$ & 19,2 & $-1,574$ & 2 & 1713,8 & F16P1_MOUSE & E9Q0T7_MOUSE & Fructose-1,6-bisphosphatase 1; & $\begin{array}{l}\text { phosphohydrolase 1;Fructose-1,6- } \\
\text { bisphosphatase isozyye 3;liver FBPase; }\end{array}$ \\
\hline LVPVTHAMMC & MNCGCDFSLTVR & n.d. & N-ter + 42.01 Da, C +57.02 Da & 11,6 & $-3,655$ & 4 & 1500,6 & FGD5_MOUSE & E9QKY4_MOUSE & FYVE, RhoGEF and PH domain-containing protein 5; & \\
\hline ENLSAKNLSD & LFTIHSVADVQTLR & n.d. & $\mathrm{N}$-ter $+42.01 \mathrm{Da}$ & 12,0 & $-8,643$ & 2 & 1640,9 & G2E3_MOUSE & E9Q7C2_MOUSE & G2/M phase-specific E3 ubiquitin-protein ligase; & \\
\hline M & SLVDLGKKLLEAAR & n.d. & $\mathrm{N}$-ter + 42.01 Da, K+34.06 Da & 41,4 & 0 & 2 & 1622,0 & GABP1_MOUSE & Q3UT69_MOUSE & GA-binding protein subunit beta-1; & GABP subunit beta-2; \\
\hline ms & ATQHKTSLPQGVR & 0,3 & $\mathrm{~N}$-ter + 42.01 Da, $\mathrm{K}+28.03 \mathrm{Da}$ & 33,4 & 4,215 & 3 & 1491,8 & Q9CRB1_MOUSE & D3Z141_MOUSE & Galectin \{ECO:0000256|RuleBase:RU102079\}; & \\
\hline RGMVPDQAGR & FHVNLLCGEEQGADAALHFNPR & n.d. & N-ter + 42.01 Da, C +57.02 Da & 44,6 & 6,498 & 3 & 2536,2 & Q9CRB1_MOUSE & LEG7_MOUSE & Galectin \{ECO:0000256|RuleBase:RU102079\}; & \\
\hline ADAALHFNPR & LDTSEVVFNTKEQGKWGR & 0,8 & $\mathrm{~N}$-ter + 42.01 Da, K+28.03 Da & 31,1 & 9,343 & 3 & 2191,2 & Q9CRB1_MOUSE & D3Z141_MOUSE & Galectin \{ECO:0000256|RuleBase:RU102079\}; & \\
\hline M & SATQHKTSLPQGVR & 0,1 & $\mathrm{~N}$-ter + 42.01 Da, K+28.03 Da & 61,5 & 0,822 & 2 & 1578,9 & Q99RB1_MOUSE & D3Z141_MOUSE & Galectin \{ECO:0000256|RuleBase:RU102079\}; & \\
\hline M & ACGLVASNLNLKPGECLKVR & n.d. & $\begin{array}{l}\text { N-ter + 42.01 Da, K+34.06 Da, } \\
\mathrm{C}+57.02 \mathrm{Da}\end{array}$ & 61,1 & 0,130 & 3 & 2308,3 & LEG1_MOUSE & & Galectin-1; & $\begin{array}{l}14 \text { kDa lectin;Beta-galactoside-binding lectin L- } \\
14-\text { - Galaptin;Lactose-binding lectin 1;Lectin } \\
\text { galactoside-binding soluble 1;S-Lac lectin 1; }\end{array}$ \\
\hline M & SATHHKTSLPQGVR & $-2,2$ & $\mathrm{~N}$-ter + 42.01 Da, K+28.03 Da & 21,0 & $-0,189$ & 3 & 1587,9 & LEG7_MOUSE & & Galectin-7; & \\
\hline NNLTAVGCSL & ALAAVFPLGLDGYHIGR & n.d. & $\mathrm{N}$-ter $+42.01 \mathrm{Da}$ & 14,3 & $-8,545$ & 3 & 1811,0 & GABR1_MOUSE & & Gamma-aminobutyric acid type B receptor subunit 1; & \\
\hline M & ASSDCEGHAGQEGETFLYFAYGSNLLTER & n.d. & N-ter + 42.01 Da, C +57.02 Da & 35,9 & $-0,369$ & 3 & 3250,4 & GGCT_MOUSE & & Gamma-glutamylcyclotransferase; & \\
\hline EGEEIGLGPN & IDTPNWSEDSDDEDAQQPLSR & n.d. & $\mathrm{N}$-ter + 42.01 Da & 14,0 & $-5,646$ & 3 & 2459,0 & GCP5_MOUSE & & Gamma-tubulin complex component 5 ; & Gasdermin-C1:Melanoma-derived leucine \\
\hline m & SYTFDWLSKDVVKKLQGR & n.d. & N-ter + 42.01 Da, K+34.06 Da & 24,3 & 4,144 & 3 & 2313,4 & GSDMC_MOUSE & & Gasdermin-C; & $\begin{array}{l}\text { zipper-containing extranuclear factor } \\
\{E C O: 0000303 \mid \text { PubMed:112223543\}; }\end{array}$ \\
\hline DLEAKMPLSA & LYGSLSFLQQLR & n.d. & $\mathrm{N}$-ter + 42.01 Da & 17,3 & $-2,521$ & 2 & 1465,8 & GSDC2_MOUSE & & Gasdermin-C2; & \\
\hline GAASRRRYLL & YDVNPPEGFNLR & n.d. & $\mathrm{N}$-ter $+42.01 \mathrm{Da}$ & 11,5 & $-2,596$ & 2 & 1461,7 & OFUT2_MOUSE & & GDP-fucose protein O-fucosyltransferase 2; & $\begin{array}{l}\text { Peptide-O-Oucosyltransferase 2; } \\
\text { General transcription factor III } 56 \text { kDa }\end{array}$ \\
\hline M & ADPDVLTEVPAALKR & n.d. & $\mathrm{N}$-ter $+42.01 \mathrm{Da}, \mathrm{K}+34.06 \mathrm{Da}$ & 23,0 & $-0,778$ & 2 & 1670,0 & T2EA_MOUSE & & General transcription factor IIE subunit 1; & $\begin{array}{l}\text { subunit; Transcription initiation factor III } \\
\text { subunit alpha; }\end{array}$ \\
\hline KGPPMQEADQ & DSEEDSEEDSEEEAEEAPR & n.d. & $\mathrm{N}$-ter + 42.01 Da & 11,1 & 0,449 & 4 & 2222,8 & GSE1_MOUSE & & Genetic suppressor element 1; & \\
\hline M & ATEGMLTNNDHQIR & n.d. & $\mathrm{N}$-ter + 42.01 Da & 47,2 & $-0,562$ & 3 & 1776,9 & GEPH_MOUSE & A0JNY3_MOUSE & 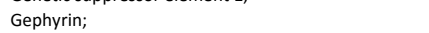 & \\
\hline M & AEGSAVSDPQHAAR & n.d. & $\mathrm{N}$-ter $+42.01 \mathrm{Da}$ & 44,2 & $-0,209$ & 2 & 1436,7 & GAN_MOUSE & F6TZU3_MOUSE & Gigaxonin \{ECO:0000312|MGI:MGI:1890619\}; & \\
\hline M & SESLVVCDVAEDLVEKLR & 0,1 & $\begin{array}{l}\text { N-ter + 42.01 Da, K+28.03 Da, } \\
\text { C +57.02 Da }\end{array}$ & 68,7 & 0,422 & 3 & 2130,1 & GMFB_MOUSE & & Glia maturation factor beta; & \\
\hline M & AVEELQSIIKR & n.d. & $\mathrm{N}$-ter + 42.01 Da, K +34.06 Da & 28,1 & 1,761 & 2 & 1360,8 & GLMN_MOUSE & Q3T9A5_MOUSE & Glomulin; & $\begin{array}{l}\text { FK506-binding protein-associated protein;FKBP- } \\
\text { associated protein; } \\
\text { Glucocorticoid-induced gene } 18\end{array}$ \\
\hline M & STASSSSSQTPHSAPQR & n.d. & N-ter $+42.01 \mathrm{Da}$ & 14,4 & 4,207 & 2 & 1756,8 & GLCI1_MOUSE & E9QKK4_MOUSE & Glucocorticoid-induced transcript 1 protein; & $\begin{array}{l}\text { protein;Testhymin; Thymocyte/spermatocyte } \\
\text { selection protein 1; }\end{array}$ \\
\hline
\end{tabular}




\begin{tabular}{|c|c|c|c|c|c|c|c|c|c|c|c|}
\hline Non Prime Site & Prime Site & $\begin{array}{l}\text { Fold change of } \\
\text { XPRESS ratio } \\
\text { (Adam17 } 7^{\Delta K C} / \mathrm{WT} \text { ) }\end{array}$ & Modifications & Hyperscore & $\begin{array}{l}\text { Mass error } \\
\text { in ppm }\end{array}$ & charge & $\begin{array}{l}\text { Precursor } \\
\text { neutral mass } \\
\text { in Da }\end{array}$ & Uniprot ID 1 & Uniprot ID 2 & Protein Name 1 & Protein Name 2 \\
\hline$\overline{\text { QDTTSVSNSL }}$ & LPEKAFSDIPSPYLR & $-5,0$ & $\mathrm{~N}$-ter + 42.01 Da, K+28.03 Da & 18,0 & $-1,995$ & 3 & 1802,0 & GFOD1_MOUSE & & $\begin{array}{l}\text { Glucose-fructose oxidoreductase domain-containing } \\
\text { protein 1; }\end{array}$ & \\
\hline IQEAIALINS & LHPELLDTNR & n.d. & $\mathrm{N}$-ter $+42.01 \mathrm{Da}$ & 24,1 & $-7,516$ & 2 & 1248,6 & GID8_MOUSE & & Glucose-induced degradation protein 8 homolog; & Two hybrid-associated protein 1 with RanBPM; \\
\hline M & AQEFVNCKIQSGKVVVFIKPTCPYCR & n.d. & $\begin{array}{l}\text { N-ter + 42.01 Da, K+28.03 Da, } \\
\text { C +57.02 Da }\end{array}$ & 17,2 & 0,368 & 4 & 3253,7 & GLRX1_MOUSE & & Glutaredoxin-1; & Thioltransferase- 1 ; \\
\hline M & AGKPVLHHFNAR & $-0,4$ & $\mathrm{~N}$-ter $+42.01 \mathrm{Da}, \mathrm{K}+28.03 \mathrm{Da}$ & 28,1 & $-0,704$ & 4 & 1415,8 & E9Q6L7_MOUSE & & $\begin{array}{l}\text { Glutathione S-transferase } \\
\text { \{ECO:0000256|RuleBase:RU004454\}; }\end{array}$ & \\
\hline M & AGKPVLHYFNAR & n.d. & N-ter + 42.01 Da, K+34.06 Da & 33,9 & $-0,414$ & 3 & 1447,8 & GSTA1_MOUSE & GSTA2_MOUSE & Glutathione S-transferase A1; & $\begin{array}{l}\text { GST class-alpha member 1;Glutathione S- } \\
\text { transferase Ya;Glutathione S-transferase Ya1; }\end{array}$ \\
\hline M & AGKPVLHYFDGR & n.d. & $\mathrm{N}$-ter + 42.01 Da, K+34.06 Da & 20,8 & 1,322 & 3 & 1434,8 & GSTA3_MOUSE & & Glutathione S-transferase A3; & $\begin{array}{l}\text { GST class-alpha member 3;Glutathione S- } \\
\text { transferase Ya3;Glutathione S-transferase Yc; }\end{array}$ \\
\hline M & AAKPKLYYFNGR & $-0,1$ & N-ter + 42.01 Da, K+28.03 Da & 23,1 & 0,916 & 3 & 1524,9 & GSTA4_MOUSE & & Glutathione S-transferase A4; & $\begin{array}{l}\text { GST A4-4;GST class-alpha member } \\
\text { 4;Glutathione S-transferase 5.7; }\end{array}$ \\
\hline M & SSKSMVLGYWDIR & n.d. & $\mathrm{N}$-ter + 42.01 Da, K +34.06 Da & 35,6 & 0,309 & 2 & 1616,9 & GSTM5_MOUSE & E9Q5L9_MOUSE & Glutathione S-transferase Mu 5; & Fibrous sheath component 2;GST class-mu 5; \\
\hline $\begin{array}{l}M \\
M\end{array}$ & $\begin{array}{l}\text { VKVGVNGFGR } \\
\text { AAAKKAVLGPIVGAVDOGTSSTR }\end{array}$ & n.d. & $\mathrm{N}$-ter + 42.01 Da, $\mathrm{K}+34.06 \mathrm{Da}$ & 15,0 & $-3,515$ & 2 & 1107,7 & G3P_MOUSE & D3YYI5_MOUSE & Glyceraldehyde-3-phosphate dehydrogenase; & Peptidyl-cysteine S-nitrosylase GAPDH; \\
\hline M & $\begin{array}{l}\text { AAAKKAVLGPLVGAVDQGTSSTR } \\
\text { AKPLTDSER }\end{array}$ & $\begin{array}{l}-0,4 \\
\text { n.d. }\end{array}$ & $\begin{array}{l}\text { N-ter + 42.201 Da, K+28.03 Da } \\
\text { N-ter + 4.2.01 Da K }+28.03 \text { Da }\end{array}$ & $\begin{array}{l}56,7 \\
18,0\end{array}$ & $\begin{array}{l}0,710 \\
0,184\end{array}$ & $\begin{array}{l}3 \\
2\end{array}$ & $\begin{array}{l}2294,3 \\
1085,6\end{array}$ & $\begin{array}{l}\text { GLPR_MOUSE } \\
\text { PYGB_MOUSE }\end{array}$ & B1ASZ3_MOUSE & $\begin{array}{l}\text { Glycerol kinase; } \\
\text { Glyyogen hhosph }\end{array}$ & ATP:glycerol 3-phosphotransferase; \\
\hline M & $\begin{array}{l}\text { ARPLTDQEKR } \\
\text { AKPTE }\end{array}$ & n.d. & N-ter + 42.01 Da, K+34.06 Da & 36,2 & $-0,154$ & 3 & 1294,8 & PYGL_MOUSE & & Glycogen phosphorylase, liver form; & \\
\hline \multirow{3}{*}{ AYHVYYRDGP } & ALLAEHLLKPLPADR & n.d. & $\mathrm{N}$-ter + 42.01 Da, K+34.06 Da & 41,0 & 1,153 & 3 & 1732,1 & GLTP_MOUSE & D3Z1H8_MOUSE & Glycolipid transfer protein; & \\
\hline & LYPVNQLR & n.d. & $\mathrm{N}$-ter $+42.01 \mathrm{Da}$ & 11,8 & 1,243 & 2 & 1043,6 & LARG2_MOUSE & Q14ATO_MOUSE & Glycosyltransferase-like protein LARGE2; & Glycosyltransferase-like 18; \\
\hline & MKVFVTGPLPAEGR & n.d. & N-ter + 42.01 Da, K+34.06 Da & 35,3 & $-0,633$ & 2 & 1576,9 & GRHPR_MOUSE & D6REG4_MOUSE & Glyoxylate reductase/hydroxypyruvate reductase; & \\
\hline MQLKIMTNRL & RGAYGGNDVDFQDASDDGSGSGSGGGCPDDTCGR & n.d. & N-ter + 42.01 Da, C +57.02 Da & 11,6 & $-0,528$ & 4 & 3407,3 & GPC1_MOUSE & & Glypican-1; & \\
\hline SLVASGKAEL & IKTHHNDTELIR & n.d. & N-ter + 42.01 Da, K+28.03 Da & 17,5 & $-5,488$ & 3 & 1545,8 & GUAA_MOUSE & & GMP synthase [glutamine-hydrolyzing]; & GMP synthetase;Glutamine amidotransferase; \\
\hline LGLKPQVQNE & ETNEIQAVGEEHQQASIQGQAVADGTR & n.d. & $\mathrm{N}$-ter + 42.01 Da & 15,0 & $-7,387$ & 3 & 2907,3 & GOLM1_MOUSE & & Golgi membrane protein 1 ; & $\begin{array}{l}\text { Golgi membrane protein GP73;Golgi } \\
\text { phosphoprotein 2; }\end{array}$ \\
\hline ERPGAEGAPP & QTPPSSAPGNGLGSGASGQQR & n.d. & $\mathrm{N}$-ter + 42.01 Da & 17,8 & 3,604 & 3 & 1995,0 & GCP60_MOUSE & & Golgi resident protein GCP60; & $\begin{array}{l}\text { Acyl-CoA-binding domain-containing protein } \\
\text { 3;Golgi complex-associated protein 1;Golgi } \\
\text { phosphoprotein 1;PBR-and PKA-associated } \\
\text { protein 7; ;eripheral benzodiazepine receptor- } \\
\text { associated protein PAP7; }\end{array}$ \\
\hline M & TQEYDNKRPVLVLQNEALYPQR & $-0,1$ & N-ter + 42.01 Da, K+28.03 Da & 46,5 & 0,874 & 3 & 2743,4 & GRHL1_MOUSE & & Grainyhead-like protein 1 homolog; & $\begin{array}{l}\text { Transcription factor CP2-like 2; Transcription } \\
\text { factor LBP-32; }\end{array}$ \\
\hline $\begin{array}{l}\text { M } \\
\text { DGSPWQPPAD }\end{array}$ & $\begin{array}{l}\text { EHLLSVEENVQPR } \\
\text { LSGLSIEEVSKSLR }\end{array}$ & $\begin{array}{l}\text { n.d. } \\
-2,5\end{array}$ & $\begin{array}{l}\text { N-ter + 42.01 Da } \\
\text { N-ter + 42.01 Da, } \mathrm{K}+28.03 \mathrm{Da}\end{array}$ & $\begin{array}{l}22,8 \\
18,9\end{array}$ & $\begin{array}{l}-7,247 \\
-0,063\end{array}$ & $\begin{array}{l}3 \\
3\end{array}$ & $\begin{array}{l}1721,8 \\
1586,9\end{array}$ & $\begin{array}{l}\text { GRM1C_MOUSE } \\
\text { GAREM_MOUSE }\end{array}$ & & $\begin{array}{l}\text { GRAM domain-containing protein } 1 C ; \\
\text { GRB2-associated and regulator of MAPK protein; }\end{array}$ & GRB2-associated and regulator of MAPK1; \\
\hline M & EDSAPDAVAAAPSGTPKSKLETLPR & $-0,2$ & $\mathrm{~N}$-ter + 42.01 Da, K+28.03 Da & 21,7 & $-0,949$ & 3 & 2736,4 & GCC2_MOUSE & B2RSU7_MOUSE & GRIP and coiled-coil domain-containing protein 2; & 185 kDa Golgi coiled-coil protein; \\
\hline M & EAIAKYDFKATADDELSFKR & 0,5 & N-ter + 42.01 Da, K+28.03 Da & 30,2 & $-0,310$ & 4 & 2574,3 & GRB2_MOUSE & B1AT92_MOUSE & Growth factor receptor-bound protein 2; & Adapter protein GRB2; $\mathrm{SH} 2 / \mathrm{SH} 3$ adapter GRB2; \\
\hline SPPPGSWRSS & ENSSSEGNALR & n.d. & $\mathrm{N}$-ter $+42.01 \mathrm{Da}$ & 14,8 & $-0,663$ & 2 & 1204,5 & MCF2L_MOUSE & E9QPM7_MOUSE & Guanine nucleotide exchange factor DBS; & $\begin{array}{l}\text { DBL's big sister;MCF2-transforming sequence- } \\
\text { like protein; }\end{array}$ \\
\hline M & SSKTASTNSIAQAR & 0,2 & N-ter + 42.01 Da, K+28.03 Da & 68,0 & $-1,005$ & 2 & 1490,8 & GBG12_MOUSE & & $\begin{array}{l}\text { Guanine nucleotide-binding protein } \mathrm{G}(\mathrm{I}) / \mathrm{G}(\mathrm{S}) / \mathrm{G}(\mathrm{O}) \\
\text { subunit gamma-12; }\end{array}$ & \\
\hline м & SGSSSVAAMKKVVQQLR & 0,1 & $\mathrm{~N}$-ter $+42.01 \mathrm{Da}, \mathrm{K}+28.03 \mathrm{Da}$ & 28,7 & 6,663 & 3 & 1873,1 & GBG5_MOUSE & & $\begin{array}{l}\text { Guanine nucleotide-binding protein } G(1) / G(S) / G(O) \\
\text { subunit gamma-5; }\end{array}$ & \\
\hline M & SELDQLRQEAEQLKNQIR & 0,6 & $\mathrm{~N}$-ter + 42.01 Da, K+28.03 Da & 39,9 & $-8,590$ & 3 & 2267,2 & GBB1_MOUSE & H3BLF7_MOUSE & $\begin{array}{l}\text { Guanine nucleotide-binding protein } G(1) / G(S) / G(T) \\
\text { subunit beta-1; }\end{array}$ & Transducin beta chain $1 ;$ \\
\hline SWVMTCAYAP & SGNFVACGGLDNICSIYSLKTR & n.d. & $\begin{array}{l}\text { N-ter + 42.01 Da, K+34.06 Da, } \\
\mathrm{C}+57.02 \mathrm{Da}\end{array}$ & 14,1 & $-3,306$ & 3 & 2507,2 & GBB2_MOUSE & D3YZX3_MOUSE & $\begin{array}{l}\text { Guanine nucleotide-binding protein } G(I) / G(S) / G(T) \\
\text { subunit beta-2; }\end{array}$ & $\begin{array}{l}G \text { protein subunit beta-2; Transducin beta chain } \\
2 ;\end{array}$ \\
\hline TDGLHENETL & ASLKSEAESLKGKLEEER & n.d. & N-ter + 42.01 Da, K+34.06 Da & 21,0 & 2,046 & 3 & 2147,3 & GBB5_MOUSE & & Guanine nucleotide-binding protein subunit beta-5; & Gbeta5; Transducin beta chain 5; \\
\hline M & ANVSKKVSWSGR & n.d. & $\mathrm{N}$-ter + 42.01 Da, K+34.06 Da & 18,4 & 3,984 & 3 & 1427,9 & CLCN7_MOUSE & & $\mathrm{H}(+) / \mathrm{Cl}(-)$ exchange transporter 7 ; & $\begin{array}{l}\text { Chloride channel } 7 \text { alpha subunit; Chloride } \\
\text { channel protein } 7 \text {; }\end{array}$ \\
\hline M & TKVKAAPEESEAQAEGCSEER & n.d. & $\begin{array}{l}\text { N-ter + 42.01 Da, K+34.06 Da, } \\
\text { C +57.02 Da }\end{array}$ & 42,3 & 0,744 & 3 & 2415,2 & NHP2_MOUSE & & H/ACA ribonucleoprotein complex subunit $2 ;$ & $\begin{array}{l}\text { Nucleolar protein family A member 2;snoRNP } \\
\text { protein NHP2; }\end{array}$ \\
\hline
\end{tabular}




\begin{tabular}{|c|c|c|c|c|c|c|c|c|c|c|c|}
\hline Non Prime Site & Prime Site & $\begin{array}{l}\text { Fold change of } \\
\text { XPRESS ratio } \\
\left.\text { (Adam17 } 7^{\Delta K C} / \mathrm{WT}\right)\end{array}$ & Modifications & Hyperscore & $\begin{array}{l}\text { Mass error } \\
\text { in ppm }\end{array}$ & Charge & $\begin{array}{l}\text { Precursor } \\
\text { neutral mass } \\
\text { in Da }\end{array}$ & Uniprot ID 1 & Uniprot ID 2 & Protein Name 1 & Protein Name 2 \\
\hline $\begin{array}{l}\text { TLVMGALDNP } \\
M\end{array}$ & $\begin{array}{l}\text { NPILRCAAGEALGR } \\
\text { PIKEAGGGAGPR }\end{array}$ & n.d. & $\begin{array}{l}\text { N-ter + 42.01 Da, C +57.02 Da } \\
\text { N-ter + 42.01 Da K +34.06 Da }\end{array}$ & $\begin{array}{l}20,5 \\
46,1\end{array}$ & $\begin{array}{l}4,281 \\
-2974\end{array}$ & $\begin{array}{l}3 \\
2\end{array}$ & \begin{tabular}{l|}
1538,8 \\
1275,6
\end{tabular} & $\begin{array}{l}\text { HTR5B_MOUSE } \\
\text { HS12A MOUSE }\end{array}$ & & $\begin{array}{l}\text { HEAT repeat-containing protein } 5 B \text {; } \\
\text { Heat }\end{array}$ & \\
\hline GIDLGTYYSC & $\begin{array}{l}\text { ADKEAGGGDAGR } \\
\text { VGVFGGKVEIIANDQGNR }\end{array}$ & $\begin{array}{l}\text { n.d. } \\
\text { n.d. }\end{array}$ & $\begin{array}{l}\text { N-ter + 42.01 Da, } \mathrm{K}+34.06 \mathrm{Da} \\
\mathrm{N} \text {-ter + 42.01 Da, } \mathrm{K}+34.06 \mathrm{Da}\end{array}$ & $\begin{array}{l}46,1 \\
17,8\end{array}$ & $\begin{array}{l}-2,794 \\
5,789\end{array}$ & 3 & $\begin{array}{l}1275,6 \\
2156,2\end{array}$ & $\begin{array}{l}\text { HS12A_MOUSE } \\
\text { HS71A_MOUSE }\end{array}$ & HS71B_MOUSE & $\begin{array}{l}\text { Heat shock } 70 \text { kDa protein } 12 \mathrm{~A} ; \\
\text { Heat shock } 70 \text { kDa protein } 1 \mathrm{~A} ;\end{array}$ & Heat shock 70 kDa protein 3;Hsp68; \\
\hline WAFTDTERL & IGDAAKNQVAMNPQNTVFDAKR & $-5,7$ & $\mathrm{~N}$-ter + 42.01 Da, K+28.03 Da & 23,1 & 9,444 & 3 & 2485,3 & HS71L_MOUSE & & Heat shock 70 kDa protein 1-like; & $\begin{array}{l}\text { Heat shock } 70 \text { kDa-like protein } 1 ; \text { Spermatid- } \\
\text { specific heat shock protein 70; }\end{array}$ \\
\hline RTDTGEPMGR & GTKVILHLKEDQTEYLEER & n.d. & $\mathrm{N}$-ter + 42.01 Da, K+28.03 Da & 17,8 & 6,744 & 4 & 2398,3 & HS9OA_MOUSE & HS90B_MOUSE & Heat shock protein HSP 90-alpha; & $\begin{array}{l}\text { Heat shock } 86 \mathrm{kDa} ; \text { Tumor-specific } \\
\text { transplantation } 86 \text { kDa antigen; }\end{array}$ \\
\hline M & TTTTFKGVDPNSR & n.d. & N-ter + 42.01 Da, K+34.06 Da & 29,1 & $-1,186$ & 2 & 1599,8 & HN1_MOUSE & & Hematological and neurological expressed 1 protein; & \\
\hline GISSSKPNRM & ASNIFGPTEEPKNIPKR & n.d. & $\mathrm{N}$-ter + 42.01 Da, K+34.06 Da & 17,2 & 7,711 & 3 & 2007,2 & HN1L_MOUSE & & $\begin{array}{l}\text { Hematological and neurological expressed 1-like } \\
\text { protein; }\end{array}$ & \\
\hline M & FQGADSQAGKSGSR & 0,0 & $\mathrm{~N}$-ter + 42.01 Da, K+28.03 Da & 58,8 & 0,751 & 2 & 1595,7 & HN1L_MOUSE & & $\begin{array}{l}\text { Hematological and neurological expressed 1-like } \\
\text { protein; }\end{array}$ & \\
\hline MV & NFTAEEKTLINGLWSKVNVEEVGGEALGR & n.d. & N-ter + 42.01 Da, K+34.06 Da & 28,0 & 1,649 & 5 & 3269,8 & HBE_MOUSE & Q9CR49_MOUSE & Hemoglobin subunit epsilon-Y2; & Epsilon-Y2-globin;Hemoglobin epsilon-Y2 chain; \\
\hline vilMYarvsp & SCTSSCTNFGEQLR & n.d. & $\mathrm{N}$-ter + 42.01 Da, C +57.02 Da & 14,1 & $-5,146$ & 3 & 1687,7 & HOST3_MOUSE & & Heparan-sulfate 6-O-sulfotransferase 3; & \\
\hline PHARSSVGHR & GSHLPPAHPR & n.d. & $\mathrm{N}$-ter $+42.01 \mathrm{Da}$ & 12,4 & $-7,197$ & 2 & 1109,6 & HNF6_MOUSE & & Hepatocyte nuclear factor $6 ;$ & One cut domain family member 1 ; One cut \\
\hline ESNKMHAING & KLYANLR & n.d. & $\mathrm{N}$-ter + 42.01 Da, $\mathrm{K}+28.03 \mathrm{Da}$ & 11,2 & 5,271 & 2 & 946,6 & HEPH_MOUSE & & Hephaestin; & \\
\hline M & ENSQLCKLFIGGLNVQTSESGLR & n.d. & $\begin{array}{l}\text { N-ter + 42.01 Da, } \mathrm{K}+34.06 \mathrm{Da}, \\
\mathrm{C}+57.02 \mathrm{Da}\end{array}$ & 74,7 & $-0,761$ & 3 & 2756,4 & ROAO_MOUSE & & Heterogeneous nuclear ribonucleoprotein $\mathrm{A} 0$; & \\
\hline M & SKSESPKEPEQLR & 0,0 & $\mathrm{~N}$-ter + 42.01 Da, K+28.03 Da & 59,2 & $-0,868$ & 2 & 1611,9 & ROA1_MOUSE & Q5EBP8_MOUSE & Heterogeneous nuclear ribonucleoprotein $\mathrm{A} 1$; & $\begin{array}{l}\text { HDP-1; Helix-destabililizing protein;Single-strand- } \\
\text { binding protein;TTopo isomerase-inhibitor } \\
\text { suppressed;hnRNP core protein A1; }\end{array}$ \\
\hline KALSKQEMQS & AGSQRGRGGGSGNFMGR & n.d. & $\mathrm{N}$-ter+ 42.01 Da & 20,7 & 8,079 & 3 & 1692,8 & ROA3_MOUSE & E9Q7H5_MOUSE & Heterogeneous nuclear ribonucleoprotein A3; & \\
\hline M & EVKPPPGRPQPDSGR & n.d. & $\mathrm{N}$-ter + 42.01 Da, $\mathrm{K}+34.06 \mathrm{Da}$ & 30,5 & 0,000 & 3 & 1823,0 & ROA3_MOUSE & A2AL12_MOUSE & Heterogeneous nuclear ribonucleoprotein $\mathrm{A} 3$; & \\
\hline M & MLGPEGGEGYVVKLR & n.d. & $\mathrm{N}$-ter + 42.01 Da, K+34.06 Da & 70,8 & 0,773 & 2 & 1679,9 & HNRPF_MOUSE & J3QMQ5_MOUSE & Heterogeneous nuclear ribonucleoprotein F; & \\
\hline YCLSGMYDHR & YGDSEFTVQSTTGHCVHMR & n.d. & $\mathrm{N}$-ter + 42.01 Da, C+57.02 Da & 17,2 & 8,644 & 3 & 2253,0 & HNRPF_MOUSE & & Heterogeneous nuclear ribonucleoprotein $F_{;}$ & \\
\hline CVHMRGLPYR & ATENDIYNFFSPLNPVR & n.d. & N-ter + 42.01 Da & 15,1 & 2,647 & 2 & 2038,0 & HNRH1_MOUSE & HNRPF_MOUSE & Heterogeneous nuclear ribonucleoprotein $\mathrm{H}$; & \\
\hline & MLGAEGGEGFV & n.d. & $\mathrm{N}$-ter + 42.01 Da, K +34.06 Da & 86,6 & 1,138 & 2 & 1754,9 & HNRH1_MOUSE & Q8C2Q7_MOUSE & Heterogeneous nuclear ribonucleoprotein $\mathrm{H}$; & \\
\hline QQRGAGKEAA & GKSSGPTSLFAVTVAPPGAR & n.d. & $\mathrm{N}$-ter $+42.01 \mathrm{Da}, \mathrm{K}+34.06 \mathrm{Da}$ & 16,8 & $-3,690$ & 3 & 1975,1 & HNRPU_MOUSE & G3XA10_MOUSE & Heterogeneous nuclear ribonucleoprotein U; & Scaffold attachment factor $\mathrm{A}$; \\
\hline M & EKTLETVPLER & $-0,1$ & $\mathrm{~N}$-ter + 42.01 Da, K+28.03 Da & 48,3 & $-0,462$ & 2 & 1514,8 & ROA2_MOUSE & & Heterogeneous nuclear ribonucleoproteins A2/B1; & \\
\hline M & ASNVTNKTDPR & n.d. & N-ter + 42.01 Da, K+34.06 Da & 15,5 & $-1,016$ & 2 & 1277,7 & HNRPC_MOUSE & & Heterogeneous nuclear ribonucleoproteins $\mathrm{C} 1 / \mathrm{C} 2$; & \\
\hline $\begin{array}{l}M \\
M\end{array}$ & $\begin{array}{l}\text { STNTDLSLSSYDEGQGSKFIR } \\
\text { SESGSKSSQPLASKQEKDGTEKR }\end{array}$ & $\begin{array}{c}\text { n.d. } \\
0,1\end{array}$ & $\begin{array}{l}\text { N-ter + 42.01 Da, } \mathrm{K}+34.06 \mathrm{Da} \\
\mathrm{N} \text {-ter + 42.01 Da, } \mathrm{K}+28.03 \mathrm{Da}\end{array}$ & $\begin{array}{l}33,4 \\
30,3\end{array}$ & $\begin{array}{c}-1,133 \\
0,229\end{array}$ & $\begin{array}{l}3 \\
4\end{array}$ & $\begin{array}{l}2380,2 \\
2617,4\end{array}$ & $\begin{array}{l}\text { HIG1A_MOUSE } \\
\text { HMGA1_MOUSE }\end{array}$ & J3QPX3_MOUSE & $\begin{array}{l}\text { HIG1 domain family member } 1 \mathrm{~A}, \text { mitochondrial; } \\
\text { High mobility group protein HMG-I/HMG-Y; }\end{array}$ & $\begin{array}{l}\text { Hypoxia-inducible gene } 1 \text { protein; } \\
\text { High mobility group AT-hook protein 1; }\end{array}$ \\
\hline M & ADEIAKAQVAQPGGDTIFGKIIR & n.d. & $\mathrm{N}$-ter + 42.01 Da, K +34.06 Da & 75,1 & $-3,944$ & 3 & 2507,4 & HINT1_MOUSE & BOR1E3_MOUSE & Histidine triad nucleotide-binding protein $1 ;$ & $\begin{array}{l}\text { Adenosine 5'-monophosphoramidase;Protein } \\
\text { kinase Cinhibitor 1;Protein kinase C-interacting } \\
\text { protein 1; }\end{array}$ \\
\hline CGSSGHPSCL & KFCPELTANVKALR & n.d. & $\begin{array}{l}\text { N-ter + 42.01 Da, K+28.03 Da, } \\
\text { C +57.02 Da }\end{array}$ & 19,2 & 4,465 & 3 & 1744,0 & KAT6B_MOUSE & & Histone acetyltransferase KAT6B; & $\begin{array}{l}\text { MOZ, YBF2/SAS3, SAS2 and TIP60 protein } \\
\text { 4; Protein querkopf; }\end{array}$ \\
\hline M & AKVSVLNVAVLENPSPFHSPFR & $-1,6$ & N-ter + 42.01 Da, K +28.03 Da & 22,5 & 1,895 & 3 & 2477,4 & ASF1B_MOUSE & & Histone chaperone ASF1B; & Anti-silencing function protein 1 homolog B; \\
\hline $\begin{array}{l}\text { M } \\
\text { TKAVAASKER }\end{array}$ & $\begin{array}{l}\text { SETAPAAPAAPAPAEKTPVKKKAR } \\
\text { SGVSLAALKKALAAAGYDVEKNNSR }\end{array}$ & $\begin{array}{l}-0,3 \\
-0,1\end{array}$ & $\begin{array}{l}\text { N-ter + 42.01 Da, K+28.03 Da } \\
\text { N-ter + 42.01 Da, K+28.03 Da }\end{array}$ & $\begin{array}{l}24,2 \\
42,9\end{array}$ & $\begin{array}{l}0,707 \\
7,587\end{array}$ & $\begin{array}{l}4 \\
4\end{array}$ & $\begin{array}{l}2540,5 \\
2658,5\end{array}$ & $\begin{array}{l}\text { H14_MOUSE } \\
\text { H14_MOUSE }\end{array}$ & H12_MOUSE & $\begin{array}{l}\text { Histone H1.4; } \\
\text { Histone H1.4; }\end{array}$ & $\begin{array}{l}\text { H1 VAR.2; } ; 1 \mathrm{e} ; \\
\text { H1 VAR.2;H1e; }\end{array}$ \\
\hline M & ADKEAAFDDAVEER & 0,0 & $\mathrm{~N}$-ter + $42.01 \mathrm{Da}, \mathrm{K}+28.03 \mathrm{Da}$ & 27,1 & $-2,809$ & 3 & 1634,7 & RBBP4_MOUSE & & Histone-binding protein RBBP4; & $\begin{array}{l}\text { Chromatin assembly factor } 1 \text { subunit } \\
\text { c;Chromatin assembly factor I p48 } \\
\text { subuniti; } 4 \text { ucleosome-remodeling factor subunit } \\
\text { RBAPA8;Retionoblastoma-binding protein } \\
\text { 4;Retinoblastoma-binding protein p48; }\end{array}$ \\
\hline M & ASKEMFEDTVEER & $-0,2$ & $\mathrm{~N}$-ter + 42.01 Da, K+28.03 Da & 60,1 & $-0,975$ & 2 & 1639,7 & RBBP7_MOUSE & A2AFJ1_MOUSE & Histone-binding protein RBBP7; & $\begin{array}{l}\text { Histone acetyltransferase type B subunit } \\
\text { 2;NNucleosome-remodeling factor subunit } \\
\text { RBAPA6;RRetinoblastoma-binding protein } \\
\text { 7;Retinoblastoma-binding protein p46; }\end{array}$ \\
\hline AGSLTGPPGS & FFPGNLALR & n.d. & $\mathrm{N}$-ter + 42.01 Da & 11,5 & 1,578 & 2 & 1075,6 & KMT2D_MOUSE & J3KMNO_MOUSE & Histone-Iysine N-methyltransferase 2D; & $\begin{array}{l}\text { ALL1-related protein;Myeloid//ymphoid or } \\
\text { mixed-lineage leukemia protein 2; }\end{array}$ \\
\hline
\end{tabular}




\begin{tabular}{|c|c|c|c|c|c|c|c|c|c|c|c|}
\hline Non Prime Site & Prime Site & $\begin{array}{l}\text { Fold change of } \\
\text { XPRESS ratio } \\
\text { (Adam17 } 7^{\text {AKC } / \text { WT) }}\end{array}$ & Modifications & Hyperscore & $\begin{array}{l}\text { Mass error } \\
\text { in ppm }\end{array}$ & charge & $\begin{array}{l}\text { Precursor } \\
\text { neutral mass } \\
\text { in Da }\end{array}$ & Uniprot ID 1 & Uniprot ID 2 & Protein Name 1 & Protein Name 2 \\
\hline QTARSVRTRR & LAAAEENLEVAR & n.d. & $\mathrm{N}$-ter + 42.01 Da & 23,4 & 0,753 & 2 & 1326,7 & ASH1L_MOUSE & & Histone-lysine N-methyltransferase ASH1L; & $\begin{array}{l}\text { ASH1-like protein;Absent small and homeotic } \\
\text { disks protein } 1 \text { homolog; }\end{array}$ \\
\hline KGNYSRFMNH & SCNPNCETQKWTVNGDVR & n.d. & $\begin{array}{l}\text { N-ter + 42.01 Da, K+34.06 Da, } \\
\mathrm{C}+57.02 \mathrm{Da}\end{array}$ & 13,2 & $-2,585$ & 4 & 2240,0 & NSD3_MOUSE & D3Z357_MOUSE & Histone-lysine N-methyltransferase NSD3; & $\begin{array}{l}\text { Nuclear SET domain-containing protein 3; Wolf- } \\
\text { Hirschhorn syndrome candidate 1-like protein } 1 \\
\text { homolog; }\end{array}$ \\
\hline EADGLEQDVA & ETEDDESPEQR & n.d. & $\mathrm{N}$-ter + 42.01 Da & 11,9 & $-3,772$ & 3 & 1375,5 & BBX_MOUSE & B8JK50_MOUSE & HMG box transcription factor BBX; & $\begin{array}{l}\text { Bobby sox homolog;HMG box-containing } \\
\text { protein } 2 \text {. }\end{array}$ \\
\hline GPGGGRLLQG & AAAAAAAAAAAAAAATATGTAGPR & n.d. & $\mathrm{N}$-ter $+42.01 \mathrm{Da}$ & 18,0 & $-7,470$ & 3 & 1938,0 & ARX_MOUSE & & Homeobox protein ARX; & $\begin{array}{l}\text { Protenni; } \\
\text { Aristaless-related homeobox; }\end{array}$ \\
\hline & HCHAELR & n.d. & $\mathrm{N}$-ter + 42.01 Da, C +57.02 Da & 17,0 & $-2,096$ & 3 & 1094,5 & BARX̄2_MOUSE & & Homeobox protein BarH-like 2; & \\
\hline PGGGGGGGSG & GLGPGTHGYAPAPLDLWLDAPR & n.d. & $\mathrm{N}$-ter $+42.01 \mathrm{Da}$ & 14,8 & $-0,906$ & 3 & 2315,2 & HXA10_MOUSE & & Homeobox protein Hox-A10; & Homeobox protein Hox-1.8; \\
\hline FQSSSIKTST & LSHSTLPPPFEQTIPSLNPGSHPR & n.d. & $\mathrm{N}$-ter $+42.01 \mathrm{Da}$ & 15,7 & $-5,639$ & 3 & 2763,4 & HXA2_MMOUSE & & Homeobox protein Hox-A2; & Homeobox protein Hox-1.11; \\
\hline HLNQNSSPSL & MFPWMRPHAPGR & n.d. & $\mathrm{N}$-ter $+42.01 \mathrm{Da}$ & 12,3 & $-1,835$ & 2 & 1523,7 & HXC8_MOUSE & & Homeobox protein Hox-C8; & $\begin{array}{l}\text { Homeobox protein Hox-3.1; Homeobox protein } \\
\text { M31; }\end{array}$ \\
\hline SGQNYNSTRQ & GCGQGKSSGSEQYGASSGQSSGCSSGQSTR & $-0,2$ & $\begin{array}{l}\text { N-ter + 42.01 Da, } \mathrm{K}+28.03 \mathrm{Da}, \\
\mathrm{C}+57.02 \mathrm{Da}\end{array}$ & 20,5 & 3,502 & 3 & 2995,2 & HORN_MOUSE & E9QNP3_MOUSE & Hornerin; & \\
\hline SEQSSGLGHY & GSTSGQTASSTRQGSGQGQASGSGR & n.d. & N-ter + 42.01 Da & 33,1 & 3,588 & 3 & 2338,1 & HORN_MOUSE & HORN_MOUSE & Hornerin; & \\
\hline TSGQNYNSTR & QGCGQGKSSGSEQYGASSGQSSGCSSGQSTR & n.d. & $\begin{array}{l}\mathrm{N} \text {-ter + 42.01 Da, } \mathrm{K}+34.06 \mathrm{Da}, \\
\mathrm{C}+57.02 \mathrm{Da}\end{array}$ & 41,4 & 6,545 & 3 & 3129,3 & HORN_MOUSE & E9QNP3_MOUSE & Hornerin; & \\
\hline GSEQSSGLGH & YGSTSGQTASSTRQGSGQGQASGSGR & n.d. & N-ter + 42.01 Da & 17,0 & $-1,957$ & 3 & 2501,1 & HORN_MOUSE & HORN_MOUSE & Hornerin; & \\
\hline MRRRGEIEM & ATEGDVELELETETSGPERPPEKPR & n.d. & $\mathrm{N}$-ter + 42.01 Da, K+34.06 Da & 39,5 & $-0,106$ & 3 & 2841,4 & HYPK_MOUSE & & Huntingtin-interacting protein $\mathrm{K}$; & Huntingtin yeast partner $\mathrm{K}$; \\
\hline LWAESTALFP & SVYLDETLASSVHSR & n.d. & N-ter $+42.01 \mathrm{Da}$ & 25,7 & $-4,043$ & 2 & 1704,8 & HYAL2_MOUSE & & Hyaluronidase-2; & Hyaluronoglucosaminidase-2; \\
\hline LYPDCHNYNV & YATNYTGSCPEEEVLR & n.d. & N-ter + 42.01 Da, C +57.02 Da & 12,4 & 2,690 & 3 & 1929,9 & HYAL4_MOUSE & & Hyaluronidase-4; & $\begin{array}{l}\text { Chondroitin sulfate endo-beta-N- } \\
\text { acetylgalactosaminidase; Chondroitin sulfate } \\
\text { hydrolase;Hyaluronoglucosaminidase-4; }\end{array}$ \\
\hline ESIERKVSVR & EHATLEETTR & n.d. & $\mathrm{N}$-ter $+42.01 \mathrm{Da}$ & 24,4 & 9,678 & 2 & 1227,6 & HYDIN_MOUSE & & Hydrocephalus-inducing protein; & Protein $\mathrm{Hy}-3$; \\
\hline M & LPNTGKLAGCTVFITGASR & 0,9 & $\begin{array}{l}\text { N-ter + 42.01 Da, } \mathrm{K}+28.03 \mathrm{Da}, \\
\mathrm{C}+57.02 \mathrm{Da}\end{array}$ & 34,2 & 0,323 & 3 & 2163,1 & HSDL2_MOUSE & & Hydroxysteroid dehydrogenase-like protein 2; & \\
\hline GLAPSLSLLC & QHEETVQPR & n.d. & $\mathrm{N}$-ter + 42.01 Da & 15,1 & 0,257 & 2 & 1164,6 & HIF3A_MOUSE & E9Q633_MOUSE & Hypoxia-inducible factor 3-alpha; & $\begin{array}{l}\text { Basic-helix-loop-helix-PAS protein MOP7; } ; \text { HF3- } \\
\text { alpha-1;inhibitory PAS domain protein; Member } \\
\text { of PAS protein } 7 \text {; }\end{array}$ \\
\hline D & IQMTQSTSSLSASLGDR & n.d. & $\mathrm{N}$-ter $+42.01 \mathrm{Da}$ & 26,0 & 9,016 & 3 & 1937,9 & KVSAD_MOUSE & & Ig kappa chain V-V region HP 123E6; & \\
\hline IVGVCLGLLC & LLACMCAGLRR & n.d. & N-ter + 42.01 Da, C +57.02 Da & 17,1 & $-9,753$ & 2 & 1361,7 & IGDC4_MOUSE & E9QAQO_MOUSE & Immunoglobulin superfamily DCC subclass member 4; & 4; Neighbor of punc e11;Protein DDM36; \\
\hline WVVTDSEVPA & VFKMMSNR & n.d. & $\mathrm{N}$-ter + 42.01 Da, K+34.06 Da & 17,1 & $-1,468$ & 2 & 1087,6 & IGS10_MOUSE & & Immunoglobulin superfamily member 10; & \\
\hline ASRTYRLRLE & AARPADAGTYR & n.d. & $\mathrm{N}$-ter $+42.01 \mathrm{Da}$ & 15,1 & 0,839 & 2 & 1189,6 & IGSF8_MOUSE & G3UYZ1_MOUSE & Immunoglobulin superfamily member 8; & $\begin{array}{l}\text { CD81 partner 3;Glu-Trp-lle EWI motif- } \\
\text { containing protein 2;Keratinocyte-associated } \\
\text { transmembrane protein 4;Prostaglandin } \\
\text { regulatory-like protein; }\end{array}$ \\
\hline M & ADNEKLDNQR & n.d. & $\mathrm{N}$-ter + 42.01 Da, K+34.06 Da & 41,1 & 3,673 & 2 & 1277,7 & IMA3_MOUSE & & Importin subunit alpha-3; & $\begin{array}{l}\text { Importin alpha Q1; Karyopherin subunit alpha- } \\
4 ;\end{array}$ \\
\hline M & AENPGLENHR & n.d. & $\mathrm{N}$-ter $+42.01 \mathrm{Da}$ & 36,9 & $-0,424$ & 2 & 1177,6 & IMA4_MOUSE & & Importin subunit alpha-4; & $\begin{array}{l}\text { Importin alpha Q2;Karyopherin subunit alpha- } \\
3 \text { importin alpha-S1;Karyopherin subunit alpha- }\end{array}$ \\
\hline M & STPGKENFR & n.d. & N-ter + 42.01 Da, K+34.06 Da & 23,9 & 1,708 & 2 & 1110,6 & IMA5_MOUSE & G3UWJ8_MOUSE & Importin subunit alpha-5; & $\begin{array}{l}\text { 1; Nucleoprotein interactor 1;RAG cohort } \\
\text { protein 2;SRP1-beta; }\end{array}$ \\
\hline M & ETMASPGKDNYR & $-0,1$ & N-ter + 42.01 Da, K+28.03 Da & 39,1 & $-1,019$ & 2 & 1568,7 & IMA7_MOUSE & & Importin subunit alpha-7; & Importin alpha-S2;Karyopherin subunit alpha-6; \\
\hline M & ELITLEKTVSPDR & 0,0 & $\mathrm{~N}$-ter + 42.01 Da, $\mathrm{K}+28.03 \mathrm{Da}$ & 46,1 & 0,386 & 2 & 1814,0 & IMB1_MOUSE & & Importin subunit beta-1; & $\begin{array}{l}\text { Karyopherin subunit beta-1; Nuclear factor } \\
\text { p97; Pore targeting complex 97 kDa } \\
\text { subunit;;SCG; }\end{array}$ \\
\hline MRQVQRAQNH & LHLGGSVATCNSEAR & n.d. & N-ter + 42.01 Da, C+57.02 Da & 14,4 & 3,426 & 4 & 1805,9 & IKKE_MOUSE & E9PYL6_MOUSE & $\begin{array}{l}\text { Inhibitor of nuclear factor kappa-B kinase subunit } \\
\text { epsilon: }\end{array}$ & Inducible I kappa-B kinase; \\
\hline SKLQTHPVCL & SPESSADNSGKELSADASR & n.d. & N-ter + 42.01 Da, K+34.06 Da & 17,2 & $-3,172$ & 3 & 1982,9 & IP3KC_MOUSE & & Inositol-trisphosphate 3-kinase C; & Inositol 1,4,5-trisphosphate 3-kinase $C_{;}$ \\
\hline M & MNKLYIGNLSPAVTADDLR & n.d. & N-ter + 42.01 Da, K+34.06 Da & 18,8 & 0,507 & 3 & 2166,2 & IF2B2_MOUSE & & Insulin-like growth factor 2 mRNA-binding protein 2; & $\begin{array}{l}\text { IGF-II mRNA-binding protein 2; VICKZ family } \\
\text { member 2; }\end{array}$ \\
\hline M & ELQKGKGTVAAAASGAAGGGGGGAGAGAPGGGR & 0,1 & $\mathrm{~N}$-ter + 42.01 Da, K+28.03 Da & 24,3 & $-9,035$ & 4 & 2851,4 & INT3_MOUSE & & Integrator complex subunit 3; & $\begin{array}{l}\text { SOSS complex subunit A;Sensor of single-strand } \\
\text { DNA complex subunit A; }\end{array}$ \\
\hline QKEVDFCISL & LRERFMECLMIGR & n.d. & N-ter + 42.01 Da, C +57.02 Da & 11,7 & 0,969 & 2 & 1751,9 & INT3_MOUSE & & Integrator complex subunit 3; & $\begin{array}{l}\text { SOSS complex subunit A;Sensor of single-strand } \\
\text { DNA complex subunit A; }\end{array}$ \\
\hline KGKGTVAAAA & SGAAGGGGGGAGAGAPGGGR & n.d. & $\mathrm{N}$-ter $+42.01 \mathrm{Da}$ & 14,6 & $-3,121$ & 2 & 1439,7 & INT3_MOUSE & & Integrator complex subunit 3; & $\begin{array}{l}\text { SOSS complex subunit A;Sensor of single-strand } \\
\text { DNA complex subunit A; }\end{array}$ \\
\hline
\end{tabular}




\begin{tabular}{|c|c|c|c|c|c|c|c|c|c|c|c|}
\hline Non Prime Site & Prime Site & $\begin{array}{l}\text { Fold change of } \\
\text { XPRESS ratio } \\
\text { (Adam17 } 7^{\text {AKC } / \text { WT) }}\end{array}$ & Modifications & Hyperscore & $\begin{array}{l}\text { Mass error } \\
\text { in ppm }\end{array}$ & Charge & $\begin{array}{l}\text { Precursor } \\
\text { neutral mass } \\
\text { in Da }\end{array}$ & Uniprot ID 1 & Uniprot ID 2 & Protein Name 1 & Protein Name 2 \\
\hline $\begin{array}{l}\text { LADAGYGEQE } \\
\text { WNGCNEDEHC }\end{array}$ & $\begin{array}{l}\text { LDANSALMELDKGLR } \\
\text { VPDLVLDAR }\end{array}$ & $\begin{array}{l}\text { n.d. } \\
\text { n.d. }\end{array}$ & $\begin{array}{l}\text { N-ter + 42.01 Da, K +34.06 Da } \\
\text { N-ter + 42.01 Da }\end{array}$ & $\begin{array}{l}19,1 \\
12,0\end{array}$ & $\begin{array}{l}2,088 \\
6,528\end{array}$ & $\begin{array}{l}3 \\
3\end{array}$ & $\begin{array}{l}1720,9 \\
1038,6\end{array}$ & $\begin{array}{l}\text { INT7_MOUSE } \\
\text { ITA11_MOUSE }\end{array}$ & & $\begin{array}{l}\text { Integrator complex subunit 7; } \\
\text { Integrin alpha-11; }\end{array}$ & \\
\hline NKNPDVLLGS & VQPMFLLTNTFR & n.d. & $\mathrm{N}$-ter + 42.01 Da & 12,1 & $-0,464$ & 2 & 1507,8 & ITAL_MOUSE & E9QNL8_MOUSE & Integrin alpha-L; & $\begin{array}{l}\text { CD11 antigen-like family member A;Leeukocyte } \\
\text { adhesion glycoprotein LFA-1 alpha } \\
\text { chain;:Lukocyte function-associated molecule } 1 \\
\text { alpha chain;:Lymphocyte antigen 15; }\end{array}$ \\
\hline M & DLFGDLPEPERAPR & n.d. & N-ter + 42.01 Da & 15,4 & 0,448 & 2 & 1783,9 & ILKAP_MOUSE & & $\begin{array}{l}\text { Integrin-linked kinase-associated serine/threonine } \\
\text { phosphatase 2C; }\end{array}$ & \\
\hline RAQAMERYPL & APDRAPRLASDFSTR & n.d. & $\mathrm{N}$-ter $+42.01 \mathrm{Da}$ & 12,7 & $-0,939$ & 3 & 1700,9 & I2BP2_MOUSE & & Interferon regulatory factor 2-binding protein 2; & \\
\hline LTLDLPGIT & RVAVGNQPADIGR & n.d. & $\mathrm{N}$-ter + 42.01 Da & 16,6 & 3,723 & 3 & 1393,8 & MX1_MOUSE & MX2_MOUSE & Interferon-induced GTP-binding protein $\mathrm{M} \times 1$ & $\begin{array}{l}\text { Influenza resistance protein;Myxoma resistance } \\
\text { protein 1;Myxovirus resistance protein 1; }\end{array}$ \\
\hline M & ASDTPGFYMDKLNKYR & 0,4 & $\mathrm{~N}$-ter + 42.01 Da, K+28.03 Da & 24,0 & 2,792 & 3 & 2003,0 & E2AK2_MOUSE & & $\begin{array}{l}\text { Interferon-induced, double-stranded RNA-activated } \\
\text { protein kinase; }\end{array}$ & $\begin{array}{l}\text { Eukaryotic translation initiation factor 2-alpha } \\
\text { kinase } 2 \text {;interferon-n-inducible RNA-dependent } \\
\text { protein kinase;P1/elf-2A protein kinase;Protein } \\
\text { kinase RNA-activated;Serine/threonine-protein } \\
\text { kinase TKK; Tyrosine-protein kinase ElF2AK2;p68 } \\
\text { kinase; }\end{array}$ \\
\hline RNASVEEARV & SLAGVEEQLR & n.d. & $\mathrm{N}$-ter + 42.01 Da & 18,7 & 1,922 & 2 & 1142,6 & IRAK2_MOUSE & & Interleukin-1 receptor-associated kinase-like 2; & \\
\hline & AKDILGEA & n.d. & $\mathrm{N}$-ter + 42.01 Da, K+34.06 Da & 80,6 & $-0,577$ & 3 & 2248,3 & IFT2O_MOUSE & & Intraflagellar transport protein 20 homolog; & \\
\hline MAVANRDMVT & AEIAYAA & $\mathrm{n}$ & $\mathrm{N}$-ter + 42.01 Da, $\mathrm{K}+34.06 \mathrm{Da}$ & 17,9 & $-7,428$ & 3 & 1679,9 & IFT80_MOUSE & & Intraflagellar transport protein 80 homolog; & WD repeat-containing protein 56 ; \\
\hline FLSIEHLKAS & FPGHNVKV & & $\mathrm{N}$-ter + 42.01 Da, K+28.03 Da & 17,0 & $-2,048$ & 4 & 2388,3 & AQR_MOUSE & A2AQA7_MOUSE & Intron-binding protein aquarius; & \\
\hline PDEASGGSYL & IFADKTPEQVR & n.d. & $\mathrm{N}$-ter + 42.01 Da, K+34.06 Da & 16,9 & $-3,474$ & 3 & 1378,8 & IQCAL_MOUSE & & IQ and AAA domain-containing protein 1-like; & Protein IQCA1P1; \\
\hline KKVKQDIYCY & DLQYYCLLVLSQDSSR & n.d. & $\mathrm{N}$-ter $+42.01 \mathrm{Da}, \mathrm{C}+57.02 \mathrm{Da}$ & 21,5 & $-4,143$ & 4 & 1951,0 & IQCB1_MOUSE & & IQ calmodulin-binding motif-containing protein 1 ; & \\
\hline QALSCYASSS & SDSCGSTPLR & n.d. & $\mathrm{N}$-ter + 42.01 Da, C+57.02 Da & 13,8 & 2,762 & 2 & 1120,5 & IQEC3_MOUSE & & 1Q motif and SEC7 domain-containing protein 3; & \\
\hline LIGSNAAAGK & SIAPQCWNCGHAR & n.d. & $\mathrm{N}$-ter + 42.01 Da, C +57.02 Da & 14,5 & 0,000 & 3 & 1597,7 & HSC2O_MOUSE & & $\begin{array}{l}\text { Iron-sulfur cluster co-chaperone protein HscB, } \\
\text { mitochondrial; }\end{array}$ & Hsc2O; \\
\hline M & AGSAWVSKVSR & n.d. & $\mathrm{N}$-ter + 42.01 Da, K+34.06 Da & 13,3 & 2,041 & 2 & 1222,7 & IDH3A_MOUSE & & $\begin{array}{l}\text { Isocitrate dehydrogenase [NAD] subunit alpha, } \\
\text { mitochondrial; }\end{array}$ & $\begin{array}{l}\text { Isocitric dehydrogenase subunit alpha; } \mathrm{NAD}(+)- \\
\text { specific ICDH subunit alpha; }\end{array}$ \\
\hline M & LGSGFKAER & 0,0 & $\mathrm{~N}$-ter + 42.01 Da, K+28.03 Da & 31,0 & 0,772 & 2 & 1164,6 & IST1_MOUSE & & IST1 homolog; & \\
\hline M & SLQMIVENVKLAR & n.d. & $\mathrm{N}$-ter $+42.01 \mathrm{Da}, \mathrm{K}+34.06 \mathrm{Da}$ & 30,1 & 1,204 & 2 & 1575,9 & KTNA1_MOUSE & E9QKG2_MOUSE & $\begin{array}{l}\text { Katanin p60 ATPase-containing subunit A1 } \\
\{\text { ECO-0000255 HAMAP-Rule-MF } 030231 \text {. }\end{array}$ & $\begin{array}{l}\text { Lipotransin;p60 katanin \{ECO:0000255 |HAMAP- } \\
\text { Rule:MF 03023\}: }\end{array}$ \\
\hline DGSCNLASVE & YYNPVTDKWTLLPTNMSTGR & $-0,2$ & $\mathrm{~N}$-ter + 42.01 Da, K+28.03 Da & 20,5 & 1,564 & 3 & 2426,2 & KLHLЗ_MOUSE & J3KMI2_MOUSE & 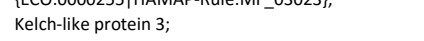 & \\
\hline VSHHGSVTIR & KSYTHIRR & n.d. & $\mathrm{N}$-ter + 42.01 Da, K+28.03 Da & 16,3 & 1,148 & 3 & 1129,6 & KLHLG_MOUSE & & Kelch-like protein 6; & $\begin{array}{l}\text { Keratin-36 } \\
\{E C O: 0000312 \text { |EMBL:CAM17771.11\}:Keratin-5 }\end{array}$ \\
\hline M & ATQICTPTFSAGSAKGLLGTSGSFSR & n.d. & $\begin{array}{l}\text { N-ter + } 42.01 \mathrm{Da}, \mathrm{K}+34.06 \mathrm{Da}, \\
\mathrm{C}+57.02 \mathrm{Da}\end{array}$ & 32,4 & 0,660 & 3 & 2724,3 & KRT36_MOUSE & & $\begin{array}{l}\text { Keratin, type I cuticular Ha6 } \\
\text { \{ECO:0000250|UniprotkB:076013\}; }\end{array}$ & 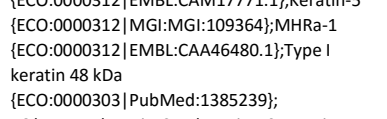 \\
\hline VLLQIDNARL & AADDFRLKYENEVTLR & n.d. & N-ter + 42.01 Da, K+34.06 Da & 24,1 & $-2,874$ & 3 & 2015,1 & K1C10_MOUSE & A2A513_MOUSE & Keratin, type I cytoskeletal 10; & $\begin{array}{l}56 \text { kDa cytokeratin;Cytokeratin-10;Keratin, type } \\
\text { I cytoskeletal } 59 \text { kDa;Keratin-10; }\end{array}$ \\
\hline ALEEQLQQIR & AETECQNAEYQQLLDIKTR & n.d. & $\begin{array}{l}\text { N-ter + 42.01 Da, K+34.06 Da, } \\
\mathrm{C}+57.02 \mathrm{Da}\end{array}$ & 23,5 & $-4,815$ & 3 & 2385,2 & K1C10_MOUSE & A2A513_MOUSE & Keratin, type I cytoskeletal 10; & $\begin{array}{l}56 \text { kDa cytokeratin;Cytokeratin-10;Keratin, type } \\
\text { I cytoskeletal } 59 \text { kDa;Keratin-10; }\end{array}$ \\
\hline KVTMQNLNDR & LASYMDKVR & n.d. & N-ter + 42.01 Da, K+34.06 Da & 27,3 & $-0,862$ & 3 & 1157,6 & K1C10_MOUSE & A2A513_MOUSE & Keratin, type I cytoskeletal 10; & $\begin{array}{l}56 \text { kDa cytokeratin;Cytokeratin-10;Keratin, type } \\
\text { I cytoskeletal } 59 \text { kDa;Keratin-10; }\end{array}$ \\
\hline YQQLLDIKTR & LENEIQTYR & n.d. & $\mathrm{N}$-ter $+42.01 \mathrm{Da}$ & 25,8 & $-2,400$ & 2 & 1206,6 & K1C10_MOUSE & A2A513_MOUSE & Keratin, type I cytoskeletal 10; & $\begin{array}{l}56 \text { kDa cytokeratin;Cytokeratin-10;Keratin, type } \\
\text { I cytoskeletal } 59 \text { kDa;Keratin-10; }\end{array}$ \\
\hline NARLAADDFR & LKYENEVTLR & n.d. & N-ter + 42.01 Da, K+34.06 Da & 18,3 & $-6,107$ & 3 & 1339,8 & K1C10_MOUSE & A2A513_MOUSE & Keratin, type I cytoskeletal 10; & $\begin{array}{l}56 \text { kDa cytokeratin;Cytokeratin-10;Keratin, type } \\
\text { I cytoskeletal } 59 \text { kDa;Keratin-10; }\end{array}$ \\
\hline DLTQLLNNMR & NQYEQLAEKNR & n.d. & N-ter + 42.01 Da, K+34.06 Da & 33,6 & 1,156 & 3 & 1467,8 & K1C10_MOUSE & A2A513_MOUSE & Keratin, type I cytoskeletal 10; & $\begin{array}{l}56 \text { kDa cytokeratin;Cytokeratin-10;Keratin, type } \\
\text { I cytoskeletal } 59 \text { kDa;Keratin-10; }\end{array}$ \\
\hline DNARLAADDF & RLKYENEVTLR & 1,4 & $\mathrm{~N}$-ter + $42.01 \mathrm{Da}, \mathrm{K}+28.03 \mathrm{Da}$ & 27,4 & 8,373 & 3 & 1489,8 & K1C10_MOUSE & A2A513_MOUSE & Keratin, type I cytoskeletal 10; & $\begin{array}{l}56 \text { kDa cytokeratin;Cytokeratin-10;Keratin, type } \\
\text { I cytoskeletal } 59 \text { kDa;Keratin-10; }\end{array}$ \\
\hline M & SVLYSSSSKOFSSSRSGGGGGGGSVR & 0,7 & $\mathrm{~N}$-ter + $42.01 \mathrm{Da}, \mathrm{K}+28.03 \mathrm{Da}$ & 43,6 & $-1,921$ & 3 & 2547,2 & K1C10_MOUSE & A2A513_MOUSE & Keratin, type I cytoskeletal 10; & $\begin{array}{l}56 \text { kDa cytokeratin;Cytokeratin-10;Keratin, type } \\
\text { I cytoskeletal } 59 \text { kDa;Keratin-10; }\end{array}$ \\
\hline M & SVLYSSSSKOFSSSR & n.d. & $\mathrm{N}$-ter + 42.01 Da, K+34.06 Da & 84,5 & $-0,058$ & 2 & 1724,9 & K1C10_MOUSE & A2A513_MOUSE & Keratin, type I cytoskeletal 10; & $\begin{array}{l}56 \text { kDa cytokeratin;Cytokeratin-10;Keratin, type } \\
\text { I cytoskeletal } 59 \text { kDa;Keratin-10; }\end{array}$ \\
\hline
\end{tabular}




\begin{tabular}{|c|c|c|c|c|c|c|c|c|c|c|c|}
\hline Non Prime Site & Prime Site & $\begin{array}{l}\text { Fold change of } \\
\text { XPRESS ratio } \\
\text { (Adam17 } 7^{\alpha \mathrm{KCC}} / \mathrm{WT} \text { ) }\end{array}$ & Modifications & Hyperscore & $\begin{array}{l}\text { Mass error } \\
\text { in ppm }\end{array}$ & Charge & $\begin{array}{l}\text { Precursor } \\
\text { neutral mass } \\
\text { in Da }\end{array}$ & Uniprot ID 1 & Uniprot ID 2 & Protein Name 1 & Protein Name 2 \\
\hline MS & VLYSSSSKRFSSSR & $-0,3$ & $\mathrm{~N}$-ter + 42.01 Da, K+28.03 Da & 20,9 & $-6,304$ & 2 & 1631,8 & K1C10_MOUSE & A2A513_MOUSE & Keratin, type I cytoskeletal 10; & $\begin{array}{l}56 \text { kDa cytokeratin;Cytokeratin-10;Keratin, type } \\
\text { I cytoskeletal } 59 \text { kDa;Keratin-10; }\end{array}$ \\
\hline CIFSGNDGGL & LSGSEKETMQNLNDR & n.d. & $\mathrm{N}$-ter + 42.01 Da, K+34.06 Da & 21,0 & 5,056 & 3 & 1796,9 & K1C12_MOUSE & & Keratin, type I cytoskeletal 12; & Cytokeratin-12;Keratin-12; \\
\hline $\begin{array}{l}\text { RMKYENELAL } \\
\text { EDLKNKIVSA }\end{array}$ & $\begin{array}{l}\text { RQTVEADINGLR } \\
\text { RVSNAQLLQIDNAR }\end{array}$ & $\begin{array}{l}\text { n.d. } \\
\text { n.d. }\end{array}$ & $\begin{array}{l}\text { N-ter + 42.01 Da } \\
\text { N-ter + 42.01 Da }\end{array}$ & $\begin{array}{l}21,2 \\
28,5\end{array}$ & $\begin{array}{l}0,424 \\
2,735\end{array}$ & $\begin{array}{l}3 \\
3\end{array}$ & $\begin{array}{l}1412,7 \\
1752,0\end{array}$ & $\begin{array}{l}\text { K1C12_MOUSE } \\
\text { K1C12_MOUSE }\end{array}$ & & $\begin{array}{l}\text { Keratin, type I cytoskeletal 12; } \\
\text { Keratin, type I cytoskeletal 12; }\end{array}$ & $\begin{array}{l}\text { Cytokeratin-12;Keratin-12; } \\
\text { Cytokeratin-12;Keratin-12; }\end{array}$ \\
\hline AQLSELRSEM & ECQNQEYKMLLDIKTR & 0,9 & $\begin{array}{l}\text { N-ter + 42.01 Da, K+28.03 Da, } \\
\mathrm{C}+57.02 \mathrm{Da}\end{array}$ & 24,7 & $-6,224$ & 3 & 2166,1 & K1C13_MOUSE & & Keratin, type I cytoskeletal 13; & 47 kDa cytokeratin;Cytokeratin-13;Keratin-13; \\
\hline DLTRVLAEMR & EQYEALAEKNR & n.d. & $\mathrm{N}$-ter + 42.01 Da, K+34.06 Da & 21,5 & $-2,380$ & 3 & 1425,7 & K1C13_MOUSE & K1C15_MOUSE & Keratin, type I cytoskeletal 13; & 47 kDa cytokeratin;Cytokeratin-13;Keratin-13; \\
\hline IKILEATTDN & NRIILEIDNAR & n.d. & $\mathrm{N}$-ter + 42.01 Da & 18,2 & $-5,183$ & 2 & 1367,8 & K1C13_MOUSE & & Keratin, type I cytoskeletal 13; & 47 kDa cytokeratin;Cytokeratin-13;Keratin-13; \\
\hline KILEATTDNN & RIILEIDNAR & n.d. & $\mathrm{N}$-ter + 42.01 Da & 16,1 & $-0,398$ & 2 & 1253,7 & K1C13_MOUSE & & Keratin, type I cytoskeletal 13; & 47 kDa cytokeratin;Cytokeratin-13;Keratin-13; \\
\hline SVEEQLAQLR & CEMEQQNQEYKIILDVKTR & n.d. & $\begin{array}{l}\text { N-ter + 42.01 Da, K+34.06 Da, } \\
\mathrm{C}+57.02 \mathrm{Da}\end{array}$ & 40,1 & 1,301 & 3 & 2534,3 & K1C14_MOUSE & K1C17_MOUSE & Keratin, type I cytoskeletal 14; & Cytokeratin-14;Keratin-14; \\
\hline MATCSRQ & FTSSSSMKGSCGIGGGSSR & n.d. & $\begin{array}{l}\text { N-ter + 42.01 Da, K +34.06 Da, } \\
\mathrm{C}+57.02 \mathrm{Da}\end{array}$ & 16,2 & 7,521 & 3 & 1924,9 & K1C14_MOUSE & K1C16_MOUSE & Keratin, type I cytoskeletal 14; & Cytokeratin-14;Keratin-14; \\
\hline SCGIGGGSSR & MSSILAGGSCR & n.d. & $\mathrm{N}$-ter + 42.01 Da, C+57.02 Da & 14,8 & 0,085 & 2 & 1179,5 & K1C14_MOUSE & K1C16_MOUSE & Keratin, type I cytoskeletal 14; & Cytokeratin-14;Keratin-14; \\
\hline TKYETELNLR & MSVEADINGLRR & n.d. & $\mathrm{N}$-ter + 42.01 Da & 17,7 & 0,356 & 3 & 1401,7 & K1C14_MOUSE & K1C17_MOUSE & Keratin, type I cytoskeletal 14; & Cytokeratin-14;Keratin-14; \\
\hline TMQNLNDRLA & TYLDKVR & n.d. & N-ter + 42.01 Da, K +34.06 Da & 17,6 & $-0,926$ & 2 & 969,6 & K1C14_MOUSE & K1C16_MOUSE & Keratin, type I cytoskeletal 14; & Cytokeratin-14;keratin-14; \\
\hline M & ATTFLQTSSTFGGSSTRGASLR & n.d. & $\mathrm{N}$-ter + 42.01 Da & 18,1 & 1,669 & 3 & 2274,1 & K1C15_MOUSE & B1AQ77_MOUSE & Keratin, type I cytoskeletal 15; & Cytokeratin-15;Keratin-15; \\
\hline M & ATTFLLTSSTFGGSSTR & n.d. & $\mathrm{N}$-ter + 42.01 Da & 16,5 & $-1,172$ & 2 & 1789,9 & K1C15_MOUSE & B1AQ77_MOUSE & Keratin, type I cytoskeletal 15; & Cytokeratin-15;Keratin-15; \\
\hline GLETQLSELR & CEMEAQNQEYNMLLDIKTR & 0,3 & $\begin{array}{l}\text { N-ter + } 42.01 \mathrm{Da}, \mathrm{K}+28.03 \mathrm{Da}, \\
\mathrm{C}+57.02 \mathrm{Da}\end{array}$ & 17,5 & $-0,163$ & 3 & 2455,1 & K1C15_MOUSE & B1AQ77_MOUSE & Keratin, type I cytoskeletal 15; & Cytokeratin-15;Keratin-15; \\
\hline $\begin{array}{l}\text { DLTRMLAEMR } \\
\text { FRLKYENELT }\end{array}$ & $\begin{array}{l}\text { EQYEAIAEKNR } \\
\text { LRQGVEADINGLR }\end{array}$ & $\begin{array}{l}\text { n.d. } \\
\text { n.d. }\end{array}$ & $\begin{array}{l}\text { N-ter + 42.01 Da, K+34.06 Da } \\
\text { N-ter + 42.01 Da }\end{array}$ & $\begin{array}{l}27,1 \\
17,0\end{array}$ & $\begin{array}{r}2,310 \\
-2,492\end{array}$ & $\begin{array}{l}3 \\
3\end{array}$ & $\begin{array}{l}1425,7 \\
1481,8\end{array}$ & $\begin{array}{l}\text { K1C15_MOUSE } \\
\text { K1C15_MOUSE }\end{array}$ & $\begin{array}{l}\text { K1C13_MOUSE } \\
\text { B1AQ77_MOUSE }\end{array}$ & $\begin{array}{l}\text { Keratin, type I cytoskeletal 15; } \\
\text { Keratin, type I cytoskeletal 15; }\end{array}$ & $\begin{array}{l}\text { Cytokeratin-15;Keratin-15; } \\
\text { Cytokeratin-15;Keratin-15; }\end{array}$ \\
\hline SVEEQLAQLR & CEMEQQSQEYNILLDVKTR & 0,3 & $\begin{array}{l}\text { N-ter + 42.01 Da, K+28.03 Da, } \\
\text { C +57.02 Da }\end{array}$ & 16,7 & 4,682 & 3 & 2453,2 & K1C16_MOUSE & & Keratin, type I cytoskeletal 16; & Cytokeratin-16;Keratin-16; \\
\hline KNHEEEMLAL & RGQTGGDVNVEMDAAPGVDLSR & n.d. & $\mathrm{N}$-ter + 42.01 Da & 45,0 & 0,393 & 3 & 2285,1 & K1C16_MOUSE & & Keratin, type I cytoskeletal 16; & Cytokeratin-16;Keratin-16; \\
\hline NKILVATVDN & ASILLQIDNAR & n.d. & $\mathrm{N}$-ter + 42.01 Da & 2 & & 2 & 1254,7 & K1C17_N & & Keratin, type I cytoskelet & Cytokeratin-17;Keratin-17; \\
\hline $\begin{array}{l}\text { LKNNILVATV } \\
\text { RSPLLGGG }\end{array}$ & $\begin{array}{l}\text { DNASILLQIDNAR } \\
\text { KATMOLNDR }\end{array}$ & n.d. & $\begin{array}{l}\text { N-ter + 42.01 Da } \\
\text { N-ter 42.01 Da K +34.06 Da }\end{array}$ & 7 & & 2 & 3,8 & $\begin{array}{l}\mathrm{K} 1117-1 \\
\mathrm{~K} 1 \mathrm{C} 17\end{array}$ & & $\begin{array}{l}\text { Kerati } \\
\text { Kerati }\end{array}$ & $\begin{array}{l}\text { Cytokeratin-17;Keratin-17; } \\
\text { cytokeratin-17.Keratin-17. }\end{array}$ \\
\hline $\begin{array}{l}\text { RSSPLLGGGG } \\
\text { KNKLVAVDD }\end{array}$ & $\begin{array}{l}\text { KATMQNLNDR } \\
\text { NASIILIDNAR }\end{array}$ & n.d. & $\begin{array}{l}\text { N-ter + 42.01 Da, K +34.06 Da } \\
\text { N-ter 42.01 Da }\end{array}$ & 3 & & 2 & 1265,7 & K1C17_MOUSE & K1C23_MOUSE & - & Cytokeratin-17;Keratin-17; \\
\hline $\begin{array}{l}\text { KNKLIVATVD } \\
\text { NIIRLOEOYY }\end{array}$ & $\begin{array}{l}\text { NASILLQIDNAR } \\
\text { DGMANAHIVYLDNAR }\end{array}$ & n.d. & $\begin{array}{l}\text { N-ter + 42.01 Da } \\
\text { N-ter 42.01 Da K +28.03 Da }\end{array}$ & 0,3 & $\begin{array}{l}1,167 \\
876\end{array}$ & 2 & 1368,7 & K1C17_MOUSE & & Keratin, type I cytoskeletal 17; & 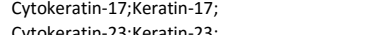 \\
\hline $\begin{array}{l}\text { NISRLQEQIV } \\
\text { M }\end{array}$ & $\begin{array}{l}\text { DGKMANAHIVVLIDNAR } \\
\text { NSSHSFNQTYSASVHSLGSTR }\end{array}$ & $\begin{array}{l}\text { n.d. } \\
\text { n.d. }\end{array}$ & $\begin{array}{l}\text { N-ter + 42.01 Da, K+28.03 Da } \\
\text { N-ter + 42.01 Da }\end{array}$ & $\begin{array}{l}18,6 \\
49,5\end{array}$ & $\begin{array}{r}8,276 \\
-5,160\end{array}$ & $\begin{array}{l}3 \\
3\end{array}$ & $\begin{array}{l}1906,0 \\
2439,1\end{array}$ & $\begin{array}{l}\text { K1C23_MOOSE } \\
\text { K1C23 MOUSE }\end{array}$ & & 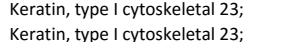 & $\begin{array}{l}\text { Cytokeratin-23; Keratin-23; } \\
\text { cytokeratin-23:Keratin-23. }\end{array}$ \\
\hline etacosaeye & CLLNIKTR & n.d. & $\begin{array}{l}\text { N-ter + 42.01 Da, K +28.03 Da, } \\
\text { C +57.02 Da }\end{array}$ & 12,3 & $-4,685$ & 2 & 1086,6 & K1C24_MOUSE & & Keratin, type I cytoskeletal 24; & Cytokeratin-24; Keratin-24;Type I keratin-24; \\
\hline SIGFLNNEPG & LFSGNEKVAMQNLNDR & 0,9 & $\mathrm{~N}$-ter $+42.01 \mathrm{Da}, \mathrm{K}+28.03 \mathrm{Da}$ & 20,5 & 3,354 & 3 & 1905,0 & K1C26_MOUSE & & Keratin, type I cytoskeletal 26; & $\begin{array}{l}\text { Cytokeratin-26;Keratin-26;Type l inner root } \\
\text { sheath-specific keratin-K25irs2; }\end{array}$ \\
\hline EEemVVLQNA & SGGNINVEMNAAPSVDLTAMLNNMR & n.d. & $\mathrm{N}$-ter $+42.01 \mathrm{Da}$ & 12,3 & $-2,966$ & 3 & 2660,2 & K1C26_MOUSE & & Keratin, type I cytoskeletal 26; & $\begin{array}{l}\text { Cytokeratin-26;Keratin-26;Type l inner root } \\
\text { sheath-specific keratin-K2Sirs2; }\end{array}$ \\
\hline EDLKRQILSM & TTCNANLVLQNDNAR & n.d. & N-ter + 42.01 Da, C +57.02 Da & 15,1 & $-3,259$ & 4 & 1744,8 & K1C26_MOUSE & & Keratin, type I cytoskeletal 26; & $\begin{array}{l}\text { Cytokeratin-26;Keratin-26;Type l inner root } \\
\text { sheath-specific keratin-K2Sirs2; } \\
\text { Cytokeratin-27:Keratin complex-1 acidic, gene }\end{array}$ \\
\hline MKYENELALH & QSVDADINGLRR & n.d. & $\mathrm{N}$-ter $+42.01 \mathrm{Da}$ & 12,8 & 2,596 & 2 & 1384,7 & K1C27_MOUSE & & Keratin, type I cytoskeletal 27 & $\begin{array}{l}\text { Cytokereatin-27;Reratin complex-1, acidic, gene } \\
\text { C29;Keratin-27; Type linner root sheath-specific } \\
\text { keratin-K25irs3; }\end{array}$ \\
\hline TIEDLRNKIL & AATIDNASIVLQIDNAR & n.d. & $\mathrm{N}$-ter $+42.01 \mathrm{Da}$ & 21,2 & 2,406 & 3 & 1826,0 & K1C42_MOUSE & & Keratin, type I cytoskeletal 42; & $\begin{array}{l}\text { Cytokeratin-42;Keratin-17n;Keratin-42;Type I } \\
\text { keratin Ka22; }\end{array}$ \\
\hline KKDVDCAYLR & KSDLEANAEALTQETDFLR & 0,4 & $\mathrm{~N}$-ter + 42.01 Da, K +28.03 Da & 18,7 & 3,059 & 3 & 2220,1 & KRT81_MOUSE & KRT83_MOUSE & $\begin{array}{l}\text { Keratin, type II cuticular Hb1 } \\
\text { \{ECO:0000250|UniProtkB:Q14533\}; }\end{array}$ & $\begin{array}{l}\text { Keratin-81 } \\
\text { \{ECOO0000303|PubMed:12399393\}; Type II hair } \\
\text { keratin Hb1; }\end{array}$ \\
\hline RRSRADVESW & YQTKYEEMR & n.d. & N-ter + 42.01 Da, K +34.06 Da & 14,4 & 5,133 & 2 & 1322,7 & KRT84_MOUSE & & Keratin, type II cuticular Hb4; & $\begin{array}{l}65 \mathrm{kDa} \text { type III keratin;:Keratin-84;Type II hair } \\
\text { keratin Hb4; Type-II keratin Kb24; }\end{array}$ \\
\hline AETFYOSKYE & ELQITAGKHGDSVR & n.d. & $\mathrm{N}$-ter + $42.01 \mathrm{Da}, \mathrm{K}+34.06 \mathrm{Da}$ & 16,3 & 2,643 & 3 & 1585,9 & K2C1_MOUSE & & Keratin, type II cytoskeletal 1; & $\begin{array}{l}67 \mathrm{kDa} \text { cytokeratin;Cytokeratin-1; Keratin- } \\
1 \text {; Type-II keratin Kb1; }\end{array}$ \\
\hline GSYGGSSGGG & GSGGSYGGGSGGGSYGGGSGGGSSGSHR & n.d. & $\mathrm{N}$-ter $+42.01 \mathrm{Da}$ & 26,9 & $-4,234$ & 3 & 2287,9 & K2C1_MOUSE & & Keratin, type II cytoskeletal 1; & $\begin{array}{l}67 \mathrm{kDa} \text { cytokeratin;Cytokeratin-1;Keratin- } \\
\text { 1;Type-II keratin Kb1; }\end{array}$ \\
\hline VRFLEQQNQV & LQTKWELLQQVDTTTR & n.d. & N-ter + 42.01 Da, K+34.06 Da & 21,0 & 0,785 & 3 & 2035,1 & K2C1_MOUSE & & Keratin, type II cytoskeletal 1; & $\begin{array}{l}67 \mathrm{kDa} \text { cytokeratin;Cytokeratin-1;Keratin- } \\
\text { 1;Type-II keratin Kb1; }\end{array}$ \\
\hline VDSLKSDQSR & MDSELKNMQDLVEEYR & 0,4 & $\mathrm{~N}$-ter + 42.01 Da, K+28.03 Da & 31,0 & 6,516 & 3 & 2069,0 & K2C1_MOUSE & & Keratin, type II cytoskeletal 1; & $\begin{array}{l}67 \text { kDa cytokeratin;Cytokeratin-1; Keratin- } \\
\text { 1; Type-II keratin Kb1; }\end{array}$ \\
\hline
\end{tabular}




\begin{tabular}{|c|c|c|c|c|c|c|c|c|c|c|c|}
\hline Non Prime Site & Prime Site & $\begin{array}{l}\text { Fold change of } \\
\text { XPRESS ratio } \\
\left.\text { (Adam17 } 7^{\Delta K c} / \mathrm{WT}\right)\end{array}$ & Modifications & Hyperscore & $\begin{array}{l}\text { Mass error } \\
\text { in ppm }\end{array}$ & Charge & $\begin{array}{l}\text { Precursor } \\
\text { neutral mass } \\
\text { in Da }\end{array}$ & Uniprot ID 1 & Uniprot ID 2 & Protein Name 1 & Protein Name 2 \\
\hline ELQQVDDTT & RTQNLDPFFENYISLR & n.d. & $\mathrm{N}$-ter + 42.01 Da & 27,6 & 5,299 & 3 & 2167,1 & K2C1_MOUSE & & Keratin, type II cytoskeletal 1 ; & $\begin{array}{l}\text { 67 KDa cytokeratin;Cytokeratin-1;Keratin- } \\
\text { 1;Type-II keratin Kb1; }\end{array}$ \\
\hline KLLEGEEIRM & SGECTPNVSVSVSTSHTSMSGSSSR & n.d. & N-ter + 42.01 Da, C +57.02 Da & 35,4 & $-5,637$ & 3 & 2569,1 & K2C1_MOUSE & & Keratin, type II cytoskeletal 1; & $\begin{array}{l}67 \text { kDa cytokeratin;Cytokeratin-1;Keratin- } \\
\text { 1; Type-II keratin Kb1; }\end{array}$ \\
\hline VKSQEREQIK & SLNDKFASFIDKVR & $-8,0$ & N-ter + 42.01 Da, K+28.03 Da & 21,3 & $-2,529$ & 3 & 1736,9 & K2C1_MOUSE & & Keratin, type II cytoskeletal 1; & $\begin{array}{l}67 \text { kDa cytokeratin;Cytokeratin-1;Keratin- } \\
\text { 1; Type-II keratin Kb1; }\end{array}$ \\
\hline SGECTPNVSV & SVSTSHTSMSGSSSR & n.d. & $\mathrm{N}$-ter $+42.01 \mathrm{Da}$ & 37,8 & $-0,454$ & 2 & 1538,7 & K2C1_MOUSE & & Keratin, type II cytoskeletal 1; & $\begin{array}{l}67 \text { kDa cytokeratin;Cytokeratin-1;Keratin- } \\
\text { 1; Type-II keratin Kb1; }\end{array}$ \\
\hline LLRDFQELMN & TKLALDMEIATYKKLLEGEEIR & n.d. & N-ter + 42.01 Da, K+34.06 Da & 16,2 & $-9,408$ & 3 & 2707,6 & K2C1_MOUSE & & Keratin, type II cytoskeletal 1; & $\begin{array}{l}67 \text { kDa cytokeratin;Cytokeratin-1;Keratin- } \\
\text { 1; Type-II keratin Kb1; }\end{array}$ \\
\hline NMQDLVEEYR & TKYEDEINKR & n.d. & N-ter + 42.01 Da, K+34.06 Da & 19,6 & 0,284 & 3 & 1404,8 & K2C1_MOUSE & & Keratin, type II cytoskeletal 1; & $\begin{array}{l}67 \text { KDa cytokeratin;Cytokeratin-1;Keratin- } \\
\text { 1; Type-II keratin Kb1; }\end{array}$ \\
\hline LLQVDDTTR & TQNLDPFFENYISLLR & n.d. & $\mathrm{N}$-ter + 42.01 Da & 25,7 & $-0,099$ & 2 & 2011,0 & K2C1_MOUSE & & Keratin, type II cytoskeletal 1; & $\begin{array}{l}67 \text { kDa cytokeratin;Cytokeratin-1;Keratin- } \\
\text { 1; Type-II keratin Kb1; }\end{array}$ \\
\hline SRGGGSGGGR & $\begin{array}{l}\text { YGGGGSYGGGSGGGSYGGSSGGGGSGGSYGGGSG } \\
\text { GGSYGGGSGGGSSGSHR }\end{array}$ & n.d. & $\mathrm{N}$-ter $+42.01 \mathrm{Da}$ & 21,1 & 9,458 & 3 & 4067,6 & K2C1_MOUSE & & Keratin, type II cytoskeletal 1 ; & $\begin{array}{l}67 \mathrm{kDa} \text { cytokeratin; Cytokeratin-1; Keratin- } \\
\text { 1; Type-II keratin Kb1; }\end{array}$ \\
\hline TAGKHGDDLK & NSKMEISELNR & n.d. & N-ter + 42.01 Da, K+34.06 Da & 32,9 & $-0,930$ & 2 & 1395,7 & K2C1B_MOUSE & & Keratin, type II cytoskeletal 1b; & $\begin{array}{l}\text { Cytokeratin-1B;Embryonic type II keratin- } \\
\text { 1;Keratin-77;Type-II keratin Kb39; }\end{array}$ \\
\hline AGKHGDDLKN & SKMEISELNR & n.d. & N-ter + 42.01 Da, K+34.06 Da & 34,1 & 0,078 & 2 & 1281,7 & K2C1B_MOUSE & & Keratin, type II cytoskeletal 1 b; & $\begin{array}{l}\text { Cytokeratin-1B;Embryonic type II keratin- } \\
\text { 1;Keratin-77; Type-II keratin Kb39; }\end{array}$ \\
\hline MQDTVEDYKS & KYEDEINKR & $-1,0$ & $\mathrm{~N}$-ter + 42.01 Da, K+28.03 Da & 20,5 & 8,110 & 3 & 1291,7 & K22E_MOUSE & K2C1B_MOUSE & Keratin, type II cytoskeletal 2 epidermal; & $\begin{array}{l}\text { Cytokeratin-2e;Epithelial keratin-2e;Keratin-2 } \\
\text { epidermis;Keratin-2e;Type-II keratin Kb2; }\end{array}$ \\
\hline SVSITSSTIS & SSVASKTGFGSGGQSSGGR & n.d. & $\mathrm{N}$-ter + 42.01 Da, K +28.03 Da & 16,2 & 0,224 & 3 & 1782,9 & K22E_MOUSE & & Keratin, type II cytoskeletal 2 epidermal; & $\begin{array}{l}\text { Cytokeratin-2e;Epithelial keratin-2e;Keratin-2 } \\
\text { epidermis;Keratin-2e;Type-II keratin Kb2; }\end{array}$ \\
\hline FLRTLYDMEL & SQIQSHVSDTSVVLSMDNNR & n.d. & $\mathrm{N}$-ter $+42.01 \mathrm{Da}$ & 25,1 & 8,182 & 3 & 2258,1 & K22O_MOUSE & & $\begin{array}{l}\text { Keratin, type II cytoskeletal } 2 \text { oral } \\
\{\text { \{CO:0000250|UniProtkB:Q01546\}; }\end{array}$ & $\begin{array}{l}\text { Keratin-76 } \\
\text { \{ECO:0000312|MGl:MG:1924305\};Type-II } \\
\text { keratin Kb9; }\end{array}$ \\
\hline DTVEDYKSKY & EDEINKR & $-1,4$ & $\mathrm{~N}$-ter + 42.01 Da, K+28.03 Da & 23,4 & $-2,668$ & 2 & 972,5 & K2C5_MOUSE & K22E_MOUSE & Keratin, type II cytoskeletal 5; & Cytokeratin-5;Keratin-5;Type-II keratin Kb5; \\
\hline MDEINFMKMF & FDAELSQMQTHVSDTSWVLSMDNNR & n.d. & $\mathrm{N}$-ter $+42.01 \mathrm{Da}$ & 18,5 & 5,753 & 3 & 2865,3 & K2C5_MOUSE & & Keratin, type II cytoskeletal 5; & Cytokeratin-5;Keratin-5;Type-II keratin Kb5; \\
\hline AGSSQGGMSF & GSGGGSGSSVKFVSTTSSSR & n.d. & N-ter + 42.01 Da, K+34.06 Da & 22,0 & $-7,173$ & 3 & 1906,9 & K2C5_MOUSE & & Keratin, type II cytoskeletal 5; & Cytokeratin-5;Keratin-5;Type-II keratin Kb5; \\
\hline M & SRQSSVSFR & n.d. & $\mathrm{N}$-ter $+42.01 \mathrm{Da}$ & 23,5 & $-1,186$ & 2 & 1094,6 & K2C5_MOUSE & & Keratin, type II cytoskeletal 5; & Cytokeratin-5;Keratin-5;Type-II keratin Kb5; \\
\hline QYEDIANRSR & TEAESWYQTKYEELQQTAGR & 0,6 & N-ter + 42.01 Da, K+28.03 Da & 24,5 & 0,964 & 3 & 2487,2 & K2C5_MOUSE & & Keratin, type II cytoskeletal 5; & Cytokeratin-5;Keratin-5;Type-II keratin Kb5; \\
\hline IQRLRSEIDN & VKKQCANLQNAIAEAEQR & n.d. & $\begin{array}{l}\text { N-ter + 42.01 Da, K+28.03 Da, } \\
C+57.02 \text { Da }\end{array}$ & 18,2 & 0,276 & 3 & 2168,1 & K2C5_MOUSE & & Keratin, type II cytoskeletal 5; & Cytokeratin-5;Keratin-5;Type-II keratin Kb5; \\
\hline PPGGIQEVTI & NQNLLTPLNVQIDPTIQR & n.d. & $\mathrm{N}$-ter $+42.01 \mathrm{Da}$ & 17,2 & 6,836 & 3 & 2118,2 & K2C6B_MOUSE & Q3UV11_MousE & Keratin, type II cytoskeletal 6B; & Cytokeratin-6B;Keratin-6-beta;keratin-6B; \\
\hline FFKCLFEAEM & AQIQSHISDMSVILSMDNNR & n.d. & $\mathrm{N}$-ter + 42.01 Da & 20,5 & 8,033 & 3 & 2300,1 & K2C71_MOUSE & & Keratin, type II cytoskeletal 71; & $\begin{array}{l}\text { Cytokeratin-6G;Cytokeratin-71;Keratin- } \\
6 G ; \text { Keratin-71; Type II inner root sheath-specific } \\
\text { keratin-KGirs1;Type-II keratin Kb34; }\end{array}$ \\
\hline TDDIKFFKVL & FEGEIAQMQSHISDTSVILSMDNNR & n.d. & $\mathrm{N}$-ter + 42.01 Da & 18,2 & $-4,710$ & 3 & 2863,3 & K2C72_MOUSE & & Keratin, type II cytoskeletal 72; & $\begin{array}{l}\text { Cytokeratin-72;Keratin-72;Type II inner root } \\
\text { sheath-specific keratin-KGirs2; Type-II keratin } \\
\text { Kb35; }\end{array}$ \\
\hline QINFYRMIYE & AELSQMQNQVSDTSVVLSMDNNR & n.d. & $\mathrm{N}$-ter + 42.01 Da & 17,6 & $-1,034$ & 3 & 2607,2 & K2C75_MOUSE & & Keratin, type II cytoskeletal 75; & $\begin{array}{l}\text { Cytokeratin-75;Keratin-6 hair follicle;Keratin- } \\
\text { 75;Type II keratin-K6h;;Type-II keratin Kb18; }\end{array}$ \\
\hline DQINFYRMIY & EAELSQMQNQVSDTSVVLSMDNNR & n.d. & $\mathrm{N}$-ter $+42.01 \mathrm{Da}$ & 29,1 & 5,293 & 3 & 2736,3 & K2C75_MOUSE & & Keratin, type II cytoskeletal 75; & $\begin{array}{l}\text { Cytokeratin-75;Keratin-6 hair follicle;Keratin- } \\
75 \text {; Type II keratin-K6hf;Type-II keratin Kb18; }\end{array}$ \\
\hline LTDQINFYRM & IYEAELSQMQNQVVSDTSVVLSMDNNR & n.d. & $\mathrm{N}$-ter $+42.01 \mathrm{Da}$ & 19,4 & $-3,315$ & 4 & 3012,4 & K2C75_MOUSE & & Keratin, type II cytoskeletal 75; & $\begin{array}{l}\text { Cytokeratin-75;Keratin-6 hair follicle;Keratin- } \\
\text { 75;Type II keratin-K6h;;Type-II keratin Kb18; }\end{array}$ \\
\hline NFYRMIYEAE & LSQMQNQVSDTSVVLSMDNNR & n.d. & $\mathrm{N}$-ter + 42.01 Da & 17,6 & $-9,336$ & 3 & 2407,1 & K2C75_MOUSE & & Keratin, type II cytoskeletal 75; & $\begin{array}{l}\text { Cytokeratin-75;Keratin-6 } 6 \text { hair follicle;Keratin- } \\
75 \text {;Type II keratin-K6hf;Type-II keratin Kb } 18 \text {; }\end{array}$ \\
\hline
\end{tabular}




\begin{tabular}{|c|c|c|c|c|c|c|c|c|c|c|c|}
\hline Non Prime Site & Prime Site & $\begin{array}{l}\text { Fold change of } \\
\text { XPRESS ratio } \\
\text { (Adam17 } 7^{\text {akC } / W T)}\end{array}$ & Modifications & Hyperscore & $\begin{array}{l}\text { Mass error } \\
\text { in ppm }\end{array}$ & Charge & $\begin{array}{l}\text { Precursor } \\
\text { neutral mass } \\
\text { in } \mathrm{Da}\end{array}$ & Uniprot ID 1 & Uniprot ID 2 & Protein Name 1 & Protein Name 2 \\
\hline RMIYEAELSQ & MQNQVSDTSVVLSMDNNR & n.d. & $\mathrm{N}$-ter+ 42.01 Da & 28,9 & $-8,886$ & 3 & 2078,9 & K2C75_MOUSE & & Keratin, type II cytoskeletal 75; & $\begin{array}{l}\text { Cytokeratin-75;Keratin-6 hair follicle;Keratin- } \\
\text { 75;Type II keratin-K6hf; Type-II keratin Kb18; }\end{array}$ \\
\hline GSSSGGFGSR & SLYNLGGKNISVSMACGASSGR & $-5,9$ & $\begin{array}{l}\text { N-ter + 42.01 Da, K+28.03 Da, } \\
\text { C+57.02 Da }\end{array}$ & 23,1 & $-4,998$ & 3 & 2298,1 & K2C79_MOUSE & & Keratin, type II cytoskeletal 79; & Cytokeratin-79;Keratin-79;Type-II keratin Kb38; \\
\hline M & ADPAECNIKVMCR & 0,0 & $\begin{array}{l}\text { N-ter + 42.01 Da, K+28.03 Da, } \\
C+57.02 \text { Da }\end{array}$ & 58,3 & $-0,795$ & 2 & 1632,7 & KINH_MOUSE & E9QAK5_MOUSE & Kinesin-1 heavy chain; & $\begin{array}{l}\text { Conventional kinesin heavy chain;Ubiquitous } \\
\text { kinesin heavy chain; }\end{array}$ \\
\hline M & AGASVKVAVR & 0,1 & $\mathrm{~N}$-ter + 42.01 Da, K+28.03 Da & 28,5 & $-0,681$ & 2 & 1026,6 & KIF1A_MOUSE & KIF1C_MOUSE & Kinesin-like protein KIF1A; & Axonal transporter of synaptic vesicles; \\
\hline M & ATSAEAPAKEAQKR & n.d. & $\mathrm{N}$-ter + 42.01 Da, K+34.06 Da & 28,2 & 1,784 & 3 & 1566,9 & KRR1_MOUSE & G3UZAO_MOUSE & KRR1 small subunit processome component homolog; & $\begin{array}{l}\text {; HIV-1 Rev-binding protein } 2 \text { homolog;KRR-R } \\
\text { motif-containing protein 1; }\end{array}$ \\
\hline $\begin{array}{l}\text { KVRSGTSGKS } \\
\mathrm{M}\end{array}$ & $\begin{array}{l}\text { GDKGNGDASPDGR } \\
\text { ASLPPHAGPATPLSPTR }\end{array}$ & $\begin{array}{l}\text { n.d. } \\
\text { n.d. }\end{array}$ & $\begin{array}{l}\text { N-ter + 42.01 Da, } \mathrm{K}+34.06 \mathrm{Da} \\
\text { N-ter + 42.01 Da }\end{array}$ & $\begin{array}{l}13,3 \\
53,6\end{array}$ & $\begin{array}{r}-1,815 \\
-1,401\end{array}$ & $\begin{array}{l}2 \\
2\end{array}$ & $\begin{array}{l}1320,6 \\
1710,9\end{array}$ & $\begin{array}{l}\text { KLF6_MOUSE } \\
\text { LMNB2_MOUSE }\end{array}$ & Q8BPQ2_MOUSE & $\begin{array}{l}\text { Krueppel-like factor 6; } \\
\text { Lamin-B2; }\end{array}$ & Core promoter element-binding protein; \\
\hline M & ATQVEPLLPAGAPLLQAEEHGLAR & n.d. & $\mathrm{N}$-ter $+42.01 \mathrm{Da}$ & 63,2 & $-0,832$ & 3 & 2522,4 & LARP1_MOUSE & J3QNB1_MOUSE & La-related protein $1 ;$ & La ribonucleoprotein domain family member 1 \\
\hline M & TSDQDAKVVAEPQAQR & n.d. & $\mathrm{N}$-ter + 42.01 Da, K+34.06 Da & 32,6 & 1,867 & 3 & 1817,9 & LAR4B_MOUSE & & La-related protein 4B; & $\begin{array}{l}\text { La ribonucleoprotein domain family member } \\
\text { AB;La ribonucleoprotein domain family } \\
\text { member } 5 \text {; La-related protein } 5 \text {; }\end{array}$ \\
\hline M & ETENQKTMEESTKR & n.d. & N-ter + 42.01 Da, K+34.06 Da & 18,4 & 0,768 & 3 & 1951,0 & LARP7_MOUSE & & La-related protein 7 ; & La ribonucleoprotein domain family member 7 \\
\hline FSWLGFDHKI & TGKOFYKQVTTVGR & n.d. & $\mathrm{N}$-ter + 42.01 Da, K+28.03 Da & 15,5 & 0,234 & 3 & 1710,0 & LAT4_MOUSE & & Large neutral amino acids transporter small subunit 4 ; & $\begin{array}{l}\text { L-type amino acid transporter 4;Solute carrier } \\
\text { ffyily } 43 \text { member 2; }\end{array}$ \\
\hline EPERIDEAQP & SDVTLPAEDASNR & n.d. & $\mathrm{N}$-ter $+42.01 \mathrm{Da}$ & 15,1 & 3,668 & 2 & 1415,7 & LST2_MOUSE & & Lateral signaling target protein 2 homolog; & Zinc finger FYVE domain-containing protein 28; \\
\hline M & EIPPTHYAASR & n.d. & $\mathrm{N}$-ter + 42.01 Da & 38,2 & $-1,272$ & 2 & 1413,7 & LXN_MOUSE & & Latexin; & $\begin{array}{l}\text { Endogenous carboxypeptidase inhibitor; Tissue } \\
\text { carboxypeptidase inhibitor; }\end{array}$ \\
\hline DMEILRTVKG & SSTGEVNVHLVAR & n.d. & $\mathrm{N}$-ter + 42.01 Da & 17,1 & $-7,857$ & 3 & 1409,7 & LMBL1_MOUSE & Q3V558_MOUSE & Lethal(3)malignant brain tumor-like protein 1; & L(3)mbt protein homolog; \\
\hline M & AELTNYKDAASNR & 0,1 & $\mathrm{~N}$-ter + 42.01 Da, K+28.03 Da & 53,8 & 1,641 & 2 & 1521,8 & LUZP1_MOUSE & E9Q9L3_MOUSE & Leucine zipper protein $1 ;$ & Leucine zipper motif-containing protein; \\
\hline M & AIVHTLPVPLEPAR & n.d. & $\mathrm{N}$-ter $+42.01 \mathrm{Da}$ & 24,5 & 1,607 & 2 & 1553,9 & LZTS2_MOUSE & & $\begin{array}{l}\text { Leucine zipper putative tumor suppressor } 2 \\
\text { \{ECO:0000255|HAMAP-Rule:MF__3026\}; }\end{array}$ & $\begin{array}{l}\text { Protein LAPSER1 \{ECO:0000255|HAMAP- } \\
\text { Rule:MF_03026\}; }\end{array}$ \\
\hline M & AELGLNEHHQNEVINYMR & n.d. & $\mathrm{N}$-ter + 42.01 Da & 38,2 & 0,407 & 3 & 2208,0 & LZTL1_MOUSE & E9Q9P1_MOUSE & Leucine zipper transcription factor-like protein 1 ; & \\
\hline TSLARACDSS & SSSSLGSGEAAGLGR & n.d. & $\mathrm{N}$-ter + $42.01 \mathrm{Da}$ & 14,7 & $-3,914$ & 3 & 1376,7 & LRFN2_MOUSE & & $\begin{array}{l}\text { Leucine-rich repeat and fibronectin type-III domain- } \\
\text { containing protein 2; }\end{array}$ & \\
\hline GISSRPGSAG & SDEVDGALKALR & n.d. & $\mathrm{N}$-ter + 42.01 Da, K+28.03 Da & 21,0 & $-3,123$ & 2 & 1342,7 & LRGUK_MOUSE & & $\begin{array}{l}\text { Leucine-rich repeat and guanylate kinase domain- } \\
\text { containing protein; }\end{array}$ & \\
\hline IPEGIPAETR & MLELSRNR & n.d. & $\mathrm{N}$-ter + 42.01 Da & 15,3 & $-8,667$ & 2 & 1059,5 & LIGO3_MOUSE & & $\begin{array}{l}\text { Leucine-rich repeat and immunoglobulin-like domain- } \\
\text { containing nogo receptor-interacting protein 3; }\end{array}$ & Leucine-rich repeat neuronal protein 6B; \\
\hline M & TSPEGAQNKEIDCLSPEAQR & n.d. & $\begin{array}{l}\text { N-ter + 42.01 Da, K+34.06 Da, } \\
\text { C +57.02 Da }\end{array}$ & 34,5 & 0,217 & 3 & 2305,1 & LRRF1_MOUSE & E9Q9T1_MOUSE & Leucine-rich repeat flightless-interacting protein 1 ; & FLL-LRR-associated protein 1;H186 FLAP; \\
\hline NQITELANTT & FRPMPNLR & n.d. & $\mathrm{N}$-ter $+42.01 \mathrm{Da}$ & 14,2 & 8,942 & 2 & 1071,6 & LRRT1_MOUSE & EOCX41_MOUSE & $\begin{array}{l}\text { Leucine-rich repeat transmembrane neuronal protein } \\
1 ;\end{array}$ & \\
\hline DAVKNYSICG & KSTTTER & n.d. & N-ter + 42.01 Da, K+34.06 Da & 11,4 & 1,001 & 2 & 897,5 & LRRT3_MOUSE & & $\begin{array}{l}\text { Leucine-rich repeat transmembrane neuronal protein } \\
3 ;\end{array}$ & \\
\hline SMPAFPLLR & SSEEKTLYSDR & n.d. & $\mathrm{N}$-ter + 42.01 Da, $\mathrm{K}+34.06 \mathrm{Da}$ & 24,5 & 1,006 & 2 & 1389,7 & LRC49_MOUSE & G5E8R5_MOUSE & Leucine-rich repeat-containing protein 49; & $\begin{array}{l}\text { Tubulin polyglutamylase complex subunit } \\
4 ; ; 79 ;\end{array}$ \\
\hline EANIFLKVLD & ISHNGFGDSGASAIGDALR & n.d. & N-ter + 42.01 Da & 17,3 & 3,124 & 3 & 1885,9 & YV012_MOUSE & & $\begin{array}{l}\text { Leucine-rich repeat-containing protein 74B } \\
\text { \{ECO:0000312|MGI:MGl:1921935\}; }\end{array}$ & \\
\hline KGSLAERASP & GAAPGPR & n.d. & $\mathrm{N}$-ter + 42.01 Da & 15,1 & $-0,898$ & 2 & 666,3 & F211A_MOUSE & & Leucine-rich repeat-containing protein 75A; & $\begin{array}{l}\text { Leucine-rich repeat-containing protein } \\
\text { FAM211A; }\end{array}$ \\
\hline FRKSNNPIKK & WGSELNKEFSPEEYR & n.d. & N-ter + 42.01 Da, K+28.03 Da & 15,2 & 1,338 & 3 & 1939,9 & POL2_MOUSE & & LINE-1 retrotransposable element ORF2 protein; & $\begin{array}{l}\text { Long interspersed element-1;Retrovirus-related } \\
\text { Pol polyprotein LNE-1; }\end{array}$ \\
\hline $\begin{array}{l}\text { M } \\
\text { LSLAPQFSSS }\end{array}$ & $\begin{array}{l}\text { SHPSWLPPKSTGEPLGHVPAR } \\
\text { GPQQASRASTASSTR }\end{array}$ & $\begin{array}{l}0,0 \\
\text { n.d. }\end{array}$ & $\begin{array}{l}\text { N-ter + 42.01 Da, } \mathrm{K}+28.03 \mathrm{Da} \\
\text { N-ter + 42.01 Da }\end{array}$ & $\begin{array}{l}38,3 \\
21,6\end{array}$ & $\begin{array}{l}0,646 \\
8,871\end{array}$ & $\begin{array}{l}4 \\
3\end{array}$ & $\begin{array}{l}2319,2 \\
1642,8\end{array}$ & $\begin{array}{l}\text { LPP_MOUSE } \\
\text { ARMC9_MOUSE }\end{array}$ & & $\begin{array}{l}\text { Lipoma-preferred partner homolog; } \\
\text { LisH domain-containing protein ARMC9; }\end{array}$ & \\
\hline M & ATVTELKAVLKDTLEKR & n.d. & N-ter + 42.01 Da, K+34.06 Da & 21,0 & $-3,444$ & 3 & 2058,3 & FOPNL_MOUSE & D3Z644_MOUSE & LisH domain-containing protein FOPNL; & $\begin{array}{l}\text { FGFR1OP N-terminal-like protein;FOP-related } \\
\text { protein of } 20 \mathrm{kDa} \text {; }\end{array}$ \\
\hline LPSSLAPESF & FGEFTDSSDSDSVPPR & n.d. & $\mathrm{N}$-ter $+42.01 \mathrm{Da}$ & 12,3 & 1,736 & 2 & 1783,8 & E9Q286_MOUSE & & Little elongation complex subunit 1; & $\begin{array}{l}\text { Interactor of little elongator complex ELL } \\
\text { subunit } 1 \text {. }\end{array}$ \\
\hline AIGLSVADLA & ESIMKNLR & 0,2 & N-ter + 42.01 Da, K+28.03 Da & 12,0 & $-0,188$ & 2 & 1059,6 & LDHA_MOUSE & G5E8N5_MOUSE & L-lactate dehyd & $\begin{array}{l}\text { subunit 1; } \\
\text { LDH muscle subunit; }\end{array}$ \\
\hline & EVHELFR & n.d. & $\mathrm{N}$-ter + 42.01 Da & 20,3 & 0,181 & 2 & 1101,5 & ACSL1_MOUSE & D3Z041_MOUSE & Long-chain-fatty-acid--CoA ligase 1; & Long-chain acyl-CoA synthetase 1 ; \\
\hline
\end{tabular}




\begin{tabular}{|c|c|c|c|c|c|c|c|c|c|c|c|}
\hline Non Prime Site & Prime Site & 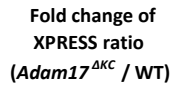 & Modifications & Hyperscore & $\begin{array}{l}\text { Mass error } \\
\text { in ppm }\end{array}$ & Charge & $\begin{array}{l}\text { Precursor } \\
\text { neutral mass } \\
\text { in Da }\end{array}$ & Uniprot ID 1 & Uniprot ID 2 & Protein Name 1 & Protein Name 2 \\
\hline M & AEVGSKSVLFVCLGNICR & n.d. & $\begin{array}{l}\text { N-ter + } 42.01 \mathrm{Da}, \mathrm{K}+34.06 \mathrm{Da}, \\
\mathrm{C}+57.02 \mathrm{Da}\end{array}$ & 27,6 & 5,031 & 3 & 2084,1 & PPAC_MOUSE & Q561M1_MOUSE & $\begin{array}{l}\text { Low molecular weight phosphotyrosine protein } \\
\text { phosphatase; }\end{array}$ & $\begin{array}{l}\text { Low molecular weight cytosolic acid } \\
\text { phosphatase; }\end{array}$ \\
\hline GRDTASKGVS & SPCSSLEFHCGSSECIHR & n.d. & N-ter + 42.01 Da, C +57.02 Da & 17,2 & $-4,786$ & 3 & 2190,9 & LDLR_MOUSE & & Low-density lipoprotein receptor; & \\
\hline SPPSTFLLFS & QKFAISR & n.d. & $\mathrm{N}$-ter + $42.01 \mathrm{Da}, \mathrm{K}+28.03 \mathrm{Da}$ & 11,0 & 7,496 & 2 & 918,5 & LRP5_MOUSE & & Low-density lipoprotein receptor-related protein 5; & $\begin{array}{l}\text { Low-density lipoprotein receptor-related } \\
\text { protein 7; Lr3; }\end{array}$ \\
\hline SSSTKGTYFP & AILNPPPSPATER & n.d. & $\mathrm{N}$-ter $+42.01 \mathrm{Da}$ & 12,2 & 2,917 & 2 & 1403,8 & LRP6_MOUSE & E9Q8KO_MOUSE & Low-density lipoprotein receptor-related protein 6; & \\
\hline GGGTTDYAVQ & IFHAAAKNGTFECNLEAGTR & n.d. & $\begin{array}{l}\text { N-ter + 42.01 Da, K+28.03 Da, } \\
\mathrm{C}+57.02 \mathrm{Da}\end{array}$ & 11,3 & 3,071 & 3 & 2276,1 & TDH_MOUSE & & L-threonine 3-dehydrogenase, mitochondrial; & \\
\hline YDENEDLSDV & EEIVSVRGFSLEEKLR & n.d. & N-ter + 42.01 Da, K+34.06 Da & 21,0 & 2,285 & 3 & 1966,1 & KDM2B_MOUSE & DЗYUE3_MOUSE & Lysine-specific demethylase 2B; & $\begin{array}{l}\text { F-box and leucine-rich repeat protein 10;F--box } \\
\text { protein FBL10; F-box/LRR-repeat protein } \\
\text { 10;jmjc domain-containing histone } \\
\text { demethylation protein 1B; [Histone-H3]-lysine- } \\
36 \text { demethylase 1B; }\end{array}$ \\
\hline M & ADAAASPVGKR & n.d. & N-ter + 42.01 Da, K +34.06 Da & 21,7 & 1,072 & 2 & 1117,6 & KDM3B_MOUSE & B9EKS2_MOUSE & Lysine-specific demethylase 3B; & $\begin{array}{l}\text { Jmjc domain-containing histone demethylation } \\
\text { protein } 2 B ; J \text { Jumonji domain-containing protein } \\
1 B ;\end{array}$ \\
\hline VHCEGCARRR & SAGLQGVVVLEQYR & n.d. & $\mathrm{N}$-ter $+42.01 \mathrm{Da}$ & 14,8 & $-7,998$ & 3 & 1559,8 & KDM6B_MOUSE & & Lysine-specific demethylase 6B; & $\begin{array}{l}\text { Jmjc domain-containing protein 3;Jumonji } \\
\text { domain-containing protein 3; }\end{array}$ \\
\hline M & ATLQESEVKVDGEQKLSKNELKR & n.d. & N-ter + 42.01 Da, K+34.06 Da & 26,9 & $-7,400$ & 4 & 2806,7 & SYK_MOUSE & & Lysine--tRNA ligase; & Lysyl-tRNA synthetase; \\
\hline AGHMVPTDKP & RAAFTMFSR & n.d. & $\mathrm{N}$-ter + 42.01 Da & 12,3 & 9,473 & 2 & 1127,6 & PPGB_MOUSE & G3X8T3_MOUSE & Lysosomal protective protein; & $\begin{array}{l}\text { Carboxypeptidase } C_{;} \text {Carboxypeptidase } \\
\text { L;Cathepsin A;Protective protein cathepsin } \\
\text { A; Protective protein for beta-galactosidase; }\end{array}$ \\
\hline M & ADTAPQLKR & 0,1 & N-ter + 42.01 Da, K +28.03 Da & 34,1 & 0,560 & 2 & 1068,6 & DCPS_MOUSE & Q3TBW9_MOUSE & m7GpppX diphosphatase; & $\begin{array}{l}\text { DCS-1;Decapping scavenger enzyme;Hint- } \\
\text { related 7meGMP-directed hydrolase;Histidine } \\
\text { triad nucleotide-binding protein 5;Histidine } \\
\text { triad protein member 5;Scavenger mRNA- } \\
\text { decapping enzyme DcPS; }\end{array}$ \\
\hline $\begin{array}{l}\text { LEGKRSTRDR } \\
\text { TFTDANHTCT }\end{array}$ & $\begin{array}{l}\text { RSPAELEGGSASEGAAR } \\
\text { NEKAYLTVEDR }\end{array}$ & $\begin{array}{l}\text { n.d. } \\
-6,5\end{array}$ & $\begin{array}{l}\text { N-ter + 42.01 Da } \\
\text { N-ter + 42.01 Da, K+28.03 Da }\end{array}$ & $\begin{array}{l}20,2 \\
12,0\end{array}$ & $\begin{array}{l}-4,915 \\
2,517\end{array}$ & $\begin{array}{l}3 \\
2\end{array}$ & $\begin{array}{l}1685,8 \\
1507,8\end{array}$ & $\begin{array}{l}\text { CSF1_MOUSE } \\
\text { MRC1_MOUSE }\end{array}$ & & $\begin{array}{l}\text { Macrophage colony-stimulating factor 1; } \\
\text { Macrophage mannose receptor 1; }\end{array}$ & \\
\hline TETLNVRVRE & STAKQQEDISKLEER & n.d. & N-ter + 42.01 Da, K +28.03 Da & 20,3 & 9,721 & 3 & 1859,0 & MSRE_MOUSE & & Macrophage scavenger receptor types I and II; & $\begin{array}{l}\text { Macrophage acetylated LDL receptor I and } \\
\text { ॥;; cavenger receptor type A; }\end{array}$ \\
\hline GAPGIRGIPG & VKGDRGQIGFPGGR & n.d. & $\mathrm{N}$-ter + 42.01 Da, $\mathrm{K}+28.03 \mathrm{Da}$ & 19,1 & 1,122 & 3 & 1512,8 & MSRE_MOUSE & & Macrophage scavenger receptor types I and $\|$; & $\begin{array}{l}\text { Macrophage acetylated LDL receptor I and } \\
\text { II;Scavenger receptor type A; }\end{array}$ \\
\hline M & YTPIPQSGSPFPASVQDPGLHIWR & n.d. & $\mathrm{N}$-ter + 42.01 Da & 50,4 & 1,274 & 3 & 2822,4 & CAPG_MOUSE & Q99LB4_MOUSE & Macrophage-capping protein; & $\begin{array}{l}\text { Actin regulatory protein CAP-G;Actin-capping } \\
\text { protein GCAP39;Myc basic motif homolog 1; }\end{array}$ \\
\hline NAVHAVLDEG & KIIIMDLEPQDIQSAR & n.d. & $\begin{array}{l}\text { N-ter + 42.01 Da, K+28.03 Da, } \\
\mathrm{C}+57.02 \mathrm{Da}\end{array}$ & 17,4 & $-6,787$ & 3 & 1986,0 & MPP4_MOUSE & D3Z0G8_MOUSE & MAGUK p55 subfamily member 4; & Discs large homolog 6; \\
\hline M & DGETAGEKGSLVPPPGALGGSALGGAPAPGVR & 0,1 & $\mathrm{~N}$-ter + $42.01 \mathrm{Da}, \mathrm{K}+28.03 \mathrm{Da}$ & 54,6 & 1,313 & 3 & 3042,6 & MAPK3_MOUSE & & MAP kinase-activated protein kinase 3; & Acid dustar notein 33. Snactic naranleoi \\
\hline LSTEAKEEMY & KLYPNAR & n.d. & N-ter + 42.01 Da, K+34.06 Da & 13,5 & 0,426 & 2 & 936,6 & SPG21_MOUSE & & Maspardin; & $\begin{array}{l}\text { autosomal recessive Mast syndrome protein } \\
\text { homolog; }\end{array}$ \\
\hline M & SKSFQQSSLGR & n.d. & $\mathrm{N}$-ter + 42.01 Da, K+34.06 Da & 37,6 & $-1,076$ & 2 & 1299,7 & MATR3_MOUSE & & Matrin-3; & \\
\hline NRRAHLK & ECFETLKR & n.d. & $\begin{array}{l}\text { N-ter }+42.01 \text { Da, } K+34.06 \text { Da, } \\
\text { C+57.02 Da }\end{array}$ & 12,0 & $-1,897$ & 2 & 1157,6 & MNT_MOUSE & Q5SWE2_MOUSE & Max-binding protein MNT; & Myc antagonist MNT;Protein ROX; \\
\hline WATSQNSKSY & GKSISIETANLR & 3,1 & $\mathrm{~N}$-ter $+42.01 \mathrm{Da}, \mathrm{K}+28.03 \mathrm{Da}$ & 21,6 & $-6,393$ & 3 & 1357,8 & MD12L_MOUSE & & $\begin{array}{l}\text { Mediator of RNA polymerase II transcription subunit } \\
\text { 12-like protein; }\end{array}$ & Mediator complex subunit 12-like protein; \\
\hline $\begin{array}{l}\text { TQDEIERTKQ } \\
\text { GEEVAAGQMT } \\
\text { WLPPELLGLW }\end{array}$ & $\begin{array}{l}\text { FSKDVVDLLR } \\
\text { AADHPAFGR } \\
\text { THCAQVPQR }\end{array}$ & $\begin{array}{l}\text { n.d. } \\
\text { n.d. } \\
\text { n.d. }\end{array}$ & $\begin{array}{l}\text { N-ter + 42.01 Da, K+34.06 Da } \\
\text { N-ter + 42.01 Da } \\
\text { N-ter + 42.01 Da, C +57.02 Da }\end{array}$ & $\begin{array}{l}19,8 \\
11,5 \\
18,0\end{array}$ & $\begin{array}{l}8,269 \\
-6,603 \\
-1,229\end{array}$ & $\begin{array}{l}3 \\
2 \\
2\end{array}$ & $\begin{array}{c}1266,8 \\
982,5 \\
1137,5\end{array}$ & $\begin{array}{l}\text { MARF1_MOUSE } \\
\text { MEII_MOUSE } \\
\text { REC8_MOUSE }\end{array}$ & F8WHB9_MOUSE & $\begin{array}{l}\text { Meiosis arrest female protein } 1 \text {; } \\
\text { Meiosis inhibitor protein } 1 ; \\
\text { Meiotic recombination protein REC8 homolog; }\end{array}$ & $\begin{array}{l}\text { Limkain-b1; } \\
\text { Meiosis defective protein 1; } \\
\text { Cohesin Rec8p; }\end{array}$ \\
\hline M & AQKPDGGAGLR & n.d. & $\mathrm{N}$-ter + $42.01 \mathrm{Da}, \mathrm{K}+34.06 \mathrm{Da}$ & 29,9 & 0,262 & 2 & 1144,6 & MAGD1_MOUSE & & Melanoma-associated antigen D1; & $\begin{array}{l}\text { Dlxin-1;MAGE-D1 antigen;Neurotrophin } \\
\text { receptor-interacting MAGE homolog; }\end{array}$ \\
\hline M & SEHAAAPGPGPNGGGGGGAAPVR & n.d. & $\mathrm{N}$-ter $+42.01 \mathrm{Da}$ & 15,6 & $-3,879$ & 3 & 1981,9 & MBRL_MOUSE & & M & Transmembrane protein 259; \\
\hline M & AAGDGDVKLSTLGSGGESGGDGSPGGAGATAAR & 0,2 & $\mathrm{~N}$-ter + $42.01 \mathrm{Da}, \mathrm{K}+28.03 \mathrm{Da}$ & 76,7 & 0,452 & 3 & 2872,4 & PGRC2_MOUSE & & $\begin{array}{l}\text { Membrane-associated progesterone receptor } \\
\text { component 2; }\end{array}$ & \\
\hline M & SAFNLLHLVTKSQPVAPR & n.d. & $\mathrm{N}$-ter + 42.01 Da, K +34.06 Da & 28,7 & 8,997 & 3 & 2053,2 & MSRB3_MOUSE & D3YUC9_MOUSE & Methionine-R-sulfoxide reductase $\mathrm{B} 3$, mitochondrial; & \\
\hline
\end{tabular}




\begin{tabular}{|c|c|c|c|c|c|c|c|c|c|c|c|}
\hline Non Prime Site & Prime Site & $\begin{array}{l}\text { Fold change of } \\
\text { XPRESS ratio } \\
\text { (Adam17 } 7^{\Delta K c} / \text { WT) }\end{array}$ & Modifications & Hyperscore & $\begin{array}{c}\text { Mass error } \\
\text { in ppm }\end{array}$ & Charge & $\begin{array}{l}\text { Precursor } \\
\text { neutral mass } \\
\text { in Da }\end{array}$ & Uniprot ID 1 & Uniprot ID 2 & Protein Name 1 & Protein Name 2 \\
\hline MLSPAM & SFLKSFPPPGSADGLR & n.d. & $\mathrm{N}$-ter + 42.01 Da, K+34.06 Da & 22,0 & $-0,913$ & 2 & 1751,0 & ICLN_MOUSE & Q923F1_MOUSE & Methylosome subunit plCIn; & $\begin{array}{l}\text { Chloride channel, nucleotide sensitive } \\
1 A ; \text {;Chloride conductance regulatory protein } \\
\text { ICln;Chloride ion current inducer protein; }\end{array}$ \\
\hline VGYadivNID & ISEVVIKQMKER & n.d. & $\mathrm{N}$-ter + 42.01 Da, K+28.03 Da & 13,7 & 5,773 & 2 & 1556,9 & MET13_MOUSE & HЗBLКЗ_MOUSE & Methyltransferase-like protein 13; & \\
\hline AQDGEASEKG & DRLDGAAGR & n.d. & $\mathrm{N}$-ter $+42.01 \mathrm{Da}$ & 15,8 & 5,239 & 2 & 971,5 & MET16_MOUSE & Q5SW15_MOUSE & Methyltransferase-like protein 16; & $\begin{array}{l}\text { Methyltransferase } 10 \text { domain-containing } \\
\text { protein; }\end{array}$ \\
\hline $\begin{array}{l}\text { M } \\
\text { ACPTREPPLG }\end{array}$ & $\begin{array}{l}\text { SVPSALMKQPPIQSTAGAVPVR } \\
\text { ASGDWPPHLSTKEEAAGR }\end{array}$ & $\begin{array}{l}-0,1 \\
n \cdot d\end{array}$ & $\begin{array}{l}\text { N-ter + 42.01 Da, } \mathrm{K}+28.03 \mathrm{Da} \\
\text { N-ter + 42.01 Da K +34.06 Da }\end{array}$ & 52,8 & $\begin{array}{r}0,130 \\
-0,655\end{array}$ & $\begin{array}{l}3 \\
2\end{array}$ & 2303,3 & MFAP1_MOUSE & & Microfibrillar-associated protein 1; & \\
\hline & $\begin{array}{l}\text { ADLSLVDALTEPPPEIEGEIKR } \\
\text { ADLER }\end{array}$ & $\begin{array}{l}\text { n.d. } \\
0,2\end{array}$ & $\begin{array}{l}\text { N-ter + 42.01 Da, K +34.06 Da } \\
\text { N-ter + 42.01 Da, K +28.03 Da }\end{array}$ & $\begin{array}{l}10,0 \\
55,5\end{array}$ & $\begin{array}{l}-0,655 \\
0,649\end{array}$ & $\begin{array}{l}2 \\
3\end{array}$ & $\begin{array}{l}1984,0 \\
2461,3\end{array}$ & $\begin{array}{l}\text { MAP1A_MOUSE } \\
\text { MAP4_MOUSE }\end{array}$ & $\begin{array}{l}\text { ALRPB_NGOUSE } \\
\text { Q78TF_MOUSE }\end{array}$ & 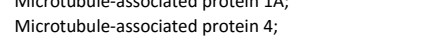 & \\
\hline KRSPSAATSS & HYLGSKVVLDQKOPR & n.d. & $\mathrm{N}$-ter + 42.01 Da, K+28.03 Da & 14,5 & $-5,579$ & 3 & 1879,1 & MAP9_MOUSE & & Microtubule-associated protein $9 ;$ & Aster-associated protein; \\
\hline TRDPFPDVVR & LEEQDSGGSNTPEQDDTSEGR & n.d. & $\mathrm{N}$-ter $+42.01 \mathrm{Da}$ & 11,8 & 1,873 & 4 & 2291,9 & MAST1_MOUSE & E9Q605_MOUSE & $\begin{array}{l}\text { Microtubule-associated serine/threonine-protein } \\
\text { kinase 1; }\end{array}$ & $\begin{array}{l}\text { Syntrophin-associated serine/threonine-protein } \\
\text { kinase; }\end{array}$ \\
\hline PLEEMAQLSS & YDSPDTPETDDSVEGR & n.d. & $\mathrm{N}$-ter + 42.01 Da & 15,0 & $-1,532$ & 4 & 1823,7 & MAST2_MOUSE & B1AST8_MOUSE & $\begin{array}{l}\text { Microtubule-associated serine/threonine-protein } \\
\text { kinase 2; }\end{array}$ & \\
\hline EHGAAWQPRI & MEEKALEVYDLLR & n.d. & $\mathrm{N}-\operatorname{ter}+42.01 \mathrm{Da}, \mathrm{K}+34.06 \mathrm{Da}$ & 23,8 & 4,982 & 2 & 1683,9 & FA96A_MOUSE & & MIP18 family protein FAM96A; & \\
\hline ALGGQSLFGQ & EVYSQLLKEGHR & n.d. & $\mathrm{N}$-ter + 42.01 Da, K +34.06 Da & 13,8 & 5,921 & 3 & 1533,9 & ALL12_MOUSE & & $\begin{array}{l}\text { Mitochondrial 10-formyltetrahydrofolate } \\
\text { dehydrogenase; }\end{array}$ & Aldehyde dehydrogenase family 1 member $\mathrm{L}$; \\
\hline LEKLLQYCET & GVQEGATLVYGGR & n.d. & $\mathrm{N}$-ter $+42.01 \mathrm{Da}$ & 11,9 & $-9,854$ & 2 & 1347,7 & ALL12_MOUSE & D3Z6Bg_MOUSE & $\begin{array}{l}\text { Mitochondrial 10-formyltetrahydrofolate } \\
\text { dehydrogenase; }\end{array}$ & Aldehyde dehydrogenase family 1 member $L 2 ;$ \\
\hline GQSLFGQEVY & SQLLKEGHR & n.d. & $\mathrm{N}$-ter + 42.01 Da, K +34.06 Da & 22,0 & 2,708 & 2 & 1142,7 & AL1L2_MOUSE & & $\begin{array}{l}\text { Mitochondrial 10-formyltetrahydrofolate } \\
\text { dehydrogenase; }\end{array}$ & Aldehyde dehydrogenase family 1 member $L 2 ;$ \\
\hline IGQSLFGQEV & YSQLLKEGHR & n.d. & $\mathrm{N}$-ter + 42.01 Da, K +34.06 Da & 25,4 & 4,588 & 2 & 1305,7 & AL1L2_MOUSE & & $\begin{array}{l}\text { Mitochondrial 10-formyltetrahydrofolate } \\
\text { dehydrogenase; }\end{array}$ & $\begin{array}{l}\text { Aldehyde dehydrogenase family } 1 \text { member L2; } \\
\text { CARD adapter inducing interferon }\end{array}$ \\
\hline M & TFAEDKTYKYIR & n.d. & N-ter + 42.01 Da, K+34.06 Da & 18,9 & $-1,032$ & 3 & 1643,9 & MAVS_MOUSE & S4R1W6_MOUSE & Mitochondrial antiviral-signaling protein; & $\begin{array}{l}\text { beta;interfereon beta promoter stimulator } \\
\text { protein } ; \text {; Virus-induced-signaling adapter; }\end{array}$ \\
\hline M & EAVLNELVSVEDLKNFER & 0,1 & $\mathrm{~N}-\mathrm{ter}+42.01 \mathrm{Da}, \mathrm{K}+28.03 \mathrm{Da}$ & 47,4 & $-0,173$ & 3 & 2304,2 & FIS1_MOUSE & & Mitochondrial fission 1 protein; & $\begin{array}{l}\text { FIS1 homolog;Tetratricopeptide repeat protein } \\
\text { 11; }\end{array}$ \\
\hline $\begin{array}{l}\text { M } \\
\text { NDEEASCLRF }\end{array}$ & $\begin{array}{l}\text { EANVTIPIWQNKPHGAAR } \\
\text { RHSLWQKKEER }\end{array}$ & $\begin{array}{l}\text { n.d. } \\
\text { n.d. }\end{array}$ & $\begin{array}{l}\text { N-ter + 42.01 Da, } \mathrm{K}+34.06 \mathrm{Da} \\
\mathrm{N} \text {-ter }+42.01 \mathrm{Da}, \mathrm{K}+34.06 \mathrm{Da}\end{array}$ & $\begin{array}{l}15,7 \\
15,9\end{array}$ & $\begin{array}{c}1,402 \\
-1,678\end{array}$ & $\begin{array}{l}3 \\
3\end{array}$ & $\begin{array}{l}2208,2 \\
1605,9\end{array}$ & $\begin{array}{l}\text { MFR1L_MOUSE } \\
\text { MTFR2_MOUSE }\end{array}$ & D3YW54_MOUSE & $\begin{array}{l}\text { Mitochondrial fission regulator 1-like; } \\
\text { Mitochondrial fission regulator 2; }\end{array}$ & DUF729 domain-containing protein 1 ; \\
\hline M & ESSTSSSGSALGAVDPQLQHFIEVETQKQR & n.d. & $\mathrm{N}$-ter + 42.01 Da, K+28.03 Da & 12,3 & $-1,170$ & 3 & 3416,7 & TIM8A_MOUSE & & $\begin{array}{l}\text { Mitochondrial import inner membrane translocase } \\
\text { subunit Tim8 A; }\end{array}$ & Deafness dystonia protein 1 homolog; \\
\hline M & $\begin{array}{l}\text { AASKPIEAAMAAAAAPGSGNGVGGGGGTAGPGSG } \\
\text { AGTLRR }\end{array}$ & 0,1 & $\mathrm{~N}$-ter + 42.01 Da, K+28.03 Da & 79,0 & 0,206 & 4 & 3388,7 & TOM70_MOUSE & & Mitochondrial import receptor subunit TOM70; & $\begin{array}{l}\text { Mitochondrial precursor proteins import } \\
\text { receptor; ;ranslocase of outer membrane } 70 \\
\text { kDa subunit; }\end{array}$ \\
\hline M & SQERPTFYR & n.d. & $\mathrm{N}$-ter + 42.01 Da & 29,7 & 0,245 & 2 & 1224,6 & MK14_MOUSE & B2KF35_MOUSE & Mitogen-activated protein kinase 14; & $\begin{array}{l}\text { CRK1;Mitogen-activated protein kinase p38 } \\
\text { alpha; }\end{array}$ \\
\hline DLLRSEGIPS & TEAVPTASPLSGSPKMSTASSR & n.d. & N-ter + 42.01 Da, K+28.03 Da & 16,6 & $-1,164$ & 3 & 2231,1 & M3K13_MOUSE & & Mitogen-activated protein kinase kinase kinase 13; & \\
\hline M & DDQQALNSIMQDLAVLHKASR & n.d. & N-ter + 42.01 Da, K+34.06 Da & 37,4 & 3,122 & 3 & 2559,3 & M3K2_MOUSE & G5E8L__MOUSE & Mitogen-activated protein kinase kinase kinase 2; & MAPK/ERR kinase kinase 2; \\
\hline PALADTPAAA & MEELRPAPPPQPEPDPECCPAAR & n.d. & $\mathrm{N}$-ter $+42.01 \mathrm{Da}, \mathrm{C}+57.02 \mathrm{Da}$ & 13,0 & $-3,906$ & 3 & 2685,2 & M3K4_MOUSE & & Mitogen-activated protein kinase kinase kinase 4; & MAPK/ERK kinase kinase 4; \\
\hline TPHVAEPAEN & EHDEQDENGAEASAELR & n.d. & $\mathrm{N}$-ter + 42.01 Da & 13,3 & $-0,309$ & 3 & 1940,8 & MOES_MOUSE & & Moesin; & Membrane-organizing extension spike protein; \\
\hline $\begin{array}{l}\text { QDSPPARWSN } \\
\text { PIPPKADUT }\end{array}$ & FSWDPLR & n.d. & N-ter $+42.01 \mathrm{Da}$ & 10,3 & 6,124 & 2 & 961,5 & MOCS1_MOUSE & G3UXN5_MOUSE & Molybdenum cofactor biosynthesis protein 1 & \\
\hline $\begin{array}{l}\text { M } \\
\text { MAADLI }\end{array}$ & $\begin{array}{l}\text { TEEELLL } \\
\text { AENNA }\end{array}$ & $\begin{array}{l}\text { n.d. } \\
-0.4\end{array}$ & $\mathrm{~K}+28.03 \mathrm{Da}$ & $\begin{array}{l}12 \\
48\end{array}$ & $-0,6,6$ & $\begin{array}{l}2 \\
3\end{array}$ & 1884,0 & $\begin{array}{l}\text { MOCS1_MOUSE } \\
\text { MSPD2MOUSE }\end{array}$ & $\begin{array}{l}\text { G3UWH1_MOUSE } \\
\text { BAAU74 MOUSE }\end{array}$ & $\begin{array}{l}\text { E Molybdenum cofactor } \\
\text { Motile sperm domain- }\end{array}$ & \\
\hline MRRGAP & QDQE & n.d. & $\mathrm{N}$-ter $+42.01 \mathrm{Da}$ & 15,8 & 6,873 & 3 & 1364 & MSPD3_MOUSE & D3Z1F3_MOUSE & Motile sperm domain-c $c$ & \\
\hline LCFSQQSGDG & TLQSIFIFLR & n.d. & N-ter $+42.01 \mathrm{Da}$ & 13,0 & $-4,525$ & 3 & 1278,7 & MP17L_MOUSE & E9Q4P9_MOUSE & Mpv17-like protein; & \\
\hline M & EGSAKASVASDPESPPGGNEPAAASGQR & 0,1 & $\mathrm{~N}$-ter + 42.01 Da, K+28.03 Da & 72,7 & 0,884 & 3 & 2824,3 & MCES_MOUSE & D3Z4K1_MOUSE & mRNA cap guanine-N7 methyltransferase; & $\begin{array}{l}\text { methyltransferase;mRNA cap } \\
\text { methyltransferase; }\end{array}$ \\
\hline M & AAAAAGGLPGKGHDISLAALR & n.d. & $\mathrm{N}$-ter + 42.01 Da, K +34.06 Da & 21,3 & 0,551 & 3 & 1992,1 & DCP1B_MOUSE & B9EIXO_MOUSE & mRNA-decapping enzyme $1 \mathrm{~B} ;$ & \\
\hline GRPPKTRRRA & EEDGEHER & n.d. & N-ter $+42.01 \mathrm{Da}$ & 14,7 & 6,709 & 2 & 1041,4 & MINT_MOUSE & A2ADBO_MOUSE & Msx2-interacting protein; & $\begin{array}{l}\text { SMART/DADAC-1-associated repressor } \\
\text { protein;SPEN homolog; }\end{array}$ \\
\hline NQQQALPIRP & KDSNKLSGKQLALR & n.d. & N-ter + 42.01 Da, K +34.06 Da & 14,9 & $-5,454$ & 4 & 1701,1 & S47A1_MOUSE & & gand toxin extrusion protein 1; & Solute carrier family 47 member 1 ; \\
\hline AEDGGPGPSP & RSFHAAAYYPAGR & n.d. & N-ter $+42.01 \mathrm{Da}$ & 20,0 & $-6,428$ & 3 & 1443,7 & MEGF8_MOUSE & & $\begin{array}{l}\text { Multiple epic } \\
\text { protein } 8 \text { : }\end{array}$ & Epidermal growth factor-like protein 4; \\
\hline M & $\begin{array}{l}\text { AEMKSPTKAEPATPAEAAQSDR } \\
\text { MKSPTKAEPATPAEAAQSDR }\end{array}$ & $\begin{array}{l}0,4 \\
\text { n.d. }\end{array}$ & $\begin{array}{l}\text { N-ter + 42.01 Da, K+28.03 Da } \\
\text { N-ter + 42.01 Da, K +34.06 Da }\end{array}$ & $\begin{array}{l}56,3 \\
17,2\end{array}$ & $\begin{array}{c}0,712 \\
-7,051\end{array}$ & $\begin{array}{l}3 \\
3\end{array}$ & $\begin{array}{l}2383,2 \\
2195,1\end{array}$ & $\begin{array}{l}\text { MBB1A_MOUSE } \\
\text { MBB1A_ MOUSE }\end{array}$ & & $\begin{array}{l}\text { Myb-binding protein } 1 \mathrm{~A} ; \\
\text { Myb-binding protein } 1 \mathrm{~A} ;\end{array}$ & $\begin{array}{l}\text { Myb-binding protein of } 160 \mathrm{kDa} \text {; } \\
\text { Myb-binding protein of } 160 \mathrm{kDa} \text {; }\end{array}$ \\
\hline
\end{tabular}




\begin{tabular}{|c|c|c|c|c|c|c|c|c|c|c|c|}
\hline Non Prime Site & Prime Site & $\begin{array}{l}\text { Fold change of } \\
\text { XPRESS ratio } \\
\text { (Adam17 } 7^{\text {akc } / \text { WT) }}\end{array}$ & Modifications & Hyperscore & $\begin{array}{l}\text { Mass error } \\
\text { in ppm }\end{array}$ & Charge & $\begin{array}{l}\text { Precursor } \\
\text { neutral mass } \\
\text { in Da }\end{array}$ & Uniprot ID 1 & Uniprot ID 2 & Protein Name 1 & Protein Name 2 \\
\hline VPSIVKEESS & $\begin{array}{l}\text { EEEIAIAATSAKELGHEPVPADLGEVR } \\
\end{array}$ & n.d. & $\mathrm{N}$-ter + 42.01 Da, $\mathrm{K}+28.03 \mathrm{Da}$ & 18,0 & 7,405 & 3 & 2900,5 & MYBB_MOUSE & & Myb-related protein B; & Myb-like protein 2; \\
\hline VRQFGQQDWK & FLASHFPNR & n.d. & $\mathrm{N}$-ter + 42.01 Da & 15,4 & $-1,060$ & 3 & 1129,6 & MYBB_MOUSE & & Myb-related protein B; & $\begin{array}{l}\text { Myb-like protein 2; } \\
\text { Amphiphysin II;Amphiphysin-like }\end{array}$ \\
\hline LWMDYHQKLV & DQALTMDTYLGQFPDIKSR & n.d. & $\mathrm{N}$-ter + 42.01 Da, K+28.03 Da & 15,4 & 1,720 & 3 & 2381,2 & BIN1_MOUSE & Q6P1B9_MOUSE & Myc box-dependent-interacting protein 1 ; & $\begin{array}{l}\text { protein;Bridging integrator } 1 ; \text { SH3 domain- } \\
\text { containing protein } 9 \text {; }\end{array}$ \\
\hline M & ADADKSEAAAGDDGSQQQPAEPR & n.d. & $\mathrm{N}$-ter + 42.01 Da, K +34.06 Da & 66,6 & 0,084 & 3 & 2389,1 & MYEF2_MOUSE & A2ATP5_MOUSE & Myelin expression factor 2; & \\
\hline M & CDFTEDQTAEFKEAFQLFDR & n.d. & $\begin{array}{l}\text { N-ter + 42.01 Da, K+34.06 Da, } \\
\mathrm{C}+57.02 \mathrm{Da}\end{array}$ & 50,6 & 5,242 & 3 & 2572,2 & MYL6_MOUSE & & Myosin light polypeptide 6; & $\begin{array}{l}17 \text { kDa myosin light chain;:Myosin light chain } \\
\text { 3;Myosin light chain akkali 3;Smooth muscle } \\
\text { and nonmuscle myosin light chain alkali } 6 \text {; }\end{array}$ \\
\hline FKEAFQLFDR & TGDGKILYSQCGDVMR & n.d. & $\begin{array}{l}\text { N-ter + 42.01 Da, K+34.06 Da, } \\
\mathrm{C}+57.02 \mathrm{Da}\end{array}$ & 14,6 & 5,272 & 3 & 1874,9 & MYL6_MOUSE & & Myosin light polypeptide 6; & $\begin{array}{l}17 \text { kDa myosin light chain;:Myosin light chain } \\
\text { 3;Myosin light chain akalal 3;Smooth muscle } \\
\text { and nonmuscle myosin light chain alkali } 6 ;\end{array}$ \\
\hline EDVIRNAFAC & FDEEATGTIQEDYLR & n.d. & $\mathrm{N}$-ter + 42.01 Da & 19,7 & 0,819 & 3 & 1827,8 & ML12B_MOUSE & & Myosin regulatory light chain 12B; & $\begin{array}{l}\text { Myosin regulatory light chain 2-B, smooth } \\
\text { muscle isoform;Myosin regulatory light chain } \\
20 \text { KDa;Myosin regulatory light chain MRLC2; }\end{array}$ \\
\hline ERNSLQDQLD & EEMEAKQNLER & n.d. & $\mathrm{N}$-ter + 42.01 Da, K +34.06 Da & 15,4 & 7,360 & 2 & 1451,7 & MYH11_MOUSE & E9QPE7_MOUSE & Myosin-11; & $\begin{array}{l}\text { Myosin heavy chain 11;Myosin heary chain, } \\
\text { smooth muscle isoform;sSMMHC; } \\
\text { Phosphatidylinositol-3,5-bisphosphate 3- }\end{array}$ \\
\hline NLPLFAFUNE & EKFNVDGWTVYNPVEEYR & n.d. & N-ter + 42.01 Da, K+28.03 Da & 20,2 & 1,338 & 3 & 2314,1 & MTM1_MOUSE & B1AW21_MOUSE & Myotubularin; & $\begin{array}{l}\text { phosphatase;Phosphatidylinositol-3-phosphate } \\
\text { phosphatase; }\end{array}$ \\
\hline EEKFNVDGWT & VYNPVEEYR & n.d. & $\mathrm{N}$-ter $+42.01 \mathrm{Da}$ & 18,9 & 2,476 & 2 & 1209,6 & MTM1_MOUSE & B1AW21_MOUSE & Myotubularin; & $\begin{array}{l}\text { phosshatidyyinositol-3,,-5idsphosphate 3- } \\
\text { phoshatase; Phosphatidylinositol-3-phosphate } \\
\text { phosphatase; }\end{array}$ \\
\hline M & EKSSSCESLGAQLPAAR & n.d. & $\begin{array}{l}\text { N-ter + } 42.01 \mathrm{Da}, \mathrm{K}+28.03 \mathrm{Da}, \\
\mathrm{C}+57.02 \mathrm{Da}\end{array}$ & 18,6 & $-0,050$ & 3 & 1991,0 & MTMR2_MOUSE & B8JJF6_MOUSE & Myotubularin-related protein 2; & $\begin{array}{l}\text { Phosphatidylinositol-3,5-bisphosphate 3- } \\
\text { phosphatase;Phosphatidylinositol-3-phosphate } \\
\text { phosphatase; } \\
\text { Aspartylglucosaminidase;Glycosylasparaginase; }\end{array}$ \\
\hline TLDAMIMDGT & AMDVGAVGGLR & n.d. & N-ter $+42.01 \mathrm{Da}$ & 20,1 & $-6,247$ & 2 & 1086,5 & ASPG_MOUSE & & N(4)-(beta-N-acetyl|glucosaminy)|-L-asparaginase; & $\begin{array}{l}\text { N4-(N-acetyl-beta-glucosaminyl)-L-asparagine; } \\
\text { amidase; }\end{array}$ \\
\hline M & AGLGHPSAFGR & n.d. & $\mathrm{N}$-ter $+42.01 \mathrm{Da}$ & 34,6 & $-0,539$ & 2 & 1110,6 & DDAH1_MOUSE & & $\begin{array}{l}\mathrm{N}(\mathrm{G}), \mathrm{N}(\mathrm{G}) \text {-dimethylarginine dimethylaminohydrolase } \\
1 ;\end{array}$ & DDAHI;Dimethylargininase-1; \\
\hline FPNASSPDVG & ATLPNDKAKTPTTVDR & n.d. & $\mathrm{N}$-ter + 42.01 Da, K+28.03 Da & 18,7 & 1,805 & 3 & 1825,0 & PGRP2_MOUSE & & $\mathrm{N}$-acetylmuramoyl-L-alanine amidase; & $\begin{array}{l}\text { Peptidoglycan recognition protein } \\
\text { 2;Peptidoglycan recognition protein long;TagL; }\end{array}$ \\
\hline M & AFPKKKLR & n.d. & $\mathrm{N}$-ter + 42.01 Da, K +34.06 Da & 25,6 & $-0,706$ & 3 & 1130,8 & NPL_MOUSE & & $\mathrm{N}$-acetylneuraminate lyase; & $\begin{array}{l}\text { N-acetylneuraminate pyruvate-lyase;N- } \\
\text { acetylneuraminic acid aldolase;Sialate } \\
\text { lyase;Sialate-pyruvate lyase;Sialic acid } \\
\text { aldolase;Sialic acid lyase; }\end{array}$ \\
\hline $\begin{array}{l}\text { MYYEGKFPCL } \\
\text { STINGYEGTG }\end{array}$ & $\begin{array}{l}\text { EEKVLETPQEIR } \\
\text { RSLSLKLQQLR }\end{array}$ & $\begin{array}{l}\text { n.d. } \\
\text { n.d. }\end{array}$ & $\begin{array}{l}\text { N-ter + 42.01 Da, } \mathrm{K}+34.06 \mathrm{Da} \\
\text { N-ter + 42.01 Da, K+28.03 Da }\end{array}$ & $\begin{array}{l}18,5 \\
15,4\end{array}$ & $\begin{array}{l}0,968 \\
-4,977\end{array}$ & $\begin{array}{l}3 \\
3\end{array}$ & $\begin{array}{l}1545,9 \\
1523,9\end{array}$ & $\begin{array}{l}\text { NAT10_MOUSE } \\
\text { NAT10_MOUSE }\end{array}$ & & $\begin{array}{l}\text { N-acetyltransferase 10; } \\
\text { N-acetyltransferase 10; }\end{array}$ & \\
\hline VLVNNSNLWS & LDLGHNILDDAGLNILDALR & n.d. & $\mathrm{N}$-ter + 42.01 Da, C +57.02 Da & 14,9 & $-7,399$ & 3 & 2362,2 & NAL14_MOUSE & & NACHT, LRR and PYD domains-containing protein 14; & Germ cell specific leucine-rich repeat NTPase; \\
\hline LAAEGMWTDT & FVFAEEALRR & n.d. & $\mathrm{N}$-ter $+42.01 \mathrm{Da}$ & 18,0 & $-9,448$ & 2 & 1278,7 & NAL4E_MOUSE & & NACHT, LRR and PYD domains-containing protein 4E; & NALP-epsilon; \\
\hline M & ELVEVLKR & $-0,1$ & $\mathrm{~N}$-ter + 42.01 Da, K+28.03 Da & 28,1 & $-0,758$ & 2 & 1185,7 & NDUAC_MOUSE & & $\begin{array}{l}\text { NADH dehydrogenase [ubiquinone] } 1 \text { alpha } \\
\text { subcomplex subunit 12; }\end{array}$ & $\begin{array}{l}\text { Complex I-B17.2;NADH-ubiquinone } \\
\text { oxidoreductase subunit B17.2; }\end{array}$ \\
\hline M & AGLLKKTTGLVGLAVCDTPHER & n.d. & $\begin{array}{l}\text { N-ter + 42.01 Da, } \mathrm{K}+34.06 \mathrm{Da} \\
\mathrm{C}+57.02 \mathrm{Da}\end{array}$ & 75,8 & $-1,348$ & 3 & 2445,4 & NDUA5_MOUSE & & $\begin{array}{l}\text { NADH dehydrogenase [ubiquinone] } 1 \text { alpha } \\
\text { subcomplex subunit } 5 \text {; }\end{array}$ & $\begin{array}{l}\text { Complex I subunit B13;Complex I-13kD-B;NADH- } \\
\text { ubiquinone oxidoreductase } 13 \text { KDa-B subunit; }\end{array}$ \\
\hline M & SGSKYKPAPLATLPSTLDPAEYDVSPETR & n.d. & N-ter + 42.01 Da, K+34.06 Da & 55,2 & $-1,468$ & 3 & 3199,7 & NDUB4_MOUSE & & $\begin{array}{l}\text { NADH dehydrogenase [ubiquinone] } 1 \text { beta } \\
\text { subcomplex subunit 4; }\end{array}$ & $\begin{array}{l}\text { Complex I-B15;NADH-ubiquinone } \\
\text { oxidoreductase B15 subunit; }\end{array}$ \\
\hline M & SGYTPDEKLR & n.d. & $\mathrm{N}$-ter + 42.01 Da, K+34.06 Da & 28,2 & 2,334 & 2 & 1240,7 & NDUB6_MOUSE & A2AP32_MOUSE & $\begin{array}{l}\text { NADH dehydrogenase [ubiquinone] } 1 \text { beta } \\
\text { subcomplex subunit } 6 \text {; }\end{array}$ & $\begin{array}{l}\text { Complex I-B17;NADH-ubiquinone } \\
\text { oxidoreductase B17 subunit; }\end{array}$ \\
\hline M & AFCAPPAYLTHQQKVLR & 0,5 & $\begin{array}{l}\text { N-ter + 42.01 Da, K+28.03 Da, } \\
\mathrm{C}+57.02 \mathrm{Da}\end{array}$ & 21,1 & 2,461 & 3 & 2069,1 & NDUB9_MOUSE & & $\begin{array}{l}\text { NADH dehydrogenase [ubiquinone] } 1 \text { beta } \\
\text { subcomplex subunit } 9 \text {; }\end{array}$ & $\begin{array}{l}\text { Complex I-B22;NADH-ubiquinone } \\
\text { oxidoreductase B22 subunit; }\end{array}$ \\
\hline
\end{tabular}




\begin{tabular}{|c|c|c|c|c|c|c|c|c|c|c|c|}
\hline Non Prime Site & Prime Site & $\begin{array}{l}\text { Fold change of } \\
\text { XPRESS ratio } \\
\text { (Adam17 10Kc / WT) }\end{array}$ & Modifications & Hyperscore & $\begin{array}{c}\text { Mass error } \\
\text { in ppm }\end{array}$ & Charge & $\begin{array}{l}\text { Precursor } \\
\text { neutral mass } \\
\text { in Da }\end{array}$ & Uniprot ID 1 & Uniprot ID 2 & Protein Name 1 & Protein Name 2 \\
\hline LTHPVEPKNS & EKMSENGVSSR & n.d. & $\mathrm{N}$-ter + 42.01 Da, $\mathrm{K}+34.06 \mathrm{Da}$ & 12,1 & $-1,999$ & 2 & 1298,6 & NAA25_MOUSE & & N-alpha-acetyltransferase 25 , NatB auxiliary subunit; & $\begin{array}{l}\text { Mitochondrial distribution and morphology } \\
\text { protein 20;N-terminal acetyltransferase B } \\
\text { complex subunit MDM M20;N-terminal } \\
\text { acetyltransferease B complex subunit NAA25; }\end{array}$ \\
\hline TMGPPEDDED & DDGEEPEAGGR & n.d. & $\mathrm{N}$-ter + 42.01 Da & 13,2 & 3,917 & 2 & 1172,5 & NANO1_MOUSE & & Nanos homolog 1; & \\
\hline M & LTLASKLKR & n.d. & $\mathrm{N}$-ter + 42.01 Da, $\mathrm{K}+34.06 \mathrm{Da}$ & 15,0 & $-0,079$ & 3 & 1269,9 & UB2FA_MOUSE & & NEDD8-conjugating enzyme UBE2F; & $\begin{array}{l}\text { NEDD8 carrier protein UBEEFFNEDDD8 protein } \\
\text { ligase UBE2F;NEDD8-conjugating enzymee } \\
\text { 2; ;Ubiquitin-conjugating enzyme E2 F; }\end{array}$ \\
\hline NKLGATDELW & APPSIASLLTAAVIDNIR & n.d. & $\mathrm{N}$-ter + 42.01 Da & 17,1 & $-4,716$ & 3 & 1863,0 & NELFA_MOUSE & & Negative elongation factor A; & $\begin{array}{l}\text { Wolf-Hirschhorn syndrome candidate } 2 \\
\text { homolog; }\end{array}$ \\
\hline KQVKTCKTAQ & ASLKTYQNEVTGLCAQGR & n.d. & $\begin{array}{l}\text { N-ter + 42.01 Da, K +28.03 Da, } \\
\text { C +57.02 Da }\end{array}$ & 18,3 & 0,774 & 3 & 2065,0 & SYNE1_MOUSE & & Nesprin-1; & $\begin{array}{l}\text { Enaptin;Myocyte nuclear envelope protein } \\
\text { 1;Nuclear envelope spectrin repeat protein } \\
\text { 1;Synaptic nuclear envelope protein } 1 \text {; }\end{array}$ \\
\hline DAKSALKSSV & TSTGNQLLR & n.d. & $\mathrm{N}$-ter+42.01 Da & 11,8 & 4,164 & 2 & 1030,6 & SYNE1_MOUSE & & Nesprin-1; & $\begin{array}{l}\text { Enaptin;Myocyte nuclear envelope protein } \\
\text { 1;Nuclear envelope spectrin repeat protein } \\
\text { 1;Synaptic nuclear envelope protein } 1 \text {; }\end{array}$ \\
\hline LHEHLKQEQQ & EVGDKPSAGASECTVAER & n.d. & $\begin{array}{l}\text { N-ter + 42.01 Da, K+34.06 Da, } \\
\mathrm{C}+57.02 \mathrm{Da}\end{array}$ & 17,0 & $-1,803$ & 3 & 1937,9 & SYNE2_MOUSE & E9QP46_MOUSE & Nesprin-2; & $\begin{array}{l}\text { Nuclear envelope spectrin repeat protein } \\
\text { 2;Nucleus and actin connecting element } \\
\text { protein;;Synaptic nuclear envelope protein 2; }\end{array}$ \\
\hline KASVRIAYLR & KNFEQDPQGR & n.d. & $\mathrm{N}$-ter + 42.01 Da, K+28.03 Da & 13,8 & $-3,179$ & 2 & 1287,6 & UNC5D_MOUSE & & Netrin receptor UNC5D; & $\begin{array}{l}\text { Protein unc-5 homolog 4; Protein unc-5 } \\
\text { homolog D; }\end{array}$ \\
\hline CDLAAAGQLG & KAGIMASDCEPALNQAESR & n.d. & $\begin{array}{l}\text { N-ter + 42.01 Da, K+28.03 Da, } \\
\text { C +57.02 Da }\end{array}$ & 18,1 & $-0,142$ & 3 & 2117,0 & NCDN_MOUSE & & Neurochondrin; & M-Sema F-associating protein of $75 \mathrm{kDa}$;Norbin; \\
\hline TRKNNMEITW & TPMNATSAFGPNLR & n.d. & $\mathrm{N}$-ter $+42.01 \mathrm{Da}$ & 15,8 & $-3,093$ & 2 & 1517,7 & NFASC_MOUSE & E9PW06_MOUSE & Neurofascin; & \\
\hline KEQLQALNDR & FAGYIDKVR & n.d. & N-ter + 42.01 Da, K+34.06 Da & 11,9 & $-0,786$ & 2 & 1143,7 & NFH_MOUSE & & Neurofilament heavy polypeptide; & $\begin{array}{l}200 \text { kDa neurofilament protein;Neurofilament } \\
\text { triplet } \mathrm{H} \text { protein; }\end{array}$ \\
\hline IFVTLSIVVT & VFVLNIHYR & n.d. & $\mathrm{N}$-ter $+42.01 \mathrm{Da}$ & 12,5 & 3,489 & 2 & 1201,7 & ACHAG_MOUSE & & Neuronal acetylcholine receptor subunit alpha-6; & \\
\hline EASRQKFRHF & QYEESAGPQKAMSQLR & n.d. & $\mathrm{N}$-ter + 42.01 Da, K+34.06 Da & 14,9 & 8,680 & 3 & 1898,0 & NRIF1_MOUSE & & Neurotrophin receptor-interacting factor 1 ; & $\begin{array}{l}\text { Neurotrophin receptor-interacting factor;zinc } \\
\text { finger protein } 110 \text {; }\end{array}$ \\
\hline SKDKKEVTIV & VLGNKCDLQEQRR & n.d. & $\begin{array}{l}\text { N-ter + 42.01 Da, K+28.03 Da, } \\
\text { C +57.02 Da }\end{array}$ & 17,4 & 1,541 & 2 & 1684,9 & KBRS2_MOUSE & FGXZA4_MOUSE & NF-kappa-B inhibitor-interacting Ras-like protein 2; & 1-kappa-B-interacting Ras-like protein 2; \\
\hline $\begin{array}{l}\text { FEEGLDRCP } \\
\text { KVELLYYESE }\end{array}$ & $\begin{array}{l}\text { APGPHPALVEGR } \\
\text { KLQEENSILR }\end{array}$ & $\begin{array}{l}\text { n.d. } \\
\text { n.d. }\end{array}$ & $\begin{array}{l}\mathrm{N} \text {-ter + 42.01 Da } \\
\mathrm{N} \text {-ter + 42.01 Da, K +28.03 Da }\end{array}$ & $\begin{array}{l}15,6 \\
21,2\end{array}$ & $\begin{array}{r}-3,133 \\
8,226\end{array}$ & $\begin{array}{l}3 \\
2\end{array}$ & $\begin{array}{l}1241,7 \\
1298,7\end{array}$ & $\begin{array}{l}\text { NAB2_MOUSE } \\
\text { NIN_MOUSE }\end{array}$ & $\begin{array}{l}\text { Q3TYF1_MOUSE } \\
\text { E9Q4S3_MOUSE }\end{array}$ & $\begin{array}{l}\text { NGFI-A-binding protein 2; } \\
\text { Ninein; }\end{array}$ & EGR-1-binding protein 2; \\
\hline SYTTHPQMQQ & ASVSSPIVAGGLR & n.d. & $\mathrm{N}$-ter + $42.01 \mathrm{Da}$ & 18,8 & 3,657 & 3 & 1254,7 & NIPBL_MOUSE & & Nipped-B-like protein; & Delangin homolog;SCC2 homolog; \\
\hline APRCVGRIQW & GKLQVFDAR & n.d. & N-ter + 42.01 Da, K+34.06 Da & 13,7 & 4,858 & 3 & 1108,7 & NOS3_MOUSE & E9Q9X4_MOUSE & Nitric oxide synthase, endothelial; & $\begin{array}{l}\text { Constitutive NOS;EC-NOS;Endothelial NOS;NOS } \\
\text { type III; }\end{array}$ \\
\hline M & QSNKAFNLEKONHTPR & 0,1 & $\mathrm{~N}$-ter + 42.01 Da, K+28.03 Da & 28,4 & 0,746 & 4 & 2140,1 & NONO_MOUSE & B1AXTO_MOUSE & $\begin{array}{l}\text { Non-POU domain-containing octamer-binding } \\
\text { protein; }\end{array}$ & \\
\hline $\begin{array}{l}\text { AWLSEGRCVR } \\
\text { DLQIALASFY }\end{array}$ & $\begin{array}{l}\text { EVTEPGALR } \\
\text { EDGGDEDIVTISQATPSSVSR }\end{array}$ & $\begin{array}{l}\text { n.d. } \\
\text { n.d. }\end{array}$ & $\begin{array}{l}\text { N-ter + 42.01 Da } \\
\text { N-ter + 42.01 Da }\end{array}$ & $\begin{array}{l}15,5 \\
14,7\end{array}$ & $\begin{array}{l}1,577 \\
1,087\end{array}$ & $\begin{array}{l}2 \\
4\end{array}$ & $\begin{array}{l}1012,5 \\
2204,0\end{array}$ & $\begin{array}{l}\text { TNK1_MOUSE } \\
\text { NSF1C_MOUSE }\end{array}$ & $\begin{array}{l}\text { Q5F2B4_MOUSE } \\
\text { A2ATO2_MOUSE }\end{array}$ & $\begin{array}{l}\text { Non-receptor tyrosine-protein kinase TNK1; } \\
\text { NSFL1 cofactor p47; }\end{array}$ & $\begin{array}{l}\text { Kinase of embryonic stem cells; } \\
\text { p97 cofactor p47; }\end{array}$ \\
\hline M & ASVDFKTYVDQACR & n.d. & $\begin{array}{l}\text { N-ter + 42.01 Da, K+28.03 Da, } \\
\mathrm{C}+57.02 \mathrm{Da}\end{array}$ & 12,5 & $-5,258$ & 2 & 1728,8 & NXT1_MOUSE & & NTF2-related export protein 1 ; & \\
\hline YMHAPKRGQF & CVFQLVSTENNR & n.d. & $\mathrm{N}$-ter $+42.01 \mathrm{Da}, \mathrm{C}+57.02 \mathrm{Da}$ & 12,0 & $-2,583$ & 2 & 1507,7 & NU160_MOUSE & & Nuclear pore complex protein Nup160; & $\begin{array}{l}160 \text { kDa nucleoporin;Gene trap locus 1-13 } \\
\text { protein;Nucleoporin Nup160; }\end{array}$ \\
\hline YCRFKKCIDV & GMKLEAVR & n.d. & N-ter + 42.01 Da, K+28.03 Da & 10,5 & 7,798 & 2 & 972,6 & NR5A2_MOUSE & Q1WLP7_MOUSE & Nuclear receptor subfamily 5 group A member 2 & Liver receptor homolog 1 ; \\
\hline PLTIDGIPVS & VDKMTQAQLR & n.d. & $\mathrm{N}$-ter + 42.01 Da, K+28.03 Da & 14,3 & $-4,283$ & 2 & 1258,7 & NRF1_MOUSE & Q999k73_MOUSE & Nuclear respiratory factor 1 ; & Alpha palindromic-binding protein; \\
\hline M & ADEGKSYNEHDDR & n.d. & N-ter + 42.01 Da, K+34.06 Da & 17,3 & 3,532 & 3 & 1610,7 & NXF1_MOUSE & & Nuclear RNA export factor 1; & $\begin{array}{l}\text { Tip-associated protein;Tip-associating } \\
\text { proteinimRNA export factor TAP. }\end{array}$ \\
\hline $\begin{array}{l}\text { MATAAS } \\
M\end{array}$ & $\begin{array}{l}\text { GPCAGGSPR } \\
\text { ADPEKQGPAESR }\end{array}$ & $\begin{array}{l}\text { n.d. } \\
\text { n.d. }\end{array}$ & $\begin{array}{l}\text { N-ter + 42.01 Da, C +57.02 Da } \\
\text { N-ter + 42.01 Da, K +34.06 Da }\end{array}$ & $\begin{array}{l}11,7 \\
21,1\end{array}$ & $\begin{array}{c}-3,439 \\
0,514\end{array}$ & $\begin{array}{l}2 \\
2\end{array}$ & $\begin{array}{l}899,4 \\
1359,7\end{array}$ & $\begin{array}{l}\text { NDC1_MOUSE } \\
\text { NP1L__MOUSE }\end{array}$ & J3QMD9_MOUSE & $\begin{array}{l}\text { Nucleoporin NDC1; } \\
\text { Nucleosome assembly protein 1-like 5; }\end{array}$ & Transmembrane protein 48; \\
\hline VGNPTPHVSW & EKDRQPVEAGAR & $-5,1$ & $\mathrm{~N}$-ter + 42.01 Da, K +28.03 Da & 18,0 & 0,420 & 3 & 1424,7 & OBSCN_MOUSE & E9QQ99_MOUSE & Obscurin; & $\begin{array}{l}\text { Obscurin-RhoGEF;Obscurin-myosin light chain } \\
\text { kinase; }\end{array}$ \\
\hline KEQGTASMDV & EISHAEVEGSWTR & n.d. & $\mathrm{N}$-ter + 42.01 Da & 15,5 & 4,923 & 2 & 1541,7 & OBSCN_MOUSE & E9QQ96_MOUSE & Obscurin; & $\begin{array}{l}\text { Obscurin-RhoGEF;Obscurin-myosin light chain } \\
\text { kinase; }\end{array}$ \\
\hline
\end{tabular}




\begin{tabular}{|c|c|c|c|c|c|c|c|c|c|c|c|}
\hline Non Prime Site & Prime Site & 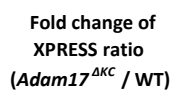 & Modifications & Hyperscore & $\begin{array}{l}\text { Mass error } \\
\text { in ppm }\end{array}$ & Charge & $\begin{array}{l}\text { Precursor } \\
\text { neutral mass } \\
\text { in Da }\end{array}$ & Uniprot ID 1 & Uniprot ID 2 & Protein Name 1 & Protein Name 2 \\
\hline $\begin{array}{l}\text { FLLIYTFGLL } \\
\end{array}$ & GNLSIITAVTR & n.d. & $\mathrm{N}$-ter + 42.01 Da & 14,7 & $-0,421$ & 2 & 1185,7 & Q5NC55_MOUSE & & $\begin{array}{l}\text { Olfactory receptor } \\
\text { \{ECO:0000256|RuleBase:RU000688\}; }\end{array}$ & \\
\hline EIGYTCSVIP & KMLQSLVSEAR & n.d. & N-ter + 42.01 Da, K +28.03 Da & 13,0 & 4,502 & 2 & 1330,7 & Q7TRJ3_MOUSE & Q8VEY1_MOUSE & $\begin{array}{l}\text { Olfactory receptor } \\
\text { \{ECO:0000256|RuleBase:RU000688\}; }\end{array}$ & \\
\hline LQPPLFLMIM & TIYTVTLVGNVGIILVRR & n.d. & $\mathrm{N}$-ter $+42.01 \mathrm{Da}$ & 18,6 & $-2,806$ & 3 & 2028,2 & Q7TR24_MOUSE & Q7TR25_MOUSE & $\begin{array}{l}\text { Olfactory receptor } \\
\{E C O: 0000256 \mid \text { RuleBase:RU000688\}; }\end{array}$ & \\
\hline GLVVLAVTRS & TSIVAPVVVLASR & n.d. & $\mathrm{N}$-ter $+42.01 \mathrm{Da}$ & 20,0 & $-1,106$ & 3 & 1352,8 & Q9WU94_MOUSE & & $\begin{array}{l}\text { Olfactory receptor } \\
\text { \{ECO:0000256|RuleBase:RU000688\}; }\end{array}$ & \\
\hline GFIELSTISG & VLVSYCYIISSVLKIR & n.d. & $\begin{array}{l}\text { N-ter + 42.01 Da, K+28.03 Da, } \\
\mathrm{C}+57.02 \mathrm{Da}\end{array}$ & 22,1 & 5,289 & 3 & 1982,1 & Q7TR42_MOUSE & Q7TR48_MOUSE & $\begin{array}{l}\text { Olfactory receptor } \\
\text { \{ECO:0000256|RuleBase:RU000688\}; }\end{array}$ & \\
\hline GFLALMLLFS & VCVSYVHIGVALR & n.d. & N-ter + 42.01 Da, C +57.02 Da & 11,8 & $-4,052$ & 2 & 1626,9 & OL148_MOUSE & & Olfactory receptor 148 & $\begin{array}{l}\text { Odorant receptor } \mathrm{M} 30 \text {; Olfactory receptor 224- } \\
\text { 4; Olfactory receptor } 7 F \text {; }\end{array}$ \\
\hline DLNLAMDALR & EVILPYSAAHCQGAPGR & n.d. & N-ter + 42.01 Da, C +57.02 Da & 14,9 & 4,813 & 3 & 1866,9 & OLG1_MOUSE & & oligodendrocyte transcription factor 1 ; & $\begin{array}{l}\text { Oligodendrocyte-specific bHLH transcription } \\
\text { factor } 1 \text {; }\end{array}$ \\
\hline M & SKPPPKPVKKGQVKKVFR & n.d. & $\mathrm{N}$-ter + 42.01 Da, K+34.06 Da & 28,4 & 4,393 & 5 & 2066,4 & OSTF1_MOUSE & & Osteoclast-stimulating factor 1 ; & SH3 domain protein 3; \\
\hline DHFFTFDGR & RYTFPATCQYILAKSR & n.d. & $\begin{array}{l}\text { N-ter + 42.01 Da, K+34.06 Da, } \\
\mathrm{C}+57.02 \mathrm{Da}\end{array}$ & 22,8 & 5,793 & 4 & 2050,1 & OTOG_MOUSE & G3UWC8_MOUSE & E Otogelin; & \\
\hline M & ADKEALPKLR & n.d. & N-ter + 42.01 Da, K+34.06 Da & 14,8 & 3,752 & 3 & 1249,8 & HTA12_MOUSE & & Oxidoreductase HTATIP2; & \\
\hline YHSVISPPES & RGAELSGSPELGGR & n.d. & $\mathrm{N}$-ter $+42.01 \mathrm{Da}$ & 17,0 & 6,085 & 3 & 1426,7 & A2A716_MOUSE & & $\begin{array}{l}\text { Oxysterol-binding protein } \\
\text { \{ECO:0000256|RuleBase:RU003845\}; }\end{array}$ & \\
\hline MKWKNKFWGK & SLEIVPVGTVNVSLPR & n.d. & $\mathrm{N}$-ter $+42.01 \mathrm{Da}$ & 18,2 & $-1,218$ & 3 & 1721,0 & A2A716_MOUSE & F8VQM4_MOUSE & $\begin{array}{l}\text { Oxysterol-binding protein } \\
\text { \{ECO:0000256|RuleBase:RU003845\}: }\end{array}$ & \\
\hline $\begin{array}{l}M \\
M\end{array}$ & $\begin{array}{l}\text { NTEAEQQLLHHAR } \\
\text { EEAELVKGR }\end{array}$ & $\begin{array}{l}\text { n.d. } \\
0,1\end{array}$ & $\begin{array}{l}\text { N-ter + 42.01 Da } \\
\text { N-ter +42.01 Da, } \mathrm{K}+28.03 \mathrm{Da}\end{array}$ & $\begin{array}{l}17,4 \\
47,2\end{array}$ & $\begin{array}{l}-0,581 \\
-4,543\end{array}$ & $\begin{array}{l}3 \\
2\end{array}$ & $\begin{array}{l}1718,8 \\
1230,6\end{array}$ & $\begin{array}{l}\text { OSBL1_MOUSE } \\
\text { PALMD_MOUSE }\end{array}$ & $\begin{array}{l}\text { Q8K2D2_MOUSE } \\
\text { Q3UVT7MOUSE }\end{array}$ & $\begin{array}{l}\text { Oxysterol-binding protein-related protein } 1 \text {; } \\
\text { Palmdel phin: }\end{array}$ & \\
\hline GAFDGLHTVE & GQYYLQICILLKCKTTNLR & n.d. & $\begin{array}{l}\text { N-ter + 42.01 Da, K +34.06 Da, } \\
\mathrm{C}+57.02 \mathrm{Da}\end{array}$ & 14,5 & 1,161 & 4 & 2494,4 & VNN1_MOUSE & & Pantetheinase; & $\begin{array}{l}\text { Pantetheine hydrolase;Vascular non- } \\
\text { inflammatory molecule } 1 ;\end{array}$ \\
\hline EDPLPHTSRP & SLEPSLR & n.d. & $\mathrm{N}$-ter + 42.01 Da & 14,3 & 0,118 & 2 & 842,5 & PTHR_MOUSE & Q924X4_MOUSE & Parathyroid hormone-related protein; & Parathyroid hormone-like protein; \\
\hline FQVYRAPFGD & FTKKLTACMSTVGLLQAASPSR & n.d. & $\begin{array}{l}\text { N-ter + 42.01 Da, K+28.03 Da, } \\
\mathrm{C}+57.02 \mathrm{Da}\end{array}$ & 17,5 & 3,891 & 3 & 2464,3 & PTHD2_MOUSE & E9QL24_MOUSE & Patched domain-containing protein 2; & RND-type protein RNDEu-2; \\
\hline SRYGRNSISS & YKQNLENLFGR & n.d. & $\mathrm{N}$-ter + $42.01 \mathrm{Da}, \mathrm{K}+34.06 \mathrm{Da}$ & 15,1 & $-1,645$ & 2 & 1456,8 & PED1B_MOUSE & & PC-esterase domain-containing protein 1B; & $\begin{array}{l}\text { Protein FAM113B; } \\
\text { START domain-containing protein }\end{array}$ \\
\hline M & EKPAASTEPQGSRPALGR & n.d. & $\mathrm{N}$-ter + $42.01 \mathrm{Da}, \mathrm{K}+34.06 \mathrm{Da}$ & 27,7 & 0,146 & 3 & 2058,1 & PCTL_MOUSE & E9PVPO_MOUSE & PCTP-like protein; & $\begin{array}{l}\text { 10;Serologically defined colon cancer antigen } \\
28 \text { homolog;StAR-related lipid transfer protein } \\
10 \text {; }\end{array}$ \\
\hline M & THSVTLR & n.d. & $\mathrm{N}$-ter + $42.01 \mathrm{Da}$ & 15,0 & 0,467 & 2 & 854,5 & PDLI4_MOUSE & Q.5SWV3_MOUSE & PDZ and LIM domain protein 4; & LIM protein RLL;Reversion-induced LIM protein; \\
\hline $\begin{array}{l}\text { VARRTQSETP } \\
\text { EGVEEDEGCC }\end{array}$ & $\begin{array}{l}\text { RHVSQDLEDSSR } \\
\text { CCEPGHLPR }\end{array}$ & $\begin{array}{l}\text { n.d. } \\
\text { n.d. }\end{array}$ & $\begin{array}{l}\text { N-ter + 42.01 Da } \\
\text { N-ter + 42.01 Da, C+57.02 Da }\end{array}$ & $\begin{array}{l}18,0 \\
12,6\end{array}$ & $\begin{array}{l}-0,612 \\
1,626\end{array}$ & $\begin{array}{l}2 \\
2\end{array}$ & $\begin{array}{l}1469,7 \\
1166,5\end{array}$ & $\begin{array}{l}\text { PCX2_MOUSE } \\
\text { PCX3_MOUSE }\end{array}$ & FGRYD8_MOUSE & $\begin{array}{l}\text { Pecanex-like protein 2; } \\
\text { Pecanex-like protein 3; }\end{array}$ & \\
\hline ITADDEPLGR & VSFELFADKVPKTAENFR & 0,9 & $\mathrm{~N}$-ter + $42.01 \mathrm{Da}, \mathrm{K}+28.03 \mathrm{Da}$ & 38,4 & 2,320 & 3 & 2195,2 & PPIA_MOUSE & & Peptidyl-prolyl cis-trans isomerase A; & $\begin{array}{l}\text { Cyclophilin A;Cyclosporin A-binding } \\
\text { protein;Rotamase A;SP18; }\end{array}$ \\
\hline M & ADEEKLPPGWEKR & n.d. & $\mathrm{N}$-ter + 42.01 Da, K+34.06 Da & 53,8 & 0,300 & 3 & 1663,9 & PIN1_MOUSE & & $\begin{array}{l}\text { Peptidyl-prolyl cis-trans isomerase NIMA-interacting } \\
1 ;\end{array}$ & Peptidyl-prolyl cis-trans isomerase Pin1; \\
\hline PVTNIRNPQV & ASTWNEVNTNSNTQCGSNNR & n.d. & N-ter + 42.01 Da, C +57.02 Da & 15,2 & 7,789 & 3 & 2295,0 & PCM1_MOUSE & & Pericentriolar material 1 protein; & \\
\hline STYDLVSSAY & VSTKDQYPYLR & n.d. & $\mathrm{N}$-ter + $42.01 \mathrm{Da}, \mathrm{K}+28.03 \mathrm{Da}$ & 13,8 & $-0,208$ & 2 & 1438,8 & PLIN2_MOUSE & B1AXR5_MOUSE & Perilipin-2; & $\begin{array}{l}\text { Adipophilin;Adipose differentiation-related } \\
\text { protein; }\end{array}$ \\
\hline PLteAeLALI & ATPPEDSDMASLQQQR & n.d. & $\mathrm{N}$-ter + 42.01 Da & 17,3 & 9,627 & 3 & 1814,8 & PLIN3_MOUSE & & Perilipin-3; & $\begin{array}{l}\text { Cargo selection protein TIP47; Mannose-6- } \\
\text { phosphate receptor-binding protein 1; }\end{array}$ \\
\hline M & ASGNAQIGKSAPDFTATAVVDGAFKEIKLSDYR & 0,6 & N-ter + 42.01 Da, K+28.03 Da & 38,9 & 0,450 & 4 & 3552,9 & PRDX2_MOUSE & D3Z4A4_MOUSE & Peroxiredoxin-2; & $\begin{array}{l}\text { Thiol-specific antioxidant protein;Thioredoxin } \\
\text { peroxidase 1;Thioredoxin-dependent peroxide } \\
\text { reductase 1; }\end{array}$ \\
\hline M & ADGKAGEEKPEKPQR & n.d. & N-ter + 42.01 Da, K+34.06 Da & 27,0 & $-0,616$ & 4 & 1783,0 & PCNP_MOUSE & Q3TM89_MOUSE & PEST proteolytic signal-containing nuclear protein; & \\
\hline LAVKIKVGSD & DLERIEARLATIEGDDPALR & n.d. & $\mathrm{N}$-ter + 42.01 Da & 20,1 & 5,006 & 3 & 2294,2 & PSD2_MOUSE & & PH and SEC7 domain-containing protein 2; & $\begin{array}{l}\text { Pleckstrin homology and SEC7 domain- } \\
\text { containing protein } 2 \text {; }\end{array}$ \\
\hline RRSRFGSRER & DWLREDVKR & n.d. & $\mathrm{N}$-ter + $42.01 \mathrm{Da}, \mathrm{K}+28.03 \mathrm{Da}$ & 19,1 & 7,605 & 3 & 1285,7 & PHLP2_MOUSE & J3QM82_MOUSE & $\begin{array}{l}\text { PH domain leucine-rich repeat-containing protein } \\
\text { phosphatase } 2 \text {; }\end{array}$ & $\begin{array}{l}\text { PH domain leucine-rich repeat-containing } \\
\text { protein phosphatase-like; } \\
49 \text { kDa cytoskeletal protein;:Beaded filament }\end{array}$ \\
\hline QELETQLRAH & LESKAKSSGGWDALR & n.d. & $\mathrm{N}$-ter + 42.01 Da, K+28.03 Da & 13,8 & 0,000 & 2 & 1701,9 & BFSP2_MOUSE & & Phakinin; & $\begin{array}{l}\text { structural protein 2; Lens fiber cell beaded } \\
\text { filament protein CP 49; }\end{array}$ \\
\hline GLYYLGKIKR & VSSPKQSCLVTFEDNSK & n.d. & $\begin{array}{l}\text { N-ter + 42.01 Da, K+34.06 Da, } \\
\mathrm{C}+57.02 \mathrm{Da}\end{array}$ & 15,5 & 0,491 & 3 & 2035,1 & PHF19_MOUSE & & PHD finger protein 19; & Polycomb-like protein 3; \\
\hline
\end{tabular}




\begin{tabular}{|c|c|c|c|c|c|c|c|c|c|c|c|}
\hline Non Prime Site & Prime Site & $\begin{array}{l}\text { Fold change of } \\
\text { XPRESS ratio } \\
\text { (Adam17 7אC / WT) }\end{array}$ & Modifications & Hyperscore & $\begin{array}{l}\text { Mass error } \\
\text { in ppm }\end{array}$ & Charge & $\begin{array}{l}\text { Precursor } \\
\text { neutral mass } \\
\text { in Da }\end{array}$ & Uniprot ID 1 & Uniprot ID 2 & Protein Name 1 & Protein Name 2 \\
\hline$\overline{\text { MLEAM }}$ & $\begin{array}{l}\text { AEPSPEDPPPTLKPETQPPEKR } \\
\end{array}$ & n.d. & N-ter + 42.01 Da, K +34.06 Da & 13,0 & 4,897 & 3 & 2549,4 & PHF23_MOUSE & & PHD finger protein 23; & $\begin{array}{l}\text { PDH-containing protein JUNE-1; } \\
\end{array}$ \\
\hline M & AKHHPDLIFCR & n.d. & $\begin{array}{l}\text { N-ter + 42.01 Da, K+34.06 Da, } \\
\text { C +57.02 Da }\end{array}$ & 19,0 & 1,495 & 3 & 1468,8 & PHF5A_MOUSE & & PHD finger-like domain-containing protein $5 \mathrm{~A}$; & Splicing factor 3B-associated 14 kDa protein; \\
\hline QGGYGSQGYK & YTWKLEEAR & n.d. & N-ter + 42.01 Da, K+34.06 Da & 14,7 & 6,286 & 2 & 1270,7 & SYFA_MOUSE & E9PWY9_MOUSE & Phenylalanine--tRNA ligase alpha subunit; & Phenylalanyl-tRNA synthetase alpha subunit; \\
\hline QPVDLLEYPD & YRDIIDTPMDFATVR & n.d. & $\mathrm{N}$-ter $+42.01 \mathrm{Da}$ & 30,5 & $-6,843$ & 2 & 1853,9 & PHIP_MOUSE & F8VQ93_MOUSE & PH-interacting protein; & $\begin{array}{l}\text { IRS-1 PH domain-binding protein;Neuronal } \\
\text { differentiation-related protein;WD repeat- } \\
\text { containing protein } 11 \text {; }\end{array}$ \\
\hline M & AADISQWAGPLCLQEVDEPPQHALR & n.d. & $\mathrm{N}$-ter $+42.01 \mathrm{Da}, \mathrm{C}+57.02 \mathrm{Da}$ & 58,6 & $-0,035$ & 3 & 2842,4 & PEBP1_MOUSE & D3Z1V4_MOUSE & Phosphatidylethanolamine-binding protein 1 ; & HCNPpp; \\
\hline M & TAIIKEIVSR & n.d. & $\mathrm{N}$-ter + 42.01 Da, K +34.06 Da & 28,2 & $-2,983$ & 2 & 1204,8 & PTEN_MOUSE & & $\begin{array}{l}\text { Phosphatidylinositol 3,4,5-rrisphosphate 3- } \\
\text { phosphatase and dual-specificity protein phosphatase } \\
\text { PTEN; }\end{array}$ & $\begin{array}{l}\text { Mutated in multiple advanced cancers } \\
\text { 1; Phosphatase and tensin homolog; }\end{array}$ \\
\hline M & DLEAVCKR & n.d. & $\begin{array}{l}\text { N-ter }+42.01 \mathrm{Da}, \mathrm{K}+34.06 \mathrm{Da}, \\
\mathrm{C}+57.02 \mathrm{Da}\end{array}$ & 34,9 & 1,335 & 2 & 1196,6 & AGM1_MOUSE & Q8BWW3_MOUSE & E Phosphoacety|glucosamine mutase \{ $\{C \mathrm{CO}: 0000305\}$ & 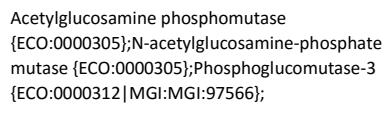 \\
\hline M & SLSNKLTLDKLDVKGKR & n.d. & N-ter + 42.01 Da, K+28.03 Da & 12,9 & $-5,694$ & 4 & 2068,3 & PGK1_MOUSE & S4R2M7_MOUSE & Phosphoglycerate kinase $1 ;$ & \\
\hline M & AAYKLVLIR & 0,0 & N-ter + 42.01 Da, K+28.03 Da & 37,4 & $-0,805$ & 2 & 1115,7 & PGAM1_MOUSE & & Phosphoglycerate mutase 1; & $\begin{array}{l}\text { BPG-dependent PGAM 1;Phosphoglycerate } \\
\text { mutase isozyme B; }\end{array}$ \\
\hline M & EPKAPCPAAVPSEER & n.d. & $\begin{array}{l}\text { N-ter + 42.01 Da, } \mathrm{K}+34.06 \mathrm{Da}, \\
\mathrm{C}+57.02 \mathrm{Da}\end{array}$ & 21,7 & 1,841 & 3 & 1843,9 & COAC_MOUSE & & Phosphopantothenoylcysteine decarboxylase; & Coac; \\
\hline M & SSISGKVQTVLGLVEPSQLGR & n.d. & $\mathrm{N}$-ter + 42.01 Da, K+34.06 Da & 14,6 & $-3,000$ & 3 & 2230,3 & PTER_MOUSE & A2AUR3_MOUSE & Phosphotriesterase-related protein; & Parathion hydrolase-related protein; \\
\hline M & ELLSTPHSIEINNITCDSFR & n.d. & $\mathrm{N}$-ter + 42.01 Da, C +57.02 Da & 22,2 & $-2,142$ & 3 & 2518,2 & PHYIP_MOUSE & EOCXJ1_MOUSE & Phytanoyl-CoA hydroxylase-interacting protein; & $\begin{array}{l}\text { Phytanoyl-CoA hydroxylase-associated protein } \\
\text { 1; }\end{array}$ \\
\hline MSYYFLHVVA & DIKASQILASR & n.d. & $\mathrm{N}$-ter + 42.01 Da, K +34.06 Da & 16,9 & $-6,642$ & 3 & 1276,8 & PIEZ2_MOUSE & S4R2SO_MOUSE & $\begin{array}{l}\text { Piezo-type mechanosensitive ion channel component } \\
2 ;\end{array}$ & Protein FAM38B; \\
\hline M & $\begin{array}{l}\text { ASSKKVTLSVLSR } \\
\text { NHSPKTSAYYFCEO }\end{array}$ & n.d. & $\mathrm{N}$-ter + 42.01 Da, K+34.06 Da & 38,6 & 0,134 & 3 & 1485,0 & PIR_MOUSE & A2AIH8_MOUSE & Pirin; & Probable quercetin 2,3-dioxygenase PIR; \\
\hline M & $\begin{array}{l}\text { NHSPLKTALAYECFQDQDNSTLALPSDQKMKTGTSG } \\
\mathrm{R}\end{array}$ & n.d. & $\begin{array}{l}\text { N-ter }+42.01 \text { Da, K+34.06 Da, } \\
\text { C+57.02 Da }\end{array}$ & 22,5 & 3,395 & 5 & 4384,2 & PKP1_MOUSE & & Plakophilin-1; & \\
\hline QLEACGKARE & RQQQEQQVTEENLR & n.d. & $\mathrm{N}$-ter $+42.01 \mathrm{Da}$ & 20,0 & $-0,765$ & 3 & 1826,9 & PLVAP_MOUSE & G3X924_MOUSE & Plasmalemma vesicle-associated protein; & $\begin{array}{l}\text { MECA-32 antigen;Plasmalemma vesicle protein } \\
1 ;\end{array}$ \\
\hline PFIIQENLNL & ALNSASAIGCHVVNIGAEDLR & n.d. & N-ter + 42.01 Da, C +57.02 Da & 17,3 & $-2,351$ & 4 & 2208,1 & PLST_MOUSE & B1AX58_MOUSE & Plastin-3; & T-plastin; \\
\hline M & SQGDSNPAAIPHAAEDIQGDDR & n.d. & $\mathrm{N}$-ter + $42.01 \mathrm{Da}$ & 63,8 & 0,737 & 2 & 2305,0 & PA1B2_MOUSE & & $\begin{array}{l}\text { Platelet-activating factor acetylhydrolase IB subunit } \\
\text { beta; }\end{array}$ & $\begin{array}{l}\text { AAF acetylhydrolase } 30 \text { kDa subunit;PAF-AH } \\
\text { subunit beta; }\end{array}$ \\
\hline M & SGEGENPASKPTPVQDVQGDGR & 0,1 & $\mathrm{~N}$-ter + 42.01 Da, K+28.03 Da & 48,8 & 1,001 & 3 & 2294,1 & PA1B3_MOUSE & Q8CA83_MOUSE & $\begin{array}{l}\text { Platelet-activating factor acetylhydrolase IB subunit } \\
\text { gamma; }\end{array}$ & $\begin{array}{l}\text { PAF acetylydrolase } 29 \text { kDa subunit;PAF-AH } \\
\text { subunit gamma; }\end{array}$ \\
\hline $\begin{array}{l}\text { M } \\
\text { EEENQPVLVL }\end{array}$ & $\begin{array}{l}\text { VEEVQKHSVHTLVFR } \\
\text { SSEEPEPGPATVDCPR }\end{array}$ & $\begin{array}{l}0,1 \\
\text { n.d. }\end{array}$ & $\begin{array}{l}\text { N-ter + 42.01 Da, } \mathrm{K}+28.03 \mathrm{Da} \\
\text { N-ter + 42.01 Da, C+57.02 Da }\end{array}$ & $\begin{array}{l}21,1 \\
14,8\end{array}$ & $\begin{array}{l}0,744 \\
4,290\end{array}$ & $\begin{array}{l}4 \\
3\end{array}$ & $\begin{array}{l}1877,0 \\
1768,8\end{array}$ & $\begin{array}{l}\text { PLRG1_MOUSE } \\
\text { PODN_MOUSE }\end{array}$ & D3Z4V1_MOUSE & $\begin{array}{l}\text { Pleiotropic regulator 1; } \\
\text { Podocan; }\end{array}$ & \\
\hline TGAGRLPCHL & IIHAVGPR & n.d. & $\mathrm{N}$-ter + 42.01 Da & 14,1 & $-4,417$ & 2 & 903,5 & PARP9_MOUSE & & Poly [ADP-ribose] polymerase 9; & $\begin{array}{l}\text { ADP-ribosyltransferase diphtheria toxin-like 9;B } \\
\text { aggressive lymphoma protein homolog; }\end{array}$ \\
\hline FQKYRHYIVL & TASASTEENHLEWVGLVESKIR & n.d. & $\mathrm{N}$-ter $+42.01 \mathrm{Da}, \mathrm{K}+34.06 \mathrm{Da}$ & 14,7 & 3,867 & 3 & 2531,3 & PAPOG_MOUSE & Q8BY05_MOUSE & Poly(A) polymerase gamma; & $\begin{array}{l}\text { Polynucleotide adenylyltransferase gamma;SRP } \\
\text { RNA 3'-adenylating enzyme; } \\
\text { Nuclear poly(A)-binding protein 1;Poly(A)- }\end{array}$ \\
\hline MAA & AAAAAAAAGAAGGRGSGPGRR & n.d. & $\mathrm{N}$-ter + 42.01 Da & 34,8 & $-7,071$ & 3 & 1764,9 & PABP2_MOUSE & G3UY42_MOUSE & Polyadenylate-binding protein 2 & $\begin{array}{l}\text { binding protein II;Polyadenylate-binding } \\
\text { nuclear protein 1; }\end{array}$ \\
\hline $\begin{array}{l}\text { HVQADHELFL } \\
\text { M }\end{array}$ & $\begin{array}{l}\text { QAFEKPTQIYR } \\
\text { EDVTLHIVERPYSGFPDASSEGPEPTQGEAR }\end{array}$ & $\begin{array}{l}\text { n.d. } \\
\text { n.d. }\end{array}$ & $\begin{array}{l}\text { N-ter + 42.01 Da, K+34.06 Da } \\
\text { N-ter + 42.01 Da }\end{array}$ & $\begin{array}{l}16,9 \\
20,6\end{array}$ & $\begin{array}{l}-3,153 \\
-4,455\end{array}$ & $\begin{array}{l}3 \\
4\end{array}$ & $\begin{array}{l}1455,8 \\
3542,6\end{array}$ & $\begin{array}{l}\text { SUZ12_MOUSE } \\
\text { PTRF_MOUSE }\end{array}$ & E9PW15_MOUSE & $\begin{array}{l}\text { Polycomb protein Suz12; } \\
\text { Polymerase I and transcript release factor; }\end{array}$ & $\begin{array}{l}\text { Suppressor of zeste } 12 \text { protein homolog; } \\
\text { Cav-p60;Cavin-1; }\end{array}$ \\
\hline M & DGIVPDIAVGTKR & 0,0 & $\mathrm{~N}$-ter + 42.01 Da, K+28.03 Da & 45,1 & $-0,583$ & 2 & 1540,8 & PTBP1_MOUSE & Q92217_MOUSE & Polypyrimidine tract-binding protein 1 & Heterogeneous nuclear ribonucleoprotein I; \\
\hline M & DGIVTEVAVGVKR & n.d. & $\mathrm{N}$-ter + 42.01 Da, K +34.06 Da & 36,2 & 5,610 & 2 & 1548,9 & PTBP2_MOUSE & & Polypyrimidine tract-binding protein 2; & $\begin{array}{l}\text { Brain-enriched polypyrimidine tract-binding } \\
\text { protein;Neural polypyrimidine tract-binding } \\
\text { protein;RRM-type RNA-binding protein brPTB; }\end{array}$ \\
\hline NMEDCEDTRE & AKGPWGTR & n.d. & N-ter + 42.01 Da, K+28.03 Da & 10,4 & $-3,498$ & 2 & 941,5 & KCNT1_MOUSE & COKTP6_MOUSE & Potassium channel subfamily T member 1 & \\
\hline REEEEEPLDS & EDQESEGPSASEGR & n.d. & $\mathrm{N}$-ter $+42.01 \mathrm{Da}$ & 13,3 & 1,447 & 2 & 1518,6 & KCNG1_MOUSE & & $\begin{array}{l}\text { Potassium voltage-gated channel subfamily G } \\
\text { member 1; }\end{array}$ & $\begin{array}{l}\text { Voltage-gated potassium channel subunit } \\
\text { Kv6.1; }\end{array}$ \\
\hline VLKGGTVLAI & LGKGDLIGCELPQR & n.d. & $\begin{array}{l}\mathrm{N} \text {-ter + 42.01 Da, K+28.03 Da, } \\
\mathrm{C}+57.02 \mathrm{Da}\end{array}$ & 12,9 & 2,643 & 2 & 1624,9 & KCNH3_MOUSE & B2RU85_MOUSE & $\begin{array}{l}\text { Potassium voltage-gated channel subfamily } \mathrm{H} \\
\text { member } 3 \text {; }\end{array}$ & $\begin{array}{l}\text { Ether-a-go-go-like potassium channel 2;Voltage- } \\
\text { gated potassium channel subunit Kv12.2; }\end{array}$ \\
\hline
\end{tabular}




\begin{tabular}{|c|c|c|c|c|c|c|c|c|c|c|c|}
\hline Non Prime Site & Prime Site & $\begin{array}{l}\text { Fold change of } \\
\text { XPRESS ratio } \\
\left.\text { (Adam17 }^{\Delta K C} / \mathrm{WT}\right)\end{array}$ & Modifications & Hyperscore & $\begin{array}{l}\text { Mass error } \\
\text { in ppm }\end{array}$ & Charge & $\begin{array}{l}\text { Precursor } \\
\text { neutral mass } \\
\text { in Da }\end{array}$ & Uniprot ID 1 & Uniprot ID 2 & Protein Name 1 & Protein Name 2 \\
\hline SSSQLTHSRS & RESLCSIR & n.d. & $\mathrm{N}$-ter $+42.01 \mathrm{Da}, \mathrm{C}+57.02 \mathrm{Da}$ & 11,4 & $-1,410$ & 2 & 1061,5 & KCNH7_MOUSE & Q8C782_MOUSE & $\begin{array}{l}\text { Potassium voltage-gated channel subfamily } \mathrm{H} \\
\text { member 7; }\end{array}$ & $\begin{array}{l}\text { Ether-a-go-go-related gene potassium channel } \\
\text { 3;Voltage-gated potassium channel subunit } \\
\text { Kv11.3; }\end{array}$ \\
\hline QQVGAKAWIM & DEEEDGEEEGAGGR & n.d. & $\mathrm{N}$-ter + 42.01 Da & 11,4 & $-1,445$ & 3 & 1519,6 & HCN4_MOUSE & B2RY58_MOUSE & $\begin{array}{l}\text { Potassium/sodium hyperpolarization-activated cyclic } \\
\text { nucleotide-gated channel } 4 \text {; }\end{array}$ & Brain cyclic nucleotide-gated channel 3; \\
\hline M & AAAKDGCGLETAAGNGR & $-0,3$ & $\begin{array}{l}\text { N-ter + 42.01 Da, K+28.03 Da, } \\
\mathrm{C}+57.02 \mathrm{Da}\end{array}$ & 72,2 & $-0,178$ & 2 & 1687,8 & PFD3_MOUSE & Q3TIR6_MOUSE & Prefoldin subunit 3; & Von Hippel-Lindau-binding protein 1; \\
\hline M & AATMKKAAAEDVNVTFEDQQKINKFAR & n.d. & $\mathrm{N}$-ter + 42.01 Da, $\mathrm{K}+34.06 \mathrm{Da}$ & 28,2 & 0,094 & 5 & 3201,8 & Q3UWL8_MOUSE & E E9Q6U4_MOUSE & $\begin{array}{l}\text { Prefoldin subunit } 4 \\
\text { \{ECO:0000256|PIRNR:PIRNR016477\}; }\end{array}$ & \\
\hline ETPSQRRATR & SGAQASSTPLSPTR & n.d. & $\mathrm{N}$-ter $+42.01 \mathrm{Da}$ & 21,0 & $-2,709$ & 2 & 1400,7 & LMNA_MOUSE & D3YUF7_MOUSE & Prelamin-A/C; & Nuclear matrix protein 200 \\
\hline M & SLICSISNEVPEHPCVSPVSNHVYER & n.d. & $\mathrm{N}$-ter $+42.01 \mathrm{Da}, \mathrm{C}+57.02 \mathrm{Da}$ & 44,6 & $-3,863$ & 4 & 3050,4 & PRP19_MOUSE & & Pre-mRNA-processing factor $19\{$ \{ECO:0000305\}; & $\begin{array}{l}\{\text { \{ECO:0000303|Ref.2\};PRP 19/PSO4 } \\
\text { homolog;Senescence evasion factor } \\
\text { \{ECO:O0000303|Ref.2\}\}; }\end{array}$ \\
\hline GIKKEKTGWD & TSESELSEGELERR & n.d. & $\mathrm{N}$-ter + 42.01 Da & 17,0 & $-6,904$ & 3 & 1662,8 & PR40B_MOUSE & F6XW22_MOUSE & Pre-mRNA-processing factor 40 homolog B; & $\begin{array}{l}\text { Huntingtin yeast partner } \mathrm{C} ; \text { Huntingtin- } \\
\text { interacting protein } ; \text {; }\end{array}$ \\
\hline M & DILKSELLR & n.d. & N-ter + 42.01 Da, K +28.03 Da & 15,5 & $-1,397$ & 2 & 1286,7 & PRP18_MOUSE & & Pre-mRNA-splicing factor 18; & $\begin{array}{l}\text { PRP18 homolog; } \\
\text { P }\end{array}$ \\
\hline M & TNEEPLPKKVR & n.d. & $\mathrm{N}$-ter + 42.01 Da, K +34.06 Da & 15,7 & $-4,990$ & 3 & 1419,9 & FL2D_MOUSE & EOCYHO_MOUSE & Pre-mRNA-splicing regulator WTAP; & $\begin{array}{l}\text { Female-lethal(2)D homolog;WT1-associated } \\
\text { protein;WWilms tumor 1-associating protein; }\end{array}$ \\
\hline GQLIYTPFTE & DTPSVGQR & n.d. & $\mathrm{N}$-ter + 42.01 Da & 12,7 & $-0,222$ & 2 & 900,4 & PSN2_MOUSE & Q3U4P5_MOUSE & Presenilin-2; & \\
\hline $\begin{array}{l}\text { DNYSEFRLVG } \\
\text { GSMEGLEVIT }\end{array}$ & ILMGLAVYNSITLDIR & n.d. & N-ter + 42.01 Da & 15,2 & 0,545 & 3 & 1833,0 & HECD2_MOUSE & B2RPZ5_MOUSE & Probable E3 ubiquitin-protein ligase HECTD2; & HECT domain-containing protein 2; \\
\hline $\begin{array}{l}\text { GSMEGLEVIT } \\
\text { THESEDNAMT }\end{array}$ & $\begin{array}{l}\text { EFOASSALADTGR } \\
\text { LGLDVTDVTMGHISNLLOSR }\end{array}$ & 色.d. & $\begin{array}{l}\mathrm{N} \text {-ter + 42.01 Da, K+34.06 Da } \\
\text { N-ter + 42.01 Da }\end{array}$ & $\begin{array}{l}15,0 \\
11,8\end{array}$ & $\begin{array}{l}3,320 \\
-0,304\end{array}$ & $\begin{array}{l}3 \\
3\end{array}$ & $\begin{array}{ll}1442,7 \\
22972\end{array}$ & $\begin{array}{l}\text { GP101_MOOSE } \\
\text { GP133 MOUSE }\end{array}$ & & $\begin{array}{l}\text { Probable G-protein coupled receptor 101; } \\
\text { Probable G-protein conpled receptor } 333 \text {. }\end{array}$ & \\
\hline $\begin{array}{l}\text { THESEDNAMI } \\
\text { DKTEVCPWEI }\end{array}$ & $\begin{array}{l}\text { LGLVDTIDTVMGHISSNLQSR } \\
\text { HSQSLLEDENR }\end{array}$ & $\begin{array}{l}\text { n.e. } \\
\text { n.d. }\end{array}$ & $\begin{array}{l}\text { N-ter + 42.01 Da } \\
\text { N-ter + 42.01 Da }\end{array}$ & 15,3 & 2,554 & 2 & 1368,6 & $\begin{array}{l}\text { GP133_MNOSE } \\
\text { GP158_MOUSE }\end{array}$ & & $\begin{array}{l}\text { Probable G-protein coupled receptor 133; } \\
\text { Probable G-protein coupled receptor 158; }\end{array}$ & G-protein coupled receppor PGR25; \\
\hline KKARKKKTIS & LATHETTDMSOSSGGR & n.d. & $\mathrm{N}$-ter $+42.01 \mathrm{Da}$ & 19,5 & $-9,816$ & 3 & 1718,7 & GPR22_MOUSE & G3X9C3_MOUSE & Probable G-protein coupled receptor 22; & \\
\hline IVPGANYGDS & YAIFEMGSKEIIGDLAAHR & n.d. & N-ter + 42.01 Da, K+28.03 Da & 15,1 & $-5,312$ & 3 & $2162,1 \quad 1$ & IDHG2_MOUSE & & $\begin{array}{l}\text { Probable isocitrate dehydrogenase [NAD] gamma 2, } \\
\text { mitochondrial; }\end{array}$ & $\begin{array}{l}\text { Isocitric dehydrogenase subunit gamma } \\
\text { 2:NAD(+)-specific ICDH subunit gamma 2; }\end{array}$ \\
\hline VVVSLVMVAL & QHFAGRWYLQIIR & n.d. & $\mathrm{N}$-ter $+42.01 \mathrm{Da}$ & 21,2 & 9,527 & 3 & 1728,9 & ATP9A_MOUSE & Q8C288_MOUSE & Probable phospholipid-transporting ATPase IIA; & ATPase class II type 9A; \\
\hline ALAICNTVVV & SAPNQPR & n.d. & N-ter $+42.01 \mathrm{Da}$ & 13,8 & 8,124 & 2 & 810,4 & AT10D_MOUSE & & Probable phospholipid-transporting ATPase VD; & $\begin{array}{l}\text { ATPase class V type 10D;P4-ATPase flippase } \\
\text { complex alpha subunit ATP10D; }\end{array}$ \\
\hline M & AQNLKDLAGR & 0,0 & $\mathrm{~N}$-ter + 42.01 Da, K+28.03 Da & 40,9 & 0,087 & 2 & 1154,6 & PHB2_MOUSE & & Prohibitin-2; & $\begin{array}{l}\text { B-cell receptor-associated protein } \\
\text { BAP37;Repressor of estrogen receptor activity; }\end{array}$ \\
\hline M & SGEDEQQEQTIAEDLVVTKYKMGGDIANR & $-0,2$ & $\mathrm{~N}$-ter + 42.01 Da, K+28.03 Da & 45,4 & 0,150 & 3 & 3321,6 & PA2G4_MOUSE & D3YVH7_MOUSE & Proliferation-associated protein 2G4; & $\begin{array}{l}\text { IRES-specific celllular trans-acting factor } 45 \\
\text { KDa;Mpp1; ;roliferation-associated protein } \\
\text { 1;Protein } \mathrm{P38}-2 \mathrm{2} 4 ;\end{array}$ \\
\hline PATANHLGLS & VPGLVSVPPR & n.d. & N-ter $+42.01 \mathrm{Da}$ & 17,8 & $-5,166$ & 3 & 1061,6 & PELP1_MOUSE & & Proline-, glutamic acid-and leucine-rich protein 1 ; & $\begin{array}{l}\text { Modulator of non-genomic activity of estrogen } \\
\text { receptor; }\end{array}$ \\
\hline $\begin{array}{l}\text { MSIRLARM } \\
\text { QALREYFNED }\end{array}$ & $\begin{array}{l}\text { ASGRPEELWEAVVGAAER } \\
\text { MECRALCEGPQR }\end{array}$ & $\begin{array}{l}\text { n.d. } \\
\text { n.d. }\end{array}$ & $\begin{array}{l}\text { N-ter + 42.01 Da } \\
\text { N-ter + 42.01 Da, C+57.02 Da }\end{array}$ & $\begin{array}{l}61,0 \\
14,1\end{array}$ & $\begin{array}{r}1,066 \\
-2,194\end{array}$ & $\begin{array}{l}3 \\
2\end{array}$ & $\begin{array}{l}1968,0 \\
1547,7\end{array}$ & $\begin{array}{l}\text { AKTS1_MOUSE } \\
\text { P3H2_MOUSE }\end{array}$ & D3Z4W3_MOUSE & $\begin{array}{l}\text { Proline-rich AKT1 substrate 1; } \\
\text { Prolyl 3-hydroxylase 2; }\end{array}$ & Leprecan-like protein 1; \\
\hline M & QPASAKWYDRR & n.d. & $\mathrm{N}$-ter + 42.01 Da, K +34.06 Da & 17,8 & 2,080 & 3 & 1583,8 & TEBP_MOUSE & D3Z7C6_MOUSE & Prostaglandin E synthase 3; & $\begin{array}{l}\text { Cytososolic prostaglandin E2 synthase;;Hs990 co- } \\
\text { chaperone;Progesterone receptor complex } \\
\text { p23;Sid 3177; Telomerase-binding protein p23; }\end{array}$ \\
\hline M & QPASAKWYDR & $-0,2$ & $\mathrm{~N}$-ter + 42.01 Da, K+28.03 Da & 40,9 & 0,492 & 2 & 1421,7 & TEBP_MOUSE & D3Z7C6_MOUSE & Prostaglandin E synthase 3; & $\begin{array}{l}\text { Cytosolic prostaglandin E2 synthase; ;sp90 co- } \\
\text { chaperone;Progesterone receptor complex } \\
\text { p23;Sid 3177; Telomerase-binding protein p23; }\end{array}$ \\
\hline IGGAISQYNR & TGPCPQGPAPEVVIYQQLR & n.d. & $\mathrm{N}$-ter + 42.01 Da, C +57.02 Da & 27,4 & 9,052 & 3 & 2151,1 & PTGR1_MOUSE & & Prostaglandin reductase 1 & $\begin{array}{l}\text { 15-oxoprostaglandin 13-reductase;NADP- } \\
\text { dependent leukotriene B4 12- } \\
\text { hydroxydehydrogenase; }\end{array}$ \\
\hline M & ASLLKVDQEVKLKVDSFR & 0,3 & $\mathrm{~N}$-ter $+42.01 \mathrm{Da}, \mathrm{K}+28.03 \mathrm{Da}$ & 31,9 & 0,726 & 3 & 2200,3 & PSME3_MOUSE & & Proteasome activator complex subunit 3; & $\begin{array}{l}11 \mathrm{~S} \text { regulator complex subunit } \\
\text { gamma; Activator of multicatalytyic protease } \\
\text { subunit } 3 ; \text {; i nuclear autoantigen; } \text {;roteasome } \\
\text { activator } 28 \text { subunit gamma; }\end{array}$ \\
\hline QKHLAGLFNS & ITSFYHPSNNGR & n.d. & $\mathrm{N}$-ter + $42.01 \mathrm{Da}$ & 17,7 & 6,264 & 3 & 1433,7 & PSME4_MOUSE & & Proteasome activator complex subunit 4 & Proteasome activator PA200; Protein TEMO; \\
\hline M & AATFFGEVVKAPCR & n.d. & $\begin{array}{l}\text { N-ter + 42.01 Da, K+34.06 Da, } \\
\text { C+57.02 Da }\end{array}$ & 53,1 & $-0,982$ & 2 & 1627,9 & PSMG1_MOUSE & D6RG59_MOUSE & Proteasome assembly chaperone $1 ;$ & $\begin{array}{l}\text { Down syndrome critical region protein } 2 \\
\text { homolog; }\end{array}$ \\
\hline HFPDSNRNNQ & AEKSNQVSIAGQPAGVESAR & n.d. & N-ter + 42.01 Da, K +28.03 Da & 11,3 & 1,400 & 3 & 2068,1 & BICC1_MOUSE & G3X856_MOUSE & Protein bicaudal C homolog 1; & \\
\hline
\end{tabular}




\begin{tabular}{|c|c|c|c|c|c|c|c|c|c|c|c|}
\hline Non Prime Site & Prime Site & 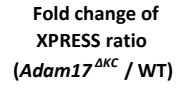 & Modifications & Hyperscore & $\begin{array}{l}\text { Mass error } \\
\text { in ppm }\end{array}$ & Charge & $\begin{array}{l}\text { Precursor } \\
\text { neutral mass } \\
\text { in Da }\end{array}$ & s Uniprot ID 1 & Uniprot ID 2 & Protein Name 1 & Protein Name 2 \\
\hline $\bar{M}$ & SAAAAPAAEGEDAPVPPSSEKEPEMPGPR & 0,2 & $\mathrm{~N}$-ter + 42.01 Da, K+28.03 Da & 63,9 & 0,583 & 3 & 2914,4 & DEK_MOUSE & E9Q8Y1_MOUSE & $\begin{array}{l}\text { Protein DEK; } \\
\end{array}$ & \\
\hline LKAEGSERLL & AKVDATEESDLAQQYGVR & 1,0 & $\mathrm{~N}$-ter + 42.01 Da, K+28.03 Da & 17,7 & 3,070 & 3 & 2049,0 & PDIA1_MOUSE & & Protein disulfide-isomerase; & $\begin{array}{l}\text { Cellular thyroid hormone-binding } \\
\text { protein;Endoplasmic reticulum resident protein } \\
59 ; \text {;rolyl 4-hydroxylase subunit bita;; } 555 ;\end{array}$ \\
\hline TVIPVDVMRR & AGIKVTVAGLAGKDPVQCSR & n.d. & $\begin{array}{l}\text { N-ter + 42.01 Da, K +28.03 Da, } \\
\mathrm{C}+57.02 \mathrm{Da}\end{array}$ & 13,0 & 7,287 & 3 & 2124,2 & PARK7_MOUSE & A2A815_MOUSE & Protein DJ-1; & Parkinson disease protein 7 homolog; \\
\hline M & ESEQMLEGQTQVAENPHSEYGLTDSVER & n.d. & $\mathrm{N}-\mathrm{ter}+42.01 \mathrm{Da}$ & 63,2 & 1,228 & 3 & 3335,5 & DPY30_MOUSE & & Protein dpy-30 homolog; & $\begin{array}{l}\text { Dpy-30-like protein; } \\
\text { Embryonic large molecule derived from yolk }\end{array}$ \\
\hline HSKIIEAFMS & QGEHKQALR & n.d. & N-ter + 42.01 Da, K+28.03 Da & 11,8 & $-4,219$ & 2 & 1135,6 & ELYS_MOUSE & & Protein ELYS; & $\begin{array}{l}\text { sac;Protein MEL-28;Putative AT-hook- } \\
\text { containing transcription factor 1; }\end{array}$ \\
\hline $\begin{array}{l}\text { SSAEEEGLRL } \\
\text { RASFCMHKRS }\end{array}$ & $\begin{array}{l}\text { FVGPGGSTTFGSHHLPNR } \\
\text { SSWGSTHP }\end{array}$ & n.d. & $\mathrm{N}$-ter $+42.01 \mathrm{Da}$ & 14,5 & $\begin{array}{r}-8,107 \\
2,454\end{array}$ & 3 & $\begin{array}{l}1908,9 \\
1057,5\end{array}$ & $\begin{array}{l}\text { F102B_MOUSE } \\
\text { F117A MOUSE }\end{array}$ & E9Q4R1_MOUSE & $\begin{array}{l}\text { Protein FAM102B; } \\
\text { PoM117; }\end{array}$ & \\
\hline $\begin{array}{l}\text { RASFCMHKRS } \\
\text { M }\end{array}$ & $\begin{array}{l}\text { ASWGSTDHR } \\
\text { DGEDDSNLVIKKR }\end{array}$ & $\begin{array}{l}\text { n.d. } \\
0,0\end{array}$ & $\begin{array}{l}\text { N-ter + 42.01 Da } \\
\text { N-ter + 42.01 Da, K +28.03 Da }\end{array}$ & $\begin{array}{l}12,9 \\
37,1\end{array}$ & $\begin{array}{l}2,454 \\
1,861\end{array}$ & $\begin{array}{l}2 \\
3\end{array}$ & $\begin{array}{l}1057,5 \\
1716,9\end{array}$ & $\begin{array}{l}\text { F117A_MOUSE } \\
\text { F192A_MOUSE }\end{array}$ & & $\begin{array}{l}\text { Protein FAM117A; } \\
\text { Protein FAM192A; }\end{array}$ & NEFA-interacting nuclear protein NIP30; \\
\hline SASASSSSAS & MVSSASSSGSSVGNSASNSSANMSR & n.d. & $\mathrm{N}$-ter + 42.01 Da & 16,5 & 4,388 & 3 & 2390,0 & F199X_MOUSE & & Protein FAM199X; & \\
\hline M & LGGLGKLAAEGLAHR & $-0,1$ & $\mathrm{~N}$-ter + 42.01 Da, K+28.03 Da & 95,0 & $-1,081$ & 2 & 1662,9 & FM25C_MOUSE & & Protein FAM25C; & \\
\hline LKKAQESGDR & VVKEVTEKVTHTITDAVTHAAEGLGR & n.d. & $\mathrm{N}$-ter + 42.01 Da, K+28.03 Da & 17,9 & 7,057 & 4 & 2858,6 & FM25C_MOUSE & & Protein FAM25C; & \\
\hline & AQYKGAASEAGR & $-0,1$ & $\mathrm{~N}$-ter + 42.01 Da, K+28.03 Da & 73,0 & $-0,078$ & 2 & 1277,6 & FA50A_MOUSE & & Protein FAM50A; & Protein XAP-5; \\
\hline HRFGGQPCQG & APGSAPCGQAGDSWSPDPHPVGGGR & n.d. & $\mathrm{N}$-ter + 42.01 Da, C +57.02 Da & 13,4 & $-3,573$ & 5 & 2458,1 & FA53B_MOUSE & Q3UOR8_MOUSE & Protein FAM53B; & \\
\hline M & AFSQVVQCLDDNHVNWR & n.d. & N-ter + 42.01 Da, C+57.02 Da & 47,7 & $-0,344$ & 3 & 2029,9 & FA83G_MOUSE & & Protein FAM83G; & \\
\hline KLRAGRPGFA & DPDDFALGAGHR & n.d. & $\mathrm{N}$-ter + 42.01 Da & 11,6 & 0,457 & 2 & 1311,6 & FA83H_MOUSE & & Protein FAM83H; & \\
\hline ESEELPKPRV & SDSESEDPQKGPASDSEAEDASR & n.d. & N-ter + 42.01 Da, K+28.03 Da & 13,9 & 9,124 & 3 & 2463,0 & IWS1_MOUSE & & Protein IWS1 homolog; & IWS1-like protein; \\
\hline RASSSPLEAL & EACLKGIPPGGSSPLQSLAISWSR & n.d. & $\begin{array}{l}\mathrm{N} \text {-ter + 42.01 Da, } \mathrm{K}+34.06 \mathrm{Da}, \\
\mathrm{C}+57.02 \mathrm{Da}\end{array}$ & 20,8 & $-3,090$ & 3 & 2586,4 & KRBA1_MOUSE & D3Z4B8_MOUSE & Protein KRBA1; & \\
\hline PNLESINKVN & GLCEDTAPSPGR & n.d. & $\mathrm{N}$-ter $+42.01 \mathrm{Da}, \mathrm{C}+57.02 \mathrm{Da}$ & 11,5 & $-0,614$ & 2 & 1300,6 & LAP2_MOUSE & B7ZNX6_MOUSE & Protein LAP2; & $\begin{array}{l}\text { Densin-180-like protein;Erbb2-interacting } \\
\text { protein; }\end{array}$ \\
\hline M & AALGEPVRLER & n.d. & $\mathrm{N}$-ter $+42.01 \mathrm{Da}$ & 20,7 & $-2,313$ & 2 & 1251,7 & LIN7C_MOUSE & & Protein lin-7 homolog C; & $\begin{array}{l}\text { Mammalian lin-seven protein 3; Vertebrate lin-7 } \\
\text { homolog 3; }\end{array}$ \\
\hline M & SGSSGTPYLGSKISLISKAQIR & 0,0 & $\mathrm{~N}$-ter + 42.01 Da, K +28.03 Da & 21,2 & 1,404 & 3 & 2347,3 & LS14B_MOUSE & & Protein LSM14 homolog B; & Protein FAM61B;RNA-associated protein 55B; \\
\hline M & TSHSTSAQCSASDSACR & n.d. & N-ter + 42.01 Da, C +57.02 Da & 14,6 & $-0,970$ & 2 & 1853,7 & MDM4_MOUSE & Q3UTC9_MOUSE & Protein Mdm4; & $\begin{array}{l}\text { Double minute } 4 \text { protein;Mdm2-like p53-- } \\
\text { binding protein;Protein Mdmx;p53-binding } \\
\text { protein Mdm4; }\end{array}$ \\
\hline LSMWVSLMAL & ADFDEPQR & n.d. & $\mathrm{N}$-ter + 42.01 Da & 14,2 & 1,470 & 2 & 1018,4 & TMM8C_MOUSE & E9QA72_MOUSE & Protein myomaker; & $\begin{array}{l}\text { Transmembrane protein } 8 \mathrm{C} ; \\
\text { Gene trap locus protein } F 3-2 ; G e n e \text { trap locus }\end{array}$ \\
\hline M & AHATPPSALEQGGPIR & n.d. & $\mathrm{N}$-ter $+42.01 \mathrm{Da}$ & 13,5 & 1,034 & 2 & 1642,8 & GTL3B_MOUSE & & Protein NATD1; & $\begin{array}{l}\text { protein F Fb; N-acetyltransferase domain- } \\
\text { cottaining protein } 1 ;\end{array}$ \\
\hline QKVTFFIHSC & MDAVQVaVR & n.d. & $\mathrm{N}$-ter + 42.01 Da & 14,4 & $-6,155$ & 2 & 1086,5 & NDNF_MOUSE & & Protein NDNF; & $\begin{array}{l}\text { Epidermacan;Neuron-derived neurotrophic } \\
\text { factor; }\end{array}$ \\
\hline VTVLLGKAGM & GKTTLAYRLR & n.d. & $\mathrm{N}-\operatorname{ter}+42.01 \mathrm{Da}, \mathrm{K}+28.03 \mathrm{Da}$ & 17,2 & 2,239 & 3 & 1247,7 & NLRC5_MOUSE & & Protein NLRC5; & \\
\hline HSANSASPEG & APTDAENGISGNVTPDQDPAR & n.d. & N-ter +42.01 Da & 11,8 & 1,153 & 2 & 2166,0 & NXP20_MOUSE & & Protein Noxp20; & $\begin{array}{l}\text { Nervous system overexpressed protein } \\
\text { 20:Protein FAM114A1: }\end{array}$ \\
\hline PASETGSENS & VSMAAHTLMILSR & n.d. & $\mathrm{N}$-ter $+42.01 \mathrm{Da}$ & 17,0 & $-7,125$ & 3 & 1470,8 & NPAT_MOUSE & & Protein NPAT; & \\
\hline SSDELMQLEL & CSKEDLDDNANPANGSGR & $-3,6$ & $\begin{array}{l}\text { N-ter + 42.01 Da, K +28.03 Da, } \\
\mathrm{C}+57.02 \mathrm{Da}\end{array}$ & 17,1 & 0,552 & 4 & 1988,9 & PPR3A_MOUSE & & Protein phosphatase 1 regulatory subunit 3A; & $\begin{array}{l}\text { Protein phosphatase } 1 \text { glycogen-associated } \\
\text { regulatory subunit;;rotein phosphatase type-1 } \\
\text { glycogen targeting subunit; }\end{array}$ \\
\hline M & SALEKSMHLGR & n.d. & N-ter + 42.01 Da, K+34.06 Da & 33,2 & $-0,995$ & 3 & 1303,7 & PPME1_MOUSE & & Protein phosphatase methylesterase 1 ; & \\
\hline DTRPEDLPGA & TPQTLPKDR & n.d. & $\mathrm{N}$-ter + 42.01 Da, $\mathrm{K}+34.06 \mathrm{Da}$ & 11,7 & $-5,121$ & 2 & 1130,7 & PCLO_MOUSE & & Protein piccolo; & $\begin{array}{l}\text { Aczonin;Brain-derived HLMN } \\
\text { protein;Multidomain presynaptic cytomatrix } \\
\text { protein; }\end{array}$ \\
\hline $\begin{array}{l}\text { KRQSEELSKL } \\
\text { M }\end{array}$ & $\begin{array}{l}\text { RQEIYSSHNQPSCGGR } \\
\text { SEGDSVGDSVHGKPSVYYR }\end{array}$ & $\begin{array}{l}\text { n.d. } \\
\text { n.d. }\end{array}$ & $\begin{array}{l}\text { N-ter + 42.01 Da, C +57.02 Da } \\
\text { N-ter + 42.01 Da, K +34.06 Da }\end{array}$ & $\begin{array}{l}14,7 \\
35,1\end{array}$ & $\begin{array}{l}1,563 \\
4,773\end{array}$ & $\begin{array}{l}3 \\
3\end{array}$ & $\begin{array}{l}1916,9 \\
2050,0\end{array}$ & $\begin{array}{l}\text { POF1B_MOUSE } \\
\text { RER1_MOUSE }\end{array}$ & & $\begin{array}{l}\text { Protein POF1B; } \\
\text { Protein RER1; }\end{array}$ & \\
\hline MPSQ & MEHAMETMMLTFHR & n.d. & $\mathrm{N}$-ter $+42.01 \mathrm{Da}$ & 24,9 & $-2,377$ & 3 & 1805,8 & S10AA_MOUSE & & Protein S100-A10; & $\begin{array}{l}\text { Calpactin I light chain;Calpactin-1 light } \\
\text { chain;Cellular ligand of annexin II;S100 calcium- } \\
\text { binding protein A10;p10 protein;p11; }\end{array}$ \\
\hline M & ANKAPSQMER & n.d. & $\mathrm{N}$-ter + 42.01 Da, K+34.06 Da & 50,9 & $-0,827$ & 2 & 1206,6 & S10A9_MOUSE & & Protein S100-A9; & 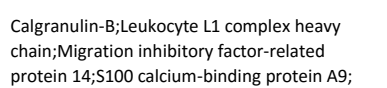 \\
\hline
\end{tabular}




\begin{tabular}{|c|c|c|c|c|c|c|c|c|c|c|c|}
\hline Non Prime Site & Prime Site & $\begin{array}{l}\text { Fold change of } \\
\text { XPRESS ratio } \\
\text { (Adam17 } 1 \text { ¿KC / WT) }\end{array}$ & Modifications & Hyperscore & $\begin{array}{c}\text { Mass error } \\
\text { in ppm }\end{array}$ & Charge & $\begin{array}{l}\text { Precursor } \\
\text { neutral mass } \\
\text { in Da }\end{array}$ & Uniprot ID 1 & Uniprot ID 2 & Protein Name 1 & Protein Name 2 \\
\hline M & VSVMNTVDTSHEDMIHDAQMDYYGTR & n.d. & $\mathrm{N}$-ter + 42.01 Da & 22,5 & $-2,354$ & 3 & 3056,3 & SEC13_MOUSE & & Protein SEC13 homolog; & SEC13-like protein 1;SEC13-related protein; \\
\hline M & SESGHSQPGLYGIER & n.d. & $\mathrm{N}$-ter + $42.01 \mathrm{Da}$ & 17,8 & $-2,772$ & 2 & 1657,8 & SMG9_MOUSE & D3Z719_MOUSE & Protein SMG9; & $\begin{array}{l}\text { Protein smg- } 9 \text { homolog; } \\
\text { SOGA family member } 1 \text {;Suppressor of glucose }\end{array}$ \\
\hline MSEFESLLDC & SPYLAGGDAR & n.d. & $\mathrm{N}$-ter + 42.01 Da & 17,6 & 0,095 & 2 & 1047,5 & SOGA1_MOUSE & A2ACV6_MOUSE & Protein SOGA1; & $\begin{array}{l}\text { by autophagy; Suppressor of glucose, autophagy- } \\
\text { associated protein } 1 \text {; } \\
\text { Coiled-coil domain-containing protein } 99\end{array}$ \\
\hline LKQKLDLQGE & LLAHKSEELR & n.d. & N-ter + 42.01 Da, K +34.06 Da & 26,7 & 2,277 & 3 & 1270,8 & SPDLY_MOUSE & & $\begin{array}{l}\text { Protein Spindly \{ECO:0000255|HAMAP- } \\
\text { Rule:MF_03041\}; }\end{array}$ & $\begin{array}{l}\text { \{ECO:0000255|HAMAP- } \\
\text { Rule:MF_03041\};Spindle apparatus coiled-coil } \\
\text { domain-containing protein } 1 \\
\text { \{ECO:0000255|HAMAP-Rule:MF_03041\}; }\end{array}$ \\
\hline IAAHQLYYYYV & ADHASSYHMKPLR & n.d. & N-ter + 42.01 Da, K+34.06 Da & 14,5 & 0,629 & 2 & 1587,8 & SZT2_MOUSE & & Protein SZT2; & $\begin{array}{l}\text { Seizure threshold } 2 \text { protein;Transcript } \\
\text { increased in glutamate resistance; }\end{array}$ \\
\hline LLEVTDKKL & QLLTYNWAPDLGAALGR & n.d. & $\mathrm{N}$-ter + 42.01 Da & 17,5 & 7,094 & 3 & 1900,0 & SZT2_MOUSE & & Protein SZT2; & $\begin{array}{l}\text { Seizure threshold } 2 \text { protein; Transcript } \\
\text { increased in glutamate resistance; }\end{array}$ \\
\hline NISLNGKADA & ALISKVSSHLVLR & n.d. & N-ter + 42.01 Da, K +28.03 Da & 15,5 & $-0,468$ & 4 & 1491,9 & TANC1_MOUSE & E9QAF9_MOUSE & Protein TANC1; & $\begin{array}{l}\text { Tetratricopeptide repeat, ankyrin repeat and } \\
\text { coiled-coil domain-containing protein } 1 \text {; }\end{array}$ \\
\hline KGVPHLYPEG & VSKQPLHVSTEAHR & n.d. & $\mathrm{N}$-ter + 42.01 Da, K +34.06 Da & 13,9 & 3,599 & 3 & 1663,9 & TANC1_MOUSE & E9QAF9_MOUSE & Protein TANC1; & $\begin{array}{l}\text { Tetratricopeptide repeat, ankyrin repeat and } \\
\text { coiled-coil domain-containing protein } 1 \text {; }\end{array}$ \\
\hline VGEMITGGQR & TPTSVKTTSTSSR & n.d. & $\mathrm{N}$-ter + 42.01 Da, K +34.06 Da & 16,0 & $-4,473$ & 3 & 1427,8 & TLLB_MOUSE & & Protein tilB homolog; & $\begin{array}{l}\text { Leucine-rich repeat-containing protein } \\
\text { 6;Leucice-rich testis-specific protein; Testis- } \\
\text { specific leucine-rich repeat protein; }\end{array}$ \\
\hline GSSHSQEFMK & FATIEAIQR & n.d. & $\mathrm{N}$-ter + 42.01 Da & 17,5 & $-1,374$ & 2 & 1089,6 & SC16B_MOUSE & F6Q2Y9_MOUSE & Protein transport protein Sec16B; & $\begin{array}{l}\text { Leucine zipper transcription regulator } \\
\text { 2;;Regucalcin gene promoter region-related } \\
\text { protein p117; } 5 \text { EC16 homolog B; } \\
\text { Leucine zipper transcription regulator }\end{array}$ \\
\hline KYKRQPPVAN & LINLTDEDWPVLSSGTR & n.d. & $\mathrm{N}$-ter $+42.01 \mathrm{Da}$ & 19,5 & $-0,510$ & 3 & 1957,0 & SC16B_MOUSE & F6Q2Y9_MOUSE & Protein transport protein Sec16B; & $\begin{array}{l}\text { 2;Regucalcin gene promoter region-related } \\
\text { protein p117;SEC16 homolog B; } \\
\text { Dendrite arborization and synapse maturation }\end{array}$ \\
\hline KAPPAFIDQP & KEEYFQEVGR & n.d. & $\mathrm{N}$-ter + $42.01 \mathrm{Da}, \mathrm{K}+34.06 \mathrm{Da}$ & 16,0 & 4,623 & 3 & 1359,7 & TUTLA_MOUSE & & Protein turtle homolog A; & $\begin{array}{l}\text { protein 1;Immunoglobulin superfamily member } \\
9 A_{;} \text {; }\end{array}$ \\
\hline $\begin{array}{l}\text { GSGAAVTEQE } \\
\text { RGGLGPSWAS }\end{array}$ & $\begin{array}{l}\text { LLALDTIRPEHVLR } \\
\text { TNSGSGGSRGFVSGGSGRGR }\end{array}$ & n.d. & $\begin{array}{l}\text { N-ter }+42.01 \mathrm{Da} \\
\text { N-ter + 42.01 Da, } \mathrm{K}+34.06 \mathrm{Da}\end{array}$ & $\begin{array}{l}15,9 \\
19,7\end{array}$ & $\begin{array}{r}0,710 \\
-1,955\end{array}$ & $\begin{array}{l}3 \\
3\end{array}$ & $\begin{array}{l}1687,0 \\
2043,0\end{array}$ & $\begin{array}{l}\text { U119B_MOUSE } \\
\text { VIR_MOUSE }\end{array}$ & E9PZY8_MOUSE & $\begin{array}{l}\text { Protein unc-119 homolog B; } \\
\text { Protein virilizer homolog; }\end{array}$ & \\
\hline M & AQGAVIHVAPEQPTHAVCVVGTATPLDVR & n.d. & N-ter + 42.01 Da, C +57.02 Da & 37,5 & 0,461 & 3 & 3034,6 & PADI4_MOUSE & & Protein-arginine deiminase type-4; & $\begin{array}{l}\text { Peptidylarginine deiminase IV;Protein-arginine } \\
\text { deiminase type VI; } \\
\text { L-isoaspartyI protein carboxyI }\end{array}$ \\
\hline LYLLRVCFAM & AWKSGGASHSELIHNLR & n.d. & N-ter + 42.01 Da, K +34.06 Da & 43,9 & $-9,166$ & 4 & 1938,0 & PIMT_MOUSE & F7D432_MOUSE & $\begin{array}{l}\text { Protein-L-isoaspartate(D-aspartate) O- } \\
\text { methyltransferase; }\end{array}$ & $\begin{array}{l}\text { methyltransferase;Protein L-isoasparty//D- } \\
\text { aspartyl methyltransferase; Protein-beta- } \\
\text { aspartate methyltransferase; }\end{array}$ \\
\hline $\begin{array}{l}\text { DKLSMVMYLS } \\
\text { M }\end{array}$ & $\begin{array}{l}\text { KFYELFR } \\
\text { SDAAVDTSSEITTKDLKEKKEVVEEAENGR }\end{array}$ & $\begin{array}{l}\text { n.d. } \\
1,3\end{array}$ & $\begin{array}{l}\text { N-ter + 42.01 Da, K+28.03 Da } \\
\text { N-ter + 42.01 Da, K+28.03 Da }\end{array}$ & $\begin{array}{l}13,9 \\
33,9\end{array}$ & $\begin{array}{l}-0,559 \\
-2,416\end{array}$ & $\begin{array}{l}2 \\
4\end{array}$ & $\begin{array}{l}1071,6 \\
3431,7\end{array}$ & $\begin{array}{l}\text { MICA2_MOUSE } \\
\text { PTMA_MOUSE }\end{array}$ & & $\begin{array}{l}\text { Protein-methionine sulfoxide oxidase MICAL2; } \\
\text { Prothymosin alpha; }\end{array}$ & Molecule interacting with CasL protein 2; \\
\hline DFYQATVSET & ATNLTQVLR & n.d. & $\mathrm{N}$-ter $+42.01 \mathrm{Da}$ & 19,4 & $-0,756$ & 2 & 1056,6 & FAT4_MOUSE & & Protocadherin Fat 4; & $\begin{array}{l}\text { FAT tumor suppressor homolog 4; Fat-like } \\
\text { cadherin protein FAT-J; }\end{array}$ \\
\hline RKEPEINIEE & KEPQISIEPTHR & n.d. & $\mathrm{N}$-ter + 42.01 Da, K+28.03 Da & 17,8 & 3,188 & 2 & 1503,8 & PCD20_MOUSE & & Protocadherin-20; & \\
\hline LAGTTVALII & LALGFLLAQVVSPR & n.d. & $\mathrm{N}-\operatorname{ter}+42.01 \mathrm{Da}$ & 16,9 & $-0,924$ & 2 & 1512,9 & MYCT_MOUSE & & Proton myo-inositol cotransporter; & $\begin{array}{l}\mathrm{H}(++ \text {-)yyo-inositol symporter;solute carrier } \\
\text { family } 2 \text { member 13; }\end{array}$ \\
\hline TLCTLPYSLL & CDYYQSKKFAGSSADGTR & n.d. & $\begin{array}{l}\text { N-ter + 42.01 Da, K+28.03 Da, } \\
\mathrm{C}+57.02 \mathrm{Da}\end{array}$ & 17,8 & $-7,707$ & 3 & 2138,0 & S45A1_MOUSE & & Proton-associated sugar transporter A; & $\begin{array}{l}\text { Deleted in neuroblastoma } 5 \text { protein } \\
\text { homolog; Solute carrier family } 45 \text { member 1; }\end{array}$ \\
\hline $\begin{array}{l}\text { KAKYSRNIVK } \\
\text { M }\end{array}$ & $\begin{array}{l}\text { KFLMYGSKPQVAEIIR } \\
\text { NHDFQALALESR }\end{array}$ & $\begin{array}{l}\text { n.d. } \\
\text { n.d. }\end{array}$ & $\begin{array}{l}\text { N-ter + 42.01 Da, } \mathrm{K}+34.06 \mathrm{Da} \\
\text { N-ter+42.01 Da }\end{array}$ & $\begin{array}{l}21,1 \\
31,7\end{array}$ & $\begin{array}{l}6,274 \\
0,064\end{array}$ & $\begin{array}{l}3 \\
2\end{array}$ & $\begin{array}{l}1989,2 \\
1572,7\end{array}$ & $\begin{array}{l}\text { KO020_MOUSE } \\
\text { PUM2_MOUSE }\end{array}$ & E9Q0P1_MOUSE & $\begin{array}{l}\text { Pumilio domain-containing protein KIAA0020; } \\
\text { Pumilio homolog 2; }\end{array}$ & \\
\hline DQAGSKLEAE & IKKLVAEAGR & n.d. & N-ter + 42.01 Da, K +28.03 Da & 11,2 & $-3,886$ & 2 & 1181,8 & S38AA_MOUSE & & $\begin{array}{l}\text { Putative sodium-coupled neutral amino acid } \\
\text { transporter 10; }\end{array}$ & Solute carrier family 38 member 10; \\
\hline M & DASLEKIADPTLAEMGKNLKEAMR & n.d. & $\mathrm{N}$-ter + 42.01 Da, K+34.06 Da & 35,0 & 0,412 & 4 & 2905,6 & PDXD1_MOUSE & D3YZA7_MOUSE & $\begin{array}{l}\text { Pyridoxal-dependent decarboxylase domain- } \\
\text { containing protein 1; }\end{array}$ & \\
\hline $\begin{array}{l}\text { IQTQQLHAAM } \\
\text { M }\end{array}$ & $\begin{array}{l}\text { ADTFFEEMCR } \\
\text { AAAPALALDPQPQEKQKDASESSELSR }\end{array}$ & $\begin{array}{l}\text { n.d. } \\
1,7\end{array}$ & $\begin{array}{l}\text { N-ter + 42.01 Da, C +57.02 Da } \\
\text { N-ter + 42.01 Da, K+28.03 Da }\end{array}$ & $\begin{array}{l}22,4 \\
36,7\end{array}$ & $\begin{array}{l}0,983 \\
0,592\end{array}$ & $\begin{array}{l}2 \\
3\end{array}$ & $\begin{array}{l}1320,6 \\
3035,5\end{array}$ & $\begin{array}{l}\text { KPYM_MOUSE } \\
\text { RABE2 MOUSE }\end{array}$ & & $\begin{array}{l}\text { Pyruvatat kinase PK; } \\
\text { Rab GTPase-binding effec }\end{array}$ & $\begin{array}{l}\text { Prruvate kinase muscle isozyme; } \\
\text { Rabatin-5beta; }\end{array}$ \\
\hline QARARQQAQE & $\begin{array}{l}\text { AAAPAATLLLPDQPQEKQKDASESSELSR } \\
\text { HEAERLIIIVIKLR }\end{array}$ & $\begin{array}{l}1, / \\
\text { n.d. }\end{array}$ & $\begin{array}{l}\mathrm{N} \text {-ter + 42.01 Da, } \mathrm{K}+28.03 \mathrm{Da} \\
\mathrm{N} \text {-ter + 42.01 Da, } \mathrm{K}+34.06 \mathrm{Da}\end{array}$ & $\begin{array}{l}36,1 \\
17,5\end{array}$ & $\begin{array}{l}0,592 \\
-1,684\end{array}$ & 4 & $\begin{array}{l}3035,5 \\
1837,1\end{array}$ & $\begin{array}{l}\text { RAAE2_MOUUE } \\
\text { RABE2_MOUSE }\end{array}$ & & $\begin{array}{l}\text { Rab GTPase-binding effector protein 2; } \\
\text { Rab GTPase-binding effector protein 2; }\end{array}$ & $\begin{array}{l}\text { Rabaptin-5beta; } \\
\text { Rabaptin-5beta; }\end{array}$ \\
\hline
\end{tabular}




\begin{tabular}{|c|c|c|c|c|c|c|c|c|c|c|c|}
\hline Non Prime Site & Prime Site & 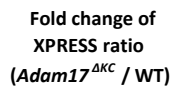 & Modifications & Hyperscore & $\begin{array}{l}\text { Mass error } \\
\text { in ppm }\end{array}$ & Charge & $\begin{array}{l}\text { Precursor } \\
\text { neutral mass } \\
\text { in Da }\end{array}$ & Uniprot ID 1 & Uniprot ID 2 & Protein Name 1 & Protein Name 2 \\
\hline YQQNHDSEEG & VTATWQDLIHETEEAISLR & n.d. & $\mathrm{N}$-ter + 42.01 Da & 14,5 & 0,044 & 3 & 2253,1 & RAE2_MOUSE & & $\begin{array}{l}\text { Rab proteins geranylgeranyltransferase component A } \\
2 ;\end{array}$ & Choroideremia-like protein;Rab escort protein \\
\hline M & ELCQPTSLSDHDQPASGPQR & n.d. & N-ter + 42.01 Da, C +57.02 Da & 13,9 & 1,877 & 3 & 2395,1 & RFIP3_MOUSE & & Rab11 family-interacting protein 3; & \\
\hline GEGTVACLQF & SVSLQTEER & n.d. & $\mathrm{N}$-ter $+42.01 \mathrm{Da}$ & 20,4 & $-0,916$ & 2 & 1089,5 & RGP1_MOUSE & Q3TDM6_MOUSE & $\begin{array}{l}\text { RABGA-GEF complex partner protein } 2 \\
\{\text { ECO:0000305\}; }\end{array}$ & $\begin{array}{l}\text { Retrograde Golgi transport protein RGP1 } \\
\text { homolog \{ECO:0000250| UniProtKB:P16664\}; }\end{array}$ \\
\hline M & FSALKKLVGSEQAPGR & n.d. & N-ter + 42.01 Da, K+34.06 Da & 49,9 & 0,311 & 3 & 1928,1 & RABL6_MOUSE & & Rab-like protein 6; & GTP-binding protein Parf;Rab-like protein 1; \\
\hline VATVTGSESA & SPVHSALGSR & n.d. & $\mathrm{N}$-ter $+42.01 \mathrm{Da}$ & 18,4 & 0,000 & 2 & 1051,5 & RGPA1_MOUSE & & Ral GTPase-activating protein subunit alpha-1; & $\begin{array}{l}\text { GAP-related-interacting partner to E12;GTPase- } \\
\text { activating RapGAP domain-like 1; Tuberin-like } \\
\text { protein 1;p240; }\end{array}$ \\
\hline PPDFPCLKQL & VAYVQLNMPGSDLER & n.d. & $\mathrm{N}$-ter $+42.01 \mathrm{Da}$ & 10,3 & 3,113 & 2 & 1732,9 & GNDS_MOUSE & Q3TWC1_MOUSE & Ral guanine nucleotide dissociation stimulator; & Ral guanine nucleotide exchange factor; \\
\hline KLSREKNSSP & GGSPGDPSSPTSSVSPGSPPSSPR & n.d. & $\mathrm{N}$-ter + 42.01 Da & 16,1 & 4,090 & 3 & 2222,0 & RGL3_MOUSE & & Ral guanine nucleotide dissociation stimulator-like 3; & $\begin{array}{l}\text { RalGDS-related defector protein of M-Ras;Ras } \\
\text { pathway modulator; }\end{array}$ \\
\hline MV & MEKPSPLLVGR & n.d. & N-ter + 42.01 Da, K+34.06 Da & 33,6 & 1,764 & 2 & 1301,8 & G3BP1_MOUSE & G3BP2_MOUSE & Ras GTPase-activating protein-binding protein 1; & $\begin{array}{l}\text { ATP-dependent DNA helicase VIII;GAP SH3 } \\
\text { domain-binding protein 1;HDH-VIII; }\end{array}$ \\
\hline $\begin{array}{l}\text { RFQPGETLTE } \\
M\end{array}$ & $\begin{array}{l}\text { ILETPATNEQEAEHQR } \\
\text { SAAEFVDGIGVVRPHYGSVLDNER }\end{array}$ & $\begin{array}{l}\text { n.d. } \\
\text { n.d. }\end{array}$ & $\begin{array}{l}\text { N-ter + 42.01 Da } \\
\text { N-ter + 42.01 Da }\end{array}$ & $\begin{array}{l}17,8 \\
38,8\end{array}$ & $\begin{array}{l}-2,251 \\
2,143\end{array}$ & $\begin{array}{l}3 \\
3\end{array}$ & $\begin{array}{l}1906,9 \\
2610,3\end{array}$ & $\begin{array}{l}\text { IOGA1_MOUSE } \\
\text { IOGA1 MOUSE }\end{array}$ & & $\begin{array}{l}\text { Ras GTPase-activating-like protein IQGAP1; } \\
\text { Ras GTPase-activating-like protein IOGAP1. }\end{array}$ & \\
\hline M & SKSLKKLVEESR & n.d. & $\mathrm{N}$-ter + 42.01 Da, K+28.03 Da & 15,7 & 5,875 & 3 & 1528,9 & RSU1_MOUSE & AZAUR7_MOUSE & $\begin{array}{l}\text { Ras suppressor protein } 1 \text {; } \\
\text {, }\end{array}$ & \\
\hline SFTRLTELVL & QAHRKELDGLR & n.d. & N-ter + 42.01 Da, K+28.03 Da & 18,3 & 3,226 & 3 & 1391,8 & RAB15_MOUSE & D3YTZ8_MOMUSE & Ras-related protein Rab-15; & \\
\hline M & NILAPVRR & n.d. & N-ter + 42.01 Da & 13,8 & $-7,370$ & 2 & 1110,6 & RAB34_MOUSE & QOPD20_MOUSE & Ras-related protein Rab-34; & $\begin{array}{l}\text { Ras-reletaded homolog;Rasar-elated protein Rab- } \\
\text { 39;Ras-related protein Rah; }\end{array}$ \\
\hline QELNFEKNKA & VQTALDEFKVER & n.d. & $\mathrm{N}$-ter + 42.01 Da, K +28.03 Da & 16,5 & 1,327 & 3 & 1503,8 & RBCC1_MOUSE & F7CC56_MOUSE & RB1-inducible coiled-coil protein $1 ;$ & $\begin{array}{l}\text { Coiled-coil-forming protein 1;FAK family kinase- } \\
\text { interacting protein of } 200 \mathrm{kDa} \text {;LXXp180; }\end{array}$ \\
\hline LEAEGKVDVY & GYVVKLRR & n.d. & $\mathrm{N}$-ter + 42.01 Da, K+34.06 Da & 12,4 & $-4,870$ & 2 & 1065,7 & PTPRC_MOUSE & S4R2V1_MOUSE & Receptor-type tyrosine-protein phosphatase C; & $\begin{array}{l}\text { Leukocyte common antigen;:Lymphocyte } \\
\text { antigen 5; T200; }\end{array}$ \\
\hline AVGSPMPYVK & WMLGAEDLTPEDDMPIGR & n.d. & N-ter + 42.01 Da & 14,3 & $-2,967$ & 3 & 2086,9 & PTPRD_MOUSE & B9EJA3_MOUSE & Receptor-type tyrosine-protein phosphatase delta; & \\
\hline LTFVRRSSAA & RMPDMGPVLVHCSAGVGR & n.d. & N-ter + 42.01 Da, C +57.02 Da & 13,9 & 5,645 & 4 & 1980,0 & PTPRG_MOUSE & F8VQD7_MOUSE & Receptor-type tyrosine-protein phosphatase gamma; & \\
\hline M & AAGGGGGSSKASSSSASSAGALESSLDR & n.d. & $\mathrm{N}$-ter + 42.01 Da, $\mathrm{K}+34.06 \mathrm{Da}$ & 36,8 & $-0,763$ & 3 & 2487,2 & RPRD2_MOUSE & & $\begin{array}{l}\text { Regulation of nuclear pre-mRNA domain-containing } \\
\text { protein 2; }\end{array}$ & \\
\hline KGTSSDGVSL & SNLTQPSLPTTDQQQEEHYR & n.d. & $\mathrm{N}$-ter + 42.01 Da & 11,9 & 1,324 & 3 & 2413,1 & RPRD2_MOUSE & & $\begin{array}{l}\text { Regulation of nuclear pre-mRNA domain-containing } \\
\text { protein 2; }\end{array}$ & \\
\hline GVPNGRMVLA & VSDGELISTAGSQAQGEGR & n.d. & $\mathrm{N}$-ter + 42.01 Da & 22,0 & $-4,066$ & 3 & 1890,9 & RGS14_MOUSE & & Regulator of G-protein signaling 14; & RAP1/RAP2-interacting protein; \\
\hline M & DSEAFQHAR & n.d. & $\mathrm{N}$-ter + 42.01 Da & 41,7 & $-1,863$ & 2 & 1232,5 & IASPP_MOUSE & D32505_MOUSE & RelA-associated inhibitor; & 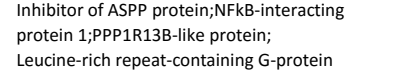 \\
\hline $\begin{array}{l}\text { KLYLSHNRIT } \\
\text { M }\end{array}$ & $\begin{array}{l}\text { FLKPGVFEDLHR } \\
\text { QAFLKGTSVSAKAQLTKDR }\end{array}$ & $\begin{array}{l}\text { n.d. } \\
\text { n.d. }\end{array}$ & $\begin{array}{l}\text { N-ter + } 42.01 \mathrm{Da}, \mathrm{K}+28.03 \mathrm{Da} \\
\text { N-ter } 42.01 \mathrm{Da}, \mathrm{K}+28.03 \mathrm{Da}\end{array}$ & $\begin{array}{l}14,4 \\
14,5\end{array}$ & $\begin{array}{l}2,092 \\
-1,126\end{array}$ & $\begin{array}{l}3 \\
4\end{array}$ & $\begin{array}{l}1526,8 \\
2305,3\end{array}$ & $\begin{array}{l}\text { RXFP1_MOUSE } \\
\text { RFC4 MOUSE }\end{array}$ & $\begin{array}{l}\text { S4R1B2_MOUSE } \\
\text { O3U184 MOUSE }\end{array}$ & $\begin{array}{l}\text { Relaxin receptor 1; } \\
\text { Replication factor C subunit } 4 \text {; }\end{array}$ & $\begin{array}{l}\text { coupled receptor 7; Relaxin family peptide } \\
\text { receptor } 1 ; \text { subunit } 4 \text { : }\end{array}$ \\
\hline M & TAAAPSQQRPAAAR & n.d. & $\mathrm{N}$-ter $+42.01 \mathrm{Da}$ & 18,4 & $-2,363$ & 2 & 1436,8 & RFC5_MOUSE & D3Z1Y6_MOUSE & Replication factor C subunit 5; & $\begin{array}{l}\text { Activator } 136 \text { kDa subunit;:Activator } 1 \text { subunit } \\
\text { 5;Replication factor C } 36 \text { kDa subunit; }\end{array}$ \\
\hline $\begin{array}{l}\text { M } \\
\text { DSSGESDDTV }\end{array}$ & EDIMQLPKAR & $\begin{array}{c}\text { n.d. } \\
-10,0\end{array}$ & $\begin{array}{l}\text { N-ter + 42.01 Da, K +34.06 Da } \\
\text { N-ter + 42.01 Da K } 28.03 \text { Da }\end{array}$ & $\begin{array}{l}41,4 \\
14,2\end{array}$ & $\begin{array}{l}-0,071 \\
0,382\end{array}$ & $\begin{array}{l}2 \\
3\end{array}$ & $\begin{array}{l}1406,8 \\
2355,2\end{array}$ & $\begin{array}{l}\text { RFA3_MOUSE } \\
\text { RTN3_MOUSE }\end{array}$ & & $\begin{array}{l}\text { Replication protein A } 14 \text { kDa subunit; } \\
\text { Reticulon-3; }\end{array}$ & Replication factor A protein 3; \\
\hline QEVASVRSLS & STSSSVPTHAAHSGDATTPR & n.d. & $\mathrm{N}-\operatorname{ter}+42.01 \mathrm{Da}, \mathrm{K}+28.03 \mathrm{Da}$ & 20,8 & $-8,205$ & 3 & 2007,9 & RHG07_MOUSE & E9QKB1_MOUSE & Rho GTPase-activating protein 7 ; & $\begin{array}{l}\text { Deleted in liver cancer } 1 \text { protein homolog; Rho- } \\
\text { type GTPase-activating protein 7;START domain- } \\
\text { containing protein 12;StAR-related lipid } \\
\text { transfer protein 12; }\end{array}$ \\
\hline VYIPAEESKP & QETTETKDPEATASR & n.d. & $\mathrm{N}$-ter + 42.01 Da, $\mathrm{K}+28.03 \mathrm{Da}$ & 15,0 & 7,897 & 2 & 1732,8 & ARHGA_MOUSE & F7BQE4_MOUSE & Rho guanine nucleotide exchange factor 10 ; & \\
\hline M & ADFGADEASSKSESPEQEDQGSEDR & $-0,1$ & N-ter + 42.01 Da, K+28.03 Da & 56,7 & 1,057 & 3 & 2740,1 & ARH37_MOUSE & E9Q5R6_MOUSE & Rho guanine nucleotide exchange factor 37 ; & \\
\hline HTVSRLEETN & SVLTKDIEMLR & 0,1 & N-ter + 42.01 Da, K +28.03 Da & 18,6 & $-0,654$ & 2 & 1373,8 & ROCK1_MOUSE & & Rho-associated protein kinase 1; & $\begin{array}{l}\text { Rho-associated, coiled-coil-containing protein } \\
\text { kinase 1;Rho-associated, coiled-coil-containing } \\
\text { protein kinase l;p160 ROCK-1; }\end{array}$ \\
\hline EVVLALDAIH & SMGLIHR & n.d. & $\mathrm{N}$-ter + 42.01 Da & 17,5 & 1,985 & 2 & 854,5 & ROCK2_MOUSE & F8VPK5_MOUSE & Rho-associated protein kinase 2; & $\begin{array}{l}\text { Rho-associated, coiled-coil-containing protein } \\
\text { kinase 2; Rho-associated, coiled-coil-containing } \\
\text { protein kinase Il;p164 ROCK-2; }\end{array}$ \\
\hline
\end{tabular}




\begin{tabular}{|c|c|c|c|c|c|c|c|c|c|c|c|}
\hline Non Prime Site & Prime Site & $\begin{array}{l}\text { Fold change of } \\
\text { XPRESS ratio } \\
\text { (Adam17 } 1 \text { ¿KC / WT) }\end{array}$ & Modifications & Hyperscore & $\begin{array}{l}\text { Mass error } \\
\text { in ppm }\end{array}$ & Charge & $\begin{array}{l}\text { Precursor } \\
\text { neutral mass } \\
\text { in Da }\end{array}$ & Uniprot ID 1 & Uniprot ID 2 & Protein Name 1 & Protein Name 2 \\
\hline M & SRPPPTGKMPGAPEAAPGDGAGAGR & n.d. & $\mathrm{N}$-ter + 42.01 Da, K+34.06 Da & 45,1 & 2,395 & 3 & 2377,2 & ROCK2_MOUSE & & Rho-associated protein kinase 2; & $\begin{array}{l}\text { Rho-associated, coiled-coil-containing protein } \\
\text { kinase 2;Rho-associated, coiled-coil-containing } \\
\text { protein kinase Il;p164 ROCK-2; }\end{array}$ \\
\hline CSARIQAYTA & ELQKSKEEIANQTGAR & n.d. & $\mathrm{N}$-ter $+42.01 \mathrm{Da}, \mathrm{K}+28.03 \mathrm{Da}$ & 21,2 & $-1,893$ & 3 & 1899,0 & RTKN2_MOUSE & E9QP76_MOUSE & Rhotekin-2; & $\begin{array}{l}\text { Pleckstrin homology domain-containing family } \\
\mathrm{K} \text { member } 1 \text {; }\end{array}$ \\
\hline $\begin{array}{l}M \\
M\end{array}$ & $\begin{array}{l}\text { KNPEEAADGKQR } \\
\text { AAAPQAPKR }\end{array}$ & $\begin{array}{l}-1,7 \\
\text { n.d. }\end{array}$ & $\begin{array}{l}\text { N-ter + 42.01 Da, } \mathrm{K}+28.03 \mathrm{Da} \\
\text { N-ter + 42.01 Da, K +34.06 Da }\end{array}$ & $\begin{array}{l}27,8 \\
17,2\end{array}$ & $\begin{array}{r}0,318 \\
-0,304\end{array}$ & $\begin{array}{l}3 \\
2\end{array}$ & $\begin{array}{l}1570,8 \\
984,6\end{array}$ & $\begin{array}{l}\text { RNH2C_MOUSE } \\
\text { RPP38_MOUSE }\end{array}$ & AZAJGO_MOUSE & $\begin{array}{l}\text { Ribonuclease H2 subunit } \mathrm{C}_{;} \\
\text {Ribonuclease P protein subunit p38; }\end{array}$ & Ribonuclease HI subunit C; \\
\hline MAW & ISYMPCCPGR & n.d. & $\mathrm{N}$-ter + 42.01 Da, C +57.02 Da & 11,8 & $-4,207$ & 2 & 1281,5 & FGRQA2_MOUSE & & $\begin{array}{l}\text { Ribosomal protein S6 kinase } \\
\{E C O: 000256 \mid \text { PIRNR:PIRNR000606\}; }\end{array}$ & \\
\hline EGHIIITDFG & LSKEATDHDKR & n.d. & $\mathrm{N}$-ter + 42.01 Da, K +28.03 Da & 15,3 & $-4,290$ & 2 & 1396,7 & KS6A2_MOUSE & & Ribosomal protein 56 kinase alpha-2; & $\begin{array}{l}90 \text { kDa ribosomal protein S6 kinase 2;MAP } \\
\text { kinase-activated protein kinase 1c;Protein- } \\
\text { tyrosine kinase Mpk-9;Ribosomal S6 kinase } \\
\text { 3;pp90RKK3; }\end{array}$ \\
\hline M & ASGCKIGPSILNSDLANLGAECLR & 0,3 & $\begin{array}{l}\mathrm{N} \text {-ter }+42.01 \mathrm{Da}, \mathrm{K}+28.03 \mathrm{Da}, \\
\mathrm{C}+57.02 \mathrm{Da}\end{array}$ & 44,1 & $-2,473$ & 3 & 2585,3 & RPE_MOUSE & B2KGFO_MOUSE & Ribulose-phosphate 3-epimerase; & Ribulose-5-phosphate-epimerase; \\
\hline SPSAAATASD & MDKNSGSNSSSASSGSSKGQQPPR & n.d. & N-ter + 42.01 Da, K +34.06 Da & 23,5 & 5,415 & 3 & 2490,2 & RNF10_MOUSE & D3Z1N2_MOUSE & RING finger protein 10; & Sid 2705; \\
\hline TMMVFKPIAE & KFGFTFNCDIKTR & n.d. & $\begin{array}{l}\text { N-ter + 42.01 Da, K+28.03 Da, } \\
\text { C+57.02 Da }\end{array}$ & 17,2 & 2,077 & 2 & 1730,9 & RTCA_MOUSE & D3Z263_MOUSE & RNA 3'-terminal phosphate cyclase; & $\begin{array}{l}\text { RNA terminal phosphate cyclase domain- } \\
\text { containing protein } 1 \text {; }\end{array}$ \\
\hline ATATAATAAA & AAAAAYSDGYGR & n.d. & $\mathrm{N}$-ter + 42.01 Da & 16,7 & 4,772 & 2 & 1213,5 & RFOX2_MOUSE & & RNA binding protein fox-1 homolog 2; & $\begin{array}{l}\text { Fox-1 homolog B;Fox-1 homolog } \\
\text { Fxh;Hexaribonucleotide-binding protein 2;RNA- } \\
\text { binding motif protein } 9 \text {;RNA-binding protein } 9 ;\end{array}$ \\
\hline GRCVRSEECY & YHWGRLR & n.d. & $\mathrm{N}$-ter $+42.01 \mathrm{Da}$ & 14,5 & $-4,561$ & 2 & 1028,5 & REX01_MOUSE & S4R246_MOUSE & RNA exonuclease 1 homolog; & $\begin{array}{l}\text { Transcription elongation factor B polypeptide 3- } \\
\text { binding protein } 1 \text {; }\end{array}$ \\
\hline M & TSASKAVELQLQVKHNAEELQDFMR & n.d. & N-ter + 42.01 Da, K+34.06 Da & 57,2 & 6,431 & 4 & 2981,6 & RPAP3_MOUSE & & RNA polymerase II-associated protein 3 ; & \\
\hline ADVPPELLNN & VGALHFR & n.d. & $\mathrm{N}$-ter + 42.01 Da & 12,4 & $-5,223$ & 2 & 840,5 & CTR9_MOUSE & & RNA polymerase-associated protein CTR9 homolog; & $\begin{array}{l}\text { SH2 domain-binding protein } \\
1 \text {; Tetratricopoptide repeat-containing, SHz- } \\
\text { binding phosphoprotein of } 150 \mathrm{kDa} \text {; }\end{array}$ \\
\hline M & VEADRPGKLFIGGLNTETNEKALEAVFGKYGR & 0,0 & N-ter + 42.01 Da, K+28.03 Da & 48,4 & 1,441 & 4 & 3604,9 & RBMX_MOUSE & RMXL1_MOUSE & RNA-binding motif protein, $\mathrm{X}$ chromosome; & Heterogeneous nuclear ribonucleoprotein $G$; \\
\hline M & AAALGAGGGAGAGDDDFDQFDKPGAER & n.d. & $\mathrm{N}$-ter + 42.01 Da, $\mathrm{K}+28.03 \mathrm{Da}$ & 13,5 & 3,719 & 3 & 2605,2 & RBM33_MOUSE & D3Z519_MOUSE & RNA-binding protein 33; & $\begin{array}{l}\text { Proline-rich protein 8;RNA-binding motif } \\
\text { protein 33; } \\
\text { Maternally-expressed hnRNP C-related }\end{array}$ \\
\hline M & SLKIQTSNVTNKNDPKSINSR & n.d. & $\mathrm{N}$-ter $+42.01 \mathrm{Da}, \mathrm{K}+28.03 \mathrm{Da}$ & 14,7 & 6,145 & 4 & 2469,4 & RALY_MOUSE & A2AU60_MOUSE & RNA-binding protein Raly; & $\begin{array}{l}\text { protein;hnRNP associated with lethal yellow } \\
\text { protein; }\end{array}$ \\
\hline EQLQRLRDQT & AASAQAQEDAQR & n.d. & $\mathrm{N}$-ter $+42.01 \mathrm{Da}$ & 11,9 & 6,907 & 2 & 1286,6 & CROCC_MOUSE & & Rootletin; & Ciliary rootlet coiled-coil protein; \\
\hline & ATVAATTKVPEIR & n.d. & $\mathrm{N}$-ter + 42.01 Da, K +34.06 Da & 64,4 & $-0,628$ & 2 & 1431,9 & RUVB2_MOUSE & D3YXQ__MOUSE & RuvB-like 2; & p47 protein; \\
\hline KEQVIKAVVP & AKYLDEDTVYHLQPSGR & n.d. & N-ter + 42.01 Da, K+34.06 Da & 12,9 & $-2,076$ & 4 & 2067,0 & METK1_MOUSE & & S-adenosylmethionine synthase isoform type-1; & Methionine adenosyltransferase 1 ; \\
\hline M & AAAAGAVVASAASGPAEGKKITELR & 0,0 & $\mathrm{~N}$-ter $+42.01 \mathrm{Da}, \mathrm{K}+28.03 \mathrm{Da}$ & 91,0 & 0,960 & 3 & 2393,3 & SLTM_MOUSE & & SAFB-like transcription modulator; & $\begin{array}{l}\text { Modulator of estrogen-induced } \\
\text { transcription;SAF-like transcription modulator; }\end{array}$ \\
\hline M & AAETVELHKLKLAELKQECLAR & n.d. & $\begin{array}{l}\text { N-ter + } 42.01 \mathrm{Da}, \mathrm{K}+28.03 \mathrm{Da}, \\
\mathrm{C}+57.02 \mathrm{Da}\end{array}$ & 20,5 & 3,283 & 5 & 2675,5 & SARNP_MOUSE & & SAP domain-containing ribonucleoprotein; & Nuclear protein Hcc-1; \\
\hline M & SDFDSNPFADPDLNNPFKDPSVTQVTR & 0,2 & N-ter + 42.01 Da, K+28.03 Da & 23,9 & $-0,743$ & 3 & 3092,4 & SCAM1_MOUSE & & Secretory carrier-associated membrane protein 1 ; & \\
\hline M & GETKIIYHLDGQETPYLVKLPLPAER & n.d. & N-ter + 42.01 Da, K+28.03 Da & 21,0 & $-9,670$ & 4 & 3077,7 & DVL__MOUSE & E9Q967_MOUSE & Segment polarity protein dishevelled homolog DVL-3; & DSH homolog 3; \\
\hline M & ATKCTKCGPGYSTPLEAMKGPR & 0,9 & $\begin{array}{l}\text { N-ter + } 42.01 \mathrm{Da}, \mathrm{K}+28.03 \mathrm{Da}, \\
\mathrm{C}+57.02 \mathrm{Da}\end{array}$ & 25,0 & 1,300 & 4 & 2535,3 & SBP1_MOUSE & DGRHN2_MOUSE & Selenium-binding protein 1 & 56 kDa selenium-binding protein; \\
\hline ARDPYCAWDG & HSCSRFYPTGKR & n.d. & $\begin{array}{l}\text { N-ter + 42.01 Da, K +34.06 Da, } \\
\text { C +57.02 Da }\end{array}$ & 15,0 & $-8,133$ & 3 & 1570,8 & SEM3C_MOUSE & & Semaphorin-3C; & Semaphorin-E; \\
\hline M & ASCDEIKEHPR & $-0,1$ & $\begin{array}{l}\mathrm{N} \text {-ter + 42.01 Da, K+28.03 Da, } \\
\mathrm{C}+57.02 \mathrm{Da}\end{array}$ & 30,2 & $-4,598$ & 3 & 1410,7 & SEP10_MOUSE & & Septin-10; & \\
\hline M & AVAVGRPSNEELR & n.d. & $\mathrm{N}$-ter $+42.01 \mathrm{Da}$ & 47,7 & $-0,347$ & 2 & 1438,8 & SEP11_MOUSE & & Septin-11; & \\
\hline AGQTGKSRKM & SKQQPTQFINPETPGYVGFANLPNQVHR & n.d. & $\mathrm{N}$-ter + 42.01 Da, K +34.06 Da & 24,6 & $-4,004$ & 4 & 3242,7 & SEPT2_MOUSE & D3Z3CO_MOUSE & Septin-2; & $\begin{array}{l}\text { Neural precursor cell expressed } \\
\text { developmentally down-regulated protein 5; }\end{array}$ \\
\hline M & ASFTVKAYLLGKEEATR & n.d. & $\mathrm{N}$-ter + 42.01 Da, K+34.06 Da & 25,5 & $-2,154$ & 3 & 1993,2 & SQSTM_MOUSE & D3YZ1_MOUSE & Sequestosome-1; & itin-binding protein p62; \\
\hline $\begin{array}{l}\text { NIGNAMFIEK } \\
\text { PGPQAPKPA }\end{array}$ & $\begin{array}{l}\text { DLQQLLEFFEKTR } \\
\text { SPKKPPGER }\end{array}$ & n.d. & $\begin{array}{l}\text { N-ter + 42.01 Da, } \mathrm{K}+28.03 \mathrm{Da} \\
\text { Neter+4201 }\end{array}$ & $\begin{array}{l}11,9 \\
108\end{array}$ & $\begin{array}{l}4,489 \\
2601\end{array}$ & 2 & $\begin{array}{ll}1668,9 & 5 \\
11897 & 5\end{array}$ & $\begin{array}{l}\text { SPABK_MOUSE } \\
\text { SRM2 MOUSE }\end{array}$ & SPA3M_MOUSE & $\begin{array}{l}\text { Serine protease inhibititr } A 3 K ; \\
\text { Serine/arginine repetitive matrix }\end{array}$ & Contrapsin;SPI-2; \\
\hline
\end{tabular}




\begin{tabular}{|c|c|c|c|c|c|c|c|c|c|c|c|}
\hline Non Prime Site & Prime Site & 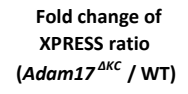 & Modifications & Hyperscore & $\begin{array}{c}\text { Mass error } \\
\text { in ppm }\end{array}$ & Charge & $\begin{array}{l}\text { Precursor } \\
\text { neutral mass } \\
\text { in Da }\end{array}$ & Uniprot ID 1 & Uniprot ID 2 & Protein Name 1 & Protein Name 2 \\
\hline M & SYGRPPPDVEGMTSLKVDNLTYR & 0,0 & $\mathrm{~N}$-ter + 42.01 Da, K+28.03 Da & 68,2 & 0,038 & 3 & 2664,3 & SRSF2_MOUSE & & Serine/arginine-rich splicing factor 2; & $\begin{array}{l}\text { Protein PR264;Putative myelin regulatory } \\
\text { factor 1;Splicing component, } 35 \text { kDa;Splicing } \\
\text { factor SC35;Splicing factor, arginine/serine-rich } \\
2 ;\end{array}$ \\
\hline WPVGSTTETS & GGPELSDSLSSGGIVAQAPLR & n.d. & $\mathrm{N}$-ter $+42.01 \mathrm{Da}$ & 15,4 & 9,489 & 3 & 2052,1 & S11IP_MOUSE & & $\begin{array}{l}\text { Serine/threonine-protein kinase 11-interacting } \\
\text { protein; }\end{array}$ & LKB1-interacting protein 1; \\
\hline IPTNPPPTFR & KPELWSDDFTDFVKK & n.d. & N-ter + 42.01 Da, $\mathrm{K}+34.06 \mathrm{Da}$ & 17,6 & 3,948 & 3 & 1998,1 & STK3_MOUSE & & Serine/threonine-protein kinase 3; & $\begin{array}{l}\text { Mammalian STE20-like protein kinase 2;STE20- } \\
\text { like kinase MST2; }\end{array}$ \\
\hline LFSEDKCWYR & CKVLKTISDDKCLVR & n.d. & $\begin{array}{l}\text { N-ter + 42.01 Da, K+34.06 Da, } \\
\mathrm{C}+57.02 \mathrm{Da}\end{array}$ & 14,6 & $-1,312$ & 3 & 1978,2 & STK31_MOUSE & E9Q260_MOUSE & Serine/threonine-protein kinase 31; & \\
\hline SFEALLAdLt & RTLSDNVNLPQGVR & n.d. & $\mathrm{N}$-ter $+42.01 \mathrm{Da}$ & 16,7 & $-1,921$ & 4 & 1609,9 & DCLK1_MOUSE & Q80VB6_MOUSE & Serine/threonine-protein kinase DCLK1; & $\begin{array}{l}\text { Doublecortin-like and CAM kinase-like } \\
\text { 1;Doublecortin-like kinase 1; }\end{array}$ \\
\hline DQSRVSTLQN & SCHSELQETLQPTPADASR & n.d. & $\mathrm{N}$-ter + 42.01 Da, C +57.02 Da & 16,8 & 3,409 & 3 & 2168,0 & LMTK2_MOUSE & & Serine/threonine-protein kinase LMTK2; & Brain-enriched kinase;Lemur tyrosine kinase 2; \\
\hline M & SNNGVDIQDKPPAPPMR & n.d. & $\mathrm{N}$-ter + 42.01 Da, K+34.06 Da & 21,5 & $-0,940$ & 3 & 1911,0 & PAK1_MOUSE & G5E884_MOUSE & Serine/threonine-protein kinase PAK 1; & $\begin{array}{l}\text { Alpha-PAK;CDC42/RAC effector kinase PAK- } \\
\text { A;p21-activated kinase 1;p65-PAK; }\end{array}$ \\
\hline M & SDNGELEDKPPAPPVR & 0,0 & N-ter + 42.01 Da, K+28.03 Da & 45,7 & 1,060 & 2 & 1789,9 & PAK2_MOUSE & & Serine/threonine-protein kinase PAK 2; & Gamma-PAK;p21-activated kinase 2 ; \\
\hline M & SDSLDNEEKPPAPPLR & n.d. & $\mathrm{N}$-ter + 42.01 Da, K+34.06 Da & 34,2 & $-0,760$ & 3 & 1839,9 & PAK3_MOUSE & A3KGC2_MOUSE & Serine/threonine-protein kinase PAK 3; & $\begin{array}{l}\text { Beta-PAK;CDC42/RAC effector kinase PAK- } \\
\text { B;p21-activated kinase 3; }\end{array}$ \\
\hline MVMEYCVCGM & QEMLDSVPEKR & n.d. & $\mathrm{N}$-ter + 42.01 Da, K+34.06 Da & 11,4 & $-2,697$ & 2 & 1406,7 & STK11_MOUSE & E9Q811_MOUSE & Serine/threonine-protein kinase STK11; & Liver kinase B1 homolog; \\
\hline M & GSGPIDPKELLKGLDFFLTR & n.d. & $\mathrm{N}$-ter + 42.01 Da, K+34.06 Da & 22,9 & 0,443 & 3 & 2252,3 & PP1RA_MOUSE & S4R164_MOUSE & $\begin{array}{l}\text { Serine/threonine-protein phosphatase } 1 \text { regulatory } \\
\text { subunit 10; }\end{array}$ & MHC class I region proline-rich protein CATS3; \\
\hline M & SEPKAIDPKLSTTDR & 0,0 & $\mathrm{~N}$-ter + 42.01 Da, K+28.03 Da & 19,1 & 0,512 & 3 & 1754,9 & PP2BA_MOUSE & & $\begin{array}{l}\text { Serine/threonine-protein phosphatase 2B catalytic } \\
\text { subunit alpha isoform; }\end{array}$ & $\begin{array}{l}\text { CAM-PRP catalytic subunit;Calmodulin- } \\
\text { dependent calcineurin A subunit alpha isoform; }\end{array}$ \\
\hline MSA & MEAADVFHR & n.d. & $\mathrm{N}$-ter $+42.01 \mathrm{Da}$ & 19,4 & $-0,179$ & 2 & 1116,5 & CPPED_MOUSE & D3Z7F7_MOUSE & Serine/threonine-protein phosphatase CPPED1; & $\begin{array}{l}\text { Calcineurin-like phosphoesterase domain- } \\
\text { containing protein } 1 ;\end{array}$ \\
\hline M & SAMEAADVFHR & n.d. & $\mathrm{N}$-ter $+42.01 \mathrm{Da}$ & 36,6 & $-0,470$ & 2 & 1274,6 & CPPED_MOUSE & D3Z7F7_MOUSE & Serine/threonine-protein phosphatase CPPED1; & $\begin{array}{l}\text { Calcineurin-like phosphoesterase domain- } \\
\text { containing protein 1; }\end{array}$ \\
\hline M & SDSEKLNLDSIIGR & n.d. & $\mathrm{N}$-ter + 42.01 Da, K +34.06 Da & 40,9 & $-5,234$ & 2 & 1621,9 & PP1A_MOUSE & & $\begin{array}{l}\text { Serine/threonine-protein phosphatase PP1-alpha } \\
\text { catalytic subunit; }\end{array}$ & \\
\hline M & ADIDKLNIDSIIQR & $-0,2$ & $\mathrm{~N}$-ter $+42.01 \mathrm{Da}, \mathrm{K}+28.03 \mathrm{Da}$ & 22,7 & 2,789 & 2 & 1682,9 & PP1G_MOUSE & & $\begin{array}{l}\text { Serine/threonine-protein phosphatase PP1-gamma } \\
\text { catalytic subunit: }\end{array}$ & Protein phosphatase $1 \mathrm{C}$ catalytic subunit; \\
\hline $\begin{array}{l}\text { DTEYSLSSFP } \\
\text { WCTGTVPIGE }\end{array}$ & $\begin{array}{l}\text { FMLLNYTSIEDFYR } \\
\text { YLVNSEDGAHR }\end{array}$ & n.d. & N-ter + 42.01 Da & 11,1 & $\begin{array}{l}-1,509 \\
-0,383\end{array}$ & $\begin{array}{l}3 \\
3\end{array}$ & $\begin{array}{l}1852,9 \\
13016\end{array}$ & ATM_MOUSE & EGUXY2 MOUSE & $\begin{array}{l}\text { Serine-protein kinase ATM; } \\
\text { Serin--roteip kianse ATM. }\end{array}$ & Ataxia telangiectasia mutated homolog; \\
\hline M & $\begin{array}{l}\text { YLVNSEUGAHK } \\
\text { DSLTAANNKFCFDFFR }\end{array}$ & 1,2 & $\begin{array}{l}\text { N-ter + 42.201 Da } \\
\text { N-ter + 42.01 Da, K+28.03 Da, }\end{array}$ & 72,4 & 0,046 & 2 & $\begin{array}{l}1301,6 \\
2153,0\end{array}$ & $\begin{array}{l}\text { ATM_MOUSE } \\
\text { SPB12 MOUSE }\end{array}$ & FGUXVL__MOUSE & $\begin{array}{l}\text { Serine-protein kinase ATM; } \\
\text { Serpin B12; }\end{array}$ & Ataxia telangiectasia mutated homolog; \\
\hline M & ALKQISSNR & n.d. & $\mathrm{N}$-ter + 42.01 Da, $\mathrm{K}+34.06 \mathrm{Da}$ & 28,6 & 0,366 & 2 & 1091,7 & ESTD_MOUSE & H3BJC6_MOUSE & S-formylglutathione hydrolase; & Esterase 10;Esterase D;Sid 478; \\
\hline & ADLKKAAGGR & n.d. & N-ter + $42.01 \mathrm{Da}, \mathrm{K}+34.06 \mathrm{Da}$ & 13,2 & $-5,370$ & 3 & 1095,7 & 3BP5L_MOUSE & & SH3 domain-binding protein 5-like; & \\
\hline RRKRQCVPLN & LTEPSLR & n.d. & $\mathrm{N}$-ter + 42.01 Da & 15,3 & $-2,446$ & 2 & 856,5 & SGOL2_MOUSE & & Shugoshin-like 2; & Shugoshin-2; \\
\hline & SGEVPPNINIKEPR & $-0,1$ & N-ter + $42.01 \mathrm{Da}, \mathrm{K}+28.03 \mathrm{Da}$ & 21,6 & $-1,789$ & 2 & 1618,9 & SFXN1_MOUSE & & Sideroflexin-1; & \\
\hline PLMRQRELQV & GIPVTDEAGQR & n.d. & $\mathrm{N}$-ter + 42.01 Da & 17,6 & 1,434 & 2 & 1183,6 & SFXN3_MOUSE & Q3U4FO_MOUSE & Sideroflexin-3; & \\
\hline RIFLLGLADN & EAAIVQAESEETKER & n.d. & $\mathrm{N}$-ter + $42.01 \mathrm{Da}, \mathrm{K}+34.06 \mathrm{Da}$ & 19,3 & $-4,415$ & 2 & 1764,9 & SRP68_MOUSE & A2AAN2_MOUSE & Signal recognition particle subunit SRP68; & Signal recognition particle 68 kDa protein; \\
\hline QRSEDSLADQ & MEPTCHLPAVSKVLPAFR & 0,2 & $\begin{array}{l}\text { N-ter + 42.01 Da, K+28.03 Da, } \\
\mathrm{C}+57.02 \mathrm{Da}\end{array}$ & 28,3 & $-1,506$ & 3 & 2122,1 & SI1L1_MOUSE & & $\begin{array}{l}\text { Signal-induced proliferation-associated 1-like protein } \\
\text { 1: }\end{array}$ & \\
\hline QEHPKRPVLG & LECPQSEVSGAR & n.d. & $\mathrm{N}$-ter + 42.01 Da, C +57.02 Da & 17,5 & 0,364 & 2 & 1373,6 & SMUG1_MOUSE & & $\begin{array}{l}\text { Single-strand selective monofunctional uracil DNA } \\
\text { glycosylase; }\end{array}$ & \\
\hline M & FAKGKGSAVPSDGQAR & n.d. & N-ter + 42.01 Da, K+34.06 Da & 22,1 & 2,199 & 3 & 1816,0 & SSBP3_MOUSE & B1AS34_MOUSE & Single-stranded DNA-binding protein 3; & $\begin{array}{l}\text { Lck-associated signal transducer;:Sequence- } \\
\text { specific single-stranded-DNA-binding protein; }\end{array}$ \\
\hline KLKKLKRLLM & ENTYEGPDSQKEEDASR & n.d. & N-ter + 42.01 Da, K+34.06 Da & 14,7 & $-0,983$ & 4 & 2029,9 & DCC1_MOUSE & D3YVY9_MOUSE & Sister chromatid cohesion protein DCC1; & $\begin{array}{l}\text { Defective in sister chromatid cohesion protein } 1 \\
\text { homolog; }\end{array}$ \\
\hline LHRMLHRDST & ISNESSQSCSSGR & n.d. & N-ter + 42.01 Da, C +57.02 Da & 14,8 & $-4,783$ & 3 & 1439,6 & SGSM1_MOUSE & B2RQR5_MOUSE & Small G protein signaling modulator 1 & RUN and TBC1 domain-containing protein 2; \\
\hline KKPSLLGAC & HPWLFIEEAAGR & n.d. & $\mathrm{N}$-ter $+42.01 \mathrm{Da}$ & 13,8 & $-3,881$ & 2 & 1466,7 & SGSM3_MOUSE & F8WIA4_MOUSE & Small G protein signaling modulator 3 ; & RUN and TBC1 domain-containing protein 3; \\
\hline M & SLLNKPKSEMTPEELQKR & n.d. & N-ter + 42.01 Da, K+34.06 Da & 57,4 & $-0,044$ & 4 & 2271,3 & SMD2_MOUSE & & Small nuclear ribonucleoprotein Sm D2; & snRNP core protein D2; \\
\hline M & SIGVPIKVLHEAEGHIVTCETNTGEVYR & 0,4 & $\begin{array}{l}\text { N-ter + } 42.01 \text { Da, } \mathrm{K}+28.03 \mathrm{Da}, \\
\mathrm{C}+57.02 \mathrm{Da}\end{array}$ & 59,8 & 0,440 & 4 & 3177,6 & SMD3_MOUSE & & Small nuclear ribonucleoprotein Sm D3; & SnRNP core protein D3; \\
\hline EKEYKEIIHR & TLEKLR & n.d. & N-ter + 42.01 Da, K+28.03 Da & 17,1 & $-3,388$ & 3 & 941,6 & UTP20_MOUSE & E9QK83_MOUSE & Small subunit processome component 20 homolog; & Down-regulated in metastasis protein; \\
\hline M & EPSPDAEEAHTVR & n.d. & $\mathrm{N}$-ter $+42.01 \mathrm{Da}$ & 18,6 & 0,496 & 2 & 1609,7 & SMTL2_MOUSE & & Smoothelin-like protein 2; & \\
\hline
\end{tabular}




\begin{tabular}{|c|c|c|c|c|c|c|c|c|c|c|c|}
\hline Non Prime Site & Prime Site & 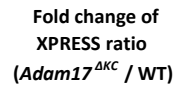 & Modifications & Hyperscore & $\begin{array}{l}\text { Mass error } \\
\text { in ppm }\end{array}$ & Charge & $\begin{array}{l}\text { Precursor } \\
\text { neutral mass } \\
\text { in Da }\end{array}$ & Uniprot ID 1 & Uniprot ID 2 & Protein Name 1 & Protein Name 2 \\
\hline MTAEQALPLG & NGKAAEEARGSETLGGGGGGAAGTR & n.d. & $\mathrm{N}$-ter + 42.01 Da, K +34.06 Da & 20,1 & $-1,949$ & 3 & 2306,1 & S6A11_MOUSE & & Sodium- and chloride-dependent GABA transporter & $\begin{array}{l}\text { Sodium- and chloride-dependent GABA } \\
\text {; transporter 4;Solute carrier family } 6 \text { member } \\
11 ;\end{array}$ \\
\hline $\begin{array}{l}\text { TIWRQQGMLG } \\
\text { ILLTDHFQYS }\end{array}$ & $\begin{array}{l}\text { LWRGVGGAVPR } \\
\text { RQGHPNSYALSR }\end{array}$ & $\begin{array}{l}\text { n.d. } \\
\text { n.d. }\end{array}$ & $\begin{array}{l}\text { N-ter + 42.01 Da } \\
\text { N-ter +42.01 Da }\end{array}$ & $\begin{array}{l}16,6 \\
21,3\end{array}$ & $\begin{array}{l}0,000 \\
-4,550\end{array}$ & $\begin{array}{l}3 \\
2\end{array}$ & $\begin{array}{l}1208,7 \\
1426,7\end{array}$ & $\begin{array}{l}\text { S2534_MOUSE } \\
\text { S35E1_MOUSE }\end{array}$ & & $\begin{array}{l}\text { Solute carrier family } 25 \text { member 34; } \\
\text { Solute carrier family } 35 \text { member E1; }\end{array}$ & \\
\hline IVVVAGENRS & RLADMNATIR & n.d. & N-ter + 42.01 Da & 12,1 & 1,080 & 2 & 1201,6 & S40A1_MOUSE & & Solute carrier family 40 member 1 & $\begin{array}{l}\text { Ferroportin-1;iron-regulated transporter } \\
1 \text {;:Metal transporter protein 1; }\end{array}$ \\
\hline $\begin{array}{l}M \\
M\end{array}$ & $\begin{array}{l}\text { AAPAKGENLSLVVHGPGDIR } \\
\text { SDTAVADTRR }\end{array}$ & $\begin{array}{l}0,3 \\
\text { n.d. }\end{array}$ & $\begin{array}{l}\text { N-ter + 42.01 Da, K +28.03 Da } \\
\text { N-ter + 42.01 Da }\end{array}$ & $\begin{array}{l}53,4 \\
24,2\end{array}$ & $\begin{array}{l}-0,868 \\
-2,027\end{array}$ & $\begin{array}{l}3 \\
2\end{array}$ & $\begin{array}{l}2070,1 \\
1132,6\end{array}$ & $\begin{array}{l}\text { DHSO_MOUSE } \\
\text { SNX12_MOUSE }\end{array}$ & Q3V2H3_MOUSE & $\begin{array}{l}\text { Sorbitol dehydrogenase; } \\
\text { Sorting nexin-12; }\end{array}$ & $\begin{array}{l}\text { L-iditol 2-dehydrogenase; } \\
\text { SDP8 protein; }\end{array}$ \\
\hline M & AANPSGQGFQNKNR & n.d. & $\mathrm{N}$-ter + 42.01 Da, $\mathrm{K}+34.06 \mathrm{Da}$ & 31,2 & 0,703 & 2 & 1563,8 & SOSSC_MOUSE & & SOSS complex subunit C; & $\begin{array}{l}\text { INTS3- and NABP-interacting protein; Sensor of } \\
\text { single-strand DNA complex subunit C;SSensor of } \\
\text { SSDNA subunit C; } C \text {;ingle-stranded DNA-binding } \\
\text { protein-interacting protein } 1 \text {; }\end{array}$ \\
\hline SFRKRRAQKM & DPSGVKVLETAEDIQER & 0,1 & $\mathrm{~N}$-ter $+42.01 \mathrm{Da}, \mathrm{K}+28.03 \mathrm{Da}$ & 59,0 & 2,299 & 2 & 2086,0 & SPTN1_MOUSE & E9Q447_MOUSE & Spectrin alpha chain, non-erythrocytic 1 ; & Alpha-II spectrin;Fodrin alpha chain; \\
\hline LEPEMNNQAS & RVAVVNQIAR & n.d. & $\mathrm{N}$-ter + 42.01 Da & 14,7 & 6,075 & 2 & 1166,7 & SPTB2_MOUSE & & Spectrin beta chain, non-erythrocytic 1; & $\begin{array}{l}\text { Beta-ll spectrin;:Embryonic liver fodrin;Fodrin } \\
\text { beta chain; }\end{array}$ \\
\hline M & ADLLGSILSSMEKPPSLGDQESR & 0,0 & N-ter + 42.01 Da, K+28.03 Da & 74,0 & 0,280 & 3 & 2499,3 & SPAG7_MOUSE & Q55X56_MOUSE & Sperm-associated antigen 7 ; & \\
\hline FSDTRQLEKF & HPCPINLNMEQFWEAVSR & n.d. & $\mathrm{N}$-ter $+42.01 \mathrm{Da}, \mathrm{C}+57.02 \mathrm{Da}$ & 17,0 & 4,267 & 4 & 2269,1 & SATL1_MOUSE & F7BXA4_MOUSE & $\begin{array}{l}\text { Spermidine/spermine N(1)-acetyltransferase-like } \\
\text { protein 1; }\end{array}$ & \\
\hline $\begin{array}{l}\text { NILLLSGDVN } \\
\text { M }\end{array}$ & $\begin{array}{l}\text { LAESDLAYTR } \\
\text { NRPLSAEAEEELEWQVASR }\end{array}$ & $\begin{array}{l}\text { n.d. } \\
\text { n.d. }\end{array}$ & $\begin{array}{l}\text { N-ter + 42.01 Da } \\
\text { N-ter + 42.01 Da }\end{array}$ & $\begin{array}{l}14,9 \\
32,9\end{array}$ & $\begin{array}{l}-0,508 \\
-1,884\end{array}$ & $\begin{array}{l}2 \\
3\end{array}$ & $\begin{array}{l}1179,6 \\
2386,1\end{array}$ & $\begin{array}{l}\text { SPSY_MOUSE } \\
\text { SSFA2_MOUSE }\end{array}$ & $\begin{array}{l}\text { E9P258_MOUSE } \\
\text { A2AOD6 MOUSE }\end{array}$ & $\begin{array}{l}\text { Spermine synthase; } \\
\text { Sperm-specific antigen } 2 \text { homolog: }\end{array}$ & $\begin{array}{l}\text { Spermidine aminopropyltransferase; } \\
\text { Ki-ras-induced actin-interacting protein; }\end{array}$ \\
\hline
\end{tabular}

